Sprachliches Wissen zwischen Lexikon und Grammatik 


\section{Institut für Deutsche Sprache Jahrbuch 2010}




\section{Sprachliches Wissen zwischen Lexikon und Grammatik}

Herausgegeben von Stefan Engelberg, Anke Holler und Kristel Proost

De Gruyter 
Redaktion: Franz Josef Berens, Melanie Steinle

ISBN 978-3-11-026232-2

e-ISBN 978-3-11-026233-9

\section{Bibliografische Information der Deutschen Nationalbibliothek}

Die Deutsche Nationalbibliothek verzeichnet diese Publikation in der Deutschen Nationalbibliografie; detaillierte bibliografische Daten sind im Internet über http://dnb.d-nb.de abrufbar.

(C) 2011 Walter de Gruyter GmbH \& Co. KG, Berlin/Boston

Gesamtherstellung: Hubert \& Co. GmbH \& Co. KG, Göttingen

$\infty$ Gedruckt auf säurefreiem Papier

Printed in Germany

www.degruyter.com 


\section{Inhalt}

Ludwig M. Eichinger: Übergänge: Grammatik im Grenzbereich ............ VII

Stefan Engelberg / Anke Holler / Kristel Proost: Zwischenräume -

Phänomene, Methoden und Modellierung im Bereich zwischen

Lexikon und Grammatik

\section{Konstruktionsidentität und Konstruktionsvarianz}

Hans C. Boas: Zum Abstraktionsgrad von Resultativkonstruktionen.... 37

Stefan Engelberg / Svenja König / Kristel Proost / Edeltraud Winkler:

Argumentstrukturmuster als Konstruktionen? Identität -

Verwandtschaft - Idiosynkrasien........................................................... 71

Wolfgang Imo: Die Grenzen von Konstruktionen: Versuch einer granularen Neubestimmung des Konstruktionsbegriffs der Construction Grammar.

\section{Regeln und Konstruktionen}

Gert Webelhuth: Paradigmenwechsel rückwärts: Die Renaissance der grammatischen Konstruktion.

Anatol Stefanowitsch: Keine Grammatik ohne Konstruktionen:

Ein logisch-ökonomisches Argument für die Konstruktionsgrammatik

Gereon Müller: Regeln oder Konstruktionen? Von verblosen

Direktiven zur sequenziellen Nominalreduplikation

Tibor Kiss: Bedingungen für den Wegfall eines Artikels:

Distribution und Interpretation von Präposition-Nomen-

Kombinationen

Beatrice Primus: Das unpersönliche Passiv - Ein Fall für die

Konstruktionsgrammatik? 


\section{Wörter und Phrasen}

Claudia Maienborn: Strukturausbau am Rande der Wörter:

Adverbiale Modifikatoren beim Zustandspassiv

Joachim Jacobs: Grammatik ohne Wörter?.

\section{Erwerb und Verarbeitung}

Heike Behrens: Grammatik und Lexikon im Spracherwerb:

Konstruktionsprozesse

Rosemarie Tracy: Konstruktion, Dekonstruktion und Rekonstruktion:

Minimalistische und (trotzdem) konstruktivistische Überlegungen zum Spracherwerb

Ina Bornkessel-Schlesewsky / Matthias Schlesewsky: Dynamische Aspekte der Argumentinterpretation: Eine neurokognitive Perspektive

\section{Korpora und lexikalische Resourcen}

Christiane Fellbaum: Klassifikation des Verblexikons in WordNet und Abgleichung mit FrameNet.

Patrick Hanks: Wie man aus Wörtern Bedeutungen macht: Semantische Typen treffen Valenzen

Annelies Häcki Buhofer: Lexikografie der Kollokationen zwischen Anforderungen der Theorie und der Praxis

Ulrich Heid: Korpusbasierte Beschreibung der Variation bei

Kollokationen: Deutschland - Österreich - Schweiz - Südtirol

Carolin Müller-Spitzer / Stefan Engelberg: Elektronische Lexikografie zwischen Grammatik und Lexikon

Sachindex.

Namensindex 
Ludwig M. Eichinger

\title{
Übergänge: Grammatik im Grenzbereich
}

\begin{abstract}
Innerhalb der Sätze, zwischen den Wörtern, gilt eher die Chemie; zwischen den Sätzen eher die Physik.

(Peter Handke: Am Felsfenster morgens. Salzburg: Residenz 1998, S. 328)
\end{abstract}

\section{Die Gelegenheit}

Die 46. Jahrestagung des Instituts für Deutsche Sprache fand vom 9. bis 13. März 2010 statt. Die rund 470 Teilnehmerinnen und Teilnehmer aus zweiunddreißig verschiedenen Nationen fanden ein Mannheim vor, das sich noch nicht recht entscheiden konnte, ob es seine Gäste eher spätwinterlich oder vorfrühlingshaft begrüßen mochte.

\section{Das Thema: Zwischenräume - zwischen Räumen}

Das Tagungsthema „Sprachliches Wissen zwischen Lexikon und Grammatik" bezieht sich auf eine ganz zentrale und grundlegende Diskussionslinie in der systemlinguistischen Diskussion der letzten Jahre. Die auf der Tagung gehaltenen Vorträge, die in diesem Band dokumentiert werden - und deren Einordnung der Einleitung der Herausgeber überlassen bleibt - loten von verschiedenen Seiten her und auf der Basis unterschiedlicher linguistischer Überzeugung aus, wie jenes „Zwischen“, von dem in der Themenformulierung die Rede ist, ausgestaltet sei, und zumindest in den grundsätzlicheren Beiträgen geht es eigentlich auch darum, ob da überhaupt ein Zwischenraum ist oder einfach bloß ein Raum dazwischen, der einen Übergang markiert. Entgegen einer in weiten linguistischen Kreisen und über lange Zeit gehegten Einschätzung, dass das Lexikon jener Ort des Idiosynkratischen sei, der der Regelhaftigkeit einer syntaktischen Grundstrukturierung bedürfe, um in grammatisch verlässlichen Sätzen in die sprachliche Welt entlassen zu werden, gewinnen in letzter Zeit Überlegungen, die bis dahin allenfalls an den Rändern des linguistischen Mainstream gedacht worden waren, an Gewicht, die zeichenhafte Entitäten unterschiedlicher Länge und Strukturiertheit unter unmittelbarem Einfluss einer lexikalischen Bindung und entsprechender paradigmatischer Optionen entstehen sehen. In paradigmatischer 
Weise ist die dadurch angedeutete Bruchzone im Areal der linguistischen Theorien abgebildet im Gegeneinander der Positionen der Universalgrammatiker auf der einen und der Konstruktionsgrammatiker auf der anderen Seite.

So ist es zweifellos der Aktualität und einer im Rahmen dieser Aktualität hinreichenden Entfaltetheit und Entwicklung der Diskussion geschuldet, dass die Behandlung von Übergangsphänomenen, deren Erklärung innerhalb einer regelorientierten Grammatik ebenso Schwierigkeiten macht wie innerhalb der zum Individuellen neigenden Erklärung innerhalb des Lexikons, zum Thema der Jahrestagung gewählt wurde. Neben diesem auf die aktuelle linguistische Diskussion bezogenen Grund, gibt es einen - wenn man so will - externen Grund, der sich bei näherem Hinsehen dann doch nicht als nur äußerlich entpuppt. Schon im letzten Jahr, als wir uns im Rahmen der Jahrestagung, die den Titel „Sprache intermedial: Stimme und Schrift, Bild und Ton" trug, ${ }^{1}$ auf den Weg in multimodale Welten und zu den Weisen ihrer Erforschung machten, war damit auch die Vorstellung eines neuen Arbeitsfeldes verbunden, das in der Abteilung Pragmatik des IDS nach dem Wechsel der Abteilungsleitung entstanden ist. So ist es auch dieses Mal, wo die Tagung von Stefan Engelberg geplant und vorbereitet wurde: Mit der angedeuteten Untersuchung der Syntax-Semantik-Schnittstelle wird auch eine entsprechende Akzentuierung im Arbeitsprogramm der Abteilung Lexik vorgestellt, deren Leiter er seit 2006 ist. Das betrifft in Sonderheit die Arbeit in dem Projekt „Polysemie und konstruktionelle Varianz", das auch mit einem Beitrag an diesem Band beteiligt ist. ${ }^{2}$

Dieser Hinweis bietet auch die Gelegenheit Anke Holler, Peter Lutzeier sowie als Kolleginnen und Kollegen aus dem Haus neben Stefan Engelberg, Kristel Proost und Kathrin Steyer für die Planung und Vorbereitung der Tagung zu danken.

\section{Im Kontext des IDS}

\subsection{Schwellen und ihre Funktion}

Es gibt seit einiger Zeit generelle Kontexte und Interessen, an denen sich die Diskussionen um die Art des Anschlusses zwischen Strukturen in Grammatik und Lexikon oder eben zu Existenz und Art des Raums entzündet, der zwischen diesen beiden Regelungsarealen anzunehmen ist. ${ }^{3}$ Wie auch

Ihre Ergebnisse können im Jahrbuch 2009 des IDS nachgelesen werden (Deppermann/Linke (Hg.) 2010).

Vgl. Engelberg et al. (in diesem Band).

Die eher theorieinternen Bezüge und Diskussionen werden in der Einleitung der Herausgeber zu diesem Band dargestellt und in eine entsprechende Ordnung gebracht (vgl. Engelberg/Holler/Proost in diesem Band). 
immer man sich bei der im letzten Satz angedeuteten Alternative entscheiden mag, offenkundig gibt es eine Reihe von Beobachtungen, die das ebenso einfache wie undifferenzierte Bild der traditionellen Binarität zwischen diesen beiden Bereichen stört.

Und wie die jeweils angenommenen Räume, ihre Ausmaße und ihre Gestalt auch beschaffen sein mögen, man kann das Gefühl haben, dass wir an der thematisierten Stelle jedenfalls auf Schwellen stoßen. Schwellen, welcher Art sie auch sein mögen, sind Entitäten, die uns auffordern, auf das, worauf wir stoßen, nachdem wir diese Schwelle passiert haben, mit einer Differenzerfahrung zu reagieren. Wenn man so die Wegmarken des Übergangs als ein Mittel der Instruktion begreift, sollte man, wenn man auf sie trifft, materiale Hinweise bekommen, dass dem so ist. Und das gilt zudem für die beiden Seiten, von denen man auf diese Schwellen stoßen kann. Noch etwas kommt dazu: Traditionell schlagen Türen an der einen Seite einer Schwelle an, der Raum, den die Schwelle einnimmt, gehört dann eigentlich zumindest eher zur anderen Seite, wenn auch irgendwie nicht so ganz; seltener und irgendwie markiert ist der Fall, dass an beiden Seiten Türen angeschlagen sind: Dann gibt es Raum dazwischen; er ist allerdings begrenzt. Die an unsere gedachte Schwelle stoßenden Räume nehmen die Grammatik und das Lexikon ein. Und von beiden Seiten her gibt es Schwellensignale, die Fragen der Neukonzeptualisierung aufrufen. Wohin deren Richtung geht, hängt ganz davon ab, auf welcher Seite man sich die Tür denkt.

\subsection{Mehrwortfügungen - Mehrwortlexeme}

Dass das Arrangement dieser Räume prinzipiell oder zumindest schon seit einiger Zeit Anlass zum Nachdenken bot, lässt sich auch an den Diskussionslagen in den letzten Jahrestagungen des Instituts ablesen. Besonders augenfällig ist das vielleicht, wenn man Thema und Problemstellungen der Jahrestagung 2003 betrachtet. ${ }^{4}$ In diesem Jahr ging es um mehr oder minder feste Wortverbindungen, und schon diese an dem Tagungstitel angelehnte Formulierung spricht eigentlich von einer Schwelle, einer Schwelle zudem, an die man sich konzeptuell von der lexikalischen Seite annähert. Das sieht man daran, dass von „Wortverbindungen“ die Rede ist, wo im anderen Zimmer vielleicht von syntaktischen Fügungen gesprochen würde. Deren mehr oder minder große Festigkeit auf der anderen Seite ist es, was zweifellos für die zentrale Regel-Grammatik, die sich dort findet, zum Problem werden könnte. In traditionellen Unterteilungen wird dies großenteils so gelöst, dass solche Fragen dem Bereich der Lexikologie zugeordnet und unter dem Obertitel der Phraseologie abgehandelt werden. Das hat auch damit zu tun, dass mit diesen Fragen eng das Problem ihrer adäquaten - und

Dokumentiert in Steyer (Hg.) (2004). 
wiederfindbaren - lexikografischen Verzeichnung und Lemmatisierung verknüpft ist. Mehr und mehr zeigte sich aber in der neueren einschlägigen Diskussion, dass die Orientierung an den Grenzen einer Frege-Semantik das Problem zu eng führt, dass vielmehr allgemeinere Prinzipien der Beschreibung von Kollokationen zu finden sind. In diesem Kontext entwickelt sich daher eine intensivere Diskussion der adäquaten Behandlung so genannter Mehrwortlexeme; ${ }^{5}$ im Anschluss daran wurden Konzepte entwickelt, um usuelle Wortverbindungen in „Verbindungsfeldern“ zu beschreiben, in der auch die morphosyntaktischen Varianten und ihre Bedeutungssystematik ihren angemessenen Beschreibungsplatz finden. Es ist offenkundig, dass man damit eine Art der Beschreibung gewählt hat, die sich gut für eine konstruktionsorientierte Deutung anbietet.

\subsection{Korpora}

Einer gewissen Systematik halber wurde bisher darauf verzichtet, darauf hinzuweisen, dass die angedeutete Entwicklung im Umfeld der Beschreibung fester Verbindungen nicht zuletzt durch die in den letzten Jahren sprunghaft vorangegangenen Fortschritte im Bereich korpuslinguistisch basierter Techniken gestützt und in gewisser Weise erst möglich wurde. Wegen eines prinzipiell lexem- bzw. wortparadigmaorientierten Herangehens an große Datenmengen wurden solche Ansätze als erstes im lexikografischen Umfeld virulent. Da seit einigen Jahren die Anzahl und Größe solcherart nutzbarer Korpora geradezu exponentiell gestiegen ist, und gleichzeitig immer mehr und leistungsfähigere Werkzeuge zu ihrer Analyse entwickelt und zur Verfügung gestellt wurden, sind nunmehr über statistische Korrelationsanalysen Kookkurrenzen erkennbar, die man vorher so nicht sehen konnte. ${ }^{6}$ In der einen oder anderen Weise hat so die Linguistik nun auch mit ihrem Deutungsinventar mit den solcherart emergierenden Strukturen umzugehen. Die Frage, wie sich diese vergleichsweise neue Datenquelle im Feld der insgesamt der Sprachwissenschaft zur Verfügung stehenden Methoden der Datengewinnung verhält und inwieweit davon auch neue Ansätze zur Konzeptualisierung empiriebasierter linguistischer Forschung ableitbar sind, spielte eine zentrale Rolle auf der Jahrestagung 2004 des IDS, die der Korpuslinguistik und dem mit ihrem Arbeiten verbundenen Erkenntnisgewinn gewidmet war. ${ }^{7}$ Entsprechende Überlegungen finden sich seither durchwegs in den einschlägigen Diskussionen auf den weiteren Jahrestagungen. ${ }^{8}$

\footnotetext{
Was in einem Teilprojekt im Umfeld von elexiko praktisch angegangen wurde.

Vgl. dazu Eichinger (2010a).

Nicht untypisch ist, dass die Tagung, die in Kallmeyer/Zifonun (Hg.) (2007) dokumentiert ist, von den Leitern der Abteilungen Grammatik und Pragmatik gemeinsam getragen wurde. Vgl. etwa Kupietz/Keibel (2009).
} 


\subsection{Wörter und Syntax}

In diesen diskursiven Kontext passt auch ganz gut, dass bei der Tagung des Jahres 2005, die sich unter dem Titel „Grammatik und darüber hinaus“ mit dem Textverstehen beschäftigte, Dinge thematisiert wurden, die man unter dem Blickwinkel unserer Überlegungen als Schwellenüberschreitung in beide Richtungen betrachten kann. ${ }^{9}$ Das zeigt sich unter anderem daran, dass das Projekt am IDS, das für die Planung jener Tagung verantwortlich war, eine Art Wörterbuch von Elementen erstellt, die dezidiert eine zentrale Rolle in der syntaktischen Strukturierung spielen. Konnektoren lassen sich auch als Grenzsignale betrachten, von denen Räume eröffnet werden, in denen bestimmte Muster mit einem bestimmten Ausmaß an lexikalischer Variation zu erwarten sind. Das zeigt sich auch, wenn man in Korpora nach Korrelationen sucht. ${ }^{10}$ So wird offenbar das Verstehen von Texten im Fall von Konnektoren durch die Indizierung argumentativer Abläufe gesteuert ${ }^{11}$ - oder unter einem anderen Blickwinkel: gefördert - und zwar nicht nur über die semantische Wegweisung durch diese Elemente, sondern darüber hinaus über Muster, die mit ihnen eröffnet werden. Diese Muster schließen auch Präferenzen für die Besetzung der lexikalischen Positionen, die in ihrem Gefolge auftreten. Wie man mit solch einer Verbindung von regelhaftstruktureller Wegweisung und lexikalisch-paradigmatischer Vernetzung umgeht, ist zweifellos eine Frage, die in die Nähe unseres Tagungsthemas führt.

In generellerer Weise kann man eine Verbindung herstellen zu der Diskussion und Weiterentwicklung von valenzgrammatischen Überlegungen, wie sie am Institut eine lange Tradition hat. ${ }^{12}$ So ist es denn kein Zufall, dass bei der Behandlung von verbalen Wortbildungen die angedeutete Schwelle unmittelbar in den Blick kommt, und es ist naheliegend, dass gerade bei bestimmten Typen der so genannten trennbaren Verben das Bewusstsein sichtbar wird, sich an einer Schwelle zu befinden, an der eine stark fixierte Syntax in der Wortbildung auf Strukturen des Lexikons stößt. ${ }^{13}$

\footnotetext{
Vgl. Blühdorn/Breindl/Waßner (Hg.) (2006).

10 Vgl. dazu Keibel/Kupietz (2009, S. 40-41); weiter kontextualisiert in Eichinger (2010a); vgl. auch Heringer (2009, S. 31).

11 Mit Pasch et al. (2003) wurde ein Handbuch vorgelegt, das die Bedeutung dieser Wortart erst so recht sichtbar macht.

12 Als derzeitiges Beispiel vgl. das elektronische Valenzwörterbuch E-Valbu (http://hypermedia2. ids-mannheim.de/evalbu/index.html) oder auch die Beteiligung einer Reihe von Autoren (Breindl, Eichinger, Engel, Hoberg, Kubczak, Pasch, Plewnia, Schumacher, Zifonun) aus dem IDS in Ágel et al. (Hg.) (2006).

13 Vgl. die syntaktische Behandlung bestimmter Bildungen bei Motsch (2004), die z.B. bei Donalies (2005) unter die Präverbbildung fallen würden; zu einer analogischen Musterbildung bei so genannten Partikelverben vgl. auch Eichinger (1989).
} 


\section{Etwas anderes: Ein Ereignis für das Institut}

Alle zwei Jahre ist die Jahrestagung des IDS auch der Rahmen und der Ort für die Verleihung des Konrad-Duden-Preises der Stadt Mannheim, der von der Stadt gemeinsam mit dem Duden-Verlag verliehen wird. Überreicht wurde 2010 der Preis für das Jahr 2009, das fünfzigste Jahr in der Geschichte des Preises. Aus diesem Anlass hat der Gemeinderat der Stadt Mannheim auf Vorschlag der Vergabejury beschlossen, den Preis, der mit $12.500 €$ dotiert ist, an das Institut für Deutsche Sprache, und damit zum ersten Mal an ein linguistisches Forschungsinstitut zu vergeben. Der Preis wurde am 10. März 2010 im Rittersaal des Schlosses durch den Oberbürgermeister der Stadt Mannheim, Dr. Peter Kurz, verliehen. Die Laudatio hielt traditionsgemäß der letzte Preisträger, Peter Eisenberg, der die Spannweite der Entwicklung aufwies, die das Institut seit seiner Gründung im Jahre 1964 mitgemacht hat. In seiner Dankrede im Namen des Instituts sprach der Direktor zum Thema „Die Liebe zur Sprache und die Sprachwissenschaft“ ${ }^{14}$ Das Institut ist der 28. Preisträger, bis dahin ging der Preis immer an Einzelpersonen, darunter auch eine große Zahl von Forscherinnen und Forschern aus dem nicht-deutschsprachigen Raum. ${ }^{15}$ Das passt insofern auch zur Preisverleihung an das IDS, als in der Begründung der Jury als ein zentraler Punkt auftaucht, das IDS sei ein zentraler Ort der Forschung für Sprachgermanisten in der ganzen Welt. Und auch die Jahrestagung findet hier ihren Platz, heißt es doch im Begründungstext: „Im Rahmen seiner Jahrestagungen bündelt das IDS die linguistische In- und Auslandsgermanistik. Die jährlich stattfindende Tagung ist ein zentrales Forum für den sprachwissenschaftlichen Austausch." Dass das auch in diesem Jahr so ist, belegen einerseits das in diesem Band dokumentierte Programm der Tagung wie die zu Beginn dieses Beitrags erwähnte Zahl und Vielfalt der nationalen Herkunft der Teilnehmer.

\section{Literatur}

Ágel, Vilmos et al. (Hg.) (2006): Dependenz und Valenz. Ein internationales Handbuch der zeitgenössischen Forschung. 2. Halbbd. (= Handbücher zur Sprach- und Kommunikationswissenschaft (HSK) 25.2). Berlin/New York.

Blühdorn, Hardarik/Breindl, Eva/Waßner, Ulrich (Hg.) (2006): Text-Verstehen. Grammatik und darüber hinaus. (= Jahrbuch des Instituts für Deutsche Sprache 2005). Berlin/New York.

14 Sie ist - gemeinsam mit der Laudatio Peter Eisenbergs - veröffentlicht in Eichinger/Institut für Deutsche Sprache (2010).

15 Im Jahr 2007, dem „Jahr der Geisteswissenschaften“ in der Bundesrepublik Deutschland bekam das Goethe-Institut einen - undotierten - Sonderpreis zuerkannt. 
Deppermann, Arnulf/Linke, Angelika (Hg.) (2010): Sprache intermedial. Stimme und Schrift, Bild und Ton. (= Jahrbuch des Instituts für Deutsche Sprache 2009). Berlin/New York.

Donalies, Elke (2005): Die Wortbildung des Deutschen. Ein Überblick. 2., überarb. Aufl. (= Studien zur deutschen Sprache 27). Tübingen.

Eichinger, Ludwig M. (1989): Raum und Zeit im Verbwortschatz des Deutschen. Eine valenzgrammatische Studie. (= Linguistische Arbeiten 224). Tübingen.

Eichinger, Ludwig M. (2010a): Der durchschnittliche Linguist und die Daten. Eine Annäherung. In: Kratochvílová, Iva/Wolf, Norbert R. (Hg.): Kompendium Korpuslinguistik. Eine Bestandsaufnahme aus deutsch-tschechischer Perspektive. (= Germanistische Bibliothek 38). Heidelberg, S. 27-51.

Eichinger, Ludwig M. (2010b): Die Liebe zur Sprache und die Sprachwissenschaft. In: Eichinger/Institut für Deutsche Sprache, S. 15-32.

Eichinger, Ludwig M./Institut für Deutsche Sprache (2010): Die Liebe zur Sprache und die Sprachwissenschaft. Rede anlässlich der Verleihung des Konrad-DudenPreises der Stadt Mannheim am 10. März 2010. (= Dudenbeiträge Heft 60). Mannheim/Zürich.

Heringer, Hans Jürgen (2009): Ist das Deutsche grammatisch zu fassen? In: Konopka/ Strecker (Hg.), S. 23-32.

Kallmeyer, Werner/Zifonun, Gisela (Hg.) (2007): Sprachkorpora - Datenmengen und Erkenntnisfortschritt. (= Jahrbuch des Instituts für Deutsche Sprache 2006). Berlin/New York.

Konopka, Marek/Strecker, Bruno (Hg.) (2009): Deutsche Grammatik - Regeln, Normen, Sprachgebrauch. (= Jahrbuch des Instituts für Deutsche Sprache 2008). Berlin/New York.

Keibel, Holger/Kupietz, Marc (2009): Approaching grammar: towards an empirical linguistic research programme. In: Minegishi, Makoto/Kawaguchi, Yuji (Hg.): Working papers in corpus-based linguistics and language education 3. Tokyo, S. 6176. Internet: http://cblle.tufs.ac.jp/assets/files/publications/working_papers_03/section/ 061-076.pdf (Stand: 01.02.2011).

Kupietz, Marc/Keibel, Holger (2009): Gebrauchsbasierte Grammatik: Statistische Regelhaftigkeit. In: Konopka/Strecker (Hg.), S. 33-50.

Motsch, Wolfgang (2004): Deutsche Wortbildung in Grundzügen. 2., überarb. Aufl. (= Schriften des Instituts für Deutsche Sprache 8). Berlin/New York.

Pasch, Renate et al. (2003): Handbuch der deutschen Konnektoren. Linguistische Grundlagen der Beschreibung und syntaktische Merkmale der deutschen Satzverknüpfer (Konjunktionen, Satzadverbien und Partikeln). (= Schriften des Instituts für Deutsche Sprache 9). Berlin/New York.

Steyer, Katrin (Hg.) (2004): Wortverbindungen - mehr oder weniger fest. (= Jahrbuch des Instituts für Deutsche Sprache 2003). Berlin/New York. 



\title{
Zwischenräume \\ Phänomene, Methoden und Modellierung im \\ Bereich zwischen Lexikon und Grammatik
}

\author{
Es war einmal ein Lattenzaun \\ mit Zwischenraum, hindurchzuschaun. \\ Ein Architekt, der dieses sah, \\ stand eines Abends plötzlich da - \\ und nahm den Zwischenraum heraus \\ und baute draus ein großes Haus. \\ Der Zaun indessen stand ganz dumm, \\ mit Latten ohne was herum. \\ (Christian Morgenstern: Der Lattenzaun)
}

\begin{abstract}
Die vorliegende Einführung in den Band diskutiert zunächst den Zusammenhang zwischen den drei Dichotomien Lexikon versus Grammatik, Wort versus Phrase und Idiosynkrasie versus Regel. Im Folgenden werden Varianten des Konstruktionsbegriffs dargestellt und hinsichtlich verschiedener Dimensionen analysiert. Einer Darstellung der im Zusammenhang mit der Lexikon-Grammatik-Abgrenzung diskutierten Phänomene und angewandten empirischen Methoden schließt sich eine Übersicht über die Aufsätze des vorliegenden Bandes an.
\end{abstract}

\section{Dichotomien}

\subsection{Lexikon und Grammatik}

Drei Dichotomien bestimmen den Gegenstand dieses Bandes, die Dichotomie zwischen Grammatik und Lexikon, die zwischen Satz und Wort und die zwischen Regeln und Idiosynkrasien. Ein einfaches Modell sprachlichen Wissens könnte dazu neigen, diese drei Dichotomien aufeinander abzubilden, so dass auf der einen Seite die Grammatik steht, die regelhaftes Wissen über den Aufbau von Sätzen enthält, und auf der anderen Seite das Lexikon, in dem idiosynkratisches Wissen über Wörter seinen Platz hat. Aber Sprachtheorien, die einer solch' simplen extensionalen Identifizierung der drei Dichotomien anhängen, sind kaum zu finden. Wir wollen die drei Dichotomien daher hier kurz beleuchten. 
Der Titel des vorliegenden Bandes, „Sprachliches Wissen zwischen Lexikon und Grammatik“, erlaubt zwei dem Gegenstand des Buches angemessene Interpretationen. In der einen Lesart verweist zwischen darauf, dass es da möglicherweise einen Zwischenraum gibt zwischen Lexikon und Grammatik, einen Bereich von Phänomenen und theoretischer Modellierung, der weder vom Lexikon noch von der Grammatik zufriedenstellend zu behandeln ist. Das ist ein Aspekt dieses Bandes, ein Aspekt, der auch stark mit den methodischen Entwicklungen in der Linguistik zu tun hat, und der - insbesondere aus der Korpuslinguistik gespeist - eine Fülle von sprachlichen Gebrauchsphänomenen ans Licht gebracht hat, deren sprachtheoretischer Status hinsichtlich der Zuordnung zum Lexikon oder zur Grammatik nicht eindeutig ist, und von denen zu zeigen ist, ob sie überhaupt etablierten lexikalischen oder grammatischen Prinzipien gehorchen oder eine eigenständige Modellierung verlangen. In diesem Sinne von zwischen gibt es vielleicht einen theoretisch zu erfassenden Bereich, der zwischen Grammatik und Lexikon liegt, so wie Liechtenstein zwischen der Schweiz und Österreich liegt, oder - je nach Annahmen über den Umfang dieses Bereichs - so wie Frankreich zwischen Luxemburg und Andorra liegt. In der anderen Lesart verweist zwischen auf eine Grenze, die zwei Bereiche teilt, ohne aber einen Zwischenraum zu implizieren, so wie man zwischen Frankreich und Deutschland hin- und herreisen kann, ohne durch ein unbekanntes Drittes zu gelangen. Dass eine solche Grenze zwischen Lexikon und Grammatik gezogen werden kann, ist von nicht konstruktionsbasierten Grammatiktheorien kaum in Frage gestellt worden. Wo die Grenze verläuft, ist dagegen Gegenstand andauernder Debatten gewesen.

Schaut man auf die letzten 50 Jahre Sprachtheorie zurück, so ist festzustellen, dass je nach Theorie dem Lexikon eine in Konzeption und Umfang deutlich unterschiedliche Rolle zugewiesen wird. Das spiegelte sich auf den verschiedenen sprachtheoretischen Ebenen, der Syntax, der Semantik, der Morphologie und der Phonologie, wider. Die Überlegungen zu Lexikon und Grammatik werden dabei getrieben von Beobachtungen zu formal oder inhaltlich komplexen sprachlichen Phänomenen, die einerseits bestimmten Regeln zu folgen scheinen, andererseits aber auch idiosynkratisches Verhalten zeigen. Die Größe des Lexikons in einer Sprachtheorie bestimmt sich damit über folgende Erwägungen:

(i) Wenn man die regelhaften Aspekte partiell idiosynkratischer Ausdrücke ausschließlich über Regeln erfasst, die unabhängig, d.h. außerhalb des Lexikons bereits motiviert sind, so kann das Lexikon regelfrei bleiben; ist das nicht der Fall, so werden spezifisch lexikalische Regeln eingeführt. Zwei Beispiele sollen dies verdeutlichen. So hat man etwa in den 60er Jahren versucht, komplexe Wörter lexikonextern über syntaktische Transformationen zu generieren (Lees 1960). Probleme aus diesem An- 
satz resultierten dann in der Annahme spezifisch lexikalischer Wortbildungsregeln (z.B. Aronoff 1976) oder kopfgetriebener Konstituentenstrukurregeln (vgl. Selkirk 1982; Höhle 1982). Argumentstrukturelles Semantik-Syntax-Mapping hat die Generative Semantik über syntaktische Transformationen herzustellen versucht (z.B. McCawley 1968). Ebenso haben Ansätze in der Folge von Hale/Kayser (1993), wie etwa Harley/Noyer (2000) für die „Distributed Morphology“, syntaktische Prinzipien zur Erklärung von Linking-Phänomenen angenommen. Auch nach Borer (2005) ist die Interpretation von Argumenten nicht lexikalisch bestimmt, sondern durch die syntaktische Position, die sie in einer reichen funktionalen Satzstruktur einnehmen und die eine bestimmte ereignisstrukturelle Interpretation erzeugt. Auf der anderen Seite werden spezifisch lexikalische Linking-Prinzipien postuliert, die semantische Argumentstrukturen auf syntaktische Kategorien abbilden (z.B. Rappaport/Levin 1988; Wunderlich 1997, vgl. dazu auch Engelberg i.Dr.).

(ii) Werden lexikalische Regeln angenommen, so können diese als Erzeugungsregeln, wie z.B. Aronoffs (1976) Wortbildungsregeln, oder - wie bei Jackendoff (1975) - als Redundanzregeln konzipiert werden, um den zahlreichen Ausnahmen und Idiosynkrasien Rechnung zu tragen. Ebenso sind Linking-Regeln einerseits für subkategoriell unterspezifizierte Verbeinträge formuliert worden (vgl. Wunderlich 1997), andererseits auch als Redundanzregeln über vollständig subkategorisierten Einträgen aufgefasst worden (siehe Dowty 1991; Blume 2000). Im jeweils zweiten Fall ist das Lexikon umfangreicher, da es zum einen Regeln enthält, zum anderen trotzdem alle Einträge voll spezifiziert sind.

(iii) Je mehr Idiosynkrasien von komplexen Ausdrücken aus spezifischen Varianten nicht-komplexer Ausdrücke projiziert werden, umso stärker tendiert das Lexikon dazu, ausschließlich nicht-komplexe Ausdrücke zu enthalten. In Bezug etwa auf Distributionsidiosynkrasien, wie sie durch lexikalische Valenzinformationen erfasst werden, ist das ein verbreitetes Verfahren. Aber es ist auch vorgeschlagen worden, die semantischen und syntaktischen Besonderheiten von festen Wendungen ähnlich zu behandeln. In diesem Fall würde den Löffel abgeben aus einer spezifischen Lesart von abgeben projiziert - unter Inkaufnahme einer entsprechenden Vermehrung der Lesarten von abgeben (vgl. Jackendoff 1995 für eine Diskussion solcher Ansätze; siehe auch Müller). ${ }^{1}$

(iv) Die Größe des Lexikons hängt auch davon ab, wie viel Vertrauen man in pragmatische Prinzipien der Bedeutungskonstitution setzt. Je mehr man das tut, umso lesartenärmer sind lexikalische Einträge und umso unspezifischer sind einzelne Lesarten von Lexemen ausgestaltet. Eine skeptischere Haltung gegenüber solchen pragmatischen Prinzipien führt

Die fettgedruckten Namen verweisen auf Beiträge in dem vorliegenden Band. 
dagegen dazu, dass Einträge lesartenreich und in den einzelnen Lesarten umfangreich spezifiziert sind. Dass pragmatische Prinzipien kontextabhängig erhebliche semantische Unterspezifizierungen (z.B. Bierwisch 1983) oder Uminterpretationen (vgl. Maienborn 2003 oder Primus) erlauben, wird von manchen angenommen, etwa in der Zwei-EbenenSemantik, von anderen aber bestritten (Engelberg 2009; Engelberg et al.).

Die Größe des Lexikons hängt in den traditionellen Grammatikmodellen also unter anderem davon ab, ob es lexikalische Regeln gibt, ob diese als Redundanzregeln fungieren, ob komplexe Ausdrücke im Lexikon vorkommen und ob lexikalische Einträge stark semantisch unterspezifiziert sind. Mit dem Auftreten der Konstruktionsgrammatik hat das Lexikon allerdings neue Konkurrenz bekommen. Je nachdem, ob man der Konstruktionsgrammatik folgt, der zu Folge alles sprachliche Wissen in Form von Konstruktionen gespeichert ist (z.B. Goldberg 1995), oder gemäßigten Ansätzen anhängt, die die Existenz von Konstruktionen neben grammatischen Regeln und traditionellen lexikalischen Einträgen einräumen (vgl. Jacobs 2008), ersetzt das Konstruktikon Lexikon und Grammatik, tritt als Drittes neben Lexikon und Grammatik oder wird als Teil eines der beiden konzipiert.

\subsection{Wort und Phrase}

An das bisher Gesagte schließen sich nun auch unsere Bemerkungen zu den beiden anderen Dichotomien an. Die Dichotomie zwischen Wort und Phrase kann unter zwei Aspekten betrachtet werden, zum einen dahingehend, ob es nicht einen Zwischenraum zwischen den Kategorien Wort und Phrase gibt, in dem Phänomene hybrider Natur ihren Platz haben. Zu diesen Phänomenen gehören bekannterweise Verben mit abtrennbaren Partikeln aber auch Wendungen wie Schule machen oder Farbe bekennen, deren nominale Elemente im Gegensatz zu dem, was in Phrasen dieser Struktur üblich ist, keine Modifikation erlauben. Jacobs führt eine Reihe weiterer Phänomene an, die die Zweiteilung zwischen Wort und Phrase in Frage stellen.

Zum anderen ist zu überlegen, wie eindeutig die Zuordnung von Phrasen zur Grammatik und Wörtern zum Lexikon aufrechterhalten werden kann. Auch hier unterscheiden sich, wie wir gesehen haben, die Grammatiktheorien. Feste Wendungen und Sprichwörter etwa werden traditionell dem Bereich des Lexikons zugerechnet. Andere Ansätze (siehe oben) projizieren idiomatische Mehrwortausdrücke grammatisch regelhaft aus spezifischen Lesarten einzelner darin vorkommender Wörter. Andere Phänomenbereiche sind bezüglich ihrer Zuordnung zum grammatischen versus lexikalischen Bereich noch umstrittener. Komplexe Wörter können als Pro- 
dukt grammatischer Regeln verstanden werden oder als lexikalische Einheiten, deren gemeinsame Eigenschaften etwa über lexikalische Redundanzregeln erfasst werden. Während es also auf der einen Seite Ansätze gibt, die das Lexikon möglichst phrasenfrei halten, spüren korpuslinguistische Methoden eine ungeahnte Vielfalt an Mehrwortausdrücken auf, die nicht-kompositionelle Eigenschaften oder doch zumindest einen hohen Grad an Usualisierung aufweisen, und die ihren Platz im Lexikon (oder Konstruktikon) zu beanspruchen scheinen.

\subsection{Idiosynkrasien und Regeln}

Schließlich wollen wir auch die Dichotomie zwischen Regel und Idiosynkrasie unter zwei Aspekten betrachten, zum einen hinsichtlich ihrer Abgrenzung voneinander, zum anderen hinsichtlich ihrer Zuordnung zur Grammatik bzw. zum Lexikon. Die geläufige Abgrenzung zwischen dem Idiosynkratischen und dem Regelhaften ist bei näherer Betrachtung gar nicht so selbstverständlich. Zum einen stellen etwa eine vermeintlich idiosynkratische Valenzangabe zu einem Verb und eine konstituentenstrukturelle Regel beide Generalisierungen über unendlich viele phrasale Strukturen dar. Zum anderen sind natürlich auch Regeln Idiosynkrasien, soweit sie nicht allgemeineren Prinzipien, wie etwa dem X-bar-Schema folgen. So wird denn auch der idiosynkratische Charakter etwa einer Phrasenstrukturregel eben dann deutlich, wenn eine solche allgemeinere Struktur entdeckt wird.

Unter diesem Blickwinkel bildet sich die Unterscheidung von Regel und Idiosynkrasie auch nur sehr undeutlich auf die zwischen Grammatik und Lexikon ab. Es stellt sich eher die Frage, in welchem Maße Abstraktionen und Generalisierungen im Lexikon Platz haben. Deren Anteil ist eben je nach Lexikontheorie sehr hoch oder eben auch sehr gering, wenn man das Lexikon als ein Gefängnis auffasst, wie es in dem bekannten Zitat aus Di Sciullo/ Williams (1987, S. 3) zum Ausdruck kommt: „The lexicon is like a prison - it contains only the lawless, and the only thing that its inmates have in common is lawlessness. " In der Konstruktionsgrammatik schließlich mit ihrer Aufgabe der Trennung zwischen Grammatik und Lexikon, löst sich die Beschreibung einer Sprache ohnehin in eine Menge von gleichermaßen idiosynkratischen, aber unterschiedlich abstrakten Konstruktionen auf.

\section{Konstruktionen}

\subsection{Konstruktionen bei der Erfassung hybrider Phänomene}

Im vorherigen Abschnitt haben wir argumentiert, dass die drei Dichotomien, die das Themenfeld dieses Bandes abstecken, in der Modellbildung 
nicht einfach aufeinander abzubilden sind, was zugleich bedeutet, dass die durch die Pole der Dichotomien markierten Zwischenräume, die die Bereiche des Übergangs zwischen Grammatik und Lexikon, zwischen Satz und Wort sowie zwischen Regeln und Idiosynkrasien umfassen, jeweils Untersuchungsgegenstände eigener Art sind. Anders ausgedrückt, auch die Übergangszonen zwischen den durch die Dichotomien festgelegten Polen beherbergen je verschiedene Phänomene und lassen sich nicht eins-zu-eins aufeinander beziehen.

Zwar wird von allen gängigen Grammatiktheorien die Existenz von Übergangsphänomenen anerkannt, allerdings unterscheiden sich die Theorien maßgeblich darin, wie viel Bedeutung sie den in den Zwischenräumen angesiedelten Phänomenen beimessen und inwieweit sie überhaupt Erklärungsansätze für diese Phänomene liefern (wollen). Während sich die Generative Grammatik Chomskyscher Prägung eher auf regelhafte Standardphänomene konzentriert, werden in der Konstruktionsgrammatik Aspekte der innersprachlichen Varianz und entsprechend auch Idiosynkrasien stärker berücksichtigt.

Bei aller Unterschiedlichkeit der drei Dichotomien und ihrer jeweils evozierten Übergangsphänomene fällt auf, dass sie offenbar konstruktionistische Herangehensweisen befördern. Das wirft aber unmittelbar die Frage auf, woran es liegen könnte, dass eine auf Konstruktionen beruhende Analyse für die Beschreibung der Übergangszonen so attraktiv erscheint. Zwei Gründe sind vermutlich dafür verantwortlich:

Zum Ersten ist das Konzept der Konstruktion gewissermaßen unscharf genug, um es sprachtheoretisch mit verschiedenartigen Phänomenen, die ein grammatisches Kontinuum adressieren, zu verknüpfen. Jenseits der vortheoretischen Verwendungsweise des traditionellen Begriffs der Konstruktion als syntaktische Beschreibungseinheit sind im Rahmen der verschiedenen Varianten der Kognitiven Grammatik und der Konstruktionsgrammatik mehrere, im Detailgrad durchaus unterschiedliche Begriffsbestimmungen für die Konstruktion ausgearbeitet worden. Allen Ansätzen ist gemeinsam, dass letztlich nur eine Anforderung maßgeblich für die Existenz einer Konstruktion ist, und zwar die direkte Zuordnung von Form- und Bedeutungsmerkmalen, manifestiert in einem sprachlichen Zeichen oder einer Klasse von sprachlichen Zeichen. Jacobs reduziert die Begriffsbestimmung für die Konstruktion sogar vollständig auf diese Bedingung:

Eine Konstruktion L ist eine direkte Festlegung von Aspekten der Form oder Aspekten der Bedeutung einer Klasse von Zeichen von L, die im Zusammenwirken mit anderen grammatischen Mechanismen zur Erzeugung dieser Zeichenklasse eingesetzt wird. (Jacobs 2008, S. 5)

Die Minimalität dieser Anforderung erlaubt es, den Konstruktionsbegriff in der Sprachbeschreibung sehr flexibel zu verwenden und Konstruktionen 
zur Beschreibung von sprachlichen Phänomenen aus drei unterschiedlichen Übergangsbereichen (Lexikon/Grammatik, Wort/Satz und Idiosynkrasie/ Regel) heranzuziehen.

Ein zweiter Grund für die recht variablen Einsatzmöglichen der Konstruktion zur Beschreibung von Übergangsphänomenen ergibt sich daraus, dass Konstruktionen (i) intern strukturiert sind und (ii) extern in Beziehungen zu anderen Konstruktionen treten. Die Kombination beider Dimensionen eröffnet eine Vielzahl von Variationsmöglichkeiten für die Modellierung sprachlichen Wissens. Je nach zu beschreibendem Phänomen und nach theoretischem Hintergrund können Konstruktionen hinsichtlich ihrer internen Strukturierung unterschiedlich komplex und unterschiedlich abstrakt angesetzt werden, was auch seinen Ausdruck in der Veränderbarkeit der so genannten Korngröße findet (vgl. Imo). Ein breites Spektrum von konstruktionalen Beschreibungsoptionen entsteht schließlich durch die Verschiedenartigkeit der möglichen Beziehungen, in die Konstruktionen untereinander eintreten können: Sie können beispielsweise über Familienähnlichkeiten, Vererbungshierarchien oder Netzstrukturen miteinander verknüpft sein. Dies, gepaart mit dem Konzept der Unterspezifikation und den (z.B. durch Schematisierung erzielten) verschiedenen Abstraktions- und Komplexitätsniveaus von Konstruktionen, erzeugt eine enorme Flexibilität für die Erfassung sprachlicher Phänomene. Daher überrascht es kaum, dass konstruktionistische Ansätze derzeit hinsichtlich der Beschreibung von Übergangsphänomenen eine so große Aufmerksamkeit erlangen. Die damit automatisch einhergehende Frage, ob dies berechtigt ist, d.h. ob konstruktionistische Ansätze tatsächlich projektionistischen Ansätzen überlegen sind oder ob sich die Sachlage nicht genau umgekehrt darstellt, wird in dem vorliegenden Band nicht endgültig geklärt. Dieser Aspekt wird aber implizit in den meisten Beiträgen des vorliegenden Bandes thematisiert, in einigen, wie z.B. in Kiss, Maienborn, Müller, Primus, Stefanowitsch und Webelhuth, wird er sogar explizit diskutiert. Weiterführende Bemerkungen zu diesem Problemfeld finden sich darüber hinaus u.a. bei Müller (2006), Fischer/ Stefanowitsch (Hg.) (2006), Jacobs (2008) und Knobloch (2009).

\subsection{Dimensionen des Konstruktionsbegriffs}

Angesichts der Verschiedenheit der im Band behandelten sprachlichen Phänomene (siehe auch Abschnitt 3), die sich zudem in je anderen Bereichen der genannten Dichotomien bewegen, überrascht die Vielfalt der im Band vertretenen Auffassungen über Konstruktionen, ihrer Beziehungen zueinander und ihrer Rolle in der Grammatik nicht. Wir wollen daher an dieser Stelle den Begriff der Konstruktion etwas näher beleuchten und dabei versuchen offenzulegen, wie er in den konstruktionistisch ausgerichteten Beiträ- 
gen des Bandes verwendet wird und welche verschiedenen Ordnungs- und Abhängigkeitsbeziehungen zwischen Konstruktionen jeweils angenommen werden.

Bei aller Verschiedenheit der einzelnen Ansätze bildet die von Fillmore/ Kay/O'Connor (1988, S. 36) eingebrachte Vorstellung der grammatischen Konstruktion als ,any syntactic pattern which is assigned one or more conventional functions in a language" den Ausgangspunkt der konstruktionsbasierten Analysen. Die in Fillmore/Kay/O'Connor (ebd.) proklamierte einfache Zuordnung von konventionalisierten Funktionen zu syntaktischen Mustern in Form-Funktionspaaren ist im Rahmen der konstruktionsgrammatischen Theoriebildung allerdings auf verschiedene Weise ausgebaut und fortentwickelt worden. Dabei haben sich mehrere (zum Teil graduelle) Dimensionen der Konstruktionsbeschreibung herausgebildet, die wir im Folgenden kurz umreißen möchten.

1) Zeichencharakter der Konstruktionen: Sowohl in formale als auch in gebrauchsbasierte Varianten der Konstruktionsgrammatik hat die Annahme Eingang gefunden, dass die Konstruktion eine symbolische Basiseinheit ist, die auf allen sprachlichen Ebenen grammatische, pragmatische und diskursfunktionale Aspekte eines Ausdrucks repräsentiert. Die ursprüngliche einfache Paarung von syntaktischen und funktionalen Eigenschaften wird erweitert auf eine direkte Zuordnung von Formkomponenten (beispielsweise auch phonologischen und morphologischen) einerseits und Bedeutungsbzw. Funktionskomponenten andererseits. Die von Lakoff eingebrachte Definition ist dafür ein repräsentatives Beispiel:

Each construction will be a form-meaning pair (F,M) where $\mathrm{F}$ is a set of conditions on syntactic and phonological form and $\mathrm{M}$ is a set of conditions on meaning and use. (Lakoff 1987, S. 467)

Demnach sind die Kontext- und Verwendungsbedingungen grammatischer Formen unmittelbarer Bestandteil des sprachlichen Zeichens. Die Vorstellung, dass Konstruktionen im Sinne von de Saussure Zeichencharakter haben, wird im Prinzip von allen konstruktionistisch ausgerichteten Aufsätzen des Bandes (Behrens, Boas, Engelberg et al., Imo, Stefanowitsch und Webelhuth) geteilt.

2) Analysierbarkeit der Konstruktionen: Als maßgebliches Kriterium für Konstruktionen wird mitunter angeführt, dass die Zuordnung zwischen den Form- und Bedeutungs- bzw. Funktionsmerkmalen einer Konstruktion in dem Sinne nicht kompositionell ist, dass ihre Gesamtbedeutung nicht vollständig aus den Bedeutungen ihrer Teile und den jeweiligen Verknüpfungsbeziehungen hergeleitet werden kann, weil jeweils eine stipulierte Konstruktionsbedeutung hinzu kommt. Goldberg formuliert: 
$\mathrm{C}$ is a construction iff $\mathrm{def}_{\mathrm{def}} \mathrm{C}$ is a form-meaning pair $\left\langle\mathrm{F}_{\mathrm{i}}, \mathrm{S}_{\mathrm{i}}\right\rangle$ such that some aspect of $\mathrm{F}_{\mathrm{i}}$, or some aspect of $\mathrm{S}_{\mathrm{i}}$ is not strictly predictable from C's component parts or from other previously established constructions. (Goldberg 1995, S. 4)

Somit haben Einheiten bzw. Strukturen, die vollständig auf andere Einheiten und Strukturen zurückgeführt werden können, keinen unabhängigen Status und gelten nicht als Konstruktionen, sondern als produktiv gebildete Epiphänomene. Goldberg (2006) dehnt den Konstruktionsbegriff allerdings auch auf transparente Muster aus, die aber nur dann als Konstruktionen gelten, wenn sie hochfrequent auftreten.

Die Annahme der partiellen Nicht-Analysierbarkeit bzw. Unvorhersagbarkeit formaler und/oder semantischer Eigenschaften als Bedingung für Konstruktionen wird vor allem von Fillmore (1988), Fillmore/Kay/ O'Connor (1988), Kay/Fillmore (1999) und Goldberg (1995) vertreten, in gewisser Hinsicht auch von Croft (2001). Langacker (1987) hingegen setzt die Nicht-Kompositionalität explizit nicht als Definitionskriterium für Konstruktionen an. Auch wenn Konstruktionen als nicht oder nicht vollständig vorhersagbare Form-Bedeutungspaare definiert werden, ist dieses nicht gleichbedeutend damit, dass die Kompositionalität generell aus der Konstruktionsgrammatik verbannt wäre. Gerade die unten in 9) dargestellten externen Verknüpfungsbeziehungen zwischen Konstruktionen setzen Kombinationsoperationen wie z.B. die Unifikation voraus. Darüber hinaus ist auch anzumerken, dass sprachliche Einheiten, die in konstruktionistischen Ansätzen als semiotisch atomar und damit intern nicht weiter kompositionell strukturiert angesehen werden, im Rahmen anderer (generativer) Theorien durchaus kompositionell behandelt werden können.

3) Frequenz als Maß für Konstruktionen: Mit Goldbergs (2006) Ausdehnung des Konstruktionsbegriffs auf transparente gebrauchsgetriebene Muster kommt Frequenz als weiteres Maß für die Identifikation von Konstruktionen ins Spiel. Zugleich wird die einfache Gleichsetzung von Konstruktionen und Mustern, wie sie noch Fillmore/Kay/O'Connor (1988) vornehmen, aufgehoben. Goldberg bezeichnet alle Vorkommensvarianten inklusive der hochfrequenten Muster als Konstruktionen:

Any linguistic pattern is recognized as a construction as long as some aspect of its form or function is not strictly predictable from its component parts or from other constructions recognized to exist. In addition, patterns are stored as constructions even if they are fully predictable as long as they occur with sufficient frequency. (Goldberg 2006, S. 5)

Stefanowitsch (2009) hingegen präzisiert das Verhältnis von partiell nichtanalysierbaren, teilweise nicht vorhersagbaren Konstruktionen einerseits und vollkommen vorhersagbaren, transparenten gebrauchsgetriebenen Mustern andererseits. Stefanowitsch schreibt: 
In Stefanowitsch (2009) schlage ich vor, den Konstruktionsbegriff für syntaktisch und/oder semantisch unvorhersagbare Strukturen zu verwenden, und Strukturen, die (z.B. aufgrund ihrer Häufigkeit) in ihrer Gesamtheit repräsentiert werden, als ,Muster ${ }^{6}$ zu bezeichnen. Es ist dann klar, dass alle Konstruktionen auch Muster sind, nicht aber umgekehrt.

Ungeklärt ist jedoch, wie nach dieser Auffassung selten auftretende Konstruktionen eingestuft werden sollen, da sie die Frequenz-Bedingung für Muster ja offensichtlich nicht erfüllen. Kay (2002, S. 7) unternimmt ebenfalls den Versuch ,echte ${ }^{6}$ Konstruktionen von bestimmten Mustern, so genannten ,non-productive, non-constructional pattern[s] of coining“ abzugrenzen. Nur zu diesem Zweck legt er das Kriterium der Produktivität an, das nach seiner Auffassung zwar von Konstruktionen, aber nicht von Mustern erfüllt wird.

4) Spezifizierungsgrad der Konstruktionen: Der Konstruktionsbegriff wird, ausgehend von den sprachlich konkreten, voll spezifizierten Fillmoreschen Konstruktionen auch auf nicht oder nur teilweise spezifizierte, abstrakte Konstruktionen, so genannte Schemata, ausgeweitet.

Grammatical patterns are analyzed as schematic symbolic units, which differ from other symbolic structures not in kind, but only in degree of specificity. (Langacker 1987 , S. 58)

Abstrakte Konstruktionen entstehen im Zuge eines Schematisierungsprozesses und drücken Generalisierungen über spezifischen Konstruktionen aus (vgl. auch Abschnitt 3).

In Abhängigkeit von den Graden der Spezifikation bzw. der Schematizität entstehen verschiedene konstruktionale Abstraktionsniveaus, vgl. Goldberg (1995, 2006), Langacker (1987, 1999, 2000), Kay/Fillmore (1999), Croft (2001) und auch Boas. Während Langacker (1987, 1999, 2000) und Croft (2001) voraussetzen, dass abstrakte Konstruktionen eine relativ spezifische Bedeutung aufweisen, lassen Kay/Fillmore (1999) auch abstrakte Konstruktionen zu, die diese Bedingung nicht erfüllen. Damit einher geht die Vorstellung, die allerdings nicht uneingeschränkt unter Konstruktionsgrammatikern geteilt wird (beispielsweise nicht von Croft 2001), dass auf der höchsten Abstraktionsstufe universell gültige Konstruktionen existieren.

5) Omnipräsenz der Konstruktionen: In Folge der ausgeweiteten Abstraktionsmöglichkeiten kann fast alles zur Konstruktion erhoben werden. Dies trifft insbesondere auch für grammatische Gesetzmäßigkeiten und Regeln zu, die als besonders stark dekontextualisiert und schematisiert gelten. Hierzu sei auch auf Abschnitt 3 verwiesen. Auch Sequenzen sprachlicher Zeichen, die über die Grenzen der Syntax hinausgehen, wie z.B. Gespräche und Texte, oder ihnen hierarchisch übergeordnete Einheiten, wie Gattungen, Textsor- 
ten, oder auch fragmentarische Ausdrücke (Günthner 2006), sind nach Auffassung einiger Konstruktionsgrammatiker als Konstruktionen repräsentierbar (vgl. Östman 2005 und auch Imo).

6) Komplexität der Konstruktionen: Sprachliche Äußerungen, die über ein Wort hinausgehen, können intern strukturiert, d.h. aus zwei oder mehreren Konstruktionen zusammengesetzt sein. Es werden grundsätzlich atomare von komplexen Konstruktionen unterschieden, vgl. u.a. Croft (2001), Jacobs (2008) und auch Abschnitt 3. Atomare Konstruktionen sind solche, die nicht weiter in kleinere konstruktionale Einheiten zerlegt werden können. Entsprechend ist jede vollspezifizierte Konstruktion auch atomar. Ebenso gelten auch Wörter, die der Form nach nicht weiter reduzierbar sind, obwohl sie semantisch komplex (z.B. dekomponiert) sind, als atomar.

Die konstruktionsgrammatischen Ansätze unterscheiden sich dahingehend, ob Morpheme oder Wörter als die kleinsten Einheiten angesetzt werden. Die Bedeutung einer komplexen Konstruktion ist entweder fest mit der jeweiligen Form verbunden (z.B. bei Idiomen) oder ergibt sich aus der Konstruktionsbedeutung der jeweils beteiligten Konstruktionen und der Bedeutung der kleinsten Teile, z.B. der Bedeutung beteiligter Morpheme oder der lexikalischen Bedeutung beteiligter Wörter sowie der Art ihrer Verknüpfung. Letzterer Fall belegt erneut, dass Kompositionalität unter bestimmten Voraussetzungen auch von den Konstruktionsgrammatikern angenommen wird.

7) Konstruktionen als Primitive der Grammatik: Die minimalen Einheiten in der Grammatik über der Wortebene sind Konstruktionen, nicht jedoch syntaktische Kategorien, Relationen oder Regeln. „Constructions, not categories and relations, are the basic, primitive units of syntactic representation" (Croft 2001, S. 46).

Während Regeln als abstrakte Schemata und damit selbst als Konstruktionen definiert werden, werden syntaktische Kategorien und Relationen, wie z.B. Nomen oder Subjekt, nicht verallgemeinert, sondern nur in Bezug auf eine konkrete Konstruktion angesetzt. Beispielsweise fungiert eine Einheit A bezogen auf eine Konstruktion X als Subjekt A von X, und eine Einheit B bezogen auf eine Konstruktion Y als Subjekt B von Y. Außerhalb der Konstruktionen sind A und B keiner eigenständigen Kategorie zugeordnet, da diese Kategorien nach Langacker (1987) und Croft (2001) außerhalb der Konstruktionen nicht existieren. Diese Sichtweise wird allerdings von den konstruktivistisch orientierten formalen Grammatiktheorien wie der „Head-Driven Phrase Structure Grammar“ (HPSG) nicht geteilt. Auch die vor allem von Langacker $(1987,1999)$ und Croft (2001) eingebrachte strikte Auffassung, dass innerhalb der Konstruktion keinerlei systematische syntaktische Beziehungen zwischen ihren Teilen bestehen, wird nur bedingt allgemein vertreten, in gewisser Hinsicht aber von Goldberg $(1995,2006)$. 
8) Kognitive Relevanz der Konstruktionen: Vor allem Vertreter gebrauchsorientierter konstruktionsgrammatischer und kognitiver Ansätze sind an Sprache als kognitivem Phänomen interessiert (vgl. Langacker 1987, 1999, 2000; Croft 2001; Goldberg 2006; Behrens). Sie sehen Konstruktionen als kognitive Einheiten, die aus allgemeinen kognitiven Kategorisierungs- und Lernprozessen resultieren. Entsprechend haben grammatische Strukturen kognitionspsychologische Relevanz. Dies bedeutet zugleich, dass eine Information redundant repräsentiert werden kann, d.h. sowohl in einer spezifischen Konstruktion als auch innerhalb einer schematischen Konstruktion. Dieser Aspekt wird auch von Boas aufgegriffen.

9) Verknüpfungsbeziehungen zwischen Konstruktionen: Die Vorstellungen über die Prinzipien der Strukturierung der Menge der Konstruktionen variieren. Lakoff (1987) ebenso wie Goldberg $(1995,2006)$ und Kay (2000) nehmen an, dass Konstruktionen in Vererbungshierarchien zueinander geordnet sind, wobei spezifischere Konstruktionen die grammatischen und pragmatischen Eigenschaften von weniger spezifischen und in diesem Sinne allgemeineren Konstruktionen übernehmen. Die Vererbung der Eigenschaften kann vollständig oder partiell geschehen. Die erste Auffassung wird z.B. von Kay (2000) vertreten und im Rahmen der HPSG unifikationsbasiert formalisiert. Lakoff (1987) und Goldberg $(1995,2006)$ hingegen plädieren dafür, auch eine partielle Vererbung (Default-Vererbung) zuzulassen, was zu netzwerkartigen Verknüpfungsbeziehungen führt.

Zwei weitere Strukturierungsprinzipien sind alternativ zur Vererbung angesetzt worden: Zum einen die taxonomische Verlinkung von untereinander verbundenen spezifischeren Konstruktionen mit einer allgemeineren schematischen Konstruktion (Langacker 1987, 1999, 2000; Croft 2001) und zum anderen die Ableitung der Eigenschaften der spezifischeren Konstruktionen aus den Eigenschaften einer Menge von protoptypischen schematischen Konstruktionen (Lakoff 1987). Beiden Prinzipien ist die Idee gemeinsam, dass Konstruktionen Kategorien bilden. Während Croft (2001) die Auffassung vertritt, dass spezifische Konstruktionen Instanzen einer konstruktionalen schematischen Kategorie sind, bestimmt Lakoff (1987) die Konstruktion als radiale Kategorie, wobei die abstrakten Schemata dem prototypischen Zentrum und die spezifizierten Konstruktionen der Peripherie zugerechnet werden.

\subsection{Konstruktionsauffassungen in den Aufsätzen dieses Bandes}

Die konstruktionistisch ausgerichteten Beiträge des Bandes greifen die einzelnen definitorischen Dimensionen der Konstruktionen auf unterschiedliche Weise auf und diskutieren jeweils verschiedene Aspekte des Konstruktionsbegriffs. 
Aus der konstruktionistisch orientierten Spracherwerbsperspektive sieht Behrens Konstruktionen als abstrahierte usualisierte Muster:

Die Konstruktion wird gefasst als schematische Einheit mit mehr oder weniger offenen Slots: Teile der Konstruktion können lexikalisch fixiert sein, oder aber produktiv und durch andere Ausdrücke ersetzbar sein.

Konstruktionen sind demnach sprachliche Form-Funktionseinheiten, die sich input- und interaktionsabhängig im Kontext wiederkehrender Entitäten, Diskurse und Situationen kognitiv entwickeln: Aus konkreten itemgestützten Schemata (auch so genannten Konstruktionsinseln) entstehen sukzessiv abstrakte Konstruktionen auf verschiedenen Abstraktionsniveaus, vgl. z.B. Tomasello (2003). Eine gebrauchsbasierte Sicht auf Konstruktionen betont den datengetriebenen Lernprozess, in dessen Verlauf bedeutungstragende sprachliche Muster induktiv abgeleitet werden. Sie ist vor allem gerichtet auf das Kontinuum zwischen einerseits regulären, produktiven und andererseits arbiträren, idiosynkratischen Einheiten und Strukturen. Entsprechend spricht man auch von mehr oder weniger festen Mustern, die sich im Gebrauch „einschleifen“. Bei dieser Betrachtungsweise steht der innere Bau der Konstruktionen, einschließlich ihres Abstraktionsgrades bzw. ihrer Komplexität im Vordergrund. Fragen der Formalisierung der Konstruktionen im Allgemeinen oder der Modellierung von grammatischen Beziehungen zwischen Konstruktionen interessieren weniger. Die gebrauchsbasierte und kognitive Herangehensweise an Konstruktionen hat nicht nur Bezüge zu Lakoff (1987) und Langacker (1987, 1999, 2000), sondern ist auch erkennbar von Crofts (2001) ablehnender Haltung gegenüber grammatischen Kategorien jeglicher Art geprägt.

Es ist naheliegend, dass der gebrauchsgestützte Begriff einer situativ und diskursiv gebundenen Konstruktion außer für die Spracherwerbsforschung auch für die Gesprächsforschung relevant ist. Der Zeichencharakter konstruktionaler Muster und die damit verbundene Form-Funktions-Zuordnung scheint auf den ersten Blick optimal geeignet zu sein, um interaktionale Aspekte der Sprachverwendung zu modellieren. Ganz so einfach liegen die Dinge aber nicht (vgl. auch Deppermann 2008). Imo argumentiert anhand der Analyse gesprochener Sprache, dass die zeichenbasierte Vorstellung der Konstruktion als einer Paarung von Form und Bedeutung bzw. Funktion bei der Erfassung gesprochener Sprache an ihre Grenzen stößt. Stattdessen bringt er das Konzept der Korngröße (Granularität) ein, das es erlaubt, flexible Grenzziehungen zwischen konstruktionalen Mustern vorzunehmen.

Ein Teil der konstruktionistisch ausgerichteten Aufsätze des Bandes beschäftigt sich anhand einzelner Phänomenbereiche vor allem mit Fragen der Erfassung bestimmter Konstruktionen bzw. Konstruktionsfamilien und der Modellierung der externen Beziehungen zwischen den identifi- 
zierten Konstruktionen (teilweise unterschiedlichen Abstraktionsgrades). Die untersuchten Phänomenbereiche umfassen z.B. die Relativsatzkonstruktion (Webelhuth), die Resultativkonstruktion (Boas, Engelberg et al.), besondere $w h$-Konstruktionen (Stefanowitsch) oder bestimmte Verbklassen, wie Geräuschverben, Verben mit inneren Objekten und Verben des Suchens (Engelberg et al.). Gemeinsam ist allen Analysen, dass jeweils durch die Etablierung von hierarchischen und/oder netzwerkartigen Verknüpfungsbeziehungen versucht wird, Abhängigkeiten zwischen spezifischen und abstrakten Konstruktionen und konstruktionalen Mustern zu modellieren und in dieser Weise Generalisierungsbeziehungen auszudrücken. Dabei werden im Prinzip zwei Wege eingeschlagen:

(i) Webelhuth beschreibt die Relativsatzkonstruktionen im Theorierahmen der HPSG und folgt damit der formal orientierten Auffassung von Konstruktionen und ihren Verknüpfungsbeziehungen im Sinne von Kay (2000). Konstruktionen sind Mengen von sprachlichen Zeichen, die als getypte Merkmalsstrukturen modelliert werden und in redundanzfreien Vererbungshierarchien zueinander geordnet sind. Die Vererbung von Information wird über Unifikation realisiert, die ihrerseits Subsumption voraussetzt. In der Hierarchie höher stehende Einheiten sind weniger spezifiziert und in diesem Sinne abstrakter als in der Hierarchie niedriger stehende Einheiten. Die Vererbungshierarchie spannt sich als partiell geordneter Verband zwischen einer maximal unterspezifizierten Einheit und den maximal spezifischen Einheiten auf. Eine spezifische Einheit E muss mindestens über diejenigen Eigenschaften verfügen, die die weniger spezifischen Einheiten aufweisen, zu denen $\mathrm{E}$ in einer Subsumptionsbeziehung steht.

(ii) Boas, Stefanowitsch sowie Engelberg, König, Proost und Winkler (Engelberg et al.) gehen stärker von gebrauchsbasierten Beziehungen zwischen Konstruktionen aus und knüpfen in dieser Hinsicht eher an Goldberg $(1995,2006)$ an. Allerdings teilen vor allem Boas und Engelberg et al. die von Goldberg $(1995,2006)$ angesetzten Ordnungsstrukturen nicht vollständig. Boas plädiert für ein Netzwerk aus Resultativkonstruktionen unterschiedlichen Abstraktionsgrades, wodurch sowohl lexikalisch-spezifische als auch abstrakt-schematische Konstruktionsinformation einheitlich repräsentiert wird. Die entsprechend spezifizierte Information wird partiell über Schnittmengenbildung verknüpft. Eine im Satz realisierte Resultativkonstruktion ergibt sich demnach aus dem Schnitt der so genannten Minikonstruktionen mit der abstrakten Schemakonstruktion, wobei die Schemakonstruktion lexikalisch spezifiziert und damit instantiiert wird. Im Gegensatz zu den strikten Vererbungshierarchien der HPSG erlauben bzw. erfordern die von Boas angedachten hierarchischen Konstruktionsnetzwerke die Repräsenta- 
tion redundanter Information. Engelberg et al. fokussieren nicht auf hierarchische Beziehungen zwischen Konstruktionen unterschiedlichen Abstraktionsgrades, sondern befassen sich mit Ähnlichkeiten zwischen (teilweise) spezifizierten konstruktionalen Mustern derselben kategorialen Ausdrucksklasse. Sie plädieren dafür, Argumentstrukturmuster als ein Netz von Familienähnlichkeiten zu repräsentieren und nicht, wie Goldberg $(1995,2006)$, als jeweils eigene polyseme Argumentstrukturkonstruktionen. Engelberg et al. decken auf, dass Goldbergs Umgang mit konstruktioneller Polysemie widersprüchlich ist: Ein und dasselbe Form-Bedeutungspaar firmiert entweder als Variante eines einzelnen konstruktionellen Zeichens, oder es wird derart separiert, dass zwangsläufig zwei homonyme eigenständige konstruktionelle Zeichen entstehen.

\section{Phänomene}

\subsection{Idiome und andere Phraseologismen}

Das Bewusstsein für die Häufigkeit fester Einheiten in der Sprache ist aus der Beschäftigung der linguistischen Forschung mit Idiomen hervorgegangen. Bereits Bolinger hat darauf hingewiesen, dass es in der Sprache weitaus mehr präfabrizierte Einheiten gibt als bis dahin gemeinhin angenommen worden war:

I want to take an idiomatic rather than an analytic view, and argue that analyzability always goes along with its opposite at whatever level, and that our language does not expect us to build everything starting with lumber, nails, and blueprint, but provides us with an incredibly large number of prefabs, which have the magical property of persisting even when we knock some of them apart and put them together in unpredictable ways. (Bolinger 1976, S. 1)

Diese Ansicht ist zumindest teilweise als Reaktion auf die damals in der Generativen Grammatik gängige Auffassung zu sehen, dass lexikalische Einheiten durch eine lexikalische Insertionsregel in die Tiefenstruktur eingefügt würden und syntaktische Strukturen erst später, und zwar kompositionell, interpretiert würden (vgl. Chomsky 1957 [1968], 1965). Wie Jackendoff (1995) später gezeigt hat, führte die Postulierung einer Insertionsregel in der Generativen Grammatik zu einer bestimmten Auffassung des Lexikons, in der Idiome grundsätzlich keinen Platz hatten: Da lexikalische Insertion ein terminales Element (eine lexikalische oder $\mathrm{X}^{0}$-Kategorie) in einem Phrasenstrukturbaum durch eine lexikalische Einheit ersetzt, kommen nur $\mathrm{X}^{0}$-Kategorien als Einheiten des Lexikons in Frage; Idiome sind aber keine $\mathrm{X}^{0}$-Kategorien. Außerdem werfe die Auffassung, dass syntaktische Strukturen kompositionell, und zwar erst nach dem Zeitpunkt der lexikalischen 
Insertion, interpretiert würden, in Bezug auf Idiome die Frage auf, wie diese zum Zeitpunkt der lexikalischen Insertion markiert sein müssen, damit sie später nicht-kompositionelle Interpretationen erhalten können (vgl. Jackendoff 1995, S. 133).

Vor diesem Hintergrund ist auch die Forderung nach einer stärkeren Berücksichtigung von Idiomatizität als Gegenstück zu Analysierbarkeit zu sehen (vgl. dazu auch Chafe 1968; Sinclair 1987), wobei von Anfang an betont wurde, dass Idiomatizität nicht nur eine Eigenschaft von Idiomen sei. Auch andere Mehrwortausdrücke wie etwa Paarformeln, kommunikative Routineformeln, Sprichwörter, bekannte Zitate und Klischees wurden als mehr oder weniger idiomatisch betrachtet (vgl. Makkai 1972; Bolinger 1976; Sinclair 1987; Burger 1998). Einige Autoren haben ebenfalls auf die Idiomatizität mancher Komposita und englischer ,phrasal verbs“ hingewiesen (vgl. Hockett 1958; Makkai 1972; Jackendoff 1995 für Komposita und „,phrasal verbs“ sowie Makkai 1972 und Cowie/Mackin 1993, S. ix-x für „phrasal verbs"). Als „Kollokationen“ werden üblicherweise schwach-idiomatische Ausdrücke bezeichnet (vgl. Burger 1998; Dobrovol'skij 1995). Dazu gehören Ausdrücke wie sich die Zähne putzen, zahlreiche Zwillingsformeln wie groß und stark sowie Funktionsverbgefüge wie eine Entscheidung treffen, deren Konventionalisierung sich darin zeigt, dass sie in formaler Hinsicht invariabel sind (vgl. " die Zähne waschen, ${ }^{\#}$ eine Entscheidung nebmen, ${ }^{*}$ stark, und groß) oder an ganz bestimmte Gebrauchskontexte gebunden sind (z.B. die Verwendung von gro $\beta$ und stark in Kontexten, in denen Kinder dazu ermuntert werden, mehr zu essen (... damit du groß und stark wirst) (vgl. Burger 1998, S. 50-51; zu Funktionsverbgefügen vgl. auch Van Pottelberge 2001 und Storrer 2007). Hausmann zählt zu den Kollokationen nur Zweierkombinationen, die der „langue“ angehören und aus einer Basis, dem determinierenden Partner, und einem Kollokator, dem determinierten Partner, bestehen, wie etwa schütteres Haar, Geld abbeben und ein Schwall von Worten (vgl. Hausmann 1985, S. 118-119). Kollokationen sind Gegenstand der Beiträge von Häcki Buhofer, Heid und Hanks in diesem Band. Häcki Buhofer beschäftigt sich mit der lexikografischen Darstellung von Kollokationen, Heid mit der korpusbasierten Beschreibung deren regionaler Variation und Hanks mit den Kollokaten von Verben. Kollokationsähnliche Phänomene sind auch die von Kiss untersuchten Präposition-Nomen-Kombinationen sowie der Diskursmarker ich mein $(e)$ und das Vergewisserungssignal weißst $D u$, die von Imo diskutiert werden.

Während einerseits die Festigkeit und die oft damit einhergehende Nicht-Kompositionalität vieler sprachlicher Einheiten betont wurden, etablierte sich andererseits etwa zur gleichen Zeit die Einsicht, dass nicht alle so genannten „festen“ Einheiten gleichermaßen fest und/oder nicht-kompositionell sind. Fraser (1970) teilte z.B. Idiome aufgrund der Anzahl der von ihnen erlaubten syntaktischen Operationen in unterschiedliche Klassen 
ein, die er in einer Festigkeitshierarchie, einer so genannten „Frozenness Hierarchy“, einordnete. Nunberg/Sag/Wasow (1994) teilen Idiome aufgrund ihrer (Nicht-)Kompositionalität in zwei Klassen ein, „Idiomatically Combining Expressions" (kompositionelle Idiome wie take advantage und pull strings) und „Idiomatic Phrases“ (nicht-kompositionelle Idiome wie kick the bucket und saw logs), und betonen, dass die meisten (englischen) Idiome zu Ersteren gehören. „Idiomatically Combining Expressions“ sind kompositionell, weil Teile ihrer Bedeutung mit Teilen ihrer lexikalischen Struktur korrespondieren; „Idiomatic Phrases“ weisen hingegen keine solche Isomorphie von Form und Bedeutung auf.

Die Beschäftigung mit Idiomen hat also gezeigt, dass die Grenze zwischen Lexikon und Grammatik als den klassischen Domänen von Wörtern bzw. Syntagmen in mehrfacher Hinsicht fließend ist, denn (i) Mehrwortausdrücke können wie Wörter Einheiten des Lexikons sein, (ii) viele Idiome und andere Phraseologismen verhalten sich insofern ähnlich wie freie Wortverbindungen, als sie häufig kompositionell sind und (iii) auch Wörter (manche Komposita und „phrasal verbs“) können als Idiome betrachtet werden. Damit ist auch die Dichotomie von Wort und Syntagma in Frage gestellt (siehe auch Abschnitt 1). In den Beiträgen von Jacobs und Maienborn wird diese Dichotomie in Bezug auf andere Phänomene problematisiert, im Beitrag von Maienborn in Bezug auf den Übergangscharakter des Zustandspassivs zwischen dem Vorgangspassiv als einem phrasalen Muster und Wortbildungsmustern, im Beitrag von Jacobs u.a. hinsichtlich der Fugen in Komposita, die schwachen Formen von Funktionswörtern (z.B. ' $n$ statt ein), die Flexion von Teilgliedern in Komposita und Zusammenrückungen, Partikelverben als Basen für die Adjektivierung mit -bar und die Nicht-Vorfeldfähigkeit der Erstglieder vieler Partikelverben. Nach Imo ist auch der Diskursmarker ich mein(e) ein Phänomen, das auf unterschiedlichen Ebenen (der lexikalischen, der syntaktischen und der der Gesprächsstruktur) zu beschreiben sei.

\subsection{Schematische Idiome}

Die Initialzündung für die Konstruktionsgrammatik lieferte die Erkenntnis von Fillmore/Kay/O'Connor, dass auch lexikalisch nicht-spezifizierte syntaktische Muster, die mit einer ganz bestimmten, nicht aus ihrer Form vorhersagbaren Bedeutung und Funktion assoziiert sind, als Idiome betrachtet werden können (vgl. Fillmore/Kay/O'Connor 1988, S. 505 f.). Idiome dieses Typs nennen sie „formal idioms“. Als Beispiele erwähnen sie u.a. die the X-er, the Y-er-Konstruktion, die let alone-Konstruktion und die MadMagazine-Konstruktion (Him, be a doctor?...). ${ }^{2}$ Andere Autoren nennen lexi-

Lexikalisch voll spezifizierte Idiome wie It takes one to know one bezeichnen Fillmore/Kay/ O'Connor (1988) als „substantive idioms“. Als „substantive idioms“ gelten allerdings auch 
kalisch nicht-spezifizierte syntaktische Muster mit fester Bedeutung und/ oder Funktion „abstract idioms“ (Williams 1994), „schematische Konstruktionen“ (Croft 2007, S. 466-468; Jacobs 2008, S. 4) oder „constructional idioms" (Jackendoff 1995, S. 154) und fassen darunter Phänomene wie die sequenzielle Nominalreduplikation $N_{x} P N_{x}$ (day by day, cheek, to cheek, layer on layer, dollar for dollar, limb from limb, ...; vgl. Williams 1994, S. 18-20), die Resultativkonstruktion (Jackendoff 1995, S. 153-154, Croft 2007, S. 468), die benefaktive Dativ-Konstruktion (der Oma den Koffer tragen, dem Freund das Auto reparieren, ...) und die In-X-setzen-Konstruktion (in Bewegung setzen, in Kenntnis setzen, ...) (beide aus Jacobs 2008, S. 6 f.) sowie die way-Konstruktion (Jackendoff 1995, S. 154-155).

In Übereinstimmung mit der bereits erwähnten Annahme der Omnipräsenz von Konstruktionen (siehe Abschnitt 2) wird jede Form grammatischen Wissens von konstruktionsgrammatischen Ansätzen als Konstruktion repräsentiert. Das gilt auch für syntaktische Regeln, Regeln der semantischen Interpretation und für die grundlegenden Satzmuster einer Sprache (vgl. Croft 2007, S. 468). Aus diesem Grund wird beispielsweise die Existenz einer Transitivkonstruktion (vgl. Goldberg 1995, S. 116-119) und einer Intransitivkonstruktion (vgl. Croft 2007, S. 468) sowie einer NP- und einer VP-Konstruktion (vgl. Goldberg 2006, S. 10 bzw. Kay/Fillmore 1999, S. 8) angenommen. Analog könnte, wie Jacobs gezeigt hat, die klassische $\mathrm{X}$-bar-Regel $\mathrm{X}^{\prime} \rightarrow \mathrm{X}^{0}$ VP als eine phonologisch wie semantisch nicht-spezifizierte Komplement-Konstruktion dargestellt werden (vgl. Jacobs 2008, S. 7). Von den bisher erwähnten Konstruktionen unterscheiden sich diese ,allgemeinen" Konstruktionen nur dadurch, dass sie maximal unterspezifiziert, d.h. hoch-schematisch sind. Jacobs nennt diese Art von phonologisch und semantisch nicht- oder nur minimal spezifizierten Konstruktionen ,abstrakt“, alle anderen bezeichnet er als „,konkret“ (vgl. ebd.). Zur Gruppe der hoch-schematischen Konstruktionen werden auch alle Satzmuster gezählt, deren formale Eigenschaften mit einer bestimmten kommunikativen Funktion einhergehen wie die Fragekonstruktion, die Passivkonstruktion, die Topikalisierungskonstruktion und die Relativsatzkonstruktion (vgl. Goldberg 2006, S. 9). Für nicht-konstruktionsgrammatische Theorien, die die Existenz von Konstruktionen anerkennen, bleiben Regeln und Gesetze weiterhin relevant. Als Konstruktionen kommen im Rahmen solcher Theorien nur Phänomene in Frage, deren Form und/oder Bedeutung bzw. Funktion nicht aufgrund von Eigenschaften ihrer konstituierenden Teile oder aufgrund von Regeln der Grammatik vorhergesagt werden kann. Jacobs betrachtet beispielsweise sowohl Konstruktionen als auch Gesetze als grammatische Regeln. Erstere erlauben bestimmte Ausdrucksklassen und er-

Idiome wie trip the light fantastic oder kick the bucket, weil diese trotz ihrer fixierten lexikalischen Besetzung ein flektierbares Verb enthalten. 
möglichen es, idiosynkratische Züge des jeweiligen Ausdrucks festzuhalten. Letztere verbieten bestimmte Ausdrucksklassen und beschreiben per definitionem Regularitäten (vgl. Jacobs 2008, S. 9 f.).

Schematische Idiome sind häufig aus einer konstruktionsgrammatischen Perspektive untersucht sowie im Rahmen anderer Ansätze als Kandidaten für eine konstruktionsgrammatische Beschreibung in Betracht gezogen worden. Das zeigt sich auch in der großen Anzahl der Beiträge dieses Buches, die solche Phänomene zum Gegenstand haben. Müller und Stefanowitsch beschäftigen sich in ihren Beiträgen mit lexikalisch partiell spezifizierten Konstruktionen, Müller aus Sicht der Generativen Grammatik mit verblosen Direktiven und den bereits erwähnten N-P-N-Verbindungen, Stefanowitsch aus Sicht der Konstruktionsgrammatik mit der Was-in-allerWelt-Konstruktion und der X-hat-seinen-Preis-Konstruktion. Argumentstrukturmuster sind Thema eines Großteils der Beiträge dieses Buches. Der Beitrag von Hanks thematisiert allgemein die Valenz von Verben, der von Bornkessel-Schlesewsky/Schlesewsky die Interpretation von Argumenten aus psycho- und neurolinguistischer Sicht und der von Behrens den Erwerb der Argumentstruktur. Einzelne Typen von Argumentstrukturen sind Thema der Beiträge von Boas (Resultativkonstruktionen), Engelberg et al. (Such-Argumentstrukturmuster, Argumentstrukturmuster mit innerem Objekt, Argumentstrukturmuster mit Geräusch-als-Bewegungsverben, Resultativ-Argumentstrukturmuster), Fellbaum (Mittelkonstruktion, Instrument-Subjekt-Alternation, Kausativ-Inchoativ-Alternation, benefaktiver Dativ, Dativ-Alternation, Lokativ-Alternation) und Primus (unpersönliches Passiv). Einige Beiträge haben klassische syntaktische Phänomene zum Gegenstand. Das trifft beispielsweise auf den Beitrag von Webelhuth zu, der deutsche und englische Relativsatztypen untersucht und zu dem Schluss kommt, dass sich diese mit den Mitteln der Konstruktionsgrammatik (Typen, Untertypen, Vererbung) adäquat erfassen lassen. Behrens und Tracy beschäftigen sich in ihren Beiträgen mit dem Erwerb der Satzklammer, Behrens aus konstruktionsgrammatischer Sicht und Tracy aus der Sicht der Generativen Grammatik und der Theorie dynamischer Systeme. Als Übergangsphänomene im Sinn von zeitweise koexistierenden und konkurrierenden Strukturen, die später auf einen einzigen Bauplan hin konvergieren, diskutiert Tracy u.a. V2- und Verbletzt- $w$-Fragen, Nebensätze mit und ohne Komplementierer sowie produktive Verbletztkonstruktionen und V2-Formeln.

\subsection{Phänomene auf der Wortebene}

Phänomene im Grenzbereich zwischen Lexikon und Grammatik existieren nicht nur auf der Ebene der Mehrwortausdrücke, sondern auch auf der der Wörter. Morphologisch komplexe Wörter werden als „morphologische 
Konstruktionen“ bezeichnet (vgl. Jacobs 2008, S. 6). Diese unterscheiden sich nur dadurch von syntaktischen Konstruktionen (schematischen Idiomen), dass ihre Elemente gebunden sind; die Elemente syntaktischer Konstruktionen sind frei (vgl. Croft 2007, S. 470). Beispiele morphologischer Konstruktionen sind die „ornative be-Verb-Konstruktion“, instanziiert etwa durch bemannen, bereifen und besoblen (vgl. Jacobs 2008, S. 6), und Konstruktionen mit unikalen Morphemen (cranberry), die $s$-Plural-Konstruktion (books) und Pluralkonstruktionen mit stammspezifischen Pluralmorphemen (brethren) (vgl. Croft 2007, S. 470). Als morphologische Konstruktionen können auch Partikelverben betrachtet werden, die von Jacobs als Phänomene erwähnt werden, die aufgrund mancher ihrer Eigenschaften Anlass dazu geben, die traditionelle Zweiteilung von Lexikon und Grammatik in Frage zu stellen.

Croft unterscheidet auch morphologische Konstruktionen nach dem Grad ihrer Schematizität: (i) Ausdrücke wie book-s sind voll spezifiziert; (ii) Konstruktionen wie book-NUMBER und NOUN-s sind teilweise spezifiziert bzw. teilweise schematisch und (iii) solche wie NOUN-NUMBER sind voll schematisch (vgl. Croft 2007, S. 470).

Morphologische Simplizia sind atomar (vgl. ebd., S. 471). Beispiele spezifizierter atomarer Konstruktionen sind this und green (ebd.), avocado, anaconda und and (vgl. Goldberg 2006, S. 5); die klassischen Bezeichnungen für Wortarten wie ,Nomen', ,Adverb' usw. sind Beispiele schematischer atomarer Konstruktionen (vgl. Croft 2007, S. 471). Ein weiteres, in diesem Buch angeführtes Beispiel ist der von Imo diskutierte Ausdruck jetət, der in einer Vielzahl unterschiedlicher Funktionen verwendet wird. Nach Imo instanziiert jetžt abhängig von der gewählten Korngröße, mit der man diesen Ausdruck betrachtet (grob, mittel, fein), unterschiedliche Konstruktionen.

Nach Croft bilden Wörter die Untergrenze für Konstruktionen: ,[...] a word is again just the limiting case of a construction" (ebd.). Nach anderen Auffassungen bilden nicht Wörter, sondern Morpheme die Untergrenze für Konstruktionen, weil weder ihre Form noch ihre Bedeutung aufgrund ihrer konstituierenden Teile (Phoneme) prädiktabel sind. Goldberg führt Morpheme wie pre- und -ing als Beispiele atomarer Konstruktionen an (vgl. Goldberg 2006, S. 5). Sogar Autoren, die die Existenz von Konstruktionen generell leugnen, erkennen den Status von Morphemen als (die einzig existierenden) Konstruktionen an (vgl. z.B. Müller).

\section{Methoden}

\subsection{Typologie empirischer Methoden}

Zur Untersuchung der im vorangehenden Abschnitt dargestellten Phänomene im Grenzbereich von Grammatik und Lexikon werden mittlerweile 
eine Vielzahl verschiedener Methoden eingesetzt, die oft, wie etwa bei psycho- und neurolinguistischen Methoden, miteinander kombiniert werden. Dazu gehören

- korpuslinguistische Methoden (Worthäufigkeitszählungen, N-Gramm-Analysen, Kookkurrenzanalysen, Analysen des diachronen Verlaufs von Ausdrucksfrequenzen etc.);

- psycholinguistische Methoden (Priming-Experimente, Lesezeitexperimente, Experimente unter Einsatz von Eye-Trackern, computergestützte Sprachsimulationsmethoden etc.);

- neurolinguistische Methoden (Methoden zur Messung ereigniskorrelierter Potenziale, Experimente unter Einsatz bildgebender Verfahren, sprachpathologische Diagnosemethoden);

- befragungsbasierte Methoden (Methoden zur Ermittlung von Spracheinstellungen, Elizitationsmethoden in der Feldforschung, sprachbiografische Interviews etc.).

Die Daten, die durch solche Methoden erzeugt werden, können wir entlang verschiedener Dimensionen typologisieren: qualitative versus quantitative Daten, Rezeptionsdaten versus Produktionsdaten, sprachliche versus metasprachliche Daten, Daten geschriebener versus gesprochener Sprache und spontane versus elizitierte Daten:

- qualitative Daten (z.B. durch Interpretation von Äußerungen aus qualitativen Longitudinalstudien, durch Auswertung von Belegsammlungen aus Korpora oder Antworten in qualitativen fragebogengestützten Erhebungen etc.) vs. quantitative Daten (z.B. aus Akzeptabilitätsurteilserhebungen, aus Messungen ereigniskorrelierter Potenziale oder aus korpusgesteuerten Kookkurrenzanalysen etc.);

- Rezeptionsdaten (z.B. aus rezeptionsbasierten psycho- und neurolinguistischen Experimenten etc.) vs. Produktionsdaten (z.B. aus der Analyse von Daten aus elektronischen Textkorpora, ${ }^{3}$ aus Erhebungen experimentell elizitierter Äußerungen oder aus der linguistischer Feldforschung etc.);

- sprachliche Daten (z.B. als Daten aus Korpora oder als elizitierte Produktions- und Rezeptionsdaten aus psycho- und neurolinguistischen Experimenten etc.) vs. metasprachliche Daten (z.B. als Akzeptabilitätsurteile, Daten aus Erhebungen zu Spracheinstellungen oder zu Kompetenzeinschätzungen etc.);

- Daten geschriebener Sprache (z.B. aus elektronischen Textkorpora, aus schriftsprachlich basierten psycho- oder neurolinguistischen Experimenten oder aus Erhebungen von Akzeptabilitätsurteilen etc.) vs. Daten gesprochener Sprache (z.B. aus Korpora gesprochener Sprache oder aus Longitudinalstudien zum Spracherwerb etc.);

Im Falle von annotierten Korpora sind die Annotationen selber wiederum Rezeptionsdaten, insofern als sie auf der Analyse von Korpustexten basieren. 
- spontane Daten (z.B. aus Textkorpora geschriebener und gesprochener Sprache oder aus nicht-teilnehmender Beobachtung sprachlichen Verhaltens etc.) versus elizitierte Daten (z.B. aus psycho- und neurolinguistischen Experimenten, aus Erhebungen von Akzeptabilitätsurteilen oder aus Fragebogenstudien etc.)

\subsection{Empirische Methoden in den Artikeln dieses Bandes}

Die Aufsätze in diesem Band basieren auf empirischen Daten, die die Ausprägungen aller fünf der hier angeführten Dimensionen widerspiegeln. Im Folgenden soll uns aber vor allem die letzte Unterscheidung, die zwischen spontanen und elizitierten Daten, interessieren, die in vergleichbarer Form schon in anderen methodologischen Arbeiten erörtert wurde (vgl. Penke/ Rosenbach 2004, S. 488 ff.; Lehmann 2004, S. 192; Gilquin/Gries 2009, S. 8 ff.).

Spontandaten entstehen dabei meist durch die qualitative oder quantitative Analyse von Äußerungen, die in der einen oder anderen Form als Sprachkorpora aufgefasst werden können. Korpora geschriebener Sprache liegen dabei den Studien von Boas, Kiss, Stefanowitsch, Hanks, Heid, Häcki Buhofer und Engelberg et al. zugrunde, Korpora gesprochener Sprache der Studie von Imo und den Spracherwerbsstudien von Behrens und Tracy.

Die qualitative Auswertung von Spontandaten, also die Analyse von Einzelbelegen aus Textkorpora, ist natürlich seit langer Zeit ein übliches Verfahren und findet sich entsprechend in vielen Arbeiten dieses Bandes, darunter auch in Arbeiten, die Korpora zudem quantitativ auswerten, wie etwa Stefanowitsch, Behrens, Boas und Engelberg et al., oder solchen, in denen auch andere empirische Methoden eingesetzt werden wie etwa bei Primus oder Maienborn. Rein qualitative Korpusauswertungen kennzeichnen Tracys Beitrag, in dem Daten aus einer Longitudinalstudie analysiert werden, sowie den Aufsatz von Imo, der Korpora gesprochener Sprache für qualitative gesprächsanalytische Datenauswertungen nutzt.

Quantitative Analysen von Textkorpora werden von einer ganzen Reihe von Autoren durchgeführt. Dabei kommen verschiedene Analysemethoden zum Einsatz. Einfache Frequenzanalysen werden zur Bestimmung der Verteilung von Ausdrücken relativ zu bestimmten Mustern und Konstruktionen eingesetzt (Behrens, Stefanowitsch, Engelberg et al.). Kookkurrenzanalysen ermitteln Wörter, die in einem bestimmten Abstand voneinander häufiger miteinander auftreten als aufgrund ihrer Gesamthäufigkeit zu erwarten wäre, und decken auf diese Weise kollokationale Zusammenhänge auf (Hanks, Heid, Häcki Buhofer). Darüber hinaus finden sich auch komplexe Variablenanalysen wie etwa die von Kiss verwendete binäre logistische Regression. 
Stefanowitschs Analyse von Konstruktionen des Deutschen (was/wer in aller Welt und $x$ bat ibren/seinen Preis) basiert unter anderem auf der Auswertung relativer Häufigkeiten der lexikalischen Füller in diesen Konstruktionen. Stefanowitsch zeigt dabei, wie diesbezügliche idiosynkratische Verteilungsasymmetrien zur Bildung von Mustern führen, die spezifische Verletzungen von Regularitäten ermöglichen (z.B. auffällig häufige Genuskongruenzverletzungen wie in Qualität hat seinen Preis), die über rein regelbasierte Ansätze nicht zu erklären seien.

In Boas' Arbeit wird demonstriert, dass Verteilungen von Daten in Korpora zeigen, dass etwa bestimmte Argumentstrukturkonstruktionen nicht uneingeschränkt produktiv sind, sondern idiosynkratischen Beschränkungen unterliegen. So unterscheidet sich die Verteilung der weitgehend bedeutungsgleichen Resultativprädikate dead und to death je nach gewähltem Verb erheblich. Solche Beobachtungen geben Anlass zu einer Hypothese über Musterbildung in der Grammatik, die eher für analogische als für derivative Prozesse spricht.

Ähnlich nutzen Engelberg, König, Proost und Winkler (Engelberg et al.) elektronische Textkorpora für die Erhebung qualitativer und quantitativer Daten im Zusammenhang mit der Untersuchung der Varianz von Verben hinsichtlich der Argumentstrukturmuster, in denen sie auftreten. Qualitative Daten in Form von Belegsammlungen werden hier verwendet, um die Bandbreite an Argumentstrukturmustern aufzudecken, in denen einzelne Verben auftreten. Quantitative Daten zeigen, dass die idiosynkratische Verteilung von Argumentstrukturmustern auf Verben als auch die inhaltliche Füllung der Argumentstrukturmuster gegen die Annahme einer einfachen Anwendung abstrakter Argumentstrukturkonstruktionen auf neue Verben spricht.

Während manche quantitative Analysen auf unannotierten Korpora beruhen, arbeiten die folgenden quantitativ-korpuslinguistischen Arbeiten mit verschiedenen Arten von Annotationen. Mit Hilfe der „Sketch Engine“ (Kilgarriff et al. 2004), die üblicherweise auf wortart-getaggten Korpora operiert, führt Hanks Kookkurrenzanalysen durch, um Korpusmuster zu Wörtern zu erhalten, wobei sich ein Korpusmuster als ein Tripel aus der syntaktischen Valenz eines Verbs, den Typen und Rollen der Argumente und deren Realisierung durch saliente Kollokate manifestiert. Aus den verschiedenen Korpusmustern eines Wortes kristallisieren sich dann die verschiedenen Lesarten des Wortes heraus.

Heid unternimmt eine variationslinguistische Untersuchung zu Kollokationen in deutschen, Schweizer und österreichischen Zeitungstexten. Dabei stehen weniger Kollokationen mit varietätentypischen Lexemen im Mittelpunkt (z.B. in der Schweiz Zustupf leisten im Sinne von ,Zuschuss geben'), sondern vielmehr typische Verbindungen mit varietätenunspezifischem Vokabular (z.B. in der Schweiz tiefer Preis statt niedriger Preis) sowie die 
morphosyntaktischen Präferenzen in solchen Kollokationen. Heid verwendet dazu wortartenspezifische kookkurrenzanalytische Verfahren. Da aufgrund der variablen Wortstellung des Deutschen diese Verfahren auf rein sequenzieller Basis problematisch sind, werden die Korpora dazu zunächst durch einen dependenzgrammatischen Parser aufbereitet.

Kiss untersucht Kombinationen aus Präposition und artikelloser Nominalprojektion mit zählbarem Substantiv im Singular als syntaktischem Kopf. Mit Hilfe von Annotation Mining und logistischer Regression wird die syntaktische Distribution solcher Kombinationen ermittelt. Die Studie basiert auf einem Zeitungskorpus, in dem für die Studie relevante Präpositionalphrasen mit reichhaltigen Annotationen versehen werden. Auf lexikalischer Ebene werden Annotationen zu Wortarten, Flexionsmorphologie, Derivationsmorphologie von Substantiven, Zählbarkeit von Substantiven, der Interpretation von Substantiven durch Wortfelder, der Interpretation von Präpositionen und der Kompositabildung bei Substantiven angefügt. Auf syntaktischer Ebene wird der Status (Adjunkt oder Komplement) der Präpositionalphrase annotiert sowie die syntaktischen Komplemente des Nomens und seine pränominalen Modifikatoren. Auf globaler Ebene wird festgehalten, ob die Phrase Bestandteil einer Überschrift, eines Titels oder eines Zitats ist. Die Annotation erfolgt teilweise automatisch mit Hilfe zweier Tagger („Regression Forest Tagger“, „Tree Tagger") und zweier lexikalischer Ressourcen (GermaNet, HaGenLex). Insbesondere die semantische Annotation der Präpositionen erfolgt manuell. Über eine multivariate Analyse binärer Merkmale werden die Merkmale bestimmt, die die Wahrscheinlichkeit des Artikelwegfalls in Präpositionalphrasen erhöhen bzw. vermindern (binäre logistische Regression). Es kann auf diese Weise die Wahrscheinlichkeit für den Wegfall des Artikels abhängig von den betrachteten Eigenschaften bestimmt werden.

Die beiden spracherwerbsbezogenen Arbeiten in diesem Band nutzen Textkorpora, die durch Longitudinalstudien entstanden sind. Tracy verfolgt in ihrem Beitrag zum Erwerb der Satzklammer im Deutschen die These, dass sich bereits in frühen Phasen des Spracherwerbs Evidenz für abstrakte Strukturen findet. Sie führt dazu qualitative Analysen von Daten aus der Longitudinalstudie durch, anhand derer argumentiert wird, dass sich die Dynamik des Spracherwerbs nicht durch Häufigkeiten im Lernerinput entfaltet, sondern dadurch, dass Lerner als mangelhaft empfundene Strukturen immer wieder dekonstruieren und auf einer neuen Ebene rekonstruieren. Demgegenüber argumentiert Behrens mit quantitativen Daten. Sie berichtet von einer Longitudinalstudie, anhand derer die Entwicklung der Argumentrealisierung bei reparieren verfolgt wird, indem die quantitative Verteilung der verschiedenen Argumentstrukturen über die Zeit ermittelt wird. Behrens betont dabei, dass gelegentliche Stichprobenerhebungen oft eine große Produktivität syntaktischer Muster suggerieren. Erstellt man 
dagegen ein großes, zeitlich dichtes Korpus über kontinuierliche Aufzeichnungen, ist eine hohe Stereotypizität und geringe Variabilität in den Daten zu beobachten, was für den in ihrem konstruktionsgrammatischen Ansatz vorhergesagten langsamen Strukturaufbau spricht.

Während Spontandaten in den meisten Fällen durch verschiedene Arten von Korpusanalysen erzeugt werden, gehen elizitierte Daten auf eine größere Vielfalt verschiedener empirischer Methoden zurück. Psycholinguistische Verfahren wie Lesezeitexperimente, Priming-Experimente, Blickbewegungsstudien und neurolinguistische Verfahren wie die Ermittlung von ereigniskorrelierten Potenzialen (EKP) auf der Basis von EEGs und Studien mit Hilfe bildgebender Verfahren sowie methodisch eher am sozialwissenschaftlichen Repertoire orientierte Fragebogenstudien und Akzeptabilitätsurteilserhebungen sind nur ein Teil der praktizierten Verfahren. In den vorliegenden Arbeiten finden sich Beispiele für Priming-Experimente (Maienborn), EKP-Studien (Bornkessel-Schlesewsky/Schlesewsky) und Akzeptabilitätsurteilserhebungen (Primus, Jacobs).

Bornkessel-Schlesewsky/Schlesewsky führen Sprachverstehensexperimente auf der Basis von EEG-Messungen durch, die ereigniskorrelierte Potenziale erfassen. Die Zeitspanne zwischen Reizdarbietung und gemessenem Effekt, die Polarität (Positivierung versus Negativierung) sowie die maximale Ausprägung des Effekts (Amplitude) erlauben in ihrer Kombination und im Kontrast mit der Kontrollbedingung Rückschlüsse auf bestimmte Verarbeitungsvorgänge. In der Studie von Bornkessel-Schlesewsky/ Schlesewsky werden damit Parameter ermittelt, die die inkrementelle semantische Verarbeitung von Argumenten bestimmen, die sequenziell vor dem Verb auftreten.

Im Rahmen ihrer Analyse ereignisbezogener adverbialer Modifikatoren beim Zustandspassiv stützt sich Maienborn auf ein von ihren Mitarbeiterinnen durchgeführtes Experiment zum semantischen Priming (Gese/ Stolterfoht 2010). In der Reaktionszeitstudie mit Wortwiedererkennungsaufgabe müssen Probanden entscheiden, ob ein präsentiertes Wort in einem vorher gelesenen Satz vorkam oder nicht. Ziel des Experiments ist es, Aufschluss zu geben über den Grad der Integriertheit eines Modifikators in einem Satz mit Zustandspassiv.

Primus geht der Frage nach, Verben welcher Klassen im unpersönlichen Passiv akzeptabel sind. Neben qualitativen Analysen von Korpusbelegen wird auch eine Akzeptabilitätsstudie durchgeführt, deren Kontextund Testsätze normierte Varianten vorgefundener Korpusbelege sind. Ziel der Studie ist es, unter Bezugnahme auf ältere Reaktionszeitexperimente mit ähnlicher Fragestellung zu zeigen, dass aspektuelle Uminterpretationen von Verben vorkommen, die diese passivfähig machen. Die Uminterpretation korrelierte dabei mit signifikant niedrigeren Akzeptabilitätswerten im Vergleich zu Verben, die ohne Uminterpretation ein unpersönliches Passiv 
erlauben. Auf der Erhebung von Akzeptabilitätsurteilen basieren auch einige Daten aus den Überlegungen von Jacobs zum Wortstatus sprachlicher Ausdrücke.

Andere Arbeiten befassen sich nicht (nur) mit der Erhebung von Daten und ihrer Anführung als Evidenz für bestimmte theoretische Behauptungen, sondern stellen Verfahren der Aufbereitung von Daten vor. Fellbaum beschreibt Methoden der Zusammenführung lexikalischer Ressourcen, hier der Abbildung des semantisch paradigmatisch ausgerichteten WordNets auf das syntagmatisch konzipierte FrameNet. Häcki-Buhofers Arbeit befasst sich mit der korpusbasierten Erhebung und lexikografischen Darstellung von Kollokationen. Hanks wiederum beschreibt das Vorgehen bei der Erstellung eines Wörterbuchs auf Basis der oben beschriebenen Korpusmusteranalysen. In dem Beitrag von Müller-Spitzer/Engelberg werden weitere lexikografische Ressourcen vorgestellt, bei deren Aufbau korpusanalytische Methoden verwendet wurden.

\subsection{Spontane versus elizitierte Daten}

Die Entscheidung für spontane versus elizitierte Daten setzt eine Analyse ihrer Vor- und Nachteile voraus (vgl. etwa Gilquin/Gries 2009, S. 8 ff.; Lemnitzer/Zinsmeister 2006, S. 19 ff.; Everaert et al. 2010, S.4 ff.; Penke/Rosenbach 2004, S. 485 ff.). Als Vorteile korpuslinguistischen Arbeitens lassen sich unter anderem die Natürlichkeit der Daten und das quantitative Ausmaß der Daten, das auch feinste Verteilungsmuster von Daten erfassbar macht und die Anwendung bestimmter quantitativer Erschließungsmethoden ermöglicht, anführen. Nachteile korpuslinguistischen Arbeitens ergeben sich aus dem Fehlen negativer Evidenz, ${ }^{4}$ aber auch aus fehlerhaften Daten in Korpora. Auch das Problem unzureichender Repräsentativität scheint trotz einer immer größer werdenden Textsortenstreuung der Daten für die Korpuslinguistik nicht prinzipiell lösbar.

Elizitierte Daten haben den Vorteil, dass sie auch bei der Untersuchung von in Korpora sehr seltenen Phänomenen eingesetzt werden können, ebenso wie zur Erzeugung negativer Evidenz. Die Möglichkeit, Variablen zu kontrollieren, erlaubt es, einzelne Phänomene im Detail im Hinblick auf theorierelevante Eigenschaften zu untersuchen. Manche Methoden der Datenelizitation ermöglichen es auch, Prozesse der Sprachverarbeitung zu beobachten, zum Teil in Echtzeit. Auf der anderen Seite können bei Experimenten immer nur sehr kleine Ausschnitte der Sprachwirklichkeit überprüft werden. Auch fordert die sehr mittelbare Art der Daten oft eine große interpretative Anstrengung, die nicht selten eine eingeschränkte Validität der Daten reflektiert.

Gegen die Behauptung, Korpora lieferten keine negative Evidenz, argumentiert allerdings

Stefanowitsch (2006). 
Über diese Unterschiede zwischen spontanen und elizitierten Daten hinaus ist zu beobachten, dass korpuslinguistische Methoden vor allem dort eingesetzt werden, wo konstruktionsgrammatische Ansätze vertreten werden oder doch zumindest mit solchen sympathisiert wird, während experimentelle Methoden dort bevorzugt werden, wo traditionelle Ansätze mit einer Trennung von Grammatik und Lexikon verfolgt werden. Die Frage nach dem Huhn und dem Ei ist hier eher eine wissenschaftshistorische. Aus dem Blick auf die gegenwärtige Forschungslandschaft ist die Paar-Bildung von Theorie und Methode durchaus schlüssig.

Die Korpuslinguistik mit ihren Analysemethoden wie Frequenzanalysen und Kookkurrenzanalysen ermöglicht eine stark induktive Theoriebildung. Indem sie ohne größeren sprachtheoretischen Input Ordnungen in großen Mengen von Daten aufdeckt, produziert sie viele Datenmuster, die entweder gängigen Vorstellungen sprachlicher Regelhaftigkeit widersprechen oder sprachtheoretisch nicht ohne Weiteres erklärbare Usualisierungsgrade aufweisen. Die sprachgebrauchsorientierte Konstruktionsgrammatik in ihrer deskriptiven Mächtigkeit kann solche Datenmuster - insbesondere Mehrwortstrukturen unterschiedlichen Abstraktionsgrads - sowohl gut erfassen als auch über sie generalisieren. Experimentelle Methoden bieten sich dagegen für eine stärker durch Deduktion geprägte Theoriebildung an. Insbesondere dort, wo Reaktionen auf sprachlichen Input experimentell gestaltet werden, ist dieser Input üblicherweise durch eine Hypothese bestimmt, die irgendeine vermutete sprachliche Regelhaftigkeit widerspiegelt. Experimente dienen also üblicherweise dazu, Aussagen aus einer Sprachtheorie zu überprüfen. ${ }^{5}$

\section{5. Überblick über die Struktur des Bandes}

\subsection{Konstruktionsidentität und Konstruktionsvarianz}

Der vorliegende Band ist in fünf größere thematische Abschnitte untergliedert. Der erste Abschnitt mit dem Titel „Konstruktionsidentität und

Dass dies natürlich nicht zwangsläufig so ist, zeigt die korpuslinguistische Analyse in dem Beitrag von Kiss, der keine konstruktionsgrammatische Erklärung verfolgt. Untypisch für gebrauchsorientierte Ansätze ist allerdings ohnehin das durch umfangreiche Annotationen geprägte Vorgehen von Kiss, das durch die Theorieabhängigkeit der Annotationen nur einen eingeschränkt induktiven, wenn auch explorativen Charakter hat. Kiss diskutiert das auch ausführlicher: „Auch eine Analyse, die auf dem Annotation Mining aufsetzt, kann nicht gänzlich unabhängig von introspektiven Urteilen durchgeführt werden. Allerdings kommt introspektiven Bewertungen hier eine indirekte und vermittelnde Rolle zu. Annotation Mining bedeutet ja nichts anderes, als Rohdaten entweder automatisch oder manuell mit Annotationen zu versehen, was wiederum voraussetzt, dass für die relevanten Ebenen linguistische Annotationsschemata entwickelt werden. Es ist nun sehr unwahrscheinlich, dass die Entwicklung eines solchen Schemas - das ja eigentlich eine Mikrogrammatik ist - ohne jedes introspektive Urteil erfolgt.“ 
Konstruktionsvarianz" umfasst drei Aufsätze, die sich mit konzeptuellen Fragen der Identifizierbarkeit, Konstituierung und Abgrenzung von Konstruktionen beschäftigen. Den Auftakt bildet der Aufsatz von Hans Boas, der sich anhand der Resultativkonstruktion mit dem Status abstrakter Konstruktionen auseinandersetzt und auf der Basis von Kollokationsanalysen und Frequenzdaten eine netzwerkartige Konstruktionshierarchie entwirft, die Konstruktionen auf unterschiedlichen Abstraktionsebenen verknüpft. Daran schließt sich der Aufsatz von Stefan Engelberg, Svenja König, Kristel Proost und Edeltraud Winkler (Engelberg et al.) an, in dem die Identität von Konstruktionen aus der Sicht von Phänomenen im Bereich der Argumentstruktur untersucht wird. Die Autorinnen und der Autor weisen nach, dass das von Goldberg (1995) vorgeschlagene Konzept der konstruktionellen Polysemie die von ihnen korpusgestützt erhobenen und grammatiktheoretisch ausgewerteten Daten nicht erfassen kann. Sie plädieren stattdessen dafür, die Ähnlichkeiten zwischen den Argumentstrukturmustern und die jeweiligen idiosynkratischen lexikalischen Kookkurrenzen über ein Netz von Familienähnlichkeiten zu beschreiben. Im Zuge ihrer Überlegungen zur angemessenen sprachtheoretischen Modellierung der Daten zeigen sie sowohl Schwächen valenzbasierter Theorien als auch Mängel konstruktionsbasierter Ansätze auf. Wolfgang Imo betrachtet in seinem Beitrag das Zeichenkonzept von Konstruktionen im Rahmen einer Granularitätstheorie. Er geht in seinem Aufsatz zunächst auf einige hinsichtlich des Konstruktionsbegriffs problematische Fälle des gesprochenen Deutsch ein und nimmt dann eine granulare Re-Analyse der vorgestellten Phänomene vor.

\subsection{Regeln und Konstruktionen}

Im zweiten Abschnitt des Bandes mit dem Titel „Regeln und Konstruktionen" sind die Beiträge versammelt, die - jeweils auf eigene methodische Weise - versuchen zu klären, ob projektionistische oder konstruktionistische Ansätze besser geeignet sind, um die Phänomene des Zwischenraumes zwischen Lexikon und Grammatik adäquat zu beschreiben. Gert Webelhuth beleuchtet in seinem Aufsatz die Arbeitsteilung zwischen Lexikon und Grammatik mit Bezug auf die Frage nach der Existenz und der Natur von grammatischen Konstruktionen und argumentiert für eine konstruktionale Analyse der Relativsätze des Deutschen und Englischen. Anatol Stefanowitsch versucht auf der Basis von Spracherwerbsdaten, der Analyse idiomatischer Strukturen und lexiko-statistischer Daten zu zeigen, dass die Konstruktionsgrammatik einen sparsameren Theorieaufbau ermöglicht als andere Grammatiktheorien. Gereon Müller hingegen will in seinem Beitrag anhand zweier auf den ersten Blick konstruktionaler Phänomene (verblose Direktive und die sequenzielle Nominalreduplikation) nachweisen, dass ein ausschließlich regelbasierter Ansatz nicht nur deskriptiv kon- 
kurrenzfähig ist, sondern darüber hinaus konstruktivistischen Ansätzen auch explanativ überlegen ist. Tibor Kiss untersucht mit dem computerlinguistischen Verfahren des Annotation Mining und der logistischen Regression die syntaktische Distribution von Präposition-Substantiv-Kombinationen ohne erwartbaren Artikel. Anhand zweier Präpositionen (ohne und unter) bestimmt er detailliert die Realisationsbedingungen dieser Kombinationen und zeigt ihre Regelhaftigkeit. Beatrice Primus befasst sich mit der Funktion semantischer Rollen in Argumentstrukturen und unterschiedlichen Dimensionen solcher rollensemantischer Informationen. Auch ihr Beitrag zu den semantischen Bedingungen des unpersönlichen Passivs zielt darauf, mit Hilfe sprachvergleichender, korpusbasierter und experimenteller Daten nachzuweisen, dass regel- bzw. beschränkungsbasierte Analysen in der Lage sind, Phänomene zu erklären, die bisher als Evidenz für die Überlegenheit konstruktionsgrammatischer Herangehensweisen galten.

\subsection{Wörter und Phrasen}

Im dritten Abschnitt des Bandes mit dem Titel „Wörter und Phrasen“ werden die beiden Aufsätze präsentiert, die sich mit dem Übergangsbereich zwischen Wort und phrasaler Struktur befassen. Claudia Maienborn diskutiert in ihrem Beitrag zum Zustandspassiv verschiedene Analyseoptionen für ereignisbezogene adverbiale Modifikatoren beim Zustandspassiv. Diese Modifikatoren treten typischerweise in verbalen Kontexten, nicht aber bei Adjektiven auf, woraus sie Rückschlüsse auf den Status des Zustandspassivs zieht. Sie argumentiert für eine kompakte strukturelle Einheit im Zwischenraum zwischen Wort und Phrase, für die sie eine strikt kompositionale Semantik entwickelt. Joachim Jacobs wendet sich in seinem Beitrag der grundsätzlichen Frage zu, ob die auch für die Lexikon-Grammatik-Unterscheidung wichtige Abgrenzung zwischen Wort und Syntagma angesichts einer Vielzahl problematischer Daten haltbar ist. Er zeigt am Beispiel des Deutschen, dass sowohl die Strategie der Aufspaltung von Wort vs. Syntagma in mehrere spezifischere Kategorienpaare als auch ihre Umdeutung als Prototypen- oder Default-Kategorien nicht geeignet ist, um die angeführten differenzierten Daten vollständig, detailliert und widerspruchsfrei zu erfassen. Er weist aber zugleich darauf hin, dass der Erhalt der Unterscheidung für bestimmte Zwecke nützlich sein kann.

\subsection{Erwerb und Verarbeitung}

Der vierte Abschnitt des Bandes mit dem Titel „Erwerb und Verarbeitung“ befasst sich mit psycho- und neurolinguistischen Aspekten des Spannungsfeldes zwischen Lexikon und Grammatik. Die beiden ersten Beiträge von Heike Behrens und Rosemarie Tracy diskutieren im Lichte aktueller 
Spracherwerbstheorien jeweils die Rolle der Konstruktionen bzw. der grammatischen Struktur im Erwerbsprozess. Während Behrens den Schwerpunkt auf den inputgesteuerten Erwerb von verbalen Form- und Funktionseinheiten legt und dabei zeigt, wie das konstruktionsgrammatische Paradigma beim Erwerb des Deutschen umgesetzt wird, weist Tracy auf der Grundlage von Daten monolingualer und bilingualer Kinder zum Erwerb der Verbzweitstellung im Deutschen nach, dass Kinder sich früh analytisch mit Sprache auseinandersetzen und auf in der Grammatik angelegte Strukturen zurückgreifen. Der dritte Aufsatz dieses Abschnittes ist im Bereich des Sprachverstehens angesiedelt. Ina Bornkessel-Schlesewsky und Matthias Schlesewsky diskutieren, wie verbale Argumente kognitiv erkannt und verarbeitet werden. Sie plädieren dafür, die durch EEG-Messungen experimentell gewonnenen Befunde durch die Interaktion von typologisch motivierten Prominenzskalen zu beschreiben.

\subsection{Korpora und lexikalische Ressourcen}

Im fünften und letzten Abschnitt des Bandes mit dem Titel „Korpora und lexikalische Ressourcen" werden vor allem statistikbasierte korpuslinguistische Analysemethoden, lexikografische Aspekte sowie Fragen der Erstellung großer semantischer Ressourcen diskutiert. Zu Beginn des Abschnittes stellt Christiane Fellbaum die beiden derzeit wohl einflussreichsten und am häufigsten eingesetzten lexikalischen Ressourcen WordNet und FrameNet einander gegenüber. Sie vergleicht ihre jeweilige Klassifikation der Verben und unternimmt einen Versuch, beide Ressourcen im Zuge einer Korpusannotation miteinander zu harmonisieren. Patrick Hanks stellt ebenfalls die Frage der Aufbereitung lexikalischer Ressourcen zur Repräsentation von Argumentstrukturen im verbalen Bereich. Er stellt insbesondere die Methode der Korpusmusteranalyse vor, mit deren Hilfe Valenzen und Kollokationen von Verben ermittelt werden. Diese werden in Beziehung zu bestimmten, empirisch ermittelten semantischen Typen gesetzt, die in einer Ontologie strukturiert organisiert sind. Erklärtes Ziel ist es, auf diese Weise die Analyse von Valenzen und semantischen Typen von Verbargumenten zusammenzubringen, um eine Ressource zu schaffen, mit deren Hilfe man Textbedeutung auf Wortverwendung abbilden kann. Annelies Häcki Buhofer beschäftigt sich in ihrem Aufsatz mit der lexikografischen Erfassung von Kollokationen. Sie hebt hervor, dass für die konkrete Wörterbucherstellung der jeweilige praktische Verwendungszweck, zum Beispiel die Sprachpraxis und damit die Lernerperspektive, für den Umgang mit Kollokationen ausschlaggebend sein muss. Der Beitrag von Ulrich Heid zielt auf die Ermittlung von varietätentypischen Kollokationen im Deutschen. Er diskutiert verschiedene korpusanalytische Verfahren und Werkzeuge zur semi-automatischen Extraktion von Kollokationskan- 
didaten aus Textkorpora. Der Abschnitt und damit auch der Band schließt mit einem Bericht von Carolin Müller-Spitzer und Stefan Engelberg über die Projektmesse zur elektronischen Lexikografie, die die Jahrestagung begleitet hat.

\section{Literatur}

Aronoff, Mark (1976): Word formation in Generative Grammar. (= Linguistic Inquiry: Monographs 1). Cambridge, MA.

Bierwisch, Manfred (1983): Semantische und konzeptionelle Repräsentation lexikalischer Einheiten. In: Ruzicka, Rudolf/Motsch, Wolfgang (Hg.): Untersuchungen zur Semantik. (= Studia Grammatica 22). Berlin, S. 48-80.

Blume, Kerstin (2000): Markierte Valenzen im Sprachvergleich: Lizenzierungs- und Linkingbedingungen. (= Linguistische Arbeiten 211). Tübingen.

Bolinger, Dwight (1976): Meaning and memory. In: Forum Linguisticum 1, 1, S. 1-14.

Borer, Hagit (2005): Structuring sense. Bd. 2: The normal course of events. Oxford u.a.

Burger, Harald (1998): Phraseologie: Eine Einführung am Beispiel des Deutschen. (= Grundlagen der Germanistik 36). Berlin.

Chafe, Wallace (1968): Idiomaticity as an anomaly in the Chomskyan paradigm. In: Foundations of Language 4, S. 109-125.

Chomsky, Noam (1957 [1968]): Syntactic Structures. Studia memoriae Nicolai Van Wijk dedicata. (= Ianua Linguarum: Series Minor 4). Den Haag/Paris. [7. Aufl. 1968].

Chomsky, Noam (1965): Aspects of the theory of syntax. 2. Aufl. (= Special Technical Report/Massachusetts Institute of Technology, Research Laboratory of Electronics 11). Cambridge, MA.

Cowie, Anthony P./Mackin, Ronald (1993): Oxford dictionary of phrasal verbs. Oxford dictionary of current idiomatic English. Bd. 1. Oxford.

Croft, William (2001): Radical Construction Grammar. Syntactic theory in typological perspective. Oxford u.a.

Croft, William (2007): Construction Grammar. In: Geeraerts, Dirk/Cuykens, Hubert (Hg.): The Oxford handbook of cognitive linguistics. Oxford u.a., S. 463-509.

Deppermann, Arnulf (2008): Lexikalische Bedeutung oder Konstruktionsbedeutungen? Eine Untersuchung am Beispiel von Konstruktionen mit verstehen. In: Stefanowitsch, Anatol/Fischer, Kerstin (Hg.): Konstruktionsgrammatik II: Von der Konstruktion zur Grammatik. (= Stauffenburg Linguistik 47). Tübingen, S. 103-133.

Di Sciullo, Anne-Marie/Williams, Edwin (1987): On the definition of word. (= Linguistic Inquiry: Monographs 14). Cambridge, MA u.a.

Dobrovol'skij, Dmitrij (1995): Kognitive Aspekte der Idiom-Semantik: Studien zum Thesaurus deutscher Idiome. (= Eurogermanistik 8). Tübingen.

Dowty, David R. (1991): Thematic proto-roles and argument selection. In: Language 67, S. 547-619. 
Engelberg, Stefan (2009): Blätter knistern über den Beton. Zwischenbericht aus einer korpuslinguistischen Studie zur Bewegungsinterpretation bei Geräuschverben. In: Winkler, Edeltraud (Hg.): Konstruktionelle Varianz bei Verben. (= OPAL Sonderheft 4/2009). Mannheim, S. 75-97. Internet: www.ids-mannheim.de/pub/laufend/opal/ privat/pdf/opal09-4_engelberg.pdf (Stand: 28.01.2011).

Engelberg, Stefan (i.Dr.): Frameworks of lexical decomposition of verbs. In: Heusinger, Klaus von/Maienborn, Claudia/Porter, Paul (Hg.): Semantics: an international handbook of natural language meaning. Bd. 1. Berlin/New York, S. 356-397.

Everaert, Martin et al. (2010): The linguistics enterprise. From knowledge of language to knowledge in linguistics. In: Everaert, Martin et al. (Hg.): The linguistics enterprise. From knowledge of language to knowledge in linguistics. (= Linguistik aktuell 150). Amsterdam/Philadelphia, S. 1-10.

Fillmore, Charles J. (1988): The mechanisms of ,construction grammar'. In: Proceedings of the Fourteenth Annual Meeting of the Berkeley Linguistic Society 14, S. $35-55$.

Fillmore, Charles J./Kay, Paul/O'Connor, Mary Catherine (1988): Regularity and idiomaticity in grammatical constructions: the case of let alone. In: Language 64, 3, S. 501-538.

Fischer, Kerstin/Stefanowitsch, Anatol (Hg.) (2006): Konstruktionsgrammatik. Von der Anwendung zur Theorie. (= Stauffenburg Linguistik 40). Tübingen.

Fraser, Bruce (1970): Idioms within a transformational grammar. In: Foundations of Language 6, S. 22-42.

Gese, Helga/Stolterfoht, Britta (2010): Processing in one step: the integration of event modifiers. Ms. Tübingen.

Gilquin, Gaëtanelle/Gries, Stefan T. (2009): Corpora and experimental methods: a state-of-the-art review. In: Corpus Linguistics and Linguistic Theory 5,1, S. 1-26.

Goldberg, Adele E. (1995). Constructions: a Construction Grammar approach to argument structure. Chicago u.a.

Goldberg, Adele E. (2006): Constructions at work: the nature of generalization in language. Oxford u.a.

Günthner, Susanne (2006): Grammatische Analysen der kommunikativen Praxis ,Dichte Konstruktionen' in der Interaktion. In: Deppermann, Arnulf/Fiehler, Reinhard/Spranz-Fogasy, Thomas (Hg.): Grammatik und Interaktion - Untersuchungen zum Zusammenhang von grammatischen Strukturen und Gesprächsprozessen. Radolfzell, S. 95-122.

Hale, Kenneth/Keyser, Samuel Jay (1993): On argument structure and the syntactic expression of lexical relations. In: Hale, Kenneth/Keyser, Samuel Jay (Hg.): The view from building 20. Essays in linguistics in honor of Sylvain Bromberger. (= Current Studies in Linguistics Series 24). Cambridge, MA, S. 53-109.

Harley, Heidi/Noyer, Rolf (2000): Formal versus encyclopedic properties of vocabulary: evidence from nominalizations. In: Peeters, Bert (Hg.): The lexicon-encyclopedia interface. (= Current Research in the Semantics - Pragmatic Interface 5). Amsterdam u.a., S. 349-375. 
Hausmann, Franz Josef (1985): Kollokationen im deutschen Wörterbuch: Ein Beitrag zur Theorie des lexikographischen Beispiels. In: Bergenholtz, Henning (Hg.): Lexikographie und Grammatik: Akten des Essener Kolloquiums zur Grammatik im Wörterbuch, 28.-30.06.1984. (= Lexicographica, Series Maior 3). Tübingen, S. $118-129$.

Hockett, Charles F. (1958): A course in modern linguistics. New York u.a.

Höhle, Tilmann N. (1982): Über Komposition und Derivation: Zur Konstituentenstruktur von Wortbildungsprodukten im Deutschen. In: Zeitschrift für Sprachwissenschaft 1, S. 76-112.

Jackendoff, Ray (1975): Morphological and semantic regularities in the lexicon. In: Language 51, S. 639-671.

Jackendoff, Ray (1995): The boundaries of the lexicon. In: Everaert, Martin et al. (Hg.): Idioms: structural and psychological perspectives. Hillsdale u.a., S. 133-165.

Jacobs, Joachim (2008). Wozu Konstruktionen? In: Linguistische Berichte 213, S. 3-44.

Kay, Paul (2000): An informal sketch of a formal architecture for Construction Grammar. In: Grammar 5, 1, S. 1-19.

Kay, Paul (2002): Patterns of coining. Plenarvortrag, gehalten auf der 2nd International Conference on Construction Grammar, Universität Helsinki, Finnland, September 2002.

Kay, Paul/Fillmore, Charles J. (1999): Grammatical constructions and linguistic generalizations: The $W$ hat's $X$ doing $Y$ ? Construction. In: Language 75, S. 1-33.

Kilgarriff, Adam et al. (2004): The Sketch Engine. In: Williams, Geoffrey/Vessier, Sandra (Hg.): Proceedings of the 11th Euralex International Congress, Lorient, France, July 6-10, 2004. Bd. 1. Lorient, S. 105-115.

Knobloch, Clemens (2009): Einladung und Einleitung. In: Zeitschrift für Germanistische Linguistik 37, 3, S. 385-401.

Lakoff, George (1987): Women, fire, and dangerous things: what categories reveal about the mind. Chicago u.a.

Langacker, Ronald W. (1987): Foundations of Cognitive Grammar: theoretical prerequisites. Stanford.

Langacker, Ronald W. (1999): Grammar and conceptualization. (= Cognitive Linguistics Research 14). Berlin u.a.

Langacker, Ronald W. (2000): A dynamic usage-based model. In: Barlow, Michel/Kemmer, Suzanne (Hg.): Usage-based models of language. Stanford, CA, S. 1-60.

Lees, Robert B. (1960): The grammar of English nominalizations. (= Indiana University Research Center in Anthropology Folklore, and Linguistics Publication 12). Bloomington.

Lehmann, Christian (2004): Data in linguistics. In: The Linguistic Review 21, S. 175 210.

Lemnitzer, Lothar/Zinsmeister, Heike (2006): Korpuslinguistik. Eine Einführung. Tübingen. 
Maienborn, Claudia (2003): Die logische Form von Kopulasätzen. (= Studia grammatica 56). Berlin.

Makkai, Adam (1972): Idiom Structure in English. (= Janua Linguarum, Series Maior 48). Den Haag u.a.

McCawley, James D. (1968): Lexical insertion in a transformational grammar without deep structure. In: Darden, Bill J./Bailey, Charles James N./Davison, Alice (Hg.): Papers from the fourth regional meeting of the Chicago Linguistic Society. Chicago, S. 71-80.

Müller, Stefan (2006): Resultativkonstruktionen, Partikelverben und syntaktische vs. lexikonbasierte Konstruktionen. In: Fischer/Stefanowitsch (Hg.), S. 177-202.

Nunberg, Geoffrey/Sag, Ivan A./Wasow, Thomas (1994): Idioms. In: Language 70, 3 , S. 491-538.

Östman, Jan Ola (2005): Construction discourse: a prolegomenon. In: Östman, JanOla/Fried, Mirjam (2005): Construction Grammars: cognitive grounding and theoretical extensions. (= Constructional Approaches to Language 3). Amsterdam u.a., S. 121-144.

Penke, Martina/Rosenbach, Anette (2004): What counts as evidence in linguistics? An introduction. In: Studies in Language 28, 3, S. 480-526.

Rappaport, Malka/Levin, Beth (1988): What to do with $\theta$-roles. In: Wilkins, Wendy (Hg.): Thematic relations. (= Syntacs and Semantics 21). San Diego u.a., S. 7-36.

Selkirk, Elisabeth O. (1982): The syntax of words. (= Linguistic Inquiry: Monographs 7). Cambridge, MA u.a.

Sinclair, John McH. (1987): Collocation: a progress report. In: Steele, Ross/Threadgold, Terry (Hg.): Language topics: essays in honour of Michael Halliday. Bd. II. Amsterdam/Philadelphia, S. 319-331.

Stefanowitsch, Anatol (2006): Negative evidence and the raw frequency fallacy. In: Corpus Linguistics and Linguistic Theory 2, 1, S. 61-77.

Stefanowitsch, Anatol (2009): Bedeutung und Gebrauch in der Konstruktionsgrammatik: Wie kompositionell sind modale Infinitive im Deutschen? In: Zeitschrift für Germanistische Linguistik 37, 3, S. 565-592.

Storrer, Angelika (2007): Corpus-based investigations on German support verb constructions. In: Fellbaum, Christiane (Hg.): Idioms and collocations: corpus-based linguistic and lexicographic studies. London, S. 164-187.

Tomasello, Michael (2003): Constructing a language: a usage-based theory of language acquisition. Cambridge, MA u.a.

Van Pottelberge, Jeroen (2001): Verbnominale Konstruktionen, Funktionsverbgefüge: vom Sinn und Unsinn eines Untersuchungsgegenstands. (= Germanistische Bibliothek 12). Heidelberg.

Williams, Edwin (1994): Remarks on lexical knowledge. In: Lingua 92, S. 7-34.

Wunderlich, Dieter (1997): Cause and the structure of verbs. In: Linguistic Inquiry 28, S. 27-68. 
Konstruktionsidentität und Konstruktionsvarianz 

Hans C. Boas

\title{
Zum Abstraktionsgrad von Resultativkonstruktionen
}

\begin{abstract}
Das in diesem Beitrag vorgeschlagene Netzwerk aus Resultativkonstruktionen unterschiedlichen Abstraktionsgrades vereinigt die Vorteile von Goldbergs (1995, 2006) und Boas' (2003a, 2005a) Analysen. So können sowohl die beim Dekodieren relevanten abstrakt-schematischen Konstruktionsinformationen als auch die beim Kodieren wichtigen lexikalisch-spezifizierten Konstruktionsinformationen über semantische, pragmatische, und syntaktische Restriktionen auf der Ebene von Ereignis-Frames in einem einheitlichen Netzwerk erfasst und analysiert werden. Diese einheitliche Analyse bietet zwei entscheidene Vorteile. Erstens vereinfacht die Beschreibung der Distribution von Konstruktionen auf primär semantisch-pragmatischer Ebene die kontrastive Analyse von gleichartigen Konstruktionen in unterschiedlichen Sprachen. Zweitens erfüllt die hier vorgeschlagene Methode auch die in neueren Veröffentlichungen zu Parallelen der Valenzgrammatik und Konstruktionsgrammatik gemachten Vorschläge, unterschiedliche Abstraktionsgrade von Konstruktionen zu berücksichtigen. Ich danke Hans Ulrich Boas, Marc Pierce, Guido Halder, Martin Hilpert, Wolfgang Imo, Klaus Welke, Gert Webelhuth, Alexander Ziem und den Herausgebern für ihre Kommentare und Anregungen.
\end{abstract}

\section{Einleitung}

Spätestens seit Goldbergs (1995) Monographie „Constructions“ sind grammatische Konstruktionen ein (wieder) etabliertes Konzept in der neueren Sprachwissenschaft. Davon zeugen zahlreiche weitere Monographien und Sammelbände, ${ }^{1}$ die Gegenstand empirischer und theoretischer Debatten sind, sowie eine eigens auf Konstruktionen und semantische Frames ausgerichtete Zeitschrift („Constructions and Frames“) und eine alle zwei Jahre stattfindende internationale Konferenz zur Konstruktionsgrammatik. ${ }^{2}$ Die Ergebnisse konstruktionsgrammatischer Forschung haben nicht nur in die Spracherwerbsforschung (Tomasello 2003; Diessel 2004; Gries/Wulff 2005; Behrens 2009) und die Computerlinguistik (Bergen/Chang 2005; van Trijp 2008; Boas (Hg.) 2009) Eingang gefunden, sondern haben mittlerweile auch andere Theorien beeinflusst. So hat z.B. Ray Jackendoff, einer der prominentesten Vertreter von Chomskys generativer Syntaxtheorie (Chomsky 1981, 1995), das Konzept der Konstruktion als einen wichtigen theoretischen Fortschritt anerkannt, ohne den sich Sprachen nicht vollständig be-

\footnotetext{
1 Vgl. Croft (2001), Boas (2003a), Östman/Fried (Hg.) (2005), Fried/Boas (Hg.) (2005), Goldberg (2006), Imo (2007), Barðdal (2008), Bergs/Diewald (Hg.) (2008), Hilpert (2008), Iwata (2008), Stefanowitsch/Fischer (Hg.) (2008) u.v.a.

2 Siehe www.constructiongrammar.org (Stand: 25.10.2010).
} 
schreiben und analysieren lassen (Jackendoff 1997, 2010). Auch die „HeadDriven Phrase Structure Grammar" (Pollard/Sag 1994) hat das theoretische Konzept der Konstruktion anerkannt und entwickelt sich hin zu einer konstruktionsbasierten Phrasenstrukturgrammatik, der so genannten ,Signbased Construction Grammar" (siehe Sag 2010; Boas/Sag (Hg.) i.Dr.). ${ }^{3}$

Eine der wichtigsten Thesen konstruktionsgrammatischer Forschung ist, dass es keine klare Trennung zwischen universalgrammatischer Kerngrammatik und Peripherie gibt. So umfasst der relativ flexible Konstruktionsbegriff alle Arten linguistischer Zeichen (d.h. konventionalisierte Paare aus Form- und Bedeutungskomponenten), unabhängig davon wie abstraktschematisch oder konkret diese sind. Dies bedeutet auch, dass es im Prinzip - anders als in anderen gängigen Grammatikmodellen - keine klare Trennung von Grammatik und Lexikon gibt, da derselbe Formalismus zur Beschreibung von Konstruktionen unterschiedlicher Abstraktionsgrade bzw. Komplexitätsstufen benutzt werden kann (vgl. Kay/Fillmore 1999; Boas 2008a, 2008b; Iwata 2008; Sag 2010; Fillmore/Lee-Goldman/Rhodes i.Dr.). Diese Paarung von Form und Bedeutung/Funktion ist in Abbildung 1 dargestellt.

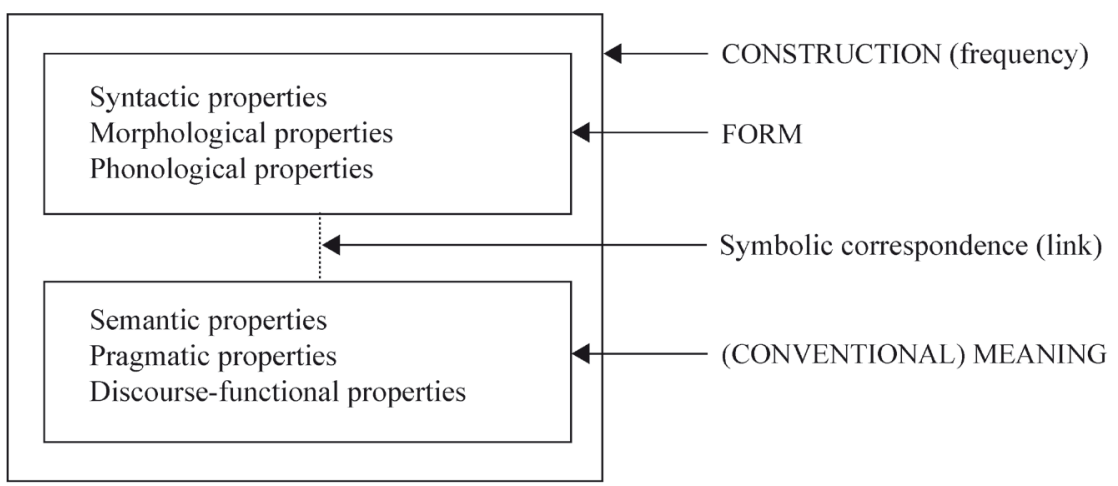

Abb. 1: Beziehung zwischen Form und Bedeutung in der Konstruktionsgrammatik (Croft 2001, S. 18)

3 Die Konstruktionsgrammatik wurde in den 1980er Jahren ursprünglich nur für die Beschreibung und Analyse des Englischen entwickelt. Ziel war zunächst eine beschreibungsadäquate Theorie einer einzelnen Sprache zu entwickeln, wie Kay/Fillmore (1999, S. 1) klarstellen: „To adopt a constructional approach is to undertake a commitment in principle to account for the entirety of each language. This means that the relatively general patterns of the language, such as the one licensing the ordering of a finite auxiliary verb before its subject in English, often known as SAI, and the highly idiomatic patterns, like kick the bucket, stand on an equal footing as data for which the grammar must account. An explicit grammar that covers the full range of constructions must represent all constructions, of whatever degree of generality or idiomaticity, in a common notation and must provide an explicit account of how each sentence of a language is licensed by a subset of the leaves of the inheritance hierarchy of constructions which constitutes the grammar of that language." Seit den 1990er Jahren sind die Ideen der Konstruktionsgrammatik auch auf andere Sprachen angewandt worden. Dabei lassen sich einzelsprachliche Studien wie z.B. Fillmore/Kay/O'Connor (1988), Lam- 
Aus dieser einheitlichen Sicht von Sprache ergibt sich, dass es Konstruktionen mit unterschiedlichem Abstraktionsgrad gibt. Der Status von abstrakten Konstruktionen in der Konstruktionsgrammatik ist aber umstritten. Goldberg $(1995,2006)$ postuliert abstrakt-schematische Konstruktionen wie die Resultativkonstruktion in Verbindung mit Lexikoneinträgen von Verben, die Resultativsätze wie z.B. Fritz, hustet den Schaum vom Bier lizenzieren sollen. ${ }^{4}$ Im Gegensatz dazu wird in Boas (2003a, 2005a) die Ansicht vertreten, dass Resultativkonstruktionen nicht immer ausreichend restringiert werden können, was zur Übergenerierung führen kann. So können von der Konstruktion Sätze lizenziert werden, die im tatsächlichen Sprachgebrauch nicht vorkommen oder nicht zulässig sind, wie z.B. Fritz inhaliert das Buch vom Regal. Nach der in Boas (2003a, 2005a) vertretenen Auffassung können die so entstehenden Probleme nur dadurch gelöst werden, dass lexikalisch spezifierte Resultativkonstruktionen auf konkret-komplexeren Ebenen postuliert werden, um so Übergenerierungen zu verhindern.

Ziel dieses Beitrags ist es zu zeigen, dass sich die beiden Analysen nicht grundsätzlich gegenseitig ausschließen, sondern dass sie sich innerhalb eines Konstruktionsnetzwerks wie bei Langacker (2000) ergänzen. Die Kombination der beiden Analysen hat zur Folge, dass je nach Konstruktion unterschiedliche Arten von konkret-spezifizierten Informationen gelistet und in einem Konstruktionsnetzwerk bzw. einer Konstruktionshierarchie festgehalten werden müssen, die auch z.T. redundante Informationen enthalten. Die so vorgeschlagene Arbeitsteilung zwischen abstrakt-schematischer Konstruktion und lexikalisch-spezifizierten Konstruktionen verbindet die Vorteile von Goldbergs und Boas' Vorschlägen.

Der Beitrag ist wie folgt gegliedert: Zunächst erläutere ich Goldbergs (1995, 2006) Analyse der (englischen) Resultativkonstruktion. Dann zeige ich, dass Goldbergs Analyse eine implizite Trennung zwischen Lexikon und Grammatik annimmt, die zur Folge hat, dass die Beschränkungen, welche die Fusionierung von abstrakten Konstruktionen mit konkreten Lexikoneinträgen von Verben regulieren sollen, nicht immer funktionieren (vgl. Boas 2003b, 2005a). Auf der Basis von Kollokationsanalysen und Frequenzdaten argumentiere ich, dass Konstruktionen auf unterschiedlichen Abstraktionsebenen existieren, die in einer Konstruktionshierarchie miteinander verbunden sind (vgl. Boas 2003a). Außerdem zeige ich, inwieweit

brecht/Lemoine (2005) und Barðdal (2008) von konstrastiven Analysen wie etwa Boas (2003a, 2010b), Hilpert (2008), Iwata (2008) unterscheiden. Zur Kritik einer sprachübergreifenden bzw. universellen Konstruktionsgrammatik siehe Croft (2001).

4 Die Lizenzierung erfolgt natürlich auch durch „Coercion“, d.h. durch einen entsprechenden Interpretationsprozess. Goldberg (1995) unterscheidet zwischen so genannten Caused-Motion-Konstruktionen (z.B. Fritz, bustet den Schaum vom Bier) und so genannten Resultativkonstruktionen (z.B. Ruth läuft sich die Füße wund). Im Gegensatz dazu folge ich Boas (2003a), der beide Sorten von Konstruktionen als Resultativkonstruktion analysiert, da sie eine parallelinterpretierbare Semantik haben, d.h., sie bezeichnen beide einen Endzustand des postverbalen Objekts. 
lexikalische Einträge von Verben valenz-relevante Informationen enthalten, die über die Lizenzierung von postverbalen Nominal- und Resultativphrasen Auskunft geben können. Ich schließe mit Überlegungen, die zeigen sollen, wie Konstruktionen von unterschiedlichem Abstraktionsgrad mit einer einheitlichen Notation erfasst werden können. Der Großteil der in diesem Beitrag diskutierten Daten stammt aus dem Englischen, weil Goldberg (1995) auf englischen Daten beruht. Um eine erweiterte konstrastive Perspektive zu bieten, bespreche ich aber auch deutsche Resultativkonstruktionen, die zeigen, inwieweit sich die auf dem Englischen basierenden Methoden auf das Deutsche übertragen lassen.

\section{Der Status abstrakter Konstruktionen}

\subsection{Der Konstruktionsbegriff in Goldbergs Theorie}

Anders als der ursprüngliche konstruktionsgrammatische Ansatz von Fillmore und Kay (Fillmore 1988; Fillmore/Kay/O'Connor 1988; Fillmore/Kay 1995; Kay/Fillmore 1999), der Konstruktionen eigentlich nur dann postuliert, wenn diese zumindest partiell unanalysiert sind, schlägt Goldberg (1995, 2006) auch solche Einheiten als Konstruktionen vor, die sowohl völlig transparent als auch partiell oder vollständig unanalysierte, nicht-kompositionelle Einheiten sind: „It's constructions all the way down“" (Goldberg 2006, S. 18). Nach dieser Auffassung sind alle Form-Bedeutungs-Paarungen als unterschiedliche Konstruktionen anzusehen, wenn sich deren Existenz nicht aus schon vorher existierenden Konstruktionen erklären lässt:

According to Construction Grammar, a distinct construction is defined to exist if one or more of its properties are not strictly predictable from knowledge of other constructions existing in the grammar: $\mathrm{C}$ is a construction iff $_{\text {def }} \mathrm{C}$ is a form-meaning pair $\left.<\mathrm{F}_{\mathrm{i}}, \mathrm{S}_{\mathrm{i}}\right\rangle$ such that some aspect of $\mathrm{F}_{\mathrm{i}}$ or some aspect of $\mathrm{S}_{\mathrm{i}}$ is not strictly predictable from C's component parts or from other previously established constructions. (Goldberg 1995, S. 4)

Any linguistic pattern is recognized as a construction as long as some aspect of its form or function is not strictly predictable from its component parts or from other constructions recognized to exist. In addition, patterns are stored as constructions even if they are fully predictable as long as they occur with sufficient frequency. (Goldberg 2006, S. 5) ${ }^{5}$

Diese Ansicht hat zur Folge, dass Konstruktionen im Prinzip das gesamte Spektrum des Syntax-Lexikon-Kontinuums abdecken, von formal vollständig kompositionellen und semantisch transparenten Strukturen bis hin zu vollständig lexikalisierten und formal fixierten Redewendungen, wie in Tabelle 1 dargestellt.

Zu Alternativdefinitionen von Konstruktionen siehe z.B. Croft (2001, S. 17-21) und Fried/ Östman (Hg.) (2004, S. 18-23). 


\begin{tabular}{|l|l|}
\hline Morpheme & e.g. pre-, -ing \\
\hline Word & e.g. avocado, anaconda, and \\
\hline Complex word & e.g. daredevil, shoo-in \\
\hline Complex word (partially filled) & e.g. [N-s] (for regular plurals) \\
\hline Idiom (filled) & e.g. going great guns, give the Devil his due \\
\hline Idiom (partially filled) & $\begin{array}{l}\text { e.g. jog < }<\text { someone's }>\text { memory, send } \\
\text { <someone }>\text { to the cleaners }\end{array}$ \\
\hline Covariational Conditional & $\begin{array}{l}\text { The Xer the Yer (e.g. the more you think } \\
\text { about it, the less you understand) }\end{array}$ \\
\hline Ditransitive (double object) & $\begin{array}{l}\text { Subj } \mathrm{V} \mathrm{Obj}, \mathrm{Obj}_{2} \text { (e.g. he gave her a fish } \\
\text { taco; he baked her a muffin) }\end{array}$ \\
\hline Passive & $\begin{array}{l}\text { Subj aux } \mathrm{VP}_{\mathrm{PP}}\left(\mathrm{PP}_{\mathrm{by}}\right) \text { (e.g. the armadillo } \\
\text { was hit by a car) }\end{array}$ \\
\hline
\end{tabular}

Tab. 1: Beispiele von Konstruktionen, die sich in Umfang und Komplexität unterscheiden (nach Goldberg 2006, S. 5)

Außer solchen Konstruktionen wie in Tabelle 1 gibt es laut Goldberg auch noch so genannte abstrakt-schematische Konstruktionen, die unabhängig existieren und eine eigene Bedeutung haben. Solche Konstruktionen verbinden ebenfalls eine bestimmte Form mit einer bestimmten Bedeutung/ Funktion, und sie können unter gegebenen Umständen Verben semantische Argumente beifügen, die sie sonst alleine nicht hätten, wie in Beispiel (1).

(1) a. He talked himself blue in the face. (Goldberg 1995, S. 189)

b. Er redete sich dumm und dusselig.

Goldberg behauptet, dass das Verb talk alleine nicht die ganze Bedeutung mitbringt, die es in (1a) zum Ausdruck bringt. Intransitive Verben wie talk haben laut Goldberg keine eigenständige Bedeutung, die den Endzustand von Personen beschreiben, die viel reden. Um dieses Problem zu lösen, postuliert sie Lexikoneinträge, welche die Grundbedeutung von Verben repräsentieren, und zwar im Format der Frame-Semantik von Fillmore (1985): „Lexical entries should make reference to world and cultural knowledge" (Goldberg 1995, S. 28). Der Lexikoneintrag des Verbs talk in (2) verfügt in Goldbergs Ansatz über eine so genannte Partizipantenrolle (den TALKER), die stellvertretend für die Beschreibung der Situation eines Sprechgeschehens steht.

(2) talk $<$ talker $>$

(Goldberg 1995, S. 189)

Die von Goldberg (2006, S. 5) gelisteten Konstruktionen sind entweder Morpheme, einzelne Wörter oder aber abstraktere morpho-syntaktische Konstruktionen, die partiell mit lexikalischen Einheiten gefüllt sind. Darüber hinaus gibt es aber auch konstruktionsgrammatische Analysen, die sich mit anderen Aspekten der Sprache beschäftigen, vgl. z.B. Boas (2004) zur Interaktion von Syntax, Morphologie und Phonologie und Östman (2005), Deppermann (2007) und Günthner (2008) zur Diskurs- bzw. Konversationsanalyse. 


\subsection{Fusionierung abstrakter Konstruktionen mit Verben}

Um die Lizenzierung von Sätzen wie in (1a) zu erklären, behauptet Goldberg (1995, S. 180-198), dass Verben wie talk in (2) mit der unabhängig existierenden abstrakt-schematischen „Resultative Construction“ fusionieren können, die eine bestimmte Bedeutung (X CAUSES Y TO BECOME Z) mit einer bestimmten Form (SUBJ, OBJ, OBL) paart. Die Variablen X, Y und Z in der Bedeutungsbeschreibung der Konstruktion sind als semantische Argumentrollen zu verstehen, d.h. X als AGENT (derjenige, der etwas tut), $\mathrm{Y}$ als PATIENT (derjenige, mit dem etwas gemacht wird) und $\mathrm{Z}$ als das RESULT-GOAL (der Endzustand, den der PATIENT nach der vom Verb bezeichneten Aktivität erreicht). Diese Bedeutungen sind, wie in Abbildung 2 dargestellt, mit bestimmten syntaktischen Relationen, nämlich Subjekt, Objekt und Oblique gepaart.

\begin{tabular}{|c|c|c|c|c|}
\hline Sem & $\begin{array}{l}\text { CAUSE-BECOME } \\
\mid\end{array}$ & $<$ agt & $\begin{array}{c}\text { pat } \\
1 \\
1\end{array}$ & $\begin{array}{c}\text { result-goal> } \\
\mid\end{array}$ \\
\hline $\begin{array}{l}\text { R: instance, } \\
\text { means }\end{array}$ & PRED & $<$ & $\downarrow$ & $\downarrow$ \\
\hline Syn & V & SUBJ & OBJ & $\mathrm{OBL}_{\mathrm{AP} / \mathrm{PP}}$ \\
\hline
\end{tabular}

Abb. 2: Resultativkonstruktion (vgl. Goldberg 1995, S. 189)

Die oberste Zeile in Abbildung 2 repräsentiert die Semantik (,Sem“) der abstrakt-schematischen Resultativkonstruktion, die eine Verursachungsbeziehung (CAUSE-BECOME) zwischen den Argumentrollen AGENT und PATIENT der Konstruktion beschreibt. Der daraus hervorgehende Resultatszustand wird durch die Argumentrolle RESULT-GOAL dargestellt. Die von oben nach unten führenden Pfeile deuten an, dass die Semantik der Konstruktion mit einer bestimmten syntaktischen Form gepaart ist, die in der untersten Zeile unter „Syn“ als Subjekt, Objekt und Oblique aufgeführt ist. Der vielleicht wichtigste Aspekt der Konstruktion ist die Zeile in der Mitte (R: instance, means), in die das Verb (PRED) unter bestimmten Umständen eingefügt werden kann. Wenn ein Verb mit einer Konstruktion fusioniert, dann geschieht das laut Goldberg dadurch, dass die Partizipantenrollen des Lexikoneintrags des Verbs in die mittlere Zeile (PRED) der Konstruktion eingefügt werden. Dieser Vorgang ist jedoch nur möglich, wenn die Partizipantenrolle(n) des Verbs mit der/den Argumentrolle(n) der Konstruktion semantisch kompatibel sind.

Wenn die Rollen des Verbs und der Konstruktion auf diese Weise abgeglichen werden können, dann findet die Fusionierung des Verbs mit der Konstruktion statt. So kann z.B. die Partizipantenrolle TALKER des Verbs 
talk in (2) in die Resultativkonstruktion in Abbildung 2 eingefügt werden, weil diese als eine spezifischere Realisierung der verallgemeinerten Argumentrolle AGENT der Konstruktion interpretiert werden kann und als solche kompatibel mit ihr ist. Somit fusionieren laut Goldberg auch gleichzeitig die Bedeutungen des Verbs und der Konstruktion. ${ }^{7}$ Immer wenn solch eine Fusionierung von Verb und Konstruktion stattfindet, hat die Konstruktion gleichzeitig die Möglichkeit, zusätzliche Argumentrollen beizusteuern. Im Falle von talk kann die Resultativkonstruktion zwei weitere semantische Argumentrollen beisteuern, den PATIENT und das RESULTGOAL. Diese werden dann, genauso wie die AGENT-Rolle, durch die Konstruktion syntaktisch realisiert. Auf diese Art und Weise erklärt Goldberg nicht nur die Lizenzierung der vom Verb nicht-subkategorisierten Konstituenten in Resultativkonstruktionen, sondern auch analoge Phänomene in einer Reihe von anderen Konstruktionen wie der „Ditransitive Construction“ und der „Way Construction“. Analoge Vorschläge lassen sich auch zur Lizenzierung von Resultativkonstruktionen im Deutschen wie in (1b) machen (siehe Boas 2002a).

Einer der Vorteile von Goldbergs Analyse liegt darin, dass Verben nur eine prototypische Grundbedeutung haben, weil abstrakte Konstruktionen den Verben durch Fusionierung zusätzliche Argumentstellen beisteuern können. Dies ermöglicht eine systematische Generalisierung auf Konstruktionsebene, wodurch gleichzeitig verhindert werden kann, dass alle syntaktischen Kontexte, in denen ein Verb vorkommen kann, in dessen Lexikoneintrag spezifiziert werden müssen (vgl. Goldberg 1995, S. 224). Ein weiterer Vorteil ist laut Goldberg, dass Konstruktionen unabhängig von der Bedeutung einzelner Verben als Form-Bedeutungs-Paare abstrahierbar sind. Dies wiederum erlaubt eine Reduzierung der Länge und Detailgenauigkeit von Lexikoneinträgen, was wiederum zu minimaleren Lexikoneinträgen führt (vgl. Goldberg 1995, S. 28-30).

\subsection{Probleme mit Goldbergs abstrakt-schematischen Konstruktionen}

Goldbergs abstrakt-schematische Konstruktionen sind zwar in der Lage, mit Verben zu fusionieren und ihnen semantische Argumentrollen beizusteuern, aber dieser Prozess kann nicht immer korrekt restringiert werden, da es nicht immer möglich ist, genaue Kriterien festzulegen, die helfen können zu entscheiden, warum ein bestimmtes Verb mit einer Konstruktion fu-

Die Fusionierung von Lexikoneinträgen mit der Resultativkonstruktion unterliegt einer Reihe von semantischen Restriktionen, vgl. Goldberg (1995, S. 193-197), sowie generellen Restriktionen wie dem „Semantic Coherence Principle“ und dem „Correspondence Principle“ (Goldberg 1995, S. 50). Diese Restriktionen sollen die Übergenerierung von nichtattestierten Sätzen verhindern, ähnlich wie so genannte Filter in der generativen Grammatik Chomskys (siehe Boas 2008a). 
sioniert, aber ein anderes bedeutungsverwandtes Verb nicht (siehe u.a. Kay 1996, 2005; Nemoto 1998, 2005; Boas 2002b, 2003a, 2007, 2008b). Dies hat u.a. mit der Struktur der Lexikoneinträge bei Goldberg zu tun, welche nicht immer detailgenau die vielen unterschiedlichen Aspekte von Wortbedeutungen mit frame-semantischen Beschreibungen darstellen. So lässt sich z.B. anhand der Lexikoneinträge einiger bedeutungsverwandter Kommunikationsverben wie in (3a)-(3f) nicht genau feststellen, welche Bedeutungsaspekte eines Verbs für die Fusionierung mit einer Konstruktion relevant sind, oder welche einer Fusionierung gar im Wege stehen könnten. Parallele Beobachtungen lassen sich für das Deutsche machen.
a. talk $<$ talker $>$
b. speak $<$ speaker $>$
c. whisper $<$ whisperer $>$
d. grumble $<$ grumbler $>$
e. murmur $<$ murmurer $>$
f. $\operatorname{sigh}<$ sigher $>$

(vgl. Boas 2003a, S. 106)

Obwohl die Lexikoneinträge in (3a)-(3f) in der Lage sind mit einigen Konstruktionen wie der Hauptsatzkonstruktion zu fusionieren (vgl. Miriam \{talked/spoke/whispered/grumbled/murmered/sighed\} (to Joe)), können nicht alle mit anderen Konstruktionen fusionieren. So zeigt z.B. Boas (2003a), dass das Verb talk in (4a) mit der Resultativkonstruktion fusionieren kann, aber nicht andere bedeutungsverwandte Kommunikationsverben wie speak, whisper, grumble, murmur und sigh in (4b)-(4f). Mit den von Goldberg postulierten allgemeinen semantischen Restriktionen, welche die Fusionierung restringieren sollen (siehe Fußnote 7 ), lassen sich unakzeptable Beispiele wie in (4b)-(4f) nicht systematisch ausschließen. Im Deutschen verhält es sich ähnlich (vgl. Boas 2003a, S. 287-289). ${ }^{8}$

(4) a. Miriam talked herself blue in the face.

b. *Miriam spoke herself blue in the face.

c. ?Miriam whispered herself blue in the face.

d. *Miriam grumbled herself blue in the face.

e. *Miriam murmured herself blue in the face.

f. ?Miriam sighed herself blue in the face. (vgl. Boas 2003a, S. 105)

Weitere Schwierigkeiten ergeben sich, wenn es darum geht, die semantische Bandbreite der von der Konstruktion beigesteuerten Argumentrollen zu bestimmen. So zeigt Boas (2003a, 2008a), dass bedeutungsverwandte

Boas (2008b) untersucht auch die Distribution der Verben in (4a)-(4f) in der „Ditransitive Construction“ und der so genannten „Way Construction“. Es wird gezeigt, dass die Distribution dieser sechs bedeutungsverwandten Verben sich auch bzgl. dieser beiden Konstruktionen stark unterscheidet, d.h., einige Verben können mit den Konstruktionen fusionieren und andere nicht. 
Verben wie eat (,essen'), chew (,kauen'), devour (,verschlingen') und swallow (,(ver)schlucken) nicht alle dieselben Kategorien von postverbalen Konstituenten zulassen. Wenn man z.B. Goldberg folgend für eat einen Lexikoneintrag wie in (5) annimmt und dieser Eintrag mit einer Reihe von unterschiedlichen Konstruktionen fusionieren kann, um so unterschiedliche Sätze wie in (6a)-(6d) zu lizenzieren, dann könnte man auch erwarten, dass die bedeutungsverwandten Verben in (7)-(9) mit denselben Konstruktionen fusionieren können, was jedoch nicht der Fall ist.

eat: $<$ eater eaten $>$ essen: $<$ Esser Gegessenes $>$

a'. Pat aß.

b'. Pat aß sein Essen.

c'. Pat aß sein Essen auf.

d'. Pat aß seinen Teller leer.

a'. Pat kaute.

b'. Pat kaute sein Essen.

c'. ?Pat kaute sein Essen auf.

d'. *Pat kaute seinen Teller leer.

a'. *Pat verschlang.

b'. Pat verschlang sein Essen.

c'. *Pat verschlang sein Essen auf.

d'. *Pat verschlang seinen Teller leer.

a'. Pat schluckte.

b'. Pat schluckte sein Essen.

c'. Pat verschluckte sein Essen.

d'. *Pat schluckte/verschluckte seinen Teller leer.

Parallele Beobachtungen lassen sich für das Deutsche machen. Die deutschen Parallelbeispiele belegen einen weiteren wichtigen Punkt, nämlich dass die Resultativphrasen zu einem gewissen Grad konventionalisiert sind und nicht anhand von generellen Prinzipien erfasst werden können. So ist es z.B. im Deutschen normalerweise nicht möglich, die Resultativphrase sauber zusammen mit essen zu verwenden, vgl. (6d'). Goldbergs Fusionierung von Verben und abstrakten Konstruktionen ist auch problematisch, wenn es um die semantisch-pragmatische Restringierung von postverbalen Konstituenten geht. So zeigt z.B. Boas (2003a), dass Verben sehr spezielle Anforderungen bzgl. der Resultativphrasen stellen, wie in den Tabellen 2 und 3 dargestellt. 


\begin{tabular}{|c|c|}
\hline Verb & No. of occur. \\
\hline shoot & 408 \\
\hline kill & 9 \\
\hline strike & 8 \\
\hline make, knock & 3 \\
\hline flatten, kick, smite & 1 \\
\hline
\end{tabular}

Tab. 2: Distribution von Verben mit dead in Resultativkonstruktionen im British National Corpus (BNC) (Boas 2003a, S. 130)

\begin{tabular}{|c|c|}
\hline Verb & No. of occur. \\
\hline stab & 114 \\
\hline beat & 74 \\
\hline batter & 39 \\
\hline frighten & 34 \\
\hline crush & 25 \\
\hline scare & 24 \\
\hline burn & 18 \\
\hline torture & 16 \\
\hline drink, starve & 15 \\
\hline bludgeon, hack & 12 \\
\hline shoot, kick & 11 \\
\hline club & 9 \\
\hline bore, knife, choke & 8 \\
\hline blast, trample, work, worry & 7 \\
\hline love & 6 \\
\hline strangle & 4 \\
\hline dash, poison, kiss & 3 \\
\hline $\begin{array}{l}\text { axe, bayonet, boil, bring, clap, suffocate, kick, } \\
\text { freeze, spear, spray, stone, suck, gun, hammer, } \\
\text { hug, knock, nag, peck, play, rape, shag, sting }\end{array}$ & 2 \\
\hline $\begin{array}{l}\text { annoy, eat, bleed, blend, bug, bully, flog, frit, } \\
\text { cudgel, curse, dance, feed, gas, flog, jog, } \\
\text { laugh, pitchfork, pound, run, schmaltz, scorch, } \\
\text { scratch, seduce, shock, sing, smother, squash, } \\
\text { squeeze, stamp, strike, suffocate, sweat, whip }\end{array}$ & 1 \\
\hline
\end{tabular}

Tab. 3: Distribution von Verben mit to death in Resultativkonstruktionen im BNC (Boas 2003a, S. 131) 
Die Tabellen 2 und 3 sind Teilergebnisse aus der umfangreichen korpusbasierten Studie von Resultativkonstruktionen in Boas (2003a). Um festzustellen, welche Arten von Resultativphrasen mit bestimmten Verben vorkommen können, habe ich die Kollokationen von fünfzig Resultativphrasen (insbesondere Adjektiv- und Präpositionalphrasen) und der damit erscheinenden Verben im British National Corpus untersucht (vgl. Boas 2003a, S. 11-18). Daten wie in Tabelle 2 und 3 belegen, dass Goldbergs abstraktschematische Resultativkonstruktion nie in der Lage ist, systematisch zu bestimmen, welche Arten von Resultativphrasen mit welchen Verben vorkommen können. Dies ist besonders offensichtlich bei Resultativphrasen, die bedeutungsverwandt sind, wie z.B. die Adjektivphrase dead (,tot') und die Präpositionalphrase to death (,zu Tode). Dies lässt sich aber auch für den Rest der von mir untersuchten Resultativphrasen feststellen. Die Datenlage im Deutschen ist sehr ähnlich (siehe Boas 2003a, S. 285-312). Aus diesen Beobachtungen schließe ich, dass abstrakte Resultativkonstruktionen, wenn sie mit Lexikoneinträgen von Verben fusionieren, nicht in der Lage sind, die relativ idiosynkratische Distribution von Resultativphrasen adäquat zu restringieren.

Zusammenfassend lässt sich bzgl. der Interaktion von abstrakt-schematischen Konstruktionen und Lexikoneinträgen Folgendes feststellen: (1) Goldbergs Ansatz ermöglicht es nicht, systematisch zu erklären, warum Resultativkonstruktionen mit einem bestimmten Verb fusionieren können, aber nicht mit einem bedeutungsverwandten Verb. Der Prozess der Fusionierung kann so zur Übergenerierung führen. (2) Der Prozess der Fusionierung von Verben und Konstruktionen ist nicht in der Lage, die semantische, pragmatische und syntaktische Bandbreite von Resultativphrasen zu restringieren, da die dazu nötigen Informationen und Mechanismen fehlen. Diese Schwierigkeiten treffen nicht nur auf Resultativkonstruktionen zu, sondern lassen sich auch bei anderen abstrakten Konstruktionen nachweisen, wie z.B. der „Caused-motion Construction“ (vgl. Boas 2002b, 2003b, 2006; Iwata 2005; Kay 2005), der „Ditransitive Construction“ (Nemoto 1998; Kay 2005; Boas 2010a), und der der Lokativalternation unterliegenden Konstruktionen (Michaelis/Ruppenhofer 2001; Boas 2006, 2009, i.Dr.; Nemoto 2005; Iwata 2008).

\section{Lexikalisch-spezifizierte Minikonstruktionen}

Um die oben dargestellten Probleme zu lösen, habe ich in einer Reihe von Publikationen vorgeschlagen, die Lexikoneinträge von Verben genauer zu spezifizieren, um so die Lizenzierung von Resultativkonstruktionen (und anderen nur partiell produktiven Konstruktionen) adäquat zu erfassen (vgl. Boas 2003a, 2005a, 2007, 2008a, 2009). Dieser Alternativansatz sieht eine 
viel bedeutendere Rolle der Frame-Semantik (Fillmore 1985) vor, in dem größerer Wert auf eine genauere Analyse einzelner Verbbedeutungen gelegt wird.

Die Frame-Semantik beruht auf der Idee, dass Wortbedeutungen (fast) immer Bezug auf strukturierte semantische Konzepte, Erfahrungen, Erinnerungen und Weltwissen nehmen - so genannte semantische Frames -, die für die korrekte Benutzung von Wörtern unabdingbar sind (vgl. Petruck 1996; Fillmore 2007; Ziem 2008). Fillmore/Atkins (1992, S. 76-77) beschreiben die zentrale Rolle von Frames wie folgt.

A word's meaning can be understood only with reference to a structured background of experience, beliefs, or practices, constituting a kind of conceptual prerequisite for understanding the meaning. Speakers can be said to know the meaning of the word only by first understanding the background frames that motivate the concept that the word encodes. Within such an approach, words or word senses are not related to each other directly, word to word, but only by way of their links to common background frames and indications of the manner in which their meanings highlight particular elements of such frames.

Als Beispiel sei hier das englische Verb talk (,reden', sprechen') genannt, welches in einer seiner Bedeutungen den so genannten Statement Frame evoziert. ${ }^{9}$ In FrameNet, einer lexikografischen Onlinedatenbank des Englischen, welche die theoretischen Prinzipien der Frame-Semantik in die Praxis umsetzt (vgl. Fillmore/Johnson/Petruck 2003; Boas 2005b; Ruppenhofer et al. 2006; Fillmore/Baker 2010) ${ }^{10}$ beschreibt der Statement Frame Szenarien mit unterschiedlichen voneinander abhängigen so genannten Frame Elements, die spezifischere Realisierungen abstrakterer semantischer Rollen wie AGENT, UNDERGOER, INSTRUMENT etc. darstellen. Die Definition des Statement Frames besteht aus den Definitionen der einzelnen Frame Elements sowie ihrer Beziehungen zueinander. Dieser Frame beschreibt Situationen, in denen ein SPEAKER (der Sprecher) einen ADDRESSEE (den Adressaten) mit einer MESSAGE (die Nachricht) anspricht, die auch indirekt durch ein TOPIC (das Thema) ausgedrückt weden kann. Der ADDRESSEE ist diejenige Person, die die Nachricht vom Sprecher erhält. Die MESSAGE ist das vom Sprecher Gesagte. Der SPEAKER ist die wahrnehmende Person, welche die Nachricht produziert, und das Frame Element TOPIC beschreibt generell den Inhalt der Nachricht (vgl. Ruppenhofer et al. 2006).

$9 \quad$ Die Frame-Semantik vertritt ein feinkörniges Konzept der Polysemie, d.h., Wörter werden entsprechend ihrer Unterbedeutungen beschrieben und analysiert. So aktiviert das Verb talk nicht nur den Statement Frame, sondern auch den Chatting und Talking_into Frame. Obwohl alle drei Frames miteinander in Beziehung stehen (alle beinhalten z.B. einen SPEAKER als Frame Element), sind sie doch unterschiedlich und werden daher auch von unterschiedlichen „Lexical Units“ (Wortbedeutungen) evoziert (vgl. Boas 2005b, Boas 2010a).

10 Siehe http://framenet.icsi.berkeley.edu/ (Stand: 25.10.2010). 
Der Statement Frame wird nicht nur vom Verb talk evoziert, sondern auch von anderen bedeutungsverwandten Verben wie z.B. claim oder contend, sowie von Substantiven wie z.B. declaration und statement. Die frame-semantische Beschreibung von Wortbedeutungen hat eine Reihe von Vorteilen. Erstens erlaubt sie eine systematische Analyse der zum Verstehen eines Wortes notwendigen Bedeutungsstrukturen und der dazugehörigen Konzepte, die auch für eine adäquate Verwendung von Wörtern notwendig sind. Zweitens ermöglicht sie es, bedeutungsverwandte Wörter mit demselben Beschreibungsinventar zu analysieren, auch über Wortartengrenzen hinweg. Drittens schafft eine solch feingegliederte Strukturierung des Lexikons auch die Grundlage, die Beziehungen zwischen der Bedeutung eines Wortes und ihrer unterschiedlichen syntaktischen Realisierung systematisch zu erfassen. So enthält z.B. der FrameNet-Eintrag des Verbs talk nicht nur Informationen über die den Statement Frame konstituierenden Frame Elements, sondern auch eine ausführliche auf Korpusdaten basierende Auflistung der syntaktischen Realisierungen der Frame Elements (siehe Fillmore 2007, 2009). ${ }^{11}$ Der Hauptgedanke dieser Art der frame-semantischen Strukturierung des Lexikons ist es, Wörter so genau wie möglich nach unterschiedlichen Bedeutungsstrukturen und Konzepten zu beschreiben und darzustellen, wie die Bedeutungen einzelner Wörter syntaktisch unterschiedlich realisiert werden können.

Wenn man nun die von Fillmore konzipierte frame-semantische Strukturierung des Lexikons mit der von Goldberg $(1995,2006)$ vergleicht, so fallen ein paar wichtige Unterschiede auf. Erstens folgt Goldberg nicht genau der detaillierten Beschreibungsmethode von Fillmore bzgl. der Struktur und Detailgenauigkeit von Frames. Zwar behauptet sie, dass lexikalische Einträge auf Weltwissen zugreifen („lexical entries should make reference to world and cultural knowledge", Goldberg 1995, S. 28), aber die Umsetzung dieses Gedankens scheint nicht sehr gelungen, wie der Lexikoneintrag des Verbs talk in (2) zeigt. Goldberg räumt zwar ein, dass es schwierig ist frame-semantisches Wissen systematisch zu beschreiben, aber ihre eigenen Lexikoneinträge gehen auch nicht über die Strukturen und Inhalte wie in (2) hinaus. Eine Interpretation der Informationen in (2) mag zwar Auskunft darüber geben, dass es eine Person gibt, die spricht (die Partizipantenrolle TALKER), aber sonst wird nichts weiter über die Situation, andere Beteiligte, oder die begleitenden Umstände gesagt. Im Vergleich zu Fillmores

$11 \quad$ Ein weiterer Vorteil frame-semantischer Analysen ist die Tatsache, dass sich die für das Englische entwickelten Frames auch weitgehend auf die Analyse von anderen Sprachen übertragen lassen. Diese Methode erlaubt konstrastive Studien auf semantischer Ebene (d.h. der Frame-Ebene), wobei die syntaktischen Realisierungen der Frame Elements als die wirklich idiosynkratischen Eigenschaften einzelner Sprachen betrachtet werden können. Siehe z.B. die in Boas (Hg.) (2009) enthaltenen Beiträge über FrameNets für Deutsch, Japanisch, Spanisch, Hebräisch und Französisch. 
etablierter Framekonzeption lässt sich hier also eine stark reduzierte Konzeption der Frame-Semantik feststellen, die sonst keine weiteren Auskünfte gibt und offen für sehr unterschiedliche Interpretationen sein mag (siehe auch Boas 2003a, 2007, 2008b; Iwata 2008).

Zweitens erscheint Goldbergs Einbeziehung der Frame-Semantik in Bezug auf Polysemiestrukturen von Wörtern problematisch (siehe auch Kay 1996; Nemoto 1998; Boas 2002b, 2008c). Im Gegensatz zu Fillmore, dessen FrameNet klar veranschaulicht, dass unterschiedliche Wortbedeutungen unterschiedliche semantische Frames aktivieren, scheint Goldberg von einer eher minimalistischen Sichtweise von Verbbedeutung auszugehen. So ist außer den Informationen über die Partizipantenrolle TALKER in (2) nichts weiter über mögliche andere Bedeutungen von talk zu erfahren, die eventuell auch für eine mögliche Fusionierung mit Konstruktionen in Frage kommen könnten. Andererseits zeigt die frame-semantische Repräsentation von talk in FrameNet, dass es außer dem Statement Frame auch noch die Frames Chatting und Talking_into aktivieren kann, welche sich trotz ihrer semantischen Verwandschaft deutlich voneinander unterscheiden (vgl. die Definitionen in FrameNet sowie die des zur Beschreibung von Frames verwendeten Formalismus, um auch Konstruktionen beschreiben zu können (siehe hierzu Fillmore 2008)). ${ }^{12}$

Goldbergs Ansatz unterscheidet sich von Fillmores Theorie in einem weiteren wichtigen Punkt. Während in der Frame-Semantik darauf geachtet wird, dass das so genannte Linking von semantischen Argumenten (den Frame Elements) zur Syntax hin zu einem gewissen Grad als eine vom Verb alleine bestimmte Eigenschaft angesehen wird (vgl. z.B. Boas 2003b, i.Dr.), schlägt Goldberg (1995, S. 28) das Gegenteil vor:

Since the mapping between semantics and syntax is done via constructions, not via lexical entries, that there should be a class of „syntactically relevant aspects of verb meaning" follows from the existence of constructions, which are independently motivated.

\subsection{Minikonstruktionen und konventionalisierte Resultative}

Um die Probleme mit Goldbergs Implementierung der Frame-Semantik zu lösen, habe ich die so genannte Ereignis-basierte Frame-Semantik (Boas 2003a, S. 159-213) entwickelt, die im Prinzip mit Goldbergs Ansatz kompatibel ist, aber mit viel größerer Genauigkeit die unterschiedlichen semantischen, pragmatischen und syntaktischen Anforderungen von einzelnen so genannten Minikonstruktionen (= Verbbedeutungen) beschreibt, die zum großen Teil die für die Lizenzierung von Resultativkonstruktionen wichtigen Informationen enthalten. Der zentrale Gedanke ist, dass jede einzelne

12 Goldberg würde wohl eine TALK-INTO-Konstruktion annehmen, in die das monoseme Verb talk eingesetzt würde. 
Bedeutung eines Verbs eine so genannte Minikonstruktion bildet, d.h. eine Paarung von Form mit Bedeutung/Funktion. Im Gegensatz zu Goldbergs Ansatz, der eine Lizenzierung von Resultativen durch die Fusionierung von abstrakt-schematischen Resultativkonstruktionen mit minimalen Lexikoneinträgen von Verben erklärt, sieht der in Boas (2003a) vertretene Alternativansatz vor, dass Resultative in der Sprachproduktion direkt von konventionalisierten Minikonstruktionen lizenziert werden, die ihre eigenen spezifisch-idiosynkratischen Restriktionen mit sich bringen. Der semantische Teil einer Minikonstruktion (der so genannte Ereignis-Frame) erfasst in größtmöglicher Genauigkeit den semantischen Frame, der von einer Verbbedeutung evoziert wird. Dieser Frame wird aber nicht nur mit den traditionell frame-semantischen Methoden wie oben dargestellt beschrieben, sondern wird um weitere relevante Informationen spezifiziert. In Anlehnung an Talmys (2000) so genannte „Force Dynamics“ werden frame-semantische Beschreibungen einzelner Verbbedeutungen um detailliertere Informationen über die einzelnen Frame Elements, ihre Beziehungen zueinander und den zeitlichen Ablauf des von der Verbbedeutung beschriebenen Geschehens erweitert.

\begin{tabular}{|c|c|c|}
\hline & GOAL & \\
\hline & $\begin{array}{c}\mathrm{Ag} \\
(\mathrm{W} \text { p2) } \\
\mathrm{Pt}(\mathrm{p} 3)\end{array}$ & \\
\hline $\mathrm{Ag}:$ & \multirow{4}{*}{\multicolumn{2}{|c|}{$\begin{array}{l}\text { Person, die Farbe auf eine Oberfläche aufträgt } \\
\text { Oberfläche oder Objekt, welches so interpretiert } \\
\text { werden kann, als ob es eine Oberfläche hätte. } \\
\text { SYN: AP oder NP } \\
\text { beschreibt eine Farbe oder eine Eigenschaft, } \\
\text { die mit dem Auftragen von Farbe verbunden ist } \\
\text { und so interpretiert werden kann. }\end{array}$}} \\
\hline Pt: & & \\
\hline p3: & & \\
\hline SEM: & & \\
\hline
\end{tabular}

Abb. 3: Ereignis-basierte frame-semantische Repräsentation der prototypischen Bedeutung von paint, inkl. Weltwissen (W) (vgl. Boas 2003a, S. 224)

Als Beispiel sei hier die prototypische Bedeutung des Verbs paint (,malen` bzw. ,bemalen') genannt, die ein Ereignis evoziert, in dem ein AGENT einem PATIENT Farbe aufträgt. Das Ereignis fängt zu einem bestimmten Zeitpunkt an (SOURCE), zieht sich über die Zeitspanne des Malens hin (PATH) und kommt letztendlich zum Abschluss, wenn das Ziel (GOAL) des Malens erreicht ist. Ein Teil der Bedeutung des Verbs ist also - neben den einzelnen Frame Elements - auch das Wissen, dass sich Ereignisse über bestimmte Zeitspannen ereignen und zu einem Endzustand führen kön- 
nen. Dieser Endzustand des von paint bezeichneten Ereignisses ist in Abbildung 3 dargestellt, wo nur der letzte Zeitpunkt des Ereignisses (GOAL) und der Endzustand der Frame Elements dargestellt ist (SOURCE und PATH, die den Zeitraum vor GOAL darstellen, sind ausgelassen, weil hier irrelevant).

Der Ereignis-Frame in Abbildung 3 repräsentiert die prototypische Bedeutung von paint bzw. den Bedeutungsteil der Minikonstruktion (der Formteil wird unten besprochen). Unterhalb des Kästchens in Abbildung 3 befinden sich genauere Spezifikationen der Frame Elements. Sie spezifizieren die semantischen und pragmatischen Selektionsrestriktionen des AGENT (Ag) und des PATIENT (Pt), sowie mögliche Endresultatszustände (p2, p3), die aus der von paint beschriebenen prototypischen Aktivität hervorgehen können. Die Spezifizierung von p3, dem Endresultatszustand des PATIENT, zeigt darüber hinaus auch, dass neben semantischen und pragmatischen Restriktionen auch syntaktische Restriktionen im Ereignis-Frame festgelegt werden können. So ist die syntaktische Kategorie der Resultativphrase auf Nominalphrasen (z.B. a light shade of blue) und Adjektivphrasen (z.B. red) festgelegt. Die eingeklammerten Elemente p3, W, und p2 sind optional, d.h., sie müssen nicht syntaktisch realisiert werden (aber sie können implizit mitverstanden werden). Die durch Minikonstruktionen erfassten Spezifikationen sind als Defaultspezifikationen zu verstehen, d.h. sie können ggf. von anderen grammatischen Konstruktionen im gegebenen Kontext modifiziert werden. W steht für das übrige Weltwissen, welches für die Benutzung des Verbs paint relevant sein kann, z.B. dass man häufig Pinsel zum Malen benutzt, oder dass man müde werden kann, wenn man sehr lange malt. Das von W repräsentierte Weltwissen ist häufig irrelevant, manchmal kann es aber relevant werden, wenn nichtprototypische Endresultatszustände von Malaktivitäten beschrieben durch p2 ausgedrückt werden sollen wie z.B. Joe painted the brush to pieces (,Joe malte die Bürste kaputt') oder Miriam painted herself tired (,Miriam malte sich müde') (vgl. Boas 2003a, S. 181-192, 224, 256-259). ${ }^{13}$

Die so durch den Ereignis-Frame bereitgestellten frame-semantischen Informationen und Kollokationsrestriktionen sind nicht nur viel detaillierter in ihren Strukturen und Inhalten als die von Goldberg vorgeschlagenen Lexikoneinträge, sondern werden auch durch generelle Linking Rules à la Dowty (1991) direkt zur syntaktischen Realisierung gebracht (vgl. Boas 2003a, S. 244, 290). So lizenziert der Ereignis-Frame in Abbildung 3 Sätze

13 Vgl. z.B. Charniak (1977), der ausführlich die beim Malen eines Bildes mitverstandenen Informationen bespricht. Es ist auch möglich festzulegen, welche Ereignis-Frames besondere Arten von Resultativsätze lizenzieren können, wie z.B. so genannte „fake object resultatives“. Diese Informationen können in den Ereignis-Frames detailliert festgelegt werden, um so die Übergenerierung wie in (4) und (6)-(9) oben zu verhindern. Die speziellen Lizenzierungsbedingungen für „fake object resultatives“ sind in Boas (2003a, S. 181-224, 240-260) dargestellt. 
wie Collin painted the barn red (,Collin malte die Scheune rot an): Der AGENT wird als Subjekt realisiert, der PATIENT als Objekt, und der optionale Endresultatszustand wird als adjektivische Resultativphrase realisiert. In diesem Zusammenhang muss klargestellt werden, dass der in Abbildung 3 dargestellte Ereignis-Frame nur einer von mehreren Ereignis-Frames von paint ist, nämlich der prototypische.

Der Vorteil dieser detaillierten Ereignis-Frames liegt darin, dass sie die oben diskutierten Probleme mit Goldbergs Ansatz lösen können. So kann nicht nur genau spezifiziert werden, welche Arten von Beziehungen es zwischen den einzelnen Frame Elements eines Ereignis-Frames gibt, sondern es ist auch möglich, dass Kollokations- und Frequenzinformationen genau spezifiziert werden können, sowohl für die postverbale Nominalphrase als auch für die Resultativphrase. Da diese Analyse auf Korpusdaten basiert, ist es möglich, die Kollokationsrestriktionen unterschiedlich genau zu fassen, d.h. von sehr generell wie „Oberfläche“ in Abbildung 3, bis zu genaueren Restriktionen wie „NP oder AP“, bis hin zu sehr detaillierten Restriktionen, die genau Auskunft darüber geben können, welche Resultativphrasen lizenziert werden können, und welche nicht (vgl. Tabelle 2 und 3 oben, sowie Boas 2003a, S. 321-343). Die Möglichkeit, genaue Spezifikationen unterschiedlicher Art und Granularität auf der Ebene der Ereignis-Frames (dem Bedeutungsteil einer Minikonstruktion) vorzunehmen, ermöglicht es auch, die unterschiedliche und z.T. idiosynkratische Verteilung von Resultativen bei bedeutungsnahen Verben zu erfassen.

\subsection{Nichtkonventionalisierte Resultative: Lizenzierung durch Analogie}

Soweit bezieht sich mein Vorschlag nur auf konventionalisierte EreignisFrames, d.h. solche, die auf Grund von attestierten Korpusdaten klar identifiziert und als solche kodifiziert werden können. Wie aber steht es mit Goldbergs Paradebeispielen wie Joe coughed the napkin off the table (,Joe hustete die Serviette vom 'Tisch'), die u.a. als Hauptargumente für die Existenz von unabhängig existierenden (abstrakten) Konstruktionen angeführt werden? In ihrer Kritik behaupten Goldberg/Jackendoff (2004, S. 562), dass meine Analyse in Boas (2003a) nicht den Daten gerecht wird, weil sie angeblich keine Möglichkeit vorsieht, neuartige nichtkonventionalisierte Resultativkonstruktionen zu lizenzieren. Sie argumentieren, dass nur eine abstrakte bedeutungstragende Resultativkonstruktion in der Lage sei, nichtkonventionalisierte Resultative durch Fusionierung mit Lexikoneinträgen zu lizenzieren. Doch in ihrer Kritik haben Goldberg/Jackendoff augenscheinlich einen wichtigen Teil übersehen, der sich eben auch mit solchen Fällen beschäftigt.

Mein Alternativvorschlag, dargestellt in Boas (ebd., S. 260-277), nimmt nämlich auch an, dass nichtkonventionalisierte Resultative auf der Basis von Analogie mit bereits existierenden konventionalisierten Resultativen lizen- 
ziert werden können. Die Idee ist, dass es z.B. Verben wie blow (,blasen) gibt, die bereits die in ihren Ereignis-Frames spezifizierten semantischen Informationen (,etwas durch blasen bewegen“) konventionell als [NP V NP PP] realisieren, wie in Joe blew the napkin off the table (,Joe blies die Serviette vom Tisch).

Genauer gesagt: Wenn es eine neue Situation gibt, in der ein Verb wie cough in einer nichtkonventionalisierten Weise verwendet wird, um Bewegung eines Objekts durch Luftausstoß auszudrücken, dann können Sprecher von bereits existierenden konventionalisierten Minikonstruktionen Gebrauch machen, die diese Bedeutung (als Ereignis-Frame dargestellt) bereits konventionalisiert auf der syntaktischen Ebene als [NP V NP PP] realisieren, in diesem Falle blow. Gibt es eine solche prototypische Minikonstruktion, so kann ein Verb wie cough auch mit nichtkonventionalisierten syntaktischen Konstituenten realisiert werden, soweit sich die kontextuellen Gegebenheiten der jeweiligen Situationen zwischen blow und cough ausreichend überlappen (genaue Einzelheiten sind in Boas 2003a, S. 270-277 dargestellt). Der springende Punkt ist, dass im Falle einer analogischen Erweiterung der Bedeutung eines Verbes wie cough die ganze Bandbreite von semantischen, pragmatischen und syntaktischen Restriktionen eines so bereits konventionalisierten Verbs mit übernommen werden. ${ }^{14}$

Dieser Vorgang kann dann auch im Laufe der Zeit zu dem von Tomasello beschriebenen Prozess der Schematisierung führen:

Wenn Menschen wiederholt ,ähnliche` Dinge in ,ähnlichen` Situationen sagen, entwickelt sich daraus mit der Zeit ein sprachliches Verwendungsmuster, das in den Köpfen der Benutzer als neue Kategorie oder Konstruktion schematisiert wird mit unterschiedlichen Abstraktionsgraden. (Tomasello 2006, S. 21)

Der auf Analogie beruhende Prozess der Bedeutungserweiterung von Verben wie blow und cough und die daraus folgende Erweiterung der syntaktischen Möglichkeiten lässt sich auch auf sprachhistorischer Ebene belegen. So paart z.B. das Verb blow laut Oxford English Dictionary seit 1382 die Bedeutung „etwas durch Luftausstoß bewegen zu können“ mit dem syntaktischen Rahmen [NP V NP PP]. Andere Verben wie cough und sneeze, die in ihrer Grundbedeutung ebenfalls wie blow starken Luftausstoß beschreiben können, sind aber (noch nicht) mit der erweiterten Bedeutung konventionalisiert. ${ }^{15}$

Ein entscheidender Vorteil meiner Methode ist, dass man so auch bei nichtkonventionalisierten Resultativen die relevanten Restriktionen auf der Ebene der Minikonstruktionen festhalten kann. Da durch analogische Asso-

14 Welke (2009a, S. 517-524) schlägt eine parallele Vorgehensweise im Rahmen der Valenzgrammatik vor, um so z.B. Valenzerweiterungen von Verben zu analysieren.

$15 \mathrm{Zu}$ ähnlichen Vorschlägen in der diachronen Sprachwissenschaft siehe u.a. Diewald (2006, 2008) und Traugott (2008). 
ziation die Restriktionen von z.B. blow weitgehend auf cough übertragen werden, ist es so möglich, auch diese nichtkonventionalisierten Resultative dementsprechend zu restringieren. Dies ist bei Goldberg/Jackendoff (2004) nicht möglich, da die von ihnen postulierten abstrakten Resultativkonstruktionen (ähnlich wie bei Goldberg 1995) nicht in der Lage sind, inakzeptable Lizenzierungen auszuschließen (siehe Boas 2005a, 2007). ${ }^{16}$

\subsection{Konstruktionsnetzwerke}

Auf den ersten Blick erscheinen die von Goldberg $(1995,2006)$ und von Boas (2003a, 2005a) vertretenen Analysen nicht miteinander kompatibel zu sein. Dem ist jedoch nicht so. Bei genauerem Hinsehen fällt auf, dass die beiden Vorschläge sehr wohl miteinander kompatibel sind. Der größte Unterschied zwischen den beiden Ansätzen liegt darin, welchen Einfluss man abstrakten Konstruktionen wie der Resultativkonstruktion zugesteht. So ergeben sich meines Erachtens aus der Perspektive des Hörers keine Probleme beim Dekodieren, d.h. dem Verstehen eines Resultativsatzes auf der Basis von Goldbergs minimalen Lexikoneinträgen in Verbindung mit der abstrakten Resultativkonstruktion. Ob konventionalisierte oder nicht konventionalisierte Resultative, alles, was ein Hörer bei der Interpretation eines unbekannten Resultativsatzes machen muss, ist, das Gehörte zu interpretieren, indem er es mit existierenden Verbeinträgen (die auch relativ minimal sein können) in Verbindung mit Resultativkonstruktion abgleicht. Die oben angesprochenen Kritikpunkte an Goldbergs Ansatz scheinen beim Verstehen von Resultativsätzen also nicht so gravierend zu sein.

Dieser Vorgang des Dekodierens unterscheidet sich jedoch prozedural und inhaltlich vom Vorgang des Kodierens, den ein Sprecher bei der Produktion eines Resultativsatzes durchführt. So sollte ein Sprecher nämlich von vornherein wissen, was für postverbale Konstituenten mit welchen Verben in Resultativsätzen erscheinen können. Auch das Wissen der bei der Produktion wichtigen Kollokationsrestriktionen ist entscheidend dafür, ob ein Sprecher einen akzeptablen Resultativsatz produzieren kann. Diese Situation ist ähnlich der eines Fremdsprachenlerners, der zwar sowohl die Einzelbedeutungen von Adjektiven wie kalt und Adverbien wie bitter als auch die Regel der Adjektivmodifikation kennen mag, um so Adjektivmodifikationen wie richtig gut, bitter kalt und schrecklich langweilig produzieren zu

16 Konventionalisierte Minikonstruktionen sind in meinem Ansatz als Basis für analogische Neubildungen zu verstehen. So kann eine Neubildung wie Es kann ja nicht angehen, dass er Ihnen das Essen vom Teller hechelt (ein Hundetrainer kommentiert das Verhalten eines Hundes) dann entstehen, wenn das Verb becheln per Analogie zu blasen oder husten im Kontext so interpretiert werden kann, dass das postverbale Objekt vom Subjekt in der jeweiligen Situation durch einen starken Luftausstoß bewegt werden kann. Ich danke Wolfgang Imo für das Korpusbeispiel. $\mathrm{Zu}$ näheren Einzelheiten der Analogiebildung auf Grundlage von konventionalisierten Minikonstruktionen und kontextuellem Hintergrundwissen siehe Boas (2003a, S. 272-284). 
können, aber dennoch gleichzeitig wissen muss, dass man im Deutschen bitter kalt sagt, und nicht salzig kalt oder sauer kalt. Mit anderen Worten: Es gibt keine abstrakte Konstruktion in der Grammatik, die einem Fremdsprachensprecher systematisch helfen könnte, alleine die richtige Adjektivmodifikation zu wählen, um so die akzeptierte Modifikation von kalt durch bitter adäquat zu produzieren. Dies hat zur Folge, dass der Fremdsprachenlerner nicht nur die Regel der Adjektivmodifikation kennen muss, sondern auch die konventionalisierte Phrase bitter kalt als konkret-spezifizierten Extraeintrag in sein Lexikon mit aufnehmen muss.

Mit der Resultativkonstruktion verhält es sich ähnlich, d.h. um einen akzeptablen Resultativsatz produzieren zu können, muss ein Sprecher wissen, welche Sorten von postverbalen Konstituenten mit welchen Verben in Resultativsätzen vorkommen können. Dazu gehört, wie oben ausgeführt, nicht nur genaues Wissen über die Situationen, die von bestimmten Einzelbedeutungen von Verben beschrieben werden können (die so genannten Ereignis-Frames), sondern auch Informationen über semantische, pragmatische und syntaktische Restriktionen in Verbindung mit genauen Kollokationsrestriktionen und Frequenzinformationen. Ohne solch ein Wissen ist es meines Erachtens nicht möglich, die oben besprochenen Schwierigkeiten bzgl. des von Goldberg überbewerteten Einflusses der abstrakt-schematischen Resultativkonstruktion zu lösen.

Diese feinen Unterschiede zwischen Dekodierung und Kodierung mögen vielleicht auf den ersten Blick unwichtig erscheinen, werden aber noch immer in der Literatur übersehen oder falsch interpretiert. So stellt z.B. Welke (2009a, S. 516) fest: „Folgerichtig geht die Verallgemeinerung bei Goldberg (1995) induktiv vor sich, von unten (bottom up), vom Einzelnen zum Allgemeinen, und nicht deduktiv, von oben (top down), vom Allgemeinen zum Einzelnen." Meine Ausführungen haben, so hoffe ich, klar gezeigt, dass dem nicht so ist: Bei Goldberg (ebd.) und Goldberg/Jackendoff (2004) findet nämlich keine wirkliche „bottom up“-Verallgemeinerung statt, die aussagekräftige Vorhersagen über die Produktion von Resultativsätzen machen kann, weil sie ,nur“ abstrakte Konstruktionen in Verbindung mit unterspezifizierten Lexikoneinträgen postulieren.

Wie kann diesen Gegensätzen nun Rechnung getragen werden? Zunächst sollte bedacht werden, dass zahlreiche Analysen der letzten zwanzig Jahre überzeugende Argumente geliefert haben, die für eine Architektur von Lexikon, Syntax, und Semantik auf Netzwerkebene plädiert haben. So schlagen u.a. Bybee $(1985,2007)$, Langacker $(1987,2000)$, Barlow/Kemmer (Hg.) (2000) und Boas (2003a) einen so genannten „usage-based“-Ansatz vor, der auf der zentralen Rolle der Sprechererfahrung bei der Kategorisierung von linguistischen Informationen beruht. Dieser Ansatz vertritt die Auffassung, dass einzelne konkrete Tokens im täglichen Sprachgebrauch benutzt werden und als solche auch abgespeichert werden können, obwohl sie eine 
konkrete Realisierung abstrakterer Types (oder Schemata) darstellen. Bei hochfrequentem Gebrauch eines bestimmten Typus von Token kann nach Bybee (2003) eine Abstraktion erfolgen, die dann zu einer Generalisierung führt, was auf einer abstrakteren Ebene zur Emergenz eines abstrakten Schemas führen kann. Der entscheidende Punkt dabei ist die Tatsache, dass abstrakte Schemata zusammen mit ihren konkreten Tokens ein Netzwerk bilden können, in dem (auch häufig redundante) Informationen auf unterschiedlichen Abstraktionsebenen festgehalten werden können. Abbildung 4, in Anlehnung an Langackers (2000, S. 34) Diskussion der „Ditransitive Construction", stellt einen Ausschnitt eines solchen Netzwerks für Resultativkonstruktionen unterschiedlichen Abstraktionsgrads dar.

Abbildung 4 repräsentiert einen Ausschnitt des Netzwerks der Resultativkonstruktion mit unterschiedlichen Abstraktsionsebenen, wobei jede konkretere Ebene Informationen von den darüber liegenden Ebenen erbt. Der Formalismus ist an Goldberg (1995) angelehnt und soll darstellen, wie die Bedeutung einer Konstruktion mit einer bestimmten Form gepaart sein kann. Ebene I repräsentiert den höchsten Grad der Konstruktionsgeneralisierung bzgl. der Resultativkonstruktion, da sie keine eigenständige Bedeutung repräsentiert (dargestellt durch die $\varnothing$ auf der Konstruktionsbedeutungsebene). Die relativ bedeutungsleere Konstruktion auf Ebene I ist auf der abstrakt-schematischsten Stufe dieses Konstruktionsnetzwerksausschnitts und verbindet ein bestimmtes synktaktisches Muster [[NP][V][NP][XP]] mit einer relativ leeren Semantik, d.h., sie spezifiziert nur, dass die erste Nominalphrase als AGENT („Ag") realisiert wird. Diese relativ unterspezifizierte Paarung von Form und Bedeutung ist eine abstrakt-schematische sekundäre Prädikationskonstruktion, die ihre Informationen nicht nur an Resultativkonstruktionen, sondern auch an andere Typen von sekundären Prädikationskonstruktionen (hier durch einen Pfeil nach rechts repräsentiert) wie Small Clause-Komplemente und -Adjunkte (vgl. Staudinger 1997) vererben kann.

Auf Ebene II befindet sich eine stärker spezifizierte Konstruktion, nämlich die abstrakt-schematische Resultativkonstruktion, die Informationen von Ebene I erbt, aber gleichzeitig auch spezifischere Angaben bzgl. der Paarung von Form und Bedeutung macht. Diese Konstruktion ist spezifischer als die auf Ebene I befindliche abstraktere Konstruktion, da ihre Semantik nicht nur die CAUSE-BECOME-Bedeutung ausdrückt, sondern auch gleichzeitig festlegt, dass die postverbale NP als PATIENT interpretiert wird, und die postverbale XP als RESULT-GOAL.

Die einzelnen Resultativkonstruktionen auf Ebene III sind wiederum konkretere Instantiierungen der abstrakteren Konstruktion auf Ebene II. Sie unterscheiden sich hauptsächlich dadurch, dass sie die unterschiedlichen syntaktischen Kategorien der Resultativphrase als AP, PP oder NP spezifizieren. Ebenen I-III bilden die abstrakt-schematischen Ebenen 
der Resultativkonstruktion, die für die Lizenzierung von Resultativsätzen (mit)verantwortlich sind. Die auf diesen Netzwerkebenen spezifizierten Resultativkonstruktionen spiegeln keine phrasenstrukturellen Hierarchien wieder, weil sie nur die Defaultsatzstellung im Hauptsatz darstellen. Andere Wortstellungsvarianten werden durch die in Abbildung 4 dargestellten Resultativkonstruktionen in Verbindung mit anderen Konstruktionen wie der Relativsatzkonstruktion oder der Passivkonstruktion lizenziert (siehe Ackerman/Webelhuth 1998).

I:

II:
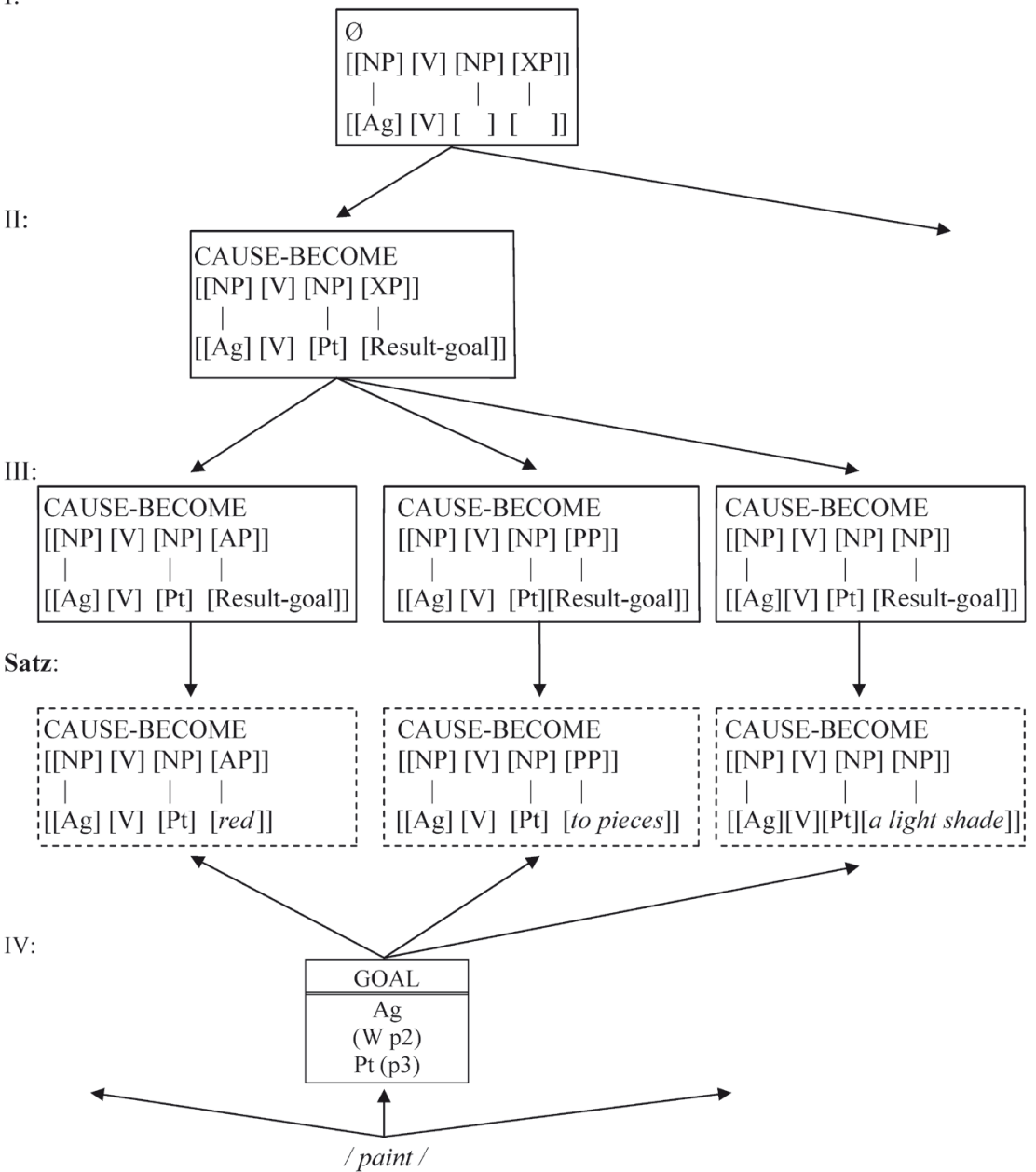

Abb. 4: Ausschnitt des Resultativkonstruktionsnetzwerks mit unterschiedlichen Abstraktionsgraden 
Auf der untersten Ebene IV finden wir eines der vielen Verben, die resultative Bedeutungen zum Ausdruck bringen können. Der Pfeil, der vom Verb paint auf den Ereignis-Frame zeigt, identifiziert die prototypische Bedeutung des Verbs, die hier durch einen die unterschiedlichen Partizipanten beschreibenden und andere Restriktionen festlegenden EreignisFrame festgehalten ist (vgl. Abbildung 2). Die anderen Pfeile zeigen auf hier nicht dargestellte nicht-prototypische Verbbedeutungen von paint, die wiederum durch andere Ereignis-Frames dargestellt werden können (vgl. Boas 2003a, S. 224-225). Ähnliche Verhältnisse lassen sich für andere Verben postulieren.

Die auf der so genannten Satzebene dargestellten Resultativkonstruktionen (mit gestrichelten Linien) zwischen Ebene III und Ebene IV bilden die Schnittmenge der auf Ebene III abstrakt-schematischen Konstruktionsschemata in Verbindung mit den durch den Ereignis-Frame lexikalisch-spezifizierten Informationen auf Ebene IV. Das bedeutet, dass durch die Satzebene lizenzierten Resultativsätze wie Joe paints the house red, Joe paints the brush to pieces und Joe paints the bouse a light shade of blue sowohl von abstrakt-schematischen Konstruktionen als auch von lexikalisch-spezifizierten EreignisFrames (Minikonstruktionen) lizenziert werden (siehe Boas 2003a, S. 240 260). ${ }^{17}$ Mit anderen Worten: Resultativsätze werden gleichzeitig von der abstrakten Resultativkonstruktion und einem lexikalisch-spezifischen Ereignis-Frame lizenziert, der die idiosynkratischen Unterschiede zwischen einzelnen Verbbedeutungen festhält. ${ }^{18}$ Die Notwendigkeit der Arbeitsteilung von verbbasierten und konstruktionsbasierten Informationen wird auch von Welke im Rahmen seines Vergleichs von konstruktionsbasierten und valenzbasierten Ansätzen hervorgehoben:

Auch wenn man den Zusammenhang von Verb und Konstruktion von der Konstruktion aus angeht, muss man beide miteinander abgleichen. Beim Verb müssen Informationen über mögliche Konstruktionen mitgegeben sein und bei der Konstruktion über mögliche Verben. (Welke 2009b, S. 88)

17 Traugott (2008, S. 8) schlägt im Rahmen einer konstruktionsbasierten Grammatikalisierungstheorie ähnliche Prinzipien zur Erfassung von Konstruktionen unterschiedlichen Abstraktheitsgrads vor, die miteinander verwandt sind. So postuliert sie z.B. auch unterschiedliche Ebenen eines hierarchischen Systems, in dem unterschiedliche Konstruktionsebenen angenommen werden müssen: ,(a) Makrokonstruktionen: abstrakte Schemata, die höchststufige Ebene, die für die gegebene Diskussion relevant ist, z.B. die Transitive Konstruktion, die Ditransitive Konstruktion, (...); (b) Mesokonstruktionen: Mengen sich ähnlich verhaltender Konstruktionen (...), (c) Mikro-Konstruktionen: Individuelle Konstruktionstypen [...]; und (d) Konstrukte: empirisch attestierte Token (,usage-based events' in der Terminologie von Langacker 1987), der Ort von Innovationen.“

18 Konstruktionsnetzwerke werden auch erfolgreich für die Analyse von Spracherwerbsdaten (Tomassello 1998, 2003; Behrens 2009) und Grammatikalisierungsprozesse (Traugott 2008; Diewald 2009) eingesetzt. 
Das hier vorgeschlagene Netzwerkmodell, das Informationen von abstraktschematischen Konstruktionen mit denen aus Ereignis-Frames (Minikonstruktionen) kombiniert, folgt meines Erachtens dem von Welke gemachten Vorschlag.

Der hier skizzierte Alternativansatz, der die Analysen von Goldberg $(1995,2006)$ und Boas $(2003 a, 2005 a)$ miteinander verbindet, mag durch die explizite Spezifizierung von Konstruktionsinformationen auf unterschiedlichen Abstraktionsebenen womöglich als zu unökonomisch kritisiert werden. So behauptet z.B. Müller (2006), dass zur Erfassung von Resultativkonstruktionen in der Konstruktionsgrammatik unnötig viele Annahmen gemacht werden müssen. Müller stützt seine Behauptung auf die Beobachtung, dass die Postulierung von komplexen Konstruktionen zu einer enormen Anzahl von weiteren konkreten komplexen Konstruktionen führen würde, um so allen Vorkommensmöglichkeiten von Resultativen in verschiedenen syntaktischen Bildungsweisen wie z.B. „Scrambling“, Passiv- und Mediumsätze, und Sätze mit Adverbialen Rechnung zu tragen (vgl. auch Jacobs 2008). Um das ganze Spektrum von Resultativen zu erfassen, müsse man mindestens 32 verschiedene komplexe Resultativkonstruktionen annehmen. Dies sei aber nicht plausibel, da man nicht davon ausgehen kann, dass Sprecher des Deutschen all diese 32 Konstruktionen einzeln im Langzeitgedächtnis gespeichert haben. Als Alternative schlägt Müller (2006) vor, dass eine einzige lexikalische Regel die von ihm besprochenen Daten erfassen könne.

Meine obigen Ausführungen stehen diesen Beobachtungen nicht unbedingt entgegen. So ist das Resultativkonstruktionsnetzwerk in Abbildung 4 so strukturiert, dass es auf abstrakt-schematischeren Ebenen wie Ebene I und II unterspezifizierte Repräsentationen gibt, die sich von anderen Ebenen unterscheiden. Auf diese Art werden bis zur Ebene III hinunter abstrakt-schematische Informationen bzgl. Resultativbildungen erfasst, die auch gleichzeitig mit Konstruktionen aus anderen Konstruktionsnetzwerken wie dem Passiv (vgl. Ackerman/Webelhuth 1998) fusionieren können, um den von Müller (2006) besprochenen syntaktischen Bildungsweisen Rechnung zu tragen. Die kognitiven Repräsentationen der unterschiedlichen Konstruktionen mögen zwar redundant sein, aber sie erfassen die umfangreichen zur Lizenzierung nötigen Spezifikationen. Die von mir als Minikonstruktionen bzw. Ereignis-Frames bezeichneten Einheiten auf der Satzebene halten demnach nur die minimalen Idiosynkrasien einzelner Verbbedeutungen fest, die unbedingt - da sie konventionalisiert sind - als eigenständige konkrete semantische, pragmatische und syntaktische Spezifizierungen von abstrakt-schematischeren Konstruktionen gelistet werden müssen (vgl. Abschnitt 2.3). Die obigen Vorschläge müssen natürlich noch verfeinert werden, besonders bzgl. der Arbeitsteilung zwischen abstraktschematischen und konkret-spezifischen Konstruktionen. 
3.4 Ereignis-Frames und abstrakte Konstruktionen im Englischen und Deutschen

Die oben dargestellten Vorschläge lassen sich auch auf das Deutsche weitgehend übertragen. ${ }^{19}$ So gibt es auch im Deutschen viele Verben, die sowohl der Struktur als auch der Bedeutung der oben besprochenen englischen Resultativbeispiele entsprechen:
a. Ursula wischte den Tisch (sauber).
b. Ulrich strich das Haus (rot).
c. Fritz lief sich müde.
a'. Ursula wiped the table (clean).
b'. Ulrich painted the house (red).
c'. Fritz ran himself tired.

(vgl. Boas 2003a, S. 286)

Doch der Schein trügt: Nicht wirklich alle deutschen Übersetzungen englischer Verben können auch im Deutschen in Resultativsätzen vorkommen. Die Beispiele in (11) und (12) zeigen, dass die deutschen Verben rennen und bungern nicht wie ihre englischen Entsprechungen run und starve in Resultativkonstruktionen vorkommen können. Das Gegenteil ist auch in (13) der Fall, wo das englische pray nicht wie beten in einer Resultativkonstruktion vorkommen kann.

(11) a. Martin ran Ed off the road.

b. *Martin rannte Ed von der Straße ab.

c. Martin drängte Ed (beim Rennen) von der Straße ab.

(12) a. The doctors starved the patients to death.

b. *Die Ärzte hungerten die Patienten zu Tode.

c. Die Ärzte ließen die Patienten verhungern.

(13) a. Der Mann betete seine Tochter gesund.

b. ?The man prayed his daughter healthy.

c. The man prayed. As a result of his praying, his daughter became healthy.

(vgl. Boas 2003a, S. 287)

Aus Daten wie in (11)-(13) lassen sich folgende Schlussfolgerungen ziehen: (1) Trotz ihrer parallelen Grundbedeutungen können nicht alle englischen Verben und ihre deutschen Entsprechungen in ihrer Defaultinterpretation in Resultativkonstruktionen vorkommen. (2) In einigen Fällen verlangen das Deutsche bzw. das Englische ein völlig anderes Verb in Verbindung mit einer anderen Konstruktion. Dies ist z.T. auf typologische Unterschiede zurückzuführen (z.B. die Tatsache, dass im Deutschen ein genauer Unterschied zwischen den Arten des Wissens, Verlassens oder Stellens gemacht

19 Zu den Einzelheiten der hierbei relevanten Aspekte der deutschen Verbalmorphologie siehe u.a. Müller (2002, 2005, 2006). 
werden muss, aber nicht im Englischen - vgl. Hawkins 1986; Leisi/Mair 1999; Boas 2003a), muss aber dennoch irgendwo in der Grammatik bzw. im Lexikon festgehalten werden. (3) Die Zusammensetzung von Verben und postverbalen Konstituenten in Resultativkonstruktionen muss im Deutschen genauso wie im Englischen durch so genannte Ereignis-Frames lizenziert werden, die als lexikalisch-spezifizierte Minikonstruktionen die einzelnen Unterbedeutungen von Verben repräsentieren und so als konkrete Spezifizierung einer abstrakten Resultativkonstruktion angesehen werden (vgl. Abschnitt 3.3). Ein weiterer Beleg für diese Sichtweise ergibt sich aus folgender Tabelle:

\begin{tabular}{|c|c|c|c|c|}
\hline & Bedeutung & $\begin{array}{c}\text { Englische } \\
\text { Resultativkonstruktion }\end{array}$ & $\begin{array}{l}\text { Deutsches } \\
\text { Äquivalent }\end{array}$ & $\begin{array}{c}\text { Deutsche } \\
\text { Resultativkonstruktion }\end{array}$ \\
\hline 1 & $\begin{array}{l}\text { To guide, con- } \\
\text { trol, or direct } \\
\text { (a vehicle) }\end{array}$ & $\begin{array}{l}\text { He drove the car into } \\
\text { the ditch/ to pieces. }\end{array}$ & fahren & $\begin{array}{l}\text { Er fuhr das Auto in } \\
\text { den Graben/kaputt. }\end{array}$ \\
\hline 2 & $\begin{array}{l}\text { To convey } \\
\text { transport in a } \\
\text { vehicle. }\end{array}$ & $\begin{array}{l}\text { She drove the kids to } \\
\text { school. }\end{array}$ & $\begin{array}{l}\text { befördern/ } \\
\text { fahren }\end{array}$ & $\begin{array}{l}\text { Sie beförderte/fuhr } \\
\text { die Kinder zur Schule. }\end{array}$ \\
\hline 3 & To penetrate & $\begin{array}{l}\text { Kim drove the stake } \\
\text { into the ground. }\end{array}$ & $\begin{array}{l}\text { (ein-)schlagen, } \\
\text { treiben }\end{array}$ & $\begin{array}{l}\text { Karla schlug den Stock } \\
\text { in den Boden (ein). }\end{array}$ \\
\hline 4 & $\begin{array}{l}\text { To repulse } \\
\text { forcefully; } \\
\text { put to flight }\end{array}$ & $\begin{array}{l}\text { We drove the attackers } \\
\text { away. }\end{array}$ & $\begin{array}{l}\text { wegjagen/ } \\
\text { vertreiben }\end{array}$ & $\begin{array}{l}\text { Wir jagten die An- } \\
\text { greifer weg./Wir ver- } \\
\text { trieben die Angreifer. }\end{array}$ \\
\hline 5 & $\begin{array}{l}\text { To throw, strike, } \\
\text { or cast }\end{array}$ & $\begin{array}{l}\text { He drove the ball into } \\
\text { the goal. }\end{array}$ & treten & $\begin{array}{l}\text { Er trat den Ball in das } \\
\text { Tor. }\end{array}$ \\
\hline 6 & $\begin{array}{l}\text { To change the } \\
\text { mental state of } \\
\text { somebody }\end{array}$ & She drove me crazy. & treiben & $\begin{array}{l}\text { Sie trieb mich in den } \\
\text { Wahnsinn. }\end{array}$ \\
\hline
\end{tabular}

Tab. 4: Englische Resultativkonstruktionen mit drive und ihre deutschen Entsprechungen (vgl. Boas 2003a, S. 295)

Das englische Verb drive verfügt über mindestens sechs unterschiedliche Bedeutungen, die alle in resultativer Verwendung vorkommen können. Im Gegensatz dazu enthalten die deutschen Übersetzungsäquivalente alle unterschiedliche Verben. Gemeinsam ist den englischen Beispielen und ihren deutschen Entsprechungen, dass die Ereignis-Frames genaue Kollokationsrestriktionen bzw. Spezifikationen bzgl. syntaktischer, semantischer und pragmatischer Restriktionen festlegen, die bei der Produktion eines Resultativsatzes nicht alleine durch eine abstrakte Resultativkonstruktion erfasst werden können (vgl. *Er fuhr das Auto grün, *Sie fubr die Kinder krank, *Sie trieb mich in die Glücklichkeit, etc.). Daraus folgt u.a., dass sich die für das Englische vorgeschlagene Analyse von Resultativkonstruktionen im Rahmen von Ereignis-Frames auch weitgehend auf das Deutsche übertragen lässt. 


\title{
4. Schlussbetrachtungen
}

Das in diesem Beitrag vorgeschlagene Netzwerk aus Resultativkonstruktionen unterschiedlichen Abstraktionsgrads vereinigt die Vorteile von Goldbergs $(1995,2006)$ und Boas' $(2003 a, 2005 a)$ Analysen. So können sowohl die beim Dekodieren relevanten abstrakt-schematischen Konstruktionsinformationen als auch die beim Kodieren wichtigen lexikalisch-spezifizierten Konstruktionsinformationen über semantische, pragmatische, und syntaktische Restriktionen auf der Ebene von Ereignis-Frames in einem einheitlichen Netzwerk erfasst und analysiert werden. Dies erfüllt auch die von Goldberg (2006, S. 18) postulierte Prämisse: „It's constructions all the way down."

Diese einheitliche Analyse bietet auch zwei weitere entscheidende Vorteile. Erstens vereinfacht die Beschreibung der Distribution von Konstruktionen auf primär semantisch-pragmatischer Ebene die kontrastive Analyse von gleichartigen Konstruktionen in unterschiedlichen Sprachen. Da der Formteil einer Konstruktion letztendlich nur sekundären Rang in der Konstruktionsbeschreibung hat, ist es so einfacher, sprachvergleichend Konstruktionen zu beschreiben, wie in Abschnitt 3.4 gezeigt wurde (vgl. dazu Boas 2003a, 2010b). Zweitens erfüllt die hier vorgeschlagene Methode auch die in neueren Veröffentlichungen zu Parallelen der Valenzgrammatik und Konstruktionsgrammatik gemachten Vorschläge, unterschiedliche Abstraktionsgrade von Konstruktionen zu berücksichtigen (vgl. Jacobs 2008; Welke 2009a, b). Welke z.B. fordert:

\begin{abstract}
Wir plädieren, was die heutige Valenz- und Konstruktionsgrammatik betrifft, nicht für einseitige Übernahmen oder Abgrenzungen, sondern für eine Integration. Valenzänderungen (einschliesslich Valenzerweiterungen und Valenzreduktion) und Valenzvererbung können als konstruktionell bedingte Änderungen erklärt werden. Grundlage ist einerseits ein am Einzelwort, andererseits ein an der Einzelkonstruktion orientiertes Vorgehen, das nicht vom Allgemeinen zum Einzelnen gelangt, sondern via prototypischer und analogischer Verallgemeinerung vom Einzelnen zum Allgemeinen. Wesentlich ist in diesem Zusammenhang die Frage nach dem jeweils erreichten Verallgemeinerungsgrad, der „Reichweite“, von Konstruktionen. (Welke 2009b, S. 541)
\end{abstract}

Meine in diesem Beitrag skizzierten Vorschläge setzen meines Erachtens die von Welke (2009b) gestellten Integrationsforderungen bzgl. Wörtern und Konstruktionen in die Tat um. Zukünftige Analysen sollten sich noch eingehender mit der genauen Arbeitsteilung von Verben und Konstruktionen beschäftigen sowie systematische Untersuchungen zu Interaktionen zwischen unterschiedlichen Konstruktionen anstellen. Darüber hinaus ist auch die Entwicklung eines noch einheitlicheren Formalismus zur Beschreibung von Konstruktionen unterschiedlichen Abstraktionsgrads wie in der „Sign-based Construction Grammar“ (Michaelis 2010; Sag 2010; Boas/Sag (Hg.) i.Dr.; Fillmore/Lee-Goldman/Rhodes i.Dr.) wünschenswert. 


\section{Literatur}

Ackerman, Farrell/Webelhuth, Gert (1998): A theory of predicates. Stanford.

Barðdal, Jóhanna (2008): Productivity: evidence from case and argument structure in Icelandic. (= Constructional Approaches to Language 8). Amsterdam/Philadelphia.

Barlow, Michael/Kemmer, Susanne (Hg.) (2000): Usage-based models of language. Stanford.

Behrens, Heike (2009): Konstruktionen im Spracherwerb. In: Zeitschrift für Germanistische Linguistik 37, S. 427-444.

Bergen, Benjamin/Chang, Nancy (2005): Embodied Construction Grammar in simulation-based language understanding. In: Östman/Fried (Hg.), S. 147-190.

Bergs, Alexander/Diewald, Gabriele (Hg.) (2008): Constructions and language change. Berlin/New York.

Boas, Hans C. (2002a): Lexical or syntactic selection of non-subcategorized nominal arguments? The case of German ,Sätzchen'. In: Rauch, Irmengard/Carr, Gerald F. (Hg.): New Insights in Germanic Linguistics III. (= Berkeley Insights in Linguistics and Semiotics 52). New York, S. 9-26.

Boas, Hans C. (2002b): On the role of semantic constraints in resultative constructions. In: Rapp, Reinhard (Hg.): Sprachwissenschaft auf dem Weg in das Dritte Jahrtausend: Akten des 34. Linguistischen Kolloquiums in Germersheim 1999 (Linguistics on the way into the new millennium). Bd. 1. Frankfurt a.M., S. 35-44.

Boas, Hans C. (2003a): A constructional approach to resultatives. Stanford.

Boas, Hans C. (2003b): A lexical-constructional account of the locative alternation. In: Carmichael, Leslie/Huang, Scott C.-H./Samiian, Vida (Hg.): Proceedings of the 2001 Western Conference in Linguistics 13, S. 27-42.

Boas, Hans C. (2004): You wanna consider a constructional approach to wanna-contraction? In: Achard, Michael/Kemmer, Suzanne (Hg.): Language, Culture, and Mind. Stanford, S. 479-491.

Boas, Hans C. (2005a): Determining the productivity of resultative constructions: a reply to Goldberg \& Jackendoff. In: Language 81, 2, S. 448-464.

Boas, Hans C. (2005b): From theory to practice: Frame semantics and the design of FrameNet. In: Langer, Stefan/Schnorbusch, Daniel (Hg.): Semantik im Lexikon. (= Tübinger Beiträge zur Linguistik 479). Tübingen, S. 129-160.

Boas, Hans C. (2006): A frame-semantic approach to identifying syntactically relevant elements of meaning. In: Steiner, Petra/Boas, Hans C./Schierholz, Stefan (Hg.): Contrastive studies and valency. Studies in honor of Hans Ulrich Boas. Frankfurt a.M./New York, S. 119-149.

Boas, Hans C. (2007): Construction Grammar in the twenty-first century. In: English Language and Linguistics 11, 3, S. 569-585.

Boas, Hans C. (2008a): Determining the structure of lexical entries and grammatical constructions in Construction Grammar. In: Annual Review of Cognitive Linguistics 6, S. 113-144. 
Boas, Hans C. (2008b): Resolving form-meaning discrepancies in Construction Grammar. In Leino, Jaakko (Hg.): Constructional Reorganization. (= Constructional approaches to language 5). Amsterdam/Philadelphia, S. 11-36.

Boas, Hans C. (2008c): Towards a frame-constructional approach to verb classification. In: Acevedo, Eulalia Sosa/Rodríguez, Francisco José Cortés (Hg.): Grammar, constructions, and interfaces. Special Issue of Revista Canaria de Estudios Ingleses 57, S. 17-48.

Boas, Hans C. (2009): Verb meanings at the crossroads between higher-level and lowerlevel constructions. In: Lingua 120, S. 22-34.

Boas, Hans C. (Hg.) (2009): Multilingual FrameNets in computational lexicography. Methods and applications. (= Trends in Linguistics: Studies and Monographs 200). Berlin/New York.

Boas, Hans C. (2010a): The syntax-lexicon continuum in Construction Grammar: a case study of English communication verbs. In: Belgian Journal of Linguistics 24, S. 57-86.

Boas, Hans C. (2010b): Comparing constructions across languages. In: Boas, Hans C. (Hg.): Contrastive studies in Construction Grammar. Amsterdam/Philadelphia, S. $1-20$.

Boas, Hans C. (i. Dr.): A frame-semantic approach to syntactic alternations: the case of build verbs. In: Guerrero Medina, Pilar (Hg.): Morphosyntactic Alternations in English. London.

Boas, Hans C./Sag, Ivan A. (Hg.) (i.Dr.): Sign-based Construction Grammar. Stanford.

Bybee, Joan (1985): Morphology: a study of the relation between meaning and form. (= Typological Studies in Language 9). Amsterdam/Philadelphia.

Bybee, Joan (2003): Mechanisms of change in grammaticalization: the role of frequency. In: Joseph, Brian D./Janda, Richard D. (Hg.): The handbook of historical linguistics. Oxford, S. 602-623.

Bybee, Joan (2007): Frequency of use and the organization of language. Oxford.

Charniak, Eugene (1977). A framed PAINTING: the representation of a common sense knowledge fragment. In: Cognitive Science 1, S. 355-395.

Chomsky, Noam (1981): Lectures on government and binding. (= Studies in Generative Grammar 9). Dordrecht.

Chomsky, Noam (1995): The minimalist program. (= Current Studies in Linguistics Series 28). Cambridge, MA u.a.

Croft, William (2001): Radical Construction Grammar: syntactic theory in typological perspective. Oxford.

Deppermann, Arnulf (2007): Grammatik und Semantik aus gesprächsanalytischer Sicht. (= Linguistik - Impulse und Tendenzen 14). Berlin/New York.

Diessel, Holger (2004): The acquisition of complex sentences. (= Cambridge Studies in Linguistics 105). Cambridge.

Diewald, Gabriele (2006): Konstruktionen in der diachronen Sprachwissenschaft. In: Fischer/Stefanowitsch (Hg.), S. 79-103. 
Diewald, Gabriele (2008): Idiomatische Konstruktionen in Grammatikalisierungsprozessen. In: Stefanowitsch/Fischer (Hg.), S. 33-58.

Diewald, Gabriele (2009): Konstruktionen und Paradigmen. In: Zeitschrift für Germanistische Linguistik 37, S. 445-468.

Dowty, David (1991): Thematic proto-roles and argument selection. In: Language 67, S. $547-619$.

Fillmore, Charles J. (1985): Frames and the semantics of understanding. In: Quadernie di Semantica 6, 2, S. 222-254.

Fillmore, Charles J. (1988): The mechanisms of Construction Grammar. In: Axmaker, Shelley/Jassier, Annie/Singmaster, Helen (Hg.): General session and parasession on grammaticalization. Proceedings of the Fourteenth Annual Meeting of the Berkeley Linguistic Society. Berkeley, S. 35-55.

Fillmore, Charles J. (2007): Valency issues in FrameNet. In: Herbst, Thomas/Götz-Votteler, Katrin (Hg.): Valency: theoretical, descriptive, and cognitive issues. (= Trends in Linguistics: Studies and Monographs 187). Berlin/New York, S. 129-160.

Fillmore, Charles J. (2008): Border conflicts: FrameNet meets Construction Grammar. Plenarvortrag, EURALEX XIII, Barcelona.

Fillmore, Charles J. (2009): A valency dictionary of English. In: International Journal of Lexicography 22, 1, S. 55-85.

Fillmore, Charles J./Atkins, Beryl T.S. (1992): Towards a frame-based organization of the lexicon: the semantics of RISK and its neighbors. In: Lehrer, Adrienne/Kittay, Eva (Hg.): Frames, fields, and contrasts: new essays in lexical organization. Hillsdale, S. 75-102.

Fillmore, Charles J./Baker, Collin (2010): A frame approach to semantic analysis. In: Heine/Narrog (Hg.), S. 313-340.

Fillmore, Charles J./Johnson, Christopher R./Petruck, Miriam R.L. (2003): Background to FrameNet. In: International Journal of Lexicography 16, 3, S. 235-250.

Fillmore, Charles J./Kay, Paul (1995): Construction Grammar. Ms. Berkeley.

Fillmore, Charles J./Kay, Paul/O'Connor, Mary (1988): Regularity and idiomaticity in grammatical constructions: The case of ,let alone'. In: Language 64, S. 501-538.

Fillmore, Charles J./Lee-Goldman, Russell/Rhodes, Russell (i.Dr.): Sign-based Construction Grammar and the FrameNet constructicon. In: Boas/Sag (Hg.).

Fischer, Kerstin/Stefanowitsch, Anatol (Hg.) (2006): Konstruktionsgrammatik. Von der Anwendung zur Theorie. (= Stauffenburg Linguistik 40). Tübingen.

Fried, Mirjam/Boas, Hans C. (Hg.) (2005): Grammatical constructions. Back to the roots. (= Constructional Approaches to Language 4). Amsterdam/Philadelphia.

Fried, Mirjam/Östman, Jan-Ola (Hg.) (2004): Construction Grammar in a cross-language perspective. (= Constructional Approaches to Language 2). Amsterdam/ Philadelphia.

Goldberg, Adele (1995): Constructions: a construction grammar approach to argument structure. Chicago. 
Goldberg, Adele (2006): Constructions at work: the nature of generalization in language. Oxford.

Goldberg, Adele/Jackendoff, Ray (2004): The English resultative as a family of constructions. In: Language 80, 3, S. 532-568.

Gries, Stefan T./Wulff, Stefanie (2005): Do foreign language learners also have constructions? Evidence from priming, sorting, and corpora. In: Annual Review of Cognitive Linguistics 3, S. 182-200.

Günthner, Susanne (2008): Die “die Sache/das Ding ist”-Konstruktion im gesprochenen Deutsch - eine interaktionale Perspektive auf Konstruktionen im Gebrauch. In: Stefanowitsch/Fischer (Hg.), S. 157-178.

Hawkins, John (1986): A comparative typology of English and German. Unifying the contrasts. London.

Heine, Bernd/Narrog, Heiko (Hg.) (2010): The Oxford handbook of linguistic analysis. Oxford.

Hilpert, Martin (2008): Germanic future constructions: a usage-based approach to language change. (= Constructional Approaches to Language 7). Amsterdam/Philadelphia.

Imo, Wolfgang (2007): Construction Grammar und Gesprochene-Sprache-Forschung: Konstruktionen mit zehn matrixsatzfähigen Verben im gesprochenen Deutsch. (= Reihe Germanistische Linguistik 275). Tübingen.

Iwata, Seizi (2005): The role of verb meaning in locative alternations. In: Fried/Boas (Hg.), S. 101-118.

Iwata, Seizi (2008): Locative Alternation. A lexical-constructional approach. (= Constructional Approaches to Language 6). Amsterdam/Philadelphia.

Jackendoff, Ray (1997): The architecture of the language faculty. (= Linguistic Inquiry Monographs 28). Cambridge, MA.

Jackendoff, Ray (2010): Meaning and the lexicon: the parallel architecture, 1975-2010. Oxford.

Jacobs, Joachim (2008): Wozu Konstruktionen? In: Linguistische Berichte 213, S. 1-44.

Kay, Paul (1996): Argument structure: causative ABC constructions. Ms. University of California. Berkeley.

Kay, Paul (2005): Argument structure constructions and the argument-adjunct distinction. In: Fried/Boas (Hg.), S. 71-100.

Kay, Paul/Fillmore Charles J. (1999): Grammatical constructions and linguistic generalizations: the ,What's X doing Y?' constructions. In: Language 75, S. 1-33.

Lambrecht, Knud/Lemoine, Kevin (2005): Definite null objects in (spoken) French: A Construction-Grammar account. In: Fried/Boas (Hg.), S. 13-56.

Langacker, Ron (1987): Foundations of Cognitive Grammar I: Theoretical prerequisites. Stanford.

Langacker, Ron (2000): A dynamic usage-based model. In: Barlow/Kemmer (Hg.), S. 1-63. 
Leisi, Ernst/Mair, Christian (1999): Das heutige Englisch: Wesenszüge und Probleme. 8., neubearb. Aufl. Heidelberg.

Michaelis, Laura (2010): Sign-based Construction Grammar. In: Heine/Narrog (Hg.), S. 139-158.

Michaelis, Laura/Ruppenhofer, Joseph (2001): Beyond alternations: a constructional model of the German applicative pattern. Stanford.

Müller, Stefan (2002): Complex predicates: verbal complexes, resultative constructions, and particle verbs in German. Stanford.

Müller, Stefan (2005): Resultative constructions: syntax, world knowledge, and collocational restrictions. Review of Hans C. Boas: A Constructional Approach to Resultatives. In: Studies in Language 29, S. 651-681.

Müller, Stefan (2006): Resultativkonstruktionen, Partikelverben und syntaktische vs. lexikonbasierte Konstruktionen. In: Fischer/Stefanowitsch (Hg.), S. 177-202.

Nemoto, Noriko (1998): On the polysemy of ditransitive save: the role of frame semantics in Construction Grammar. In: English Linguistics 15, S. 219-242.

Nemoto, Noriko (2005): Verbal polysemy and frame semantics in Construction Grammar. In: Fried/Boas (Hg.), S. 118-136.

Östman, Jan-Ola (2005): Construction discourse: a prolegomenon. In: Östman/Fried (Hg.), S. 121-144.

Östman, Jan-Ola/Fried, Mirjam (Hg.) (2005): Construction Grammars. Cognitive grounding and theoretical extensions. (= Constructional Approaches to Language 3). Amsterdam/Philadelphia.

Petruck, Miriam (1996): Frame semantics. In: Verschueren, Jef et al. (Hg.): Handbook of pragmatics. Amsterdam/Philadelphia, S. 1-13.

Pollard, Carl/Sag, Ivan (1994): Head-driven phrase structure grammar. Stanford.

Ruppenhofer, Joseph et al. (2006): FrameNet II: extended theory and practice. Internet: http://framenet.icsi.berkeley.edu (Stand: 01.11.2010).

Sag, Ivan (2010): English filler-gap constructions. In: Language 86, 3, S. 486-545.

Staudinger, Bernd (1997): Sätzchen: Small clauses im Deutschen. (= Linguistische Arbeiten 363). Tübingen.

Stefanowitsch, Anatol/Fischer, Kerstin (Hg.) (2008): Konstruktionsgrammatik II: Von der Konstruktion zur Grammatik. (= Stauffenburg Linguistik 47). Tübingen.

Talmy, Len (2000): Toward a cognitive semantics. Cambridge, MA.

Tomasello, Michael (1998): The return of constructions. In: Journal of Child Language 75, S. 431-447.

Tomasello, Michael (2003): Constructing a language: a usage-based theory of language acquisition. Cambridge, MA.

Tomasello, Michael (2006): Konstruktionsgrammatik und früher Erstspracherwerb. In: Fischer/Stefanowitsch (Hg.), S. 19-38. 
Traugott, Elisabeth (2008): Grammatikalisierung, emergente Konstruktionen und der Begriff der „Neuheit“. In: Stefanowitsch/Fischer (Hg.), S. 5-32.

van Trijp, Remi (2008): Argumentstruktur in der Fluid Construction Grammar. In: Stefanowitsch/Fischer (Hg.), S. 223-246.

Welke, Klaus (2009a): Valenztheorie und Konstruktionsgrammatik. In: Zeitschrift für Germanistische Linguistik 37, S. 81-124.

Welke, Klaus (2009b): Konstruktionsvererbung, Valenzvererbung und die Reichweite von Konstruktionen. In: Zeitschrift für Germanistische Linguistik 37, S. 514-543.

Ziem, Alexander (2008): Frames und sprachliches Wissen: kognitive Aspekte der semantischen Kompetenz. (= Sprache und Wissen 2). Berlin/New York. 



\title{
Argumentstrukturmuster als Konstruktionen? Identität - Verwandtschaft - Idiosynkrasien
}

\begin{abstract}
Aus den Argumentstrukturen von Verben lassen sich vielfach eigenständige Argumentstrukturmuster mit idiosynkratischen formalen oder inhaltlichen Eigenschaften abstrahieren. Der Artikel zeigt, dass sich Ähnlichkeiten zwischen solchen Mustern nicht, wie von Goldberg (1995) vorgeschlagen, über das Konzept polysemer Argumentstrukturkonstruktionen erfassen lassen, sondern adäquater über ein Netz von Familienähnlichkeiten modelliert werden können. Die einzelnen Argumentstrukturmuster zeigen dabei eine Vielzahl von idiosynkratischen lexikalischen Kookkurrenzen, die spezifisch für die je einzelnen Argumentstrukturmuster sind und in einer implikativen Beziehung zu diesen stehen. Überlegungen zur angemessenen sprachtheoretischen Modellierung der Daten zeigen dabei sowohl Schwächen valenzbasierter Theorien als auch Mängel konstruktionsbasierter Ansätze auf. ${ }^{1}$
\end{abstract}

\section{Argumentstrukturmuster}

\subsection{Argumentstrukturmuster auf verschiedenen Abstraktionsebenen}

Die Argumentstruktur eines Lexems bringt zum Ausdruck, inwieweit seine idiosynkratischen Eigenschaften die Art der syntaktischen und semantischen Einbindung der das Lexem im Satz umgebenden Phrasen bestimmen. Unter Argumentstruktur verstehen wir dabei die einem Lexem zugeordnete Liste von Argumentstellen mit ihren syntaktischen und ihren semantischen Beschränkungen, also Beschränkungen hinsichtlich des Phrasentyps, der Kasus, der Präpositionswahl etc. einerseits und Beschränkungen hinsichtlich semantischer Rollen bzw. lexikalischer Dekomposition sowie Selektionsbeschränkungen andererseits. ${ }^{2}$ Nun lassen sich im Bereich der Argumentstrukturen von Verben auf verschiedenen Abstraktionsebenen auffällige Kovorkommen von Ausdrucksklassen beobachten, die lexemübergreifend mit bestimmten Bedeutungen einhergehen. Das legt die Vermutung nahe,

1 Die hier vorgestellten Überlegungen und Untersuchungen sind in dem Forschungsprojekt „Polysemie und konstruktionelle Varianz“ am Institut für Deutsche Sprache entstanden (www.ids-mannheim.de/lexik/PolysemieVarianz/). Für Kommentare zu dem vorliegenden Aufsatz danken wir Anke Holler.

2 Diese Auffassung von Argumentstruktur lehnt sich an ein Konzept von Valenz an, das - wie in Jacobs (1994) - syntaktische und semantische Dimensionen der Valenz unterscheidet. 
dass die Liste der semantischen und die der syntaktischen Beschränkungen lexikalischer Argumentstrukturen oft nicht in lexemspezifischer Weise aufeinander abgebildet werden, sondern wiederkehrende allgemeine Muster konstituieren wie in (1).
a. $\mathrm{X}_{\mathrm{NPnom}} \mathrm{V} \mathrm{Y}_{\mathrm{NPdat}} \mathrm{Z}_{\mathrm{NPakk}}, X$ transferiert $Z$ an $Y^{\mathrm{c}}$ z.B.: er gibt ibr das Buch/sie schickt ibm einen Brief
b. $\mathrm{X}_{\mathrm{NPnom}} \mathrm{V} \mathrm{Y}_{\mathrm{PP} \text { nach }}, X$ versucht $Y$ zu finden/zu erlangen ${ }^{6}$ z.B.: sie forscht nach alten Papieren / sie jagt nach Gold
c. $\mathrm{X}_{\mathrm{NPnom}} \mathrm{V} \mathrm{Y}_{\mathrm{NPakk}} Z_{\mathrm{PP} n a c b}, X$ versucht $Z$ in $Y$ zu finden/zu erlangen z.B. sie durchsucht die Taschen nach Geld/sie bören den Himmel nach Signalen intelligenter Wesen ab
d. $\mathrm{X}_{\mathrm{NPnom}} \mathrm{V}_{\text {folgen }} \mathrm{Y}_{\mathrm{NPdat}}, X$ folgt $Y^{6}$ sie folgte dem Dieb/sie folgen einer Spur
e. $\mathrm{X}_{\mathrm{NPnom}} \mathrm{V}_{\mathrm{V}[\text { Geräusch] }} \mathrm{Y}_{\mathrm{PP}[\mathrm{dir}]}, X$ macht das in $V$ beschriebene Geräusch und bewegt sich entlang $Y^{6}$
z.B.: die Babn quietscht um die Ecke/ der Trecker rumpelt durch die Stadt
f. $\mathrm{X}_{\mathrm{NPnom[Motorrad]}} \mathrm{V}_{\text {knattern }} \mathrm{Y}_{\mathrm{PP} \text { durrbl } / \text { iber }}$, Motorrad knattert und bewegt sich über/durch $Y^{\circ}$
z.B.: das Motorrad knattert durch die Stadt/die Harleys knattern über den Highway
g. $\mathrm{X}_{\mathrm{NPnom}} \mathrm{V}_{\text {reden }} \mathrm{Y}_{\text {Refl-akk }} \mathrm{Z}_{\mathrm{PP} \text { in Rage }}, X$ redet und gerät dadurch in Rage z.B.: der Minister redet sich in Rage / du redest dich in Rage

Die konkreteren solcher Kovorkommen (z.B. $V_{\text {knattern }} \mathrm{Y}_{\mathrm{PP} \text { durrb/uiber }}$ in (1f)) lassen sich meist unmittelbar als statistisch signifikante Kookkurrenzen aus Korpora ermitteln, die kategorial bestimmten argumentstrukturbezogenen Kovorkommen (z.B. $V_{\mathrm{V}[\text { Geräusch }]} \mathrm{Y}_{\mathrm{PP}[\mathrm{dir}]}$ in (1e)) ergeben sich aus Abstraktionen über konkreten Kovorkommen. Solche argumentstrukturbezogenen, kategorial bestimmten und semantisch stabilen Kovorkommen wollen wir im Folgenden als Argumentstrukturmuster (ASM) bezeichnen.

Der Aufsatz befasst sich mit der Frage, wie sich die Identität solcher Argumentstrukturmuster bestimmen lässt und wie sie repräsentiert werden müssen. Der Umgang mit Argumentstrukturen und Argumentstrukturmustern unterscheidet sich je nach sprachtheoretischen Grundannahmen erheblich. Die meisten Grammatiktheorien sind in der einen oder anderen Weise valenz- bzw. subkategorisierungsbasiert und projizieren Argumentstrukturen aus lexikalischen Verbeinträgen. ${ }^{3}$ Die hier als Argumentstrukturmuster bezeichneten nicht-idiosynkratischen Aspekte von Argumentstrukturen werden dabei in verschiedener Weise berücksichtigt. Müller (2006) zeigt am Beispiel von Resultativkonstruktionen, wie diese Aspekte in Form lexikalischer Regeln erfasst werden können. Form-Bedeutungs-Abhängig-

In Jacobs (1992) ist schön zu sehen, wie in einem valenzgrammatischen Ansatz solche Projektionsprinzipien operieren. 
keiten in Argumentstrukturen hat man in verschiedenen Ansätzen auch über die Etablierung von Linking-Prinzipien zu erfassen versucht (z.B. Wunderlich 2006). In radikaleren Ansätzen werden aber auch andere Strategien verfolgt. So wird etwa in der Distribuierten Morphologie (z.B. Harley/Noyer 2000) ganz auf lexikalische Repräsentationen von Argumentstrukturen verzichtet, indem Argumentstrukturen, integriert in Dekompositionen, unmittelbar in der Syntax erzeugt werden. Argumentstrukturen können aber auch als eigenständige syntaktisch-semantische Entitäten betrachtet werden, so wie es die Konstruktionsgrammatik tut, die insofern für die hier Argumentstrukturmuster genannten Kookkurrenzen eigene „Argumentstrukturkonstruktionen" postuliert (Goldberg 1995, 2006). In der traditionell valenztheoretisch geprägten germanistischen Linguistik hat sich in jüngster Zeit eine Debatte entsponnen, die den Platz von Konstruktionen in valenzbasierten Grammatiken zum Gegenstand hat (Jacobs 2008, 2009; Müller 2006; Welke 2009; Handwerker 2008; Engelberg 2007, 2009).

Ziel dieses Aufsatzes ist es, ein Konzept für die Repräsentation von Argumentstrukturmustern zu entwickeln, in dem erstens fein differenzierte Argumentstrukturmuster über ein Netz von Familienähnlichkeiten verbunden sind, und zweitens idiosynkratische lexikalische Kookkurrenzen innerhalb solcher Muster repräsentiert werden können. Der Aufsatz wirft zunächst in Abschnitt 1.2 einen kritischen Blick auf die Praxis konstruktionsgrammatischer Ansätze zur Behandlung von Argumentstrukturmustern. Insbesondere werden wir Widersprüche und konzeptionelle Schwächen des Polysemiekonzepts für Argumentstrukturkonstruktionen aufdecken. Dem stellen wir in Abschnitt 2.1 Überlegungen zu einem Netz von Familienähnlichkeiten zur Repräsentation von Beziehungen zwischen Argumentstrukturmustern entgegen, die wir auf der Basis von zwei sehr unterschiedlichen Familien von Argumentstrukturmustern, den Such-Argumentstrukturmustern (Abschnitt 2.2) und den Argumentstrukturmustern mit inneren Objekten (Abschnitt 2.3) korpusbasiert unterfüttern. Darauf folgend wird zunächst am Beispiel von Bewegungslesarten von Geräuschverben (Abschnitt 3.1), dann anhand von Resultativkonstruktionen (Abschnitt 3.2) und schließlich am Beispiel von lexikalisch stark restringierten propositionalen Mustern (Abschnitt 3.3) gezeigt, dass lexikalische Kookkurrenzen innerhalb eines Argumentstrukturmusters die Repräsentation einer Vielzahl idiosynkratischer Spezifizierungen eines solchen Musters erforderlich machen.

\subsection{Die Identität von Argumentstrukturmustern}

In einer inzwischen schon als klassisch zu bezeichnenden Arbeit definiert Goldberg (1995, S. 4) Konstruktionen wie folgt: 
$\mathrm{C}$ is a CONSTRUCTION iff $_{\text {def }} \mathrm{C}$ is a form-meaning pair $\left\langle\mathrm{F}_{i}, \mathrm{~S}_{i}\right\rangle$ such that some aspect of $\mathrm{F}_{i}$ or some aspect of $\mathrm{S}_{i}$ is not strictly predictable from $\mathrm{C}^{\prime}$ s component parts or from other previously established constructions. ${ }^{4}$

Dabei macht die Konstruktionsgrammatik bekanntermaßen keinen Unterschied zwischen atomaren (nicht komplexen) und komplexen Konstruktionen. Wörter und syntaktische Regelhaftigkeiten konstituieren also gleichermaßen Konstruktionen und werden in einem einheitlichen Format repräsentiert (Goldberg 1995, S. 7). Allgemeine Eigenschaften, die das Inventar atomarer Konstruktionen - also etwa Wörter - strukturieren, sollten daher auch im Inventar komplexer Konstruktionen - also etwa Argumentstrukturkonstruktionen - wirken. Und genau das wird in Goldberg (ebd.) auch angenommen. Zu den inventarstrukturierenden Eigenschaften, die wir aus dem Lexikon kennen, gehören Homonymie und Polysemie. Im Falle von Polysemie werden mehrere Form-Bedeutungseinheiten, deren Formen identisch und deren Bedeutungen verwandt sind, einem Zeichen zugeordnet. Im Falle von Homonymie werden dagegen Form-Bedeutungseinheiten, deren Formen identisch und deren Bedeutungen nicht miteinander verwandt sind, verschiedenen Zeichen zugewiesen. Goldberg beobachtet, dass Polysemie im Lexikon sehr häufig, Homonymie dagegen selten auftritt. Eine solche Verteilung sollte daher auch im Bereich komplexer Konstruktionen zu erwarten sein (Goldberg 2009). Goldberg (1995, S. 33 f.) illustriert dies am Beispiel von Argumentstrukturkonstruktionen, von denen sie annimmt, dass sie typischerweise polysem sind. Sie konstituieren damit ein Zeichen mit mehreren Bedeutungen. ${ }^{5}$ So hat etwa die Ditransitiv-Konstruktion neben der zentralen Transferbedeutung, die durch Verben wie give exemplifiziert wird, eine Reihe weiterer Bedeutungen, u.a. die durch die folgenden Beispiele repräsentierten:
a. Chris baked Jan a cake.
b. Bill promised his son a car.
c. Joe allowed Bill a popsicle.
d. Joe refused Bill a raise in salary.

Jede dieser Lesarten der Ditransitivkonstruktion weist nach Goldberg eine semantische Idiosynkrasie auf und ist auf die zentrale Lesart bezogen. ${ }^{6}$ In (2a) ist der durch die zentrale Lesart gegebene Transfer nicht impliziert, sondern lediglich intendiert, in (2b) ist er in die Glückensbedingungen des

4 In Goldberg (2006, S. 5) wird der Konstruktionsbegriff dahingehend erweitert, dass nicht nur formale oder semantische Idiosynkrasien, sondern auch Gebrauchshäufigkeiten die Existenz von Konstruktionen begründen können.

5 Goldberg spricht von „senses“, wobei wir „sense“ hier im Sinne von „Lesart“ als eine von mehreren miteinander verwandten Bedeutungen eines Zeichens verstehen.

6 Die zentrale Lesart wird dadurch identifiziert, dass sie konkret ist, diachron primär und Grundlage für metaphorische Erweiterungen (Goldberg 1995, S. 33). 
Verbs eingebunden, in (2c) wird er lediglich ermöglicht, und in (2d) negiert (Goldberg 1997, S. 392). Aufgrund der Polysemieannahme sind alle Sätze in (2) Exemplifikationen der gleichen Konstruktion. Gegenüber dem häufigen Auftreten von Polysemie bei Argumentstrukturkonstruktionen sei Homonymie dagegen, so Goldberg (2006, S. 36), bei Argumentstrukturkonstruktionen sehr selten. Als eines der seltenen Beispiele führt sie forAdverbial-Konstruktionen an, die mit den beiden nicht aufeinander bezogenen Bedeutungen in (3) verknüpft sind. Hier haben wir es nach Goldberg also mit zwei verschiedenen Konstruktionen zu tun.

a. The statue stood for three hours.

b. He exchanged the socks for a belt.

Die Annahme konstruktioneller Polysemie beinhaltet allerdings sowohl theorieinterne Widersprüche als auch konzeptionelle Schwächen. Das zentrale Argument Goldbergs (1995, S. 34) für die Zurückführung von Beispielen wie (2a) auf eine eigene Bedeutungsvariante $\left[F, B_{2}\right]$ der Ditransitivkonstruktion basiert darauf, dass die spezifischen Eigenschaften dieser Variante nicht semantisch regelhaft ableitbar oder pragmatisch zu erschließen, sondern bis zu einem gewissen Maße unprädiktabel seien. Die Nicht-Prädiktabilität eines Aspekts eines Form-Bedeutungspaares wie $\left[F, B_{2}\right]$ ist aber gemäß der einleitenden Definition von Konstruktionen das zentrale Kriterium für das Vorliegen einer eigenständigen Konstruktion. Das ist ein offensichtlicher Widerspruch. Will man also Polysemie von Konstruktionen zulassen, muss man auch die Definition von Konstruktionen revidieren und wie folgt rekonstruieren:

\section{Konstruktionsidentität unter der Annahme konstruktioneller Polysemie}

$K$ ist eine Konstruktion $\operatorname{gdw}_{\text {def }}$ gilt: $K$ ist ein Paar $\left[F,<B_{1}, \ldots, B_{n}>\right]$ aus genau einer Form $F$ und einer nicht leeren Liste von Bedeutungen $\left\langle B_{1}, \ldots, B_{n}>\right.$, wobei

(i) mindestens ein Aspekt von $F$ oder mindestens ein Aspekt von jedem $B$ aus $<B_{1}, \ldots, B_{n}>$ nicht strikt aufgrund der konstituierenden Teile von $K$ oder aufgrund anderer, bereits etablierter Konstruktionen prädiktabel ist, und

(ii) jede Bedeutung $B$ aus $<B_{1}, \ldots, B_{n}>$ semantisch auf die zentrale Bedeutung $B_{1}$ bezogen ist.

Kein Paar $[F, B]$ für ein beliebiges $B$ aus $<B_{1}, \ldots, B_{n}>$ ist eine Konstruktion.

Der letzte Punkt ergibt sich daraus, dass ansonsten zwei Paare, $\left[F, B_{2}\right]$ und $\left[F, B_{3}\right]$, eigenständige Konstruktionen wären und aufgrund ihrer Formidentität damit homonym. Und genau das wird ja explizit von Goldberg negiert. 
Schaut man sich nun den weiteren Umgang Goldbergs mit konstruktioneller Polysemie an, so muss man feststellen, dass die von ihr als Lesarten bestimmten Varianten von Konstruktionen tatsächlich oft wie eigenständige Konstruktionen behandelt werden. Das zeigt sich vor allem im Umgang mit den für Konstruktionsgrammatiken üblichen Vererbungsprinzipien, die es erlauben, geteilte Informationen verschiedener Konstruktionen zentral zu repräsentieren und von dort quasi zu verteilen. Vererbung wird dabei aufgefasst als eine Relation zwischen Konstruktionen (Goldberg 1995, S. 73). Bezüglich der Lesarten von Argumentstrukturkonstruktionen wird nun so verfahren, dass die Bedeutung der einzelnen Lesarten mit der zentralen Lesart über so genannte „polysemy links“ verknüpft ist, die die jeweils spezifische Art der semantischen Beziehung beschreiben. Die syntaktischen Eigenschaften der einzelnen Lesarten werden dagegen von der zentralen Lesart vererbt (Goldberg 1995, S. 75). Dass die Lesarten (semantische „extensions") nun plötzlich wie eigenständige Konstruktionen behandelt werden, macht Goldberg dann auch explizit:

Each of the extensions constitutes a minimally different construction, motivated by the central sense; that is, each sense can be represented by a construction that is minimally different from that of the central sense. (Goldberg 1995, S. 76)

Damit werden Form-Bedeutungs-Paare wie $\left[F, B_{2}\right]$ und $\left[F, B_{3}\right]$ nun gleichzeitig als Varianten eines einzelnen polysemen konstruktionellen Zeichens behandelt wie auch als zwei homonyme konstruktionelle Zeichen. Neben diesem Widerspruch hat Goldbergs Auffassung von konstruktioneller Polysemie unseres Erachtens aber auch eine konzeptionelle Schwäche. Diese resultiert aus der Überstrapazierung der Parallelen zwischen atomaren und komplexen Konstruktionen. Goldbergs von uns rekonstruierte Auffassung von Konstruktionsidentität unter der Annahme konstruktioneller Polysemie fordert strikte Formidentität der Form-Bedeutungspaare, aber keine strikte Bedeutungsidentität. Die Ditransitivkonstruktion konstituiert also in ihren semantischen Varianten genau ein abstraktes Zeichen und nicht mehrere. Dagegen führt Bedeutungsidentität bei divergierenden Formen nicht zu Konstruktionsidentität, sondern zur Annahme von verschiedenen synonymen bzw. semantisch eng verwandten Konstruktionen. ${ }^{7}$ Man mag sich fragen, warum das so ist. Schauen wir uns Fälle von Synonymie bzw. enger Bedeutungsverwandtschaft im Lexikon an, so stellen wir fest, dass synonyme Zeichen wie Orange und Apfelsine gewöhnlich in ihrer Form stark divergieren. Das motiviert bei aller Bedeutungsgleichheit oder - in anderen Fällen - enger Bedeutungsverwandtschaft die Annahme, dass es sich um unterschiedliche Zeichen handelt. Nun ist im Bereich von Argumentstruk-

Goldberg (1995, S. 67) nimmt ein „Principle of No Synonymy“ an, demzufolge es keine echte Synonymie gibt. Das im Folgenden angeführte Argument gilt aber auch, wenn verschiedene Formen nicht die gleiche, aber doch eine sehr ähnliche Bedeutung haben. 
turkonstruktionen allerdings manchmal festzustellen, dass synonyme bzw. semantisch eng verwandte Konstruktionen anders als im Fall von Orange und Apfelsine formal nur partiell differieren.

a. Sie untersuchten die Daten nach Schlüsselwörtern.

b. Sie untersuchten die Daten auf Schlüsselwörter.

Die beiden zugrundeliegenden Argumentstrukturmuster unterscheiden sich nur an einer Stelle, nämlich in der Wahl der Präposition: ${ }^{8}$

a. $\mathrm{X}_{\mathrm{NPnom}} \mathrm{V} \mathrm{Y}_{\mathrm{NPakk}} Z_{\mathrm{PP} \text { nacb }}, X$ versucht $Z$ in $Y$ zu erlangen

b. $\mathrm{X}_{\mathrm{NPnom}} \mathrm{V} \mathrm{Y}_{\mathrm{NPakk}} Z_{\mathrm{PP} a u f}, X$ versucht $Z$ in $Y$ zu erlangen ${ }^{6}$

Da die beiden Muster auch in der Bedeutung kaum differieren, ${ }^{9}$ stellt sich hier unter der Annahme, dass Konstruktionen prinzipiell mehrere Varianten in sich vereinigen können, die Frage, warum ein Goldbergscher Ansatz es nicht zulässt, (5a) und (5b) als formale Varianten einer Konstruktion aufzufassen.

Wir haben gesehen, dass Goldbergs Zeichenkonzept in Bezug auf Argumentstrukturkonstruktionen durch Widersprüche gekennzeichnet ist. Ein und dieselbe partiell idiosynkratische Form-Bedeutungseinheit wird einmal als Konstruktion in homonymer Relation zu anderen Konstruktionen aufgefasst, einmal als Lesart einer Konstruktion in Relation zu anderen Lesarten. Zudem erscheint die Polysemieannahme auch dahingehend ad hoc, dass Form-Bedeutungseinheiten mit gleichen Formen und ähnlichen Bedeutungen einer Konstruktion zugeordnet werden, solche mit gleichen Bedeutungen und ähnlichen Formen aber verschiedenen Konstruktionen. Beides lässt das Polysemiekonzept von Konstruktionen fragwürdig erscheinen.

Wir wollen daher im Folgenden auf der Basis empirischer Korpusstudien zeigen, dass Varianten von Argumentstrukturmustern sich eher als ein Netz von Familienähnlichkeiten zwischen granular modulierten einzelnen Argumentstrukturmustern konstituieren denn als ein hierarchisierendes Polysemiekonzept.

\section{Familienähnlichkeiten bei Argumentstrukturmustern}

\subsection{Familienähnlichkeiten statt konstruktioneller Polysemie}

Angelehnt an Wittgensteins (2001) Konzept von Familienähnlichkeiten konzipieren wir Strukturen innerhalb der Menge von Argumentstruk-

Vgl. die Belege in Proost (2009).

Es sei dahingestellt, ob die beiden Varianten synonym sind oder geringfügige Bedeutungsdifferenzen aufweisen. Sie stehen sich semantisch aber sicherlich näher als die Varianten der Ditransitivkonstruktion. 
turmustern als Familien. Dabei gehen wir davon aus, dass eine Familie sich als ein Netz von Familienmitgliedern konstituiert, die anderen, aber nicht allen Mitgliedern der Familie hinreichend ähnlich sein müssen.

\section{Familienähnlichkeit bei Argumentstrukturmustern}

Für $\mathrm{R}$ als eine Relation hinreichender Ähnlichkeit bezüglich der Werte einer Menge von Attributen $\{\alpha, \beta, \gamma, \ldots\}$ und A als eine Menge von $n$ Argumentstrukturmustern gilt:

$A$ ist in Bezug auf $R$ eine Familie, $\mathfrak{F}^{A R}$, gdw. es für jede Teilmenge $A^{T} \subset A$ und ihre Komplementmenge ${ }^{10} \mathrm{~A}^{\mathrm{K}} \subset \mathrm{A}$ mindestens ein Paar von Argumentstrukturmustern, $a^{t} \in A^{T}$ und $a^{k} \in A^{K}$, gibt, die in einer Relation hinreichender Ähnlichkeit, $\mathrm{R}\left(\mathrm{a}^{\mathrm{t}}, \mathrm{a}^{\mathrm{k}}\right)$, stehen.

Die Ähnlichkeitsrelation $\mathrm{R}$ wird in Bezug auf bestimmte Attribute definiert, in unserem Fall also etwa die Anzahl der Argumente in einem Muster, die formalen Eigenschaften der Argumentstellen in den einzelnen Mustern, die semantischen Eigenschaften zur Charakterisierung der Muster etc. Wann zwei Argumentstrukturmuster in Bezug auf die Werte dieser Attribute als hinreichend ähnlich gelten, lässt diese Definition offen. Das ist gesondert zu operationalisieren.

Die formulierte Bedingung für Familienähnlichkeit stellt zum einen sicher, dass jedes Argumentstrukturmuster mindestens einem anderen Muster der Familie ähnlich ist. Das ergibt sich daraus, dass (i) jedes einzelne Argumentstrukturmuster eine Teilmenge konstituiert und dieses dann zu einem Muster in der Komplementmenge in Beziehung steht und (ii) in jeder Teilmenge, die mehrere einander ähnliche Argumentstrukturmuster enthält, wiederum mindestens ein Argumentstrukturmuster eine Ähnlichkeitsbeziehung ins Komplement dieser Teilmenge unterhält. Die Bedingung stellt damit sicher, dass alle Argumentstrukturmuster in das Ähnlichkeitsnetz eingebunden sind, das die Familie konstituiert. Wäre lediglich verlangt, dass jedes Muster in A zu mindestens einem anderen Muster in A in einer Ähnlichkeitsbeziehung steht, so wäre diese Bedingung in einer Menge von 4 Mustern schon durch $\mathrm{R}\left(\mathrm{a}^{1}, \mathrm{a}^{2}\right)$ und $\mathrm{R}\left(\mathrm{a}^{3}, \mathrm{a}^{4}\right)$ erfüllt. Das ist zur Konstituierung einer Familie aber sicherlich nicht hinreichend, denn $a^{1}$ und $a^{2}$ einerseits und $\mathrm{a}^{3}$ und $\mathrm{a}^{4}$ andererseits sind nun ihrerseits nicht mehr miteinander verbunden. Der Umweg über die Partitionierung von A in sämtliche mögliche Teilmengen und ihre Komplemente verhindert das.

Die Relation $R$ ist hier als binäres kategoriales Merkmal \pm HINREICHEND_ÄHNLICH konzipiert. Als solches ist sie reflexiv, da jedes Argumentstrukturmuster sich natürlich selbst hinreichend ähnlich ist, und sym-

${ }_{10} \quad \mathrm{~A}^{\mathrm{T}}$ und $\mathrm{A}^{\mathrm{K}}$ sind also disjunkt, d.h. ihre Schnittmenge ist leer, und sie schöpfen A aus, d.h. ihre Vereinigungsmenge ist gleich A. 
metrisch, da dann, wenn ein Argumentstrukturmuster a ${ }^{1}$ einem Muster $\mathrm{a}^{2}$ ähnlich ist, umgekehrt $\mathrm{a}^{2}$ auch $\mathrm{a}^{1}$ ähnlich ist. $\mathrm{R}$ ist aber nicht transitiv. ${ }^{11} \mathrm{Das}$ heißt, wenn $\mathrm{a}^{1}$ und $\mathrm{a}^{2}$ hinreichend ähnlich sind und $\mathrm{a}^{2}$ und $\mathrm{a}^{3}$ ebenfalls, so folgt daraus nicht, dass auch $\mathrm{a}^{1}$ und $\mathrm{a}^{3}$ hinreichend ähnlich sind. Das ergibt sich daraus, dass die Ähnlichkeit zwischen $\mathrm{a}^{1}$ und $\mathrm{a}^{2}$ auf anderen Attributen basieren kann als die zwischen $\mathrm{a}^{2}$ und $\mathrm{a}^{3}$. So ist (6a) (6b) semantisch hinreichend ähnlich, und (6b) ist (6c) formal hinreichend ähnlich. Daraus folgt aber nicht, dass (6a) und (6c) einander hinreichend ähnlich sind.
a. $\quad \mathrm{X}_{\mathrm{NPakk}} \mathrm{V}, \mathrm{X}$ macht die in $V$ ausgedrückte psychische Erfahrung (z.B. ihn friert)
b. $\mathrm{X}_{\mathrm{NPnom}} \mathrm{V}, X$ macht die in $V$ ausgedrückte psychische Erfahrung (z.B. er friert)
c. $\mathrm{X}_{\mathrm{NPnom}} \mathrm{V}, \mathrm{X}$ führt die in $V$ ausgedrückte psychische Handlung aus ${ }^{6}$
(z.B. er denk.t nach)

Die Nicht-Transitivität der Ähnlichkeitsbeziehung ist unserer Auffassung nach ein wichtiger Bestandteil eines Wittgensteinschen Konzepts von Familienähnlichkeiten, das nur sehr abstrakte notwendige Bedingungen für die Familienzugehörigkeit verlangt - in dem hier vorliegenden Fall lediglich, dass alle Familienmitglieder Argumentstrukturmuster sind. Die Nicht-Transitivität der Ähnlichkeitsbeziehung beinhaltet, dass es darüber hinaus keine weiteren Eigenschaften gibt, die notwendigerweise von allen Familienmitgliedern geteilt werden.

Das vorgestellte Konzept von hinreichender Ähnlichkeit entspricht als kategoriales Konzept natürlich nicht einem intuitiven Konzept von Ähnlichkeit als gradueller Eigenschaft. Ein Familienähnlichkeitskonzept für Argumentstrukturmuster könnte natürlich auch auf einer ordinalen Konzeption von Ähnlichkeit beruhen, in der auf der Basis der Anzahl der Übereinstimmungen in den Attributwerten zu $\{\alpha, \beta, \gamma, \ldots\}$ Stufen von Ähnlichkeit unterschieden werden. Diese Abstufung könnte gegebenenfalls durch die unterschiedliche Gewichtung einzelner Merkmale ergänzt werden.

Über die Menge der Ähnlichkeitsrelationen zu anderen Mitgliedern der Familie und gegebenenfalls über die Stärke dieser Ähnlichkeitsrelationen können schließlich zentrale Muster innerhalb einer Familie von Argumentstrukturmustern ermittelt werden. Letztlich lässt das vorgestellte Konzept erwarten, dass sich alle Argumentstrukturmuster in einer großen Familie wiederfinden werden. Rund um die zentralen Muster werden sich eng miteinander verwandte Argumentstrukturmuster zu clusterähnlichen Teilfamilien gruppieren.

$11 \quad$ Reflexivität: $\mathrm{R}\left(\mathrm{a}^{1}, \mathrm{a}^{1}\right)$. Symmetrie: $\mathrm{R}\left(\mathrm{a}^{1}, \mathrm{a}^{2}\right) \Rightarrow \mathrm{R}\left(\mathrm{a}^{2}, \mathrm{a}^{1}\right)$. Transitivität: $\mathrm{R}\left(\mathrm{a}^{1}, \mathrm{a}^{2}\right) \& \mathrm{R}\left(\mathrm{a}^{2}, \mathrm{a}^{3}\right) \Rightarrow$ $\mathrm{R}\left(\mathrm{a}^{1}, \mathrm{a}^{3}\right)$. 
Ein Beispiel für eine über Familienähnlichkeiten strukturierte Menge von Argumentstrukturmustern findet sich im Anhang. Dort werden die Ähnlichkeiten innerhalb der Familie der Such-Argumentstrukturmuster, die im folgenden Abschnitt 2.2 ausschnittsweise näher beschrieben wird, tabellarisch und als Abbildung dargestellt.

Es sei hier darauf hingewiesen, dass auch Goldberg den Terminus ,Familienähnlichkeit` für das Beziehungsgefüge von Argumentstrukturkonstruktionen für sich beansprucht:

[...] we have treated resultatives as a sort of ,family' of constructions (we might call them subconstructions of the resultative), sharing important properties but differing in certain specifics, including their degree of productivity. By, family' we have in mind the sort of family resemblances recognized to exist in non-linguistic categories (Rosch \& Mervis 1975, Wittgenstein 1955). Our strategy here is first to establish a taxonomy of the subconstructions, then to state which of their properties can be explained by the construction as a whole, and finally pick out some of the differences. (Goldberg/Jackendoff 2004, S. 535 f.)

Ein Unterschied zu unserem Konzept ergibt sich hier daraus, dass Goldberg und Jackendoff auf der einen Seite von Familienähnlichkeiten im Sinne Wittgensteins sprechen, auf der anderen Seite jedoch (i) die Notwendigkeit bestimmter, sehr eng gefasster, „important properties“ konstatieren, die allen Familienmitgliedern gemein sein müssen, und (ii) zudem die Konstruktionen in einer ,taxonomy of the subconstructions" organisieren. Insbesondere Letzteres entspricht allerdings gerade nicht dem Begriff der Familienähnlichkeit bei Wittgenstein, der auf der Annahme beruht, dass die Mitglieder einer Familie eben nicht notwendig offensichtliche zentrale Eigenschaften miteinander teilen, sondern in feineren Netzen der oben beschriebenen Art miteinander verknüpft sind. Gerade der Schwierigkeit der taxonomischen Erfassung und Hierarchisierung von Begriffen ist Wittgensteins Ansatz der Familienähnlichkeit geschuldet, der sich eben solcher Mengen von Ausdrücken annimmt, die unklare Grenzen aufweisen. Das im vorliegenden Aufsatz beschriebene Konzept von Familienähnlichkeit unterscheidet sich jedenfalls in dieser Hinsicht von dem Konzept, das Goldberg/Jackendoff (2004) entwickeln.

In den folgenden beiden Abschnitten werden wir zwei sehr unterschiedliche Familien von Argumentstrukturmustern auf der Basis von Korpusdaten näher betrachten. Sie sollen das Konzept einer granularen Auffassung von Argumentstrukturmustern illustrieren, die in ihren formalen und inhaltlichen Ähnlichkeiten Familien im oben beschriebenen Sinn konstituieren. ${ }^{12}$

12 Die Daten entstammen alle dem unter COSMAS II zugänglichen Deutschen Referenzkorpus am Institut für Deutsche Sprache (www.ids-mannheim.de/kl/projekte/korpora/). 


\subsection{Die Familie der Such-Argumentstrukturmuster}

Such-Argumentstrukturmuster sind Argumentstrukturmuster, mit denen ausgedrückt wird, dass der Subjektreferent den Referenten der in der PP eingebetteten NP zu finden, zu erlangen oder zu ermitteln versucht (vgl. Die Touristen suchen nach ibren Reisedokumenten. Der Jurist forscht nach Motiven für die Tat.). Insgesamt lassen sich vier Typen von Such-Argumentstrukturmustern unterscheiden: (i) Such-Argumentstrukturmuster mit Bezug auf nicht-mentale, nicht-sprachliche Handlungen, (ii) Such-Argumentstrukturmuster mit Bezug auf mentale Handlungen, (iii) Such-Argumentstrukturmuster mit Bezug auf sprachliche Handlungen und (iv) Such-Argumentstrukturmuster mit Bezug auf Einstellungen. Da jedem dieser vier Typen von Such-Argumentstrukturmustern verschiedene Varianten zugeordnet werden können, entsprechen den Typen (i)-(iv) vier Subfamilien der Familie der Such-Argumentstrukturmuster. Jedes Such-Argumentstrukturmuster kann insofern als separates Argumentstrukturmuster betrachtet werden, als es der Definition für Argumentstrukturmuster genügt:

\section{Argumentstrukturmuster}

Eine Kombination von überzufällig stark kookkurrierenden sprachlichen Einheiten ist genau dann ein Argumentstrukturmuster, wenn (i) diese Einheiten aus einem verbalen Element und seinen Argumenten bestehen, (ii) mindestens eine dieser Einheiten nicht durch lexikalische Elemente, sondern durch kategoriale semantische und/oder syntaktische Spezifizierungen gekennzeichnet ist, und (iii) mindestens ein formaler oder semantischer Aspekt dieser Spezifizierung nicht aus allgemeinen Festlegungen der Grammatik (Syntax/Morphologie/Semantik/Pragmatik) oder des Lexikons hergeleitet werden kann.

Die Nicht-Prädiktabilität von Aspekten eines Argumentstrukturmusters kann dabei die Realisierungsform und Anzahl der Argumente, die Bedeutung des Musters oder die Klassen von Verben betreffen, die in ihm auftreten. Die Definition erlaubt die Unterscheidung abstrakter von konkreten Argumentstrukturmustern durch den Grad der Generalität der kategorialen Beschreibung.

Im Folgenden wird am Beispiel einiger ausgewählter Such-Argumentstrukturmuster gezeigt, dass diese bestimmte Idiosynkrasien aufweisen, die dafür sprechen, sie als separate Argumentstrukturmuster anzusehen. Argumentstrukturmuster werden dabei als Paare bestehend aus einer Form F und einer Bedeutung B dargestellt, so dass beispielsweise das Muster ASM1 als eine Verbindung einer Form1, $\mathrm{F}_{1}$, und einer Bedeutung1, $\mathrm{B}_{1}$, ASM2 als eine Verbindung von $\mathrm{F}_{2}$ und $\mathrm{B}_{2}$ usw. repräsentiert wird. Insgesamt lassen sich 13 Such-Argumentstrukturmuster unterscheiden. In diesem Beitrag werden nur 8 von diesen diskutiert; die übrigen sind in der Übersichtsgra- 
fik im Anhang aufgeführt. (Die Nummerierung der im Text diskutierten Such-Argumentstrukturmuster bezieht sich auf die Nummerierung in dieser Übersichtsgrafik.)

\subsubsection{Such-Argumentstrukturmuster mit Bezug auf nicht-mentale, nicht-sprachliche Handlungen}

Das semantisch zentrale Muster der Familie der Such-Argumentstrukturmuster stellt das Muster ,nach etwas suchen' (ASM1) dar. Es stellt semantisch den Ausgangspunkt für die Konstitution der Familie dar und nimmt Verben (wie z.B. suchen) auf, die der Argumentstrukturmusterbedeutung sehr nahe stehen. Es steht nicht in einer übergeordneten taxonomischen Relation zu den anderen Argumentstrukturmustern der Familie. ASM1 besteht in formaler Hinsicht aus einem Verb, einer NP im Nominativ und einer PP mit nach und hat die Bedeutung , $X$ versucht, $Y$ durch Ausführen der Handlung $V$ zu finden, zu erlangen, zu ermitteln', vgl.:

ASM1: nach etwas suchen

$\mathrm{F}: \mathrm{X}_{\mathrm{NPnom}} \mathrm{V} \mathrm{Y}_{\mathrm{PP} \text { nacb }}$

B: $X$ versucht, $Y$ durch Ausführen der Handlung $V$ zu finden, zu erlangen, zu ermitteln.

Das Muster ASM1 wird durch den folgenden Beleg aus den IDS-Korpora exemplifiziert:

(7) [Die Verlage, die überleben wollen $]_{\mathrm{X}}$, [suchen $]_{\mathrm{V}}$ händeringend [nach literarischer Ware $]_{Y}$ (Der Spiegel, 17.01.1994; ERZÄHLER MÜSSEN HER [S. 147])

ASM1 ist in semantischer Hinsicht zunächst insofern unprädiktabel, als es nur mit Verben bestimmter semantischer Klassen vorkommt (Such-Verben, Verben wie graben, Bewegungsverben, Verben wie kratzen, Wahrnehmungsverben und Verben des Anstrengens und Bemühens) und nicht beispielsweise mit Herstellungsverben wie bauen oder mit Verben, mit denen auf mentale Aktivitäten Bezug genommen wird, wie etwa lesen. Denkbare Äußerungen wie z.B. Er baut nach einem neuen Haus oder Sie liest nach einer Lösung kommen in den Korpora nicht vor. Dass durch die Verbindung des ASM1 mit Verben der oben erwähnten Klassen die Interpretation, suchen ${ }^{\circ}$ bzw. , auf eine bestimmte Art und Weise suchen' evoziert wird (vgl. nach neuen Klamotten schauen bzw. nach Zeichen von Verscbütteten lauschen), ist ebenfalls unprädiktabel. Ähnlich argumentiert Goldberg mit Bezug auf die Ditransitiv-Konstruktion, dass es keinen erkennbaren Grund dafür gibt, warum diese Konstruktion nur mit Verben bestimmter semantischer Klassen vor- 
kommt und warum durch ihre Verbindung mit Verben bestimmter semantischer Klassen eine ganz bestimmte Interpretation entsteht (vgl. Goldberg 1995, S. 34). Über die bereits erwähnten Gründe hinaus ist ASM1 auch deswegen unprädiktabel, weil es innerhalb der Klassen von Verben, die dieses Muster prinzipiell erlauben, immer auch eine beträchtliche Anzahl von Verben gibt, die in den Korpora nicht mit ASM1 vorkommen (z.B. ergründen und eruieren in der Klasse der Such-Verben, gaffen und kosten in der Klasse der Wahrnehmungsverben, wetzen und eilen in der Klasse der Bewegungsverben usw. (vgl. Proost 2009, S. 17 f.).

Das Argumentstrukturmuster ASM2 (,auf etwas angeln') unterscheidet sich nur darin von ASM1, dass das Argument der Entität, die der Subjektreferent zu erlangen versucht, nicht als PP mit nach, sondern als PP mit auf realisiert wird. Dementsprechend kann dieses Muster folgendermaßen dargestellt werden: ${ }^{13}$

ASM2: auf etwas angeln

$\mathrm{F}: \mathrm{X}_{\mathrm{NPnom}} \mathrm{V} \mathrm{Y}_{\mathrm{PP} \text { auf }}$

B: $X$ versucht, $Y$ durch Ausführen der Handlung $V$ zu erlangen.

ASM2 wird durch Belege wie (8) illustriert:

(8) Eigentlich [hat $]_{\mathrm{V}}[\text { Frank Pauli }]_{\mathrm{X}}[\text { auf Aal }]_{\mathrm{Y}}[\text { geangelt }]_{\mathrm{V}}$, stand im Mondlicht geduldig am Neckar-Schifffahrtskanal und wartete auf einen dicken Fisch, der wollte jedoch nicht anbeißen. (Mannheimer Morgen, 01.07.2004; Mondschein-Geschichte mit Haaren und Haken)

Da ASM2 sich in formaler Hinsicht von ASM1 unterscheidet und im Gegensatz zu diesem nur mit einigen wenigen Verben (angeln, fischen, jagen und gehen) vorkommt, stellt es ein eigenes Argumentstrukturmuster dar. Unprädiktabel ist vor allem, warum die anderen Such-Verben, die mit ASM1 auftreten, nicht als Teil von ASM2 vorkommen.

Eine dritte Variante der Such-Argumentstrukturmuster, das Muster ASM3 (etwas nach etwas durchsuchen'), unterscheidet sich sowohl formal als auch im Hinblick auf seine Bedeutung von den beiden bereits erwähnten Such-Argumentstrukturmustern. Zusätzlich zu den beiden Argumenten, die auch ASM1 und ASM2 enthalten (derjenige, der sucht, und die gesuchte Entität) enthält das Muster ASM3 das Argument der Entität, in der gesucht wird. Dementsprechend hat ASM3 die Bedeutung, $X$ versucht, $Y$ durch Ausführen der Handlung $V$ in $Z$ zu finden, zu erlangen, zu ermitteln'. ASM3 kann folgendermaßen als ein Form-Bedeutungspaar dargestellt werden:

13 Es ist außerdem zu beobachten, dass das in die PP eingebettete Substantiv in manchen Fällen im Singular artikellos steht. 
ASM3: etwas nach etwas durchsuchen

$\mathrm{F}: \mathrm{X}_{\mathrm{NPnom}} \mathrm{V} \mathrm{Z}_{\mathrm{NPakk}} \mathrm{Y}_{\mathrm{PP} \text { nach }}$

B: $X$ versucht, $Y$ durch Ausführen der Handlung $V$ in $Z$ zu finden, zu erlangen, zu ermitteln.

ASM3 wird durch Belege wie (9) illustriert:

(9) Am Sonntag brachen unbekannte Täter ebenfalls tagsüber in eine Erdgeschoßwohnung in der Welserstraße ein. [Sie $]_{\mathrm{X}}$ [durchsuchten $]_{\mathrm{V}}$ [alles $]_{Z}[\text { nach Wertgegenständen }]_{Y}$. (Mannheimer Morgen, 07.05.1996, Lokales; Hundehalterin nach Unfallflucht gesucht)

Obwohl man die Bedeutung von ASM3 als eine Erweiterung der Bedeutung von ASM1 (,nach etwas suchen') ansehen könnte, ist die Tatsache, dass dieses Argumentstrukturmuster ein zusätzliches Argument in einer bestimmten semantischen Rolle enthält und sich damit formal von ASM1 und ASM2 unterscheidet, ein Grund, es als ein eigenständiges Argumentstrukturmuster zu betrachten.

Das Argumentstrukturmuster ASM4 (,etwas auf etwas durchsuchen) unterscheidet sich nur darin von ASM3 (,etwas nach etwas durchsuchen), dass das Argument der gesuchten Entität nicht als PP mit nach, sondern als PP mit auf realisiert wird, vgl.:

ASM4: etwas auf etwas durchsuchen

$\mathrm{F}: \mathrm{X}_{\mathrm{NPnom}} \mathrm{V} \mathrm{Z}_{\mathrm{NPakk}} \mathrm{Y}_{\mathrm{PP} \text { auf }}$

B: $X$ versucht, $Y$ durch Ausführen der Handlung $V$ in $Z$ zu finden, zu erlangen, zu ermitteln.

ASM4 ist durch das folgende Beispiel belegt:

(10) Wer dennoch eine Impfung scheut, $[\mathrm{der}]_{\mathrm{X}}[\text { sollte }]_{\mathrm{V}}$ wenigstens geschlossene Kleidung tragen und [seine Sachen $]_{Z}$ zuhause nach der Tour [auf Zecken] $]_{\mathrm{Y}}$ [durchsuchen] $]_{\mathrm{V}}$. (Frankfurter Rundschau, 04.06. 1999, S. 36, Ressort: HESSEN; Zwei Krankheiten und ihre Verbreitungsgebiete)

Das Argumentstrukturmuster ist insofern unprädiktabel, als es nicht mit allen Verben vorkommt, die als Teil von ASM3 auftreten können. Durchwühlen kann beispielweise als Teil von ASM3, aber nicht als Teil von ASM4 vorkommen. 


\subsubsection{Such-Argumentstrukturmuster mit Bezug auf mentale Handlungen}

Während die Argumentstrukturmuster ASM1-ASM4 ausdrücken, dass der Subjektreferent den Referenten der in der PP eingebetteten NP zu finden, zu erlangen oder zu ermitteln versucht, indem er eine bestimmte Handlung ausführt, drücken die Muster ASM6 (,nach etwas sinnen ) und ASM7 (,auf Rache/Revanche/Vergeltung sinnen') aus, dass der Subjektreferent den Referenten der in der PP eingebetteten NP durch Ausführen einer mentalen Handlung zu ermitteln versucht. Das Argumentstrukturmuster ASM6, das durch Beispiel (11) illustriert ist, kann folgendermaßen dargestellt werden:

ASM6: nach etwas sinnen

F: $X_{\text {NPnom }} V Y_{\text {PP nach }}$

B: $X$ versucht, $Y$ durch Ausführen der mentalen Handlung $V$ zu ermitteln.

(11) Olaf Nicolai freute sich, dass seine raumfüllende „Flamme der Revolution, liegend (in Wolfsburg)" noch einmal aufgestellt wurde. Ohne Sponsoren und das Engagement der Städtischen Galerie wäre das nicht möglich gewesen, und deshalb $[\mathrm{sann}]_{\mathrm{V}}[\mathrm{er}]_{\mathrm{X}}$ gestern schon [nach Ideen, wie er der Galerie etwas Gutes tun könnte] ${ }_{Y}$. (Braunschweiger Zeitung, 02.03.2007; Zum fröhlichen Künstler-Bahnhof im Schloss)

ASM6 ist insofern idiosynkratisch, als es zwar mit Verben des Denkens (brüten, grübeln, rätseln, sich den Kopf / das Hirn zermartern, sinnen und sinnieren), nicht aber mit denken und überlegen vorkommt. Dass durch die Verbindung der Verben dieser Klasse mit dem Muster ASM6 die Interpretation ,durch Überlegen suchen' hervorgerufen wird, ist ebenfalls nicht vorhersagbar.

ASM7 (,auf Rache/Revanche/Vergeltung sinnen') (vgl. Beleg (12)) ist eine idiomatische Spezialisierung von ASM6 (,nach etwas sinnen). In formaler Hinsicht unterscheidet es sich darin von Letzterem, dass das Argument der gesuchten Entität als PP mit auf realisiert wird und die Menge der in der PP eingebetteten nominalen Ausdrücke auf einige wenige Nomina wie Rache, Revanche und Vergeltung beschränkt ist. Dementsprechend ist die Bedeutung von ASM7 spezifischer als die von ASM6. Das Argumentstrukturmuster ASM7 kann als die folgende Verbindung von Form und Bedeutung dargestellt werden: 
ASM7: auf Rache/Revanche/Vergeltung sinnen

F: $\mathrm{X}_{\mathrm{NPnom}} \mathrm{V} \mathrm{Y}_{\mathrm{PP} \text { auf }}$

B: $X$ versucht, $Y$ (eine Möglichkeit der Rache) durch Ausführen der mentalen Handlung $V$ zu ermitteln.

(12) Das Sozialzentrum habe ihm die Wohnung gekündigt und ihn nicht weiter beschäftigen wollen. [Der psychisch labile Mann $]_{\mathrm{X}}[\text { habe }]_{\mathrm{V}}$ deshalb [auf Rache] $]_{\mathrm{Y}}$ [gesonnen] $]_{\mathrm{V}}$. (Berliner Zeitung, 31.08.2004; ExMitarbeiter steckte jüdisches Zentrum an [S. 6])

Obwohl die Bedeutung von ASM7 als eine Spezialisierung der Bedeutung von ASM6 betrachtet werden kann, hat ASM7 aufgrund seiner formalen Idiosynkrasien den Status eines eigenständigen Argumentstrukturmusters.

\subsubsection{Such-Argumentstrukturmuster mit Bezug auf sprachliche Handlungen}

Im Gegensatz zu den Mustern ASM6 und ASM7, die Bezug auf mentale Handlungen haben, drückt das Muster ASM9 (,nach etwas schreien') aus, dass der Subjektreferent den Referenten der in der PP eingebetteten NP für sich oder für eine dritte Person zu erlangen versucht, indem er einen impliziten Adressaten dazu auffordert, etwas zu tun, um das Gewünschte zu beschaffen. Form und Bedeutung von ASM9 können folgendermaßen dargestellt werden:

ASM9: nach etwas schreien

$\mathrm{F}: \mathrm{X}_{\mathrm{NPnom}} \mathrm{V} \mathrm{Y}_{\mathrm{PP} \text { nacb }}$

B: $X$ versucht, $Y$ zu erlangen, indem er einen impliziten Adressaten mit der sprachlichen Handlung $V$ dazu auffordert, etwas zu tun, damit $X$ oder eine 3. Person $Y$ bekommt.

ASM9 wird durch Belege wie (13) instanziiert:

(13) Wirtschafts- und Finanzbehörde lehnen ein solches staatliches Sponsoring aus grundsätzlichen Erwägungen ab, der Senat mag ebenfalls nicht einsehen, daß [ein privatwirtschaftliches Unternehmen] $]_{X}$ immer dann [nach staatlicher Hilfe $]_{\mathrm{Y}}\left[\right.$ schreit $_{\mathrm{V}}$, wenn's ökonomisch nicht so prächtig funktioniert hat. (die tageszeitung, 13.04.1991, S. 28; Kein Herz für den Hamburger SV)

Das Vorhandensein eines impliziten Adressaten als Komponente der Bedeutung von ASM9 stellt eine semantische Idiosynkrasie dar, die dafür spricht, ASM9 als ein eigenes Argumentstrukturmuster innerhalb der Familie der Such-Argumentstrukturmuster zu betrachten. 


\subsubsection{Such-Argumentstrukturmuster mit Bezug auf Einstellungen}

Schließlich könnten auch Argumentstrukturmuster wie ASM13 (auf etwas hoffen) (vgl. Beleg (14)) zur Familie der Such-Argumentstrukturmuster gerechnet werden. Im Gegensatz zu allen bereits diskutierten Mustern haben solche wie ASM13 Bezug auf Einstellungen. Obwohl ASM13 den Mustern ASM1-ASM10 formal sehr ähnlich ist, unterscheidet es sich im Hinblick auf seine Bedeutung deutlich von ihnen. Semantisch ist ASM13 den bereits diskutierten Mustern nur insofern ähnlich, als der Subjektreferent den Referenten der in der PP eingebetteten NP antizipiert, ohne selber dabei handelnd in Erscheinung zu treten. ASM13 kann als die folgende Verbindung von Form und Bedeutung dargestellt werden:

ASM13: auf etwas hoffen

F: $\mathrm{X}_{\mathrm{NPnom}} \mathrm{V} \mathrm{Y}_{\mathrm{PPauf}}$

B: $X$ antizipiert $Y$ in der durch $V$ ausgedrückten Weise.

(14) „Was ist das gemeinsame von Gasometer und Aktentasche?" [Der Ausbildungsplatzanwärter, der diese Frage beim Intelligenz-Struktur-Test im Rahmen seiner Bewerbung richtig beantwortet $]_{\mathrm{X}}$, [kann $]_{\mathrm{V}}$ [auf Beschäftigung $]_{\mathrm{Y}}$ [hoffen $]_{\mathrm{V}}$. (die tageszeitung, 24.10.1986, S. 9; Bildungspolitische Notizen)

ASM13 kommt nur mit einigen wenigen Verben (boffen und warten) vor.

Die beobachteten Idiosynkrasien der Muster ASM1-ASM13 haben gezeigt, dass diese nach der Definition der Identität von Argumentstrukturmustern separate Argumentstrukturmuster sind. Da keine der Bedeutungen $B_{1}-B_{13}$ als zentrale Lesart angesehen werden kann, auf die alle anderen Bedeutungen bezogen werden könnten, können die Muster ASM1ASM13 nicht als Varianten einer einzigen polysemen Konstruktion betrachtet werden. Stattdessen strukturieren sich die Muster in Form von Familienähnlichkeiten, so wie es in der Tabelle und den Grafiken im Anhang dargestellt ist. Tabelle 1 zeigt, welche semantischen und formalen Ähnlichkeiten zwischen Such-ASM1-13 bestehen. Die Ähnlichkeiten wurden jeweils für ein Paar von Such-ASM (ASMx-ASMy, vgl. Spalte 1) aufgeführt. Als semantische Ähnlichkeiten wurden das Vorkommen beider ASM in der gleichen Subfamilie sowie ihre Übereinstimmung im Hinblick auf die Anzahl ihrer Argumente (beide 2 Argumente vs. beide 3 Argumente) berücksichtigt (Spalten 2-4). Als formale Ähnlichkeiten zählten die Übereinstimmung der beiden ASM hinsichtlich ihrer Präposition (beide nach, beide auf oder beide um) sowie die Übereinstimung der in ihnen vorkommenden phrasalen Kategorien (d.h. ob beide eine NPnom und/oder beide eine NPakk enthalten) (vgl. Spalten 5-9). Übereinstimmung zwischen zwei ASM in Bezug auf 
eines oder mehrere dieser Merkmale wurde mit „,““ markiert. Aus der Anzahl der übereinstimmenden Merkmale wurde das Maß der Ähnlichkeit zwischen den ASM eines Paares errechnet, wobei alle Merkmale als gleichwertig angesehen wurden. Insgesamt variierte der Grad der Ähnlichkeit der ASM zwischen 1 und 4, d.h. es gibt ein bis vier gemeinsame Merkmale (vgl. letzte Spalte). In Abbildung 13 wird der Grad der Ähnlichkeit von Such-ASM exemplarisch für ASM1, ASM3, ASM4 und ASM9 dargestellt. Der Grad der semantischen und formalen Ähnlichkeit zwischen diesen ASM wird durch die unterschiedliche Linienstärke repräsentiert.

\subsection{Die Familie der Argumentstrukturmuster mit inneren Objekten}

Die Gemeinsamkeiten und Unterschiede zwischen den Varianten von Argumentstrukturmustern, die innere Objekte enthalten, lassen sich ebenfalls über Familienähnlichkeiten beschreiben. Es gibt sowohl Varianten, die die gleiche Form, aber eine andere Bedeutung haben, als auch Varianten, die bedeutungsgleich oder bedeutungsähnlich sind, und eine unterschiedliche Form haben. Alle diese Argumentstrukturmuster bestehen aus einem verbalen Argument und seinen Elementen, wobei meistens mehrere dieser Einheiten durch kategoriale semantische und /oder syntaktische Spezifizierungen gekennzeichnet sind, und auch mindestens ein formaler oder semantischer Aspekt dieser Spezifizierungen nicht prädiktabel ist aufgrund von allgemeinen Festlegungen der Grammatik oder des Lexikons. So stellt das innere Objekt in den meisten Fällen eine Argumenterweiterung bei normalerweise intransitiv verwendeten Verben dar, die nicht aus allgemeinen Festlegungen herleitbar ist. Auch die morphologische und etymologische Verwandtschaft des Objektnomens mit dem Verb, die häufig vorhanden ist, ist nicht auf diese Weise prädiktabel. Außerdem sind alle hier besprochenen Varianten von Argumentstrukturmustern, die innere Objekte enthalten, insofern idiosynkratisch, als in keiner Weise vorhersagbar ist, mit welchen Verben sie vorkommen können oder nicht (vgl. zu den einzelnen Varianten Winkler 2009).

Das Muster ASM14 ist das wohl häufigste und wird im Allgemeinen sowohl unter formalen als auch unter semantischen Gesichtspunkten mit inneren Objekten assoziiert (vgl. u.a. Baron 1971; Matsumoto 1996; Höche 2009).

ASM14: ein Lächeln lächeln

$\mathrm{F}: \mathrm{X}_{\mathrm{NPnom}} \mathrm{V} \mathrm{Y}_{\mathrm{NPakk}}$

B: $X$ führt eine Handlung $V$ aus, und $V$ ist mit dem von $Y$ Bezeichneten ereignis-identisch. 
ASM14 wird durch Belege wie (15) illustriert:

(15) An die Adresse der Rebellen gerichtet sagte Rice: „Sie sollten wissen, dass $[\text { wir }]_{\mathrm{X}}$ [diesen Kampf $]_{\mathrm{Y}}[\text { kämpfen werden }]_{\mathrm{V}}$, bis er siegreich beendet ist." Die Sicherheitslage in Afghanistan hat sich in den vergangenen Monaten dramatisch verschlechtert. (dpa, 28.06.2006; (Zusammenfassung 1545 - neu: Panzerfaustbeschuss statt Sprengsatz) Wieder Anschlag auf Bundeswehr in Kundus - drei Soldaten verletzt)

Das Objektsnomen in ASM14 ist morphologisch, etymologisch und / oder semantisch mit dem Verb verwandt und wird in den meisten Fällen durch Modifikation noch weiter spezifiziert. Diese Form des inneren Objekts kommt in der Mehrzahl der Fälle bei intransitiven Verben vor und stellt dann eine Argumenterweiterung dar. ASM14 kommt bei Verben wie gehen, hassen, kämpfen, lachen, lächeln, laufen, leben, schlafen, spielen, sterben, tanzen oder tun vor.

Das Muster ASM15 ist ebenso wie das folgende ASM16 auf das Verb sterben festgelegt, was auch in der Formkomponente der Beschreibung bereits angegeben ist. Bis auf diese Festlegung ist ASM15 formgleich mit ASM14; das Objektsnomen ist aber nur semantisch mit dem Verb verwandt und wird stets durch Modifikation weiter spezifiziert.

ASM15: einen qualvollen Tod sterben

F: $\mathrm{X}_{\mathrm{NPnom}} \mathrm{V}_{[\text {sterben] }} \mathrm{Y}_{\mathrm{NPakk}}$

B: $X$ befindet sich in dem Prozess $V$, und $Y$ ist das Resultat von $V$.

ASM15 ist durch einen Beleg wie (16) illustriert:

(16) [Mehr als 5.000 Menschen, überwiegend Frauen und Kinder $]_{\mathrm{X}}$, [starben $]_{V}$ in der irakischen Stadt [einen qualvollen Tod $]_{Y}$. Die Produktion des Giftgases wurde im Irak erst durch Lieferungen deutscher Firmen ermöglicht. (die tageszeitung, 17.03.1989, S. 2; Giftgasopfer - schon vergessen?)

Die Variante ASM16 von Argumentstrukturmustern mit innerem Objekt ist mit ASM15 bedeutungsgleich und unterscheidet sich durch einen einzelnen Formaspekt von diesem: Das innere Objekt wird hier in einem anderen Kasus realisiert als in ASM15, nämlich im Genitiv statt im Akkusativ:

ASM16: eines natürlichen Todes sterben

$\mathrm{F}: \mathrm{X}_{\mathrm{NPnom}} \mathrm{V}_{[\text {sterben }]} \mathrm{Y}_{\mathrm{NPgen}}$

B: $X$ befindet sich in dem Prozess $V$, und $Y$ ist das Resultat von $V$. 
ASM16 wird durch Beispiele wie (17) belegt:

(17) Vor kurzem warteten Wissenschaftler aus Bozen mit einer weiteren dramatischen Neuigkeit auf: [«Ötzi»] $]_{\mathrm{X}}[\mathrm{starb}]_{\mathrm{V}}$ möglicherweise [eines gewaltsamen Todes $]_{Y}$ - eine steinerne Pfeilspitze steckt in seinem Oberkörper. (St. Galler Tagblatt, 25.09.2001, Ressort: TB-WIS (Abk.); Russ in der Lunge, Flöhe im Kleid)

Auch ASM16 tritt nur bei dem Verb sterben auf, das semantisch mit dem Objektsnomen verwandt ist und durch Adjektive modifiziert wird. Sowohl ASM15 als auch ASM16 unterscheiden sich durch die Ereignisqualität von den anderen Argumentstrukturmustern, sterben ist keine Handlung, sondern ein Prozess.

Die Variante ASM17 der Argumentstrukturmuster, die innere Objekte enthalten, ist formgleich mit ASM14, hat aber eine von diesem deutlich unterschiedene Bedeutung, die so nicht prädiktabel ist. Die Ereignisidentität, die bei ASM14 gegeben ist, kann hier nicht angenommen werden, da der Objektreferent lediglich eines von mehreren möglichen Handlungsmustern bezeichnet, gemäß dem die Handlung ausgeführt wird. Die Variante kommt bei Verben wie tanzen, singen oder spielen vor.

ASM17: einen Tango tanzen

F: $\mathrm{X}_{\mathrm{NPnom}} \mathrm{V} \mathrm{Y}_{\mathrm{NPakk}}$

B: $X$ führt eine Handlung $V$ gemäß dem durch $Y$ bezeichneten Handlungsmuster aus.

ASM17 wird durch Belege wie (18) instanziiert:

(18) $[\mathrm{Wir}]_{\mathrm{X}}[\operatorname{tanzen}]_{\mathrm{V}}[\text { Cha Cha Cha, Rumba und Jive }]_{\mathrm{Y}}$ (lateinamerikanische Tänze), außerdem [Langsamen Walzer, Tango und Quick Step $]_{Y}$ (Standardtänze). Jive und Tango sind meine Lieblingstänze, Quick Step dagegen mag ich nicht so sehr. (Nürnberger Nachrichten, 04.08.2001; Manchmal tun die Füße ganz schön weh - Kerstin Vierkant und Thomas Reichelsdorfer bereiten sich gerade auf Bayerische Meisterschaft vor)

Das Objektsnomen ist in den meisten Fällen mit dem Verb weder morphologisch noch etymologisch verwandt, es besteht allerdings eine enge semantische Verwandtschaft. Nur wenn das Objektsnomen in Form eines Kompositums realisiert wird, kann zumindest beim Grundwort auch etymologische und morphologische Verwandtschaft vorliegen.

In dem Argumentstrukturmuster ASM18 wird ein dem inneren Objekt verwandter Ausdruck in Form einer Präpositionalphrase realisiert: 
ASM18: mit der Laubsäge sägen/mit Meersalz salzen

F: $\mathrm{X}_{\mathrm{NPnom}} \mathrm{V}\left(\mathrm{Z}_{\mathrm{NPakk}}\right) \mathrm{Y}_{\mathrm{PP} \text { mit }}$

B: $X$ führt die Handlung $V$ mit Hilfe von $Y$ in Bezug auf $Z$ aus.

ASM18 wird durch Belege wie in (19) illustriert:

a. Auch das Basteln stand gestern noch einmal hoch im Kurs. [Vincent $]_{\mathrm{X}}(7)[\text { sägte }]_{\mathrm{V}}[\mathrm{mit} \text { der Laubsäge }]_{\mathrm{Y}}[$ ein echtes australisches Schwirrholz $]_{Z}$. (Mannheimer Morgen, 13.10.2001, Ressort: Lokal Lampertheim; Ein letztes Mal erklingen die bunten Didgeridoos)

b. In einem schweren Mörser habe ich mittlerweile schwarze Pfefferkörner und Kümmel zerstoßen; je 1/2 TL für zwei Portionen: über die Kartoffeln streuen und $[\text { diese }]_{Z}[\text { mit Meersalz }]_{Y}[$ salzen $]_{\mathrm{V}}$. Den Rest besorgt die Pfanne. (Die Zeit (Online-Ausgabe), 16.01.2003, Nr. 4, Ressort: Leben; Es gibt Bratkartoffeln!)

Die Nominalphrase im Akkusativ bezeichnet in ASM18 das Patiens. Diese Argumentstrukturmustervariante (vgl. auch Levin 1993) tritt entweder bei instrumentinkorporierenden Verben wie sägen, feilen oder bämmern auf oder aber bei Verben, deren Bedeutung sich als „etwas auf/in etwas tun“ paraphrasieren lässt, wie zuckern, salz̨en oder buttern. Die Präpositionalphrase enthält eine Modifikation des normalerweise im Verb inkorporierten Objekts, also in anderer Weise auch eine Argumenterweiterung. Es erfolgt entweder eine Spezifikation des Instruments, mit dessen Hilfe die Handlung ausgeführt wird, oder eine Spezifikation der Substanz, die in oder auf etwas getan wird. Die Nominalphrase in der durch mit eingeleiteten Präpositionalphrase wird hier fast ausnahmslos durch ein Kompositum realisiert, dessen zweiter Bestandteil morphologisch mit dem Verb verwandt ist. Das Nomen der Präpositionalphrase hat die gleiche Referenz wie ein Teil der lexikalischen Paraphrase des Verbs (z.B. für sägen: x zerteilt z mit Hilfe von $y \& \operatorname{SÄGE}(y)$; für salzen: $x$ tut $y$ in/auf $z \& \operatorname{SALZ}(y))$. Das ist eine andere Art von Identität als sie für das erste Muster (ASM14) mit inneren Objekten angenommen wurde. Es besteht also sowohl auf der Form- als auch auf der Bedeutungsseite lediglich eine geringe Ähnlichkeit mit ASM14.

Das letzte Argumentstrukturmuster im Bereich der inneren Objekte, ASM19, ist formgleich mit ASM14 und steht darüber und über die weiteren Eigenschaften der Nominalphrase im Akkusativ in einer Ähnlichkeitsbeziehung zu ASM14. 
ASM19: einen Traum träumen

F: $\mathrm{X}_{\mathrm{NPnom}} \mathrm{V} \mathrm{Y}_{\mathrm{NPakk}}$

B: $X$ ist Träger des von $V$ beschriebenen mentalen Zustands oder Ereignisses, und $Y$ bezeichnet den Inhalt oder die Ausprägung des mentalen Zustands.

ASM19 ist durch Beispiele wie (20) belegt:

(20) [Einen Traum $]_{\mathrm{Y}}$ aber [träumte $]_{\mathrm{V}}[\text { Hans Castorp }]_{\mathrm{X}}$ sogar zweimal in dieser Nacht, und zwar beide Male genau in derselben Form, - das letztemal gegen Morgen. (T. Mann: Der Zauberberg. Roman, (1. Buchausg. 1924), In: [Gesammelte Werke in zwölf Bänden mit einem Ergänzungsband], Bd. 3. Frankfurt a.M.: S. Fischer Verlag, 1960)

Die Nominalphrase im Akkusativ ist dabei morphologisch, etymologisch und semantisch mit dem Verb verwandt. Die Verben, die diese Variante von inneren Objekten zulassen, bezeichnen Zustände oder mentale Ereignisse (wie träumen oder glauben) und können sowohl transitiv als auch intransitiv verwendet werden. Auch ASM19 unterscheidet sich durch die Ereignisqualität von den anderen Argumentstrukturmustern. Die Objekts-NP bezeichnet im ASM19 eine komplexe Proposition, nämlich den ganz bestimmten, spezifischen Inhalt des Träumens oder Glaubens, nicht diesen mentalen Zustand oder dieses mentale Ereignis als solches. In einer Variante der betreffenden Verben kann diese komplexe Proposition ja auch unmittelbar als propositionales Objektsatzkomplement realisiert werden (er träumte/glaubte, dass ...). Anders, als das bei normalen Transitivkonstruktionen mit zufälliger formaler Ähnlichkeit zu ASM19 der Fall ist, bezeichnet die Objekts-NP im ASM19 nicht den Gegenstand, auf den die mit dem Verb beschriebene Handlung bezogen ist. ${ }^{14}$

Die Beschreibung der Argumentstrukturmuster ASM14-ASM19 hat gezeigt, dass es sich jeweils um separate Argumentstrukturmuster handelt, die in Relationen von Familienähnlichkeiten zueinander stehen. Keine der Bedeutungen der einzelnen Argumentstrukturmuster kann als die zentrale angesehen werden, auf die alle anderen bezogen sind. ASM14, ASM15, ASM17 und ASM19 haben die gleiche Form, aber unterschiedliche Bedeutungen.

14 Es gibt daneben solche normalen Transitivkonstruktionen, die durch die zufällige morphologische Verwandtschaft des Akkusativobjekts mit dem Verb eine formale Ähnlichkeit mit inneren Objekten aufweisen, wie in dem folgenden Beispiel:

Ich habe keine Ressentiments, ich verzeihe, aber ich vergesse nicht! [Ich $]_{\mathrm{X}}[\text { hasse }]_{\mathrm{V}}$ [den $\mathrm{Haß}]_{\mathrm{Y}},[i c h]_{\mathrm{X}}[\text { liebe }]_{\mathrm{V}}[\text { die Liebe }]_{\mathrm{Y}}$ und ich weiß, wie schwer es ist, ein Held zu sein. (Die Presse, 20.05.1992; Kreativ in vielen Kunstgärten)

Die Nominalphrase im Akkusativ entspricht in solchen Sätzen dem normalen valenzgeforderten Objekt des Verbs, die formale Ähnlichkeit ist nur zufällig. Das wäre auch nach Goldberg ein Fall von Homonymie. Solche formalen Ähnlichkeiten mit inneren Objekten können bei Verben wie lieben, hassen oder träumen auftreten. 
ASM15 und ASM16 haben die gleiche Bedeutung, unterscheiden sich aber in formaler Hinsicht. Die einzelnen Argumentstrukturmuster stehen jeweils in unterschiedlichen, direkten oder vermittelten Ähnlichkeitsbeziehungen zueinander. Anders als bei der Familie der Such-Argumentstrukturmuster kristallisieren sich bei den Argumentstrukturmustern mit inneren Objekten aber keine Subfamilien heraus.

\section{Idiosynkrasien der lexikalischen Füllung von Argumentstrukturmustern}

\subsection{Die Bewegungsvariante von Geräuschverben}

Argumentstrukturmuster sind also als recht feinkörnige, durch Familienähnlichkeiten verknüpfte Entitäten konzipiert und können damit relativ viele Idiosynkrasien des Sprachgebrauchs auffangen. In den Eingangsbeispielen in (1) war allerdings auch von Kookkurrenzen im Argumentstrukturbereich die Rede, die sich auf einer tieferen Abstraktionsebene abspielen. Darum soll es in diesem und den beiden folgenden Abschnitten gehen. In diesem Abschnitt wollen wir am Beispiel der Bewegungslesarten von Geräuschverben demonstrieren, welche Probleme solche Kovorkommen für klassische konstruktionistische Ansätze aufwerfen (vgl. dazu Engelberg 2009).

(21) a. [Die Harleys] $]_{X}[\text { knattern }]_{V}$ gemütlich [über die märkischen Landstraßen $]_{Y}$, die sportlicheren Maschinen jagen gefährlich um die Kurven. (Berliner Zeitung, 24.06.2000 [S. 35])

b. Weithin hörbar [quietscht $]_{\mathrm{V}}[\mathrm{sie}]_{\mathrm{X}}$ mit atemberaubender Erbarmungslosigkeit [um die Kurve] $]_{Y}$, und meine Zähne klappern nun nicht mehr vor Kälte, sondern weil ich sie ob des schmerzenden Geräusches zusammenbeißen muß. (die tageszeitung, 06.03.1990, S. $28 ;,, \ldots$ wejen de Gleise ...")

c. [Pferdedroschken und Straßenbahnen $]_{\mathrm{X}}[\text { rumpelten }]_{\mathrm{V}}$ [über den einst ruhigen Wittenbergplatz $]_{Y^{\prime}}$ (die tageszeitung, 07.07.1990, S. 39; Inszenierte Rekorde)

Beispiele wie die in (21) werden in konstruktionsgrammatischen Ansätzen im Idealfall so erklärt, dass Verben wie knattern, quietschen oder rumpeln lediglich als einstellige Verben im Lexikon repräsentiert sind, die ihre Bewegungslesart dadurch erhalten, dass sie in eine intransitive Bewegungskonstruktion eingepasst werden, die die PP und die Bewegungslesart lizenziert (Goldberg 2006, S. 100; Welke 2009). Die Erwartung ist dann natürlich, dass sich Geräuschverben in dieser Konstruktion ähnlich wie Bewegungsverben verhalten. Da nun gerade die Direktionalphrase der Indikator für die Bewegungslesart ist, ist zu überprüfen, ob Geräusch- und Bewegungsverben diesbezüglich ein ähnliches Verhalten zeigen. In einer Korpusstudie auf der 


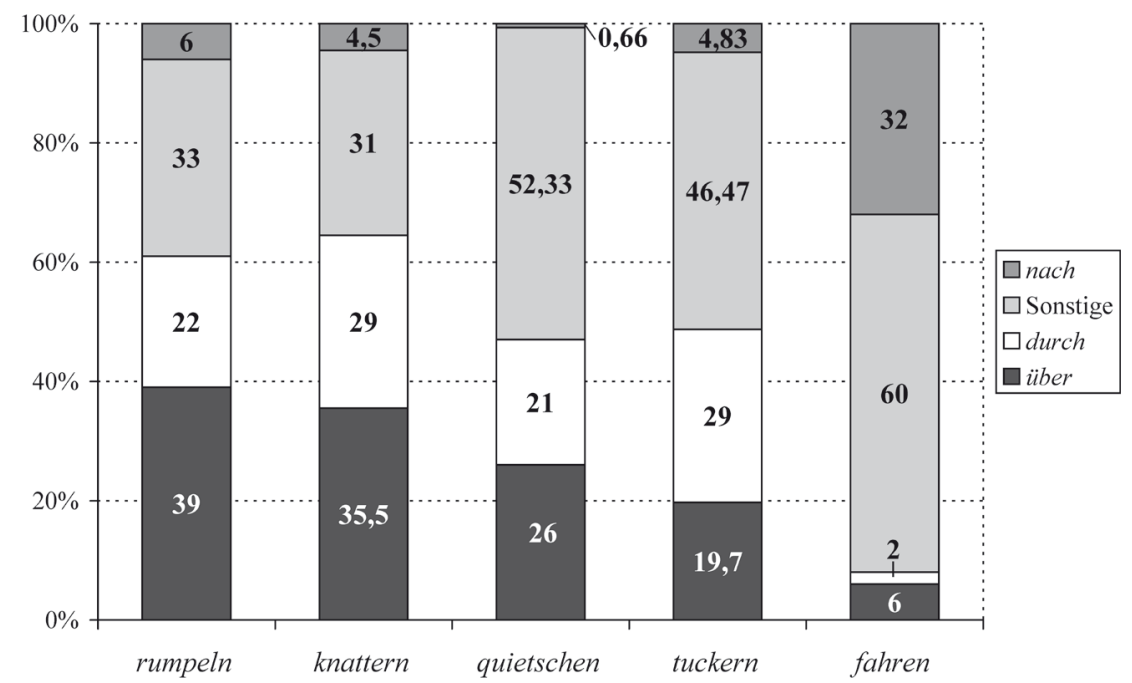

Abb. 1: Verteilung der Präpositionen über, durch und nach bei Geräusch-als-Bewegungsverben und bei fahren

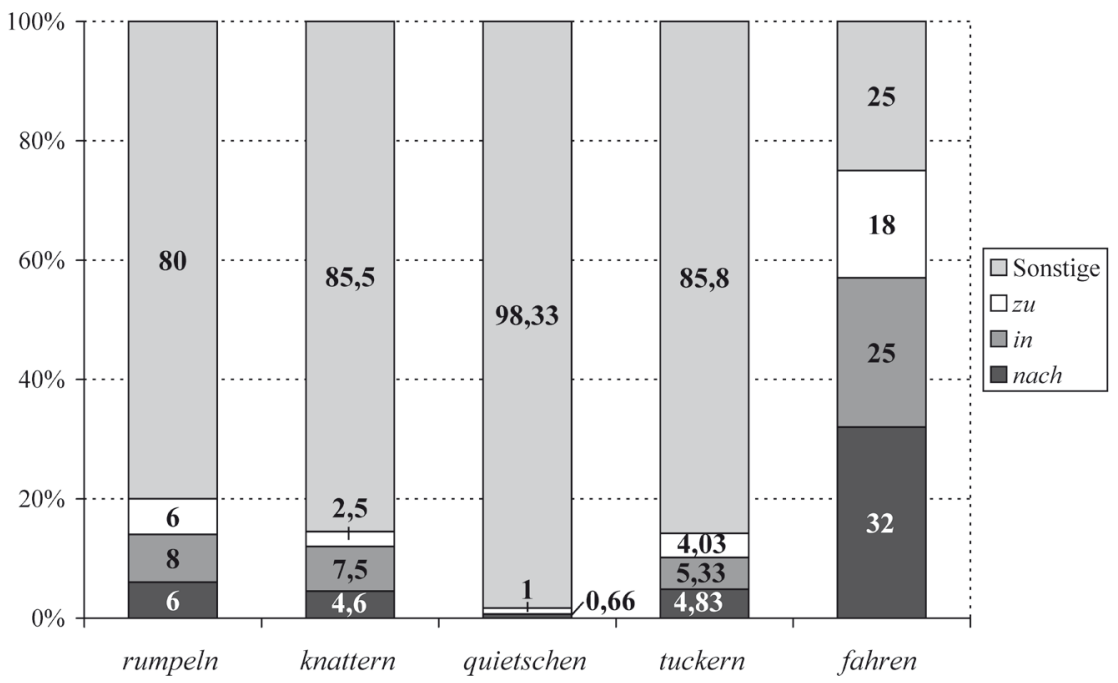

Abb. 2: Verteilung der Präpositionen nach, in und $₹ u$ bei Geräusch-als-Bewegungsverben und bei fahren

Basis von 200 Zufallsbelegen pro Verb zeigte sich allerdings, dass unter den ca. 25 Direktionalausdrücken, die mit einem Geräusch-als-Bewegungsverb kookkurrieren, immer zwei quantitativ herausstechen, nämlich PPs eingeleitet durch über oder durch (siehe auch Engelberg 2009). Sie machen zusam- 
men im Schnitt über 50\% der Belege aus. Die Erwartung, dass auch ein genuines Bewegungsverb wie fahren eine solche Verteilung zeigt, erfüllt sich allerdings nicht (siehe Abbildung 1).

Das Bewegungsverb fabren zeigt ganz andere Präferenzen, nämlich solche für die Präpositionen nach, in und ₹u (siehe Abbildung 2).

Im Gegensatz zu den Geräuschverben, die in ihrer Bewegungslesart wegspezifizierende PPs bevorzugen, zeigt fahren also eine Präferenz für zielspezifizierende PPs. Das lässt etwa Welkes Annahme unplausibel erscheinen, die Bewegungslesart käme ad hoc aufgrund der konzeptuellen Anpassung von Geräuschverben an eine an genuinen Bewegungsverben ausgebildete Bewegungskonstruktion zustande. Die Daten legen vielmehr nahe, von einem eigenen Argumentstrukturmuster für die Bewegungsvariante von Geräuschverben auszugehen, das sich durch besondere Präferenzen für die Realisierung der Direktionalphrase auszeichnet:

ASM20: durchs Dorf knattern

F: $\mathrm{X}_{\mathrm{NPnom}} \mathrm{V} \mathrm{Y}_{\mathrm{PP} / \mathrm{AdvP}[\text { Präererenz für PPdurrb und PP iber }]}$

B: $X$ bewegt sich entlang des in $Y$ spezifizierten Pfades und erzeugt dabei das durch $V$ denotierte Geräusch.

In Goldberg/Jackendoff (2004, S. 540 f.) wird für die Bewegungsvariante von Geräuschverben eine eigene Subkonstruktion (,sound-emission path resultative") der Resultativkonstruktion angenommen, allerdings aus einem ganz anderen Grund. Die intransitive Bewegungskonstruktion wird hier durch Ausdrücke wie (22a) exemplifiziert, in der roll die Art und Weise der Bewegung spezifiziert. Bei Geräuschverben wie in (22b) geht es natürlich nicht darum, die Art und Weise der Bewegung auszudrücken, sondern „that the motion causes the sound to be emitted".

a. The ball rolled down the hill.

b. The trolley rumbled down the tunnel.

Über diese semantische Besonderheit rechtfertigen Goldberg/Jackendoff (2004) die Annahme einer eigenen Subkonstruktion..$^{15}$ Dazu ist zu sagen, dass die angenommene semantische Besonderheit eine Erklärung in der bekannten Beschränkung finden könnte, dass zwei Teilereignisse, die über ein einzelnes Verb ausgedrückt werden (also hier Bewegung und Geräuschemission), kausal oder über kausalitätsähnliche Relationen (Kaufmann 1995; Maienborn 1994; Engelberg 2000) verknüpft sein müssen. So kann man nicht sagen er pfeift in den Raum (in der Bedeutung, er kommt pfeifend in den Raum), da Geräusch und Bewegung nicht ursächlich verknüpft sind. Wenn

15 In Goldberg (1995) hätte eine ähnliche Überlegung möglicherweise zur Annahme konstruktioneller Polysemie bei der intransitiven Bewegungskonstruktion geführt. 
man diese Beschränkung so versteht, dass die kausale Verknüpfung nicht unmittelbar sein muss, sondern auch vermittelt sein kann über einen gemeinsamen Verursacher, so ist sie datenadäquater als Goldberg/Jackendoffs (2004) semantische Analyse, die nur für Fälle wie rumpeln und quietschen gilt, nicht aber für Fälle wie knattern und tuckern, bei denen ja nicht die Bewegung das Geräusch hervorruft, sondern sowohl die Bewegung als auch das Geräusch im Normalfall durch die Aktivität des Motors hervorgerufen werden. Die kausale Verknüpfung ist also hier eine andere als die von Goldberg/Jackendoff (2004) angenommene.

Die eigentliche Idiosynkrasie, die zu repräsentieren ist, besteht in der Präferenz der Geräuschverben für die beiden Präpositionen über und durch. Diese ist im Übrigen auch nicht durch semantisch-pragmatische Erfordernisse vollständig zu erklären. Man könnte vermuten, dass gleichzeitige Modusspezifizierung (Geräusch) und Zielspezifizierung dispräferiert sind. Aber selbst wenn das so ist, bleibt die Frage offen, warum unter den vielen wegspezifizierenden Ausdrücken (entlang, vorbei, herum, vorüber, daher, auf, um etc.) ausgerechnet diese beiden bevorzugt werden. Auch die Annahme, dass die räumliche Konfiguration, die die PP beschreibt, ursächlich an der Geräuschentwicklung beteiligt sein muss, führt letztlich nicht zum Ziel. So ist es zwar die räumliche über-Relation zwischen Lastwagen und Straßenbelag, die relevant für die Erzeugung des Geräuschs ist, wenn ein Laster über die Straße rumpelt, aber wenn ein Motorrad durch die Stadt knattert, ist die durch-Relation irrelevant für die Geräuscherzeugung. Die Präpositionspräferenz bleibt also eine Idiosynkrasie des spezifischen Argumentstrukturmusters bzw. der spezifischen Verbklasse der Geräuschverben als Bewegungsverben. Goldberg/Jackendoff (2004) haben demzufolge durchaus recht, wenn sie eine eigene Konstruktion für Geräuschverben als Bewegungsverben ansetzen, aber es gibt schlagkräftigere Argumente als die von ihnen angeführten.

\subsection{Idiosynkrasien bei Resultativprädikaten}

Neben solchen verbklassenspezifischen Restriktionen bei Argumentstrukturmustern sind auch verbspezifische Besonderheiten festzustellen. So arbeiten Stefanowitsch/Gries (2003) heraus, dass Argumentstrukturmuster gewöhnlich eine Präferenz für bestimmte Verben zeigen. Es soll in diesem Abschnitt am Beispiel der subjektreflexiven sowie der transitiven Resultativkonstruktion gezeigt werden, dass darüber hinaus auch die Füllungen weiterer Slots innerhalb eines Argumentstrukturmusters durch Idiosynkrasien geprägt sind.

So bevorzugen beispielsweise Resultativkonstruktionen nicht nur bestimmte Verben, sondern es zeigen sich auch klare Vorlieben bezüglich der Kombination von Verb und Resultativprädikat. Eine korpusbasierte Unter- 
Es ist nun erkennbar, dass Verben, wenn sie in der subjektreflexiven Resultativkonstruktion vorkommen, Präferenzen haben hinsichtlich des Resultativprädikats, mit dem sie auftreten. Für das Verb reden in der subjektreflexiven Resultativkonstruktion führte eine Korpusuntersuchung zu dem in Abbildung 3 dargestellten Ergebnis.

Diese zu beobachtende auffällige Präferenz des Verbs reden (ohne Verbpartikel) für das sekundäre Prädikat in Rage lässt sich nicht durch die absolute Häufigkeit der Wortform in Rage oder des Lemmas Rage erklären. So kommt Rage außerhalb der Resultativkonstruktion im Vergleich zu anderen Ausdrücken zur Zustandsbeschreibung eines heftigen durch Ärger hervorgerufenen Gefühls deutlich seltener vor: Eine Korpusuntersuchung für die Lemmata Rage, Wut, Zorn, wütend und zornig ergab, dass Rage eindeutig am seltensten im Korpus vertreten ist (Abbildung 4).

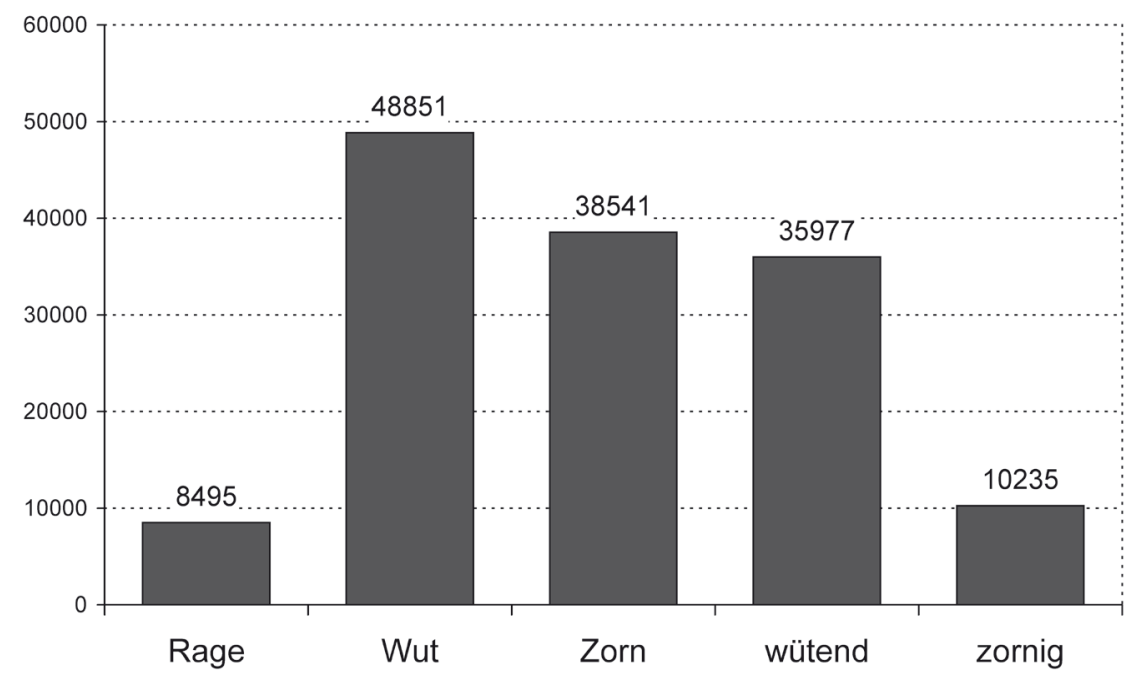

Abb. 4: Vorkommen der Lemmata Rage, Wut, Zorn, wütend und zornig

Auch der feste Ausdruck in Rage kommt verglichen mit den Ausdrücken bzw. Wortformen in Wut, in/im Zorn, wütend und zornig nicht am häufigsten vor (Abbildung 5).

Im Hinblick auf diese quantitativen Ergebnisse ist es umso bemerkenswerter, dass die genannten semantisch ähnlichen Ausdrücke als Resultativprädikate für subjektbezogenes reflexives reden in verschwindend geringer Anzahl vorkommen (Abbildung 6). 


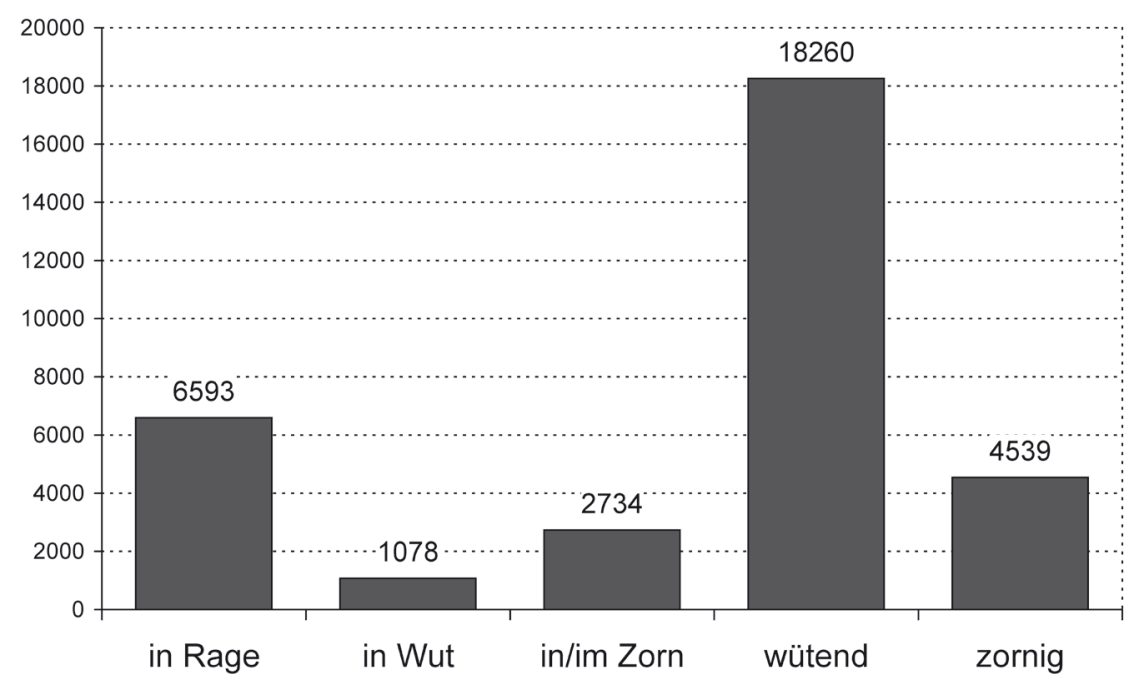

Abb. 5: Vorkommen der Ausdrücke bzw. Wortformen in Rage, in Wut, in Zorn, wütend und zornig

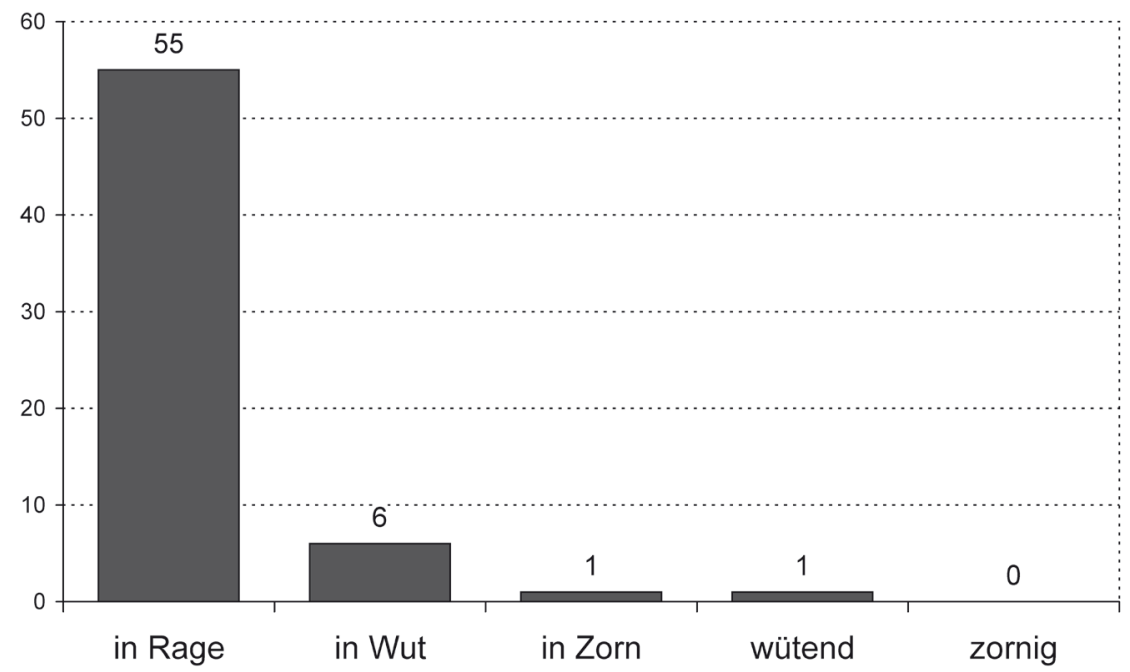

Abb. 6: In Rage, in Wut, in Zorn, wütend und zornig als Resultativprädikate für subjektbezogenes reflexives reden

Weder die Bedeutung von Rage noch allgemeine lexikalische Häufigkeiten oder Weltwissen können erklären, warum subjektreflexives-resultatives reden das Resultativprädikat in Rage so eindeutig präferiert. Gestaltet man nun die Korpusabfrage genau anders herum, untersucht also die Verteilung von Verben aus Sicht des Resultativprädikats, so ergibt sich folgendes Bild: 


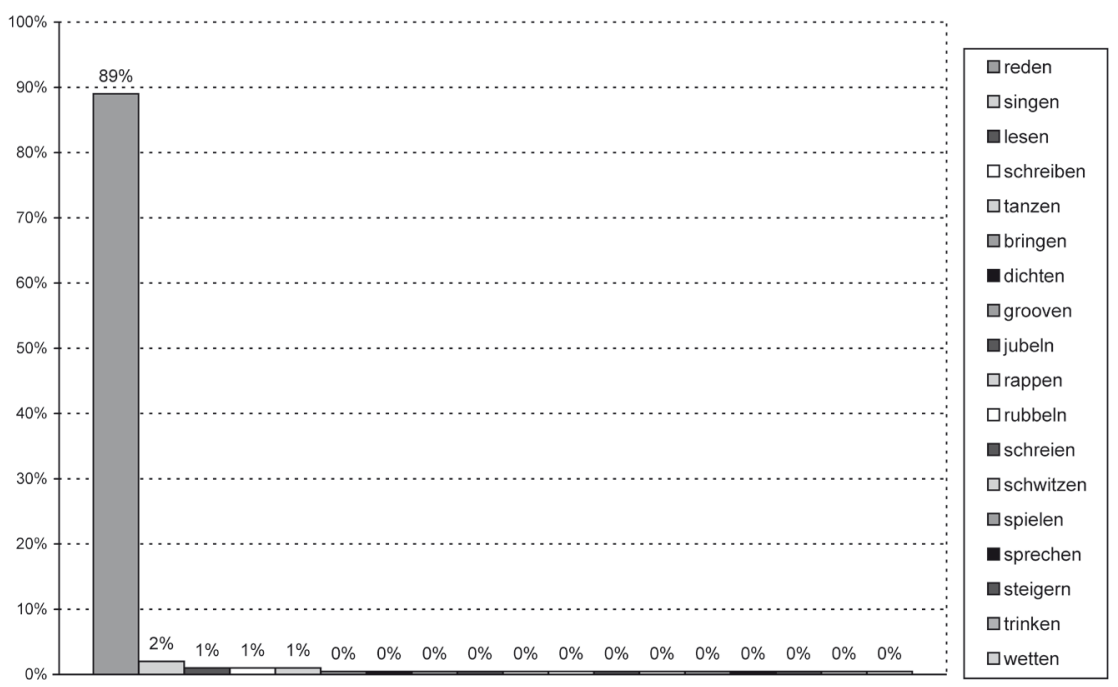

Abb. 7: Verteilung der Verben, die in der subjektreflexiven Resultativkonstruktion mit dem Resultativprädikat in Rage vorkommen (Prozentwerte gerundet)

Hier zeigt sich, dass in Rage mit reden im Rahmen der subjektreflexiven Resultativkonstruktion mit $89 \%$ der Vorkommen deutlich am häufigsten auftritt. Diese starke Präferenz lässt sich wiederum nicht aus generellen Häufigkeiten erklären. So ist reden bei weitem nicht das frequenteste Verb im Korpus. Dass dieses Ergebnis weder auf eine generelle Kollokation von reden und in Rage zurückzuführen ist, noch in Bezug auf eine allgemeine Resultativkonstruktion Gültigkeit hat, zeigt sich in den folgenden Untersuchungen. Zum einen wird bei einer statistischen Kookkurrenzanalyse (Belica 2001-2007) für reden bzw. Rage deutlich, dass beide Lemmata andere Kookkurrenzpartner haben, die (zum Teil wesentlich) häufiger mit ihnen vorkommen. So steht zum Beispiel Rage erst an 47. Stelle der Kookkurrenzpartner von reden, reden als Kookkurrenzpartner für Rage immerhin an fünfter. Zum anderen ergibt sich bereits bei der syntaktisch sehr ähnlichen, in ASM22 dargestellten, nicht-reflexiven transitiven Resultativkonstruktion für das Resultativprädikat in Rage ein abweichendes Bild, wie im Folgenden gezeigt werden soll.

ASM22: jemanden krankenhausreif prügeln

F: $\mathrm{X}_{\mathrm{NPnom}} \mathrm{V} \mathrm{Y}_{\mathrm{NPakk}} \mathrm{Z}_{\mathrm{PP} / \mathrm{AP}}$

B: $X$ führt eine Handlung $V$ aus, die dazu führt, dass $Y$ in den von $Z$ beschriebenen Zustand gerät. 
ASM22 wird durch Belege wie (24) instanziiert:

(24) Mitten auf dem Golfplatz lauerte [das Känguruh $]_{\mathrm{X}}$ [sic!] dem Buben auf, fiel über ihn her und [prügelte $]_{\mathrm{V}}[\mathrm{ihn}]_{\mathrm{Y}}[\text { krankenhausreif }]_{\mathrm{Z}}$. (Salzburger Nachrichten, 30.10.1998, Ressort: LOKALES; Salzburg im Känguruh-Fieber: Alle suchen den Ausreißer)

Erhebt man nun im Korpus die Daten für das Resultativprädikat in Rage unmittelbar gefolgt von $i h n$, so erhält man für die vorkommenden Verben folgende Verteilung:

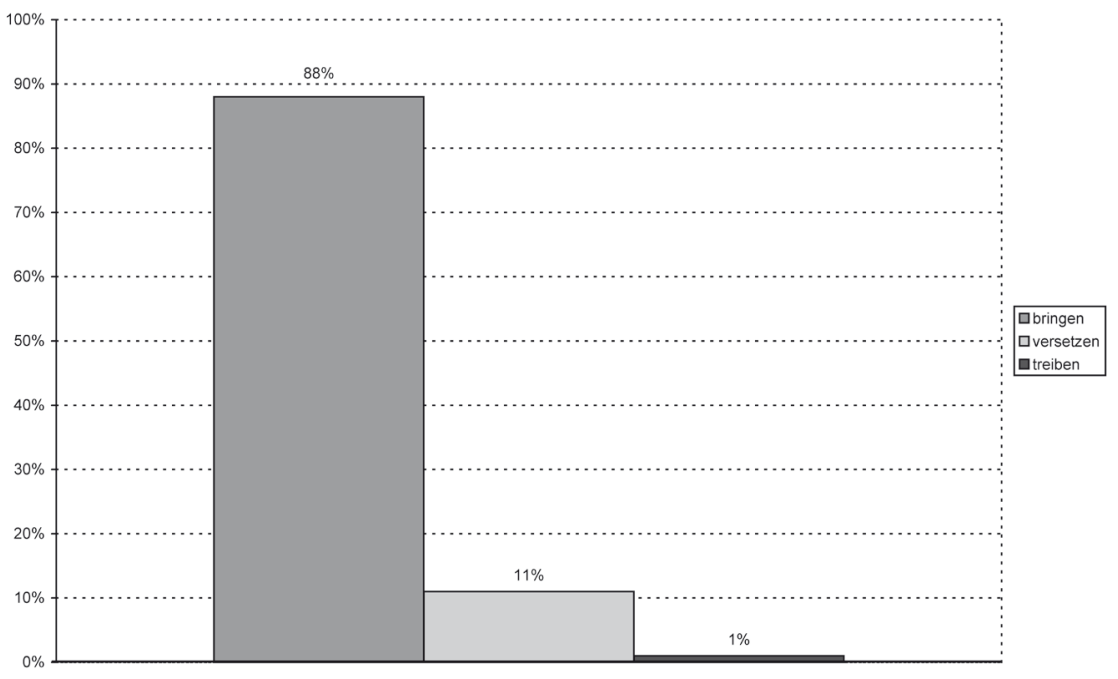

Abb. 8: Verteilung der Verben, die in der transitiven Resultativkonstruktion mit dem Resultativprädikat in Rage vorkommen

In der transitiven Resultativkonstruktion kommt also das Verb bringen am häufigsten mit dem sekundären Prädikat in Rage vor, reden hingegen tritt im Rahmen dieser Untersuchung kein einziges Mal auf. Das Resultativprädikat in Rage zeigt also in den verschiedenen Typen von Resultativkonstruktionen unterschiedliche idiosynkratische Präferenzen für das Verb innerhalb des jeweiligen Argumentstrukturmusters.

\subsection{Idiosynkratische propositionale Muster}

Wir haben in den vergangenen Abschnitten gesehen, dass und wie idiosynkratische Präferenzen für die Füllung von Argumentstellen relativ zu bestimmten Argumentstrukturmustern zustande kommen: Argumentstrukturmuster präferieren bestimmte Verben, Verbklassen präferieren bestimmte Präpositionen in bestimmten Argumentstrukturmustern, Füller von Ar- 
gumentstellen präferieren in bestimmten Argumentstrukturmustern bestimmte Verben. Darüber hinaus ist aber zu beobachten, dass nicht nur Präferenzen für die Füllung einzelner Argumentstellen repräsentiert werden müssen, sondern auch ganze propositionale Muster (vgl. Engelberg 2009). Unter einem propositionalen Muster wollen wir hier die idiosynkratische Kookkurrenz der Argumentstellen eines Verbs in einem Argumentstrukturmuster hinsichtlich der semantischen Klassen verstehen, denen die Ausdrücke angehören, die die Argumentstellen spezifizieren. Das wird im Folgenden an einem Beispiel aus dem Bereich der Bewegungsvariante von Geräuschverben (siehe oben ASM20) erläutert.

Klassifiziert man die Füller der verschiedenen Argumentstellen, die in einer zufälligen Auswahl von 100 Belegen für die Bewegungsvariante von knattern auftreten, so ergibt sich das in Abbildung 9 und 10 dargestellte Bild für die Füllung der Subjekts-NP und die der PP-internen NP. Die Präferenz für bestimmte Präpositionen ist oben in Abbildung 2 ersichtlich.

\begin{tabular}{|l|l|c|}
\hline \multicolumn{1}{|c|}{ Semantische Klasse } & \multicolumn{1}{|c|}{ Lexeme } & Anzahl \\
\hline MOTORRAD & $\begin{array}{l}\text { Simson-Roller, Krafträder, Harley, } \\
\text { Mopeds, Mofa, Wespen etc. }\end{array}$ & 35 \\
\hline LUFTFAHRZEUG & $\begin{array}{l}\text { BH-Hubschrauber, Helikopter, } \\
\text { Fokker, DC-3, Hubschrauber } \text { etc. }\end{array}$ & 25 \\
\hline AUTO & $\begin{array}{l}\text { Ente, Bus, Trabbis, Miniautos, LKW } \\
\text { etc. }\end{array}$ & 17 \\
\hline ANDERE FAHRZEUGE & Schneemobil, Cart etc. & 14 \\
\hline sonstige & Krähen, Akteure, Filmmagazine etc. & 9 \\
\hline
\end{tabular}

Abb. 9: Füllung der Subjekts-NP bei der Bewegunglesart von knattern

\begin{tabular}{|l|l|c|}
\hline \multicolumn{1}{|c|}{ Semantische Klasse } & \multicolumn{1}{|c|}{ Lexeme } & Anzahl \\
\hline $\begin{array}{l}\text { GEOGRAFISCHES } \\
\text { GEBIET }\end{array}$ & $\begin{array}{l}\text { Dörfer, Kramsach, Insel, Wohn- } \\
\text { gebiet, Tal, Wiesen, Oberlausitz, } \\
\text { Hamburg, Wüste etc. }\end{array}$ & 44 \\
\hline STRASSE & $\begin{array}{l}\text { Rennstrecke, Gleise, Unter den Lin- } \\
\text { den, Ring, Allee etc. }\end{array}$ & 19 \\
\hline GEBÄUDE(TEIL) & Kino, Bühne etc. & 11 \\
\hline sonstige & $\begin{array}{l}\text { Projektoren, Fest, Hand, Lastwagen, } \\
\text { Story, Ruhe etc. }\end{array}$ & 26 \\
\hline
\end{tabular}

Abb. 10: Füllung der PP-internen NP bei der Bewegunglesart von knattern

Nun ist zu beobachten, dass sich die Füller der Subjektstelle, der Präposition und der PP-internen NP präferiert zu propositionalen Mustern verbinden. Die fünf häufigsten Muster aus knattern, einer bestimmten Präposition, 
einer bestimmten semantischen Klasse der Subjekts-NP und einer der PPinternen NP decken, wie in dem innersten Rechteck in Abbildung 11 ersichtlich, bereits 35\% aller Belege ab. Generalisiert man das Muster nach und nach geringfügig, werden bereits $90 \%$ aller Belege erfasst. Das heißt, in $90 \%$ aller Belege für knattern in Bewegungslesart geht es darum, dass ein Fahrzeug auf einem Pfad relativ zu einem Gebiet, einer Straße, einem Gebäude oder einem Gegenstand knattert.

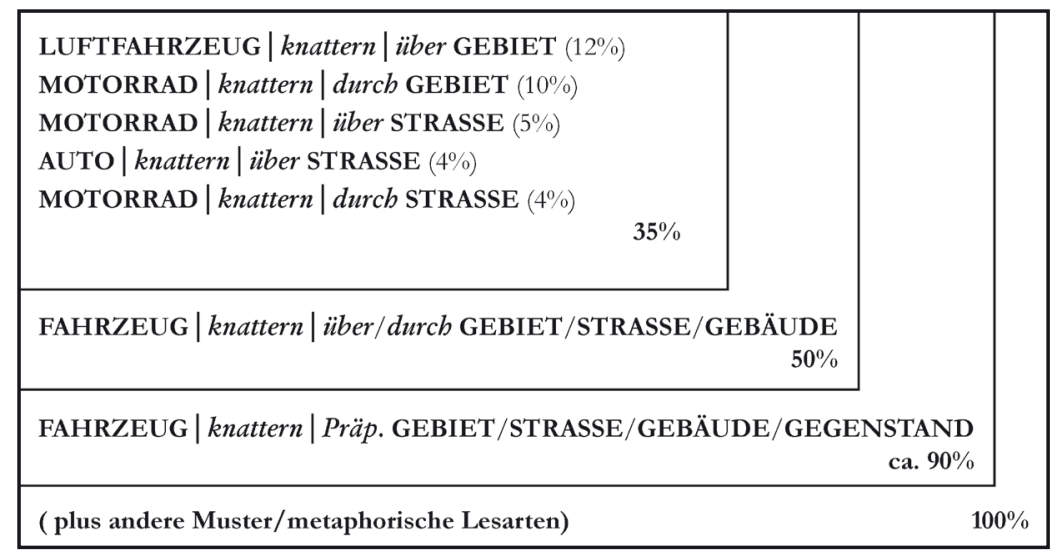

Abb. 11: Propositionale Muster bei knattern in Bewegungslesart

Die restlichen 10\% der Belege sind dagegen weit gestreut und zeigen, dass durchaus auch ganz andere Sachverhalte durch die Bewegungslesart von knattern ausgedrückt werden können:

(25) a. [Salven aus Kalaschnikows $]_{\mathrm{X}}[\text { knatterten }]_{\mathrm{V}}$ [über die Köpfe der außer Rand und Band geratenen Menschenmassen $]_{\mathrm{Y}}[\ldots]$... (Salzburger Nachrichten, 26.11.1991; Belgrad verhöhnt Friedensbemühung von UNO und $\mathrm{EG}$ )

b. Gegen Ende der zwölfminütigen Diaschau zur Explosion des Parthenon [knattern $]_{\mathrm{V}}$ [die Bildmagazine $]_{\mathrm{X}}$ wie verrückt [durch die Projektoren $]_{Y},[\ldots]$. (die tageszeitung, 16.07.1990, S. 25; Schuß in die Pulverkammer)

c. [Knapp 16.500 Bikes $]_{\mathrm{X}}[\text { knatterten }]_{\mathrm{V}} 1994$ [über den Ladentisch $]_{\mathrm{Y}}$, heuer werden es weit mehr als 17.000 sein. (Neue Kronen-Zeitung, 04.04.1995, S. 5)

Die Typen in (25a) und (25b) sind in der Stichprobe jeweils durch zwei ähnliche Beispiele vertreten. Sie konstituieren jeweils eigene propositionale Muster (vgl. Engelberg 2009):

(26) a. SALVE | knattern | ̈̈ber KOPF/PERSON (2\% der Belege)

b. FILM | knattern | durch PROJEKTOR (2\% der Belege) 
Die deutliche Präferenz für Motorräder gegenüber Schüssen in Subjektposition bei der Bewegungsvariante von knattern ist auch über Weltwissen oder thematische Präferenzen nicht zu erklären. Zumindest treten unabhängig von ihrem Vorkommen mit knattern als Bewegungsverb Schüsse in großen Textkorpora nicht seltener auf als Motorräder.

Der Beleg in (25c) wiederum ist ein gutes Indiz für die Existenz des dominierenden 90\%-Musters. Er ist in seiner stilistischen Funktion ohne die Vertrautheit mit Sätzen dieses Musters wohl kaum zu verstehen. Nur auf dieser Basis kann die Amalgamierung von ,über [Straßen] knattern" und „über den Ladentisch [gehen]" ihre Funktion entfalten.

Wir konstatieren also, dass offenbar ganze propositionale Muster als Spezifizierung bestimmter Argumentstrukturmuster kognitiv präsent sind. Das steht auch in Einklang mit neueren psycholinguistischen Tests, die zeigen, dass Sprecher offenbar ganze propositionale Einheiten aus Gesprächen über längere Zeit memorieren (Gurevich/Johnson/Goldberg 2010).

Die in den Abschnitten 3.1 bis 3.3 beschriebenen Phänomene stellen alle verschiedenen Arten von idiosynkratischen lexikalischen Kookkurrenzen innerhalb einzelner Argumentstrukturmuster dar, die offenbar Teil unseres sprachlichen Wissens sind. Als Spezifizierungen eines Argumentstrukturmusters berühren sie die generellen Eigenschaften eines solchen Musters nicht. Sie lassen sich über implikative Beziehungen oder Vererbungshierarchien mit der allgemeinen Repräsentation des Musters in Beziehung setzen.

\section{Theoretische Einordnung der Befunde}

Ziel dieses Artikels war es zu zeigen, wie Argumentstrukturmuster identifiziert werden können, wie die Beziehungen, in denen sie zueinander stehen, erfasst werden können, und welche Arten idiosynkratischer lexikalischer Kookkurrenzen innerhalb solcher Muster repräsentiert werden müssen. Das Unterfangen war im Wesentlichen um deskriptive Adäquatheit bemüht, verbunden mit angemessenen Generalisierungen. Es war nicht beabsichtigt zu zeigen, wie die beschriebenen Restriktionen und Regelhaftigkeiten solcher Muster sprachtheoretisch angemessen erfasst werden können. In dem $\mathrm{Zu}-$ sammenhang ist zu sagen, dass natürlich auch nicht behauptet werden sollte, dass alle deskriptiven Elemente in den dargestellten Mustern idiosynkratischer Natur sind. Die jeweils repräsentierte Nominalphrase im Nominativ ist ja keine Idiosynkrasie eines einzelnen Argumentstrukturmusters. Jedes der angeführten Muster enthält allerdings Idiosynkrasien, von denen wir annehmen, dass sie nicht aus anderen Festlegungen der Grammatik vollständig herleitbar sind.

In weiten Bereichen ist der Auffassung Jacobs' (2009) zuzustimmen, dass Kookkurrenzen im Bereich von Argumentstrukturen deskriptiv adä- 
quat über konstruktionsbasierte oder valenzbasierte projektionistische Modelle beschrieben werden können. $\mathrm{Ob}$ also die Argumentstrukturmuster als Konstruktionen beschrieben werden, die bestimmte Verben selegieren, oder aber als lexikalische Redundanzregeln, die über argumentstrukturell voll spezifizierten lexikalischen Einträgen operieren, ist unter dem Aspekt deskriptiver Adäquatheit für die meisten der betrachteten Phänomene unerheblich. Es hat sich aber auch gezeigt, dass manche Präferenzen für die Füllung von Argumentstellen sich nicht an valenz- und damit weitgehend kopforientierte Beschreibungen von Umgebungsrestriktionen anbinden lassen. Wie etwa im Fall der Idiosynkrasien bei Resultativkonstruktionen zu sehen war, gehen Selektionspräferenzen in bestimmten Argumentstrukturmustern nicht unbedingt vom Kopf des Musters aus. Auch die Indizien für das Vorliegen ganzer propositionaler Muster kommen valenzbasierten Vorstellungen, dass einzelne Argumente unabhängig voneinander durch das Verb semantisch beschränkt werden, nicht unbedingt nahe.

In noch stärkerem Maße widersprechen die Daten allerdings solchen konstruktionsbasierten Theorien, die annehmen, dass Verben bestimmter Bedeutungsklassen (z.B. Geräuschverben) in quasi fremde Argumentstrukturmuster (z.B. eine intransitive Bewegungskonstruktion) eingesetzt werden können und im Zuge einer konzeptuellen Anpassung dort ad hoc im Sprachverarbeitungsprozess verfügbar gemacht werden. Die beobachteten Idiosynkrasien sprechen hier eindeutig gegen solche pragmatisch gesteuerten konstruktionsgrammatischen Ansätze. Wenn eine konstruktionsgrammatische Modellierung dieser Phänomene angestrebt ist, dann muss sie von pragmatisch gesteuerten Ad-hoc-Prozessen weitgehend absehen, und stattdessen von einer Vielzahl äußerst feinkörnig gestalteter Konstruktionen als Bestandteil unseres gespeicherten syntaktisch-semantischen Wissens ausgehen.

Unsere Daten legen insbesondere nahe, dass der Konstruktionsgedanke vor allem auf einer sehr ausdrucksnahen, konkreten Ebene greift. Die idiosynkratischen Präferenzen für bestimmte Argumentstellen in bestimmten Argumentstrukturmustern suggerieren, dass solche Idiosynkrasien in Form konstruktionsähnlicher Gebrauchsmuster kognitiv präsent sind. Auf der abstrakteren Ebene scheint uns die Frage nach der sprachtheoretischen Angemessenheit von konstruktionsbasierten versus regelgesteuerten valenzbasierten Modellen noch nicht beantwortet. Es ist zumindest deutlich geworden, dass die Varianz bei Argumentstrukturmustern erheblich größer ist, als dies im Allgemeinen angenommen wird, und dass jedes dieser hier recht granular erfassten Muster Idiosynkrasien in Form und/oder Bedeutung einerseits und bezüglich der Einsetzbarkeit von Verben andererseits aufweist. Zumindest auf einer deskriptiven Ebene konstituieren solche Muster eher ein Netz von Familienähnlichkeiten als eine rein taxonomische oder polysemiebasierte Struktur. 


\section{Anhang}

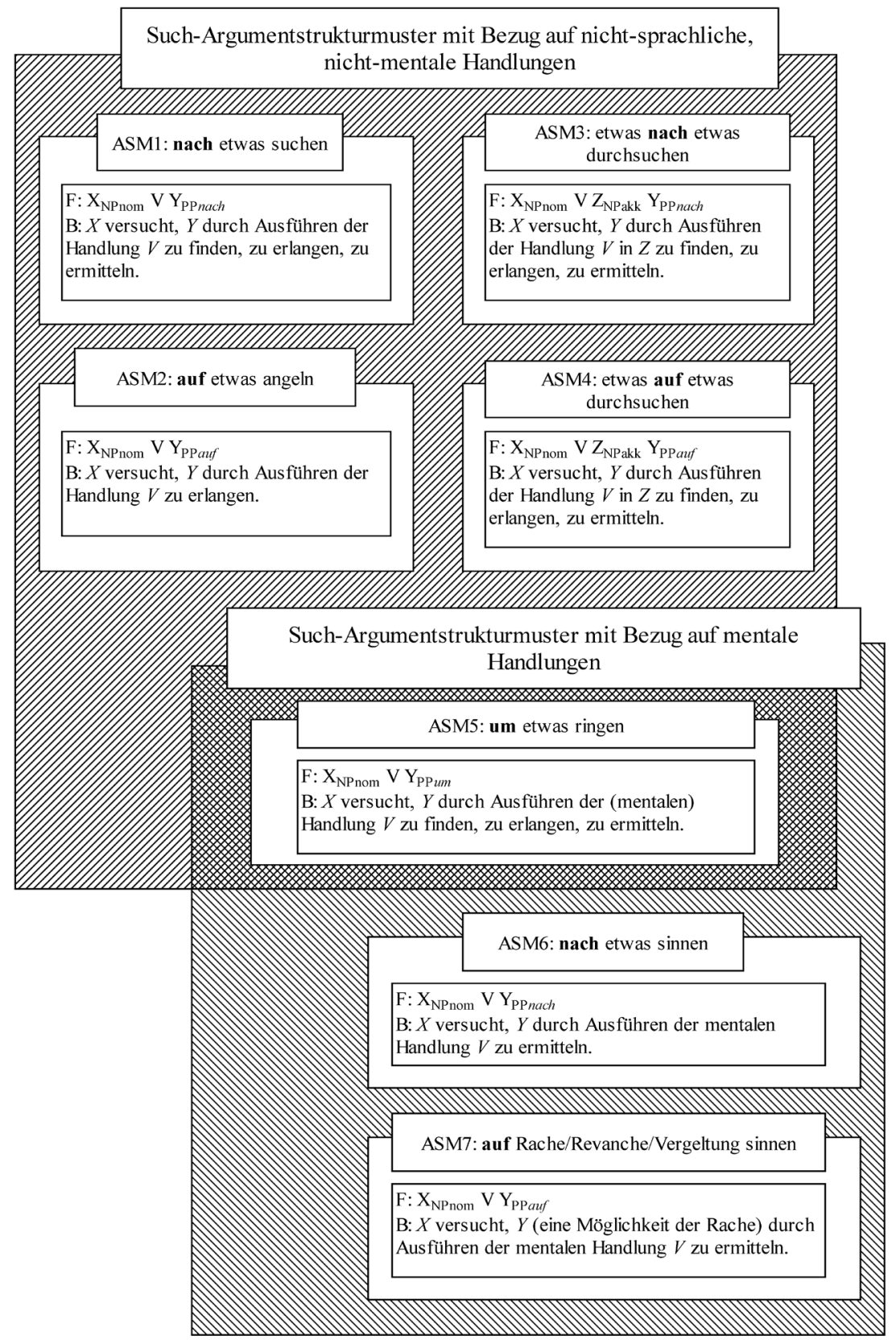



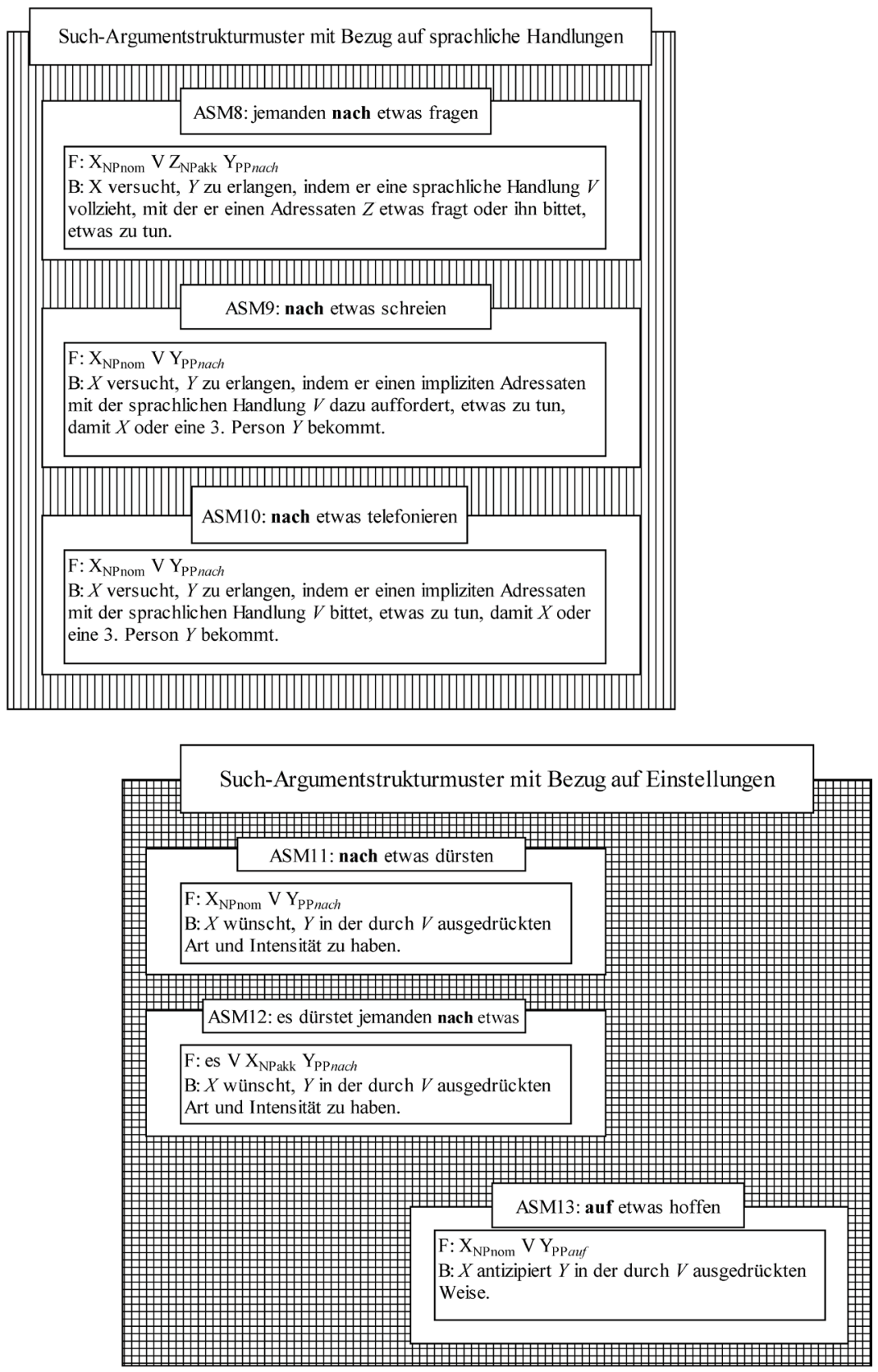

Abb. 12: Die Familie der Such-Argumentstrukturmuster 


\begin{tabular}{|c|c|c|c|c|c|c|c|c|c|}
\hline \multirow[t]{3}{*}{ ASM } & \multicolumn{3}{|c|}{ Semantische Ähnlichkeiten } & \multicolumn{5}{|c|}{ Formale Ähnlichkeiten } & \multirow{3}{*}{$\begin{array}{c}\text { Ausmaß } \\
\text { der } \\
\text { Ähn- } \\
\text { lichkeit }\end{array}$} \\
\hline & \multirow{2}{*}{$\begin{array}{l}\text { Vorkom- } \\
\text { men in } \\
\text { gleicher } \\
\text { Subfamilie }\end{array}$} & \multicolumn{2}{|c|}{$\begin{array}{c}\text { Übereinstimmung } \\
\text { Anzahl der } \\
\text { Argumente }\end{array}$} & \multicolumn{3}{|c|}{$\begin{array}{l}\text { Übereinstimmung } \\
\text { Präposition }\end{array}$} & \multicolumn{2}{|c|}{$\begin{array}{c}\text { Übereinstimmung } \\
\text { phrasaler } \\
\text { Kategorien } \\
\end{array}$} & \\
\hline & & 2 Arg. & 3 Arg. & PPnach & PPauf & PРиm & NPnom & NPakk & \\
\hline ASM1/ASM2 & * & * & & & & & * & & 3 \\
\hline ASM1/ASM3 & $*$ & & & $*$ & & & $*$ & & 3 \\
\hline ASM1/ASM4 & * & & & & & & * & & 2 \\
\hline ASM1/ASM5 & & $*$ & & & & & $*$ & & 2 \\
\hline ASM1/ASM6 & & $*$ & & $*$ & & & $*$ & & 3 \\
\hline ASM1/ASM7 & & * & & & & & $*$ & & 2 \\
\hline ASM1/ASM8 & & & & $*$ & & & $*$ & & 2 \\
\hline ASM1/ASM9 & & * & & $*$ & & & $*$ & & 3 \\
\hline ASM1/ASM10 & & * & & * & & & * & & 3 \\
\hline ASM1/ASM11 & & $*$ & & $*$ & & & * & & 3 \\
\hline ASM1/ASM12 & & $*$ & & $*$ & & & & & 2 \\
\hline ASM1/ASM13 & & $*$ & & & & & $*$ & & 2 \\
\hline ASM2/ASM3 & $*$ & & & & & & $*$ & & 2 \\
\hline ASM2/ASM4 & $*$ & & & & $*$ & & $*$ & & 3 \\
\hline ASM2/ASM5 & & * & & & & & $*$ & & 2 \\
\hline ASM2/ASM6 & & * & & & & & $*$ & & 2 \\
\hline ASM2/ASM7 & & $*$ & & & $*$ & & $*$ & & 3 \\
\hline ASM2/ASM8 & & & & & & & * & & 1 \\
\hline ASM2/ASM9 & & $*$ & & & & & $*$ & & 2 \\
\hline ASM2/ASM10 & & $*$ & & & & & $*$ & & 2 \\
\hline ASM2/ASM11 & & * & & & & & $*$ & & 2 \\
\hline ASM2/ASM12 & & $*$ & & & & & & & 1 \\
\hline ASM2/ASM13 & & $*$ & & & $*$ & & $*$ & & 3 \\
\hline ASM3/ASM4 & * & * & & & & & $*$ & $*$ & 4 \\
\hline ASM3/ASM5 & & & & & & & * & & 1 \\
\hline ASM3/ASM6 & & & & $*$ & & & $*$ & & 2 \\
\hline ASM3/ASM7 & & & & & & & $*$ & & 1 \\
\hline ASM3/ASM8 & & & * & $*$ & & & * & $*$ & 4 \\
\hline ASM3/ASM9 & & & & $*$ & & & $*$ & & 2 \\
\hline ASM3/ASM10 & & & & $*$ & & & $*$ & & 2 \\
\hline ASM3/ASM11 & & & & $*$ & & & $*$ & & 2 \\
\hline ASM3/ASM12 & & & & $*$ & & & & $*$ & 2 \\
\hline ASM3/ASM13 & & & & & & & $*$ & & 1 \\
\hline ASM4/ASM5 & & & & & & & $*$ & & 1 \\
\hline ASM4/ASM6 & & & & & & & $*$ & & 1 \\
\hline ASM4/ASM7 & & & & & & & $*$ & & 1 \\
\hline ASM4/ASM8 & & & $*$ & & & & $*$ & $*$ & 3 \\
\hline ASM4/ASM9 & & & & & & & $*$ & & 1 \\
\hline ASM4/ASM10 & & & & & & & $*$ & & 1 \\
\hline ASM4/ASM11 & & & & & & & $*$ & & 1 \\
\hline
\end{tabular}




\begin{tabular}{|c|c|c|c|c|c|c|c|c|c|}
\hline \multirow{3}{*}{ ASM } & \multicolumn{3}{|c|}{ Semantische Ähnlichkeiten } & \multicolumn{5}{|c|}{ Formale Ähnlichkeiten } & \multirow{3}{*}{$\begin{array}{c}\text { Ausmaß } \\
\text { der } \\
\text { Ähn- } \\
\text { lichkeit }\end{array}$} \\
\hline & \multirow{2}{*}{\begin{tabular}{|c|} 
Vorkom- \\
men in \\
gleicher \\
Subfamilie
\end{tabular}} & \multicolumn{2}{|c|}{$\begin{array}{c}\text { Übereinstimmung } \\
\text { Anzahl der } \\
\text { Argumente }\end{array}$} & \multicolumn{3}{|c|}{$\begin{array}{l}\text { Übereinstimmung } \\
\text { Präposition }\end{array}$} & \multicolumn{2}{|c|}{$\begin{array}{c}\text { Übereinstimmung } \\
\text { phrasaler } \\
\text { Kategorien } \\
\end{array}$} & \\
\hline & & 2 Arg. & 3 Arg. & PPnach & PPauf & PРum & NPnom & NPakk & \\
\hline ASM4/ASM12 & & & & & & & & * & 1 \\
\hline ASM4/ASM13 & & & & & * & & * & & 2 \\
\hline ASM5/ASM6 & * & * & & & & & * & & 3 \\
\hline ASM5/ASM7 & $*$ & $*$ & & & & & * & & 3 \\
\hline ASM5/ASM8 & & & & & & & * & & 1 \\
\hline ASM5/ASM9 & & $*$ & & & & & * & & 2 \\
\hline ASM5/ASM10 & & * & & & & & $*$ & & 2 \\
\hline ASM5/ASM11 & & $*$ & & & & & $*$ & & 2 \\
\hline ASM5/ASM12 & & * & & & & & & & 1 \\
\hline ASM5/ASM13 & & $*$ & & & & & * & & 2 \\
\hline ASM6/ASM7 & * & * & & & & & $*$ & & 3 \\
\hline ASM6/ASM8 & & 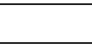 & & $*$ & & & $*$ & & 2 \\
\hline ASM6/ASM9 & & $*$ & & $*$ & & & $*$ & & 3 \\
\hline ASM6/ASM10 & & * & & $*$ & & & * & & 3 \\
\hline ASM6/ASM11 & & * & & $*$ & & & * & & 3 \\
\hline ASM6/ASM12 & & $*$ & & $*$ & & & - & & 2 \\
\hline ASM6/ASM13 & & $*$ & & & & & $*$ & & 2 \\
\hline ASM7/ASM8 & & & & & & & * & & 1 \\
\hline ASM7/ASM9 & & $*$ & & & & & $*$ & & 2 \\
\hline ASM7/ASM10 & & $*$ & & & & & $*$ & & 2 \\
\hline ASM7/ASM11 & & $*$ & & & & & $*$ & & 2 \\
\hline ASM7/ASM12 & & * & & & & & & & 1 \\
\hline ASM7/ASM13 & & $*$ & & & * & & $*$ & & 3 \\
\hline ASM8/ASM9 & $*$ & - & & $*$ & & & $*$ & & 3 \\
\hline ASM8/ASM10 & $*$ & 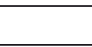 & & $*$ & & & $*$ & & 3 \\
\hline ASM8/ASM11 & & & & $*$ & & & $*$ & & 2 \\
\hline ASM8/ASM12 & & & & * & & & & * & 2 \\
\hline ASM8/ASM13 & & & & & & & $*$ & & 1 \\
\hline ASM9/ASM10 & $*$ & $*$ & & $*$ & & & $*$ & & 4 \\
\hline ASM9/ASM11 & & $*$ & & $*$ & & & $*$ & & 3 \\
\hline ASM9/ASM12 & & * & & $*$ & & & & & 2 \\
\hline ASM9/ASM13 & & $*$ & & - & & & $*$ & & 2 \\
\hline ASM10/ASM11 & & $*$ & & $*$ & & & $*$ & & 3 \\
\hline ASM10/ASM12 & & $*$ & & $*$ & & & & & 2 \\
\hline ASM10/ASM13 & & $*$ & & & & & $*$ & & 2 \\
\hline ASM11/ASM12 & $*$ & $*$ & & $*$ & & & & & 3 \\
\hline ASM11/ASM13 & $*$ & $*$ & & & & & $*$ & & 3 \\
\hline ASM12/ASM13 & $*$ & $*$ & & & & & & & 2 \\
\hline
\end{tabular}

Tab. 1: Semantische und formale Ähnlichkeiten von Such-Argumentstrukturmustern 


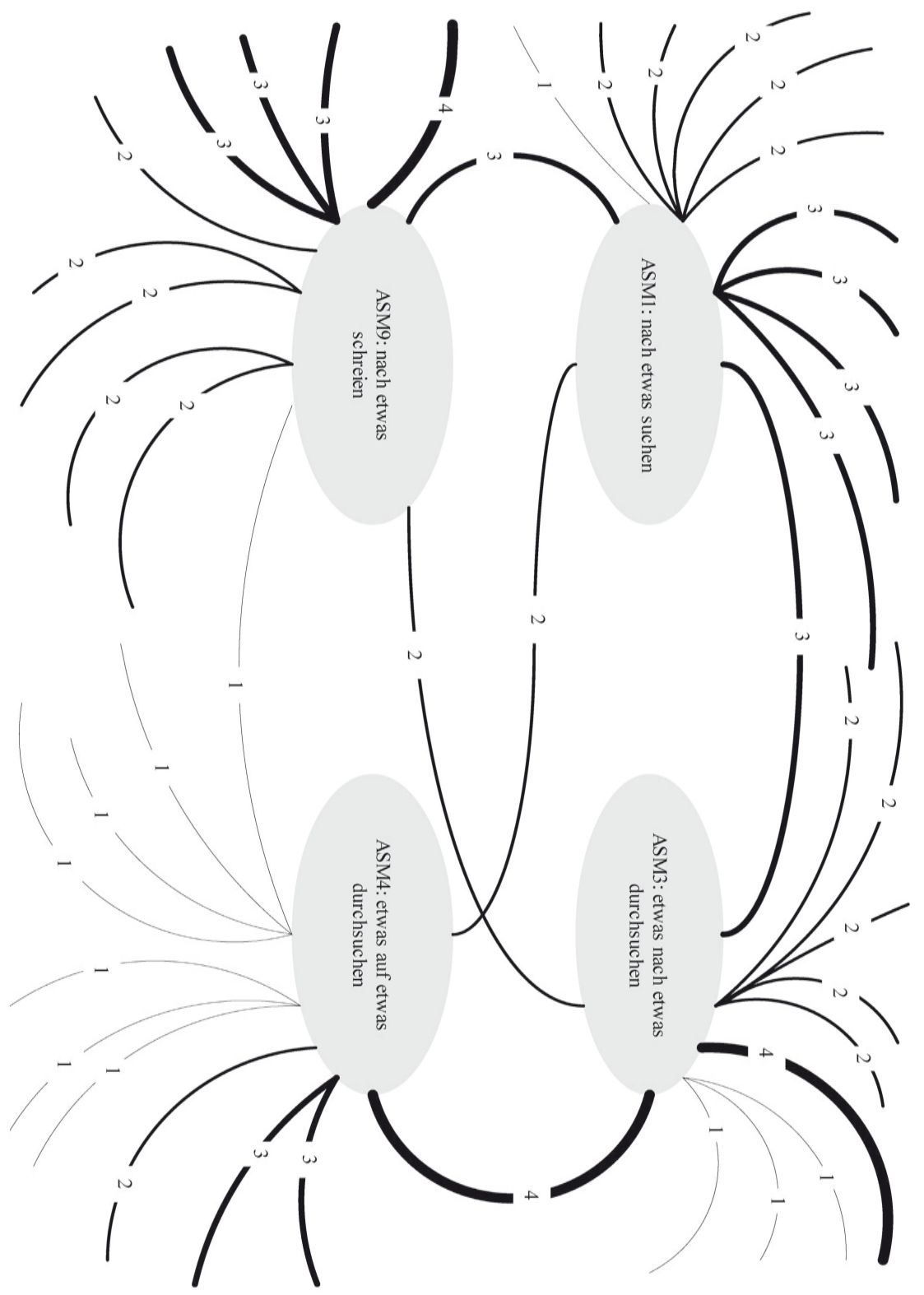

Abb. 13: Grad der Ähnlichkeit von Such-Argumentstrukturmustern 


\section{Literatur}

Baron, Naomi (1971): On defining cognate object. In: Glossa 0017-1271, S. 71-98.

Belica, Cyril (2001-2007): Kookkurrenzdatenbank CCDB - V3.2. Eine korpuslinguistische Denk- und Experimentierplattform. Institut für Deutsche Sprache, Mannheim.

Engelberg, Stefan (2000): Verben, Ereignisse und das Lexikon. (= Linguistische Arbeiten 414). Tübingen.

Engelberg, Stefan (2007): Konstruktionelle Varianten zwischen Wörterbuch und Grammatik. In: Germanistische Mitteilungen 66, S. 11-27.

Engelberg, Stefan (2009): Blätter knistern über den Beton. Zwischenbericht aus einer korpuslinguistischen Studie zur Bewegungsinterpretation bei Geräuschverben. In: Winkler (Hg.), S. 75-97. Internet: www.ids-mannheim.de/pub/laufend/opal/privat/pdf/opal09-4 _engelberg.pdf (Stand: 08.11.2010).

Goldberg, Adele E. (1995): Constructions: a Construction Grammar approach to argument structure. Chicago.

Goldberg, Adele E. (1997): The relationships between verbs and constructions. In: Verspoor, Marjolijn H./Kee Dong, Lee/Sweetser, Eve (Hg.): Lexical and syntactical constructions and the construction of meaning. (= Current Issues in Linguistic Theory 150). Amsterdam/Philadelphia, S. 383-398.

Goldberg, Adele E. (2006): Constructions at work. The nature of generalization in language. Oxford.

Goldberg, Adele E. (2009): The nature of generalization in language. In: Cognitive Linguistics 20, S. 93-127.

Goldberg, Adele E./Jackendoff, Ray (2004): The English resultative as a family of constructions. In: Language 80, 3, S. 532-568.

Gurevich, Olga/Johnson, Matthew A./Goldberg, Adele E. (2010): Incidental verbatim memory for language. In: Language and Cognition 21, S. 45-78.

Handwerker, Brigitte (2008): Lernbasis Lexikon. - Das Verb und die Lizenz zu konstruieren. In: Fandrych, Christian/Thonhauser, Ingo (Hg.): Fertigkeiten und Kompetenzen - separiert oder integriert? Zur Neubewertung der Fertigkeiten und Kompetenzen im Fremdsprachenunterricht. Wien, S. 35-54.

Harley, Heidi/Noyer, Rolf (2000): Formal versus encyclopedic properties of vocabulary: evidence from nominalizations. In: Peeters, Bert (Hg.): The lexicon-encyclopedia interface. (= Current Research in the Semantics-Pragmatics Interface 5). Amsterdam, S. 349-375.

Höche, Silke (2009): Cognate object constructions in English: a cognitive-linguistic account. (= Language in Performance 41). Tübingen.

Jacobs, Joachim (1992): Bewegung als Valenzvererbung, Teil I. In: Linguistische Berichte 138 , S. 85-122.

Jacobs, Joachim (1994): Kontra Valenz. Trier. 
Jacobs, Joachim (2008): Wozu Konstruktionen? In: Linguistische Berichte 213, S. 3-44.

Jacobs, Joachim (2009): Valenzbindung oder Konstruktionsbindung? Eine Grundfrage der Grammatiktheorie. In: Zeitschrift für germanistische Linguistik 37, 3, S. 490513.

Kaufmann, Ingrid (1995): Konzeptuelle Grundlagen semantischer Dekompositionsstrukturen. Die Kombinatorik lokaler Verben und prädikativer Komplemente. (= Linguistische Arbeiten 335). Tübingen.

Levin, Beth (1993): English verb classes and alternations. A preliminary investigation. Chicago.

Maienborn, Claudia (1994): Kompakte Strukturen: Direktionale Präpositionalphrasen und nicht-lokale Verben. In: Felix, Sascha W./Habel, Christopher/Rickheit, Gert (Hg.): Kognitive Linguistik. Repräsentation und Prozesse. Opladen, S. 229-249.

Matsumoto, Masumi (1996): The syntax and semantics of the cognate object construction. In: English Linguistics (Journal of the English Linguistic Society of Japan) 13, S. $190-220$.

Müller, Stefan (2006): Resultativkonstruktionen, Partikelverben und syntaktische vs. lexikonbasierte Konstruktionen. In: Fischer, Kerstin/Stefanowitsch, Anatol (Hg.): Konstruktionsgrammatik. Von der Anwendung zur Theorie. (= Stauffenburg Linguistik 40). Tübingen, S. 177-202.

Proost, Kristel (2009): Warum man nach Schnäppchen jagen, aber nicht nach Klamotten bummeln kann. Die nach-Konstruktion zwischen Lexikon und Grammatik. In: Winkler (Hg.), S. 10-41. Internet: www.ids-mannheim.de/pub/laufend/opal/privat/ pdf/opal09-4_proost.pdf (Stand: 08.11.2010).

Stefanowitsch, Anatol/Gries, Stefan T. (2003): Collostructions: Investigating the interaction of words and constructions. In: International Journal of Corpus Linguistics 8, 2, S. 209-243.

Welke, Klaus (2009): Valenz und Konstruktionsgrammatik. In: Zeitschrift für Germanistische Linguistik 37, S. 81-124.

Winkler, Edeltraud (2009): Anna lächelte ibr freundliches Lächeln. Syntaktischer und semantischer Status von inneren Objekten im Deutschen. In: Winkler (Hg.), S. 126145. Internet: www.ids-mannheim.de/pub/laufend/opal/privat/pdf/opal09-4_winkler.pdf (Stand: 08.11.2010).

Winkler, Edeltraud (Hg.) (2009): Konstruktionelle Varianz bei Verben. (= OPAL - Online publizierte Arbeiten zur Linguistik Sonderheft 4/2009). Mannheim. Internet: www.ids-mannheim.de/pub/laufend/opal/privat/opal09-4.html (Stand: 08.11.2010).

Wittgenstein, Ludwig (2001): Philosophische Untersuchungen. Kritisch-genetische Edition. Hrsg. v. Joachim Schulte. Frankfurt a.M.

Wunderlich, Dieter (2006): Towards a structural typology of verb classes. In: Wunderlich, Dieter (Hg.): Advances in the theory of the lexicon. (= Interface Explorations 13). Berlin/New York, S. 57-166. 
Wolfgang Imo

\title{
Die Grenzen von Konstruktionen: Versuch einer granularen Neubestimmung des Konstruktionsbegriffs der Construction Grammar
}

\begin{abstract}
In der letzten Zeit wurde aus verschiedenen linguistischen Teildisziplinen heraus versucht, die Anwendbarkeit der Construction Grammar in Bereichen wie beispielsweise der Gesprächsforschung zu überprüfen. Für die Gesprächsforschung bzw. Interaktionale Linguistik bietet die Construction Grammar einen viel versprechenden theoretischen Rahmen, da sich viele der Grundannahmen von Construction Grammar und Interaktionaler Linguistik/Gesprächsanalyse decken. Trotz dieser positiven Übereinstimmungen führt das zeichenbasierte Konzept der Konstruktionsgrammatik zu Problemen bei der Analyse gesprochener Sprache. Häufig können bestimmte Phrasen, Satzmuster oder Wörter nicht bestimmten Konstruktionen eindeutig zugeordnet werden, da zu ihrem Verständnis Kontextinformationen nötig sind oder da sie die Merkmale mehrerer Konstruktionen teilen. Anhand dreier problematischer Fälle aus dem gesprochenen Deutsch, bei denen das Konzept, Konstruktionen als Zeichen zu betrachten, zu Problemen führt, werden die Grenzen des konstruktionsgrammatischen Modells aufgezeigt. In einem zweiten Schritt wird als Lösungsvorschlag die Theorie der Granularität nach Bittner/Smith (2001a und b, 2003) vorgestellt, die dazu entwickelt wurde, „to map vague concepts onto crisp portions of reality" (Bittner/Smith 2011a, S 1). Zuletzt wird eine granulare Re-Analyse der eingangs vorgestellten Problemfälle vorgenommen.
\end{abstract}

\section{Einleitung}

In den letzten Jahren ist eine „Familie“ von grammatiktheoretischen Ansätzen, die unter den Begriffen Construction Grammar oder manchmal auch Cognitive Grammar zusammengefasst werden, in den Blick von ForscherInnen unterschiedlicher linguistischer Teildisziplinen geraten, die diese Ansätze auf ihre Anwendbarkeit beispielsweise in der Historiolinguistik, Korpuslinguistik, Gesprächslinguistik oder Computerlinguistik untersuchen (Fischer/Stefanowitsch 2006; Stefanowitsch/Fischer (Hg.) 2008; Fried/ Östman 2005). Gerade für die theoriescheue Gesprochene-Sprache-Forschung (vgl. Hennig 2006) scheint sich die Construction Grammar anzubieten, da deren Grundannahmen - Grammatik als symbolisches Inventar, Grammatik als im Gebrauch erworbene und emergente Struktur, holistische Beschreibung von Konstruktionen, die auch prosodische Eigenschaften, Kollokationen oder den Gebrauchskontext umfasst und gebrauchsbasierte Analyse von Sprache - mit den empirisch gewonnenen Ergebnissen 
der Gesprochene-Sprache-Forschung auf den ersten Blick kompatibel erscheinen (vgl. Deppermann 2006; Günthner/Imo 2006; Imo 2007a). Die Idee, „dass Konstruktionen als Form-Bedeutungs-Einheiten in der kommunikativen Praxis entstehen" (Günthner/Imo 2006, S. 8), entspricht dabei der Sichtweise in der Gesprächsforschung, dass Handlungen, Rollen und Sinn generell interaktiv erzeugt werden.

Immer deutlicher wird allerdings, dass die Annahme, bei Konstruktionen handele es sich um feste Form-Bedeutungs-Paare bzw. Form-Funktions-Paare (im Folgenden werde ich den Begriff „Bedeutung“ so verwenden, dass er die „Funktion“ einer Konstruktion mit umfasst), der Realität nicht standhält. So finden sich Ausdrücke, die bei gleicher Form - wobei hier nicht nur die (Morpho-)Syntax, sondern auch Prosodie, äußere Syntax und Kontext zur Form zählen - unterschiedliche Bedeutungen innehaben. Bei anderen Ausdrücken wiederum können mehrere Bedeutungen gleichzeitig aktiviert sein oder es besteht eine Unentscheidbarkeit in Bezug auf die Bedeutung. Letzeres ist beispielsweise der Fall bei so genannten ,garden path"-Strukturen (Imo i.Dr.a) oder bei Ausdrücken, die sich in einem Grammatikalisierungs-, Pragmatikalisierungs- oder Lexikalisierungsprozess befinden (vgl. Günthner/Imo 2003). Das folgende Beispiel aus Günthner (2009) soll kurz illustrieren, weshalb eine auf Zeichen basierende Grammatik in Schwierigkeiten kommen kann. Günthner (ebd.) untersucht „Adjektiv + dass-Satz-Konstruktionen" im gesprochenen Deutsch und stellt dabei fest, dass es nicht ein festes Muster gibt, sondern eine Reihe von ähnlichen Strukturen, die sie in folgender Tabelle zusammenfasst:

\begin{tabular}{|c|c|l|c|l|}
\hline Vorfeld & Finitum & \multicolumn{1}{|c|}{ Mittelfeld } & Infiniter VK & \multicolumn{1}{|c|}{ Nachfeld } \\
\hline es & Ist & gUt, & - & $\begin{array}{l}\text { dass es so gekOm- } \\
\text { men ist, }\end{array}$ \\
\hline & Is & ja KLAR? & - & $\begin{array}{l}\text { dass der kontakt } \\
\text { !NACH!lässt; }(.)\end{array}$ \\
\hline & s= & echt SCHAde, & - & $\begin{array}{l}\text { dass das jetzt nicht } \\
\text { geKLAPPT hat- }\end{array}$ \\
\hline & & schön & - & $\begin{array}{l}\text { dass die so schön } \\
\text { SCHWER is; }\end{array}$ \\
\hline
\end{tabular}

Tab. 1: Adjektiv + dass-Satz-Konstruktionen (Günthner 2009, S. 172)

Wie man sehen kann, handelt es sich auf der formalen Seite um vier unterschiedliche Realisierungsweisen, die allerdings eng miteinander verbunden sind. Wenn nun auf der Bedeutungs- oder Funktionsebene alle diese Varianten identisch wären oder, umgekehrt, alle eine klar erkennbare eigene Funktion hätten, wäre eine Analyse als Zeichen machbar. Leider ist das nicht der Fall: 
Auch funktional scheinen sich die verschiedenen Formen zu unterscheiden: Während die „Adjektiv + dass-Satz“-Konstruktionen häufig als (resümierende) Kommentare eingesetzt werden, die aktuelle Gesprächsthemen abschließen, ist dies bei Extrapositionen [gemeint sind Muster wie das erste in der Tabelle, Anm. d. Verf.] eher nicht der Fall. (Günthner 2009, S. 172)

Auf beiden Seiten - formal und inhaltlich - liegen Variationen vor, die weder trennscharf genug sind, um für eine Zeichenkonstitution herhalten zu können (die einschränkenden Ausdrücke „häufig“" und „eher nicht“ weisen darauf hin) noch umgekehrt einfach ignoriert werden können.

Hopper (2004) stellt angesichts solcher Befunde - die GesprocheneSprache-Forschung kann mit Unmengen weiterer Beispiele aufwarten - die Frage, ob es überhaupt Sinn hat, den zeichenbasierten Konstruktionsbegriff der Construction Grammar zu verwenden, oder ob im Sprachgebrauch nicht immer nur „fragments of constructions“ verwendet werden. Wenn das der Fall ist, was bedeutet das für den Konstruktionsbegriff bzw. für die Kategorienbildung generell? Sind Konstruktionen lediglich ad hoc abstrahierte Muster auf der Basis von ständig divergierenden Verwendungsweisen? Oder existieren sie schon vor der Produktion von Äußerungen als abstrakte Zeichen, an denen sich SprecherInnen orientieren? Im Folgenden werde ich zunächst den Konstruktionsbegriff der Construction Grammar vorstellen, dann auf die Vorteile eingehen, die eine auf dem Zeichenbegriff und nicht einem Regelbegriff aufgebaute Grammatiktheorie bietet und zuletzt die Frage stellen, welche Möglichkeiten bestehen, das Zeichenkonzept auszubauen und so dessen Vorteile beizubehalten. Eine solche Möglichkeit könnte darin bestehen, eine ,granulare“ Perspektive (vgl. Bittner/Smith 2001a, b, 2003; Schegloff 2000) in das Zeichenkonzept der Construction Grammar einzuführen.

\section{Die Definition von Konstruktionen aus der Sicht der Construction Grammar}

Trotz aller inhaltlichen und methodischen Divergenzen zwischen den unterschiedlichen Ansätzen, die zum Bereich der Construction Grammar gerechnet werden können (wie z.B. kognitive Ansätze ${ }^{1}$ (Langacker 1987, 1999 oder Taylor 2002), Spracherwerbsansätze (Tomasello 2003, 2006; Wong-Fillmore 1979) oder unterschiedliche gebrauchsbasierte und korpusbasierte Ansätze (Goldberg 1995, 1996, 1998; Fischer/Stefanowitsch 2006; Stefanowitsch/ Fischer (Hg.) 2008; Günthner/Imo 2006; Imo 2007a), herrscht im Allge-

Die Nähe vieler Ansätze der Cognitive Grammar und der Construction Grammar führt dazu, dass beide oft zusammen behandelt werden. Goldberg (1998, S. 205) sieht beide Begriffe sogar als austauschbar an: „Within the theory of Construction Grammar (also Cognitive Grammar) [...]“. Vgl. auch Fischer/Stefanowitsch (2006, S. 5). 
meinen der Konsens, dass es sich bei Konstruktionen um „Zeichen“ in einem saussureanischen Sinn handelt. So definiert Fillmore (1988, S. 36) eine grammatische Konstruktion als ,any syntactic pattern which is assigned one or more conventional functions in a language“.2 Fischer/Stefanowitsch (2006, S. 3) stellen fest, die Konstruktionsgrammatik sei „derzeit (noch) keine einheitliche Theorie, sondern eher eine Familie von Theorien, die durch die Einsicht miteinander verbunden sind, dass die menschliche Sprache auf allen sprachlichen Ebenen aus Zeichen“" bestehe. Dabei ist die „Annahme, dass Grammatik umfassend und ausschließlich als Inventar linguistischer Zeichen verstanden werden kann“ das „,konstituierende Element einer Konstruktionsgrammatik und findet sich folglich in allen Versionen“" (ebd.). Auch in der kognitiven Grammatik bildet der Zeichenbegriff den zentralen Ausgangspunkt. Nach Taylor (2002, S. 20) stellt die Konzeption der Grammatik vom Begriff des Zeichens her - Taylor spricht von der „symbolic thesis“ - sogar die Hauptmotivation der Cognitive Grammar dar:

What is special about the Cognitive Grammar approach is that syntax itself is regarded as inherently symbolic, and therefore handled in terms of symbolic relations between phonological and semantic structures.

Die „symbolic thesis“ als Triebkraft für die Construction Grammar und Cognitive Grammar hatte ihren Ursprung in dem von Fillmore/Kay/ O'Connor (1988) formulierten Unbehagen an einer nur schwer begründbaren und entsprechend unscharfen Trennung zwischen Lexikon und Syntax. Als Alternative wurde vorgeschlagen, beides als Symbolinventar zu betrachten, so dass ein Kontinuum entsteht, ,whose full and proper characterization reduces to assemblies of symbolic structures" (Langacker 1995, S. 153). ${ }^{3}$ Dabei unterscheidet sich ein Wort (das als ,klassisches“ Zeichen in einem Lexikon einen Eintrag erhält, der Form und Bedeutung spezifiziert) von einer grammatikalischen Struktur (vormals einer syntaktischen Regel) lediglich darin, dass letztere schematisch und abstrakt ist, ersteres dagegen spezifisch: „Grammatical patterns are analyzed as schematic symbolic units, which differ from other symbolic structures not in kind, but only in degree of specificity" (Langacker 1987, S. 58). Zusammen mit der formalen Ausweitung des Zeichenbegriffs von phonologischen Strukturen über die Wortoder Phraseologismusebene bis hin zu einer abstrakten Ebene, die auch syntaktische Muster umfasst, fand eine zweite, parallele Ausweitung des Inhaltsbegriffs von Zeichen statt. So enthalten konstruktionale Zeichen nicht

\footnotetext{
$2 \quad$ Vgl. auch Goldberg (1996, S. 68): „A construction is defined to be a pairing of form with meaning/use such that some aspect of the form or some aspect of the meaning/use is not strictly predictable from the component parts or from other constructions already established to exist in the language."

3 Kritisch äußert sich Jacobs (2008) zu einer Umstellung von Lexikon + Regeln auf Konstruktionen, wobei er aber die Vorteile des Konstruktionsbegriffs als zu groß einstuft, als dass man völlig auf ihn verzichten solle.
} 
nur die Bedeutung im engeren Sinn, sondern auch die Funktion, die prosodische Realisierung (sofern sie für die Konstruktion relevant ist), Informationen über den Kontext, in dem eine Konstruktion auftritt, Kollokationen, und vieles mehr - kurz: „facts about the use of entire constructions, including facts about registers, restricted dialect variation, etc. are stated as part of the construction" (Goldberg 1996, S. 69). Der Grund, weshalb sich die Construction Grammar als attraktive Theorie gerade für empirisch arbeitende Forschungsfelder der Linguistik anbietet (z.B. quantitative und qualitative Korpuslinguistik oder Spracherwerbsforschung), liegt nicht zuletzt in dieser Ausweitung des Zeichenkonzepts.

\section{Die Vorteile, Konstruktionen als Zeichen zu konzipieren}

Der Zeichenbegriff der Construction Grammar stellt für die Gesprächsforschung einen attraktiven Anknüpfungspunkt zur Verfügung, da durch ihn sowohl eine umfassende Beschreibung auf allen relevanten linguistischen Ebenen zugleich ermöglicht wird als auch der Tatsache Rechnung getragen werden kann, dass (wie empirische Untersuchungen gesprochener Sprache gezeigt haben) sprachliche Einheiten ,rich in information“ (vgl. Norén/ Linell 2007, S. 413) sind, dass also z.B. auch kontextuelle und sequenzielle Informationen elementare Bestandteile von grammatischen Strukturen sind:

\footnotetext{
Gesprächsanalyse und Construction Grammar lehnen es beide ab, Syntax als autonom organisiertes Modul zu betrachten und entsprechend zu analysieren. Es ist gerade ein Leitsatz der Interaktionalen Linguistik, den holistischen Zusammenhang von Syntax, Semantik, Prosodie, Pragmatik, Multimodalität und sequenziellkontextuellen Aspekten grammatischer Strukturen im Gespräch zu rekonstruieren und dabei den Kontingenzen des empirischen Datums in all seiner Reichhaltigkeit Rechnung zu tragen. (Deppermann 2006, S. 59)
}

Der holistische Ansatz der Gesprächsanalyse führte dazu, dass zahlreiche Phänomene beschrieben wurden, für die in klassischen Grammatiktheorien „kein Platz“ war (vgl. Fillmore/Kay/O'Connors (1988, S. 504) Begriff des „appendix to the grammar") und die mit Hilfe der Construction Grammar ihren Platz in einer Grammatiktheorie finden konnten. Im Folgenden soll nun die Attraktivität der Construction Grammar anhand einiger Beispielanalysen aus der gesprochenen Sprache gezeigt werden.

3.1 Einheiten, die „zwischen“ traditionellen linguistischen Beschreibungsebenen verortet sind

Typisch für die gesprochene Sprache sind Einheiten, die „dazwischen“ stehen - entweder zwischen Lexik und Syntax oder zwischen Syntax und Gesprächsstruktur/Textstruktur. Ein gutes Beispiel hierfür sind die so genannten 
Diskursmarker (manchmal auch pragmatic markers, Operatoren oder Diskurspartikeln genannt). ${ }^{4}$ Ein solcher Diskursmarker im Deutschen ist ich mein(e), der von Günthner/Imo (2003) und Imo (2007a) analysiert wurde. Das folgende Beispiel aus einer Radio-Beratungssendung zeigt eine typische Verwendungsweise von ich mein $(e):^{5}$

\section{Beispiel 1: Von Mensch zu Mensch Einsamkeit}

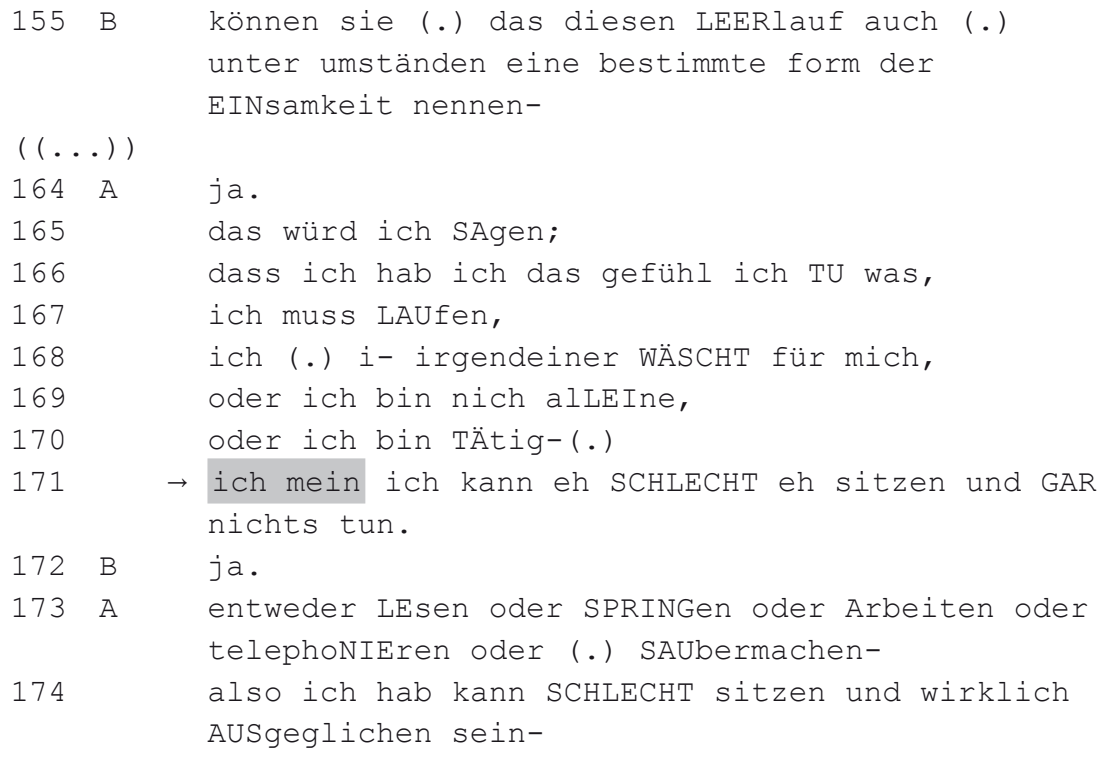

Es fällt sofort auf, dass ich mein (Z. 171) hier nicht als traditioneller Matrixsatz verwendet wird. Vielmehr wird durch den zur Formel verkürzten Ausdruck eine Aktivität (die Aufzählung, die die Anruferin in Z. 166 startet) unterbrochen und eine neue Aktivität (eine Zusammenfassung bzw. ein Fazit in Z. 171) eingeleitet. Wenn man sich nun die Frage stellt, wie ich mein (e) grammatikalisch zu beschreiben ist, so sind drei Ebenen festzustellen: (1) Man könnte ich mein(e) ausschließlich als Vertreter der Wortart „Diskursmarker" beschreiben, ähnlich wie ja auch als dass als komplexe Konjunktion gewertet werden kann. Insofern bliebe man hier auf der Ebene des Lexikons. (2) Gleichzeitig handelt es sich bei einem Diskursmarker aber nicht um eine bloße Wortart, sondern um einen Teil einer komplexen syntaktischen Konstruktion. Insofern ist der Begriff der Operator-SkopusStrukturen, den Barden/Elstermann/Fiehler (2001) vorschlagen, aussage-

$4 \quad$ Vgl. u.a. Brinton (1996), Fraser (1990), Schiffrin (1987), Barden/Elstermann/Fiehler (2001), Gohl/Günthner (1999), Günthner/Imo (2003), Auer/Günthner (2005).

5 Eine ausführliche Analyse des Beispiels findet sich in Imo (2007a, S. 190 f.). 
kräftiger: Diskursmarker befinden sich vor einer Äußerung - typischerweise im Vor-Vorfeld eines Satzes - und projizieren eben diesen nachfolgenden Satz. Ähnlich wie bei der Kategorie „Matrixsatz“ handelt es sich also bei Diskursmarkern nicht (nur) um eine Wortart, sondern vor allem um ein syntaktisches Muster. (3) Auf einer dritten Ebene muss nicht nur das Folgesyntagma mit in die Analyse einbezogen werden, sondern auch die gesamte Gesprächsstruktur. Wie Günthner/Imo (2003) herausstellen, besteht die Funktion von Diskursmarkern darin, einen „Aktivitätswechsel“ zu markieren. In die Beschreibung der Diskursmarker-Konstruktion gehört also auch der Kotext, d.h. das sequenzielle Umfeld. Hier wird der Bereich der Syntax verlassen und die Ebene der Gesprächsstruktur erreicht.

Das Dilemma der Gesprächsanalyse war bislang, dass eine Anbindung der Forschungsergebnisse an Syntaxtheorien an deren Beschränkung auf die hier unter Punkt (2) dargestellte mittlere Ebene scheiterte. Wird das Konzept „Diskursmarker“ im Rahmen der Construction Grammar - und somit als komplexes Zeichen - beschrieben, lösen sich diese Probleme: Die Beschreibung der Funktion, der sequenziellen Platzierung und der projektiven Kraft gehört mit in die Konstruktion „Diskursmarker“. 6

\subsection{Einheiten, bei denen die sequenziellen und prosodischen}

Realisierungsweisen einen wichtigen Informationsanteil stellen

Eine den Diskursmarkern verwandte Konstruktion, die allerdings nicht äuBerungsinitial, sondern prototypisch äußerungsfinal positioniert wird, ist die des Vergewisserungssignals (bzw. der tag question). Obwohl es zahlreiche Vergewisserungssignale im Deutschen gibt (gell?, nicht wahr?, ne?, verstehste?, weißt $d u$ ? etc.), werden sie in Grammatiken nur sehr stiefmütterlich, meist unter dem Abschnitt „Interjektionen“ (Zifonun et al. 1997) oder (Gesprächs)partikeln (Duden 2005; Weinrich 2005) behandelt. Während bei Diskursmarkern die prosodische Realisierung relativ variabel ist, wird bei Vergewisserungssignalen über die Prosodie zwischen einem Vergewisserungssignal im engen Sinne und einem lediglich aufmerksamkeitsheischenden und gesprächsstrukturierenden Signal unterschieden:

Eine steigende Intonationskontur und eine abgesetzte Realisierung aktivieren dabei die Gestalt eines Vergewisserungssignals, eine fallende oder gleich bleibende Tonhöhe in Bezug auf die Umgebungsäußerungen und die prosodische Anbindung an diese Äußerungen sind dagegen mit der eines aufmerksamkeitsheischenden Signals verbunden. (Imo 2007a, S. 335)

Ein weiteres, ganz ähnlich gelagertes Phänomen sind die von Günthner (2006) beschriebenen „dichten Konstruktionen“, wie z.B. „ICH - NIX wie WEG“. Als dichte Konstruktionen gelten „fragmentarische Formen - wie ,uneigentliche Verbspitzenstellungen im narrativen Präsens', ,Infinitkonstruktionen', ,Subjektlose Infinitkonstruktionen' sowie ,Minimale Setzungen““ (ebd., S. 95), die im Rahmen von Alltagserzählungen auftreten. 
Die argumentative, d.h. gesprächsstrukturierende Funktion ist sehr gut in Beispiel 2 zu sehen, während Beispiel 3 „weißt du“ als Vergewisserungssignal im engeren Sinne zeigt. Beide Ausschnitte stammen aus schwäbischen Familiengesprächen (S1 und S2 sind die beiden Söhne, T die Tochter):

Beispiel 2: Schwaben Gott

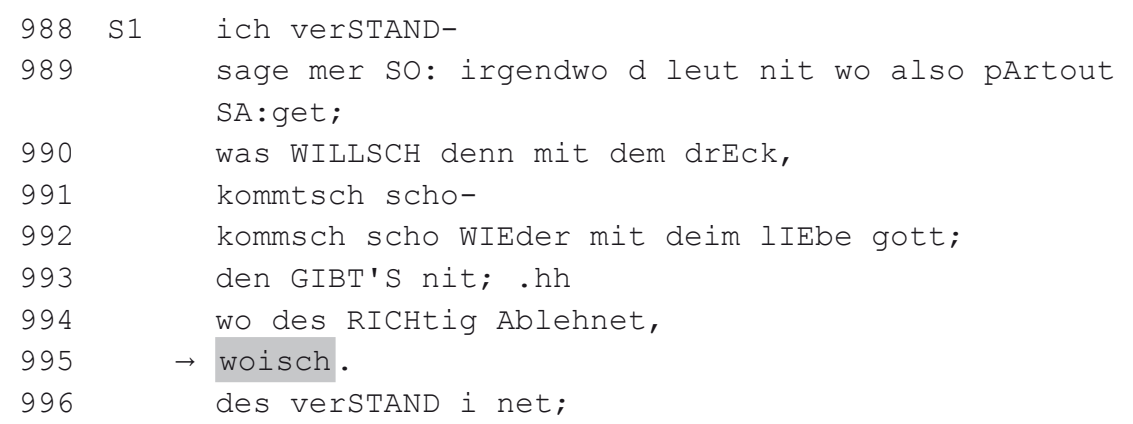

Beispiel 3: Schwaben SS

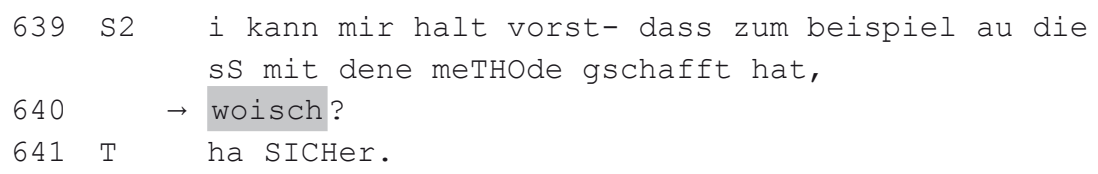

Beide Verwendungsweisen unterscheiden sich darin, dass einmal woisch. (Z. 995) als eine Art Interpunktion funktioniert, also als Marker, der die Darstellung der Leute, die nicht an Gott glauben (Z. 989 bis 994) von der Bewertung des verSTAND i net (Z. 996) trennt. Dagegen dient das woisch? in Z. 640 klar der Einforderung einer Rückmeldung (präferiert einer bestätigenden Rückmeldung).

Auch hier ist die holistische Beschreibung für die Konstruktionsbeschreibung unerlässlich: Sowohl die sequenzielle Platzierung (d.h. ob auf die sprachliche Einheit eine Reaktion eines Gesprächsteilnehmers folgt oder ob der momentane Sprecher fortfährt) als auch die Prosodie liefern notwendige Informationen zum Verständnis des Gebrauchs von Vergewisserungssignalen. ${ }^{7}$

\subsection{Lexikon - Syntax - Gesprächsstruktur - Gattung}

Die fließende Skala von Konstruktionen macht nicht an der Grenze der Syntax oder spätestens der Sequenz halt, auch kommunikative Gattungen oder Textsorten können als Konstruktionen beschrieben werden, wie Öst-

Zu einer kritischen Einschätzung der Rolle der Prosodie im Rahmen der Construction Grammar siehe allerdings Imo (i.Dr.b). 
man (2005) mit seinem Begriff des „Construction Discourse“ betont. Die Möglichkeit, auch solche großen Einheiten als Zeichen zu konzipieren, erweist sich insofern als Vorteil, als ein Sprung der Beschreibungsebenen vermieden werden kann und somit eine einheitliche und konsistente Beschreibung möglich wird. Ein Beispiel für eine solche Interaktion von Gattung und syntaktisch-gesprächsstrukturierendem Muster wurde bereits oben mit Günthners (2006) dichten Konstruktionen erwähnt: Die kommunikative Gattung „Alltagserzählung“ enthält - wenn man sie als Zeichen konzeptualisiert - als einen möglichen Eintrag, dass zu ihrer Herstellung „dichte Konstruktionen“ eingesetzt werden können. Umgekehrt enthalten die „dichten Konstruktionen“ den Eintrag, dass sie in der Gattung „Alltagserzählung" verwendet werden.

Die Faktoren der Gebrauchsbasiertheit und der Sedimentierung - d.h. die Entstehung von mehr oder weniger festen Mustern als Resultat eines langsamen Einschleifens durch den Gebrauch bewährter Lexik, Syntax, Prosodie, Sequenzstruktur etc. - spielten in der Gattungsanalyse von Anfang an eine große Rolle. Tomasello stellt für den Erstspracherwerb fest, dass diese Prozesse für das Erlernen von im engeren Sinne syntaktischen und lexikalischen Konstruktionen genau denselben Stellenwert haben:

Wenn Menschen wiederholt, ähnliche' Dinge in ,ähnlichen' Situationen sagen, entwickelt sich daraus mit der Zeit ein sprachliches Verwendungsmuster, das in den Köpfen der Benutzer als neue Kategorie oder Konstruktion schematisiert wird - mit unterschiedlichen Abstraktionsgraden. (Tomasello 2006, S. 21)

Die Verknüpfung von Sedimentierung und der Entstehung von Zeichen - denn nichts anderes bedeutet es, wenn „ähnliche Dinge“ (Form) in „ähnlichen Situationen“ (Bedeutung/Funktion) gesagt werden - scheint sich tatsächlich als kognitive Strategie von SprachnutzerInnen zu bestätigen. Eine Grammatiktheorie, die auf diesem Prinzip aufbaut, hat also klare empirische Vorteile, wenn es um die Beschreibung gesprochener Sprache geht.

Doch trotz aller hier skizzierten Vorteile, die das Zeichenkonzept der Construction Grammar mit in die „Ehe“ mit der Gesprochene-SpracheForschung gebracht hat, handelt man sich auch einige Nachteile ein, wie im folgenden Abschnitt erläutert wird.

\section{Probleme und Grenzen des Zeichenbegriffs}

Die semiotisch orientierte Forschung zur Konstitution und Verwendung von Zeichen hat mit vielen Problemen zu kämpfen. So stellt Eco (1977, S. 189) fest, dass auf Grund der Tatsache, dass „das semantische System sich verändert" und es ,nur teilweise und in Reaktion auf konkrete Kommunikationsereignisse" beschrieben werden kann, mit Hilfe von Zeichen bestenfalls Möglichkeiten formuliert, nicht aber Ergebnisse postuliert wer- 
den können. Das bedeutet, dass die Reichweite einer Interpretation von Zeichen sehr begrenzt ist und entweder am konkreten Kontext orientiert sein muss oder zu einer Auflistung von Interpretationspotenzialen führt. In einem abrupten Sprung über Jahrzehnte der Forschung zu Zeichen sei kurz auf ein aktuelles Modell - die integrationale Zeichenkonzeption von Harris (2008) - hingewiesen, das versucht, trotz der von Eco konstatierten Probleme einen gangbaren Weg der Kombination von Zeichen und Kontext/ Situation zu formulieren: „Für Integrationisten ist ein Zeichen erst dann ein Zeichen, wenn es kontextualisiert ist: Der Akt der Kontextualisierung und der Erschaffung eines Zeichens sind ein und dasselbe." (ebd., S. 20). Statt der Akzeptanz der Vagheit von Zeichen soll so durch die Einbindung des Kontextes der Knoten gelöst werden. Dabei müssen vier integrative Aspekte nach Harris (ebd., S. 20) beachtet werden: Die ,interpersonale Integration“, die „Umweltintegration“, die „transmodale Integration“ und die „temporale Integration“ .8 Doch trotz dieser so geordnet und strukturiert klingenden Ausweitung des Zeichenbegriffs bleibt das Grundproblem der Vagheit von Zeichen ungelöst:

Die Vorstellung, dass alle Zeichen radikal unbestimmt sind, besagt, dass die Vorstellung semiologischer Bestimmtheit selbst illusorisch ist. Das liegt daran, dass alternative Kontextualisierungen immer möglich sind. Sogar wenn eine Interpretation eines Zeichens als sehr wahrscheinlich empfunden wird, gibt es keinen Weg, alle anderen Kandidaten auszuschließen. (Harris 2008, S. 27)

Trotz aller Bemühungen, das Zeichenkonzept anwendbar und gangbar zu machen, bleibt das zentrale Problem der nicht-Eindeutigkeit von Zeichen bestehen - ein Problem, mit dem die Construction Grammar in der einen oder anderen Weise umgehen muss.

Im Folgenden möchte ich nun zunächst an einigen konkreten Fällen exemplarisch zeigen, inwiefern die semiologische Unbestimmtheit von Zeichen bei der Beschreibung von Konstruktionen eine Rolle spielt.

\subsection{Eine Form - unterschiedliche oder parallele Bedeutungen}

Das Problem bei dieser Kombination - dass eine phonologische Form mehrere Funktionen oder Bedeutungen tragen kann - besteht darin, dass man nicht immer dafür argumentieren kann, dass es sich dabei um verschiedene Konstruktionen (also Zeichen) handelt, sondern dass diese Bedeutungen

\footnotetext{
„Die Integration eigener Aktivitäten mit denen eines anderen Individuums oder anderer Individuen. Das ist interpersonale Integration. [...] Die Integration der Aktivitäten eines Individuums mit Objekten und Ereignissen in der physischen Welt. Das ist Umweltintegration [...]. Die Integration verbaler mit nichtverbaler Kommunikation, visueller mit oraler Kommunikation. Das ist transmodale Integration. [...] Die Integration der Gegenwart mit der Vergangenheit und der Zukunft. Das ist temporale Integration.“ (Harris 2008, S. 20).
} 
„irgendwie“ alle Bestandteil der „Konstruktion“ sein können und je nach Kontext aktiviert oder deaktiviert sind. Es handelt sich dabei um solche Formen, die viele differenzierte Bedeutungsvarianten aufweisen, die aber selten oder nie in einer reinen, prototypischen Form vorkommen, sondern immer in unterschiedlichen Anteilen gemischt sind, also in der aktuellen Verwendung selbst Polysemie aufweisen. Dieses Problem lässt sich nicht dadurch lösen, dass man der Konstruktionsbeschreibung mehr Einträge zu weiteren Informationstypen zugesteht: Auch durch zusätzliche Informationen über Kontext, Prosodie o.Ä. lassen sich keine systematisch strukturierten Konstruktionen erstellen - bestenfalls würde eine höchst detaillierte Ausweitung dazu führen, dass Konstruktionen zu Konstrukten (,constructs“) werden, d.h. also dass nur noch die "Tokens“ gezählt aber keine „Types“ mehr erstellt werden können. Eine Grammatik, die nur noch aus „Tokens“ besteht, ist aber keine mehr. An zwei Beispielen möchte ich diese Problematik erläutern:

\subsubsection{Die Facetten von jetrt}

Wenn man sich Verwendungsweisen des Wortes jetzt betrachtet, so wird deutlich, dass es in einer Reihe höchst unterschiedlicher Funktionen gebraucht wird. Eine detaillierte Untersuchung von jetzt in gesprochener Sprache (Imo 2010) zeigt, dass jetz̧ vorkommt (1) als Element, das Handlungsoder Geschehenszeit und Sprechzeit direkt engführt, d.h. mit der Äußerung von jetət geschieht gleichzeitig etwas, (2) als ein Element, das auf eine in Kürze stattfindende Handlung oder ein Ereignis hinweist, also temporal projizierend wirkt, (3) als ein Element, das auf eine vor kurzem stattgefundene Handlung oder ein Ereignis verweist, also temporal rückblickend ist, (4) als ein Element, das - im Sinne einer „Deixis am Phantasma“ (Bühler 1982 [1934], S. 123) - dazu dient, Erzählungen zu strukturieren und (5) als ein Element, das als Gesprächspartikel z.B. dazu verwendet wird, eine Liste zu strukturieren. Diese Bedeutungsnuancen sind so stark von konkreten Konstellationen aus Kotext und Kontext abhängig, dass es nicht möglich ist, jeweils eigene Konstruktionen zu erstellen. Dazu kommt noch, dass beispielsweise der Unterschied zwischen einer direkten Engführung von Äußerung und Handlung/Ereignis sowie einem projizierenden oder rückblickenden Gebrauch graduell ist, so dass eine Grenzziehung nicht funktionieren kann, wie folgendes Beispiel zeigt:

$9 \quad$ Weshalb beispielsweise bei Zifonun et al. (1997, S. 340) der Begriff „Nahzeitraum“ als Bedeutung von jetzt verwendet wird, der es ermöglicht, diese feinen Unterschiede ignorieren zu können. 
Beispiel 4: Big Brother back to basic

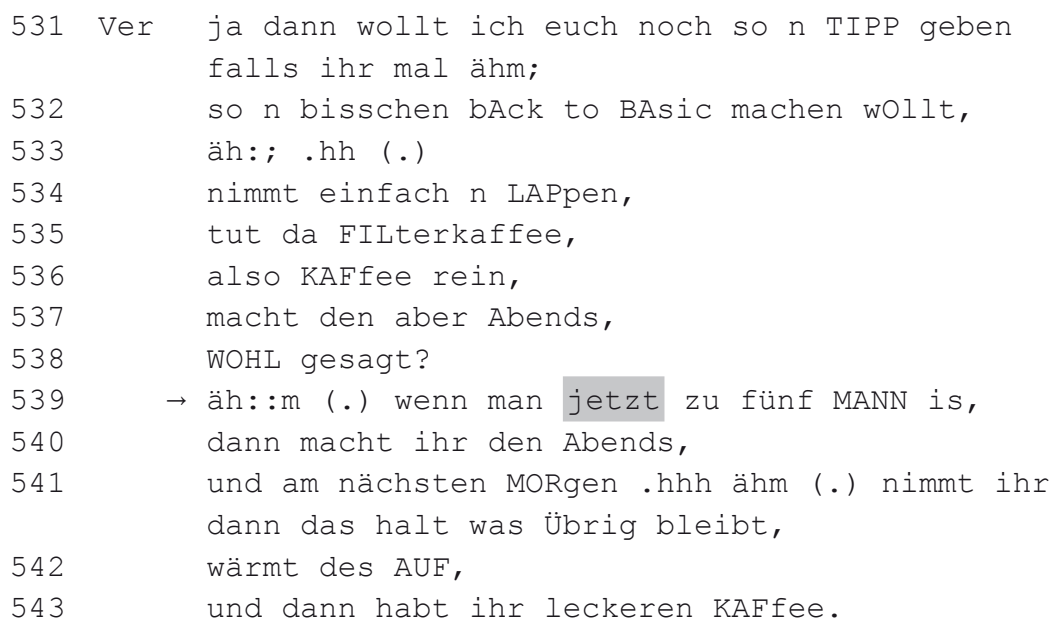

Exemplarisch kann das unklare Bedeutungs- und Funktionsgemenge von jetz̨t an Beispiel 4 aus einer Folge von „Big Brother“ erläutert werden. Verena macht einen Vorschlag, wie das Konzept bAck to BAsic (Z. 532), also ein spartanisches Leben im Big Brother-Container, umgesetzt werden könnte. Zunächst schildert sie allgemein das Vorgehen, den Kaffee zu kochen und springt dann auf die konkrete Situation der fünf MANN (Z. 539) im Container. Um was für eine Konstruktion handelt es sich bei jetzt? Zeitdeixis ist durchaus vorhanden, man könnte sogar für zwei zeitdeiktische Aspekte plädieren: Einerseits verweist jetz̧ auf die aktuelle Situation, in der Verena sich befindet, nämlich eine Situation im Big Brother-Haus mit fünf Personen. Andererseits verweist jeţ̨ auf eine temporal nächste Stufe ihres Erzählvorgangs: Zunächst wird das Kaffeekochen geschildert, dann die Kaffeekonsumenten in die von Verena entworfene Situation eingeführt und zuletzt die Handlungsanweisung für diese Konsumenten geliefert. Eine dritte Bedeutung/Funktion hängt mit dieser zweiten zusammen: Neben einer temporalen Strukturierung der Szenerie, die Verena entwirft, hat jetzt auch gesprächsorganisierende Wirkung; es dient als eine Art Hinweissignal, dass ein weiterer Gesprächsschritt folgt.

\subsubsection{Matrixsatz, Diskursmarker oder beides - ich mein $(e)$}

Während bei jetøt kaum eine begründbare Trennlinie gezogen werden kann, die unterschiedliche jetzt-Konstruktionen voneinander abgrenzen kann, ist es für ich mein(e) (Günthner/Imo 2003; Imo 2007a) so, dass tatsächlich zwei Konstruktionen als Prototypen angegeben werden können, die auch entsprechend häufig eindeutig vorkommen und daher stark salient sind. Es 
handelt sich auf der einen Seite um ich mein(e) als Matrixsatz, dem ein eingeleiteter Nebensatz, uneingeleiteter Nebensatz oder abhängiger Hauptsatz folgt und auf der anderen Seite um ich mein(e) als Diskursmarker, dem eine unabhängige Äußerung folgt. Doch auch hier tauchen Probleme auf: In manchen Fällen, bei denen nach ich mein(e) ein abhängiger Hauptsatz realisiert wird, ist es nicht zu entscheiden, ob die Konstruktion „Matrixsatz + abhängiger Hauptsatz" als zugrundeliegendes Zeichen aktiviert ist oder eher „Diskursmarker + Äußerung“. Beispiel 5 stammt aus einer RadioBeratungssendung („Von Mensch zu Mensch"); die Anruferin A leidet unter einem Waschzwang, der sich darin äußert, dass sie täglich die Waschmaschine anstellt. Der Berater (B) fragt sie, ob sich der Waschzwang auch auf ,traditionelle“ Weise (Händewaschen, Duschen) zeigt:

Beispiel 5: Von Mensch zu Mensch duschen

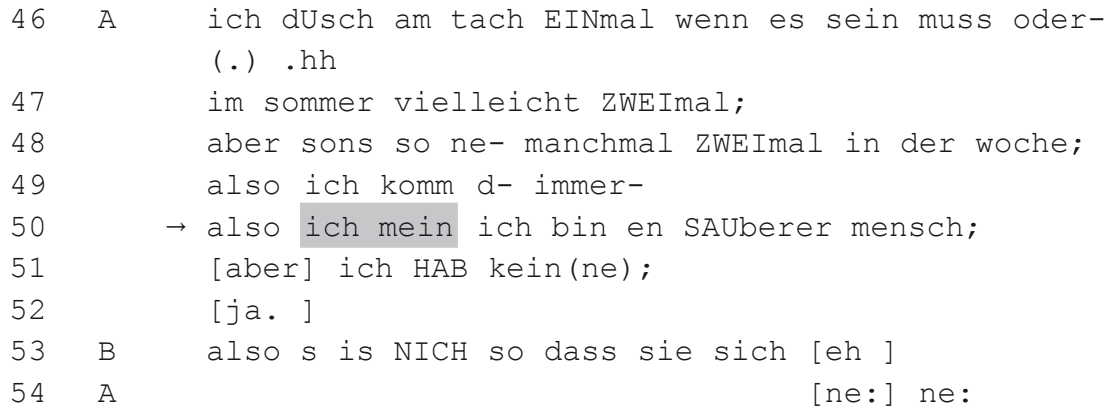

Die Frage des Psychologen (B), ob sich der Waschzwang auch in übertriebener körperlicher Reinlichkeit äußert, wird von der Anruferin vehement zurückgewiesen. Ab Z. 46 schildert sie, wie oft sie sich duscht, und in Z. 50 (bzw. mit dem Abbruch in Z. 49) leitet sie durch also eine Zusammenfassung ein. Dabei kann ich mein entweder als Matrixsatz gewertet werden (im Sinne von „meiner Meinung nach bin ich ein sauberer Mensch, habe aber keinen Waschzwang") oder als Diskursmarker, der zusammen mit dem „also“ den Aktivitätsbruch von der Schilderung des Duschverhaltens zum Fazit durchführt - nämlich der Antwort auf die Frage des Beraters, ob sie auch unter einem ,traditionellen“ Waschzwang leidet. Wie geht man mit diesen Fällen um? Handelt es sich um zwei Zeichen, die gleichzeitig und sich überlagernd aktiviert sind? Handelt es sich um ein drittes Zeichen, das eine Mischung aus „Matrixsatz“ und „Diskursmarker“ ist? Oder handelt es sich mal um einen Matrixsatz, mal um einen Diskursmarker, wie in einem Vexierbild? Die Ambiguität von ich mein(e) wird jedenfalls nicht aufgelöst, eine Festlegung auf eine Bedeutung ist nicht möglich, es gibt keine Anhaltspunkte, welche Interpretation ,korrekt" ist bzw. ob überhaupt eine Festlegung nötig ist. 


\subsection{Eine Form? - Eine Bedeutung?}

Ein zweites großes Problem, mit dem man zu kämpfen hat, wenn man Alltagsinteraktion untersucht, besteht darin, dass jeweils zu entscheiden ist, wann etwas als eigene Konstruktion gewertet werden soll, d.h. wann man von einer Form-Funktions-Verbindung ausgehen kann. Da in den meisten Grammatiken viele Phänomene überhaupt nicht erwähnt sind, die in der gesprochenen Sprache vorkommen, steht man hier oft vor der Aufgabe, neue Kategorien zu bilden (wie zum Beispiel die Kategorie „Diskursmarker").

Ein gutes Beispiel für ein Muster, bei dem nicht klar ist, ob es als Konstruktion in den Bestand der Grammatik aufgenommen werden kann, sind Sätze, bei denen ein Element nachgeliefert wird, d.h. beispielsweise nach der rechten Satzklammer produziert wird, obwohl es „eigentlich“ im Mittelfeld hätte stehen müssen. Diese Strukturen haben eine gewisse Ähnlichkeit mit Rechtsversetzungen (Altmann 1981), es liegt aber kein Korrelat vor, das den Platzhalter für die nachgelieferte Konstituente bildet. Solche Muster werden in letzter Zeit verstärkt unter dem Blickwinkel der „Inkremente“ und „Expansionen“ diskutiert (vgl. Couper-Kuhlen/Ono 2007 und Auer 1996, 2007). Auer (2007, S. 653) führt als Beispiel den Satz „hier wird ORdentlich gegessen heute" an und stellt die Frage, ob es sich dabei wirklich um ein rein prozessuales Phänomen handelt - nämlich einen Inkrementierungsprozess, bei dem, bedingt durch den „on line“-Charakter gesprochensprachlicher Syntax (vgl. Auer 2000, 2008), eine Konstituente nicht nachträglich und rückwirkend an ihrem typischen Ort eingefügt werden kann - oder ob dieser Inkrement- bzw. Expansionscharakter nur durch einen durch traditionelle normative Grammatik verstellten Blick zu Stande kommt: „It could be argued that they are constructions specific to German, i.e. that the ,expansion' looks like an expansion from the normative point of view of written language only." (Auer 2007, S. 653). Der Autor lässt offen, ob er einer solchen Reanalyse tatsächlich zustimmen würde. Ein ähnlich gelagertes Beispiel findet sich bei Intensifikatoren, die im gesprochenen Deutsch - vor allem in emphatischen Situationen - oft nachgestellt werden.

\section{Beispiel 6: Big Brother Brötchen}

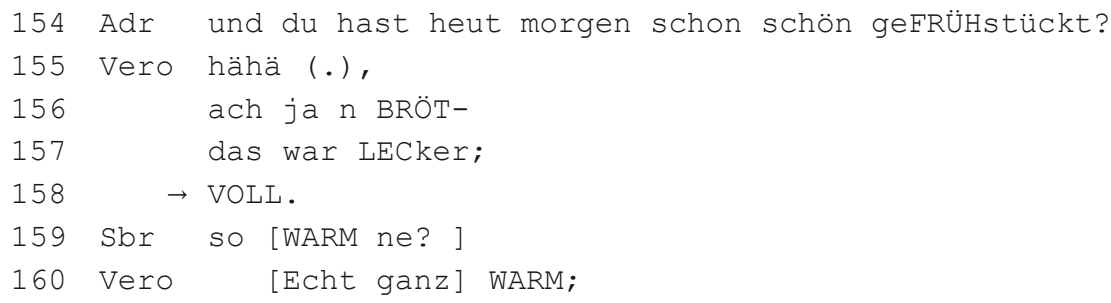


Andrea fragt Verona, die am Abend zuvor erst als Gast in den Big BrotherContainer eingezogen ist, ob sie schon schön geFRÜHstückt (Z. 154) hat. Verona bejaht dies, benennt, was sie gefrühstückt hat (n BRÖT-; Z. 156) und fügt dann eine Evaluation (das war LECker;) nach. Diese Evaluation wird in einer abgeschlossenen Intonationskontur produziert: Der Akzent liegt auf LECker und die Tonhöhe fällt zum Ende hin ab. In Z. 158 wird dann aber - ebenfalls betont und mit fallender Intonation - die Gradpartikel VOLL . nachgeliefert, die nach Helbig (1999, S. 99) eigentlich in keiner anderen Position stehen dürfte als vor dem Adjektiv, auf das sie sich bezieht. Nach Couper-Kuhlen/Ono (2007, S. 515) könnte man dieses VOLL. als ein „,insertable“ bezeichnen: „Insertables“ sind Inkremente, die ihre kanonische Position innerhalb einer Äußerung haben und - bedingt durch den prozessualen Charakter gesprochener Sprache - nachgeliefert werden. Eine alternative Erklärung wäre, dass die Struktur, Intensifikatoren nachzuliefern, als feste Konstruktion in der Sprache vorliegt: Es macht nämlich einen Unterschied, ob Verona sagt „das war VOLL LECker“ oder „das war LECker ... VOLL“. Im zweiten Fall rückt „VOLL“ als eigenständiges Element stärker in das Zentrum der Aufmerksamkeit, ist also salienter und der emphatische Charakter wird verstärkt. Aus dem Blickwinkel der Construction Grammar könnte man also durchaus auch ein Zeichen postulieren, das auf der formalen Seite den Eintrag „AdjP + Intensifikator“ und auf der inhaltlichen Seite „starke Emphase“ hat (Imo i.Dr.c). Ob es sinnvoll ist, eine solche Struktur anzunehmen, sei dahingestellt - in jedem Fall muss aber entschieden werden, inwieweit man Muster der Inkrementierung lediglich als Prozesse fasst (die Frage ist dann, welchen Status ein solcher Prozess im Vergleich zu einer Konstruktion hat und ob/wie solche Prozesse in die Construction Grammar integriert werden sollen), oder inwieweit man das Zeichenpostulat ernst nimmt und auf alle Bereiche der Sprachverwendung ausdehnt.

\section{Mögliche Antworten auf die Probleme}

Eine erste mögliche Reaktion auf die Probleme, die sich durch die Anwendung des Zeichenbegriffs für die Grammatiktheorie und die Erforschung der Strukturen gesprochener Sprache ergeben, besteht darin, dem Zeichenkonzept eine Absage zu erteilen und generell auch gegen eine einheitliche Theorie zu plädieren. Einen solchen Weg schlägt Hopper (1992, 2001, 2004) ein, der auf der einen Seite den Zeichenbegriff ablehnt, wenn er konstatiert, dass in der Sprachverwendung bestenfalls „fragments of constructions" zur Anwendung kommen, die mehr oder weniger lose aneinandergereiht werden (Hopper 2001), und auf der anderen Seite auch gegen eine einheitliche Theorie zu Gunsten eines „Anarcho-Syndikalismus“ (Hopper 1992, S. 236) von Forschungspraktiken argumentiert, die jeweils nur mit begrenztem Umfang kleine Probleme angehen. Der Konstruktionsbegriff 
wäre dann nur noch ein Begriff unter vielen, dem aber keine besondere Stellung im Pool der Konzepte, Methoden und Theorien gegeben würde. Ein anderer Vorschlag stammt von Linell (2006). Linell stellt sich die Frage, ob und wie ,interactional language“ formalisiert werden kann und kommt nach zahlreichen Einschränkungen zu dem Ergebnis, dass man den Blick auf formalisierbare Operationen richten müsse, nicht auf Zeichen:

This leads to a conception of formalization as pertaining to operations rather than constituent structure. Operations are actions or methods by which the language user does something: $\mathrm{x}$ is made into $\mathrm{y}[\ldots]$ in a given contextual matrix. (Linell 2006, S. 60)

Trotz einiger Vorschläge, wie eine solche Formalisierung von Operationen aussehen könnte, bleibt es unklar, wie man dabei konkret vorgehen könnte. Ein dritter Ansatz zielt auf die prinzipielle Beibehaltung der Konzepte der Construction Grammar, wobei allerdings ein anderer, offenerer Konstruktionsbegriff gefordert wird. Im Bereich der Gesprochene-Sprache-Forschung greifen Linell (1998) und Norén/Linell (2007) die Idee der Bedeutungspotenziale („meaning potentials“) auf, die an die Stelle der exakt angebbaren Bedeutungen treten. Deppermann/Elstermann (2008, S. 128-129) stellen fest, dass für die Beschreibung von Konstruktionen mit dem Verb ,,verstehen“ eine „lexikalische Basisbedeutung“, eine „grammatische Konstruktionsbedeutung", eine ,sequenzielle Bedeutungskonstitution“ und letztendlich ein allgemeines „Hintergrundwissen“ notwendig sind. Dies entspricht einer deutlichen Absage an die Relevanz des Zeichenkonzepts der Construction Grammar:

In Bezug auf den ,Konstruktions'-Begriff spricht diese Untersuchung also dafür, dass eine Eins-zu-eins-Zuordnung von Form und Funktion empirisch unhaltbar ist. Das gilt bei den untersuchten Konstruktionen selbst für die Fälle, in denen tatsächlich eine konstruktionsspezifische Interpretation ausgemacht werden kann, die aber eben auch nur unter bestimmten, nicht in der Konstruktion selbst liegenden Kontextbedingungen zweifelsfrei aktualisiert wird. Ein weiteres Mal zeigt die korpusbasierte Untersuchung, dass die Hypostasierung von klar abgrenzbaren Konstruktionen empirisch oft nicht sachhaltig ist. (Deppermann/Elstermann 2008, S. 130$)^{10}$

Eine vierte Möglichkeit, eine Proliferation an Konstruktionen zu vermeiden, könnte das Arbeiten mit variabler „Korngröße“ bzw. „Granularität“ sein. Schegloff (2000) beschreibt in einem Aufsatz mit dem Titel „On Granularity" programmatisch und im Sinne eines Forschungsdesiderats einige Aspekte des Operierens von Granularität sowohl im Alltag als auch

10 Zum gleichen Schluss kommt auch Günthner (2009, S. 180): „Statt also von starren, fixierten Form-Funktionspaaren als mentalen Konzeptualisierungen, die dann im konkreten Diskurs aktualisiert werden, auszugehen, scheinen Konstruktionen bzw. Fragmente von Konstruktionen eher als Orientierungsmuster zu fungieren, deren Instantiierung eine gewisse Flexibilität und Dynamik aufweist." Vgl. auch Imo (2007a, b). 
in der Forschung und sieht Granularität als eine Methode, wie Menschen mit Erfahrungen und Wahrnehmungen umgehen. ${ }^{11}$ Granularität liefert einen kognitiv realistischen Zugang ,to the terms in which the world is observed, noticed, and experienced by members of a society in the range of settings in which they live their lives“ (Schegloff 2000, S. 718). So betrachtet, kommt dem Konzept der Granularität kognitiv ein ähnlich hoher Stellenwert zu wie dem der holistischen Wahrnehmung, die von kognitiven Ansätzen der Construction Grammar für das Zeichenmodell ins Feld geführt werden. $\mathrm{Ob}$ diese These zu halten ist, muss an einem stärker ausgebauten Konzept der Granularität als dem, das Schegloff darstellt, überprüft werden. Als maßgeblich sind hierfür die Arbeiten von Bittner und Smith (2001a und b, 2003) anzusehen.

\section{Die „theory of granularity“ nach Bittner und Smith}

Das Ziel der Granularitätstheorie nach Bittner/Smith (2001a, S. 1) besteht darin, ein ,general framework within which we can understand the relation between vague terms and concepts and the corresponding crisp portions of reality“ zu entwickeln. Als Ausgangspunkt dient die Hypothese, dass bei Urteilen über die Realität stets zwei Operationen notwendig sind, nämlich ein Sortieren der beobachteten Daten und deren Merkmale in solche, die im Vordergrund der Aufmerksamkeit stehen und solche, die ausgeblendet werden und, damit verbunden, eine granulare Betrachtung der Gegenstände. Granular ist die Betrachtung deshalb, weil im Alltag nie eine vollständige Repräsentation angestrebt wird (noch angestrebt werden kann) und daher immer Aspekte ausgeklammert werden müssen, und weil auch die Grenzziehung zwischen dem, was ein Objekt ausmacht, und dem, was als „Rest“ ausgeklammert wird, immer granulare Merkmale aufweist: ${ }^{12}$

Our fundamental idea is that every use of language to make a judgment about reality brings about a certain granular partition. Already every act of singular reference and every act of perception effects a partition of reality into a foreground domain, within which the object of reference is located, and a background domain, which comprehends all the entities beyond. (Bittner/Smith 2001a, S. 6)

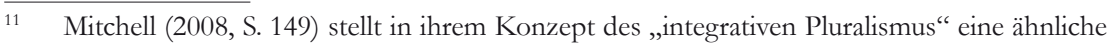
Frage: „Wann soll man eine fein gerasterte Darstellung verwenden, und wann reicht ein gröberes Raster aus? Beide geben die Welt, die wir darstellen wollen, ,richtig' und ,genau' wieder. Was bestimmt darüber, welche Abstraktionsebene oder welches Raster pragmatisch ist? Diese Frage lässt sich nur im Zusammenhang mit dem jeweiligen Kontext und den wissenschaftlichen Zielen beantworten.“

12 „A granular partition is granular in virtue of the fact that it can recognize an object without recognizing all its parts. The theory of granular partitions can thus provide the basis for understanding the selective focus of our maps and classifications and above all their ability to trace over parts below a certain level. To impose a partition on a given domain of reality is to foreground certain objects and features in that domain and to trace over others." (Bittner/ Smith 2001b, S. 34). 
Bei der Verwendung von Sprache - und selbstverständlich noch viel stärker bei der Analyse von Sprache durch WissenschaftlerInnen - spielen die Begründung und Art der Grenzziehung und die Strategien der Aufteilung der Fakten in solche, die in den Vordergrund rücken und solche, die in den Hintergrund rücken, eine zentrale Rolle. In den Hintergrund gerückte Merkmale sind nicht verloren, sondern lediglich für die Wahl einer bestimmten Korngröße irrelevant, können aber sozusagen „herangezoomt“" werden. Bittner/Smith (2001a, S. 6) nennen diesen Prozess „ontological zooming“" und illustrieren dessen Operieren am Beispiel eines leergetrunkenen Bierglases. Für den Gast, der das Bier getrunken hat, ist das Bierglas leer - die wenigen Schaumflocken und Tropfen sind nicht relevant. Für einen Gesundheitsinspektor dagegen wäre das Glas nicht leer, wenn er ein solches Glas im Schrank finden würde - sein ontologisches ,Zooming" ist weitaus schärfer als das des Gastes. Ähnlich geht es darum, dass man, wenn man sagt, man geht nach „Berlin“, die Stadt als Ganzes fokussiert und Einzelteile (Straßen, Gebäude, Bäume etc.) für die Kategorie nicht relevant sind: „For to say that partitions are granular is to say that they do not recognize parts beneath a certain size.“ (ebd., S. 6). Genau dieses Phänomen, dass wir beim alltäglichen wie wissenschaftlichen Kategorienbilden immer Merkmale unter den Tisch fallen lassen, die uns für die Kategorienbildung in einem bestimmten Kontext nicht relevant erscheinen, nimmt die Theorie der Granularität in den Blick. Während solche Fälle, in denen eine scharfe Grenzziehung möglich ist, unproblematisch, aber leider selten sind, benötigt man eine Theorie der Granularität vor allem für die Bereiche, bei denen man mit unscharfen Grenzen operieren muss. Gerade im Bereich der Sprache sind Grenzen notorisch unscharf. Das ist für die „naiven Sprechpartner“ (Bühler 1982 [1934], S. 102) auch kein Problem:

Contexts where judging subjects have the authority and the need to bring a precise boundary into existence are, it must be admitted, very rare. Fortunately however there is in most contexts no need for the high degree of precision which such contexts represent. In most contexts, that is to say, we get along with a created boundary that is just precise enough. [...] In most cases, therefore, it will manifest a certain degree of vagueness, and the actual degree of vagueness (or the degree of precision) will depend on context. Where vagueness is involved indeterminate cases threaten to arise. (Bittner/Smith 2001a, S. 19)

Das Vorgehen, Grenzen zu ziehen und, daraus abgeleitet, Kategorien zu bilden, die gerade präzise genug sind, um damit operieren zu können, reicht im Alltag in den meisten Fällen aus. Vor allem in Streitsituationen merkt man aber, dass die Vagheit von Sprache immer vorhanden ist und dass darauf zurückgegriffen werden kann, um beispielsweise einer Aufforderung aus dem Weg zu gehen etc. (vgl. Imo 2010, wo gezeigt wird, wie die Vagheit von jetzt in einem Streitgespräch ausgenutzt werden kann, um alle Versuche 
des Gesprächspartners ins Absurde zu führen, mit der Aufforderung ,gib mir jetzt die Brille" diese Brille auch tatsächlich zu erhalten). Es gilt also zu zeigen, wie genau solche vagen Demarkierungen möglich sind und wie gewährleistet werden kann, dass je nach Absicht und Kontext mal gröbere und mal feinere Demarkationslinien gezogen werden.

Besonders eindrucksvoll illustrieren Bittner und Smith das Problem der Demarkationslinien von Objekten, die man als Einheit konstruieren will, am Beispiel des Himalaya: Man erkennt dort zwar einzelne Berge, wenn man aber angeben will, bis wohin beispielsweise der Mount Everest reicht, wird das immer schwerer, je feiner die Auflösung gewählt wird. Eine Grenzziehung wird im Endeffekt unmöglich, niemand kann die Frage beantworten „Where does the mountain stop?“" „As will by now be clear, there is no generally applicable and context-independent stop condition that can be inferred from a general concept such as mountain." (Bittner/Smith 2001a, S. 18). Bittner und Smith illustrieren dieses Fehlen einer Stop-Regel an den benachbarten Bergen „Lhotse“ und „Mount Everest“. Wenn man eine Luftaufnahme der Berge betrachtet, so sieht man zunächst einmal, dass in der Tat zwei Berge existieren (also zwei Kategorien gebildet werden müssen). Auf einen ersten, alltagsklassifikatorischen Schritt hin könnte man um diese Berge eine Linie ziehen; beide Berge wiederum sind in die noch größere granulare Zelle des Himalaya eingebettet:
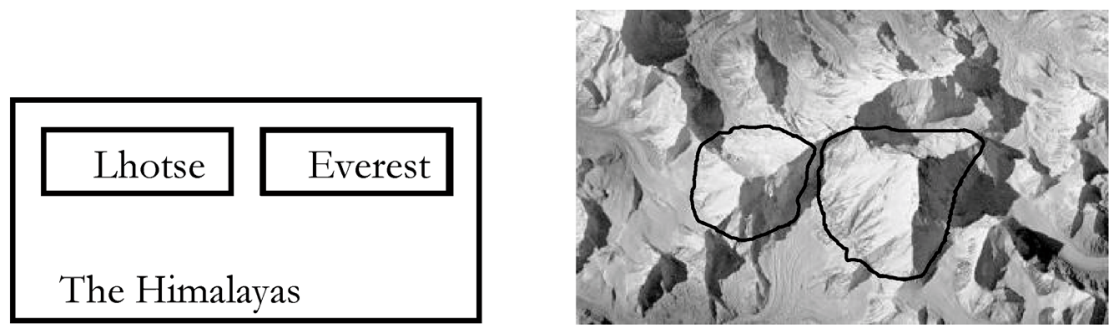

Abb. 1: Beispiel für eine Partition: Auf der linken Seite die Zellen Lhotse, Everest und Himalaya, auf der rechten Seite eine Satellitenfotografie der Region. (Bittner/Smith 2001a, S. 3)

Wenn man also vom Weltall aus langsam heranzoomt, wird man zunächst „Gebirgszug" als Zelle von umgebenden Zellen (wie „Ebene“ oder „Meer") abtrennen. Zoomt man näher heran, lassen sich einzelne Berge abtrennen. Zoomt man noch näher heran, wird es nötig, die Grenzlinie zu begründen. Dabei taucht das Problem auf, dass die Begründbarkeit der Grenzlinie nicht gegeben ist: Aus einer Reihe von Punkten können diejenigen ausgeschlossen werden, die zweifellos dem einen oder anderen Berg zugerechnet werden können (z.B. die jeweiligen Gipfel). Dazwischen gibt es jedoch eine Serie von möglichen Grenzen, die alle in Frage kommen. Bittner und Smith veranschaulichen dies an folgender Grafik: 


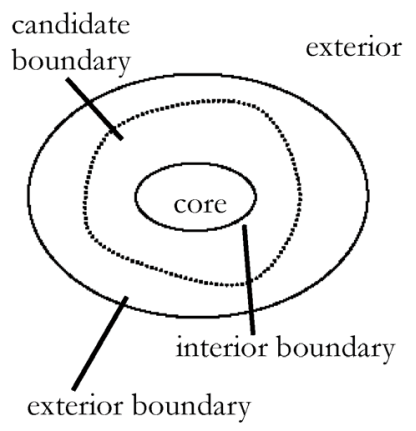

Abb. 2: Grenzkandidaten (Bittner/Smith 2001a, S. 24)

Ab einem gewissen Punkt (der ,äußeren Grenze“) beginnen mögliche Kandidaten für eine Grenzziehung. Ab einem zweiten Punkt, der ,inneren Grenze", hört die Kandidatenliste auf. Innerhalb dieser inneren und äußeren Grenzen (die ihrerseits wieder nicht eindeutig bestimmbar sind), kommen eine ganze Serie von „Grenzkandidaten“ in Frage.

Stellt man eine Sammlung solcher Grenzkandidaten auf, erhält man ein neues Bild, nämlich keine scharfe Trennlinie (wie in Abb. 1), sondern eine vage Linie:
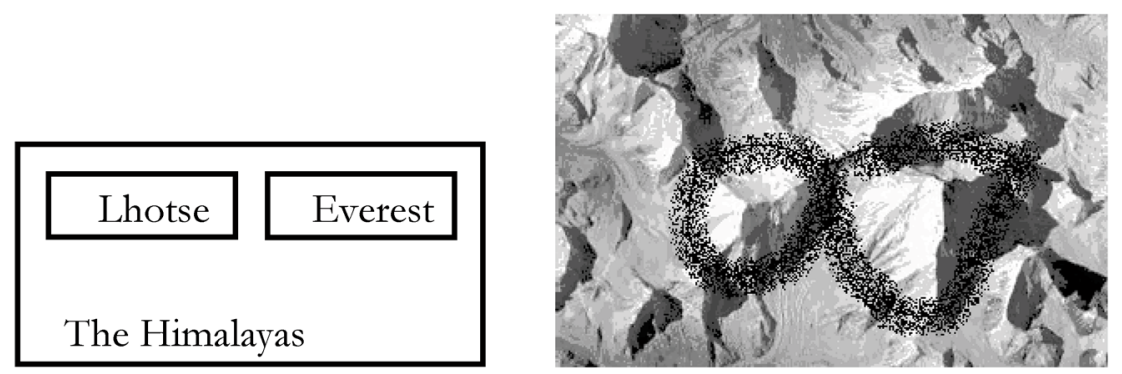

Abb. 3: Vage Grenzen der Berge Lhotse und Everest (nach Bittner/Smith 2001a, S. 6)

Jeder der Punkte kommt als mögliche Grenzlinie in Betracht und dennoch kann keiner der Punkte tatsächlich als Grenze gewertet werden - eine scharfe Trennung ist absolut unmöglich. Wenn zwei Bergsteiger vom Lhotse zum Mount Everest unterwegs wären, könnten sie bestenfalls irgendwann sagen „wir sind gerade auf der Grenze zwischen Lhotse und Mount Everest", aber nie ,in diesem Moment überqueren wir die Grenze zwischen Lhotse und Mount Everest“. Eine solche Äußerung klänge absurd:

It is pragmatically impossible to invoke crisp partitions in contexts where both speaker and audience know that vague partitions are the best that can be achieved. Corresponding attempts to make judgments will not be taken seriously. (Bittner/ Smith 2001a, S. 22) 
Ein etwas anders gelagertes Beispiel einer Grenzziehung, die - anders als ein Bergmassiv - menschengemachte Kategorien als Ausgangspunkte hat, ist nach Bittner/Smith (2001a, S. 20-21) ein Café mit einer Raucherzone. Mit Hilfe eines Striches auf dem Boden kann zwar hier eine eindeutige, scharfe Grenze gezogen werden, so dass man tatsächlich sagen könnte, „ich überquere nun die Grenze von der Nichtraucher- in die Raucherzone“. Granularität spielt aber auch hier eine Rolle, nämlich in Bezug auf die Objekte, für die die Grenze gilt. So ist es möglich, Tische, Stühle und Menschen als Teil der Raucherzone zu betrachten. Die Frage, ob auch ein einzelnes Nikotin- oder Sauerstoffmolekül jeweils Teil der einen oder anderen Zone ist, ist für die Grenzziehung nicht relevant: Die Korngröße von „Raucherzonen" richtet sich nicht an solche kleinen Objekte, sie fallen durch das Betrachtungsraster:

[A] judgment of the form ,This nicotine molecule is part of the smoking zone cannot be uttered in the given context, since the unity condition U4 does not admit molecules as parts of smoking (or non-smoking) zones. A judgment of this form reflects an illegitimate mixing of granularities. If judgments of the given form are to be judgeable, then more precise specifications of the relevant boundaries would needed to be made [sic] by those involved, and this would mean creating a new context. (Bittner/Smith 2001a, S. 20) ${ }^{13}$

Somit sind bereits zwei Komponenten der Theorie der Granularität identifiziert: Zum einen beschäftigt sie sich mit der Frage, wie Grenzziehung angesichts empirisch gegebener Daten möglich ist (z.B. zwischen Bergen) und schlägt die Wahl der Korngröße als Ausweg vor. Zum anderen erörtert sie den Aspekt, welche Aussagen bei einer willkürlich getroffenen Grenzziehung (Raucher- und Nichtraucherbereich) zulässig sind bzw. warum manche Aussagen pragmatisch unsinnig sind.

Folgende Komponenten beinhaltet die Theorie der Granularität:

1) Aufteilung (,partition“): Das Aufteilen von empirisch vorliegenden wahrnehmbaren Dingen in Einheiten und das Klassifizieren dieser Einheiten ist ein grundlegendes menschliches Verhalten. Dieses Vorgehen ist kognitiv verankert: „Partitions are the cognitive devices designed and built by human beings to fulfill these various listing, mapping and classifying purposes.“ (Bittner/Smith 2003, S. 1).

13 Vgl auch: „Consider a simple biological partition of the animal kingdom including a cell projecting on the species dog (Canis familiaris). Our definition of the domain of a partition and our constraint on functionality of projection implies that, besides the species dog also your dog Fido, and also Fido's DNA-molecules, proteins, and atoms are parts of the domain of this partition. But the latter are of course not recognized by the partition itself. It is cases such as this which illustrate why mereology requires supplementation by a theory like the one presented here. Partition theory allows us to define a new, restricted notion of parthood that takes granularity into account [...].“ (Bittner/Smith 2003, S. 19). 
2) Der Akt des Aufteilens führt zu einer Matrix von beschrifteten Zellen (vgl. die schematische Darstellung von „Himalaya“ als größere Zelle, die die kleineren Zellen „Lhotse“ und „Mount Everest“ enthält): „Such a grid of labeled cells is an example of what we shall call a granular partition." (Bittner/Smith 2003, S. 4).

3) Die Struktur, jeweils Zellen in andere Zellen einzubetten, führt dazu, dass zwei Komponenten bedacht werden müssen, nämlich „(A) a theory of the relations between cells, subcells, and the partitions in which they are contained“ und „(B) a theory of the relations between partitions and objects in reality". Die Zellen werden durch ihre Position innerhalb einer Partition und durch Beziehungen zu anderen Zellen definiert und werden wiederum auf die Realität projiziert: „Briefly, we can think of cells as being projected onto objects in something like the way in which flashlights are projected upon the objects which fall within their purview." (Bittner/Smith 2003, S. 5).

Eine granulare Analyse bietet sich daher für die Kombination mit dem Konstruktionsbegriff geradezu an. Eine der Schwächen der Construction Grammar war bislang, dass Einbettungsstrukturen v.a. in Hinblick auf Vererbungsprozesse analysiert wurden (in rein formalem Kontext wie z.B. dem Berkeley-Formalismus; vgl. Fillmore/Kay 1995 oder Kay 2000) und dass die Stellung der Konstruktionen zueinander sowie ihre Abbildung auf die Realität kaum problematisiert wurden. Im Folgenden soll nun gezeigt werden, wie die Theorie granularer Kategorisierung für die Zwecke einer Grammatik verwendet werden könnte.

\section{Granularität - Zeichen - Konstruktionen}

Ansätze zu einer Einbindung von Granularität in die Analyse sprachlicher Einheiten lassen sich bereits - wenn auch ohne expliziten Rekurs auf das Konzept, sondern eher als Alltagskonzept im Sinne von Schegloff (2000) in zahlreichen Ansätzen finden. So plädiert beispielsweise Traugott (2008, S. 8) für eine differenziertere Aufteilung von Konstruktionen in „Makrokonstruktionen“, die als „abstrakte Schemata“ die „höchststufige Ebene, die für die gegebene Diskussion relevant ist", stellen. Darunter kommen die „Mesokonstruktionen“, die als „Mengen sich ähnlich verhaltender Konstruktionen“ beschrieben werden. Noch geringeren Umfang haben die „individuellen Konstruktions-Typen“, die „Mikro-Konstruktionen“ genannt werden, und am Ende der Skala finden sich dann die „Konstrukte“ als „empirisch attestierte Token“. Eine solche Unterteilung - die von Traugott eher als heuristisches Mittel und nicht im Sinne einer strikten Vererbungsrelation intendiert ist - geht sicher in die richtige Richtung, wobei allerdings in der 
Definition der Mesokonstruktionen bereits vorausgesetzt wird, dass man die Mikrokonstruktionen (oder Makrokonstruktionen?) schon beschrieben hat, die dann als sich ähnlich verhaltend zusammengruppiert werden können. Dennoch ist gerade der Satz „die für die gegebene Diskussion relevant ist" (Traugott 2008, S. 8) aufschlussreich: Die „Stufigkeit“ der Ebene ist abhängig von dem Zweck und Einsatzort, es handelt sich also um die granulare Argumentation, Aspekte eines Zeichens oder einer Kategorie auszublenden, die im gegebenen Kontext nicht relevant sind. Im Folgenden soll gezeigt werden, inwiefern sich eine granulare Perspektive bei der Beschreibung der oben dargestellten problematischen Konstruktionen als hilfreich erweisen kann.

\subsection{Eine granulare Reanalyse von jetzt}

Wenn man jetzt granular reinterpretiert, so muss zunächst festgestellt werden, dass mindestens drei große Schritte der Feinjustierung der konstruktionalen Beobachtungslinse möglich sind. Zunächst einmal kann man mit Bühler (1982 [1934], S. 102) jetæt als „Augenblicksmarke“ sehen. Auf diese Weise erhält man eine Konstruktion, die auf der einen Seite die Lautform von jetzt und auf der anderen Seite den semantisch-funktionalen Eintrag „Augenblicksmarke“ hat, also den RezipientInnen mitteilt, dass irgendetwas gerade passiert - um was es sich dabei genau handelt, ist nicht Bestandteil der Konstruktion bei dieser Korngröße, wie eben auch die Nikotinmoleküle bei der Kategorisierung in „Raucherzone“ und „Nichtraucherzone“ nicht betrachtet werden. Dass diese grobe - im Sinne einer groben Korngröße - Kategorie jetz̨t tatsächlich eine sprachliche Realität hat, wird bereits von Bühler (1982 [1934], S. 102) festgestellt, wenn er sagt, dass die Aufforderung von jetzt, nämlich ,schau auf mich Klangphänomen und nimm mich als Augenblicksmarke“ von dem „naiven Sprechpartner“ internalisiert wurde und dieser die Aufforderung auch völlig unproblematisch versteht - er „nimmt sie auch so.“ Wenn eine feinere Korngröße zur Betrachtung gewählt wird, könnte man feststellen, dass sich vielleicht zwei kleinere Konstruktionen aus der Makrokonstruktion (im Sinne Traugotts 2008, S. 8) herausschälen: Einerseits eine „temporale Augenblicksmarke“ und andererseits eine ,gesprächsstrukturierende Augenblicksmarke“. Dabei hat die erste eine klare temporale Semantik (was man durch die Überprüfung der Akzeptabilität von Paraphrasen wie „das geschieht in genau diesem Moment“ oder „in diesem Zeitraum“ überprüfen kann), die zweite dagegen ist semantisch entleert („temporale ,Entwertung“" nach Hennig 2000, S. 190). Dass diese reduzierte Korngröße möglicherweise für die Interagierenden, in jedem Fall aber für die kategorienbildenden ForscherInnen relevant sein muss, zeigt eine Gegenüberstellung der folgenden beiden Beispiele: 
Beispiel 7: Autotour Lüfter

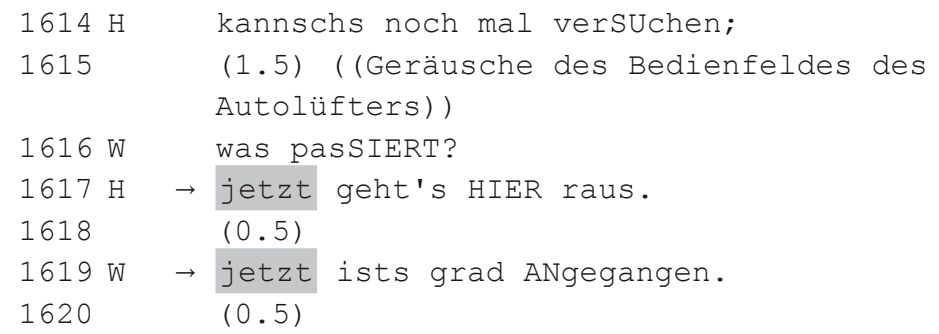

Beispiel 8: Big Brother vermissen

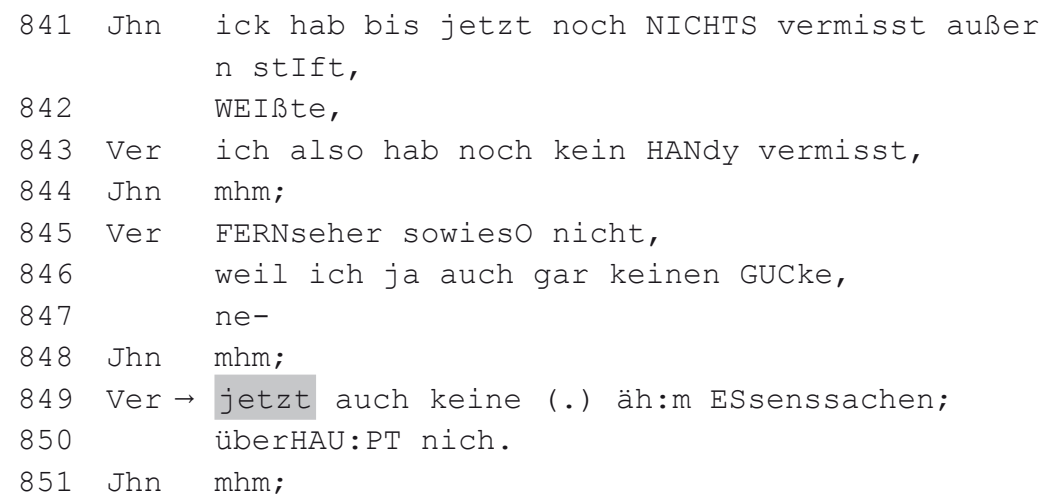

In Beispiel 7 aus einer aufgezeichneten Unterhaltung zweier Freunde beim Autofahren versuchen $\mathrm{H}$ und $\mathrm{W}$ die Heizung im Auto anzustellen. Das jetzt (Z. 1617, 1619) verweist jeweils direkt temporal auf die Ereignisse (den austretenden Luftstrom und die anspringende Heizung). In Beispiel 8 unterhalten sich Verena und John aus dem Big Brother-Container darüber, dass sie entgegen ihrer Erwartungen keine Dinge dort vermissen. Verena setzt mit einer Liste an, unterbricht diese in Z. 846 durch eine begründende Seitensequenz und knüpft in Z. 849 durch jetz̧ wieder an diese Liste an. Eine temporale Semantik ist hier nicht mehr feststellbar, die „Augenblicksmarke“ (d.h. die Funktion der Makrokonstruktion) bezieht sich auf das sequenzielle Strukturieren von Gesprächseinheiten.

Die Wahl einer noch feineren Korngröße führt dazu, dass die oben erwähnten Bedeutungs- und Funktionsnuancen von jetzt in dessen Beschreibung mit aufgenommen werden können. Es wird dann möglich, zu spezifizieren, ob jetžt projizierend, rückblickend, in der Funktion der Anknüpfung an eine Listenstruktur, in der Funktion der Präsentation einer Geschichte etc. verwendet wird. Bei einer solch feinen Korngröße werden der Ko- und Kontext immer wichtiger. Fragen wie: „In welchem Tempus oder Modus steht das Verb?“, „Welche Handlung wird gerade durchgeführt?“, „Handelt 
es sich um einen reaktiven oder eigenständigen Turn?" sind notwendig, da zum Beispiel ein Verb im Präteritum oder im Konjunktiv II ein Indiz dafür sein kann, dass jetžt rückblickend eingesetzt wird. Bei dieser Korngröße stößt die Grammatiktheorie an ihre Grenzen und muss zu Gunsten von anderen, an der detaillierten Rekonstruktion von Einzelfällen orientierten Ansätzen (Gesprächsforschung, Textlinguistik) aufgegeben werden. Ähnlich wie bei der Betrachtung eines impressionistisch oder pointilistisch gemalten Bildes geht ab einer bestimmten Entfernung die Gesamtstruktur verloren und die Bezüge verschwinden. Übertragen auf die Grammatik heißt das, dass das Konzept von Konstruktionen (d.h. von Kategorien) verloren geht zu Gunsten der momentanen, situativen, reichen Formen-, Bedeutungs- und Funktionsvielfalt sprachlicher Einheiten.

Durch die Einführung von Granularität in die Grammatiktheorie verlagert sich das Problem von der Fragestellung, wie viele Informationen in einer Konstruktion enthalten sein müssen, hin zu der Frage, in welchem Kontext welche Korngröße und somit welche Beschreibungsdichte gewählt werden muss. Anders ausgedrückt: Die Einbeziehung der Granularität in die Grammatiktheorie führt dazu, dass die Abstraktionsebene zu jeder Beschreibung jeweils offengelegt werden muss. Nach Knobloch (persönliche Kommunikation) müsste die Construction Grammar also „fallweise die gewählte Korngröße semiotisch begründen“. Ob ein solches Begründungsinstrumentarium tatsächlich funktionieren kann, bleibt zu überprüfen.

\subsection{Eine granulare Reanalyse von ,ich mein“}

Gerade im Fall von ich mein(e), das jeweils um abstrahierte Konstruktionen wie „Matrixsatz“ und „Diskursmarker“ herum Cluster bildet, stellt sich besonders deutlich die Frage: „Where does the mountain stop?“ Die Fälle, die sowohl als Matrixsätze als auch als Diskursmarker analysiert werden können, verschwinden, wenn eine gröbere Korngröße verwendet wird. Ähnlich wie bei jetžt kann man für ich mein $(e)$ mit einer groben Rasterung die zwei Konstruktionen „Diskursmarker“ und „Matrixsatz“ annehmen, die in Grammatiken aufgenommen werden sollten. Für die Fälle, die zwischen diesen schematischen Konstruktionen liegen, gilt ähnlich wie bei jetrt, dass sie erst ab einer bestimmten feinen Korngröße relevant werden. Auch dann stellen sich wieder Fragen wie: „Welche Aktivität liegt vor?“, „Wie reagieren die GesprächspartnerInnen auf eine Äußerung, die mit ich mein $(e)$ beginnt?", „Was genau leistet ich mein $(e)$ in der aktuellen Verwendung, die in einer konkreten Äußerung vorliegt?" etc. Übertragen in das Beispiel von Bittner/ Smith (2001a, S. 16) mit den Grenzen der Berge „Mount Everest“ und „Lhotse“, die mit einer groben Rasterung (,vague partitions") getrennt werden, verhalten sich die Matrixsätze, Diskursmarker und unentscheidbaren Mischfälle mit $i c h$ mein $(e)$ wie folgt zueinander: 


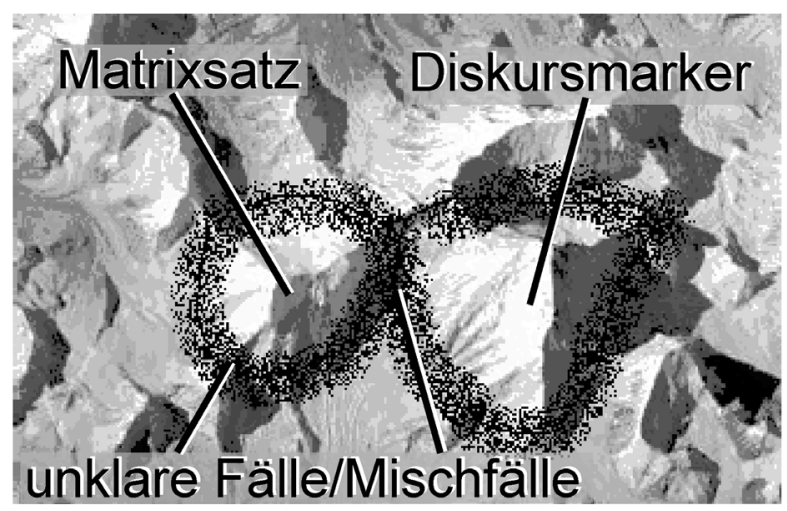

Abb. 4: Sprachliche Kategorien mit vagen Grenzen

(Bildgrundlage basierend auf Bittner/Smith 2001a, S. 6)

Was hier als eine Sammlung von Punkten erscheint, sind die empirisch in feiner Korngröße gewonnenen Fälle, die nicht eindeutig als Matrixsätze zu klassifizieren sind. Darunter fallen einerseits die Mischfälle, die auf der Grenze der beiden „Berge“ „Matrixsatz“ und „Diskursmarker“ liegen, aber auch sonstige unklare Fälle, wie sie z.B. nach einem Konstruktionsabbruch (Anakoluth) entstehen. Die Mischfälle können nicht eindeutig zugeordnet werden: Wie in der Analyse von Beispiel 5 gezeigt wurde, gibt es keine Möglichkeit, eine der beiden Interpretationen als die richtige auszuwählen, beide Bedeutungen bleiben nebeneinander stehen. Zusammengenommen und mit einer groben Korngröße betrachtet, wirken sie wie ein mit dickem Filzstift gezogener Trennstrich: Sie fallen aus dem Raster der Konstruktionsbetrachtung heraus und die Konstruktionen selbst werden auf die unproblematischen Fälle ,in der Mitte“ reduziert. Die Theorie der Granularität legt so den Dekontextualisierungsprozess offen, der zu Kategorien und zu Grenzziehungen führt. Auch hier stellt sich natürlich wieder die Frage der Begründung der Korngröße, wenn auch durch die zwei starken Pole „Matrixsatz“ und „Diskursmarker“ - anders als bei jetət, wo es mindestens drei Schritte mit immer größerer Genauigkeit gibt - der Akt der Korngrößenwahl auf zwei Schritte reduziert werden kann und somit die Wahl binär auf die zwischen Abstraktion und detailgetreuer qualitativer Analyse reduziert wird. Dies ermöglicht die Zuschreibung von Aufgaben für unterschiedliche linguistische Ansätze: Für die Betrachtung aus dem Blickwinkel der Grammatiktheorie heraus wird die hohe Abstraktionsebene gewählt, für die Betrachtung aus einer Theorie wie der Gesprächsanalyse heraus kann die Detailebene gewählt werden. 
7.3 Versuche einer granularen Begründung des Zeichenstatus von grammatischen Mustern

Für die Reanalyse von sprachlichen Einheiten, die je nach Detaillierungsgrad unterschiedliche Zeichenqualitäten haben, eignet sich der granulare Ansatz gut. Kann das Konzept der Granularität aber auch dabei helfen, zu entscheiden, wann etwas als Zeichen wahrgenommen wird? Hier stehen wir vor dem in Abschnitt 3.2 dargestellten Befund, dass empirische Untersuchungen gesprochener Sprache belegen, dass manche Inkrementstrukturen häufig vorkommen und es daher zu überlegen ist, ob sie tatsächlich als dem „on line“-Charakter (Auer 2000, 2008) der konzeptionell gesprochenen Sprache geschuldete Prozesse betrachtet werden sollten, oder ob sie nicht vielmehr als Konstruktionen reanalysiert werden müssen. Der „on line“Aspekt alleine reicht nicht aus, um zu sagen, dass es sich nicht um eine Konstruktion handelt, sondern um einen mehr oder weniger ungeplanten Prozess: Auch im Konzept der Diskursmarker ist die Projektion einer Nachfolgeäußerung als Merkmal integriert (vgl. in diesem Zusammenhang auch das Konzept der „Projektorkonstruktionen“ (Günthner 2008a, b, c; Bücker 2008), von einer Konstruktion wie „Verbzweitsatz“ mit seiner (potenziellen) projizierenden Klammerstruktur ganz zu schweigen.

In einem ersten Schritt kann festgestellt werden, dass nach der Granularitätstheorie alleine die Benennung und Beschreibung von so etwas wie „Inkrement“ oder „Expansion“ bereits als Prozess der Herstellung einer granularen Grenzziehung gewertet werden muss: „We shall argue that granular partitions are involved in all naming, listing, sorting, counting, cataloguing and mapping activities." (Bittner/Smith 2003, S. 18). Wenn man eine Grenze gezogen hat - selbst wenn diese Grenze vage im Sinne der Granulariätstheorie ist - folgt daraus, dass sowohl „Zellen“ als auch „Domänen“ aus dieser Grenzziehung heraus entstehen: „Each partition has a certain domain, which we can define as that portion of reality upon which its maximal cell is projected." (Bittner/Smith 2001a, S. 11). Das Objekt, auf das eine Zelle projiziert wird, kann nach Bittner/Smith (ebd., S. 11) eine Menge konkreter Einheiten ebenso beinhalten wie auch Abstraktionen solcher Einheiten. Wozu führt nun eine Grenzziehung um so etwas wie „Inkremente"? Zunächst entsteht nichts weiter als eine Zelle, die alle tatsächlich vorliegenden und als solche identifizierten Inkrementstrukturen enthält. Wenn man nun die Körnigkeit der Beschreibung zurücknimmt und nicht mehr alle Formen und Funktionen im Detail betrachtet, entsteht auf Grund der Abstraktionsleistung ein Zeichen: Zelle und abstrahierte Domäne ergeben eine Konstruktion. Je nach der gewählten Korngröße lässt sich nun überlegen, ob zwischen den beiden Extrempolen der absoluten Abstraktion und der Einzelfallbeschreibung sinnvollerweise Zwischenebenen einzuziehen sind, d.h. neue Partitionen gesetzt und so neue Zellen gebildet werden 
müssen. Eine Möglichkeit bestünde z.B. in der Trennung zwischen „Adj.P + Intensifikator“ („das war lecker, voll“) und „Verbzweitsatz + nachgeliefertes Mittelfeldelement" (,hier wird ordentlich gegessen heute"). Angesichts der Tatsache, dass das Gebiet der Inkremente noch zu wenig erforscht ist, als dass darüber bereits gesicherte Aussagen möglich sind (vgl. Auer 2007; Imo i.Dr.c), bleibt es ein Forschungsdesiderat, über solche weiteren Grenzziehungen nachzudenken. Der Ansatz der Granularität vermag aber immerhin, einen gangbaren Weg aufzuzeigen, der bei der Arbeit helfen könnte, die „elusive objects“, wie Auer (2007, S. 1) die Inkremente nennt, zu analysieren.

\section{Fazit}

Was kann die Theorie der Granularität nun in Bezug auf das Dilemma zeichenbasierter Konstruktionen leisten? Kann das Konstruktionsmodell aufrechterhalten und mit Hilfe eines granularen Ansatzes ausgebaut werden? Muss die Konstruktionsgrammatik nicht vor der Reichhaltigkeit an Informationen und Merkmalen von Sprache-in-Interaktion kapitulieren?

Eine eindeutige Antwort auf die letzte Frage besteht darin, dass Repräsentationen (d.h. also auch Konstruktionen) zugleich korrekt und unvollständig sein können: ${ }^{14}$

A correct representation, as we see, is not necessarily a complete representation. Indeed, since partitions are cognitive devices, and cognition is not omniscient, it follows that no partition is such as to recognize all objects. (Bittner/Smith 2003, S. 18)

Das liegt daran, dass Partitionen ,do not recognize parts which fall beneath a certain size“ (Bittner/Smith 2003, S. 18). Eine Abstraktion, die für die Bildung von Kategorien bzw. Konstruktionen notwendig ist, ist also möglich, sofern eine granulare Feineinstellung mit bedacht wird. Das wirft dabei allerdings die Frage auf, ob eine Begründung für die gewählte Korngröße für jeden konkreten Fall für eine Grammatiktheorie überhaupt machbar sei. Mit Bittner/Smith (2001a, S. 37) ist das durchaus der Fall. Durch die prinzipiell unendlichen Variationsmöglichkeiten, die sprachliche Mittel in ihrer Verwendung in je konkreten Kontexten haben, kann nicht die Annahme der Vagheit von Konstruktionen an sich die Lösung sein, sondern das Prinzip, mit dem Interagierende im Alltag mit dieser Vagheit umgehen. Die Einbindung von Granularität in die Grammatiktheorie ist eine mögliche Lösung:

$14 \quad$ Vgl. auch Diewald (2008, S. 87), die im Kontext der diachronen Analysen von Sprache ebenfalls die Notwendigkeit granularer Ansätze betont: „Durch die in der Konstruktionsgrammatik mögliche flexible Granularität der Analyse bieten sich hier formale Lösungsoptionen, die nicht zu unbegründbaren Kategorisierungsentscheidungen zwingen, sondern die es erlauben, mit der formalen Analyse den tatsächlichen Kenntnisstand abzubilden." 
$[\mathrm{T} t$ is insufficient to consider the vagueness of names and predicates in a contextfree fashion. Rather, vague names and predicates must be evaluated as they appear within judgments actually made in natural contexts. We then argued that judgments add context to sentences in a way that helps to resolve the dilemma posed by vagueness. Note that this does not mean that vagueness is somehow eliminated. Vague names and predicates are still as vague as they always were. Rather, we showed that the framework of granular partitions can provide the framework for understanding how, in real-world contexts, judgments with indeterminate truthvalues are systematically avoided. (Bittner/Smith 2001a, S. 37)

Fragen der konkreten Einbindung des Ansatzes der Granularität in die Grammatiktheorie müssen durch zukünftige Analysen geklärt werden.

\section{Literatur}

Altmann, Hans (1981): Formen der Herausstellung im Deutschen: Rechtsversetzung, Linksversetzung, freies Thema und verwandte Konstruktionen. (= Linguistische Arbeiten 106). Tübingen.

Auer, Peter (1996): On the prosody and syntax of turn-continuations. In: Couper-Kuhlen, Elizabeth/Selting, Margret (Hg.): Prosody in conversation: interactional studies. (=Studies in Interactional Sociolinguistics 12). Cambridge, S. 57-100.

Auer, Peter (2000): On line-Syntax - oder: Was es bedeuten könnte, die Zeitlichkeit der mündlichen Sprache ernst zu nehmen. In: Sprache und Literatur 85, S. 43-56.

Auer, Peter (2007): Why are increments such elusive objects? An afterthought. In: Pragmatics 17, 4, S. 647-658.

Auer, Peter (2008): On-line syntax: thoughts on the temporality of spoken language. In: Language Sciences 31, 1, S. 1-13.

Auer, Peter/Günthner, Susanne (2005): Die Entstehung von Diskursmarkern im Deutschen - ein Fall von Grammatikalisierung?. In: Leuschner, Torsten/Mortelmans, Tanja (Hg.): Grammatikalisierung im Deutschen. (= Linguistik - Impulse \& Tendenzen 9). Berlin/New York, S. 335-362.

Barden, Birgit/Elstermann, Mechthild/Fiehler Reinhard (2001): Operator-Skopus-Strukturen in gesprochener Sprache. In: Liedtke, Frank/Hundsnurscher, Franz (Hg.): Pragmatische Syntax. (= Beiträge zur Dialogforschung 23). Tübingen, S. 197-232.

Bittner, Thomas/Smith, Barry (2001a): A unified theory of granularity, vagueness, and approximation. In: Proceedings of the 1st Workshop on Spatial Vagueness, Uncertainty, and Granularity (SVUG01), S. 1-39. Internet: www.cs.northwestern.edu/ bittner/BittnerSmithSVUG01.pdf (Stand: 03.08.2010).

Bittner, Thomas/Smith, Barry (2001b): A taxonomy of granular partitions. In: Lecture Notes in Computer Science 22, 05, S. 28-42.

Bittner, Thomas/Smith, Barry (2003): A theory of granular partitions. Internet: http:// citeseerx.ist.psu.edu/viewdoc/summary?doi=10.1.1.17.2458 (Stand: 17.11.2010). 
Brinton, Laurel J. (1996): Pragmatic markers in English: grammaticalization and discourse functions. (= Topics in English Linguistics 19). Berlin/New York.

Bücker, Jörg (2008): Elf Freunde sollt ibr sein? Von wegen! - nicht-präpositionale Spielarten mit von wegen als Projektorkonstruktionen in der deutschen Gegenwartssprache. In: Arbeitspapierreiche GIDI (Grammatik in der Interaktion) 17, S. 1-33. Internet: http://noam.uni-muenster.de/gidi/ (Stand: 03.08.2010).

Bühler, Karl (1982 [1934]): Sprachtheorie: die Darstellungsfunktion der Sprache. Ungekürzter Neudruck der Ausgabe Jena 1934. Stuttgart.

Couper-Kuhlen, Elizabeth/Ono, Tsuyoshi Ono (2007): ,Incrementing' in conversation. A comparison of practices in English, German and Japanese. In: Pragmatics 17, 4, S. 513-552.

Deppermann, Arnulf (2006): Construction Grammar - eine Grammatik für die Interaktion? In: Deppermann/Fiehler/Spranz-Fogasy (Hg.), S. 43-66.

Deppermann, Arnulf/Fiehler, Reinhard/Spranz-Fogasy, Thomas (Hg.) (2006): Grammatik und Interaktion - Untersuchungen zum Zusammenhang von grammatischen Strukturen und Gesprächsprozessen. Radolfzell.

Deppermann, Arnulf/Elstermann, Mechthild (2008): Lexikalische Bedeutung oder Konstruktionsbedeutungen? Eine Untersuchung am Beispiel von Konstruktionen mit verstehen. In: Stefanowitsch/Fischer (Hg.), S. 103-133.

Diewald, Gabriele (2008): Konstruktionen in der diachronen Sprachwissenschaft. In: Stefanowitsch/Fischer (Hg.), S. 79-103.

Duden (2005): Der Duden in 12 Bänden. Bd. 4: Die Grammatik. Hrsg. v. der Dudenredaktion. 7., völlig neu erarb. u. erw. Aufl. Mannheim u.a.

Eco, Umberto (1977): Zeichen: Einführung in einen Begriff und seine Geschichte. Frankurt a.M.

Fillmore, Charles J. (1988): The Mechanisms of ,Construction Grammar'. In: Proceedings of the Annual Meeting of the Berkeley Linguistics Society 14, S. 35-55.

Fillmore, Charles J./Kay, Paul (1995): Construction Grammar. Unveröff. Ms. Berkeley.

Fillmore, Charles J./Kay, Paul/O'Connor, Mary C. (1988): Regularity and idiomaticity in grammatical constructions: the case of let alone. In: Language 64, S. 501-538.

Fischer, Kerstin/Stefanowitsch, Anatol (2006): Konstruktionsgrammatik: Ein Überblick. In: Fischer/Stefanowitsch (Hg.), S. 3-17.

Fischer, Kerstin/Stefanowitsch, Anatol (Hg.) (2006): Konstruktionsgrammatik: Von der Anwendung zur Theorie. (= Stauffenburg Linguistik 40). Tübingen.

Fraser, Bruce (1990): An approach to discourse markers. In: Journal of Pragmatics 14, S. 383-395.

Fried, Mirjam/Östman, Jan-Ola (2005): Construction Grammar: A thumbnail sketch. In: Östman/Fried (Hg.), S. 11-86.

Gohl, Christine/Günthner, Susanne (1999): Grammatikalisierung von weil als Diskursmarker in der gesprochenen Sprache. In: Zeitschrift für Sprachwissenschaft 18, 1, S. 39-75. 
Goldberg, Adele (1995): Constructions: a Construction Grammar approach to argument structure. Chicago.

Goldberg, Adele (1996): Construction Grammar. In: Brown, Keith/Miller, Jim (Hg.): Concise encyclopedia of syntactic theories. Oxford, S. 68-70.

Goldberg, Adele (1998): Patterns of experience in patterns of language. In: Tomasello, Michael (Hg.): The new psychology of language. Mahwah, S. 203-219.

Günthner, Susanne (2006): Grammatische Analysen der kommunikativen Praxis - ,Dichte Konstruktionen' in der Interaktion. In: Deppermann/Fiehler/Spranz-Fogasy (Hg.), S. 95-122.

Günthner, Susanne (2008a): Projektorkonstruktionen im Gespräch: Pseudoclefts, die Sache ist-Konstruktionen und Extrapositionen mit es. In: Gesprächsforschung Online Zeitschrift zur verbalen Interaktion 9, S. 86-114. Internet: http://www. gespraechsforschung-ozs.de/heft2008/ga-guenthner.pdf (Stand: 17.11.2010).

Günthner, Susanne (2008b): Das Ding ist/die Sache ist ... - Die „N be that"-Konstruktion im gesprochenen Deutsch. In: Stefanowitsch/Fischer (Hg.), S. 157-178.

Günthner, Susanne (2008c): ,Die Sache ist ...': eine Projektorkonstruktion im gesprochenen Deutsch. In: Zeitschrift für Sprachwissenschaft 27, 1, S. 39-72.

Günthner, Susanne (2009): ,Adjektiv + dass-Satz'-Konstruktionen als kommunikative Ressourcen der Positionierung. In: Günthner, Susanne/Bücker, Jörg (Hg.): Grammatik im Gespräch: Konstruktionen der Selbst- und Fremdpositionierung. (= Linguistik - Impulse \& Tendenzen 33). Berlin/New York, S. 149-184.

Günthner, Susanne/Imo, Wolfgang (2003): Die Reanalyse von Matrixsätzen als Diskursmarker: ich mein-Konstruktionen im gesprochenen Deutsch. In: Orosz, Magdolna/Herzog, Andreas (Hg.): Jahrbuch der ungarischen Germanistik. Budapest, S. 181-216.

Günthner, Susanne/Imo, Wolfgang (2006): Konstruktionen in der Interaktion. (= Linguistik - Impulse \& Tendenzen 20). Berlin/New York.

Harris, Roy (2008): Die integrationale Zeichenkonzeption. In: Semiotik 30, 1-2, S. $11-$ 28.

Helbig, Gerhard (1999): Deutsche Grammatik: Grundfragen und Abriß. 4., unveränd. Aufl. München.

Hennig, Mathilde (2000): Tempus und Temporalität in geschriebenen und gesprochenen Texten. (= Linguistische Arbeiten 421). Tübingen.

Hennig, Mathilde (2006): Grammatik der gesprochenen Sprache in Theorie und Praxis. Kassel.

Hopper, Paul J. (1992): Times of the sign: discourse, temporality and recent linguistics. In: Time \& Society 1, 2, S. 223-238.

Hopper, Paul J. (2001): Grammatical constructions and their discourse origins: prototype or family resemblance?. In: Pütz, Martin/Niemeier, Susanne/Dirven, René (Hg.): Applied cognitive linguistics I: theory and language acquisition. New York, S. 109-129. 
Hopper, Paul J. (2004): The openness of grammatical constructions. In: Chicago Linguistic Society 40, S. 239-256.

Imo, Wolfgang (2007a): Construction Grammar und Gesprochene-Sprache-Forschung: Konstruktionen mit zehn matrixsatzfähigen Verben im gesprochenen Deutsch. (= Reihe Germanistische Linguistik 275). Tübingen.

Imo, Wolfgang (2007b): Der Zwang zur Kategorienbildung: Probleme der Anwendung der Construction Grammar bei der Analyse gesprochener Sprache. In: Gesprächsforschung - Online Zeitschrift zur verbalen Interaktion 8, S. 22-45. Internet: www. gespraechsforschung-ozs.de/heft2007/ga-imo.pdf (Stand: 17.11.2010).

Imo, Wolfgang (2010): Das Adverb jetžt zwischen Zeit- und Gesprächsdeixis. In: Zeitschrift für Germanistische Linguistik 38, 1, S. 25-58.

Imo, Wolfgang (i.Dr.a): On-line changes of syntactic gestalts in spoken German. Or: Do garden path sentences exist in everyday conversation? In: Auer, Peter/Pfänder, Stefan (Hg.): Constructions: emerging and emergent. Berlin u.a.

Imo, Wolfgang (i.Dr.b): How holistic can construction grammar get? On the problems of integrating prosodic information into constructions.

Imo, Wolfgang (i.Dr.c): Ad-hoc-Produktion oder Konstruktion? Verfestigungstendenzen bei Inkrement-Strukturen im gesprochenen Deutsch. In: Lasch, Alexander/ Ziem, Alexander (Hg.): Konstruktionsgrammatik III. Tübingen.

Jacobs, Joachim (2008): Wozu Konstruktionen?. In: Linguistische Berichte 213, S. 3-44.

Kay, Paul (2000): An informal sketch of a formal architecture for Construction Grammar. In: Fillmore, Charles/Kay, Paul: Berkeley Construction Grammar. Internet: www. icsi.berkeley.edu/ kay/bcg/ConGram.html (Stand: 03.08.2010).

Langacker, Ronald W. (1987): Foundations of cognitive grammar. Stanford.

Langacker, Ronald W. (1995): Viewing in cognition and grammar. In: Davis, Philipp W. (Hg.): Descriptive and theoretical modes in the alternative linguistics. (= Amsterdam Studies in the Theory and History of Linguistic Science: Series 4, Current Issues in Linguistic Theory 102). Amsterdam u.a., S. 152-212.

Langacker, Ronald W. (1999): Grammar and conceptualization. (= Cognitive Linguistics Research 14). Berlin/New York.

Linell, Per (1998): Approaching dialogue: talk, interaction and contexts in dialogical perspective. (= Impact: Studies in Language and Society 3). Amsterdam u.a.

Linell, Per (2006): Can we formalise the linguistic resources of interactional language? In: Suominen, Mickael et al. (Hg.): A man of measure: Festschrift in honour of Fred Karlsson on his 60th birthday. Special Supplement to SKY Journal of Linguistics 19, S. 56-65.

Mitchell, Sandra (2008): Komplexitäten. Warum wir erst anfangen, die Welt zu verstehen. (= Edition Unseld 1). Frankfurt a.M.

Norén, Kerstin/Linell, Per (2007): Meaning potentials and the interaction between lexis and contexts: an empirical substantiation. In: Pragmatics 17, 3, S. 387-416. 
Östman, Jan-Ola (2005): Construction discourse: a prolegomenon. In: Östman/Fried (Hg.), S. 121-144.

Östman, Jan-Ola/Fried, Mirjam (Hg.) (2005): Construction Grammars: cognitive grounding and theoretical extensions. (= Constructional Approaches to Language 3). Amsterdam u.a.

Schegloff, Emanuel A. (2000): On granularity. In: Annual Review of Sociology 26, S. 715-720.

Schiffrin, Deborah (1987): Discourse markers. (= Studies in Interactional Sociolinguistics 5). Cambridge.

Stefanowitsch, Anatol/Fischer, Kerstin (Hg.) (2008): Konstruktionsgrammatik II: Von der Konstruktion zur Grammatik. (= Stauffenburg Linguistik 47). Tübingen.

Taylor, John R. (2002): Cognitive grammar. Oxford.

Tomasello, Michael (2003): Constructing a language: a usage-based theory of language acquisition. Cambridge, MA.

Tomasello, Michael (2006): Konstruktionsgrammatik und früher Erstspracherwerb. In: Fischer/Stefanowitsch (Hg.), S. 19-38.

Traugott, Elizabeth C. (2008): Grammatikalisierung, emergente Konstruktionen und der Begriff der ,Neuheit'. In: Stefanowitsch/Fischer (Hg.), S. 5-32.

Weinrich, Harald (2005): Textgrammatik der deutschen Sprache. 3., rev. Aufl. Hildesheim/Zürich/New York.

Wong-Fillmore, Lily (1979): Individual differences in second language acquisition. In: Fillmore, Charles J./Kempler, Daniel/Wang, William S.Y. (Hg.): Individual differences in language ability and language behavior. New York u.a., S. 203-228.

Zifonun, Gisela/Hoffmann, Ludger/Strecker, Bruno (1997): Grammatik der deutschen Sprache. 3 Bde. (= Schriften des Instituts für Deutsche Sprache 7). Berlin/New York. 

Regeln und Konstruktionen 



\title{
Paradigmenwechsel rückwärts: Die Renaissance der grammatischen Konstruktion ${ }^{1}$
}

\begin{abstract}
Im letzten halben Jahrhundert hat in der formalen Grammatikforschung eine intensive Diskussion über die Natur syntaktischer und lexikalischer Information stattgefunden. Während die frühe Generative Grammatik der traditionellen Grammatik folgte und konstruktionsspezifische Regeln anerkannte, gibt es seit den achtziger Jahren extreme Formen des Lexikalismus, die die Existenz von Konstruktionen bestreiten. Als Reaktion auf diese Entwicklungen leitete die „Berkeley Construction Grammar" eine Renaissance der grammatischen Konstruktion ein. Der vorliegende Aufsatz untersucht anhand deutscher Relativsatztypen, ob diese besser rein lexikalisch oder mit Hilfe von Konstruktionen zu analysieren sind. Der Befund ist eindeutig: Die empirischen Daten treiben die rein lexikalische Theorie vor sich her und erweisen sie als unmotiviert und unüberzeugend. Im Gegensatz dazu kann die konstruktionale Theorie mit „,intelligenten“ Werkzeugen wie Typen, Untertypen und Vererbung Generalisierungen über deutsche Relativsätze auf allen Ebenen elegant und effizient erfassen. Der Vorschlag Chomskys, Konstruktionen aus der Grammatik zu verbannen, erweist sich somit konzeptuell und empirisch als wissenschaftliche Fehlentscheidung.
\end{abstract}

\section{Die Natur syntaktischer und lexikalischer Information}

Seit einem halben Jahrhundert findet in der formalen Grammatikforschung eine intensive Diskussion über die Natur syntaktischer und lexikalischer Information und über die Arbeitsteilung zwischen dem Lexikon und der Syntax statt. In den fünfziger und sechziger Jahren wurden Lexikoneinträge als relativ informationsarm angesehen. Dem gegenüber stand eine große Menge von Regeln zur Erzeugung von Phrasenstrukturen. Für das Deutsche könnte man u.a. exemplarisch folgende Regeln (vgl. Tabelle 1) ansetzen:

Ich möchte mich bei Anke Holler, Stefan Müller, Stefan Engelberg und Kristel Proost für ihre detaillierten Kommentare und Verbesserungsvorschläge zu einem ersten Entwurf dieses Artikels bedanken! Ich bin mir sicher, ich werde es bereuen, nicht allen ihren Vorschlägen gefolgt zu sein. Auch von den Fragen und Kommentaren des Publikums nach meinem Vortrag bei der IDS-Jahrestagung habe ich sehr profitiert. Für alle verbleibenden Fehler und Probleme zeichne ich allein verantwortlich. 


$$
\begin{array}{lll}
\mathrm{VP} \rightarrow \mathrm{V} & \mathrm{AP} \rightarrow \mathrm{A} & \mathrm{NP} \rightarrow \mathrm{N} \\
\mathrm{VP} \rightarrow \mathrm{NP} \mathrm{V} & \mathrm{AP} \rightarrow \mathrm{NP} \mathrm{A} & \mathrm{NP} \rightarrow(\text { Det }) \mathrm{N} \mathrm{NP} \\
\mathrm{VP} \rightarrow \mathrm{PP} \mathrm{V} & \mathrm{AP} \rightarrow \mathrm{PP} \mathrm{A} & \mathrm{NP} \rightarrow \text { (Det) N PP } \\
\mathrm{VP} \rightarrow \mathrm{NP} \mathrm{PP} \mathrm{V} & & \mathrm{NP} \rightarrow \text { (Det) N NP PP } \\
\mathrm{VP} \rightarrow \mathrm{PP} \mathrm{PP} \mathrm{V} & & \mathrm{NP} \rightarrow \text { (Det) N PP PP } \\
\mathrm{VP} \rightarrow \mathrm{S} \mathrm{V} & \mathrm{AP} \rightarrow \mathrm{S} \mathrm{A} & \mathrm{NP} \rightarrow \text { (Det) N S }
\end{array}
$$

Tab. 1: Regeln zur Erzeugung von Phrasenstrukturen

Diese Art von Regelapparat kann als formale Entsprechung der Satzbaupläne der traditionellen Grammatik aufgefasst werden, insofern die Regeln (im Zusammenspiel mit Transformationen) gemeinsam die Satzmuster einer Sprache charakterisieren.

In der weiteren Forschung wurde schnell deutlich, dass Regelsysteme wie in Tabelle 1 Schwächen haben, von denen ich hier nur zwei nennen möchte. Erstens ist es mit dem Vokabular, mit dem die oben genannten für das Deutsche nützlichen Regeln geschrieben sind, genauso leicht möglich, unnützliche Regeln zu formulieren. So sind die folgenden beiden unerwünschten Regeln vollständig aus Komponenten zusammengesetzt, die auch in den sinnvollen Regeln in Tabelle 1 erscheinen:

$$
\begin{aligned}
& * \mathrm{VP} \rightarrow \mathrm{A} \mathrm{PP} \\
& * \mathrm{AP} \rightarrow \mathrm{NP} \mathrm{PP}
\end{aligned}
$$

Selbst wenn wir solche Regeln auf irgendeine Weise ausschließen, übergeneriert das Regelsystem in (1) noch massiv, da z.B. nicht jedes Verb in jeder Regel vorkommen kann, die auf der rechten Seite die Kategorie V erwähnt. So dürfen in der Verbposition der ersten Regel nur Verben stehen, die strikt intransitiv gebraucht werden können, wie die folgenden Beispiele zeigen:

(1) dass Maria starb.

(2) *dass Maria bekochte.

(3) *dass Maria stellte.

Die beiden genannten Probleme können durch eine geänderte Arbeitsteilung der lexikalischen und phrasenstrukturellen Mechanismen gleichzeitig gelöst werden. Für die Verben in (1)-(3) können wir demgemäß Lexikoneinträge postulieren, die außer der syntaktischen Kategorie des Eintrags auch Argumentstrukturinformationen ${ }^{2}$ (d.h. Valenz) enthalten. ${ }^{3}$ Die fol-

\footnotetext{
In den sechziger Jahren sprach man in der Generativen Grammatik eher von „Subkategorisierung", siehe u.a. Chomsky (1965) und Bechert et al. (1970).

3 Hier und im Folgenden beschränke ich die Diskussion aus Platz- und Relevanzgründen auf Komplemente und vermeide eine Diskussion der Repräsentation von Subjekten und Spezifizierern.
} 
genden Einträge besagen, dass das Verb sterben kein Komplement erfordert, bekochen ein NP-Komplement selegiert und dass stellen sich mit zwei Komplementen - einer NP und einer PP - verbindet:

(4) sterben, V, Comps: []

(5) bekochen, V, Comps: [NP]

(6) stellen, V, Comps: [NP, PP]

Die Lexikalisierung der syntaktischen Kontexte, in denen Wörter vorkommen können, ermöglicht nun eine dramatische Reduktion der Phrasenstrukturkomponente. Die Regeln in Tabelle 1 können durch das folgende allgemeine Strukturschema, die so genannte X-bar-Theorie, ersetzt werden: ${ }^{4}$

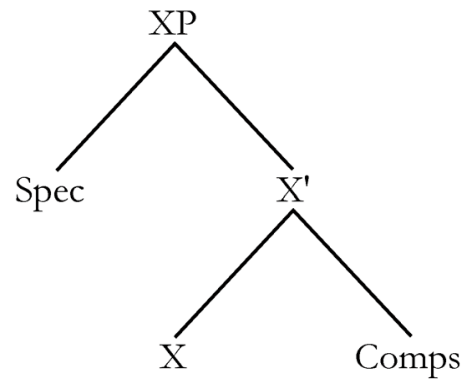

(7) Die X-bar-Theorie

Die Erzeugung der Phrasenstruktur in der X-bar-Theorie ist kopfgetrieben, in dem Sinne, dass die Ausbildung des Strukturschemas in (7) Bezug auf die lexikalische Information des Kopfes der Phrase nimmt: (i) die Kategorie der Phrase entspricht der lexikalischen Kategorie ihres Kopfes und (ii) die Anzahl und Art der Komplemente innerhalb von X' wird von der Argumentstruktur des Kopfes bestimmt. Auf der Basis der Lexikoneinträge in (8)-(10) lizenziert das allgemeine X-bar-Schema in (5) daher die drei Strukturen in (11), ohne die drei Regeln in der ersten Zeile von (1) zu benötigen:

(8) sterben, V, Comps: []

(9) müde, A, Comps: []

(10) Johanna, N, Comps: []

$4 \quad$ Der Ursprung der X-bar-Theorie findet sich in Harris (1951). Durch Chomsky (1970) wurde sie der breiten generativen Öffentlichkeit bekannt. Siehe auch Jackendoff (1977), Kornai/ Pullum (1990) und Chomsky (1995b). 


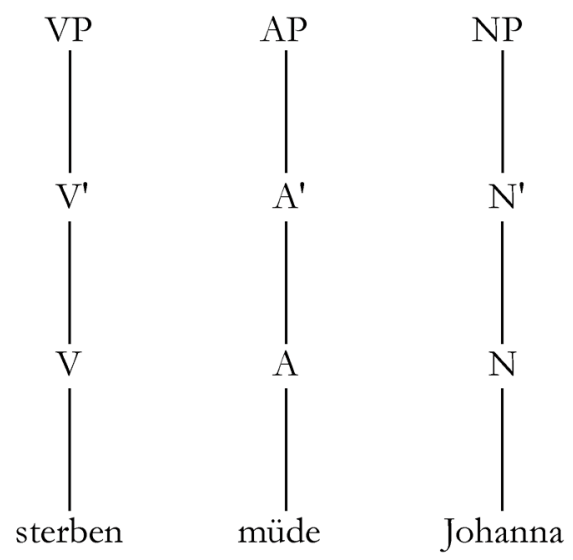

(11) Phrasen mit einem Kopf ohne Komplement

Entsprechend werden die Phrasen in (15) korrekt aus den folgenden Lexikoneinträgen projiziert:

(12) sprechen, V, Comps: [PP]

(13) verärgert, A, Comps: [PP]

(14) Verärgerung, $\mathrm{N}$, Comps: [PP]

Es ergeben sich drei verschiedene Phrasenkategorien, die jeweils aus dem Kopf der Kategorie und einer Präpositionalphrase bestehen:
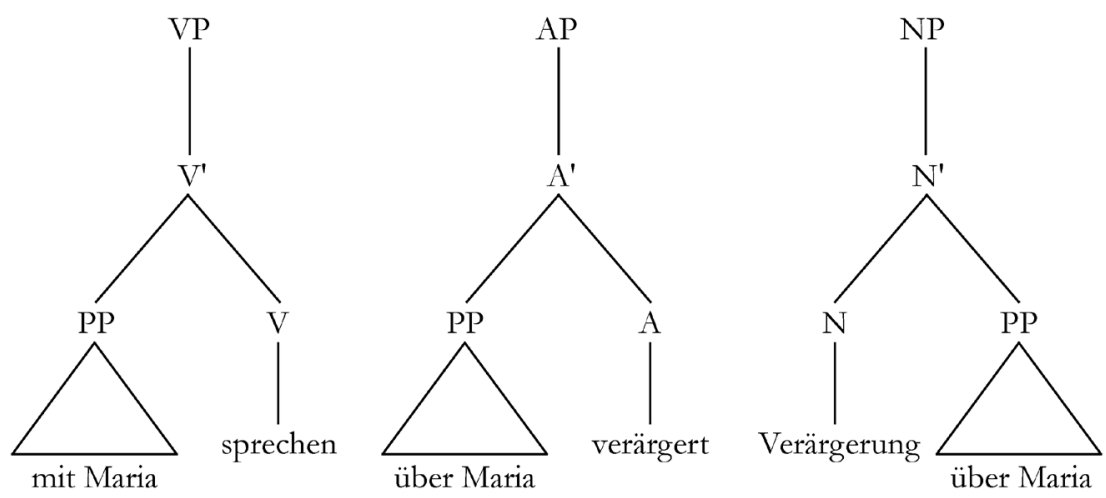

(15) Phrasen mit einem Kopf und einem PP-Komplement

Somit können wir ein erstes Fazit ziehen: Durch die Verlagerung von Informationen aus Phrasenstrukturregeln in das Lexikon ist es möglich, die Anzahl und die Komplexität der Phrasenstrukturregeln zu reduzieren. 
Der Erfolg der X-bar-Theorie führte zu einem Siegeszug des Lexikalismus in den achtziger Jahren des vorigen Jahrhunderts: Der Projektionismus, d.h. das Konzept, syntaktische Strukturen aus Lexikoneinträgen zu projizieren, wurde zu einer führenden Idee nahezu aller Ausprägungen der Generativen Grammatik. Zwei Theorien, die „Lexical-Functional Grammar“ ${ }^{\text {“5 }}$ (LFG) und die „Head-Driven Phrase Structure Grammar ${ }^{\text {“6 }}$ (HPSG) setzten die Idee auf besonders konsequente Weise um und eliminierten den Mechanismus der Transformation, der zum Beginn der Generativen Grammatik noch den einzigen grammatischen Regeltyp darstellte, mit dem systematische Beziehungen zwischen Sätzen formal erfasst werden konnten. In den genannten Theorien entstanden systematisch lexikalische Gegenanalysen zu vormals mit Transformationen arbeitenden Herangehensweisen an viele grammatische Phänomene. Als ein Beispiel sei das Passiv genannt. Bresnan (1982) entwickelte eine Analyse für das Englische, ${ }^{7}$ die Passivpartizipien im Lexikon erzeugt (was wegen der morphologischen Irregularitäten vieler Partizipien auf der Basis der Lexikalistischen Hypothese von Chomsky (1970) ohnehin motiviert ist) und in der Lage ist, mit Hilfe der X-bar-Theorie die essenziellen syntaktischen Eigenschaften von Passivsätzen aus den Lexikoneinträgen der passivierten Verben zu projizieren. Bresnan (2001) argumentiert, dass durch die Verbesserung der lexikalischen Instrumente in der Analyse des Passivs Transformationsregeln nicht nur überflüssig geworden sind, sondern dass eine lexikalische Analyse des Passivs einer transformationellen auch empirisch und konzeptuell überlegen ist. So sagt sie zum Beispiel automatisch vorher, dass der Anwendungsbereich der Passivoperation auf die Argumente eines Verbs beschränkt ist und dass der lexikalische Output der Operation als Grundlage weiterer lexikalischer Operationen dienen kann, unter anderem der Derivation von Adjektiven.

Mit der LFG und der HPSG sind somit Theorien entstanden, die man als Ausprägungen eines „Konkreten Lexikalismus“ bezeichnen könnte. Dessen Hauptziele sind unter anderem:

- Die Anzahl sprachspezifischer Phrasenstrukturregeln soll minimiert werden.

- Die Theorie ist oberflächenorientiert, d.h. sie strebt die größtmögliche Vermeidung unsichtbarer Elemente wie Tiefenstrukturen oder leere Kategorien an.

- Die deskriptive Adäquatheit soll nicht einer vermeintlichen explanatorischen Adäquatheit geopfert werden.

Siehe Bresnan (Hg.) (1982) und (2001).

Siehe Pollard/Sag (1994) und Ginzburg/Sag (2000).

Höhle (1978) legt die Grundlagen für eine lexikalische Analyse der deutschen Passive. 
Die Mechanismen, mit denen diese Ziele erreicht werden sollen, weichen von denen der klassischen, stark auf das eine Instrument der strukturverändernden syntaktischen Transformation fixierten Generativen Grammatik ab und machen Transformationen durch ein ganzes Portfolio von lexikalischen Instrumenten überflüssig:

- Informationsreiche Lexikoneinträge;

- Verwendung von Typenhierarchien und Vererbung gemäß der Annahme, dass das Lexikon kein Gefängnis von Gesetzlosen (Di Sciullo/ Williams 1987), sondern ein intelligentes, hoch strukturiertes System ist, das lexikalisches Wissen durch Typen, Untertypen, Vererbung und Lexikonregeln strukturiert.

Die Verwendung von Typenhierarchien und Vererbung hat darüber hinaus den Vorteil, dass sie kognitiv umsonst kommen, insofern es starke Evidenz dafür gibt, dass diese Instrumente auch in anderen Bereichen der Wissensrepräsentation Anwendung finden und mit ihrer Hilfe auf elegante Weise die Interaktion von allgemeinen und idiosynkratischen Eigenschaften erfasst werden kann, die für die natürliche Sprache so typisch ist.

Eine andere Form des Lexikalismus, den ich als „Atomistischen Lexikalismus der Satzstruktur" bezeichnen möchte, entstand in der Folge von Chomsky (1981). Dieses Werk setzt sich von dem bis dahin vorherrschenden Konzept einer Generativen Grammatik als einer Regelmenge von unbestimmter Größe ab und konzipiert eine Generative Grammatik im Gegensatz dazu als eine Menge von Prinzipien und Parametern. Dies führt zur folgenden extremen antikonstruktionalen Position:

In a Principles-and-Parameters approach, ,[t]he notion of grammatical construction is eliminated, and with it, construction-particular rules". (Chomsky 1993, S. 4)

Die Prinzipien des Ansatzes, zu denen unter anderem die X-bar-Theorie gehört, sollen eine kleine Anzahl „parametrischer“ Wahlmöglichkeiten enthalten, die in der Universalgrammatik aufgelistet sind und in Einzelgrammatiken unterschiedlich „fixiert“" werden können. ${ }^{8}$

In Chomsky (1981) wird davon ausgegangen, dass ein universelles Prinzip die Hierarchie der Satzstruktur wie folgt festlegt:

Die Anhänger der Prinzipien- und Parametertheorie stimmen darin überein, dass Prinzipien und Parameter wissenschaftliche Erklärungskraft besitzen, weil sie Teil einer zu entdeckenden Universalgrammatik sind. Über den Ursprung der Universalgrammatik besteht allerdings keine Einigkeit: Während Chomsky nach wie vor biologisch argumentiert (Chomsky 2007), vertritt Baker (1996, S. 514) die Ansicht, dass eine kreationistische Begründung der Universalgrammatik der biologischen Hypothese überlegen ist: „Linguistic diversity results from a direct act of God.“ 


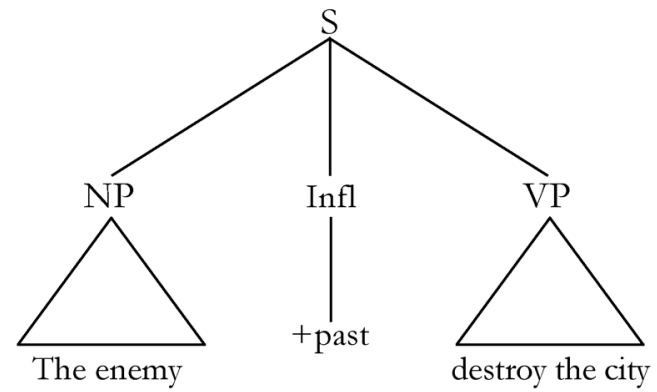

(16) Die universelle Satzhierarchie nach Chomsky (1981)

Wie in der Grafik gezeigt, besteht ein Satz aus drei Teilen, dem Subjekt, einem INFL-Knoten (u.a. für Flexionselemente) und einer Verbalphrase. Die Abfolge der Subkonstituenten des Satzes sowie deren phonologische Realisierung in Einzelsprachen sollen Resultate von parametrischen Entscheidungen (Linearisierungsparameter, Pro Drop) sein. Chomsky (1981) analysiert primär das Englische und ausgewählte Bereiche des Italienischen und Chinesischen. In der Folge wurde recht schnell deutlich, dass die in (16) gegebene Satzstruktur nicht ausdifferenziert genug war, um eine Anzahl parametrischer Optionen zur Verfügung zu stellen, die ausreichen würde, die einzelsprachlichen syntaktischen Unterschiede mit den Mechanismen der Prinzipien- und Parametertheorie auch nur annähernd zu erfassen. Als Konsequenz erweiterte Chomsky (1986) die vorgeblich universelle Satzhierarchie um eine $\mathrm{C}$ (omplementizer)-Kategorie und schlug vor, dass diese wie die schon in Chomsky (1981) postulierte I(nfl)-Kategorie voll in die $\mathrm{X}$-bar-Theorie integriert ist und eine eigene Phrase projiziert:

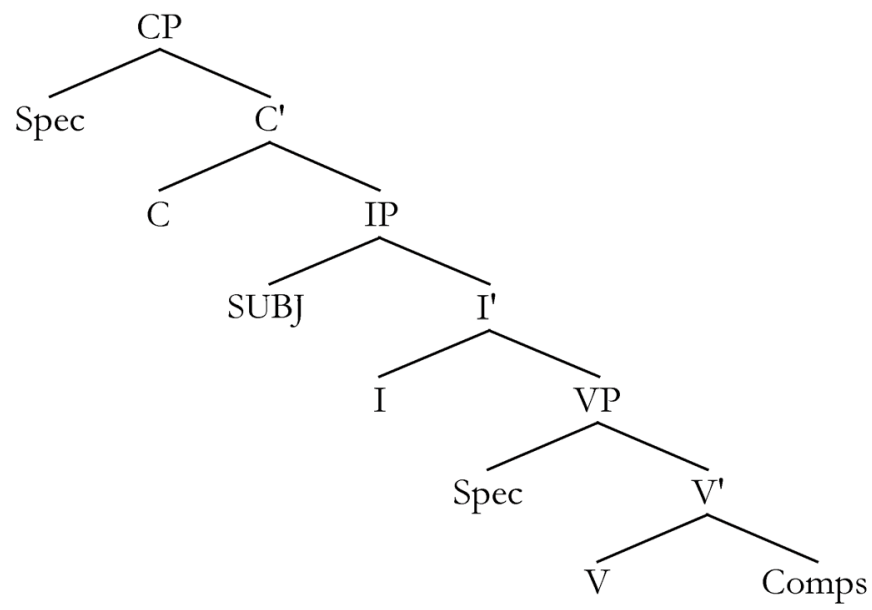

(17) Die universelle Satzhierarchie nach Chomsky (1986) 
Pollock (1989) analysiert das Wortstellungsverhalten von Auxiliaren, Verben und verschiedenen Adverbklassen im Französischen und Englischen und kommt zu dem Schluss, dass eine weitere Aufspaltung der I-Kategorie in $\mathrm{T}$ (ense) und $\operatorname{Agr}($ eement) notwendig ist, um die Daten zu erfassen:?

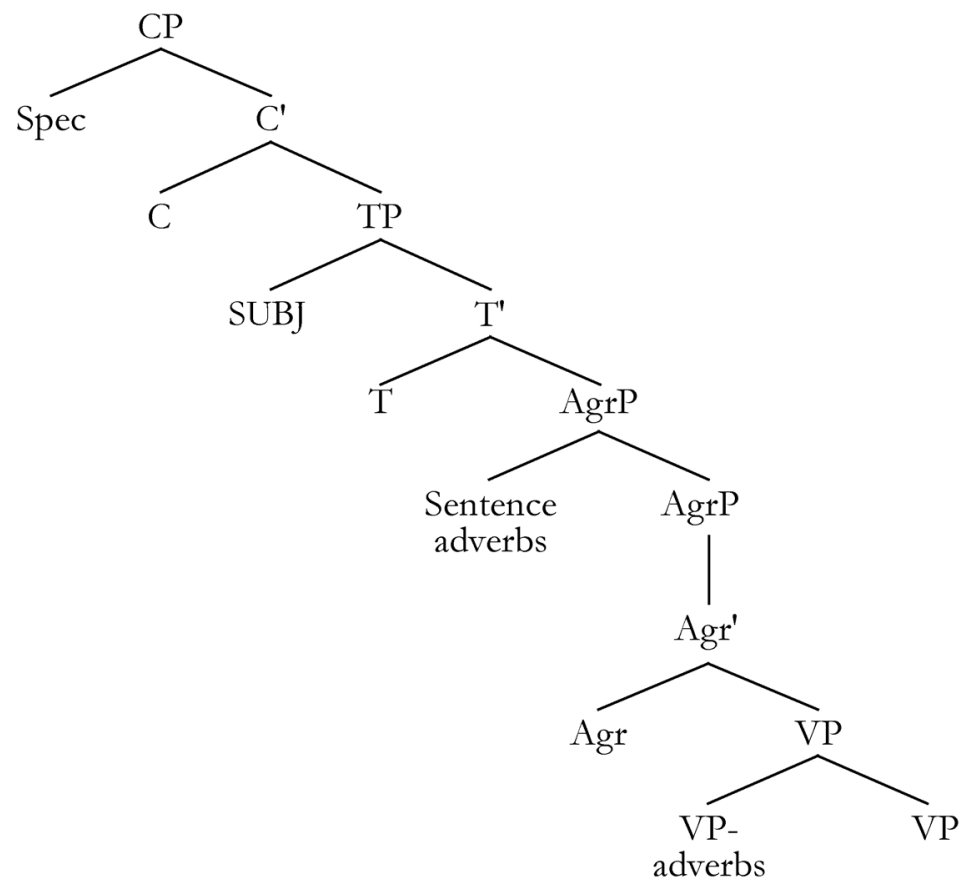

(18) Die universelle Satzhierarchie nach Pollock (1989)

Cinque (1999) erweitert die Anzahl funktionaler Köpfe allein in der IP-Domäne auf $32:^{10}$
a. Mood $_{\text {Speech Act }}$
d. Mood $_{\text {Epistemic }}$
b. Mood $_{\text {Evaluative }}$
e. $\mathrm{T}_{\text {(Past) }}$
g. Mood $_{\text {Irrealis }}$
h. Mod $_{\text {Necessity }}$
k. Mod $_{\text {Obligation }}$
j. $\quad$ Mod $_{\text {Volitional }}$
n. Asp $P_{\text {Repetetive(I) }}$
m. Asp $\mathrm{Aabitual}$
p. Asp Celerative(I)
q. $\mathrm{T}_{\text {(Anterior) }}$
c. Mood $_{\text {Evidential }}$
f. $T_{\text {(Future) }}$
i. Mod $_{\text {Possibility }}$
1. $\operatorname{Mod}_{\text {Ability/permiss. }}$
O. Asp Frequentative(I)
r. Asp Terminitive

$9 \quad$ Iatridou (1990) zeigt, dass die von Pollock vorgestellten Daten die theoretischen Konsequenzen, die er aus ihnen zieht, nicht wirklich stützen. Aber der Zug war nicht mehr aufzuhalten: Die quasi unbeschränkten deskriptiven Möglichkeiten, die durch die „Explosion“ der Satzstruktur geschaffen wurden, stellten eine Versuchung dar, der die meisten Anhänger der Prinzipien- und Parametertheorie nicht mehr widerstehen konnten. Trotz des Mangels an Motivation wurden Pollocks Strukturannahmen unkritisch übernommen und Iatridous Argumente ignoriert.

10 Diese Zusammenstellung ist Newmeyer (2004, S. 194) entnommen. 

s. Asp Continuative
v. Asp proximative
t. Asperfect(?)
w. Asp Durative
z. Asp SgCompletive(I)
y. Asp Prospective
bb. Voice
cc. Asp Celerative(II)
ff. Asp Frequentative(II)
u. Asp Retrospective
x. Asp $\mathrm{P}_{\text {Generic/progress. }}$
aa. Asp PICompletive
dd. Asp SgComplet. (II) $_{\text {. }}$
ee. Aspepetitive(II)

Aber damit nicht genug. Newmeyer (2004, S. 194) sammelt weitere lexikalische Kopfkategorien, die in der Literatur der Prinzipien- und Parametertheorie bzw. des Minimalistischen Programms vorgeschlagen worden sind (die Hervorhebungen stammen von mir):

On the basis of a look at fifteen languages, fourteen of them Indo-European (from only four subfamilies), Longobardi (2003) proposes 30 binary parameters for DP. Cinque (1994) divides Adjective Phrase into at least five separate maximal projections encoding Quality, Size, Shape, Color, and Nationality. Beghelli and Stowell (1997) break down Quantifier Phrase into projections headed by $W h, N e g$, Distributive, Referential, and Share. CP has also been split into a dozen or more projections, including ForceP, FocusP, and an indefinite number of Topic Phrases (Rizzi 1997). Facts pertaining to clitic inversion and related phenomena in some northern dialects of Italian have led to the positing of Left Dislocation Phrase, Number Phrase, Hearer Phrase, and Speaker Phrase (Poletto 2000). Damonte (2004) proposes projections corresponding to the set of thematic roles, including Reciprocal, Benefactive, Instrumental, Causative, Comitative, and Reversive Phrases. We have seen Verb Phrase split into two projections, one headed by $V$ and the other by $v^{6}$ (Chomsky 1995). Zanuttini (2001) posits four distinct Negative Phrase projections for Romance alone [...].

Cinque und Rizzi - zwei Hauptvertreter des Minimalismus - gehen in einer Veröffentlichung im Rahmen des kartografischen Ansatzes aus dem Jahr 2010 von mindestens 400 universellen Kopfkategorien aus, von denen alle in jeder natürlichen Sprache existieren sollen, „egal, ob sich dafür offene Evidenz in der Sprache finden lässt oder nicht" (Cinque/Rizzi 2010, S. 55 und 57).

Als Reaktion gegen diesen extremen Lexikalismus entstand Ende der achtziger Jahre an der amerikanischen Westküste als Gegenbewegung die Konstruktionsgrammatik (Fillmore/Kay/O'Connor 1988; Kay/Fillmore 1999), die ein ausgewogeneres Verhältnis zwischen dem Lexikon und der Syntax anstrebt. Typisch für diesen Ansatz ist die Analyse des folgenden Satzparadigmas in Fillmore (1999) (die Hervorhebungen stammen von mir):

(19) Englische Satzarten mit Subjekt-Auxiliar-Inversion

Fragesatz: Was she here this morning?

Wunschsatz: May she live forever.

Exklamativsatz: Boy, was I stupid.

Negierter Imperativsatz: Don't you even touch that!

Konditionalsatz: Were they here now, we wouldn't have this problem.

Korrelativer Konditionalsatz: The faster you finish eating those hot dogs, the sooner will we be able to claim the prize. 
Fillmore weist darauf hin, dass diese Sätze trotz ihrer zum Teil enormen semantischen, pragmatischen und auch syntaktischen Unterschiede alle ein Formmerkmal teilen: Sie weichen alle von der Standardwortstellung SVO des Englischen ab und erfordern ein (overtes) Auxiliar im Satz, auf das unmittelbar das Subjekt und dann das Komplement des Auxiliars folgen. Fillmore sieht die Eigenschaften der oben genannten Sätze als ein Zusammenspiel ihres unterschiedlichen lexikalischen Gehalts mit einer grammatischen Konstruktion, die ihnen allen gemeinsam ist, der Subject-Auxiliary-Inversion-Konstruktion, die ich für die Zwecke des vorliegenden Artikels wie folgt informell darstellen möchte:

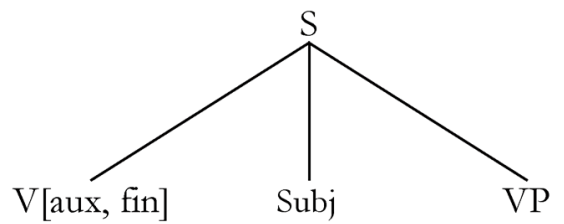

(20) Die Subject-Auxiliary-Inversion-Konstruktion (SAI)

Die Konstruktion definiert eine syntaktische Satzstruktur mit drei Tochterkonstituenten: ein finites Auxiliar, gefolgt vom Subjekt und der VP. Diese Konstruktion existiert in einem komplexen Netzwerk von Konstruktionen, das Teil der Grammatik des Englischen ist. Um die Gemeinsamkeiten und Unterschiede zwischen den Satztypen in (19) zu erfassen, postuliert Fillmore für jeden Satztyp eine Unterkonstruktion von (20):

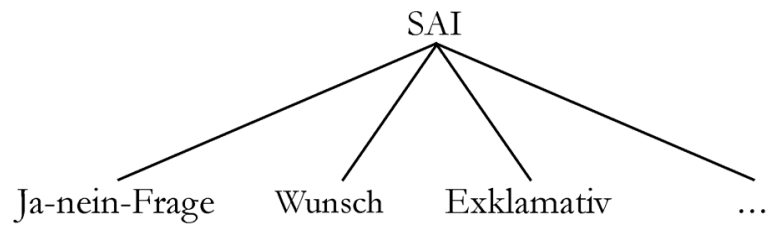

(21) Einige Unterkonstruktionen der Subject-Auxiliary-Inversion-Konstruktion

Jede Unterkonstruktion erbt alle Eigenschaften der Subjekt-Auxiliary-Inversion-Konstruktion, fügt diesen Eigenschaften aber ihre konstruktionsspezifischen Eigenschaften hinzu. Die Gesamtheit der grammatischen Eigenschaften der Einzelkonstruktionen ist somit die Summe von ererbten allgemeinen und konstruktionsspezifischen idiosynkratischen Faktoren, die technisch durch Konstruktionstypen, Untertypen und Vererbung modelliert werden.

Die Tagung, aus der der vorliegende Aufsatz hervorgegangen ist, stand unter dem Motto „Sprachliches Wissen zwischen Lexikon und Grammatik“. 
Das vorbereitende Exposé, das den eingeladenen SprecherInnen zur Verfügung gestellt wurde, enthielt folgenden Passus:

[Wir sehen dort,] wo wir früher eine strikte Grenze postulierten zwischen der Grammatik als dem Ort des syntaktisch-semantisch Regelhaften und dem Lexikon als dem Repositorium des syntaktisch-semantisch Idiosynkratischen, mittlerweile ein immer breiter werdendes Feld von semiproduktiven Prozessen, graduellen Kategoriezuordnungen, frequenzgesteuerten Usualisierungen eigentlich regelhafter Strukturen, und fein differenzierten, nur schwer generalisierbaren Konstruktionsmustern. Das strikt Regelhafte und das rein Idiosynkratische drohen daneben nur noch als Randbereiche des sprachlichen Wissens zu erscheinen.

Im Folgenden werde ich mich angesichts der oben beschriebenen Pendelbewegung der generativen Grammatiktheorien zwischen den lexikalischen und konstruktionalen Polen mit der Frage beschäftigen, ob es auch im Deutschen die im Exposé angesprochene Situation gibt und wie am besten mit ihr umzugehen ist.

\section{Deutsche Relativsätze}

\section{$2.1 \quad$ Überblick}

Der genannten Frage möchte ich anhand der folgenden deutschen Relativsatz- und relativsatzartigen Konstruktionen nachgehen:

Restriktive Relativsätze

(22) Das Auto, das vor der Tür steht, gehört Johanna.

Appositive Relativsätze

(23) Das Auto, das übrigens gerade vor der Tür steht, gehört Johanna.

Cleft-Relativsätze

(24) Es ist Petra, die den Vorschlag machte.

Verb-zweit-Relativsätze (Gärtner 2001)

(25) Das Blatt hat eine Seite, / die ist ganz schwar:

Freie Relativsätze (Müller 1999b)

(26) Wer schläft, sündigt nicht.

Weiterführende $w$-Relativsätze (Holler 2005)

(27) Anna gewann die Schachpartie, was Peter maßlos ärgerte. 


\subsection{Distinktive Eigenschaften der Einzelkonstruktionen}

Es ist leicht, zu zeigen, dass die oben angeführten Satztypen alle ein individuelles Eigenschaftsprofil haben und es sich somit um sechs verschiedene Konstruktionen handeln muss. Eine systematische Untersuchung von vier Eigenschaften genügt, um dies zu dokumentieren. Beginnen wir mit der Eigenschaft, ob der Relativsatz ein externes Bezugselement haben muss und falls ja, ob dieses Bezugselement nominal ist.

Kriterium 1: Hat der Relativsatz ein nominales Bezugselement? ${ }^{11}$

(28) Die Frau, [die Gedanken lesen kann], kommt auch.

(29) Die Frau, [die (übrigens) Gedanken lesen kann], kommt auch.

(30) Es ist die Frau, [die Gedanken lesen kann].

(31) Ich habe eine Frau kennengelernt, (/) [die kann Gedanken lesen].

(32) Ich habe, [was man mir aufgetragen hat], schon erledigt.

(33) Petra kennt Johanna, [was mich (übrigens) überrascht].

Die Beispiele zeigen, dass ein nominales Bezugselement bei restriktiven, appositiven, Cleft-Relativsätzen und Verb-zweit(V2)-Relativsätzen vorliegt, aber nicht bei freien Relativsätzen, (die gar kein Bezugselement haben) und bei weiterführenden $w$-Relativsätzen (deren Bezugselement ein Satz oder ein Prädikat ist).

Ein zweites Unterscheidungskriterium bildet die Frage, ob das Bezugselement einen singulären Term denotiert. Da die letzten beiden Relativsatztypen kein nominales Bezugselement haben, ist dieses Kriterium nur auf die ersten vier Typen anwendbar. Bekanntermaßen muss das semantische Bezugselement von restriktiven Relativsätzen restringierbar sein und darf daher keinen singulären Term denotieren. Gärtner (2001, S. 114f.) zeigt, dass V2-Relativsätze ein indefinites Bezugselement erfordern. Die anderen Relativsätze mit nominalem Bezugselement weichen hiervon ab und erlauben einen singulären Term:

Kriterium 2: Kann das Bezugselement einen singulären Term denotieren?

(34) *Petra, [die Gedanken lesen kann], kommt auch.

(35) Petra, [die (übrigens) Gedanken lesen kann], kommt auch.

(36) Es ist Petra, [die Gedanken lesen kann].

(37) *Ich habe Petra kennengelernt, (/) [die kann Gedanken lesen].

Die sechs Relativsatztypen, die uns beschäftigen, unterscheiden sich auch hinsichtlich ihres Verhaltens gegenüber dem Satztypoperator, in dessen Skopus das (semantische) Bezugselement des Relativsatzes liegt. Dies zeigen die folgenden Beispiele:

$11 \quad$ Trotz der kursiven Darstellung der gesamten NP in (28)-(31) möchte ich mich nicht darauf festlegen, ob das Bezugselement durch die gesamte NP oder nur einen Teil derselben gebildet wird. 
Kriterium 3: Fällt der Relativsatz in den Skopus des Satztypoperators seines (semantischen) Bezugselements? ${ }^{12}$

(38) Kommt die Frau, [die Gedanken lesen kann], auch? Int(Die Frau, [die Gedanken lesen kann], kommt auch.)

(39) Kommt die Frau, [die (übrigens) Gedanken lesen kann], auch? Int(Die Frau kommt auch.) ^ Ass([Sie kann Gedanken lesen].)

(40) Ist es die Frau, [die Gedanken lesen kann]? Int(Es ist die Frau, [die Gedanken lesen kann].)

(41) *Hast du eine Frau kennengelernt, (/) [die kann Gedanken lesen]?

(42) Hast du, [was man dir aufgetragen hat], schon erledigt? Int(Du hast, [was man dir aufgetragen hat], schon erledigt.)

(43) Ist Petra Lehrerin, [was ich selbst gern werden möchte]? Int(Petra ist Lehrerin $) \wedge$ Ass ([Das möchte ich selbst gern werden].)

Das Bezugselement des restriktiven Relativsatzes in (38) fällt in den Skopus des interrogativen Satztypoperators Int und dies gilt auch für den Relativsatz: ob die Frau Gedanken lesen kann, ist Teil der Frage, wie in der schematischen logischen Form in der zweiten Zeile dargestellt. Der appositive Relativsatz (39) verhält sich anders: Er ist nicht Teil der Frage, sondern drückt eine eigene Assertion aus. Der Cleft-Relativsatz verhält sich wie der restriktive, er liegt im Skopus desselben Satztypoperators wie sein Bezugselement. Die Ungrammatikalität von (41) zeigt, dass der V2-Relativsatz gar nicht mit Fragesätzen kombinierbar ist. Will man das gegenwärtige Kriterium auf den freien Relativsatz (42) überhaupt anwenden, dann macht es am meisten Sinn, das ihn regierende Verb als Bezugselement zu betrachten. Unter dieser Annahme fällt der freie Relativsatz unter denselben Satztypoperator wie sein Bezugselement. Der weiterführende Relativsatz (43), schließlich, verhält sich wie der appositive in (39).

Als viertes und letztes Kriterium zur Klassifizierung der deutschen Relativsatztypen bietet sich die Wortstellung an. Mit Ausnahme des V2-Relativsatzes sind alle anderen Relativsätze des Deutschen Verb-letzt-Sätze.

Tabelle 2 fasst die Ergebnisse unserer kontrastiven Untersuchung der deutschen Relativsätze zusammen.

Wie angekündigt, hat jede Relativsatzkonstruktion ein individuelles Eigenschaftsprofil. Die V2-Relativsätze unterscheiden sich von allen anderen durch ihre Wortstellung. Die freien Relativsätze haben kein externes Bezugselement und fallen unter den Skopus des Satztypoperators des minimalen Satzes, der sie enthält. Die restriktiven Relativsätze haben eine andere Wortstellung als V2-Relativsätze, können im Gegensatz zu freien und weiterführenden $w$-Relativsätzen ein nomimales Bezugselement haben, das aber im 12 Im Folgenden steht Int für einen interrogativen und Ass für einen assertiven Satztypoperator. 
Gegensatz zu appositiven und Cleft-Relativsätzen keinen singulären Term denotieren darf. Der Cleft-Relativsatz hat ein nominales Bezugselement, unterscheidet sich aber von allen anderen derartigen Relativsätzen, insofern dieses Bezugselement einen singulären Term denotieren darf und der Relativsatz gleichzeitig im Skopus des Satztypoperators seines Bezugselements liegt. Der appositive und der weiterführende $w$-Relativsatz teilen zwar die Skopuseigenschaften des V2-Relativsatzes, haben aber eine andere Wortstellung als dieser. Voneinander unterscheiden sie sich in der Existenz eines nominalen Bezugselements, so dass auch sie jeweils individuelle Eigenschaftsprofile aufweisen.

\begin{tabular}{|l|c|c|c|c|}
\hline & $\begin{array}{c}\text { Nominales } \\
\text { Bezugselement }\end{array}$ & $\begin{array}{c}\text { Singulärer } \\
\text { Term }\end{array}$ & $\begin{array}{c}\text { im Skopus des } \\
\text { Satztypoperators } \\
\text { des (semantischen) } \\
\text { Bezugselements }\end{array}$ & $\begin{array}{c}\text { Verbzweit- } \\
\text { Wortstellung }\end{array}$ \\
\hline Restriktiver RS & + & - & + & - \\
\hline Appositiver RS & + & + & - & - \\
\hline Cleft-RS & + & + & + & - \\
\hline V2-RS & + & - & - & + \\
\hline Freier RS & - & $\mathrm{n} / \mathrm{a}$ & + & - \\
\hline Weiterf. $w$-RS & - & $\mathrm{n} / \mathrm{a}$ & - & - \\
\hline
\end{tabular}

Tab. 2: Eigenschaften der deutschen Relativsatzkonstruktionen im Überblick

\subsection{Die grammatische Analyse der Relativsatzkonstruktionen}

Auf der Basis der vorgestellten empirischen Systematisierung der deutschen Relativsatzkonstruktionen kehren wir nun zurück zur theoretischen Hauptfrage, die uns in diesem Artikel beschäftigt: Gibt es im Deutschen phrasale Konstruktionen oder lassen sich im Einklang mit einem extremen Lexikalismus die Eigenschaften aller Phrasentypen des Deutschen auf natürliche Weise aus den Eigenschaften ihrer Kopfkonstituenten im Zusammenspiel mit allgemeinen Prinzipien ohne Bezug auf einzelsprachliche Konstruktionen ableiten?

Ein Relativsatz besteht im Deutschen im minimalen Fall aus zwei Wörtern, einem Relativpronomen und einem einstelligen Verb:

(das Haus) [RS das brennt]

Wenn die Eigenschaften des gesamten Relativsatzes aus den Eigenschaften seines Kopfes projiziert werden sollen, so gibt es nur drei logische Möglichkeiten für die Wahl eines solchen Kopfes: 
a. das Relativpronomen bzw. die Relativphrase

b. das Verb

c. ein drittes, oberflächensyntaktisch nicht realisiertes Element, z.B. ein unsichtbarer Relativkomplementierer.

Sollte sich keine der drei Optionen als sinnvoll erweisen, dann bleibt nur noch die konstruktionale Analyse. Im Folgenden werde ich zeigen, dass dies in der Tat die beste Lösung ist.

\subsubsection{Das Relativpronomen bzw. die Relativphrase als Projektor der Relativsatzeigenschaften}

Die Annahme, dass das Relativelement die Eigenschaften des gesamten Satzes projiziert, führt zu einer falschen Gruppierung der Relativsatztypen. Sie würde nämlich vorhersagen, dass sich einerseits die Sätze in (45a)-(48a) und andererseits die Beispiele in (45b)-(48b) gleich verhalten, denn die Sätze im jeweiligen Block enthalten dasselbe Relativelement, das nominale Relativpronomen die im ersten Fall und die ein Relativpronomen einbettende Präpositionalphrase an die im zweiten:

(45a) Die Frau, [die Gedanken lesen kann], kommt auch.

(46a) Die Frau, [die (übrigens) Gedanken lesen kann], kommt auch.

(47a) Es ist die Frau, [die Gedanken lesen kann].

(48a) Ich habe eine Frau kennengelernt, (/) [die kann Gedanken lesen].

(45b) Die Frau, [an die ich gerade denke], kommt auch.

(46b) Die Frau, [an die ich (übrigens) gerade denke], kommt auch.

(47b) Es ist die Frau, [an die ich gerade denke].

(48b) Ich habe eine Frau kennengelernt, (/) [an die denke ich gerade].

Unabhängig von der Kategorie des Relativelements verhalten sich die Sätze in (a) und (b) mit derselben Nummer identisch, zum Beispiel sind die beiden Sätze in (45) restriktive Relativsätze. Punkt a (vgl. Kapitel 2.3) sagt das Gegenteil voraus und ist somit nicht überzeugend. ${ }^{13}$

\subsubsection{Das finite Verb als Projektor der Relativsatzeigenschaften}

Ein ähnliches Argument zeigt, dass die distinktiven Eigenschaften der deutschen Relativsatzkonstruktionen ihren Ursprung auch nicht im finiten Verb

13 Stefan Müller hat mich darauf aufmerksam gemacht, dass das Relativelement in der Kategorialgrammatik als Kopf analysiert wird, siehe z.B. Morrill (1995, S. 204) und Steedman/ Baldridge (2006, S. 614). Pollard (1988, S. 409 ff.) und Müller (2010, S. 183 ff.) zeigen Schwierigkeiten für solche Analysen auf. Die minimalistische Analyse von freien Relativsätzen in Chomsky (2008), die ebenfalls dem Relativelement Kopfstatus zuerkennt, wird in Müller (2010, S. 94 ff.) als empirisch defizient kritisiert. 
haben können. Wie die folgenden Beispiele nachweisen, ist es keine allgemeine Eigenschaft von finiten Verben, einen Relativsatz projizieren zu müssen:

(49) Es schneit.

(50) Schneit es?

(51) Schneit es, dann machen wir den Ausflug nicht.

(52) Ich behaupte, dass es schneit.

(53) Ich frage mich, ob es mal wieder schneit.

(54) Obwohl es schneit, wollen wir einen Ausflug machen.

Statt um Relativsätze handelt es sich bei den obigen Beispielen jeweils um einen selbstständigen deklarativen (49), einen selbstständigen interrogativen (50), einen Konditional- (51), einen abhängigen deklarativen (52), einen abhängigen interrogativen (53) und um einen Adverbialsatz (54).

Will man um jeden Preis das finite Verb für die Eigenschaften der Relativsatzkonstruktionen verantwortlich machen, so ist dies technisch machbar. Man müsste dann angesichts der Befunde in Tabelle 2 allerdings sechs verschiedene, homophone Lexikoneinträge für jedes finite Verb annehmen, einen für jeden Relativsatz, und darüber hinaus weitere Einträge für Deklarativsätze, Interrogativsätze, Adverbialsätze, etc. Diese Annahme ist extrem künstlich, da man die Differenzen zwischen den Konstruktionen nicht an sichtbaren Unterschieden zwischen ihnen festmacht, sondern an systematisch homophonen Verben, wie die folgende Tabelle für Sätze mit dem Kopf stört zeigt:

\begin{tabular}{|l|c|c|c|c|c|}
\hline & $\begin{array}{c}\text { Nominales } \\
\text { Bezugselement }\end{array}$ & $\begin{array}{c}\text { Singulärer } \\
\text { Term }\end{array}$ & $\begin{array}{c}\text { im Skopus des } \\
\text { Satztypoperators } \\
\text { des (semantischen) } \\
\text { Bezugselements }\end{array}$ & V2 & Kopf \\
\hline Restriktiver RS & + & - & + & - & $\underline{\text { stört }}$ \\
\hline Appositiver RS & + & + & - & - & $\underline{\text { stört }}$ \\
\hline Cleft-RS & + & + & + & - & $\underline{\text { stört }}$ \\
\hline V2-RS & + & - & - & + & $\underline{\text { stört }}$ \\
\hline Freier RS & - & $\mathrm{n} / \mathrm{a}$ & + & - & $\underline{\text { stört }}$ \\
\hline Weiterf. $w$-RS & - & $\mathrm{n} / \mathrm{a}$ & - & - & $\underline{\text { stört }}$ \\
\hline
\end{tabular}

Tab. 3: Sechs verschiedene Relativsatzkonstruktionen mit sechs verschiedenen, aber homophonen Kopfkonstituenten

Da es willkürlich wäre, eine Obergrenze für die Anzahl von homophonen Lexikoneinträgen pro Grammatik zu setzen, sehe ich nicht, wie man die Hypothese widerlegen könnte, dass die sechs deutschen Relativsatzkonstruk- 
tionen von sechs verschiedenen, aber homophonen Verbeinträgen pro Verb projiziert werden. Allerdings sehe ich an der Theorie auch nichts Gewinnbringendes oder Überzeugendes! $!^{14}$

\subsubsection{Relativkomplementierer als Projektoren der Relativsatzeigenschaften}

Somit verbleibt aus der Liste der Möglichkeiten (vgl. Kapitel 2.3) nur noch die dritte lexikalische Option: die Annahme, dass deutsche Relativsätze unsichtbare Relativkomplementierer enthalten, die als Kopfkonstituenten die Eigenschaften der einzelnen Relativsatztypen via X-bar-Projektion determinieren. Als mögliches Motiv für diese Strategie mag wie bei Option b der Wunsch ausschlaggebend sein, in der Grammatiktheorie Konstruktionen um jeden Preis zu vermeiden. Gleichzeitig existiert in allen gegenwärtig gängigen Grammatik-Frameworks die technische Maschinerie zur kopfgetriebenen Phrasenprojektion und es hindert uns als GrammatikerInnen nichts daran, abstrakte Köpfe zu formulieren..$^{15}$ Angesichts der zum Beginn dieses Artikels skizzierten Entwicklung hin zu lexikalischen Lösungen und den Nachteilen der anderen beiden lexikalischen Ansätze (vgl. Kapitel 2.3) überrascht es nicht, dass sich in der Literatur Vorschläge für abstrakte Relativkomplementierer finden.

Brandt et al. (1999, S. 131) schlagen in ihrem Textbuch Analysen für restriktive und appositive Relativsätze im Deutschen vor, die von der folgenden Annahme ausgehen:

Je nach ,Vorwissens-Kontext ${ }^{\complement}[\ldots]$ darf ein und derselbe Satzkomplex [...] einmal als Beispiel für einschränkende, ein andermal für erläuternde Attribution gelten [...] Während restriktive Attribute wegen ihrer engen Korrespondenz mit D als Adjunkte an $\mathrm{D}^{1} \mathrm{zu}$ interpretieren sind, stellen wir erläuternde [...] als Adjunkte an $\mathrm{N}^{1}$ dar [...].

Für einen restriktiven Relativsatz geben die Autoren folgende Struktur, die einen unsichtbaren Relativkomplementierer enthält (ebd., S. 131):

14 Ich möchte darauf hinweisen, dass die Unplausibilität der Annahme, dass sich alle Eigenschaften der deutschen Relativsatzkonstruktionen aus ihren verbalen Köpfen projizieren lassen, keinen Anlass zu der Schlussfolgerung bietet, dass diese Sätze kopflose Phrasen sind und dass insbesondere die in ihnen obligatorisch vorkommenden finiten Verben diese Kopffunktion nicht wahrnehmen! Ein analoges Argument gilt für die Sätze in (49)-(54): Nur weil sich nicht alle Eigenschaften dieser Sätze aus ihrem Verb projizieren lassen, folgt daraus nicht, dass gar keine Eigenschaften des Satzes vom Verb kommen. Eine konstruktionale Analyse aller hier diskutierten Satztypen ist mit dem Kopfstatus des finiten Verbs kompatibel, solange Konstruktionen Eigenschaften der Mutterphrase determinieren können, die ihr Kopf unbeschränkt lässt!

15 Mit dem Vokabular, mit dem man phonologisch realisierte Lexikoneinträge schreiben kann, ist es genauso möglich, willkürlich viele Lexikoneinträge zu formulieren, die keinen sichtbaren Beitrag zur Satzphonologie leisten. Vergleiche die entsprechende Diskussion des Regelsystems in (1) am Beginn des Artikels. 


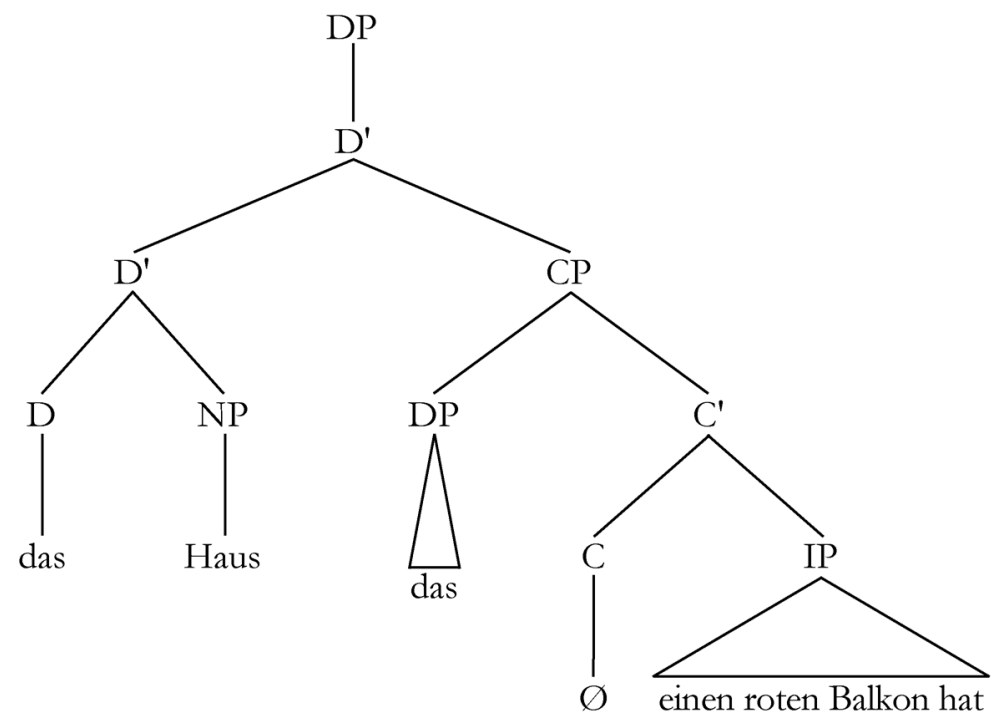

(55) Restriktive Relativsätze nach Brandt et al. (1999, S. 131)

Appositive Relativsätze haben einen anderen Adjunktionsort, enthalten allerdings ebenfalls einen leeren Relativkomplementierer (ebd.):

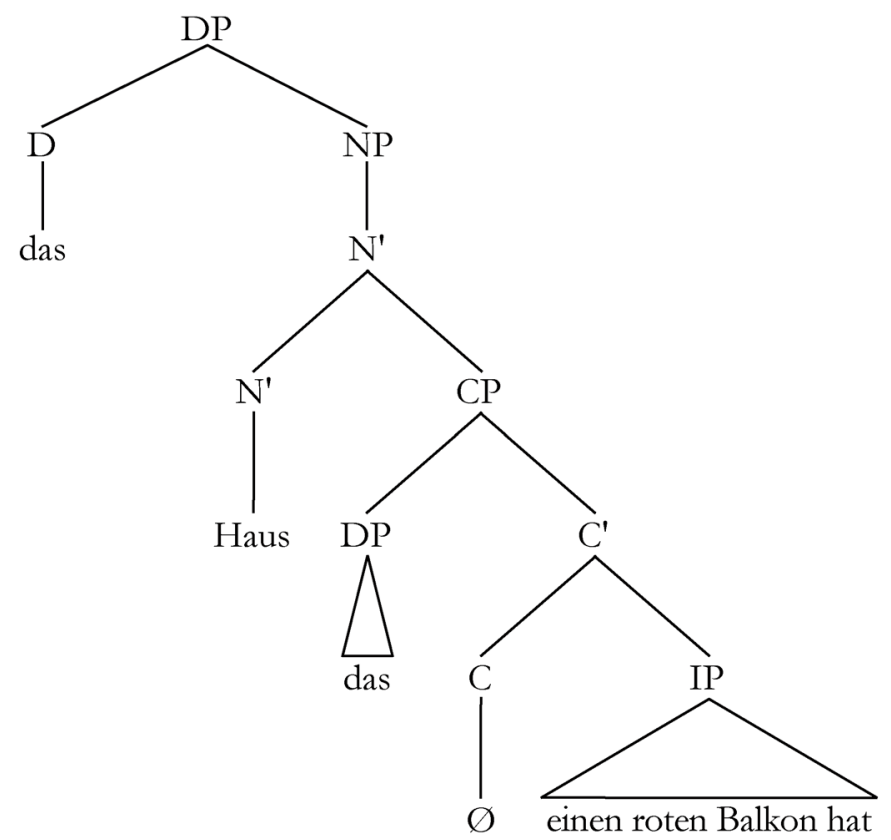

(56) Appositive Relativsätze nach Brandt et al. (1999, S. 131) 
Grewendorf/Poletto (1991, S. 200) machen im Zusammenhang mit ihrer Analyse von Cleft-Sätzen folgende Aussage:

Es ist klar, dass der identifizierende Nebensatz einer Cleft-Konstruktion im Deutschen nur die Form eines Relativsatzes hat, dass er in Wirklichkeit aber nicht als echter Relativsatz anzusehen ist, da er keinem der uns bekannten Typen von Relativsätzen entspricht.

Der Artikel enthält keinen expliziten Strukturbaum für deutsche CleftSätze, aus dem sich der Kopfstatus eindeutig entnehmen lässt. Im selben Zeitraum gibt Grewendorf (1988, S. 92) allerdings die Struktur in (57) für restriktive Relativsätze des Deutschen an, die einen unsichtbaren Relativkomplementierer enthält:

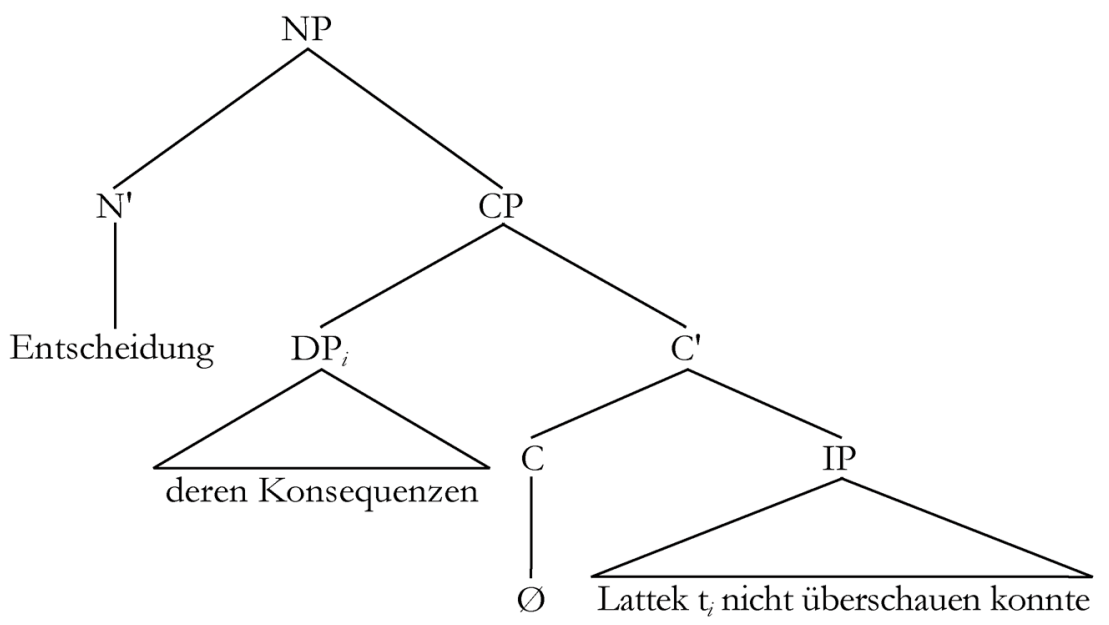

(57) Restriktive Relativsätze nach Grewendorf (1988, S. 92)

Angesichts der Aussage im oben genannten Zitat, dass Cleft-Sätze zwar keine echten Relativsätze sind, aber deren Form teilen, ist davon auszugehen, dass Grewendorf/Poletto (1991) auch für Cleft-Sätze einen leeren Komplementierer annahmen.

Gärtner (2001), das Hauptwerk zu V2-Relativsätzen des Deutschen, analysiert diese mit Hilfe eines unsichtbaren Komplementierers, wie folgt (ebd., S. 105): ${ }^{16}$

16 Gärtner bezeichnet die Konstruktion als „Integrated Verb Second Construction (IV2)“. Über die Ansetzung der nicht unabhängig motivierten unsichtbaren Kopfkategorie in seiner Analyse scheint der Autor selbst nicht völlig glücklich zu sein, denn er schreibt: ,the multiplication of empty heads is not fully satisfactory" (Gärtner 2001, S. 106). Die Kombination von syntaktischen, phonologischen und semantischen Eigenheiten, die Gärtner sorgfältig für V2Relativsätze etabliert, schreit geradezu nach einer konstruktionalen Analyse, die der Autor allein mit der Begründung vermeidet, dass ,,in line with the tenet of generative grammar [...] constructions should be regarded as epiphenomena [...]“ (ebd., S. 108). 


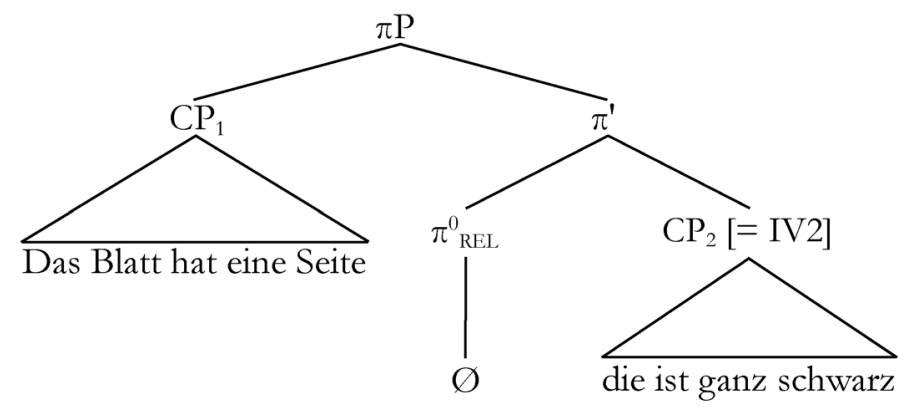

(58) V2-Relativsätze nach Gärtner (2001, S. 105)

Für freie Relativsätze im Deutschen postuliert Sternefeld (2006, S. 391) einen unsichtbaren Relativierer:

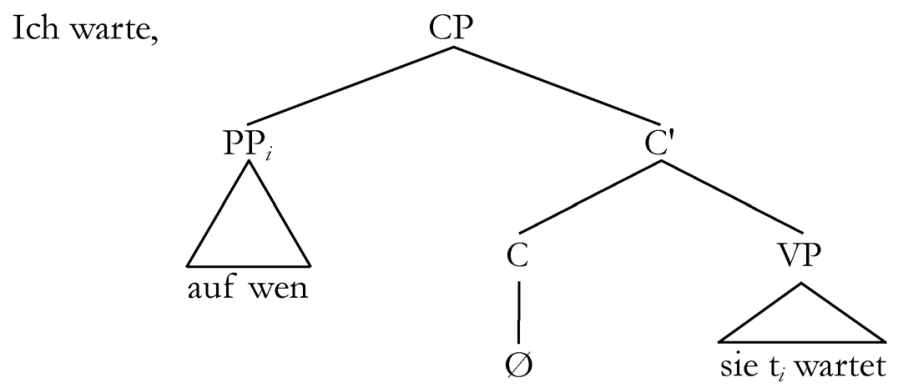

(59) Freie Relativsätze nach Sternefeld (2006, S. 391)

Auch für den letzten hier diskutierten Relativsatztyp - den weiterführenden $w$-Relativsatz - findet sich in der Literatur eine Analyse mit einem phonologisch leeren Komplementierer als Kopf, nämlich in Holler (2005, S. 293):

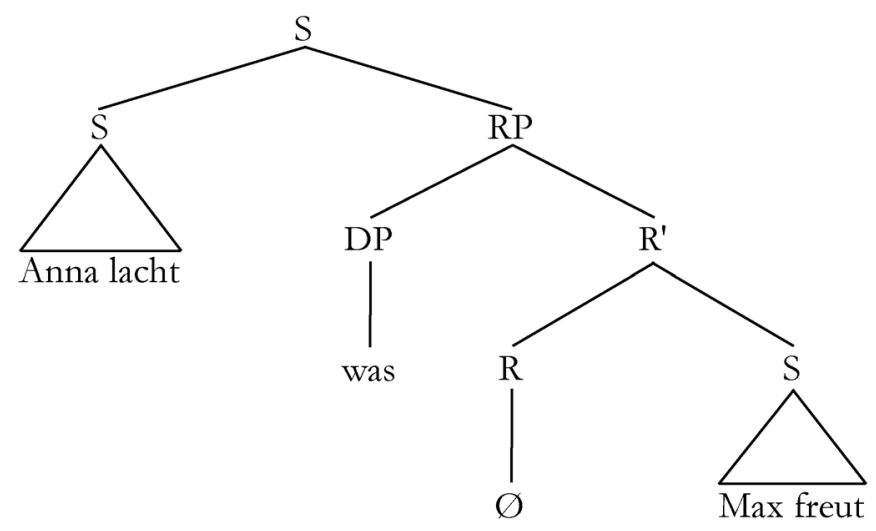

(60) Weiterführende $w$-Relativsätze nach Holler (2005, S. 293) 


\subsubsection{Bewertung der Relativkomplementierer-Hypothese}

Im letzten Abschnitt wurde gezeigt, dass für jeden Relativsatztyp des Deutschen eine lexikalische Analyse mit einem unsichtbaren Relativkomplementierer vorgeschlagen worden ist, um eine Relativsatzkonstruktion zu vermeiden.

Wir haben die anderen lexikalischen Optionen (vgl. Kapitel 2.3) schon als nicht überzeugend kritisiert und deshalb verworfen. Ich sehe nicht, dass die dritte Version eine Verbesserung gegenüber den ersten beiden darstellt. Zunächst erbt sie alle Probleme der Theorie, die versucht, die Eigenschaften der verschiedenen Relativsatztypen aus dem finiten Verb als Kopf zu projizieren, insbesondere die Unnatürlichkeit, die beobachtbaren Unterschiede zwischen den Relativsatztypen zu ignorieren und deren unterschiedliches Verhalten aus Kopfelementen zu projizieren, die sich selbst nicht sichtbar voneinander unterscheiden. Dass die sechs zu postulierenden Relativkomplementierer nicht nur homophon, sondern darüber hinaus ausnahmslos unsichtbar sein müssten, macht die Relativkomplementierer-Hypothese schließlich noch unüberzeugender als die Verbtheorie.

Die Situation, die wir hier vorfinden, ist in Culicover (1999, S. 6) in anderem Zusammenhang wie folgt diagnostiziert worden: „notice that what we have done here is simply to encode the superficial difference with an abstract but extensionally equivalent feature difference".

Ich teile die Meinung von Culicover, dass die Unterscheidung von phonologisch leeren Kopfelementen, die allein dazu dient, beobachtete Oberflächenunterschiede eins-zu-eins in einen abstrakten Grammatikformalismus zu übersetzen, keinen Erklärungsstatus sondern lediglich einen abstrakten Beschreibungsstatus besitzt.

\subsubsection{Weitere Probleme für die Homophonieansätze}

Wir haben bisher gezeigt, dass die Homophonieansätze - sowohl in der Verb- als auch der Komplementierer-Version - unmotiviert und unexplanatorisch sind, da sie sechs oberflächlich verschiedene Phänomene aus ebenso vielen Kopfkonstituenten ableiten wollen, die aber jeweils dieselbe Form haben. Es kommt allerdings noch schlimmer für diese Theorien. Bisher habe ich den Fokus auf die Beobachtung gelegt, dass jede der sechs Relativsatzkonstruktionen, die hier diskutiert werden, von den anderen unterschieden werden muss, was bei einem extremen lexikalischen Ansatz die Postulierung von sechs verschiedenen Köpfen für die Relativsätze erzwingt. Dies ist aber nur die eine Seite der Medaille, denn wie ich nun zeigen werde, haben die deutschen Relativsätze oder zumindest natürliche Klassen von deutschen Relativsätzen auch grammatische Eigenschaften gemein. Eine Grammatik, die dies nicht effektiv zu erfassen in der Lage ist, verliert linguistisch 
signifikante Generalisierungen über das Deutsche. Es wird deutlich werden, dass das vollständige Datenmuster die Homophonieansätze in ein Dilemma bringt: Um die distinktiven Eigenschaften der jeweiligen Konstruktionen zu erfassen, postulieren sie sechs verschiedene Kopfelemente - aber dann ist es ein purer Zufall, dass alle oder einige dieser Köpfe bestimmte grammatische Eigenschaften gemeinsam haben!

Beginnen wir mit der Konstituentenstruktur: Unabhängig von den Unterschieden zwischen ihnen haben alle deutschen Relativsätze zwei Tochterkonstituenten, die darüber hinaus über alle Relativsatzkonstruktionen hinweg die folgenden Beschränkungen erfüllen:

a. Die linke Tochter muss ein Relativpronomen enthalten.

b. Die rechte Tochter muss ein saturierter finiter Satz sein.

In dem Bestreben, dem offensichtlichen analytischen Offenbarungseid durch die Hinzufügung von identischen Beschränkungen zu den sechs verschiedenen hypothetischen Kopfkonstituenten für deutsche Relativsätze zu entgehen, könnte die lexikalische Theorie Hilfe von der Universalgrammatik erhoffen: Wären die beiden Beschränkungen universal, dann wären sie motiviert.

Diese Hoffnung wird allerdings allein schon durch das Englische zerstört, das Relativkonstruktionen besitzt, in denen die Beschränkungen a und b verletzt werden. So zeigt Sag (1997, S. 468), dass so genannte „Bare Relative Clauses" ihrem Namen entsprechend kein Relativpronomen in der linken Peripherie enthalten und somit a als Universal nicht in Frage kommt:

(61) Everything ${ }_{\text {RS }} \varnothing$ you like] is on the table.

Die Bedingung in $\mathrm{b}$ wird durch nicht-finite $W h$-Relativsätze des Englischen falsifiziert. (62) enthält ein Beispiel aus Sag (1997): ${ }^{17}$

17 Der Satztyp in (62) zeigt noch andere interessante Eigenheiten, z.B. muss das Relativelement eine Präpositionalphrase sein, was u.a. den Standardfall eines nominalen Relativelements ausschließt:

(i) * the baker [Rs who to buy bread from]

(ii) * the baker [Rs whose bread to buy]

Diese Beschränkung ist konstruktionsspezifisch und keine allgemeine Eigenschaft von $W_{b}$ Infinitiven im Englischen. So lassen nicht-finite Interrogativsätze sowohl NPs als auch PPs als „Filler" zu:

(iii) I wonder $\left[_{\mathrm{RS}}\right.$ in whom to place my trust] now.

(iv) I wonder [ ${ }_{\mathrm{RS}}$ who to place my trust in] now.

Analog zur Diskussion des Deutschen im vorliegenden Artikel zeigt Sag (1997), dass es im Englischen einerseits Eigenschaften gibt, die individuellen Relativsatzkonstruktionen zukommen und andererseits Eigenschaften, die von mehreren Relativsatzkonstruktionen geteilt werden. Eine reine lexikalische Analyse ist daher für englische Relativsätze ebenso wenig überzeugend wie für deutsche. 
(62) the baker [RS in whom to place your trust]

Über die Konstituentenstruktur hinaus haben die deutschen Relativsätze andere Eigenschaften gemeinsam. So findet sich in allen Relativsatztypen der Rattenfängereffekt mit Nominalphrasen und Präpositionalphrasen:

Restriktive Relativsätze

(63) Das Auto, [ ${ }_{\mathrm{NP}}$ dessen Windschutzscheibe] ich gerade gereinigt habe, gehört Petra.

(64) Das Auto, [pp an dem] ich interessiert bin, gehört Petra.

Appositive Relativsätze

(65) Das Auto, [NP dessen Windschutzscheibe] ich übrigens gerade gereinigt habe, gehört Petra.

(66) Das Auto, [pp an dem] ich übrigens interessiert bin, gehört Petra.

Cleft-Relativsätze

(67) Es ist das Auto, [ ${ }_{N P}$ dessen Windschutzscheibe] ich gerade gereinigt habe.

(68) Es ist das Auto, [PP an dem] ich interessiert bin.

V2-Relativsätze

(69) Ich hatte mal ein Auto, [ ${ }_{N P}$ dessen Windschutzscheibe] war getönt.

(70) Ich hatte mal ein Auto, [pp um das] haben mich alle beneidet.

Freie Relativsätze

(71) [NP Wessen Birne] noch halbwegs in der Fassung steckt, pflegt solcherlei Erloschene zu meiden; schließlich ist die eigene Intaktheit sehr fragil. ${ }^{18}$

(72) Ich warte [pp auf wen] sie wartet. ${ }^{19}$

Weiterführende $w$-Relativsätze

(73) Hans bat Anna, den Fußboden zu putzen, [NP welche Bitte] sie entschieden ablehnte. ${ }^{20}$

(74) Die Elbe hat Hochwasser, [ ${ }_{\mathrm{PP}}$ aus welchem Grund] die meisten StraBen gesperrt sind.

Weiterhin haben alle deutschen Relativsätze gemeinsam, dass sie Extraktionsinseln bilden. Nach von Stechow/Sternefeld (1988, S. 371), die sich auf eine empirische Studie von Anderson/Kvam (1984) beziehen, wird im Deutschen die lange Bewegung von Nicht-Argumenten viel häufiger akzeptiert als die von Argumenten, und zwar auch von Sprechern des Standarddeutschen. Dies erklärt, warum (75) akzeptabler ist als (76):

\footnotetext{
Zitiert aus Müller (1999b, S. 134).

Zitiert aus Sternefeld (2006, S. 391).

20 (73) und (74) zitiert aus Holler (2005, S. 101).
} 
(75) [Auf diesem Gebiet $]_{i}$ wünsche ich nicht, dass dies $t_{i}$ geschieht.

(76) *[Auf diesen Namen $]_{i}$ glaube ich schon, dass ich $t_{i}$ gestoßen bin.

Wenn man nun allerdings versucht, ein Nicht-Argument aus einem der sechs Relativsatztypen des Deutschen zu extrahieren, die in diesem Artikel diskutiert werden, dann erhält man in jedem Falle einen ungrammatischen Satz:

(77) *[Auf diesem Gebiet $]_{i}$ kenne ich keinen Linguisten, der $t_{i}$ gearbeitet hat.

(78) *[Auf diesem Gebiet $]_{i}$ kenne ich den Linguisten Chomsky, der $t_{i}$ gearbeitet hat.

(79) *[Auf diesem Gebiet $]_{\mathrm{i}}$ kenne ich einen Linguisten, (/) der hat $\mathrm{t}_{\mathrm{i}}$ gearbeitet.

(80) *[Auf diesem Gebiet $]_{\mathrm{i}}$ ist es der Linguist, der $\mathrm{t}_{\mathrm{i}}$ gearbeitet hat.

(81) *[Auf diesem Gebiet $]_{i}$ will ich, was bisher $t_{i}$ veröffentlicht worden ist, lesen.

(82) *[Auf diesem Gebiet $]_{\mathrm{i}}$ sollten alle Prüfungen auf Chinesisch stattfinden, welcher Vorschlag $t_{i}$ noch nicht gemacht worden ist.

Als nächste konstruktionsumfassende Eigenschaft der deutschen Relativsätze ist zu nennen, dass sie alle im Nachfeld stehen können:

(83) Gestern habe ich eine Frau kennengelernt, [die Gedanken lesen kann].

(84) Gestern habe ich Petra Müller kennengelernt, [die übrigens Gedanken lesen kann].

(85) Es ist die Frau gewesen, [die Gedanken lesen kann].

(86) Ich habe eine Frau kennengelernt, (/) [die kann Gedanken lesen].

(87) Ich habe schon erledigt, [was man mir aufgetragen hat].

(88) Petra kennt Johanna, [was mich (übrigens) überrascht].

Die Verschiebung des Relativsatzes ins Vorfeld ohne sein Bezugselement ist dagegen verboten. Daher können nur die freien Relativsätze, die ja kein Bezugselement besitzen, allein im Vorfeld stehen:

(89) *[Die Gedanken lesen kann $]_{i}$, habe ich gestern eine Frau $t_{i}$ kennengelernt.

(90) *[Die übrigens Gedanken lesen kann], habe ich gestern Petra Müller $\mathrm{t}_{\mathrm{i}}$ kennengelernt.

(91) *[Die Gedanken lesen kann $]_{\mathrm{i}}$ ist es die Frau gewesen, $\mathrm{t}_{\mathrm{i}}$.

(92) *[Die kann Gedanken lesen], habe ich eine Frau kennengelernt, $(/) t_{\mathrm{i}}$.

(93) [Was man mir aufgetragen $]_{i}$ hat, habe ich schon $t_{i}$ erledigt.

(94) *[Was mich (übrigens) überrascht $]_{i}$, kennt Petra Johanna, $t_{i}$.

Wir haben bisher dokumentiert, dass es Eigenschaftsbündel gibt, die im Deutschen sechs verschiedene Relativsatztypen voneinander unterscheiden, 
dass all diese Relativsatztypen allerdings auch Eigenschaften gemeinsam haben. Nun zeige ich schließlich noch, dass es auch Generalisierungen auf der mittleren Ebene gibt, d.h. Eigenschaften, die von mehr als einem aber nicht von allen Relativsätzen geteilt werden.

Eine solche Eigenschaft findet sich in der Kongruenz: Wie die folgenden Sätze zeigen, kongruieren diejenigen $d$-Relativpronomen, die ein Bezugsnomen haben, mit diesem im grammatischen Numerus:

(95) Der Junge $\mathrm{sg}_{\mathrm{sg}}$, $\left[\mathrm{den}_{\mathrm{sg}} / * \operatorname{die}_{\mathrm{pl}}\right.$ du kennst], heißt Peter.

(96) Der Junge ${ }_{\mathrm{sg}},\left[\mathrm{den}_{\mathrm{sg}} / *\right.$ die $_{\mathrm{pl}}$ du übrigens kennst], heißt Peter.

(97) Es ist der Junge ${ }_{\mathrm{sg}},\left[\mathrm{den}_{\mathrm{sg}} / * \mathrm{die}_{\mathrm{pl}}\right.$ du kennst].

(98) Ich kenne einen Jungen ${ }_{\mathrm{sg}},\left[\mathrm{den}_{\mathrm{sg}} / *\right.$ die $_{\mathrm{pl}}$ kennst du auch].

In Bezug auf das grammatische Genus scheint eine andere Bedingung zu gelten. Es ist bekannt, dass anaphorische Pronomina sich satzübergreifend auf das Genus oder den Sexus des Bezugsnomens bzw. dessen Denotat beziehen können:

(99) Schau mal, das kleine Mädchen neut $! \mathrm{Es}_{\text {neut }} / \mathrm{Sie}_{\text {fem }}$ heißt Lucy.

Dasselbe scheint für den V2-Relativsatz zu gelten:

(100) Ich kenne ein Mädchen $n_{\text {neut }}$ [ das $_{\text {neut }} /$ die $_{\text {fem }}$ heißt Lucy].

In allen anderen Relativsätzen muss das $d$-Relativpronomen, das ein Bezugsnomen hat, sich aber auf das grammatische Genus beziehen: ${ }^{21}$

(101) Das Mädchen neut , $\left[\mathrm{das}_{\text {neut }} / * \mathrm{die}_{\text {fem }}\right.$ neben mir saß], heißt Maria.

(102) Das Mädchen neut $_{\text {, }}\left[\mathrm{das}_{\text {neut }} / * \operatorname{die}_{\text {fem }}\right.$ übrigens neben mir saß], heißt Maria.

(103) Es ist das Mädchen $n_{\text {neut }},\left[\mathrm{das}_{\text {neut }} / * \mathrm{die}_{\mathrm{fem}}\right.$ neben mir saß].

Die Satztypen in (101)-(103) bilden eine natürliche Klasse: Sie sind alle Verb-letzt-Relativsätze mit einem $d$-Relativpronomen. Für diese Klasse kann eine entsprechende Kongruenzregel für das grammatische Genus formuliert werden.

\section{Bilanz der Untersuchung und theoretische Bewertung}

Bevor wir eine Bilanz ziehen, erinnere ich nochmals an die theoretische Frage, die die Jahrestagung „Sprachliches Wissen zwischen Lexikon und Grammatik" bestimmte und die hier vorgelegte Studie zu den deutschen Relativsatztypen motivierte (vgl. Kapitel 1):

$21 \quad$ Müller (1999a, S. 417 f.) zitiert Beispiele, in denen keine Genuskongruenz vorliegt, z.B. „Es ist ein junges Mädchen, die auf der Suche nach CDs bei Bolzes reinschaut“" (taz, 13.03.1996, S. 11). Diese sind nach meinem Urteil inakzeptabel, weisen aber möglicherweise auf die Existenz von dialektaler und/oder ideolektaler Variation in diesem grammatischen Bereich hin. 
[Wir sehen dort,] wo wir früher eine strikte Grenze postulierten zwischen der Grammatik als dem Ort des syntaktisch-semantisch Regelhaften und dem Lexikon als dem Repositorium des syntaktisch-semantisch Idiosynkratischen, mittlerweile ein immer breiter werdendes Feld von semiproduktiven Prozessen, graduellen Kategoriezuordnungen, frequenzgesteuerten Usualisierungen eigentlich regelhafter Strukturen, und fein differenzierten, nur schwer generalisierbaren Konstruktionsmustern. Das strikt Regelhafte und das rein Idiosynkratische drohen daneben nur noch als Randbereiche des sprachlichen Wissens zu erscheinen.

Anhand der deutschen Relativsätze wollten wir testen, ob es auch im Deutschen die oben beschriebene Situation gibt und wie am besten mit ihr umzugehen ist.

Unsere Untersuchung hat folgende Ergebnisse erbracht:

1) Es gibt im Deutschen mindestens sechs Relativsatzkonstruktionen, die sich durch ihre grammatischen Eigenschaften voneinander unterscheiden.

2) Die Relativphrase ist kein plausibler Kopf der Konstruktion, weil diese Annahme z.B. inkorrekt vorhersagt, dass sich alle Relativsätze mit einer NP-Relativphrase identisch verhalten sollten.

3) Das finite Verb oder ein abstrakter Komplementierer bilden ebenfalls keine überzeugenden Kopfkonstituenten, aus denen alle Eigenschaften der verschiedenen Relativsätze projiziert werden können (Homophonieproblem, Atomismusproblem).

Als Fazit ist zu ziehen, dass der Versuch, die Eigenschaften deutscher Relativsätze aus ihren lexikalischen Köpfen zu projizieren, weder gewinnbringend noch überzeugend ist!

Ein zweites Fazit lautet, dass ein konstruktionsbasierter Ansatz zu deutschen Relativsätzen nicht den unnatürlichen und stipulativen Charakter der lexikalischen Theorie teilt. Mit seinen ,intelligenten“ Werkzeugen, d.h. Typen, Untertypen und Vererbung, ist es möglich, Relativsatzeigenschaften auf mehreren Ebenen gleichzeitig zu erfassen: Auf der Ebene aller deutschen Relativsätze, der Ebene der einzelnen Relativsatztypen und der Ebene dazwischen, d.h. natürliche Klassen von Relativsätzen. ${ }^{22}$

Dem deutschen Satztyp Relativsatz können mit diesen Mitteln alle folgenden grammatischen Eigenschaften zugeschrieben werden:

22 Durch die Anwendung von Vererbung könnte der lexikalische Ansatz im Prinzip weniger stipulativ gemacht werden. Der Ansatz würde dadurch aber konzeptuell nicht weniger künstlich, denn es bleibt dabei, dass er beobachtbare Unterschiede von Phrasen eins zu eins in Kopfelemente „rückprojiziert“, die sich nicht auf beobachtbare Weise voneinander unterscheiden und allein zu dem Zweck postuliert werden, Konstruktionen zu vermeiden. Die lexikalische Theorie würde daher eine eklatante Verletzung des fundamentalen wissenschaftstheoretischen Prinzips von Ockhams Rasiermesser darstellen. 
- Die linke Tochter muss ein Relativpronomen enthalten.

- Die rechte Tochter muss ein saturierter finiter Satz sein.

- Er zeigt einen Rattenfängereffekt.

- Er ist eine Extraktionsinsel.

- Er kann im Nachfeld stehen.

Der Untertyp Nicht-Argument-Relativsatz erbt die oben genannten Eigenschaften und unterliegt zusätzlich der folgenden Beschränkung:

- Er kann nicht ohne sein Bezugselement im Vorfeld stehen.

Der Untertyp $d$-Relativsatz, wird durch eine zusätzliche Kongruenzeigenschaft beschränkt:

- Das Relativpronomen kongruiert mit seinem Bezugsnomen im grammatischen Numerus.

Verb-letzt-d-Relativsatz ist gleichzeitig Untertyp von Nicht-Argument-Relativsatz und von d-Relativsatz. Außer den Eigenschaften, die er von diesen Satztypen (und deren Obertyp Relativsatz) erbt, erfüllt er eine weitere Kongruenzbedingung:

- Das Relativpronomen kongruiert mit seinem Bezugsnomen im grammatischen Genus.

Die hier dargelegte konstruktionale Analyse ist konkret und hocheffizient: Sie kommt ohne systematische Homophonie und unsichtbare lexikalische Köpfe aus und ist dennoch bzw. gerade deshalb in der Lage, die empirischen Eigenschaften der deutschen Relativsatztypen auf jeder Generalisierungsebene mit einer einzigen Beschränkungsformulierung zu erfassen.

Im Gegensatz zur konstruktionalen Analyse sind die lexikalischen Theoriealternativen unmotiviert und ad-hoc: Für jeden neu diskutierten Relativsatztyp stipulieren sie einen neuen lexikalischen Kopf mit neuen grammatischen Eigenschaften. Dass der neue Kopf systematisch homophon mit den schon vorher postulierten ist, bleibt reiner Zufall. Zudem müssen die Eigenschaften, die allen deutschen Relativsätzen oder natürlichen Klassen gemeinsam sind, mehrfach formuliert werden, für jeden der hypothetischen Köpfe einzeln.

\section{Zusammenfassung}

Wir fassen zusammen: Wie die traditionelle Grammatik war die frühe generative Syntaxtheorie konstruktional, indem sie Phrasenstrukturregeln und Transformationsregeln für einzelne Konstruktionen formulierte. Beginnend mit Chomskys „Remarks on Nominalization“ (Chomsky 1970) wurden 
zunehmend Eigenschaften der Phrasenstruktur aus Phrasenstrukturregeln ins Lexikon verlagert und mit Hilfe der X-bar-Theorie in die Syntax projiziert. Ähnliche Entwicklungen gab es in der Folge von Ross (1967) im Bereich der Transformationsregeln. Das Projektionsprinzip in Chomskys „Lectures on Government and Binding“ (Chomsky 1981) sowie die Entstehung stark lexikalisch geprägter Grammatiktheorien wie der LFG und der HPSG entwickelten die Lexikalisierung der Syntax weiter, so dass Mitte der achtziger Jahre in der Generativen Grammatik ein theoretischer Konsens darüber bestand, dass die interne Struktur von nicht-funktionalen Phrasenkategorien aus den Argumentstrukturen ihrer Kopfelemente projiziert wird. Chomsky formulierte die extreme Hypothese, dass es weder Konstruktionen noch konstruktionsspezifische Regeln gibt. Zur Erlangung deskriptiver Adäquatheit ist die extreme lexikalistische Version, die die Prinzipien- und Parametertheorie (Chomsky 1981) und den Minimalismus (Chomsky 1995a) bestimmen, allerdings auf zusätzliche theorieintern postulierte lexikalische Kopfkategorien angewiesen, deren Zahl seit Ende der achtziger Jahre prinzipienlos anwächst und gegenwärtig unplausibel im dreistelligen Bereich liegt, ohne dass ein Ende des Wachstums absehbar ist.

Gegen Ende der achtziger Jahre entsteht an der amerikanischen Westküste eine konstruktionale Gegenbewegung zum extremen Lexikalismus (Fillmore/Kay/O'Connor 1988 und Kay/Fillmore 1999). Diese „Berkeley Construction Grammar" sieht sich nicht in der Opposition zum Lexikalismus an sich, sondern zu einem unausgewogenen Lexikalismus, der im Gegensatz zur traditionellen Grammatik und der frühen Generativen Grammatik per Definition keinen Raum mehr für Konstruktionen lässt, stattdessen aber grenzenlos lexikalische Homophonie stipuliert.

Vor dem Hintergrund dieser fachgeschichtlichen Entwicklung und der theoretischen Kontroverse um die richtige Grenzziehung zwischen lexikalischen und konstruktionalen Werkzeugen habe ich in diesem Artikel auf der Basis einer Systematisierung der Eigenschaften einiger deutscher Relativsatztypen demonstriert, dass eine rein lexikalische Analyse deutscher Relativsätze stipulativ und unüberzeugend ist: Mit jedem neuen Relativsatztyp muss ein neuer homophoner Kopf postuliert werden, nur um schließlich die Generalisierungen über alle oder natürliche Klassen von Relativsatztypen Kopf für Kopf formulieren zu müssen. An der lexikalischen Theorie ist nichts elegant oder explanativ: Die empirischen Fakten treiben die Theorie vor sich her und zwingen sie dazu, die Anzahl unsichtbarer lexikalischer Kategorien und abstrakter Ableitungsmechanismen prinzipienlos zu erweitern.

Ein konstruktionaler Ansatz ist dagegen in der Lage, die Eigenschaften deutscher Relativsätze auf allen notwendigen Ebenen elegant zu erfassen: (i) alle deutsche Relativsätze, (ii) natürliche Klassen von Relativsätzen und (iii) die sechs Einzelkonstruktionen. Die skizzierte Theorie ist gleichzeitig 
vollständig kompatibel mit der Projektion von Phrasen aus den Argumentstrukturen von lexikalischen Köpfen und realisiert somit die plausible theoretische Mittelposition der „Berkeley Construction Grammar“, die die Grammatik auf zwei ebenbürtige Pfeiler stellt: lexikalische Köpfe und phrasale Konstruktionen.

\section{Literatur}

Anderson, Sven-Gunnar/Kvam, Sigmund (1984): Satzverschränkung im heutigen Deutsch: eine syntaktische und funktionale Studie unter Berücksichtigung alternativer Konstruktionen . (= Studien zur deutschen Grammatik 24). Tübingen.

Baker, Mark (1996): The polysynthesis parameter. New York u.a.

Bechert, Johannes et al. (1970): Einführung in die generative Transformationsgrammatik: ein Lehrbuch. (= Linguistische Reihe 2). München.

Brandt, Patrick et al. (1999): Sprachwissenschaft. Ein roter Faden für das Studium. Köln/Weimar/Wien.

Bresnan, Joan (1982): The passive in lexical theory. In: Bresnan (Hg.), S. 3-86.

Bresnan, Joan (Hg.) (1982): The mental representation of grammatical relations. Cambridge, MA.

Bresnan, Joan (2001): Lexical functional syntax. (= Blackwell Textbooks in Linguistics 16). Malden.

Chomsky, Noam (1965): Aspects of the theory of syntax. 2. Aufl. Cambridge, MA.

Chomsky, Noam (1970): Remarks on nominalization. In: Jacobs, Roderick A./Rosenbaum, Peter S. (Hg.): Readings in English Transformational Grammar. Waltham, MA, S. 184-221.

Chomsky, Noam (1981): Lectures on government and binding. (= Studies in Generative Grammar 9). Dordrecht u.a.

Chomsky, Noam (1986): Barriers. (= Linguistic Inquiry Monographs 13). Cambridge, MA u.a.

Chomsky, Noam (1993): A minimalist program for linguistic theory. In: Hale, Kenneth/ Keyser, Samuel Jay (Hg.): The view from building 20. Essays in linguistics in honor of Sylvain Bromberger. (= Current Studies in Linguistics Series 24). Cambridge, MA, S. 1-52.

Chomsky, Noam (1995a): The minimalist program. (= Current Studies in Linguistics Series 28). Cambridge, MA u.a.

Chomsky, Noam (1995b): Bare phrase structure. In: Webelhuth, Gert (Hg.): Government and binding theory and the minimalist program: principles and parameters in syntactic theory. (= Generative Syntax 1). Oxford u.a., S. 383-439.

Chomsky, Noam (2007): Biolinguistic explorations: design, development, evolution. In: International Journal of Philosophical Studies 15, 1, S. 1-21. 
Chomsky, Noam (2008): On phases. In: Freidin, Robert/Otero, Carlos P./Zubizarreta, Maria Luisa (Hg.): Foundational issues in linguistic theory. Essays in honor of JeanRoger Vergnaud. Cambridge, MA u.a., S. 133-166.

Cinque, Guglielmo (1999): Adverbs and functional heads: a cross-linguistic perspective. New York u.a.

Cinque, Guglielmo/Rizzi, Luigi (2010): The cartography of syntactic structures. In: Heine, Bernd/Narrog, Heiko (Hg.): The Oxford handbook of linguistic analysis. Oxford u.a., S. 51-65.

Culicover, Peter (1999): Syntactic nuts: hard cases, syntactic theory, and language acquisition. (= Foundations of Syntax 1). Oxford u.a.

Di Sciullo, Anna Maria/Williams, Edwin (1987): On the definition of word. (= Linguistic Inquiry Monographs 14). Cambridge, MA u.a.

Fillmore, Charles J. (1999): Inversion and constructional inheritance. In: Webelhuth, Gert/Koenig, Jean-Pierre/Kathol, Andreas (Hg.): Lexical and constructional aspects of linguistic explanation. Stanford, S. 113-128.

Fillmore, Charles J./Kay, Paul/O'Connor, Mary Katherine (1988): Regularity and idiomaticity in grammatical constructions: the case of let alone. In: Language 64, S. $501-538$.

Gärtner, Hans-Martin (2001): Are there V2 relative clauses in German? In: The Journal of Comparative Germanic Linguistics 3, S. 97-141.

Ginzburg, Jonathan/Sag, Ivan (2000): Interrogative investigations: the form, meaning, and use of English interrogatives. Stanford.

Grewendorf, Günther (1988): Aspekte der deutschen Syntax: eine Rektions-BindungsAnalyse. (= Studien zur deutschen Grammatik 33). Tübingen.

Grewendorf, Günther/Poletto, Cecilia (1991): Die Cleft-Konstruktion im Deutschen, Englischen und Italienischen. In: Fanselow, Gisbert/Felix, Sascha (Hg.): Strukturen und Merkmale syntaktischer Kategorien. (= Studien zur deutschen Grammatik 39). Tübingen, S. 174-216.

Harris, Zellig (1951): Methods in structural linguistics. Chicago.

Höhle, Tilman N. (1978): Lexikalistische Syntax: Die Aktiv-Passiv-Relation und andere Infinitkonstruktionen im Deutschen. (= Linguistische Arbeiten 67). Tübingen.

Holler, Anke (2005): Weiterführende Relativsätze. Empirische und theoretische Aspekte. (= Studia grammatica 60$)$. Berlin.

Iatridou, Sabine (1990): About AgrP. In: Linguistic Inquiry 21, S. 551-577.

Jackendoff, Ray S. (1977): X-bar syntax: a study of phrase structure. (= Linguistic Inquiry Monographs 2). Cambridge, MA.

Kay, Paul/Fillmore, Charles J. (1999): Grammatical constructions and linguistic generalizations: the What's $X$ doing $Y$ ? construction. In: Language 75, S. 1-33.

Kornai, András/Pullum, Geoffrey K. (1990): The X-bar theory of phrase structure. In: Language 66, S. 24-50. 
Morrill, Glyn (1995): Discontinuity in categorial grammar. In: Linguistics and Philosophy 18,2 , S. 175-219.

Müller, Stefan (1999a): Deutsche Syntax deklarativ. Head-Driven Phrase Structure Grammar für das Deutsche. (= Linguistische Arbeiten 394). Tübingen.

Müller, Stefan (1999b): An HPSG-analysis of free relative clauses in German. In: Grammars 2, 1, S. 53-105.

Müller, Stefan (2010): Grammatiktheorie: Von der Transformationsgrammatik zur beschränkungsbasierten Grammatik. (= Stauffenburg Einführungen 20). Tübingen.

Newmeyer, Frederick J. (2004): Against a parameter-setting approach to language variation. In: Linguistic Variation Yearbook 4, S. 181-234.

Pollard, Carl J. (1988): Categorial grammar and phrase structure grammar: an excursion on the syntax-semantics frontier. In: Oehrle, Richard/Bach, Emmon/Wheeler, Deirdre (Hg.): Categorial grammars and natural language structures. (= Studies in Linguistics and Philosophy 32). Dordrecht u.a., S. 391-415.

Pollard, Carl J./Sag, Ivan A. (1994): Head-driven phrase structure grammar. Stanford.

Pollock, Jean-Yves (1989): Verb movement, universal grammar, and the structure of IP. In: Linguistic Inquiry 20, S. 365-424.

Ross, John Robert (1967): Constraints on variables in syntax. Diss., Massachusetts Institute of Technology. [Veröffentlicht als: Ross, John Robert (1986): Infinite syn$\operatorname{tax}$ ! Norwood.]

Sag, Ivan A. (1997): English relative clause constructions. In: Journal of Linguistics 33, 2, S. 431-484.

Steedman, Mark J./Baldridge, Jason (2006): Combinatory categorial grammar. In: Brown, Keith (Hg.): The encyclopedia of language and linguistics. 2. Aufl. Amsterdam u.a., S. 610-621.

Stechow, Arnim von/Sternefeld, Wolfgang (1988): Bausteine syntaktischen Wissens. Ein Lehrbuch der generativen Grammatik. Opladen.

Sternefeld, Wolfgang (2006): Syntax. Eine morphologisch motivierte generative Beschreibung des Deutschen. (= Stauffenburg Linguistik 31). Tübingen. 



\title{
Keine Grammatik ohne Konstruktionen: Ein logisch-ökonomisches Argument für die Konstruktionsgrammatik ${ }^{1}$
}

\begin{abstract}
Es ist unbestritten, dass in allen natürlichen Sprachen nicht-kompositionelle FormBedeutungspaare verschiedener Komplexitäts- und Abstraktionsgrade existieren. Uneinigkeit besteht dagegen bezüglich der Frage, ob diese Form-Bedeutungspaare als Teil der Grammatik oder gar, wie in der Konstruktionsgrammatik postuliert, als grundsätzliches grammatisches Organisationsprinzip zu verstehen sind. In meinem Beitrag argumentiere ich für eine zentrale Rolle von Konstruktionen bei der Repräsentation sprachlichen Wissens: Da Menschen offensichtlich in der Lage sind, Konstruktionen (im oben genannten Sinne) zu erwerben und zu verarbeiten, muss ein entsprechendes, konstruktionsverarbeitendes System existieren. Dieses kann auch nicht-idiomatische (regelhafte) Strukturen verarbeiten. Umgekehrt kann aber ein regelverarbeitendes System nicht ohne Weiteres idiomatische Strukturen verarbeiten. Das Sparsamkeitsprinzip sagt uns, dass eine Grammatik mit nur einem System einer mit zwei Systemen vorzuziehen ist, wenn es keine zwingenden Gründe für die Annahme eines zweiten Systems gibt. Neben diesem logisch-ökonomischen Argument diskutiere ich abschließend die Möglichkeit, anhand der systematischen Verletzung scheinbar allgemeingültiger grammatischer Regeln die Plausibilität eines konstruktionsverarbeitenden Systems empirisch zu untermauern.
\end{abstract}

\section{Einleitung}

Jede grammatiktheoretische Strömung innerhalb der modernen Sprachwissenschaft erkennt die Existenz von nicht-kompositionellen Form-Bedeutungspaaren mit verschiedenen Komplexitäts- und Abstraktionsgraden in allen natürlichen Sprachen an. Diese Form-Bedeutungspaare werden unter einer Vielzahl von Begriffen wie „Phraseologismus“, „Phrasem“, „Phraseolexem“, „festes Syntagma“, „(konstruktionelles) Idiom“, „Formel“, „Flos-

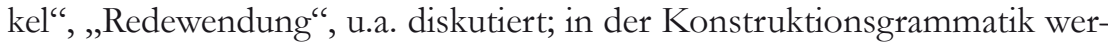
den sie alle unter dem Begriff der „Konstruktion“ zusammengefasst.

Für interessante Fragen und Anregungen danke ich den Teilnehmer/innen der 46. Jahrestagung des Instituts für Deutsche Sprache, insbesondere Heike Behrens, Stefan Engelberg, Joachim Jacobs, Tibor Kiss, Gereon Müller, Beatrice Primus, Peter Uhrig, Gert Webelhuth und Alexander Ziem. Ferner danke ich den Herausgeber/innen für ihre aufmerksame Lektüre des Manuskripts und ihre konstruktive Kritik. Ein besonderer Dank gebührt Juliana Goschler für ausführliche Diskussionen und Verbesserungsvorschläge während der Entstehung dieser Arbeit. 
Uneinigkeit besteht dagegen bezüglich der Frage, welche Rolle diese Konstruktionen in der Grammatik natürlicher Sprachen spielen. In den meisten Theorien geht man davon aus, dass diese Konstruktionen Ausnahmeerscheinungen sind, die außerhalb des eigentlichen, regelgestützten Kerns der Grammatik - etwa im Lexikon - aufgelistet werden müssen. In der Konstruktionsgrammatik werden sie dagegen als grundsätzliches grammatisches Organisationsprinzip verstanden.

Im vorliegenden Beitrag werde ich für eine zentrale Rolle von Konstruktionen bei der Repräsentation sprachlichen Wissens argumentieren. Dabei gehe ich zunächst von einem deduktiven, logisch-ökonomischen Argument aus, das seit den Anfängen der Konstruktionsgrammatik immer wieder mehr oder weniger explizit in die Theoriediskussion eingebracht worden ist, das aber bisher keine ausreichende Beachtung gefunden hat.

Dieses Argument lässt sich etwa wie folgt zusammenfassen: Menschen sind offensichtlich in der Lage, Konstruktionen (im konstruktionsgrammatischen Sinne) zu erwerben und zu verarbeiten; es muss also in den Köpfen der Sprecher/innen ein entsprechendes, konstruktionsverarbeitendes System existieren. ${ }^{2}$ Ein System, das irreguläre und idiomatische Strukturen verarbeiten kann, ist logischerweise auch in der Lage, regelhafte und kompositionelle Strukturen zu verarbeiten, denn letztere unterscheiden sich von ersteren ja nur durch ihre weniger starken syntaktischen und semantischen Beschränkungen. Umgekehrt kann aber ein regelverarbeitendes System nicht ohne weiteres idiomatische Strukturen verarbeiten. Ockhams Rasiermesser sagt uns, dass eine Grammatik mit nur einem System einer mit zwei Systemen ceterus paribus vorzuziehen ist.

Der Beitrag ist wie folgt strukturiert: Im zweiten Unterkapitel stelle ich zunächst in allgemeiner Form die Grundannahmen der Konstruktionsgrammatik dar und diskutiere dann speziell den Kernbegriff der Konstruktion als nicht-kompositionelles Form-Bedeutungspaar und seine potenzielle Reichweite in der Architektur natürlichsprachlicher Grammatiken. Im dritten Unterkapitel folgt eine ausführliche Diskussion des logischen Arguments für die Existenz von Konstruktionen sowie eine Fallstudie des Ausdrucks $[W H$ in aller Welt], die zeigen soll, dass Konstruktionen bis weit in die Bereiche der Grammatik hineinwirken, die traditionell als „Kerngrammatik“ bezeichnet werden. Im vierten Unterkapitel stelle ich das ökonomische Argument für die Konstruktion als einziges Organisationsprinzip natürlicher Sprachen vor und präsentiere eine Fallstudie des grammatischen Musters

\footnotetext{
Es sei hier dahingestellt, ob es sich bei diesem System um ein sprachspezifisches kognitives „Modul“ zur Verarbeitung von Konstruktionen handelt, oder um eine allgemeinere kognitive Fähigkeit. In der Konstruktionsgrammatik dürfte derzeit die zweite Möglichkeit eher konsensfähig sein (vgl. z.B. Langacker 1987; Lakoff 1987; Goldberg 2006), aber keines der Kernprinzipien der Konstruktionsgrammatik schließt die erste Möglichkeit kategorisch aus. Für das im Folgenden dargestellte Argument spielt diese Frage keine Rolle: Entscheidend ist nur, dass ein kognitives System existiert, das Konstruktionen verarbeiten kann.
} 
$\left[\mathrm{NP}_{\mathrm{i}}\right.$ hat $\mathrm{POSS}_{\mathrm{i}}$ Preis], die zeigen soll, dass die Verletzung von Regeln Hinweise auf die konstruktionsgestützte Natur unseres sprachlichen Wissens geben kann.

\section{Theoretischer Hintergrund}

\subsection{Grundannahmen der Konstruktionsgrammatik}

Unter der Bezeichnung „Konstruktionsgrammatik“ wird eine recht heterogene Klasse von grammatiktheoretischen Ansätzen zusammengefasst, deren kennzeichnendes Merkmal es ist, dass sie das sprachliche Zeichen im saussureschen Sinne - in der Konstruktionsgrammatik „Konstruktion“ genannt - als zentrales oder sogar einziges Organisationsprinzip natürlichsprachlicher Grammatiken betrachten. Der Zeichenbegriff der Konstruktionsgrammatik geht dabei insofern über das klassische saussuresche Verständnis hinaus, als dass darunter Strukturen mit beliebigem Komplexitäts- und Abstraktionsgrad erfasst werden, solange sie arbiträre, nicht aus ihren Bestandteilen ableitbare Eigenschaften besitzen.

Eine häufig zitierte und in der Konstruktionsgrammatik akzeptierte Definition des Begriffs „Konstruktion“ findet sich in Goldberg (1995):

$\mathrm{C}$ ist eine Konstruktion dann und nur dann, wenn $\mathrm{C}$ ein Form-Bedeutungspaar $<\mathrm{F}_{i}, \mathrm{~S}_{i}>$ ist, dergestalt, dass irgendein Aspekt von $\mathrm{F}_{i}$ oder irgendein Aspekt von $\mathrm{S}_{i}$ sich nicht auf der Grundlage der Komponenten von C oder von bereits etablierten Konstruktionen vorhersagen lässt. (Goldberg 1995, S. 4, übers. Fischer/Stefanowitsch 2006, S. 5)

Der Begriff „Bedeutung“ ist dabei in dieser Definition weit zu fassen, er bezieht sich nicht nur auf wahrheits- oder framesemantische Eigenschaften einer Konstruktion, sondern auch auf Aspekte des Gebrauchs wie Diskursfunktion, Informationsstruktur usw. ${ }^{3}$

Die Konstruktionsgrammatik geht davon aus, dass sprachliches Wissen vollständig in Form von Konstruktionen von unterschiedlicher Komplexität und unterschiedlichem Abstraktionsgrad repräsentiert ist (die Gesamtheit aller Konstruktionen einer Sprache wird dabei als „Konstruktikon“ bezeichnet). Anders als Kritiker der Konstruktionsgrammatik manchmal behaupten (z.B. Leiss 2009), bedeutet das aber nicht, dass das sprachliche Wissen als unstrukturierte Ansammlung strukturloser sprachlicher Zeichen verstanden werden soll. Konstruktionen sind sowohl intern als auch in Bezug zueinander strukturiert, sodass regelhafte Aspekte einer Konstruktion auf einer höheren Abstraktionsebene repräsentiert sind und von

Eine seltener zitierte, aber nicht weniger akzeptierte Definition in Lakoff (1987) macht dies explizit: „Eine Konstruktion sei ein Form-Bedeutungspaar (F, M), wobei F eine Menge von Bedingungen bezüglich der syntaktischen und phonologischen Form und M eine Menge von Bedingungen bezüglich Bedeutung und Verwendung ist" (Lakoff 1987, S. 467, übers. Fischer/Stefanowitsch 2006, S. 5, eigene Hervorhebung). 
mehreren Konstruktionen geteilt werden können. ${ }^{4}$ Auf der höchsten Abstraktionsebene finden sich dann Konstruktionen, die traditionellen Regeln ähneln (etwa die Subjekt-Prädikat-Konstruktion oder die Modifikator-Kopf-Konstruktion, siehe unten). Die Form und die Bedeutung von Konstruktionen ist also systemintern durch Verknüpfungen mit Konstruktionen ähnlicher Form und Bedeutung motiviert; sie ist zudem beschränkt durch allgemeine Eigenschaften des kognitiven Systems (vgl. z.B. Langacker 1987) und durch die kommunikativen Funktionen, die die Konstruktionen erfüllen müssen.

Über diese gemeinsamen Grundannahmen hinaus unterscheiden sich konstruktionsgrammatische Theorien teilweise drastisch voneinander (eine Diskussion der wichtigsten Unterschiede und Gemeinsamkeiten findet sich in Fischer/Stefanowitsch 2006). Im Rahmen der vorliegenden Arbeit spielen die meisten dieser Unterschiede keine große Rolle, da es hier um die grundsätzliche Frage gehen soll, ob und in welchem Umfang es plausibel ist, Konstruktionen als Teil des sprachlichen Wissens zu betrachten.

Ein entscheidender Unterschied muss aber noch erwähnt werden: Einige Versionen der Konstruktionsgrammatik betrachten das Kriterium der Nicht-Kompositionalität nicht mehr als notwendige Voraussetzung, um einer Struktur Konstruktionsstatus zuzuschreiben. Alternativ legen sie eine Art allgemeines Repräsentationskriterium zugrunde:

Als Konstruktion wird jedes sprachliche Muster akzeptiert, wenn irgendein Aspekt seiner Form oder Funktion sich auf der Grundlage seiner Komponenten oder anderer, bereits bekannter Konstruktionen nicht genau vorhersagen lässt. Außerdem werden auch Muster, die voll vorhersagbar sind, als Konstruktionen gespeichert, wenn sie ausreichend bäufig sind. (Goldberg 2006, S. 5, übers. Stefanowitsch, eigene Hervorhebung)

Dieser Konstruktionsbegriff stammt aus einer Strömung innerhalb der Kognitiven Linguistik, die als „gebrauchsgestütztes Modell“ (Usage-Based Model) bekannt ist und die die Konventionalität sprachlicher Zeichen in den Mittelpunkt stellt (vgl. Langacker 1987). Die Idee, dass häufige Strukturen auch dann in ihrer Gesamtheit gespeichert werden (können), wenn sie voll regelhaft und kompositionell sind, ist natürlich grundsätzlich mit der Idee eines strukturierten Konstruktikons vereinbar. Es ist nachvollziehbar, dass die hohe Gebrauchshäufigkeit einer sprachlichen Struktur dazu führt, dass diese Struktur mental als Einheit repräsentiert und in der Sprachverarbeitung nicht mehr für jedes Gebrauchsereignis aus seinen Einzelteilen zusammengesetzt bzw. in diese zerlegt wird. Trotzdem sollten die zwei Arten mentaler Einheiten terminologisch unterschieden werden. In Stefanowitsch (2009) schlage ich vor, den Konstruktionsbegriff für syntaktisch und/oder semantisch nicht vorhersagbare Strukturen zu verwenden, und

Vgl. z.B. Kay (1997, S. 129), der als Beispiel die englische „Left-Isolation Construction“ nennt, die von allen Konstruktionen geteilt wird, bei denen eine Konstituente nicht in ihrer kanonischen Position, sondern am linken Rand des Satzes steht, also etwa bei $W h$-Sätzen und verschiedenen Arten von Topikalisierungsstrukturen. 
Strukturen, die (z.B. aufgrund ihrer Häufigkeit) in ihrer Gesamtheit repräsentiert werden, als „Muster“ zu bezeichnen. Es ist dann klar, dass alle Konstruktionen auch Muster sind, nicht aber umgekehrt.

\subsection{Reichweite des Konstruktionsbegriffs}

Eine Konstruktion ist also jede sprachliche Struktur in einer Sprache, deren formale oder funktionale Eigenschaften sich nicht auf der Grundlage bereits vorhandener Konstruktionen in dieser Sprache vorhersagen lassen.

Dazu gehören zunächst natürlich monomorphemische Wörter und Flexions- und Derivationsmorpheme wie trink-/,„Flüssigkeit in den Mund nehmen und schlucken" oder $\left[\mathrm{V}_{\mathrm{x}^{-}}\right.$er $] /$,,jemand, der regelmäßig $\mathrm{X}$ tut"; Morpheme sind ja dadurch charakterisiert, dass sie nicht-vorhersagbare Form-Bedeutungspaare sind.

Aber auch morphologisch komplexe Wörter können Konstruktionen in diesem Sinn sein, sofern sich ihre Form oder Bedeutung nicht aus ihren Einzelteilen vorhersagen lässt. Kombiniert man z.B. trink- und $\left[\mathrm{V}_{\mathrm{X}}-\mathrm{er}\right]$, so lässt sich die Form Trinker vorhersagen. Auf der Bedeutungsseite ergibt sich dagegen die erwartete Bedeutung ,,jemand, der Flüssigkeit in den Mund nimmt und schluckt", die nur einen Teil der tatsächlichen Bedeutung des Wortes erfasst: Ein Trinker ist ,jemand, der regelmäßig und zwanghaft alkoholbaltige Flüssigkeit in den Mund nimmt und schluckt". Da die kursiv gesetzten zusätzlichen Bedeutungskomponenten nicht vorhersagbar sind, muss auch das Wort Trinker als Konstruktion betrachtet werden.

Umgekehrt ist zum Beispiel die Bedeutung der im Deutschen Referenzkorpus des Instituts für Deutsche Sprache belegten Komposita Apfelgetränk, Orangengetränk, Beerengetränk, Erdbeergetränk, Hafergetränk und Gerstengetränk vorhersagbar. Auf der Formseite unterscheiden sich die Wörter dagegen auf unvorhersagbare Weise. So steht beim Apfelgetränk das Erstglied im Singular und zwischen den Kompositionsgliedern steht kein Fugenelement; beim Orangengetränk und beim Beerengetränk dagegen steht das Erstglied im Plural (bzw. die Kompositionsglieder sind durch ein Fugen- $n$ verbunden). Bei einem Getränk aus Erdbeeren würde man entweder das Wort *Erdbeeregetränk (analog zu Apfelgetränk) oder das Wort *Erdbeerengetränk (analog zu Orangengetränk) erwarten, stattdessen findet sich im Deutschen Referenzkorpus ausschließlich das Wort Erdbeergetränk mit dem auf Komposita beschränkten Erstglied Erdbeer, und auch eine Google-Suche fördert keine Treffer für die anderen beiden Formen zutage, die eindeutig deutschen Muttersprachlern zuzuordnen sind. Schließlich muss beim nicht-zählbaren Erstglied Gerste ein Fugen- $n$ stehen, während das ebenfalls nicht-zählbare Erstglied Hafer kein Fugenelement benötigt. ${ }^{5}$

Die Erstglieder verhalten sich nicht nur in Komposition mit dem Zweitglied Getränk so, wie hier dargestellt, sondern typischerweise in allen Komposita. Das ändert aber nichts an der 
An dieser Stelle lässt sich der Unterschied zwischen Konstruktionen und Mustern demonstrieren. Das Wort Esser (,,jemand, der isst") lässt sich, anders als das Wort Trinker, sowohl in seiner Form als auch in seiner Bedeutung vorhersagen. Trotzdem ist die Annahme gerechtfertigt, dass das Wort im mentalen Lexikon deutscher Muttersprachler als Muster repräsentiert ist und nicht jedes Mal aus seinen Bestandteilen erzeugt wird. Dafür spricht unter anderem, dass es konventionalisierte Kollokationen mit diesem Wort gibt (ein guter/schlecbter Esser ist ein konventioneller Ausdruck, ein guter/scblechter Essender nicht; gleiches gilt für ein Esser mehr/zu viel im Vergleich zu ein Essender mehr/ zu viel).

Dass einzelne Morpheme ebenso wie formal oder semantisch unregelmäßige komplexe Wörter der oben zitierten Konstruktionsdefinition entsprechen, dürfte unstrittig sein, sie werden in allen Theorien im Lexikon verortet. Dass sie als „Konstruktionen“ bezeichnet werden, mag ungewohnt sein, ist aber eine oberflächliche terminologische Frage (und nicht alle konstruktionsgrammatischen Ansätze folgen dieser Terminologie ${ }^{6}$ ).

Es dürfte auch weitgehend Einigkeit darüber bestehen, dass der Begriff der Konstruktion auf Syntagmen mit nicht vorhersagbaren formalen oder semantischen Eigenschaften anwendbar ist, wie z.B. jmd. unter den Tisch trinken oder einen über den Durst trinken. Semantisch unvorhersagbare Eigenschaften dieser Syntagmen sind z.B., dass sie (wie auch das Kompositum Trinker) auf die Einnahme alkoholischer Getränke beschränkt sind, dass jemand, der unter den Tisch getrunken worden ist, nicht tatsächlich unter dem Tisch liegen muss, und dass jemand, der einen über den Durst getrunken hat, immer noch Durst haben kann. Formal unvorhersagbar ist, dass die sprachliche Flexibilität dieser Syntagmen eingeschränkt ist, man also z.B. nicht *jmd. unter den Stubl trinken oder *einen unter den/dem Durst trinken kann. Problematisch ist an solchen Syntagmen höchstens die Tatsache, dass sie partiell semantisch transparent und partiell syntaktisch flexibel sind, dass sie also in einer Form im Lexikon repräsentiert werden müssen, die allgemeinen Regeln in beschränktem Umfang zugänglich ist, doch entsprechende Lösungen existieren in verschiedenen regelgestützten Theorien (vgl. z.B. Tree-Adjoining Grammar, Abeillé/Schabes 1989; HPSG (Head-driven Phrase Structure Grammar), Sailer 2003).

Die Einigkeit bröckelt vermutlich bei Ausdrücken wie auf NP trinken, die variable Leerstellen enthalten, in die jeder formal passende Ausdruck eingesetzt werden kann (ein weiteres Beispiel wären die modalen Infinitive im Deutschen, vgl. Stefanowitsch 2006, 2010); sie endet spätestens bei Kon-

Tatsache, dass es sich um nicht-vorhersagbare Formen handelt - die Nicht-Vorhersagbarkeit ist lediglich auf einer allgemeineren Ebene anzusiedeln.

$6 \quad$ Siehe z.B. Langacker (1987), der das, was in anderen konstruktionsgrammatischen Ansätzen „Konstruktion“ heißt, als „symbolische Einheit“ (symbolic unit) bezeichnet und den Begriff „Konstruktion“ (construction) auf komplexe Strukturen aus mindestens zwei solchen symbolischen Einheiten beschränkt. 
figurationen abstrakter grammatischer Kategorien, die lexikalisch nicht mehr spezifiziert sind, wie etwa Resultativen (vgl. z.B. Müller 2006), verblosen Direktiven (Jacobs 2008) oder Relativsätzen (Webelhuth in diesem Band). In der Konstruktionsgrammatik werden auch solche Strukturen als Konstruktionen verstanden; ihnen gilt das Hauptinteresse konstruktionsgrammatischer Theoriebildung. Ihr Status ergibt sich aus den oben zitierten Definitionen: Es handelt sich um Form-Bedeutungspaare, die nicht-vorhersagbare formale oder semantische Eigenschaften haben.

Nehmen wir den Fall der Resultativkonstruktion, die sich in Beispielen wie den folgenden manifestiert:
a. Sie trank ihren Calvados leer. [WAM]
b. Norina trank ihr Glas leer. [M02]
c. [Er] trank die Minibar leer. [RHZ02]

Bei (1a) mag es zunächst aussehen, als handle es sich um die regelhafte Erweiterung des transitiven Satzes (2a) um eine Adjunkt-AP leer:

(2) a. Sie trank ihren Calvados.

b. ? Norina trank ihr Glas (vgl. [Sie machten] an jeder Gastwirtschaft stopp ... und tranken ein Gläschen. [RHZ04])

c. ${ }^{*}$ Er trank die Minibar.

Diese Analyse ist bei (1b) schon problematischer, da der Status des dafür notwendigen transitiven (2b) zweifelhaft ist. Bei (1c) schließlich ist die Analyse ausgeschlossen, da der Satz ungrammatisch ist. Ebenso ist es problematisch, dass bei (1b-c) tatsächlich das Glas bzw. die Minibar leer ist, bei (1a) der Calvados jedoch nicht leer, sondern nicht mehr da wäre. Schließlich bliebe unklar, woher die resultative Lesart kommt; sie ergibt sich keinesfalls zwingend aus dem Anfügen einer AP an einen transitiven Satz, wie die nichtresultativen Lesarten von Sätzen wie Sie trank ibren Calvados eiskalt oder Sie trank ibren Calvados pur zeigen.

Stefan Müller (2006) zeigt im Rahmen einer HPSG-Analyse, dass diese Strukturen durch eine Lexikonregel erzeugt werden können, die die beobachtete Valenz erzeugen und gleichzeitig eine resultative Lesart hinzufügen kann. Dass Müller von „Regeln“ spricht, darf hier nicht darüber hinwegtäuschen, dass diese unter den oben genannten Konstruktionsbegriff fallen: Lexikonregeln in der HPSG sind formale Operationen, die einen ohne die Regel nicht vorhersagbaren semantischen Effekt haben können; wo dies der Fall ist, handelt es sich um Form-Bedeutungspaare im Sinne des oben genannten Konstruktionsbegriffs.

Konstruktionsgestützte Analysen solcher abstrakten grammatischen Strukturen werden von Gegnern der Konstruktionsgrammatik häufig mit dem Argument angegriffen, man könne sie durchaus aus allgemeinen (möglicherweise sogar universellen) Regeln ableiten. So leitet Gereon Müller (in diesem Band) verblose Direktive (Zur Hölle mit dieser Regierung, In den Müll mit 
diesen Klamotten) aus Regeln ab, die in anderen Sprachen für Antipassivbildung und stilistische Voranstellung zuständig sind. Solche Analysen sind interessant (ob man ihnen im Einzelnen folgen mag oder nicht), aber als Gegenargumente einer konstruktionsgrammatischen Perspektive werden sie erst dann relevant, wenn sich zeigen lässt, dass sich auch ihre Bedeutung aus der Anwendung allgemeiner Regeln ableiten lässt. Dies ist im Allgemeinen nicht der Fall; Müller gelingt seine Herleitung der verblosen Direktiven nur deshalb, weil er deren semantische Eigenschaften außer Acht lässt. Damit zeigt er im besten Fall, dass die Form von verblosen Direktiven sich innerhalb des in natürlichen Sprachen möglichen Formeninventars bewegt, nicht aber, dass die Form verbloser Direktive vorhersagbar oder deren Bedeutung aus ihrer Form ableitbar wäre. Auch unter seiner Analyse bleiben sie Konstruktionen.

Abschließend sei darauf hingewiesen, dass in der Konstruktionsgrammatik konsequent auch die abstraktesten grammatischen Strukturen als Konstruktionen analysiert werden; Beispiele sind die englischen Argumentstrukturkonstruktionen (Goldberg 1995), die Subjekt-Prädikat-Konstruktion (Kay/Fillmore 1999) und die deutsche Verberst-Konstruktion (Diessel 1997).

\subsection{Regeln vs. Konstruktionen}

Wie in der Diskussion der Resultativkonstruktion bereits angedeutet, charakterisiert der Konstruktionsbegriff bestimmte Eigenschaften sprachlichen Wissens, er sagt jedoch für sich genommen weder etwas darüber aus, wie dieses sprachliche Wissen mental repräsentiert sein könnte, noch darüber, wie es am besten darzustellen ist.

Natürlich lässt sich jede Konstruktion im konstruktionsgrammatischen Sinne auch als Regel darstellen, sofern man ein Modell annimmt, in dem Regeln semantische und pragmatische Informationen enthalten dürfen; umgekehrt lässt sich auch eine rein formale Regel als Konstruktion darstellen, sofern man ein Modell annimmt, in dem die Bedeutungsseite einer Konstruktion leer bleiben darf (so wie in Langackers Kognitiver Grammatik). Die Argumentstrukturkonstruktionen in Goldberg (1995) lassen sich z.B. als lexikalische Regeln darstellen und die lexikalischen Regeln in Müller (2006) als Konstruktionen, ohne dass allein durch die Darstellung eine grundsätzlich andere Art sprachlichen Wissens angenommen würde. Im Rahmen einer theoriebildenden Diskussion ist jedoch eben die Frage entscheidend, welche Art von sprachlichem Wissen in einer solchen Konstruktion oder Regel erfasst werden darf: Die Unterschiede zwischen den Theorien ergeben sich aus der Antwort auf diese Frage (und natürlich aus der Gesamtarchitektur, in die die „Regeln“ oder „Konstruktionen“ eingebettet sind).?

Vgl. in diesem Zusammenhang Jacobs (2009), der darauf hinweist, dass die Unterschiede zwischen konstruktionistischen und projektionistischen Analysen in vielen Fällen nur durch 
Mangelnde Klarheit über den Unterschied zwischen Notation und theoretischer Substanz kann leicht dazu führen, dass die Darstellung von Konstruktionen als Regel fälschlicherweise als ein Gegenargument zu einer konstruktionsgestützten Analyse oder gar als Beweis der Nichtexistenz von Konstruktionen gewertet wird. Wenn eine „Regel“ ein Form-Bedeutungspaar spezifiziert, ist sie eine Konstruktion, deren Notation manipuliert wurde, um sie wie eine Regel aussehen zu lassen. Eine solche „Regel“ mag in der Darstellung elegant und nützlich sein, aber auf ihrer Grundlage lässt sich der philosophische Überbau der generativen Grammatik Chomskyschen Zuschnitts (etwa die Idee einer wie auch immer gearteten Universalgrammatik) nicht rechtfertigen.

\section{Das logische Argument: Konstruktionen als Teil der Sprachkompetenz}

Akzeptiert man die Existenz nicht-kompositioneller und/oder formell idiosynkratischer sprachlicher Strukturen, so lässt sich folgendes logisches Argument für die Existenz eines - wie auch immer gearteten - konstruktionsverarbeitenden Systems konstruieren:

Alle Sprachen enthalten nicht-kompositionelle und/oder syntaktisch nicht voll regelhafte Strukturen.

Sprecher können diese Strukturen produzieren und verarbeiten.

Daraus folgt: Es existiert ein System, das nicht-kompositionelle und/ oder syntaktisch nicht voll regelhafte Strukturen repräsentieren und zur Verarbeitung bereitstellen kann.

Das logische Argument besagt zunächst nur, dass es keine mentale Grammatik geben kann, die nicht auch Konstruktionen verarbeiten kann, oder die durch ein entsprechendes System ergänzt wird. Wie in Abschnitt 2.2 oben dargestellt, ist dies im Falle von Wörtern und idiomatischen Redewendungen auch relativ unstrittig und führt dazu, dass Wörter und idiomatische Syntagmen im Lexikon verortet werden und die syntaktische Komponente einzig für kerngrammatische Strukturen zuständig ist.

Das Problem an dieser Sichtweise ist allerdings, dass idiomatische Syntagmen häufig eng mit scheinbar kerngrammatischen Strukturen und Regeln interagieren. Das hat Konsequenzen für die Plausibilität einer Trennung von Kerngrammatik und Lexikon (Fillmore 1985). Bevor ich diese Konsequenzen diskutiere, möchte ich die Interaktion von Konstruktionen mit kerngrammatischen Regeln am Beispiel eines deutschen Ausdrucks zeigen,

die Darstellung bedingt sind, und dass konstruktionistische Analysen auf Phänomene begrenzt werden sollten, in denen eine projektionistische Analyse unmöglich oder zumindest unmotiviert ist. 
dessen englisches Äquivalent Charles Fillmore in seiner ersten Arbeit zur Konstruktionsgrammatik (Fillmore 1985) kurz anreißt. Dieser Ausdruck besteht aus einem Interrogativpronomen gefolgt von der PP in aller Welt:
a. Was in aller Welt will Frau Merkel erreichen, ist sie völlig naiv und vergesslich (Stichwort Kosovo)? [NUZ08]
b. Warum in aller Welt sollte man ein Rockkonzert besuchen? Die Luft ist schlecht, der Sound meist ebenso. [NUZ07]
c. Inzenberg, wo in aller Welt liegt das denn? [A97]
d. Aber wie in aller Welt wird es ausgesprochen? [R97]

In Wörterbüchern wird die Bedeutung dieses Ausdrucks mit „Steigerungspartikel“ (Grimm/Grimm 1854-1960) oder „Formel zur Intensivierung“ (Wahrig-Burfeind (Hg.) 2006) angegeben. Die Beispiele in (3) zeigen aber, dass damit nur ein Teil der Bedeutung erfasst ist; zusätzlich drückt das Idiom Unverständnis des Sprechers bezüglich der Proposition des Satzes aus, in dem sie auftritt. Weder die Intensivierung noch der Ausdruck von Unverständnis ergeben sich kompositionell aus der Bedeutung der einzelnen Konstituenten des Ausdrucks oder lassen sich aus allgemeinen pragmatischen Prinzipien herleiten, wie eine Ersetzung durch synonyme Wörter und Ausdrücke zeigt:
a. *Was überall will Frau Merkel erreichen?
b. *Was in/auf der ganzen Welt will Frau Merkel erreichen?
c. *Was in allen Ländern der Erde will Frau Merkel erreichen?

Die Sätze in $(4 a-c)$ sind zunächst ungrammatisch, da keiner der kursiv gesetzten Ausdrücke direkt hinter dem $W$ h-Pronomen stehen kann. Selbst, wenn man dies ignoriert, hätten $(4 a-c)$ aber nicht dieselbe Bedeutung wie die entsprechenden Sätze mit in aller Welt, sondern könnten nur wörtlich interpretiert werden. Damit fällt der Ausdruck [WH in aller Welt aus semantischen und formalen Gründen unter den konstruktionsgrammatischen Konstruktionsbegriff; ich werde ihn im Folgenden als WhIAW-Konstruktion oder kurz als WhIAW bezeichnen.

Die WhIAW-Konstruktion gehört zu einer Klasse von formal ähnlichen Konstruktionen, die alle eine intensivierende Bedeutung haben, die sich aber darüber hinaus semantisch klar unterscheiden und die auch bezüglich ihres syntaktischen Verhaltens keine homogene Gruppe bilden; zu dieser Gruppe gehören z.B. WH zur Hölle, WH zum Teufel, WH um Himmelswillen, WH in Dreiteufelsnamen u.a. Um in jedem Falle entscheiden zu können, ob eine bestimmte formale oder semantische Eigenschaft spezifisch für WhIAW ist, oder ob sie für all diese intensivierenden PPs gilt, müsste man jeden dieser Ausdrücke detailliert analysieren; dies kann hier aber nicht geleistet werden, sodass ich im Folgenden nur punktuell auf Unterschiede zwischen WhIAW und anderen Ausdrücken eingehen werde. Dies birgt das 
Risiko einer Zuschreibung von Eigenschaften an die WhIAW-Konstruktion, die besser bei allgemeineren Konstruktionen verortet wären; das ändert aber nicht grundsätzlich etwas an der konstruktionellen Natur der betreffenden Eigenschaften. ${ }^{8}$

Die WhIAW-Konstruktion kann nicht nur in interrogativen Hauptsätzen stehen, sondern auch in indirekten Fragesätzen als Komplement von Verben und Substantiven: ${ }^{\prime}$

a. Jeder andere würde sich an seiner Stelle fragen, wo in aller Welt er jetzt noch Freunde suchen soll. [NUN96]

b. Zudem werfen sie die Frage auf, was in aller Welt der Wiener Exzentriker [Falco] mit Wolfgang Amadeus Mozart gemeinsam haben soll. [HZ04]

Im Deutschen Referenzkorpus kommt WhIAW in indirekten Fragesätzen mit den Verben und Verbkomplexen in (6a) und den Substantiven in (6b) vor:
a. fragen (9-mal), wissen (2-mal), sich fragen, darüber nachdenken, binterfra- gen, rätseln, überlegen, sagen, erklären (je 1-mal)
b. Frage (9-mal), Bemerkung (1-mal)

Die Verwendung von WhIAW in indirekten Fragesätzen unterliegt dabei subtilen Beschränkungen, bei denen es zunächst so scheinen mag, als ob sie lexikalischer Natur seien. Tatsächlich aber lassen sie sich alle auf die folgende allgemein semantisch-pragmatische Beschränkung zurückführen: ${ }^{10}$

$8 \quad$ So ließe sich beispielsweise einwenden, dass die Position von in aller Welt direkt hinter dem Wh-Pronomen nicht unvorhersagbar ist, da intensivierende PPs grundsätzlich in dieser Position stehen können. Dies ließe sich durch eine allgemeinere Regel erfassen, die besagen würde, dass $w$ h-Pronomen durch intensivierende PPs (und nur durch diese) modifiziert werden dürfen. Diese „Regel“ müsste aber Bezug auf die Bedeutung der PP nehmen (denn nur so lässt sich feststellen, ob diese ,intensivierend“ ist) und wäre somit selbst eine Konstruktion im hier angenommenen Sinne. Interessant ist aber, dass in aller Welt, anders als alle anderen intensivierenden PPs, nahezu kategorisch auf diese Position beschränkt ist (siehe unten); dies ließe sich nicht aus einer allgemeinen Konstruktion [Wh [ $\left.\left.\mathrm{PP}_{\text {intens }}\right]\right]$ herleiten, müsste also doch auf der Ebene der WhIAW-Konstruktion spezifiziert werden.

9 Außerdem kann WhIAW auch in Wh-Verbletzt-Sätzen auftreten; das Deutsche Referenzkorpus enthält zwar kein entsprechendes Beispiel, aber Beispiele wie Was in aller Welt Falco wobl mit Mozart zu tun hat? scheinen akzeptabel.

10 Diese Beschränkung ist nicht so zu verstehen, dass WhIAW nur in Sätzen auftreten kann, die zum Vollzug des illokutionären Akts einer Frage dienen - es muss keine Aufforderung vorliegen, die offene Frage im Kopf des Sprechers oder des Referenten des Matrixsatzes zu beantworten. Die Beispiele in $(9 \mathrm{a}-\mathrm{c})$ unten zeigen dies: Keins von ihnen stellt direkt oder indirekt eine solche Aufforderung dar. Man kann die Beschränkung in (7) deshalb nicht ohne Weiteres mit der Unterscheidung zwischen Fragesemantik und Fragepragmatik erfassen, die vor allem in der klassischen generativen Grammatik häufig getroffen wird (vgl. z.B. Holler 2007 für eine zusammenfassende Darstellung dieser Unterscheidung). 
(7) WhIAW kann nur verwendet werden, wenn das $W h$-Pronomen Teil eines Satzes ist, dessen Proposition als unbeantwortete Frage im Kopf des Sprechers oder des Referenten eines Arguments im Matrixsatz interpretiert werden muss.

Diese Beschränkung erklärt zunächst natürlich, warum WhIAW in interrogativen Hauptsätzen auftritt, denn diese stellen ihre Proposition ja direkt als offene Frage dar. Sie erklärt auch, warum WhIAW in indirekten Fragesätzen mit Abstand am häufigsten mit den Köpfen Frage und (sich) fragen auftritt, und auch das Vorkommen mit den Verben sich fragen, darüber nachdenken, binterfragen, rätseln und überlegen ergibt sich daraus.

Das Vorkommen mit den Verben wissen, sagen und erklären sowie mit dem Substantiv Bemerkung scheint die Beschränkung dagegen zu verletzen, und tatsächlich ist die Verwendung von WhIAW mit diesen und ähnlichen Verba Dicendi und Sentiendi nicht ohne Weiteres akzeptabel:
a. *Maria sagte, was in aller Welt Falco mit Mozart zu tun hatte.
b. *Maria erklärte Hans, was in aller Welt Falco mit Mozart zu tun hatte.
c. *Maria wusste, was in aller Welt Falco mit Mozart zu tun hatte.

Das ändert sich aber in Kontexten, in denen die Verwendungsweise des jeweiligen Verbs voraussetzt, dass eine unbeantwortete Frage im Kopf des Sprechers oder eines der Referenten des Matrixsatzes existiert; auf diese Weise erklären sich die Vorkommen im Deutschen Referenzkorpus:

(9) a. Wer kann sagen, warum in aller Welt ein Eisenbahntunnel schädlich für ein Gebirge sein soll ... [N98]

b. [Ich] grübelte, wie ich meinen Eltern erkläre, warum in aller Welt ich eine hundsmiserable Note mit nach Hause gebracht hatte. [BRZ07]

c. Doch die Tatsache, dass bisher nur ein paar Insider wissen, wer in aller Welt denn bitteschön Füsun ist, könnte sich bald ändern ... [NUZ08]

Die Phrase wer kann sagen in (9a) zeigt, dass die anschließende Frage aus Sicht des Sprechers unbeantwortet (und unbeantwortbar) ist, und die Verwendung von erklären in (9b) setzt voraus, dass die erwähnten Eltern des Sprechers eine entsprechende Frage im Kopf haben werden, wenn der Sohn ihnen von der schlechten Note erzählt. Das Beispiel in (9c) ist komplexer: Hier werden diejenigen, die eine entsprechende Frage haben, nicht direkt genannt, sondern bilden die nicht-genannte Kontrastmenge zu den genannten Insidern. ${ }^{11}$

$11 \quad$ Die Verwendung von WhIAW mit diesen Verben wird auch dann akzeptabel, wenn diese in Interrogativ- oder Imperativsätzen auftreten, die eine Frage im Kopf des Sprechers voraussetzen, vgl. Weißt du/Sagst du mir bitte/Erklär mir, was in aller Welt Falco mit Mozart zu tun hat. 
Abschließend sei darauf hingewiesen, dass die Interpretation eines Satzes die Existenz einer „offenen Frage“ zwingend voraussetzen muss, damit WhIAW verwendet werden kann; es genügt nicht, dass ein Verb eine kommunikative Handlung oder einen mentalen Zustand bezeichnet, der eine solche Frage voraussetzen kann. Dies erklärt Unterschiede zwischen semantisch ähnlichen Verben wie denen in (10a-b) und (11a-b):

(10) a. Hans rätselte, was in aller Welt Falco mit Mozart zu tun haben könnte.

b. *Hans riet, was in aller Welt Falco mit Mozart zu tun haben könnte.

(11) a. Maria überlegte, was in aller Welt Falco mit Mozart zu tun haben könnte.

b. *Maria erforschte/ untersuchte, was in aller Welt Falco mit Mozart zu tun haben könnte.

Das Verb rätseln impliziert semantisch, dass sein Agens eine offene Frage im Kopf hat, das Verb raten tut dies nicht (vgl. 12a); ebenso impliziert überlegen eine solche Frage semantisch, während ähnliche Verben wie erforschen und untersuchen dies nicht tun (vgl. 12b):

a. Hans riet/*rätselte, was Falco mit Mozart zu tun haben könnte, ohne darüber nachzudenken.

b. Maria erforschte/ untersuchte/*überlegte, was Falco mit Mozart zu tun haben könnte, ohne darüber nachzudenken.

Wenn Verben wie raten, überlegen und erforschen in einem Satzzusammenhang auftreten, in dem die offene Frage im Kopf eines Partizipanten des Matrixsatzes durch andere Mittel zu einem zwingenden Teil der Bedeutung gemacht wird, können sie auch mit WhIAW verwendet werden:

a. Hans versuchte wieder und wieder zu raten, was in aller Welt Falco mit Mozart zu tun haben könnte.

b. Maria untersuchte voller Neugier, was in aller Welt Falco mit Mozart zu tun haben könnte.

Alle scheinbaren verbspezifischen Unregelmäßigkeiten bei der Verwendung von WhIAW in indirekten Fragesätzen ergeben sich also aus der allgemeinen Beschränkung in (7). Die Beschränkung selbst ergibt sich nicht unmittelbar aus der intensivierenden oder Unverständnis anzeigenden Funktion der Konstruktion, muss also als Teil der Konstruktionsbedeutung postuliert werden.

Auch formal hat WhIAW eine Reihe von Eigenschaften, die sich nicht aus allgemeinen Regeln ableiten lassen und die auch nicht durch die Funktion der Konstruktion motiviert sind. Die auffälligste Eigenschaft ist dabei 
die Position der PP in aller Welt, die gemeinsam mit dem $W h$-Pronomen eine NP zu bilden scheint. Dies ist deshalb unerwartet, weil - wie eingangs angedeutet - PPs normalerweise in dieser Position nicht stehen können: ${ }^{12}$
a. *Was im Briefkasten ist denn das?
b. Was ist denn das im Briefkasten?

Aus der Funktion „Intensivierung und Unverständnis“ lässt sich das Auftreten der PP in dieser Position nicht erklären, denn dann müssten auch andere Adverbiale mit einer solchen Funktion dort stehen können. Dies ist aber nicht der Fall:
a. *Was schon wieder ist denn das?
b. Was ist denn das schon wieder?

Das Auftreten der PP in aller Welt ließe sich auf der Grundlage allgemeiner Regeln nur dann vorhersagen, wenn man sie als Apposition analysieren würde. Für eine solche Analyse würde oberflächlich sprechen, dass diese PP im Schriftdeutschen manchmal durch Kommata vom $W$-Pronomen abgesetzt wird (vgl. 16a), wobei das allerdings in der großen Mehrzahl der Belege nicht der Fall ist (vgl. 16b):
a. Was, in aller Welt, soll das sein? [RHZ97]
b. Was in aller Welt soll das alles? [SOZ06]

Gegen eine Analyse der PP als appositiv spricht aber auch, dass ihr klare Beispiele von Appositionen immer folgen (vgl. 17a). Wenn es sich um eine appositive PP handeln würde, müsste sie auch vor dem $W$ h-Pronomen oder hinter einer weiteren Apposition stehen können, doch das ist - anders als bei der semantisch und strukturell ähnlichen PP zum Teufel - nicht der Fall (vgl. 17b-c):

a. Was in aller Welt, mag man sich fragen, hat Freud mit der „Analytischen Philosophie des Geistes" zu tun? [R97]

b. *In aller Welt, was hat Freud mit ... zu tun? (vgl. Zum Teufel, warum ist sie ausgerechnet hierher gekommen? [MK2])

c. *Was, mag man sich fragen, in aller Welt, hat Freud mit ... zu tun?

Ein weiteres Argument gegen eine Analyse als Apposition besteht darin, dass in aller Welt - wiederum anders als z.B. zum Teufel - nur in $W$ h-Interrogativsätzen auftreten kann: ${ }^{13}$

Es gibt noch andere PPs, die in dieser Position stehen können, z.B. von NP (Wer von den beiden
lïgt?) oder an NP (Was an dem Film bat dir am besten gefallen?) (vgl. Holler 2007, Kap. 4.5.2).
Allerdings können diese PPs, anders als in aller Welt, auch in den kanonischen PP-Positionen
stehen (Wer lïgt von den beiden? Was hat dir an dem Film am besten gefallen?).
Betrachtet man die sprachgeschichtliche Entwicklung dieser Ausdrücke, so stellt man fest,
dass auch in aller Welt ursprünglich syntaktisch noch relativ frei auftritt. So finden sich noch 
(18) a. Wenigstens dann, zum Teufel/*in aller Welt, müssen doch die Emotionen endlich hochkochen! [RHZ04]

b. Javitt, zum Teufel/*in aller Welt, melde dich! [MK2]

Eine Analyse als Apposition ist damit ausgeschlossen. Dagegen lassen sich vereinzelte Beispiele finden, in denen die PP in aller Welt in kanonischen PP-Positionen steht:

a. Jetzt wirst Du mich wieder fragen: was mich denn in aller Welt dazu getrieben hätte, diese merkwürdige Wallfahrt anzutreten? [N94]

b. ... und das Problem erhob sich für die fleißigen Kameraleute: Was tun damit in aller Welt? [M95]

In einem entsprechenden Grammatikmodell ließe sich also argumentieren, dass die PP in aller Welt in einer kanonischen PP-Position generiert und dann durch eine Bewegungsregel in ihre typische Position direkt hinter dem $W$ hPronomen bewegt wird. Dagegen spräche die Tatsache, dass Beispiele wie (19a-b) extrem selten sind (es sind die einzigen Beispiele ihrer Art im Deutschen Referenzkorpus). Außerdem müsste man eine solche Regel speziell für die PP in aller Welt postulieren, denn schon ähnliche PPs wie zum Teufel und um Himmelswillen verhalten sich anders. Eine solche Regel könnte also bestenfalls einen Mechanismus zur Verfügung stellen, der die PP an ihre typische Position in der Oberflächenstruktur bringt; sie würde nichts daran ändern, dass in aller Welt sich unvorhersagbar verhält und damit auch dem formalen Kriterium des Konstruktionsbegriffs entspricht.

Eine weitere unvorhersagbare formale Eigenschaft ist die, dass in aller Welt nicht mit allen Interrogativformen verwendet werden kann. Ausgeschlossen ist welch-, sowohl als Determiner (20a) als auch als Pronomen (20b):

a. *Welche Verbindung in aller Welt besteht zwischen Falco und Mozart?

b. A: Es gibt eine Verbindung zwischen Falco und Mozart.

$\mathrm{B}: *$ Und welche in aller Welt ist das?

Auch diese Beschränkung gilt nicht für alle emphatischen PPs (vgl. z.B. Nun muß ich nur noch mit dem richtigen Bein aufstehen. Welches, zum Teufel, war denn das? [L98]).

Mitte des 18. Jahrhunderts Belege, in denen gar kein $W h$-Pronomen auftritt (i-ii), und auch Ende des 18. Jahrhunderts, als die Verwendung bereits auf $W h$-Interrogative eingeschränkt war, tritt in aller Welt noch in klar appositiven Verwendungen (iii) und in kanonischen Adjunktpositionen (iv) auf:

(i) Ist es in aller Welt möglich? [G.E. Lessing, 1755]

(ii) Sagen Sie mir doch in aller Welt, Pater, ob Sie haben wollen, daß wir Christum verlassen, und seinem Widersacher dienen sollen? [F. Ambrosius, 1771]

(iii) Aber in aller Welt, was soll daraus werden und giebt es denn gar kein Mittel dagegen? [L. Tieck, 1796]

(iv) Wie hätt' ich denn in aller Welt darauf kommen sollen? [C.L. Heyne, 1786] 
Schließlich kann die PP in aller Welt ausschließlich mit $W$ h-Pronomen auftreten, die im Vorfeld eines Interrogativsatzes oder indirekten Fragesatzes stehen. Sie kann also nicht in Exklamativsätzen (21a), bei $W$ b-Pronomen in Deklarativsätzen (21b) stehen, und auch nicht bei einem $W$ h-Pronomen in einem Interrogativsatz, wenn dieses nicht im Vorfeld steht (22b, c):

(21) a. *Was in aller Welt für eine Naivität!

b. *Ich soll WAS in aller Welt tun?

a. Offensichtlich werden wieder Aktien gekauft. Aber wer kauft was? [A98]

b. ... Aber wer in aller Welt kauft was?

c. ...*Aber wer kauft was in aller Welt?

Es lässt sich argumentieren, dass (21a) aufgrund der semantischen Beschränkung in (7) ausgeschlossen ist, da Exklamativsätze keine offene Frage im Kopf des Sprechers voraussetzen. Bei (21b) greift ein solches Argument aber nur bedingt: Kontrastiv betonte $W h$-Pronomen in situ können zwar ebenfalls exklamativ gebraucht werden, der Satz ist aber auch dann inakzeptabel, wenn er tatsächlich als Frage gemeint ist. Bei (21b) handelt es sich um eine Echo-Frage, von der man möglicherweise sagen könnte, dass sie ebenfalls keine offene Frage im Kopf des Sprechers voraussetzt; bei (22c) handelt es sich aber eindeutig um eine echte Frage.

Auch in Relativsätzen kann WhIAW nicht auftreten (23a, b), selbst dann nicht, wenn inhaltlich identische indirekte Fragesätze völlig akzeptabel sind (vgl. 24a, b):

(23) a. *Die Regierenden dachten über das nach, was in aller Welt man tun könnte, um das Konsumklima im Land zu verbessern.

b. *Ich wüsste nichts, was in aller Welt Bands wie den Fleshtones „helfen“ könnte.

(24) a. ... wenn sich nun ... die Regierenden am Kopf kratzen und laut darüber nachdenken, was in aller Welt man wohl tun könne, um das Konsumklima im Land zu verbessern. [HAZ08]

b. Ich wüsste nicht, was in aller Welt Bands wie den Fleshtones ,,helfen" könnte. [www.flying-revolver.net]

Die Eigenschaften der WhIAW-Konstruktion können wie folgt zusammengefasst werden:

(25) WhIAW-Konstruktion
a. $\mathrm{S}_{\mathrm{i}}\left[\left[_{\mathrm{Np}}[\mathrm{N} W\right.\right.$ h $][\mathrm{pp}$ in aller Welt $\left.] \mathrm{VP}\right]$
b. $S_{i}$ ist ein Hauptsatz oder
c. Argument eines Matrixprädikats $\mathrm{S}_{i}$
d. $\mathrm{S}_{\mathrm{i}}$ ist eine offene Frage im Kopf von $\mathrm{X}$
e. X drückt Intensivierung und Unverständnis bezüglich $\mathrm{p}\left(\mathrm{S}_{\mathrm{i}}\right)$ aus 
f. $\mathrm{X}$ ist Sprecher von $\mathrm{S}_{\mathrm{i}}$ oder

g. Referent eines Arguments von $\mathrm{S}_{j}$

Die Notation spielt dabei keine Rolle, entscheidend ist, dass in (25) das Wissen erfasst ist, das ein Sprecher haben muss, um WhIAW angemessen zu verwenden und um angemessene Verwendungen zu erkennen. ${ }^{14}$ Dieses Wissen beinhaltet Informationen bezüglich der syntaktischen (Oberflächen-)Struktur des Satzes, in dem das $W h$-Pronomen mit der intensivierenden PP steht, sowie ggf. über die Struktur des Matrixsatzes; es beinhaltet Informationen über die Bedeutung von WhIAW und über die Bedeutung des Satzes, in dem es auftritt, und es beinhaltet Informationen über den Sprecher und/oder die Referenten der Argumente des Matrixsatzes.

Aus grammatiktheoretischer Sicht ist nun die Frage entscheidend, wo ein syntaktisch-semantisch-pragmatischer Wissenskomplex wie der in (25) in einem Grammatikmodell verortet werden soll. Im Prinzip gibt es auf diese Frage vier mögliche Antworten: (a) in der Syntax, (b) im Lexikon, (c) im Weltwissen, oder (d) verteilt über Syntax, Lexikon und Weltwissen. Betrachten wir die Konsequenzen jeder dieser Möglichkeiten für ein Grammatikmodell:

(a) Wenn (25) in der Syntax verortet wird, lässt man damit semantisches und enzyklopädisches Wissen in der Syntax zu; die Syntax wird damit zu einem Konstruktikon im Sinne der Konstruktionsgrammatik. Ein Lexikon würde in diesem Fall überflüssig, da auch Wörter in einem solchen Konstruktikon repräsentiert werden können.

(b) Wenn (25) im Lexikon verortet wird, bedeutet dies, dass das Lexikon syntaktische Strukturen enthalten kann. Das Lexikon wird damit zu einem Konstruktikon im Sinne der Konstruktionsgrammatik. Eine Syntax würde in diesem Fall überflüssig, denn auch abstraktere syntaktische Strukturen könnten im Lexikon repräsentiert werden.

(c) Wenn (25) im Weltwissen verortet wird, bedeutet dies, dass das Weltwissen sowohl lexikalisches als auch syntaktisches Wissen beinhalten kann. Damit würden sowohl Syntax als auch Lexikon überflüssig; außerdem

14 Dabei spielt es keine Rolle, in welchem Format die Zusammenfassung der Eigenschaften von WhIAW vorgenommen wird. Es wäre z.B. vorstellbar, eine Transformationsregel für eine (optionale) ,intensivierende W-Expansion“ zu formulieren, die, analog zu traditionellen phonologischen Regeln, die Bedingungen beschreibt, unter denen diese Expansion möglich ist: $[\mathrm{NP}[\mathrm{N} \mathrm{WH}]] \rightarrow\left[\mathrm{NP}[\mathrm{N} \mathrm{WH}][\mathrm{PP}\right.$ in aller Welt $] / \mathrm{S}_{\mathrm{i}}[\# \ldots \mathrm{VP}]$

$\mathrm{S}_{\mathrm{i}}$ ist ein Hauptsatz oder ein Argument eines Matrixsatzes $\mathrm{S}_{\mathrm{i}}$

$\mathrm{S}_{\mathrm{i}}$ ist eine offene Frage im Kopf von $\mathrm{X}$

$\mathrm{X}$ drückt Unverständnis bezüglich $\mathrm{p}\left(\mathrm{S}_{\mathrm{i}}\right)$ aus

$\mathrm{X}$ ist Sprecher von $\mathrm{S}_{\mathrm{i}}$ oder

Referent eines Arguments von $\mathrm{S}_{\mathrm{i}}$

Diese „Regel“ wäre aber inhaltlich identisch mit der Darstellung in (25) und sie würde unter den konstruktionsgrammatischen Konstruktionsbegriff fallen, da sie ein Form-Bedeutungspaar mit nicht-vorhersagbaren formalen und semantischen Eigenschaften beschreibt. 
bestünde keine kategorische Grenze mehr zwischen sprachlichem und nicht-sprachlichem Wissen (eine Vorstellung, die nicht notwendigerweise von allen Konstruktionsgrammatikern geteilt wird, die sich aber in der Ideengeschichte der Konstruktionsgrammatik wiederholt findet).

(d) Wenn (25) über Syntax, Lexikon und Weltwissen verteilt repräsentiert wird, müssen spezifische, nicht-vorhersagbare Verknüpfungen zwischen diesen Bereichen zugelassen werden. Damit entstünde wiederum ein Konstruktikon im Sinne der Konstruktionsgrammatik, das sich allenfalls durch seine modulare Struktur von der holistischeren Lösung in (c) unterscheide. Da diese modulare Struktur aufgrund der nicht-vorhersagbaren Verknüpfungen keine Autonomie der einzelnen „Module“ zulassen würde, wäre dies aus konstruktionsgrammatischer Sicht wenig problematisch.

Unabhängig davon, welche Lösung man wählt, entsteht also ein Konstruktikon im Sinne der Konstruktionsgrammatik. Damit ist man nicht notwendigerweise gezwungen, die klassische Trennung von Syntax und Lexikon/ Weltwissen aufzugeben: Die Lösung in (b), die wohl am ehesten der Lösung entspricht, die in traditionellen generativen Modellen gewählt würde, erlaubt es natürlich theoretisch, eine unabhängige syntaktische Komponente beizubehalten, in der dann nur diejenigen syntaktischen Prozesse verortet werden, die völlig regelmäßig und vorhersagbar sind. Das logische Argument für die Existenz von Konstruktionen in der Grammatik bliebe davon unberührt, da es ja nur besagt, dass es Konstruktionen gibt, nicht, dass Konstruktionen die einzige Form sprachlichen Wissens darstellen. Allerdings entfällt die Notwendigkeit einer separaten syntaktischen Komponente. Eine solche Notwendigkeit lässt sich auch nicht ohne Weiteres aus der Idee einer Trennung zwischen Kerngrammatik und Peripherie ableiten, da die „unregelmäßigen" Strukturen im Lexikon, wie die WhIAW-Konstruktion zeigt, in den Bereich der Kerngrammatik fallen können (zumindest dann, wenn man annimmt, dass Fragesätze in einer solchen Kerngrammatik zu verorten sind). Aus dieser Tatsache ergibt sich ein starkes Argument dafür, Konstruktionen als einzige Repräsentationsform grammatischen Wissens zu betrachten.

\section{Konstruktionen als einzige Repräsentationsform grammatischen Wissens}

\subsection{Das ökonomische Argument}

Ein konstruktionsverarbeitendes System, also ein Mechanismus, der es erlaubt Strukturen mit idiosynkratischen Eigenschaften zu verarbeiten, kommt auch mit regelhaften Strukturen (also mit solchen obne idiosynkratische Ei- 
genschaften) zurecht (vgl. z.B. Fillmore 1985; Kay 1997). Regelhafte Strukturen sind ja nichts grundsätzlich anderes als idiosynkratische Strukturen, sie sind nur weniger spezifiziert und damit in mehr Zusammenhängen verwendbar. Ein System, das beispielsweise die WhIAW-Konstruktion in (25) verarbeiten kann, kann auch die Struktur in (26) verarbeiten:
$W$ h-Konstruktion ${ }^{15}$
a. $\mathrm{S}_{\mathrm{i}}\left[\left[_{\mathrm{NP}}[\mathrm{N} W\right.\right.$ b] $\mathrm{VP}]$
b. $\mathrm{S}_{\mathrm{i}}$ ist ein Hauptsatz oder
c. Argument eines Matrixsatzes $\mathrm{S}_{j}$

Die $W$ h-Konstruktion in (26) unterscheidet sich von der WhIAW-Konstruktion in (25) nur dadurch, dass letztere zusätzliche formale, semantische und pragmatische Informationen enthält.

Ein regelverarbeitendes System kann dagegen ausschließlich regelhafte Strukturen verarbeiten, da es keine Möglichkeit zur Verfügung stellt, Idiosynkrasien zu berücksichtigen. Natürlich kann man ein System postulieren, das einen Wh-Satz wie Was hast du getan (vgl. 27a) nach allgemeinen Regeln aus (27b) ableitet:

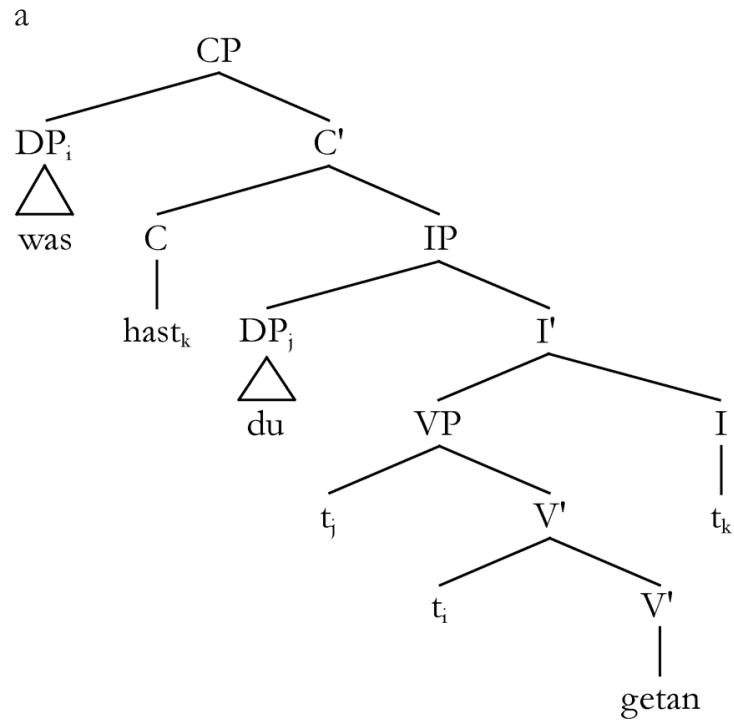

15 Natürlich soll (26) keine auch nur annähernd vollständige Darstellung der Eigenschaften von $W$ h-Sätzen im Deutschen sein, sondern lediglich einen Versuch darstellen, die Gemeinsamkeiten aller $W h$-Sätze in einer Form zu erfassen, die (25) ähnelt. Dabei werden die Unterschiede zwischen Verbzweit- und Verbletztstellung ebenso ignoriert wie die Tatsache, dass das $W$ h-Pronomen das Subjekt oder ein VP-internes Argument sein kann. Diese und weitere Unterschiede lassen sich aber grundsätzlich auf dieselbe Weise darstellen, z.B. $\mathrm{S}_{\mathrm{V} 2}\left[\left[_{\mathrm{NP}}\right.\right.$ $\left.\left.\left[\mathrm{N} W h_{\mathrm{DAT}}\right]\right] \mathrm{VP}_{-\mathrm{DAT}}\right], \mathrm{S}_{\mathrm{Vfin}}\left[\left[_{\mathrm{NP}}\left[\mathrm{N} W h_{\mathrm{AKK}}\right]\right] \mathrm{VP}_{-\mathrm{AKK}}\right]$ usw. (vgl. in diesem Zusammenhang z.B. die HPSG-Analyse von deutschen Fragesätzen in Feldhaus 1999). 
b.

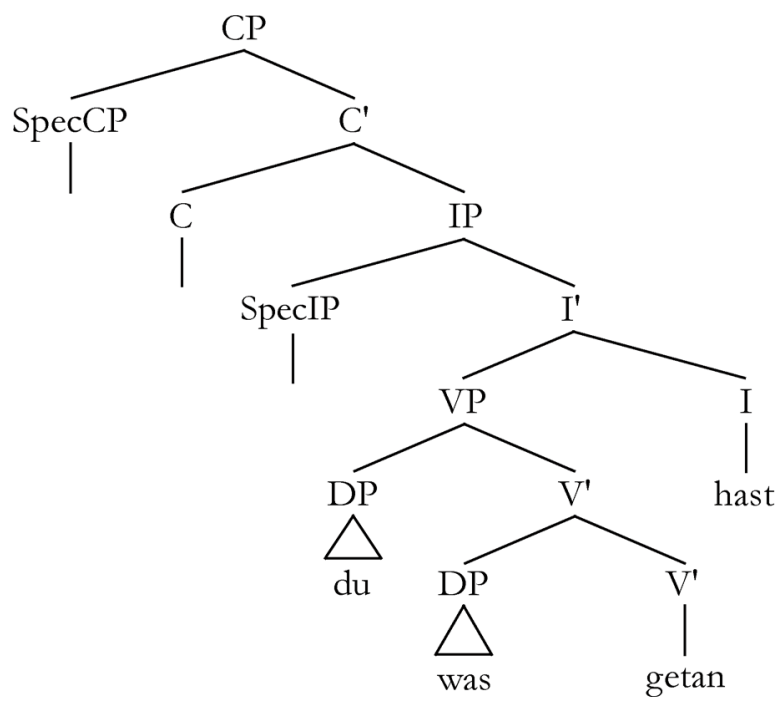

Allerdings kann dieses System den analogen Satz Was in aller Welt hast du getan nicht mit demselben Regelapparat erzeugen, da sich die formalen, semantischen und pragmatischen Beschränkungen nicht aus den in diesem Apparat vorhandenen Regeln ergeben. Es müssten also zusätzliche Regeln eingeführt werden, die eine Einbindung von semantischen und pragmatischen Informationen erlauben. Solche „Regeln“ wären aber von grundsätzlich anderer Art als die Regeln, die (27a) aus (27b) erzeugen und könnten nicht als Teil desselben Systems dargestellt werden; sie würden also ein separates System erfordern (z.B. ein mit den notwendigen Mechanismen versehenes Lexikon). ${ }^{16}$

Aus einem Modell, das von der Existenz eines regelverarbeitenden Systems ausgeht, ergibt sich deshalb notwendigerweise eine Grammatik mit (mindestens) zwei Systemen. Dagegen kommt ein Modell, das von vorneherein ein konstruktionsverarbeitendes System annimmt, mit einem einzigen System aus.

$\overline{16}$ In der generativen Literatur werden einige der formalen Eigenschaften des englischen $[W H$ the bell] damit erklärt, dass das emphatische the hell kein „D-Linking“ zulässt, also nicht auf einen im Diskurs erwähnten bzw. anderweitig identifizierbaren Referenten verweisen darf (Pesetsky 1987). Es ist jedoch nicht klar, inwiefern the bell tatsächlich kein D-Linking erlaubt oder inwiefern die syntaktischen Eigenschaften dieses Ausdrucks aus dieser Tatsache folgen (vgl. Szabolcsi/Zwarts 1993). Ob sich WhIAW und ähnliche deutsche Ausdrücke von $W$ hPronomen im Allgemeinen überhaupt durch D-Linking unterscheiden, will ich nicht beurteilen (siehe dazu Wiltschko 1997); in jedem Fall scheint es nicht möglich, die spezifischen semantischen und syntaktischen Beschränkungen aus dieser Eigenschaft abzuleiten. 
Akzeptiert man also grundsätzlich die Existenz von Konstruktionen im sprachlichen Wissen, verlangt es das Sparsamkeitsprinzip, ein rein konstruktionsverarbeitendes System anzunehmen:

Jedes System, das nicht-kompositionelle und/oder syntaktisch nicht voll regelhafte Strukturen repräsentieren und zur Verarbeitung bereitstellen kann, kann auch kompositionelle und syntaktisch regelhafte Strukturen repräsentieren und zur Verarbeitung bereitstellen.

In den Köpfen von Sprechern existiert ein System, das nicht-kompositionelle und/oder syntaktisch nicht voll regelhafte Strukturen repräsentieren und zur Verarbeitung bereitstellen kann.

Es wäre unökonomisch, zusätzlich zu diesem ohnehin vorhandenen System ein zweites, regelverarbeitendes System anzunehmen.

Hier wird angenommen, dass eine Grammatik mit zwei Systemen (oder eine Grammatik, die um ein nicht-grammatisches System zur Verarbeitung von Konstruktionen ergänzt werden muss), weniger ökonomisch ist als eine Grammatik, die aus einem einzigen (nicht notwendigerweise sprachspezifischen) System besteht. Sparsamkeit wird also anhand der Anzahl benötigter Systeme gemessen. Dagegen ließe sich einwenden, dass eine Lösung mit zwei Systemen dann sparsamer sein könnte, wenn das eine System mit einer kleinen Zahl von Regeln den größten Teil der Grammatik beschreiben könnte, während das andere System nur einige wenige irreguläre Strukturen enthält. In diesem Fall wären die beiden Systeme gemeinsam möglicherweise deutlich kleiner als ein gemeinsames, das das gesamte sprachliche Wissen in Form von Konstruktionen erfasst. Bedenkt man aber, dass selbst das Lexikon im klassischen Sinne (d.h. als reiner Wortspeicher verstanden) für einen durchschnittlichen Sprecher selbst nach konservativen Schätzungen allein 17.000 Lexeme enthalten und zur sofortigen Verarbeitung bereitstellen muss (Goulden et al. 1990), so ist klar, dass Einsparungen von einigen Hundert Konstruktionen nicht ins Gewicht fallen würden, selbst wenn sie möglich sein sollten. ${ }^{17}$

Damit ist natürlich nicht bewiesen, dass die menschliche Sprachfähigkeit tatsächlich auf einem rein konstruktionsverarbeitenden System beruht, denn das Sparsamkeitsprinzip führt nicht notwendigerweise zum realistischsten Modell, aber es legt eine Forschungsstrategie nahe, die solange von einem rein konstruktionsverarbeitenden System ausgeht, bis dieses System an empirischer Evidenz scheitert.

\footnotetext{
Ein zwei-systemiges Modell wäre nicht nur deshalb unökonomisch, weil es gegen das wissenschaftstheoretische Sparsamkeitsprinzip verstoßen würde und damit nur schwer zu rechtfertigen wäre, sondern auch, weil unklar wäre, wie ein solches System evolutionär entstehen sollte, da es zunächst nur eine ohnehin vorhandene Fähigkeit replizieren und damit keinen Selektionsvorteil bringen würde.
} 


\subsection{Empirische Evidenz}

Die Frage nach der grundlegenden Architektur natürlichsprachlicher Grammatiken kann natürlich nicht auf der Grundlage des hier diskutierten logischökonomischen Arguments entschieden werden. Ökonomieargumente können leicht zu falschen Ergebnissen führen, sie müssen deshalb, wie jedes andere Argument, durch empirische Evidenz aus möglichst vielen Phänomenbereichen abgesichert werden.

Empirische Evidenz für ein konstruktionsverarbeitendes System als einziges (oder wenigstens dominantes) System natürlichsprachlicher Grammatiken findet sich aber in einer Reihe von Phänomenbereichen, vom Spracherwerb über die Untersuchung syntaktischer Irregularitäten auch in scheinbar regelgeleiteten Bereichen der Grammatik (die mindestens seit Lakoff 1970 und Ross 1967 einen festen Platz in syntaxtheoretischen Diskussionen haben sollte) bis hin zur Idiomforschung und Phraseologie.

Ich möchte hier nicht näher auf diese Bereiche eingehen, da sie die ausführliche Diskussion, die ihnen zusteht, an anderer Stelle erhalten. Die Forschung zum konstruktionsgestützten Spracherwerb wird ausführlich z.B. in Tomasello (2003) zusammengefasst; speziell zum Deutschen vgl. z.B. Behrens (2009, in diesem Band), vgl. auch die ausführliche Darstellung des Erwerbs von $W h$-Sätzen im Deutschen in Steinkrauss (2009). Die Erforschung syntaktischer Phänomene im Kernbereich der Grammatik, die sich nicht auf allgemeine Regeln reduzieren lassen, hat in Bezug auf das Deutsche gerade erst begonnen, siehe z.B. Jacobs (2008, 2009), Stefanowitsch (2006, 2009) und Webelhuth (in diesem Band). Als Einstieg in die neuere empirische Idiomforschung sei z.B. Wulff (2008) empfohlen, die u.a. zeigt, dass (wie in der Konstruktionsgrammatik erwartbar) ein fließender Übergang zwischen (scheinbar) idiomatischen und (scheinbar) kompositionellen Strukturen besteht.

Stattdessen möchte ich hier auf einen Phänomenbereich eingehen, der bisher wenig Beachtung gefunden hat: die systematische Nichtanwendung scheinbar allgemeiner „Regeln“. In einem regelgestützten System dürfte es zwar eine gelegentliche unsystematische Nichtanwendung von Regeln (im Sinne von „Performanzfehlern“) geben, aber wenn Regeln systematisch nicht angewendet würden, wäre das ein klarer Hinweis auf holistisch repräsentierte Muster, bei deren Abruf und Kombination mit anderen Mustern und Konstruktionen keine allgemeinen Regeln zur Anwendung kommen (oder, um es konstruktionsgrammatisch auszudrücken: kein Abgleich mit allgemeineren, abstrakteren Konstruktionen stattfindet).

Ich werde das am Beispiel des Ausdrucks seinen/ihren Preis haben zeigen, der in (28a) schematisch in seiner häufigsten syntaktischen Form dargestellt ist (typische Beispiele sind 28b-c): 
(28) a. $\mathrm{NP}_{\mathrm{i}}$ hat POSS $_{\mathrm{i}}$ Preis

b. Luxus hat seinen Preis: Ein Whirlpool von guter Qualität kostet rund 7000 bis 8000 Euro. [RHZ06]

c. Doch Qualität hat ibren Preis, kostet viel Geld. [RHZ07]

d. $\mathrm{NP}_{\mathrm{i}}$ hat $\mathrm{POSS}_{\mathrm{i}}$ Ursache/n, Grund/Gründe, Stärken, Schwächen, Febler, Tücken, Risiken, Probleme, Ordnung, Ricbtigkeit, Berechtigung, Gutes, Positives, Vorteile, Nachteile, Für und Wider, zwei Seiten, Grenze/n, Ausnahmen, Zeit, Stunde, Ende, ...

Der Ausdruck ist eine spezielle Variante eines abstrakteren Musters [NP hat $\operatorname{POSS}_{\mathrm{i}} \mathrm{NP}_{\mathrm{j}}$ ], für das in (28d) weitere typische Beispiele aufgeführt sind. Dieses abstraktere Muster scheint nicht voll produktiv zu sein; so sind z.B. die Ausdrücke sein/ihr Gutes/Positives/Schönes haben sehr konventionell, während sich die Antonyme sein/ibr Schlechtes/Negatives/Hässliches baben sehr selten bis nie finden. Dies ist keine allgemeine Beschränkung auf positiv konnotierte Wörter, da z.B. sowohl das Wort Vorteile als auch sein Antonym Nachteile häufig in dem Muster auftreten. Andererseits ist das Muster nicht auf die häufig darin verwendeten Substantive beschränkt, es kann problemlos auf ungewöhnlichere Substantive ausgedehnt werden:

(29) a. Aber alles hat seine Nachwirkungen, ich nehme das Zeug seit 12 Monaten, mir geht's soweit gut. [www.ht-mb.de]

b. 100V oder 110V ist dem Netzteil erfahrungsgemäß egal. Alles hat so seine Toleranzen. [www.embjapan.de]

c. Jedes System hat seine Bugs. [forum.ts.fujitsu.com]

Das Muster ist trotz seiner möglicherweise leicht eingeschränkten Produktivität semantisch weitgehend kompositionell: Es bedeutet so etwas wie „X hat eine Eigenschaft Y, die für X typisch/erwartbar ist", wobei die Komponente „für X typisch/erwartbar" sich aus der Verwendung des Possessivpronomens ableiten lässt. Dafür spricht auch, dass sich ein analog konstruiertes Muster mit derselben Bedeutung auch in anderen Sprachen findet:

a. For Americans, getting sick has its price [washingtonpost.com] „Für Amerikaner hat das Krankwerden seinen Preis.“

b. Reste que virer Pellegrini a son prix: 4 millions d'euros selon les calculs du quotidien sportif espagnol AS. [sportune.fr] „Aber Pellegrini zu entlassen hat seinen Preis: 4 Millionen Euro, nach den Berechnungen der spanischen Sportzeitschrift AS.“

Das Muster ist außerdem syntaktisch regelhaft, es lässt sich z.B. mit allen regulär erwartbaren Wortstellungen und in allen Tempora verwenden, das Verb und das Possessivpronomen der Objekt-NP kongruieren mit dem Subjekt usw. 
In einer regelgestützten Grammatik gäbe es deshalb keinen Grund, das Muster in seiner Gesamtheit zu repräsentieren, es könnte bei Bedarf mit den vorhandenen Regeln aus den vorhandenen Lexikoneinträgen erzeugt werden. In der Konstruktionsgrammatik könnte das Muster aus allgemeineren Konstruktionen im Konstruktikon erzeugt werden, aufgrund des einheitlichen Repräsentationsformats im Konstruktikon könnte es bei entsprechender Gebrauchshäufigkeit aber auch einen eigenen Eintrag (oder mehrere Einträge für verschiedene häufige Varianten) erhalten.

Auf der Grundlage introspektiver Beispiele lassen sich diese beiden Möglichkeiten nicht unterscheiden, aber in Gebrauchsdaten wäre, wie oben angedeutet, ein Unterschied zu erwarten: Wenn das Muster für jedes Gebrauchsereignis aus allgemeinen Regeln erzeugt würde, müssen diese Regeln (von vereinzelten unsystematischen Versprechern abgesehen) vollständig zur Anwendung kommen. Wenn das Muster dagegen holistisch repräsentiert wird und nur diese holistische Repräsentation abgerufen wird, könnten dabei einzelne allgemeinere Regeln systematisch verletzt werden, da sie in einem konkreten Gebrauchsereignis nicht zur Anwendung kommen müssten.

Genau das ist bei dem Muster in (28a) der Fall: Im Deutschen Referenzkorpus findet sich eine beträchtliche Zahl von Beispielen wie den folgenden, bei denen das Possessivpronomen der Objekt-NP nicht mit dem femininen Subjekt kongruiert, sondern stattdessen in der maskulinen/neutralen Form steht:

(31) a. Das aber bedeutet: Qualität hat seinen Preis - bei MTB's kann der in Regionen von 5000 Mark und mehr gehen. [M95]

b. ... denn die anwaltliche Vertretung vor zwei Instanzen hat seinen Preis. [A99]

c. Aber Boppard ist nicht irgendeine Kleinstadt, sondern Tourismusmetropole und Mittelzentrum. Diese Doppelfunktion hat seinen Preis. [RHZ99]

d. Doch die harte Selektion auf dem Weg zum bayerischen Gymnasium hat seinen Preis ... [NUZ05]

Wenn diese Sätze, wie durch ein regelgestütztes Grammatikmodell vorhergesagt, unsystematische Versprecher („Performanzfehler") wären, würden wir erwarten, dass auch der umgekehrte Fall von maskulinen/neutralen Subjekt-NPs mit femininem Possessivpronomen in der Objekt-NP existiert. Und tatsächlich finden sich derartige Beispiele:

a. Der Zugang zur letzten Meile hat ibren Preis [NUZ06]

b. Der Einstieg ins ernsthafte journalistische Geschäft bei der ARD hat ibren Preis. [NUZ06] 
c. Schon nach nur wenigen Stunden Unterricht kann Billie mit auswendig gelernten Floskeln wie „Eine Supermacht zu sein, hat ibren Preis" etwa die brisante Frage nach der Notwendigkeit der Anwesenheit der US-Soldaten im Nahen Osten beantworten. [RHZ05]

Bei der Verteilung der inkongruenten Possessivpronomen zeigt sich aber ein drastisches Missverhältnis zwischen maskulinen/neutralen und femininen Possessivpronomen. Von insgesamt 1.085 Beispielen dieses Musters mit maskulinen oder neutralen Subjekt-NPs finden sich nur drei, in denen das Possessivpronomen feminin ist. Bei den insgesamt 1.129 Beispielen mit femininen Subjekt-NPs finden sich dagegen 40, in denen das Possessivpronomen maskulin/neutral ist (vgl. Tabelle 1, in der die statistisch erwarteten Zufallswerte in Klammern angegeben sind). Dieser Unterschied ist höchst signifikant (Fisher-Yates, $\mathrm{p}<0.001) .{ }^{18}$

\begin{tabular}{|l|c|c|c|}
\hline & Mask./Neut. & Fem. & Gesamt \\
\hline Kongruent & $\begin{array}{c}1082 \\
(1064)\end{array}$ & $\begin{array}{c}1089 \\
(1107)\end{array}$ & 2171 \\
\hline Inkongruent & 3 & 40 & 43 \\
& $(21)$ & $(22)$ & \\
\hline Gesamt & 1085 & 1129 & 2214 \\
\hline
\end{tabular}

Tab. 1: Häufigkeit von kongruenten und nicht-kongruenten Possessivpronomen im Muster $\left[\mathrm{NP}_{\mathrm{i}}\right.$ hat POSS $_{\mathrm{i}}$ Preis] (Deutsches Referenzkorpus, Archiv W)

In einem konstruktionsgestützten Grammatikmodell erklärt sich diese Asymmetrie auf natürliche Weise, wenn man annimmt, dass im Konstruktikon die Muster mit dem maskulinen/neutralen bzw. dem femininen Possessivpronomen jeweils eigene Repräsentationen haben (d.h., dass es getrennte (aber natürlich verknüpfte) Einträge für $\left[\mathrm{NP}_{\mathrm{i}}\right.$ hat seinen Preis] und [ $\mathrm{NP}_{\mathrm{i}}$ bat ihren Preis] gibt), und dass die Variante mit dem maskulinen/neutralen Pronomen kognitiv stärker verankert wäre. Von diesen Repräsentationen würde dann normalerweise diejenige ausgewählt, die zum Genus der in die Leerstelle eingefügten Subjekt-NP passt. Da aber keine allgemeine Kongruenzregel zur Anwendung käme, würde die $\left[\mathrm{NP}_{\mathrm{i}}\right.$ hat seinen Preis] aufgrund ihrer stärkeren Verankerung auch dann manchmal gewählt werden, wenn die SubjektNP feminin ist.

$18 \quad$ Tatsächlich ist die Asymmetrie zwischen den Kongruenzfehlern mit maskulinen und denen mit femininen Possessivpronomen noch größer als der statistische Unterschied suggeriert. Die Beispiele für letztere, die in (32) komplett aufgelistet sind, lassen sich in zwei Fällen klar als Flüchtigkeitsfehler analysieren, bei denen eine feminine NP (Meile, ARD) zwischen dem Subjekt und dem Possessivpronomen steht und zur femininen Kongruenz verleitet; der dritte Fall ist sogar ein direktes nicht-muttersprachliches Zitat. Bei den maskulinen Kongruenzfehlern gibt es manchmal eine entsprechende maskuline NP zwischen Subjekt und Possessivpronomen (Gymnasium in [31d]), aber in den meisten Fällen ist dies dort nicht der Fall. 
Diese Erklärung kann natürlich nur dann überzeugen, wenn es einen unabhängigen Grund für die Annahme gibt, dass die maskuline/neutrale Variante des Musters stärker verankert ist. Ein solcher Grund könnte entweder in der jeweiligen Häufigkeit der beiden Varianten oder in der Häufigkeitsverteilung von Wörtern innerhalb jeder der Varianten liegen.

Die Häufigkeit der beiden Varianten unterscheidet sich nicht sehr stark: Die Variante mit dem maskulinen/neutralen Pronomen macht 51 Prozent aus, die mit dem femininen 49 Prozent. Ein solcher Unterschied dürfte kaum reichen, um das eine Muster sehr viel stärker zu verankern als das andere. Und tatsächlich ist ohnehin nicht anzunehmen, dass die reine Häufigkeit einer Struktur die hier beobachtete Art von Kongruenzfehlern beeinträchtigen könnte, da in diesem Fall zu erwarten wäre, dass solche Fehler überall dort auftreten müssten, wo eine Variante eines sprachlichen Ausdrucks häufiger als die anderen Varianten ist.

Anders sieht es bei der Verteilung der Subjekt-NPs innerhalb der beiden Varianten aus: Diese zeigt eine Asymmetrie, die potenziell eine Erklärung für die beobachteten Kongruenzfehler liefern könnte. Tabelle 2 zeigt die jeweils zehn häufigsten Substantive in der Subjektposition der beiden Muster.

\begin{tabular}{|l|c|l|c|}
\hline \multicolumn{2}{|c|}{$\left[\mathrm{NP}_{i}\right.$ hat seinen $_{i}$ Preis $]$} & \multicolumn{2}{c|}{$\left[\mathrm{NP}_{i}\right.$ hat ibren $_{i}$ Preis] } \\
\hline Subjekt-NP & Häufigkeit & Subjekt-NP & Häufigkeit \\
\hline das & 213 & Qualität & 201 \\
\hline alles & 121 & Sicherheit & 48 \\
\hline Erfolg & 31 & Schönheit & 30 \\
\hline das alles & 25 & Freiheit & 27 \\
\hline Luxus & 25 & Arbeit & 23 \\
\hline Qualität & 14 & Technik & 22 \\
\hline der & 14 & die & 18 \\
\hline Aufwand & 13 & Exklusivität & 18 \\
\hline Angebot & 12 & Demokratie & 15 \\
\hline Fortschritt & 11 & Leistung & 15 \\
\hline
\end{tabular}

Tab. 2: Die zehn häufigsten Subjekte im Muster $\left[\mathrm{NP}_{\mathrm{i}}\right.$ hat seinen/ihren Preis] (DeReKo, Archiv W)

Bei der Variante mit maskulinem/neutralem Possessivpronomen machen die beiden häufigsten Muster das hat seinen Preis und alles hat seinen Preis (unter Einbeziehung des kombinierten das alles hat seinen Preis) fast ein Drittel $\left(31,8\right.$ Prozent) aller Vorkommen des Musters $\left[\mathrm{NP}_{i}\right.$ bat seinen ${ }_{i}$ Preis $]$ aus. Zwischen diesen beiden Mustern und dem nächsthäufigsten Ausdruck Erfolg hat seinen Preis fällt die Häufigkeit auf ein Zehntel ab. 
Bei der Variante mit femininem Possessivpronomen gibt es dagegen keinen Ausdruck, der sich in seiner Häufigkeit derartig stark absetzt. Der häufigste Ausdruck Qualität hat ibren Preis macht zwar immerhin ein Fünftel

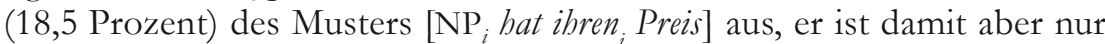
viermal häufiger als die nächsthäufigste Subjekt-NP Sicherheit.

Angesichts dieser klaren Häufigkeitsunterschiede ist es durchaus plausibel anzunehmen, dass die Variante [(das/alles) hat seinen Preis] aufgrund ihrer relativen Häufigkeit ein stark verankertes eigenständiges Muster darstellt und so dazu beiträgt, dass auch das abstraktere Muster $\left[\mathrm{NP}_{\mathrm{i}}\right.$ bat seinen $_{\mathrm{i}}$ Preis $]$ insgesamt stärker verankert ist als die Variante mit dem femininen Possessivpronomen. Auf dieses stärker verankerte Muster greifen Sprecher/innen dann häufig auch in den Fällen zu, in denen sie eine feminine Subjekt-NP in die Leerstelle einsetzen. Die Kongruenz von Subjekt-NP und Possessivpronomen wird dabei nicht überprüft. Solche systematischen Regelverletzungen im Kontext spezifischer grammatischer Muster stellen klare empirische Evidenz für ein konstruktionsgestütztes Grammatikmodell dar, vor allem, wenn sich weitere ähnliche Fälle finden; ob dies der Fall ist, bleibt abzuwarten.

\section{Schlussbemerkungen}

Die Grammatik natürlicher Sprachen wird in der Sprachwissenschaft ganz selbstverständlich als regelverarbeitendes System betrachtet. Diese Sichtweise hat eine lange Tradition, deren Ursprünge weit vor der Entstehung generativer Grammatiktheorien liegen; vor dem Aufkommen beschränkungsgestützter Modelle ist sie nie ernsthaft infrage gestellt worden (und auch in Letzteren liegt das Hauptaugenmerk auf der Modellierung von Regularitäten). Die Selbstverständlichkeit dieser Tradition führt dazu, dass schon bei der Suche nach theorierelevanter Evidenz, und erst recht bei deren Interpretation, Phänomene bevorzugt werden, die sich in eine regelgestützte Sichtweise einfügen.

Angesichts dieser Tradition ist nicht zu erwarten, dass sich eine alternative Sichtweise schnell durchsetzen wird, selbst wenn sie, wie die Konstruktionsgrammatik, durch eine wachsende Zahl methodisch ausgereifter empirischer Studien aus dem Spracherwerb, der Psycholinguistik, der diachronen Linguistik, der Typologie und zunehmend auch der Syntaxtheorie gestützt wird.

Die Diskussion um die Architektur menschlicher Sprachen wäre aber wissenschaftlich sauberer, wenn das logisch-ökonomische Argument als syntaxtheoretische Nullhypothese etabliert würde. Anstatt davon auszugehen, dass Sprache regelgestützt ist, und alles, was diesen Regeln nicht gehorcht, ins Lexikon abzuschieben, sollte die Sprachwissenschaft mit der einfacheren 
Hypothese anfangen, dass die kognitiven Prozesse, die uns die Verarbeitung von idiosynkratischen Konstruktionen auf den verschiedensten Komplexitäts- und Abstraktionsstufen ermöglichen, auch zur sehr viel weniger anspruchsvollen Verarbeitung regelhafter Strukturen befähigen.

Die beiden hier vorgestellten Fallstudien zeigen, wie theoretisch motivierte empirische Analysen einzelner Muster und Konstruktionen dazu beitragen können, die Reichweite eines konstruktionsgestützten Grammatikmodells zu erforschen. Die erste Studie zeigt, wie unvorhersagbare formale und semantische Eigenschaften einzelner Konstruktionen direkt in die Kerngrammatik hinein wirken und deshalb nicht als randständige Ausnahmefälle betrachtet werden können. Die zweite Studie zeigt anhand systematischer Regelverletzungen, dass auch vollständig (oder weitgehend) vorhersagbare Syntagmen in ihrer Gesamtheit repräsentiert und verarbeitet werden können. Diese Art von Regelverletzung stellt möglicherweise die beste Evidenz für ein konstruktionsgestütztes Grammatikmodell dar, die sich außerhalb der Spracherwerbsforschung und der Psycholinguistik finden lässt.

Fallstudien einzelner Konstruktionen sind natürlich potenziell immer dem Vorwurf einer geringen Reichweite ausgesetzt. Sie sind aber für die konstruktionsgrammatische Forschung in der aktuellen Phase der Theoriefindung und Theoriediskussion unvermeidlich; sieht man von der HPSG ab, so bieten Konstruktionsgrammatiken derzeit kein Grammatikmodell, das zur vollständigen Beschreibung einer bestimmten Sprache taugt. Konstruktionsgrammatiken sollen auch eher Theorien darüber sein, wie sprachliches Wissen erlernt, repräsentiert und in der Sprachproduktion angewendet wird. Bei allen unüberbrückbaren theoretischen Differenzen zur traditionellen Generativen Grammatik, wie sie im vorliegenden Band etwa von Gereon Müller vertreten wird, ist dies interessanterweise eine Eigenschaft, die die beiden theoretischen Lager eint: Auch die traditionelle Generative Grammatik hat nie eine auch nur annähernd vollständige Beschreibung einer Sprache hervorgebracht - unser Wissen über Sprache und notwendige sprachwissenschaftliche Fragestellungen hat sie trotzdem, und trotz ihrer deskriptiven Grenzen, ein großes Stück vorangebracht.

\section{Daten}

Deutsches Referenzkorpus (DeReKo) am Institut für Deutsche Sprache, Mannheim, Archiv der geschriebenen Sprache (W-öffentlich). Internet: www.ids-mannheim.de/ kl/projekte/korpora (Stand: 20.12.2010). 


\section{Literatur}

Abeillé, Anne/Schabes, Yves (1989): Parsing idioms in lexicalized TAGs. In: Proceedings of the Fourth Conference on European Chapter of the Association For Computational Linguistics. Morristown, NJ, S. 1-9.

Behrens, Heike (2009): Konstruktionen im Spracherwerb. In: Zeitschrift für Germanistische Linguistik 37, S. 427-444.

Behrens, Heike (in diesem Band): Die Grenzen des lexikalischen Lernens: Konstruktionsprozesse im Spracherwerb.

Diessel, Holger (1997): Verb-first constructions in German. In: Verspoor, Marjolijn/ Dong, Lee Kee/Sweetser, Eve (Hg.): Lexical and syntactical constructions and the construction of meaning: proceedings of the bi-annual ICLA meeting in Albuquerque, July 1995. Amsterdam u.a., S. 51-68.

Feldhaus, Anke (1999): German Wh-interrogatives in HPSG. In: Kordoni, Valia (Hg.): Tübingen studies in head-driven phrase structure grammar. (= Arbeitspapiere des SFB 340, 132). Tübingen, S. 459-493.

Fillmore, Charles J. (1985): Syntactic intrusions and the notion of grammatical construction. In: Proceedings of the Eleventh Annual Meeting of the Berkeley Linguistic Society, S. 73-86.

Fischer, Kerstin/Stefanowitsch, Anatol (2006): Konstruktionsgrammatik: Ein Überblick. In: Fischer/Stefanowitsch (Hg.), S. 3-17.

Fischer, Kerstin/Stefanowitsch, Anatol (Hg.) (2006): Konstruktionsgrammatik. Von der Anwendung zur Theorie. (= Stauffenburg Linguistik 40). Tübingen.

Goldberg, Adele E. (1995): Constructions: a Construction Grammar approach to argument structure. Chicago.

Goldberg, Adele E. (2006): Constructions at work. The nature of generalization in language. Oxford u.a.

Goulden, Robin/Nation, Paul/Read, John (1990): How large can a receptive vocabulary be? In: Applied Linguistics 11, S. 341-363.

Grimm, Jacob/Grimm, Wilhelm (1854-1960): Deutsches Wörterbuch. 16 Bde. Leipzig.

Holler, Anke (2007): Interrogativum. In: Hoffmann, Ludger (Hg.): Handbuch der deutschen Wortarten. Berlin/New York, S. 445-482.

Jacobs, Joachim (2008): Wozu Konstruktionen? In: Linguistische Berichte 213, S. 3-44.

Jacobs, Joachim (2009): Valenzbindung oder Konstruktionsbindung? Eine Grundfrage der Grammatiktheorie. In: Zeitschrift für Germanistische Linguistik 37, S. 490 513.

Kay, Paul (1997): Construction grammar. In: Kay, Paul (Hg.): Words and the grammar of context. (= CSLI Lecture Notes 40). Stanford, S. 123-131.

Kay, Paul/Fillmore, Charles (1999): Grammatical constructions and linguistic generalizations: the What's X doing Y construction. In: Language 75, S. 1-34. 
Lakoff, George (1970). Irregularity in syntax. New York u.a.

Lakoff, George (1987): Women, fire, and dangerous things: what categories reveal about the mind. Chicago u.a.

Langacker, Ronald W. (1987): Foundations of Cognitive Grammar: theoretical prerequisites. Stanford.

Leiss, Elisabeth (2009): Sprachphilosophie. Eine Einführung in die Axiomatik. Berlin.

Müller, Gereon (in diesem Band): Regeln oder Konstruktionen? Von verblosen Direktiven zur sequenziellen Nominalreduplikation.

Müller, Stefan (2006): Resultativkonstruktionen, Partikelverben und syntaktische vs. lexikonbasierte Konstruktionen. In: Fischer/Stefanowitsch (Hg.), S. 177-202.

Pesetsky, David (1987): Wh-in-situ: movement and unselective binding. In: Reuland, Eric J./ter Meulen, Alice G. B. (Hg.): The representation of (in)definiteness. (= Current Studies in Linguistics Series 14). Cambridge, MA u.a., S. 98-129.

Ross, John R. (1967): Constraints on variables in syntax. Diss. Cambridge, MA. [Neu aufgelegt als Ross, John R. (1986): Infinite syntax! Norwood, NJ.]

Sailer, Manfred (2003): Combinatorial semantics and idiomatic expressions in headdriven phrase structure grammar. (= Arbeitspapiere des SFB 340,161). Stuttgart/ Tübingen.

Stefanowitsch, Anatol (2006): Konstruktionsgrammatik und Korpuslinguistik. In: Fischer/Stefanowitsch (Hg.), S. 151-176.

Stefanowitsch, Anatol (2009): Bedeutung und Gebrauch in der Konstruktionsgrammatik: Wie kompositionell sind modale Infinitive im Deutschen? In: Zeitschrift für Germanistische Linguistik 37, S. 565-592.

Steinkrauss, Rasmus (2009): Frequency and function in WH-question acquisition. A usage-based case study of German L1 acquisition. Diss. Groningen.

Szabolcsi, Anna/Zwarts, Frans (1993): Weak islands and an algebraic semantics of scope taking. In: Natural Language Semantics 1, S. 235-284.

Tomasello, Michael (2003): Constructing a language: a usage-based theory of language acquisition. Cambridge, MA u.a.

Wahrig-Burfeind, Renate (Hg.) (2006): Wahrig Deutsches Wörterbuch. 8., vollst. neu bearb. u. aktual. Aufl. Gütersloh.

Webelhuth, Gerd (in diesem Band): Paradigmenwechsel rückwärts: die Renaissance der grammatischen Konstruktion.

Wiltschko, Martina (1997): D-linking, scrambling, and superiority in German. In: Groninger Arbeiten zur Germanistischen Linguistik 41, S. 107-142.

Wulff, Stefanie (2008): Rethinking idiomaticity: a usage-based approach. London/New York. 
Gereon Müller

\title{
Regeln oder Konstruktionen?
}

\author{
Von verblosen Direktiven zur sequenziellen Nominalreduplikation ${ }^{1}$
}

\begin{abstract}
Linguistische Ausdrücke, die offensichtlich aus kleineren Teilen zusammengesetzt sind, deren formale oder funktionale Eigenschaften jedoch nicht auf der Basis dieser kleineren Teile bestimmt werden können, kann man als Konstruktionen bezeichnen. Eine Standardannahme in regelbasierten Grammatikmodellen ist, dass komplexe linguistische Ausdrücke ins Lexikon gehören, wenn sie Konstruktionen sind, und in einem regelbasierten Bereich der Grammatik erfasst werden, wenn sie keine Konstruktionen sind. Dies führt zu einer inhomogenen und konzeptuell daher wenig attraktiven Theorie, die zwei mögliche Quellen für komplexe linguistische Ausdrücke vorsieht: Lexikon und Grammatik. Grundsätzlich gibt es zwei Auswege aus diesem Dilemma: Zum einen kann man die Rolle von Konstruktionen stärken, so dass Konstruktionen viel oder sogar alles von dem abdecken, was traditionell von grammatischen Regeln behandelt wird. Zum anderen kann man aber auch versuchen, die Rolle von Regeln zu stärken, so dass Regeln viel oder sogar alles von dem abdecken, wofür man typischerweise Konstruktionen bemüht. In diesem Aufsatz möchte ich anhand von zwei Phänomenen in der Grammatik des Deutschen, die auf den ersten Blick wie Musterexemplare für Konstruktionen aussehen, argumentieren, dass ein ausschließlich regelbasierter Ansatz nicht nur deskriptiv konkurrenzfähig ist, sondern darüber hinaus auch explanativ überlegen. Die untersuchten Phänomene sind verblose Direktive (wie in „Her mit dem Geld!") einerseits und sequenzielle Nominalreduplikation (wie in "Jahr für Jahr") andererseits. Die allgemeine Konklusion ist, dass es vermutlich (außer, trivialerweise, Morphemen) gar keine Konstruktionen gibt.
\end{abstract}

\section{Hintergrund}

\section{$1.1 \quad$ Konstruktionen}

Der Begriff der Konstruktion lässt sich wie in (1) fassen. ${ }^{2}$

(1) Konstruktion: Ein sprachlicher Ausdruck $\Gamma$ ist eine Konstruktion, wenn (a) und (b) gilt.

\footnotetext{
Dank für hilfreiche Hinweise an Sebastian Bank, Manfred Bierwisch, Petr Biskup, Sandra Döring, Stefan Engelberg, Juliana Goschler, Lutz Gunkel, Fabian Heck, Joachim Jacobs, Alexander Jahraus, Stefan Keine, Tibor Kiss, Guido Mensching, Stefan Müller, Karl Mund, Sandra Pappert, Beatrice Primus, Kristel Proost, Uli Sauerland, Anatol Stefanowitsch, Wolfgang Sternefeld, Jochen Trommer, Bernd Wiese, Edeltraud Winkler, Gisela Zifonun und Peter Uhrig; und allgemein an die Teilnehmer der Ringvorlesung ,Algorithmen und Muster Strukturen in der Sprache" an der FU Berlin (Mai 2009), der GGS-Tagung an der Universität Leipzig (Mai 2009) sowie der Jahrestagung „Sprachliches Wissen zwischen Lexikon und Grammatik“ des Instituts für Deutsche Sprache Mannheim (März 2010).
} 
a. Es gibt Evidenz dafür, dass $\Gamma$ aus kleineren Teilen $\alpha_{1}-\alpha_{\mathrm{n}}$ zusammengesetzt ist.

b. Die formalen oder funktionalen Eigenschaften von $\Gamma$ können nicht allein auf der Grundlage der Eigenschaften von $\alpha_{1}-\alpha_{n}$ bestimmt werden.

Unter dieser Perspektive werden Phraseme (d.h., idiomatische Ausdrücke) oft als prototypische Konstruktionen betrachtet. Komplexe syntaktische Kategorien, deren formale und funktionale Eigenschaften systematisch auf der Basis der Eigenschaften ihrer Teile berechnet werden können, sind dagegen keine Konstruktionen im Sinne von (1). Es ist eine Standardannahme in regelbasierten Grammatikmodellen, dass Konstruktionen systematisch einen anderen Status haben als Nicht-Konstruktionen: Sprachliche Ausdrücke, die Konstruktionen sind, gehören ins (mentale) Lexikon: Die unvorhersagbaren Eigenschaften von Konstruktionen müssen durch besondere lexikalische Regeln (Chomsky 1980) oder durch die Postulierung gelisteter syntaktischer Objekte (Di Sciullo/Williams 1987; Jackendoff 1997) erfasst werden. Sprachliche Ausdrücke, die keine Konstruktionen sind, werden demgegenüber in einem regelbasierten Teil der Grammatik generiert: Wenn die Eigenschaften eines sprachlichen Ausdrucks auf der Grundlage der Eigenschaften seiner Teile vorhersagbar sind, dann existiert der sprachliche Ausdruck nicht im Lexikon; vielmehr wird er durch grammatische Regeln (in den strukturaufbauenden Komponenten Morphologie und Syntax) abgeleitet.

Diese systematische Zweiteilung in regelbasierten Grammatiken ist wie gesagt eine Standardannahme. Sie führt allerdings unmittelbar zu einem konzeptuellen Problem, denn es entsteht so eine uneinheitliche Theorie mit zwei möglichen Quellen für komplexe sprachliche Ausdrücke, nämlich (i) Lexikon und (ii) System der grammatischen Regeln. Es gibt zwei einigermaßen radikale (und daher potenziell interessante) Auswege aus diesem Dilemma. Zum einen kann man die Rolle von Konstruktionen stärken (vgl. Jackendoff 1997, 2002; Culicover/Jackendoff 2005), so dass Konstruktionen das meiste (oder sogar alles) von dem, was traditionell durch regelbasierte Systeme abgeleitet wird, abdecken können (vgl. hierzu neben anderen Ackerman/Webelhuth 1998, Goldberg 2003, 2006 und Tomasello 2003). Zum anderen kann man aber auch die Rolle von Regeln stärken, so dass Regeln das meiste (oder sogar alles) von dem, was üblicherweise durch Bezug auf

(1) repräsentiert eine von zwei Versionen des Konstruktionsbegriffs im Rahmen der Konstruktionsgrammatik. Alternativ dazu ist auch ein schwächeres (und im Folgenden ignoriertes) Konzept der Konstruktion postuliert worden, demzufolge ein sprachlicher Ausdruck ungeachtet der Frage der Vorhersagbarkeit seiner Eigenschaften auf der Basis der Eigenschaften seiner Teile bereits dann als Konstruktion gilt, wenn er hinreichend häufig ist bzw. hinreichend tief im Sprachsystem verwurzelt ist; vgl. Goldberg (2006) und Stefanowitsch (2009) zur Diskussion dieser Alternativen. 
Konstruktionen erfasst wird, abdecken können. Für Letzteres möchte ich in diesem Aufsatz argumentieren. Konkret möchte ich zeigen, dass die Detailanalyse von scheinbaren Konstruktionen häufig ergibt, dass regelbasierte Ansätze letztlich doch erfolgreich sein können, vorausgesetzt, dass grammatische Regeln hinreichend abstrakt sein können. Wenn dieses Ergebnis verallgemeinert werden kann, ist die Rolle von Konstruktionen vielleicht minimal: Nur Morpheme sind Konstruktionen (und müssen demgemäß im Lexikon abgespeichert werden); alle anderen sprachlichen Ausdrücke werden durch grammatische Regeln in der Morphologie oder in der Syntax abgeleitet.

Warum sind Morpheme notwendigerweise Konstruktionen? Die Antwort ergibt sich aus der Betrachtung einer der zentralen konstitutiven Eigenschaften aller natürlichen Sprachen (neben Rekursion; vgl. dazu u.a. Chomsky 1957; Hauser et al. 2002; Friederici et al. 2006 und Roeper 2007), und zwar der doppelten Artikulation (vgl. Martinet 1964; Eisenberg 2000; Williams 2005). Doppelte Artikulation beschreibt den Umstand, dass sprachliche Ausdrücke auf zwei unterschiedlichen Ebenen kodiert sind: Sie können aufgespalten werden in einerseits minimale Einheiten, die Bedeutung tragen (Morpheme), und andererseits minimale Einheiten, die Bedeutung unterscheiden (Phoneme). Doppelte Arikulation stellt sicher, dass diskrete Unendlichkeit sprachlicher Ausdrücke auf der Basis eines sehr kleinen Inventars irreduzibler Elemente erreicht werden kann, und trägt so wesentlich zu Ökonomie und Eleganz von Grammatiken natürlicher Sprachen bei. Allerdings sind alle Morpheme (die aus mehr als einem Phonem bestehen) per se Konstruktionen, weil die Eigenschaften eines Morphems nicht vorhergesagt werden können auf der Grundlage der Eigenschaften seiner Teile (der Phoneme), auch wenn grammatische Regeln die Kombination von Phonemen zu Morphemen beschränken (über ein System phonologischer Regeln). Morpheme müssen also im mentalen Lexikon abgespeichert sein. Die Hypothese, die ich im Folgenden zu Grunde legen werde, ist daher, dass nur Morpheme Konstruktionen sind, sonst nichts (vgl. zu dieser Position auch Marantz 1998, mit „Wurzel“ anstelle von „Morphem“).

Aus dieser Perspektive muss man argumentieren, dass sowohl formale, als auch interpretative Eigenschaften scheinbar irregulärer sprachlicher Ausdrücke letztlich doch immer als systematisch erwiesen werden können. Ich werde über den zweiten Punkt in diesem Aufsatz nichts Neues zu sagen haben und beschränke mich daher auf den Hinweis, dass es in der Literatur verschiedene wohletablierte Techniken für eine kompositionelle Interpretation von Phrasemen (auch wenn diese zunächst einmal vollkommen opak erscheinen) gibt; vgl. Ruhl (1975), Chomsky (1980), Pesetsky (1985), Gazdar et al. (1985), Everaert (1991), Nunberg et al. (1994), Marantz (1998), Sailer (2003), Wunderlich (2004) zu unterschiedlichen Ansätzen und Komplikationen. So kann man etwa vereinfacht ein Phrasem wie das englische 
spill the beans kompositionell so interpretieren, dass spill ,ausplaudern' heißt im Kontext von beans, und beans, Information' bedeutet im Kontext von spill. Nicht immer muss dabei einem Teil eines Phrasems selbst ein nichttrivialer semantischer Beitrag attribuiert werden. Für kick the bucket kann man z.B. annehmen, dass kick, sterben' heißt im Kontext von bucket, dass bucket aber ein Expletivum ist im Kontext eines solchen Verbs kick (oder hier die Identitätsfunktion denotiert); the wäre dann ein Expletivum im Kontext eines Expletivums. ${ }^{3}$ Dies vermehrt dann selbstverständlich (aber auf harmlose Art und Weise) die Zahl der Lexikoneinträge.

Ob derartige Techniken, scheinbar nicht-kompositionelle komplexe sprachliche Ausdrücke kompositionell zu interpretieren, aus (von der Vermeidung von Konstruktionen gemäß (1) unabhängiger) konzeptueller Perspektive attraktiver (weil möglicherweise, wie Marantz 1998 behauptet, restriktiver) sind, ist eine offene (und kontrovers diskutierte) Frage, deren Beantwortung für die gegenwärtigen Zwecke aber nicht notwendig ist. Fest steht, dass mit Hilfe solcher Techniken in allen relevanten Fällen (außer wie gesagt bei Morphemen) empirisch im Wesentlichen äquivalente Ergebnisse erzielt und ein Konstruktionsstatus komplexer sprachlicher Ausdrücke (im Sinne von (1)) vermieden werden kann. ${ }^{4}$ Damit bleibt die entscheidende Frage, wie es um formale Eigenschaften komplexer sprachlicher Ausdrücke in der deutschen Grammatik bestellt ist, die sich regelbasierten Analysen zu widersetzen scheinen. Um diese Frage bzgl. der beiden ausgewählten Phänomene (verblose Direktive und sequenzielle Nominalreduplikation) zu klären, ist es zunächst notwendig, einige Grundannahmen über die Grammatiktheorie darzulegen, vor deren Hintergrund die Analyse dann erfolgen kann.

\subsection{Grundannahmen}

Vorausgesetzt wird im Folgenden eine derivationelle minimalistische Grammatik (vgl. Chomsky 1995, 2001, 2008) mit einer postsyntaktischen phonologischen Realisierung syntaktischer Köpfe (Distribuierte Morphologie; vgl. Halle/Marantz 1993, 1994; Noyer 1992; Halle 1997; Harley/Noyer 2003; Embick/Noyer 2001).

3 Dass Artikel semantisch leer sein können, ist ja an sich nichts Ungewöhnliches; das ist z.B. der naheliegende Schluss für Artikel in Kontexten mit Prädikatsnomina wie Er ist (ein) Lehrer.

$4 \quad$ Wenn so alle Phraseme letztlich kompositionell interpretiert werden, kann man die selektive transformationelle Defektivität von Phrasemen (vgl. Fraser 1970) nicht mehr durch eine Unterscheidung in kompositionelle und nichtkompositionelle Phraseme ableiten, wie das Nunberg et al. (1994) tun. Tatsächlich erweist sich die Datenlage in diesem Bereich bei genauerer Analyse als viel komplizierter. Relevante Faktoren sind der involvierte Transformationstyp und der Grad der semantischen Opazität des Phrasems; letzterer ist bestimmbar mit Hilfe einer komplexen Skala, die die Qualität und Quantität der Annahmen kodiert, die für eine kompositionelle Analyse gemacht werden müssen; vgl. Müller (2000), auf der Basis von Cernyševa (1970) und Šanskij (1972). 
Die Grammatik ist dabei wie folgt aufgebaut. Am Anfang steht das (mentale) Lexikon. Dieses umfasst eine Liste von (abstrakten) Morphemen und eine Liste von (konkreten) Vokabularelementen; es beinhaltet aber keine Regeln (und ist somit keine generative Komponente der Sprachfähigkeit). Dem Lexikon nachgeordnet ist die Numeration. Hier werden für die Derivation komplexer sprachlicher Objekte (Sätze) Morpheme zunächst aus dem Lexikon selegiert und dann mit nicht-inhärenten Merkmalen angereichert (z.B. Kasus-und Numerusinformation bei Nomina, Tempus-, Modus-, Person-und Numerusinformation bei Verben). Darauf folgt als dritte Komponente die Syntax: Im Laufe der syntaktischen Derivation operieren elementare syntaktische Operationen wie Verkettung (merge), Bewegung (move) und Abgleich (agree). Schließlich folgt am Ende (also postsyntaktisch) die phonologische Realisierung, in deren Verlauf u.a. die von der Syntax manipulierten und zu immer größeren Kategorien verketteten abstrakten Morpheme durch Vokabulareinsetzung (d.h., erneuten Zugriff auf das Lexikon, nur eben eine andere Liste) konkret realisiert werden (ebenso folgt - irrelevanterweise für alles Weitere - die semantische Interpretation).

Vorausgesetzt sei, dass es zwei Typen von Merkmalen gibt, die syntaktische Operationen auslösen (vgl. Heck/Müller 2007; aufbauend auf Adger 2003; Roberts/Roussou 2002; Sternefeld 2006): Auf der einen Seite sind das strukturaufbauende Merkmale (sog. Randmerkmale sowie Subkategorisierungsmerkmale), die eine externe respektive interne Verkettung (d.h. Basisverkettung oder Bewegung) auslösen. Diese werden wie folgt notiert: [•F•] (mit F als Variable über diversen Merkmalstypen, z.B. Kategoriemerkmalen bei der Subkategorisierung). Auf der anderen Seite sind das Sondenmerkmale (probes), die einen Abgleich auslösen (z.B. bei Phänomenen wie Kasuszuweisung oder Kongruenz), und die als [*F*] notiert werden. Strukturaufbauende Merkmale $([\bullet \mathrm{F} \bullet])$ und Sondenmerkmale $([* \mathrm{~F} *])$ bedürfen jeweils eines entsprechenden Merkmals [F] auf einer Zielkategorie, um die von ihnen kodierte syntaktische Operation auszulösen; nach der Operation werden sie gelöscht (bzw. als abgearbeitet markiert und für die weitere syntaktische Derivation unzugänglich gemacht). Die Rolle von strukturaufbauenden Merkmalen und Sondenmerkmalen in der syntaktischen Derivation wird durch zwei komplementäre Beschränkungen erfasst. Das Ökonomieprinzip in (2a) verlangt, dass jede syntaktische Operation merkmalsgetrieben ist; die Merkmalsbedingung in (2b) besagt demgegenüber, dass alle operationsauslösenden Merkmale auf einem lexikalischen Element (einem Morphem aus dem Lexikon, das vorher in der Numeration angereichert wurde) abgearbeitet werden müssen, bevor die Projektion des Elements als Phrase (XP) selbst wiederum Gegenstand weiterer Verkettungsoperationen mit neuen Köpfen sein kann.

(2) a. Ökonomieprinzip: Jede syntaktische Operation muss entweder $[\bullet \mathrm{F} \bullet$ oder $[* \mathrm{~F} *]$ abarbeiten. 
b. Merkmalsbedingung: Ein Merkmal $[\bullet F \bullet]$ oder $[* F *]$ auf einer syntaktischen Kategorie X muss abgearbeitet werden, bevor XP eingebettet werden kann (oder ein finaler Wurzelknoten wird).

Vor diesem Hintergrund kann nun in den Abschnitten 2 respektive 3 die Frage angegangen werden, ob eine regelbasierte, den Konstruktionsstatus leugnende Analyse von verblosen Direktiven und sequenzieller Nominalreduplikation im Deutschen möglich und vielleicht sogar vorteilhaft ist.

\section{Verblose Direktive}

\subsection{Daten}

Jacobs (2008) beobachtet, dass verblose Direktive im Deutschen (mit einer „direktional-resultativen Prädikation") Eigenschaften haben, die sich einer regelbasierten Analyse zu widersetzen scheinen; verblose Direktive sehen, zumindest auf den ersten Blick, wie klare Fälle von Konstruktionen aus. Typische Beispiele, wie sie von Jacobs diskutiert werden, sind die Adverb,mit'-Direktiv-Konstruktion in (3) und die PP-,mit'-Direktiv-Konstruktion in (4). In beiden Fällen ist deutlich, dass die dem mit folgende nominale Konstituente beliebig komplex sein kann; ${ }^{5}$ dem mit voran geht entweder ein Adverb (vgl. (3)) oder eine PP (wie in (4)).

(3) a. Her mit \{dem Geld / dem gestohlenen Geld / dem Geld, das du mir gestohlen hast $\}$ !

b. Weg mit dem \{Krempel / dem alten Krempel / dem alten Krempel auf dem Speicher\}!

c. Nieder mit \{den Studiengebühren / den sozialfeindlichen Studiengebühren / den sozialfeindlichen Studiengebühren für Erstsemester\}!

(4) a. In den Müll mit \{diesen Klamotten / diesen geschmacklosen Klamotten / diesen Klamotten von H\&M $\}$ !

b. Zur Hölle mit \{dieser Regierung / dieser unfähigen Regierung / dieser Regierung, die keines ihrer Versprechen gehalten hat $\}$ !

Wie Jacobs (2008) zeigt, werfen solche Daten zunächst einmal Probleme für einen regelbasierten Ansatz auf. ${ }^{6}$

Jacobs nimmt an, dass die nominalen Konstituenten DPs sind. Im Unterschied dazu werde ich unter Einbeziehung der Argumente in Bruening (2009) und Georgi/Müller (2010) bei den regelbasierten Analysen unten immer davon ausgehen, dass nominale Konstituenten NPs sind (mit DP als Spezifikator). Daran hängt allerdings für die vorgebrachten Analysen nicht viel.

6 Jacobs betrachtet noch einen ähnlichen Typ von komplexem sprachlichem Ausdruck, den er "Adverb-PP-Direktiv-Konstruktion" nennt und der in (i) illustriert ist. 


\subsection{Probleme für einen regelbasierten Ansatz}

Jacobs (2008) konstatiert folgendes Dilemma für die Erfassung von Daten wie denen in (3) und (4) in einem regelbasierten Ansatz. Einerseits scheint eine Ableitung von mit-Direktiv-Konstruktionen über Ellipse nicht möglich, weil die Quelle ungrammatisch ist. Andererseits scheint aber auch eine Ableitung von mit-Direktiven nur über strukturaufbauende Regeln nicht möglich, weil hierfür unplausible Annahmen notwendig wären. Da diese beiden Optionen den Spielraum für Analysen von mit-Direktiven mit Hilfe allgemeiner Regeln der Grammatik erschöpfen, bleibt für Jacobs somit nur noch als letzte Möglichkeit übrig, dass mit-Direktive Konstruktionen sind (und also nicht über generelle Regeln erfasst werden können).

Die zwei ins Dilemma führenden Optionen seien nun der Reihe nach betrachtet. Die Ellipsenanalyse und ihr Scheitern fasst Jacobs (2008, S. 26) in der folgenden Beobachtung zusammen:

Diese Probleme lassen sich übrigens nicht dadurch aus der Welt schaffen, dass man verblose Direktiva auf zugrundliegende vollständige Sätze zurückführt, indem man ein unhörbares Imperativ-Verb postuliert. Dagegen spricht unter anderem die Inkompatibilität aller in Frage kommenden Verben mit als Thema-Argument interpretierten mit-Phrasen.

Für die Adverb-mit-Direktive in (3) würde die Ellipsenanalyse zum Beispiel besagen, dass es Quellen geben müsste wie die in (5); diese Sätze sind aber nun selbst nicht wohlgeformt.
a. *Gib (geh, trag, bring, ...) her mit dem Geld!
b. *Bring (...) weg mit dem Krempel!
c. *Mach (...) nieder mit den Studiengebühren!

Ebenso sähen die Quellen für die PP-,mit'-Direktive in (4) unter der Ellipsenanalyse wie in (6) aus; wiederum sind dies aber keine möglichen Sätze des Deutschen. Aus diesem Grund schließt Jacobs, dass eine Analyse von mit-Direktiven, derzufolge hier Tilgung involviert ist, scheitern muss. ${ }^{7}$

(i) a. Raus aus \{meinem Haus / meinem frisch renovierten Haus\}!

b. Hinein ins \{Vergnügen / große Badevergnügen / große Badevergnügen in der Kurtherme Bad Sassendorf $\}$ !

Ich werde diesen Fall jedoch im Folgenden ignorieren, weil er nicht die Probleme für eine regelbasierte Analyse erzeugt, wie es die anderen beiden Fälle tun; (i-ab) können z.B. ohne Schwierigkeiten mit Hilfe von Ellipse analysiert werden, wie (ii-ab) zeigt.

(ii) a. Geh raus aus meinem Haus!

b. Spring hinein ins Vergnügen!

Dies kann allerdings letztlich kein entscheidendes, sondern bloß ein Plausibilitätsargument sein. Denn dass die Anwendung einer syntaktischen Operation (wie hier z.B. Tilgung) nur zu einem wohlgeformten Satz führen kann, wenn der auch ohne Anwendung dieser Operation schon wohlgeformt ist, ist ein Postulat, das tatsächlich die allermeisten Grammatiktheorien nicht respektieren: Üblicherweise wird angenommen, dass Zwischenstufen in der Ableitung 
(6) a. *Schmeiß (...) in den Müll mit diesen Klamotten!

b. *Schick (...) zur Hölle mit dieser Regierung!

Die Alternative zur Tilgung ist eine Analyse verbloser Direktive über minimalen Strukturaufbau (und ohne Ellipse). Vor dem Hintergrund der in Abschnitt 1.2 gemachten Annahmen kann man die lexikalischen Einträge in (7) annehmen, die die von Jacobs ins Auge gefasste Analyse vollständig replizieren.
a. raus: $\{[\mathrm{Adv}],[$ Wurzel], $[\bullet \mathrm{P}:$ mit $\bullet]\}$
b. mit: $\{[\mathrm{P}],[\bullet \mathrm{N} \bullet],[* \mathrm{dat} *]\}$
c. dem: $\{[\mathrm{D}],[\mathrm{dat}]\}$
d. Köter: $\{[\mathrm{N}],[\mathrm{dat}],[\bullet \mathrm{D} \bullet],[* \mathrm{dat} *]\}$

(7a) besagt, dass es einen Lexikoneintrag für raus gibt, demzufolge das ein Adverb ist, das als Wurzelknoten (also als Kopf des Gesamtsatzes) fungieren kann und eine PP mit dem Kopf mit subkategorisiert. Nach (7b) ist mit eine Präposition, die eine NP subkategorisiert und Dativ zuweist (bzw. genauer, als Sonde mit einem geeigneten Ziel überprüft). Gemäß (7c) ist dem eine D-Kategorie im Dativ, das eine DP subkategorisiert und selbst wieder Dativ zuweist. ${ }^{89}$ Köter schließlich ist nach (7d) ein Nomen, das Dativ trägt (die Anreicherung mit dem Dativ-Merkmal ist in der Numeration erfolgt). Mit Hilfe der allgemeinen Prinzipien Verkettung und Abgleich kann auf der Grundlage von (7) der komplexe sprachliche Ausdruck in (8) entstehen.

von sprachlichen Ausdrücken fehlerhaft sein können; vgl. u.a. Zwicky (1974) zu relevanter Diskussion. Im Prinzip könnte es also sein, dass Strukturen wie die in (5) und (6) doch die Quellen für die wohlgeformten verblosen Direktive sind; Ellipse würde dann hier applizieren, um eine ansonsten nicht wohlgeformte Struktur zu retten. Es ist allerdings nicht leicht zu sehen, wie eine solche Analyse überzeugend begründet werden könnte (es müsste ja ein Verb ein atypisches Subkategorisierungsverhalten zeigen, das nur dann erlaubt werden kann, wenn das Verb am Ende getilgt wird); daher bleibt in jedem Fall ein Plausibilitätsargument gegen die Ellipsenanalyse.

8 Diese Mehrfachfunktion pränominaler Elemente - Empfänger wie Zuweiser von Kasus reflektiert den Umstand, dass Abgleich (wenn nicht weitere, komplizierende Annahmen gemacht werden) eine binäre Operation, pränominale Kasus-bzw. ø-Merkmalkongruenz aber prätheoretisch eine Eins-zu-Viele-Beziehung ist.

9 All dies ist eine Vereinfachung, da ich ja oben angenommen habe, dass die Syntax nur abstrakte Merkmalsbündel manipuliert und phonologische Realisierung erst postsyntaktisch erfolgt. Statt z.B. dem müsste es genauer heißen: dasjenige Merkmalsbündel, das durch das Vokabularelement dem (oder, bei morphologischer Subanalyse wie in Pike 1965; Wiese 2001; Fischer 2006, die Vokabularelemente $d$, em) post-syntaktisch realisiert wird. An dieser Stelle ist die Unterscheidung allerdings noch nicht von Bedeutung. 
(8)

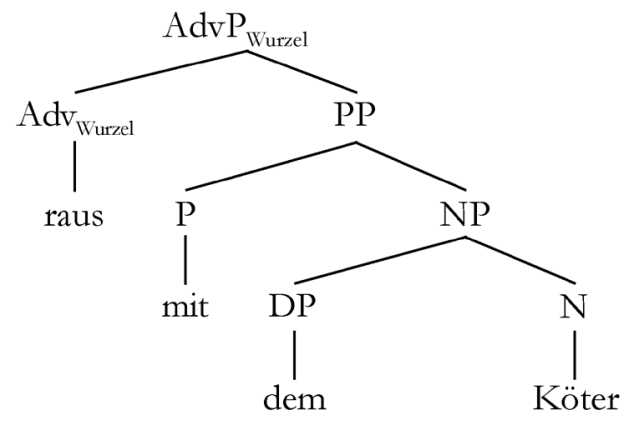

Jacobs zeigt nun aber, dass auch eine solche Analyse über regulären Strukturaufbau zum Scheitern verurteilt ist. Konkret identifiziert er vier Probleme: (i) irreguläres Verhalten von Adverbien bzgl. Subkategorisierung; (ii) Probleme mit dem Illokutionspotenzial; (iii) irreguläre Bedeutungszuweisung bei Adverbien; sowie (iv) Probleme mit dem erzwungenen Kopfstatus von Adverbien. Jacobs' Argumente können wie folgt zusammengefasst werden. Problem (i) besteht darin, dass Adverbien normalerweise andere Elemente nicht subkategorisieren (also insbesondere auch keine PPs). In (9) ist die Wahl von P frei. Dies zeigt, dass das Adverb keine Subkategorisierung bzw. Selektion durchführt.

(9) dass Peter \{raus auf die Wiese / in den Garten / zur Haltestelle\} lief

Problem (ii) ergibt sich daraus, dass es in einer kompositionellen Interpretation das Adverb sein muss, das den Direktiv-Operator (DIR) beiträgt, der das Illokutionspotenzial kodiert. Dies ist aber unvereinbar mit der Annahme, dass ein und dasselbe lexikalische Element nicht sowohl lexikalische Bedeutung tragen, als auch die Quelle des Illokutionspotenzials sein kann. (Gäbe es hier einen C-Kopf, würde das Problem verschwinden; aber es gibt in (8) ja keinen.)

Das nächste Problem (iii) ist, dass die Interpretation, die dem Adverb in (8) zugewiesen werden muss (nämlich $\lambda \mathrm{w}$.[RAUS(w)] für eine propositionale Interpretation des Gesamtausdrucks), sich unterscheidet von der Interpretation, die es normalerweise erhalten muss. Intuitiv sollte es aber seine alte Bedeutung auch bei einer Kombination mit der mit-Phrase weiter tragen.

Problem (iv) schließlich besteht darin, dass Adverbien üblicherweise keinen Kopf-Status haben. (Genauer gesagt gilt, dass Satzprojektionen normalerweise keine Projektionen von adverbialen Köpfen sind.) In (8) muss eben dieses jedoch der Fall sein.

Jacobs' (2008) Konklusion aus alldem ist, dass eine konstruktionsbasierte Analyse von mit-Direktiven benötigt wird. Diese Analyse nimmt z.B. für die verblosen Direktive der Art in (3) (wie raus mit dem Köter) und (4) (wie in in den Müll mit den Klamotten) die Konstruktionen in (10) bzw. (11) an (hier steht „U“ für obligatorischen Wurzelstatus). 
(10) raus mit dem Köter

a. Phon: /X mit Y/

b. Cat: [U $\left.\mathrm{X}_{\mathrm{Adv} \text {, dir }}\left[\mathrm{pp}_{\mathrm{Pp}} \mathrm{mit}_{\mathrm{P}} \mathrm{Y}_{\mathrm{NP}, \mathrm{dat}}\right]\right]$

c. Sem: $\operatorname{DIR}_{\text {ill }}(\mathrm{sp}, \operatorname{adr},[\mathrm{GO}-\mathrm{END}(\mathrm{x}, \mathrm{w}) \& \mathrm{Y}(\mathrm{x}) \& \mathrm{X}(\mathrm{w})])$

(11) in den Müll mit den Klamotten

a. Phon: /X mit Y/

b. Cat: $\left[\mathrm{X}_{\mathrm{PP}, \mathrm{dir}}\left[{ }_{\mathrm{pP}}\right.\right.$ mit $\left.\left.\mathrm{Y}_{\mathrm{P}, \mathrm{dat}}\right]\right]$

c. Sem: $\operatorname{DIR}_{\mathrm{ill}}(\mathrm{sp}, \operatorname{adr},[\mathrm{GO}-\mathrm{END}(\mathrm{x}, \mathrm{w}) \& \mathrm{Y}(\mathrm{x}) \& \mathrm{X}(\mathrm{w})])$

Diese Analyse umgeht die Schwierigkeiten (i)-(iv): Die (ansonsten merkwürdigen) Eigenschaften verbloser Direktive sind nunmehr als Eigenschaften der Konstruktion identifiziert; sie sind nicht mehr Eigenschaften von lexikalischen Elementen. Da Konstruktionen quasi per Definition irregulär sind (vgl. dazu, sowie zu möglichen Einschränkungen, Fischer/Stefanowitsch 2006), gibt es keine Probleme mehr: Der Anspruch ist geringer, und somit auch die Gefahr des Scheiterns. ${ }^{10}$

\subsection{Analyse}

Ein genauerer Blick legt nahe, dass die Konklusion, dass ein regelbasierter Ansatz nicht zielführend sein kann, vorschnell war. Es erweist sich, dass eine Ellipsenanalyse problemlos verfügbar ist, wenn man einen etwas abstrakteren Ansatz in der Syntax verfolgt: Man muss die naive Idee aufgeben, dass eine einfache Hinzufügung von lexikalischen Elementen aus einem mit-Direktiv eine wohlgeformte Kette macht. Ich möchte demgegenüber vorschlagen, dass die Verb-basierten Paraphrasen, nach denen man bei verblosen Direktiven wie denen in den folgenden drei (a)-Beispielen suchen muss, nicht die in den (b)-Beispielen sind (wie von Jacobs vorausgesetzt), sondern die in den entsprechenden (c)-Beispielen.
a. Nieder mit den Studiengebühren!
b. *Mach(t) (...) nieder mit den Studiengebühren!
c. Macht(t) (...) die Studiengebühren nieder!

10 So, wie die syntaktischen Aspekte (,Cat') der Konstruktionen in (10) und (11) kodiert sind (vgl. Jacobs 2008, S. 16-17), ist die Analyse noch nicht zufriedenstellend, auch aus konstruktionsgrammatischer Perspektive nicht. Der Grund ist, dass absolut regelhafte Eigenschaften in (10) und (11) als Teil der Konstruktion stipuliert worden sind: Insbesondere hat die Präposition mit im Deutschen regelhaft eine NP als Komplement, und sie weist ihrem NP-Komplement regelhaft den Dativ zu; dass dies in (10) und (11) speziell vermerkt wird, stellt somit eine Redundanz dar, auf die man sicherlich in einem verfeinerten konstruktionsgrammatischen Ansatz mit Vererbungsmechanismen verzichten möchte. Allgemein gilt wohl, dass Präpositionen in (auf den ersten Blick) nicht regelhaften komplexen sprachlichen Ausdrücken dennoch ihr Rektionsverhalten üblicherweise nicht ändern; eine solche Änderung wird aber konstruktionsgrammatisch als völlig unproblematisch und geradezu erwartbar klassifiziert nichts Grundsätzliches spräche dagegen, dass z.B. in (10) mit den Akkusativ zuwiese oder ein VP-Komplement nähme. 
(13) a. In den Müll mit diesen Klamotten!

b. *Schmeiß(t) (...) in den Müll mit diesen Klamotten!

c. Schmeiß(t) (...) die Klamotten in den Müll!

(14) a. Zum Teufel mit dir!

b. *Scher zum Teufel mit dir!

c. Scher dich zum Teufel!

Genauer ist die Hypothese, dass mit-Direktive das Ergebnis einer die grammatische Funktion verändernden Operation sind, die sich ergibt aus der Hinzufügung eines Antipassiv-artigen Kopfs zur verbalen Kategorie v. ${ }^{11}$ Antipassiv ist eine Diathese, die prototypisch in Sprachen mit ergativischer Argumentkodierung auftritt. Ein einschlägiges Beispiel für die Antipassiv-Alternation ist das folgende Paar von Beispielen aus der paläosibirischen Sprache Chukchi (siehe Comrie 1979).
a. Yemronə-na qərir-ərkən-in Yemron-ERG ${ }_{1}$ suchen-PRS-3.SG $.3 . \mathrm{SG}_{2}$ Sohn-ABS 2 ,Yemron sucht seinen Sohn.
b. Yemron ine-lqərir-ərkən (akka-gtə)

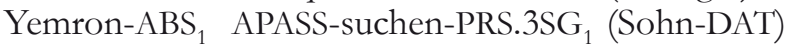
,Yemron sucht (nach seinem Sohn).

(15a) ist ein einfacher transitiver Satz (mit Ergativmarkierung auf dem externen Argument und Absolutivmarkierung auf dem internen Argument des Verbs); (15b) ist die korrespondierende Antipassiv-Variante. Antipassiv hat die folgenden Eigenschaften (vgl. u.a. Baker 1988 und Bittner/Hale 1996): Ein Antipassiv-Morphem (hier glossiert als APASS) kann ans Verb angefügt werden. APASS macht die Zuweisung von strukturellem Kasus an ein direktes Objekt (des Absolutivs in Ergativsystemen) unmöglich; das Verb wird intransitiv. Das direkte Objekt wird zurückgestuft (,Demotion'): Es wird entweder weggelassen oder als oblique Phrase realisiert. Das Subjekt (also das externe Argument) ist normalerweise nicht betroffen, aber es ändert in Ergativsystemen seinen Kasus: Es trägt dann Absolutiv (weil es kein direktes Objekt mit strukturellem Kasus mehr gibt). Die Argumentabsenkung in mit-Direktiven, wie sie sich z.B. aus der Alternation in (12a) und

\footnotetext{
$11 \quad$ Es sei darauf hingewiesen, dass die vorliegende Analyse nicht der einzige und nicht der erste Versuch ist, verblose Direktive regelbasiert zu erfassen. Wilder (2008) schlägt eine Analyse vor, die wesentlich auf der Postulierung zweier abstrakter Elemente beruht, und zwar (i) einem funktionalen Kopf IMP und (ii) einem leeren Verb GO; in Sprachen mit verblosen Direktiven „lizensiert IMP ein leeres Verb GO“. Wilders Analyse hat anders als der zu entwickelnde Ansatz keinen unmittelbaren Diathesenbezug (abgesehen von Spekulationen, die auf eine mögliche Verwandtschaft von verblosen Direktiven und der spray/load-Alternation rekurrieren, und Letzteres wird ja häufig in der Literatur als Applikativ analysiert, vgl. Wilder 2008, S. 245) - siehe unten; und sie ist auch ansonsten recht anders aufgebaut. Ein detaillierter Vergleich würde im vorliegenden Rahmen aber zu weit führen.
} 
(12c) ergibt, hat nun ganz ähnliche Eigenschaften. Dieser Umstand mag es gerechtfertigt erscheinen lassen, sich auf verblose Direktive im Deutschen als Antipassiv-artige Sätze zu beziehen. ${ }^{12}$

Bevor die Antipassiv-Analyse von verblosen Direktiven ausgeführt wird, lässt sich schon eine Vorhersage ableiten: Wenn mit-Direktive Antipassivartige Konstruktionen sind, sollte es möglich sein, das zurückgestufte direkte Objekt (also die mit-Phrase) wegzulassen. Diese Vorhersage wird bestätigt; vgl. das Fehlen einer mit-Phrasen-Realisierung in Adverb-Kontexten in (16) sowie das Fehlen einer mit-Phrasen-Realisierung in PP-Kontexten in (17) (jeweils bei (ii)). Umgekehrt kann das initiale Adverb bzw. die initiale PP nicht weggelassen werden (jeweils unter (iii)).

a. (i) Weg mit dem Krempel!

(ii) Weg!

(iii) $*$ Mit dem Krempel!

b. (i) Nieder mit den Studiengebühren!

(ii) Nieder!

(iii) *Mit den Studiengebühren!

a. (i) In den Müll mit diesen Klamotten!

(ii) In den Müll!

(iii) $*$ Mit diesen Klamotten!

$12 \quad$ Wie sich zeigen wird, weichen verblose Direktive in Details dann doch von kanonischen Antipassiv-Sätzen in Ergativsprachen ab. Letztlich hängt an der Terminologie nichts; wichtig für die folgende Analyse wird sein, dass ein Merkmal [apass] existiert (das aber auch anders genannt werden kann), das wiederum bestimmte syntaktische Prozesse auslöst, wie sie sich auch bei klassischer Antipassivbildung in Ergativsystemen finden. Nichtsdestoweniger ergibt sich unter der im Text entwickelten Analyse eine weit größere Affinität von verblosen Direktiven zum Antipassiv als zum Applikativ (vgl. die vorangehende Fußnote). Erstens bietet Antipassivierung, nicht aber Applikativbildung eine mögliche Erklärung für geändertes Subkategorisierungsverhalten des Verbs bzgl. des externen Arguments (und dies wird sich als wichtig erweisen; siehe unten). Zweitens bleibt bei Applikativbildung struktureller Objektkasus erhalten - er wechselt lediglich das Argument (vgl. (i-ab), unter der Annahme, dass (i-b) eine Applikativbildung instantiiert); in verblosen Direktiven gibt es jedoch keinen strukturellen Akkusativ.

(i) a. Sie lädt Heu auf den Wagen.

b. Sie belädt den Wagen mit Heu.

Drittens wird die Begründung für das Fehlen einer sichtbaren Verbform in verblosen Direktiven in der Analyse unten darauf Bezug nehmen, dass es keine geeignete morphologische Realisierung (kein Vokabularelement) für Antipassiv im Deutschen gibt; unter der Annahme, dass be-in (i-b) eine morphologische Realisierung für Applikativ ist (vgl. etwa Stechow 1992; Wunderlich 1993), kann man eine solche einfache Erklärung für die Verblosigkeit verbloser Direktive nicht vorbringen. Viertens schließlich können die bei verblosen Direktiven beteiligten Verben an der Applikativbildung im Deutschen (marginal) partizipieren, und das auch in Imperativen; das Ergebnis ist dann aber formal wie von der Interpretation her vollkommen unterschiedlich; vgl. (i-abc).

(ii) a. Schmeiß die Klamotten auf den Stapel!

b. \#Beschmeiß den Stapel mit den Klamotten!

c. Auf den Stapel mit den Klamotten! 
b. (i) Zur Hölle mit dieser Regierung!

(ii) Zur Hölle!

(iii) *Mit dieser Regierung!

Die Konstruktionsanalyse hat nichts über diese Asymmetrien zu sagen; und sie muss zusätzliche Konstruktionen für die (ii)-Beispiele in (16) und (17) stipulieren.

Nun zur Analyse: Am Anfang steht ein abstraktes Antipassiv-artiges Merkmal [apass]. Dieses Merkmal kann optional in der Numeration auf der prädikativen Kategorie $\mathrm{v}$ instantiiert werden (Alternativen hierzu sind die Ausstattung von v mit [aktiv] oder [passiv], was zu regulären Aktiv- respektive Passivsätzen führt). Das lexikalische Element v subkategorisiert zunächst einmal eine VP (mit dem Verb als Kopf sowie mit internen Argumenten) und führt dann als Spezifikator (sofern vorhanden) ein externes Argument ein; vgl. Chomsky (1995, 2008). Entscheidend ist nun die Annahme, dass das Merkmal [apass] auf $\mathrm{v}$ dazu führt, dass $\mathrm{v}$ nicht mehr (wie sonst üblich) strukturellen Kasus zuweisen kann; es absorbiert im Deutschen das Akkusativüberprüfungsmerkmal [*akk*] von v. Dieser Effekt kann durch eine Merkmalskookkurrenzbeschränkung (vgl. Gazdar et al. 1985) implementiert werden; vgl. (18).

$$
\text { [apass }] \supset \sim[* a k k *]
$$

Ein VP-internes Objekt muss daher als ein Obliquus realisiert werden (oder es wird gar nicht realisiert, wie in (ii) in (16) und (17)); diejenige Präposition, die zur direktional-resultativen Bedeutung der involvierten Verben passt, ist mit (vgl. Baker 1988 zur Frage, welche Präpositionen mit welchen Veränderungen der grammatischen Funktion kompatibel sind). In Ergativsystemen ergeben sich bei Antipassivierung für das externe Argument (außer einem Kasuswechsel wegen Intransitivierung) keine weiteren Konsequenzen. In Akkusativsystemen jedoch impliziert dann Burzios Generalisierung (gemäß der Akkusativzuweisungsfähigkeit und Einführung eines externen Arguments aneinander gekoppelt sind), dass ein normales externes Argument nicht mehr von v subkategorisiert werden kann (ein Passiv-artiger Effekt). Hier möchte ich allerdings vorschlagen, dass als Konsequenz von [apass] auf v das Subkategorisierungsmerkmal für das externe Argument nicht vollständig verschwinden muss; es erfolgt eine Verarmung des Subkategorisierungsmerkmals: Aus $[\bullet \mathrm{N} \bullet$ ] auf $\mathrm{v}$ wird ein defektives Merkmal $[\bullet \mathrm{X} \bullet$, das nicht ausreicht, um externe Verkettung einer NP mit einer eigenen thematischen Rolle auszulösen; $[\bullet \mathrm{X} \bullet]$ kodiert eine allgemeine EPP-Eigenschaft, d.h., die Generierung eines Spezifikators von v mit Hilfe eines beliebigen verfügbaren Elements. ${ }^{13}$ Damit kann das externe Argument nicht mehr syntaktisch realisiert werden.

13 Zum EPP („Extended Projection Principle“) vgl. Chomsky (1982, 1995, 2008). 
Eine zentrale Annahme der vorliegenden Untersuchung ist wie erwähnt, dass die morphologische Realisierung aller syntaktischen Kategorien (Funktions- wie Inhaltswörter) postsyntaktisch stattfindet (vgl. Marantz 1995, 1998). Vokabularelemente wie geb-, bring-, mach-, schmeiß-, schick- usw. werden zusammen mit der angemessenen Flexionsendung normalerweise postsyntaktisch in $\mathrm{V}+\mathrm{v}$-Kontexte eingesetzt, die durch syntaktische Kopfbewegung von $\mathrm{V}$ zu v geschaffen worden sind; hier sei vorausgesetzt, dass jeweils der Verbstamm in V eingesetzt wird, die Endung in v (vgl. z.B. Adger 2003). Die Vokabulareinsetzung folgt generell einem Teilmengenprinzip: Das Vokabularelement muss durch eine Teilmenge der Merkmale gekennzeichnet sein, die sich im Einsetzungskontext finden (vgl. Halle/Marantz 1993; Halle 1997; Trommer 1999 u.v.m.). Und genau an dieser Stelle ergibt sich ein Problem: Im Einsetzungskontext findet sich nun mit [apass] ein Merkmal, das durch die Flexionsmorphologie des Deutschen nicht realisiert werden kann - es gibt in dieser Sprache keinen Antipassivmarker (anders als z.B. im Chukchi, vgl. ine in (15b)). ${ }^{14}$ Das an sich wäre noch nicht fatal, da ja Vokabularelemente nur eine Teilmenge der Merkmale des syntaktischen Kontexts realisieren müssen. Da nun aber die verschiedenen Genera Verbi einander ausschließen und deutsche Verbmorphologie (außer beim lassen-Passiv) eindeutig für Genus Verbi spezifiziert ist (nämlich für [aktiv] oder, analytisch, [passiv]), kommt es bei [apass] auf v unweigerlich zu einer Merkmalsinkompatibilität, die die Einsetzung von Flexionselementen verhindert. Damit bliebe ein eingesetzter Verbstamm in $\mathrm{V}$ ohne jede (auch leere) Flexionsendung; unter der Annahme, dass so etwas nicht möglich ist, wird in [apass]-Kontexten daher auch die Einsetzung eines Verbstamms unmöglich. Als Konsequenz bleibt die Gesamtkategorie $\mathrm{V}+\mathrm{v}$ phonologisch ohne Realisierung.

Damit sind wesentliche Eigenschaften von verblosen Direktiven abgeleitet; es fehlt allerdings noch die Wortstellung. Diese ergibt sich wie folgt. Die EPP-Eigenschaft von v (also das strukturaufbauende Merkmal $\left[\bullet X^{\bullet}\right]$ ) löst Bewegung aus: Die Subjektposition Specv muss gefüllt werden. Es bewegt sich interessanterweise von den beiden Kandidaten (z.B. mit den Klamotten und in den Müll) das tiefere Element (also z.B. in den Müll) - entgegen dem, was man eigentlich unter der Annahme einer allgemeinen Minimalitätsbedingung erwarten würde, vgl. Fanselow (1991); Chomsky (2001). Eine mögliche Erklärung hierfür ist die, dass, wenn sich das höhere Element (das zurückgestufte Objekt) bewegt, das defektive Subkategorisierungsmerkmal von $\mathrm{v}([\bullet \mathrm{X} \bullet])$ immer noch ausreichend ist, um eine Verletzung des $\theta$-Kriteriums auszulösen (weil ein Argument dann mit zwei thematischen Rollen assoziiert wäre). Eine andere mögliche Erklärung könnte darauf Bezug neh-

$14 \quad$ Man beachte im Übrigen, dass mit der Postulierung eines [apass]-Merkmals im Deutschen (ohne morphologische Realisierung) und im Chukchi (mit morphologischer Realisierung) nicht die weiterreichende Behauptung verbunden ist, dass die Verfügbarkeit von [apass] in allen Sprachen der Welt gegeben ist. 
men, dass die durch [apass] auf $\mathrm{v}$ bedingte Objekt-Demotion tatsächlich phrasenstrukturell ist, wie bei Larsons (1988) Analyse von Doppel-ObjektKonstruktionen, so dass bei [apass] auf $\mathrm{v}$ in den Müll tiefer in der VP steht als mit den Klamotten und dann die Minimalitätsbedingung korrekt vorhersagt, dass erstere XP die EPP-Eigenschaft von $\mathrm{v}$ saturiert. Welche Erklärung auch immer hierfür gewählt wird: In beiden Ansätzen ist nun sichergestellt, dass das interne Argument nicht nach Specv bewegt werden kann; daher kann es auch nicht Nominativ-Kasus von T erhalten, wie in gewöhnlichen Passivkontexten. ${ }^{15}$

Hiermit ist zwar die korrekte Abfolge aller lexikalisch realisierten Elemente in verblosen Direktiven abgeleitet. Zur Erfassung funktionaler Beschränkungen für die Verwendung von verblosen Direktiven möchte ich jedoch des Weiteren noch annehmen, dass [apass] auf $\mathrm{v}$ im Deutschen obligatorische Verb-Zweit-Bewegung von V $+v$ zur Satzkategorie C bewirkt. Dies setzt voraus, dass der Auslöser für Verb-Zweit-Bewegung nicht wie üblicherweise angenommen eine Eigenschaft der C-Kategorie ist, sondern sich auf dem Verb $(=\mathrm{V}+\mathrm{v})$ in situ selbst befindet. Tatsächlich werden in Fanselow (2008) und Müller (2010) Argumente dafür vorgebracht, dass dem so ist. In diesen Arbeiten wird dann postuliert, dass ein Sondenmerkmal $[* T *]$ auf $\mathrm{v}$ der Auslöser ist, das Bewegung von $(\mathrm{V}+) \mathrm{v}$ in eine $\mathrm{T}$ c-kommandierende Position erzwingt (wobei TP zwischen CP und vP interveniert und für die gegenwärtigen Zwecke angenommen werden kann, dass ein an $\mathrm{C}$ bewegtes $\mathrm{V}+\mathrm{v}$ die TP (also auch T) c-kommandieren kann). ${ }^{16}$

Diese Kopplung von [apass] an [*T*] lässt sich wiederum einfach mit einer Merkmalskookkurrenzbeschränkung fassen: $[* T *]$ ist normalerweise optional auf v, aber (19) macht dieses bewegungsauslösende Merkmal obligatorisch bei verblosen Direktiven.

$$
\text { [apass }] \supset[* T *]
$$

Topikalisierung kann allerdings anders als in sonstigen Verb-Zweit-Kontexten (genauer: $\mathrm{V}+\mathrm{v}$-nach-C-Kontexten) nicht applizieren, weil diese Operation im Deutschen eine sichtbare Verbform in C erfordert (außer bei Gapping). Es folgt somit, dass die resultierenden Strukturen letztlich VerbErst-Sätze sein müssen, und somit wie deutsche Verb-Erst-Sätze generell als Imperative oder als Entscheidungsfragen interpretiert werden können (nicht aber als Deklarativsätze). Insgesamt ergibt sich (20) als Struktur von verblosen Direktiven.

\footnotetext{
15 Nebenbei sei bemerkt, dass die Bewegung des Adverbs bzw. der PP in die ansonsten externen Argumenten vorbehaltene Position Specv somit sehr ähnlich ist zur stilistischen Voranstellung (,stylistic fronting) im Isländischen (gemäß Holmbergs (2000) Analyse).

16 Vgl. etwa Baker (1988) zu einem relevanten C-Kommando-Konzept. Letztlich wird in Fanselow (2008) und Müller (2010) davon ausgegangen, dass ein bewegtes Verb selbst reprojiziert und in Verb-Zweit-Sätzen CP gar nicht vorhanden ist. Dieser Unterschied ist für die gegenwärtige Argumentation aber nicht von Belang.
} 


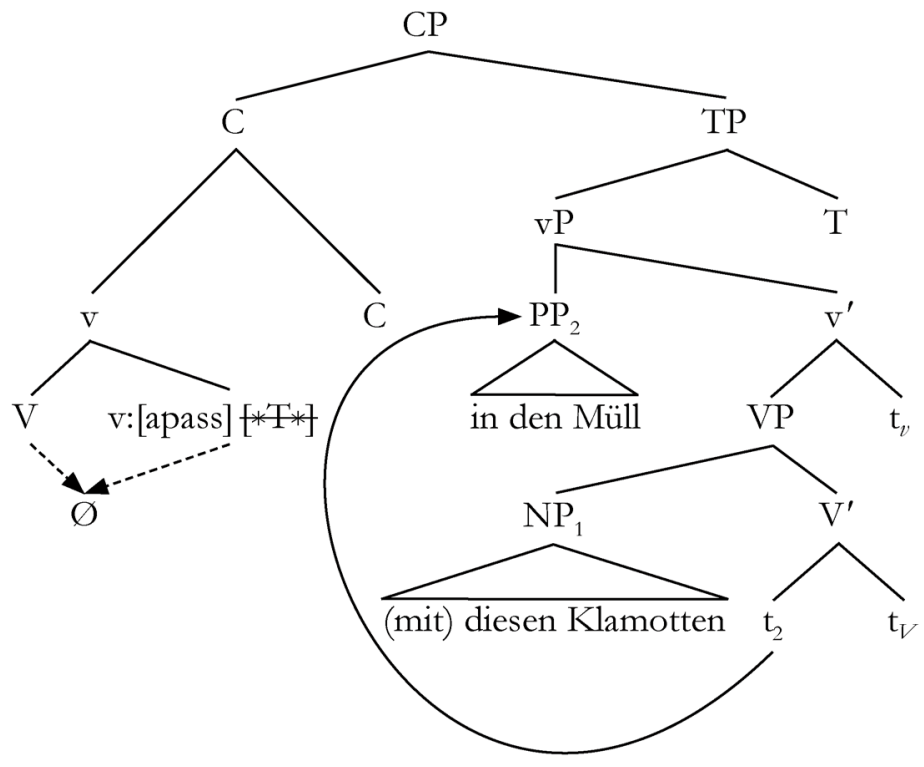

Damit sind die von Jacobs herausgearbeiteten Probleme für regelbasierte Analysen gelöst. Problem (i) betraf den Umstand, dass normalerweise Adverbien nicht andere Elemente subkategorisieren. Dieses Problem verschwindet, denn in der vorgestellten Analyse subkategorisieren Adverbien nichts. Problem (ii) war, dass ein und dasselbe lexikalische Element nicht sowohl lexikalische Bedeutung als auch Illokutionspotenzial haben kann. Auch dieses Problem ist gelöst: Das Adverb (bzw. die PP) trägt lexikalische Bedeutung; Illokutionspotenzial ist auf der Satzkategorie C verankert. Problem (iii) resultierte daraus, dass die Interpretation, die man dem Adverbial zuweisen muss, sich von der Interpretation unterscheidet, die es normalerweise bekommen muss; intuitiv sollte es aber seine alte Bedeutung auch bei einer Kombination mit der mit-Phrase weiter tragen. Hierzu ist zu sagen, dass in der vorgestellten Analyse die Interpretation des Adverbs (bzw. der PP) bei verblosen Direktiven dieselbe ist wie in anderen Sätzen. Problem (iv) schließlich war, dass Satzprojektionen normalerweise nicht Projektionen von adverbialen Köpfen sind; und tatsächlich projiziert das Adverb (bzw. die PP) in (20) ja auch keinen Satz.

Abgesehen davon, dass der hier entwickelte Ansatz den vier Kernproblemen für eine regelbasierte Analyse von verblosen Direktiven entgeht, stellt sich heraus, dass er auch noch einige interessante Vorhersagen macht. Die erste betrifft externe Argumente. Es sollte sich nach dem oben Gesagten so verhalten, dass in mit-Direktiven externe Argumente überhaupt nicht realisiert werden können, obschon externe Argumente an sich im Deutschen 
in Imperativen optional auftreten können. Wie die Beispielgruppen in (21) und (22) zeigen, wird diese Vorhersage durch die empirische Evidenz bestätigt.

(21) a. Macht (ihr) die Studiengebühren nieder!

b. *Nieder ihr mit den Studiengebühren!

c. *Nieder mit den Studiengebühren von euch!

(22) a. Schmeiß (du) die Klamotten in den Müll!

b. *In den Müll du die Klamotten!

c. *In den Müll die Klamotten von dir!

Eine weitere Konsequenz betrifft den Satzmodus. Nichts in der vorgestellten Analyse erzwingt per se eine Imperativ-Interpretation. Das Einzige, was gefordert wird, ist, dass der resultierende Satz Verb-Erst-Stellung hat. Als Vorhersage ergibt sich somit, dass, wenn nicht weitere Beschränkungen eingeführt werden, verblose Direktive kompatibel sein sollten sowohl mit einer Interpretation als Imperativsatz, als auch mit einer Interpretation als (Ja/ Nein-)Interrogativsatz. Auch diese Vorhersage wird bestätigt; vgl. (23).

a. In den Müll mit den Klamotten? (Oder was wird damit gemacht?)

b. Zur Hölle mit ihm?

c. Also was jetzt: Nieder mit den Studiengebühren oder nicht?

Unter der vorgestellten Analyse folgt dies ohne Weiteres aus der Tatsache, dass mit-Direktive Verb-Erst-Sätze sind. In einer konstruktionsbasierten Analyse muss man demgegenüber zur Erfassung von (23) die existierenden Konstruktionen duplizieren, und dabei minimal in einer der beiden Mengen die Imperativsemantik zu einer Interrogativsemantik ändern.

Drittens ergibt sich eine weitere Konsequenz bzgl. der syntaktischen Aktivität von mit-Direktiven. Der regelbasierte Ansatz sagt voraus, dass, wenn alle Beschränkungen, die sich aus der Analyse ergeben, respektiert werden, verblose Direktive sich genau wie alle anderen syntaktischen Ausdrücke verhalten sollten. Speziell sollten sie für weitere externe und interne Verkettungsoperationen zugänglich sein. Der konstruktionsbasierte Ansatz sagt dagegen voraus, dass verblose Direktive unzugänglich für weitere syntaktische Operationen sein sollten (es sei denn, man stipuliert Erweiterungen der Analyse, die den Ansatz dann aber nah an einen regelbasierten Ansatz bringen). Die Evidenz zeigt, dass Ersteres der Fall ist: Die interne Struktur von mit-Direktiven ist zugänglich für weitere syntaktische Operationen. ${ }^{17}$ So funktioniert z.B., wie Jacobs (2006) beobachtet, die Modifikation von verblosen Direktiven genau wie in regulären transitiven Sätzen; die Beschränkungen sind, wie (24) (reguläre transitive Imperativsätze) im Vergleich mit

$17 \quad$ Vgl. auch Müller, St. (2006) zu Resultativkonstruktionen. 
(25) (verblose Direktive) zeigt, identisch. Dies ist ein potenzielles Problem für den konstruktionsbasierten Ansatz, aber es folgt unmittelbar aus dem hier entwickelten regelbasierten Ansatz.

(24) a. Schmeiß den Krempel weg!

b. Schmeiß den Krempel schnell weg!

c. ? *Schmeiß den Krempel sorgfältig weg!

(25) a. Weg mit dem Krempel!

b. Schnell weg mit dem Krempel!

c. ? *Sorgfältig weg mit dem Krempel!

Ebenso verhält es sich bei syntaktischer Bewegung. In Varietäten des Deutschen, die an sich P(ostpositions)-Stranden (auf der Basis eines Pronominaladverbs) erlauben, können mit-Direktive (zumindest marginal) auch P-Stranden involvieren. Das ist unter dem regelbasierten Ansatz erwartet; es ist unerwartet unter dem konstruktionsbasierten Ansatz (der hier in jedem einzelnen Fall noch eine weitere Konstruktion vorsehen müsste). Dies veranschaulichen die Beispiele in (26a-b) (Bewegung und P-Stranden in transitiven Sätzen) und (26c-d) (analoge Fälle von P-Stranden in verblosen Direktiven) für die PP da rein, sowie die Beispiele in (27) für die PP dabin.

a. Wirf die Klötze da rein!

b. Wirf da die Klötze rein!

c. Da rein mit den Klötzen!

c. Da mit den Klötzen rein!

(27) a. Bring den Krempel dahin!

b. Bring da den Krempel hin!

c. Dahin mit dem Krempel!

d. Da mit dem Krempel hin!

Als Konklusion ergibt sich, dass eine regelbasierte Analyse von verblosen Direktiven nur so lange problematisch ist, wie angenommen wird, dass ein Ellipsenansatz naiv sein muss (so dass die bloße Hinzufügung von lexikalischem Material in wohlgeformten Sätzen resultiert). Dagegen erweist sich eine regelbasierte Analyse als unproblematisch, wenn verblose Direktive als vollständige CPs analysiert werden, in deren Kopf ein Verb mit einem hinzugefügten Antipassiv-Morphem steht, dessen phonologische Realisierung null ist, und das Demotion des Objekts auslöst. Damit ist sämtliche Gegenevidenz entkräftet (d.h., Jacobs' vier Probleme sind gelöst). Darüber hinaus sind ohne Zusatzannahmen weitere Effekte ableitbar, über die der konstruktionsbasierte Ansatz wenig zu sagen hat. ${ }^{18}$

18 Die vorgestellte Analyse wirft die Frage auf, wie Alternationen wie Sie sucht den Schliussel gegenüber Sie sucht nach dem Schlïssel zu behandeln sind, die auch Objektabsenkung zu involvieren scheinen. Klar ist, dass es sich hier nicht um eine [apass]-gesteuerte Operation handeln 
Für die übergreifende Thematik (Regeln oder Konstruktionen?) lässt sich festhalten: Es gibt Evidenz dafür, dass verblose Direktive im Deutschen aus kleineren Einheiten zusammengesetzt sind. Diese Evidenz betrifft den Wortstatus der Teile und die Zugänglichkeit für syntaktische Regeln, die auf die interne Struktur zugreifen. Tatsächlich können aber die Eigenschaften von verblosen Direktiven allein auf der Grundlage der Eigenschaften der einzelnen lexikalischen Elemente determiniert werden: Ein regelbasierter Ansatz ist möglich, und darüber hinaus gut begründet, weil so syntaktische Effekte vorhergesagt werden, die unter einem konstruktionsbasierten Ansatz geheimnisvoll bleiben müssen. Verblose Direktive im Deutschen sind damit keine Konstruktionen.

\section{Sequenzielle Nominalreduplikation}

\subsection{Daten}

Es gibt in vielen Sprachen N-P-N-Konstruktionen wie in (28) im Deutschen; das Muster ist durchweg produktiv (vgl. u.a. Malkiel 1959, S. 131; Burger 1973, S. 46; Williams 1994, S. 18 ff.; Fleischer 1997, S. 108; Goldberg 2006, S. 14; Jacobs 2008, S. 27 und Jackendoff 2008, S. 10). Burger und Fleischer behandeln diese sprachlichen Ausdrücke als Sonderfälle von Binomialen (Paarformeln), also formelhaften und-Verbindungen wie kurz und gut, fix und fertig, Kind und Kegel (vgl. hierzu u.a. Müller 1997 und Eisenberg 2000). Binomiale haben aber vollkommen andere Eigenschaften. Der zu untersuchende Ausdruckstyp involviert (anders als Binomiale) eine Präposition in der Mitte (aus einer kleinen Menge von morphologisch einfachen Präpositionen des Deutschen), die (anders als bei Binomialen) zu beiden Seiten flankiert wird von ein und demselben $\mathrm{N}$, einhergehend mit (wiederum anders als bei Binomialen, die häufig semantisch opak sind) im weiteren Sinne sequenzieller (iterativer, intensivierender) Interpretation. Ich werde mich im Folgenden auf Beispiele wie die in (28) mit dem Begriff, sequenzielle Nominalreduplikation' beziehen, der (wie ,verblose Direktive) den funktionalen und den formalen Aspekt gleichermaßen in den Vordergrund stellt (wenn auch in umgekehrter Reihenfolge). ${ }^{19}$

kann; konkret möchte ich hier (ohne weitere Argumentation) annehmen, dass eine präsyntaktische, durch ein anderes Merkmal ausgelöste Operation verantwortlich ist, die die Subkategorisierungseigenschaften des Verbs verändert.

19 Vgl. Beck/Stechow (2006) (sowie die dort angegebene Literatur) zur Präzisierung der Semantik der sequenziellen Nominalreduplikation. Für Fälle wie (den adverbialen Gebrauch von) book after book im Englischen wird argumentiert, dass die Bedeutung sowohl auf Sequenzen von Teilereignissen, als auch auf Sequenzen von Individuen (hier: Büchern) rekurriert. Das zentrale Konzept der Sequenz kann vermutlich auf alle Fälle in (28) generalisiert werden, auch wenn es nicht immer in offensichtlicher Weise um die temporale Aufeinanderfolge von Teilereignissen gehen kann (wie bei für, um und auf), sondern auch lokale Sequenzialität 
(28) a. Jahr für Jahr, Tag für Tag, Minute für Minute, Buch für Buch, Mann für Mann, Tisch für Tisch, Seite für Seite, Schublade für Schublade, Stück für Stück

b. Schulter an Schulter, Tür an Tür, Kopf an Kopf, Mensch an Mensch, Buch an Buch

c. Stunde um Stunde, Buch um Buch

d. Hand in Hand, Auge in Auge, Buch in Buch

e. Schlag auf Schlag, Buch auf Buch, Mensch auf Mensch, Plan auf Plan, Anfrage auf Anfrage

Sequenzielle Nominalreduplikationen haben eine Reihe von interessanten Eigenschaften. Eigenschaft (i) ist die vielleicht offensichtlichste: $\mathrm{N}$ und $\mathrm{N}$ dürfen nicht distinkt sein. Obligatorische N-N-Identität zeigt (29). ${ }^{20}$
a. Buch für Buch
b. *Buch für Zeitung

Eigenschaft (ii) ist, dass die sequenzielle Nominalreduplikation XP-Status hat. Dies zeigt sich unter anderem daran, dass solche Ausdrücke vorfeldfähig sind: vgl. (30).

(30) a. Seite für Seite ist er durchgegangen.

b. Buch auf Buch hat er gelesen.

Drittens darf N nicht z.B. durch ein pränominales Adjektiv erweitert werden; die obligatorische Minimalität der Projektion zeigen die Beispiele in (31) (Eigenschaft (iii)). ${ }^{21}$

(31) a. Ich bin (*schriftliche) Anfrage auf (*schriftliche) Anfrage die Akten durchgegangen.

eine Rolle spielt (bei in und an); vgl. auch Jackendoff (2008, S. 26). Auf formaler Seite soll der Begriff der Nominalreduplikation an dieser Stelle ebenfalls noch prätheoretisch sein; tatsächlich wird sich aber das Konzept der Reduplikation am Ende als Kern der Analyse erweisen.

20 Eine mögliche Ausnahme ist Hals über Kopf (vgl. auch allüberall); aber über verhält sich, wie sich auch weiter unten noch zeigen wird, etwas anders als die anderen bei der sequenziellen Nominalreduplikation beteiligten Präpositionen und wird im Folgenden außerhalb der Betrachtung bleiben. Sebastian Bank (pers. Mit.) weist darauf hin, dass Google-Suchen Daten zutage fördern wie (Sie sollen sich) Brust an Rücken (zusammenstellen). oder (Wir lagern Ibre Reifen) Sommer auf Winter. Wenn solche Fälle von Nicht-Identität der beiden Ns gleichberechtigt, produktiv und mehr als eine Ansammlung von Ausnahmen sind, müsste die unten vorgestellte Analyse stark modifiziert werden.

21 Diese Beschränkung scheint im Englischen nicht in dieser strikten Form zu gelten; Jackendoff (2008, S. 21) geht davon aus, dass im Englischen das zweite N unabhängig modifiziert werden kann, das erste dagegen nicht; allerdings sei simultane Modifikation beider Ns wieder möglich; vgl. (i).

(i) a. day by miserable day

b. miserable day by miserable day

c. *awful day by day 
b. Er hat (*neues) Jahr für (*neues) Jahr die gleichen Kurse unterrichtet.

Eigenschaft (iv) ist besonders interessant: Eine sequenzielle Nominalreduplikation kann alternativ adverbial oder als Argument (mit dann beliebiger grammatischer Funktion) verwendet werden. Die Multifunktionalität wird durch die Beispiele in (32) illustriert.
a. *Ich bin durchgegangen.
b. Ich bin Seite für Seite durchgegangen.
$(*$ in der relevanten Lesart)
c. Ich bin Seite für Seite den Aufsatz durchgegangen.
(Argument)

d. Mann für Mann wurde gerettet.

(Adverbiale Best.)

e. Mann für Mann wurde die Besatzung gerettet. (Adverbiale Best.)

(32a) zeigt, dass durchgehen (im Sinne von lesen) obligatorisch transitiv ist und ein internes Argument verlangt. Die sequenzielle Nominalreduplikation Seite für Seite in (32b) kann offensichtlich diese Anforderung erfüllen; aber ebenso kann Seite für Seite, wie (32c) deutlich macht, auch als adverbiale Bestimmung interpretiert werden, die zu einem internen Argument (hier: den Aufsatz) hinzutritt. Derselbe Kippfigureffekt ergibt sich in (32d-e): Mann für Mann kann alternativ Argument von gerettet und adverbiale Bestimmung sein.

Eine weitere Beobachtung (Eigenschaft (v)) ist, dass N nicht (oder zumindest nicht gut) flektiert werden kann. Das Flexionsverbot zeigen die Beispiele in (33); (33a) für Pluralflexion, (33b) für Kasusflexion. ${ }^{22}$

a. *Sie hat Bücher für Bücher gelesen.

b. *Die Arbeiten lagen Buch in Buche verschränkt auf dem Tisch.

Die letzte hier zu betrachtende Eigenschaft (vi) schließlich ist, dass die Konstruktion eine Insel für Bewegung und Referenz ist. Den Inselstatus für Bewegung veranschaulichen die Beispiele in (34) (wo der Reihe nach das erste $\mathrm{N}$, das zweite $\mathrm{N}, \mathrm{P}$ und das zweite $\mathrm{N}$ sowie $\mathrm{P}$ und das erste $\mathrm{N}$ topikalisiert wurden).

(34) a. *Jahr bin ich für Jahr dorthin gefahren.

b. *Jahr bin ich Jahr für dorthin gefahren.

c. *Für Jahr bin ich Jahr dorthin gefahren.

d. *Jahr für bin ich Jahr dorthin gefahren.

$22 \quad$ Wie oben schon angedeutet, verhält sich über anders als andere Präpositionen; vgl. (Er stellt) Fragen über Fragen mit Pluralmarkierung. Im Bereich der Kasusflexion könnte alles in allem eine Ausnahme sein. Außerdem sei auf Gallmann (1996) verwiesen, der zeigt, dass das Dativ-e der deutschen Nominalflexion ohnehin nur auftreten kann, wenn auch noch ein pränominales, sichtbar kasusflektiertes Element vorhanden ist; vgl. *aus Holze gegenüber aus bartem Holze; gerade dieser Kontext ist aber wegen Eigenschaft (iii) unabhängig nicht verfügbar. 
Der Inselstatus für Referenz wird deutlich auf der Basis von Beispielen wie (35).

(35) Pass für Pass habe ich durchgesehen, und dann *war er/?* waren sie weg.

\subsection{Probleme für einen regelbasierten Ansatz}

Sequenzielle Nominalreduplikation scheint auf den ersten Blick viele Eigenschaften zu haben, die ansonsten nicht für die Grammatik (des Deutschen oder anderer Sprachen) etabliert sind; die im vorangegangenen Abschnitt aufgeführten Eigenschaften (i)-(vi) sehen zunächst einmal unerwartet aus, wenn man sie abgleicht mit den ansonsten in der Grammatik des Deutschen geltenden Regeln. Es ergibt sich somit eine Herausforderung für regelbasierte Ansätze. Jacobs (2008, S. 27) konstatiert (ohne das allerdings weiter durch eine genauere Analyse von sequenziellen Nominalreduplikationen zu begründen), dass „,die Probleme für projektionistische [d.h., regelbasierte, Anm. d. Verf.] Analysen parallel zu denen bei verblosen Sätzen“ sind. Ähnlich stellt Jackendoff (2008, S. 8) für das Englische fest: „The internal syntactic structure of NPN violates standard principles of phrase structure"; ein regelbasierter Ansatz benötige zwei Dinge:

first, a general way of generating phrases of the form NPN, with all the constraints on their internal structure; and second, a special sense of the preposition [...] that means multiple succession and that can be inserted only in a structure where it is surrounded by identical nouns. (ebd., S. 15).

Jacobs wie Jackendoff kommen auf dieser Grundlage zum Schluss, dass nur eine konstruktionsbasierte Analyse erfolgversprechend sein kann, in der alle problematischen Eigenschaften von sequenziellen Nominalreduplikationen als Eigenschaften der Konstruktion stipuliert werden (vgl. Jackendoff 2008, S. 26).

Tatsächlich sind bestehende regelbasierte Ansätze noch nicht sehr überzeugend. So notiert Williams (1994, S. 19) bzgl. der sequenziellen Nominalreduplikation im Englischen: „One is at first hard-pressed to identify what structures these are well-formed instances of, but I think the most likely candidate is the compound prepositional phrases, as from ... to" (vgl. auch schon Malkiel 1959, S. 131 zu Überlegungen in diese Richtung). Williams' Quelle für sequenzielle Nominalreduplikationen im Englischen wie z.B. minute to minute oder station to station ist also ein Satz vom Typ in (36); der Ansatz ist damit eine Ellipsenanalyse.

John went from Paris to New York. 
Beck/Stechow (2006) fassen ebenfalls eine Ellipsenanalyse ins Auge. Sie erwägen, dass dog after dog durch Tilgung auf der Basis von one dog after the other dog entsteht. Solche Analysen leiten jedoch, wenn nicht noch sehr viel mehr gesagt wird, kaum eine der Eigenschaften von sequenzieller Nominalreduplikation im Deutschen oder Englischen ab: Identität (i), Minimalität (iii), Verwendbarkeit als Argument (iv), Flexionsverbot (v) und Inselstatus (vi) bleiben unerklärt. Dazu kommt, dass es in beiden Ansätzen für beinahe alle Fälle von sequenzieller Nominalreduplikation im Deutschen keine derartige Quelle geben kann, die über Tilgung eine sequenzielle Nominalreduplikation produziert - wie sollte z.B. Seite für Seite durch Tilgung abgeleitet werden (vgl. z.B. *von Seite für Seite, *eine Seite für die andere Seite)? Auch eine abstraktereEllipsenanalyse (wie oben für die verblosen Direktive vorgeschlagen) führt hier nicht zum Ziel.

Dabei ist nicht nur die Ellipsenanalyse zum Scheitern verurteilt; auch eine Analyse über regulären Strukturaufbau wird unmittelbar mit Problemen konfrontiert. Zwei naheliegende einfache Strukturen für die sequenzielle Nominalreduplikation sind die in (37) und (38).

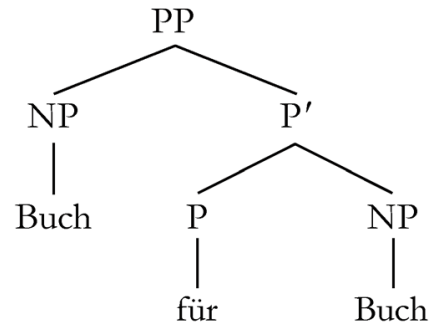

(38)

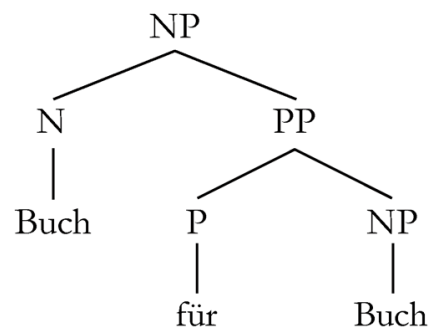

Aus beiden Strukturen ergibt sich die Konstituenz von sequenziellen Nominalreduplikationen (Eigenschaft (ii)). Die anderen Eigenschaften folgen allerdings nicht. Nichts erzwingt die Identität der beiden Ns (i). Durch nichts wird die Minimalität der beiden Ns gewährleistet (iii). Es bleibt unklar, warum $\mathrm{N}$ nicht flektiert werden kann (v). Auch der Inselstatus (vi) bleibt wenigstens zum Teil geheimnisvoll: In (37) würde man erwarten, dass (bei Argumentstatus des sprachlichen Ausdrucks) der Spezifikator von P bewegbar sein sollte (in Analogie zum P-Stranden bei Pronominaladverbien); in (38) sollte demgegenüber (wiederum bei Argumentstatus der gesamten Konstituente) die PP als Komplement von N bewegbar sein (analog zu Über die Liebe habe ich ein Buch gelesen). Die Multifunktionalität (iv) schließlich folgt ebenfalls nicht in naheliegender Weise. Macht man die Standardannahme, dass Kernargumente des Verbs (externes Argument, internes Argument), die strukturellen Kasus erhalten, im Deutschen immer NPs sein müssen (und nicht PPs sein können), ergibt sich aus dem PP-Status in (37) ein Problem bei der argumentalen Verwendung einer sequenziellen Nominalreduplikation. Um- 
gekehrt wirft der in (38) postulierte NP-Status dann die Frage auf, warum die sequenzielle Nominalreduplikation problemlos und in vielen Kontexten als adverbiale Bestimmung verwendet werden kann; normalerweise (abgesehen von wenigen, streng abgegrenzten Fällen wie den ganzen Tag) ist so etwas im Deutschen nicht gut möglich. ${ }^{23}$

Das Hauptproblem mit (37) und (38) ist nun aber gar nicht einmal, dass die Eigenschaften von sequenziellen Nominalreduplikationen im Deutschen nicht abgeleitet werden können. Es ist vielmehr der Umstand, dass weder (37), mit einer NP als Spezifikator von P, noch (38), mit einer NichtArgument-PP als Komplement von N, sonst in der Grammatik des Deutschen mögliche Strukturen sind (vgl. u.a. Bierwisch (1988) zum ersten Fakt). In (38) müsste darüber hinaus ein bloßes Appellativum als Kopf z.B. einer Objekt-NP eines Verbs fungieren (wie in (39a)), was sonst nicht geht; vgl. (39b).

(39) a. Ich habe Buch für Buch durchgelesen.

b. *Ich habe Buch durchgelesen.

Und in (37)? Hier muss ein bloßes Appellativum als Komplement von P auftreten können. Auch dies erscheint zunächst einmal ungewöhnlich (darauf weist auch Williams (1994, S. 20) für das Englische hin): Bloße singularische Appellativa kommen nach weit verbreiteter Meinung normalerweise nicht als Argumente von Präpositionen vor. Aus dieser Perspektive ist es nicht nur überraschend, dass Minimalität (iii) notwendig ist; es ist ebenso überraschend, dass Minimalität überhaupt möglich ist. Diese Argumentation beruht jedoch auf einer falschen Prämisse: Wie Kiss (2008, in diesem Band) und Keßelmeier et al. (2009) zeigen, sind bloße Appellativa als Argumente von Präpositionen im Deutschen durchaus üblich; vgl. (40). Dieser Aspekt von (37) ist also unproblematisch.

(40) auf Anfrage, ohne Vorwarnung, ohne Ausbildungsplatz, mit Vorbehalt

Diese Präposition-Nomen-Kombinationen (PNKs) zeigen aber noch nicht alle typischen Eigenschaften von sequenziellen Nominalreduplikationen. Erstens sind PNKs erweiterbar; Minimalität (iii) gilt also nicht; vgl. (41a). Zweitens sind PNKs distributionell eindeutig als PPs erwiesen; sie kommen nicht als Kernargumente des Verbs vor; vgl. (41b). Multifunktionalität (iv) liegt also auch nicht vor. Drittens gilt das Flexionsverbot nicht (v); vgl. (41c). Und schließlich kann N häufig (d.h., wenn andere Präpositionen vorliegen als in (28)) auch nicht verdoppelt werden in einer PNK; vgl. (41d).

23 Das Problem der korrekten Kategoriezuweisung bleibt im Übrigen auch in einer konstruktionsbasierten Analyse bestehen; vgl. die inkonklusive Diskussion bei Jackendoff, an deren Ende festgestellt wird: "There is no standard category into which they fit comfortably“ (Jackendorf 2008, S. 19). 
(41) a. auf schriftliche Anfrage

b. *Ich bin auf Anfrage durchgegangen.

c. wegen schwerwiegenden Verstoßes gegen das Parkverbot

d. *Sie haben Vorwarnung ohne Vorwarnung geschossen.

\subsection{Analyse}

Nichtsdestoweniger möchte ich vorschlagen, dass die PNK die Quelle für die sequenzielle Nominalreduplikation ist. Hinzu kommt lediglich eine durch ein designiertes auf P instantiiertes Merkmal ausgelöste Operation - nämlich Reduplikation. Dass es sich hier um echte Reduplikation handelt, ist aus übereinzelsprachlicher Perspektive geradezu zu erwarten. Sequenzielle Nominalreduplikation ist eine Instanz eines typologisch weit verbreiteten, sehr produktiven Musters von Reduplikation, das Stolz (2009) ,totale syndetische Reduplikation' nennt. Nicht zuletzt ist der beobachtete interpretative Effekt (Sequenzialität bzw. Iterativität/Intensivierung) eine typische Instanz von Reduplikationssemantik.

Die Analysestrategie wird nun wie folgt aussehen: In der Syntax ist nur eine aus $\mathrm{P}$ und folgendem $\mathrm{N}$ bestehende Konstituente vorhanden (als Instanz einer PNK). Postsyntaktisch wird N redupliziert. Dies genügt, um die charakteristischen Eigenschaften von sequenzieller Nominalreduplikation im Wesentlichen abzuleiten. ${ }^{24}$ Was vor diesem Hintergrund benötigt wird, ist eine postsyntaktische, rein phonologische Theorie der Reduplikation, die mit den in Abschnitt 1.2 gemachten Grundannahmen verträglich ist. Eine solche Theorie ist von Frampton (2009) entwickelt worden. Im Folgenden skizziere ich zunächst einige Grundzüge von Framptons Ansatz; auf dieser Grundlage entwickele ich danach eine rein regelbasierte Analyse der sequenziellen Nominalreduplikation.

\subsubsection{Framptons Theorie der Reduplikation}

Framptons Grundannahme ist (wie auch im vorliegenden Aufsatz bisher durchweg vorausgesetzt), dass es eine strikt derivationelle, minimalistische Syntax-Komponente gibt, die lediglich abstrakte Merkmalsbündel als syntaktische Kategorien manipuliert, und dass phonologische Realisierung durch postsyntaktische Operationen wie Vokabulareinsetzung erfolgt. Eine spezielle Annahme über Reduplikation ist, dass das reduplizierte Element syntaktisch abwesend ist und postsyntaktisch entweder in das Reduplikation auslösende Morphem integriert wird oder strukturell sogar ins Nichts wandert; unter beiden Optionen wird keine neue syntaktische Struktur geschaffen. Reduplikation wird als eine komplexe, jeweils durch ein designiertes Affix

24 Vgl. auch Jackendoff (2008, S. 26), der ein derartiges Auseinanderklaffen von Phonologie und Syntax kurz erwägt, aber letztlich verwirft. 
gesteuerte Operation betrachtet, die aus drei Teilen besteht: (i) Am Anfang steht die Vorbereitung der autosegmentalen Struktur, die eine Taktschicht (,timing tier') und eine Segmentschicht (,segmental tier') beinhaltet. Das Vorhandensein der Segmentschicht zu diesem Zeitpunkt impliziert, dass postsyntaktische Vokabulareinsetzung vor postsyntaktischer Reduplikation passiert sein muss. In der Vorbereitungskomponente erfolgt nun zunächst (i-a) die Auswahl der Duplikationsdomäne mit Hilfe der Zuweisung von so genannten Duplikationsfugen ([ und ]); danach (i-b) kommt die Auswahl der Kürzungsdomäne darin, die über die Zuweisung von so genannten Kürzungsfugen $(<$ und $>$ ) durchgeführt wird. (Duplikationsfugen und Kürzungsfugen zusammen werden unter dem Begriff der T(ranskriptions)-Fugen subsumiert.) Und schließlich (i-c) erfolgt gegebenenfalls eine prosodische Anpassung. Dies beendet die Vorbereitung der Struktur. Als zweiter Teil der komplexen Operation Reduplikation folgt (ii) die Transkription; dieser Prozess schafft überkreuzende Äste. Diese überkreuzenden Äste werden von der Grammatik nicht dauerhaft toleriert; im abschließenden dritten Teil (iii) wird daher eine Reparatur durchgeführt, die die durch (ii) geschaffenen überkreuzenden Äste durch Kopieren der Segmente wieder auflöst.

Frampton wendet die Transkriptionstheorie auf praktisch sämtliche gut untersuchten Reduplikationsphänomene der Sprachen der Welt an. Ein vergleichsweise unkompliziertes Beispiel ist die tasty-shmasty-Reduplikation im Englischen (vgl. Frampton 2009, S. 55-56), die in mancherlei Hinsicht der sequenziellen Nominalreduplikation vergleichbar ist und im Wesentlichen Modell für ihre Analyse steht. Das die Reduplikation auslösende Element ist ein postsyntaktisch eingesetztes Vokabularelement shm, das hinten an ein ebenfalls postsyntaktisch eingesetztes Vokabularelement (das z.B. eine Adjektivposition füllt) tritt, von Kürzungsfugen umgeben ist (d.h., selbst nicht mit transkribiert und redupliziert wird), eine rechte Duplikationsfuge ] bereits mitbringt und am Anfang ein Wortgrenzensymbol \# aufweist; vgl. (42a). Weiterhin ist damit die Duplikationsfugenregel in (42b) assoziiert, die eine linke Duplikationsfuge [ vor dem ersten Vokal einfügt (bei Regelanwendung von links nach rechts).
a. Auslösendes Vokabularelement: $<\#$ shm $>$ ]
b. Duplikationsfugenregel: $\varnothing \rightarrow[/ \ldots V$

Für tasty-shm zeigt (43), wie postsyntaktische Reduplikation durch Transkription und Reparatur zustande kommt und tasty-shmasty erzeugt. ${ }^{25}$ Im Vorbereitungsteil (43a) wird die linke Duplikationsfuge [ vor dem ersten Vokal von tasty in die Struktur eingefügt; die rechte Duplikationsfuge ] und die Kürzungsfugen $<$, $>$ in der Duplikationsdomäne hat das Vokabularelement $s h m$ selbst schon in die Struktur mitgebracht. Im Transkriptionsteil (43b)

$25 \quad$ Statt der eigentlich korrekten phonematischen wird der Einfachheit halber hier wie bei Frampton eine graphematische Repräsentation der Segmente angenommen. 
erfolgt dann die Transkription: Alle Segmente, die nicht in der Kürzungsdomäne liegen, werden der Reihe nach rechts von $s h m$ mit neu erzeugten Taktpositionen (x) der Taktschicht verbunden; sie werden somit multidominiert. ${ }^{26}$ Die Segmente bilden somit keine lineare Ordnung mehr und können nicht ausgesprochen werden; dieser Mangel wird behoben durch die Reparatur im letzten Teil (43c): Die Segmente werden der Reihe nach nach hinten kopiert, so dass jedes Segment nur noch von genau einer Taktposition (x) dominiert wird.

a. Vorbereitung:

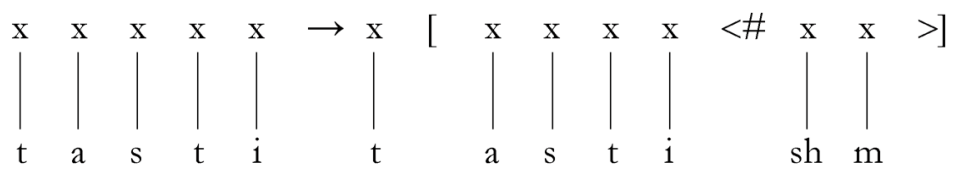

b. Transkription:

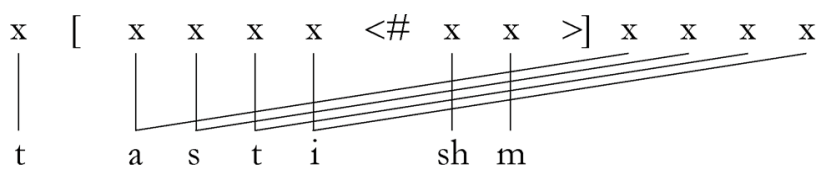

c. Reparatur:

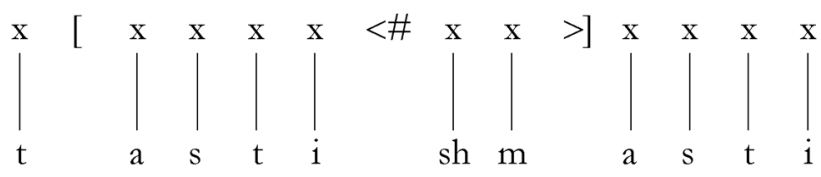

\subsubsection{Sequenzielle Nominalreduplikation als Reduplikationsphänomen}

Nun liegen alle Bausteine für eine regelbasierte Analyse sequenzieller Nominalreduplikationen vor. Gemäß dem angenommenen Modell der Grammatik ist die Analyse über drei Bereiche zu verteilen: präsyntaktische Numeration, Syntax, postsyntaktische phonologische Realisierung.

Wie bei den verblosen Direktiven nehme ich auch bei den sequenziellen Nominalreduplikationen an, dass alles, was passiert, auf ein einziges Merkmal zurück geht, das in der Numeration einem lexikalischen Element zugewiesen wird. Konkret sei vorausgesetzt, dass am Anfang in der Numeration ein P-Morphem $\in\{f \ddot{r}$, an $, u m, i n$, auf, ... $\}$ ausgewählt werden kann (genauer: ein P-Morphem, dass in der Menge der entsprechenden Merkmalsmatritzen noch ohne phonologische Realisierung liegt, die dann postsyntaktisch als für, an etc. realisiert werden). Dieses P-Element erhält optional in der Numeration das Reduplikationsmerkmal [red]. Das Merkmal [red]

26 Ob die Transkription nach rechts oder links geht, ist nicht per se festgelegt, sondern kann von Reduplikationsaffix zu Reduplikationsaffix verschieden sein. 
muss letztlich für die (kompositionelle) Interpretation unter Bezug auf das Konzept der (generalisierten) Sequenz verantwortlich sein. Weiter sei angenommen, dass zu einem abstrakten P:[red] passende Vokabularelemente (die postsyntaktisch in P:[red] eingesetzt werden können) auch das Merkmal [red] tragen können; wenn sie eingesetzt werden, müssen sie es auch tragen.

Ebenfalls wie bei der Analyse der verblosen Direktive sind mit dem auslösenden Merkmal direkt weitere Effekte verbunden, und wie vorher lässt sich dies durch eine Merkmalskookkurrenzbeschränkung für Numerationen (vgl. Gazdar et al. 1985) einfach fassen; vgl. (44): Das Reduplikationsmerkmal ist inkompatibel mit der Präsenz des Merkmals N (mit Wert + oder -).

$$
[\mathrm{red}] \supset \sim[ \pm \mathrm{N}]
$$

Dies setzt eine Dekomposition der kategorialen Merkmale N, V, P und A in Kombinationen primitiver Merkmale voraus, wie sie häufig angenommen wird, um natürliche Klassen von Kategorien abzuleiten (vgl. Bierwisch 1967; Chomsky 1981): Wenn gilt, dass $\mathrm{V}=[+\mathrm{V},-\mathrm{N}], \mathrm{N}=[+\mathrm{N},-\mathrm{V}], \mathrm{A}=[+\mathrm{V},+\mathrm{N}]$ und $\mathrm{P}=[-\mathrm{V},-\mathrm{N}]$, dann bilden z.B. $\mathrm{N}$ und $\mathrm{P}$ auf der einen sowie $\mathrm{V}$ und $\mathrm{A}$ auf der anderen Seite eine natürliche Klasse, und man kann z.B. die Linearisierungsregeln des Deutschen sehr ökonomisch so formulieren, dass [+V]Kategorien rechts von ihren Komplementen verkettet werden, während [-V]-Kategorien links von ihren Komplementen verkettet werden (vgl. Grewendorf 1988). Die Konsequenz von (44) ist nun, dass $[-\mathrm{N}]$ bei einer Präposition mit dem Merkmal [red] präsyntaktisch getilgt werden muss und so eine kategorial unterspezifizierte nicht-verbale Kategorie entsteht.

Analog zur Merkmalskookkurrenzbeschränkung (44) für das abstrakte P-Element gibt es nun noch eine Regel, die dafür sorgt, dass ein mit dem Merkmal [red] versehenes präpositionales Vokabularelement postsyntaktisch Reduplikation in der intendierten Weise bewirkt. Diese Regel lässt sich für das Vokabularelement auf wie folgt fassen. ${ }^{27}$

$$
[\text { red }]+/ \text { auf } / \rightarrow /[<\# \text { auf }>/
$$

Es ergibt sich damit, dass präsyntaktisch P-Elemente mit dem Merkmal [red] existieren, die bzgl. kategorialer Information unterspezifiziert sind, sowie separat dazu passende Vokabularelemente mit dem Merkmal [red], die postsyntaktisch totale Reduplikation des Komplements unter Auslassung von sich selbst $(<>)$ in den Bereich links des P-Elements (mit \# als Wortgrenze) auslösen werden.

27 Die anderen an der sequenziellen Nominalreduplikation beteiligten Präpositionen erfahren die gleiche Behandlung. Dies wird letztlich so zu implementieren sein, dass die Regel in (45) verallgemeinert wird und /auf/ durch $/ \mathrm{P}_{\alpha} /$ ersetzt wird, wobei $\alpha$ die Zugehörigkeit eines Vokabularelements zur Menge derjenigen Vokabularelemente P kodiert, die [red] tragen können. 
In der Syntax hat die unterspezifizierte und noch nicht phonologisch realisierte Präposition [-V]:[red] wie alle transitiven Präpositionen ein Subkategorisierungsmerkmal, das zu Verkettung mit einer NP und Projektion einer [-V]P führt. Gemäß der angenommenen Dekomposition kategorialer Merkmale ist dieses Subkategorisierungsmerkmal nunmehr statt als $[\bullet \mathrm{N} \bullet]$ als $[\bullet+\mathrm{N},-\mathrm{V} \bullet$ zu notieren. (Man beachte, dass (44) für Subkategorisierungsmerkmale keine Konsequenzen hat.) Weitere Verkettung findet in $[-\mathrm{V}] \mathrm{P}$ nicht statt. In diesem Bereich verhalten sich die an der sequenziellen Nominalreduplikation beteiligten Präpositionen exakt wie andere Präpositionen des Deutschen: Die strukturaufbauenden Merkmale der ursprünglich voll spezifizierten Präposition sind von kategorialer Unterspezifikation ja nicht affiziert. Dies löst bereits das Hauptproblem im Zusammenhang mit (37) und (38); wie (46) zeigt, ist die syntaktische Struktur für sequenzielle Nominalreduplikation vollkommen regulär.

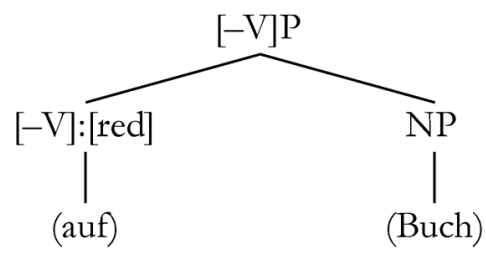

Die unterspezifizierte [-V]P kann sowohl in adverbialen (PP), als auch in Argumentkontexten (NP) auftreten. Die Voraussetzung hierfür wurde bereits eingeführt: Die Dekomposition kategorialer Merkmale zur Erfassung natürlicher Klassen von Kategorien ist auch in Subkategorisierungsmerkmalen reflektiert. So kann [-V]P die Subkategorisierungsforderung von z.B. einem $\mathrm{V}$ mit dem Merkmal $[\bullet+\mathrm{N},-\mathrm{V} \bullet]$ erfüllen und in Argumentkontexten auftreten, anders als eine voll spezifizierte PP $([-\mathrm{N},-\mathrm{V}])$, die eine Merkmalsinkompatibilität auslöst. Ebenso kann $[-\mathrm{V}] \mathrm{P}$ in adverbialen Kontexten auftreten, wo ein kategoriales Merkmal $[+\mathrm{N}]$ normalerweise nicht toleriert wird. ${ }^{28}$

28 Es stellt sich die Frage, wie die unterspezifizierte [-V]P als externes Argument an SubjektVerb-Kongruenz partizipieren kann. Dafür eröffnen sich zwei Möglichkeiten. Zum einen könnte immer Default-Kongruenz involviert sein, denn der Numerusmarker auf dem Verb trägt hier immer das Default-Merkmal [singular] (wie in (32d)); vgl. etwa (i-ab).

(i) a. Buch auf Buch wurde durchgelesen.

b. *Buch auf Buch wurden durchgelesen.

Zum anderen könnte Bezug genommen werden auf das Konzept des relativierten Kopfes (vgl. Williams 1981), wie es ohnehin für Koordination benötigt wird, wenn die oberste Schicht einer Konstituente mit zwei koordinierten NPs eine \&P (mit \& als Kopf) ist: Ist der höchste Kopf für $\Phi$ - oder Kasusmerkmale nicht spezifiziert, blockiert er auch nicht die Weitergabe der relevanten Information von unten. Da in [-V]Ps syntaktisch nur eine einzige NP vorhanden ist, und die im Singular steht, ergibt sich (anders als bei der Koordination) Singularkongruenz. 
Nach der Syntax erfolgt schließlich die phonologische Realisierung. Zunächst werden die Vokabularelemente in die syntaktischen Positionen eingesetzt. Dann erfolgt die Reduplikation durch Transkription und Reparatur. Angenommen, das abstrakte P-Morphem mit dem Merkmal [red] wird durch das Vokabularelement $/[<\#$ auf $>/$ realisiert, und das nominale Komplement durch das Vokabularelement /bu:x/. Dann stellt sich die Vorbereitung der Reduplikation wie in (47a) dar (die rechte Duplikationsfuge wird per Default am rechten Rand eingesetzt). Transkription generiert wie in (47b) gezeigt iterativ links neue Taktpositionen, die jeweils der Reihe nach mit einem zu reduplizierenden Segment assoziiert werden; die Segmente des Vokabularelements / [<\#auf $>$ / liegen in der Kürzungsdomäne und werden daher von der Transkription nicht affiziert. Am Ende gibt es dann den Reparaturprozess, der die Segmente der Reihe nach kopiert, um phonologische Realisierung zu ermöglichen, und der somit Reduplikation bewirkt; vgl. (47c).

a. Vorbereitung

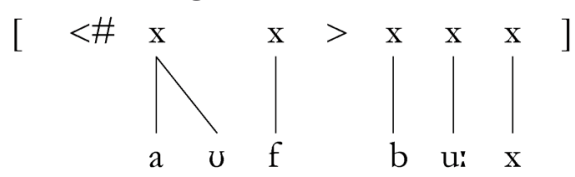

b. Transkription

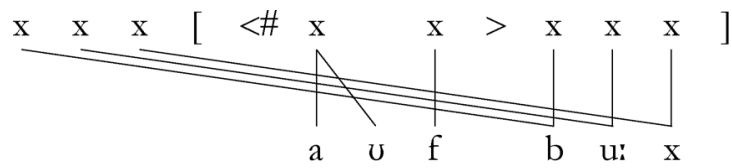

c. Reparatur

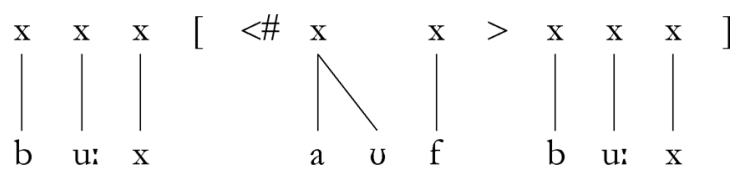

$\mathrm{Zu}$ zeigen ist nun noch, ob und, wenn ja, wie die vorgestellte strikt regelbasierte Analyse von sequenziellen Nominalreduplikationen die problematischen Eigenschaften in (i)-(vi) ableiten kann. Ich fange mit der Identität an (i): Dies folgt unmittelbar aus der Annahme, dass hier echte Reduplikation im Spiel ist.

Der Status von sequenziellen Nominalreduplikationen als Konstituenten, die z.B. syntaktisch bewegt (intern verkettet) werden können (ii), ergibt sich ohne Weiteres aus der syntaktischen Analyse als [-V]P (auch wenn das, was bewegt wird, das nach links reduplizierte Element noch nicht aufweist). 
Was die Minimalität (iii) betrifft, so ist es eine ganz allgemeine Eigenschaft von Reduplikation, dass der Prozess immer nur höchstens Wort-große Elemente affizieren kann. Konkret kann man auf der Basis der hier angenommenen Reduplikationstheorie davon ausgehen, dass zwar mindestens ein Segment transkribiert werden muss, wenn eine Transkriptionsdomäne vorliegt; dass danach aber, wenn die Transkriptionsdomäne zu groß gewählt wurde, die Transkription nach dem Einlesen eines \#-Symbols stoppt, ergebnislos abbricht und zum Zusammenbruch der Derivation führt.

Die Multifunktionalität (iv) folgt wie gezeigt aus der syntaktischen Analyse als unterspezifizierte $[-\mathrm{V}] \mathrm{P}$.

Das Flexionsverbot (v) wird dagegen zunächst einmal nicht automatisch abgeleitet. Hier wären möglicherweise weitere Annahmen erforderlich. Vermutlich hat die Restriktion im Deutschen aber andere, unabhängige Ursachen (vgl. Fußnote 22), die mit Beschränkungen für Kasusmarker auf bloBen Ns oder auch der Semantik des Plurals zu tun haben könnten. In dieses Bild fügt sich auch gut ein, dass andere indoeuropäische Sprachen zwar ebenfalls sequenzielle Nominalreduplikation zulassen, aber kein Flexionsverbot kennen; vgl. etwa šag za šagom (,Schritt für Schritt $_{\text {instr }}{ }^{\circ}$ ) im Russischen oder kousek po kousku (,Stück für Stück ${ }_{\mathrm{lok}}$ ) im Tschechischen. Solche Beispiele könnten der vorgestellten Analyse durch die Annahme akkomodiert werden, dass die Duplikationsdomäne hier vor dem Kasusmarker endet. ${ }^{29}$

Die letzte abzuleitende Eigenschaft (Inselstatus; (vi)) ergibt sich im Fall der Bewegung entweder daraus, dass es das bewegte Element in der Syntax noch gar nicht gibt und spätere Reduplikation strikt lokal ist (vgl. (34a), hier wiederholt als (48a)); oder aber daraus, dass Komplemente von P im Deutschen (außer im Fall der Pronominaladverbien, siehe oben) nicht bewegt werden können und abgesehen davon die Bewegung das zu reduplizierende Element vor einer möglichen Reduplikation weggeschoben hat (vgl. (34b), wiederholt als (48b)); oder daraus, dass es das zurückgelassene Element in der Syntax noch gar nicht gibt und, wiederum, Reduplikation strikt lokal ist (vgl. (34c), wiederholt als (48c)); oder schließlich daraus, dass eine NichtKonstituente bewegt wurde und darüber hinaus wieder der Kontext für post-syntaktische Reduplikation zerstört ist; vgl. (34d), wiederholt als (48d).
a. *Jahr bin ich für Jahr dorthin gefahren.
b. *Jahr bin ich Jahr für dorthin gefahren.
c. *Für Jahr bin ich Jahr dorthin gefahren.
d. *Jahr für bin ich Jahr dorthin gefahren.

29 Andererseits beobachtet Jackendoff (2008, S. 27), dass zumindest im Polnischen Erst-und Zweitglied unterschiedliche Kasusmarker tragen können. Dies ist unter der gegenwärtigen Analyse zunächst einmal unerwartet. Es mag ein Indiz dafür sein, dass die Phänomene (oder zumindest Teile davon) in slawischen Sprachen doch ganz anders analysiert werden müssen (und vielleicht letztlich auch nicht Phrasemstatus haben); alternativ bräuchte man eine relativ komplexe Analyse, mit einer Komplikation des Reduplikationsmechanismus und unter Umständen auch einem postsyntaktischen Zugang zu Kasus. 
Was den Inselstatus für Referenz betrifft, so setze ich ohne weitere Argumentation voraus, dass das aus der Interpretation von [red] folgt; vgl. (49) $(=(35))$.

(49) Pass für Pass habe ich durchgesehen, und dann *war er/?? waren sie weg.

Hiermit ist der konstruktive Teil der Analyse im Wesentlichen abgeschlossen. Ich möchte jedoch noch auf zwei interessante Konsequenzen hinweisen, die sich aus dem Reduplikationsansatz ergeben könnten. Erstens kann Reduplikation total oder partiell sein; bisher habe ich vorausgesetzt, dass bei der sequenziellen Nominalreduplikation nur Ersteres möglich ist. Bei optional enger gewählter Duplikationsdomäne (z.B. ]-Einfügung nach der ersten Wurzel des Komplements von P) könnte im Prinzip auch partielle Reduplikation entstehen. Das Datenmuster, das damit vorausgesagt würde, ergibt sich aus (50).

a. (i) \#Ich habe Hand-für Handbuch durchgesehen.

(ii) *Ich habe -Buch für Handbuch durchgesehen.

(iii) *Ich habe Handbuch für -buch durchgesehen.

(iv) *Ich habe Handbuch für Hand- durchgesehen.

b. (i) \#Sie haben Tagungs- auf Tagungsmappe ausgegeben.

(ii) *Sie haben -Mappe auf Tagungsmappe ausgegeben.

(iii) *Sie haben Tagungsmappe auf -mappe ausgegeben.

(iv) *Sie haben Tagungsmappe auf Tagungs- ausgegeben.

Die (i)-Beispiele, die dann jeweils als einzig mögliche Fälle von partieller Reduplikation von der Analyse vorhergesagt würden, mögen ungewöhnlich und markiert sein. Es hat sich jedoch bei informellen Befragungen einer ganzen Reihe von Sprechern herausgestellt, dass sie durchweg als wesentlich akzeptabler eingestuft werden als die entsprechenden Varianten in (ii), (iii) und (iv); und dieser Kontrast wäre abgeleitet. ${ }^{30}$

Zweitens kann das bei der (totalen) sequenziellen Reduplikation im Deutschen involvierte $\mathrm{N}$ morphologisch beliebig komplex sein; vgl. Handbuch für Handbuch, Reisesser auf Reisesser, Hundesucherbeobacbter auf Hundesucherbeobachter. Damit kann man aber im Deutschen Muster generieren, die sehr ähnlich denen sind, die Culy (1985) im Bambara beobachtet hat, und auf deren Basis eines der zentralen Argumente gegen die Kontextfreiheit natürlicher

30 Eine mögliche alternative Erklärung für das beobachtete Muster könnte auf eine Tilgungsoperation wie in $A n$ - und Abreise Bezug nehmen; der präpositionale Kontext wäre allerdings ungewöhnlich (denn die einschlägige Tilgungsregel, Right Node Raising, appliziert typischerweise nur unter Koordination). Abgesehen davon sei noch darauf hingewiesen, dass partielle Reduplikation möglicherweise einen Ansatz bieten könnte, die ansonsten nicht ohne Weiteres erfassbaren Beispiele mit pränominaler Modifikation im Englischen zu analysieren; vgl. Fußnote 21. Insbesondere die zu (50) analoge Asymmetrie zwischen (i-a) und (i-c) in Fußnote 21 könnte suggestiv sein. 
Sprachen etabliert worden ist. Bambara hat zwei morphologische Operationen, die miteinander kombiniert werden können und so zu nicht kontextfreien Mustern wie $\mathrm{a}^{\prime \prime} \mathrm{b}^{n} \mathrm{a}^{\prime \prime} \mathrm{b}^{n}$ führen. Dies ist zum einen die N-o-N-Bildung wie in (51a), zum anderen die $\mathrm{N}+\mathrm{V}_{\text {trans }}+l a$-Bildung (Komposition plus Derivation) in (51b). Ein Resultat der freien Kombinierbarkeit der beiden Operationen findet sich in (51c). Wie bei der sequenziellen Nominalreduplikation im Deutschen gilt eine Identitätsbedingung; vgl. (51d). Dies und der Umstand, dass der semantische Effekt (,welcher ... auch immer) für Reduplikation prototypisch ist, könnten Anlass geben, die Daten im Bambara auf eine vergleichbare Art und Weise per Reduplikation zu erfassen. Insgesamt ergäben sich dann möglicherweise interessante Konsequenzen für das Argument gegen Kontextfreiheit auch auf der Basis des Deutschen. ${ }^{31}$
a. wulu o wulu Hund o Hund ,welcher Hund auch immer
b. wulu nyini la Hund such er ,Hundesucher
c. wulu nyini lam filè lan o wulu nyini lat" file $\quad \mathrm{a}^{n}$ Hund such er beobacht er o Hund such er beobacht er ,welcher Hundesucherbeobachter auch immer
d. *wulu nyini la o wulu filè la Hund such er o Hund beobacht er

Was auch immer der Status von Beispielen wie denen in (50) und (51) in Bezug auf die sequenziellen Nominalreduplikationen des Deutschen letztlich ist: Zusammenfassend lässt sich festhalten, dass es Evidenz dafür gibt, dass sequenzielle Nominalreduplikationen eine interne Struktur haben. Die Evidenz betrifft den Wortstatus der Teile und, nicht zuletzt, die Produktivität (anders als bei den verblosen Direktiven gibt es jedoch mit der möglichen Ausnahme der Kongruenzregeln keine Regeln, die auf die interne Struktur zugreifen). Es gibt einige auf den ersten Blick zum Teil ungewöhnlichen Eigenschaften der sequenziellen Nominalreduplikationen. Hierfür muss aber nicht angenommen werden, dass es sich um Konstruktionen handelt; die Eigenschaften ergeben sich fast vollständig ohne Weiteres aus dem hier entwickelten regelbasierten Ansatz. Tatsächlich sind umgekehrt unter einem konstruktionsbasierten Ansatz sämtliche Eigenschaften der sequenziellen Nominalreduplikation zunächst einmal rätselhaft; sie können gesondert stipuliert, aber nicht einheitlich abgeleitet werden. Die Konklusion ist daher wie bei den verblosen Direktiven: Sequenzielle Nominalreduplikationen im Deutschen sind keine Konstruktionen.

31 Culy (1985) zeigt auf der Basis von Tonverteilungen, dass die wohlgeformten Beispiele in (51) phonologisch Wortstatus haben; dies bleibt ein Unterschied, denn das erste $\mathrm{N}$ in der sequenziellen Nominalreduplikation des Deutschen bildet fraglos ein eigenes phonologisches Wort. 


\section{Zusammenfassung und Ausblick}

Ich habe argumentiert, dass verblose Direktive und sequenzielle Nominalreduplikationen im Deutschen zwar auf den ersten Blick klare Kandidaten für Konstruktionen sein mögen, dass sie sich aber bei genauerem Hinsehen einer regelbasierten Analyse ohne konstruktionsspezifische Annahmen nicht widersetzen und eine solche Analyse sogar stützen, weil dadurch jeweils charakteristische Eigenschaften auf einfache Weise abgeleitet werden können (und nicht gesondert stipuliert zu werden brauchen, wie in konstruktionsbasierten Ansätzen). In beiden Fällen sind sämtliche scheinbaren Idiosynkrasien rückführbar auf die Eigenschaften eines einzigen Merkmals (nämlich [apass] bzw. [red]), das in der Numeration einem lexikalischen Element beigefügt werden kann (nämlich $\mathrm{v}$ bzw. P) und dann im weiteren Verlauf der Derivation drastische Konsequenzen hat und massive Abweichungen gegenüber dem ohne das Merkmal Erwartbaren bewirkt. Verblose Direktive sind unter dieser Perspektive keine Konstruktionen, sondern VerbErst-Sätze mit regelhaftem (nur eben nicht morphologisch realisierbarem) Antipassiv; sequenzielle Nominalreduplikationen sind ebenfalls keine Konstruktionen, sondern einfache Präposition-Nomen-Verbindungen mit regelhafter Reduplikation. Wenn dieses Ergebnis auch für andere komplexe sprachliche Ausdrücke mit scheinbar ungewöhnlichen Eigenschaften generalisiert werden kann, dann drängt sich die weitergehende Vermutung auf, dass es vielleicht (außer Morphemen) gar keine Konstruktionen gibt.

\section{Literatur}

Ackerman, Farrell/Webelhuth, Gerth (1998): A theory of predicates. Stanford.

Adger, David (2003): Core syntax: a minimalist approach. Oxford u.a.

Baker, Mark C. (1988): Incorporation. A theory of grammatical function changing. Chicago u.a.

Beck, Sigrid/Stechow, Arnim von (2006): Dog after dog revisited. Ms. Tübingen.

Bierwisch, Manfred (1967): Syntactic features in morphology: general problems of socalled pronominal inflection in German. In: To honor Roman Jakobson: essays on the occasion of his 70th birthday, 11 October 1966. Bd. 1. (= Janua linguarum: Series maior 31). Den Haag u.a., S. 239-270.

Bierwisch, Manfred (1988): On the grammar of local prepositions. In: Bierwisch, Manfred (Hg.): Syntax, Semantik und Lexikon: Rudolf Rủžička zum 65. Geburtstag. (= Studia grammatica 29). Berlin, S. 1-65.

Bittner, Maria/Hale, Ken (1996): The structural determination of case and agreement. In: Linguistic Inquiry 27, S. 1-68. 
Bruening, Benjamin (2009): Selectional asymmetries between CP and DP suggest that the DP hypothesis is wrong. In: MacKenzie, Laurel (Hg.): Proceedings of the 32th Annual Penn Linguistics Colloquium. (= Penn Working Papers in Linguistics 15.1). Philadelphia, S. 26-35.

Burger, Harald (1973): Idiomatik des Deutschen. (Unter Mitarbeit von Harald Jaschke). (= Germanistische Arbeitshefte 16). Tübingen.

Cernyševa, Irina I. (1970): Frazeologija sovremennogo nemeckogo jazyka. Moskau.

Chomsky, Noam (1957): Syntactic structures. (= Janua Linguarum: Series Minor 4). Den Haag.

Chomsky, Noam (1980): Rules and representations. Oxford.

Chomsky, Noam (1981): Lectures on government and binding. (= Studies in Generative Grammar 9). Dordrecht.

Chomsky, Noam (1982): Some concepts and consequences of the theory of government and binding. (= Linguistic Inquiry Monographs 6). Cambridge, MA u.a.

Chomsky, Noam (1995): The minimalist program. (= Current Studies in Linguistics Series 28). Cambridge, MA u.a.

Chomsky, Noam (2001): Derivation by phase. In: Kenstowicz, Michael (Hg.): Ken Hale. A life in language. (= Current Studies in Linguistics 36). Cambridge, MA u.a., S. 1-52.

Chomsky, Noam (2008): On phases. In: Freidin, Robert/Otero, Carlos/Zubizarreta, Maria Luisa (Hg.): Foundational issues in linguistic theory. Essays in honor of Jean-Roger Vergnaud. (= Current Studies in Linguistics 45). Cambridge, MA u.a., S. 133-166.

Comrie, Bernard (1979): Degrees of ergativity: some Chukchee evidence. In: Plank, Frans (Hg.): Ergativity. Towards a theory of grammatical relations. London u.a., S. 219-240.

Culicover, Peter/Jackendoff, Ray (2005): Simpler syntax. Oxford u.a.

Culy, Christopher (1985): The complexity of the vocabulary in Bambara. In: Linguistics and Philosophy 8, S. 345-351.

Di Sciullo, Anna Maria/Williams, Edwin (1987): On the definition of word. (= Linguistic Inquiry Monographs 14). Cambridge, MA u.a.

Eisenberg, Peter (2000): Grundriß der deutschen Grammatik. Bd. 1: Das Wort. Stuttgart.

Embick, David/Noyer, Rolf (2001): Movement operations after syntax. In: Linguistic Inquiry 32, S. 555-595.

Everaert, Martin (1991): The lexical representation of idioms and the morphologysyntax interface. Ms. Utrecht.

Fanselow, Gisbert (1991): Minimale Syntax. Habil. Passau.

Fanselow, Gisbert (2008): Bootstrapping verb movement and the clausal architecture of German (and other languages). Ms. Potsdam. [Auch erschienen in: Alexiadou, Artemis et al. (Hg.) (2009): Advances in comparative Germanic syntax. Amsterdam u.a., S. 85-117]. 
Fischer, Kerstin/Stefanowitsch, Anatol (2006): Konstruktionsgrammatik: Ein Überblick. In: Fischer, Kerstin/Stefanowitsch, Anatol (Hg.): Konstruktionsgrammatik I: Von der Anwendung zur Theorie. (= Stauffenburg Linguistik 40). Tübingen, S. 3-17.

Fischer, Silke (2006): Zur Morphologie der deutschen Personalpronomina - eine Spaltungsanalyse. In: Müller, Gereon/Trommer, Jochen (Hg.): Subanalysis of argument encoding in distributed morphology. (= Linguistische Arbeitsberichte 84). Leipzig, S. 77-101.

Fleischer, Wolfgang (1997): Phraseologie der deutschen Gegenwartssprache. 2., durchges. u. erg. Aufl. Tübingen.

Frampton, John (2009): Distributed reduplication. (= Linguistic Inquiry Monograph 52). Cambridge, MA.

Fraser, Bruce (1970): Idioms within a transformational grammar. In: Foundations of Language 6, S. 22-42.

Friederici, Angela et al. (2006): The brain differentiates human and non-human grammars: functional localization and structural connectivity. In: Proceedings of the National Academy of Sciences of the United States of America (PNAS) 103, S. 24582463.

Gallmann, Peter (1996): Die Steuerung der Flexion in der DP. In: Linguistische Berichte 164, S. 283-314.

Gazdar, Gerald et al. (1985): Generalized phrase structure grammar. Oxford.

Georgi, Doreen/Müller, Gereon (2010): Noun phrase structure by reprojection. In: Syntax 13, 1, S. 1-36.

Goldberg, Adele E. (2003): Constructions: a new theoretical approach to language. In: Trends in Cognitive Sciences 7, S. 219-224.

Goldberg, Adele E. (2006): Constructions at work: the nature of generalization in language. Oxford u.a.

Grewendorf, Günther (1988): Aspekte der deutschen Syntax: eine Rektions-BindungsAnalyse. (= Studien zur deutschen Grammatik 33). Tübingen.

Halle, Morris (1997): Distributed morphology: impoverishment and fission. In: Bruening, Benjamin/Kang, Yeonyung/McGinnis, Martha (Hg.): Papers at the Interface. (= MIT Working Papers in Linguistics 30). Cambridge, MA, S. 425-449.

Halle, Morris/Marantz, Alec (1993): Distributed morphology and the pieces of inflection. In: Hale, Kenneth L./Keyser, Samuel J. (Hg.): The view from building 20. Essays in linguistics in honor of Sylvain Bromberger. (= Current Studies in Linguistics Series 24). Cambridge, MA u.a., S. 111-176.

Halle, Morris/Marantz, Alec (1994): Some key features of distributed morphology. In: Carnie, Andreas/Harley, Heidi/Bures, Tony (Hg.): Papers on phonology and morphology. (= MIT Working Papers in Linguistics 21). Cambridge, MA, S. 275-288.

Harley, Heidi/Noyer, Rolf (2003): Distributed morphology. In: Cheng, Lisa/Sybesma, Rint (Hg.): The second GLOT international state-of-the-article book. (= Studies in Generative Grammar 61). Berlin u.a., S. 463-496. 
Hauser, Marc/Chomsky, Noam/Fitch, W. Tecumseh (2002): The faculty of language: what is it, who has it, and how did it evolve? In: Science 298, S. 1569-1579.

Heck, Fabian/Müller, Gereon (2007): Extremely local optimization. In: Brainbridge, Erin/Agbayani, Brian (Hg.): Proceedings of WECOL 2006. Fresno, S. 170-183.

Holmberg, Anders (2000): Scandinavian stylistic fronting: how any category can become an expletive. In: Linguistic Inquiry 31, S. 445-483.

Jackendoff, Ray (1997): The architecture of the language faculty. (= Linguistic Inquiry Monographs 28). Cambridge, MA, u.a.

Jackendoff, Ray (2002): Foundations of language: brain, meaning, grammar, evolution. Oxford u.a.

Jackendoff, Ray (2008): Construction after construction and its theoretical challenges. In: Language 84, S. 8-28.

Jacobs, Joachim (2006): Ein kleines Skopus-Rätsel für Manfred. In: Gärtner, Hans-Martin et al. (Hg.): Between 40 and 60 puzzles for Krifka. Berlin. Internet: www.zas.gwz -berlin.de/fileadmin/material/40-60-puzzles-for-krifka/pdf/jacobs.pdf (Stand: 10.02.2011).

Jacobs, Joachim (2008): Wozu Konstruktionen? In: Linguistische Berichte 213, S. 3-44.

Keßelmeier, Katja/Kiss, Tibor/Müller, Antje/Roch, Claudia/Stadtfeld, Tobias/Strunk, Jan (2009): Developing an annotation scheme and a reference corpus for preposition senses in German. Ms. Bochum.

Kiss, Tibor (2008): Towards a grammar of preposition-noun combinations. In: Müller, Stefan (Hg.): Proceedings of the 15th International Conference on Head-Driven Phrase Structure Grammar. Stanford, S. 116-130.

Kiss, Tibor (in diesem Band): Bedingungen für den Wegfall eines Artikels: Distribution und Interpretation von Präposition-Nomen-Kombinationen.

Larson, Richard (1988): On the double object construction. In: Linguistic Inquiry 19, S. 335-391.

Malkiel, Yakov (1959): Studies in irreversible binomials. In: Lingua 8, S. 113-160.

Marantz, Alec (1995): ,Cat' as a phrasal idiom: consequences of late insertion in distributed morphology. Ms. Cambridge, MA.

Marantz, Alec (1998): No escape from syntax: don't try morphological analysis in the privacy of your own lexicon. In: Dimitriadis, Alexis et al. (Hg.): Proceedings of Penn Linguistics Colloquium 21. Philadelphia, S. 201-225.

Martinet, André (1964): Elements of general linguistics. Chicago.

Müller, Gereon (1997): Beschränkungen für Binomialbildung im Deutschen. In: Zeitschrift für Sprachwissenschaft 16, S. 5-51.

Müller, Gereon (2000): Idioms and transformations. Vortragshandout, GGS-Tagung in Potsdam. Internet: www.uni-leipzig.de/ muellerg/mu51.pdf (Stand: 07.01.2011).

Müller, Gereon (2010): Movement from verb-second clauses revisited. In: Hanneforth, Thomas/Fanselow, Gisbert (Hg.): Language and logos: studies in theoretical and computational linguistics. Festschrift for Peter Staudacher on his 70th Birthday. (= Studia grammatica 72$)$. Berlin. 
Müller, Stefan (2006): Phrasal or lexical constructions? In: Language 82, 4, S. 850-883.

Noyer, Rolf (1992): Features, positions, and affixes in autonomous morphological structure. Diss. Cambridge, MA.

Nunberg, Geoffrey/Sag, Ivan/Wasow, Thomas (1994): Idioms. In: Language 70, 3 , S. 491-538.

Pesetsky, David (1985): Morphology and logical form. In: Linguistic Inquiry 16, S. 193 246.

Pike, Kenneth L. (1965): Non-linear order and anti-redundancy in German morphological matrices. In: Zeitschrift für Mundartforschung 31, S. 193-221.

Roberts, Ian/Roussou, Anna (2002): The extended projection principle as a condition for the tense-dependency. In: Svenonius, Peter (Hg.): Subjects, expletives, and the EPP. New York u.a., S. 125-156.

Roeper, Thomas (2007): 'The prism of grammar. Cambridge, MA u.a.

Ruhl, Charles (1975): ,Kick the Bucket' is not an idiom. (= Interfaces 2.4). Washington, DC.

Sailer, Manfred (2003): Combinatorial semantics and idiomatic expressions in HeadDriven Phrase Structure Grammar. Diss. Tübingen. Internet: http://w210.ub.uni -tuebingen.de/dbt/volltexte/2003/916/ (Stand: 07.01.2011).

Šanskij, Nikolaj M. (1972): Leksikologija sovremennogo russkogo jazyka. Moskau.

Stechow, Arnim von (1992): Kompositionsprinzipien und grammatische Struktur. In: Suchsland, Peter (Hg.): Biologische und soziale Grundlagen der Sprache. Interdisziplinäres Symposium des Wissenschaftsbereiches Germanistik der FriedrichSchiller-Universität Jena, 17.-19. Oktober 1989. (=Linguistische Arbeiten 280). Tübingen, S. 175- 248.

Stefanowitsch, Anatol (2009): Bedeutung und Gebrauch in der Konstruktionsgrammatik. Wie kompositionell sind modale Infinitive im Deutschen? In: Zeitschrift für Germanistische Linguistik 37, S. 565-592.

Sternefeld, Wolfgang (2006): Syntax. Eine morphologisch motivierte generative Beschreibung des Deutschen. 2 Bde. (= Stauffenburg-Linguistik 31). Tübingen.

Stolz, Thomas (2009): Total reduplication: syndetic vs. asyndetic patterns in Europe. In: Grazer Linguistische Studien 71, S. 99-113.

Tomasello, Michael (2003): Constructing a language. A usage-based theory of language acquisition. Cambridge, MA u.a.

Trommer, Jochen (1999): Morphology consuming syntax' resources. In: Procceedings of the ESSLI Workshop on Resource Logics and Minimalist Grammars. Nijmegen, S. 37-55.

Wiese, Bernd (2001): Pronominale Deklination. Handout, Institut für Deutsche Sprache. Mannheim. 
Wilder, Christopher (2008): The PP-with-DP construction. In: Witkoś, Jacek/Fanselow, Gisbert (Hg.): Elements of slavic and germanic grammars: a comparative view. (= Polish Studies in English Language and Linguistics 23). Frankfurt a.M. u.a., S. 235-253.

Williams, Edwin (1981): Argument structure and morphology. In: The Linguistic Review 1, S. 81-114.

Williams, Edwin (1994): Remarks on lexical knowledge. In. Lingua 92, S. 7-34.

Williams, Edwin (2005): What is beyond explanatory adequacy? Ms. Princeton.

Wunderlich, Dieter (1993): Diathesen. In: Jacobs, Joachim et al. (Hg.): Syntax. Ein internationales Handbuch zeitgenössischer Forschung. Halbbd. 1. (Handbücher zur Sprach- und Kommunikationswissenschaft 9). Berlin u.a.

Wunderlich, Dieter (2004): Emanzipation der Linguistik in Deutschland. Ein Interview mit Dieter Wunderlich. In: Linguistische Berichte 200, S. 427-450.

Zwicky, Arnold (1974): Taking a false step. In: Language 50, S. 215-242. 



\title{
Tibor Kiss
}

\section{Bedingungen für den Wegfall eines Artikels: Distribution und Interpretation von Präposition-Nomen-Kombinationen ${ }^{1}$}

\begin{abstract}
Kombinationen aus Präposition und artikelloser Nominalprojektion, deren syntaktischer Kopf ein zählbares Substantiv im Singular ist, fristeten lange Zeit ein Schattendasein in der Grammatikschreibung. Sie wurden ignoriert oder als Ausnahmen beschrieben, obwohl sie offenkundig regelhaft gebildet werden. Im vorliegenden Aufsatz verwenden wir computerlinguistische Verfahren, insbesondere „Annotation Mining“" und logistische Regression, um die syntaktische Distribution dieser Kombinationen zu charakterisieren und anhand zweier Präpositionen (obne und unter) detailliert die Realisationsbedingungen zu bestimmen.
\end{abstract}

\section{Einleitung}

Präpositionen werden in vielen Sprachen mit Nominalphrasen kombiniert. Viele Präpositionen gestatten jedoch auch eine Kombination mit einem artikellosen Substantiv oder einer artikellosen Substantivgruppe, wie die Beispiele in (1) zeigen.

(1) auf parlamentarische Anfrage, bei absolut klarer Zielsetzung, in untertreibender Anspielung, mit schwer beladenem Rucksack, ohne mündliche Vorwarnung, unter sanfter Androhung

Die Beispiele in (1) sind bemerkenswert, denn die Substantive werden als zählbar analysiert und dürften infolge dieser Eigenschaft eigentlich nicht ohne Artikel realisiert werden. So stellt der Duden (2005, S. 337) in der Regel 442 fest, dass „Substantive mit Merkmalkombination ,zählbar“ plus Singular ... grundsätzlich immer ein Artikelwort bei sich [haben], und wenn es als letzte Möglichkeit der indefinite Artikel ist."

1 Die vorliegenden Ergebnisse entstammen dem Projekt „Erfassung und Analyse syntaktischer und semantischer Eigenschaften von Präposition-Substantiv-Sequenzen unter Verwendung computerlinguistischer Analyseverfahren", das seit 2009 freundlicherweise von der DFG gefördert wird. Die Ergebnisse hätten ohne die Mitarbeit von Antje Müller, Claudia Roch, Tobias Stadtfeld, Katja Keßelmeier und Jan Strunk nicht erzielt werden können. Ich danke ihnen herzlich. Durch die Einladung zur IDS-Jahrestagung 2010 hatte ich die Gelegenheit, Teile des vorliegenden Aufsatzes zu präsentieren. Ich danke Stefan Engelberg und den Organisatoren für die Einladung, Anke Holler, Kristel Proost und Stefan Engelberg für ihre Kommentare und den Zuhörern für die Diskussionsbeiträge. 
Deutlich wird anhand der Beispiele in (1) auch, dass es sich hierbei nicht um Paarbildungen aus Präposition und Substantiv handelt: Pränominale Modifikation ist in vielen Fällen möglich, teilweise auch durchaus komplex. Dieser Eindruck wird noch dadurch verstärkt, dass postnominale Komplemente nicht ausgeschlossen sind:

(2) Riess-Passer unterstrich die Forderung nach Stilllegung des grenznahen Atomkraftwerks Temelin in Tschechien. (NZZ, AUSLAND, 30.11.2001)

Das Beispiel in (2) zeigt darüber hinaus, dass die fraglichen Konstruktionen nicht nur adverbial verwendet werden können, sondern auch in regierten Kontexten erscheinen - die Präposition nach wird in (2) durch Forderung regiert.

In der Folge bezeichne ich Kombinationen wie die in (1) und (2) als Präposition-Nomen-Kombinationen (PNKen). PNKen wurden in der Grammatikschreibung lange ignoriert oder als Ausnahmen charakterisiert; in den letzten Jahren hat sich die Position aber sowohl aus typologischer als auch aus grammatiktheoretischer Perspektive gewandelt. Himmelmann (1998, S. 316) hat beobachtet, dass PNKen in vielen Sprachen auftreten, deren Grammatiken verlangen, dass zählbare Substantive im Singular durch einen Artikel ergänzt werden müssen. Stvan (1998) bietet für das Englische eine Analyse an, die auf den semantischen Eigenschaften des Substantivs basiert; Baldwin et al. (2006) entwickeln eine Typologie der Konstruktion für das Englische und stellen beiläufig fest, dass es sich zumindest in Teilen um eine produktive - im syntaktischen Sinne also regelhafte - Konstruktion handelt, die durch Ausnahmen nicht erfasst werden kann. ${ }^{2}$ Während der Duden (2005) die Konstruktion als Ausnahme beschreibt, zeigen Kiss (2007) und Dömges et al. (2007) anhand eines quantitativen Produktivitätsmaßes, dass es sich um eine produktive und somit regelhafte Kombination handelt, die somit auch durch die Grammatik erfasst werden muss.

PNKen stellen so die Grammatikschreibung vor eine Herausforderung: Es besteht einerseits weitgehender Konsens darüber, dass die Kombination in vielen Sprachen regelhaft ist; andererseits ist aber auch klar, dass die zugrunde liegenden Regeln offensichtlich nicht dem Typ entsprechen, der etwa die Kombination einer DP mit einem transitiven Verb steuert. ${ }^{3}$ Introspektive Urteile zur Kombinierbarkeit von Präposition und artikelloser

\footnotetext{
Studien zu PNKen im Englischen bezeichnen die Konstruktion als „,determinerless PPs“ (Baldwin et al. 2006) oder „bare PPs“ (Le Bruyn et al. 2009).

Ich gehe im Folgenden davon aus, dass vollständige Nominalprojektionen, die einen Artikel enthalten, DPen sind. Die Festlegung ist im vorliegenden Kontext schon deswegen nützlich, weil so Nominalprojektionen mit Artikel notationell von Nominalprojektionen ohne Artikel unterschieden werden können. Eine Festlegung auf eine bestimmte Analyse der Nominalprojektion ist damit nicht verbunden.
} 
Nominalgruppe werden nur zögerlich getroffen. Sprecher sind ebenfalls sehr zögerlich, neue PNKen zu bilden. Dem steht wiederum die quantitative Produktivität des Prozesses gegenüber - eigentlich ein Widerspruch!

Zur Identifikation der Grammatikalitätsbedingungen der PNKen wähle ich das „Annotation Mining“ (vgl. Chiarcos et al. 2008). Es bietet sich als exploratives Analyseverfahren an, in dem auf die Identifikation oder Elizitation von Grammatikalitätsurteilen verzichtet wird. Grammatikalitätsbedingungen werden aus umfangreichen Sprachdaten abgeleitet, die wiederum durch Merkmale auf den unterschiedlichen linguistischen Ebenen annotiert sind (Lexikon, Syntax, Semantik, globaler Kontext). Die annotierten Daten werden Klassifikationsverfahren aus der inferentiellen Statistik unterworfen, im vorliegenden Fall insbesondere der binären logistischen Regression (Harrell 2001), die in Abschnitt 4.2 vorgestellt wird.

Anhand von zwei Pilotstudien zur Syntax der Präpositionen obne und unter möchte ich erläutern, warum die Ansätze von Stvan (1998) und Baldwin et al. (2006) grundsätzlich in die richtige Richtung weisen, aber dann eben doch nicht auf das Deutsche übertragbar sind. Die Modelle zeigen, dass es unabhängig von der Interpretation der Präpositionen strukturelle Faktoren gibt, die die Weglassbarkeit des Artikels zumindest partiell bedingen.

\section{Zur Grammatik von Präposition-Nomen-Kombinationen}

Die hier verwendete Definition von PNKen ist gegenüber anderen Definitionen durch eine interne und eine externe Bedingung eingeschränkt. Obwohl diese Einschränkungen eigentlich zwingend mitgedacht werden müssen, zeigen etwa die Untersuchungen von Le Bruyn et al. (2009) - in denen weder die interne noch die externe Bedingung eine Rolle spielt - dass es erforderlich ist, die Bedingungen explizit zu machen. Die interne Bedingung sieht vor, dass unter PNKen nur solche Kombinationen aus einer Präposition und einer artikellosen Nominalprojektion fallen, in denen das Substantiv ein zählbares Substantiv im Singular ist. Weder PPen mit Massentermen noch PPen mit nackten Pluralia fallen so unter die PNKen. Da im Deutschen Artikel bei Massentermen nicht obligatorisch sind - Indefinita sind sogar untersagt - und bei Pluralia zumindest optional, entstehen durch den Wegfall des Artikels in diesen Phrasen keine Probleme. Betrachtete man die Distribution des Artikels bei Pluralia und Massentermen in anderen Sprachen, etwa den romanischen Sprachen (vgl. Espinal/McNally ersch. 2011), so würden andere Definitionen erforderlich.

Für das Deutsche liegt zunächst aber die Tücke im Detail: Zum einen setzt die Definition ein Konzept der Zählbarkeit voraus, zum anderen handelt es sich hierbei bestenfalls um eine notwendige Definition, nicht aber um eine hinreichende, denn es kann nicht jede Präposition mit jedem Substantiv in einer PNK kombiniert werden. 
Die externe Bedingung setzt voraus, dass es sich bei der Präposition in der PNK um eine Präposition handelt, die überhaupt DP-Objekte besitzen kann. Auch diese Annahme ist nicht trivial. Sie führt dazu, dass wir nicht alle Präpositionen des Deutschen untersuchen, sondern nur einen Teilbereich der Präpositionen, nämlich solche, die DP-Objekte besitzen und in PNKen auftreten können. Diese Präpositionen sind in (3) aufgeführt; in Abschnitt 2.3 werde ich anhand der Präposition per das Verhalten von Kombinationen aus Präposition und Substantiv erläutern, die dieser Bedingung nicht genügen. Die in (3) aufgeführten Präpositionen erfüllen das externe Kriterium, allerdings nicht mit jedem zählbaren Substantiv im Singular, wie anhand von (4) verdeutlicht werden kann. ${ }^{4}$

(3) an, auf, bei, dank, durch, für, gegen, gemäß, hinter, in, mit, mittels, nach, neben, ohne, seit, über, um, unter, vor, während, wegen

(4) *Riess-Passer wehrt sich gegen Forderung nach Stilllegung des grenznahen Atomkraftwerks Temelin in Tschechien.

Das Beispiel in (4) ist eine Variation des Beispiels in (2), bei der auch auf den wohl tatsächlich obligatorischen Artikel vor Forderung verzichtet wurde. Auch semantisch sehr nahe Substantive können in PNKen nicht einfach ausgetauscht werden, wie anhand der Substantive Voraussetzung und Prämisse in (5) und (6) gezeigt werden kann.

(5) Auch Philipp Egli besteht auf einer eigenen Handschrift - unter der Voraussetzung/der Prämisse des Einverständnisses des Ensembles.

(6) Auch Philipp Egli besteht auf einer eigenen Handschrift - unter Voraussetzung/*Prämisse des Einverständnisses des Ensembles.

Während Voraussetzung mit und ohne Artikel als Komplement von unter erscheinen kann, ist bei Prämisse nur die Realisation in einer PP möglich.

Der Kontrast zwischen (5) und (6) könnte durch zwei Muster erklärt werden: entweder durch einen Rekurs auf eine entsprechende Definition der Zählbarkeit, oder durch den Nachweis tatsächlich fehlender Regelhaftigkeit der Konstruktion. Diese Punkte wollen wir in den folgenden Abschnitten diskutieren.

\subsection{Das Problem der Zählbarkeit}

Die Dudenregel 442 (Duden 2005, S. 337) besagt, dass zählbare Substantive im Singular im Deutschen durch einen Artikel begleitet werden müssen. Daraus folgt nicht nur, dass die Beispiele in (1) und (2) ungrammatisch sein

\footnotetext{
Das Beispiel (4) wird dann nicht als ungrammatisch empfunden, wenn es als Überschrift eines Zeitungsartikels verwendet würde. In Überschriften findet man häufig PNKen, die außerhalb von Überschriften als deutlich abweichend bewertet werden.
} 
sollten. Die Regel setzt auch voraus, dass die Eigenschaft der Zählbarkeit definiert wird. Zählbarkeit könnte als lexikalische Abbildung von Worten auf Denotate verstanden werden. Sind die Denotate zählbar, dann ist auch das Wort zählbar. Diese Definition geht zumindest auf Jespersen (1924) zurück und fand auch in der generativen Grammatik Rückhalt. Sie führt aber zu einer Vielzahl von Problemen. Hier ist nicht der Raum, um dies auch nur andeutungsweise zu erläutern, es soll nur erwähnt werden, dass es nach dieser Definition keine Synonyme $S_{1}$ und $S_{2}$ in einer Sprache geben sollte, bei denen $S_{1}$ zählbar ist, $S_{2}$ aber nicht. ${ }^{5}$ Darüber hinaus kommt diese Definition in Schwierigkeiten, wenn ein zählbares Wort nicht zählbar verwendet wird, man vergleiche hierzu die Verwendung von Fahrzeug vs. Fahrzenge in (7).

(7) Hier bekommen Sie mehr Fahrzeug/Fahrzeuge für ihr Geld.

Allan (1980) hat eine alternative Sichtweise des Konzepts der Zählbarkeit in der Sprachwissenschaft etabliert, die in jüngeren Arbeiten von Borer (2005) und Bale und Barner (2009) auch formal expliziert wurde. Nach dieser Auffassung ist Zählbarkeit keine lexikalische Eigenschaft, sondern eine Eigenschaft nominaler Phrasen, die durch kontextuelle formale Merkmale zugewiesen wird, etwa die Pluralmorphologie oder die Realisation eines Artikels.

Dieser Idee folgend ist ein Nomen zunächst einmal weder zählbar noch nicht zählbar. Wird dieses Nomen in einen entsprechenden Kontext eingesetzt, ist die resultierende Phrase aber zählbar oder nicht. Für das Beispiel (7) bedeutet dies, dass Fahrzeng, wenn es weder flektiert noch durch einen Artikel begleitet wird, als Massenterm analysiert wird. Das Pluralmorphem bildet hingegen einen Kontext für Zählbarkeit, ebenso würde ein solcher Kontext durch einen singularischen oder pluralischen Artikel geschaffen. Für die Syntax der PNKen ergibt sich nun allerdings eine merkwürdige Konsequenz: Da hier die relevanten Substantive in Kontexten realisiert werden, in denen weder ein Artikel noch ein Pluralmorphem vorliegt, wird die Dudenregel 442 schon deswegen nicht verletzt, weil ein Substantiv in einer PNK nach dieser Definition nicht zählbar ist. Dies bedeutet aber auch, dass jedes Substantiv in einer PNK realisiert werden können müsste, weil hier nie ein Kontext für Zählbarkeit vorliegt. Konsequenterweise sollten sich PNKen also wie PPen verhalten und ihre besondere Betrachtung wäre überflüssig. Dass die Schlussfolgerung nun wieder voreilig ist, folgt aus zwei Beobach-

Der Leser erwartet hier sicherlich eine Stellungnahme, aus der hervorgeht, ob es nun entsprechende Synonyme gibt oder nicht. Anhand von Beispielen wie Fußbekleidung vs. Schube könnte man diese Frage nun positiv beantworten. Allerdings ist zu berücksichtigen, dass das Konzept der Synonymie nicht präzise definiert ist. Es wird jedoch auch niemand bestreiten wollen, dass es sehr schwer werden würde, Modelle zu konstruieren, in denen die Aussagen (i) und (ii) unterschiedliche Wahrheitswerte besitzen:

(i) Du hast aber viele Schuhe.

(ii) Du hast aber viel Fußbekleidung. 
tungen: Zum einen haben wir bereits gesehen, dass nicht jedes Substantiv in einer PNK realisiert werden kann; zum anderen würde dies auch bedeuten, dass jedes Substantiv in einer PNK die Interpretation eines Massenterms erhält. Dies ist aber ebenfalls falsch, wie man an Beispielen wie (8) und (9) sieht.

(8) Milosevic unterschrieb auch unter $\varnothing /$ der/einer Androhung von NATO-Bombardementen nicht.

In (8) können wir beobachten, dass bei den Realisationen als PP (DP mit definitem oder indefinitem Artikel) oder PNK die gleiche Interpretation hervorgerufen wird. Es ist dann die plausiblere Überlegung, dass bei einer Realisation mit Artikel tatsächlich Zählbarkeit vorliegt und somit Bedeutungsgleichheit auch bei einer Realisation ohne Artikel. Die gleiche Argumentation gilt auch für die mit durch eingeleitete Phrase in (9).

(9) Ursprünglich war der Artikel als Verbot der Beleidigung jeder Religion eingeführt worden, der Diktator engte ihn jedoch nach 1980 auf die Beleidigung des Islam ein, und er verschärfte ihn durch ø/eine/ die Androhung der Todesstrafe. (NZZ, AUSLAND, 25.02.1995, Originalbeleg mit definitem Artikel)

Es zeigt sich hier, dass die kontextuelle Definition der Zählbarkeit ebenso problematisch ist wie die lexikalistische Position. Darüber hinaus ist es notwendig zu erläutern, was im Rahmen einer automatischen Klassifikation eigentlich unter einem zäblbaren Substantiv zu verstehen ist, wenn weder gänzlich auf den Typ noch gänzlich auf die einzelnen Vorkommen rekurriert werden kann.

Die Zählbarkeitsklassifikation basiert auf zwei statistischen Klassifikatoren, die einerseits das Verhältnis von Plural und Singularvorkommen betrachten und andererseits syntaktische Kontexte (die durch die POS-Tags vor dem zu klassifizierenden Nomen identifiziert werden, vgl. Abschnitt 3). Diese Klassenbildung erfolgt nach einem Vorschlag von Stadtfeld (i.Vorb.), basierend auf den Arbeiten von Gillon (1999) und Barner/Snedeker (2005). Stadtfeld unterscheidet fünf Zählbarkeitsklassen (I = zählbar, II = Pluralia tantum, III = echt ambig, IV und V = nicht zählbar), die nach drei Kriterien identifiziert werden können.

Das erste Kriterium - der Messmodus - erfasst, ob ein Singularvorkommen eines Nomens mit mehr hinsichtlich einer definierten Quantität verglichen werden kann. Für echt zählbare Substantive (Klasse I) ergibt dies keinen Sinn, für nicht-zählbare Substantive der Klasse IV ist ein Vergleich hinsichtlich Quantität von Individuen oder einer anderen Messgröße möglich, für nicht-zählbare Substantive der Klasse V ist ein Vergleich nur hinsichtlich einer nicht auf Individuen quantifizierenden Messgröße möglich. 
(10) a. ??New York hat mehr Hochhaus als Berlin.

b. Paul hat mehr Besteck als Peter.

c. Paul hat mehr Reis als Peter.

Das Beispiel (10a) ist auch dann inakzeptabel, wenn vorher bekannt ist, dass das Gesamtgewicht der Hochhäuser in New York höher ist als das Gewicht aller Hochhäuser Berlins (Beispiele dieses Typs findet man eigentlich nur in Werbekontexten). Das Beispiel (10b) kann wahr sein, wenn es tatsächlich insgesamt mehr individuelle Besteckelemente gibt oder wenn Paul mehr Bestecktypen als Peter besitzt. Das Beispiel (10c) setzt voraus, dass die MessgröBe das Gesamtvolumen ist. Individuen sind also bei Klasse V ausgeschlossen, führen aber bei Klasse IV zu einer Typ- bzw. Behälterinterpretation.

Der zweite Test überprüft verborgene Typlesarten bei Pluralrealisationen. Auch hier erfolgt eine Einbettung des Nomens unter mehr, das Nomen wird nun aber im Plural realisiert. Bei Massentermen der Klasse V ist der Test nicht anwendbar, weil sie keine Pluralrealisation besitzen, bei Massentermen der Klasse IV ergibt die explizite Realisation einer Numeralkonstruktion eine äquivalente Lesart (11), bei zählbaren Substantiven der Klasse I ist diese Äquivalenz nicht gegeben (12).

(11) a. Paul hat mehr Weine als Peter.

b. Paul hat mehr Sorten Wein als Peter.

(12) a. Ulrich vertreibt mehr Weingläser als Paul.

b. Ulrich vertreibt mehr Sorten Weinglas als Paul.

Der letzte Test betrifft die Verwendung des indefiniten Artikels in Kontexten der Form indefiniter Artikel $+N$ ist ein Hyperonym. Bei zählbaren Substantiven der Klasse I ist der indefinite Artikel obligatorisch (13), die Klassen IV und V gestatten die Realisation des indefiniten Artikels nicht (14), es kommt zu Typlesarten, die im vorgegebenen Kontext blockiert sind, weil hier allgemeine Eigenschaften das Prädikat bilden.

(13) a. Ein Auto ist ein Fahrzeug.

b. *Auto ist ein Fahrzeug.

(14) a. Stahl ist eine Metalllegierung.

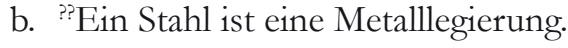

(15) a. Besteck ist ein Werkzeug.

b. ??Ein Besteck ist ein Werkzeug.

Ein wesentlicher Vorteil dieses feineren Klassifikationsansatzes ist die Identifikation echt mehrdeutiger Substantive, die in der bislang nicht diskutierten Klasse III erfasst werden. Diese Substantive sind für eine binäre Klassifikation insofern problematisch, als sie Eigenschaften von zäblbaren und nicht- 
zäblbaren Nomina aufweisen. So gestatten Substantive wie Kuchen oder Fisch die Verwendung des indefiniten Artikels (16a), was für die Zählbarkeit spricht, zugleich kann aber der Artikel auch weggelassen werden, wie (16b) zeigt.

a. Ein Kuchen ist ein Grundnahrungsmittel.

b. Kuchen ist ein Grundnahrungsmittel, meinte schon MarieAntoinette.

PNKen, in denen das Substantiv der Klasse III angehört, werden zunächst aus der Analyse ausgeschlossen, bis die Charakteristika dieser Klasse genauer bestimmt sind. Erfreulicherweise enthält diese Klasse wenige Elemente.

In der folgenden Analyse (vgl. Abschnitte 3 und 4) bezeichne ich die in den PNKen auftretenden Substantive als zäblbar, weil sie der Klasse I und als nicht-zäblbar, wenn sie den Klassen IV oder V zugeordnet werden konnten. Eine binäre Opposition wird damit nicht behauptet.

\subsection{Produktivität}

Wir können somit davon ausgehen, dass zählbare Substantive in PNKen realisiert werden, ein Verweis auf eine mögliche Analyse als Massenterme ist falsch und hilft nicht weiter. Aus dieser Annahme würde folgen, dass Nomina in PNKen grundsätzlich als Massenterme interpretiert würden - und somit PNKen auch grundsätzlich grammatisch sein sollten, wobei eine Unakzeptabilität dann entstünde, wenn eine Massenterminterpretation inkompatibel wäre.

Ein weiterer Ausweg könnte natürlich darin bestehen, die Konstruktionen insgesamt als Ausnahmen zu analysieren. Dies ist etwa der Stand der Dinge im Duden: Die Regel 395 zählt die Ausnahmen auf, die die Realisation eines zählbaren Substantivs ohne Artikel gestatten. Kurz gesagt: Die hier bislang vorgestellten, zweifelsfrei grammatischen Beispiele zählen nicht dazu.

Diese Feststellung soll aber nicht genügen. Basierend auf dem morphologischen Produktivitätsmaß P aus Baayen (2001) und dessen Verallgemeinerung für syntaktische Kontexte in Evert (2004) legen Dömges et al. (2007) eine quantitative Analyse der Produktivität von PNKen mit unter vor, aus der hervorgeht, dass es sich bei PNKen um regelhafte Kombinationen handelt. Ohne die technischen Details dieser Analyse hier zu diskutieren, kann der Ansatz wie folgt zusammengefasst werden: Wenn man davon ausgehen würde, dass eine syntaktische Kombination tatsächlich eine aufzählbare Ausnahme ist, dann müsste es auch endlich viele Instanzen dieser Ausnahme geben. Dies wiederum würde bedeuten, dass bei einem genügend großen Korpus irgendwann einmal beobachtet werden könnte, dass das Vokabular für diese spezielle Konstruktion nicht mehr anwächst (während 
bei regelhaften, nicht aufzählbaren Konstruktionen ein stetiges Vokabularwachstum beobachtbar ist). Wenn wir nun eine Präposition als Beispiel auswählen, dann besteht das fragliche Vokabular aus Substantiven, die mit dieser Präposition kombiniert werden können. In diesem Sinne wächst das Vokabular von unter Androbung auf unter sanfter Androbung nicht an, weil wir hier das Substantiv Androbung bereits gesehen haben. Anders ist dies, wenn wir zunächst unter Androbung und dann unter Auswertung oder auch unter Gewaltandrobung beobachten würden.

Konkretisiert auf eine Präposition wie unter bedeutet dies, dass zu irgendeinem Zeitpunkt $\mathrm{t}_{1}$ die Wahrscheinlichkeit, dass zu Folgezeitpunkten $\mathrm{t}_{1+\mathrm{n}}$ noch neue Instanzen von unter + Substantiv beobachtet werden können, unter einen kritischen Wert fällt, wenn der Prozess nicht produktiv ist.

Diese Annahme kann nun von beobachteten Daten auf nicht beobachtete Daten, also von bereits sehr umfangreichen empirischen Korpora auf wesentlich umfangreichere nicht beobachtete Korpora übertragen werden, wenn man so genannte LNRE-Modelle (,large number of rare events“, Baayen 2001) zur Vorhersage nicht beobachteter Daten verwendet. Dömges et al. (2007) haben gezeigt, dass das plausibelste Modell für unter+Substantiv dasjenige Modell ist, das unendlich viele Instanzen dieser Kombination vorhersagt. Somit ist die Kombination regelhaft, auch der Weg über die Ausnahme ist verbaut.

\subsection{Eine Analyse des Englischen, die im Deutschen nicht funktioniert}

Für PNKen im Englischen schlägt Stvan (1998) vor, dass es einen Prozess der N-Selektion gibt. Nach dieser Analyse werden PNKen durch die in ihnen enthaltenen Substantive lizenziert, wie anhand der Substantive school, jail und prison in (17) illustriert werden kann.

$$
\text { from school, at school, in jail, from jail, in prison, from prison ... }
$$

Baldwin et al. (2006) legen Stvans Analyse zugrunde und argumentieren, dass es sich bei den hier realisierten Substantiven häufig um solche handelt, die auch in anderen syntaktischen Kontexten ohne Artikel realisiert werden können, obwohl die Artikelrealisation eigentlich obligatorisch ist. Als Beispiel mag hier das Substantiv scbool in (18) dienen, das als Subjekt eines kategorischen Satzes artikellos realisiert wird. Baldwin et al. (2006) bezeichnen solche Substantive als „defective nouns“ und charakterisieren sie semantisch als ,institutional nouns“.

(18) School is over.

Stvan (1998) beobachtet nun weiterhin, dass bei einer Realisation eines Substantivs in einer PNK eine pragmatische Bedeutungserweiterung erfolgt. So bedeutet das Beispiel in (19a) nicht allein, dass Marys Gatte im Gefängnis 
verortet ist, sondern vor allem, dass Marys Gatte in diesem Gefängnis eine Haftstrafe verbüßt; (19b) bedeutet nicht nur, dass John in einer Schule lokalisiert werden kann, sondern, dass eine institutionell geprägte kontextuell näher zu explizierende Beziehung zwischen John und der Schule besteht.

a. Mary's husband is in prison.

b. John is at school.

Baldwin et al. (2006) schlagen neben PNKen mit N-Selektion auch PNKen mit P-Selektion vor. Sie charakterisieren solche PNKen durch semantische Selektionsregeln die den Typ des nominalen Komplements einschränken, wobei sie implizit davon ausgehen, dass durch die semantische Selektion auf das Substantiv auch die Polysemie der Präposition reduziert wird (zur Polysemie siehe Abschnitt 3). Diese Selektion ergibt dann produktive Muster wie die in (20) und (21).

(20) by train, by plane, by bus, by pogo stick, by hydro-foil, ...

(21) on disc, on CD, on DVD, on tape, on stick, on memory card, ...

Es stellt sich dann natürlich die Frage, ob die Vorschläge von Stvan (1998) und Baldwin et al. (2006) vom Englischen auf das Deutsche übertragen werden können.

Zunächst ist hier festzuhalten, dass es produktive Muster des Typs (20) und (21) auch im Deutschen gibt, aber gerade nicht bei PNKen, so wie ich sie definiert habe. Eine Übersetzung von by ins Deutsche würde wahrscheinlich die Präposition per ergeben, die dann in der Tat Muster wie in (22) zeigt.

(22) per Zug, per Flugzeug, per Bus, per Skateboard, ...

Hier greift nun die externe Bedingung der Definition der PNKen: Die Präposition per besitzt im Deutschen überhaupt keine DP-Objekte, d.h. Objekte mit realisiertem Artikel. Somit handelt es sich bei den Beispielen in (15) nicht um PNKen. Muster des Typs (20) werden im Deutschen durch DPen mit definitem Artikel realisiert, so wie man in (23a) sehen kann. Diese Kombination ist auch ohne Präposition verwendbar, wie (23b) zeigt, aber niemals ohne Artikel (24). ${ }^{6}$

Hier mag man einwenden, dass Fabren wir doch mit Bus und Babn grammatisch ist und somit der oben genannten Behauptung widerspricht. Der Grammatikalität dieses Beispiels liegt allerdings eine andere Regularität zugrunde: Mit oder oder und verknüpfte artikellose Aufzählungen von Substantiven sind nach Präpositionen des Deutschen grundsätzlich möglich. Obwohl dieser Teilbereich der Grammatik der Präpositionen noch nicht gut untersucht ist, so kann man doch festhalten, dass Aufzählungen zu einer Pluraldenotation führen und somit die Beschränkungen für artikellose Pluralia im Deutschen gelten. Ich denke, dass ein Beispiel wie etwa Ich fubr mit Bus und Babn nicht wahr sein kann, wenn ich tatsächlich nur mit dem Bus (oder nur mit der Bahn) gefahren bin. 
(23) a. Fahren wir mit dem Bus/mit dem Zug/mit dem Skateboard?

b. Nehmen wir den Bus/den Zug/das Skateboard?

a. *Fahren wir mit Bus/mit Zug/mit Skateboard?

b. *Nehmen wir Bus/Zug/Skateboard?

In den Beispielen in (23) wird keineswegs vorausgesetzt, dass ein Fahrzeug salient, einzigartig oder vorerwähnt wäre.

Die von Stvan (1998) beobachtete pragmatische Bedeutungserweiterung findet sich im Deutschen nicht oder kaum in PNKen, wohl aber in Verschmelzungsformen wie (25).

(25) Er ist im Gefängnis.

Dass PNKen nicht notwendigerweise mit Bedeutungsveränderungen korrespondieren, hatten wir bereits anhand der Beispiele in (8) und (9) verdeutlicht, auf die hier nochmals verwiesen werden soll. Wenn hier eine artikellose Phrase eine Bedeutung besitzt, die nahezu identisch zu einer Phrase mit Artikel ist, dann kann eine pragmatische Bedeutungserweiterung kein konstitutiver Bestandteil von PNKen im Deutschen sein.

Auch nicht beobachten lassen sich „defekte Nomina“. Beispiele des Typs (18) verlangen im Deutschen einen definiten Artikel. Bereits in Hinsicht auf die so genannten ,institutional nouns“ (school, church) stellen Baldwin et al. (2006, S. 170) unter Verweis auf Himmelmann (1998) fest, dass „the fact that institutional nouns can occur without determiners in these environments is, however, a peculiarity of English; related Germanic languages such as German or Swedish require the definite article here."

Die Schule ist aus.

Während wir für die hier diskutierten Beispiele wohl zurecht behaupten können, dass eine einfache Übertragung der Ansätze von Stvan (1998) und Baldwin et al. (2006) auf das Deutsche nicht möglich ist, sollte die generelle Idee, dass die Semantik der beteiligten Elemente eine konstitutive Rolle spielt, weiter überprüft werden. Tatsächlich haben wir in unserer Analyse die Einbeziehung der Semantik der Präposition und der Semantik des Substantivs vorgesehen und es wird sich zeigen, dass beide eine Rolle spielen.

Wir schließen aber zunächst unsere Betrachtung mit der Schlussfolgerung, dass Analysen dem Phänomenbereich nicht gerecht werden, die entweder das Substantiv als nicht zählbar oder PNKen insgesamt als Ausnahmen klassifizieren. Ebenso wenig ist eine einfache Übertragung der Analysen aus dem Englischen auf das Deutsche möglich. Und schließlich muss auch nochmals betont werden, dass sich die Konstruktionen der reinen Introspektion entziehen. Eine Alternative bietet hier das Annotation Mining. 


\section{Annotation Mining}

Die vorliegende Analyse basiert auf einem Zeitungskorpus, den Jahrgängen von 1993 bis 1999 der Neuen Zürcher Zeitung mit einem Umfang von ca. 230 Millionen Wörtern. Die gesamte Annotation basiert auf einem XMLStand-Off-Format, Daten und Annotate sind also strikt voneinander getrennt, was sowohl die Extraktion einzelner Annotationsebenen als auch ihr Hinzufügen deutlich vereinfacht. Als Werkzeug für die manuelle Annotation insbesondere der Präpositionsbedeutungen verwenden wir MMAX2 (Müller/Strube 2006).

$\mathrm{Da}$ es das Ziel der vorliegenden Analyse ist, konstitutive Bedingungen für die Realisation von PNKen insbesondere auch in Abgrenzung zur Realisation in der Form einer PP zu identifizieren, werden nicht nur PNKen annotiert, sondern auch PPen, für die gilt, dass Präposition und Substantiv in einer PNK aufgetreten sind, und PPen, die ein zählbares Substantiv einbetten, das nicht in einer PNK mit derselben Präposition aufgetreten ist.

Für diese Daten sehen wir die folgenden Annotationen vor:

- Lexikalische Ebene: Part-of-Speech-Tags (kategoriale Etikettierung), Flexionsmorphologie, Derivationsmorphologie von Substantiven, Zählbarkeit von Substantiven, Interpretation von Substantiven durch GermaNet-Tops (d.h. Wortfelder), Interpretation von Präpositionen, Kompositabildung bei Substantiven.

- Syntaktische Ebene: Einbettungsmodus (Adjunkt oder Komplement) der PP/PNK, syntaktische Komplemente des Nomens, pränominale Modifikatoren des Nomens.

- Globale Ebene: Ist die Phrase Bestandteil einer Überschrift, eines Titels oder eines Zitats? Ist die Phrase idiomatisch?

In Überschriften, Titeln und Zitaten könnten PNKen nicht aufgrund syntaktischer oder semantischer Bedingungen realisiert werden, sondern weil Überschriften und Titel typische Orte für extragrammatische Kürzungen sind. Rein idiomatische PNKen mögen Kombinationsregeln folgen, die von den generellen Bedingungen für die Realisation von PNKen abweichen. Die Annotation dient auf der globalen Ebene also wesentlich dazu, bei der Analyse einzelne Fälle auszuschließen.

Große Teile der Annotation erfolgen automatisch mit der Hilfe der folgenden Werkzeuge: Der „Regression Forest Tagger“ (Schmid/Laws 2008) wird verwendet für die kategoriale Etikettierung und die flexionsmorphologische Analyse (der Tagger enthält die morphologische Analysekomponente SMOR, vgl. Schmid 2004), der „Tree Tagger“ (Schmid 1995) wird für die flache syntaktische Verarbeitung („Chunk Parsing tionsmorphologische Kategorien werden aus IMSLex (Lezius et al. 2000) abgeleitet. 
Die Analyse verwendet zwei Ressourcen, um die Interpretation der Substantive festzulegen. Die erste Ressource ist GermaNet (Kunze/Lemnitzer 2002), die deutsche Version des WordNet (vgl. http://wordnet.princeton.edu/). Wir verwenden die 23 Top-Level-Kategorien und jedes Substantiv wird mit allen Kategorien annotiert, denen es angehört. Top-Level-Kategorien entsprechen Wortfeldern. Darüber hinaus verwenden wir das Lexikon HaGenLex (Hartrumpf et al. 2003). HaGenLex kodiert spezifische sortale Informationen, die in einer formalen Ontologie strukturiert sind.

Die Klassifikation der Zählbarkeit erfolgt so, wie in Abschnitt 2.1 bereits beschrieben wurde. Zählbare Substantive sind solche, die der Klasse I angehören, nicht-zählbare Substantive sind solche, die den Klassen IV bzw. $\mathrm{V}$ angehören.

Durch automatische Annotationsverfahren liegen somit Informationen vor, die bei den Nomina von der internen Struktur über die syntaktische Distribution bis hin zur sortalen Klassifikation reichen. Leider liegt ein entsprechendes Verfahren für die Interpretation der Präpositionen nicht vor. Obwohl der Grad der Polysemie der Präpositionen nahezu ein Gemeinplatz ist, sind bislang keine Versuche unternommen worden, Kategorieninventare („,Sense Tags') für Präpositionsinterpretationen zu entwickeln. Sowohl die manuelle als auch die automatische Annotation setzen aber ein vordefiniertes Kategorieninventar voraus. Dieses Inventar muss natürlich die Polysemie der Präpositionen abbilden, darf aber nicht so komplex sein, dass die manuelle Annotation unmöglich wird. So legt etwa Schröder (1986) eine umfangreiche merkmalsbasierte Bestimmung der Interpretation von Präpositionen vor, die aber auf ca. 200 Merkmalen basiert und daher nicht für die Annotation eingesetzt werden kann. Dennoch ist Schröders Lexikon eine wertvolle Ressource.

Unsere Analyse basiert neben Schröder (1986) auf drei weiteren, im Wesentlichen gebrauchsorientierten Ressourcen zur Beschreibung der Präpositionsinterpretationen des Deutschen: der Analyse von Helbig und Buscha (2001), der Darstellung im Duden „Deutsch als Fremdsprache“ (2002) und der Arbeit von Durrell und Brée (1993) zur Interpretation temporaler Präpositionen.

$\mathrm{Zu}$ diesen Ressourcen ist zu sagen, dass sie sehr heterogen und teilweise eher beispielorientiert als regelbasiert sind. Vor einer Anwendung ist also zunächst eine Angleichung und kritische Überprüfung der genannten Inventare erforderlich. Dies führt häufig zu einer Erweiterung von Interpretationsmerkmalen für einzelne Präpositionen und nicht selten zu einer Neudefinition von Teilen des Inventars. Das Annotationsschema für Präpositionsinterpretationen wird in Müller et al. (i.Dr.) detailliert beschrieben; ich möchte es an dieser Stelle anhand der wesentlichen Merkmale charakterisieren: 
- Es gibt ein Inventar von insgesamt 27 Merkmalen, wobei die fünf Merkmale spatial, temporal, kausal, modal und Vorhandensein in einer Merkmalshierarchie organisiert sind, also Unterkategorien besitzen. Die Unterkategorien für temporale Merkmale entstammen der Analyse von Durrell und Brée (1993), die Untermerkmale der Kategorie spatial der Analyse von Schröder (1986) - zu Vorbandensein vgl. die Abschnitte 4.3 und 5.

- Annotatoren bewegen sich mittels Entscheidungsbaumverfahren durch die jeweiligen Hierarchien und haben auch die Möglichkeit, anstelle eines maximal spezifischen Interpretationstyps ein Hyperonym als Interpretation anzugeben. In den Analysen in Abschnitt 4 machen wir nur von den jeweiligen Obertypen Gebrauch.

- Zusätzlich zu den fünf Bedeutungen mit Unterbedeutungen gibt es 22 Bedeutungen ohne Unterbedeutung: Zustand, komitativ, Quantitätsdifferen₹, Beteiligung, Unterordnung, Zuordnung, Wechselbeziehung, Rangfolge, Über-/Unterscbreitung, Bezugspunkt, Thema, Empfänger, Stellvertretung/Ersatz, Austausch, komparativ, restriktiv, kopulativ, adversativ, distributiv, Stellungnahme, Agens, Realisation. Im Gegensatz zu den Merkmalen mit Untertypen mögen viele der hier genannten Merkmale eher abstrakt erscheinen. Wir werden die Merkmale anhand der Fallstudien in Abschnitt 4 genauer erläutern.

- Es besteht die Möglichkeit der Kreuzklassifikation, so dass bestimmte Eigenschaften der Interpretation nicht als Merkmale dargestellt werden; dies gilt insbesondere für die Repräsentation direktionaler Interpretationen. Direktionalität sollte bei sämtlichen lokalen Bedeutungsvarianten verfügbar sein, würde aber bei der Mehrzahl von Präpositionen mit einer lokalen Interpretation zu einer vorhersagbaren Hinzufügung des Merkmals direktional führen, das zudem nahezu immer aus dem Kasus des Objekts abgeleitet werden kann.

- Pro Präposition liegen zwischen einer und siebzehn Bedeutungen vor.

Das Vorkommen der Präpositionen im NZZ-Korpus in PNKen und PPen (PPen mit $\mathrm{N}$ aus PNK und PPen mit $\mathrm{N}$ nicht in PNK) ist im Folgenden dargestellt; die Präpositionen sind hierbei nach ihrer Polysemie geordnet:

\begin{tabular}{|l|c|c|r|r|r|}
\cline { 1 - 2 } & $\begin{array}{c}\text { Bedeu- } \\
\text { tungen }\end{array}$ & $\begin{array}{c}\text { Unterbedeu- } \\
\text { tungen }\end{array}$ & PNK & $\begin{array}{c}\text { PP } \\
\text { (N in PNK) }\end{array}$ & $\begin{array}{c}\text { PP } \\
\text { (N nicht in PNK) }\end{array}$ \\
\cline { 1 - 2 } gemäß & 1 & 1 & 455 & 418 & 177 \\
\cline { 1 - 1 } seit & 1 & 2 & 297 & 1.298 & 1.065 \\
\cline { 1 - 1 } während & 1 & 3 & 24 & 356 & 2.507 \\
\cline { 1 - 1 } hinter & 1 & 8 & 61 & 343 & 1.532 \\
\cline { 1 - 1 } dank & 2 & 3 & 171 & 246 & 741 \\
\cline { 1 - 1 } mittels & 2 & 3 & 195 & 75 & 154 \\
\hline
\end{tabular}




\begin{tabular}{|c|c|c|c|c|c|}
\hline & $\begin{array}{l}\text { Bedeu- } \\
\text { tungen }\end{array}$ & $\begin{array}{l}\text { Unterbedeu- } \\
\text { tungen }\end{array}$ & PNK & $\begin{array}{c}\text { PP } \\
\text { (N in } \mathrm{PNK})\end{array}$ & $\begin{array}{c}\text { PP } \\
\text { (N nicht in PNK) }\end{array}$ \\
\hline wegen & 2 & 3 & 1.091 & 886 & 1.409 \\
\hline neben & 3 & 4 & 164 & 253 & 1.790 \\
\hline durch & 5 & 6 & 1.970 & 7.674 & 6.311 \\
\hline vor & 5 & 15 & 786 & 8.756 & 4.688 \\
\hline bei & 5 & 24 & 2.340 & 11.679 & 6.280 \\
\hline um & 6 & 7 & 701 & 5.549 & 6.765 \\
\hline ohne & 6 & 11 & 4.632 & 473 & 218 \\
\hline gegen & 6 & 16 & 1.687 & 4.831 & 3.857 \\
\hline an & 6 & 29 & 2.916 & 22.563 & 11.174 \\
\hline nach & 7 & 11 & 5.364 & 18.942 & 4.750 \\
\hline in & 7 & 20 & 20.425 & 93.434 & 16.871 \\
\hline über & 7 & 17 & 1.168 & 9.880 & 9.275 \\
\hline auf & 7 & 29 & 27.011 & 59.938 & 16.131 \\
\hline für & 8 & 10 & 3.885 & 31.029 & 11.612 \\
\hline unter & 10 & 22 & 1.864 & 3.605 & 1.497 \\
\hline mit & 11 & 18 & 13.981 & 43.833 & 13.262 \\
\hline Summe & & & 91.188 & 326.061 & 122.066 \\
\hline
\end{tabular}

Tab. 1: Vorkommen von PNK und PP

Tabelle 1 verdeutlicht, dass nicht alle Präpositionen zwingend polysem sind - und somit eine P-selektionale Analyse, die in jedem Fall die Polysemie der Präposition voraussetzt, nicht tragen würde. Die nicht-polysemen Präpositionen sind gemäß und während. Die größte Gruppe besteht aus Präpositionen, die zwischen zwei und sechs Bedeutungen besitzen. Schließlich gibt es auch eine Gruppe hochpolysemer Präpositionen, die sieben und mehr Interpretationen besitzen.

Betrachtet man die Gruppe bis zur Präposition neben (mit drei Bedeutungen), so ließe sich der Schluss formulieren, dass niedrige Polysemie auf der Typ-Ebene auch ein geringeres Vorkommen der Präposition nach sich zieht. Diese Annahme bestätigt sich allerdings nicht, wenn die weiteren Präpositionen betrachtet werden. So gehört unter zu den hochpolysemen Präpositionen, es werden aber verhältnismäßig wenig PNKen/PPen mit unter realisiert. Die grafische Darstellung der Tabelle 1 in Abbildung 1 verdeutlicht dies nochmals. Die erste Reihe markiert hier die Häufigkeit der PNKen, die zweite die der PPen mit denselben Substantiven und die dritte die der PPen mit anderen zählbaren Substantiven. 


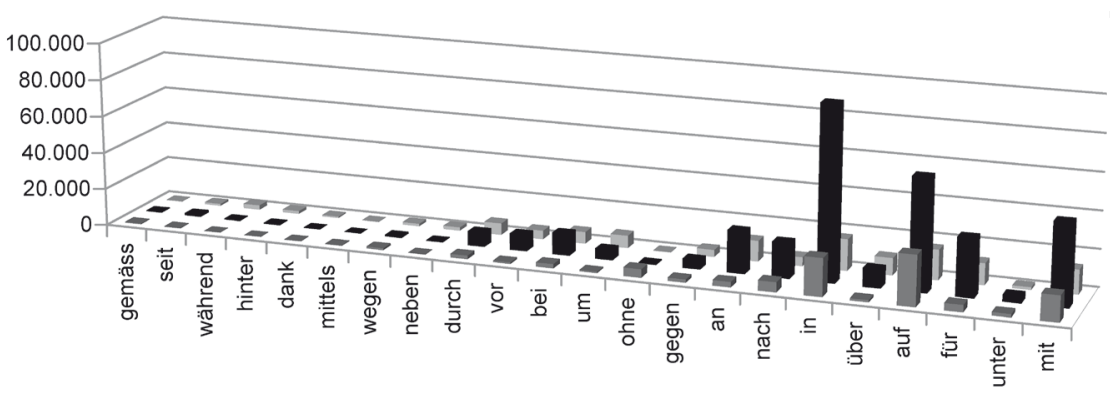

Abb. 1: Vorkommen von PNK und PP (dunkelgrau: PNK, schwarz: PP mit N in PNK, hellgrau: PP mit $\mathrm{N}$ nicht in PNK)

Die Präposition obne besitzt die Besonderheit, dass hier mehr Vorkommen in PNKen zu beobachten sind als in PPen.

\section{Pilotstudie: Bedingungen für die Realisation artikelloser Phrasen als Komplemente von obne und unter}

\subsection{Grundannahmen}

Auch eine Analyse, die auf dem Annotation Mining aufsetzt, kann nicht gänzlich unabhängig von introspektiven Urteilen durchgeführt werden. Allerdings kommt introspektiven Bewertungen hier eine indirekte und vermittelnde Rolle zu. Annotation Mining bedeutet ja nichts anderes, als Rohdaten entweder automatisch oder manuell mit Annotationen zu versehen, was wiederum voraussetzt, dass für die relevanten Ebenen linguistische Annotationsschemata entwickelt werden. Es ist nun sehr unwahrscheinlich, dass die Entwicklung eines solchen Schemas - das ja eigentlich eine Mikrogrammatik ist - ohne jedes introspektive Urteil erfolgt. Die Zuordnung einzelner Elemente zu einem Schema kann ohne introspektive Befragung und insbesondere ohne einen bereits vorliegenden Kriterienkatalog nicht erfolgen. Ein zweiter Faktor betrifft die relative Unabhängigkeit der Annotationsschemata. Diese werden typischerweise unabhängig voneinander und auch von unterschiedlichen Personen entwickelt. Somit werden hier introspektive Urteile, wenn diese bei der Entwicklung eine Rolle spielten, auf eine breite Basis gestellt. Gerade vor dem Hintergrund dieser Überlegungen ist das Annotation Mining das Mittel der Wahl, wenn es um explorative Analysen geht. Die einleitenden Überlegungen haben gezeigt, dass PNKen regelhaft sind. Die explorative Analyse dient nun dazu, möglichst objektiv (d.h. auf eine breite empirische Basis gestützt) die Bedingungen dieser Regelhaftigkeit zu identifizieren. Insbesondere, wenn es sich um multikausale Bedingungen handelt, ist ein solches Vorgehen einer traditionellen, durch Introspektion geleiteten Analyse überlegen. 
Für den vorliegenden Fall bieten sich nun Analysetechniken an, die es gestatten, die zugrunde liegende Fragestellung möglichst genau nachzuvollziehen. Eine solche Analysetechnik ist die binäre logistische Regression (Harrell 2001). Die Verletzung der Dudenregel 442 erfährt dabei die folgende Deutung: Die Realisation eines Artikels darf als regelhafte Grundbedingung gelten, d.h. wenn keine anderen Bedingungen erfüllt sind, ist die Realisation des Artikels der Normalfall. Wir betrachten den Wegfall des Artikels als vorherzusagende Eigenschaft und verwenden die im Annotation Mining identifizierten Merkmale von PNKen und PPen als Prädiktoren. Eine binäre logistische Regression identifiziert nun in den Merkmalen solche, die die Wahrscheinlichkeit eines Artikelwegfalls anheben und solche, die eine Anhebung absenken, so dass insgesamt eine Wahrscheinlichkeit für den Artikelwegfall in Abhängigkeit der vorhandenen Eigenschaften formuliert werden kann.

In den Analysen ist somit die abhängige Variable der Faktor DET, der die beiden Werte no und yes annehmen kann. Die folgenden Eigenschaften werden durch 92 Merkmale repräsentiert:

- Welche Interpretation besitzt die Präposition?

- Zu welchen Wortfeldern wird das Substantiv gezählt?

- Welche interne syntaktische Struktur besitzt das nominale Komplement: Wird es adjektivisch modifiziert, besitzt es ein Komplement, welche Kategorie besitzt das Komplement?

- Handelt es sich beim Kopf des nominalen Komplements um ein Kompositum oder nicht?

- Handelt es sich beim Nomen um ein abgeleitetes Nomen?

- Welche externe syntaktische Einbettung für die PNK/PP liegt vor: Handelt es sich um einen adverbialen Modifikator oder ein regiertes Komplement?

Bevor die Daten auf diese Weise analysiert wurden, war zunächst eine Bereinigung der Daten erforderlich: PNKen, die in Überschriften, Titeln und Zitaten vorkamen, wurden ebenso wenig für die Analyse berücksichtigt wie idiomatische Ausdrücke. Der Grund für die Elimination von PNKen in Überschriften, Titeln und Zitaten ist, dass diese Textstrukturen die extragrammatische Verknappung eines Textes in besonderem Maße fördern und somit hier auftretende PNKen möglicherweise eine grammatische Analyse verzerren würden. Ähnliches gilt auch für idiomatische Ausdrücke, die die Form einer PNK, aber ebenso häufig auch die Form einer PP annehmen. Deswegen wurden auch sie eliminiert. Insgesamt liegen den beiden Pilotanalysen die Daten in Tabelle 2 zugrunde:

Die Daten in Tabelle 2 unterscheiden sich nicht nur deswegen von den Daten aus Tabelle 1, weil PNKen in bestimmten Textstrukturen nicht berücksichtigt worden sind. Weiterhin aus- 


\begin{tabular}{|l|c|c|c|}
\hline Präposition & $\Sigma$ & PP & PNK \\
\hline ohne & 3.750 & 591 & 3.159 \\
\hline unter & 5.181 & 4.334 & 857 \\
\hline
\end{tabular}

Tab. 2: Artikelrealisation bei den Präpositonen obne und unter

Zur Bewertung der eigentlichen Analyse ist es zunächst erforderlich, eine automatische Klassifikation durchzuführen, die davon ausgeht, dass alle Merkmale, die relevant sein könnten, auch tatsächlich relevant sind (einen so genannten „full model fit"). Einem ,full model fit" liegen im vorliegenden Fall 92 Merkmale zugrunde, die die oben genannten Eigenschaften abbilden. Auf der Basis dieses „,full model fits“ werden Merkmale, deren Relevanz als gering eingeschätzt wird, entweder automatisch oder manuell verworfen und darauf erneut ein Modell entwickelt. Ein Merkmal wird dann verworfen, wenn es einen Modellkoeffizienten besitzt, der sich mit einer bestimmten Wahrscheinlichkeit nicht von 0 unterscheidet. Wenn ein Modellkoeffizient (= Gewichtung des Klassifikationsmerkmals) den Wert 0 besitzt, dann bedeutet dies nichts anderes, als dass das betreffende Merkmal für die vorliegende Analyse keine Bedeutung besitzt. Bei einer automatischen Analyse, etwa durch „fast backward elimination“, geht man davon aus, dass nur Merkmale erhalten bleiben, für deren Koeffizienten mit einer bestimmten Wahrscheinlichkeit (typischerweise wenigstens 95\%) angenommen werden kann, dass sie von 0 verschieden sind (vgl. Baayen 2008, S. 186). Dies kann aber dazu führen, dass einflussreiche Merkmale eliminiert werden, weswegen Harrell (2001, S. 56) auch von einem automatischen Verfahren abrät. Als Faustregel kann man angeben, dass ein Prädiktor entfernt wurde, wenn die Wahrscheinlichkeit, dass der Wert ungleich 0 ist, niedriger lag als 20\%. Für Prädiktoren, die nicht in den Bereich zwischen 20 und 95\% fielen, wurde jeweils ein Modell ohne und mit dem Prädiktor konstruiert und die Modellparameter wurden miteinander verglichen.

\subsection{Binäre logistische Regression}

Für die binäre logistische Regression gehen wir davon aus, dass es eine dichotome abhängige Variable gibt, beispielsweise eine Kategorie mit zwei

geschlossen werden automatisch, aber falsch annotierte Daten (etwa aufgrund von Tippfehlern), unvollständige Sätze, Postpositionen, die falsch als P gechunkt wurden, Zählbarkeitsfehler wegen Synkretismus, nicht erkannter Pluralform oder nominalisiertem Infinitiv, PPen in Funktionsverbgefügen, PP mit postnominalem Adverb, Verbpartikel, die fälschlich als Präposition klassifiziert und eine fehlerhafte syntaktische Analyse nach sich ziehen, sowie Numerativkonstruktionen. 
Werten, die intern durch die Werte 0 und 1 kodiert werden kann. Die unabhängigen Variablen können metrische oder kategoriale Werte besitzen. Im vorliegenden Modell ist die abhängige Kategorie die Zugehörigkeit zu den Kategorien PNK und PP; die unabhängigen Variablen besitzen kategoriale Werte, aus denen wir ableiten wollen, ob ein Artikel realisiert wird (= PP) oder nicht (= PNK). Es sind die in Abschnitt 4.1 vorgestellten Merkmale.

Die binäre logistische Regression bestimmt allerdings nicht, ob eine Phrase zu einer der beiden Kategorien gehört, sondern gibt die Wahrscheinlichkeit an, mit der die Phrase zu den genannten Kategorien gehört. Man spricht hier von logistischer Regression, weil im Gegensatz zur linearen Regression nicht die gewichtete Summe der Prädiktoren gebildet wird, sondern die logistische Funktion der gewichteten Summe der Prädiktoren: $\operatorname{logit}(p)=\log (p / 1-p)$. Die gewichtete Summe der Prädiktoren kann Werte zwischen $+\infty$ und $-\infty$ annehmen. Die inverse logit-Funktion bildet diese Werte aber auf das Intervall [0; 1] ab und stellt somit sicher, dass die resultierende Funktion tatsächlich eine Wahrscheinlichkeitsfunktion ist, die eine S-Form annimmt. Im Modell liegt somit eine Abbildung von Eigenschaften auf 92 Merkmale vor, von denen diejenigen Merkmale als Prädiktoren verwendet werden, die die Chancen beeinflussen, dass ein Vorkommen der einen oder anderen Kategorie zugeordnet wird.

In den folgenden Modellen wird jeweils die Wahrscheinlichkeit für die Realisation eines Artikels bestimmt. Die unabhängigen Variablen (= Prädiktoren) können hierbei positive und negative Werte annehmen. Negative Werte sprechen gegen die Realisation eines Artikels, positive Werte für die Realisation. Betrachten wir ein vereinfachtes Beispiel, in dem es nur einen Prädiktor gibt, der den Wert -1,386 annimmt. Für einen solchen Wert ergibt die Anwendung des inversen logit den Wert 0,2, d.h. die Wahrscheinlichkeit für die Weglassbarkeit des Artikels liegt in der betreffenden Konstruktion bei $1-0,2=0,8$.

\subsection{Logistische Modelle für den Wegfall des Artikels bei obne und unter}

Das in Abschnitt 4.1 beschriebene Verfahren der Elimination von Prädiktoren führte zu Modellen für den Wegfall des Artikels bei den Präpositionen obne und unter, die auf 13 (für obne) bzw. 22 (für unter) Merkmalen aus den ursprünglichen 92 Merkmalen basieren.

\subsubsection{Das Modell für obne}

Wir betrachten zunächst das Modell für obne, das in Tabelle 3 dargestellt wird. 


\begin{tabular}{|l|c|c|c|}
\hline & Koeffizient & S.E. & p \\
\hline ACHSENABSCHNITT & $-2,40$ & 0,11 & 0,000 \\
\hline UNG-NOMINALISIERUNG & $-1,36$ & 0,19 & 0,000 \\
\hline ADJ IN N' & 1,14 & 0,12 & 0,000 \\
\hline KAUSAL & 1,21 & 0,13 & 0,000 \\
\hline KOMITATIV & 2,28 & 0,52 & 0,000 \\
\hline BETEILIGUNG & 3,40 & 0,49 & 0,000 \\
\hline VORHANDENSEIN & $-0,78$ & 0,15 & 0,000 \\
\hline DEP-S & 5,08 & 1,05 & 0,000 \\
\hline DEP-NP & 2,97 & 0,17 & 0,000 \\
\hline DEP-PP & 2,20 & 0,15 & 0,000 \\
\hline GN-RELATION & $-1,03$ & 0,41 & 0,011 \\
\hline GN-ATTRIBUT & $-1,35$ & 0,30 & 0,000 \\
\hline GN-EREIGNIS & $-0,84$ & 0,14 & 0,000 \\
\hline GN-ARTEFAKT & $-0,41$ & 0,16 & 0,008 \\
\hline
\end{tabular}

Tab. 3: Koeffizienten für ein logistisches Modell für den Wegfall des Artikels bei ohne

Tabelle 3 gibt die relevanten Faktoren mit ihren Koeffizienten, d.h. Gewichtungen an, den Standardfehler der Schätzung (engl. „standard error“, d.h. S.E.) und die Wahrscheinlichkeit, dass die Gewichtung nur zufällig von 0 abweicht. Der Standardfehler kann genutzt werden, um nach der Daumenregel (Koeffizienz \pm S.E. $\times 2$ ) 95-\%-Konfidenzintervalle für die Koeffizienten zu bestimmen. Selbst bei den Werten für GN-RELATION und GNARTEFAKT macht diese Berechnung deutlich, dass die Konfidenzintervalle 0 nicht einschließen.

Würden die Intervalle den Wert 0 einschließen, dann wäre es durchaus denkbar, dass der Wert eines Koeffizienten tatsächlich 0 beträgt, was bedeuten würde, dass dieser Koeffizient für den Wegfall eines Artikels keine Rolle spielt. Der Wert für den Achsenabschnitt kann in jedem Fall vernachlässigt werden: Er ergibt sich als Artefakt aus der Modellierung der jeweils binären Werte für die unabhängigen Variablen und der Form der internen Kodierung dieser Werte.

Die identifizierten Eigenschaften können wie folgt interpretiert werden: Die Merkmale KAUSAL, KOMITATIV und BETEILIGUNG (zur Definition dieser Eigenschaften siehe Abschnitt 5) besitzen positive Werte. Sie verschieben somit die Gewichte zugunsten einer Realisation eines Artikels. Eine erste naheliegende Interpretation dieser Werte könnte lauten: Wenn 
eine Präposition die Merkmale KAUSAL, KOMITATIV oder BETEILIGUNG besitzt, dann ist die Realisation einer NP (mit Artikel) wahrscheinlicher als die Realisation einer Nominalprojektion ohne Artikel. Beispiele für die Zuweisungen dieser Interpretationen sind in (27) bis (29) gegeben. ${ }^{8}$

(27) KAUSAL:

a. Sämtliche kurdischen Politiker sind davon überzeugt, dass obne einen Machtwechsel in Bagdad die Kurdenfrage des Iraks nicht zu lösen sei. (NZZ, AUSLAND, 28.01.1993)

b. Und für eigentliche Verhandlungen hat Frau Kumaratunga nach eigenen Aussagen obne absolute Parlamentsmebrheit noch kein klares Mandat. (NZZ, AUSLAND, 14.09.1994)

(28) KOMITATIV:

a. Ein mobiles Einsatzkommando überwältigte nach Polizeiangaben den aus Tunesien stammenden Geiselnehmer, als er obne das Kind den Gerichtssaal verließ. (NZZ, VERMISCHTE MELDUNGEN, 14.07.1993)

b. Kessler, der obne Anwalt vor Gericht erschien, betonte in seinem umfangreichen Plädoyer, seine anerkanntermaßen scharfe Kritik richte sich nicht gegen die Juden als Religionsgemeinschaft. (NZZ, ZÜRICH UND REGION, 11.03.1998)

(29) BETEILIGUNG:

a. Die abschließende Verhandlung der Nevada State Athletic Commission fand obne den Angeklagten statt, dem außerdem die Kosten des Verfahrens aufgebrummt wurden. (NZZ, SPORT, 10.07.1997)

b. (keine Beispiele im Korpus gefunden)

Die Beispiele verdeutlichen - zumindest für die Merkmale KAUSAL und KOMITATIV, dass die Bedingungen für die Realisation nicht absolut zu werten sind, und insbesondere auch nicht isoliert. Ansonsten dürften die Belege (27b) und (28b) gar nicht existieren.

Das Merkmal VORHANDENSEIN besitzt demgegenüber einen negativen Wert. Liegt diese Interpretation vor, dann ist die Wahrscheinlichkeit der Realisation einer Nominalprojektion ohne Artikel höher als die Wahrscheinlichkeit der Realisation einer NP (mit Artikel). Beispiele finden sich in (30).

(30) VORHANDENSEIN:

a. Was weiter als positives Zeichen zu deuten wäre, Fragezeichen betreffend Stabilität und Solidität gegen die Portugiesen zum Trotz, ist die Serie von acht Partien obne Niederlage, die längste seit 1924. (NZZ, SPORT, 02.04.1993)

8 Die Interpretation KAUSAL subsumiert die Interpretation KONDITIONAL, die in Beispiel (27a) vorliegt. 
b. Die Anklage wirft dem ersten von drei Angeklagten, einem 32jährigen Mann obne Beruf, die Mitwirkung an allen drei Tötungsdelikten vor. (NZZ, STADT UND KANTON ZÜRICH, 14.04.1993)

Wir sehen hier also zwei unterschiedliche Interpretationsgruppen für die Präposition obne. Besitzt die Präposition die Interpretationen KAUSAL, KOMITATIV oder BETEILIGUNG, wird die Realisation eines Artikels bevorzugt. Bei der Interpretation VORHANDENSEIN hingegen wird die Weglassbarkeit des Artikels suggeriert. In Abschnitt 5 werden wir auf diese Verteilung zurückkommen und fragen, ob die hier dargestellte Verteilung der Interpretationen der Präposition obne nicht eine zugrunde liegende Eigenschaft dieser Konstruktion verbirgt.

Die mit DEP beginnenden Merkmale spezifizieren syntaktische Komplemente des Nomens in einer PNK, d.h. selegierte Sätze, postnominale NPen und postnominale PPen. Die drei Merkmale suggerieren, dass die Realisation eines Komplements die Realisation eines Artikels fördert. Das Merkmal ADJ IN N' legt fest, ob das Nomen in einer PNK adjektivisch modifiziert wurde. Das Merkmal besitzt wie DEP-S, DEP-NP und DEP-PP einen positiven Koeffizienten, d.h. fördert die Realisation eines Artikels. Allen vier Merkmalen ist gemein, dass sie die syntaktische Komplexität der Nominalprojektion erhöhen, so dass man den Schluss ziehen könnte, dass eine höhere syntaktische Komplexität der Nominalprojektion auch die Wahrscheinlichkeit eines Artikelwegfalls deutlich absenkt. Wir werden aber bei der Analyse von unter sehen, dass eher die Realisation eines Komplements als die syntaktische Komplexität die Wahrscheinlichkeit eines Artikelwegfalls beeinflusst.

Deverbale Nomina, die mit -ung gebildet werden, unterstützen ebenfalls den Wegfall des Artikels. Hier bedarf es einer genaueren Untersuchung bzw. auch der Integration von Verfahren zur Disambiguierung ung-nominalisierter Substantive, so wie dies etwa in Eberle (2009) vorgeschlagen wird. Es ist allerdings festzuhalten, dass eine Präferenz des Artikelwegfalls bei deverbalen Nomina eine übergreifende Eigenschaft zu sein scheint - sie begegnet uns erneut im Modell für unter.

Bei den Merkmalen GN-RELATION, GN-ATTRIBUT, GN-EREIGNIS und GN-ARTEFAKT handelt es sich um eine Zuordnung der Substantive zu Wortfeldern aus GermaNet (vgl. Kunze/Lemnitzer 2002). Diese Merkmale bezeichnen also semantische Eigenschaften der Substantive. Nomina, die mehr als einem Wortfeld angehören, sind polysem oder homonym. Für solche Substantive wird in der vorliegenden Klassifikation keine Disambiguierung durchgeführt.

Da die Zuordnung typbasiert erfolgt, besitzt ein mehrdeutiges Nomen in jeder PNK bzw. PP jede Zuordnung aus GermaNet. Die Definition der für das Modell für obne relevanten Kategorien in WordNet ist in (31) gegeben. 
(31) a. relation: an abstraction belonging to or characteristic of two entities or parts together.

b. attribute: an abstraction belonging to or characteristic of an entity.

c. event: something that happens at a given place and time.

d. artefact: a man-made object taken as a whole.

Beispiele für Substantive des Wortfelds relation sind in (32a) gegeben, Beispiele des Wortfelds attribute in (32b), des Wortfelds event in (32c) und des Wortfelds artefact in (32d).
a. Kameradschaft, Heiratsantrag
b. Tonhöhe, Gefühllosigkeit
c. Diffusion, Konjunkturprogramm
d. Klosteranlage, Schutzvorrichtung

Diese Wortfelder unterstützen den Wegfall des Artikels, was erneut am negativen Vorzeichen erkennbar ist. Dies ist auch deswegen interessant, weil unter diese Wortfelder keineswegs nur Abstrakta fallen, die in der Literatur ebenso oft wie fälschlich den Massentermen zugeordnet werden (vgl. etwa Bale/Barner 2009), sondern auch Konkreta. Dass diese Annahme nicht korrekt sein kann, sieht man an Substantiven wie Gefühllosigkeit oder Kameradschaft, die jeweils als zählbar klassifiziert werden können.

Diese Merkmale können als Selektionsrestriktionen interpretiert werden für substantivische Komplemente von obne, wenn obne die Interpretation VORHANDENSEIN besitzt. Bei mehr als 50\% der Vorkommen von obne mit der Interpretation VORHANDENSEIN wird ein Substantiv ausgewählt, das in wenigstens eines der oben genannten Wortfelder fällt, wobei 51,5\% der Substantive das Merkmal GN-EVENT besitzen und nur 35,3\% der Substantive das Merkmal GN-ARTEFACT.

\subsubsection{Das Modell für unter}

In Tabelle 4 findet sich das Modell für unter, das mit seinen 22 Merkmalen nahezu doppelt so viele relevante Merkmale aufweist wie das Modell für obne. Dass hier doppelt so viele Merkmale erforderlich sind, liegt sicherlich insbesondere daran, dass unter wesentlich mehr Bedeutungen als obne aufweist und damit mehr Interpretationen der Präposition und auch mehr Wortfelder der Substantive berücksichtigt werden müssen.

Hier fällt nun allerdings als erstes auf, dass alle Koeffizienten für die Präpositionsbedeutungen ein positives Vorzeichen besitzen. Es werden zwar fünf der zehn Oberbedeutungen von unter im Modell berücksichtigt, aber diese Oberbedeutungen legen jeweils eine Blockade des Artikelwegfalls nahe. Es gibt keine Bedeutungen, die unmittelbar für den Artikelwegfall stehen, so wie etwa VORHANDENSEIN für obne. Hier zeigt sich ein Muster, dass 
ich in Kiss (2007) bereits für spatiale Interpretationen beobachtet habe. In Kiss (ebd.) stellte ich fest, dass spatiale Interpretationen bei PNKen mit unter sehr selten auftreten, um nicht zu sagen nie. Vergleicht man den Koeffizienten des Merkmals SPATIAL mit den Werten für die anderen Bedeutungen, so wird erwartet, dass insbesondere UNTERORDNUNG, ZUORDNUNG und ÜBER-/UNTERSCHREITUNG die Realisation von PNKen blockieren. Wir finden also weniger lizenzierende Merkmale für den Artikelwegfall als Bedeutungen, die den Wegfall behindern.

\begin{tabular}{|l|c|c|c|}
\hline & Koeffizient & S.E. & p \\
\hline ACHSENABSCHNITT & $-0,4379$ & 0,1657 & 0,008 \\
\hline UNG-NOMINALISIERUNG & $-0,8346$ & 0,2259 & 0,000 \\
\hline ADJ IN N' & $-1,0177$ & 0,1432 & 0,000 \\
\hline KOMPOSITUM & 2,1719 & 0,2538 & 0,000 \\
\hline REGIERT & 1,9894 & 0,3017 & 0,000 \\
\hline SPATIAL & 2,3237 & 0,2044 & 0,000 \\
\hline KAUSAL & 1,3047 & 0,2272 & 0,000 \\
\hline UNTERORDNUNG & 3,0529 & 0,2559 & 0,000 \\
\hline ZUORDNUNG & 3,4228 & 0,1861 & 0,000 \\
\hline ÜBER-/UNTERSCHREITUNG & 4,4186 & 0,3677 & 0,000 \\
\hline DEP-S & 8,4717 & 4,0734 & 0,037 \\
\hline DEP-NP & 0,8551 & 0,1436 & 0,000 \\
\hline DEP-PP & 0,3043 & 0,2170 & 0,161 \\
\hline GN-GRUPPE & 0,5241 & 0,2563 & 0,041 \\
\hline GN-KOMMUNIKATION & $-0,9149$ & 0,1443 & 0,000 \\
\hline GN-ORT & 2,2704 & 0,6208 & 0,000 \\
\hline GN-RELATION & $-2,1161$ & 0,6022 & 0,000 \\
\hline GN-BESITZ & $-0,8482$ & 0,3665 & 0,021 \\
\hline GN-ATTRIBUT & $-2,2847$ & 0,2741 & 0,000 \\
\hline GN-ARTEFAKT & 0,4169 & 0,1601 & 0,009 \\
\hline GN-MENSCH & 1,8870 & 0,4999 & 0,000 \\
\hline HL-AD & $-1,0253$ & 0,1888 & 0,000 \\
\hline HL-AS & 0,3804 & 0,000 \\
\hline
\end{tabular}

Tab. 4: Koeffizienten für ein logistisches Modell für den Wegfall des Artikels bei unter 
Einige Muster aus dem Modell für obne sind auch im Modell für unter wieder vorhanden. Insbesondere ist beobachtbar, dass die Merkmale DEP-S, DEPNP und DEP-PP erneut gegen den Wegfall des Artikels sprechen. ${ }^{9}$ Das Merkmal ADJ IN N' besitzt bei unter allerdings einen negativen Koeffizienten. Dies bedeutet, dass das Vorhandensein von Adjektiven in nominalen Komplementen von obne die Weglassbarkeit des Artikels erschwert, dieselbe Bedingung bei Komplementen von unter aber den Wegfall des Artikels begünstigt.

Darüber hinaus scheint zunächst auch die externe Distribution der Phrase eine Rolle zu spielen: Das Merkmal REGIERT bestimmt, ob die PP bzw. PNK von einem Substantiv oder Verb regiert wird. Es spielt bei obne keine Rolle, während es bei unter den Wegfall des Artikels erschwert. Der Grund hierfür kann aber auch sein, dass es nur sehr wenige Verben oder Nomina gibt, die die Präposition obne regieren. Präpositionalobjekte, deren Kopf die Präposition unter ist, sind dagegen nicht so selten. Im vorliegenden Korpus werden nur 1,2\% der Vorkommen von obne überhaupt regiert, aber $3,6 \%$ der Vorkommen von unter, wobei unter ja auch insgesamt häufiger vorkommt. Dennoch kann man festhalten, dass Präpositionalobjekte mit unter den Wegfall des Artikels nicht unterstützen.

Weiterhin können wir beobachten, dass nominale Komplemente von unter, die als Komposita analysiert werden können, den Wegfall des Artikels ebenfalls nicht unterstützen. Es wird allerdings weiterer Untersuchungen bedürfen, um herauszufinden, warum dies so ist. Schließlich erkennen wir beim Modell für unter ebenso wie beim Modell für obne den Einfluss der Nominalisierung durch -ung. Nominalisierungen unterstützen den Wegfall des Artikels.

Die einzelnen Merkmale für die Wortfelder des Substantivs zeigen ein heterogenes Bild, das zu weiteren Untersuchungen einlädt: Es ist zunächst einmal auffällig, das GN-ORT einen hohen positiven Wert besitzt. GN-ORT dürfte Ausdruck einer Selektionsrestriktion bei spatialen Interpretationen von unter sein. Andere GN-Merkmale unterstützen hingegen den Wegfall des Artikels. Das Modell für unter macht aber nicht nur Gebrauch von Wortfeldern aus GermaNet, sondern auch von der ontologischen Klassifikation in HaGenLex. Die beiden relevanten Merkmale HL-AS (HaGenLex, abstrakt und statisch) und HL-AD (HaGenLex, abstrakt und dynamisch) sind Beschreibungen für abstrakte dynamische bzw. abstrakte statistische Sachverbalte aus der Sortenhierarchie von HaGenLex (vgl. Hartrumpf et al. 2003). Beispiele für die beiden Gruppen finden sich in (33).

Die Irrtumwahrscheinlichkeit bei DEP-PP liegt oberhalb von 0,05. Dieser Faktor wurde dennoch weiter im Modell gehalten, weil sich die Modellparameter insgesamt verschlechtern, wenn auf diesen Faktor verzichtet wird, vgl. Harrell (2001, S. 56). 
(33) a. abstrakte dynamische Sachverhalte (Merkmal HL-AD): Lauf, Diebstahl, Veränderung

b. abstrakte statistische Sachverhalte (Merkmal HL-As): Umstand, Notlage, Auslastung

Wie auch anhand der Beispiele in (38) erkennbar ist, liegen abstrakte Nomina vor, die aber zählbar sind. Wir sehen somit einen weiteren Grund, an der Annahme zu zweifeln, dass Abstrakta nicht zählbar sein dürfen. Substantive, die als abstrakte statische oder dynamische Konzepte in HaGenLex charakterisiert sind, fördern den Artikelwegfall bei unter.

\subsection{Die Güte der Modelle}

Die Güte logistischer Modelle kann einerseits anhand bestimmter Kennzahlen bemessen werden, andererseits auch durch unterschiedliche Evaluationsverfahren bestimmt werden. Harrell (2001) empfiehlt für die Evaluation logistischer Modelle die so genannte ,bootstrap validation“, die im Gegensatz zu anderen Validierungsverfahren, insbesondere im Gegensatz zur „crossvalidation“ den Vorteil hat, dass alle Daten zum Training und zum Testen verwendet werden können. Mittels „bootstrap validation“ kann bestimmt werden, ob das Modell zu sehr an den gesehenen Daten orientiert ist (ein „overfit“ vorliegt) oder ob hier plausibel von gesehenen auf nicht gesehene Daten generalisiert werden kann und somit ein Modell mit Vorhersagekraft vorliegt. Bei einer ,bootstrap validation“" wird aus einem Datensatz mit $n$ unterschiedlichen Elementen durch Auswahl mit Zurücklegen eine Menge von Datensätzen mit $m(=n)$ nicht-unterschiedlichen Elementen gebildet. Eine Auswahl mit Zurücklegen bedeutet hier, dass ein Datensatz in die Stichprobe übernommen wird, aber auch danach nochmals für die Stichprobe zur Verfügung steht. Die Trainingsmenge enthält also genau so viele Elemente wie der ursprüngliche Datensatz, aber durch das Zurücklegen werden einzelne Daten häufiger als einmal in der Trainingsmenge erscheinen. Die Testmenge besteht dann gerade aus denjenigen Elementen des ursprünglichen Datensatzes, die nie ausgewählt wurden. Ebenso wie bei einer „crossvalidation“ wird dieser Prozess mehrfach wiederholt. Erfreulicherweise legen bereits die reinen Kenndaten der beiden Modelle den Schluss nahe, dass hier Modelle mit Vorhersagekraft entwickelt wurden, und diese Annahme wird auch durch die ,bootstrap validation“ bestätigt.

\begin{tabular}{|l|c|c|c|c|}
\hline & Model L.R. & $\mathrm{p}$ & $\mathrm{C}$ & $\mathrm{D}_{\mathrm{xy}}$ \\
\hline ohne & $1.063,5$ & 0 & 0,876 & 0,753 \\
\hline unter & $2.245,6$ & 0 & 0,937 & 0,874 \\
\hline
\end{tabular}

Tab. 5: Grundlegende Kenndaten der Modelle 
Der Wert „Model L.R.“ (= „Model Likelihood Ratio“) gibt an, wie sehr sich die Vorhersagekraft des Modells von einem Modell mit willkürlich gewählten Prädiktoren (bzw. eben ohne jede Prädiktoren) verändert. Unter „p“ wird erneut die Wahrscheinlichkeit bestimmt, mit der diese Veränderung nur zufällig sein könnte - sie liegt bei 0 .

Besonders relevant sind die hohen Werte für „C“ und „Somers $D_{x y}$ “ in Tabelle 5. Der Somers $\mathrm{D}_{\mathrm{xy}}$-Wert bestimmt das Verhältnis von konkordanten zu nicht-konkordanten Übereinstimmungen bei einem Modell, dessen abhängige Variable die Werte 0 und 1 annehmen kann. Eine Übereinstimmung ist konkordant, wenn im Modell die vorhergesagten Wahrscheinlichkeiten mit den tatsächlichen Klassenzugehörigkeiten korrespondieren. Der Wert für $D_{x y}$ entspricht $(C-0,5) \times 2$. C und $D_{x y}$ beschreiben eigentlich denselben Sachverhalt, aber $\mathrm{D}_{\mathrm{xy}}$ ist eine Abbildung des C-Wertes auf eine Wahrscheinlichkeitsskala. Ein willkürliches Modell ohne Vorhersage besitzt einen CWert von $0,5\left(D_{\mathrm{xy}}=0\right)$. Werte für $\mathrm{C}$ über $0,8\left(\mathrm{D}_{\mathrm{xy}}=0,6\right)$ legen den Schluss nahe, dass das Modell Vorhersagekraft besitzt; wir sehen bei dem Modell für unter sogar einen Wert deutlich über 0.9.

Das Ergebnis der „,bootstrap validation“ für die beiden Modelle ist in Tabelle 6 dargestellt; hierbei wurden jeweils 200 Iterationen durchgeführt, d.h. 200 Trainingssätze wurden erstellt, mit den Modellparametern trainiert und mit den verbleibenden Daten evaluiert.

a. obne

\begin{tabular}{|l|c|c|c|c|c|}
\hline & Alle Daten & Training & Test & Optimismus & Korrektur \\
\hline $\mathrm{D}_{\mathrm{xy}}$ & 0,7526 & 0,7570 & 0,7500 & 0,0070 & 0,7456 \\
\hline $\mathrm{E}_{\max }$ & 0,0000 & 0,0000 & 0,0096 & 0,0096 & 0,0096 \\
\hline
\end{tabular}

b. unter

\begin{tabular}{|l|c|c|c|c|c|}
\hline & Alle Daten & Training & Test & Optimismus & Korrektur \\
\hline $\mathrm{D}_{\mathrm{xy}}$ & 0,8736 & 0,8744 & 0,8692 & 0,0052 & 0,8684 \\
\hline $\mathrm{E}_{\max }$ & 0,0000 & 0,0000 & 0,0055 & 0,0055 & 0,0055 \\
\hline
\end{tabular}

Tab. 6a und b: „Bootstrap validation“ der Modelle

Die Zeilen geben darüber Auskunft, dass der ursprüngliche $\mathrm{D}_{\mathrm{xy}}$-Wert für das Modell für ohne bei 0,7526 lag; die Differenz zwischen dem Training bei der „bootstrap validation“ und dem Test betrug allerdings $(0,757-0,750)=0,007$. Dieser Wert charakterisiert den Optimismus des Modells, d.h. denjenigen Anteil an der Vorhersagekraft, der einer zu starken Ausrichtung an die Trainingsdaten geschuldet ist. Entsprechend muss der ursprüngliche Wert um dieses $\mathrm{Ma} \beta$ an Optimismus nach unten korrigiert werden, so dass sich nach Validierung ein Wert von 0,7456 ergibt. Entsprechendes gilt in der zweiten Zeile für die durchschnittliche maximale Fehlerrate. Im Ursprungsmodell 
liegt diese bei 0 , weil mit allen Daten trainiert wurde. Der Testfall zeigt aber eine durchschnittliche Veränderung von 0,0096, so dass dieser Wert nun als Fehlerrate angesetzt wird. Die Ergebnisse für unter wiederholen die für obne.

Vor dem Hintergrund dieser Evaluation erfahren die Koeffizienten der oben genannten Modelle die folgende Interpretation: Je höher ein Wert ausfällt, desto einflussreicher ist der Wert, desto deutlicher wird die Wahrscheinlichkeit für die Weglassbarkeit des Artikels in Richtung des Vorzeichens des Koeffizienten modifiziert. So ist etwa der Wert für DEP-S in beiden Modellen sehr hoch und positiv, so dass wir davon ausgehen können, dass die Realisation eines sententialen Komplements des Nomens die Weglassbarkeit des Artikels extrem erschwert. Liegen aber - etwa bei obne - keine Dependenten des Substantivs vor, dafür aber die entsprechenden Interpretationen von Präposition und Substantiv, dann ist die Weglassbarkeit des Artikels sehr wahrscheinlich. Wir wollen im folgenden Abschnitt der Frage nachgehen, ob es sich nun tatsächlich um rein semantische Faktoren handelt oder hier zugrunde liegende strukturelle Faktoren eine Rolle spielen könnten.

\section{Externe Faktoren beim Artikelwegfall mit obne?}

Eine Interpretation der Präposition ohne - VORHANDENSEIN - unterstützt die Weglassbarkeit des Artikels, während die Interpretationen KAUSAL, KOMITATIV und BETEILIGUNG die Weglassbarkeit eher erschweren.

Hier stellt sich die Frage, ob die Verteilung aus intrinsischen Faktoren der Interpretationen erklärt werden kann, oder ob nicht doch andere, verborgene Faktoren eine Rolle spielen könnten. Wenn wir die Interpretationsmöglichkeiten von obne insgesamt betrachten, fällt auf, dass die Interpretationen zumindest in Teilen ein syntaktisches Korrelat besitzen, somit also eine Abbildung zwischen Interpretationen, syntaktischen Korrelaten und der Artikelweglassbarkeit denkbar ist.

Hierzu erscheint es zunächst sinnvoll, die Bedeutung der Präposition ohne zusammen mit der Bedeutung der Präposition mit zu betrachten. Das Annotationsschema für Präpositionsbedeutungen trägt den Besonderheiten dieser beiden Präpositionen - und der Tatsache, dass zumindest einige Interpretationen der einen in Opposition zu Interpretationen der anderen zu charakterisieren sind - dadurch Rechnung, dass bestimmte Kategorien nur von diesen Präpositionen besetzt werden können.

Die Präposition obne teilt mit der Präposition mit die Interpretation VORHANDENSEIN mit den Untertypen SYNTHETISCH und ANALYTISCH. Während diese Interpretation bei mit angibt, dass etwas vorhanden ist, drückt sie bei obne einen Mangel aus. Die Binnendifferenzierung von VORHANDENSEIN, SYNTHETISCH und ANALYTISCH kann leichter anhand von mit erläutert werden: 
VORHANDENSEIN bezeichnet im Schema das Vorhandensein (bzw. bei obne: Fehlen) einer Sache, eines Merkmals oder einer Eigenschaft; SYNTHETISCH bezeichnet das Vorhandensein einer Sache, eines Merkmals oder einer Eigenschaft, die als einer anderen zugehörig betrachtet werden kann, ohne im Sinne einer Mereologie Teil des anderen zu sein; man kann diese Art der Zugehörigkeit auch als eine betrachten, die durch synthetische Urteile erfasst werden kann, z.B. eine Flasche mit Verzierung. Demgegenüber beschreibt ANALYTISCH eine Zugehörigkeit, die tatsächlich als Teil-Ganzes-Beziehung beschrieben werden kann bzw. entsprechend durch ein analytisches Urteil ausgedrückt wird. Die analytische Form der Urteile hat den Charakter des Pleonastischen und deswegen findet man typische analytische Interpretationen von mit nur dann, wenn das Teil in bestimmter Weise modifiziert wird, wie die Kontraste in (34) zeigen:

(34) a. eine Hand mit Fingern vs. eine Hand mit drei Fingern

b. ein Auto mit Motor vs. ein Auto mit Dieselmotor

Für obne gilt dies natürlich nicht in gleichem Maße, weil das Fehlen eines ansonsten mitgedachten Teils einen höheren Informationsgehalt besitzt als das Vorhandensein dieses mitgedachten Teils:
a. ein Auto ohne Motor
b. eine Hand ohne Daumen

Die drei Merkmale VORHANDENSEIN, SYNTHETISCH und ANALYTISCH können die Präpositionen mit und ohne nur annehmen, wenn sie nominale Phrasen modifizieren.

Zusätzlich zu diesen Merkmalen besitzt obne die in (36) aufgeführten Interpretationen:

$$
\begin{aligned}
& \text { KAUSAL, KONDITIONAL, ART UND WEISE, BEGLEITUMSTAND, } \\
& \text { BETEILIGUNG, KOMITATIV, INSTRUMENTAL }
\end{aligned}
$$

Diese Interpretationen treten typischerweise auf, wenn obne entweder eine verbale Projektion modifiziert oder eine nominale Projektion, deren Kopf eine Ereignisnominalisierung ist. Weiterhin muss hierbei berücksichtigt werden, dass die Merkmale BETEILIGUNG und KOMITATIV ebenso wie das oben bereits angesprochene Merkmal VORHANDENSEIN nur durch die Präpositionen obne und mit besetzt werden kann. Wir können somit sagen, dass zumindest eine Kernbedeutung der Präpositionen obne und mit durch die Merkmale VORHANDENSEIN, BETEILIGUNG und KOMITATIV bestimmt wird, wobei die Verteilung dieser Merkmale entweder tatsächlich einer externen syntaktischen Distribution (welches Element wird syntaktisch modifiziert) oder einer durch die syntaktische Distribution vermittelten Interpretationsbedingung (wird ein Ereignis oder etwas Dinghaftes modifiziert) geschuldet ist. 
Diese beiden Bedingungen können für die Interpretation BETEILIGUNG wie folgt illustriert werden: In (37) liegen verbale Modifikationen vor, in (38) nominale Modifikationen mit ereignisartigem Charakter.

a. Die Hauptverhandlung wird am 8. Februar notfalls auch ohne den Angeklagten fortgesetzt. (NZZ, AUSLAND, 28.01.1993)

b. Der Viertelmeilen-Sprint, einer der anfänglich vermuteten Höhepunkte, entwickelte sich ohne den großen Abwesenden von Zürich, Michael Johnson, zum erwarteten US-Duell zwischen Antonio Pettigrew und Jerome Young, dem Zweit- und dem Drittschnellsten der Saison. (NZZ, SPORT, 12.08.1999)

(38) a. Nur akademische Miles-Davis-Forscher dürften von diesem detaillierten Einblick in die Werkstatt profitieren und stundenlang abgebrochene Versuche oder gar Durchläufe ohne den Solisten mitverfolgen. (NZZ, PHONO-SPEKTRUM, 02.10.1996)

b. Denn eine Siegerehrung ohne die Amerikanerin ist letztlich doch wohl (noch) ebenso die Ausnahme von der Regel wie die Durchführung einer Abfahrt in zwei Läufen. (NZZ, SPORT, 16.12.1995)

Es scheint also insgesamt so zu sein, dass die einen Interpretationen von obne nur in verbalen syntaktischen Kontexten realisiert werden können (bzw. in nominalen syntaktischen Kontexten, in denen das Substantiv ereignisartig interpretiert werden muss), während die anderen in nominalen Kontexten realisiert werden. Nominale Kontexte scheinen nun die Artikelweglassbarkeit zu bevorzugen, verbale Kontexte (bzw. Nomina mit Ereignislesart) hingegen blockieren die Artikelweglassbarkeit. Wenn wir nun nochmals das Modell für die Artikelweglassbarkeit bei ohne in Tabelle 3 betrachten, so könnte die Präferenz für die Weglassbarkeit bei der Interpretation VORHANDENSEIN bzw. die Präferenz für die Artikelrealisation bei den Interpretationen KAUSAL, KOMITATIV und BETEILIGUNG auch so gedeutet werden, dass die Präposition obne dann ein artikelloses Komplement besitzen kann, wenn die Präposition eine Nominalprojektion modifiziert, aber nicht, wenn sie eine verbale Projektion modifiziert. Dies muss nicht notwendigerweise bedeuten, dass die Analyse der PNK konstruktional sein muss, denn die verbale bzw. nominale Modifikation könnte formal auch durch den semantischen Typ des zu modifizierenden Elements erfasst werden, was dann wiederum auch den Einschluss von Daten wie (38) gestatten würde.

Da nun wiederum die Bedeutungen VORHANDENSEIN, KOMITATIV und BETEILIGUNG diese Verteilung aufweisen und nur als Bedeutungen für die Präpositionen ohne und mit verwendet werden, läge der Schluss nahe, auch das Annotationsschema für die Interpretationen so zu modifizieren, dass etwa das Merkmal [ \pm EREIGNIS] als Kreuzklassifikator verwendet wird, um das Merkmal VORHANDENSEIN von den anderen beiden zu trennen, und entsprechend das Schema nochmals zu vereinfachen. 


\section{Schlussfolgerungen}

Die grundlegende Idee hinter dem „Annotation Mining“ ist, eine möglichst hohe Zahl potenziell relevanter linguistischer Eigenschaften bei einer möglichst hohen Zahl einzelner Daten zu repräsentieren und aus den Daten durch Klassifikationsverfahren diejenigen Eigenschaften zu extrahieren, die für die syntaktische Distribution etwa der PNKen relevant sein können. Anhand der Präpositionen obne und unter haben wir zeigen können, welche Merkmale die Weglassbarkeit des Artikels bedingen. Für die Präposition ohne konnten wir zunächst ableiten, dass bestimmte Interpretationen der Präposition für die Weglassbarkeit des Artikels beim Komplement sprechen, andere hingegen dagegen. Eine genauere Betrachtung zeigte dann aber bereits, dass strukturelle Faktoren (was wird modifiziert) hier eine Rolle spielen könnten. Strukturelle Faktoren müssen neben den interpretatorischen Faktoren ohnehin angesetzt werden, um zu erklären, welchen Einfluss die Realisation eines postnominalen Komplements auf die Weglassbarkeit des Artikels hat.

Für die Präposition unter konnten wir diesen letzten Aspekt erneut sichtbar machen, so dass man von einer allgemeinen strukturellen Eigenschaft sprechen kann: Besitzt ein Substantiv ein postnominales Komplement, dann wird die Weglassbarkeit des Artikels erschwert, wobei dies insbesondere gilt, wenn das Komplement ein Satz ist.

Besonders auffällig ist im Vergleich der Präpositionen, dass bei obne eine Kernbedeutung den Artikelwegfall erleichtert, eine andere hingegen den Wegfall erschwert, während es bei unter nur Interpretationsmerkmale gibt, die den Artikelwegfall erschweren. Vor einer Betrachtung weiterer Präpositionen ist es sicherlich zu früh, diese Beobachtung zu werten. Dennoch bietet sich hier eine Spekulation an: Bei obne stehen die Kernbedeutungen somit für bzw. wider den Artikelwegfall (möglicherweise auch in Verbindung mit weiteren strukturellen oder interpretativen Bedingungen), bei unter ist aber ähnliches (noch) nicht zu beobachten. Ist dies vielleicht der Grund dafür, dass der Artikelwegfall bei obne schon der Regelfall ist, bei unter aber noch die Ausnahme?

\section{Literatur}

Allan, Keith (1980): Nouns and countability. In: Language 56, 3, S. 541-567.

Baayen, R. Harald (2001): Word frequency distributions. (= Text, Speech and Language Technology 18). Dordrecht u.a.

Baayen, R. Harald (2008): Analyzing linguistic data. A practical introduction to statistics using R. Cambridge u.a.

Baldwin, Timothy et al. (2006): In search of a systematic treatment of determinerless PPs. In: Saint-Dizier, Patrick (Hg.): Syntax and semantics of prepositions. (= Text, Speech and Language Technology 29). Dordrecht, S. 163-179. 
Bale, Alan/Barner, David (2009): The interpretation of functional heads: using comparatives to explore the mass/count distinction. In: Journal of Semantics 26, S. 217-252.

Barner, David/Snedeker, Jesse (2005): Quantity judgements and individuation: evidence that mass nouns count. In: Cognition 97, S. 41-66.

Borer, Hagit (2005): Structuring sense. Bd. I: In name only. Oxford u.a.

Chiarcos, Christian et al. (2008): A flexible framework for integrating annotations from different tools and tagsets. In: Traitement Automatique des Langues. Special Issue Platforms for Natural Language Processing (ATALA), 49, 2, S. 271-291.

Dömges, Florian et al. (2007): Measuring the productivity of determinerless PPs. In: Costello, Fintan/Kelleher, John/Volk, Martin (Hg.): Proceedings of the 4th ACLSIGSEM Workshop on Prepositions. Prag, S. 31-37. Internet: www.linguistics.ruhr-uni-bochum. de/ kiss/publications/DKMR_3.pdf (Stand: 13.01.2011).

Duden (2002): Duden. Standardwörterbuch Deutsch als Fremdsprache. Hrsg. v. Wissenschaftlichen Rat der Dudenredaktion. Mannheim u.a.

Duden (2005): Duden. Bd. 4: Die Grammatik. Hrsg. v. Wissenschaftichen Rat der Dudenredaktion. 7., völlig neu erarb. u. erw. Aufl. Mannheim u.a.

Durell, Martin/Brée, David (1993): German temporal prepositions from an English perspective. In: Zelinsky-Wibbelt, Cornelia (Hg.): The semantics of prepositions. From mental processing to natural language processing. (= Natural Language Processing 3). Berlin/New York, S. 295-325.

Eberle, Kurt (2009): - ung nominalizations of verbs of saying in German: events and propositions. In: Proceedings of CHRONOS 9 - 9th International Conference on Tense, Aspect and Modality. Paris.

Espinal, M. Teresa/McNally, Louise (ersch. 2011): Bare singular nominals and incorporating verbs in Spanish and Catalan. In: Journal of Linguistics.

Evert, Stefan (2004): A simple LNRE model for random character sequences. In: Le poids des mots. Actes des 7es Journées Internationales d'Analyse Statistique des Données Textuelles. Louvain-la-Neuve, S. 411-422.

Gillon, Brendan S. (1999): The lexical semantics of English count and mass nouns. In: Viegas, Evelyne (Hg.): Breadth and depth of semantic lexicons. (= Text, Speech, and Language Technology 10). Dordrecht u.a., S. 19-37.

Harrell, Frank E. (2001): Regression modeling strategies: with applications to linear models, logistic regression, and survival analysis. New York u.a.

Hartrumpf, Sven/Helbig, Hermann/Oswald, Rainer (2003): The semantically based computer lexicon HaGenLex - structure and technological environment. In: Traitement automatique des langues 44, 2, S. 81-105.

Helbig, Gerhard/Buscha, Joachim (2001): Deutsche Grammatik. Ein Handbuch für den Ausländerunterricht. Berlin u.a.

Himmelmann, Nikolaus (1998): Regularity in irregularity: article use in adpositional phrases. In: Linguistic Typology 2, S. 315-353. 
Jespersen, Otto (1924): The philosophy of grammar. London.

Kiss, Tibor (2007): Produktivität und Idiomatizität von Präposition-Substantiv-Sequenzen. In: Zeitschrift für Sprachwissenschaft 26, 2, S. 317-345.

Kunze, Claudia/Lemnitzer, Lothar (2002): GermaNet - representation, visualization, application. In: Proceedings of the Language Resources and Evaluation Conference (LREC) 2002. Bd. V. Las Palmas de Gran Canaria, S. 1485-1491.

Le Bruyn, Bert/de Swart, Henriëtte/Zwarts, Joost (2009): Bare PPs across languages. Workshop on bare nouns. Paris.

Lezius, Wolfgang/Dipper, Stefanie/Fritschen, Arne (2000): IMSLex - representing morphological and syntactic information in a relational database. In: Heid, Ulrich et al. (Hg.): Proceedings of the 9th EURALEX International Congress. Stuttgart, S. 133-139.

Müller, Antje et al. (i.Dr.): Annotation of preposition senses in German. In: Stolterfoht, Britta/Featherston, Sam (Hg.): Proceedings of Linguistic Evidence 2009. Berlin.

Müller, Christoph/Strube, Michael (2006): Multilevel annotation of linguistic data with MMAX2. In: Braun, Sabine/Kohn, Kurt/Mukherhjee, Joybrato (Hg.): Corpus technology and language pedagogy: new resources, new tools, new methods. (= English Corpus Linguistics 3). Frankfurt a.M. u.a., S. 197-214.

Schmid, Helmut (1995): Improvements in part-of-speech tagging with an application to German. In: Proceedings of the EACL SIGDAT Workshop. Dublin, S. 47-50.

Schmid, Helmut/Fitschen, Arne/Heid, Ulrich (2004): SMOR: A German computational morphology covering derivation, composition, and inflection. In: Proceedings of the Language Resources and Evaluation Conference (LREC) 2004. Lissabon, S. 1263-1266.

Schmid, Helmut/Laws, Florian (2008): Estimation of conditional probabilities with decision trees and an application to fine-grained POS tagging. In: Proceedings of 22nd International Conference on Computational Linguistics (COLING), 18-22 August 2008, Manchester, UK. Stroudsburg, S. 465-472.

Schröder, Jochen (1986): Lexikon deutscher Präpositionen. Leipzig.

Stadtfeld, Tobias (i.Vorb.): Classifying countability. Diss. Bochum.

Stvan, Laurel S. (1998): The semantics and pragmatics of bare singular noun phrases. Diss., Evanston/Chicago. 



\title{
Beatrice Primus
}

\section{Das unpersönliche Passiv - Ein Fall für die Konstruktionsgrammatik?}

\begin{abstract}
Dieser Beitrag thematisiert semantische Bedingungen des unpersönlichen Passivs im Deutschen und in anderen Sprachen. Traditionellerweise nimmt man an, dass nur atelische und agentivische Verben im unpersönlichen Passiv akzeptabel sind. Ich werde die empirischen Hypothesen der bisherigen Forschung auf der Grundlage von Akzeptabilitätsstudien und einer breiteren korpusbasierten Datenmenge revidieren. Die hier behandelten semantischen Aspekte wurden in einflussreichen Arbeiten als Evidenz für die Überlegenheit einer konstruktionsgrammatischen Herangehensweise gewertet. Ich werde diese Evidenz in Frage stellen und beschränkungsbasierte Alternativen präsentieren. ${ }^{1}$
\end{abstract}

\section{Einleitung}

Der in diesem Beitrag berücksichtigen Forschungsliteratur folgend werde ich Konstruktionen wie in den Beispielen (1)-(2), nicht jedoch solche wie in (3), die ein Kasusobjekt im Passiv behalten, als unpersönliche Passive zur Diskussion stellen:

(1) Dass auch bei der Aufführung kräftig gelacht wird, da kann man sicher sein.

[Mannheimer Morgen, 04.07.2001; „Außer Geld haben wir nichts kaputt gemacht" ]

(2) Gespielt, gesungen und getanzt wird ab zirka $12 \mathrm{Uhr}$ bis $17 \mathrm{Uhr}^{2}$ [Mannheimer Morgen, 25.03.1998; Kunterbunter Kindertag]

(3) Gedankt wird auf diese Art Bürgern der Gemeinde.

[Mannheimer Morgen, 24.05.2008; Gemeinde ehrt erfolgreiche Bürger]

Traditionellerweise nimmt man zwei semantische Bedingungen für das unpersönliche Passiv an: Nur atelische und agentivische Verben bzw. Lesarten sind im unpersönlichen Passiv akzeptabel. Solche Verwendungen sind in

$1 \quad$ Für Hinweise und Verbesserungsvorschläge bedanke ich mich bei den Bandherausgebern, für Hilfe bei der statistischen Auswertung bei Markus Linnemann und Martin Evertz, für Unterstützung bei den niederländischen Daten bei Joost Kremers, Daan Huntjens und Marieke de Winkel.

2 Die Verben in (2) können auch transitiv gebraucht werden (Skat spielen, ein Lied singen, einen langsamen Walzer tanzen). Zur Diskussion steht hier nur ihre intransitive Valenzvariante. 
(1)-(2) illustriert. Der folgende Abschnitt behandelt die ereignisstrukturelle ${ }^{3}$ Telizitätsrestriktion und der Abschnitt 3 die Bedingung der Agentivität und Belebtheit. Abschnitt 4 fasst die Ergebnisse dieses Beitrags zusammen.

\section{Telizität beim unpersönlichen Passiv}

\subsection{Lexembezogene Ansätze}

Eine erste Gruppe von Ansätzen geht davon aus, dass eine bestimmte Klasse intransitiver Verblexeme nicht passivierbar ist (z.B. Perlmutter 1978; Perlmutter/Postal 1983; Burzio 1986; Grewendorf 1989; Kaufmann 1995; Zifonun et al. 1997; Carnie/Harley 2005; Abraham/Leiss 2006). Das Hauptaugenmerk dieser Arbeiten liegt auf dem Deutschen oder Niederländischen. Die folgenden niederländischen Beispiele und deren Akzeptabilitätsbewertung stammen von Perlmutter/Postal (1983, S. 108, 112):

a. In dit weeshuis groeien de kinderen erg snel.

"In diesem Waisenhaus wachsen die Kinder sehr schnell.

b. *In dit weeshuis wordt (er) door de kinderen erg snel gegroeid. "In diesem Waisenhaus wird schnell gewachsen.

a. In dit ziekenhuis sterven de patienten dikwijls. ,In diesem Krankenhaus sterben die Patienten oft.'

b *In dit ziekenhuis wordt (er) door de patienten dikwijls gestorven. ,In diesem Krankenhaus wird oft gestorben.

Die in (4)-(5) illustrierten niederländischen Verben sterven und groeien und ihre deutschen Entsprechungen sterben und wachsen gehören zur Klasse der so genannten ,unakkusativen' oder ,ergativen' intransitiven Verben, die sich von den ,unergativen' intransitiven Verben wie arbeiten und tanzen durch mehrere syntaktische und semantische Eigenschaften unterscheiden. $\mathrm{Zu}$ den hier relevanten Unterscheidungskriterien gehören die Auxiliarselektion (sein, niederländisch zijn für Unakkusativa, haben, niederländisch bebben für Unergativa), die Passivierbarkeit, die Ereignisstruktur und die semantische Rolle des Subjekts. Die Verben sterben und wachsen und ihre niederländischen Entsprechungen sterven und groeien weisen den genannten Autoren zufolge alle Eigenschaften auf, die Unakkusativa charakterisieren: Sie selegieren sein bzw. zijn als Auxiliar im Perfekt, haben eine - weiter unten genauer spezifizierte - telische Ereignisstruktur, weisen ihrem Argument keine Agens-Rolle $\mathrm{zu}$ und sind - nach Einschätzung der oben genannten Autoren - nicht passivierbar.

In diesem Beitrag wird der Terminus „Ereignisstruktur“ und seine Adjektivierung anstelle von „Aspekt" und „Aktionsart“ verwendet, da letztere in der Forschung sehr uneinheitlich gebraucht werden. 
Perlmutter und Postal (1983) erklären die von ihnen angenommene Nicht-Passivierbarkeit der Unakkusativa im Rahmen der Relationalen Grammatik auf syntaktischer Ebene, die meisten neueren Arbeiten suchen hingegen nach semantischen Erklärungen, die in diesem Beitrag näher berücksichtigt werden.

Die Arbeiten, die sich mit der Telizitätsbeschränkung des Passivs näher befassen (z.B. Keller/Sorace 2003; Carnie/Harley 2005; Abraham/Leiss 2006), verwenden einen relativ weiten Telizitätsbegriff, der Verbbedeutungen umfasst, die ereignisstrukturell aus mindestens zwei Phasen bestehen, wobei in den meisten Fällen ein punktueller oder allmählicher Zustandswechsel impliziert wird. So bezeichnet sterben einen Zustandswechsel von Nicht-tot-Sein zu Tot-Sein, während wachsen ein allmähliches Größer-Werden denotiert, bei dem Anfangszustand und Endzustand lexikalisch unspezifiziert sind (vgl. Dowty 1979, S. 88 f.; Kennedy/Levin 2008). Ein Verb wie bleiben zeichnet sich dadurch aus, dass in der Anfangsphase und in der Endphase derselbe Zustand vorliegt und somit kein Zustandswechsel stattfindet (vgl. Engelberg 2000, S. 56). ${ }^{4}$

Für den Kernbereich der telischen Verben, die einen Wechsel zwischen einem Anfangs- und einem Endzustand denotieren (d.h. ausgenommen Verben wie wachsen und bleiben), gibt es einige recht zuverlässige Identifikationskriterien.

Erstens sind telische Ereignisse nicht divisiv. Dies bedeutet, dass nicht alle Teile eines telischen Ereignisses unter das Denotat des Verbalausdrucks fallen. Wenn es beispielsweise für ein bestimmtes Zeitintervall zutrifft, dass Peter einschlief, dann fallen nicht alle Teile seines Einschlafens darunter. Insbesondere der Anfang (Nicht-Schlafen) und das Ende (Schlafen) sind nicht mit Einschlafen gleichzusetzen. Anders verhalten sich atelische $\mathrm{Zu}-$ stände, Prozesse und Handlungen wie lieben, schlafen und arbeiten. Wenn es für ein bestimmtes Zeitintervall zutrifft, dass Peter schläft, so fallen alle echten Teile seines Schlafens darunter. Das Kriterium der Divisivität ist nicht streng anwendbar, da es durchaus Teile von Schlafen-Ereignissen geben kann, die nicht unter schlafen fallen (z.B. ein Wechsel der Körperlage oder ein Augenzucken).

Terminologisch herrscht große Uneinheitlichkeit für die hier beschriebenen Ereignisstrukturen. „Telizität“ ist in der anglo-amerikanischen Forschung der weiteste und häufigste Terminus, dem die Termini „Resultativität", „Perfektivität“, „Inchoativität“ und „Terminativität“ am nächsten stehen. Bei Vendler (1957) fallen unakkusative Verben unter ,achievements“ oder ,accomplishments“. Alle genannten Termini suggerieren einen Wechsel von einem spezifizierbaren Anfangszustand zu einem spezifizierbaren Endzustand. In Ermangelung einer besseren Terminologie bleibe ich bei der Bezeichnung „telisch“ und beziehe mich damit - wie im Text weiter oben angegeben - auf komplexe Ereignisstrukturen, die mindestens zwei (nicht notwendigerweise verschiedene) Phasen beinhalten, die hinsichtlich Anfangs- und Endzustand lexikalisch nicht notwendigerweise spezifiziert sind. 
Zweitens sind telische Ereignisse nicht kumulativ. Wenn ein telisches (genauer genommen inchoatives) Ereignis wie Peters Einschlafen für ein Zeitintervall zutrifft, beispielsweise von 17:33 bis 17:35 Uhr, und wenn man diesem Ereignis Peters Einschlafen in einem benachbarten Zeitintervall, etwa von 17:35 bis 17:37 Uhr, hinzufügt, so resultiert daraus kein singuläres Ereignis, das man mit Peter schlief von 17:33 bis 17:37 Ubr ein beschreiben kann. Wir erhalten ggf. zwei Ereignisse, d.h. eine repetitive Bedeutung. Atelische Verbbedeutungen hingegen sind kumulativ. Wenn man Peters Schlafen von fünf bis sieben Uhr ein ansonsten identisches Schlafen von Peter von sieben bis neun Uhr hinzufügt, so erhält man ein singuläres Ereignis, das man mit Peter schlief von fünf bis neun Uhr beschreiben kann.

Ein oft verwendeter Telizitätstest bedient sich des Unterschieds zwischen Zeitdauer- bzw. Durativangaben wie drei Tage lang und Zeitspannenangaben wie in drei Tagen. Vgl. (6)-(7):

a. Maria scblief in drei Minuten ein.

b. "Maria schlief drei Minuten lang ein.

a. Maria schlief drei Tage lang.

b. "Maria schlief in drei Tagen.

Hier und im Folgenden notiert das Doppelkreuz \# weder Ungrammatikalität noch generelle semantische Inakzeptabilität, sondern eine semantische Anomalie in der diskutierten Lesart bzw. den Ausschluss einer bestimmten, zur Diskussion stehenden Lesart. Meistens sind für die mit Doppelkreuz notierten Sätze alternative Lesarten möglich, auf die ich manchmal, aber nicht immer verweisen werde. Zur Diskussion steht für (6)-(7) die Lesart als singuläres (d.h. nicht repetitives) Ereignis. Die Zeitangaben sollen sich dabei hier und im Folgenden auf das Zeitintervall beziehen, in dem das Ereignis stattfindet. Diese Lesart ist bei einem telischen Ereignis mit Durativangaben ausgeschlossen, wie in (6b) gezeigt. Der Satz (6b) kann nicht so verstanden werden, dass Marias Einschlafen innerhalb eines Zeitintervalls von drei Minuten stattfand. Anders verhält sich das atelische Ereignis in (7). Die zur Diskussion stehende Lesart ist mit einer Durativangabe verträglich. Mit einer Zeitspannenangabe ist sie hingegen blockiert, wie in (7b) gezeigt. (Möglich, wenn auch ungewöhnlich ist eine inchoative Lesart von (7b), in welcher Maria drei Tage später zu schlafen anfing.)

Nachdem der Telizitätsbegriff für unsere Zwecke hinreichend geklärt wurde, widmen wir uns den empirischen Problemen, mit denen die lexembezogene Hypothese, dass telische intransitive Verblexeme nicht passivierbar sind, konfrontiert wird. Zugleich sollen alternative, empirisch angemessenere lexikonbasierte Ansätze vorgestellt werden. 


\subsection{Lesartenbezogene Ansätze}

Spätestens seit der Arbeit von Zaenen (1993) beginnt sich die Ansicht durchzusetzen, dass Unakkusativa atelisch gebraucht und in diesem Fall passiviert werden können. Vgl. Zaenens Beispiele und Akzeptabilitätsbewertungen in (8):
a. *Er werd opgestegen.
,Da wird abgeflogen.
b. Vanaf Schiphol wordt er de hele dag opgestegen. ,Von Schiphol wird den ganzen Tag abgeflogen.

Der Unterschied in der Akzeptabilität von (8b) gegenüber der Inakzeptabilität von (8a) liegt Zaenen zufolge an der Durativangabe de bele dag ,den ganzen Tag' in (8b), die eine repetitive, d.h. pluralische oder habituative, Ereignislesart dieses Satzes erzwingt. Repetitive Lesarten telischer Verblexeme haben eine atelische Ereignisstruktur, wie bereits die Durativangabe in (8b) demonstriert. Durativangaben sind, wie anhand von (6) und (7) weiter oben besprochen, zuverlässige Testmittel für die diskutierte ereignisstrukturelle Unterscheidung. Außerdem erfüllt (8b) in der repetitiven Lesart die Kriterien der Divisivität und Kumulativität, was ich hier mit Verweis auf den vorigen Abschnitt nicht eigens demonstriere.

Auch für das Deutsche lässt sich dieselbe Beobachtung wie für das Niederländische machen. Unakkusativa lassen sich bei repetitiver Lesart passivieren (vgl. Rapp 1997, S. 152). Vgl. die Korpusbelege in (9)-(11):

(9) Unserer Gesellschaft wird vorgeworfen, den Tod und das Sterben zu tabuisieren. Gestorben wird meistens im Krankenhaus. Nachbarn fragen sich erst nach einiger Zeit, wo eigentlich die alte Dame aus dem Nachbarhaus geblieben ist.

[Mannheimer Morgen, 19.11.2005; Der Tod als Berufsalltag]

(10) Gewachsen wird nachts. Lange Zeit hielten Ärzte die Klagen von Kindern über Wachstumsschmerzen für jugendliche Hypochondrie. [Der Spiegel online 10/2005, 07.03.2005]

(11) Und daß die Schutzkleidung zum Fahren gehört wie die Rollen zu den Skatern. Denn ohne kann das Stürzen ziemlich schmerzhaft ausfallen. Gefallen wird nämlich immer nach vorn, in dieser Richtung fangen stabile Schützer an Knien, Ellenbogen und Handflächen das Gewicht ab.

[Mannheimer Morgen, 28.04.1999; Bremsen lernen gehört dazu]

Auch im Türkischen, das von Perlmutter und Postal (1983) neben dem Niederländischen und Deutschen behandelt wird, werden passivierte unakkusative Verben in einer atelischen habituativen Lesart gebraucht (vgl. Özkaragöz 1980; van Schaik-Rădulescu 2009). 
Als Fazit ist festzuhalten: Entgegen der Annahme einiger lexembasierter Ansätze sind telische intransitive (unakkusative) Verblexeme passivierbar, wenn sie in einer atelischen, repetitiven Lesart gebraucht werden.

\subsection{Die konstruktionsgrammatische Sicht}

Die im vorigen Abschnitt besprochene lesartenbezogene Hypothese wird von Adele Goldberg als Argument gegen lexikonbasierte Ansätze und für die Konstruktionsgrammatik bewertet. Ich zitiere (Goldberg 1995, S. 15):

Thus the constraint on the impersonal passive seems to be a constraint on the aspect of the entire expression, rather than one directly on the Aktionsart of the main verb. However, this being the case, the construction cannot be said to be lexically governed: the constraint must be associated with the construction as a whole.

Goldberg begründet dies im Einzelnen wie folgt. Zaenen sei zu der Annahme einer lexikalischen Ambiguität, d.h. einer atelischen und einer telischen lexikalisch-semantischen Lesart unakkusativer Verben, gezwungen, um die Passivierbarkeit zu erklären. Die Auxiliarselektion sein, niederländisch zijn, kann im Gegensatz zur Passivierbarkeit nicht durch Zeitangaben, die eine atelische repetitive Lesart festlegen, beeinflusst werden. Goldbergs Kritik greift diesen Punkt auf (1995, S. 15): „A theory which posited two lexical items, with opposite Aktionsart specifications, would not be able to predict these facts about auxiliary selection."

Die alternative Annahme von Goldberg ist, dass die Telizitäts- bzw. Unakkusativitätsbeschränkung beim unpersönlichen Passiv Teil der Konstruktionsbedeutung ist. Goldberg (ebd., S. 4) definiert den Konstruktionsbegriff wie in (12):

$\mathrm{C}$ is a CONSTRUCTION iff def $_{\mathrm{C}} \mathrm{C}$ is a form-meaning pair $\left\langle\mathrm{F}_{\mathrm{i}}, \mathrm{S}_{\mathrm{i}}\right\rangle$ such that some aspect of $F_{i}$ or some aspect of $S_{i}$ is not strictly predictable from C's component parts or from other previously established constructions.

Demzufolge handelt es sich bei Konstruktionen um Form-Bedeutungspaare, bei denen irgendein Form- oder Bedeutungsaspekt nicht aus den Komponenten der Konstruktion oder aus anderen Konstruktionen strikt vorhersagbar ist. Im hier besprochenen Fall ist der relevante Bedeutungsaspekt die Atelizität der Konstruktion. Dieser Bedeutungsaspekt ist Goldberg zufolge weder aus den anderen Komponenten des unpersönlichen Passivs, etwa aus dem Lexikoneintrag des Verbs, noch aus anderen Konstruktionen strikt vorhersagbar.

Bei der Bewertung der konstruktionsgrammatischen Sicht gehe ich in mehreren Schritten vor. Bevor ich in Abschnitt 2.5 weiter unten zeigen werde, dass eine vergleichbare Telizitätsbeschränkung in mehreren Konstruktionen auftritt, werde ich im nächsten Abschnitt Goldbergs Kritik, 
dass lexikonbasierte Ansätze zur Annahme einer lexikalischen Ambiguität gezwungen sind, hinterfragen. Die attraktivere und zugleich empirisch angemessenere Alternative, die Goldberg nicht berücksichtigt, ist die Annahme einer ereignisstrukturellen Reparatur.

\subsection{Ereignisstrukturelle Reparatur}

Im Zuge einer pragmatischen (d.h. kontextinduzierten) Uminterpretation kann die lexikalisch festgelegte Ereignisstruktur eines Verbs im Kontext von Zeitangaben oder anderen ereignisstruktursensitiven Ausdrücken, die mit der ereignisstrukturellen Bedeutung des Verblexems inkompatibel sind, verschoben werden (vgl. Pustejovsky/Bouillon 1996). Vgl. (13):

Das Mädchen ist drei Stunden lang gesprungen.

Im Kontext der Durativangabe drei Stunden lang kann das Prädikat ist gesprungen nicht als singulärer Zustandswechsel, d.h. telisch, interpretiert werden. Ein zuverlässiges Indiz für die lexikalisch festgelegte telische Ereignisstruktur ist das Auxiliar sein im Perfekt. Die semantische Anomalie in (13) wird aufgelöst, indem die lexikalische Verbbedeutung so uminterpretiert wird, dass sie zur Durativangabe passt. Die bevorzugte Reparatur ist eine repetitive Lesart des Prädikats. Wie bereits erwähnt, sind repetitive Lesarten telischer Verben atelisch. Mit einer atelischen Lesart ist eine Durativangabe verträglich.

Psycholinguistische Studien belegen die Reparaturhypothese (z.B. Todorova et al. 2000; Pylkkänen/McElree 2006). So wurden erhöhte Reaktionszeiten in Experimenten registriert, in denen Sätze mit einer Inkompatibilität zwischen punktueller, telischer Verbbedeutung und Durativangabe hinsichtlich ihrer Sinnhaftigkeit bewertet wurden. Als Kontrollfälle fungierten Sätze mit einer ereignisstrukturell passenden Zeitangabe wie etwa ist gestern gesprungen, in denen keine Reparatur nötig war. Der registrierte höhere Verarbeitungsaufwand für telische Verblexeme mit Durativangabe gegenüber den Kontrollsätzen mit passender Zeitangabe wäre bei der Annahme, dass unakkusative Verben wie in (13) zwei Lesarten, eine telische und eine atelische, haben, nicht zu erwarten. ${ }^{5}$

Die Reparaturhypothese lässt sich auch in Akzeptabilitätsexperimenten belegen. An dem hier beschriebenen Experiment beteiligten sich $82 \mathrm{Stu}-$ denten der Universität zu Köln. Die Versuchspersonen wurden gebeten, schriftlich präsentierte Sätze hinsichtlich ihrer Akzeptabilität zu bewerten.

Man könnte versuchen, die psycholinguistischen experimentellen Befunde und die weiter unten präsentierten Ergebnisse der Akzeptabilitätstests mit der Ambiguitätshypothese in Einklang zu bringen, indem man eine Lesartenasymmetrie annimmt, derzufolge die atelische repetitive Lesart gegenüber der telischen nicht-repetitiven Lesart dispräferiert ist. Todorova et al. (2000) weisen allerdings nach, dass eine atelische repetitive Lesart nicht von vornherein dispräferiert ist (vgl. auch Pylkkänen/McElree 2006). 
Dieser Test unterscheidet sich von anderen Experimenten (z.B. Keller/Sorace 2003) unter anderem dadurch, dass die passivierten Testsätze in einem natürlichen Kontext erscheinen, um die Lesart des Passivs besser zu kontrollieren. Kontext- und Testsätze sind normierte Varianten vorgefundener Korpusbelege. Da aufgrund der Korpusabfragemöglichkeiten alle Korpusbelege nur benachbarte Partizip- und Auxiliarvorkommen aufweisen, wurden nur solche Konstruktionen getestet. Alle Testitems weisen eine markierte Voranstellung des Partizips auf, was möglicherweise die absoluten, nicht jedoch die relativen Akzeptabilitätswerte beeinflusst. (14) illustriert ein Testitem:

(14) Hauptunfallort der Pausenunfälle ist der Schulhof, auf dem man miteinander raufen und um die Wette laufen kann. Hingefallen wird dann auch dementsprechend oft.

Vorgegeben wurde eine fünfgliedrige Akzeptabilitätsskala von A = ,sprachlich völlig normal und sehr gut verständlich“ bis $\mathrm{E}=$,sprachlich völlig missglückt und unverständlich“. Dem Wert A entspricht bei der statistischen Auswertung der Zahlenwert 5, dem Wert B der Zahlenwert 4 usf. Um einen eventuell auftretenden Lern- oder Gewöhnungseffekt zu kontrollieren, wurden die Testitems der Hälfte der Probanden in der umgekehrten Abfolge präsentiert, so dass sich zwei spiegelbildliche Abfolgeversionen ergaben.

Der Test inkludiert unpersönliche Passive a) mit nicht-volitionalen telischen Verben (z.B. ausrutschen, einschlafen, hinfallen, sterben und wachsen), b) mit nicht-volitionalen atelischen Verben (z.B. bibbern, gäbnen, niesen, schnarchen und träumen), c) mit volitionalen atelischen Verben (z.B. lachen, rauchen, schunkeln und tanzen) sowie d) Fillersätze mit nicht-passivierten Verben. Die durchschnittlichen Akzeptabilitätswerte sind in Abbildung 1 zusammengefasst.

Wenn man die Fillersätze mit nicht-passivierten Verben außen vor lässt, so kann man festhalten, dass die Akzeptabilität in Abhängigkeit vom Verbtyp sinkt. Unpersönliche Passive mit volitionalen atelischen Verben erreichen mit 3,91 den höchsten Mittelwert, gefolgt von den Passivsätzen mit nicht-volitionalen atelischen Verben (3,70). Den niedrigsten Mittelwert erreichen die Passivsätze mit nicht-volitionalen telischen Verben $(3,33)$. Alle Verbtypen erreichen trotz markierter Wortstellung (Partizipvoranstellung) und markiertem Genus verbi (Passiv statt Aktiv) einen hohen Akzeptabilitätsmittelwert relativ zu den Füllsätzen im Aktiv mit unmarkierter Wortstellung (4.65). Alle Testitems, auch diejenigen mit nicht-volitionalen telischen Verben (Unakkusativa), wurden somit als akzeptabel gewertet, wenn man von der plausiblen Annahme ausgeht, dass ein Wert von 3,33 und höher auf einer Skala zwischen 1 (sprachlich völlig missglückt und unverständlich) und 5 (sprachlich völlig normal und sehr gut verständlich) im Bereich des Akzeptablen liegt. 


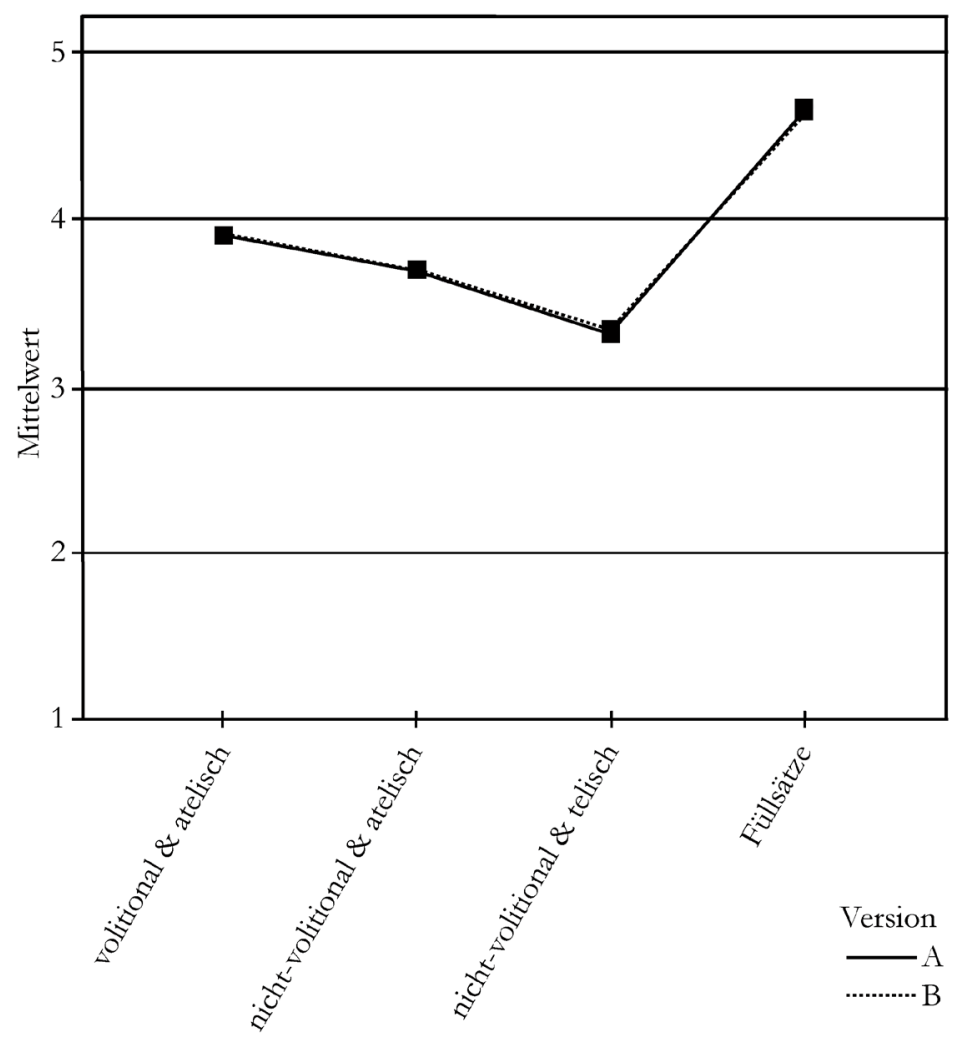

Abb. 1: Akzeptabilitätsmittelwerte der Kölner Studie

Um zu überprüfen, ob sich die oben genannten vier Satz- bzw. Verbtypen statistisch signifikant unterscheiden, wurden eine mehrfaktorielle Varianzanalyse und t-Tests durchgeführt. ${ }^{6} \mathrm{Da}$ die Reihenfolge der Testitems variiert, wurde die Abfolgeversion, A oder B in Abbildung 1, als Zwischensubjektfaktor in die Analyse aufgenommen, so dass sich eine $2 \times 4$-faktorielle Varianzanalyse (Abfolgeversion $\times$ Satztyp) ergibt. Es zeigt sich ein signifikanter Haupteffekt für den Satztyp $(\mathrm{F}(3,240)=122,88$; $\mathrm{p}<, 001$; partielles $\left.\eta^{2}=, 606\right)$, d.h. die Satz- bzw. Verbtypen unterscheiden sich stark voneinander. T-Tests zeigen, dass sich alle Satztypen paarweise hoch signifikant voneinander unterscheiden: volitional atelisch zu nicht-volitional atelisch $\mathrm{p} \leq, 003$; nicht-volitional atelisch zu nicht-volitional telisch $\mathrm{p} \leq, 000$; volitional atelisch zu nicht-volitional telisch $\mathrm{p} \leq, 000$.

6 Die statistische Analyse hat Markus Linnemann (Universität zu Köln) durchgeführt. Ihm sei an dieser Stelle gedankt. 
Wie bereits erwähnt, wurde ein eventuell auftretender Lern- oder Gewöhnungseffekt dadurch kontrolliert, dass die Testitems der Hälfte der Probanden in der umgekehrten Abfolge präsentiert wurden, so dass sich zwei spiegelbildliche Abfolgeversionen ergaben (A und B in Abbildung 1). Der Haupteffekt „Abfolgeversion“ erwies sich als nicht signifikant. Dies bedeutet, dass die verschiedenen spiegelbildlichen Abfolgen der Testitems keine Wirkung auf die Gesamtmittelwerte haben $(\mathrm{F}(1,80)=0,0 ; \mathrm{p}>$,995; partielles $\left.\eta^{2}=, 000\right)$. Zudem haben die verschiedenen Abfolgeversionen keinen unterschiedlichen Einfluss auf die vier Satztypen. Dies zeigt der nichtsignifikante Interaktionseffekt Satztyp $\times$ Abfolgeversion $(F(3,240)=0,1$; $\mathrm{p}>$,956; partielles $\left.\eta^{2}=, 001\right)$.

Ich fasse zusammen: Es gibt einen deutlichen Akzeptabilitätsabfall von nicht-volitionalen atelischen Verben zu nicht-volitionalen telischen Verben im unpersönlichen Passiv. Die Erklärung dafür ist, dass telische intransitive Verben (Unakkusativa) nur im Zuge einer pragmatischen Koerzion passiviert werden können. Lexikonbasierte Ansätze sind nicht zur Annahme einer lexikalischen Ambiguität für Unakkusativa gezwungen. Ereignisstrukturelle Koerzion ist die empirisch und theoretisch attraktivere Lösung. Damit entfällt ein wichtiges Argument gegen lexikonbasierte und für konstruktionsgrammatische Ansätze. Allerdings ist die Koerzionshypothese mit Goldbergs Annahme kompatibel, dass Atelizität eine unvorhersagbare Eigenschaft der unpersönlichen Passivkonstruktion darstellt. Diese Annahme wird im nächsten Abschnitt in Frage gestellt.

\subsection{Eine beschränkungsbasierte Alternative: Atelizität als Folge referenzieller Argumentdemotion}

Prima facie spricht der Unterschied zwischen unpersönlichem und persönlichem Passiv dafür, dass Atelizität ein nicht vorhersagbarer Bedeutungsaspekt der unpersönlichen Konstruktion ist. Ein persönliches Passiv unterliegt keiner Telizitätsbeschränkung, wie in (15) anhand des Zeitangabentests in der Lesart als singuläres Ereignis gezeigt wird:

a. Der Kuchen wurde in einer Stunde gegessen.

b. \#Der Kuchen wurde zwei Stunden lang gegessen.

Charakteristisch für das persönliche Passiv ist die Präsenz eines Subjektarguments, das meist definit ist, wie in (15). Wie ich im Folgenden zeigen werde, ist die Präsenz und der referenzielle Status dieses Arguments für den ereignisstrukturellen Unterschied zwischen persönlichen und unpersönlichen Passivsätzen verantwortlich.

Meine Argumentation basiert auf der unkontroversen konstruktionsgebundenen Beschränkung in (16), der semantischen konstruktionsspezifischen Beschränkung in (17) und der konstruktionsunabhängigen Beschränkung in (18) (vgl. auch Primus 2010a, b): 
(16) In der Passivvariante eines Verblexems wird die erste semantische Rolle $^{7}$ syntaktisch nicht als Subjektargument realisiert.

(17) Das syntaktisch unterdrückte erste Argument ist semantisch nichtgequantelt. D.h., es hat keine referenzbezogene funktionale Struktur.

(18) Ein nicht-gequanteltes ereignisstruktursensitives Argument kann keine telische Ereignisstruktur etablieren.

Ich nenne Verbargumente, die am Phasenwechsel beteiligt sind, „ereignisstruktursensitive Argumente“. Bevor ich mich dem Passiv widme, werde ich den Begriff der Quantelung ${ }^{8}$ und den Zusammenhang zwischen der Telizität verbaler Ausdrücke und dem referenziellen Status bestimmter Argumente anhand anderer Konstruktionen näher erläutern.

$\mathrm{Zu}$ den nicht-gequantelten nominalen Ausdrücken gehören artikellose Nominalphrasen im Plural wie Bücher und Stoff- oder Massennomen wie Apfelmus, die traditionellerweise als nicht-zählbar eingestuft werden. Gequantelte nominale Ausdrücke wie ein Buch oder das Buch denotieren hingegen spatio-temporal begrenzte und mithin zählbare Entitäten. Diesen Unterschied in der Referenzweise nominaler Ausdrücke kann man mithilfe der Kriterien der Divisivität und Kumulativität präzisieren. Nicht-gequantelte nominale Ausdrücke sind kumulativ und divisiv. Wenn man Objekte, die unter Bücher fallen, mit Objekten, die unter Bücher fallen, summiert, ergibt die Summe wieder Bücher (Kumulativität). Wenn Objekte unter Bücher fallen, so kann ein echter Teil dieser Objekte unter Bücher fallen (Divisivität). Gequantelte nominale Ausdrücke wie ein Buch oder das Buch sind nicht kumulativ und nicht divisiv. Wenn ein Objekt unter ein Buch fällt, so kann kein echter Teil davon unter ein Buch fallen (Nicht-Divisivität). Wenn man ein Objekt, das unter ein Buch fällt, mit einem anderen Objekt, das unter ein Buch fällt, summiert, erhält man nicht etwas, was unter ein Buch fällt (Nicht-Kumulativität).

Der Zusammenhang zwischen der Telizität verbaler Ausdrücke und der Referenzweise bestimmter Argumente ist anhand mehrerer Konstruktionen im Aktiv gründlich erforscht. ${ }^{9}$ Die Beispiele in (19) illustrieren diesen Zusammenhang an einem transitiven Satz mit einem inkrementellen Thema:
a. Peter las in fünf Stunden / \#fünf Stunden lang ein Buch.
b. Peter las \# in fünf Stunden / fünf Stunden lang Bücher.

Die erste semantische Rolle erscheint in der Rollenstruktur eines Verbs in der hierarchisch höchsten Position (vgl. u.a. Rapp 1997). Damit ist nicht gemeint, dass sie in eine ,externe Argumentposition in die Syntax projiziert werden muss.

8 Carlson (1998) folgend wird in einigen Arbeiten von Individuierung anstelle von Quantelung gesprochen.

$9 \quad$ Vgl. u.a. Verkuyl (1972), Mittwoch (1982), Krifka (1989), Tenny (1994), Hay/Kennedy/ Levin (1999), Rothstein (2004). 
Die Referenzweise eines inkrementellen Themas korreliert mit der temporalen Referenzweise der Verbalphrase. Wie weiter oben besprochen, können auch Ereignisstrukturen durch die Kriterien der Divisivität und $\mathrm{Ku}-$ mulativität charakterisiert werden. Atelische Verbalphrasen sind divisiv und kumulativ und können somit als nicht-gequantelt angesehen werden. Telische Verbalphrasen sind nicht divisiv und nicht kumulativ und können als gequantelt betrachtet werden. Wenn telische Verbalphrasen als gequantelt, atelische als nicht-gequantelt rekonstruiert werden, sind auch die Unterschiede zwischen Bücher lesen und ein Buch lesen erklärbar (vgl. Krifka 1989). Offenbar überträgt sich hier die Referenzweise des Objekts auf die Referenzweise des Gesamtausdrucks. Diese Übertragung wird dadurch ausgelöst, dass das Objektargument die semantische Rolle eines inkrementellen Themas trägt. Bei einem inkrementellen Thema wird das Argumentdenotat Teil für Teil dem vom Verbalausdruck denotierten Ereignis unterzogen, wie beispielsweise bei einen Kuchen essen, ein Bier trinken, ein Gedicht schreiben. Dabei entsprechen sich jeweils ein Teil des Objekts und ein Teil des Ereignisses.

Überträgt man diese Ergebnisse auf das persönliche Passiv in (15), so lässt sich erklären, warum diese Konstruktion im Gegensatz zum unpersönlichen Passiv ohne weiteres (d.h. ohne Reparatur) telisch interpretiert werden kann. Beim persönlichen Passiv erscheinen inkrementelle Themen typischerweise als gequantelte, meist definite Argumente, wie in (20a) gezeigt. Dadurch kann das Prädikat telisch interpretiert werden, wie die Zeitspannenangabe in (20a) demonstriert. Wenn man hingegen, wie in (20b, c), ein nichtgequanteltes Argument verwendet, kann das Prädikat nur atelisch gelesen werden. Dies illustriert die akzeptable Durativangabe in (20b) und die inakzeptable Zeitspannenangabe in (20c).

(20) a. Der Kuchen wurde in einer Stunde gegessen.

b. Es wurden zwei Stunden lang Kuchen gegessen.

c. "Es wurden in zwei Stunden Kuchen gegessen.

Eine andere Konstruktion, in welcher die nominale Referenzweise die verbale Ereignisstruktur beeinflusst, ist die so genannte, indefinite Objektellipse‘. Das syntaktisch unterdrückte ereignisstruktursensitive Argument verhält sich wie eine nicht-gequantelte Nominalphrase (vgl. Mittwoch 1982). Vgl. (21b) mit (19b) weiter oben:

a. Peter las in fünf Stunden / \#fünf Stunden lang ein Buch.

b. Peter las "in fünf Stunden / fünf Stunden lang.

Relevant für die laufende Argumentation ist die Beobachtung, dass die Argumentunterdrückung in (21b) die telische Lesart des Prädikats blockiert (eine Reparaturmöglichkeit besteht auch hier, vgl. (25) weiter unten).

Einen vergleichbaren Telizitätseffekt beobachtet man auch bei expletiven Subjekten, die semantisch leer sind. Vgl. (22): 
(22) a. Es regnete / raschelte \#in einer Stunde / eine Stunde lang.

b. Es dämmerte / taute ${ }^{*}$ in einer Stunde / eine Stunde lang.

c. Es kühlte in einer Stunde / " eine Stunde lang um zehn Grad ab.

Von besonderem Interesse sind die Prädikate in (22b), die auf Zustandsveränderungen und mithin auf telische Ereignisse in der Welt verweisen. Dennoch können solche Prädikate semantisch nicht telisch interpretiert werden, da sie keinen Begleiter haben, an dem sich die Zustandsveränderung vollzieht. In (22c) liefert die Maßangabe um zehn Grad den Referenzrahmen für die Zustandsveränderung, womit eine zeitliche Begrenzung des Ereignisses erst möglich wird.

Der diskutierte Telizitätseffekt lässt sich auch bei nicht-passivierten Unakkusativa wie in (23) beobachten (vgl. Rothstein 2004, S. 154):

(23) a. Das / Ein Kind ertrank in wenigen Minuten.

b. ${ }^{\#}$ Kinder ertranken in wenigen Minuten.

Von Relevanz ist (23b) in der Lesart, in der eine unspezifizierte Anzahl von Kindern in wenigen Minuten in einem singulären Ereignis ertrank. Diese Lesart ist blockiert, andere Lesarten entstehen über Reparatur (vgl. (26) weiter unten).

Zusammenfassend lässt sich sagen, dass man die beim unpersönlichen Passiv beobachtete Telizitätsbeschränkung auch bei anderen Konstruktionen findet, in denen die Referenzweise bestimmter Verbbegleiter die Ereignisstruktur des Satzes bestimmt. Diesen Zusammenhang findet man bei aktiven und passivierten Sätzen mit inkrementellen Themen, bei ,indefiniter Objektellipse', bei unpersönlichen Wetterkonstruktionen, die einen Zustandswechsel bezeichnen, bei Unakkusativa im Aktiv und - so meine Hypothese - auch bei Unakkusativa im Passiv.

Zaenens Daten und Akzeptabilitätsurteile in (8), die Adele Goldberg als wichtige empirische Evidenz für die Konstruktionsgrammatik anführt, greife ich in (24) wieder auf und leite sie auf der Grundlage der Beschränkungen in (16)-(18) ab.

a. Er werd opgestegen. (lt. Zaenen und Goldberg ungrammatisch) ,Da wird abgeflogen.

b. Vanaf Schiphol wordt er de hele dag opgestegen. "Von Schiphol wird den ganzen Tag abgeflogen.

In beiden Sätzen führt die referenzielle Argumentdemotion dazu, dass die Verbalphrase mit dem inhärent telischen Verblexem abfliegen nicht telisch interpretiert werden kann (vgl. (18)). Dies liegt daran, dass der Zustandswechsel, der von Abfliegen bzw. Starten impliziert wird, einen Referenzrahmen braucht, den ein gequanteltes Verbargument bereitstellt. So bemisst sich die allmählich größer werdende Distanz von der Erdoberfläche, die ein 
Abfliegen impliziert, am Argumentdenotat, aber nur falls das Denotat gequantelt und mithin individuiert ist. An einer unspezifizierten Masse von Flugzeugen, also in der nicht-gequantelten Interpretation, können keine Ereignisphasen abgelesen werden. Dies bedeutet, dass aufgrund der referenziellen Argumentdemotion keiner der Sätze in (24) ein singuläres telisches Ereignis bezeichnen kann. Allerdings sind beide Sätze in einer atelischen repetitiven Lesart grammatisch, wie die Akzeptabilitätsbefragung von Muttersprachlern des Niederländischen sowie Korpusbelege demonstrieren (vgl. Primus 2010a, b). Dies liegt daran, dass eine pragmatische Reparatur in solchen Fällen immer möglich ist. Allerdings ist dieser Weg ohne reparaturauslösende Durativangabe, wie in (24a), erschwert. Dies erklärt den von Zaenen und Goldberg angenommenen Akzeptabilitätsunterschied zum Satz (24b) mit Durativangabe. Wie erwähnt ist dieser Unterschied aufgrund meiner Befragungen nicht so ausgeprägt, wie Zaenen und Goldberg annehmen.

Die repetitive oder habituelle und mithin atelische Lesart passivierter Unakkusativa ist, wie weiter oben in Abschnitt 2.4 näher erläutert, eine ereignisstrukturelle Reparatur. Es gibt jedoch auch einen selteneren Reparaturweg, bei dem das unterdrückte Argument in bestimmten Kontexten gequantelt (z.B. definit) interpretiert wird. In solchen Fällen kommt die Telizität des Verblexems zum Tragen und der gesamte Satz kann telisch interpretiert werden. Vgl. (25)-(27):

(25) John usually spends a lot of time for lunch, but today he ate in half an bour.

(Rothstein 2004, S. 157)

(26) Erwachsene konnten sich retten. Kinder ertranken in wenigen Minuten.

(27) In fünf Minuten wird ins Bett gegangen!

In fünf Minuten wird aufgestanden!

In (25) wird die im zweiten Teilsatz auftretende Objektellipse kontextuell als , his lunch today', also definit, interpretiert. In (26) wird eine gequantelte Menge von Kindern impliziert: Jedes der in der Situation gegebenen Kinder ertrank in wenigen Minuten (distributive Lesart) oder die in der Situation gegebene Gruppe der Kinder ertrank in wenigen Minuten (kollektive Lesart). In (27) handelt es sich um eine Aufforderungslesart des unpersönlichen Passivs. In einer solchen Lesart bezieht sich das unterdrückte Argument auf den kontextuell individuierten Adressaten.

Eine referenzielle Argumentdemotion findet im Passiv auch bei Unergativa wie in (28) statt.

(28) Hier wurde aber schnell gearbeitet!

Ereignis-Individuierung bzw. -quantelung hängt in allen Verbkonstruktionen in entscheidendem Maße von Argument-Quantelung ab (vgl. Carlson 
1998; Kratzer 2008). Prädikate ohne gequanteltes Argument, wie in (28), sind typischerweise nicht-gequantelt. In der kontextuell unmarkierten Lesart bezieht sich (28) daher auf eine nicht-gequantelte ,Masse“ von Arbeitenden und mithin auf eine nicht-gequantelte ,Masse ${ }^{6}$ von Arbeitsereignissen. Eine ereignisstrukturelle Reparatur ist nicht nötig, da arbeiten von vornherein atelisch ist. Die Absenz der ereignisstrukturellen Reparatur und die Agentivität solcher Verben erklären, warum ihre Passivierung relativ zur Passivierung von Unakkusativa viel häufiger vorkommt und in Akzeptabilitätstests besser abschneidet (vgl. Abschnitt 2.4 weiter oben und Abschnitt 3.3 weiter unten). Es gibt allerdings für (28) den selteneren Reparaturweg, der zur Argumentindividuierung führt. Meint man mit (28) eine bestimmte Person, so kann man damit ein gequanteltes (beispielsweise singuläres) Ereignis beschreiben, um besondere Zusatzeffekte wie Tadel oder Ironie zu erzielen.

Ich fasse die Ergebnisse dieses Abschnitts zusammen. Beim Passiv unakkusativer Verben muss weder lexikalische Ambiguität noch Konstruktionsbedeutung postuliert werden. Die bevorzugt atelische Interpretation solcher Passivkonstruktionen geht auf eine konstruktionsunabhängige Telizitätsbeschränkung zurück, die einen gequantelten Ausdruck in einer ereignisstruktursensitiven Funktion fordert. Diese Beschränkung ist durch das allgemeinere Kompositionalitätsprinzip motiviert, demzufolge die temporale Referenzweise eines Ereignisses durch die Referenzweise des Verblexems und die Referenzweise bestimmter ereignisstruktursensitiver Argumente und Angaben bestimmt wird. Das unterdrückte Argument beim unpersönlichen Passiv ist semantisch nicht-gequantelt. D.h.: Semantisch findet beim unpersönlichen Passiv eine referenzielle Agensdemotion statt. Durch diese Interpretation des unterdrückten Arguments kann keine telische Lesart entstehen. Es kann allerdings eine pragmatische Reparatur stattfinden, die das Ereignis pluralisiert und auf diesem Weg eine atelische Lesart erzeugt. Experimentelle Daten belegen die Plausibilität dieser Annahme. Ein seltenerer Reparaturweg ist die pragmatische Individuierung des unterdrückten Arguments, bei dem die Telizität des Verblexems erhalten bleibt.

\section{Belebtheit und Agentivität beim unpersönlichen Passiv}

\subsection{Die konstruktionsgrammatische Sicht}

Die zweite semantische Restriktion beim unpersönlichen Passiv betrifft die Belebtheit und Agentivität des unterdrückten Arguments. Die Duden-Grammatik (2005, S. 554) beschreibt sie in Einklang mit der Mehrheitsmeinung wie folgt: 
Das Verb muss im Aktivsatz eine Handlung oder Aktivität bezeichnen, der Subjektaktant also ein „echtes“ Agens sein. In dem Passivsatz wird man dementsprechend, auch wenn keine Agensphrase vorhanden ist, ein belebtes Agens hineininterpretieren: Da wurde laut gequietscht kann nicht bedeuten ,da quietschte die Tür laut ${ }^{6} .^{10}$

Diese Charakterisierung kann bereits als Argument für eine konstruktionsbasierte Herangehensweise gewertet werden. Bei Verben wie quietschen, die bezüglich der Belebtheit bzw. Agentivität des Argumentreferenten ambig oder unterspezifiziert sind, wird im Passiv ein belebtes Agens hineininterpretiert. Die Interpretation als belebtes Agens wird nicht vom Verblexem, sondern von der Konstruktion beigesteuert. Vgl. auch (29):

(29) Hier wird geraucht.

Auch in (29) wird ein belebtes Agens hineininterpretiert, womit sich dieser Satz auf rauchende Personen, aber nicht auf rauchende Schlote oder Öfen beziehen kann.

Wie die Duden-Grammatik (2005, S. 554) gleich im Anschluss festhält, hat diese Beschränkung , keine absolute Gültigkeit. Unter geeigneten Umständen sind immer wieder Abweichungen zu registrieren, die sicherlich nicht jeder als unakzeptabel bewerten würde." Solche Abweichungen liefern für Dowty ein weiteres Argument für die Konstruktionsgrammatik (1991, S. 609):

Certain grammatical constructions have certain meanings associated with them [...] [T] he class of predicates permitted to appear in constructions specific for one class of verbs can be extended beyond the normal class in certain contexts, for some fanciful, metaphorical, humorous, or otherwise nonliteral effect [...] the Dutch impersonal passive construction is just such a case [...] German impersonal passives also have a volitional implicature and permit extended use with nonagentive verbs for humorous effect.

\subsection{Belebtheit und Agentivität unter der Lupe}

Folgende Korpusbelege zeigen unpersönliche Passive, deren unterdrücktes Argument nicht als Agens im strikten, d.h. Volitionalität bzw. Intentionalität implizierenden, Sinne interpretiert werden kann. Die letzten beiden Belege, (33) und (34), beziehen sich auf unbelebte Entitäten.

(30) Oft bleiben da Touristen auch länger und verheizen das ganze Holz, sodass im Ernstfall dann gefroren wird.

[www.outdoorseiten.net/forum/archive/index.php/t-21406.html, Stand: 13.06.2007]

10 Vgl. für das Deutsche auch Kaufmann (1995), Rapp (1997), Zifonun et al. (1997), Keller/ Sorace (2003); sprachvergleichend Frajzyngier (1982), Siewierska (1984), Shibatani (1998). $\mathrm{Da}$ die meisten Unakkusativa ein nicht-agentivisches Subjekt selegieren, wird diese Beschränkung oft im Zusammenhang mit der Nicht-Passivierbarkeit von Unakkusativa behandelt. 
(31) Besondere Sensibelchen aus der Duftabteilung rümpfen immer die Nase. Weil im Sommer so viel gestunken wird.

[Kleine Zeitung, 22.06.1998, Ressort: Lokal; Sechs „Halbe“ für den Hitzkopf im Schwitzdampf]

(32) Aber geblüht wird nur, wenn die Pflanze auch etwas älter ist. Es dauert einige Jahre, bis die Blüten kommen.

[www.wer-weiss-was.de/theme91/article1254810.html, Stand: 12.01.2008]

(33) Problem: Das Bremsenquietschen setzt erst 1-2 Sekunden nach Stillstand ein, dabei ist es egal, welcher Wert in CV 107 eingestellt wird. [Antwort:] CV 107 hat auf dieses Verhalten leider keinerlei Einfluss. Weder bei CV $107=100$ noch bei CV $107=255$ verändert sich das Verhalten. Gequietscht wird immer erst nach Stillstand. [http://zimoforum.huebsch.at/Lists/Tran\%20Sound/Allitems.aspx, Stand:

25.12.2007, über ein Problem bei einem Geräuschprogramm für Modelleisenbahnen]

(34) Mit einem Schalter am Armaturenbrett kann der Fahrer jederzeit auf Benzinbetrieb umschalten. Wenn der Gasdruck auf einen zu niedrigen Wert sinkt, wird automatisch umgeschaltet.

[http://www.autobild.de/artikel/fiat-panda-panda-2007-_59215.html, Stand: 03.02.2008]

Entgegen der Vorhersage von Dowty (1991) werden die obigen Belege weder metaphorisch noch humoristisch verwendet. Man vergleiche (35a), eine Aktivvariante des Belegs (34), mit der humoristisch deutbaren Personifikation in (35b):
a. Der Schalter schaltet um.
b. Der Schalter grübelte.

Nicht attestiert im Passiv ist das Fallen von Steinen, das Quietschen von Türen, das Funkeln von Sternen, Regnen oder Schneien.

Als Ergebnis halten wir fest, dass es offenbar eine Beschränkung gibt, die von der bisherigen Forschung zu strikt formuliert wurde. Die von mir aufgestellte Beschränkung für das Deutsche ist (36) (vgl. Primus 2010a):

(36) Das unterdrückte Argument im unpersönlichen Passiv muss mindestens eines der folgenden Agentivitätsmerkmale aufweisen: ein Kontrollmerkmal, Sentience oder selbstinduzierte Bewegung, wie weiter unten spezifiziert.

Diese Beschränkung setzt einen mehrdimensionalen Agensbegriff voraus, wie er u.a. von Lakoff (1977) und Dowty (1991) eingeführt wurde. Er umfasst nach Auffassung Dowtys Volitionalität, Verursachung, Sentience (d.h. Wahrnehmung, Empfindung, Emotion oder andere mentale Zustände) und 
Bewegung. Da Verursachung in Dowtys strikter Auffassung nur für Prädikate mit zwei Argumenten anwendbar ist, lasse ich diese Dimension hier außen vor.

Kontrolle (alternativ auch Volitionalität oder Intentionalität) ist neueren Erkenntnissen folgend ebenfalls mehrdimensional. So umfasst der Intentionalitätsbegriff bei Tomasello et al. (2005) folgende drei Dimensionen:

1) die ereignisspezifische Zielgerichtetheit eines an einem Ereignis beteiligten Systems, wobei auch die Herbeiführung des Ereignisses oder dessen Unterlassung als Ziel gilt;

2) die Wahl der Mittel, dieses Ziel zu erreichen, bzw. ein Handlungsplan;

3) das perzeptuelle Monitoring des Handlungsziels. Damit kontrolliert das System, ob das Handlungsziel erreicht wurde oder nicht. Im ersten Fall wird die Aktivität unterbunden, im letzten Fall fortgeführt.

Der traditionell angenommene Begriff des freien Willens, d.h. u.a. die bewusste Initiierung eines Ereignisses, spielt in der neueren Forschung eine untergeordnete Rolle. Kennzeichnend für menschliche Handlungen ist jedoch ihre bewusste Unterdrückbarkeit (vgl. Libet 2004).

Die Handlung eines Automaten wie etwa eines Thermostats, Sprinklers oder Schalters, für die der Automat hergestellt wird, erfüllt das Kriterium der Zielgerichtetheit und des perzeptuellen Monitoring. Automaten verfügen über ein rudimentäres ereignisbezogenes Wahrnehmungssystem, d.h. sie reagieren auf Umweltveränderungen, die für das Ereignis relevant sind. Dieses Wahrnehmungssystem löst das Ereignis aus und steuert seine Durchführung. Die Aktivität eines Automaten ist zielgerichtet, da es für die selbsttätige Herbeiführung (mindestens) eines bestimmten Ereignisses oder für dessen Unterlassung hergestellt wird. Während Menschen für eine Vielzahl verschiedener Ereignisse als kontrollierende Agenten fungieren können, kontrollieren Automaten nur diejenigen Aktivitäten selbsttätig, für die sie hergestellt werden. Dies erklärt den Unterschied zwischen (33)-(34) und (35a) einerseits und (35b) andererseits. So wurde das in (33) beschriebene Geräuschprogramm für Bremsenquietschen programmiert (wobei es aufgrund eines Defekts nicht vor dem Bremsen, sondern danach quietscht, was den Anlass für die Internetdiskussion liefert). Der in (34) und (35) thematisierte Schalter ist für den Wechsel der Treibstoffart programmiert und kontrolliert diesen Vorgang nach Maßgabe der oben genannten Kriterien der Zielgerichtetheit und des perzeptuellen Monitorings in (34) und (35a). Der diskutierte Schalter wurde nicht für Grübel-Ereignisse programmiert. Aus diesem Grund ist (35b) im Gegensatz zu (35a) eine Personifikation und eignet sich dafür, einen humoristischen Effekt auszulösen.

Über zielgerichtete biologische Programme für den Ablauf eines Ereignisses verfügen auch einfache Lebewesen, wie etwa blühende Pflanzen. Für manche speziesspezifische Ereignisse - wie etwa das Blühen für blühende 
Pflanzensorten - erfüllen einfache Lebewesen das Kriterium der Zielgerichtetheit, womit ich Passivbelege wie in (32) erkläre. Zielgerichtete biologische Programme laufen auch bei Körperreaktionen wie Sterben, Wachsen, Frieren und Stinken ab, womit Passivbelege wie in (9), (10), (30) und (31) erklärt werden können. An solchen Ereignissen beteiligte Personen sind insoweit agentivischer als einfache Lebewesen, als sie solche Vorgänge wahrnehmen können. Sie können somit Dowtys Agentivitätskriterium „Sentience" erfüllen. ${ }^{11}$ Des Weiteren können Personen im Gegensatz zu einfachen Lebewesen Maßnahmen ergreifen, die zur Veränderung oder Unterdrückung solcher Vorgänge oder deren Folgen führt. Auf diese Weise erklärt sich auch der Unterschied zwischen dem Fallen von Personen, das im Passiv belegt ist, vgl. (11) weiter oben, und dem Fallen von Steinen, das als unpersönliches Passiv nicht belegt ist. In Beleg (11) geht es gerade darum, die Folgen eines Sturzes durch Schutzkleidung zu kontrollieren.

Dowtys Kriterium der Bewegung wende ich strikter an. Um ein agentivisches Merkmal handelt es sich nur, wenn die Bewegung aufgrund einer eigenen Energiequelle selbstinduziert erfolgt (vgl. Gelman et al. 1995; Scholl/ Tremoulet 2000; Tremoulet/Feldman 2000; Opfer 2002). Das Fallen von Steinen, das im unpersönlichen Passiv nicht belegt ist, erfüllt dieses Kriterium nicht.

Als Zwischenfazit lässt sich für das Deutsche festhalten, dass das unterdrückte Argument im unpersönlichen Passiv mindestens ein Agentivitätsmerkmal aufweisen muss. Diese Beschränkung setzt einen mehrdimensionalen Agentivitätsbegriff voraus. Die in der Literatur eigens postulierte Beschränkung auf Personen ergibt sich aus dieser Agentivitätsbeschränkung.

\subsection{Skalenbezogene Beschränkungen und rollensemantische Informativitätsverstärkung}

Man könnte die Ergebnisse des letzten Abschnitts als Argument für die Konstruktionsgrammatik deuten, unter der Voraussetzung, dass das Agentivitätskriterium eine unvorhersagbare Eigenschaft des unpersönlichen Passivs im Deutschen ist. Die Kölner Akzeptabilitätsstudie sowie sprachvergleichende Befunde deuten allerdings darauf hin, dass sich das Deutsche nahtlos in ein typologisches Variationsmuster einordnen lässt, dass ich für die Performanz in (37i) und für die Grammatikvariation in (37ii) zusammenfasse:

(37) i) Das Passiv stark agentivischer Verben ist in der Performanz gegenüber dem Passiv schwächer agentivischer Verben präferiert.

ii) Die Passivierbarkeit schwach agentivischer Verben impliziert die Passivierbarkeit stärker agentivischer Verben. Es gibt Sprachen, in denen nur stark agentivische Verben passivierbar sind.

$11 \quad$ Neurowissenschaftlich gut untersucht ist inzwischen die menschliche Fähigkeit, Empfindungen und Emotionen zu kontrollieren (vgl. Ochsner/Gross 2005; Gross (Hg.) 2007; Mauss et. al. 2007; Goldin et al. 2008). 
(37i) manifestiert sich in der weiter oben erläuterten Kölner Akzeptabilitätsstudie (vgl. Abb. 1). Diese Studie enthält auch den Faktor der Agentivität als Variable. Zwei Gruppen von Testitems mit atelischen Verben variierten nur bezüglich Kontrolle. Passivierten Verben, deren unterdrücktes Argument alle oder die meisten Kontrollkriterien erfüllen (z.B. lachen, rauchen, schunkeln, tanzen) wurden passivierte schwach agentivische Verben gegenübergestellt (z.B. bibbern, gähnen, schnarchen, träumen). (38a) und (38b) illustrieren jeweils ein Testitem aus diesen Verbklassen:

(38) a. Einem internationalen Wissenschaftlerteam ist es gelungen, überraschende Erkenntnisse aus der Frühgeschichte der Kunst zu rekonstruieren: Getanæt wird seit über zehntausend Jahren.

b. Im Schnitt, so berichtet die Apotheken-Umschau, hat ein 70-Jähriger in seinem Leben rund 600.000 Stunden mit Schlafen verbracht: Geträumt wird dabei reichlich.

Testitems wie (38a) mit stark agentivischen (,volitionalen') Verben erreichten eine durchschnittliche Akzeptabilität von 3,91. Testitems wie (38b) mit schwach agentivischen (,nicht-volitionalen') Verben fallen mit einem Mittelwert von 3,71 deutlich ab. Alle getesteten Unakkusativa sind schwach agentivisch, so dass sich der Telizitäts- und Agentivitätsfaktor kumulieren (vgl. ein Testitem mit binfallen in (14) weiter oben). Dies erklärt, warum sie den niedrigsten Mittelwert haben (3,33). Diese Mittelwert-Unterschiede sind statistisch signifikant (vgl. Abschnitt 2.4 weiter oben). Somit ist eine Präferenz für die Passivierung stark agentivischer Intransitiva gegenüber schwach agentivischer Intransitiva festzustellen.

Die Präferenz (37i) spiegelt sich im Sprachenvergleich auf der Ebene der Grammatik wider, wie in (37ii) festgehalten (vgl. Primus 2010a). Die Passivierung stark agentivischer Intransitiva ist in allen Sprachen grammatisch, die über ein unpersönliches Passiv verfügen. Es gibt jedoch Sprachen wie das Isländische, in denen nur stark agentivische Intransitiva passivierbar sind. Am anderen Ende des Spektrums gibt es Sprachen wie das Litauische, in denen so gut wie alle Intransitiva, also auch die litauischen Entsprechungen von regnen und schneien, passivierbar sind. Das Deutsche und das Niederländische, in denen stark und schwach agentivische Verben, nicht jedoch nicht-agentivische Verben wie regnen oder schneien, passiviert werden können, nehmen eine mittlere Position ein. Die Passivierbarkeit schwach agentivischer Verben impliziert in den untersuchten Sprachen die Passivierbarkeit stärker agentivischer Verben.

Die passivbezogenen Generalisierungen in (37ii) sind Manifestationen eines umfassenderen skalenbezogenen Beschränkungsschemas. In vielen Bereichen sind sprachliche Objekte (z.B. die Elemente oder Subkategorien einer Kategorie) skalar angeordnet. So gibt es eine Sonoritätsskala in der Phonologie (vgl. Prince/Smolensky 2004), eine Kasushierarchie in der Mor- 
phologie und Syntax (vgl. Woolford 2001), eine nominale Individuierungsskala (vgl. Aissen 2003), eine Telizitätsskala (vgl. Keller/Sorace 2003; Legendre 2007) und die hier diskutierte Agentivitätsskala. Solche Skalen sind unterschiedlich motiviert. Der Sonoritätsskala in der Phonologie entspricht ein graduelles Konzept der Schallfülle, die Kasushierarchie korrespondiert mit einem kasusbezogenen Markiertheitsgefälle, die hier diskutierte Agentivitätsskala basiert auf der semantischen Implikation, d.h. auf logischer Stärke. Stärkere Agentivität entsteht durch eine Konjunktion von Agensmerkmalen, beispielsweise Kontrolle, Sentience und selbstinduzierte Bewegung. Wenn alle drei Merkmale zutreffen, so treffen auch alle Kombinationen zweier dieser Merkmale und jedes einzelne dieser Merkmale zu. Linguistischen Skalen entsprechen implikationale Beschränkungen, die ich in (37ii) für das Passiv relativ zur Agentivitätsskala formuliert habe. Für die anderen genannten Skalen wurden implikationale Generalisierungen in der jeweils angegebenen Literatur formuliert. ${ }^{12}$

Die Tatsache, dass die Passivierbarkeit mit der Agentivitätsstärke zunimmt und mit schwächeren Agensausprägungen abnimmt, ist funktional betrachtet Grammatikalisierung im Sinne einer semantischen Verblassung. Der Beginn eines solchen Grammatikalisierungspfades ist die in der Performanz und im Sprachvergleich beobachtete Präferenz für eine stark agentivische Interpretation des unpersönlichen Passivs. Diese Interpretation ergibt sich durch ein abduktives Schlussverfahren als generalisierte pragmatische Implikatur auf der Grundlage des Prinzips der Informativitätsverstärkung (vgl. Levinson 2000). Aufgrund dieses Prinzips nimmt der Adressat die logisch stärkste Lesart eines Diskursbeitrags an, die mit den Hintergrundannahmen dieses Diskurses kompatibel ist. Diese Tendenz ist konstruktionsunabhängig. Für das unpersönliche Passiv wie für beliebige Aktivkonstruktionen impliziert die zur Diskussion stehende logisch stärkste Lesart alle Agensdimensionen. Da Menschen die einzige ontologische Kategorie sind, die alle Agensdimensionen für eine Vielzahl von Verbdenotaten erfüllen können, werden menschliche Referenten aufgrund des beschriebenen abduktiven Schlussverfahrens als Indikatoren von maximaler Agentivität aufgefasst, was traditionellerweise mit Volitionalität oder Intentionalität gleichgesetzt wird (vgl. Minkoff 1997; Engelberg 2005; Goldberg 2006, S. 185 f.). ${ }^{13}$ Vgl. die Beispiele von Engelberg (2005, S. 64) in (39):
a. Der Brief half Jamaal.
b. Das Korrekturlesen half Jamaal.
c. Rebecca half Jamaal.

12 Alle in diesem Absatz genannten Arbeiten mit Ausnahme von Keller/Sorace (2003) formulieren sprachenübergreifende implikationale Generalisierungen auf der Grundlage einer optimalitätstheoretischen Beschränkungshierarchie.

13 In den genannten Arbeiten findet man die Präferenz für die Interpretation einer lexikalisch unterspezifizierten Subjektrolle als Agens, nicht jedoch die hier angebotene Erklärung. 
Wie Engelberg (2005) mit Beispielen wie in (39a, b) demonstriert, selegiert das Verb helfen kein volitionales Agens. Menschliche Referenten wie in (39c) werden allerdings im Normalfall als volitional handelnde Helfer interpretiert. Diese Interpretation ist weder Teil der lexikalischen Verbbedeutung noch Teil der Konstruktionsbedeutung, sondern ergibt sich als generalisierte pragmatische Implikatur. Beim unpersönlichen Passiv gibt es ein ähnliches Schlussverfahren. Verben, die für Agentivität unterspezifiziert sind, wie beispielsweise quietschen und rauchen, werden im unpersönlichen Passiv abduktiv als maximal agentivisch und somit mit einem menschlichen Referenten in der Rolle des unterdrückten Arguments interpretiert (vgl. (29) weiter oben). Dies ist die in den meisten Kontexten passende und mithin präferierte Interpretation. Wie der Beleg (33) für Bremsenquietschen demonstriert, ist sie jedoch nicht die einzige mögliche Deutung.

Aufgrund solcher Überlegungen lässt sich Präferenz für stark agentivische Lesarten des unpersönlichen Passivs als pragmatische Verstärkung erklären. Unter der Annahme, dass grammatische Beschränkungen mit performanzbedingten Präferenzen korrelieren (vgl. Levinson 2000; Bresnan et al. 2001; Hawkins 2004), handelt es sich bei (37ii) um das sprachvergleichende Grammatikalisierungsmuster der Präferenz (37i).

\section{Zusammenfassende Betrachtungen}

Bei der zusammenfassenden Betrachtung der Ergebnisse beginne ich mit der Telizitätsbedingung. Eine erste Gruppe von lexikonbasierten Ansätzen geht davon aus, dass nur atelische Verblexeme im unpersönlichen Passiv akzeptabel sind. Entgegen dieser Annahme sind telische intransitive (unakkusative) Verblexeme passivierbar, was ich durch Korpusbelege mit einer atelischen, repetitiven Lesart belegt habe (z.B. Gefallen wird nämlich immer nach vorn). Lexikonbasierte Ansätze, die eine lexikalische Ambiguität der unakkusativen Verblexeme postulieren, um den Passivgebrauch zu erfassen, stoßen angesichts der Auxiliarselektion dieser Verben (sein statt haben im Perfekt) auf Probleme. Die Auxiliarwahl kann im Gegensatz zum Passiv nicht durch Zeitangaben, die eine atelische repetitive Lesart festlegen, beeinflusst werden. Dies ist die lexikonbasierte Herangehensweise, die Adele Goldberg (1995) aus konstruktionsgrammatischer Sicht kritisiert. Allerdings ist die Annahme einer ereignisstrukturellen Reparatur für lexikonbasierte Ansätze die attraktivere und zugleich empirisch angemessenere Alternative, die Goldberg nicht berücksichtigt. Psycholinguistische Experimente und Akzeptabilitätsstudien deuten darauf hin, dass bei Passivsätzen mit telischen Verben Reparatur vorliegt. Solche Sätze sind zwar grammatisch, aber in der Performanz dispräferiert. Die Reparaturhypothese ist auch dadurch gestützt, dass beim unpersönlichen Passiv zwei Reparaturmöglichkeiten existieren. 
In der bevorzugten, ereignisstrukturellen Reparatur wird eine repetitive, pluralische und mithin atelische Ereignislesart erzeugt. Der seltenere Reparaturweg ist die kontextuelle Individuierung des unterdrückten Arguments, womit das Ereignis als singulär und telisch interpretiert werden kann (z.B. In fünf Minuten wird ins Bett gegangen). Mit der Reparaturhypothese entfällt ein wichtiges Argument gegen lexikonbasierte und für konstruktionsgrammatische Ansätze.

Außerdem habe ich nachgewiesen, dass die beim unpersönlichen Passiv beobachtete Telizitätsbeschränkung auch bei anderen Konstruktionen vorliegt, in denen die Referenzweise bestimmter Verbbegleiter die Ereignisstruktur des Satzes bestimmt. Dieser Zusammenhang besteht bei aktiven und passivierten Sätzen mit inkrementellen Themen, bei, indefiniter Objektellipse $e^{\varsigma}$, bei unpersönlichen Wetterkonstruktionen, die einen $\mathrm{Zu}-$ standswechsel bezeichnen, und bei Unakkusativa im Aktiv und Passiv. Die Telizitätsbeschränkung beim unpersönlichen Passiv lässt sich aus der konstruktionsunabhängigen Generalisierung ableiten, dass ein nicht-gequanteltes ereignisstruktursensitives Argument keine telische Ereignisstruktur etablieren kann. Die einzige konstruktionsspezifische Annahme ist, dass beim Passiv eine referenzielle Agensdemotion stattfindet. Im Passiv ist das Agens syntaktisch nicht als Subjekt realisiert und darüber hinaus semantisch nichtgequantelt. Somit ist die Telizitätsbeschränkung aus der referenziellen Agensdemotion und der ereignisstrukturellen Komposition ableitbar. Sie ist konstruktionsunabhängig und sprachenübergreifend gültig.

Die zweite semantische Restriktion beim unpersönlichen Passiv ist die Belebtheit und Agentivität des unterdrückten Arguments. Dieses Argument muss laut Mehrheitsmeinung ein „echtes“ belebtes Agens sein, wobei man darunter traditionellerweise einen volitional bzw. intentional Handelnden versteht. Bei Verben wie quietschen und rauchen, die bezüglich der Belebtheit und Agentivität des Argumentreferenten ambig oder unterspezifiziert sind, wird im Passiv ein belebtes Agens hineininterpretiert. Diese Beobachtung sowie abweichende Verwendungen liefern für Dowty (1991) ein Argument für eine konstruktionsgrammatische Behandlung. Abweichende Verwendungen erzeugen seiner Meinung nach bestimmte zusätzliche metaphorische oder humoristische Effekte. Ich konnte auf der Grundlage von Korpusbelegen und einer Akzeptabilitätststudie nachweisen, dass diese Beschränkung - auch aufgrund des traditionellen auf Volitionalität bzw. Intentionalität beschränkten Agensbegriffs - zu strikt formuliert wurde. Im Deutschen muss das unterdrückte Argument im unpersönlichen Passiv mindestens ein Agentivitätsmerkmal aufweisen. Diese Beschränkung setzt einen mehrdimensionalen Agentivitätsbegriff voraus, der mindestens verschiedene Kontrolldimensionen, Sentience und selbstinduzierte Bewegung zusammenfasst. Die in der Literatur eigens postulierte Beschränkung auf Personen ergibt sich aus dieser Agentivitätsbeschränkung. Menschen erfüllen im Ge- 
gensatz zu einfacheren Lebewesen oder unbelebten Entitäten mehrere Agentivitätskriterien für eine Vielzahl von Verbdenotaten. Einfachere Lebewesen und manche unbelebte Entitäten, insbesondere Automaten, können nur für bestimmte Ereignistypen und nur hinsichtlich einiger Kriterien als Agens fungieren. Unter der Voraussetzung, dass sie mindestens ein Agenskriterium erfüllen, kann man auf solche Entitäten im unpersönlichen Passiv Bezug nehmen, ohne damit metaphorische oder humoristische Effekte zu erzeugen. Nicht attestiert sind im Deutschen unpersönliche Passive, bei denen keines der oben genannten Agentivitätskriterien erfüllt ist.

Die Kölner Akzeptabilitätsstudie sowie sprachvergleichende Befunde deuten darauf hin, dass die Agentivitätsbeschränkung im Deutschen nahtlos in ein typologisches Variationsmuster passt, das auf einer Agentivitätsskala und der Präferenz basiert, stark agentivische Verben in der Performanz zugunsten schwächer agentivischer Verben zu passivieren. Diese Präferenz spiegelt sich im typologischen Sprachvergleich als skalenbezogenes implikationales Grammatikalisierungsmuster wider: Die Passivierbarkeit schwach agentivischer Verben impliziert die Passivierbarkeit stärker agentivischer Verben und es gibt Sprachen, in denen nur stark agentivische Verben passivierbar sind. Diese passivbezogenen Generalisierungen sind Manifestationen eines umfassenderen skalenbezogenen Beschränkungsschemas, das in vielen sprachlichen Bereichen Anwendung findet.

Die Tatsache, dass die Passivierbarkeit mit der Agentivitätsstärke zunimmt und mit schwächeren Agensausprägungen abnimmt, ist funktional betrachtet Grammatikalisierung im Sinne einer semantischen Verblassung. Das obere, sprachenübergreifend stabilste Ende dieses Grammatikalisierungspfades ist die in der Performanz und im Sprachvergleich beobachtete Präferenz für eine stark agentivische Interpretation des ersten Arguments. Diese Interpretation ergibt sich durch ein abduktives Schlussverfahren als generalisierte pragmatische Implikatur auf der Grundlage des Prinzips der Informativitätsverstärkung. Aufgrund dieses Prinzips nimmt der Adressat die logisch stärkste Lesart eines Diskursbeitrags an, die mit den Hintergrundannahmen dieses Diskurses kompatibel ist. Diese Tendenz ist konstruktionsunabhängig und sprachenübergreifend. Im vorliegenden Beitrag wurden beide semantischen Bedingungen des unpersönlichen Passivs, die ereignisstrukturelle und die rollensemantische, mit der Agensdemotion in Verbindung gesetzt.

Für eine konstruktionsgrammatische Behandlung des unpersönlichen Passivs ergeben sich daraus folgende Konsequenzen. Es gibt keine zwingenden empirischen Gründe für die Annahme, dass Atelizität und Agentivität unvorhersagbare Aspekte der Konstruktion sind. Lexikonbasierte Ansätze haben keine Probleme damit, unakkusative Verblexeme nach Maßgabe der Auxiliarselektion als eindeutig telisch zu kategorisieren und Reparatur anstelle von Ambiguität anzunehmen. Sie haben auch keine Probleme da- 
mit, die Agentivitätsimplikatur von unterspezifizierten Verben wie quietschen und rauchen abzuleiten. Auch trifft das konstruktionsgrammatische Argument nicht zu, dass bei abweichenden Verwendungen metaphorische oder humoristische Effekte entstehen.

Konstruktionsgrammatische Modelle haben allerdings Schwierigkeiten, die hier aufgezeigten sprachvergleichend operativen Beschränkungen und Präferenzen zu modellieren. Strikt konstruktionistische Modelle, die ohne Beschränkungen und abstrakte Konstruktionen auskommen wollen, führen grammatikexterne Beschränkungen für sprachvergleichend attestierte Muster an (vgl. Croft 2001; Goldberg 2006). Diese beziehen sich vornehmlich auf die kognitiven Bedingungen oder die kommunikative Funktion von Konstruktionen. Die strikte Trennung zwischen grammatikinternen und grammatikexternen Erscheinungen ist eine wichtige Voraussetzung für den Anspruch konstruktionistischer Ansätze, lexikonbasierten Mischmodellen dadurch überlegen zu sein, dass sie konsistent konstruktionsbasiert vorgehen und ohne Beschränkungen auskommen. Den lexikonbasierten Modellen wird vorgeworfen, dass sie für lexikonbezogene Erscheinungen den Konstruktionsbegriff und für grammatikbezogene Erscheinungen Prinzipien oder Beschränkungen in Anspruch nehmen. Es stellt sich allerdings die Frage, ob die strikte Trennung zwischen grammatikinternen und grammatikexternen Erscheinungen haltbar ist. Es gibt immer mehr Linguisten, die davon ausgehen, dass ein Großteil der grammatischen Erscheinungen von Sprachverarbeitungsmechanismen determiniert wird (vgl. u.a. Bresnan et al. 2001; Hawkins 2004; Newmeyer 2005).

Diese Kritik trifft auf konstruktionsgrammatische Mischmodelle, wie etwa das von Fillmore und Kay (Kay/Fillmore 1999), die auch mit Beschränkungen arbeiten, nicht zu. Solche Modelle stellen neben Beschränkungen auch einen Vererbungsmechanismus bereit. Stärker spezifizierte Konstruktionen ,erben' Eigenschaften von weniger stark spezifizierten Konstruktionen, die als sprachenübergreifend stabileres Schema fungieren. Alle Merkmale des Schemas bzw. der übergeordneten Konstruktion müssen ebenfalls in den untergeordneten Instanzen enthalten sein. Höhere Instanzen sind also informations- bzw. merkmalärmer als untere Instanzen. Anders verhält es sich mit dem skalenbasierten Schema beim unpersönlichen Passiv. Das Beschränkungsschema für das unpersönliche Passiv ist funktional betrachtet Grammatikalisierung im Sinne einer semantischen Verblassung entlang einer Agens-Prototypikalitäts- bzw. Stärkeskala. Der sprachvergleichend stabile Bedeutungskern ist die merkmalreichste, d.h. logisch stärkste AgensLesart. Innersprachliche und intersprachliche Variation und nachlassende Grammatikalität gibt es bei merkmalsärmeren Agens-Lesarten. Dieses Muster ist dem Variationsmuster, das durch Vererbung oder Schemabildung vorhergesagt wird, entgegengesetzt. Die hier angesprochenen Effekte der Schemabildung, Vererbung, Prototypikalität und Informativitätsverstärkung sind 
allgemeinere kognitive Organisationsprinzipien, die allerdings sprachliche Manifestationen haben und nicht einfach mit den allgemeineren kognitiven Operationen, die sie motivieren, gleichzusetzen sind. Für die Konstruktionsgrammatik bedeutet dies, dass sie mehrere Typen von Beschränkungen und konstruktionsübergreifenden Beziehungen als bisher angenommen berücksichtigen muss, wenn sie das ganze Spektrum genuin sprachlicher Muster und Generalisierungen erklären möchte.

\section{Literatur}

Abraham, Werner/Leiss, Elisabeth (2006): Personal and impersonal passives: definite vs. indefinite diatheses. In: Transactions of the Philological Society 104, S. 230-259.

Aissen, Judith (2003): Differential object marking: iconicity vs. economy. In: Natural Language and Linguistic Theory 21, S. 435-483.

Bresnan, Joan/Dingare, Shipra/Manning, Christopher D. (2001): Soft constraints mirror hard constraints: voice and person in English and Lummi. Proceedings of the Lexical Functional Grammar Conference 2001. Stanford.

Burzio, Luigi (1986): Italian syntax: a government-binding approach. (= Studies in Natural Language and Linguistic Theory 1). Dordrecht u.a.

Carlson, Gregory N. (1998): Thematic roles and the individuation of events. In: Rothstein, Susan (Hg.): Events and grammar. (= Studies in Linguistics and Philosophy 70). Dordrecht u.a., S. 35-51.

Carnie, Andrew/Harley, Heidi (2005): Existential impersonals. In: Sudia Linguistica 59, S. 46-65.

Croft, William (2001): Radical Construction Grammar: syntactic theory in typological perspective. Oxford u.a.

Dowty, David R. (1979): Word meaning and Montague grammar. The semantics of verbs and times in generative semantics and in Montague's PTQ. (=Synthese Language Library 7). Dordrecht u.a.

Dowty, David R. (1991): Thematic proto-roles and argument selection. In: Language 67, S. 547-619.

Duden-Grammatik (2005): Der Duden. Bd. 4: Die Grammatik: unentbehrlich für richtiges Deutsch. Hrsg. von der Dudenredaktion. 7., völlig neu erarb. u. erw. Aufl. Mannheim u.a.

Engelberg, Stefan (2000): Verben, Ereignisse und das Lexikon. (= Linguistische Arbeiten 414). Tübingen.

Engelberg, Stefan (2005): Stativity, supervenience, and sentential subjects. In: Maienborn, Claudia/Wöllstein, Angelika (Hg.): Event arguments. Foundations and applications. (= Linguistische Arbeiten 501). Tübingen, S. 45-68.

Frajzyngier, Zygmunt (1982): Indefinite agent, passive and impersonal passive. A functional study. In: Lingua 58, S. 267-290. 
Gelman, Rochel/Durgin, Frank/Kaufman, Lisa (1995): Distinguishing between animates and inanimates: not by motion alone. In: Sperber; Dan/Premack, David/Premack, Ann J. (Hg.): Causal cognition. A multidisciplinary debate. Oxford, S. 150-184.

Goldberg, Adele E. (1995): Constructions: a construction grammar approach to argument structure. Chicago u.a.

Goldberg, Adele E. (2006): Constructions at work: the nature of generalization in language. Oxford u.a.

Goldin, Philippe R. et al. (2008): The neural bases of emotion regulation: reappraisal and suppression of negative emotion. In: Biological Psychiatry 63, 6, S. 577-586.

Grewendorf, Günther (1989): Ergativity in German. (=Studies in Generative Grammar 35). Dordrecht u.a.

Gross, James J. (Hg.) (2007): Handbook of emotion regulation. New York u.a.

Hawkins, John A. (2004): Efficiency and complexity in grammars. Oxford.

Hay, Jennifer/Kennedy, Christopher/Levin, Beth (1999): Scalar structure underlies telicity in degree achievements. In: Matthews, Tanya/Strolovitch, Devon (Hg.): Proceedings from semantics and linguistic theory (SALT) IX. Ithaca, S. 127-144.

Kaufmann, Ingrid (1995): Konzeptuelle Grundlagen semantischer Dekompositionsstrukturen: die Kombinatorik lokaler Verben und prädikativer Komplemente. (= Linguistische Arbeiten 335). Tübingen.

Kay, Paul/Fillmore, Charles (1999): Grammatical constructions and linguistic generalizations: the What's X Doing Y? construction. In: Language 75, S. 1-33.

Keller, Frank/Sorace, Antonella (2003): Gradient auxiliary selection and impersonal passivization in German: an experimental investigation. In: Journal of Linguistics 39, S. $57-108$.

Kennedy, Christopher/Levin, Beth (2008): Measure of change: the adjectival core of degree achievements. In: McNally, Louis/Kennedy, Christopher (Hg.): Adjectives and adverbs: syntax, semantics and discourse. (= Oxford Studies in Theoretical Linguistics 20). Oxford, S. 156-182.

Kratzer, Angelika (2008): The plurality of verbs. In: Dölling, Johannes/Heyde-Zybatow, Tatjana/Schäfer, Martin (Hg.): Event structure in linguistic form and interpretation. (= Language, Context, and Cognition 5). Berlin/New York, S. 269-300.

Krifka, Manfred (1989): Nominalreferenz und Zeitkonstitution: Zur Semantik von Massentermen, Pluraltermen und Aktionsarten. (= Studien zur theoretischen Linguistik 10). München.

Lakoff, George (1977): Linguistic gestalts. In: Papers from the $13^{\text {th }}$ Regional Meeting of the Chicago Linguistic Society, S. 236-287.

Legendre, Géraldine (2007): On the typology of auxiliary selection. In: Lingua 117, 9, S. 1522-1540.

Levinson, Steven C. (2000): Presumptive meanings. The theory of generalized conversational implicature. Cambridge, MA u.a. 
Libet, Benjamin (2004): Haben wir einen freien Willen? In: Geyer, Christian (Hg.): Hirnforschung und Willensfreiheit. Zur Deutung der neuesten Experimente. Frankfurt a.M., S. 268-289.

Mauss, Iris B./Bunge, Silvia A./Gross, James J. (2007): Automatic emotion regulation. In: Social and Personality Psychology Compass 1, S. 146-167.

Minkoff, Seth A. (1997): Argument structure and animacy entailment. In: Mendikoetxea, Amaya/Uribe-Etxebarria, Myriam (Hg.): Theoretical issues at the morphologysyntax interface. Supplements of the International Journal of Basque Linguistics and Philology XL, S. 285-324.

Mittwoch, Anita (1982): On the difference between eating and eating something: activities versus accomplishments. In: Linguistic Inquiry 13, S. 113-122.

Newmeyer, Frederick (2005): Possible and probable languages: a generative perspective on linguistic typology. Oxford u.a.

Ochsner, Kevin N./Gross, James J. (2005): The cognitive control of emotion. In: Trends in Cognitive Science 9, S. 242-249.

Opfer, John. E. (2002): Identifying living and sentient kinds from dynamic information: the case of goal-directed versus aimless autonomous movement in conceptual change. In: Cognition 86, S. 97-122.

Özkaragöz, Ýnci (1980): Evidence from Turkish for the unaccusative hypothesis. In: Proceedings of the $6^{\text {th }}$ Annual Meeting of the Berkeley Linguistics Society, S. 411-422.

Perlmutter, David M. (1978): Impersonal passives and the unaccusative hypothesis. In: Proceedings of the $4^{\text {th }}$ Annual Meeting of the Berkley Linguistics Society, S. 157-189.

Perlmutter, David M./Postal, Paul M. (1983): Toward a universal characterization of passivization. In: Perlmutter, David M. (Hg.): Studies in Relational Grammar 1. Chicago u.a., S. 3-29.

Primus, Beatrice (2010a): Animacy and telicity: semantic constraints on impersonal passives. In: Lingua Special Issue 121, 1: Semantic Aspects of Case Variation, S. 80-99. Internet: http://dx.doi.org/10.1016/j.lingua.2010.07.009 (Stand: 14.02.2011).

Primus, Beatrice (2010b): Event-structure and individuation in impersonal passives. In: Brandt, Patrick/García García, Marco (Hg.): Transitivity. Form, meaning, acquisition, and processing. Amsterdam, S. 209-233.

Prince, Alan/Smolensky, Paul (2004): Optimality theory. Constraint interaction in generative grammar. Malden u.a.

Pustejovsky, James/Bouillon, Pierrette (1996): Aspectual coercion and logical polysemy. In: Pustejovsky, James/Boguraev, Branimir (Hg.): Lexical semantics. The problem of polysemy. Oxford, S. 133-162.

Pylkkänen, Liina/McElree, Brian (2006): The syntax-semantics interface: online composition of sentence meaning. In: Traxler, Matthew/Gernsbacher, Morton A. (Hg.): Handbook of psycholinguistics. 2. Aufl. Amsterdam u.a., S. 539-580. 
Rapp, Irene (1997): Partizipien und semantische Struktur. Zur passivischen Konstruktion mit dem 3. Status. (= Studien zur deutschen Grammatik 54). Tübingen.

Rothstein, Susan (2004): Structuring events: a study in the semantics of lexical aspect. (= Explorations in Semantics 2). Malden u.a.

Scholl, Brian J./Tremoulet, Patrice D. (2000): Perceptual causality and animacy. In: Trends in Cognitive Sciences 4, S. 299-309.

Shibatani, Masayoshi (1998): Voice parameters. In: Kulikov, Leonid/Vater, Heinz (Hg.): Typology of verbal categories. Papers presented to Vladimir Nedjalkov on the occasion of his $70^{\text {th }}$ birthday. (= Linguistische Arbeiten 382). Tübingen, S. 117-138.

Siewierska, Anna (1984): The passive. A comparative linguistic analysis. London.

Tenny, Carol (1994): Aspectual roles and the syntax-semantics interface. (= Studies in Linguistics and Philosophy 52). Dordrecht u.a.

Todorova, Marina et al. (2000): Aspectual coercion and the online computation of sentential aspect. In: Proceedings of the $22^{\text {nd }}$ Annual Conference of the Cognitive Science Society, S. 3-8.

Tomasello, Michael et al. (2005): Understanding and sharing intentions: the origins of cultural cognition. In: Behavioral and Brain Sciences 28, S. 675-691.

Tremoulet, Patrice D./Feldman, Jacob (2000): Perception of animacy from the motion of a single object. In: Perception 29, S. 943-951.

van Schaik-Rădulescu, Mara (2009): Unaccusatives in the Turkish impersonal passive: a semantic analysis. Paper presented at the Cologne Linguistic Colloquium, 27th of May 2009.

Vendler, Zeno (1957): Verbs and times. In: The Philosophical Review 66, S. 143-160.

Verkuyl, Henk J. (1972): On the compositional nature of the aspects. (= Foundations of Language: Supplementary Series 15). Dordrecht.

Woolford, Ellen (2001): Case patterns. In: Legendre, Géraldine/Grimshaw, Jane B./ Vikner, Sten (Hg.): Optimality-theoretic syntax. Cambridge, MA u.a., S. 509-543.

Zaenen, Annie (1993): Unaccusativity in Dutch: Integrating syntax and lexical semanguistics and Philosoptics. In: Pustejovsky, James (Hg.) Semantics and the lexicon. (= Studies in Linhy 49). Dordrecht u.a., S. 129-161.

Zifonun, Gisela/Hoffmann, Ludger/Strecker, Bruno (1997): Grammatik der deutschen Sprache. 3 Bde. (= Schriften des Instituts für Deutsche Sprache 7). Berlin/New York. 



\section{Wörter und Phrasen}



Claudia Maienborn

\title{
Strukturausbau am Rande der Wörter: Adverbiale Modifikatoren beim Zustandspassiv ${ }^{1}$
}

\begin{abstract}
Der Beitrag bietet einen Analysevorschlag für ereignisbezogene adverbiale Modifikatoren beim Zustandspassiv, der ihr Auftreten in verbaler Umgebung mit der adjektivischen Natur des Zustandspassivs in Einklang bringt. Grundlage hierfür ist eine empirisch breit abgesicherte Argumentation für das Vorliegen einer besonderen strukturellen Nähe zwischen Modifikator und Partizip, die gleichsam eine kompakte Einheit in der Übergangszone zwischen Wort und Phrase bilden. Diese Sicht macht den Weg frei für eine strikt kompositionale Semantik des Zustandspassivs samt adverbialen Modifikatoren.
\end{abstract}

\section{Das Problem}

Mit dem Tagungsthema „Sprachliches Wissen zwischen Lexikon und Grammatik" leuchtet der vorliegende Band den Unschärfebereich zwischen Wort und Phrase aus. Dieser Beitrag bietet eine Studie zu einem Ausdruckstyp, der bislang noch nicht in diesem Feld verortet wurde, gleichwohl aber - so meine Argumentation - hier anzusiedeln ist. Es handelt sich um die Kombination des Zustandspassivs mit bestimmten adverbialen Modifikatoren vom Typ (1a). Diese werfen Probleme für aktuelle adjektivische Zustandspassivanalysen auf. Eine Würdigung des besonderen strukturellen und interpretativen Status dieses Ausdruckstyps in Abgrenzung von klaren verbalen Konstruktionen wie dem Vorgangspassiv in (1b) und in Relation zu Wortbildungsmustern wie in (1c) eröffnet einen neuen Erklärungsansatz für die problematischen Zustandspassivdaten als Strukturausbau am Rande der Wörter und liefert zugleich eine verbreiterte empirische Basis zur Bestimmung der Strukturbildungsbedingungen und -optionen in der Übergangszone zwischen Lexikon und Grammatik.

(1) a. Das Manuskript ist von Chomsky zitiert.

b. Das Manuskript ist von Chomsky zitiert worden.

c. Das Manuskript ist Chomsky-zitiert.

Für Brigitte! Dieser Beitrag ist im Rahmen des DFG-Projekts A1 „Kombinatorische Bedeutungsvariation an der Semantik/Pragmatik-Schnittstelle“ im Tübinger SFB 833 „Bedeutungskonstitution" entstanden. Ich danke meinen Projektmitarbeiterinnen, Helga Gese und Britta Stolterfoht, sowie Stefan Engelberg, Werner Frey, Torgrim Solstad und dem Auditorium der IDS-Jahrestagung 2010 für anregende Diskussionen und hilfreiche Kommentare. 
Die heutige Sicht auf das so genannte „Zustandspassiv“ ist weitgehend durch die Annahme geprägt, dass es sich hierbei um die Verbindung der Kopula sein mit einem adjektivierten Partizip II (und somit um kein Genus verbi) handle (siehe insbesondere Kratzer 1994, 2000; Rapp 1996, 1997; Maienborn 2007, 2009). Problematisch für diese Adjektiv-Auffassung ist das Auftreten bestimmter adverbialer Modifikatoren, die typischerweise in verbalen Kontexten, nicht aber bei Adjektiven auftreten. So finden sich beim Zustandspassiv insbesondere Agens- (2), Instrumental- (3) und Lokalangaben (4). ${ }^{2}$ Reine Adjektiv-Konstruktionen lassen derartige Erweiterungen typischerweise nicht zu.

(2) a. Die Zeichnung ist von einem Kind angefertigt. Agensangabe b. * Die Zeichnung ist von einem Kind schön.

(3) a. Der Brief war mit roter Tinte geschrieben. Instrumentalangabe b. * Der Brief war mit roter Tinte leserlich.

a. Die Birnen waren in Rotwein gedünstet.

b. * Die Birnen waren in Rotwein weich.

Lokalangabe

Offensichtlich beziehen sich die Modifikatoren der a-Sätze in (2)-(4) auf das vom Basisverb bezeichnete Ereignis. Dieses scheint als Andockstelle für den Modifikator noch zugänglich zu sein. Das Partizip schüttelt also seine verbale Herkunft offenbar nicht vollständig ab.

Allerdings ist in der Literatur auch immer wieder darauf hingewiesen worden (z.B. in Vaagland 1983; Litvinov/Nejalkov 1988; Rapp 1996), dass derartige Kombinationen keinesfalls systematisch verfügbar seien, sondern vielmehr massiven Beschränkungen unterliegen, wie sie im Vergleich dazu etwa beim Vorgangspassiv nicht auftreten; siehe die Beispiele in (5).
a. * Die Tür ist von ihm geöffnet.
(Vaagland 1983, S. 194)
b. * Ihre Haare sind mit einem goldenen Kamm gekämmt.
c. * Sie ist im Nachbarwald umgebracht.
(Rapp 1996, S. 257)

(Litvinov/Nedjalkov 1988, S. 139)

Dies macht eine Erklärung der zulässigen Kombinationen in (1a)-(4a) umso schwieriger. Im Folgenden soll ein Vorschlag im Rahmen der Adjektivierungsanalyse des Zustandspassivs entwickelt werden, der die Zulässigkeit der Konstellation vom Typ (1a) auf die mit der Adjektivierung einhergehenden besonderen Interpretationsbedingungen des Zustandspassivs zurückführt und ihren Platz bestimmt zwischen der unzweifelhaft phrasalen Verbindung von Vorgangspassiv und adverbialem Modifikator in (1b) und dem Wortbildungsmuster in (1c).

Siehe auch die umfangreiche Aufstellung in Litvinov/Nedjalkov (1988, Kap. 5). 
Der folgende Abschnitt 2 bietet eine kurze Rekapitulation der Gründe, die für eine Adjektivierungsanalyse des Zustandspassivs sprechen. Abschnitt 3 geht auf bisherige Vorschläge, adverbiale Modifikatoren in diesem Rahmen zu erfassen, ein. In Abschnitt 4 lege ich meine Annahmen zur Semantik des Zustandspassivs dar. Dies bildet die Grundlage für die Diskussion der strukturellen Integration adverbialer Modifikatoren beim Zustandspassiv und den daraus abzuleitenden Schlussfolgerungen in den Abschnitten 5 und 6.

\section{Zur Adjektivierungsanalyse des Zustandspassivs}

In den zurückliegenden Jahren hat eine intensive Auseinandersetzung mit der Frage nach der grammatischen Einordnung des so genannten Zustandspassivs stattgefunden. Überblicke und Bewertungen der in Betracht gezogenen Optionen finden sich in Rapp (1996) und Maienborn (2007). Ich will die Argumente für und wider verschiedene Analysen hier nicht wiederholen, sondern lediglich mithilfe einiger ausgewählter Diagnostiken die heute in der Forschungsliteratur übliche, wenn auch in den meisten Grammatiken noch nicht reflektierte Sicht ${ }^{3}$ auf das Zustandspassiv als Kombination aus der Kopula sein mit einem adjektivierten Partizip begründen (siehe z.B. auch Wunderlich 1997; von Stechow 1998; Schlücker 2005).

Ein klares Indiz für die Kategorisierung des Partizips als Adjektiv ist die Möglichkeit der un-Affigierung (siehe Lenz 1993). Im Deutschen ist das Negationsaffix un- (von der beschränkten Präfigierung von Nomina abgesehen) nur mit Adjektiven, nicht aber mit Verben kombinierbar. Das heißt, dass die Partizipien in (6) als Basen für die un-Affigierung bereits adjektiviert sein müssen. ${ }^{4}$

(6) a. Oft waren diese Regeln ungeschrieben, aber sie waren trotzdem jedem klar.

(TIGER s11406)

b. Mein Haar ist ungekämmt und mein Hemdkragen ungebügelt. (COSMAS M89/909.31688)

Für die adjektivische Natur des Partizips beim Zustandspassiv spricht auch die Möglichkeit zur Graduierung wie in (7).

Siehe aber die Abwägung der Argumente in Zifonun et al. (1997, S. 1822 f.). Letztlich ist das Auftreten adverbialer Modifikatoren eines der Hauptargumente für Zifonun et al., trotz aller Adjektiv-Evidenz an der traditionellen Analyse des Zustandspassivs als Verbform festzuhalten.

4 Angaben zu elektronischen Korpusbelegen beziehen sich auf das morphosyntaktisch annotierte TIGER-Korpus (www.ims.uni-stuttgart.de/projekte/TIGER) und die COSMAS-Korpora des IDS Mannheim (www.ids-mannheim.de/cosmas2, beide Stand: 06.12.2010). 
(7) a. ... Autor, der viel berühmter und vor allem weitaus gelesener war als Goethe.

(Die Zeit, 29.07.1999)

b. Am bedrohtesten sind die Landtage.

(COSMAS V98/MAI.21411)

Zwar ist Graduierung beim Zustandspassiv nur begrenzt möglich - dies ist einer der Gründe, warum Zifonun et al. (1997, S. 1822f.) letztlich doch an der Auffassung, es handle sich um Verbformen, festhalten -, wegen der verbalen Herkunft der betreffenden Partizipien und der damit einhergehenden Beschränkungen und Besonderheiten gegenüber genuinen Adjektiven ist dies aber auch gar nicht anders zu erwarten, zumal auch unzweifelhafte Adjektive Graduierung nicht immer zulassen (siehe Lübbe 2004). Schon die Existenz von offenbar regulär gebildeten Fällen vom Typ (7) muss als ein gewichtiges Argument für eine dem Zustandspassiv zugrunde liegende Adjektivierung des Partizips gewertet werden.

Ein weiteres Adjektivierungsindiz liefern Koordinationsdaten wie in (8). Koordiniert werden kann nur Gleichartiges (siehe Lang 1984). In Verbindung mit einem genuinen Adjektiv kann die sein-Form nicht zugleich Kopula und Auxiliar sein, sondern ist auf die Kopula-Variante festgelegt.

a. Seine Schuhe waren sauber und poliert.

b. Entlassungen seien weder geplant noch nötig.

(TIGER s3166)

Und schließlich finden sich beim Zustandspassiv typisch adjektivische Wortbildungen vom Typ in (9), die im verbalen Bereich wiederum ausgeschlossen sind. ${ }^{5}$

(9) a. Dieses Geschäft ist videoüberwacht.

b. Alle Mitglieder des rot-roten Senats sind stasi-überprüft.

c. Leider waren unsere Gäste noch etwas kaufblockiert.

(Schwäbisches Tagblatt 15.10.2009)

Die Produktivität dieses Wortbildungsmusters zeigt sich auch an okkasionellen Bildungen wie Chomsky-zitiert sein in (1c) oder schwedentrainiert sein in (10). Ich werde darauf im Zusammenhang mit der Interpretation des Zustandspassivs zurückkommen.

(10) Ich hatte Sorge, wie der Japaner das Oktoberfest finden würde, aber es stellte sich heraus, dass er schwedentrainiert war.

(Hörbeleg 11/2007)

An dieser Stelle sei darauf hingewiesen, dass der attributive Gebrauch von Partizipien kein verlässlicher Indikator für ihren adjektivischen Status ist (siehe hierzu insbesondere Wunderlich 1997). Die Aussagen des vorliegenden Artikels betreffen allein den prädikativen Gebrauch des Partizips II, d.h. das Zustandspassiv. 
Die Liste der Adjektivierungsindizien ließe sich fortsetzen und auch psycholinguistisch untermauern. So zeigen Stolterfoht et al. (2010) im Rahmen eines Lesezeitexperiments, dass beim Partizip in der Zustandspassivkonstellation eine zeitliche Verzögerung gegenüber der Vorgangspassivkonstellation auftritt. Diesen Verarbeitungsmehraufwand werten sie als Evidenz für die mit dem Zustandspassiv verbundene zusätzliche Adjektivierung des verbalen Partizips.

In der Summe stützen die zusammengetragenen Diagnostiken zur kategorialen Bestimmung des Zustandspassivs übereinstimmend die Adjektivierungshypothese, derzufolge sich hinter dem Zustandspassiv die Kombination der Kopula sein mit einem adjektivierten Partizip verbirgt. Vor diesem Hintergrund ist die Redeweise vom „Zustandspassiv" unglücklich gewählt; es handelt sich gerade nicht um eine passive Verbform als sozusagen „kleine Schwester" des Vorgangspassivs. ${ }^{6}$ Da dieser Terminus zur Bezeichnung des in Rede stehenden Phänomens sich inzwischen aber in der deutschsprachigen Literatur etabliert hat, werde ich ihn hier auch weiterhin verwenden.

\section{Lösungsvorschläge für das Auftreten adverbialer Modifikatoren}

Wenn nun aber die hier diskutierten ,sein plus Partizip“-Kombinationen gar keine analytischen Verbformen sind, wie lässt sich dann das Auftreten typischer adverbialer Modifikatoren wie die Agens-, Instrumental- oder Lokalangaben in (2)-(4) erklären?7 Hierzu bietet Kratzer (1994, 2000) einen interessanten Vorschlag an. ${ }^{8}$ Danach gibt es zwei Möglichkeiten, das verbale Partizip in ein Adjektiv zu konvertieren (in (11) umgesetzt über die Annahme eines adjektivischen Nullaffixes). Die Adjektivierung kann entweder auf lexikalischer Ebene stattfinden oder aber auf phrasaler Ebene. Gegenstand der Adjektivierung ist im letzteren Fall nicht allein das Verb, sondern eine komplette Verbalphrase inklusive aller innerhalb der VP auftretenden Modifikatoren; vgl. die (vereinfachte) Darstellung in (11):

$6 \quad$ Siehe hierzu auch Gese et al. (2009, i.Dr.), die u.a. anhand der oben aufgeführten Diagnostiken zeigen, dass auch unakkusative Verben diesen Konstruktionstyp eingehen. Ein Satz wie (i) hat demnach neben der Perfekt-Lesart, bei der das Perfektauxiliar sein mit einem verbalen Partizip auftritt, auch eine „Zustandspassiv“-Lesart in Gestalt der Kombination aus Kopula sein plus adjektiviertem Partizip. Zu den damit einhergehenden semantischen Unterschieden siehe Maienborn (2009).

(i) Meine Nachbarin ist verreist.

Zum Ausschluss von ereignisbezogenen Modifikatoren in Kopula-Konstruktionen siehe Maienborn (2003a, 2005).

$8 \quad$ Kratzers Vorschlag zur phrasalen Adjektivierung wurde verschiedentlich aufgegriffen und weiterentwickelt (siehe z.B. von Stechow 1998; Anagnostopoulou 2003; Embick 2004; Alexiadou/Anagnostopoulou 2007). 
(11) a. Lexikalische Adjektivierung

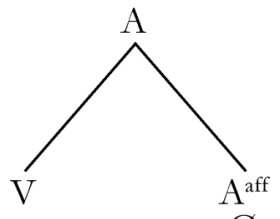

$\oslash$

b. Phrasale Adjektivierung

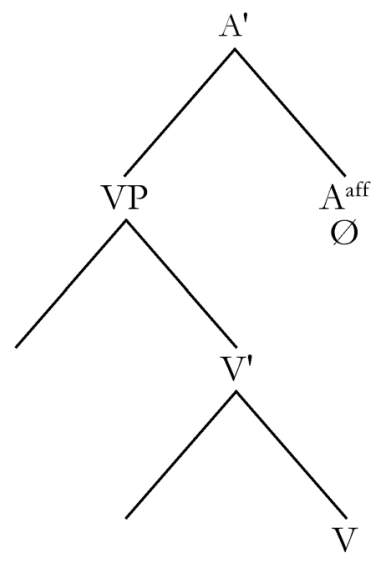

Das Auftreten adverbialer Modifikatoren beim Zustandspassiv kann damit in Kratzers Modell über die phrasale Adjektivierung erfasst werden. ${ }^{9}$ Allerdings wäre unter diesen Vorzeichen auch zu erwarten, dass via phrasale Adjektivierung beliebige innerhalb einer VP auftretende Modifikatoren auch beim Zustandspassiv grundsätzlich zulässig sein sollten. Dies trifft, wie die Beispiele in (5) zeigen, nicht zu.

Um diesem Problem der Übergenerierung zu begegnen, formuliert Rapp (1996, S. 257) die Zusatzbedingung, dass bei der phrasalen Adjektivierung nur solche adverbialen Modifikatoren zugelassen sind, die neben dem vom Basisverb bezeichneten Ereignis auch dessen Resultatszustand charakterisieren. So lässt sich beispielsweise beim Schreiben eines Briefes am Ergebnis in der Regel nicht mehr erkennen, ob der Brief langsam oder schnell geschrieben wurde, wohl aber, ob dabei rote Tinte zum Einsatz kam; vgl. (12a) vs. (12b).

Der Vorschlag erfasst zugleich die Inkompatibilität zwischen ereignisbezogenen Modifikatoren und der auf lexikalische Adjektivierung beschränkten un-Affigierung:

(i) * Das Hemd war von der Mutter ungebügelt.

(ii) $*$ Die Truhe war mit einem Brecheisen ungeöffnet.

Siehe hierzu aber die Gegenevidenz in Schlücker (2005). 
(12) a. * Der Brief war langsam geschrieben.

b. Der Brief war mit roter Tinte geschrieben.

Rapps Zusatzbedingung liefert einen ersten Hinweis auf die relevanten Lizensierungsbedingungen für adverbiale Modifikatoren beim Zustandspassiv, der im Weiteren inhaltlich und strukturell weiter präzisiert werden soll. Eine Übertragung auf unser Eingangsbeispiel (1a) (Das Manuskript ist von Chomsky zitiert.) macht deutlich, dass diese Bedingungen weiter geschärft werden müssen. Im Falle des Manuskript-Zitierens etwa ist nicht ohne weiteres klar, worin ein Resultatszustand des Zitiert-Seins genau besteht und inwiefern ein solcher Resultatszustand, in dem sich das Manuskript befindet, durch die zusätzliche Agensangabe von Chomsky näher charakterisiert würde. An dieser Stelle bedarf es eines genaueren Blicks auf die Interpretation des Zustandspassivs.

\section{Interpretation des Zustandspassivs}

Der in Maienborn (2007, 2009) entwickelte Vorschlag zur Semantik des Zustandspassivs setzt bei dessen grammatischer Einordnung an und leitet daraus die Grundzüge einer semantischen Analyse ab. Wenn das Zustandspassiv syntaktisch nichts anderes ist, als der Spezialfall einer Kopula-Konstruktion mit adjektivischem Prädikativ, so sollte dies auch für seine Semantik gelten. Wie bei allen anderen Kopula-Konstruktionen auch besteht sein semantischer Beitrag damit in der Zuschreibung einer Eigenschaft an den Subjektreferenten (siehe Maienborn 2003a, 2005). Die zweifellos besonderen Interpretationsbedingungen des Zustandspassivs wären demnach zurückzuführen auf die semantische Umsetzung des mit der Adjektivierung des verbalen Partizips einhergehenden grammatischen Kategorienwechsels und dessen pragmatischer Deutung.

Meine These zum Zustandspassiv ist, dass die Adjektivierung des Partizips zur Bildung einer pragmatisch salienten Ad hoc-Eigenschaft dient, welche aus dem vom Basisverb bezeichneten Ereignis resultiert. Während also Standard-Kopulasätze mit adjektivischem Prädikativ dem Subjektreferenten eine lexikalisch kodierte Eigenschaft zuschreiben, die einen fest vorgegebenen Platz im Eigenschaftsraum des Subjektreferenten einnimmt, ist das Zustandspassiv ein grammatisches Ausdrucksmittel, um (mehr oder weniger) ad hoc potenziell neue, ereignisbasierte Eigenschaften zu bilden, deren genaue Kontur und Platzierung im Eigenschaftsraum durch Kontext und Weltwissen mitbestimmt wird.

Was ist gemeint mit „ereignisbasierten Ad hoc-Eigenschaften“? Betrachten wir dazu zunächst Beispiel (13):

Das Manuskript ist eingereicht. 
Satz (13) besagt mehr, als dass es ein in der Vergangenheit liegendes Ereignis des Manuskript-Einreichens gibt. Aufgrund unseres reichen Hintergrundwissens über Entstehungsstadien und Bewertungen von Publikationen im Wissenschaftsbetrieb können wir (13) unter Heranziehung potenzieller Alternativen als Aussage nicht nur über den Zustand, sondern auch die Güte des Manuskripts verstehen. Für eine Projektbegutachtung beispielsweise macht es einen qualitativen Unterschied, ob ein Manuskript angefangen, eingereicht, begutachtet, akzeptiert, zurückgezogen oder abgelehnt ist. Ein akzeptiertes Manuskript ist allemal besser als ein bloß eingereichtes. Ereignisbezogene Modifikatoren - dies kann an dieser Stelle schon vorweggenommen werden - erlauben eine weitergehende Spezifizierung und Differenzierung solcher Ad hoc-Eigenschaften.
a. Das Manuskript ist bei Nature eingereicht.
b. Das Manuskript ist von Chomsky zitiert.
c. Das Manuskript ist in einer Nacht geschrieben.

Die Modifikatoren in (14) aktivieren jeweils Portionen von Hintergrundwissen, die Schlussfolgerungen auf die Art von Manuskript, mit der wir es zu tun haben, zulassen. In (14a) können wir beispielsweise aus der Reputation der Zeitschrift Nature schließen, dass das Manuskript von hoher Qualität sein muss - zumindest nach Ansicht des Autors, andernfalls hätte er nicht die Einreichung erwogen. Ähnliches gilt für (14b): Von Chomsky zitiert zu werden, gilt innerhalb weiter Teile der linguistischen Gemeinschaft als Ritterschlag. Und in (14c) lässt sich für ein Manuskript, das in einer Nacht geschrieben wurde, beispielsweise schlussfolgern, dass es aus einem Guss ist - oder aber ausgesprochen schlampig geschrieben.

Die Diskussion der Beispiele in (14) zeigt, dass die jeweiligen Schlussfolgerungen auf die dem Manuskript letztlich zuzuschreibende Eigenschaft je nach Kontext, Weltwissen und subjektiven Einstellungen erheblich variieren können. Eine vollständige Rekonstruktion dieser Zusammenhänge gehört sicher nicht zu den Aufgaben der Linguistik. Der mir wichtige Punkt hierbei ist, dass die Semantik des Zustandspassivs so angelegt ist, dass wir eine solche Ad hoc-Eigenschaft erschließen müssen: Dem Subjektreferenten wird eine Eigenschaft zugewiesen, die in einer über Weltwissen festgelegten Beziehung zu dem vom Verb bezeichneten Ereignis steht. Diese Eigenschaft erschöpft sich nicht darin, am Nachzustand oder Resultatszustand des betreffenden Ereignisses beteiligt zu sein. Die Gegenüberstellung von Zustandspassiv und Perfekt des Vorgangspassivs in (15) kann dies verdeutlichen.

a. Das Manuskript ist von Chomsky zitiert.

b. Das Manuskript ist von Chomsky zitiert worden.

Mit (15a) wird dem Subjektreferenten eine semantisch unterbestimmte Ad hoc-Eigenschaft zugeschrieben, die aus einem (in der Regel) vergangenen 
Ereignis resultiert. Das Manuskript wird als zur Klasse der von Chomsky zitierten Schriften gehörig kategorisiert, wobei der Hörer gefordert ist, die charakteristischen Merkmale dieser Klasse zu bestimmen. Mit (15b) wird ausgedrückt, dass wir uns im Nachzustand eines Zitierensereignisses befinden, an dem Chomsky als Agens und das Manuskript als Thema beteiligt waren. Mehr nicht. Daraus können wir selbstverständlich weitere Schlüsse über das Manuskript ziehen, die Semantik des Ausdrucks erzwingt dies aber nicht.

Die Differenz tritt umso deutlicher zutage, wenn Chomsky durch einen uns in diesem Zusammenhang unbekannten Eigennamen ersetzt wird.

a. ? Das Manuskript ist von Sandberger zitiert.

b. Das Manuskript ist von Sandberger zitiert worden.

In diesem Fall ist kein Weltwissen abrufbar, das eine geeignete Schlussfolgerung anstoßen könnte, mit was für einer Klasse von Manuskript wir es zu tun haben. Die Perfekt-Variante (15'b) bleibt davon unberührt. Der Satz drückt analog zu (15b) aus, dass es ein Zitierensereignis gab, an dem ein Individuum namens Sandberger als Agens und das Manuskript als Thema beteiligt waren. Die Zustandspassiv-Variante (15'a) hingegen ist in dieser Konstellation pragmatisch abweichend, weil es uns nicht gelingt, auf eine plausible, aus dem Ereignis resultierende Eigenschaft des Manuskripts zu schließen.

Im Folgenden will ich kurz den Kern einer formal-semantischen Modellierung dieses Analysevorschlags für das Zustandspassiv vorstellen; siehe Maienborn (2009). Den Ausgangspunkt bildet die in Maienborn (2003a, 2005) entwickelte Semantik der Kopula. Ein Kopulasatz wie (16) erhält danach die semantische Repräsentation in (17). Der Satz bezeichnet einen Zustand s, in dem die Eigenschaft NEU auf das Manuskript zutrifft. ${ }^{10}$

$$
\text { Das Manuskript ist neu. }
$$

$\exists \mathrm{s}$ [NEU (def-manuskript, s)]

Parallel dazu nehme ich für das Zustandspassiv in (18) die semantische Repräsentation (19) an. Danach bezeichnet (18) einen Zustand, in dem auf das Manuskript eine semantisch unterbestimmte Eigenschaft Q zutrifft,

$10 \quad$ Für die Zwecke des vorliegenden Beitrags nehme ich eine vereinfachte Zustandsanalyse an und gehe nicht auf Einzelheiten zum ontologischen Typ des Zustandsarguments ein (siehe aber Maienborn 2003a, 2005). Ferner gehe ich auch nicht auf eine weitere Differenzierung der beiden Lesarten des Zustandspassivs, Nachzustandslesart (i) und Charakterisierungslesart (ii), ein (siehe hierzu Kratzer 2000; Maienborn 2007). Maienborn (2009) macht einen Vorschlag, wie diese beiden Lesarten als konzeptuelle Spezifizierungen aus der in (20) angegebenen semantischen Struktur abgeleitet werden können.

(i) Das Manuskript ist eingereicht ... Jetzt können wir uns an den Projektantrag machen.

(ii) Das Manuskript ist eingereicht ... aber nicht angenommen.

Ausgeblendet wird ebenfalls der Beitrag des Tempus. 
wobei dieser Zustand als Resultat eines Ereignisses des Manuskript-Einreichens ausgewiesen ist. ${ }^{11}$ (20) gibt den korrespondierenden Beitrag der auf das verbale Partizip anzuwendenden Adjektivierungsoperation an.

(18) Das Manuskript ist eingereicht.

(19) $\exists \mathrm{s} \exists \mathrm{e}[\mathrm{Q}$ (def-manuskript, s) \& RESULT (e, s) \& EINREICH (def-manuskript, e)]

(20) Adjektivierungsoperation:

$\lambda \mathrm{P} \lambda \mathrm{x} \lambda \mathrm{s} \exists \mathrm{e}[\mathrm{Q}(\mathrm{x}, \mathrm{s}) \& \operatorname{RESULT}(\mathrm{e}, \mathrm{s}) \& \mathrm{P}(\mathrm{x}, \mathrm{e})]$

Der Vergleich der beiden semantischen Repräsentationen in (17) und (19) zeigt, dass die Semantik des Zustandspassivs dem Muster regulärer Kopulakonstruktionen folgt. Der Unterschied gegenüber adjektivischen Basisprädikaten besteht lediglich darin, dass das Zustandspassiv dem Subjektreferenten eine intern komplexe und semantisch unterbestimmte Eigenschaft zuweist. Um interpretierbar zu sein, muss die freie Variable Q innerhalb des gegebenen Kontexts einen geeigneten Wert erhalten. Dies kann z.B. über ein abduktives Schlussverfahren à la Hobbs et al. (1993) modelliert werden. Die ökonomischste/beste Spezifizierung der freien Variable Q ist diejenige, die mit den wenigsten kontextuell nicht lizensierten Zusatzannahmen auskommt. Wenn, wie im Falle resultativer Verben, eine entsprechende Eigenschaft bereits lexikalisch eingeführt ist, so ist diese ein bevorzugter Belegungskandidat für Q; siehe (21). Demgegenüber bedarf es eines erheblich größeren pragmatischen Aufwandes, um z.B. für die okkasionelle Bildung schwedentrainiert sein aus (10) eine geeignete Belegung für Q zu erschließen. Ein entsprechendes Räsonnement könnte etwa als Resultat aus dem Training in Schweden die Eigenschaft, große Mengen Alkohol zu vertragen sprich: Trinkfestigkeit -, ableiten; siehe (22).

(21) a. Der Brief ist geöffnet.

b. $\exists$ s $\exists$ e [Q (def-brief, s) \& RESULT (e, s) \& ÖFFNEN (def-brief, e)] mit $\mathrm{Q}=$ OFFEN

(22) a. Der Japaner war schwedentrainiert.

b. $\exists$ s $\exists$ e [Q (def-jap, s) \& RESULT (e, s) \& TRAINIEREN (def-jap, e) $\& \operatorname{IN}(e$, schweden)] mit $\mathrm{Q}=$ TRINKFEST

Während also der erforderliche pragmatische Aufwand zur Belegung der freien Variable Q erheblich variieren kann von nahezu vollständiger Vorhersagbarkeit der zu erschließenden Eigenschaft aus dem lexikalischen Gehalt des Basisverbs bis zu massiver Einbeziehung von Kontext und Welt-

11 RESULT (e, s) impliziert, dass der resultierende Zustand s zeitlich unmittelbar an das verursachende Ereignis e anschließt. 
wissen, so ist doch der grundlegende Interpretationsmechanismus derselbe. Dies erklärt die hohe Produktivität und systematisch gegebene Bildbarkeit des Zustandspassivs im Deutschen.

In dem Maße, in dem die zu erschließende Eigenschaft durch die lexikalische Semantik des korrespondierenden Basisverbs festgelegt ist, reduziert sich natürlich der Ad hoc-Charakter des Zustandspassivs. Dies gilt insbesondere für die kausativen Verben wie öffnen, schließen, leeren usw., bei denen Q vollständig lexikalisch determiniert scheint. Allerdings sind selbst hier abweichende kontextuelle Spezifizierungen für Q möglich. Dies zeigen die Beispiele in (23) für geöffnet sein.

a. Die Filmschachtel ist geöffnet, aber wieder zugeklebt.

b. Das Spiel ist geöffnet, aber unbespielt und absolut neuwertig.

Die konzeptuelle Belegung für Q ist hier nicht - wie in (21) - OFFEN, sondern geöffnet sein drückt hier vielmehr aus, dass der Subjektreferent nicht mehr im Originalverpackungszustand ist. Wie (23a) zeigt, kann eine Filmschachtel zugleich geöffnet und zugeklebt (also nicht offen) sein. Bei Ersetzung des Partizips durch das Primäradjektiv offen käme es hingegen zu einem semantischen Widerspruch.

Soweit die Skizze einer formalen Umsetzung der hier vertretenen $\mathrm{Zu}$ standspassiv-Analyse: Die Adjektivierung des Partizips bietet ein produktives Ausdrucksmittel für die Konzeptualisierung einer prinzipiell beliebig komplexen, ereignisbasierten Ad hoc-Eigenschaft zur kontextuell induzierten Kategorisierung des Subjektreferenten.

\section{Strukturelle Integration adverbialer Modifikatoren}

Vor dem Hintergrund der oben angestellten Überlegungen zur Interpretation des Zustandspassivs können wir uns nun erneut den adverbialen Modifikatoren zuwenden. Die obigen Ausführungen zu (14) haben schon angedeutet, worin ihr Beitrag besteht, nämlich in einer weitergehenden Spezifizierung und Konturierung der pragmatisch zu erschließenden Ad hocEigenschaft. Was sind die strukturellen Grundlagen hierfür?

Kratzers Analysevorschlag der Integration ereignisbezogener Adverbiale über den Weg der phrasalen Adjektivierung behandelt die betreffenden Modifikatoren in Verbindung mit dem Zustandspassiv als völlig reguläre VP-Modifikatoren wie sie auch in Kombination mit verbalen Partizipien auftreten; vgl. (11b). Die Modifikatoren in (24a)/(25a) sind demnach strukturell identisch mit den Vorgangspassiv-Fällen in (24b)/(25b).

(24) a. Der Brief war mit Wachs versiegelt.

b. Der Brief wurde mit Wachs versiegelt. 
(25) a. Annas Untermieter war von der Polizei gesucht.

b. Annas Untermieter wurde von der Polizei gesucht.

Dies halte ich für falsch. Die beim Zustandspassiv auftretenden adverbialen Modifikatoren sind keine beliebigen VP-Modifikatoren, sondern sie sind prosodisch, syntaktisch und semantisch besonders ausgewiesen. In der Terminologie von Jacobs $(1993,1999)$ sind die betreffenden Modifikatoren in den Verbalkomplex integriert, d.h. sie formen zusammen mit dem Verb eine informationelle Einheit. Für klassische VP-Modifikatoren gilt dies nicht. Diese kompakte informationelle Einheit aus Partizip und Modifikator ist die Voraussetzung dafür, dass der Modifikator von der Adjektivierung erfasst werden und einen Beitrag zur Ableitung einer komplexen Ad hoc-Eigenschaft leisten kann, und sie charakterisiert den hier aufscheinenden Übergangsbereich zwischen Wort und Phrase.

Im Weiteren werde ich Daten aus dem Bereich von Prosodie, Syntax und Semantik vorstellen, die Hinweise auf diesen besonderen strukturellen Status der Verbindung von Partizip und Modifikator beim Zustandspassiv in Abgrenzung zum Vorgangspassiv liefern, und die den für das Zustandspassiv charakteristischen Strukturausbau zwischen Wort und Phrase weiter beleuchten.

\subsection{Prosodische Integration}

Betrachten wir zunächst die Prosodie. Beim Zustandspassiv wird neutraler Satzakzent mittels Hauptakzent auf dem verbadjazenten Modifikator gekennzeichnet; siehe (26a). ,Mit Wachs versiegelt sein' wird dabei als eine kompakte Informationseinheit präsentiert. Hauptakzent auf dem Partizip - und damit ,informationelle Separierung“ (Jacobs 1999) des Modifikators ist abweichend bzw. erfordert starken Kontrastfokus; siehe (26b). Zieht man zum Vergleich die Vorgangspassiv-Variante heran, so wird hier neutraler Satzakzent am Partizip gekennzeichnet; siehe (27b). Der Vorgang des Versiegelns und das dabei eingesetzte Wachs werden als zwei unabhängige Informationseinheiten dargestellt. Hauptakzent auf dem Modifikator, wie in (27a), ist nur mit Kontrastfokus vereinbar. (Hauptakzent wird durch Fettdruck angezeigt.)

(26) Anna sah sofort, ...

a. dass der Brief an Leo [vp mit Wachs versiegelt war] neutraler Satzakzent

b. ?? dass der Brief an Leo [vp mit Wachs versiegelt war] (und nicht verschmiert) 
(27) Anna sah sofort, ...

a. dass der Brief an Leo [vp gerade mit Wachs versiegelt wurde] (statt mit Siegellack)

b. dass der Brief an Leo [ ${ }_{v p}$ gerade mit Wachs versiegelt wurde] neutraler Satzakzent

Die Beispiele (28) und (29) zeigen dasselbe Muster bei Agens-Angaben:

(28) Anna vermutete, ...

a. dass ihr Untermieter [vp von der Polizei gesucht war] neutraler Satzakzent

b. ?? dass ihr Untermieter [ ${ }_{\mathrm{vp}}$ von der Polizei gesucht war] (und nicht versteckt)

(29) Anna vermutete, ...

a. dass ihr Untermieter [vp gerade von der Polizei gesucht wurde] (und nicht von der Mafia)

b. dass ihr Untermieter [vp gerade von der Polizei gesucht wurde] neutraler Satzakzent

Während adverbiale Modifikatoren beim Zustandspassiv also mit dem Partizip eine informationelle Einheit bilden müssen, ist dies beim Vorgangspassiv nicht der Fall. Die in (26)-(29) sichtbar werdenden prosodischen Unterschiede liefern erste Evidenz dafür, dass die betreffenden Modifikatoren strukturell nicht identisch sind.

Betrachten wir weiterhin Daten wie (30), die vom Typ der in der Literatur als nicht wohlgeformt bewerteten Modifikationsfälle sind; siehe die Diskussion zu (5).

(30) a. ?? Die Kartoffeln sind von Otto gekauft.

b. ?? Das Manuskript ist mit der Leselupe gelesen.

Wird, wie in (31), durch die prosodische Struktur eine Komplexbildung angezeigt, so zwingt uns die Grammatik geradezu, eine geeignete pragmatische Deutung zu finden - in Gestalt einer aus dem gegebenen Ereignis unter Rekurs auf Kontext und Weltwissen abgeleiteten, plausiblen Ad hocEigenschaft.

(31) a. Die Kartoffeln sind von Otto gekauft. (Die schmecken bestimmt lecker, denn Otto versteht was von Kartoffeln.)

b. Das Manuskript ist mit der Leselupe gelesen. (Da ist kein Tippfehler mehr drin.)

Die Sätze in (30) würde man out of the blue vermutlich als nicht wohlgeformt zurückweisen. Wenn die Prosodie allerdings die Integration des Modifikators anzeigt, wie in (31), dann lassen sich selbst Ausdrücke wie von Otto gekauft 
sein oder mit der Leselupe gelesen sein als sinnvolle Ad hoc-Eigenschaftszuschreibungen an den Subjektreferenten deuten, etwa entlang der in Klammern hinzugefügten kontextuellen Explizierungen. Das Fazit hieraus ist, dass Sätze wie (30) oder (5) keineswegs als grammatisch nicht wohlgeformt zu bewerten sind, sondern - die strukturelle Integration des Modifikators vorausgesetzt - allenfalls einen höheren pragmatischen Aufwand zur Erschließung einer plausiblen Ad hoc-Eigenschaft erfordern. Die genuin grammatischen Kombinationsbeschränkungen für adverbiale Modifikatoren beim Zustandspassiv erweisen sich damit als viel liberaler und weniger idiosynkratisch als dies der erste Augenschein vermuten lässt. ${ }^{12}$

\subsection{Syntaktische Integration}

Wenden wir uns als nächstes der syntaktischen Evidenz für die unterschiedliche strukturelle Einbindung adverbialer Modifikatoren beim Zustandspassiv gegenüber dem Vorgangspassiv zu. Wenn die ereignisbezogenen Modifikatoren beim Zustandspassiv eine besonders enge strukturelle Bindung mit dem verbalen Partizip eingehen, dann ist zu erwarten, dass sie sich, anders als ihre Pendants beim Vorgangspassiv, durch eine gewisse Ortsfestigkeit auszeichnen. Die folgenden Daten zur Vorfeldbesetzung und zum Scrambling bestätigen diese Vorhersage.

(32) a. Die Erdbeeren sind / wurden im Bioladen gekauft.

b. Im Bioladen wurden die Erdbeeren gekauft.

c. ?? Im Bioladen sind die Erdbeeren gekauft.

(33) a. Das Lamm-Curry ist / wird mit Ingwer abgeschmeckt.

b. Mit Ingwer wird das Lamm-Curry abgeschmeckt.

c. ?? Mit Ingwer ist das Lamm-Curry abgeschmeckt.

(34) a. Oliver ist / wurde von Zsa Zsa Gabor adoptiert.

b. Von Zsa Zsa Gabor wurde Oliver adoptiert.

c. ?? Von Zsa Zsa Gabor ist Oliver adoptiert.

Während eine Topikalisierung des Lokal-, Instrumental- oder Agensadverbials beim Vorgangspassiv ohne besondere (konstruktionsspezifische) Auflagen ${ }^{13}$ möglich ist (siehe die b.-Varianten in (32)-(34)), führt im Falle des Zustandspassivs eine Herauslösung des betreffenden Adverbials aus seiner VP-internen Basisposition zu einer grammatisch markierten Struktur. Die c.-Varianten in (32)-(34) sind nur bei klarer Kontrastakzentsetzung z.B. auf

$12 \mathrm{Zu}$ den vergleichsweise wenigen grammatischen Ausschlusskriterien für die Bildbarkeit des Zustandspassivs siehe Maienborn (2007, S. 102 ff.).

13 Bezüglich der für die Vorfeldbesetzung generell geltenden informationsstrukturellen Implikationen siehe Frey $(2004,2006)$. 
dem Adverbial zulässig. ${ }^{14}$ Dies zeigt, dass es sich um markierte Strukturen handelt, die allenfalls über den letzten Ausweg Kontrast gerettet werden können. Die Adverbiale beim Vorgangspassiv sind hingegen nicht auf solch massive kontrastive Stützung angewiesen.

Ein analoger Befund ergibt sich beim Vergleich des Scramblingverhaltens der Modifikatoren. Als obligatorisch in den Verbalkomplex integrierte Elemente lassen sich die beim Zustandspassiv auftretenden ereignisbezogenen Adverbiale nur schlecht scrambeln; für ihre nicht-integrierten Pendants beim Vorgangspassiv gilt dies nicht. (35)-(38) illustrieren das Verhalten von Instrumentaladverbialen, (39)-(42) das von durch-PPn. (Zum Ereignisbezug von durch-Adverbialen siehe Solstad 2007.)

(35) Maria hat gesagt, ...

a. dass der Weg mit Mosaiksteinen gefliest wird.

b. dass mit Mosaiksteinen der Weg gefliest wird.

(36) Maria hat gesagt, ...

a. dass der Weg mit Mosaiksteinen gefliest ist.

b. ?? dass mit Mosaiksteinen der Weg gefliest ist.

(37) Der Dozent hat bemerkt, ...

a. dass die Tafel mit einem Edding beschmiert wird.

b. dass mit einem Edding die Tafel beschmiert wird.

(38) Der Dozent hat bemerkt, ...

a. dass die Tafel mit einem Edding beschmiert ist.

b. ?? dass mit einem Edding die Tafel beschmiert ist.

(39) Die Sekretärin hat angedeutet, ...

a. dass der Politiker durch Drohanrufe verunsichert wird.

b. dass durch Drohanrufe der Politiker verunsichert wird.

(40) Die Sekretärin hat angedeutet, ...

a. dass der Politiker durch Drohanrufe verunsichert ist.

b. ?? dass durch Drohanrufe der Politiker verunsichert ist.

(41) Der Trainer hat versichert, ...

a. dass die Skifahrerin durch einen Helm geschützt wird.

b. dass durch einen Helm die Skifahrerin geschützt wird.

(42) Der Trainer hat versichert, ...

a. dass die Skifahrerin durch einen Helm geschützt ist.

b. ? dass durch einen Helm die Skifahrerin geschützt ist.

14 Oder aber es stellt sich eine Listeninterpretation ein; vgl. (i) für (33c):

(i) Mit Ingwer ist das Lamm-Curry abgeschmeckt, mit Koriander das Huhn, ... 
Die in (35)-(42) angegebenen Bewertungen wurden im Rahmen einer Beurteilungsstudie überprüft und empirisch erhärtet (siehe Gese/Stolterfoht 2010). Während die Testsätze in der Abfolge Subjekt < Modifikator in der Vorgangspassiv- und der Zustandspassiv-Version von den Probanden als gleich gut bewertet wurden, zeigt sich in der gescrambelten Abfolge Modifikator < Subjekt ein deutlicher Unterschied: Die gescrambelte Abfolge wird beim Zustandspassiv signifikant schlechter bewertet als beim Vorgangspassiv. Dies kann als weiterer Hinweis darauf gewertet werden, dass der strukturelle Status des Modifikators beim Zustandspassiv ein anderer ist als beim Vorgangspassiv.

\subsection{Semantische Integration}

Semantische Reflexe der Bildung einer kompakten Informationseinheit aus Modifikator und Partizip beim Zustandspassiv zeigen sich z.B. im Hinblick auf die anaphorische Wiederaufnahme. Durch die Integration in den Verbalkomplex verliert der Modifikator seine referenzielle Unabhängigkeit und ist als Teil des Prädikats nicht mehr in vollem Umfang referenziell zugänglich. Der Vergleich der Verhältnisse beim Zustandspassiv mit denen beim Vorgangspassiv einerseits und denen entsprechender Komposita andererseits erhellt den Übergangscharakter der Zustandspassivkonstellation zwischen Wort und Phrase: Im Vorgangspassiv-Fall (43a), vom TÜV geprüft werden, bildet der Agens eine eigenständige, referenziell unabhängige Informationseinheit, die im Folgenden anaphorisch wiederaufgenommen werden kann. Beim Kompositum TÜV-geprïft in (43c) hingegen ist der Agens vollständig in das Wort integriert und damit referenziell nicht mehr zugänglich. Der Zustandspassiv-Fall (43b), vom TÜV geprüft sein, ist zwischen diesen beiden Extremen angesiedelt: Die anaphorische Wiederaufnahme ist zwar nicht vollständig blockiert, wie bei (43c), allerdings ist sie auch nicht völlig reibungslos möglich wie in (43a); vielmehr führt der Versuch einer anaphorischen Wiederaufnahme zu einem Konflikt, der allenfalls partiell durch die nachträgliche Rekonstruktion eines entsprechenden Diskursrefenten gelöst werden kann.

(43) a. Mein Auto ist vom TÜV geprüft worden. Er hatte nichts zu beanstanden.

b. Mein Auto ist vom TÜV geprüft. ? Er hatte nichts zu beanstanden.

c. Mein Auto ist TÜV-geprüft. * Er hatte nichts zu beanstanden.

Mit der sukzessiven Aufgabe der referenziellen Eigenständigkeit korrespondiert eine weitere semantische Beobachtung. So besagt die Interpretation von (43a) lediglich, dass das Auto der Sprecherin Gegenstand einer 
vom TÜV durchgeführten Prüfung war. (43a) ist damit mit der Annahme kompatibel, dass diese Prüfung negativ ausfiel; siehe (44a). Dies ist im Falle des Kompositums ausgeschlossen. Aus dem Zutreffen des komplexen Prädikats TÜV-geprüft folgt, dass die Prüfung bestanden wurde; (44c) ist damit semantisch widersprüchlich (gekennzeichnet mit \#).

a. Mein Auto ist vom TÜV geprüft worden. Leider ist die Prüfung negativ ausgefallen.

b. ?? Mein Auto ist vom TÜV geprüft. Leider ist die Prüfung negativ ausgefallen.

c. \# Mein Auto ist TÜV-geprüft. Leider ist die Prüfung negativ ausgefallen.

Was ist mit der Zustandspassiv-Konstellation (43b)? Auch hier gibt es eine starke Präferenz für die Folgerung, dass die Prüfung erfolgreich absolviert wurde; siehe (44b). Ich würde dennoch dafür plädieren, dieser Schlussfolgerung den Status einer konversationellen Implikatur einzuräumen, die - bei allerdings nur massiver kontextueller Stützung - aufhebbar ist. Ein solcher Kontext könnte z.B. eine groß angelegte Evaluation sein, bei der der TÜV selbst auf dem Prüfstand steht und gegen eine konkurrierende PrüferOrganisation antritt. In einem solchen kontrastiven Setting ließe sich sagen: Diese Autos sind vom TÜV geprüft, ohne damit zu implizieren, dass die Prüfung in jedem Fall erfolgreich verlief. Hingegen würde die Aussage Diese Autos sind TÜV-geprüft auch in diesem Kontext weiterhin nur die Klasse der vom TÜV positiv getesteten Wagen identifizieren. Auch für die konzeptuellbasierten Schlussfolgerungen gilt somit - wie oben bereits für die lexikalisch-semantisch induzierten Schlüsse am Beispiel des kausativen Verbs öffnen diskutiert - je stärker sie kontextübergreifend mit dem jeweiligen Ereigniskonzept assoziiert sind (etwa: PRÜFEN - PRÜFUNG-BESTEHEN), desto weniger ad hoc erscheint die erschlossene Eigenschaft.

Die in Abschnitt 4 entwickelte Semantik des Zustandspassivs kann die obige Beobachtung unmittelbar erfassen. Zunächst einmal geht sie mit der Forderung nach einer semantisch unterbestimmten Eigenschaft, die als Resultat des von dem Verb bezeichneten Ereignisses ausgewiesen ist, über das betreffende Ereignis hinaus. Anders als im Vorgangspassivfall reicht es also bei (43b)/(44b) nicht, dass das Auto Gegenstand einer TÜV-Prüfung war. Vielmehr muss eine daraus resultierende, kontextuell saliente Eigenschaft erschlossen werden, und zwar unter geringstmöglichem pragmatischen Aufwand. Dies ist im Default-Fall die an den erfolgreichen Abschluss der Prüfung gebundene Verkehrstauglichkeit. Nur sehr spezielle Kontexte, wie etwa der oben geschilderte, legen eine andere Instantiierung der Eigenschaftsvariable nahe. Der (mehr oder weniger ausgeprägte) Ad hoc-Charakter der Eigenschaftszuschreibung beim modifizierten Zustandspassiv 
ist damit in der kompositional angelegten semantischen Unterbestimmtheit verankert und setzt auf der engen strukturellen Bindung zwischen Modifikator und Partizip auf.

Ich will die Liste der Evidenzen für eine strukturelle Integration adverbialer Modifikatoren beim Zustandspassiv mit dem Hinweis auf ein psycholinguistisches Experiment abschließen, welches die Frage der referenziellen Eigenständigkeit bzw. Integration des Modifikators zum Gegenstand hat (siehe Gese/Stolterfoht 2010). Es handelt sich um eine an Ratcliff/McKoon (1978) angelehnte Reaktionszeitstudie mit Wortwiedererkennungsaufgabe, die von der Annahme ausgeht, dass integrierte Elemente schneller wiedererkannt werden, wenn sie nach dem integrierenden Element präsentiert werden. Konkret geht es um die Frage, ob der Modifikator beim Zustandspassiv, nicht aber beim Vorgangspassiv, durch das zuvor präsentierte Partizip "gebahnt" wird (semantisches Priming). Die Versuchspersonen bekamen in diesem Experiment Vorgangs- und Zustandspassivsätze vom Typ (45a) präsentiert und mussten im Anschluss für zwei aufeinander folgende Wörter jeweils entscheiden, ob diese im zuvor gelesenen Satz vorkamen. In den relevanten Bedingungen waren dies zunächst das Partizip und darauf folgend entweder das Modifikator-Nomen oder das Subjekt-Nomen; siehe (45b). Unsere Hypothesen waren: (H1) eine schnellere Wiedererkennung des Modifikator-Nomens nach dem Zustandspassivsatz und (H2) keine Reaktionszeitunterschiede zwischen Vorgangspassiv und Zustandspassiv bei der Wiedererkennung des Subjekt-Nomens; siehe (45c).

(45) Reaktionszeitstudie mit Wortwiedererkennungsaufgabe (Gese/Stolterfoht 2010)

a. Testsätze:

(ZP) Der Weg ist mit Mosaiksteinen gefliest.

(VP) Der Weg wird mit Mosaiksteinen gefliest.

b. Aufgabe: „Kam das Wort im zuvor gelesenen Satz vor?“ (mod) GEFLIEST [ja/nein] MOSAIKSTEINE [ja/nein] (subj) GEFLIEST [ja/nein] WEG [ja/nein]

c. Hypothesen:

H1: $\quad$ ZPmod $<$ VPmod

H2: $\quad$ ZPsubj $=$ VPsubj

Beide Hypothesen wurden bestätigt. Es zeigte sich eine signifikant schnellere Wiedererkennung des Modifikator-Nomens in der Zustandspassiv-Bedingung gegenüber der Vorgangspassiv-Bedingung. Hingegen zeigten sich keine signifikanten Reaktionszeitunterschiede für das Subjekt-Nomen. Aus diesen Ergebnissen lässt sich schließen, dass das Modifikator-Nomen beim 
Zustandspassiv (und nur dort) durch das Partizip semantisch gebahnt wird; siehe Gese/Stolterfoht (2010) zu weiteren Details zu Durchführung des Experiments, Vorstudien zum Satzmaterial und statistischer Auswertung.

Die Befunde wurden durch eine weitere Kontrollstudie erhärtet, bei der mit identischem Testmaterial lediglich die Wiedererkennung von Modifikator- oder Subjekt-Nomen ohne vorherige Präsentation des Partizips getestet wurde; siehe (46b). Hier erwarten wir keine Reaktionszeitunterschiede für das Modifikator-Nomen (H3); siehe (46c). Auch diese Hypothese wurde bestätigt.

(46) Kontrollstudie

a. Testsätze:

(ZP) Der Weg ist mit Mosaiksteinen gefliest.

(VP) Der Weg wird mit Mosaiksteinen gefliest.

b. Aufgabe: „Kam das Wort im zuvor gelesenen Satz vor?“ (mod) MOSAIKSTEINE [ja/nein]

(subj) WEG [ja/nein]

c. Hypothesen:

H3: $\quad$ ZPmod $=$ VPmod; $Z$ Psubj $=$ VPsubj

Die Ergebnisse der Kontrollstudie unterstützen damit die Deutung des beobachteten Effekts in der ersten Reaktionszeitstudie als Priming-Effekt: Beim Zustandspassiv wird das integrierte Element (= Modifikator-Nomen) durch das integrierende Element (= Partizip) semantisch gebahnt. Dies zeigt, dass Modifikator und Partizip beim Zustandspassiv eine besonders enge Bindung eingehen. Im Falle des Vorgangspassivs kommt es hingegen zu keiner entsprechenden Bahnung. Dies ist mit der Annahme kompatibel, dass in diesem Fall reguläre VP-Modifikatoren vorliegen.

\section{Fazit}

Die oben präsentierten Daten wurden mit verschiedenen Methoden gewonnen: Introspektion, Beurteilungsstudien, experimentelle Studien; und sie betreffen verschiedene Ebenen der grammatischen Strukturbildung: Prosodie, Syntax, Semantik. Somit ist eine breite empirische Fundierung für theoretische Schlussfolgerungen gegeben. Die Befunde zeigen übereinstimmend, dass die strukturelle Einbindung ereignisbezogener Modifikatoren beim Zustandspassiv nicht identisch mit derjenigen beim Vorgangspassiv ist. Sie widerlegen damit Kratzers Ausgangshypothese für ihren Vorschlag der Einbeziehung der betreffenden Modifikatoren über phrasale Adjekti- 
vierung; siehe die Diskussion zu (11) und (24)/(25). Vielmehr unterstützen die Befunde die Annahme einer besonderen strukturellen Nähe von Partizip und Modifikator beim Zustandspassiv. Beide bilden gemeinsam ein komplexes Prädikat, aus dem qua Adjektivierungsoperation eine resultierende Ad hoc-Eigenschaft abgeleitet wird; siehe (20).

Ich will an dieser Stelle auf einen Punkt in der Analyse von Schlücker (2005) noch etwas näher eingehen. Schlücker folgt im Wesentlichen dem hier vertretenen Ansatz einer verbnahen Platzierung der betreffenden Modifikatoren und entwickelt auf dieser Grundlage eine konzeptuelle Analyse ereignisbezogener Modifikatoren beim Zustandspassiv im Rahmen von Barsalous (1992) „Frame“-Konzept. Allerdings stellt sie für die Klasse der von-Adverbiale fest, dass es hier neben den obligatorisch in den Verbalkomplex integrierten von-PPn einen zweiten Typus von von-PPn gibt, der nicht dem in Abschnitt 5 geschilderten prosodischen und syntaktischen Muster folgt, sondern sich wie die beim Vorgangspassiv auftretenden regulären VPModifikatoren verhält. Schlückers Musterfall ist (47a). Neutraler Satzakzent etwa wird hier nicht auf dem Modifikator, sondern auf dem Partizip realisiert; siehe (47b) vs. (47c) sowie Schlückers weitere syntaktische Indizien.

a. Peter ist von dem Gejammer genervt.

b. weil Peter von dem Gejammer genervt ist. nur kontrastiv

c. weil Peter von dem Gejammer genervt ist. neutraler Satzakzent

Als Erklärung für diesen Befund greift Schlücker auf Kratzers Mechanismus der phrasalen Adjektivierung zurück, den sie allerdings allein für entsprechende von-PPn reserviert. Ich folge ihr in diesem Schritt nicht und vertrete vielmehr die Auffassung, dass Schlückers von-PPn vom Typ (47a) keine Agens-Angaben sind und auch keinen anderweitigen Ereignisbezug haben, sondern in Solstads (2007) Redeweise einen Grund als kausal involvierten Sachverhalt benennen (siehe auch Solstad 2010). Die betreffenden Modifikatoren haben folglich keinen Ereignisbezug, sondern Sachverhaltsbezug und sind entsprechend strukturell höher angesiedelt. In der Tat finden sich derartige Kausalangaben nicht nur beim Zustandspassiv, sondern auch bei einfachen prädikativen Adjektiven:

a. Paul war von der Reise müde.

b. Die Straße war vom Regen nass.

c. Der Rasen war von dem Rumgerenne platt.

d. Ihr Gesicht war von der Sonne rot.

Das heißt, die von-Modifikatoren vom Typ (47) liegen außerhalb des Skopus der Adjektivierungsoperation; es handelt sich, gleich den (48)-Fällen, um Modifikatoren des Kopula-Prädikativ-Komplexes. Mit dem Zustandspassiv im engeren Sinne haben sie nichts zu tun. ${ }^{15}$

15 Ich danke Torgrim Solstad für unsere Diskussion dieser Daten. 
Zurück zum Zustandspassiv. Wie soll das spezifische Strukturverhältnis zwischen Partizip und Modifikator syntaktisch umgesetzt werden? Schaut man sich nach möglichen Vorbildern um, so sind ähnliche Formen der Komplexbildung aus anderen Zusammenhängen und Sprachen wohlbekannt siehe etwa die durch Baker (1988) angestoßene Inkorporationsdiskussion sowie die Überlegungen zum Verbalkomplex im Deutschen z.B. bei Bierwisch (1988), Steinitz (1989), Frey/Tappe (1992), Frey (1993) und Haider (1993, 2010). In zweierlei Hinsicht weicht die hier betrachtete Konstruktion von klassischen Inkorporationsfällen ab. Erstens sind die integrierten Elemente in unserem Fall klare Modifikatoren, und keine Argumente des verbalen Kopfs, für die Bakers Inkorporationstheorie - und im Übrigen auch Jacobs' Theorie der Integration - ausgelegt ist. ${ }^{16}$ Und zweitens sind die integrierten Elemente eindeutig phrasale Konstituenten.

Dies genau ist die Crux für eine geradlinige strukturelle Umsetzung der obigen Befunde. Naheliegend nach dem bisher Gesagten wäre eine Modellierung über Kopf-Adjunktion wie in (49) (siehe den Vorschlag in Maienborn 2007). ${ }^{17}$ Dies erfasst die unmittelbare strukturelle Nähe zwischen Modifikator und Partizip, und es erlaubt, für das Zustandspassiv eine alleinige, auf lexikalischer Ebene angesiedelte Adjektivierungsoperation anzunehmen. Strukturell höher angesiedelte VP-Modifikatoren werden von der lexikalischen Adjektivierung nicht erfasst und sind deshalb beim Zustandspassiv ausgeschlossen.

16 Jacobs (2004) erwägt für die hier in Rede stehenden ereignisbezogenen Modifikatoren - in seiner Terminologie „Zirkumstantien“ - eine Neubewertung als Argumente. Damit ließe sich, angewandt auf unseren Fall, ihre Integration in den Verbalkomplex unter Beibehaltung der ursprünglichen Argumentrestriktion erfassen. Allerdings scheint mir eine solche Aufgabe der klassischen Argument/Modifikator-Unterscheidung zu weitreichend zu sein. Es sind in unserem Fall auch nicht Zirkumstantien per se, die als Integrationskandidaten in Frage kommen, sondern nur solche in besonderer struktureller Nähe zum Verb. Im Zusammenhang mit einer Analyse lokaler Modifikatoren habe ich in Maienborn $(1996,2001)$ argumentiert, dass die verbnah, unterhalb des direkten Arguments basisgenerierten Modifikatoren vom Typ auf den Fingern pfeifen, in der Pfanne braten, an den Füßen kitzeln grammatisch den Status von Adjunkten haben; die vorgeschlagene semantische Analyse läuft aber darauf hinaus, ihnen konzeptuell durchaus Argumentstatus zuzubilligen: Sie aktivieren und füllen funktional eingebundene Slots innerhalb der konzeptuellen Ereignisstruktur, ohne dass dies allerdings Auswirkungen auf die lexikalisch fixierte Argumentstruktur der involvierten Verben hätte (siehe hierzu insbesondere die konzeptuelle Detailanalyse in Maienborn 2003b). Die betreffenden Ausdrücke - dies gilt für die verbnahen lokalen Modifikatoren ebenso wie für die hier besprochenen ereignisbezogenen Adverbiale beim Zustandspassiv - sind demnach Zwitter: Grammatisch handelt es sich um frei hinzutretende Modifikatoren; konzeptuell greifen sie tief in die Ereignisstruktur ein und bilden gemeinsam mit dem Verb ein komplexes Prädikat. Unter dieser Perspektive wären dann allerdings die strukturellen Voraussetzungen für das Zustandekommen von Integration entsprechend zu liberalisieren.

17 Siehe auch Haiders (2010, S. 335 ff.) aktuellen Vorschlag zur Syntax von V0 -Clustern als $\mathrm{X}^{0}$-Adjunktionsstrukturen, in den er die Einbindung von so genannten „co-predicates“ (insbesondere resultative und direktionale Prädikate) explizit einbezieht. 
(49) Strukturelle Integration via Kopf-Adjunktion

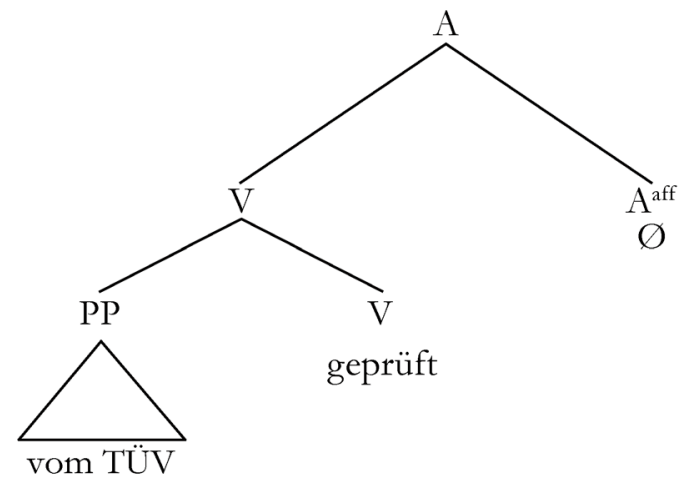

Das Lexikon wäre damit weiterhin als Domäne für morphologische Operationen, wie eben auch den hier diskutierten lexikalischen Kategorienwechsel beim Partizip, ausgewiesen, und das mächtige Instrument der phrasalen Adjektivierung wäre verzichtbar. Der Preis dafür allerdings ist, dass wir mit (49) behaupten, der komplexe Ausdruck vom TÜV geprïft sei ein Wort. Dies ist er nach unserem Standardverständnis von Wörtern und ihrer Abgrenzung gegenüber Syntagmen sicher nicht. Die Beiträge in diesem Band zeigen allerdings, dass eine solche Abgrenzung und eine entsprechend disjunkte Einteilung „Wort oder Syntagma?“ bei allem heuristischen Nutzen nicht haltbar ist (siehe insbesondere den Beitrag von Jacobs). Jacobs' Liste von Übergangserscheinungen zwischen Wort und Syntagma wäre nach dem hier Gesagten also um einen weiteren Ausdruckstypus zu ergänzen, der zwar auf syntaktischer Komplexbildung beruht, dessen interne Struktur jedoch für bestimmte syntaktische und semantische Prozesse nicht mehr zugänglich ist.

(50) X' als Integrationsdomäne

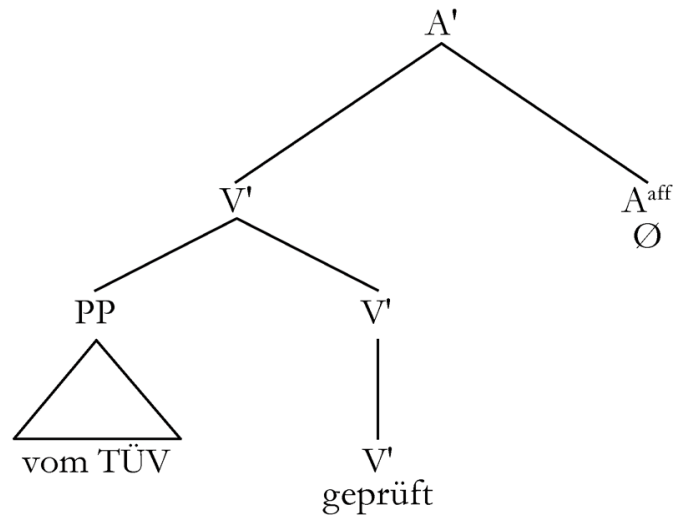


Sicher sind Alternativen zu (49) denkbar, etwa im Sinne von Frey/Tappe (1992) und Frey (1993) die Reservierung einer Zwischenprojektionsstufe X' als die relevante Domäne für Integration, innerhalb derer eine besondere strukturelle Nähe zum Kopf besteht. Nicht-integrierte Argumente und die Standard-Modifikatoren sind in diesem Modell auf VP-Ebene angesiedelt. Damit wäre $V^{\prime}$ als die strukturell höchste Position auszuweisen, auf der die Adjektivierung verbaler Partizipien (samt integrierter Modifikatoren) erfolgen kann; siehe (50).

Ob eine solche $\mathrm{X}^{\prime}$-Zwischenprojektion als strukturelles Auffangbecken für diese und andere Grenzerscheinungen zwischen Wort und Syntagma sich letztlich als tragfähig und nützlich erweist, bleibt zu zeigen - ebenso wie Theorieansätze, die von vorneherein die Unterscheidung zwischen Lexikon und Syntax aufgeben, eine Erklärung für die in Abschnitt 5 gezeigten Unterschiede der Modifikatoren bei Zustandspassiv und Vorgangspassiv bieten müssen.

Die genauen strukturellen Grundlagen für die im Grenzbereich von Wort- und Phrasenstruktur angesiedelte Form der Komplexbildung bedürfen dringend weiterer Klärung. Allerdings will ich abschließend auch unterstreichen, dass der hier vorgestellte Ansatz eine strikt kompositionale Semantik für die ereignisbezogenen Modifikatoren beim Zustandspassiv bietet, die mit beiden Strukturoptionen (49) und (50) (oder möglichen Varianten) kompatibel ist. Dies wird in (51) noch einmal exemplarisch durchgespielt. Ich lege dabei zur leichteren Lesbarkeit eine neo-davidsonsche Darstellung der betreffenden Lexikoneinträge mittels thematischer Rollen zugrunde; siehe (51a/b). Verbnah platzierte Modifikatoren werden entsprechend der Davidsonschen Grundeinsicht als zusätzliche Prädikate über den verbalen Ereignisreferenten konjunktiv angeschlossen (51c). ${ }^{18}$

Das Auto ist vom TÜV geprüft.

a. geprüft: $\quad \lambda y \lambda$ e [PRÜF (e) \& THEMA $(y, e)]$

b. vom TÜV: $\lambda \mathrm{e}^{\prime}$ [AGENS (TÜV, e')]

c. [v vom TÜV geprüft]: $\lambda$ y $\lambda$ e [PRÜF (e) \& THEMA (y, e) \& AGENS (TÜV, e)]

Die in (51c) angegebene verbale Struktur fällt unter den Skopus des in (20) vorgeschlagenen Adjektivierungsoperators, hier wiederholt in (51d). Das Resultat der Anwendung des Adjektivierungsoperators auf (51c) ist in (51e) angegeben.

$$
\begin{aligned}
& \text { d. } A^{\text {Aff: }} \quad \lambda \mathrm{P} \lambda \mathrm{x} \lambda \mathrm{s} \exists \mathrm{e}[\mathrm{Q}(\mathrm{x}, \mathrm{s}) \& \operatorname{RESULT}(\mathrm{e}, \mathrm{s}) \& \mathrm{P}(\mathrm{x}, \mathrm{e})] \\
& \text { e. [A [v vom TÜV geprüft]]: } \lambda \mathrm{x} \lambda \mathrm{s} \exists \text { e [Q }(\mathrm{x}, \mathrm{s}) \& \operatorname{RESULT}(\mathrm{e}, \mathrm{s}) \&
\end{aligned}
$$

18 Die verantwortliche Kompositionsregel für die Verbindung des semantischen Beitrags des Modifikators P mit dem des Modifikanden Q ist in (i) angegeben.

(i) $\lambda \mathrm{P} \lambda \mathrm{Q} \lambda \mathrm{e}[\mathrm{Q}(\mathrm{e}) \& \mathrm{P}(\mathrm{e})]$ 
Das Resultat (51e) wird als komplexes Prädikat in die Kopula eingespeist (51f/g).

\author{
f. Kopula: $\lambda \mathrm{P} \lambda \mathrm{x} \lambda \mathrm{s}[\mathrm{P}(\mathrm{x}, \mathrm{s})]$ \\ g. vom TÜV geprüft sei-: $\lambda \mathrm{x} \lambda \mathrm{s} \exists \mathrm{e}[\mathrm{Q}(\mathrm{x}, \mathrm{s}) \& \operatorname{RESULT}(\mathrm{e}, \mathrm{s}) \&$ \\ PRÜF (e) \& THEMA (x, e) \& AGENS (TÜV, e)]]
}

Nach Sättigung des Subjektarguments $(51 \mathrm{~h} / \mathrm{i})$ und existentieller Bindung des Zustandsarguments (via Finitheit) ergibt sich als kompositional-semantische Struktur (51j).
h. das Auto:
DEF x [AUTO (x)]
abgekürzt mit: a
i. das Auto vom TÜV geprüft sei-: $\lambda \mathrm{s} \exists \mathrm{e}[\mathrm{Q}(\mathrm{a}, \mathrm{s}) \& \operatorname{RESULT}(\mathrm{e}, \mathrm{s})$ $\&$ PRÜF (e) \& THEMA (a, e) \& AGENS (TÜV, e)]]
j. Das Auto ist vom TÜV geprüft: (Kompositionale Semantik) $\exists$ s $\exists$ e [Q (a, s) \& RESULT (e, s) \& PRÜF (e) \& THEMA (a, e) \& AGENS (TÜV, e)]]

In Prosa besagt (51j), dass es einen Zustand s gibt, in dem die unterbestimmte Eigenschaft Q auf ein im Diskurs eindeutig identifizierbares Auto a zutrifft. Dieser Zustand ist als Result eines Prüfereignisses e ausgewiesen, an welchem der TÜV als Agens und das Auto als Gegenstand der Prüfung beteiligt waren. Eine plausible konzeptuelle Spezifikation für Q folgt in (51k). Danach wird die kompositional unterbestimmt verbliebene Eigenschaft Q als Fahrtauglichkeit identifiziert. ${ }^{19}$ In Prosa: Das im Diskurs eindeutig identifizierbare Auto a befindet sich, resultierend aus einer Prüfung durch den TÜV, im Zustand der Fahrtauglichkeit.

$$
\begin{aligned}
& \text { k. Das Auto ist vom TÜV geprüft: (Konzeptuelle Spezifikation) } \\
& \text { ヨs ヨe [FAHRTAUGLICH (a, s) \& RESULT (e, s) \& PRÜF (e) \& } \\
& \text { THEMA (a, e) \& AGENS (TÜV, e)]] }
\end{aligned}
$$

Die in der Adjektivierungsoperation kompositional angelegte semantische Unterbestimmtheit, vermittelt über die freie Variable Q, ist es, die die für das Zustandspassiv charakteristische Einbeziehung von Kontext und Weltwissen ermöglicht und die Ableitung einer kontextuell salienten Ad hoc-Eigenschaft sicherstellt. Auf diese Weise ergibt sich als Ergebnis der konzeptuellen Belegung der freien Variable Q beispielsweise die Deutung in (51k).

Wenn man von so etwas wie einer ,konstruktionsspezifischen Bedeutung" des Zustandspassivs inklusive etwaiger ereignisbezogener Modifikatoren sprechen möchte, so ist sie genau hier, in der Semantik der Adjektivierungsoperation als Auslöser für den Wortartenwechsel, angelegt - und zwar völlig transparent und kompositional.

19 Das Prädikat FAHRTAUGLICH in (51k) dient selbstverständlich lediglich als Etikett für sehr viel komplexere Konzeptstrukturen, die im Zuge der konzeptuellen Spezifikation von Q aktiviert werden; siehe hierzu Maienborn (2003b). 


\section{Literatur}

Alexiadou, Artemis/Anagnostopoulou, Elena (2007): Structuring participles. Ms. Univ. Stuttgart/Univ. Kreta.

Anagnostopoulou, Elena (2003): Participles and voice. In: Alexiadou, Artemis/Rathert, Monika/Stechow, Arnim von (Hg.): Perfect explorations. (= Interface Explorations 2). Berlin u.a., S. 1-36.

Baker, Mark C. (1988): Incorporation: a theory of grammatical function changing. Chicago u.a.

Barsalou, Lawrence W. (1992): Frames, concepts, and conceptual fields. In: Lehrer, Adrienne/Kittay, Eva Feder (Hg.): Frames, fields, and contrasts: new essays in semantic and lexical organization. Hillsdale, NJ, S. 21-74.

Bierwisch, Manfred (1988): A puzzling domain in German clause structure. Ms. Berlin.

Embick, David (2004): On the structure of resultative participles in English. In: Linguistic Inquiry 35, S. 355-392.

Frey, Werner (1993): Syntaktische Bedingungen für die Interpretation: Über Bindung, implizite Argumente und Skopus. (= Studia grammatica 35). Berlin.

Frey, Werner (2004): The grammar-pragmatics interface and the German prefield. In: Sprache \& Pragmatik 52, S. 1-39.

Frey, Werner (2006): Contrast and movement to the German prefield. In: Molnár, Valéria/Winkler, Susanne (Hg.): Architecture of focus. (= Studies in Generative Grammar 82). Berlin/New York, S. 235-264.

Frey, Werner/Tappe, Hans Thilo (1992): Zur Interpretation der X-bar-Theorie und zur Syntax des Mittelfeldes. Ms. Univ. Stuttgart.

Gese, Helga/Maienborn, Claudia/Stolterfoht, Britta (i.Dr.): Adjectival conversion of unaccusatives in German. In: Journal of Germanic Linguistics.

Gese, Helga/Stolterfoht, Britta/Maienborn, Claudia (2009): Context effects in the formation of adjectival resultatives. In: Winkler, Susanne/Featherston, Sam (Hg.): The fruits of empirical linguistics. Bd. 2: Product. (= Studies in Generative Grammar 102). Berlin u.a., S. 231-262.

Gese, Helga/Stolterfoht, Britta (2010): Processing in one step: the integration of event modifiers. Ms. Univ. Tübingen.

Haider, Hubert (1993): Deutsche Syntax - generativ. Vorstudien zur Theorie einer projektiven Grammatik. (= Tübinger Beiträge zur Linguistik 325). Tübingen.

Haider, Hubert (2010): The syntax of German. Cambridge u.a.

Hobbs, Jerry J. et al. (1993): Interpretation as abduction. In: Artificial Intelligence 63, S. 69-142.

Jacobs, Joachim (1993): Integration. In: Reis, Marga (Hg.): Wortstellung und Informationsstruktur. (= Linguistische Arbeiten 306). Tübingen, S. 63-116. 
Jacobs, Joachim (1999): Informational autonomy. In: Bosch, Peter/van der Sandt, Rob (Hg.): Focus: linguistic, cognitive, and computational perspectives. Cambridge u.a., S. 56-81.

Jacobs, Joachim (2004): Zirkumstantien sind Argumente. Ms. Univ. Wuppertal.

Kratzer, Angelika (1994): The event argument and the semantics of voice. Ms. Amherst.

Kratzer, Angelika (2000): Building statives. (= Berkeley Linguistic Society 26). Internet: http://semanticsarchive.net/Archive/GI5MmIOM/kratzer.building.statives.pdf (Stand: 07.12.2010).

Lang, Ewald (1984): The semantics of coordination. (= Studies in Language Companion Series 9). Amsterdam.

Lenz, Barbara (1993): Probleme der Kategorisierung deutscher Partizipien. In: Zeitschrift für Sprachwissenschaft 12, S. 39-76.

Litvinov, Viktor P./Nedjalkov, Vladimir P. (1988): Resultativkonstruktionen im Deutschen. (= Studien zur deutschen Grammatik 34). Tübingen.

Lübbe, Anja (2004): Was heißt Lexikalisierung? Dargestellt am Beispiel deutscher Partizipien. Magisterarbeit. Humboldt-Universität Berlin.

Maienborn, Claudia (1996): Situation und Lokation: Die Bedeutung lokaler Adjunkte von Verbalprojektionen. (= Studien zur deutschen Grammatik 53). Tübingen.

Maienborn, Claudia (2001): On the position and interpretation of locative modifiers. In: Natural Language Semantics 9, 2, S. 191-240.

Maienborn, Claudia (2003a): Die logische Form von Kopula-Sätzen. (= Studia grammatica 56). Berlin.

Maienborn, Claudia (2003b): Event-internal modifiers: semantic underspecification and conceptual interpretation. In: Lang, Ewald/Maienborn, Claudia/Fabricius-Hansen, Cathrine (Hg.): Modifying adjuncts. (= Interface Explorations 4). Berlin/New York, S. $475-509$.

Maienborn, Claudia (2005): On the limits of the Davidsonian approach: the case of copula sentences. In: Theoretical Linguistics 31, S. 275-316.

Maienborn, Claudia (2007): Das Zustandspassiv: Grammatische Einordnung - Bildungsbeschränkungen - Interpretationsspielraum. In: Zeitschrift für Germanistische Linguistik 35, S. 83-114.

Maienborn, Claudia (2009): Building event-based ad hoc properties: on the interpretation of adjectival passives. In: Riester, Arndt/Solstad, Torgrim (Hg.): Proceedings of Sinn und Bedeutung 13. Stuttgart, S. 35-49.

Rapp, Irene (1996): Zustand? Passiv? - Überlegungen zum sogenannten Zustandspassiv. In: Zeitschrift für Sprachwissenschaft 15, S. 231-265.

Rapp, Irene (1997): Partizipien und semantische Struktur. Zu passivischen Konstruktionen mit dem 3. Status. (= Studien zur deutschen Grammatik 54). Tübingen. 
Ratcliff, Roger/McKoon, Gail (1978): Priming in item recognition: evidence for the propositional structure of sentences. In: Journal of Verbal Learning and Verbal Behavior 17, S. 403-417.

Schlücker, Barbara (2005): Event-related modifiers in German adjectival passives. In: Maier, Emar et al. (Hg.): Proceedings of Sinn und Bedeutung 9. Nijmegen, S. 417 430.

Solstad, Torgrim (2007): Mehrdeutigkeit und Kontexteinfluss: Die Spezifikation kausaler Relationen am Beispiel von durch. Dissertationsschrift. Univ. Oslo.

Solstad, Torgrim (2010): Some new observations on ,because (of)'. In: Aloni, Maria et al. (Hg.): Logic, language, and meaning. 17th Amsterdam Colloquium, Amsterdam, The Netherlands, December 16-18, 2009, Revised Selected Papers. (= Lecture Notes in Computer Science 6042). Berlin/Heidelberg, S. 426-445.

Stechow, Arnim von (1998): German Participles II in Distributed Morphology. Ms. Univ. Tübingen.

Steinitz, Renate (1989): $V^{\mathrm{u}}, \mathrm{I}^{\mathrm{y}}$ und $\mathrm{I}^{\mathrm{z}}$ : Überlegungen zum Prädikativ. (= Linguistische Studien des ZISW Reihe A, Arbeitsberichte 194). Berlin (Ost), S. 210-234.

Stolterfoht, Britta/Gese, Helga/Maienborn, Claudia (2010): Word category conversion causes processing costs: evidence from adjectival passives. In: Psychonomic Bulletin \& Review 17, 5, S. 651-656.

Vaagland, Erling M. (1983): Zur Agensangabe im sein-Passiv. In: Askedal, John Ole et al. (Hg.): Festschrift für Laurits Saltveit. Oslo, S. 194-200.

Wunderlich, Dieter (1997): Participle perfect and passive in German. (= Arbeiten des SFB 282 „Theorie des Lexikons“ 99). Düsseldorf.

Zifonun, Gisela/Hoffmann, Ludger/Strecker, Bruno (1997): Grammatik der deutschen Sprache. 3 Bde. (= Schriften des Instituts für Deutsche Sprache 7). Berlin/New York. 

Joachim Jacobs

\title{
Grammatik ohne Wörter?
}

\begin{abstract}
Am Beispiel des Deutschen wird gezeigt, dass verschiedene Strategien, die traditionelle Unterscheidung zwischen Wörtern und Syntagmen so zu modifizieren, dass sie die sehr differenzierten Daten vollständig, detailliert und widerspruchsfrei erfasst, zur inhaltlichen Entleerung dieser Unterscheidung führen und sie damit letztlich überflüssig machen. Das gilt sowohl für die populäre Aufspaltung von Wort vs. Syntagma in mehrere spezifischere Kategorienpaare (phonologisches Wort vs. phonologisches Syntagma, graphematisches Wort vs. graphematisches Syntagma usw.) als auch für ihre Umdeutung als Prototypen- oder Default-Kategorien. Allerdings kann man an der Unterscheidung zwischen Wörtern und Syntagmen festhalten, wenn man Einschränkungen der Vollständigkeit, Detailtreue oder Widerspruchsfreiheit in Kauf nimmt (wie in der linguistischen Praxis oft unumgänglich). Diese Überlegungen übertragen sich auf die auf Wort vs. Syntagma beruhenden Abgrenzungen linguistischer Teilgebiete, wie Syntax vs. Morphologie vs. Phraseologie, z.T. auch Grammatik vs. Lexikon. ${ }^{1}$
\end{abstract}

\section{Einleitung}

Das Verhältnis des Lexikons zur Grammatik ist nach gängigen Annahmen nicht nur durch den Gegensatz idiosynkratisch vs. regelhaft geprägt, sondern auch durch die Unterscheidung zwischen Wörtern und Syntagmen. Tatsächlich werden die beiden Gesichtspunkte \pm Regelhaftigkeit und \pm Wortstatus bei der Abgrenzung des Lexikons von der Grammatik oft nicht genau getrennt. Linguisten gehen häufig bequemerweise davon aus, dass das Lexikon als das Inventar der nicht vollständig regelhaften Einheiten aus Wörtern besteht - also eben der Wortschatz ist - und dass die Grammatik die Domäne der Syntagmen ist.

Deshalb erscheint es angebracht, im Rahmen einer Diskussion des Verhältnisses des Lexikons zur Grammatik noch einmal einen Blick auf die Unterscheidung von Wörtern und Syntagmen zu werfen, zumal diese ja weiteren umstrittenen Kompartmentalisierungen der Sprachwissenschaft

\footnotetext{
Für einsichtsvolle und anregende Kommentare danke ich den Teilnehmern der Jahrestagung des IDS. Viele interessante Hinweise erhielt ich auch an den Universitäten München und Tübingen, wo ich Vorfassungen des Textes vortragen durfte. Zu danken habe ich schließlich den Herausgebern dieses Bandes, die eine Reihe von nützlichen Verbesserungsvorschlägen gemacht haben. Nach Abschluss des Manuskripts brachte mir Martin Haspelmath seinen Aufsatz „The indeterminacy of word segmentation and the nature of morphology and syntax" zur Kenntnis, der in Folia Linguistica erscheinen wird. Haspelmath kommt aus allgemein-sprachwissenschaftlicher Sicht zu ähnlichen Ergebnissen wie ich. Leider konnte ich diese wichtige Arbeit hier nicht mehr berücksichtigen.
} 
zugrunde liegt, insbesondere der Einteilung in Morphologie (als Lehre von den komplexen Wörtern), Syntax (als Lehre von den Syntagmen) und Phraseologie (als Lehre von den verfestigten Syntagmen).

Dass die Trennung der Wörter von den Syntagmen schwierig ist, ist nicht neu. Es ist geradezu ein linguistischer Gemeinplatz, dass man keine klare Grenze zwischen diesen Ausdrucksstufen ziehen kann. In auffallendem Gegensatz dazu spielt jedoch die Unterscheidung zwischen Wörtern einerseits und Syntagmen oder Phrasen andererseits bis heute in der linguistischen Praxis und in vielen theoretischen Modellen eine zentrale Rolle. Das gilt sogar für konstruktionsgrammatische Ansätze, die, wie der von Croft (2001), nicht nur die Grenzziehung zwischen Grammatik und Lexikon, sondern auch viele andere traditionelle Einteilungen (etwa bei den Wortarten) in Frage stellen - aber eben nicht die Kategorien WORT und SYNTAGMA. ${ }^{2}$ Diese werden in Crofts Buch vielmehr als selbstverständlich vorausgesetzt. Eine andere repräsentative konstruktionsgrammatische Arbeit (Goldberg 2003) stellt sogar ausdrücklich fest, dass auch bei Aufhebung einer strikten Grenzziehung zwischen Lexikon und Grammatik ,the classical distinctions [nämlich zwischen Wörtern und Syntagmen, Anm. d. Verf.] retain their force“" (ebd., S. 119).

Es stellt sich aber die Frage, ob sie das wirklich tun. Ich möchte das hier, wenn auch mit Einschränkungen, verneinen. Ich werde zu zeigen versuchen, dass die einschlägigen Daten so differenziert sind, dass die Kategorien WORT und SYNTAGMA unter strengen wissenschaftlichen Standards nicht zu halten sind. Verschiedene Strategien, an ihnen festzuhalten, führen zu ihrer inhaltlichen Entleerung und damit dazu, dass sie überflüssig werden. - Am Ende will ich aber auch kurz deutlich machen, dass das Begriffspaar unter anderen Voraussetzungen durchaus brauchbar bleibt.

Insgesamt möchte ich also die theoretischen Konsequenzen der notorischen Abgrenzungsprobleme zwischen Wörtern und Syntagmen genauer herausarbeiten und dabei auch diese Probleme selbst noch einmal unter die Lupe nehmen, wobei ich mich auf Ergebnisse eines Wuppertaler DFGProjekts zur Grenzziehung zwischen Wörtern und Syntagmen stütze.

Den methodologischen Hintergrund bildet ein weiterer linguistischer Gemeinplatz, nämlich, dass die Brauchbarkeit einer Kategorisierung davon abhängt, was man mit ihr machen will. Wenn man also über Probleme der Unterscheidung von Wörtern und Syntagmen nachdenkt, sollte man sich zuerst klar machen, wozu man diese Unterscheidung verwenden will. Da gibt es mehrere Möglichkeiten, von denen ich hier nur eine, allerdings eine zentrale, genauer betrachte: Ich konzentriere mich auf die Rolle, die WORT und SYNTAGMA als metasprachliche Kategorien in einer empirisch adäquaten Beschreibung von Sprachsystemen spielen können. Dabei verstehe ich unter einer empirisch adäquaten Beschreibung des Systems einer Sprache L

Linguistische Kategorien schreibe ich in Großbuchstaben. 
eine, die sich zum Ziel setzt, die Ausdrücke von L vollständig und detailliert zu erfassen, und die sich dabei an allgemeinen wissenschaftsmethodologischen Standards, wie Widerspruchsfreiheit und Beschreibungsökonomie, orientiert. Diesem Konzept von empirischer Adäquatheit entspricht eine Norm, der sich viele Linguisten unterwerfen, indem sie bereit sind, Aussagen zu Sprachsystemen zu revidieren, wenn diese die Verhältnisse offensichtlich unvollständig, zu wenig detailliert, widersprüchlich oder unökonomisch wiedergeben. „Empirisch adäquate Sprachsystembeschreibung“ kürze ich durch „EAS“ ab.

\section{WORT und SYNTAGMA als reiche Kategorien}

Attraktiv, aber dann auch problematisch für EAS-Analysen sind WORT und SYNTAGMA vor allem deshalb, weil sie idealiter reiche Kategorien sind, also solche, aus deren Zutreffen sich eine große Zahl weiterer Eigenschaften vorhersagen lässt. So verbindet man mit der Kategorisierung eines sprachlichen Ausdrucks X als Wort die Erwartung, dass damit ein ganzes Cluster von Eigenschaften von X erfasst wird, anders als bei Kategorien wie STIMMHAFT oder MASKULINUM, die kaum mehr als eine einzige Eigenschaft festhalten. Dass solche reichen Kategorien in EAS-Zusammenhängen attraktiv sind, ergibt sich einerseits aus dem Streben nach detaillierter Beschreibung, andererseits aus dem Ziel, ökonomisch vorzugehen, erlauben es solche Kategorien doch, viele Fliegen mit einer Klappe zu schlagen. Bei den Kategorien WORT und SYNTAGMA kommt noch ein weiterer im EAS-Kontext wünschenswerter Zug dazu. Sie erfassen zusammen mit wenigen anderen Ausdrucksstufen (wie AFFIX) idealiter die Gesamtheit aller Ausdrücke der untersuchten Sprache: Wenn ein Ausdruck kein Wort ist, ist er, wenn er ,kleiner' ist, ein Affix, wenn er größer' ist, ein Syntagma.

Der Eindruck, man könne mit wenigen Stufenkategorien die Ausdrücke der jeweiligen Sprache vollständig abdecken und dabei gleichzeitig auf ökonomische Weise viele ihrer Details erfassen, ist wohl auch ein Grund dafür, dass man gerade diese Kategorien zur Definition ganzer Teilgebiete der Sprachbeschreibung - Morphologie, Syntax, Phraseologie - heranzieht. Zusätzlich manifestiert sich darin aber auch die Annahme, dass sich die Ausdrücke auf diesen verschiedenen Stufen so deutlich voneinander unterscheiden, dass es sich lohnt, sie separaten Teilgebieten zuzuweisen.

Konkretisieren wir das an den Stufenkategorien WORT und SYNTAGMA, so wie sie auf das Deutsche angewandt werden. Mit diesen Kategorien assoziieren Grammatiker des Deutschen mindestens die in dem Stufensystem 1 angegebenen Eigenschaften, wobei die Abkürzungen für die darunter folgenden Eigenschaftsdimensionen stehen: ${ }^{3}$

Die Eigenschaften sind in der Regel auf Wortformen, nicht auf Wortlexeme (also Zusammenfassungen von Wortformen mit gemeinsamen Eigenschaften) zu beziehen. 


\begin{tabular}{|l|c|c|}
\cline { 2 - 3 } \multicolumn{1}{c|}{} & WORT & SYNTAGMA \\
\hline DO-SYLB & + & - \\
\hline DO-PHONOTAKT & + & - \\
\hline DO-MINPHON & + & + \\
\hline INTERN-SPAT & - & + \\
\hline INTERN-MAJUSK & - & - \\
\hline DO-MINGRAPH & + & + \\
\hline FLEX-TEIL & - & - \\
\hline DERIV-BAS & + & - \\
\hline KOMP-BAS & + & + \\
\hline LK-TEIL & - & + \\
\hline VF-TEIL & - & + \\
\hline E-TEIL & - & \\
\hline
\end{tabular}

Tab. 1: System 1

$\begin{array}{ll}\text { DO-SYLB: } & \text { Domäne für Syllabierung } \\ \text { DO-PHONOTAKT: } & \text { Domäne für die phonotaktischen Beschränkungen B1 } \\ \text { DO-MINPHON: } & \text { Domäne für die phonologischen Minimalitätsbedingungen B2 } \\ \text { INTERN-SPAT: } & \text { Interne Spatien } \\ \text { INTERN-MAJUSK: } & \text { Interne Majuskeln } \\ \text { DO-MINGRAPH: } & \text { Domäne für die graphematischen Minimalitätsbedingungen B3 } \\ \text { FLEX-TEIL: } & \text { Separate Flexion von Teilgliedern } \\ \text { DERIV-BAS: } & \text { Basis für die Derivationsmuster M1 } \\ \text { KOMP-BAS: } & \text { Basis für die Kompositionsmuster M2 } \\ \text { LK-TEIL: } & \text { In die linke Satzklammer bewegbares Teilglied } \\ \text { VF-TEIL: } & \text { In das Vorfeld bewegbares Teilglied } \\ \text { E-TEIL: } & \text { Durch Modifikation, Komplementierung, Determination oder } \\ & \text { Koordination erweiterbare Teilglieder }\end{array}$

Die in System 1 angegebenen Werteverteilungen in diesen Eigenschaftsdimensionen entsprechen gängigen Annahmen über Unterschiede zwischen den Stufen: In den ersten drei Dimensionen kommt zum Ausdruck, dass Wörter eine andere lautliche Form haben als Syntagmen. Die nächsten drei halten Entsprechendes für die schriftliche Form fest. Es folgen drei Dimensionen, denen zufolge Flexion Teilausdrücke von Syntagmen, aber nicht von Wörtern betreffen kann und Derivations- und Kompositionsprozesse auf Wörtern, aber nicht auf Syntagmen operieren. Die Werteverteilungen in den folgenden drei Eigenschaftsdimensionen bringen zum Ausdruck, dass auch Bewegungs- und Erweiterungsprozesse keine Wortteile, sondern nur Teile von Syntagmen betreffen können. Diese Annahme ist unter der 
Bezeichnung „lexikalische Integrität“ populär (ein schöner Beleg für die eingangs erwähnte Tendenz, die Begriffe ,lexikalisch“ und ,auf Wörter bezogen' gleichzusetzen).

Jedem der vier Blöcke ließen sich weitere Eigenschaftsdimensionen hinzufügen, dem letzten etwa die Möglichkeit weiterer Dislozierungprozesse oder das Auftreten interner Rektions- oder Kongruenzbeziehungen. Es fehlen auch Eigenschaften, die zu keinem der Blöcke gehören, wie die lexikalische Reihenbildung, ${ }^{4}$ die in ausgeprägter Form nur bei Wörtern, nicht bei Syntagmen aufzutreten scheint. Auch semantische Eigenschaften, die als diagnostisch für die Unterscheidung von Wörtern und Syntagmen betrachtet werden, fehlen in der Liste, etwa die Referentialität von Teilgliedern. Und schließlich verzeichnet die Liste keine Eigenschaften, durch die sich typische Wörter und Syntagmen nicht voneinander, sondern gemeinsam von anderen Stufen, insbesondere von Affixen, unterscheiden. Dazu gehört die variable Stellung im Satz.

Dennoch ist die Liste umfangreich genug, um an ihr Glanz und Elend der Stufenkategorien sichtbar zu machen. Zunächst zum Glanz: Man sieht sofort, dass die Kategorien WORT und SYNTAGMA tatsächlich eine Fülle von Eigenschaften ihrer Elemente festhalten. Und weil die vielen Eigenschaftsdimensionen je gegensätzliche Werte annehmen, werden die Elemente der Kategorien als deutlich verschieden beschrieben, wie es sein sollte, wenn man Wörtern und Syntagmen eigene Teilgebiete der Sprachwissenschaft widmen will. Dafür, dass sich eine solche Gebietstrennung lohnt, spricht auch, dass jede der beiden Kategorien anscheinend eine riesige Menge von Ausdrücken umfasst, was in (1) nur an ganz wenigen Beispielen angedeutet ist:
a. WORT: sprechen, spricht, besprechen, Sprache, ...
b. SYNTAGMA: laut sprechen, mit Gerda sprechen, lebendige Sprache, ...

Man erkennt aber auch gleich Einschränkungen. So ist klar, dass die Kategorien WORT und SYNTAGMA, wie sie hier spezifiziert sind, nicht auf alle Sprachen übertragbar sind. Viele Sprachen haben ja kein Schriftsystem mit Spatien oder gar mit satzinternen Majuskeln, und auch die linke Satzklammer und das Vorfeld sind spezifisch deutsch. Die in der allgemeinen Sprachwissenschaft lang diskutierte Frage, ob die Kategorien WORT und SYNTAGMA universell sind, muss also hinsichtlich der konstitutiven Eigenschaftsdimensionen - und erst recht im Hinblick auf die konkreten Eigenschaften - verneint werden. Allenfalls gibt es Universalität auf der Ebene der Blöcke, zu denen wir die Dimensionen zusammengefasst haben.

Darunter verstehe ich die Aufnahme einer größeren Zahl von nach demselben Muster gebildeten Ausdrücken in den Bestand der usualisierten Ausdrücke der jeweiligen Sprache. 
Vielleicht haben also alle Sprachen Wörter im Sinn von Einheiten mit einer sprachspezifischen lautlichen und gegebenenfalls schriftlichen Form, die Basis für die in der Sprache möglichen morphologischen Prozesse sind, aber den möglichen Umstellungs- und Erweiterungsprozessen den Zugang verwehren, und die sich durch diese Eigenschaften von anderen Einheiten, eben denen, die in der Sprache als Syntagmen gelten, unterscheiden. ${ }^{5}$ Diese Relativierung auf die Spezifika einzelner Sprachen impliziert, dass das Wort in Sprachen, in denen nicht alle Eigenschaftsdimensionen einschlägig sind, blassere Konturen hat. So ist das Wort im Chinesischen, das keine Schrift mit Worttrennungszeichen und kaum morphologische Prozesse hat, wohl keine sehr prägnante Einheit. ${ }^{6}$

Das Problem fehlender Einschlägigkeit von Stufenkriterien, wie ich die Eigenschaftsdimensionen auch nenne, manifestiert sich aber nicht nur im Sprachvergleich, sondern auch in der Analyse von Einzelsprachen. Mehrere unserer Kriterien lassen sich ja nicht auf alle Ausdrucksklassen des Deutschen anwenden: Das Majuskelkriterium läuft leer, wenn es keine nominalen Teilausdrücke gibt, das Flexionskriterium kann man nicht auf Partikeln anwenden, die Bewegungskriterien sind nur für verbale Projektionen einschlägig, usw. Wie geht man z.B. mit einem Ausdruck wie sehr gern um, für den, weil er nur aus Partikeln besteht, weder das Majuskelkriterium noch das Flexionskriterium, noch die Bewegungskriterien einschlägig sind? Üblicherweise zieht man in solchen Fällen nur die einschlägigen Kriterien in Betracht, hier etwa das Spatienkriterium und die phonologischen Kriterien, nach denen sehr gern ein Syntagma ist. Um keine zu exotische Position einzunehmen, schließe ich mich dieser Praxis an. Festzuhalten ist aber, dass die Kategorien WORT und SYNTAGMA infolge der Nicht-Einschlägigkeit von Kriterien bei vielen Ausdrücken nicht so viele Eigenschaften voraussagen, wie man das angesichts der umfangreichen Liste in Tabelle 1 zunächst erwarten würde.

\section{Abweichungen vom Idealfall und die Aufspaltung des Stufensystems}

Doch auch, wenn man nur die je einschlägigen Kriterien betrachtet, findet man häufig nicht die idealen Werteverteilungen aus System 1. Ich betrachte nun einige Hauptgruppen dieser nicht-idealen Fälle und stelle gleichzeitig eine naheliegende und (bis zu einem bestimmten Punkt) populäre Strategie des Umgangs mit solchen Fällen vor.

Einen guten Überblick über den heutigen Stand der allgemein-sprachwissenschaftlichen Diskussion über den Status der Kategorie WORT gibt Dixon/Aikhenvald (Hg.) (2002).

Tatsächlich ist für das Chinesische bestritten worden, dass es überhaupt Wörter hat, vgl. z.B. Hockett (1944). 
3.1 Abweichungen im Hinblick auf phonologische Kriterien

Viele Ausdrücke weichen in phonologischen Stufenkriterien vom Idealfall ab. Dazu gehören sämtliche Komposita. Diese verhalten sich in der Fuge hinsichtlich der Syllabierung und oft auch phonotaktisch so, wie es von Syntagmen zu erwarten wäre, obwohl sie nach vielen anderen Kriterien Wörter sind, vgl. im Hinblick auf die Syllabierung z.B. [blu:t. so:t] vs. *[blur.tво:t] blutrot, im Hinblick auf die Phonotaktik z.B. [ba:n.kundə] vs. *[ba:n.kundə] Babnkunde. ${ }^{7}$

Das Problem ist bekannt, und bekannt ist auch eine bestimmte Lösung: Man spaltet von den ursprünglichen Stufenkategorien neue Kategorien ab, in denen die phonologischen Kriterien von den anderen Kriterien getrennt werden. Mit diesen neu definierten Stufen PHONOLOGISCHES WORT und PHONOLOGISCHES SYNTAGMA (P-WORT bzw. P-SYNTAGMA) kann unser Stufensystem wie in Tabelle 2 erweitert werden:

\begin{tabular}{|l|c|c|}
\cline { 2 - 3 } \multicolumn{1}{c|}{} & WORT & SYNTAGMA \\
\hline INTERN-SPAT & - & + \\
\hline INTERN-MAJUSK & - & + \\
\hline DO-MINGRAPH & + & + \\
\hline FLEX-TEIL & - & - \\
\hline DERIV-BAS & + & - \\
\hline KOMP-BAS & + & + \\
\hline LK-TEIL & - & + \\
\hline VF-TEIL & - & + \\
\hline E-TEIL & - & - \\
\hline
\end{tabular}

\begin{tabular}{|l|c|c|}
\cline { 2 - 3 } \multicolumn{1}{c|}{} & P-WORT & P-SYNTAGMA \\
\hline DO-SYLB & + & - \\
\hline DO-PHONOTAKT & + & - \\
\hline DO-MINPHON & + & - \\
\hline
\end{tabular}

Tab. 2: System 2

Ein wichtiges Merkmal dieses Kategorisierungssystems ist, dass die neuen mit den ursprünglichen Stufen widerspruchsfrei kombinierbar sind, da die beiden Kategorienpaare auf unterschiedlichen Kriterien beruhen. Das System erlaubt also Kreuzklassifikationen wie in (2):

Auch viele deutsche Präfix-Bildungen verhalten sich phonologisch wie Syntagmen, nach den meisten anderen Kriterien aber wie Wörter. 
(2) a. WORT, P-WORT: sprechen, befragen, Frage, ...

b. WORT, P-SYNTAGMA: Babnkunde, blutrot, ...

c. SYNTAGMA, P-SYNTAGMA: schnell sprechen, Gerda befragen, viele schwierige Fragen, ...

Die Vorteile dieser Lösung, die sie so beliebt gemacht haben, liegen auf der Hand: Man kann, soweit bisher erkennbar, nun auch die Problemfälle vollständig und widerspruchsfrei einordnen und dabei ihre Eigenschaften detailliert erfassen.

Für diese Vorteile von System 2 muss man allerdings eine Verarmung der einzelnen Stufen in Kauf nehmen. Jede der Stufen in System 2 sagt weniger Eigenschaften voraus als die beiden ursprünglichen Stufen in System 1.

Diese geringere Prädiktivität der Stufen hat Linguisten aber kaum zu denken gegeben, denn dass man für lautliche Zusammenhänge separate Kategorien braucht, erscheint ganz normal. Tatsächlich wird die Kategorie P-WORT oft völlig losgelöst von der ursprünglichen Kategorie WORT betrachtet, nämlich als rein prosodische Einheit, die hierarchisch über den prosodischen Einheiten Silbe und Fuß steht. ${ }^{8}$ Aus dieser Sicht ist allerdings kaum erklärlich, warum die Grenzen von P-Wörtern immer Morphemgrenzen sind, ${ }^{9}$ während Silben und Füße nicht mit inhaltlichen Grenzen koinzidieren müssen. Wenn man dagegen im Blick behält, dass das P-Wort aus dem ursprünglichen Wortbegriff abgespalten wurde, um bestimmte nicht-ideale Fälle zu erfassen, versteht man das: Die Grenzen von Wörtern - seien sie nun ideal oder nicht - sind ja stets Morphemgrenzen (weil Wörter auf jeden Fall Ausdrücke sind, also aus mindestens einem Morphem bestehen). ${ }^{10}$

Ein weiterer unschöner Zug des erweiterten Stufensystems ist, dass die phonologischen Eigenschaften, die das P-Wort nach System 2 definieren, manchmal nicht in der vorgesehenen Kombination auftauchen. So verhalten sich schwache Formen vieler Funktionswörter hinsichtlich der Syllabierung wie P-Wörter, im Hinblick auf Minimalitätsbedingungen jedoch nicht: Es heißt [n.?i:gəl] ' $n$ Igel und nicht [ni:.gəl], der schwache Artikel ist also eine eigene Syllabierungsdomäne. Sein Umfang erreicht jedoch nicht das für P-Wörter (nach Hall 1999b) erforderliche Minimum einer zweimorigen Silbe.

\footnotetext{
Vgl. z.B. Hall (1999a).

Ebd., S. 2.

Auf die gleiche Weise kann man erklären, dass auch die Grenzen von P-Syntagmen stets mit Morphemgrenzen koinzidieren.
} 
Dieses Problem ist Phonologen durchaus bekannt, ${ }^{11}$ es gibt aber keine allgemein akzeptierte Lösung. Manche Forscher ordnen problematische Fälle wie die schwachen Funktionswörter gar keiner Stufe mehr zu, ${ }^{12}$ geben also die Idee preis, dass das Stufensystem die Ausdrücke der Sprache vollständig abdecken soll. Andere führen für die Problemfälle zusätzliche Stufen ein, etwa P-KLITIKON, ${ }^{13}$ womit sie Vollständigkeit sichern, aber die einzelnen Stufen in ihrer Aussagekraft weiter reduzieren.

\subsection{Abweichungen im Hinblick auf graphematische Kriterien}

Probleme dieser Art treten auch auf der graphematischen Ebene auf. Divergenzen zwischen dem Spatienkriterium und anderen Stufenkriterien sind schon lange bemerkt, aber erst vor kurzem detailliert analysiert worden. ${ }^{14}$ Sie betreffen u.a. die phonologischen Kriterien: $\mathrm{Ob}$ wir zwischen zwei Ausdrücke ein Spatium setzen, ist weitgehend unabhängig davon, ob zwischen ihnen eine P-Wort-Grenze liegt. Oft fehlen Spatien, wo es P-Wort-Grenzen gibt, so bei sämtlichen Komposita, und manchmal setzen wir ein Spatium, wo nur einer der separierten Ausdrücke ein zweifelsfreies P-Wort ist, so im erwähnten Beispiel <'n Igel> (vs. ${ }^{*}<$ 'nigel $>$ ). Aber auch vielen nicht-phonologischen Kriterien läuft die Spatiensetzung zuwider, etwa den Bewegungskriterien. So schreiben wir Partikelverben ohne Spatium, obwohl ihre Teile durch Bewegung in die linke Satzklammer getrennt werden können, z.B. $<$ abspricht $>$ vs. $*<$ ab spricht $>$.

Diese vielen Divergenzen beruhen darauf, dass die deutsche Spatiensetzung, so wie sie sich historisch herausgebildet hat, bei weitem nicht für alle anderen Wortkriterien sensitiv ist. Das gilt besonders für die Getrenntschreibung, die überhaupt nicht auf Wortkriterien beruht, sondern auf dem Prinzip, dass zwischen Ausdrücken, also bedeutungshaltigen Abschnitten aller Art, ein Spatium zu setzen ist. Dieses Prinzip wird nur dann durch Zusammenschreibung von Ausdrücken verletzt, wenn deren Verbindung sich nach einigen wenigen Kriterien wie ideale komplexe Wörter verhält, etwa nach den morphologischen Kriterien. Für viele andere Kriterien, etwa die phonologischen oder die Bewegungskriterien, ist die Zusammenschreibung dagegen völlig blind. ${ }^{15}$

$11 \quad$ Vgl. z.B. Hall (1999a), Raffelsiefen (1999).

12 So Hall (1999b), bei dem die schwachen (und auch die starken) Formen von Funktionswörtern direkt von einer höheren prosodischen Stufe (der P-Phrase, entspricht unserem P-Syntagma) dominiert werden, ohne selbst einer Stufe zugeordnet zu werden.

Vgl. z.B. Nespor/Vogel (1986).

Vgl. Jacobs (2005), Fuhrhop (2007).

Vgl. Jacobs (2005, Kap. 4.1.4.). Im Lichte dieser Überlegungen ist der Behauptung von Nübling et al. (2008) zu widersprechen, dass es in der Geschichte des deutschen Schriftsystems Entwicklungen gegeben habe, die dazu beitrugen „dass die Grenzen des phonologischen Worts auch in der Schrift erkennbar sind“ (ebd., S. 40). Nübling et al. erwähnen in diesem 
Was nun die daraus resultierenden Probleme für das Stufensystem betrifft, gehen viele Linguisten von einer Lösung aus, wie wir sie schon bei den phonologischen Kriterien gesehen haben: Man lagert die graphematischen Kriterien in ein zusätzliches Stufenpaar GRAPHEMATISCHES WORT vs. GRAPHEMATISCHES SYNTAGMA (G-WORT bzw. G-SYNTAGMA) aus, so wie in System 3: ${ }^{16}$

\begin{tabular}{|l|c|c|}
\cline { 2 - 3 } \multicolumn{1}{c|}{} & WORT & SYNTAGMA \\
\hline FLEX-TEIL & - & + \\
\hline DERIV-BAS & + & - \\
\hline KOMP-BAS & + & + \\
\hline LK-TEIL & - & + \\
\hline VF-TEIL & - & + \\
\hline E-TEIL & - & + \\
\hline
\end{tabular}

\begin{tabular}{|l|c|c|}
\cline { 2 - 3 } \multicolumn{1}{c|}{} & P-WORT & P-SYNTAGMA \\
\hline DO-SYLB & + & - \\
\hline DO-PHONOTAKT & + & - \\
\hline DO-MINPHON & + & - \\
\hline
\end{tabular}

\begin{tabular}{|l|c|c|}
\cline { 2 - 3 } \multicolumn{1}{c|}{} & G-WORT & G-SYNTAGMA \\
\hline INTERN-SPAT & - & + \\
\hline INTERN-MAJUSK & - & + \\
\hline DO-MINGRAPH & + & - \\
\hline
\end{tabular}

Tab. 3: System 3

Diese Lösung hat die gleichen Vorteile wie die Einführung des P-Worts: Die mit ihr mögliche Kreuzklassifikation, wie in (3), scheint eine widerspruchsfreie und vollständige Erfassung auch der problematischen Fälle zu ermöglichen und hält deren Eigenschaften genau fest:

Zusammenhang allerdings nicht die Spatiensetzung, sondern die Konstantschreibung von Morphemen und die Substantivgroßschreibung. Beide Phänomene haben jedoch wenig mit phonologischen Wörtern zu tun. (Wenn es in der Spatiensetzung einen historischen Trend zur besseren Kennzeichnung von P-Wörtern gegeben hätte, wäre u.a. der Aufbau einer konsequenten Getrenntschreibung von Kompositionsgliedern und Präfixen zu erwarten gewesen. Faktisch ist jedoch gerade die Zusammenschreibung solcher Elemente gestärkt worden.)

16 Eine repräsentative Arbeit zum graphematischen Wort ist Fuhrhop (2008). 
(3) a. WORT, P-WORT, G-WORT: sprechen, befragen, Frage, ...

b. WORT, P-SYNTAGMA, G-WORT:

Babnkunde, blutrot, ...

c. SYNTAGMA, ${ }^{17}$ P-SYNTAGMA, G-WORT:

absprechen, aussprechen, ...

d. SYNTAGMA, P-SYNTAGMA, G-SYNTAGMA:

schnell sprechen, Gerda befragen, viele schwierige Fragen, ...

System 3 geht aber auch mit einer weiteren Verarmung der Stufen einher. Vor allem die Basisstufen WORT und SYNTAGMA sagen nun noch weniger Eigenschaften voraus als in System 2. Auch hier könnte man sich aber damit trösten, dass das Schriftsystem eine eigene Repräsentationsebene ist, die eigene Kategorien erfordert, wie das für die graphematische Silbe etwa in Primus (2003) gezeigt wurde.

Leider erfassen die neuen Stufen aber wieder nicht alle Fälle. Divergenzen zwischen graphematischen Wortkriterien gibt es z.B. bei Akronymen wie $<$ DDR $>$, $<$ DFG $>$, $<$ SPD $>$. Nach dem Spatienkriterium sind sie G-Wörter, nach dem Majuskelkriterium nicht, und sie verletzen auch die Bedingung, dass G-Wörter (nach Fuhrhop 2008) minimal aus einer graphematischen Vollsilbe bestehen. Um angesichts solcher Fälle eine vollständige und widerspruchsfreie Stufenzuordnung zu sichern, bietet es sich an, die Kategorie G-WORT weiter aufzuspalten, aber das führt dann zu einer noch weiteren Verarmung der einzelnen Stufen.

\subsection{Abweichungen im Hinblick auf morphologische Kriterien}

Eine weitere Verarmung ist aber bei Beibehaltung der Aufspaltungsstrategie ohnehin nicht zu vermeiden, wie die vielfältigen Probleme mit den Kriterien FLEX-TEIL, DERIV-BAS und KOMP-BAS zeigen. So gibt es separate Flexion von Teilgliedern nicht nur bei eindeutigen Syntagmen, sondern auch bei Ausdrücken, die nach vielen anderen Kriterien Wörter sind, etwa bei $\mathrm{V}+\mathrm{V}$-Komposita, wie gefriertrocknen oder rübrbraten, bei denen das Partizip-IIPräfix ge- und die Infinitiv-Markierung zu wie bei verbalen Syntagmen am Zweitglied ansetzen, vgl. gefrierzutrocknen vs. ?? zu gefriertrocknen, rübrgebraten vs. *gerührbraten. Noch bekanntere Beispiele für wortartige Ausdrücke mit separater Flexion von Teilen (also mit Binnenflexion) findet man unter den Zusammenrückungen, wie der Hohepriester, die Hohenpriester.

Dieses Phänomen kann man nicht auf fehlenden phonologischen Wortstatus zurückführen. Zwar sind bei separater Flexion meist mehrere phonologische Wörter im Spiel, aber bei weitem nicht alle Ausdrücke, die mehr als

17 Die Einordnung von Partikelverben als Syntagmen wird noch zu überdenken sein, siehe unten. 
ein phonologisches Wort umfassen, zeigen separate Flexion. So werden Präfixverben, auch wenn sie aus zwei phonologischen Wörtern bestehen, als ganze flektiert, vgl. zu überlassen vs. *überzulassen.

Es bleibt also im Rahmen der bisher angewandten Strategie nichts anderes übrig, als ein weiteres Stufenpaar FLEXIONSMORPHOLOGISCHES WORT vs. FLEXIONSMORPHOLOGISCHES SYNTAGMA (F-WORT bzw. F-SYNTAGMA) anzunehmen, wie in System 4:

\begin{tabular}{|l|c|c|}
\cline { 2 - 3 } \multicolumn{1}{c|}{} & WORT & SYNTAGMA \\
\hline DERIV-BAS & + & - \\
\hline KOMP-BAS & + & - \\
\hline LK-TEIL & - & + \\
\hline VF-TEIL & - & + \\
\hline E-TEIL & - & + \\
\hline
\end{tabular}

\begin{tabular}{|l|c|c|}
\cline { 2 - 3 } \multicolumn{1}{c|}{} & P-WORT & P-SYNTAGMA \\
\hline DO-SYLB & + & - \\
\hline DO-PHONOTAKT & + & - \\
\hline DO-MINPHON & + & - \\
\hline
\end{tabular}

\begin{tabular}{|l|c|c|}
\cline { 2 - 3 } \multicolumn{1}{c|}{} & G-WORT & G-SYNTAGMA \\
\hline INTERN-SPAT & - & + \\
\hline INTERN-MAJUSK & - & + \\
\hline DO-MINGRAPH & + & - \\
\hline
\end{tabular}

\begin{tabular}{|l|c|c|}
\cline { 2 - 3 } \multicolumn{1}{c|}{} & F-WORT & F-SYNTAGMA \\
\hline FLEX-TEIL & - & + \\
\hline
\end{tabular}

Tab. 4: System 4

In dem System in Tabelle 4 bringen wir unsere Problemfälle unter, wie (4) andeutet:

(4) a. WORT, P-WORT, G-WORT, F-WORT: sprechen, befragen, Frage, ...

b. WORT, P-SYNTAGMA, G-WORT, F-WORT:

Babnkunde, blutrot, ...

c. WORT, P-SYNTAGMA, G-WORT, F-SYNTAGMA:

rührbraten, Hohepriester, ... 
d. SYNTAGMA, P-SYNTAGMA, G-WORT, F-SYNTAGMA: absprechen, aussprechen, ...

e. SYNTAGMA, P-SYNTAGMA, G-SYNTAGMA, F-SYNTAGMA: schnell sprechen, Gerda befragen, viele schwierige Fragen, ...

Aber natürlich zahlen wir wieder den Preis einer weiteren Verarmung einzelner Stufen, hier schon bis zu dem Extrem, dass ein Stufenpaar, eben F-WORT vs. F-SYNTAGMA, nur noch eine einzige Eigenschaft voraussagt.

Vielleicht ist das ein Grund dafür, dass das F-WORT als eigene Stufe zwar tatsächlich vorgeschlagen wurde, ${ }^{18}$ sich aber, anders als das P- und das G-WORT, nicht durchgesetzt hat. Dabei dürfte auch eine Rolle gespielt haben, dass sich die Flexionsmorphologie im Gegensatz zur Phonologie und zur Graphematik kaum als separate Repräsentationsebene konzipieren lässt, für die ohnehin eigene Kategorien anzunehmen sind. (Flexionsmorphologische Kategorien sind ja nach üblichem Verständnis eng verwoben mit der Syntax und auch mit anderen morphologischen Kategorien.)

Man kann das Problem auch nicht dadurch verkleinern, dass man statt der bettelarmen Kategorie des F-WORTS eine etwas reichere annimmt, die wenigstens noch die anderen morphologischen Kriterien, DERIV-BAS und KOMP-BAS, umfasst. Dagegen sprechen die Daten: Als Basen für einschlägige Derivations- und Kompositionsprozesse können auch Ausdrücke mit separat flektierten Teilen dienen, etwa viele Partikelverben, die z.B. bei der Adjektivierung mit -bar einen großen Teil der Basen ausmachen, vgl. anfechtbar, auffindbar, einstellbar.

Dieses Beispiel zeigt im Übrigen, dass die stammbildungsmorphologischen Kriterien nicht nur mit dem flexionsmorphologischen, sondern auch mit syntaktischen Stufenkriterien konfligieren können, denn Partikelverben sind auch nach Bewegungskriterien Syntagmen. Also müssen wir auch die stammbildungsmorphologischen Kriterien von den syntaktischen trennen und bei Beibehaltung der bisherigen Strategie für sie ein weiteres spezielles Stufenpaar einführen. Damit erhalten wir das Stufensystem in Tabelle 5 und die in (5) illustrierte Kreuzklassifikation.

Und wieder sinkt die Aussagekraft der einzelnen Stufen. Dass die neuen Stufen WB-WORT und WB-SYNTAGMA nur geringes prädiktives Potenzial haben, hat im Übrigen einen weiteren Grund, der in der einleitenden Formulierung der entsprechenden Stufenkriterien schon angedeutet ist: Nicht alle Derivationsmuster sind sensitiv dafür, ob ihre Basen Worteigenschaften haben. Es gibt solche wie die -bar-Adjektivierung, die tatsächlich außer Partikelverben nichts als Basis akzeptieren, was deutliche Züge eines Syntagmas hat. Selbst Inkorporationsbildungen, die sonst den Partikelverben ähneln, können nach Untersuchungen in dem erwähnten Wuppertaler Forschungsprojekt kaum adjektiviert werden, z.B. ??bloßstellbar, ??kennenlernbar,

18 So in Wurzel (2000). 
*gefangennehmbar ${ }^{19}$ Es gibt aber auch Muster wie die -er-Nominalisierung, die bereitwillig Syntagmen als Basen akzeptieren, man denke an bekannte Beispiele wie Schwarsseher oder Wichtigtuer, bei denen man die Annahme eines Syntagmas als Basis nur durch die Ad-hoc-Postulierung einer vorgängigen Konversion des Zweitglieds vermeiden kann.

\begin{tabular}{|l|c|c|}
\cline { 2 - 3 } \multicolumn{1}{c|}{} & WORT & SYNTAGMA \\
\hline LK-TEIL & - & + \\
\hline VF-TEIL & - & + \\
\hline E-TEIL & - & + \\
\hline
\end{tabular}

\begin{tabular}{|l|c|c|}
\cline { 2 - 3 } \multicolumn{1}{c|}{} & P-WORT & P-SYNTAGMA \\
\hline DO-SYLB & + & - \\
\hline DO-PHONOTAKT & + & - \\
\hline DO-MINPHON & + & - \\
\hline
\end{tabular}

\begin{tabular}{|l|c|c|}
\cline { 2 - 3 } \multicolumn{1}{c|}{} & G-WORT & G-SYNTAGMA \\
\hline INTERN-SPAT & - & + \\
\hline INTERN-MAJUSK & - & + \\
\hline DO-MINGRAPH & + & - \\
\hline
\end{tabular}

\begin{tabular}{|l|c|c|}
\cline { 2 - 3 } \multicolumn{1}{c|}{} & F-WORT & F-SYNTAGMA \\
\hline FLEX-TEIL & - & + \\
\hline
\end{tabular}

\begin{tabular}{|l|c|c|}
\cline { 2 - 3 } \multicolumn{1}{c|}{} & WB-WORT & WB-SYNTAGMA \\
\hline DERIV-BAS & + & - \\
\hline KOMP-BAS & + & - \\
\hline
\end{tabular}

Tab. 5: System 5

(5) a. WORT, P-WORT, G-WORT, F-WORT, WB-WORT: sprechen, befragen, Frage, ...

b. WORT, P-SYNTAGMA, G-WORT, F-WORT, WB-WORT: Babnkunde, blutrot, ...

c. WORT, P-SYNTAGMA, G-WORT, F-SYNTAGMA, WB-WORT: ${ }^{20}$ rührbraten, Hohepriester, ...

$19 \quad$ Entsprechende Beispiele wurden von kaum mehr als 30\% unserer Informanten für sprachlich richtig gehalten, im Gegensatz zu Partikelverben mit -bar (wie vorlesbar), die über 80\% erreichten.

20 Die Zuordnung der genannten Beispiele zur Kategorie WB-WORT ist nicht ganz sicher. Dazu müsste genauer geprüft werden, bei welchen Derivations- und Kompositionsprozessen die Beispiele als Basen fungieren können. 
d. SYNTAGMA, P-SYNTAGMA, G-WORT, F-SYNTAGMA, WB-WORT: absprechen, aussprechen, ...

e. SYNTAGMA, P-SYNTAGMA, G-WORT, F-SYNTAGMA, WB-SYNTAGMA: gefangen nebmen, kennenlernen, ...

f. SYNTAGMA, P-SYNTAGMA, G-SYNTAGMA, F-SYNTAGMA, WB-SYNTAGMA: schnell sprechen, Gerda befragen, viele schwierige Fragen, ...

Deshalb habe ich oben das Kriterium DERIV-BAS nicht als Basisfähigkeit für Derivation allgemein, sondern als Basisfähigkeit für bestimmte Derivationsmuster formuliert, die, wie die -bar-Adjektivierung, sensitiv für Stufenkriterien sind.

Die gleiche Einschränkung gilt für die Komposition und das Kriterium KOMP-BAS. Manche Kompositionsmuster akzeptieren tatsächlich keine Basen mit Merkmalen von Syntagmen. Dazu gehören alle koordinativen Muster, vgl. Dichter-Komponist vs. * Gefeierter-Dichter-Komponist (vs. gefeierter DichterKomponist). Andere Kompositionsmuster lassen dagegen für ihr Erstglied solche Basen zu, wie die deswegen so genannte Phrasenkomposition, z.B. Graue-Schläfen-Effekt, Trimm-dich-Pfad. ${ }^{21}$ Syntagmen als Basen kommen aber z.B. auch bei der Verbstamm+N-Komposition vor, z.B. Kennenlern-Aktion, Schnellsprech-Wettbewerb. Wir haben es also auch bei der Komposition nur mit Basisfähigkeit für bestimmte Muster zu tun, und bei den über Basisfähigkeit definierten neuen Stufen WB-WORT und WB-SYNTAGMA mit Kategorien, die nicht nur auf Stammbildungsphänomene beschränkt sind, sondern diese auch nur sehr punktuell abdecken.

\subsection{Abweichungen im Hinblick auf syntaktische Kriterien}

Aufmerksame Leser ahnen schon, wie es weitergeht: Die Basisstufen WORT und SYNTAGMA, die inzwischen nur noch die Bewegungs- und Erweiterungseigenschaften LK-TEIL, VF-TEIL und E-TEIL festhalten, müssen noch weiter aufgespalten werden, wenn man an der bisherigen Strategie festhalten will. So haben nicht alle verbalen Komplexe mit einem Zweitglied, das in die linke Klammer bewegt werden kann, ein Erstglied, das man ins Vorfeld rücken kann. Vor allem bei Partikelverben divergieren diese Kriterien oft, vgl. (6)-(8) für aussprechen, einstellen und wiederkehren: ${ }^{22}$

(6) a. Er sprach sich nicht gegen diese Lösung aus.

b. * Aus sprach er sich gegen diese Lösung nicht.

$21 \quad$ Vgl. z.B. Meibauer (2003).

22 Die Akzeptabilitätsmarkierungen beruhen auf entsprechenden Informantenbefragungen. 
(7) a. Wir stellen Bewerber erst nach Absolvierung eines Eignungstests ein.

b. ??Ein stellen wir Bewerber erst nach Absolvierung eines Eignungstests.

(8) a. Eine Krise dieses Ausmaßes kehrt nicht so schnell wieder.

b. *Wieder kehrt eine Krise dieses Ausmaßes nicht so schnell.

Nun könnte man vermuten, dass es unabhängige Gründe für die geringe Akzeptabilität der b-Beispiele gibt. Dann wäre das Kriterium VF-TEIL für die fraglichen Verben möglicherweise gar nicht einschlägig, und ihre Einstufung als Syntagmen würde nicht zu einem Widerspruch mit anderen Kriterien führen. ${ }^{23}$

Wir haben in dem erwähnten Forschungsprojekt nach möglichen unabhängigen Gründen für die Nicht-Vorfeldfähigkeit der Erstglieder vieler Partikelverben gesucht, wurden aber nicht fündig. Naheliegende Vermutungen, etwa Blockaden wegen fehlender Kontrastfähigkeit oder mangelnder semantischer Transparenz, erwiesen sich als nicht stichhaltig, vgl. Heine/Jacobs/ Külpmann (2010).

Dass man fehlende Vorfeldfähigkeit in Fällen wie in (6b)-(8b) nicht auf solche Faktoren zurückführen kann, zeigt sich auch darin, dass es viele funktional ähnliche Partikelverben gibt, bei denen die beiden Bewegungskriterien nicht divergieren. Man betrachte die auf Korpusbelegen beruhenden Beispiele (9)-(11):

(9) a. Es fing alles am 2. Januar 1889 an.

b. An fing alles am 2. Januar 1889.

(10) a. Es fällt auf, dass im Schreiben der Stadtverwaltung ausdrücklich darauf bingewiesen wurde, dass ...

b. Auf fällt, dass im Schreiben der Stadtverwaltung von gestern ausdrücklich darauf hingewiesen wurde, dass ...

(11) a. Es geht schon nächste Woche los.

b. Los geht es schon nächste Woche.

Hier haben wir es mit semantisch nicht-transparenten Verben zu tun, und in naheliegenden Verwendungskontexten wird weder das Verb noch sein Erstglied mit anderen Elementen kontrastiert. Dennoch sind die b-Varianten von (9)-(11) deutlich akzeptabler als die von (6)-(8).

Das Kriterium der Vorfeldfähigkeit scheint also für Partikelverben durchaus einschlägig zu sein, muss dann aber wegen Fällen wie (6)-(8) im Stufensystem von dem anderen Bewegungskriterium LK-TEIL getrennt werden.

Und leider muss man VF-TEIL auch von dem Kriterium E-TEIL trennen, denn nicht alles, was ins Vorfeld bewegt werden kann, erlaubt auch Modifikation, Komplementierung etc. Das zeigt sich an Beispielen wie (12)-(13):

23 Vgl. die Bemerkungen in Kapitel 2 zum Umgang mit nicht einschlägigen Kriterien. 
(12) a. dass dieses Verfahren dann [sehr schnell [Schule machte]]

b. ??dass dieses Verfahren dann [sehr schnelle Schule] machte

c. Schule machte dieses Verfahren dann sehr schnell.

(13) a. dass er im Streitpunkt Studiengebühren [deutlich [Farbe bekennen]] muss

b. *dass er im Streitpunkt Studiengebübren [ deutliche Farbe] bekennen muss

c. Farbe bekannte er auch im Streitpunkt Studiengebühren.

Die nominalen Erstglieder von Schule machen und Farbe bekennen können nicht durch ein Adjektiv modifiziert werden, obwohl sie ins Vorfeld bewegt werden können. Das kann man übrigens nicht mit der mangelnden Referentialität dieser Erstglieder erklären. Nicht-referenzielle Nomina können in anderen Fällen durchaus modifiziert werden, vor allem, wenn der Modifikator, wie in (12)-(13), Skopus über den ganzen Komplex aus $\mathrm{N}$ und $\mathrm{V}$ hat. ${ }^{24}$

Wir landen nun also bei dem Stufensystem in Tabelle 6 und bei Klassifikationen wie in (14):

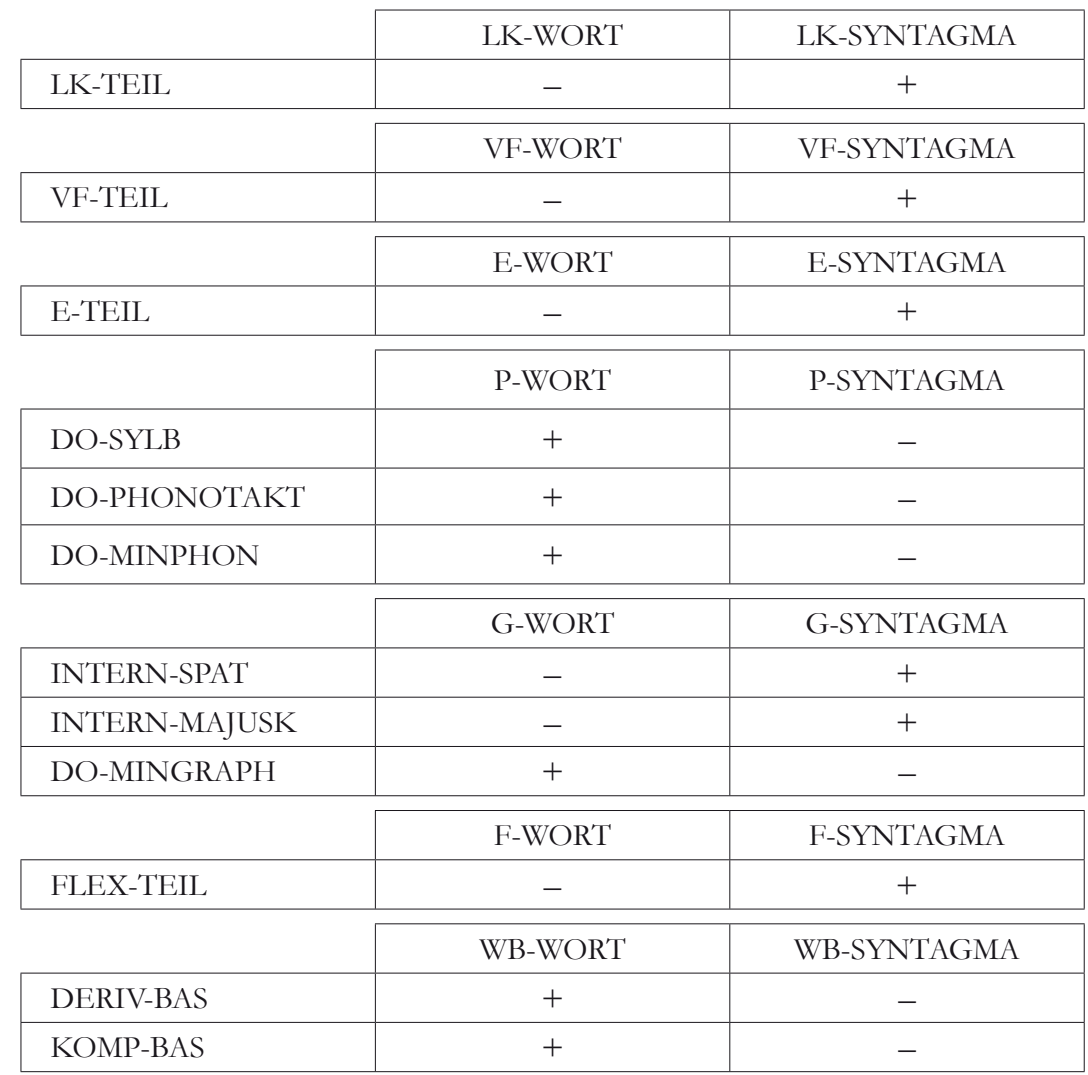

Tab. 6: System 6

$24 \quad$ Vgl. z.B. dass sie tief/ tiefen Atem bolen musste. 
(14) a. LK-WORT, VF-WORT, E-WORT, P-WORT, G-WORT, F-WORT, WB-WORT: sprechen, befragen, Frage, ...

b. LK-WORT, VF-WORT, E-WORT, P-SYNTAGMA, G-WORT, F-WORT, WB-WORT:

Babnkunde, blutrot, ...

c. LK-WORT, VF-WORT, E-WORT, P-SYNTAGMA, G-WORT, F-SYNTAGMA, WB-WORT: ${ }^{25}$ rübrbraten, Hohepriester, ...

d. LK-SYNTAGMA, VF-WORT, E-WORT, P-SYNTAGMA, G-WORT, F-SYNTAGMA, WB-WORT: absprechen, aussprechen, einstellen, ...

e. LK-SYNTAGMA, VF-SNYTAGMA, E-WORT, P-SYNTAGMA, G-WORT, F-SYNTAGMA, WB-SYNTAGMA: anfangen, gefangen nebmen, kennenlernen, ...

f. LK-SYNTAGMA, VF-SYNTAGMA, E-WORT, P-SYNTAGMA, G-SYNTAGMA, F-SYNTAGMA, WB-SYNTAGMA: Farbe bekennen, Schule machen, ...

g. LK-SYNTAGMA, VF-SYNTAGMA, E-SYNTAGMA, P-SYNTAGMA, G-SYNTAGMA, F-SYNTAGMA, WB-SYNTAGMA: schnell sprechen, Gerda befragen, viele schwierige Fragen, ...

Hier wird nun endgültig das Dilemma der Aufspaltungsstrategie deutlich: Wenn man alles widerspruchsfrei, vollständig und im Detail erfassen will, wird die Zahl der Stufen annähernd so groß wie die der grammatischen Eigenschaften, die durch sie festgehalten werden sollen. Dabei ist das Bild, das System 6 zeichnet, noch zu schön: Man erinnere sich, dass man auch das P-WORT und das G-WORT weiter aufspalten muss, um internen Divergenzen in der Phonologie bzw. Graphematik Rechnung zu tragen (vgl. 3.1 bzw. 3.2). Damit hätte man neun Stufenpaare zur Kodierung von zwölf Eigenschaftsdimensionen. Und es geht weiter in Richtung eines Eins-zu-EinsVerhältnisses zwischen Stufen und Eigenschaften, wenn man einige der Kriterien genauer betrachtet. Dann zeigt sich z.B., dass das Kriterium E-TEIL in drei Kriterien aufgespalten werden muss, da Koordinierbarkeit, Modifizierbarkeit/Komplementierbarkeit und Determinierbarkeit divergieren können. ${ }^{26}$ Und sogar das Vorfeldkriterium VF-TEIL muss differenziert werden, da die Vorfeldfähigkeit in Fällen wie (9)-(11) nur eine bedingte ist, nämlich voraussetzt, dass das zur Vorfeldpartikel gehörende verbale Zweitglied un-

25 Ob diese Beispiele WB-Wörter sind, ist nicht ganz sicher. Eine genauere Klärung muss hier jedoch unterbleiben.

26 Vgl. für Modifizierbarkeit vs. Determinierbarkeit z.B. Tiefen Atem holen vs. *Einen Atem holen. 
mittelbar in der linken Satzklammer folgt. ${ }^{27}$ Die Verteilung dieser weiter differenzierten Kriterien auf die Daten ist so, dass wieder neue Stufenpaare nötig werden. Dann hätten wir 12 Stufenpaare zur Kodierung von 14 Eigenschaftsdimensionen, d.h. jede einzelne Stufe würde im Durchschnitt weniger als 1,2 Eigenschaften voraussagen.

\section{Alternativen zur Aufspaltung des Stufensystems}

Ich fasse zusammen: Die ursprünglich mit den Ausdrucksstufen WORT und SYNTAGMA verbundene Annahme, man könne mit wenigen Kategorien die Ausdrücke der Sprache vollständig erfassen und dabei auf ökonomische Weise viele ihrer Details festhalten (vgl. 2.), scheitert an der Differenziertheit der Daten. Wenn man dieses Problem, in Anlehnung an Vorschläge aus der Forschung, durch eine Aufspaltung des Stufensystems zu lösen versucht, bekommt man eine große Zahl von Stufen, die jeweils kaum mehr als eine einzige Eigenschaft voraussagen. Bei dieser Strategie erweisen sich die Ausdrucksstufen also nicht, wie erhofft, als reiche Kategorien, sondern als sehr arme, die keinen erkennbaren Mehrwert gegenüber einer Beschreibung haben, die auf sie verzichtet. Welchen Vorteil hat z.B. die Einordnung eines Ausdrucks in die Kategorie F-WORT gegenüber einer, die ihn als Ausdruck ohne separat flektierbare Teile klassifiziert? Offensichtlich keinen, wenn F-WORT eben nichts anderes bedeutet als, Ausdruck ohne separat flektierbare Teile! Es wäre sogar unökonomisch, zusätzlich zur Kategorie AUSDRUCK OHNE SEPARAT FLEKTIERBARE TEILE noch eine Kategorie F-WORT anzunehmen, wenn beide völlig deckungsgleich sind.

\subsection{WORT und SYNTAGMA als Prototypenkategorien}

Aber gibt es nicht Alternativen zur Aufspaltungsstrategie, die es erlauben, an den ursprünglichen reichen Kategorien WORT und SYNTAGMA festzuhalten und trotzdem allen Daten widerspruchsfrei Rechnung zu tragen? Ein naheliegender Vorschlag wäre die Umdeutung der Stufen WORT und SYNTAGMA als Prototypen-Kategorien. Dann müsste ein Ausdruck, um als Wort zu gelten, nicht alle in System 1 genannten Eigenschaften haben, sondern nur so viele, dass er idealen Wörtern hinreichend ähnlich ist. Problemfälle wie Komposita oder Partikelverben könnten dann ohne Widersprüche in die Kategorie WORT eingeordnet werden, aber eben, weil sie nicht alle Eigenschaften idealer Fälle haben, nicht in das Zentrum, sondern in die Peripherie dieser Kategorie.

$27 \quad$ Vgl. z.B. (9b) vs. * An hat alles am 2. Januar gefangen. Näheres in Heine/Jacobs/Külpmann (2010). 
Dass sich diese Lösung in der Forschungspraxis nicht durchgesetzt hat, ${ }^{28}$ hat teilweise ideologische Gründe, ist aber aus der EAS-Perspektive durchaus rational. Wenn man Ausdrücke wie Babnkunde oder einstellen detailliert analysieren will, kann man es ja nicht bei der Feststellung belassen, sie seien periphere Wörter. Man muss vielmehr sagen, welche Worteigenschaften sie haben und welche nicht, z.B. dass sie aus zwei Syllabierungsdomänen bestehen, keine internen Spatien haben, dass Teile von einstellen, aber nicht von Babnkunde für syntaktische Prozesse zugänglich sind usw. Eine solche detaillierte und differenzierende Beschreibung folgt einfach nicht aus der Einordnung als peripheres Wort.

Das gilt übrigens auch, wenn man das Maß der Peripheralität nichtidealer Fälle komparativ oder quantitativ genauer spezifiziert, etwa nach der Zahl der Eigenschaften, die sie mit idealen Fällen gemeinsam haben.$^{29}$ Auch wenn wir wissen, dass Babnkunde und einstellen zum Grad 0,8 bzw. 0,65 Wörter sind, wissen wir noch lange nicht, welche Worteigenschaften sie haben und durch welche sie sich unterscheiden.

\subsection{WORT und SYNTAGMA als Defaultkategorien}

Es gibt aber andere flexible Kategorisierungsstechniken, mit denen man auch nicht-ideale Fälle den ursprünglichen Kategorien WORT und SYNTAGMA subsumieren kann, ohne die Details unter den Tisch zu kehren. So kann man diese Kategorien als oberste Elemente eines Typsystems mit Default-Vererbung betrachten, in dem untergeordnete Typen von den übergeordneten nur die Eigenschaften übernehmen, die bei ihnen nicht ausdrücklich anders spezifiziert sind. ${ }^{30}$ Zudem kann ein untergeordneter Typ Eigenschaften mehrerer übergeordneter Typen erben, wenn das nicht zu widersprüchlichen Eigenschaftskombinationen führt. In einem solchen Kategorisierungssystem würden z.B. die Ausdrücke in (14a) und in (14g) genau die Eigenschaften der Kategorien WORT bzw. SYNTAGMA erben, die Ausdrücke in $\mathrm{b}-\mathrm{f}$ jeweils nur die Teilmenge der Eigenschaften von WORT und SYNTAGMA, die bei ihnen nicht anders spezifiziert sind, wobei es zu einer Mischung von WORT- und SYNTAGMA-Eigenschaften käme, ohne dass die Stufen dazu aufgespalten werden müssen.

28 Fuhrhop (2007) stellt ihrer Arbeit zwar ein Bekenntnis zur Prototypentheorie voran (ebd., Kap. 1.2), ihre konkreten Untersuchungen zielen aber eher darauf ab, Problemfälle klassischen Kategorien zuzuordnen (etwa wenn sie fragt, ob rad in radfabren ein selbstständiges Substantiv, ein substantivisches Erstglied oder eine Verbpartikel ist; ebd., S. 55).

29 Ágel (2004) stellt eine auf der Zahl der zutreffenden Worteigenschaften beruhende Methode zur Ermittlung des quantitativen Grads der „Wortigkeit“ nicht-idealer Fälle vor. Aarts (2004) möchte mit einem ähnlichen Verfahren generell die komparative Peripheralität von Grenzfällen bestimmen.

$30 \quad$ Vgl. z.B. Müller (2007, Kap. 7.5.1). 
Aber auch bei dieser Modellierung hätten die Ausdrucksstufen kaum noch prädiktive Kraft. So könnte man daraus, dass ein Ausdruck X in der Vererbungshierarchie unter der Stufe WORT steht, noch keine einzige Eigenschaft von X sicher voraussagen. Man müsste ja stets zusätzlich prüfen, welche WORT-Eigenschaften X erbt und welche nicht. Während also bei der Aufspaltungsstrategie die Aussagekraft der Stufen dadurch schwindet, dass sie kaum mehr als eine Eigenschaft vorhersagen, geschieht das bei der Default-Strategie dadurch, dass die Zuordnung zu einer Stufe allein nicht ausreicht, um die konkreten Eigenschaften eines Ausdrucks vorherzusagen. ${ }^{31}$

Eine weitere Strategie zur Rettung der traditionellen Ausdrucksstufen sei hier nur noch kurz erwähnt: Duale Analysen, wie sie für Partikelverben (unter sehr verschiedenen theoretischen Prämissen) von Goldberg (2003), Zeller (2003) und Kremers (2009) vorgeschlagen wurden, postulieren, dass ein und derselbe Ausdruck hinsichtlich bestimmter Aspekte und/oder in bestimmten Konstruktionen als Wort, hinsichtlich anderer Aspekte bzw. Konstruktionen als Syntagma einzustufen ist. Im Hinblick auf die Prädiktivität der Ausdrucksstufen hat das den gleichen unerwünschten Effekt wie die Default-Strategie: Aus den Ausdrucksstufen allein lassen sich keine sicheren Aussagen darüber mehr ableiten, welche der mit ihnen assoziierten Eigenschaften in konkreten Fällen tatsächlich vorliegen.

\subsection{Verzicht auf WORT und SYNTAGMA als metasprachliche Kategorien: Grammatik ohne Wörter}

Angesichts dieser Probleme sollte man durchaus erwägen, auf die traditionellen Ausdrucksstufen WORT und SYNTAGMA bzw. auf differenzierte oder aufgeweichte Versionen dieser Stufen ganz zu verzichten. Man könnte ja für alle Ausdrücke spezifizieren, welchen Wert die Eigenschaftsdimensionen DO-SYLB, INTERN-SPAT, FLEX-TEIL, LK-TEIL usw. bei ihnen annehmen, ohne zu versuchen, diese Eigenschaften zu Ausdrucksstufen zusammenzufassen. Dazu muss man diese Eigenschaften nicht für jeden Ausdruck einzeln notieren. Die allermeisten Ausdrücke folgen ja Mustern oder Regeln, an denen man die fraglichen Eigenschaften festmachen könnte. So muss man die spezifische Werteverteilung der Eigenschaftsdimensionen bei $\mathrm{N}+\mathrm{N}$-Determinativkomposita nicht bei jedem einzelnen Kompositum festhalten (was gar nicht möglich ist, die Klasse ist ja offen), sondern kann sie als Charakteristikum des Musters der $\mathrm{N}+\mathrm{N}$-Komposition repräsentieren.

$31 \quad$ Die Herausgeber des vorliegenden Bandes merkten hier an, dass die Default-Kategorisierung gegenüber der Aufspaltungsstrategie den Vorteil habe, zum Ausdruck zu bringen, dass bestimmte Stufeneigenschaften dazu neigen, zu kookkurrieren. Zu der Frage, ob das wirklich ein Vorteil ist, vgl. die in 4.3 folgenden Überlegungen zur Erfassung von Abhängigkeiten zwischen den Stufeneigenschaften. 
Im Hinblick auf Vollständigkeit, Detailliertheit und Widerspruchsfreiheit wäre eine solche Grammatik obne Wörter der Prototypenstrategie überlegen und der Aufspaltungs- und Defaultstrategie ebenbürtig. Im Hinblick auf Beschreibungsökonomie hätte sie nur gegenüber der Defaultstrategie einen gewissen Nachteil, nämlich bei den Ausdrücken, die die ideale Verteilung der Eigenschaften aufweisen und deswegen bei dieser Strategie ohne weitere Angaben als WORT bzw. SYNTAGMA eingestuft werden können.

Einen Vorteil gegenüber allen anderen Strategien sehe ich darin, dass der Verzicht darauf, die Eigenschaften von vorneherein zu größeren Gruppen zusammenzufassen, den Blick freimacht auf die tatsächlichen Zusammenhänge zwischen ihnen. Bei 12 binären Eigenschaftsdimensionen wären $2^{12}$ Werteverteilungen rechnerisch möglich. Die Zahl der in den Daten belegten Verteilungen scheint jedoch weit niedriger zu sein. ${ }^{32}$ Es muss also Abhängigkeiten zwischen den Dimensionen geben, die es zu identifizieren und zu erklären gilt. In (A1)-(A4) sind einige Hypothesen über solche Abhängigkeiten aufgeführt:

$$
\begin{aligned}
& (\mathrm{A} 1)+\text { VF-TEIL } \rightarrow+\text { LK-TEIL } \\
& (\text { A2) }+ \text { DO-SYLB } \rightarrow \text { ( }- \text { LK-TEIL } \&-\text { VF-TEIL } \&-\text { E-TEIL }) \\
& (\text { A3) }+ \text { E-TEIL } \rightarrow+\text { INTERN-SPAT } \\
& \text { (A4) }+ \text { DERIV-BAS } \rightarrow-\text { INTERN-SPAT }
\end{aligned}
$$

Ad (A1): Wie es scheint, setzt die Möglichkeit, einen Teil eines Komplexes K ins Vorfeld zu bewegen, voraus, dass K einen in die linke Satzklammer bewegbaren verbalen Teilausdruck enthält. So können die Erstglieder mancher Partikelverben ins Vorfeld bewegt werden (siehe 3.4), nie jedoch die Erstglieder von Präfixverben, die keinen beweglichen verbalen Teil enthalten. ${ }^{33}$

32 Sie ist jedoch sicher größer als die sechs in (14) belegten Verteilungen. Wesentlich wächst die Zahl der belegten Kombinationen von Stufeneigenschaften zudem, wenn man die hier ausgeblendeten Eigenschaftsdimensionen (siehe Kap. 2) und die innerhalb der betrachteten Eigenschaftsdimensionen erforderlichen Differenzierungen (etwa beim Kriterium E-TEIL, 3.4) berücksichtigt. Nach bisherigen Ergebnissen unseres Projekts wächst damit die Zahl der belegten Kombinationen auf deutlich über 20. - Damit wird übrigens eine weitere denkbare Klassifizierungsstrategie unplausibel, nämlich die, jede einzelne belegte Kombination zu einer Stufe zusammenzufassen. Ein Klassifizierungssystem mit über 20 sich ausschließenden Stufen widerspricht den in Kapitel 2 dargelegten Grundgedanken der Annahme von Ausdrucksstufen nicht weniger als das in Tabelle 6 dargestellte System mit sieben sich überkreuzenden Stufenpaaren.

33 Außerdem macht (A1) die Voraussage, dass Teilausdrücke von Komplexen, die überhaupt kein Verb enthalten, nie ins Vorfeld bewegt werden können. Das berührt die altbekannte Problematik allgemeiner Beschränkungen für Bewegungsprozesse, im Hinblick auf die (A1) präzisiert werden müsste. Das würde hier jedoch den Rahmen sprengen. 
Ad (A2): Ein Ausdruck, der eine Syllabierungsdomäne ist, hat nie bewegliche oder syntaktisch erweiterbare Teile. Anders gesagt: Um für interne syntaktische Prozesse offen zu sein, müssen Ausdrücke aus mehr als einer Syllabierungsdomäne bestehen. ${ }^{34}$

Ad (A3): Komplexe mit syntaktisch erweiterbaren Teilausdrücken werden getrennt geschrieben. ${ }^{35}$ Das ist insofern bemerkenswert, als, wie schon in 3.2 bemerkt, die Zugänglichkeit von Teilausdrücken für syntaktische Prozesse ansonsten für die Getrennt-/Zusammenschreibung irrelevant ist.

Ad (A4): Komplexe, die als Basen für relevante Derivationsprozesse, etwa Adjektivierung mit -bar, fungieren können, scheinen stets zusammengeschrieben zu werden. ${ }^{36}$

In dem Maß, wie solche Abhängigkeiten gesichert werden können, können sie im Rahmen einer Grammatik ohne Wörter auch zu einer ökonomischeren Darstellung der einschlägigen Eigenschaften herangezogen werden. Wenn sich etwa (A2) bestätigt, kann man sich darauf beschränken, diesen Zusammenhang einmal in der Grammatik festzuhalten, und muss die Unzugänglichkeit für interne syntaktische Prozesse nicht bei jedem einzelnen der vielen Ausdrücke, die nur eine Syllabierungsdomäne umfassen, wiederholen. Zusammen mit der schon erwähnten Möglichkeit, die fraglichen Eigenschaften an Mustern oder Regeln festzumachen (siehe oben), lässt das hoffen, dass eine Grammatik ohne Wörter weit übersichtlicher sein könnte, als man zunächst vermuten würde.

Darüber hinaus würde ein solcher Rahmen die hier diskutierten Eigenschaften in eine wesentlich reichere deduktive Struktur ${ }^{37}$ einbinden als alle Strategien, die an einem Kategorisierungssystem festhalten, das Teilmengen der Eigenschaften zu Ausdrucksstufen zusammenfasst. Diese Strategien haben außerdem, im Gegensatz zu der skizzierten Grammatik ohne Wörter, das empirische Problem, dass sie bidirektionale Abhängigkeiten zwischen den jeweils zusammengefassten Eigenschaften erwarten lassen, nämlich eben die Kookkurrenz der Eigenschaften. Zusammenhänge wie (A1)-(A4) gelten jedoch anscheinend immer nur unidirektional. Keine der folgenden bidirektionalen Varianten von (A1)-(A4) trifft zu:

$34 \quad$ Das ist eine Fassung des Prinzips der , lexikalischen Integrität` (vgl. Kap. 2), die dessen Geltungsbereich gegenüber Fassungen, die auf dem Wortbegriff beruhen, erheblich einengt.

35 Vgl. Jacobs (2005, Kap. 4.1.4.2), wo auch einige wenige Gegenbeispiele zu dieser Implikation diskutiert werden.

36 Das gilt mit der Einschränkung, dass manche Resultativkomplexe, wie klein schneiden, deren Adjektivierung mit -bar nach den Untersuchungen in unserem Forschungsprojekt recht akzeptabel ist, erst in der neuesten Fassung der deutschen Orthographie zusammengeschrieben werden können.

37 Der Reichtum der deduktiven Struktur erhöht sich noch dadurch, dass einige der festgestellten Abhängigkeiten wohl aus noch allgemeineren Zusammenhängen folgen, etwa (A1) aus Beschränkungen für Bewegungsprozesse. 
$\left(\mathrm{A} 1^{\prime}\right)+$ VF-TEIL $\leftrightarrow+$ LK-TEIL

$\left(\mathrm{A} 2^{\prime}\right)+$ DO-SYLB $\leftrightarrow(-$ LK-TEIL $\&-$ VF-TEIL $\&-$ E-TEIL $)$

$\left(\mathrm{A}^{\prime}\right)+$ E-TEIL $\leftrightarrow+$ INTERN-SPAT

(A4') + DERIV-BAS $\leftrightarrow-$ INTERN-SPAT

(A1') ist falsch, weil es unter Partikelverben (vgl. 3.4.), Inkorporationsbildungen und Rückbildungen, aber auch unter Komplexen der Form Partikel + Verb (wie nicht zustimmen) viele ohne vorfeldfähiges Erstglied, aber mit beweglichem verbalen Zweitglied gibt. (A2') ist falsch, weil es viele Ausdrücke gibt, die mehr als eine Syllabierungsdomäne umfassen, aber syntaktisch inert sind (z.B. sämtliche Komposita). (A3') ist falsch, weil Getrenntschreibung auch bei Komplexen möglich ist, die keine syntaktisch erweiterbaren Teile haben, so bei manchen Zusammenrückungen, z.B. $<$ an Stelle>, < auf Grund>, < bei weitem>. Und (A4') trifft nicht zu, weil es zusammengeschriebene Komplexe gibt, die nicht als Basis für relevante Derivationsprozesse, etwa -bar-Adjektivierung, fungieren können, z.B., wie schon in 3.3 gesagt, Inkorporationsbildungen wie bloßstellen.

Dieser Nachteil der Zusammenfassung der Eigenschaften zu Ausdrucksstufen mag vermeidbar erscheinen, wenn man sich damit begnügt, die Eigenschaften, die mit einer gewissen Wahrscheinlichkeit kookkurrieren, anzugeben. So könnte man die Subsumierung der Eigenschaften unter die Kategorie WORT einsetzen, um festzuhalten, dass ein Ausdruck, der, sagen wir, die Eigenschaft - INTERN-SPAT hat, mit einer gewissen Wahrscheinlichkeit auch die Eigenschaften + DO-SYLB, - E-TEIL usw. hat. (Eine solche probabilistische Deutung der Ausdrucksstufen liegt auch nahe, wenn man sie als Default-Kategorien betrachtet, vgl. 4.2) Ein Problem bei dieser Sicht ist allerdings, dass für die Kookkurrenz der verschiedenen Eigenschaften in der Regel unterschiedliche Wahrscheinlichkeiten gelten. So kookkurriert - INTERN-SPAT mit hoher Wahrscheinlichkeit mit - E-TEIL, während die Kookkurrenz mit + DO-SYLB angesichts der Unzahl der zusammengeschriebenen Komposita sicher weniger wahrscheinlich ist. Solche Unterschiede könnte man auch im Rahmen einer probabilistischen Deutung der Stufenkategorien nur durch eine Aufspaltung dieser Kategorien erfassen - und geriete letztlich wieder in das in Kapitel 3 beschriebene Dilemma.

\section{Was wird aus den stufenbezogenen Kompartmentalisierungen?}

Mit den klassischen Ausdrucksstufen gerät auch die auf ihnen beruhende Kompartmentalisierung der Grammatik in Morphologie vs. Syntax vs. Phraseologie ins Wanken. Egal, ob man die Stufen in viele spezifischere Katego- 
rien aufspaltet, sie nur noch in abgeschwächten Versionen oder überhaupt nicht mehr verwendet: Man wird die Idee, dass sich die Organisation unseres sprachlichen Wissens an der Unterscheidung WORT vs. SYNTAGMA orientiert, entweder ganz aufgeben oder zumindest so stark differenzieren müssen, dass die ursprüngliche Hoffnung, man könne auf dieser Grundlage ein einfaches Bild von der Architektur von Sprachsystemen zeichnen, auf jeden Fall aufgegeben werden muss. Das betrifft auch die Abgrenzung des Lexikons von der Grammatik, soweit diese auf der Unterscheidung von Wörtern und Syntagmen beruht (vgl. Kap. 1).

Dieser Verlust an Übersichtlichkeit wird jedoch mehr als kompensiert durch einen Zugewinn an Realismus. Außerdem öffnen sich einer Linguistik, die sich von zu simplen Vorstellungen über die stufenbasierte Gliederung von Sprachsystemen verabschiedet, Wege zur Beendigung diverser verfahrener Abgrenzungsdebatten, etwa der über den Grenzverlauf zwischen einer nach dem Kriterium „Polylexikalität“ identifizierten Phraseologie und einer auf „Monolexikalität“ beruhenden Morphologie, die solche Begriffskrücken wie den „Einwortphraseologismus“ hervorgebracht hat. ${ }^{38}$

\section{Wozu noch Wörter?}

Wir müssen uns also im Hinblick auf die Ausdrucksstufen und die auf ihnen basierenden Einteilungen des Sprachsystems von liebgewordenen Gewohnheiten verabschieden, wenn wir an den EAS-Zielen Vollständigkeit, Detailtreue und Widerspruchsfreiheit festhalten wollen. Aber natürlich orientieren sich Sprachwissenschaftler nicht immer an diesen Maximalzielen. So betrachten sie oft nicht die Gesamtheit der Ausdrücke einer Sprache, sondern nur bestimmte Teilklassen. Und dabei können dann die Abgrenzungsprobleme zwischen Wörtern und Syntagmen irrelevant werden. Wenn man z.B. - aus welchen Gründen auch immer - nur Ausdrücke betrachtet, die in Beispielreihe (14) in die Zeilen a oder g gehören, kann man ohne Ungereimtheiten mit den ursprünglichen Stufen WORT und SYNTAGMA arbeiten. Man kann diese Kategorien aber auch dann ohne Differenzierung oder Aufweichung verwenden, wenn man geringere Ansprüche an die Detailtreue stellt. Oft interessiert man sich z.B. als Phonologe nicht für syntaktische oder morphologische Eigenschaften, d.h. man betrachtet nur die ersten drei Eigenschaftsdimensionen in unserem ursprünglichen System (Tabelle 1). Wenn man das tut, kann man ohne Probleme alle Ausdrücke, die in (14) unter $\mathrm{b}$ bis $\mathrm{g}$ aufgeführt sind, als Syntagmen betrachten und muss sich nicht um Unterschiede etwa zwischen Nominalkomposita und Partikelverben kümmern. Und auch bei der Widerspruchsfreiheit machen wir oft Kompro-

38 Vgl. Ágel (2004), Heine (2010), Szczek (2004). 
misse. Wenn es z.B. nur um die grobe Zusammenfassung von Ausdrücken geht, die irgendwelche Worteigenschaften haben, aber nicht notwendigerweise alle und nicht alle dieselben, ist die Verwendung der Kategorie WORT trotz aller Abgrenzungsprobleme unschädlich. Und natürlich kann man Einschränkungen der Vollständigkeit mit solchen der Detailtreue oder der Widerspruchsfreiheit verbinden, und hat dann erst recht mit der Unterscheidung von WÖRTERN und SYNTAGMEN keine Probleme mehr.

Darin liegt wohl ein wesentlicher Grund dafür, dass sich diese Unterscheidung in der sprachwissenschaftlichen Praxis so hartnäckig hält. In dieser Praxis sind Einschränkungen der Vollständigkeit, der Detailtreue (oder ,Granularität ${ }^{\circ}$ ) und der Widerspruchfreiheit ja gang und gäbe. Und das ist auch nicht generell zu kritisieren, sondern oft sehr sinnvoll, insbesondere vor dem Hintergrund bestimmter Anwendungsziele. Wenn man z.B. Lehrwerke für Deutsch als Fremdsprache entwirft, ist eine Einschränkung auf bestimmte gängige Ausdrucksklassen und die Wahl eines nicht allzu hohen Detailliertheitsgrades in der Regel unerlässlich. In einem solchen Arbeitskontext kann dann aus den genannten Gründen auch die Verwendung der traditionellen Unterscheidung von Wörtern und Syntagmen ungefährlich, ja sogar sehr praktisch sein.

Legitim kann der Einsatz dieser klassischen Begriffe auch sein, wenn man sie nicht als metasprachliche Kategorien im Rahmen der Sprachsystemanalyse, sondern in anderen Zusammenhängen verwendet. So bleibt es trotz - oder gerade wegen - der aufgezeigten Probleme eine interessante Frage, von welchem mentalen Konzept Grammatiker einer bestimmten Schule oder auch Laien einer bestimmten Sprachgemeinschaft ausgehen, wenn sie Ausdrücke als Wörter oder Nicht-Wörter klassifizieren. Interessant ist das etwa im Hinblick darauf, welche der in System 1 genannten Eigenschaftsdimensionen dieses mentale Wortkonzept am stärksten prägen. (Es ist oft vermutet worden, dass das die graphematischen Dimensionen sind, dass der Wortbegriff also wesentlich schriftinduziert ist.)

Kurz: Das Überleben der aus EAS-Sicht hochproblematischen Unterscheidung von Wörtern und Syntagmen erscheint nicht nur erklärlich, sondern sogar gerechtfertigt vor dem Hintergrund der Tatsache, dass die Sprachwissenschaft ein arbeitsteiliges Unterfangen mit unterschiedlichen methodischen Standards und unterschiedlichem Gegenstandsbezug ist. Entsprechendes gilt für die auf den klassischen Stufen aufbauende Abgrenzung von Syntax, Morphologie und Phraseologie, und auch für die von Lexikon und Grammatik. 


\section{Literatur}

Aarts, Bas (2004): Modelling linguistic gradience. In: Studies in Language 28, 1, S. 1-50.

Ágel, Vilmos (2004): Polylexikalität oder am Anfang waren mindestens zwei Wörter. Über eine Grundfrage (nicht nur) der Phraseologie. In: Brdar-Szabó, Rita/KnipfKomlósi, Elisabeth (Hg.): Lexikalische Semantik, Phraseologie und Lexikografie. Abgründe und Brücken. Festgabe für Regina Hessky. Frankfurt a.M., S. 21-50.

Croft, William (2001): Radical construction grammar. Syntactic theory in typological perspective. Oxford.

Dixon, Robert M.W./Aikhenvald, Alexandra Y. (Hg.) (2002): Word. A cross-linguistic typology. Cambridge.

Fuhrhop, Nanna (2007): Zwischen Wort und Syntagma. Zur grammatischen Fundierung der Getrennt- und Zusammenschreibung. (= Linguistische Arbeiten 513). Tübingen.

Fuhrhop, Nanna (2008): Das graphematische Wort (im Deutschen): Eine erste Annäherung. In: Zeitschrift für Sprachwissenschaft 27, 2, S. 189-228.

Goldberg, Adele E. (2003): Words by default: The Persian complex predicate construction. In: Francis, Elaine J./Michaelis, Laura A. (Hg.): Mismatch: Form-function incongruity and the architecture of grammar. Stanford, S. 117-146.

Hall, Tracy Alan (1999a): The phonological word: A review. In: Hall/Kleinhenz (Hg.), S. 1-22.

Hall, Tracy Alan (1999b): Phonotactics and the prosodic structure of German function words. In: Hall/Kleinhenz (Hg.), S. 99-132.

Hall, Tracy Alan/Kleinhenz, Ursula (Hg.) (1999): Studies on the phonological word. (= Current Issues in Linguistic Theory 174). Amsterdam.

Heine, Antje (2010): Wieviel Polylexikalität braucht ein Phraseologismus? In: Korhonen, Jarmo et al. (Hg.): Europhras 2008. Beiträge zur internationalen Phraseologiekonferenz 2008 in Helsinki. Helsinki, S. 11-18.

Heine, Antje/Jacobs, Joachim/Külpmann, Robert (2010): Quer zu den Feldern - Zur Topologie von Partikelverben. In: Linguistische Berichte 221, S. 37-60.

Hockett, Charles F. (1944): Rezension von „Linguistic Interludes and Morphology: the descriptive analysis of words" von Eugene A. Nida. In: Language 20, S. 252-255.

Jacobs, Joachim (2005): Spatien - Zum System der Getrennt- und Zusammenschreibung im heutigen Deutsch. (= Linguistik - Impulse \& Tendenzen 8). Berlin/New York.

Kremers, Joost (2009): A prosodic approach to particle verbs. In: Linguistische Berichte 217, S. 81-96.

Meibauer, Jörg (2003): Phrasenkomposita zwischen Wortsyntax und Lexikon. In: Zeitschrift für Sprachwissenschaft 22, S. 153-188.

Müller, Stefan (2007): Head-driven phrase structure grammar. Eine Einführung. (= Stauffenburg Einführungen 17). Tübingen. 
Nespor, Marina/Vogel, Irene (1986): Prosodic phonology. (= Studies in Generative Grammar 28). Dordrecht u.a.

Primus, Beatrice (2003): Zum Silbenbegriff in der Schrift-, Laut- und Gebärdensprache. In: Zeitschrift für Sprachwissenschaft 22, 1, S. 3-55.

Nübling, Damaris et al. (2008): Historische Sprachwissenschaft des Deutschen. Eine Einführung in die Prinzipien des Sprachwandels. 2. Aufl. Tübingen.

Raffelsiefen, Renate (1999): Diagnostics for prosodic words revisited: The case of historically prefixed words in English. In: Hall/Kleinhenz (Hg.), S. 133-202.

Szczek, Joanna (2004): Einwortphraseologismen und ihr Verhältnis zur Phraseologie (am Beispiel des Deutschen und des Polnischen). In: Földes, Csaba/Wirrer, Jan (Hg.): Phraseologismen als Gegenstand sprach- und kulturwissenschaftlicher Forschung. Baltmannsweiler, S. 75-85.

Wurzel, Wolfgang Ulrich (2000): Was ist ein Wort? In: Thieroff, Rolf et al. (Hg.): Deutsche Grammatik in Theorie und Praxis. Tübingen, S. 29-42.

Zeller, Jochen (2003): Moved preverbs in German: Displaced or misplaced? In: Booji, Geert/van Marle, Jaap (Hg.) (2003): Yearbook of Morphology 2003. Dordrecht, S. 179-212. 
Erwerb und Verarbeitung 



\author{
Heike Behrens
}

\title{
Grammatik und Lexikon im Spracherwerb: Konstruktionsprozesse ${ }^{1}$
}

Putting together novel expressions is something that speakers do, not grammars. It is a problemsolving activity that demands a constructive effort and occurs when linguistic convention is put to use in specific circumstances.

(Langacker 1987, S. 65)

\begin{abstract}
Der Konstruktionsbegriff hielt seinen Einzug in die Spracherwerbsforschung durch gebrauchsbasierte Lerntheorien, nach denen sprachliche Strukturen als Form-Funktionseinheiten aus dem Input abgeleitet werden, Sprache somit ein emergentes System ist (Tomasello 1998a und b; Behrens 2009a und b). Die Abstraktionseinheit für das Kind ist dabei die Äußerung in ihrer situativen Gebundenheit und ihrer Diskursfunktion, mithin die Konstruktion. Die Konstruktion wird gefasst als schematische Einheit mit mehr oder weniger offenen Slots: Teile der Konstruktion können lexikalisch fixiert oder aber produktiv und durch andere Ausdrücke ersetzbar sein. Der Kontrast zum Valenzbegriff bzw. dem der Argumentstruktur in seiner formaleren Definition liegt darin, dass die lexikalischen Eigenschaften der Wörter die Syntax nicht projizieren, sondern dass sowohl die Eigenschaften der Lemmas als auch die der Morphosyntax aus ihrem Vorkommen in konkreten Sätzen abgeleitet werden.

Empirisch konzentriert sich die Forschung auf die Ermittlung der Generalisierungsprozesse und auf deren Basis im Input, dem Sprachangebot. Erwerbsrelevant ist insbesondere der Input in seinen usualisierten Mustern in typischen Interaktionssituationen. Hier wird vor allem der Grad der Produktivität kindlicher Äußerungen analysiert. Bislang weniger untersucht, aber zunehmend im Fokus sind die Generalisierungsprozesse selbst und damit die generative Kraft des Konstruktionsbegriffs. Sobald Aspekte einer Konstruktion abstrahiert worden (= produktiv) sind, sollten sie auf neue Situationen übertragen werden können, und gilt es zu ermitteln, welche formalen, funktionalen und distributionellen Faktoren die Abstraktion sprachlichen Wissens fördern.

In dem Paradigma der gebrauchsbasierten Konstruktionsgrammatik wird die modulare Trennung zwischen Wörtern und Regeln aufgehoben. Somit kann innerhalb eines einheitlichen theoretischen Rahmens sowohl der Erwerb regelhafter als auch der stärker idiosynkratischer Strukturen erklärt werden.
\end{abstract}

Die Arbeit an diesem Aufsatz wurde unterstützt durch eine Senior Fellowship am Freiburg Institute for Advanced Studies (FRIAS). 


\section{Lexikon und Grammatik im Spracherwerb}

Die Rolle des Lexikons im Syntaxerwerb aus konstruktivistischer Perspektive möchte ich anhand von zwei unterschiedlichen Themenfeldern beleuchten: Erstens dem Erwerb der Argumentstruktur, der sich direkt auf den Tagungstitel „Sprachliches Wissen zwischen Lexikon und Grammatik“ bezieht. Hier untersucht man den Zusammenhang zwischen den semantisch bedingten Subkategorisierungseigenschaften des Verbs und der syntaktischen Realisierung der Argumente, insbesondere den Einfluss dieser Beziehung auf den Spracherwerbsprozess. Zweitens dem Erwerb der Satzklammer im Deutschen mit den unterschiedlichen Positionen für finite und nichtfinite Verben je nach Satztyp. Dieses Phänomen scheint zunächst unabhängig vom Lexikon zu sein, weil prinzipiell alle Verben dieser Wortstellungssystematik unterliegen, auch wenn es statistische Unterschiede dahingehend geben mag, wie oft die einzelnen Verbformen für einzelne Verben realisiert werden. Ich möchte zeigen, wie konstruktivistische, gebrauchsbasierte Ansätze zum Spracherwerb den Erwerb eher semantik- und eher morphosyntax-gesteuerter Phänomene in einem homogenen Framework erklären können.

\section{Der Erwerb der Argumentstruktur}

Das Erkenntnisziel der Spracherwerbsforschung ist, zu erklären, wie Kinder Sprache in ihrer Totalität erwerben. Um dieses Ziel zu erreichen, braucht man eine (Arbeits)definition dessen, was Sprache ist (Durch welche Kategorien ist sie instantiiert? Wie ist sie mental repräsentiert?), Hypothesen zu den Lernprozessen (Mittels welcher kognitiven Prozesse lernt das Kind Sprachstruktur und Sprachfunktion?) und schließlich ein methodisches Instrumentarium, mit dem sich die Hypothesen, die sich aus den Grundannahmen ableiten können, testen lassen.

Mit diesem Forschungsprogramm unterscheidet sich die Spracherwerbsforschung von anderen Gebieten der Linguistik, insofern als sie nicht nur die Stadien der Sprachentwicklung adäquat beschreiben, sondern auch erklären können muss, wie die postulierten Strukturen gelernt werden können. Spracherwerbsmodelle sind also notwendigerweise auch kognitive Modelle.

Innerhalb nativistischer Ansätze zum Spracherwerb steht die Arbeitsteilung zwischen Wörtern und Grammatik im Vordergrund. In „words and rules"-Modellen (z.B. Pinker 1989) werden Wörter nach Regeln der Grammatik zu Sätzen zusammengesetzt. Diese Sichtweise ist charakteristisch für die Sicht der generativen Grammatik auf den Spracherwerb, sowohl in Hinblick auf den Erwerb von grammatischen Regeln, die als unabhängig von semantischen oder funktionalen Faktoren konzeptualisiert werden, als auch 
in einer stärkeren lexikalistischen Ausrichtung in Studien zum Erwerb der Argumentstruktur. Sofern substantielle (repräsentationelle) Universalien angenommen werden, muss hier das Linking-Problem gelöst werden. LinkingProzesse müssen stattfinden, um die angeborenen Strukturen mit den Elementen der Zielsprache zu verknüpfen. Lernen durch Linking ist dann eindeutig, wenn alle Verben durch ein solches Regelsystem erfasst werden können. Sofern ein Teil des Lexikons bzw. der Argumentstruktur nicht durch solche Regeln vorhersagbar ist und idiosynkratisch im Lexikon gespeichert werden müsste, wirkt sich das auf den Lernprozess aus, denn nun muss erklärt werden, wie diese idiosynkratischen Eigenschaften gelernt werden (hier wird in der Regel Gebrauchsbasiertheit angenommen), und wie die sprachliche Repräsentation aussieht, die als universal und angeboren vorausgesetzt wird. D.h., man endet bei hybriden Modellen, die einerseits inputbasiert, andererseits aber gebrauchs- und sprachunabhängig sind (vgl. Hohenberger/Pelzer-Karpf 2009). Diese Hybridität löst das Erwerbs- und Linking-Problem jedoch nicht, sondern verschärft es noch, denn es erfordert zwei Lernprozesse (Linking und Generalisierung). Zudem muss erklärt werden, auf welcher Basis das Kind wissen könnte, wann es den einen oder anderen Prozess zu aktivieren hat, wann es auf die Generalisierung über den Sprachgebrauch verzichten kann, weil dieser Aspekt der Sprache gebrauchsunabhängig erworben wird. Generell gibt es keine Evidenz dafür, dass durch die Universalgrammatik (UG) kontrollierte Aspekte früher oder schneller gelernt werden als nicht-UG-gebundene; die Tatsache dass Spracherwerb zügig und zielgerichtet voranschreitet, ist keine Evidenz für Angeborenheit (Behrens 2009b).

In gebrauchsbasierten Ansätzen werden Form-Funktionsbeziehungen aus dem Sprachangebot abstrahiert. Dies impliziert, dass resultierende Kategorien sprachspezifisch und gebrauchsspezifisch sein sollten. Operationalisiert wird dieser Ansatz z.B. im Paradigma der „Preferred Argument Structure" (PAS), das einzelsprachlich und sprachvergleichend die diskurspragmatischen Faktoren, die zur Realisierung oder Ellipsis von Argumenten führen, analysiert (vgl. Allen/Skarabela/Hughes 2008 sowie die Artikel in Bowerman/Brown (Hg.) 2008). In der Sprachtypologie ist umstritten, ob es tatsächlich universale Muster der Argumentstrukturen gibt, nach denen eine bestimmte Bedeutung generell mit bestimmten Subkategorisierungsmustern einhergeht (vgl. Bowerman/Brown 2008). Gebrauchspräferenzen in der Argumentrealisierung wirken sich auch auf den Erwerb der Wortarten aus: In der Debatte, ob Nomen prinzipiell vor Verben erworben werden, weil sie referenziell sind, Verben jedoch relationale Informationen ausdrücken (Gentner 1978), erweist sich, dass in Sprachen mit starker Subjekt- und/oder Objekt-Ellipsis Verben automatisch frequenter und salienter auftreten. Zudem kann der Verbwortschatz auf lexikalisch sehr spezifischen Verben bestehen wie im Tzeltal mit einem sehr reichen Vokabular an Verben 
des Essens, des Bewegens usf., oder aus semantisch eher leeren und eher wenigen Verben wie in einigen australischen Aboriginal-Sprachen. Die semantische Spezifik sowie die perzeptuelle Prominenz durch Frequenz und Salienz erleichtern den Einstieg in die Kategorie Verb (Brown 1998).

Im Folgenden werden die Prozesse des Erwerbs der Argumentstruktur, wie sie sich aus nativistischer „top down“- bzw. gebrauchsbasierter „bottom up"-Perspektive ergeben, kontrastiert.

\subsection{Universales Linking als Einstieg in den Syntaxerwerb}

In der Spracherwerbsforschung der achtziger und neunziger Jahre galten die Subkategorisierungseigenschaften von Verben als verbindende Kraft zwischen formalen und funktionalen Aspekten des Sprachsystems und damit als eine Schnittstelle im Erwerb der Syntax und Semantik, die in den so genannten Bootstrapping-Ansätzen konzeptualisiert wurde. Bahnbrechend wirkten die Überlegungen Steven Pinkers (Pinker 1987, 1989, 1994; Gropen et al. 1991), der postulierte, dass die Semantik Kindern zum Einstieg in die Syntax verhilft. Innerhalb deduktiver generativer Ansätze muss erklärt werden können, wie das angeborene syntaktische Wissen aktiviert werden kann, da die Einheiten der Objektsprache nicht erkennbar ausgezeichnet sind. Anders formuliert: Wenn man annimmt, dass dem Kind Wissen über spezifisch sprachliche Repräsentationen angeboren ist, muss dieses Wissen in der Sprache eindeutig aktivierbar sein, da man ja explizit davon ausgeht, dass dieses sprachliche Wissen nicht durch Abstraktion über den Input konstruiert wird. Pinker löst das Linking-Problem, indem er annimmt, dass die Semantik eines Verbs die nötigen Kategorien liefert, um sein syntaktisches Verhalten eindeutig vorherzusagen: „Sentences conform to the demands of words in them because of general principles" (Pinker 1989, S. 4).

Pinker folgt hier lexikalistischen Theorien wie LFG (Lexical Functional Grammar) und HPSG (Head-Driven Phrase Structure Grammar), die ein intelligentes, hochstrukturiertes Lexikon annehmen (siehe auch Webelhuth, in diesem Band). Damit die Subkategorisierungseigenschaften eines Verbs ihre steuernde Kraft ausüben können, muss sowohl die Semantik des Verbs als auch der Zusammenhang zwischen Semantik und der Argumentstruktur erworben worden sein. Innerhalb generativer Ansätze geht man davon aus, dass ein entsprechendes Linking systematisch ist: Levin (1993) identifiziert 48 Gruppen mit 192 Unterkategorien für die Verben des Englischen auf Basis ihrer Semantik, sowie 79 Kategorien auf Basis ihrer Teilnahme an syntaktischen Alternierungen (Levin 1993, siehe auch Levin/ Rappaport Hovav 1995). Pinker $(1989,1994)$ stellte für den Spracherwerb die Hypothese auf, dass Linking-Regeln angeboren sein könnten. Das Kind müsste nur die semantischen Kasusrollen aus der Interaktion mit der Umwelt abstrahieren und könnte dann eine erfahrungsbasierte Kategorie wie 
„Agens“ mit der syntaktischen Realisierung „Subjekt“ verknüpfen. Nach dieser Hypothese bilden die semantisch zentralen Verben den Einstieg in den Syntaxerwerb, besonders der Argumentstruktur. In Anlehnung an die Arbeiten von Beth Levin und Malka Rappaport-Hovav (1995) wurde der englische Verbwortschatz in semantische Klassen eingeteilt, die jeweils mit syntaktischen Eigenschaften korrelieren, indem sie kraft ihrer Semantik die Syntax projizieren. Mit dem Konzept der semantischen Feineinteilung sollte insbesondere das schwer zu erklärende Phänomen der syntaktischen Alternierungen systematisch erfasst werden: Wieso alternieren einige Verben, obwohl semantisch eng verwandte Verben eine der Varianten nicht erlauben? Im Spracherwerb manifestiert sich dieses Problem durch Übergeneralisierungen (z.B. I disappeared the ball ,Ich verschwinde den Ball' für die Kausativ-Alternierung). In diesem Modell stehen Syntax und Semantik in einer direkten Abhängigkeitsbeziehung: Eine systematische Verknüpfung beider Ebenen erfolgt über das Linking von semantischen zu syntaktischen Rollen. Wenn diese Beziehung als eine systematische gedacht wird, ist es möglich, dass dem Kind das Wissen über eine Repräsentationsebene den Einstieg in die jeweils andere Struktur-Ebene erleichtert wird. Dies ist in den so genannten Theorien des Bootstrapping operationalisiert worden. Dieser aus der Informatik stammende Terminus bezeichnet den Prozess, mit dem ein Steuerungssystem einen komplexeren Prozess startet (Lindner/Hohenberger 2009, S. 230). Übertragen auf den Spracherwerb nahm man an, dass Wissen über einen Aspekt des Systems quasi als Starthilfe Lernprozesse auf einer anderen Ebene anstoßen kann. So wurde postuliert, dass die prosodische Gliederung systematische Hinweise auf die Grenzen syntaktischer Einheiten liefert, oder aber dass die Semantik den Einstieg in die syntaktische Repräsentation von Argumentstrukturen ermöglicht (siehe Höhle 2009 zur Diskussion verschiedener Formen des Bootstrapping). Im semantischen Bootstrapping sagten Gropen et al. (1991) für den Erwerb eine durch UG-Prinzipien gesteuerte Abfolge von „broad to narrow range rules“ vorher. Sie unterscheiden dabei zwischen „breiten“ Linking-Regeln, die als notwendige Bedingungen das syntaktische Verhalten semantischer Grobklassen wie Bewegungsverben steuern, und „engen“, stärker lexikalisierteren Linking-Regeln, die als hinreichende Bedingungen das syntaktische Verhalten bezogen auf feine semantische Unterschiede spezifizieren, z.B. bestimmte Arten der Bewegung. Mit Hilfe dieser Feinklassifizierung der Linking-Regeln sollte ein fehlerfreier Erwerb der Argumentstruktur möglich sein. Die Erwerbsaufgabe besteht in diesem Ansatz im Lernen der spezifischen Verbsemantik, die dann zur Anwendung der jeweils korrekten Linking-Regeln führen würde. Jedoch zeigten Analysen des Spracherwerbs englischer Kinder die postulierten Präferenzen für Verben mit kanonischer Argumentstruktur nicht, stattdessen finden sich früh Verben mit schwachem semantischen Gehalt (Bowerman 1990). 


\subsection{Argumentstruktur als Hilfe zum Erlernen der Verbsemantik}

Wenn eine enge Beziehung zwischen Syntax und Semantik besteht, sollte das Verhältnis auch umgekehrt nutzbar gemacht werden können. Im syntaktischen Bootstrapping geht man davon aus, dass das Verfolgen der syntaktischen Strukturen, in denen ein Verb benutzt wird, dem Kind hilft, die Verbbedeutung einzugrenzen bzw. zwischen verschiedenen Verbbedeutungen zu unterscheiden (Naigles 2002). Landau/Gleitmann (1985) fanden, dass selbst blinde Kinder die Bedeutung von Verben wie look und see aus ihrem Auftreten in bestimmten syntaktischen Kontexten abstrahieren können, obwohl ihnen die Wahrnehmungsdomäne nicht zugänglich ist.

Sowohl semantisches als auch syntaktisches Bootstrapping können nur dann funktionieren, wenn die Beziehungen zwischen Syntax und Bedeutung a priori und universal eindeutig spezifiziert sind:

The first proviso to the semantic usefulness of syntactic analysis for learning purposes is that the semantic/syntactic relations have to be materially the same across languages. Otherwise, depending on the exposure language, different children would have to perform completely different syntactic analyses to derive aspects of the meaning. (Gleitman 1990, S. 35)

Genau dies macht beide Ansätze inkompatibel (vgl. Bowerman/Brown 2008, S. 7-13), denn im semantischen Bootstrapping wäre die genaue Repräsentation der Semantik die Voraussetzung für den Erwerb der Syntax, während im syntaktischen Bootstrapping die Semantik erst gelernt werden muss, und die syntaktische Repräsentation als durch die Universalgrammatik angeboren angenommen wird. Zudem ließen sich die postulierten Syntax-Semantik-Verbindungen in sprachvergleichenden Untersuchungen nicht bestätigen (siehe die Artikel im Sammelband Bowerman/Brown (Hg.) 2008). In der Nachfolge dieser Diskussion ist Bootstrapping mehr und mehr zu einer Metapher für implizite Lernprozesse geworden (siehe Höhle 2009). Jedoch kann man die sehr differenzierten Argumente in dieser Diskussion nur dann wirklich evaluieren, wenn man sie in ihrer definitorischen Schärfe betrachtet: Pinker sah seine Theorie der Linking-Regeln als einen Mechanismus mit notwendigen und hinreichenden Bedingungen, nicht als technisierende Metapher für allgemeine Generalisierungsprozesse. Als Alternative zu den Bootstrapping-Mechanismen haben sich in der Folge konstruktivistische und gebrauchsbasierte Ansätze entwickelt, die den Erwerb der Argumentstruktur als graduellen Erwerbsprozess sehen.

\subsection{Lexikon und Grammatik in konstruktivistischen Ansätzen}

Konstruktivistischen Ansätzen liegt eine andere Hypothese von Erwerbsverläufen zu Grunde. Da sie nicht annehmen, dass spezifisches semantisches oder syntaktisches Wissen angeboren ist, muss beides aus dem Sprachge- 
brauch im weiteren Sinne, d.h. einschließlich seiner situativen und diskursiven Gebundenheit, abstrahiert werden. Ausgangspunkt sind Lernmechanismen, die auch in anderen Domänen attestiert sind. Der Vorteil ist, dass man keinen Dualismus zwischen angeborenen Erwerbsmechanismen und Lernmechanismen für den nicht angeborenen Rest annehmen muss (Tomasello 1998a; Behrens 2009b). Diese Grundannahmen implizieren anfangs graduelle, kontextspezifische Lernprozesse, da gerade nicht angenommen wird, dass mit einem Item die Kategorie als Ganzes aktiviert wird. Im Laufe der Entwicklung wird über die Wahrnehmung konkreter und relationaler Analogien das sprachliche Wissen stets abstrakter und generalisierter. Dies führt dazu, dass in der späteren Entwicklung auch sehr schnelle Lernprozesse möglich sind, sofern die zu lernende Struktur, etwa das Passiv, genügend Unterstützung durch bereits erworbene Konstruktionen, etwa die Perfekt-Konstruktionen, hat (Abbot-Smith/Behrens 2006).

Sprachtheoretisch beruht dieser Ansatz auf Grundannahmen der Kognitiven Linguistik (Langacker 1987), nach der alle sprachlichen Strukturen Form-Funktionspaare, also symbolische Einheiten sind. Dieses Konzept ist in den gebrauchsbasierten Versionen der Konstruktionsgrammatik umgesetzt. Das Verhältnis von Lexikon und Grammatik wird daher nicht als modular gesehen, wie im „words and rules“-Ansatz, sondern als Kontinuum, bei dem konkrete Morpheme und Wörter auf der einen Seite stehen, grammatisch abstrakte (lexikalisch variable) auf der anderen (Tabelle 1):

\begin{tabular}{|l|l|l|}
\hline \multicolumn{1}{|c|}{ Konstruktion } & \multicolumn{1}{|c|}{ Form/Beispiel } & \multicolumn{1}{|c|}{$\begin{array}{c}\text { Bedeutung (sofern } \\
\text { nicht transparent) }\end{array}$} \\
\hline Morphem & zer-, vor-, anti-, -lich, -te & \\
\hline Wort & Avocado, Anaconda, und & \\
\hline komplexes Wort & Feuerteufel, sit-in & Den Vogel abschießen \\
\hline Idiom (fixiert) & $\begin{array}{l}\text { In die X kriegen } \\
\text { (X= Haare, Wolle) }\end{array}$ & sich streiten \\
\hline Idiom (partiell fixiert) & je X desto Y & verbindet Variablen \\
\hline $\begin{array}{l}\text { Covariational conditional } \\
\text { construction }\end{array}$ & $\begin{array}{l}\text { Form: Subj V Dat-Obj } \\
\text { Akk-Obj / Er backte ibr } \\
\text { einen Kuchen }\end{array}$ & $\begin{array}{l}\text { Transfer (beabsichtigt } \\
\text { oder aktuell) }\end{array}$ \\
\hline ditransitive Konstruktion & $\begin{array}{l}\text { Form: Subj werden (PP) } \\
\text { Partizip Perfekt / Das } \\
\text { Gürteltier wurde von einem } \\
\text { Auto überfahren }\end{array}$ & $\begin{array}{l}\text { Diskursfunktion: einen } \\
\text { Patiens (undergoer) zum } \\
\text { Topic oder einen Agens } \\
\text { zum nicht-Topik machen }\end{array}$ \\
\hline Passiv &
\end{tabular}

Tab. 1: Beispiele für Konstruktionen verschiedener Größe und Komplexität (nach Goldberg 2003, S. 220) 
Dass grammatische Strukturen Bedeutungsträger sein können, zeigt sich eindrücklich am Phänomen der coercion (Zwang, Nötigung), wenn die Bedeutung der Konstruktion die Lesart des Lexems verändert und die „eigentliche" Argumentstruktur des Verbs verletzt (z.B. I dreamt him a cappucino ,ich (er)träumte ihm einen Cappuccino' oder he sneezed the napkin off the table ,er nieste die Serviette vom Tisch'). Trotz der Verletzung der Argumentstruktur des Verbs sind diese Sätze nicht nur eindeutig und relativ mühelos interpretierbar, sondern auch produktiv (er bustete das Bällchen aus der Röbre). Die präferierten Lesarten sind veränderbar und frequenzabhängig: Analysen des tatsächlichen Sprachgebrauchs zeigen in vielen Domänen statistische Schieflagen (,statistical skewing", ,,statistical biases"), insofern als nicht alle Zellen eines Paradigmas gleichmäßig besetzt sein müssen, und insofern als statistische Gebrauchspräferenzen die Lesart einer prinzipiell polysemen Struktur beeinflussen können. So wird im Englischen die Konstruktion „Verb - Objekt - lokative PP“ mit der Bedeutung der verursachten Bewegung im konkreten und metaphorischen Sinn assoziiert (he got he ball over the fence), während die Doppel-Objekt-Konstruktion mit der Bedeutung „konkreter" oder „metaphorischer Transfer“ assoziiert ist (be got him a cake) (Goldberg 2006).

Für den Spracherwerb bedeuten diese Grundannahmen, dass man ihn holistisch konzeptualisieren kann: Kinder erwerben nicht die Wörter mit ihren Subkategorisierungseigenschaften, die dann bestimmen, an welchen syntaktischen Strukturen ein Wort partizipieren kann, sondern sie erwerben das Lexikon durch und in den Konstruktionen, in denen sie auftauchen. In konstruktivistischen Theorien werden Syntax-Semantik-Beziehungen auf Basis einer Reihe von allgemeinen Generalisierungsmechanismen abstrahiert:

constructionist theories propose that mapping generalizations are learned from the input, with learning constrained by pragmatics, categorization principles, attentional biases, and other domain-general factors. (Boyd/Gottschalk/Goldberg 2009, S. 65)

Studien zu intransitiven, transitiven, ditransitiven und anderen Argumentstrukturrelationen haben gezeigt, dass der Erwerb einer Struktur zunächst an einzelne oder wenige Lexeme gebunden ist, dass es weder in der Distribution natürlicher Erwerbsdaten noch in Experimenten weite Generalisierungen über den attestierten Gebrauch hinaus gibt (Tomasello 2003). Im Laufe der Entwicklung lockert sich die Bindung von Strukturen an bestimmte lexikalische Einheiten oder Cluster. Der Erwerb von Syntax und Lexikon ist im Sinne der gebrauchsbasierten Konstruktionsgrammatik also als eine Abstraktion über Gebrauchsereignisse zu sehen.

Dies impliziert auch eine Loslösung vom klassischen Konzept des Valenzrahmens, da dieser keine Aussagen über deren konkrete syntaktische Realisierung macht (Behrens 2009a). Allen/Skarabela/Hughes (2008) zeigen, dass die konkrete Argumentrealisierung sprachtypologische Variation zeigt und auch durch diskurspragmatische Faktoren bedingt ist. 


\section{Konstruktionen als Einstieg in den Syntaxerwerb}

In der gebrauchsbasierten Variante der Konstruktionsgrammatik geht man davon aus, dass sprachliche Kategorien im Spracherwerbsprozess auf Basis des sprachlichen Inputs und der Interaktion geformt und gelernt werden. Dies schließt nicht aus, dass angeborenes oder in der vorsprachlichen Phase erworbenes Wissen eine Rolle spielen kann, jedoch ist das Forschungsziel, die Prozesse der sprachlichen (Über)Formung von Konzepten zu ermitteln. ${ }^{2}$

Bezogen auf das Sprachsystem heißt dies, dass sowohl die semantischen und formalen Eigenschaften der lexikalischen Einheiten als auch die Struktur des Sprachsystems aus dem Sprachgebrauch abstrahiert werden müssen. Die Konstruktionsgrammatik bringt dabei auch „top down“-Prozesse holistischen Lernens ins Spiel, wenn das Kind mit größeren Einheiten (,,chunks") beginnt und diese erst im Verlauf der Sprachentwicklung analysiert. Holistische Lernprozesse sind auch früher schon postuliert worden, galten aber als nicht-produktiv in einem Lernprozess, der im Wesentlichen als „,bottom up“-Strategie von einfachen zu komplexen Strukturen gesehen wurde. Auf den ersten Blick scheint die Tatsache, dass die meisten Kinder mit Einwortäußerungen beginnen und peu à peu Zwei- und Dreiwortstrukturen erlernen, den holistischen Strategien zu widersprechen. Jedoch sind die Einwortäußerungen und die darauffolgenden frühen Zweiwortäußerungen syntaktisch nicht komplex, sondern - funktional gesehen - strukturell ungebundene Aneinanderreihungen von Wörtern (Papa weg, Mama Socke). Distributionsanalysen der frühen Wortkombinationen zeigen, dass diese Kombinationen nur eine geringe Variabilität aufweisen, also wenig produktiv sind. Dies zeigt sich daran, dass eine sehr geringe Anzahl von Wortkombinationen den weitaus größten Teil der Tokens einer bestimmten Struktur ausmacht. Pine/Lieven/Rowland (1998) ermittelten, dass die fünf häufigsten Subjekt-Verb-Schemata (z.B. go + X oder Mommy + X) durchschnittlich 75,7\% der Kombinationen bei zwölf zwei- bis dreijährigen englischen Kindern stellten.

\subsection{Lexikalisch-spezifische Erwerbsverläufe}

Michael Tomasellos Studie zum Verb-Erwerb seiner Tochter „T“ (First verbs, 1992) bildete den Ausgangspunkt für die modernen konstruktivistischen Ansätze. Er führte eine detaillierte Tagebuchstudie zum syntaktischen

\footnotetext{
Zur Kategorienbildung vgl. insbesondere die Forschung zur sprachlichen Relativität: Hier zeigen Experimente, dass Kinder im Alter von 9-14 Monaten an unterschiedliche räumliche Kategorisierungen habituiert werden können, jedoch Kinder mit 2-3 Jahren die Kategorisierung ihrer Muttersprache übernommen haben und andere nicht mehr wahrnehmen (McDonough/Choi/Mandler 2003). Analog zu Befunden in der Lautdiskriminierung werden diese Resultate so gewertet, dass Kinder eine sehr flexible Lernfähigkeit haben, die unterschiedliche Kategorisierungen sprachlicher und nicht-sprachlicher Stimuli erlaubt.
} 
Gebrauch von Verben und stellte fest, dass sich die Syntagmen der verschiedenen Verben, die sie im zweiten Lebensjahr produzierte, kaum überschneiden, sondern jedes Verb einen eigenen Strukturaufbau zu durchlaufen schien und eine Insel der Entwicklung bildete. Tomasello prägte für diesen lexikalisch-spezifischen Entwicklungsverlauf die Metapher der Verb-Inseln. Spätere Studien zeigten, dass auch Pronomina eine solche Insel-Funktion ausüben können (Pine/Lieven/Rowland 1998). Die „,verb island“-Нypothese präjudiziert also nicht, dass den Verben per se eine privilegierte Rolle im Strukturaufbau zukommt, und sollte daher allgemeiner als lexem-spezifisches („lexically-specific“) Lernen gefasst werden, ${ }^{3}$ um zu betonen, dass das sich entwickelnde sprachliche Wissen zunächst um das konkrete Item selbst, nicht um seine Kategorie aufgebaut wird. So besteht der Valenzrahmen des Verbs kiüssen zunächst aus den Aktanten kisser/kissee statt der abstrakteren Relationen Agens/Patiens oder Subjekt/Objekt. Evidenz für diese Annahme ist die Distribution in natürlichsprachlichen Daten: Wenn Verben mit der gleichen Argumentstruktur mit unterschiedlichen Argumenten realisiert werden, gilt dies als Indiz dafür, dass die Verben nicht miteinander vernetzt sind. Der Inselstatus ist auch experimentell überprüfbar, indem man testet, inwieweit und unter welchen Bedingungen Kinder Strukturen mit ,nonce“-Wörtern, also solchen, die sie nie vorher gehört haben, auf neue Situationen übertragen: In Trainingsstudien zeigt sich, dass Kinder anfänglich nicht in der Lage sind, über den dargebotenen Subkategorisierungsrahmen hinauszugehen, sondern Strukturen so reproduzieren, wie sie sie gehört haben (siehe Tomasello 2003 für eine Übersichtsdarstellung). Wenn transitive Verben intransitiv präsentiert werden (Look! He's meeking!), findet anfangs keine Generalisierung von intransitiv zu transitiv statt (What's he doing? He's meeking. Aber nicht: He's meeking the cow). Derselbe Effekt findet sich im umgekehrten Paradigma (Training mit transitiven Strukturen, Zielstruktur intransitiv, Tomasello/Brooks 1998). In so genannten „weird word order"-Experimenten werden neue Verben zu transitiven Aktionen in ungrammatischer Wortstellung präsentiert (im Englischen SOV oder VSO). Vierjährige korrigieren die Wortstellung zuverlässig auf SVO, Zweijährige halten sich überwiegend an die jeweils gehörte Wortstellung (Akthar 1999). Auch Priming-Experimente zeigen verb-spezifische Entwicklungen. Priming bezeichnet den Effekt, dass das Hören des Wortes oder einer Struktur die Wiederauftretenswahrscheinlichkeit im Sinne einer Bahnung erhöht, weil das Wort oder die Struktur bereits aktiviert ist. Wenn Vierjährige eine erhöhte Input-Frequenz an Passiv-Sätzen erhalten, produzieren sie danach auch mehr Passivsätze, aber nur solche mit dem gleichen lexikalischen Material.

Das Konzept des Lexikons wird auch auf noch unanalysierte „chunks“ erweitert, die als Ganzes eine bestimmte Funktion enkodieren, z.B. Fragestrukturen wie where's the X oder wo's $\operatorname{der} X$. 
Bei Sechsjährigen hingegen findet sich auch strukturelles Priming: Hier erhöht sich die Zahl der Passivsätze auch mit anderem lexikalischen Material. Dies gilt als Evidenz für strukturelles Priming (Savage et al. 2003).

Die Forschung zur Produktivität und zum Grad der Generalisierung zeigt, dass Kinder in den frühen Phasen weit weniger generativ und kreativ sind als bislang postuliert. Der Eindruck, dass Kinder Sprache effizient und weitgehend fehlerfrei erlernen, lässt sich durchaus durch konservative Lernmechanismen erklären: Kinder experimentieren gerade nicht, indem sie mit bekanntem lexikalischen Material regelgeleitet neue Strukturen produzieren, sondern sie halten sich im Wesentlichen an die Modelle, die sie im Input vorfinden, um die kommunikativen Anforderungen zu bewältigen. Sie scheitern zunächst jedoch, wenn sie bekannte Strukturen auf neue Wörter übertragen müssen. Tomasello (2000) zeigte, dass selbst die syntaktisch vermeintlich einfachen Strukturen transitiver Sätze erst mit neun Jahren in allen Aspekten gemeistert werden.

\subsection{Der Erwerb der Verbzweit-Wortstellung}

Wie lassen sich Phänomene im Strukturaufbau durch die Konstruktionsgrammatik erfassen, die in ihrer Regelmäßigkeit Lexem-übergreifend sind? Als ein solches Phänomen kann die deutsche (und niederländische) Satzklammer gelten: eine syntaktische Eigenschaft, die unabhängig vom Lexikon zu sehen ist, denn prinzipiell unterliegen alle Verben diesem Strukturprinzip. Im frühen Sprachgebrauch deutscher und niederländischer Kinder gibt es jedoch Unterschiede zwischen Kopula- und Modalverben, die fast ausschließlich in finiter Form vorliegen (Jordens 1990), und den Vollverben, die je nach Flexion in V1/V2 oder verbfinaler Position auftreten. Während der Erwerb der Satzklammer den meisten Zweitsprachlernern Probleme bereitet, finden sich im Erstspracherwerb sich normal entwickelnder Kinder wenig Fehler bezogen auf den Zusammenhang von Finitheit und V1/V2Stellung (siehe Tracy in diesem Band). Doch vollzieht sich in den frühen Phasen des Spracherwerbs ein Wandel von einem System, das weitgehend durch infinite Vollverben gekennzeichnet ist (aufmachen; Katze holen etc.), zu einem finiten System.

Aus gebrauchsbasierter und konstruktionsgrammatischer Perspektive ergeben sich also die folgenden Forschungsfragen: Wie ist die Evidenz im Input für die Satzklammer? Zu unterscheiden wären Sätze mit einfachem, finiten Verb und solche mit zweiteiligem Verb, in denen die Satzklammer sichtbar wird. Weiterhin ist die Nebensatzstellung zu beachten. In welchen Entwicklungsschritten vollzieht sich der Wortstellungserwerb?

Das V2-Phänomen ist ein „Klassiker“ der Spracherwerbsforschung zum Deutschen und Niederländischen. Im Fokus steht die Korrelation zwischen Finitheitsmarker und Verbstellung. Die Erwerbsreihenfolge ist in der nor- 
malen Sprachentwicklung so, dass anfangs vor allem nicht-finite Verbformen auftreten, zunächst vorwiegend in Isolation, später in Kombination mit meist vorangestellten Objekten, Subjekten oder anderen Elementen (Negation, Adverbien) (siehe Beleg (1)). Finite Formen sind selten, hier treten - im Niederländischen - anfänglich vor allem Kopula, Modalverben und statische Verben auf (Jordens 1990) sowie eher formelhafte und imitierte Konstruktionen (2), wobei es verschiedene Thesen zur funktionalen Unterscheidung finiter und nicht-finiter Formen gibt (vgl. Blom 2008). Wenige Monate nach den ersten Infinitiven tauchen erste komplexe Verbformen auf, sowohl getrennte Partikelverbkombinationen, modale Infinitive und Perfektkonstruktionen mit Auxiliar und Partizip Präsens (3). ${ }^{4}$
a. Leo 2;0.1 schließen
b. Leo 2;0.20 Tisch decken
a. Leo 2;0.3 passt durch
b. Leo 2;0.5 guck.t müde
a. Leo 2;2.7 kann man Zug bauen
b. Leo 2;2.15 da is(t) durchgefahren

Bemerkenswert ist, dass es nach anfänglichen Schwankungen kaum Fehler in der Wortstellung gibt. In der „Principles \& Parameters“-Version der generativen Grammatik war der V2-Effekt ein potenzieller Parameter, unter der Annahme, dass bestimmte morphologische Merkmale des Verbs (z.B. Tempus, Aspekt, Modalität) die Bewegung des finiten Verbs aus der VerbletztPosition lizenzieren (siehe Lasser 2002 und Blom 2008 zu den diskutierten Varianten). Jedoch gibt es bislang keinen Konsens über die genaue Spezifikation eines solchen Parameters.

Auf den ersten Blick scheint sich die Erwerbsreihenfolge auch nicht aus dem Input erklären zu lassen, denn reine Infinitive oder Partizipien sind in der Erwachsenensprache selten und nur unter bestimmten Bedingungen möglich (Stillgestanden! Alle aufwachen!) (vgl. Lasser 1997). Infinitive und Partizipien sind aber nur dann selten, wenn man sie als eigenständige Struktur mit strikt spezifizierter morphologischer Repräsentation sieht, nicht als Teil komplexer Verbformen. Eine Auszählung des morphologisch kodierten Teilkorpus (500.000 Wörter) aus dem Leo-Korpus ergab, dass rund 35\% aller im Input vorkommenden Verben mehrteilig sind (z.B. modale Infinitive und

4 Die hier und im Folgenden zitierten Kindersprachdaten entstammen der Leipziger „,dense database“ zum Erwerb des Deutschen. Die Sprachentwicklung des monolingualen Jungen Leo wurde vom 2. bis zum 5. Lebensjahr mit einer erhöhten Aufnahmefrequenz von 5 Stunden/Woche im 3. Lebensjahr und 5 Stunden/Woche während einer Woche pro Monat im 4. und 5. Lebensjahr dokumentiert. Die Gesamtgröße des Korpus umfasst ca. 500.000 Wörter von Leo, 1.3 Millionen Wörter im Input (siehe Behrens 2006 für weitere Details). Die Altersangaben sind in JJ.MM.TT angegeben. Das Korpus ist Teil des öffentlich zugänglichen CHILDES-Archivs (MacWhinney 2000). 
Perfektkonstruktionen). Da Verben eine frequente Kategorie sind und diese Formen an salienter finaler Position stehen, ist wahrnehmungspsychologisch durchaus zu begründen, warum diese Formen als erstes aufgegriffen werden: Sie sind inhaltstragend, perzeptuell salient und frequent (Behrens 1993). In einer Reihe von Computersimulationen eines konstruktivistischen Lernmodells (MOSAIC $=$ „Model of Syntax Acquisition In Children“) simulierten Freudenthal/Pine/Gobet (2006, 2009) und Freudenthal et al. (2007) den Verbstellungserwerb im Deutschen, Englischen, Niederländischen, Französischen und Spanischen, indem sie nur die Wahrnehmungsfaktoren Position und Länge des nötigen Arbeitsspeichers modifizierten. Theoretische Basis sind die „less is more“- (Newport 1990) und die „starting small“Hypothese (Elman 1993), nach denen der begrenzte Arbeitsspeicher von Kleinkindern ihnen hilft, in das Sprachsystem einzusteigen, weil es ihre Aufmerksamkeit natürlicherweise auf die Relation zwischen eng zusammenstehenden Elementen lenkt, während sie zunächst scheitern, Relationen zwischen diskontinuierlichen Elementen zu erkennen (Newport/Aslin 2004). Das MOSAIC-Modell arbeitet ohne vorspezifiziertes sprachliches Wissen auf Basis von Input-Daten aus dem CHILDES-Archiv (MacWhinney 2000). Unter der Annahme, dass das zuletzt Gehörte in der Wahrnehmung am aktivsten ist, verarbeitet das Modell den Input von rechts nach links und produziert als Output Äußerungen zunehmender Länge. Dieser Output kann dann mit kindersprachlichen Erwerbsdaten gleicher Äußerungslänge verglichen werden. Es zeigt sich, dass das Modell Strukturen produzierte, die mit den natürlichen Erwerbssequenzen in diesen Sprachen hochgradig übereinstimmen. Die sprachspezifische Passung konnte noch erhöht werden, indem man einen zweiten Aufmerksamkeitsfokus auf den ebenfalls salienten Äußerungsbeginn modellierte, um den unterschiedlichen Wortstellungsmustern der analysierten Sprachen Rechnung zu tragen. Auch dies entspricht natürlichen Erwerbsverläufen, weil Kinder lernen müssen, wo welche Information enkodiert ist, und welche Positionen in ihrer Sprache besonders relevant sind.

Diese Computersimulationen konkreter Erwerbsverläufe in verschiedenen Sprachen zeigen also, dass wenige Faktoren (Modifikation der Aufmerksamkeitsspanne und des Aufmerksamkeitsfokus) ausreichen, um den Erwerb der Wortstellung vorherzusagen, auch ohne angeborenes Wissen annehmen zu müssen. Dieser Ansatz, der den Spracherwerb mit nur wenigen, psychologisch plausiblen Grundannahmen erklärt, wird kontrastiert mit generativen Ansätzen, die den Verbzweit-Erwerb als inkrementelle Aktivierung angeborener Parameter ansehen. Hier wären nicht die semantischen Eigenschaften des Verbs entscheidend für den Syntaxaufbau wie in der Argumentstruktur, sondern die morphologischen Merkmale des Verbs, die die Bewegung aus der verbfinalen Position lizenzieren (siehe Lasser 1997 und 2002 für eine Zusammenschau der vorgeschlagenen Repräsentationen). 
Lässt sich der Aufbau der Wortstellungsmuster auch in natürlichen Erwerbsdaten beobachten? Die Analyse des großen Leo-Korpus (siehe Fußnote 4) scheint dies zu bestätigen. Behrens (2010) analysierte den Erwerb der Wortstellung aus der Perspektive des Aufbaus des Mittelfelds. Da frühe Kindersprache durch kurze Äußerungen charakterisiert ist, ist die attestierte korrekte Wortstellung nicht überraschend, denn bei Äußerungslängen von 2-3 Wörtern ist die Wahrscheinlichkeit von Wortstellungsfehlern gering, insbesondere dann, wenn man wie im gebrauchsbasierten Paradigma davon ausgeht, dass sich Kinder zunächst konservativ an attestierte Strukturen halten. Von daher scheint es erhellender, Äußerungen zu analysieren, bei denen das Mittelfeld besetzt ist, so dass es im Prinzip eine höhere Wahrscheinlichkeit von Wortstellungsfehlern gibt. Die Auswertungen zeigen, dass das Mittelfeld nur sehr langsam aufgebaut wird: Zunächst stehen Verb und Partikel oder Auxiliar - Partizip/Infinitiv nebeneinander (4), bevor Klitika oder kurze Einzelwörter eingeschoben werden (5):
a. Leo
$1 ; 11.27$
komm mit
b. Leo
$2 ; 00.29$
nicht pack weg
Leo $\quad 2 ; 01.10$
mach mach mach Auge auf

Größere Konstituenten tauchen in Kombination mit komplexen Verben erst drei Monate nach dem Auftreten in Isolation auf (6). Da das Deutsche eine relativ große Variation in der Wortstellung erlaubt, findet man kaum explizite Wortstellungsfehler (4b), sondern eher Strukturen, die pragmatisch unangemessen sind (7).

$$
\text { Leo 2;02.5 }
$$

ein Zug fährt ab

Leo $\quad 2 ; 02.29$

\section{Tilli und Bärbel baben getroffen}

,wir haben Tilli und Bärbel (beim Spaziergang) getroffen ${ }^{6}$

Auf den ersten Blick scheinen die Daten also den schnellen und fehlerfreien Erwerb der deutschen Wortstellung zu bestätigen. Allerdings kommt die Fehlerfreiheit auch dadurch zustande, dass die betreffenden Strukturen in den ersten Monaten des Auftretens extrem selten sind: Im Alter von 2;3 etwa enthalten circa 3.000 der knapp 11.000 aufgezeichneten Äußerungen ein Verb, davon sind 348 komplexe Verbformen. Daneben sind 1.900 komplexe, minimal aus zwei Wörtern bestehende Nominalphrasen attestiert. Aber nur in 53 Äußerungen, also 5 Promille der Gesamtäußerungen, kommen mehrteilige Verben und komplexe NPs zusammen vor. Im Alter von 2;5 beträgt der Anteil solcher komplexen, aber im Deutschen durchaus üblichen Strukturen 2\%. Zieht man die Kürze der meisten Äußerungen in Betracht, bieten sich kaum Möglichkeiten, überhaupt Fehler zu machen. Insofern sind die Daten mit Theorien kompatibel, die von einer langsamen Genera- 
lisierung ausgehen, zumal der Input hinreichende Evidenz für dieses komplexe, aber hochfrequente Phänomen liefert. Im Sinne des „competition model“" (MacWhinney 2004) hätten wir es hier also mit hohen Verarbeitungskosten („,high cue cost"), aber auch hoher Verfügbarkeit („,cue availability") zu tun, zumal der hohe Verarbeitungsaufwand nur dann vorliegt, wenn man das Gesamtsystem betrachtet. Sobald perzeptive Filter wie etwa im MOSAIC-Modell angenommen werden, reduziert sich die Komplexität der jeweils zu verarbeitenden Information erheblich.

Für künftige Forschungen an spontanen Korpora wäre es aufschlussreich, nicht nur nach attestierten Strukturen und ihrer Produktivität zu schauen, sondern auch das Fehlende zu ermitteln. Welche Aspekte des Systems sind noch nicht erworben, obwohl einzelne oder alle Aspekte dieser Strukturen bereits in einigen Kontexten gemeistert werden? Dieses Verfahren lässt sich exemplarisch am Aufbau lexemspezifischen Wissens demonstrieren.

\subsection{Aufbau von Verb-Grammatiken}

Das Verb reparieren ist ein einfaches transitives Verb mit konkreter Bedeutung und von einiger Relevanz im Leben eines Kleinkindes. Die 421 Belege im Leo-Korpus konzentrieren sich dann auch auf die frühen Phasen, als Leo zu reparierende Dinge benennt oder aber Andere zur Reparatur auffordert. Betrachtet man die Argumentrealisierung der einzelnen Wortformen, stellt man folgende Unterschiede fest: Die mit Abstand häufigste Form ist der reine Infinitiv (Abbildung 1), in der Regel ohne weitere Argumente gebraucht, ab 2;3 jedoch auch in Kombination mit Objekt-NP (8a-d) und - seltener - Subjekt-NP (8e) jedoch nie mit Subjekt und Objekt. Die Wortstellung ist dabei variabel $(8 \mathrm{a}-\mathrm{b})$. Die Funktion ist vor allem die einer Bitte oder einer Aufforderung.

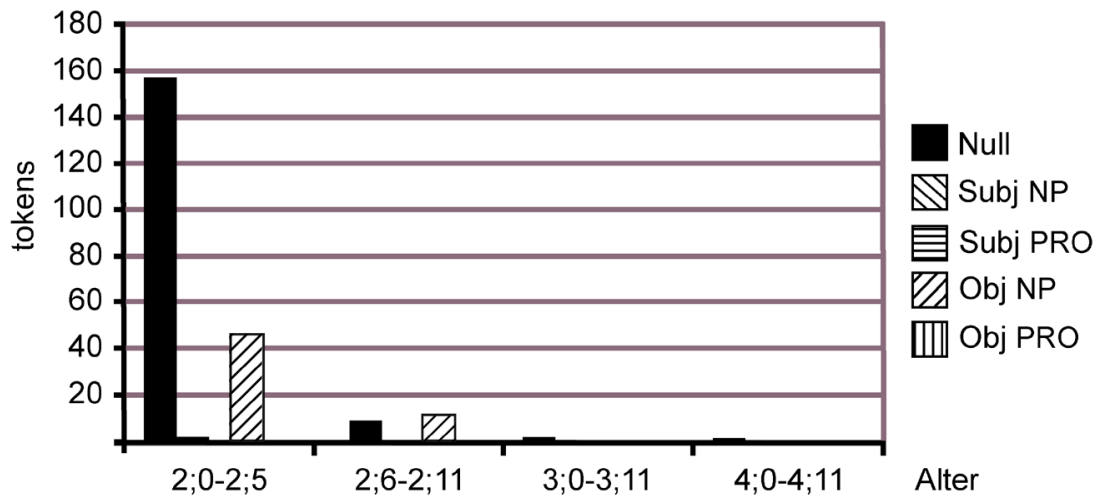

Abb. 1: Argumentrealisierung bei reparieren als Infinitiv 

a. Leo 2;3.8 Brücke reparieren
b. Leo 2;3.8 reparieren die Brücke
c. Leo 2;3.6 I+C+E reparieren
d. Leo 2;3.6 Bus reparieren
e. Leo 2;3.7 Mama reparieren

Als modaler Infinitiv (Abbildung 2) tritt reparieren auch mit Aufforderungsoder Verpflichtungscharakter auf (9), wobei zunächst nur der Agens enkodiert wird.
a. Leo
$2 ; 2.14$
ich muss auch reparieren
b. Leo $2 ; 2.25$
beute xxx, Papa Arb(eit), Papa Papa muss reparieren

Erst mit knapp 2;5 finden sich erste Belege mit Besetzung von Subjekt und Objekt, wobei das Subjekt typischerweise mit einem Eigennamen, das Objekt pronominal realisiert ist $(10 \mathrm{a}, \mathrm{b})$. Der in den elterlichen Tagebuchnotizen attestierte Beleg (10c) über den Wiederaufbau der Dresdner Frauenkirche stellt den Höhepunkt an Komplexität der Belege dar, wobei auch hier die Argumentrealisierung von reparieren selbst wenig komplex ist.
a. Leo $2 ; 4.24$
Ernie soll den reparieren
b. Leo $2 ; 4.23$
muss Ernie den reparieren
c. Leo $2 ; 9.24$ wenn man sieht, dass eine Franenkirche kaputt ist, muss man die sofort reparieren (Tagebuchnotiz)

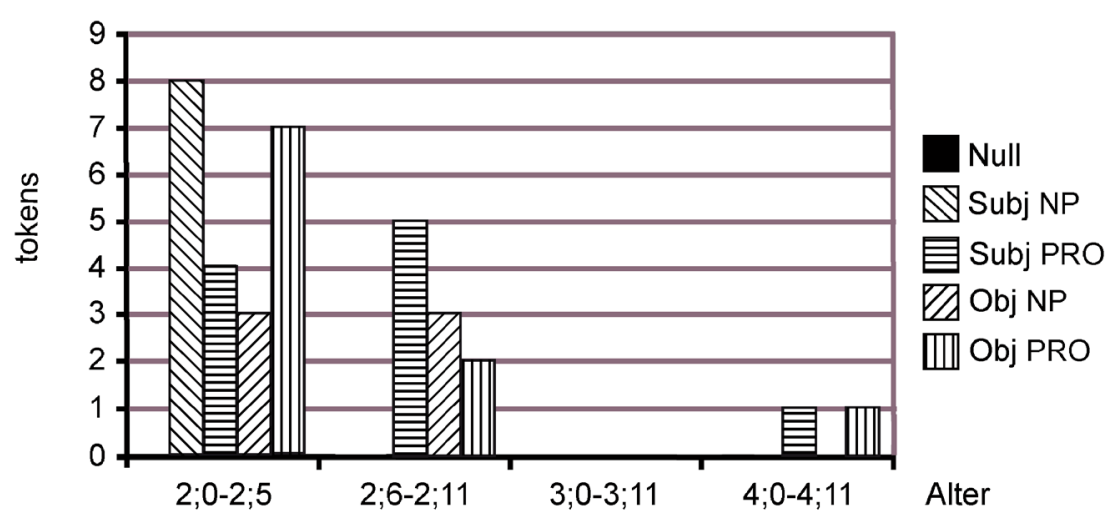

Abb. 2: Argumentrealisierung bei reparieren als Modalinfinitiv

Präsensformen treten ab 2;1.27 auf (Abbildung 3), auch sie zunächst in reduzierter Argumentstruktur (11), bis ab 2;3.20 volle Argumentstrukturen auftreten (12)..$^{5}$ Im Präsens kommentiert Leo vornehmlich die aktuelle Aktivität.

\footnotetext{
In den Abbildungen sind nur die jeweiligen Realisierungen von Subjekt und Objekt erfasst, nicht deren Kombinatorik, was zu einer zu großen Komplexität in der Darstellung geführt hätte.
} 


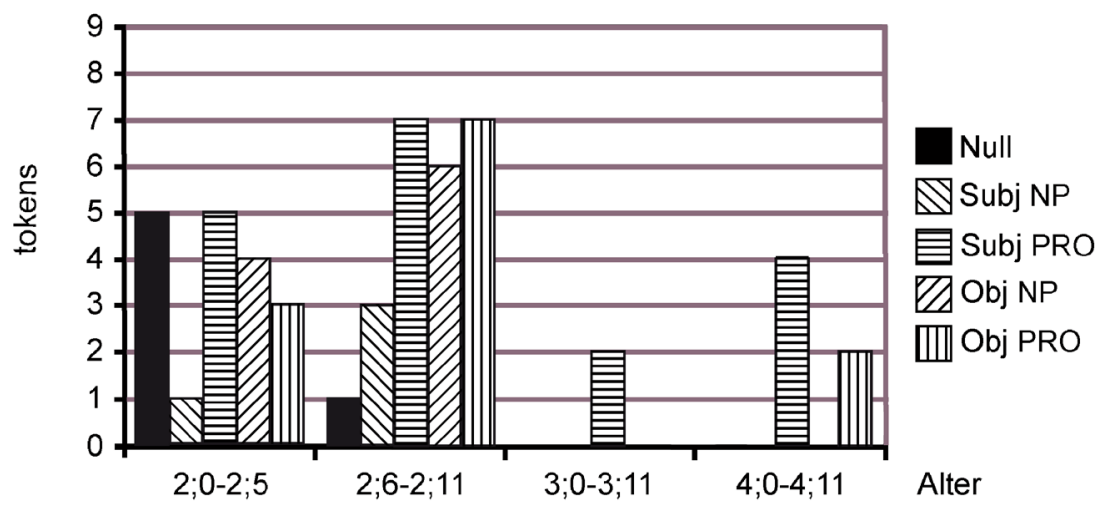

Abb. 3: Argumentrealisierung bei reparieren im Präsens
a. Leo
$2 ; 1.27$
reparierst
b. Leo
2.2 .16
Papa repariert
c. Leo
$2 ; 3.19$
den Tunnel repariert
a. Leo
$2 ; 3.20$
b. Leo
$2 ; 3.29$
ich repariere die Lampe! (Tagebuchnotiz)
c. Leo 2;6.24
der re(pa)riert ein Auto
und die reparieren ein Lichtschalter, die reparieren ein neuen Lichtschalter

Wie schon bei den Daten zum Erwerb der Verbzweitstellung zeigt sich auch hier ein sehr langsamer Strukturaufbau und eine geringe Variabilität der verwendeten Strukturen, sobald man Lexeme oder Strukturen im Detail analysiert. Analysen dieser Art sind mit traditionellen Korpora (30 oder 60 Minuten Aufnahme alle drei oder vier Wochen) kaum durchführbar, da einzelne Items nicht oft genug attestiert sind. So entsteht fast zwangsläufig der Eindruck großer Produktivität, da fast jeder der oft weit auseinander liegenden Belege neue Strukturen und Kookkurenzpartner zeigt. Wenn die Hypothese vom graduellen Strukturaufbau zutrifft, sollte also die Stereotypizität der Äußerungen in größeren Korpora stärker hervortreten, obwohl die Wahrscheinlichkeit der Variabilität in großen Datenmengen größer ist (zum Zusammenhang zwischen Sampling-Rate und der Wahrscheinlichkeit des Auftretens einer Struktur siehe Rowland/Fletcher/Freudenthal 2008).

Eine Methode zur Messung des Grads der syntaktischen Produktivität ist das so genannte „Traceback“" Hier werden die Daten eines Tages dahingehend analysiert, wie groß der Anteil der vorher noch nicht im Korpus attestierten Äußerungen ist, und in welchem Grade und in welcher Art die neuen Äußerungen von den bereits attestierten Äußerungen abweichen (Lieven et al. 2003). Bei einem englischen Kind waren 63\% der mit 2;1.11 attestierten Äußerungen in genau dieser Form schon in einem der Transkripte der 
sechs vorhergehenden Wochen belegt. Von den verbleibenden 109 neuen Äußerungen wiesen über 70\% nur eine minimale Modifikation zu vorher attestierten Äußerungen auf (Einfügen, Weglassen oder Ersetzen eines Wortes oder einer Wortgruppe). Die Übereinstimmung mit vorher Gesagtem wird noch größer, wenn man die Funktion einbezieht (Dąbrowska/Lieven 2005). Die Validität dieses Fortgehens lässt sich testen, indem man die Modifikationsschritte des vorherigen Sprachgebrauchs nimmt, um daraus Vorhersagen über die nächsten Entwicklungsschritte abzuleiten und zu testen (,traceforward“, Bannard/Lieven 2009).

\section{Wie umfassend erklärt Konstruktionsgrammatik den Spracherwerb?}

In dem theoretisch polarisierten Feld der Spracherwerbsforschung ist die am häufigsten gehörte Frage in Diskussionen die, ob Konstruktionsgrammatik tatsächlich Grammatikerwerb erklären könne, der sich in der generativen Sichtweise weitgehend unabhängig von Sprachgebrauch und Bedeutung manifestiert. Aus der Sichtweise gebrauchsbasierter Modelle stellt sich diese Frage nicht, denn grammatische Strukturen werden aus dem Sprachgebrauch abstrahiert, bestehen nicht a priori in irgendeiner mentalen Repräsentation, die nur noch aktiviert bzw. reifen muss. Im Gegenzug stellt sich die Frage, was denn das generative Modell noch erklärt. Im Modell der Prinzipienund Parametertheorie wurden sehr spezifische Vorhersagen zur Universalität sprachlicher Strukturen und zum Spielraum sprachspezifisch zu setzender Parameter gemacht, die aber letztendlich nicht bestätigt werden konnten. Der Minimalismus macht sehr viel sparsamere Vorhersagen zu universalen Strukturen, so dass der Raum dessen, was sprachspezifisch gelernt werden muss, eher größer als kleiner geworden ist. Wie oben angedeutet, ist die praktisch und theoretisch unbefriedigende Konsequenz, dass man so nicht nur zwei Erwerbsmechanismen annehmen muss (einen für die Aktivierung angeborener Strukturen, einen klassischen Lernmechanismus für den Rest), sondern dem Kind ja auch irgendein Wissen angeboren sein muss in Bezug darauf, welchen Mechanismus es denn auf welche Struktur anzuwenden hat. Bezogen auf den Wortstellungserwerb im Deutschen lässt sich sagen, dass das Phänomen der Satzklammer hochgradig regelmäßig und hochgradig präsent ist, insofern als es sich in jedem deutschen Satz mit Verb manifestiert. In der Terminologie des „,competition model“ hätte es also eine hohe „cue strength“ (MacWhinney 2004), gemessen an der Verfügbarkeit (Frequenz) und Verlässlichkeit (Eindeutigkeit). Dass Kinder dieses Phänomen recht zielsicher erwerben, ist also nicht verwunderlich. Dass es ein langwieriger und in seinen Einzelheiten ein eher lokaler als globaler Prozess ist, im Lichte der UG schon. Die oben gezeigten Befunde zum Einstieg in ein- 
fache Satzstrukturen gelten auch für den Erwerb komplexer Sätze: Auch hier ist die Wortstellung in Abhängigkeit des Komplementierers nur ein Aspekt des Erwerbs von Nebensatzkonstruktionen. Genauere Analysen zeigen, dass es einen graduellen Erwerb der Funktion und der an dieser Struktur beteiligten Lexeme gibt: Bei Verben des Meinens und Denkens etwa fungieren erste Strukturen wie I think im Englischen oder ich glaub im Deutschen eher als Satzadverbiale denn als Verben, die einen Komplementsatz subkategorisieren (vgl. Diessel 2004; Brandt/Diessel/Tomasello 2008). Konstruktivistische und konstruktionsgrammatische Ansätze zum Spracherwerb erlauben es, die Funktion der Strukturen in konkreten Diskurssituationen einzubeziehen. Grundlage für den Erwerb sprachlicher Strukturen ist die Äußerung in ihrer kontextgebundenen Manifestation.

\section{Literatur}

Abbot-Smith, Kirsten/Behrens, Heike (2006): How known construction influence the acquisition of other constructions: the German periphrastic passive and future constructions. In: Cognitive Science 30, S. 995-1026.

Akhtar, Nameera (1999): Acquiring basic word order: evidence for data-driven learning of syntactic structure. In: Journal of Child Language 26, S. 339-356.

Allen, Shanley/Skarabela, Barbora/Hughes, Mary (2008): Using corpora to examine discourse effects in syntax. In: Behrens (Hg.), S. 99-137.

Bannard, Colin/Lieven, Elena (2009): Repetition and reuse in child language learning. In: Corrigan, Roberta et al. (Hg.): Formulaic language. Bd. II: Acquisition, loss, psychological reality, functional explanations. Amsterdam/Philadelphia, S. 297-321.

Behrens, Heike (1993): Temporal reference in German child language: form and function of early verb use. Diss. Univ. Amsterdam.

Behrens, Heike (2006): The input-output relationship in first language acquisition. In: Language and Cognitive Processes 21, S. 2-24.

Behrens, Heike (Hg.) (2008): Corpora in language acquisition research: Finding structure in data. (= Trends in Language Acquisition Research 6). Amsterdam/Philadelphia.

Behrens, Heike (2009a): Konstruktionen im Spracherwerb. In: Zeitschrift für Germanistische Linguistik 20, S. 427-444.

Behrens, Heike (2009b). Usage-based and emergentist approaches to language acquisition. In: Linguistics 47, S. 383-411.

Behrens, Heike (2010): Acquiring the middle field: word-order in German. Ms. Basel.

Blom, Elma (2008): The acquisition of finiteness. (= Studies in Generative Grammar 94). Berlin/New York.

Bowerman, Melissa (1990): Mapping thematic roles onto syntactic functions: are children helped by linking rules? In: Linguistics 28, S. 1253-1290. 
Bowerman, Melissa/Brown, Penelope (2008): Introduction. In: Bowerman/Brown (Hg.), S. 1-26.

Bowerman, Melissa/Brown, Penelope (Hg.) (2008): Crosslinguistic perspectives on argument structure: implications for language acquisition. Mahwah.

Boyd, Jeremy K./Gottschalk, Erin A./Goldberg, Adele E. (2009): Linking rule acquisition in novel phrasal constructions. In: Language Learning 59, S. 64-89.

Brandt, Silke/Diessel, Holger/Tomasello, Michael (2008): The acquisition of German relative clauses: a case study. In: Journal of Child Language 35, S. 325-348.

Brown, Penelope (1998): Children's first verbs in Tzeltal: evidence from the early verb category. In: Linguistics 36, S. 713-753.

Dąbrowska, Ewa/Lieven, Elena (2005): Towards a lexically specific grammar of children's question constructions. In: Cognitive Linguistics 16, S. 437-474.

Diessel, Holger (2004): The acquisition of complex sentences. (= Cambridge Studies in Linguistics 105). Cambridge u.a.

Elman, Jeffrey L. (1993): Learning and development in neural networks: the importance of starting small. In: Cognition 48, S. 71-99.

Freudenthal, Daniel/Pine, Julian M./Gobet, Fernand (2006): Unifying cross-linguistic and within-language patterns of finiteness marking in MOSAIC. In: Sun, Ray/ Miyake, Naomi (Hg.): Proceedings of the 28th Annual Meeting of the Cognitive Science Society. Mahwah, S. 232-236.

Freudenthal, Daniel et al. (2007): Modelling the developmental patterning of finiteness marking in English, Dutch, German and Spanish using MOSAIC. In: Cognitive Science 31, S. 311-341.

Freudenthal, Daniel/Pine, Julian M./Gobet, Fernand (2009): Simulating the referential properties of Dutch, German and English Root Infinitives in MOSAIC. In: Language Learning and Development 5, S. 1-29

Gentner, Dedre (1978): On relational meaning: the acquisition of verb meaning. In: Child Development 49, S. 988-998.

Gleitman, Lila R. (1990): The structural sources of verb meaning. In: Language Acquisition 1, S. 3-55.

Goldberg, Adele E. (2003): Constructions: a new theoretical approach to language. In: Trends in Cognitive Sciences 7, S. 219-224.

Goldberg, Adele E. (2006): Constructions at work: the nature of generalization in language. Oxford.

Gropen, Jess et al. (1991): Affectedness and direct objects: the role of lexical semantics in the acquisition of verb argument structure. In: Cognition 41, S. 153-195.

Hohenberger, Annette/Peltzer-Karpf, Annemarie (2009): Language learning from the perspective of nonlinear dynamic systems. In: Linguistics 47, S. 481-511.

Höhle, Barbara (2009): Bootstrapping mechanisms in first language acquisition. In: Linguistics 47, S. 359-382. 
Jordens, Peter (1990): The acquisition of verb placement in Dutch and German. In: Linguistics 28, S. 1407-1448.

Landau, Barbara/Gleitman, Lila R. (1985): Language and experience: evidence from the blind child. Cambridge, MA.

Langacker, Ronald W. (1987): Foundations of cognitive grammar. Bd. 1: Theoretical prerequisites. Stanford.

Lasser, Ingeborg (1997): Finiteness in adult and child German. (= MPI Series in Psycholinguistics 8). Nijmegen.

Lasser, Ingeborg (2002): The roots of root infinitives: remarks on infinitival main clauses in adult and child language. In: Linguistics 40, S. 767-796.

Levin, Beth (1993): English verb classes and alternations: a preliminary analysis. Chicago.

Levin, Beth/Rappaport-Hovav, Malka (1995): Unaccusativity: at the the syntax-lexical semantics interface. (= Linguistic Inquiry Monographs 26). Cambridge, MA.

Lieven, Elena et al. (2003): Early syntactic creativity: a usage-based approach. In: Journal of Child Language 30, S. 333-370.

Lindner, Katrin/Hohenberger, Annette (2009): Introduction: concepts of development, learning, and acquisition. In: Linguistics 47, S. 211-239.

MacWhinney, Brian (2000): The CHILDES Project: tools for analyzing talk. 2 Bde. 3. Aufl. Mahwah.

MacWhinney, Brian (2004): New directions in the competition model. In: Slobin, Dan I./ Tomasello, Michael (Hg.): Beyond nature-nurture: essays in honor of Elizabeth Bates. Mahwah, S. 81-110.

McDonough, Laraine/Choi, Soonja/Mandler, Jean M. (2003): Understanding spatial relations: flexible infants, lexical adults.In: Cognitive Psychology 46, S. 229-259.

Naigles, Letitia R. (2002): Form is easy, meaning is hard: resolving a paradox in early child language. In: Cognition 86, S. 157-199.

Newport, Elissa L. (1990): Maturational constraints on language learning. In: Cognitive Science 14, S. 11-28.

Newport, Elissa L./Aslin, Richard N. (2004): Learning at a distance: I. Statistical learning of non-adjacent dependencies. In: Cognitive Psychology 48, S. 127-162.

Pine, Julian M./Lieven, Elena V.M./Rowland, Caroline F. (1998): Comparing different models of the English verb category. In: Linguistics 36, S. 807-830.

Pinker, Steven (1987): The bootstrapping problem in language acquisition. In: MacWhinney, Brian (Hg.): Mechanisms of language acquisition. Hillsdale, S. 399-441.

Pinker, Steven (1989): Learnability and cognition: the acquisition of argument structure. Cambridge, MA.

Pinker, Steven (1994): How could a child use verb syntax to learn verb semantics? In: Lingua 92, S. 377-410. 
Rowland, Caroline F./Fletcher, Sarah L./Freudenthal, Daniel (2008): How big is big enough? Assessing the reliability of data from naturalistic samples. In: Behrens (Hg.), S. 1-24.

Savage, Ceri et al. (2003): Testing the abstractness of children's linguistic representation: lexical and structural priming of syntactic constructions in young children. In: Developmental Science 6, S. 557-567.

Tomasello, Michael (1992): First verbs: a case study of early grammatical development. Cambridge.

Tomasello, Michael (1998a): Introduction: a cognitive-functional perspective on language structure. In: Tomasello, Michael (Hg.): The new psychology of language: cognitive and functional approaches to language structure. Mahwah, S. vii-xxiii.

Tomasello, Michael (1998b): The return of constructions. Review Essay on: Goldberg, A. (1995). ,Constructions: a construction grammar approach to argument structure'. In: Journal of Child Language 25, S. 443-484.

Tomasello, Michael (2000): Do young children have adult syntactic competence? In: Cognition 74, S. 209-253.

Tomasello, Michael (2003): Constructing a language: a usage-based account of language acquisition. Cambridge, MA.

Tomasello, Michael/Brooks, Patricia J. (1998): Young children's earliest transitive and intransitive constructions. In: Cognitive Linguistics 9, S. 379-395. 
Rosemarie Tracy

\title{
Konstruktion, Dekonstruktion und Rekonstruktion: Minimalistische und (trotzdem) konstruktivistische Überlegungen zum Spracherwerb
}

\begin{abstract}
Der kindliche Spracherwerb zeichnet sich sowohl durch seine Robustheit als auch durch eine spezifische Dynamik aus. Im Mittelpunkt des Beitrags stehen frühe Phasen des Erwerbs des Deutschen, in denen Konstruktionen unterschiedlicher Analysetiefe koexistieren, die im Laufe der Zeit immer wieder reanalysiert werden und schließlich auf einen einzigen abstrakten Bauplan hin konvergieren. Anhand von Daten aus diversen Fallstudien wird gezeigt, dass die Entwicklung der so genannten „Satzklammer“ des Deutschen als Konvergenzprozess verstanden werden kann, bei dem Lerner bewährte Teilsysteme, darunter anfängliche teilproduktive Formeln, dekonstruieren und auf abstrakterer Ebene rekonstruieren. Untersucht werden typische Übergangsphänomene, u.a. die Verwendung von Platzhaltern und Doppelbesetzungen, die diese Konstruktionsleistung erkennen lassen. Argumentiert wird, dass sich generative und dynamische, konstruktivistische Ansätze bei dem Bemühen um eine Erklärung dieses Erwerbsprozesses sinnvoll ergänzen.
\end{abstract}

\section{Einleitung}

In seinem 2008 erschienenen Buch „Kluge: The Haphazard Construction of the Human Mind“ konfrontiert uns Gary Marcus auf humorvolle Weise mit diversen Mängeln unserer Spezies, von der suboptimalen Konstruktion unserer Wirbelsäule und unseres Auges bis zu den Pannen unseres Gehirns, das entgegen besseren Wissens allerlei Vorurteilen, Suggestionen und Fehlschlüssen aufsitzt. Aus Sicht eines Ingenieurs ist ein Kluge - [klu:d3] ausgesprochen - ,a clumsy or inelegant solution to a problem“, so die Definition auf der Titelseite des Buches. Elegant oder nicht, vermutlich haben nicht wenige Kluges Leben gerettet. ${ }^{1}$

Wie Marcus ausführt, passt die menschliche Sprache samt beteiligter Organe in den Reigen der von ihm diskutierten Kluges: Ambiguität, Vagheit, Redundanzen und Idiosynkrasien sprechen für diese Sicht, denn Sprache „was built, rapidly, on a haphazard patchwork of mechanisms that originally evolved for other purposes“ (Marcus 2008, S. 122). Richtet man andererseits - ein Gesichtspunkt, den Marcus ebenfalls würdigt - den Blick

Marcus (2008, S. 2) erinnert an die ad hoc-Konstruktion des lebensrettenden $\mathrm{CO}_{2}$-Filters, den sich das Team der Apollo 13-Mission im Weltraum aus einer Plastiktüte, Klebeband, einem Karton und einer Socke bastelte. 
auf abstrakte Prinzipien und Ebenen der Repräsentation, so versteht man, warum Chomsky behaupten kann, dass der Architektur natürlicher Sprachen in mancher Hinsicht ein nahezu perfektes minimales Design zugrundeliegt (Chomsky 2000, S. 93). Mithin stellt sich die Frage, wie man sich die Genese dieses Designs vorzustellen hat: Hat es überhaupt, auf die Entwicklung des Kindes bezogen, eine Entstehungsgeschichte oder handelt es sich um einen universellen, angeborenen Bauplan, der nur noch von relevanter Erfahrung ausgelöst werden muss? Anliegen meines Beitrags ist es, zu zeigen, dass sich Evidenz für einen idealtypischen abstrakten Bauplan bereits in den frühsten Phasen des Spracherwerbs nachweisen lässt. Den Wandel, den wir an Lernergrammatiken - präziser: an dem Output dieser Grammatiken - feststellen, interpretiere ich dabei als aktive Konstruktionsleistung in Richtung eines minimalen, abstrakten Schemas. Strukturen innerhalb von Lernersystemen, die anfänglich verbindungslos koexistieren, geraten mit der Zeit sozusagen in den „Sog“ eines Attraktors. Sie werden als Instantiierungen eines und desselben Bauplans reanalysiert, d.h. de- und rekonstruiert. Bei den Strukturen, die sich einer solchen Metamorphose unterziehen, handelt es sich interessanterweise um Konstruktionen, die sich nicht selten in der Kommunikation bewährt hatten und möglicherweise im eigenen Output zeitweise sogar besonders frequent waren. Mit anderen Worten: Es handelt sich um sprachliche Kluges, also um passable Lösungen, die sich aber aufgrund einer veränderten Lernerperspektive als verbesserungswürdig erweisen.

Den theoretischen Rahmen meiner Ausführungen bildet die Vorstellung von einer universalgrammatisch gestützten Selbstorganisation, in der sowohl die Erkenntnisse der generativen Linguistik als auch der Theorie dynamischer Systeme eine wichtige Rolle spielen (vgl. Elman 1993, 2002; Thelen/Smith 1994; Hohenberger 2002; Hohenberger/Peltzer-Karpf 2009). Wegen seiner Affinität zum minimalistischen Programm unterscheidet sich der hier vorgestellte Ansatz prinzipiell von rein gebrauchsbasierten Erklärungen des Spracherwerbs (Tomasello 2003; Goldberg 2006; Behrens in diesem Band). Andererseits bildet meine Position, die erhebliche Reorganisation von Lernersystemen zulässt, auch ein Kontrastprogramm zu starken Kontinuitätshypothesen (vgl. den Überblick in Eisenbeiß 2009).

Abschnitt 2 beginnt mit allgemeinen Ausführungen zum Erwerb des Deutschen, das sich gerade im Hinblick auf die Frage, wie scheinbare Inkonsistenz und Inkompatibilität im Input Lerner herausfordern könnten, als eine besonders interessante Zielsprache erweist. In Abschnitt 3 folgt eine relativ unkontroverse Zusammenstellung relevanter Beobachtungen über den typischen Erwerbsverlauf und im Anschluss eine Diskussion von Übergangsphänomenen, die ich im Sinne von De- und Rekonstruktionsprozessen interpretiere. Dabei geht es um die Ausdifferenzierung von Vorläufern der linken Satzperipherie, die Konkurrenz um syntaktische Positionen, Platz- 
halterphänomene, und um einen Versuch, Form und Funktion konsistent zu alignieren. In Abschnitt 4 betrachte ich den monolingualen Erstspracherwerb aus dem Blickwinkel des bilingualen Erwerbs, d.h. unter besonderer Berücksichtigung der „Artenvielfalt" koexistierender Konstruktionen, bevor ich auf den „echten“ "bilingualen Spracherwerb eingehe. Mein Anliegen ist es, zu verdeutlichen, wie früh Kinder dazu in der Lage sind, mit koexistierenden grammatischen Optionen umzugehen, und wie sie ihre bilingualen Ressourcen nutzen, um Lücken der einen Sprache mit Hilfe der anderen auszugleichen. Im Diskussionsteil (Abschnitt 5) gehe ich dann der Frage nach, welche Prinzipien Rekonstruktions- und Konvergenzprozesse steuern könnten.

Im Folgenden spreche ich immer wieder in einem eher neutralen, umgangssprachlichen Sinne von Konstruktionen. Sofern es allerdings um analytische und deskriptiv relevante Aspekte geht, orientiere ich mich an der von Jacobs (2008) getroffenen Unterscheidung von atomaren und komplexen Konstruktionen. Letztere schließen „hochschematische“ (S. 7), abstrakte Konstruktionen ein, z.B. die Kopf-Komplement-Relation der X-barTheorie, die in meinen Überlegungen eine zentrale Rolle spielen wird.

\section{Spracherwerb im Allgemeinen und Deutsch als Erwerbsziel}

Ein oft hervorgehobenes und unstrittiges Charakteristikum des Erstspracherwerbs ist seine Robustheit. Typischerweise, d.h. in gut 95\% aller Fälle, eignen sich Kinder, sofern sie in einer normalen sprechenden oder gebärdenden Umgebung aufwachsen, in den ersten drei bis vier Lebensjahren die wichtigsten strukturellen Eigenschaften der Grammatiken ihrer Erstsprachen an. Der Plural in „Grammatiken“ und „Erstsprachen“ ist aus zwei Gründen berechtigt: Erstens kann ein Kind von Geburt an problemlos mit mehr als einer Erstsprache aufwachsen, und zweitens spielt die Koexistenz verschiedener Grammatiken möglicherweise auch beim (vermeintlich) monolingualen Erwerb eine nicht zu unterschätzende Rolle - ein Gesichtspunkt, auf den ich in Abschnitt 4 zurückkomme.

Unstrittig ist ferner die Annahme, dass die Sprachfähigkeit und damit der Spracherwerb auf einer humanspezifischen genetischen Grundausstattung beruhen. Kontroversen entzünden sich vor allem bezüglich der Natur dieser Initialausstattung und hinsichtlich der Details der Interaktion mit der Umwelt. Gibt es domänenspezifische, auf Sprache spezialisierte, angeborene Prinzipien oder reichen allgemeine kognitive Mechanismen und Strategien des Menschen aus, um die Komplexität natürlicher Sprachen hervorzubringen (vgl. Chomsky 1986, 2000; Newmeyer 2000 einerseits und Tomasello 2003; Goldberg 2006 andererseits)? Welche Funktion kommt dem Input zu und dabei insbesondere der Auftretenshäufigkeit bestimmter Phänomene (vgl. dazu auch Behrens in diesem Band)? 
In dem von mir im Folgenden entworfenen Bild des Spracherwerbs spielen neben einem minimalen, minimalistischen Apriori - allem voran die Unterscheidung von Kopf und Nicht-Kopf - und dem sprachlichen Angebot der Umgebung als dritte Instanz im Bunde generelle kognitive Strategien der Informationsverarbeitung und der Selbstorganisation eine wichtige Rolle (vgl. auch Yang 2009; Hohenberger/Peltzer-Karpf 2009). Vielen gebrauchsbasierten Theorien zufolge leisten solche allgemeinen, nicht auf Sprache spezialisierte Mechanismen ohnehin alles (vgl. Tomasello 2003). Aber auch nativistische Ansätze benötigen ihre Unterstützung, damit sprachliche Bereiche erworben werden können, die außerhalb des kerngrammatischen Bereichs liegen, wie die idiosynkratische Peripherie von Einzelsprachen, aber auch um das so genannte Bootstrapping-Problem zu lösen (vgl. Pinker 1984; Höhle 2009). Universalgrammatische Beschränkungen gelten typischerweise für abstrakte, segmentierte Symbolketten. Bevor sich aus solchen Beschränkungen Nutzen ziehen lässt, müssen elementare Segmentations- und Klassifikationsentscheidungen schon getroffen worden sein. Allein die Segmentier- und Klassifikationsaufgabe setzt ein gewisses Maß an statistischem Lernen voraus (vgl. Yang 2009; Elman 1993, 2002). Zugleich wissen wir, dass der Erwerb des Wortschatzes nicht vieler Wiederholungen bedarf und dass einige Aspekte des Fast Mapping, z.B. eine erste, rudimentäre Hypothese über die Verknüpfung von Bezeichnung und Referent, auch anderen Spezies zugänglich sind (Kaminski/Call/Fischer 2004). Wie Roeper (2007) unterstreicht, erwerben Kinder sehr präzises Wissen über Strukturen, die im Input ausgesprochen selten sind, wie rekursive multiple Possessiva. Englischsprachige Kinder erkennen im Alter von 4-6 Jahren, dass Daddy's uncle's car's wheels zulässig ist. ${ }^{2}$ Eine entsprechende Einbettungstiefe ist in deutschen possessiven DPs prinzipiell nicht möglich (vgl. Papas Onkels Auto, aber *Papas Onkels Autos Räder). Das heißt, dass sich Sprachen auch bei universell verfügbaren Prinzipien wie der Rekursion im Detail der Implementierung voneinander unterscheiden und dass die Entscheidung „möglich/unmöglich“ im Verlauf des Spracherwerbs beiläufig und auf der Basis sehr limitierter Erfahrung getroffen werden muss.

Die Fähigkeit zur rekursiven Satzeinbettung ist jedenfalls in der Sprachproduktion deutschsprachiger Kinder bei schnellen LernerInnen bereits vor dem dritten Geburtstag gut belegt, vgl. (1)-(4). ${ }^{3}$

2 Roeper (2007, S. 112) schreibt: „Vanishingly few recursive possessives occur in either adult or child English. Numbers are hard to obtain, but it would surprise me if 1 percent of possessives involve recursion. That is, there are probably 99 cases like John's car for every case like John's friend's car. [...] It must be that a few examples trigger recognition that a structure can embed itself." Vgl. auch Roeper/Pérez-Leroux (eingereicht).

3 Die im Folgenden angeführten Daten stammen aus einer Longitudinalstudie von Tracy (1991), in der in einem Zeitraum von anderthalb Jahren wöchentliche Aufnahmen von vier Kindern gemacht wurden, sowie aus zwei DFG-Projekten der Verfasserin („Erwerb der komplexen 
(1) Valle 2;2 da ist die tür, wo man immer raus und rein kann

(2) Lisa 2;3 valle hat mich gefragt am telefon, ob ich auch inə kindergruppe gehen kann

(3) Stefanie 2;9 weil sie nicht anziehn will, darum weint's

(4) Julia 2;9 wenn die julia futter reintut, dann fressen die vögeln alles auf

Das grammatische Kenntnissystem, über das diese Kinder verfügen, lässt sich mit Hilfe des folgenden topologischen Schemas erfassen (vgl. Höhle 1986; Zifonun/Hoffmann/Strecker 1997).

\begin{tabular}{|c|c|c|c|}
\hline & Vorfeld & Mittelfeld & Nachfeld \\
\hline Hauptsatz: & $\mathrm{X}$ & 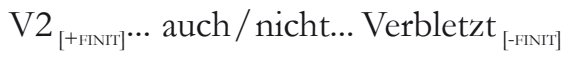 & Y \\
\hline Nebensatz: & & $\begin{array}{c}\text { Kompl. ...auch / nicht... Verbletzt } \\
\Uparrow{ }_{[+ \text {FINIT }} \\
\text { SATZKLAMMER }\end{array} \overbrace{}$ & $\mathrm{Y}$ \\
\hline
\end{tabular}

Wer das Deutsche erwirbt, muss folgende Eigenschaften dieses Bauplans für sich rekonstruieren: Das finite (tempus- und kongruenzmarkierte) Verb steht im deutschen Hauptsatz in der zweiten Position (V2), der linken Satzklammer. Nicht-finite Verbteile (Partizipien, Infinitive, Verbpartikeln) treten am Satzende (Verbletzt) in der rechten Satzklammer auf (Ich hab mein Herz in Heidelberg verloren). Im Vorfeld (X) finden sich (fast) beliebige Konstituenten (In Heidelberg hab ich ..., Mein Herz, bab ich ...). Das Nachfeld (Y) nimmt Relativsätze oder Konstituenten auf, die aus stilistischen Gründen oder auch um eines Reims Willen (...verloren (,) in einer lauen Sommernacht) verschoben wurden. Bleibt das Vorfeld unbesetzt (wie im Ja/Nein-Interrogativsatz, einem Imperativ oder am Anfang eines Konditionalsatzes wie diesem), erscheint das finite Verb in Spitzenstellung (Verberst). Im Fall eines eingebetteten Nebensatzes (Ich weiß ja, dass du dein Herz in Heidelberg verloren hast. Ich weiß, was du lieber getan bättest), der von einem Komplementierer oder einem Interrogativpronomen eingeleitet wird, tritt das finite Verb nach allen nicht-finiten Verbteilen in der rechten Satzklammer auf (Verbletzt). Links vor dem Vorfeld finden sich nebenordnende Elemente, u.a. denn, weil in denn-Lesart, aber, sondern (denn/weil du bast schon zum wiederbolten Mal dein Herz, in Heidelberg verloren).

Ich nehme an, dass die Kinder aus (1)-(4) bereits (implizit) wissen, dass ihren Haupt- und Nebensätzen - aus einer etwas abstrakteren Perspektive betrachtet - eine Konstituentenstruktur zugrunde liegt, deren einzelne Schichten von lexikalischen und funktionalen Köpfen projiziert werden: V (Verb), FP (= Fokuspartikel), Inflection (Tempus- und Kongruenzmerkmale) und C (Komplementierer). Der minimale Bauplan jeder einzelnen

Syntax des Deutschen“, „Child Languages in Contact“), in der vier monolinguale (Deutsch) und vier bilinguale Kinder (Deutsch und Englisch) ein Jahr lang alle zwei Wochen und danach einmal monatlich aufgenommen wurden. 
Schicht besteht aus einer binären Verzweigung von Kopf und Komplement, in einem weiteren binären Schritt erweitert um einen Spezifikator (X-bar-Schema, vgl. Radford 2009). Das Ergebnis ist eine hierarchische Struktur aus fraktalen, selbst-identischen Strukturschichten. ${ }^{4}$

(6) [CP [IP [ [

Was haben die zitierten Kinder im Verlauf ihres Spracherwerbs bis hin zu ihren komplexen Satzstrukturen gemacht? Haben sie anfänglich Fragmente eben solcher Äußerungen aus dem Input aufgegriffen, ganzheitlich abgespeichert und allmählich erweitert, wie es gebrauchsbasierte Ansätze erwarten lassen (Goldberg 2006; Tomasello 2003; Behrens in diesem Band)? Ich werde im Folgenden versuchen, zu zeigen, dass selbst die Fragmente, die Kinder anfänglich dem Sprachgebrauch ihrer Umgebung entnehmen, sehr schnell auf ihre Passung zu einem Prinzip binärer Strukturbildung hin überprüft und gegebenenfalls restrukturiert werden.

\section{Entwicklungsphasen und Entwicklungskrisen}

\subsection{Verben als Schrittmacher}

Wie systematisch Kinder sich die deutsche Satzstruktur erschließen, erkennt man gut anhand der Platzierung von Verben und Verbpartikeln. Schon in Zweiwortkombinationen kann man aufgrund ihrer Positionierung Vorboten der rechten Satzklammer erkennen (Ball weg, Ball reinmachen). Zugleich manifestiert sich in dieser Abfolge bereits das minimale Design aus Kopf und Nichtkopf, mit konsequenter finaler Positionierung des nicht-finiten Kopfes. Typischerweise ist die Realisierung der Argumentstruktur einige Wochen lang beschränkt: Wir finden Wagen rein und Julia rein, aber nicht Julia Wagen rein (vgl. Tracy 1991).

Für die Treffsicherheit, mit der Kinder gerade die Verbpartikeln aufgreifen, gibt es viele gute Gründe: Sie sind platzfest, morphologisch invariant und meistens morphologisch einfach, und sie kodieren wichtige semantische Teilereignisse (Schulz/Wymann/Penner 2001). Als abtrennbarer Bestandteil eines Verbkompositums (HINgehen, WEGgerannt) tragen sie den Wortakzent. Zudem besetzen sie am Satzende ohnehin eine besonders saliente Position (vgl. Slobin 1973). Hinzu kommt, dass sie mit diesen Vorzügen eine über alle Ebenen hinweg ,perfekte“ minimale Gestalt bilden und zwar

Die Bezeichnung und Menge der für das Deutsche postulierten Strukturschichten ist an dieser Stelle nicht relevant. Für eine Auseinandersetzung innerhalb der Spracherwerbsforschung mit unterschiedlichen Theorien zur Struktur deutscher Sätze vgl. die Aufsätze in Dimroth/Jordens (Hg.) (2009), Meisel (Hg.) (1992), Tracy/Lattey (Hg.) (1994) sowie Weissenborn (2000). 
im Hinblick auf den von Newport (1990) und Elman (1993) betonten Vorteil des „starting small“: Kinder richten ihre Aufmerksamkeit auf einen kleinen Ausschnitt des im Prinzip Verfügbaren.

Bereits in Zweiwortäußerungen finden sich weitere formal invariante und betonbare Partikeln: die Fokuspartikeln auch, noch, wieder, nicht etc., die sich in der Zielsprache fast ausschließlich innerhalb des Mittelfelds bewegen (vgl. Dimroth 2009; Nederstigt 2004; Penner/Tracy/Wymann 1999; Penner/Tracy/Weissenborn 2000; Winkler 2009) und die in Mehrwortkonstruktionen ihrerseits folgende VPs bzw. VP-Fragmente als Komplement nehmen, vgl. (7b).

Die Entwicklung von ersten Konstruktionen, die man bereits als Projektionen eines verbalen Kopfes analysieren kann, im Alter von etwa anderthalb Jahren bis hin zu Nebensätzen im Alter von drei bis dreieinhalb Jahren wird in (7) erfasst. Die Phase der Einwortäußerungen wird an dieser Stelle ausgespart, ebenso die detaillierte Binnenstruktur der einzelnen Konstruktionen. Auf einige Besonderheiten, vor allem im Zusammenhang mit den Fokuspartikeln, komme ich später zurück.

(7) (a) Bus weg, Bus reingehn

(b) Mama auch Bus reingehn

(c) Da geht Mama auch Bus rein Will Mama auch Bus reingehn?

(d) ... dass Mama auch Bus reingeht

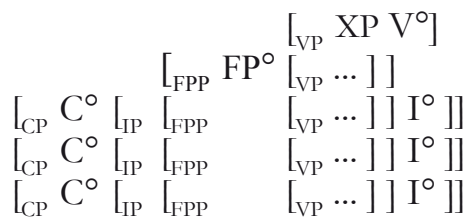

Das Auftreten von Vollverben in der Verbzweitposition korreliert beim ungestörten Erstspracherwerb sehr schnell mit der korrekten Finitheitsmarkierung der Verben (vgl. Clahsen 1988; Clahsen/Penke 1992). Mit dem Erscheinen von Komplementierern in der linken Satzklammer bzw. $\mathrm{C}^{\circ}$ treten finite Verben in der rechten Satzklammer auf (Rothweiler 1993; vgl. Fritzenschaft et al. 1990 und Gawlitzek-Maiwald/Tracy/Fritzenschaft 1992 für eine Diskussion inter-individueller Variation). Obgleich im Anschluss an diese Entwicklung hinsichtlich paradigmatischer Details (Ausdifferenzierung von Wortklassen, Verwendung von Artikeln, Präpositionen, Kasus- und Genusmarkierung) noch viel geschehen muss, steht ${ }^{`}$ zu diesem Zeitpunkt der formale Bauplan deutscher Sätze im Sinne von (5) und (6), und zwar unabhängig von den illokutiven Funktionen der jeweiligen Strukturen.

Wir wissen, dass sich auch Zweitsprachlerner, die zum ersten Mal im Alter von drei bis vier Jahren mit dem Eintritt in eine Kindertagesstätte in Kontakt mit dem Deutschen kommen, die Grundstruktur deutscher Hauptsätze (V2-Effekte, Subjekt-Verb-Kongruenz) innerhalb von sechs bis zwölf Monaten erschließen können und die Strukturen des Nebensatzes im Laufe des zweiten Kontaktjahres erwerben (Rothweiler 2006; Wenzel/ Schulz/Tracy 2009; Thoma/Tracy 2006). Dies ist angesichts des im Kindergartenkontext oft suboptimalen Inputs bemerkenswert. Wenig überra- 
schend ist hingegen, dass Zweitsprachlerner, denen es gelingt, schnell ein umfangreiches Verblexikon aufzubauen, auch zügig die Satzklammer und damit den abstrakten Bauplan deutscher Sätze entdecken (vgl. Tracy/Thoma 2009).

Das bisher skizzierte Erwerbsszenario stützte sich auf kindliche Produktionsdaten und ließ frühe Diskriminations- und Verstehensleistungen von Kindern außer Acht (vgl. Weissenborn et al. 1998; Höhle 2009). Die zeitliche Abfolge (7a)-(d) muss sich nicht bei allen Kindern overt manifestieren, d.h. sowohl bei wenig redefreudigen als auch bei sehr schnellen Lernern lässt sich möglicherweise kein klar abgrenzbares Nacheinander von Erwerbsschritten belegen. Im nächsten Abschnitt geht es um weitere Konstruktionstypen und um Übergangslösungen, die bisher ebenfalls ausgeblendet blieben, die aber für ein Verständnis der Emergenz von Verbzweitkonstruktionen essenziell sind.

\subsection{V2-Vorläufer}

Zeitgleich mit frühen verbbasierten Zwei- und Mehrwortäußerungen des Typs (7a)-(b) verfügen Kinder typischerweise über ein mehr oder weniger umfangreiches Repertoire teilproduktiver, lexem-spezifischer Schemata und gänzlich holistischer Ausdrücke (vgl. für das Deutsche Kaltenbacher 1990; für Englisch Peters 1977, 1983, 2001 und Tomasello 2000, 2003), die sich formal bereits als Vorläufer der linken Peripherie deutscher Hauptsätze deuten lassen, vgl. die Belege in (8) mitsamt plausibler zielsprachlicher Modelle auf der rechten Seite.

(8) (a) [nmaxta]

(Was) Macht der?

(b) [takœətə] (beim Legen von Puzzleteilen)

Da gehört (d)er hin

(c) [(ta) komtə], [ [te:tə], [zitsta]

Da kommt/steht/sitst (d)er

(d) [ge:tIç], [klaptIç]

Geht nicht. Klappt nicht.

(e) [atomis] (immer beim Runterspringen)

Da komm ich!

(f) [vosə] X?, [dasə] X

Wo ist X? Da ist X.

Die Kopula-basierten Platzhalter der linken Peripherie in (8f), [vosə] und [dasə] und ihre Varianten, treten dabei mit Nomen oder NPs beliebigen Genus sowie mit Singular- und Pluralformen auf, vgl. (9).

$$
\begin{array}{ll}
\text { Stefanie } 1 ; 10 & \text { (a) }[\text { vosə] də leine ? } \\
& \text { (b) [vodə] noch ein Zapfen? } \\
\text { Julia 2;0 } & \text { (c) }[\text { dazə] Leute }
\end{array}
$$

Äußerungen dieser Art wirken auf ZuhörerInnen solange unauffällig, wie sich die mit dem Verb kookkurierenden Elemente voneinander unterscheiden. In diesem Fall interpretiert man sie automatisch analog zu da komm $(t)$-die, die komm $(t)$-da etc. Man findet aber auch perfekt symmetrische Syntagmen, 
z.B. da komm $(t)-d a$, do komm $(t)-d ə$, bei denen sich pronominale Argumente und Adverbien nicht identifizieren lassen. Es handelt sich, sofern man überhaupt von einer motivierten Segmentation ausgehen kann, bestenfalls um Platzhalter, ein Phänomen, auf das ich in Abschnitt 3.4 zurückkomme.

Bei entsprechender Erhebungsdichte lässt sich bei besonders gängigen Strukturen eine allmähliche Reanalyse gut erfassen. (10) zeigt dies für Kopulaformeln exemplarisch anhand der Daten eines Kindes. Die Prozentangaben in Klammern geben den Anteil an Äußerungen an, die aus Zwei- und Mehrwortkombinationen bestehen; der MLU-Wert (mittlere Äußerungslänge) wurde anhand von Morphemen ermittelt.

(10) (a) Julia 1;8 MLU $1.11(10 \%)$ da X, [dasə] X

(b) 2;0 MLU $2.01(53 \%)$ da X, [dasə] X, [vosə] X? (mit Varianten)

(c) 2;2 MLU 2.4 (45\%) [isə] taschentuch?, da [isə] brei, da [zins] noch kinder, hier [zinzo], drüben [isə] haifisch die fuß da is $\backslash$ (streckt einen Fuß aus)

(e) 2;4 MLU 3.5 (57\%) wo is das junge is?

$2 ; 5$

$2 ; 6$ wo də anere is? wo's də anere is? wo's meine mülltonne is?

Zunächst (vgl. (10a)-(b)) wachsen über mehrere Monate hinweg die Varianten des deiktischen Schemas und der Kombination mit [vosə] an, ohne dass eine durch das Genus oder Numerus des folgenden Nomens determinierte Systematik erkennbar wäre (vgl. [voz̧] leute?).

Zwei Monate später, vgl. (10c)-(d), sind der Status der Kopula als unabhängiges Lexem und die Numerusdifferenzierung klar zu erkennen, auch wenn das Ergebnis noch nicht immer bis ins Detail zielsprachlich ist (vgl. da [zins] noch kinder). Wie man an (10d) sieht, gibt es einen ersten Beleg für eine Kopula in der rechten Satzklammer. Gelegentliche Verbverdopplungen (vgl. (10e)) belegen, dass beide Satzklammerpositionen nun für die gleichen Verben zur Verfügung stehen.

Bemerkenswert sind Äußerungsfolgen, in denen sich Kinder spontan selbst verbessern, indem sie eine bewährte Vorläuferkonstruktion durch eine aktuellere Variante ersetzen, vgl. (11)-(12). Die Reparatur deutet darauf hin, dass das holistische [dazo]/][dasa] auch aus Lernerperspektive nurmehr ein Relikt ist, das allerdings auf der Ebene artikulatorischer Planung - u.a. als präferierter trochäischer $\mathrm{Fu}$ - wahrscheinlich noch als Routine aktiviert werden kann. Man beachte, dass die isolierte Kopula nun innerhalb eines neuen Trochäus realisiert wird.

2;4 [daza] ball ... da [ISdas] ball [dasa] schava ... da [Isi] vogelscheuche

$2 ; 8$ ndas für farbe? ... * das is für farbe? 
In (11b) wird in mehrfacher Hinsicht korrigiert, da Julia auch Schava (= Scheuche) durch das vollständige zielsprachliche Kompositum Vogelscheuche ersetzt. In (12) kommt es sogar zu einer Hyperkorrektur, d.h. zu einem abweichenden Rekonstruktionsergebnis. Dieser Fall belegt deutlich den Druck, der durch die nun verfügbare fortschrittlichere Grammatik ausgeübt wird.

In dieses Bild fügt sich die Herausbildung von Auxiliaren und Modalverben ein. Sie fehlen zunächst völlig (vgl. Tür abomacht, Mama Bus malt, statt gemalt, auf eine vergangene Malaktion der Mutter referierend) und werden dann, z.T. in Form fehlsegmentierter Platzhalter; links der VP adjungiert (vgl. diesbezüglich auch Hoekstra/Jordens 1994 für das Niederländische). In (13) sieht man den Unterschied zwischen haben als Hauptverb, dessen Finitheitsstatus bereits klar ist, und dem bislang indifferenzierten Proto-Auxiliar [hədə], das noch, wie oben die Kopula, auf einer Fehlsegmentierung beruht. Dies zeigt sich besonders deutlich in (13b), da dort eigentlich sind gefordert wäre und die folgende DP nochmals einen Artikel aufweist. Alle Belege stammen von Julia aus der gleichen Aufnahme (Tracy 1991, S. 221).

(13) (a) 2;2 oh hab ich ein Pflaster [hədə] mami geschenk

(b) [hədə] deine ohrn abgegang (in klagendem Ton zu einem Stofftier ohne Ohren)

(c) ach də weint [hədə] eis runtergeschmisse (Junge auf Bild hat Eis verloren)

Zu dem Zeitpunkt, zu dem sich bei Julia die Reanalyse der ursprünglich ganzheitlichen deiktischen und interrogativen Platzhalter im Wesentlichen vollzogen hat, kann man von einer generalisierten V2-Konstruktion ausgehen, da nun auch diejenigen Verben, die zunächst ausschließlich in der rechten Satzklammer anzutreffen waren, als klar finite Verben in der linken Satzklammer vertreten sind. Im Alter 2;2 sind die 65 von Julia in einer Stunde produzierten V1/V2-Konstruktionen ausnahmslos finit (ich sitz auf schaukelpferd, ich bau ein turm mit ein ubr, vgl. Tracy 1991, S. 213 ff.). In 19 Strukturen steht das Verb ausschließlich in der rechten Satzklammer, meistens wie bisher - in nicht-finiter Form, aber viermal auch finit. Der Beleg die fuß da is aus (10d) ist einer dieser Fälle.

Insgesamt kann man festhalten, dass sich im Laufe von acht Monaten - bei einigen Kindern geschieht dies schneller, bei anderen langsamer als bei Julia - gewissermaßen aus zwei Richtungen eine konvergente Entwicklung hin zu thematisch vollständigen einfachen Sätzen vollzieht. Verben, die anfänglich in linksperipheren Platzhalterelementen „versteckt" waren (wie die Kopula), und andere Kandidaten der V2-Mimikry-Liste in (8) sowie diejenigen Verben, die vormals vor allem am rechten Satzrand auftraten (wie die frühen Verbletzt-Konstruktionen), sind nun in beiden Positionen anzutreffen. Damit existiert eine generalisierte Satzklammer, mit der Konsequenz, dass beide Positionen auch redundant besetzt werden können, wie in wo is der andere is, ein Punkt, auf den ich an späterer Stelle zurückkomme. 


\subsection{Wettbewerb der besten Köpfe}

Reanalysen von Konstruktionen, die sich an der linken Satzperipherie orientieren, lassen sich als eine Art von Degrammatikalisierungsprozess auffassen, durch den anfängliche Fehlsegmentierungen korrigiert werden (vgl. auch Harnisch 2004). Dabei kann es zu temporären Zwischenlösungen kommen, die weniger progressiv wirken und weniger zielsprachlich klingen als ihre Vorgängerkonstruktionen. So zeichnete sich beispielsweise für Julia beim Aufbrechen der Fragepartikel [vosə]/[vozə] eine Reanalyse ab, bei der segmentierte w-Operatoren und die Kopula um ein und dieselbe Position rivalisierten. (14) zeigt die frühere, holistische Variante, (15a) und (b) den auf die erste Reanalyse folgenden Wettbewerb um die gleiche Kopfposition.
(b) wo

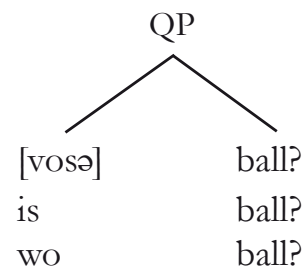

Erst in einem weiteren Schritt entsteht oberhalb und links des aktuellen Kopfes eine Spezifikatorposition, [wo [is ball]], und damit die Möglichkeit, sowohl Kopula als auch w-Operator unterzubringen, auch wenn diese Repräsentation noch von der Ableitungsgeschichte abweicht, die man bei entsprechenden Strukturen des Erwachsenensystems veranschlagen würde, nämlich eine Basisgenerierung der Kopula in einer kopf-finalen VP. Dabei sollte die Aufstockung der Struktur in (15) um einen Spezifikator durch die Erkenntnis unterstützt werden, dass wo letztlich eine maximale Phrase vertritt und somit keinen Zugang zu einer Kopfposition erhalten kann. Diese Erkenntnis geht folgerichtig mit einer weiteren De- und Rekonstruktion einher, im Zuge derer der Operatorcharakter von w-Phrasen im zielsprachlichen Sinne (d.h. durch Rekonstruktion einer Ableitungsgeschichte) erkannt wird. Damit ist der formelhafte Charakter der frühen deiktischen und interrogativen Fragmente endgültig überwunden.

Eine vergleichbare und im Grunde noch spannendere Rivalität als die zeitweilige Konkurrenz von w-Operator und Kopula findet sich aber auch noch an anderer Stelle. Innerhalb der frühen Zwei- und Mehrwortkonstruktionen gehören Kombinationen mit Fokuspartikeln wie auch ab, das auch zu den syntaktisch fortschrittlichsten und sicher auch in semantischer und pragmatischer Hinsicht zu den besonders anspruchsvollen Konstruktionen (vgl. Nederstigt 2004; Dimroth 2009; Winkler 2009). In Penner/Tracy/Wymann (1999) und Penner/Tracy/Weissenborn (2000) analysieren wir die Partikel als Kopf einer eigenen Projektion. Sobald die Fokuspartikel (FP) qua Kopf eine nichtfinite VP als Komplement selegiert, entsteht oberhalb der VP eine 
neue Strukturschicht mit einer Spezifikatorposition, gewissermaßen ein auchVorfeld, in die sich beliebige Konstituenten, aber eben immer nur eine, bewegen kann, vgl. die folgende Episode in (16) und die entsprechende Analyse in (17) (vgl. auch Penner/Tracy/Wymann 1999, S. 237). Pro repräsentiert nicht-realisierte Argumente, in (17a) das Subjekt, in (b) das Komplement.

(16) Julia (1;11) legt Spielsachen in Kiste. EINräum\

Erwachsene

Einräumen willst du sie wieder?

J. legt Ziegenfigur in Kiste.
(a) $\quad[\mathrm{FPP}$
(b) $\quad[\mathrm{FPP}$
Ziege $_{i}\left[_{F p}\right.$ AUCH
Tracy $_{j}\left[_{F P}\right.$ AUCH ziege $\mathrm{AUCH} /$... tracy AUCH einräum/

Bemerkenswert ist nun, dass zu einem späteren Zeitpunkt, wenn V2-Strukturen längst produktiv sind, Konstruktionen mit auch und nicht eher rückschrittlich wirken. Tritt nämlich eine Fokuspartikel hinzu, werden Verben ausgelassen (alle baben augen, sabine auch augen), sind nicht finit (julia auch buch angucken), oder aber sie erscheinen in finiter Form am Satzende, nicht in V2, wie in Eichhörnchen auch noch mehr steht, das passt rein, das nicht passt). Penner/ Tracy/Wymann (1999) und Penner/Tracy/Weissenborn (2000) nehmen an, dass sich zwischen finitem Verb und auch / nicht ein temporärer Konflikt um Kopfpositionen links der VP abzeichnet, der erst durch die Konstruktion neuer Strukturschichten und damit verbundenen Landeplätzen in die sich das Verb oberhalb der FPP an der linken Peripherie bewegen kann, behoben wird. Die Tatsache, dass die rechte Satzklammer zu diesem Zeitpunkt bereits von finiten Verben besetzt werden kann, obwohl es sich dabei nicht um Nebensätze handelt, belegt die Existenz einer Kopf-finalen IP. Insgesamt gäbe es dann folgendes Spektrum von Strukturformaten mit Fokuspartikeln aber ohne V2.

FPP sabine auch augen $\varnothing$

julia auch buch angucken

(b) IP eichhörnchen auch noch mehr steht

\subsection{Dummies und Doppelgänger}

Schon Stern/Stern (1928) stellten fest, dass (ihre eigenen) Kinder die Position von Konjunktionen mit undifferenzierten „Universallauten“ füllten. In der aktuellen Forschung spricht man diesbezüglich von Platzhaltern, Dummies oder Fillern (Peters 2001). Die Beobachtungen der Sterns wurden in neueren Studien in Bezug auf Komplementierer, aber auch eine ganze Reihe weiterer funktionaler Kategorien bestätigt (vgl. d'Avis/Gretsch 1994; Fritzenschaft et al. 1990; Hohenberger 2002; Müller 1993; Müller/Penner 1996; Penner 1994; Rothweiler 1993). Das Phänomen ist auch aus dem Zweit- 
spracherwerb bekannt (vgl. van de Craats 2009; Haberzettl 2003). Da ich im Folgenden vor allem auf verbale Platzhalter eingehe, sei an dieser Stelle daran erinnert, dass die zuvor bereits im Zusammenhang mit den V2-Vorläufern besprochenen formelhaften Konstruktionen z.T. bereits pronominale oder adverbiale Platzhalter enthielten. Diese Äußerungen klangen insgesamt rhythmisch und syntaktisch recht fortschrittlich, wenngleich die Funktion von $d a$, da und Varianten nicht immer geklärt werden kann, vgl. die folgenden Beispiele. In (19b) war es möglich, auf Grund des Kontextes (und des Verhaltens der Gesprächspartner), do als Platzhalter einer w-Phrase zu analysieren. In (19c) ist der Status von wer als Default (für was) klar erkennbar.

(19) (a) Mirko 1;10

(b) Julia 2;2

(c) Stefanie 2;0 də ben də ben də feuerwehr (da? / die? brenn die? Feuerwebr...)

də mach də mädchen, mami?, də machen die?, de $\operatorname{mach}(\mathrm{t})$ də kind? (für w-Operator was)

wer machen die? $(2 \mathrm{x})$, wer mach $(\mathrm{t})$ der da?, wer $\operatorname{mach}(\mathrm{t})$ die? Etc.

Insgesamt erscheint es sinnvoll, von einem Platzhalter-Kontinuum auszugehen, an dessen einem Ende spezifische Lücken, d.h. leere Kategorien anzusiedeln sind, wie beispielsweise die fehlenden Komplementierer der entsprechend benannten „,präkonjunktionalen“ Phase (Rothweiler 1993) beim Erwerb von Nebensätzen, vgl. (20a). Die Aufnahme leerer Kategorien in die Liste möglicher Platzhalter lässt sich für das Deutsche theoretisch durch damit einhergehende Strukturmerkmale rechtfertigen, vor allem durch den konsequenten Verbleib finiter Verben in der rechten Satzklammer.

(20) (a) Valle 2;2 valle will dann weiter malen $\varnothing$ valle fertig malen hat ... Ø valle fertig badet hat

(b) $\operatorname{Max} 3 ; 2$ [ənənə] laut is

(auf warum-Frage)

(c) $\operatorname{Max} 3 ; 6$ [ən] hier den räuber drin is (auf Frage: Was glaubst

(d) Stani 2;5 du auch?) das darf man if man will

In (20b) und (c) sehen wir phonologische Filler im Sinne Sterns, und in (d) die spontane Entlehnung eines englischen Äquivalents durch ein deutschenglisch bilinguales Kind. (20a) ist auch deswegen besonders aufschlussreich, weil Valle hier einen eigenen Perseverationsversprecher (malen ... malen) sowie Details der Verbform korrigiert (malen $\rightarrow$ (ge) badet). Aus der Tatsache, dass Valle bei der Reparatur die gleiche präkonjunktionale Nebensatzstruktur nutzt wie im ersten Anlauf kann man schließen, dass er zu diesem Zeitpunkt an der konjunktionslosen Konstruktion per se (noch!) nichts auszusetzen hat. 
Platzhalterphänomene lassen sich typischerweise mit speziellen Erwerbsaufgaben in Verbindung bringen, z.B. mit der Schaffung neuer Strukturschichten. Für das Englische wurden entsprechende Phänomene vor allem im Zusammenhang mit der Emergenz von Inversionsstrukturen dokumentiert, z.T. als Vorläufer des do-supports. Die Beispiele in (21) stammen aus Roeper (1992), (22) von Akmajian/Heny (1975) und (23) aus den in Tracy/ Gawlitzek-Maiwald (2005) diskutierten Daten eines deutsch-englisch bilingualen Kindes.

(21) (a) are you put this on me?

(b) are you get this down?

(c) are you know Lucy's name is?

(22) (a) is I can do that?

(b) is you should eat the applecake?

(23) Adam 5;5

(a) are you ə are you understand German?

(b) are you want that?

Für das Englische ist zu diesem Zeitraum auch die anfängliche Adjunktion von wh-Phrasen typisch (where I can go), die ebenfalls darauf hindeutet, dass es noch an ausreichend differenzierten Strukturschichten fehlt, um gleichzeitig Operatoren und verbale Köpfe strukturerhaltend zu bewegen bzw. durch „binäres Merging“ zu integrieren (vgl. Radford 2009). Man beachte hier auch die Konstruktionsleistung der Kinder, denen genau solche Konstruktionen im Input nicht begegnen.

Andererseits: Wie sehr sich Kinder bemühen, ihr Sprachangebot ernst zu nehmen, zeigt sich besonders nachdrücklich, wenn sie etwas aufgreifen, was im Gebrauch Erwachsener einen völlig anderen grammatischen Status hat, wie zum Beispiel die folgenden Interjektionen (aus Tracy 1991, vgl. auch Hoekstra/Jordens 1994; Jordens 2009; Roeper 1992).

(24) Mirko 2;1-2;2 (a) oh mach ein SCHÖN schön turm \

(b) oh də DA wohntə biber drin $\backslash \ldots$ oh DA də biber wohnt $\backslash$

(c) oh das də WEHgetat $\backslash$

(d) oh is'n DAS?

(e) oh is das ein BAUer?

(f) oh [izə] da HÜHner drin?

(g) oh [izə] də bäcker HEISST? (= wie heißt der Bäcker?)

(g) oh is WINter $\backslash$ 
Auch die folgende Episode (25) - mit Das, ist das ein(e) X? als naheliegendes Vorbild - zeigt, dass sich der Erwachsenengebrauch und die von Mirko rekonstruierte Version in prosodischer und funktionaler Hinsicht deutlich unterscheiden.

(25) Mirko 2;2 und seine erwachsene Gesprächspartnerin schauen sich

Fotos an. Er deutet jeweils auf die von ihm erwähnten Bildausschnitte.

(a) das is das ein julia $\backslash$

(b) dəs is dəs eine dicke mann $\backslash$

(c) das is das ein große stock $\backslash$

(d) das is də junge heißt uwe $\backslash$

In diesen Äußerungen steigt die Intonation weder auf dem einleitenden Demonstrativum noch am Ende, und es gibt auch keine Pause oder einen prosodischen Bruch nach dem Pronomen. Außerdem zielt Mirko nicht auf eine Bestätigung seitens seiner Gesprächspartnerin ab. Die illokutive Kraft der gesamten Konstruktion ist assertiv. Er verweist auf Bilder, die seine Gesprächspartnerin noch nicht gesehen hat.

Die in diesem Abschnitt angeführten Phänomene passen zu der Unterscheidung von „errors of omission“ und ,errors of comission“, die in der Spracherwerbsforschung erstmals gezielt von Menyuk (1972) untersucht wurden. Die frühen Phasen des Spracherwerbs zeichnen sich dabei durch systematische Auslassungen aus, die oft gerade besonders frequente und obligatorische Kategorien der Zielsprache betreffen, z.B. Subjekte, Artikel, Präpositionen, Flexionsmerkmale. In die Gruppe der „,commissions“ gehören Strukturen, die durch die Wahl oder - wie im Falle der phonologischen Filler - durch die eigene „Erfindung“ von Dummies auffallen. Letzteren kann man auch solche Phänomene zuordnen, die durch die „Übererfüllung“" eines Plans, also durch strukturelle Redundanzen zustande kommen. Die daraus resultierenden Strukturen wirken einerseits wie (normale) Kontaminationsversprecher, in denen unterschiedliche Konstruktionen gleichzeitig realisiert werden. Andererseits unterscheiden sie sich dadurch von Versprechern, dass sie typischerweise in bestimmten Entwicklungsphasen besonders häufig auftreten und daher Aufschluss dahingehend geben, an welchen Erwerbsproblemen Lerner gerade arbeiten. Im Rahmen derivationeller Theorien hat man diese Phänomene als eine nur partiell vollzogene Bewegungsoperation bezeichnet: „Copy without deletion“ (vgl. Radford 2009), wobei Fuß und Kopf einer Kette gleichzeitig realisiert werden, vgl. (26) aus Tracy (1991), (a)-(e) von monolingualen Kindern im Alter von zwei bis zweieinhalb Jahren, (f) von einer Zweitsprachlernerin, die im Alter von drei Jahren und vier Monaten $(3 ; 4)$ mit dem Deutschen in Kontakt kam. 
(26) (a) julia brauch ich das

(b) ich spiel das ... das spiel ich's auch

(c) wo is der andere is?

(d) der will das mal toben

(e) ich hab das mal meine bausteine ausgeschüttet

(f) hab nich angst habe

Wie man in (a), (d) und (e) sehen kann, handelt es sich dabei durchaus nicht immer um perfekte Kopien eines Antezedens. Vielmehr können Merkmale teilweise kopiert oder gelöscht werden (vgl. diesbezüglich auch van de Craats 2009, S. 66 f.). Hinzu kommt, dass LernerInnen auch online mit möglichen Konkurrenten um Positionen zu kämpfen haben, d.h. mit einem Potenzial, das dann tatsächlich auch zu Versprechern führen und entsprechende Selbstreparaturen auslösen kann, vgl. (27). Man beachte allerdings, dass die Wiederholung in (27b) den Versprecherstatus wiederum in Zweifel zieht.

(27) (a) Julia 2;2 deutet auf Bild und DANN sind die blätter $\backslash \ldots$ DA sind die blätter $\backslash$

(b) Mirko 2;5 deutet auf spitzen Gegenstand das kann man die FINGer mit reißen

Kurz darauf ... DA kann man die finger mit reißen\ GUCKma \das kann man FINGer mit $\backslash$ reißen \

Insgesamt belegen Dummy-Phänomene und Doppelbesetzungen das Bedürfnis von LernerInnen, abstrakte Strukturen möglichst vollständig auszubuchstabieren und strukturelle Abhängigkeiten lokal zu erhalten. Sie liefern uns sowohl Evidenz für abstrakte Konstruktionen an sich als auch für die Menge paradigmatischer Optionen, die Kindern zur Auswahl zur Verfügung stehen.

\subsection{Versuch einer stringenten Form-Funktions-Alignierung}

Trotz ihres verschiedenen Ursprungs und unabhängig von ihren illokutiven Funktionen konvergieren beim Erwerb des Deutschen sämtliche Strukturformate auf die Struktur von (5) bzw. (6). Dabei gibt es aber auch LernerInnen, die zeitweise versuchen, bestimmte Strukturen für spezifische Funktionen zu „reservieren“. Ein einschlägiges Beispiel stammt aus einer longitudinalen Studie zu den w-Fragen des Lerners Valle (vgl. Tracy 1994). Die wichtigsten Entwicklungsschritte lassen sich wie folgt zusammenfassen.

Phase A 1;11 Lückenformate: Äußerungen ohne w-Phrasen und (meist) steigender Intonation, deren Status als Entscheidungs- oder Informationsfragen sich nur anhand des Kontextes erschlieBen lässt. Die Strukturen sind oft sogar zu kurz, um bezüglich der Verbstellung eine klare Aussage zu treffen. 
(a) mama is/

(b) der mann macht/

Phase B 2;0 Konsequente Realisierung von w-Fragen in Verbletztstrukturen unter gelegentlicher Auslassung des w-Pronomens (vgl. (29b)). Der einzige Beleg für eine V2-Frage unter insgesamt $18 \mathrm{w}$-Fragen ist $(29 \mathrm{e})$.
(a) wo das haus mit dem feuer ist?
(b) wo die jetza hingehn?, die jetza hingehn?
(c) wo der eimer ist?
(d) wo der peter hinsitzt?
(e) wie geht das auf?

$\mathrm{Zu}$ diesem Zeitpunkt werden Assertionen bereits in Strukturen von beeindruckender Komplexität realisiert, inklusive Passivkonstruktionen und vollständiger Nebensätze, vgl. (30).
(a) viele dächer soll das haben
(b) aber bei dem schneeauto kann man das lenkrad nicht sehn
(c) das motorrad wird dort ganz gemacht
(d) valle badet weiter, bis du fertig malt hast

Phase C 2;3 Kurze Periode der Alternanz, in der w-Fragen sowohl in Form von V2- als auch in Form von Verbletzt-Strukturen auftreten. Man findet auch Äußerungen, die auf einen Wettbewerb bei der Produktion hindeuten, vgl. (31e).
(a) was der gerne will?
(b) was will der denn?
(c) was das ist?
(d) was ist das?
(e) was is denn da weggegangen ... da weggegangen is?

Phase D 2;4 Einheitliches V2-Hauptsatzschema. Der einzige Rückgriff auf die frühere Verbletztkonstruktion im Zusammenhang mit einer direkten Frage ist ein Gespräch in (33), das ich in vollem Umfang zitiere. Es zeigt sehr deutlich, dass die unten durch Unterstreichung gekennzeichnete - Verbletztfrage mit hoher Wahrscheinlichkeit durch den unmittelbaren Input, einen (ebenfalls unterstrichenen) Verbletzt-Relativsatz, provoziert wurde.
(a) warum kann dann sein, dass ich rausfall?

(b) wer trocknet die mama ab? 
Valle schaut auf das Tonbandgerät, das seine Gesprächspartnerin (Erw.) gerade angemacht hat.

V. is da JETZT was drauf/

Erw. Nee, jetzt ist da noch nix drauf, aber nachher, nach ner Weile.

V. DANN is da dann was drauf/

Erw. Da bist dann du drauf.

V. was SAG ich dann immer/

Erw. Das, was du jetzt auch sagst.

V. was ich dadrin SAG/

Erw. Da hört man dann das, was wir jetzt gespielt haben.

Valles ursprüngliche Präferenz für w-Verbletzt-Fragen überrascht nicht, da ihm permanent Evidenz in Form von eingebetteten indirekten Fragen und Relativsätzen wie (33) zur Verfügung stehen dürfte. Interessant ist eher, dass er seine Vorliebe aufgibt, obwohl ihm das Muster im Input weiterhin begegnet und obwohl er sich dessen mehrere Monate ausgiebig bedient hat. Sein Verzicht auf die Korrelation von Form und Funktion geht einher mit der Emergenz von Nebensätzen. Offensichtlich hat Valle längst erkannt, dass seine Verbletztkonstruktionen anderweitig bzw. sehr generell benötigt werden.

Die folgende Sequenz (34) (aus Tracy 1994) zeigt einerseits die Modellierung (hier sogar doppelt!) in Form von Rückversicherungsfragen (Echofragen) der Gesprächspartnerinnen von Valle und zugleich seine eigene Verwendungsweise, inklusive der Optionalität der w-Phrase. Es handelt sich dabei übrigens um eine der seltenen Episoden, in denen Valles direkte Frage nicht sofort als solche verstanden wurde.

(34) Valle (2;1), M (= Mutter) und eine weitere Erwachsene (= Erw) betrachten Bilder von Kindern.

$\mathrm{V}: \quad$ wo die jetza hingehn?

M: Bitte?

$\mathrm{V}: \quad$ die jetza hingehn?

M: Wo die jetzt hingehn?

Erw.: Wo die hingehn?

$\mathrm{V}: \quad \mathrm{ja} \backslash$

M: Vielleicht gehn sie ins Kinderzimmer.

\section{Mehrsprachigkeit als Prinzip}

Anhand der bisher getroffenen Auswahl typischer Übergangsphänomene konnte man erkennen, dass Kinder früh in der Lage sind, mit koexistierenden Optionen und manchen Ungereimtheiten umzugehen. Die geschilderten Restrukturierungsprozesse, die immer wieder zum Aufbrechen von Formeln und auch zum Aufbau neuer Strukturschichten führen, benötigen 
Zeit und wahrscheinlich in vielen Fällen ein nicht zu unterschätzendes Maß an positiver Evidenz. Dabei erweisen sich Kinder sowohl als risikofreudig (wie wir bei den Dummies gesehen haben) als auch als konservativ, d.h. sie scheuen vor allzu schnellen Generalisierungen zurück (vgl. Tomasello 2000; Elman 2002). Wahrscheinlich ist es nicht nur klug, vorschnelle Generalisierungen zu vermeiden, weil sie vielleicht schwer zu korrigieren wären (vgl. Randall 1990). Gewisse Zurückhaltung ist auch deshalb geboten, weil LernerInnen eines mit Sicherheit nicht a priori wissen können: mit wie vielen Sprachen sie es nach ihrer Geburt zu tun haben.

Die zeitweise Koexistenz und Konkurrenz von funktional äquivalenten Strukturschemata (z.B. [dasa] Ball, das ist der Ball, von V2- und Verbletzt-wFragen, von Nebensätzen mit und ohne Komplementierer etc.) sowie die Koexistenz von Bauplänen, die in syntaktischer Hinsicht zunächst nichts gemein zu haben scheinen (produktive Verbletztkonstruktionen vs. V2-Formeln) legen die Vermutung nahe, dass sich auch monolinguale Kinder zunächst wie bilinguale Lerner verhalten sollten, wenn sie mit - aus ihrer Sicht - konfligierenden Strukturen konfrontiert werden, wie beispielsweise mit Verben oder Argumenten in unterschiedlichen Positionen. Die Entwicklung separater Grammatiken wäre in diesem Fall eine vernünftige und geradezu ,sichere“ Lernerstrategie. Dass dies auch als permanente Lösung nicht abwegig ist, legt Roeper (1999) im Zusammenhang mit seiner These des universalen Bilingualismus dar, wenn er darauf hinweist, dass Sprachen über zahlreiche Nischen (Roeper spricht hier von „pockets“, ebd., S. 183) für Konstruktionstypen früherer Sprachstufen verfügen, das Englische beispielsweise über residuelle Verbzweitmuster wie das nicht länger produktive Well may be complain oder ein nur noch mit bestimmten Verben verbundenes Inversionspotenzial („Incredible“, said the man). Auf das positive Konfliktpotenzial, das die Koexistenz von inkompatiblen Repräsentationen in sich birgt, gehe ich in der Diskussion ein.

Einen in jeder Hinsicht authentischen separaten Strukturaufbau vollziehen „echte“ doppelte Erstsprachlerner (vgl. Döpke 2000; Meisel 1989, 2004; Müller at al. 2007; Gawlitzek-Maiwald 1997; Cenoz/Genesee 2001; Genesee/Nicoladis/Paradis 1995; Genesee/Nicoladis 2007; Paradis/Genesee 1996). Die frühe Sprachentrennung zeigt sich besonders deutlich dann, wenn sich die beteiligten Sprachen mit unterschiedlicher Geschwindigkeit entwickeln, z.B. deswegen, weil sich ein System als komplexer erweist als das andere (vgl. Bernardini/Schlyter 2004; Gawlitzek-Maiwald/Tracy 1996; Müller et al. 2007; Tracy/Gawlitzek-Maiwald 2005; Tracy 1996).

Bei Kindern, die mit Deutsch und Englisch als doppelten Erstsprachen aufwachsen, lässt sich bereits anhand minimaler VP-Fragmente nachweisen, dass sie wichtige Wortstellungskontraste, z.B. Unterschiede in der Serialisierung von Kopf und Komplement, zwischen den Sprachen erkannt haben, vgl. (35). 
(35) Hannah 2;2

(a) Mama picking flowers inə garden

$\left[{ }_{\mathrm{vp}}\right.$ Mama $[\mathrm{v}$, picking flowers ...] $]=\mathrm{VO}$

(b) ich das lesen

[vp ich [ $\quad$ v, das lesen $]] \quad=$ OV

Eine systematische Trennung der grammatischen Systeme bedeutet aber keineswegs, dass es nicht zeitweise aufgrund von strukturellen Ähnlichkeiten zu Interferenzen und damit sowohl zu Verzögerungen als auch zu Beschleunigungen (Bootstrapping-Effekten) kommen kann (vgl. Müller et al. 2007; Gawlitzek-Maiwald/Tracy 1996). Darüber hinaus findet sich auch Evidenz für momentane Unsicherheiten bezüglich des Abgleichs von Syntax und Lexikon bei der Online-Planung. Episoden wie (36), in denen Kinder sich aktiv um Reparaturen, also um die schrittweise De- und Rekonstruktion eigener Äußerungen bemühen, sind in diesem Zusammenhang besonders aufschlussreich. ${ }^{5}$

(36) Hannah (2;2) versucht, eine Puppe in einem Puppenwagen festzuschnallen.
(a) die dolly einstrappen
(b) die dolly eintrap
(c) das einstrap in ... die puppe
(d) die einstrap in ... die dolly
(e) die Mama helf mir tap it in
(f) Mama tap it in ... die dolly

Hannah beginnt in (36a) mit einem nicht-finiten $V^{6}$-Fragment mit der für das Deutsche typischen OV-Serialisierung. In (b) eliminiert sie das deutsche Suffix -en. Mit der Partikel in erscheint in (c) und (d) ein (wenngleich von der Form her ambiges) englisches funktionales Äquivalent des deutschen Präfixes ein-. In (e) und (f) tritt das englische direkte Objekt it in seiner zielsprachlichen postverbalen Position hinzu, und das deutsche Objekt verschwindet. Die schließlich in (f) folgende Variante entspricht mit Ausnahme des Artikels in der nachgeschickten DP einer englischen VP, mit angemessener VO-Serialisierung. Die gesamte Abfolge selbst-initiierter Reparaturschritte zeigt, dass Hannah (implizit) weiß, wie sich deutsche und englische Verbalphrasen syntaktisch und morphologisch voneinander unterscheiden und wie man letztlich ein beliebiges Verblexem an die Anforderungen beider Grammatiken anpassen kann. Sie ist sich lediglich unsicher hinsichtlich der lexikalischen Zugehörigkeit von strap.

Der simultane Erstspracherwerb verrät uns nicht nur viel über die frühe Fähigkeit von Kindern, mit Konstruktionen umzugehen, die sich in zentralen Eigenschaften widersprechen, wie OV und VO. Als besonders auf-

Das englische „strap“ (anschnallen) wurde dabei z.T. als [træp] und [thap] realisiert. 
schlussreich erweisen sich Situationen, in denen sich die beteiligten Sprachen trotz prinzipiell verfügbaren Inputs nicht gleich schnell entwickeln. Im Fall des bilingualen Kindes Hannah, spiegelten die Mischäußerungen bis zum Alter 2;9 eine interessante Diskrepanz wider (vgl. Gawlitzek-Maiwald/ Tracy 1996; Tracy/Gawlitzek-Maiwald 2005), vgl. (37).

(37) Hannah
(a) $2 ; 4$
Kannst du move a bit?
(b) $2 ; 6$
Soll ich bit it?
(c) $2 ; 7$
Sie haben gone away.
(d) $2 ; 7$
Mama kannst du do it up?

In diesem Zeitraum gibt es in Hannahs rein englischen Konstruktionen weder Belege für eine produktive Subjekt-Verb-Kongruenz noch für Hilfsund Modalverben (vgl. Mama picking flowers ina garden, Papa doing?), während sie im Deutschen schon vollständige finite Hauptsätze produziert. Die von ihr in dieser Phase der Asynchronie produzierten Mischäußerungen sind daher ein perfekter individueller Kluge: Indem Hannah englische VP-Fragmente in eine ansonsten deutsche Satzstruktur hinein zitiert, kann sie nicht nur mehr zum Ausdruck bringen, als es ihr zu diesem Zeitpunkt allein auf Englisch möglich wäre. Sie kommt damit zugleich dem Wunsch ihrer Gesprächspartnerin, d.h. ihrer Mutter, sie auf Englisch anzusprechen, so nahe, wie es ihr überhaupt möglich ist.

Die Diskrepanz zwischen dem Deutschen und Englischen dieses Zeitraums lässt sich durch die größere Komplexität des Englischen erklären, das es seinen LernerInnen schwer macht, die reduzierte Subjekt-Verb-Kongruenz (3. Ps. Sg. Präsens, ein leicht erweitertes Inventar im Fall des suppletiven Paradigmas der Kopula und des Hilfsverbs be) und Hilfs- und Modalverben in ihren kanonischen Oberflächenpositionen zwischen Subjekt und Verb zu entdecken. Umgangssprachliche Klitisierungen und daraus folgende strukturelle Ambiguitäten erschweren die Rekonstruktion (vgl. He's seen it, He's sick ..., He's being questioned ..., She'd tell you ...). Es kann also nicht überraschen, dass auch monolinguale, Englisch lernende Kinder eine Weile brauchen, um sich die entsprechenden Merkmale zu erschließen (Hyams 1986). Umso interessanter ist es, dass mehrsprachige Kinder dazu in der Lage sind, temporäre Asynchronien durch eine sprachübergreifende Anleihe auszugleichen.

Vom doppelten Erstspracherwerb verspricht sich die Forschung Antworten auf Fragen, die uns der monolinguale Erwerb nicht oder nur schwerlich geben kann (vgl. Genesee/Nicoladis/Paradis 1995; Gawlitzek-Maiwald/ Tracy 1996). Dies betrifft beispielsweise Fragen nach der Rolle der Reifung, denn bei ausreichendem Input sollten sich vergleichbare Strukturen in etwa zur gleichen Zeit manifestieren. Man verspricht sich auch Antworten auf die Frage, welches System sich trotz gleichermaßen verfügbaren Inputs durch 
höhere Verarbeitungskomplexität auszeichnet (vgl. diesbezüglich bereits Slobin 1973). Ein Beispiel für die größere Komplexität des Englischen im Vergleich mit dem Deutschen wurde eben erläutert. Aus Sicht gebrauchsbasierter Erwerbstheorien wäre es natürlich besonders relevant, zu klären, wie sich relative Häufigkeiten oder die Intensität des Kontakts mit dem Input in beiden Sprachen niederschlagen. Im Falle des doppelten Erstspracherwerbs verteilt sich der Input schließlich auf zwei Sprachen, die in der Umgebung des Kindes sicherlich nicht in jeder Hinsicht völlig ausgewogen repräsentiert sein dürften. Von daher darf man sich von künftigen Vergleichen von Erwerbstypen neue Erkenntnisse über die Rolle von Vorkommenshäufigkeiten versprechen.

Von besonderer Bedeutung ist aber nicht nur die Frage nach dem relativen Kontakt mit den jeweiligen Inputsprachen. Interessant sind dabei insbesondere solche Fälle, in denen Kinder ihre Sprachen zeitweise intensiv mischen, obwohl sie nicht in eine Code-Switching-Gemeinschaft hineingeboren wurden und obwohl ihnen keine gebrauchsbasierte Vorlage für Mischkonstruktionen zur Verfügung steht. Im Übrigen: Falls es im Fall von Hannah dieses Modell gegeben hätte, wäre es umso unverständlicher, dass ihre Mischäußerungen gegen Ende ihres dritten Lebensjahres von etwa 20\% auf einen Anteil von unter 3\% aller Äußerungen sinken.

\section{Diskussion}

Nicht alles, was wir an verbalem Output produzieren, gehört in die Verantwortung einer Grammatik. Man denke an unser Repertoire von Routineformeln und Versatzstücken, mit deren Hilfe wir uns in anderen Sprachen verständlich machen könnten. Auch Kinder verfügen früh über eine stattliche Sammlung ganzheitlich memorierter Formeln, vgl. das von Echols (2001, S. 255) zitierte Kind im Alter 1;7: $\Lambda$-ə-b-b-di-a-i-ai für up-above-the-world-so-bigh (aus Twinkle, Twinkle, Little Star), oder Stefanie $(1 ; 11)$ mit fuks-du-da-na-sto-lan, basierend auf Fuchs, du hast die Gans gestoblen (Tracy 1991). Es gibt keinen Grund zu der Annahme, dass (abgesehen von der Länge) Begrenzungen der Menge von Mustern existieren, die wir uns jenseits aller Grammatik aneignen könnten.

Gebrauchsbasierte Erwerbstheorien legen den Schluss nahe, dass der Spracherwerb überwiegend, wenn nicht ausschließlich aus dem schrittweisen, induktiven Aufbau eines Repertoires von item-basierten Konstruktionen, spezifischen Frames und „pivotal constructions“ (Braine 1971, 1976) besteht und dass sich abstrakte Strukturschemata erst allmählich (wenn überhaupt) herausbilden. Aus Sicht Tomasellos bedarf es auch für das zielsprachliche Erwachsenensystem keiner konstruktionsübergreifenden Grammatik, i.e.: 
the adult endpoint of language acquisition comprises nothing other than a structured inventory of linguistic constructions, a much closer and more child-friendly target than previously believed. (Tomasello 2003, S. 7; vgl. auch Behrens in diesem Band).

Wie in der generativen Spracherwerbsforschung gibt es unter gebrauchsbasierten Ansätzen eine Fülle unterschiedlicher Perspektiven, darunter auch solche, in denen durchaus die Notwendigkeit gesehen wird, den Hypothesenraum eines lernenden Systems zu beschränken, eine Vorstellung, die den Annahmen einer Universalgrammatik schon etwas näher kommt, vgl. z.B. Elman (2002, S. 175):

I am a firm believer in usage-based approaches. But I believe that usage has its place. We do more than simply record our past experiences, say, in some table of frequencies or probabilities. Rather, our experience forms the basis for generalization and abstraction. So induction is the name of the game. But it is also important to recognize that induction is not unbridled or unconstrained. Indeed, decades of work in machine learning makes abundantly clear that there is no such thing as a general purpose learning algorithm that works equally well across domains. Induction may be the name of the game, but constraints are the rules that we play it by.

Eine minimale und zugleich minimalistische Hypothese, mit der sich Lerner ihrer Umgebungssprache nähern, bestünde nach der in meinem Beitrag entwickelten Perspektive in der Annahme, dass syntaktische Baupläne ausnahmslos einem Prinzip des binären Strukturaufbaus gehorchen, beginnend in jeder Schicht mit einer Unterscheidung von Kopf und Nichtkopf. Zu dieser Annahme passen auch Strukturen, die immer mal wieder als Randerscheinungen auffallen, weil sie zielsprachlich abweichend sind, aber zugleich aus etwas abstrakterer, kategorienneutraler Perspektive als „Instantiierung in Reinform“ eines minimalen Bauplans gedeutet werden können. Dies gilt für das ist für farbe (vgl. (12) oben) ebenso wie für (38). Zu diesem Zeitpunkt verfügte Hannah weder im Deutschen noch im Englischen über finite Verben.
Mutter nach einem Puzzle. You did it
Hannah 1;8 möchte weiter machen MORE did it [more [did it] ]

Die von mir diskutierten Phänomene und die damit verbundene Erwerbsdynamik legen den Schluss nahe, dass es zu Beginn des produktiven Spracherwerbs in der Tat Evidenz für eine Reihe von unabhängigen Konstruktionen gibt, wobei jede einzelne dieser Konstruktionen beim ersten Verdacht auf Kompositionalität unter den Druck eines binären Strukturbildungsprinzips geraten sollte und auf neuer Ebene der Abstraktion rekonstruiert würde.

Bei der hier vorgenommenen Schilderung des Spracherwerbs erwiesen sich zwei Zonen deutscher Satzbaupläne als besonders relevant: die rechte und die linke Satzperipherie. Es fehlte lediglich in den ersten Monaten des 
Spracherwerbs die Integration beider in ein einheitliches Satzschema, das es erlaubt, sämtliche Satzbaupläne abzuleiten. Dieser Schritt kann vollzogen werden, sobald Kinder einen Verdacht dahingehend entwickeln, dass die tragenden Elemente beider Konstruktionstypen (V2-Vorläufer und Verbletztkandidaten) auf lexikalischer und damit auch thematischer Ebene identisch sein könnten. Dabei sollte die wachsende Einsicht in die Argumentstruktur derjenigen Verben, die anfänglich auf die VP beschränkt sind, dazu beitragen, die linksperipheren Formeln zu reanalysieren. D.h. die thematische Analyse von Mama Treppe gehen legt es nahe, auch in da ge:t-die, do ge:t do nach Argumenten zu suchen. Auf diese Weise wird der pronominale Status der Platzhalter, die zunächst in freier Variation auftraten, erkannt, und schließlich lässt sich so auch die ketteninterne Kovariation der Subjekt-Verb-Kongruenz (re-)konstruieren.

Des Weiteren sollte der Verdacht, dass gleiche lexikalische Elemente in (oberflächlich betrachtet) verschiedenen Positionen auftreten könnten (XV wie in da feuer auch brennen und XVY, do brenn $(t) d a$ zu einer hilfreichen Krise führen und Lerner dazu anregen, ein System zu konstruieren, das es ermöglicht, Ableitungsbeziehungen zwischen diversen Strukturformaten herzustellen (vgl. dazu Tracy 2002). Spezifisch linguistische Beschränkungen könnten dabei diktieren, dass Strukturen nur binär aufgebaut werden dürfen und dass es pro Strukturschicht nur einen Kopf geben kann, dessen Position zudem festgelegt ist. Ein Konflikt zwischen OV und VO ließe sich somit nicht im Sinne eines laissez faire erledigen. Vielmehr gibt es nur zwei universalgrammatisch mögliche Lösungen: (a) Es muss möglich sein, die beiden als „verbal“ erkannten Köpfe in unterschiedlichen Schichten unterzubringen (z.B. in einer VP und einer höheren IP), und (b) Es handelt sich um die VPs unterschiedlicher Sprachen, z.B. Deutsch und Englisch.

Gebrauchsbasierte Theorien lenken unsere Aufmerksamkeit zu Recht auf die Sprachverwendung in der Umgebung eines Kindes und damit auf die positive Evidenz, die Lernern prinzipiell zur Verfügung steht. Aber gerade wenn man sich die Häufigkeit bestimmter Phänomene, z.B. funktionaler Kategorien, ansieht, fehlt es an einer Erklärung dafür, warum besonders häufige und obligatorische Elemente des Inputs in frühen Phasen des Spracherwerbs von Lernern systematisch ausgeblendet werden oder warum der Erwerb nicht wesentlich gradliniger verläuft, sondern immer wieder durch Reorganisationsphasen gekennzeichnet ist. Zu klären bleibt auch, wie es sein kann, dass Konstruktionen, die über einen längeren Zeitraum intensiv gebraucht wurden und die sich in der Kommunikation bestens bewährt haben, obsolet werden, auch wenn sich zeitgleich am eigentlichen Input im Grunde wenig ändert. Was sich hingegen kontinuierlich ändert, ist der Lerner selbst, nicht zuletzt dadurch, dass ihn seine wachsende Verarbeitungs- 
kapazität und der beständige Wissenszuwachs immer wieder aufs Neue zu einer Erweiterung seines Blickwinkels und damit zur Berücksichtigung neuer Daten anregt (vgl. auch Elman 1993, S. 78 ff.).

Der hier entwickelten Perspektive zufolge liegt das Verdienst des Sprachgebrauchs, mit dem ein Lerner konfrontiert wird, nicht primär darin, ihm ein kopierfähiges Modell anzubieten, sondern vielmehr darin, immer wieder Problemlösestrategien auszulösen, u.a. durch die Notwendigkeit, im Input wahrgenommene Variation durch ,abstraktes Gleichmachen“ (Riedl 1980) konzeptuell berechenbar zu machen. Eines der Prinzipien, das sowohl aus universalgrammatischer Sicht als auch aus der Perspektive der allgemeinen Kognition einen Sinn ergibt, wäre demnach eine elementare Konstanzerwartung, dank derer die immer wieder anwachsende Unordnung, d.h. das Variationsspektrum im Input und im eigenen Output durch Reorganisation reduziert werden kann. Dass es bei den Konstruktionsleistungen, die ein Kind dabei vollbringt, zu Hypothesen kommen kann, die aus universalgrammatischer wie auch aus gebrauchsbasierter Perspektive unerwartet und letztlich sogar irrelevant sind, zeigen die beiden abschließenden Beispiele.

Auf die Tatsache, dass sich englischsprachige Kinder mit der Markierung der Subjekt-Verb-Kongruenz schwer tun, wurde bereits hingewiesen. Beobachtete Inkonsistenzen werden i.d.R. durch die Annahme einer Phase der Optionalen Infinitive (vgl. Hyams 1986) erklärt. Demuth/McCullough (2009) konnten in einer Studie zeigen, dass englische Verben dann mit dem -s der 3. Ps. Sg. versehen wurden, wenn sie offene Silben (see, show) aufwiesen. Im Fall einer Koda mit Konsonanten (bit, find, want) wurde hingegen eine Suffigierung vermieden, und die Verben wurden als Stamm-/Infinitivformen realisiert. Ein morphosyntaktisches Rätsel erwies sich als Schnittstellenphänomen und erfuhr durch Berücksichtigung der Silbenstruktur eine frappierend elegante Erklärung. Ähnliches zeigte sich bei der Artikelverwendung im Englischen ebenso wie im Sesotho, einer Bantusprache. Artikel wurden von den untersuchten Kindern im Alter von 1-2 Jahren vor allem dann realisiert, wenn sie in einen prosodischen, trochäischen Fuß (stark-schwach) integriert werden konnten, z.B. in [it's a $]_{\mathrm{FT}}$ lamb]. Obwohl die Kinder im Prinzip bereits über entsprechendes morphosyntaktisches Wissen verfügten, beugten sie sich einer prosodischen Beschränkung (Prosodic Licensing Hypothesis). Die Kinder hatten für sich in den aus ihrer Perspektive offensichtlich unordentlichen Sprachgebrauch ihrer Umgebung eine Ordnung hineingebracht, die ihnen - wenngleich nicht korrekt im Sinne der Zielsprache - als vorübergehend nützlicher Kluge die systematische Lösung eines Problems erlaubte.

Ein vergleichbares Phänomen konnten wir für ein Kind (Adam) feststellen, das mit Deutsch und Englisch als doppelten Erstsprachen aufwuchs (Tracy/Gawlitzek-Maiwald 2005). Sein Spracherwerb zeichnete sich durch 
eine ausgeprägte Asynchronie zu Ungunsten des Englischen aus. Während er in seiner stärkeren Sprache, dem Deutschen, bereits über zielsprachliche Haupt- und Nebensätze verfügte, blieben seine englischen Äußerungen lange sehr rudimentär und vor allem nicht-finit. Sein Englisch entwickelte sich aber nicht nur wesentlich langsamer als das Deutsche, sondern die Art und Weise, in der sich die Subjekt-Verb-Kongruenz schließlich manifestierte, ließ auf eine bemerkenswerte Rekonstruktionsleistung schließen, vgl. (39). Man beachte dabei, dass don't in (d)-(e) zu diesem Zeitpunkt den Status einer unanalysierten Negationspartikel hat und dass keine der aufgeführten Äußerungen auf vergangene Ereignisse referiert.
Adam 3;10
(a) Erw. So let's see if that little grey horse fits into your bed. Adam a little one's fits ... he's fits
(b) agnes knows that ... she's knows it
(c) this one's goes here
(d) is don't fit in the little beds
(e) no's don't got batteries inside
(f) he's want to sit on there
(g) in the night he's wake up
(h) he's want to be warm
(i) I swimming in there

Ähnlich wie bei Demuth/McCullough zeichnet sich hier die Tendenz ab, die 3. Ps. Sg. nur dann als Suffix zu markieren, wenn das Verb keine stimmlosen Konsonanten in der Koda aufweist (vgl. goes, knows vs. fit, got, want, wake). Die einzige Ausnahme ist das zweimalige fits in (39a), möglicherweise als Folge eines Priming durch die vorangegangene Äußerung der Erwachsenen. Zugleich sehen wir, dass Adam eine Position für einen abstrakten INFL-Kopf konstruiert hat, ausbuchstabiert durch $i /$ 's. Dieser Platzhalter tritt nur im Kontext von Subjekten der 3. Ps. Sg. auf, also immer dann, wenn eigentlich das Verb selbst overt markiert werden müsste. Außerdem kennzeichnet Adam in Fällen, in denen es die Silbenstruktur erlaubt, die Kongruenz doppelt. Keine dieser Kreationen findet ein direktes Modell im Input. Aus universalgrammatischer Sicht hingegen ist es nur konsequent, wenn Adam für die von ihm wahrgenommene Kovariation von Subjekt und Verb einen neuen funktionalen Kopf schafft.

\section{Fazit}

Ziel meines Beitrags war es, zu zeigen, dass sich eine Reihe von Strukturveränderungen, die wir im Laufe des Spracherwerbs beobachten, als systematische Bemühungen um eine Überarbeitung aktueller Konstruktions- 
typen seitens der Kinder interpretieren lassen. Diese von mir als De- und Rekonstruktion bezeichneten Prozesse setzen voraus, dass sich Lerner aktiv und kritisch mit dem Sprachgebrauch ihrer Umgebung, aber vor allem auch mit dem Spektrum ihrer eigenen Konstruktionen auseinandersetzen. Während sich einerseits gute Argumente für eine anfängliche Koexistenz unterschiedlicher Konstruktionstypen finden, kann man zugleich zeigen, dass diese im Fall des Deutschen innerhalb weniger Monate auf einen einzigen abstrakten Bauplan hin konvergieren. Dabei kann es zwischenzeitlich zu Abweichungen von der zielsprachlichen Grammatik kommen, die sich nicht unmittelbar aus dem Sprachgebrauch erklären lassen. Im Fall bilingualer Kinder beobachten wir zudem mehr oder weniger intensive Mischungen, die ebenfalls nicht auf direkte Modelle im Input zurückzuführen sind.

In ihrer Erläuterung der Vorteile gebrauchsbasierter Konstruktionsgrammatiken unterstreicht Goldberg (2006, S. 10): ,what you see is what you get". Daten aus dem Spracherwerb deuten darauf hin, dass sich Kinder nicht damit begnügen, zu übernehmen, was sie sehen oder hören. Vielmehr fühlen sie sich dazu herausgefordert, den Input zu reanalysieren und zu reparieren. Kinder leisten damit, was Bartlett im Zusammenhang mit seiner Erforschung kognitiver Schemata vor langer Zeit wie folgt formulierte: „An organism has somehow to acquire the capacity to turn around upon its own schemata and to construct them afresh" (1964 [1932], S. 207). In diesem Sinne wären auch sprachliche Konstruktionen keine ein für alle Male festen Einheiten, sondern Zwischenlösungen, die sich in der Auseinandersetzung mit neuen Daten und neuen Herausforderungen dekonstruieren und optimieren lassen.

\section{Literatur}

Akmajian, Adrian/Heny, Frank (1975): An introduction to the principles of transformational syntax. Cambridge, MA.

Bartlett, Frederic C. (1964 [1932]): Remembering: a study in experimental and social psychology. Neudruck der Ausgabe 1932. Cambridge.

Bernardini, Petra/Schlyter, Suzanne (2004): Growing syntactic structure and codemixing in the weaker language: the ivy hypothesis. In: Bilingualism. Language and Cognition 7, S. 49-69.

Braine, Martin D.S. (1971): On two types of models of the internalization of grammars. In: Slobin, Dan I. (Hg.): The ontogenesis of grammar. New York, S. 153-186.

Braine, Martin D.S. (1976): Children's first word combinations. (= Monographs of the Society for Research in Child Development 41/1). Chicago.

Cenoz, Jasone/Genesee, Fred (2001): Trends in bilingual acquisition. (= Trends in Language Acquisition Research 1). Amsterdam/Philadelphia. 
Chomsky, Noam (1986): Knowledge of language: its nature, origin, and use. New York.

Chomsky, Noam (2000): Minimalist inquiries: the framework. In: Martin, Roger/Michaels, David/Uriagereka, Juan (Hg.): Step by step: essays on minimalist syntax in honor of Howard Lasnik. Cambridge, MA, S. 89-155.

Clahsen, Harald (1988): Normale und gestörte Kindersprache. Amsterdam/Philadelphia.

Clahsen, Harald/Penke, Martina (1992): The acquisition of agreement morphology and its syntactic consequences: new evidence on German child language from the Simone Corpus. In: Meisel (Hg.), S. 181-223.

D'Avis, Franz-Joseph/Gretsch, Petra (1994): Variations on ,variation': on the acquisition of complementizers in German. In: Tracy/Lattey (Hg.), S. 59-109.

Demuth, Katherine/McCullough, Elizabeth (2009): The prosodic (re)organization of early English articles. In: Journal of Child Language 36, 1, S. 173-200.

Dimroth, Christine (2009): Stepping stones and stumbling blocks. Why negation accelerates and additive particles delay the acquisition of finiteness in German. In: Dimroth/Jordens (Hg.), S. 135-168.

Dimroth, Christine/Jordens, Peter (Hg.) (2009): Functional categories in learner language. (= Studies on Language Acquisition 37). Berlin/New York.

Döpke, Susanne (2000): Cross-linguistic structures in simultaneous bilingualism. (= Studies in bilingualism 21). Amsterdam/Philadelphia.

Echols, Catharine H. (2001): Comment on Ann Peters' „Filler syllables: what is their status in emerging grammar?". In: Journal of Child Language 28, 1, S. 254-256.

Eisenbeiß, Sonja (2009): Generative approaches to language learning. In: Linguistics 47, 2, S. 273-310.

Elman, Jeffrey (1993): Learning and development in neutral networks: the importance of starting small. In: Cognition 48, S. 71-99.

Elman, Jeffrey (2002): Generalization from sparse input. In: Proceedings of the 38th Annual Meeting of the Chicago Linguistics Society. (= CLS 38). Chicago, S. 175-199.

Fritzenschaft, Agnes et al. (1990): Wege zur komplexen Syntax. In: Zeitschrift für Sprachwissenschaft 9, S. 52-134.

Gawlitzek-Maiwald, Ira (1997): Der monolinguale und bilinguale Erwerb von Infinitivkonstruktionen: Ein Vergleich von Deutsch und Englisch. (= Linguistische Arbeiten 370). Tübingen.

Gawlitzek-Maiwald, Ira/Tracy, Rosemarie (1996): Bilingual bootstrapping. In: Müller, Natascha (Hg.): Two languages: studies in bilingual first and second language development. Sonderheft Linguistics 34, 5, S. 901-926.

Gawlitzek-Maiwald, Ira/Tracy, Rosemarie/Fritzenschaft, Agnes (1992). Language acquisition and competing linguistic representations: the child as arbiter. In: Meisel (Hg.), S. 139-179.

Genesee, Fred/Nicoladis, Elena (2007): Bilingual first language acquisition. In: Hoff, Erik/Shatz, Marilyn (Hg.): Blackwell handbook of language development. Oxford, S. 324-342. 
Genesee, Fred/Nicoladis, Elena/Paradis, Johanne (1995): Language differentiation in early bilingual development. In: Journal of Child Language 22, 3, S. 611-631.

Goldberg, Adele E. (2006): Constructions at work. The nature of generalization in language. Oxford.

Haberzettl, Stefanie (2003): „Tinkering“ with chunks: Form-oriented strategies and idiosyncratic utterance patterns without functional implications in the IL of Turkish speaking children learning German. In: Dimroth, Christine/Starren, Marianne (Hg.): Information structure and the dynamics of language acquisition. (= Studies in $\mathrm{Bi}$ lingualism 26). Amsterdam u.a., S. 35-63.

Harnisch, Rüdiger (2004): Verstärkungsprozesse. Zu einer Theorie der „Sekretion“ und des „Re-konstruktionellen Ikonismus“. In: Zeitschrift für germanistische Linguistik 32, S. 210-232.

Hoekstra, Teun/Jordens, Peter (1994): From adjunct to head. In: Hoekstra, Teun/ Schwartz, Bonnie (Hg.): Language acquisition studies in generative grammar. (= Language Acquisition and Language Disorders 8). Amsterdam u.a., S. 119-150.

Hohenberger, Annette (2002): Functional categories in language acquisition. Self-organization of a dynamical system. (= Linguistische Arbeiten 456). Tübingen.

Hohenberger, Annette/Peltzer-Karpf, Annemarie (2009): Language learning from the perspective of nonlinear dynamic systems. In: Linguistics 47, 2, S. 481-511.

Höhle, Barbara (2009): Bootstrapping mechanisms in first language acquisition. In: Linguistics 47, 2, S. 359-382.

Höhle, Tilman (1986): Der Begriff „Mittelfeld“: Anmerkungen über die Theorie der topologischen Felder. In: Weiss, Walter E./Wiegand, Herbert E./Reis, Marga (Hg.): Textlinguistik contra Stilistik. Akten des VII. Internationalen Germanisten-Kongresses Göttingen 1985. Tübingen, S. 329-340.

Hyams, Nina (1986): Language acquisition and the theory of parameters. (= Studies in Theoretical Psycholinguistics 3). Dordrecht/Lancaster.

Jacobs, Joachim (2008): Wozu Konstruktionen? In: Linguistische Berichte 213, S. 3-44.

Jordens, Peter (2009): The acquisition of functional categories in child L1 and adult L2 Dutch. In: Dimroth/Jordens (Hg.), S. 45-96.

Kaltenbacher, Erika (1990): Strategien beim frühkindlichen Syntaxerwerb: eine Entwicklungsstudie. (= Tübinger Beiträge zur Linguistik: Series A, Language Development 12). Tübingen.

Kaminski, Juliane/Call, Josep/Fischer, Julia (2004): Word learning in a domestic dog: evidence for „Fast Mapping“. In: Science 304, S. 1682-1683.

Marcus, Gary (2008): Kluge. The haphazard construction of the human mind. Boston/ New York.

Meisel, Jürgen M. (1989): Early differentiation of languages in bilingual children. In: Hyltenstam, Kenneth/Obler, Lorraine (Hg.): Bilingualism across the lifespan: aspects of acquisition, maturity and loss. Cambridge u.a., S. 13-40. 
Meisel, Jürgen M. (Hg.) (1992): The acquisition of verb placement: functional categories and V2 phenomena in language acquisition. (= Studies in Theoretical Psycholinguistics 16). Dordrecht u.a.

Meisel, Jürgen M. (2004): The bilingual child. In: Bhatia, Tej K./Ritchie, William C. (Hg.): The handbook of bilingualism. (= Blackwell Handbooks in Linguistics 15). Malden, S. 91-113.

Menyuk, Paula (1972): Sentences Children Use. Cambridge, MA.

Müller, Natascha (1993): Komplexe Sätze. Der Erwerb von COMP und Wortstellungsmustern bei bilingualen Kindern. (= Tübinger Beiträge zur Linguistik: Series A, Language Development 16). Tübingen.

Müller, Natascha et al. (2007): Einführung in die Mehrsprachigkeitsforschung: Deutsch, Französisch, Italienisch. 2., durchges. u. aktual. Aufl. Tübingen.

Müller, Natascha/Penner, Zvi (1996): Early subordination: the acquisition of free morphology in French, German, and Swiss German. In: Linguistics 34, S. 133-164.

Nederstigt, Ulrike (2004): Auch and noch in child and adult German. (=Studies on Language Acquisition 23). Berlin u.a.

Newmeyer, Frederick J. (2000): Language form and language function. 2. Aufl. Cambridge, MA.

Newport, Elizabeth L. (1990): Maturational constraints on language learning. In: Cognitive Science 14, S. 11-28.

Paradis, Johanne/Genesee, Fred. (1996): Syntactic acquisition in bilingual children: autonomous or independent? In: Studies in Second Language Acquisition 18, S. $1-25$.

Penner, Zvi (1994): Possible domains for individual variation in early developmental stages. In: Tracy/Lattey (Hg.), S. 35-58.

Penner, Zvi/Tracy, Rosemarie/Weissenborn, Jürgen (2000): Where scrambling begins: triggering object scrambling in early language acquisition. In: Powers, Susan M./ Hamann, Claudia (Hg.): The acquisition of scrambling and cliticization. (= Studies in Theoretical Psycholinguistics 26). Dordrecht u.a., S. 127-164.

Penner, Zvi/Tracy, Rosemarie/Wymann, Karin (1999): Die Rolle der Fokuspartikel AUCH im frühkindlichen Lexikon. In: Rothweiler, Monika/Meibauer, Jörg (Hg.): Das Lexikon im Spracherwerb. Tübingen/Basel, S. 229-251.

Peters, Ann M. (1977): Language learning strategies: does the whole equal the parts? In: Language 53, S. 560-573.

Peters, Ann M. (1983): The units of language acquisition. (= Cambridge Monographs and Texts in Applied Psycholinguistics 1). Cambridge, MA u.a.

Peters, Ann M. (2001): Filler syllables: what is their status in emerging grammar? In: Journal of Child Language 28, 1, S. 229-242.

Pinker, Stephen (1984): Language learnability and language development. (= Cognitive Science Series 7). Cambridge, MA. 
Radford, Andrew (2009): Analysing English sentences: a minimalist approach. Cambridge.

Randall, Janet (1990): Catapults and pendulums: the mechanics of language acquisition. In: Linguistics 28, S. 1381-1406.

Riedl, Rupert (1980): Biologie der Erkenntnis. Die stammesgeschichtlichen Grundlagen der Vernunft. Berlin/Hamburg.

Roeper, Tom (1992): From the initial state to V2: acquisition principles in action. In: Meisel (Hg.), S. 333-370.

Roeper, Tom (1999): Universal bilingualism. In: Bilingualism, Language and Cognition 2, 3, S. 169-186.

Roeper, Tom (2007): The prism of grammar. How child language illuminates humanism. Cambridge, MA u.a.

Roeper, Tom/Pérez-Leroux, Ana T. (eingereicht): Simplicity and complexity in child language and its explanation. In: Infancia y Aprendizaje.

Rothweiler, Monika (1993): Der Erwerb von Nebensätzen im Deutschen: eine Pilotstudie. (= Linguistische Arbeiten 302). Tübingen.

Rothweiler, Monika (2006): The acquisition of V2 and subordinate clauses in early successive acquisition of German. In: Lleó, Conxita (Hg.): Interfaces in multilingualism: acquisition and representation. (= Hamburg Studies on Multilingualism 4). Amsterdam, S. 91-113.

Schulz, Petra/Wymann, Karin/Penner, Zvi (2001): The early acquisition of verb meaning in German by normally developing and language impaired children. In: Brain and Language 77, S. 407-418.

Slobin, Dan I. (1973): Cognitive prerequisites for the acquisition of grammar. In: Ferguson, Charles A./Slobin, Dan I. (Hg.): Studies of Child Language Development. New York, S. 175-208.

Stern, Clara/Stern, William (1928): Die Kindersprache: eine psychologische und sprachtheoretische Untersuchung. Darmstadt.

Thelen, Esther/Smith, Linda B. (1994): A dynamic systems approach to the development of cognition and action. Cambridge, MA/London.

Thoma, Dieter/Tracy, Rosemarie (2006): Deutsch als frühe Zweitsprache: zweite Erstsprache? In: Ahrenholz, Bernt (Hg.): Kinder mit Migrationshintergrund - Spracherwerb und Fördermöglichkeiten. Freiburg i.Br., S. 58-79.

Tomasello, Michael (2000): Do young children have adult syntactic competence? In: Cognition 74, S. 209-253.

Tomasello, Michael (2003): Constructing a language: a usage-based theory of language acquisition. Cambridge, MA u.a.

Tracy, Rosemarie (1991): Sprachliche Strukturentwicklung: Linguistische und kognitionspsychologische Aspekte einer Theorie des Erstspracherwerbs. (= Tübinger Beiträge zur Linguistik: Series A, Language Development 13). Tübingen. 
Tracy, Rosemarie (1994): Raising questions: formal and functional aspects of the acquisition of wh-questions in German. In: Tracy/Lattey (Hg.), S. 1-34.

Tracy, Rosemarie (1996): Vom Ganzen und seinen Teilen: Überlegungen zum doppelten Erstspracherwerb. In: Sprache und Kognition 15, 1/2, S. 70-92.

Tracy, Rosemarie (2002): Growing (clausal) roots: all children start out (and may remain) multilingual. In: Linguistics 40, 4, S. 653-686.

Tracy, Rosemarie/Gawlitzek-Maiwald, Ira (2005): The strength of the weak: asynchronies in the simultaneous acquisition of German and English. In: Zeitschrift für Literaturwissenschaft und Linguistik 35, 139, S. 28-53.

Tracy, Rosemarie/Lattey, Elsa (Hg.) (1994): How tolerant is universal grammar: essays on language, learnability and language variation. (= Linguistische Arbeiten 309). Tübingen.

Tracy, Rosemarie/Thoma, Dieter (2009): Convergence on finite V2 clauses in L1, bilingual L1 and early L2 acquisition. In: Dimroth/Jordens (Hg.), S. 1-43.

van de Craats, Ineke (2009): The role of is in the acquisition of finiteness by adult Turkish learners of Dutch. In: Studies in Second Language Acquisition (SSLA) 31, S. 59-92.

Weissenborn, Jürgen (2000): Der Erwerb von Morphologie und Syntax. In: Grimm, Hannelore (Hg.): Enzyklopädie der Psychologie. Bd. 3: Sprachentwicklung. Göttingen u.a., S. 141-169.

Weissenborn, Jürgen et al. (1998): Children's sensitivity to word-order violations in German: evidence for every early parameter setting. In: Proceedings of the 22nd Annual Boston Conference on Language Development. Somerville, S. 756-767.

Wenzel, Ramona/Schulz, Petra/Tracy, Rosemarie (2009): Herausforderungen und Potential der Sprachstandsdiagnostik - Überlegungen am Beispiel von LiSe-DaZ. In: Reich, Hans H./Roth, Hans-Joachim (Hg.): Dokumentation der FörMig-Herbsttagung 2007: Von der Sprachdiagnose zur Sprachförderung. Münster, S. 45-70.

Winkler, Steffi (2009): The acquisition of syntactic finiteness in L1 German. A structurebuilding approach. In: Dimroth/Jordens (Hg.), S. 97-134.

Yang, Charles (2009): Three factors in language variation. In: Lingua 120, 5, S. 1160 1177.

Zifonun, Gisela/Hoffmann, Ludger/Strecker, Bruno (1997): Grammatik der deutschen Sprache. 3 Bde. (= Schriften des Instituts für Deutsche Sprache 7). Berlin/New York. 


\title{
Dynamische Aspekte der Argumentinterpretation: Eine neurokognitive Perspektive
}

\begin{abstract}
Der vorliegende Aufsatz beschäftigt sich mit der Frage, wie Argumente während des Sprachverstehens erkannt werden, welche Eigenschaften einem Argument in Abwesenheit des Verbs zugeschrieben werden und welche Art von Vorhersagen mit der Argumentinterpretation verbunden sind. Ausgehend von der Annahme, dass beim Sprachverstehen in Echtzeit jedes Wort so maximal wie möglich interpretiert wird, werden wir argumentieren, dass die zugrunde liegenden, sprachübergreifend zu findenden Mechanismen durch die Interaktion von typologisch motivierten Prominenzskalen (z.B. Belebtheitshierarchie) beschrieben werden sollten. Diese gestatten nicht nur eine Erklärung bestehender Befunde, sondern besitzen das Potenzial, zentrale Aspekte der Sprachverstehensarchitektur modelltheoretisch abzuleiten. Experimentell liegt der Fokus des Aufsatzes auf der Erfassung elektrophysiologischer-neuronaler Aktivierungsmuster, da diese uns im Gegensatz zu Urteilen oder Korpusverteilungen einen unmittelbaren Einblick in die Verarbeitung im Echtzeitbereich gestatten.
\end{abstract}

\section{Einleitung}

Betrachtet man Sprache aus einer Echtzeitperspektive, so wird schnell deutlich, dass in vielen Sprachen der Welt Nomen und Präpositionalphrasen interpretiert werden müssen, ohne dass man weiß, welche Rolle diese in der später durch das Verb festzulegenden Handlung besitzen. Nun könnte man der dadurch entstehenden Unsicherheit entgehen, wenn man diese Nomen und Präpositionalphrasen nicht sofort versucht hinsichtlich ihrer Funktion zu interpretieren, sondern einfach bis zum Auftreten des Verbs wartet. Doch so arbeitet unser Sprachverstehenssystem genau nicht. Durch den unmittelbaren Versuch der maximalen Interpretation bei gleichzeitiger Inkaufnahme einer möglichen späteren Revision vermeiden wir, dass die präverbale Information sich unstrukturiert möglicherweise so sehr ansammelt, dass wir Teile davon einfach vergessen, wenn der Moment kommt, an dem das den Rahmen aufspannende Verb auftritt (für die Idee, dass unmittelbare maximale Strukturierung Arbeitsgedächtniskosten reduziert, siehe Frazier/Fodor 1978). Doch was bedeutet maximale Interpretation? Welche Art von Merkmalen aus dem Lautstrom oder aus einem geschriebenen Satzfragment nutzen wir für die Festlegung, ob es sich um ein mögliches Verbargument handelt und welche Rolle es im zu erwartenden Verbrahmen einnimmt? Der vorliegende 
Beitrag versucht, mit Hilfe ausgewählter Daten aus der neurokognitiven Sprachforschung darauf Antworten zu geben. Er wird darüber hinaus zeigen, wie sprachübergreifend ähnliche Mechanismen genutzt werden, um Argumente hinsichtlich ihrer Funktion zu determinieren und von anderen potenziellen Argumenten möglichst eindeutig abzugrenzen.

\section{Inkrementalität: Phänomen und Konsequenzen}

Beginnen wir mit dem eigentlichen Grundproblem. Bis auf wenige Ausnahmen (z.B. Pritchetts ,head driven processing account" $(1988,1992))$ ist man seit den Anfängen der psycholinguistischen Forschung stets davon ausgegangen, dass während des Sprachverstehens die Bedeutungskonstitution sich kontinuierlich, d.h. zyklisch und maximal an jedem aus dem Lautstrom identifizierten Segment, entfaltet und dabei - was wesentlich ist - unabhängig vom Verb ablaufen kann. Dieses als „Inkrementalität“ bekannte Phänomen konnte durch zahlreiche Studien empirisch untermauert werden (siehe insbesondere Crockers „principle of incremental comprehension“ 1994, aber auch Marslen-Wilson 1973). Man kann es sich anhand eines sehr einfachen Beispiels verdeutlichen. Wenn das Satzfragment Alle börten, dass der König von Thailand ... gelesen oder gehört wird, dann gibt es mit der Verarbeitung der Präpositionalphrase von Thailand bereits bestimmte Annahmen über die syntaktische Struktur, die dann sichtbar werden, wenn mit dem nächsten Wort erfuhr der Satz beendet wird. Muttersprachler des Deutschen sind beim Auftreten des Verbs in der Regel überrascht und haben für einen kurzen Moment den Eindruck, dass der Satz nicht interpretierbar ist. Erst durch eine Reanalyse, d.h. durch die Uminterpretation der Präpositionalphrase zum Argument des Verbs erfabren, kommt die korrekte Interpretation zustande. Die Notwendigkeit zur Reanalyse zeigt aber auch, dass wir bereits zum Zeitpunkt der Verarbeitung der Präposition (bzw. der Präpositionalphrase) eine Entscheidung zugunsten der das Nomen modifizierenden Lesart getroffen haben müssen, die es nun zu korrigieren gilt (für experimentelle Befunde und ihre theoretische Einordnung siehe Konieczny et al. 1997).

Mit dem nächsten Beispiel soll hier - wiederum rein deskriptiv - eine weitere Eigenschaft des Sprachverstehens vorgestellt werden. Dabei geht es nun bereits um eine Frage der Argumentinterpretation und ihrer Tiefe. Hören oder lesen deutsche Muttersprachler den Satz Alle wussten, dass Martin Lehrerinnen widersprechen, so gibt es auch hier wieder einen kurzen Moment einer für Holzwegsätze dieser Art oft zu beobachtende Überraschung, die messbar zu einem Absinken der Akzeptabilität führt bzw. in Veränderungen in neurophysiologischen/-anatomischen Mustern resultiert (siehe Haupt et al. 2008 für einen aktuellen Überblick). Diese entsteht, wenn das Verb nicht wie erwartet im Singular auftritt, sondern durch die Pluralmarkierung eine 
Objekt-vor-Subjekt-Abfolge (OS) erzwingt. Der Effekt, der übrigens auch zu beobachten ist, wenn man den Satz in einen Kontext einbettet, der die OS-Lesart stützt, ${ }^{1}$ basiert darauf, dass bei der Verarbeitung von Martin diesem ambigen Argument lokal die grammatische Rolle des möglicherweise einzigen syntaktischen Argumentes zugewiesen wird (siehe Bornkessel-Schlesewsky/Schlesewsky 2009), welches den Annahmen der deutschen Syntax folgend damit die grammatische Funktion des Subjekts zugeschrieben bekommt. ${ }^{2}$ Eine Reanalyse wird aber dann notwendig, wenn das Verb nicht

1 Gelegentlich hört man das Argument, dass derartige OS-Abfolgen im Deutschen ja eigentlich nicht gebräuchlich sind und in Isolation wohl niemals zu beobachten sein werden. Ein Beispiel aus dem „Fränkischen Tag“, einer Tageszeitung Oberfrankens, zeigt das Gegenteil zu dieser Annahme. Dort fand sich die folgende Titelzeile: Trotz. Frost sollten Rehe Wanderer nicht füttern. Weitere Belege lassen sich ohne Probleme auch in anderen Registern finden.

2 In Schlesewsky/Bornkessel (2004) haben wir basierend auf einem Vergleich zwischen kasusambigen und eindeutig kasusmarkierten Argumenten im Deutschen argumentiert, dass die inkrementelle Interpretation kasusambiger Nominalphrasen tatsächlich nur bis zur Zuweisung der grammatischen Funktion geht. Von dort wird nicht auf die thematische Rolle, wie Agens, geschlossen. Das bedeutet nicht, dass thematische Rollenzuweisungen keine Bedeutung besitzen, doch lassen sich Effekte, die sich auf diese zurückführen lassen, nur dann beobachten, wenn eine eindeutige Kasusmarkierung oder Verbinformationen vorliegen. Hinsichtlich der Verbinformation lässt sich aber auch zeigen, dass deren thematische Eigenschaften im Kontext der Verarbeitung kasusambiger Argumente lokal vernachlässigt werden können, wenn sie mit der Zuweisung der grammatischen Funktion konfligieren. In Bornkessel/Schlesewsky (2006a) berichten wir einen Reanalyseeffekt auf der nominativmarkierten NP in Sätzen mit dem finiten Verb an zweiter Position, wie Wabrscheinlich gefällt Maria der Mann ... Unter der Annahme, dass das Verb gefallen seine höchste thematische Rolle an das erste mögliche Argument vergibt, sollte diesem Argument die grammatische Funktion des Objektes zugewiesen werden. Der Reanalyseeffekt zeigt aber, dass das offenbar nicht geschieht.

Basierend auf diesen Arbeiten haben wir auch dafür argumentiert, dass es während des inkrementellen Sprachverstehens keine automatische Kasuszuweisung an ein kasusambiges Argument gibt, d.h. ein Argument kann zwar die grammatische Funktion des Subjektes zugewiesen bekommen und damit die Erwartung für ein bestimmtes Kongruenzmerkmal auf dem Verb auslösen, diese Zuweisung der grammatischen Funktion löst aber keine Zuweisung eines Kasusmerkmals aus. Dieser experimentell motivierte Unterschied zwischen Kasus und grammatischer Funktion wird im Folgenden für die Argumentation zentral sein. Ebenfalls sei an dieser Stelle angemerkt, dass wir bereits in Bornkessel/Schlesewsky/Friederici (2003), aber insbesondere in Schlesewsky/Bornkessel (2004) und in Bornkessel/Schlesewsky (2006) argumentiert haben, dass es mit dem Konzept der generalisierten semantischen (thematischen) Rolle im Sinne von Primus (1999) und van Valin (Foley/van Valin 1984) zumindest aus der psycho-/neurolinguistischen Perspektive eine dritte Dimension gibt, die sich nicht automatisch aus den beiden anderen hier genannten Dimensionen (Kasus und grammatische Funktion) ableiten lässt. Generalisierte semantische Rollen erlauben aus der experimentellen Perspektive Vorhersagen und Erklärungen für die zu beobachtenden Effekte, die sich mit den anderen zuvor genannten Dimensionen und mit klassischen thematischen Rollenkonzeptionen (z.B. Jackendoff 1972; Grimshaw 1990) nicht ableiten lassen. Während wir in der ursprünglichen Motivation (Bornkessel et al. 2003) auf der Grundlage der Protorollenkonzeption von Dowty (1991) und Primus (1999) argumentierten, erscheint seit unseren Arbeiten ab 2006 zu diesem Thema ein Modell, welches auf der Actor/UndergoerKonzeption von van Valin beruht und den Dependenzansatz zwischen diesen Rollen im Sinne von Primus (1999) in die Relation der generalisierten semantischen Rollen integriert (Bornkessel/Schlesewsky 2006a, b). 
mit diesem Nomen kongruiert. Dass die Notwendigkeit zur Korrektur nicht nur messbar, sondern auch bewusst nachvollziehbar ist, zeigt der kognitive Aufwand, den Sprecher des Deutschen benötigen, um zur richtigen Interpretation zu gelangen (siehe Sturt/Crocker 1996 für ein Modell zur Effektstärke bei syntaktischen Reanalysen). Bemerkenswert ist in diesem Kontext aber auch, dass die Subjektspräferenz (Frazier/Flores d'Arcais 1989; Schriefers/Friederici/Kühn 1995), ${ }^{3}$ d.h. die Zuweisung der Subjektsfunktion an das erste mögliche Argument, mitunter so schnell (und einfach) reanalysiert werden kann, dass man den Aufwand nicht mehr bewusst „erlebt“. Akzeptabilitätsstudien zeigen, dass im Gegensatz zu dem zuvor genannten Beispiel der Satz Alle wussten, dass Martin Lehrerinnen gefallen signifikant besser bewertet wird (Schlesewsky/Bornkessel 2003; Bornkessel et al. 2004). Das ist einerseits überraschend, da auch hier - sollten die Prozesse der Zuweisung grammatischer Funktionen genauso automatisch ablaufen - die SO-Abfolge auf dem Verb revidiert werden muss. Andererseits ist die höhere Akzeptabilität sofort einsichtig, da das so genannte Objektexperiencerverb gefallen bei der Reanalyse durch seine inverse thematische Rollenzuweisung, d.h. die hierarchisch höhere thematische Rolle wird dem syntaktisch hierarchisch niedrigeren Argument zugewiesen und vice versa (Primus 1999; Wunderlich 1997; Fanselow 2000), den Weg zur korrekten OS-Interpretation weist. Dieser Prozess läuft nun aber so schnell ab, dass wir den kognitiven Nachteil, der durch die Reanalyse ausgelöst wird, in dem Moment, an dem uns die Bedeutung des Satzes bewusst wird, nicht mehr wie im Kontext der oben beschriebenen Sätze nachempfinden können.

An dieser Stelle entfalten nun Messmethoden ihren Vorteil, die neurophysiologisch oder neuroanatomisch Aktivierungsveränderungen direkt am oder im Kortex messen. Insbesondere Messungen mit Hilfe des Elektroenzephalogramms (EEG) sollen im Folgenden im Vordergrund stehen. Diese haben den Vorteil, dass sie im Bereich von Millisekunden Aktivierungsveränderungen erfassen können. ${ }^{4}$ EEG-Messungen bestätigen nun, dass sich

Für das Deutsche wurde die Präferenz, ambige initiale Nominalphrasen als Subjekt zu interpretieren, erstmals systematisch in Hemforth (1993) für Deklarativsätze und in Farke (1994) für $w h$-Strukturen untersucht.

4 Im Jahre 1929 veröffentlichte der Jenaer Neurologe Hans Berger eine Arbeit, in der er zeigte, dass sich an der menschlichen Schädeloberfläche elektrische Hirnströme ableiten lassen, die durch kognitive Aufgaben, wie das Lösen einer arithmetischen Aufgabe, moduliert werden können (Berger 1929). Diese Veränderungen der elektrischen Aktivität lassen sich mit Hilfe so genannter ereigniskorrelierter Potenziale (EKPs) untersuchen und charakterisieren. EKPs sind hirnelektrische Potenzialverschiebungen, die in einem zeitlich exakt fixierbaren Zusammenhang zu einem sensorischen, motorischen oder kognitiven Ereignis stehen. Die Messung von EKPs erfolgt durch die Ableitung von Synapsenpotenzialen an der Schädeloberfläche. Der Tradition folgend, wurden die ersten Sprachuntersuchungen mittels EKPs auf die Analyse von Unterschieden zwischen den Hemisphären beschränkt. Obwohl diese Arbeiten die Linkslateralisierung von Sprache, wie sie aus Patientenstudien bereits bekannt war, bestätigten, erbrachten sie keine wesentlichen Fortschritte im Hinblick auf ein erweitertes Ver- 
die beiden genannten Beispiele hinsichtlich des auftretenden Konfliktes vollkommen gleich verhalten, da sie die identische neurophysiologische Reaktion zeigen (Bornkessel et al. 2004). Die dort zu beobachtende Negativierung zwischen 300 und 500 ms wird als N400 bezeichnet und reflektiert die Notwendigkeit der Korrektur der grammatischen Funktion der beiden zuvor verarbeiteten Argumente. ${ }^{5}$ Es zeigt sich also, dass in beiden Sätzen das Auftreten eines Verbs mit einer unerwarteten Numerusflexion zu erhöhten neuronalen Aktivierungen führt und daher die Annahme abzuleiten gestattet, dass der eigentliche Konflikt identisch ist.

Abbildung 1 zeigt die neurophysiologischen Veränderungen, die man beobachten kann, wenn - wie hier - bei der Verarbeitung des Verbs (y-Achse signalisiert den zeitlichen Beginn der Wortpräsentation) die notwendige Korrektur zur OS-Abfolge deutlich wird. Sichtbar ist, dass sich beide Verbtypen

ständnis sprachlicher Prozesse. Erst mit den Arbeiten von Kutas und Hillyard (1980) wurde ein Weg beschritten, der einen direkten Zugang zu den hier beschriebenen sprachlichen Phänomenen erlaubt. Da die „natürliche“, zufällig entstehende Grundaktivität des Gehirns $(50-150 \mu \mathrm{V})$ um ein Vielfaches größer ist als die durch den kognitiven Reiz ausgelöste Aktivierung $(1-8 \mu \mathrm{V})$, werden die kritischen Reize mehrfach wiederholt und anschließend gemittelt. Durch dieses Verfahren wird einerseits die Grundaktivität eliminiert, während andererseits gleichzeitig die reizrelevante Aktivität isoliert werden kann. Die aus der Mittelung resultierenden, digitalisierten Daten lassen sich als Kurven visuell darstellen. Die in Sprachverstehensexperimenten zu beobachtenden Effekte (Komponenten) werden aus einer funktionalen Perspektive interpretiert, d.h. es werden stets zwei Bedingungen miteinander verglichen, die nur einen minimalen, genau definierten und theoretisch motivierten Unterschied besitzen. Die aus dem Vergleich resultierenden Differenzen werden auf der Basis ihrer Latenz (die Zeitspanne zwischen der Darbietung des Reizes und dem Auftreten des Effektes), Topografie (an welchen Elektrodenpositionen der Effekt auftritt, siehe Abbildung 6), Polarität (die Richtung der Auslenkung gegenüber der Kontrollbedingung, d.h. positiv oder negativ) und Amplitude (der Grad der maximalen Ausprägung) klassifiziert. Im Laufe der vergangenen Jahre ist es gelungen, eine Reihe von sprachrelevanten Komponenten zu identifizieren, die Aussagen über die Art der sprachlichen Architektur und ihren sprachübergreifenden Charakter gestatten (für einen Überblick siehe Bornkessel-Schlesewsky/Schlesewsky 2009a).

$5 \quad$ An dieser Stelle scheint eine Anmerkung zu den hier vorgestellten Komponenten notwendig. Während man bis zur Mitte der 90er Jahre des vergangenen Jahrhunderts - und basierend auf ersten Befunden vor allem aus dem Englischen - hoffte, mit Hilfe von EEG-Komponenten zwischen sprachlichen Domänen unterscheiden zu können (Garnsey 1993), geht man in der aktuellen Forschung davon aus, dass ein solcher Schluss nicht mehr aufrechterhaltbar ist (z.B. Urbach/Kutas 2010). So ist die Annahme, dass man durch das Auftreten einer N400 auf lexikalische oder semantische Prozesse schließen könnte, während das Vorkommen einer P600 (Positivierung mit einem Maximum um 600 ms) syntaktische Prozesse reflektiert, empirisch momentan nicht mehr zu rechtfertigen (siehe Bornkessel-Schlesewsky/Schlesewsky 2009a, Kapitel 16 für eine zusammenfassende Darstellung des Problems). Beispiele, die dieses Problem illustrieren, sind die so genannte ,semantische P600“ (At breakfast, the eggs would eat, Kuperberg et al. 2003; Kolk et al. 2003); Positivierungen bei Verletzung von Antonymrelationen (Das Gegenteil von schwarz ist gelb, Roehm et al. 2007) bzw. bei niedrigfrequenten Nomen am Satzanfang (Welche Trappistin/Lebrerin ..., Bergmann 2010) oder N400Effekte bei morphosyntaktischen (split-Ergativitäts-)Verletzungen im Hindi (Choudhary et al. 2009) oder bei regelbasierten phonologischen Prozessen (Janssen et al. 2006). 
gleich verhalten. Man beachte, dass die Kurven niemals absolut, d.h. im Koordinatensystem interpretiert werden, sondern nur relativ zueinander (siehe Fußnote 4).

A. Aktiv-Verben

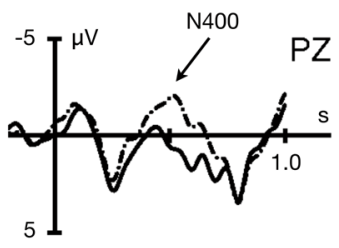

\section{B. Objektexperiencer-Verben}
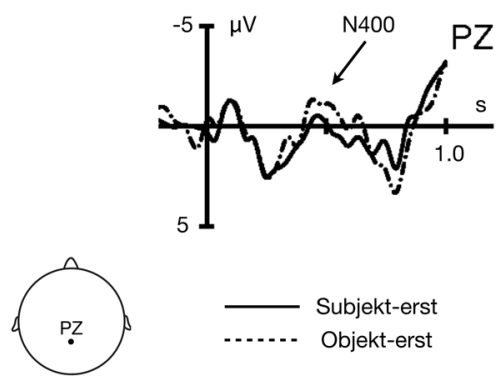

Subjekt-erst

Objekt-erst

Abb. 1: Verarbeitung von lokal ambigen NP-NP-V-Strukturen (Abbildung adaptiert aus Bornkessel et al. 2004)

Dass sich neurophysiologisch die beiden Verbtypen in ihrem Reanalyseverhalten nicht unterscheiden, dass aber behaviorale Unterschiede im Antwortverhalten zu finden sind, bestätigt die zuvor bereits beschriebene Annahme, dass es nicht der Konflikt selbst, d.h. die Erwartungsverletzung, ein singular markiertes Verb zu finden, sondern die Korrektur der Zuweisung der grammatischen Funktion ist, die die Akzeptabilitätsunterschiede verursacht (Bornkessel et al. 2004).

Lässt man die bis zu diesem Zeitpunkt angeführten Argumente Revue passieren, so kann man festhalten, dass das menschliche Sprachverarbeitungssystem a) inkrementell, d.h. kontinuierlich, die zu jedem Zeitpunkt angebotenen Informationen verarbeitet, b) im Falle von Mehrdeutigkeiten (Ambiguitäten) eine Lesart präferiert, c) aus der Lesart Erwartungen über nachfolgende Informationen (z.B. Numerus des Verbs) ableitet, und d) die Fähigkeit zur Korrektur (Reanalyse) besitzt, die unterschiedlich schwer sein kann und somit das Spektrum von unbewusster Reanalyse bis hin zur Nichtinterpretierbarkeit aufspannt (siehe die zuvor zitierten Referenzen und Bornkessel-Schlesewsky/Schlesewsky (2009a, Kapitel 7) für eine aktuelle Darstellung). Alle diese Eigenschaften bilden die Grundlage der im Folgenden zu beschreibenden Phänomene.

\section{Argumente im Wettbewerb}

Um zu verstehen, was „Argumente im Wettbewerb“ wirklich bedeutet, stellen wir uns zunächst einmal die folgende Situation aus dem wirklichen Leben vor, die viele von uns einfach nachvollziehen können. Nehmen wir 
also an, dass an einer Universität und in einer philologischen Fakultät eine Professur für Sprachwissenschaft ausgeschrieben wurde. Ein erster Aspekt, der die Besetzung dieser Stelle wesentlich beeinflusst, ist die Berufungskommission und deren Zusammensetzung. Selbst unter der Annahme, dass diese Kommission alle ethischen und moralischen Grundsätze bei einer solchen Arbeit wahrt, hat sie trotzdem bestimmte Präferenzen bzw. befindet sich im Spannungsfeld verschiedener Präferenzen der Kommissionsmitglieder. Ebenso wichtig ist der Aspekt des Bewerberfeldes. Hier gibt es zwei Seiten. Sollte es - und hier weichen wir mal ein wenig von der Realität ab nur einen Bewerber oder eine Bewerberin geben, dann sind dessen/deren wissenschaftlichen Eignungen und Präferenzen zwar nicht unwichtig, sie entfalten ihre eigentliche Bedeutung aber erst in der Konkurrenz. Solange man der alleinige Vertreter/Bewerber ist, kann man sich schon Schwächen leisten, denn objektive Zwänge (z.B. die Sicherstellung der Lehre und der Prüfungen) lassen eine gewisse Toleranz zu. In dem Moment - hier sind wir wieder in der Realität -, bei dem das Bewerberfeld aus mehreren Personen besteht, erlangen die Merkmale jedes einzelnen Bewerbers durch die kompetitive Situation eine neue Qualität. Jetzt geht es nicht mehr darum, was man selbst ist, sondern in welcher Relation, d.h. mit welchen distinktiven Eigenschaften, man zu dem oder den anderen steht. Eine einfache Entscheidung ist nur dann möglich, wenn die Bewerber klar voneinander unterscheidbar sind. Ähneln sie sich zu sehr, dann wird die Entscheidung nicht nur schwieriger, sondern der Ausgang des Verfahrens besitzt eine höhere Wahrscheinlichkeit zur Zufälligkeit.

Wenn sich nun der geneigte Leser etwas irritiert fragt, was dieses Beispiel mit Sprachverstehen und Argumentverarbeitung aus einer dynamischen Perspektive zu tun hat, dann können die Autoren ihm/ihr versichern, dass er/sie soeben die Grundeigenschaft der Argumentinterpretation und der Verarbeitung dieser kennen gelernt hat. Denn so unwahrscheinlich, wie es auf den ersten Blick erscheinen mag: Sprachübergreifende Studien (siehe die Referenzen zu den Abbildungen 3-5) deuten darauf hin, dass wir während des Sprachverstehens exakt so vorgehen, wie in der oben geschilderten Situation (Bornkessel-Schlesewsky/Schlesewsky 2009b). Dabei reflektieren Verben die Eigenschaften und Anforderungen der jeweiligen Berufungskommission und die Argumente sind die Kandidaten, die um den ersten Platz auf der zu erstellenden Liste konkurrieren. Vernachlässigen wir der Einfachheit halber zuerst einmal das Verb, da es uns nicht um die spezifischen Anforderungen des Stellenprofils geht, sondern nur um die Tatsache, dass am Ende eine Liste erstellt wird, die einen Erstplatzierten besitzt. Damit besitzen für die folgenden Ausführungen die Bewerber die zentrale Rolle. Wir beschränken uns zunächst auf das Deutsche und illustrieren hier alle wesentlichen Aspekte. In einem weiteren Schritt (Abschnitt 4) werden wir dann zeigen, dass sich diese auch sprachübergreifend beobachten lassen. 
Betrachten wir also zunächst einmal aus einer inkrementellen Perspektive die Verarbeitung eines satzinitialen kasusmarkierten Argumentes (welcher Jäger) in dem Satzfragment Jane fragte sich, welcher Jäger ... Welche Annahmen resultieren aus der Verarbeitung des nominativmarkierten Argumentes? Auf dem aktuellen Argument lässt sich selbst noch nicht viel beobachten, überraschenderweise auch dann nicht, wenn das Nomen unbelebt ist und einen eher suboptimalen Handlungsverursacher darstellt. Dies steht im Gegensatz zu Beobachtungen aus dem Englischen, da bereits an dieser Stelle suboptimale, d.h. unbelebte Subjekte zu Verarbeitungskosten führen (Weckerly/Kutas 1999, siehe Fußnote 10). Erweitert man aber den Satz durch ein zweites Argument (Jane fragte sich, welcher Jäger den Gärtner ...) zu einer transitiven Lesart, so lässt sich im EEG auf dem zweiten Argument erhöhte Aktivierung messen, die man als Kosten der Erweiterung von einer ursprünglich intransitiven auf eine transitive Lesart interpretieren kann (Bornkessel/Fiebach/Friederici 2004).

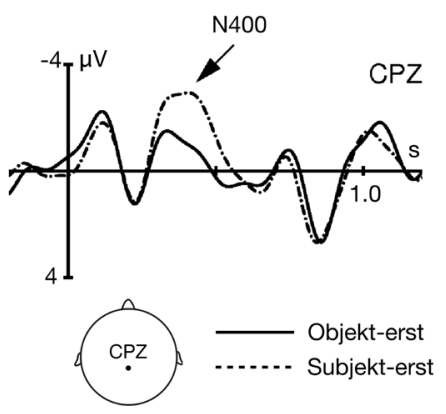

Abb. 2: Negativierung für die Verarbeitung eines zweiten (nicht vorhersagbaren) Argumentes im Vergleich zu einem zweiten Argument, welches durch einen initialen Akkusativ (Jane fragte sich, welchen Jäger ...) erwartbar ist (Abbildung adaptiert aus Bornkessel/Fiebach/Friederici 2004). Man beachte, dass die Kurven niemals absolut, d.h. im Koordinatensystem interpretiert werden, sondern nur relativ zueinander (siehe Fußnote 4).

Bei Abbildung 2 zeigt sich also eine erste Präferenz, die an das oben genannte Bewerbungsbeispiel anschließt und auf die wir bereits im Kontext der Verarbeitung kasusambiger Argumente kurz hingewiesen haben. Ohne explizite Evidenz wird auch ein initialer Nominativ so verarbeitet, als ob er das einzige Argument eines (somit intransitiven) Ereignisses oder Zustandes darstellt. Dabei ist es lokal unerheblich, ob er viele prototypische Merkmale (z.B. die genannte Belebtheitseigenschaft) besitzt oder sich eher suboptimal hinsichtlich dieser Anforderungen verhält. Wichtig ist einzig, dass weder weitere Argumente angenommen noch spezifische Anforderungen an Verbeigenschaften gestellt werden. Es wird zu diesem Zeitpunkt nur angenommen, dass der Nominativ das einzige Argument des Ereignisses ist und dass das obligatorisch zu erwartende Verb nur ein syntaktisch zu realisie- 
rendes Argument benötigt. ${ }^{6}$ Muss die Annahme der „Einzigartigkeit" revidiert werden, geht das mit messbaren Kosten einher (Bornkessel-Schlesewsky/Schlesewsky 2009b). Einerseits führt die Erweiterung von einer intransitiven zu einer transitiven Relation zu Erweiterungskosten (siehe Abschnitt 4 sowie die Diskussion zu Abbildung 4). Momentan wichtiger ist aber, dass durch das Auftreten eines zweiten Handlungsteilnehmers die lexikalischen Eigenschaften des Nominativs plötzlich bedeutsam werden. Dieses zeigt sich in der im Folgenden dargestellten Studie, in der es um die Vorhersage eines noch zu verarbeitenden Nominativarguments geht. Diese kann durch ein initiales Akkusativargument erzeugt werden, da das Objekt i.d.R. zur Erstellung einer syntaktisch korrekten Form nicht nur ein Verb sondern auch ein Nominativargument erfordert. Obwohl - wie oben kurz erwähnt - ein initialer unbelebter Nominativ keine Verarbeitungsnachteile zeigt, lassen sich auf diesem dann erhöhte Aktivierungen beobachten, wenn er wie in Jane fragte sich, welchen Gärtner der Zweig streifte einem zuvor verarbeiteten Akkusativ folgt. Dies illustriert Abbildung 3 aus einer Studie von Roehm und Kollegen aus dem Jahre 2004.

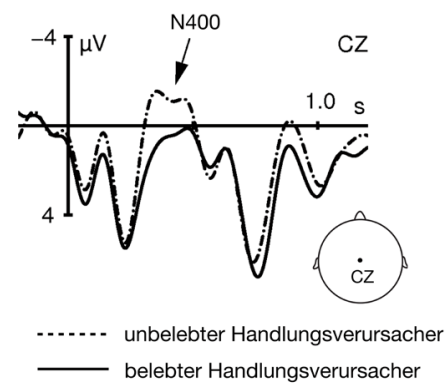

Abb. 3: Negativierung für die Verarbeitung eines unbelebten, durch einen vorangehenden Akkusativ vorhergesagten Nominativarguments im Vergleich zu einem belebten Nominativ (der Zweig im Vergleich zu der Jäger), gemessen auf dem kritischen Argument (Abbildung adaptiert aus Roehm et al. 2004). Man beachte, dass die Kurven niemals absolut, d.h. im Koordinatensystem interpretiert werden, sondern nur relativ zueinander (siehe Fußnote 4).

Wichtig ist es in diesem Zusammenhang zu erkennen, dass es weniger die Kombination von Nominativ und Belebtheit ist, die diesen Effekt verursacht, sondern die durch die Verarbeitung eines Akkusativarguments ausgelöste Erwartung für die Eigenschaften dieses Nominativarguments. Aus der Perspektive des Objektes und als Konsequenz der inkrementellen Interpre-

Das hier verwendete Konzept der Transitivität basiert auf den Arbeiten von DeLancey (1981), Comrie (1989) und de Swart (2007, für die Hörerperspektive). Transitivität ist demnach nicht nur eine dem Verb inhärente Eigenschaft, die syntaktisch und/oder semantisch die Zahl der Handlungsteilnehmer festlegt, sondern bestimmt sich auch durch die Eigenschaften der Handlungsteilnehmer selbst (siehe auch Hopper/Thompson 1980 für einen ähnlichen Ansatz). 
tation soll dieses nun nicht nur die entsprechende morphologische Markierung haben, sondern es soll auch die Eigenschaft eines optimalen Handlungsverursachers besitzen, nämlich belebt sein. Wird diese Eigenschaft nicht erfüllt, zeigt die erhöhte Aktivierung die Erwartungsverletzung und damit einen Verarbeitungsnachteil. ${ }^{7}$

An dieser Stelle wollen wir einmal kurz über die EEG-Messungen hinausgehen und uns einen Befund aus einer Studie anschauen, bei dem mit Hilfe bildgebender Verfahren (funktionelle Magnetresonanztomographie, fMRT) Aktivierungsänderungen sichtbar gemacht werden können. In Grewe et al. (2007) wurden transitive Sätze verglichen, bei denen das Akkusativargument entweder als belebt (Der Pirat hat die Prinzessin geraubt.) oder als unbelebt (Der Pirat hat den Schatz, geraubt.) realisiert wurde. Eine bestimmte anatomische Region (der linke Sulcus temporalis superior, siehe Abbildung 6) zeigt im direkten Vergleich der beiden Satzrealisierungen erhöhte Aktivierung, wenn beide Handlungsteilnehmer belebt sind. Die Autoren interpretieren diese Aktivierung ganz im Sinne des hier dargestellten Wettbewerbsprinzips. Werden die Argumente in den Verbrahmen eingefügt, konkurrieren sie um die Position des höchsten Argumentes (des Handlungsverursachers). Je ähnlicher sich die Argumente in ihren Eigenschaften sind, desto höher ist der Aufwand der Zuweisung der Argumente zu den Leerstellen. Dieser Aufwand ist, wenn man den Autoren folgen möchte, kostenträchtig und messbar. Einfach ist die Verarbeitung also dann, wenn beide sich in allen relevanten Dimensionen - welche das sind, werden wir gleich noch näher betrachten - klar voneinander unterscheiden. ${ }^{8}$ Dabei deutet die Studie darauf hin, dass neben einem eindeutigen morphologischen Merkmal (Kasus) auch Belebtheit eine wichtige Rolle im Verarbeitungsverhalten zukommt. Wie bereits kurz angedeutet, kann man neben den beiden Eigenschaften noch weitere identifizieren, die die sprachliche Informationsverarbeitung vereinfachen. In Bornkessel-Schlesewsky/Schlesewsky (2009b) haben wir basierend auf einer Reihe von Sprachverstehensdaten, auf die hier aufgrund von Platzbeschränkungen nicht näher eingegangen werden soll, die folgende Hypothese aufgestellt:

\footnotetext{
Daten aus dem Chinesischen stützen die Annahme, dass die zu beobachtenden Effekte auf die Erwartungsverletzung zurückgehen und nicht auf die Merkmalskombination (Philipp et al. 2008). Da ein Subjekt - unter der Annahme, dass das Chinesische die grammatische Funktion Subjekt besitzt - bzw. ein Handlungsverursacher in Abwesenheit expliziter Verbinformationen keine Vorhersage eines zweiten Argumentes gestattet, sollte die Verarbeitung eines Objektes bzw. Handlungserleidenden lokal keine Unterschiede hinsichtlich einer Belebtheitsvariation zeigen. Tatsächlich gibt es keine messbaren Kosten im EEG.

8 Diese Idee der wettbewerbsbasierten Konkurrenz findet sich in der psycholinguistischen Literatur an mehreren Stellen und wird i.d.R. unter interferenzbasierten Ansätzen zusammengefasst (z.B. Vosse/Kempen 2000; Gordon et al. 2001; Lewis/Vasishth 2005; van Dyke/ McElree 2006).
} 
The interface hypothesis of incremental argument interpretation:

Incremental argument interpretation (i.e. role identification and assessment of role prototypicality) is accomplished by the syntax-semantics interface, that is, with reference to a cross-linguistically defined set of prominence scales and their languagespecific weighting.

The relevant prominence scales are:

a. morphological case marking (nominative $>$ accusative / ergative $>$ nominative)

b. argument order (argument $1>$ argument 2$)^{9}$

c. animacy (+animate $>-$ animate)

d. definiteness/specificity ( + definite/specific $>-$ definite/specific)

e. $\operatorname{person}(1 \mathrm{st} / 2 \mathrm{nd}$ person $>3 \mathrm{rd}$ person)

(Bornkessel-Schlesewsky/Schlesewsky 2009b, S. 28)

Dem sich in der typologischen Literatur heimisch fühlenden Leser wird natürlich sofort auffallen, dass das Konzept der Prominenzskalen hier nicht neu eingeführt wurde, sondern, dass es zu diesem Thema bereits zahlreiche Arbeiten gibt (z.B. Silverstein 1976; Comrie 1989; Aissen 2003; Croft 2003 für eine Auswahl). Darüber hinaus gibt es mit DeLancey (1981) bereits aus der Typologie hervorgehende Annahmen, dass Informationsverarbeitung dann einfach ist, wenn die Argumente in einer unmarkierten transitiven Relation zueinander stehen, d.h. auf möglichst vielen dieser oben genannten Skalen eindeutig distinkt sind. Spannend ist nun, dass es immer mehr Daten aus dem Bereich der Psycho- und Neurolinguistik gibt, die darauf hin deuten, dass den Prominenzskalen bei der Argumentinterpretation nicht nur eine erhebliche Bedeutung zukommt, sondern dass diese auch das Potenzial zu besitzen scheinen, die Architektur des menschlichen Sprachverstehenssystems bzw. die der menschlichen Sprache überhaupt in bedeutendem Maße zu formen. Doch davon mehr im folgenden Abschnitt.

In der Literatur zur Abfolge der Argumente im deutschen Mittelfeld haben unter anderem Lenerz (1977), Uszkoreit (1986) und Müller (1999) darauf hingewiesen, dass neben der grammatischen Funktion auch Belebtheit, Pronominalität etc. sequenzrelevant sind. Mit dem vorliegenden Modell wollen wir diese Ansätze erweitern. Die genannten Merkmale sind nicht nur relevant für die Abfolge, sondern für die Verarbeitung sprachlicher Informationen generell. Neurolinguistisch lässt sich diese Position wunderbar illustrieren. Die Verletzung der z.B. durch Lenerz (1977) formulierten Abfolgeregeln führt zu Aktivierungsveränderungen im Pars opercularis des linken Gyrus frontalis inferior, der sich im linken Frontallappen befindet und der Teil des bekannten Broca-Areals ist. So zeigt nicht nur die Verletzung der Regel Nom > Akk (,, " bedeutet steht linear vor) Aktivierungsmodulationen (Friederici et al. 2006; Röder et al. 2002), sondern auch Abfolgeprinzipien, wie Pronomen > nichtpronominale NPs (Grewe et al. 2005), höhere thematische Rolle > niedrigere thematische Rolle (Bornkessel et al. 2005), belebt > unbelebt (Grewe et al. 2006), definit/spezifisch > indefinit/unspezifisch (Bornkessel et al. 2009) korrelieren mit Aktivitätsveränderungen in dieser Region am $\mathrm{Fuß}$ der dritten Stirnwindung. Informationstrukturelle Variationen führen hingegen zu Aktivierungen im pars triangularis (Bornkessel-Schlesewsky et al. 2010) und generelle Aspekte der Einfachheit der Informationsverarbeitung im bereits oben genannten Sulcus temporalis superior (Grewe et al 2007). Abbildung 6 illustriert die hier genannten neuroanatomischen Regionen. 


\section{Argumentverarbeitung aus einer sprachübergreifenden Perspektive}

Basierend auf den Erfolgen der generativen Grammatik im 20. Jahrhundert, ist es nicht verwunderlich, dass auch die in dieser Zeit entstehende Psycho- und Neurolinguistik sich an diesem theoretischen Ansatz orientierte und selbst Vertreter der Psycholinguistik, die die Grundannahmen der „Kompetenz-Performanz-Distinktion“ (Chomsky/Miller 1963), „Modularität" (Fodor 1983) oder „Serialität“ (Frazier/Fodor 1978) ablehnten, wesentliche Konsequenzen der Theorie - wenn auch möglicherweise unbewusst - mittrugen. So findet sich beispielsweise das Konzept der syntaktischen Strukturierung im Sinne der Annahmen Chomskys auch bei Vertretern interaktiver Ansätze (MacDonald et al. 1994). Wesentlich bedeutsamer für die Frage nach der Natur der Argumentinterpretation und ihren zugrunde liegenden Mechanismen war und ist die der Sprachwissenschaft der zweiten Hälfte des 20. Jahrhunderts innewohnende Überzeugung, dass man aus Beobachtungen zum Verarbeitungsverhalten im Englischen auf universelle Eigenschaften der Sprachverstehensarchitektur schließen könnte. Das hat zwei Konsequenzen: Einerseits bestimmte die frühe Verfügbarkeit des Verbs die Theoriebildung im Kontext der Argument-Verb-Relationen. Hier - wie zu Beginn des Aufsatzes bereits kurz erwähnt - gingen einzelne Arbeiten so weit anzunehmen, dass in verbfinalen Sprachen die Argumente bis zum Auftreten des Verbs entweder nur unverarbeitet gespeichert werden (Pritchett 1988, 1992) oder eine Art Vorverarbeitung stattfindet, bei der wesentliche Eigenschaften unterspezifiziert bleiben (Townsend/Bever 2001). Andererseits führte die Dominanz der Wortstellung für die inkrementelle Interpretation der Argumente zu einer Überbetonung dieser Eigenschaft als primärem Verarbeitungskonzept. Wortstellung, d.h. die lineare Position der Argumente zueinander, ist für das Englische mit Sicherheit bedeutsam (MacWhinney/Bates/Kliegl 1984), ${ }^{10}$ da es in der Regel die unmittelbare Identifizierung der grammatischen Funktion gestattet. Aus einer sprachübergreifenden Perspektive stellt die Dominanz der Wortstellung eine Ausnahme dar. Hier kommen Faktoren wie Belebtheit (Fore: Scott 1978), thematische Rolle (Belhare: Bickel 2006) oder Topikalität (Chinesisch: Li/Thompson 1981; Bisang 2006) eine deutlich bestimmendere Rolle bei der Argumentinterpretation zu.

Welche Anforderungen bestehen aber an eine Sprachverstehensarchitektur, wenn diese über die Einzelsprache hinaus Generalisierungen zur inkrementellen Argumentinterpretation erlauben soll? Verbfinalität und freie

$10 \quad$ Englisch ist gegenwärtig die einzige Sprache, die auf einem initialen unbelebten Argument einen lokalen Verarbeitungsnachteil im EEG in Form einer N400 zeigt (Weckerly/Kutas 1999). Hier ist tatsächlich die strukturelle Position, in dem ein Argument steht, bestimmend für dessen Interpretation und Handlungsprototypikalität. 
Wortstellung sind sicher wichtige Faktoren, die hier bereits genannt und besprochen wurden. Hinzu kommen aber noch Faktoren, die bisher keine Beachtung gefunden haben. Viele Sprachen der Welt erlauben die Auslassung von Argumenten (pro-drop Sprachen) und machen von dieser Möglichkeit auch regen Gebrauch (z.B. wird im Japanischen oder Türkischen zu 70\% das Subjekt in Sätzen mit transitiven Verben weggelassen). Andere Sprachen (z.B. Chinesisch) haben die Tendenz, als erstes Argument ein Topik zu präsentieren und dabei der grammatischen Funktion eher nachgeordnete Bedeutung zuzuordnen. Und was machen wir in Sprachen mit einem Ergativ-Absolutiv-Kasussystem, wenn wir ein Argument im Absolutiv verarbeiten?

Hier erweisen sich nun die in Abschnitt 3 anhand deutscher Beispiele eingeführten Interpretationsstrategien als ausgesprochen hilfreich. In den bisher untersuchten Sprachen scheint es demnach unabhängig von der Sprachfamilie so zu sein, dass das erste Argument, falls es keine Gegenevidenz gibt (z.B. Verbinformation oder Kasus), als einziges Argument einer intransitiven Relation interpretiert wird (Japanisch: Wolff et al. 2008; Türkisch: Demiral et al. 2008; Chinesisch: Wang et al. 2009; Hindi: Choudhary 2010). Diese Intransitivitätsannahme wird weder in pro-drop Sprachen (z.B. Türkisch: Demiral et al. 2008) noch in Topik-prominenten Sprachen (Chinesisch: Wang et al. 2009) durch Merkmale moduliert, die den Handlungsverursacher (Actor) in seinen prototypischen Eigenschaften absenken (z.B. Belebtheit). Eine notwendige Erweiterung zu einer transitiven Relation ist ebenso wie im Deutschen kostenträchtig (Japanisch: Wolff et al. 2008). Sollte während dieser Erweiterung deutlich werden, dass das erste Argument nicht Subjekt, d.h. das kongruierende Argument, oder Actor, der thematisch höchste Handlungsteilnehmer, ist, dann treten erhöhte Aktivierungen im neurophysiologischen Signal auf, die auf eine notwendig gewordene Reanalyse hindeuten (siehe Abbildung 4).

Der Versuch, einen Handlungsverursacher auch in Abwesenheit eines Verbs so schnell wie möglich zu identifizieren, scheint dabei die treibende Kraft zu sein. Insofern ist es auch nicht erstaunlich, dass - falls er durch ein anderes Element in seiner Existenz vorhergesagt wird - eine Verletzung von prototypischen Eigenschaften stets mit messbaren Aktivierungsveränderungen einhergeht.

Auch hier haben Wortstellung, Weglassbarkeit von Argumenten und Kasussystem keinen modulierenden Einfluss, wie man an den Beispielen in Abbildung 5 sehen kann.

Dass es dabei aber nicht nur um eine reine Bewertung der Güte eines Handlungsverursachers geht, sondern der Wettbewerb um diese Rolle das Verarbeitungsverhalten bestimmt, zeigen zwei weitere Beobachtungen aus dem Chinesischen (Philipp et al. 2008) und dem Tamil (Muralikrishnan et al. 2008). Während im Chinesischen und im Tamil, wie man in Abbildung 5 
Deutsch

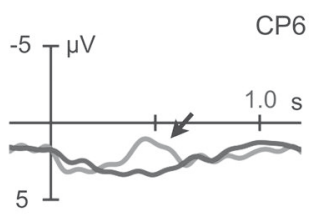

Chinesisch

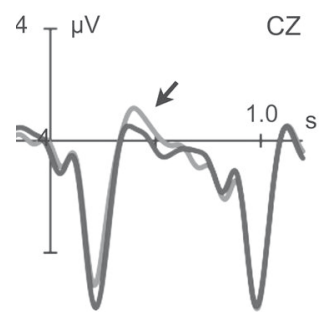

Deutsch

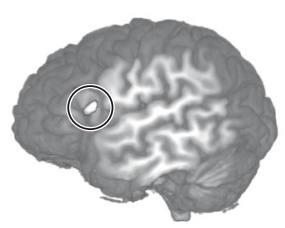

Hindi

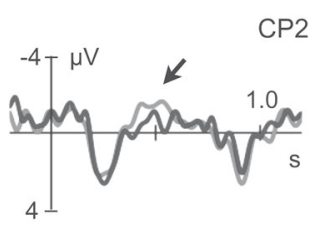

Türkisch

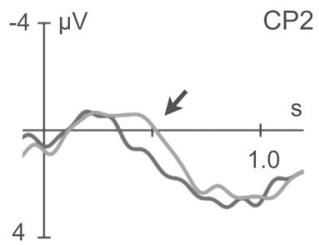

Japanisch

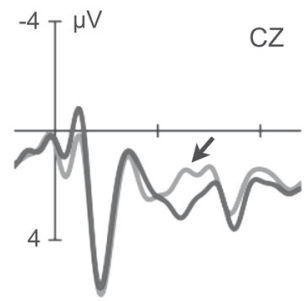

Abb. 4: Korrektur einer initialen Präferenz für ein handlungsverursachendes Actor-Argument (Deutsch: Bornkessel et al. 2005; Haupt et al. 2008; Türkisch: Bornkessel-Schlesewsky et al. (einger.), siehe ebenfalls Demiral et al. 2008; Chinesisch: Wang et al. 2009; Hindi: Choudhary et al. 2010; Japanisch: Wolff et al. 2007). Die EEGs stellen die Aktivitätsveränderungen am disambiguierenden Verb dar (Elektrodenpositionen sind in Abbildung 6 illustriert). Helle Linie: nicht-Actor-erst-Struktur; Dunkle Linie: Actor-erst-Struktur. Man beachte, dass die Kurven niemals absolut, d.h. im Koordinatensystem interpretiert werden, sondern nur relativ zueinander (siehe Fußnote 4). Die neuroanatomische Darstellung der korrespondierenden Aktivierungsmodulation im Deutschen zeigt den Pars opercularis (siehe Abbildung 6).

sehen kann, eine Negativierung im N400-Zeitfenster zu beobachten ist, wenn der Actor die Erwartungen an seine Eigenschaften (hier Belebtheit) nicht erfüllt, verändert sich in beiden Sprachen das neurophysiologische Aktivierungsmuster im Kontext von zwei unbelebten Handlungsteilnehmern qualitativ. Im Chinesischen zeigt sich nun nicht mehr eine N400, sondern eine Positivierung und im Tamil verändert sich die Latenz der Komponente, d.h. sie tritt nun signifikant früher auf. Wäre es eine reine Bewertung der argumentinhärenten Eigenschaften, so würde man nur eine Veränderung der Stärke der mit dieser verbundenen Komponente rechnen (Veränderung der Größe der N400). Das Auftreten eines anderen Musters, welches - und das muss man sich noch einmal kurz verdeutlichen - nur entstehen kann, wenn andere neuronale Netze miteinander interagieren, deutet darauf hin, dass der Wettbewerb von zwei unbelebten Argumenten 

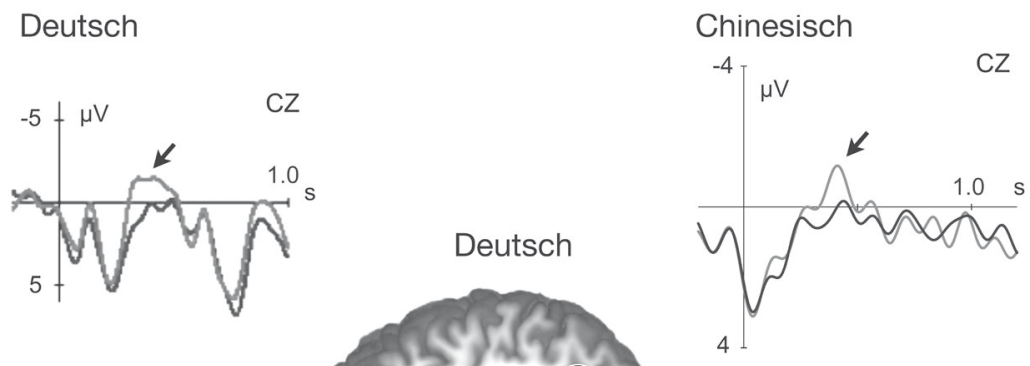

Hindi

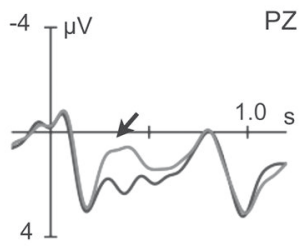

Chinesisch

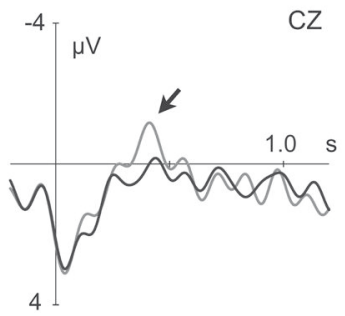

Tamil

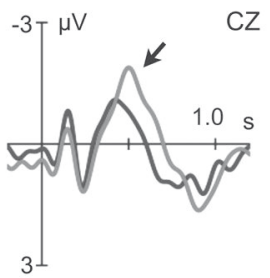

Abb. 5: Korrektur einer Erwartung für einen prototypischen Actor (Deutsch: Roehm et al. 2004, siehe ebenfalls Frisch/Schlesewsky 2001; Chinesisch: Philipp et al. 2008; Hindi: Schlesewsky et al. (ersch.); Tamil: Muralikrishnan et al. 2008). Alle Strukturen folgen dem gleichen Muster: Eine initiale NP signalisiert eine später folgende NP, die Handlungsverursacher ist. Ist die zweite NP unbelebt, erfolgt eine messbare Korrektur der Erwartung. Die Elektrodenpositionen sind in Abbildung 6 illustriert. Helle Linie: unbelebter Handlungsverursacher; Dunkle Linie: belebter Handlungsverursacher. Man beachte, dass die Kurven niemals absolut, d.h. im Koordinatensystem interpretiert werden, sondern nur relativ zueinander (siehe Fußnote 4). Die neuroanatomische Darstellung der korrespondierenden Aktivierungmodulation im Deutschen zeigt den Sulcus temporalis superior (siehe Abbildung 6).

eine völlig andere Qualität besitzt als die von zwei belebten Handlungsteilnehmern. Während im zweiten Fall der Actor prototypische Eigenschaften besitzt, kann dies im ersten Fall durch die Unbelebtheit beider Argumente zu keinem Zeitpunkt der Fall sein. Das führt - so deuten die Muster an zu alternativen Wettbewerbsstrategien. Wie sich Letzteres genau modellieren lässt, ist momentan noch nicht klar und bedarf weiterer Untersuchungen. Bringt man alle Beobachtungen zusammen, so ist das zentrale Konzept hinter allen bisherigen Daten offenkundig der Versuch, einem Argument sofort eine Actor-Rolle zuzuweisen und - sollte mehr als ein Argument vorhanden sein - diese hinsichtlich der Zuweisung dieser Rolle klar voneinander zu trennen. Alle Abweichungen führen zu erhöhter Verarbeitungskomplexität (z.B. durch Interferenz) und damit zu höheren Kosten. 
Doch hat die klare Identifizierung eines Actors und die Vermeidung von Interferenzen zwischen Argumenten nicht nur Vorteile hinsichtlich der Einfachheit der Informationsverarbeitung aus der Perspektive der Argument-Argument-Relation und der Integration dieser in den Verbrahmen. Wie Daten aus dem Türkischen zeigen (Demiral et al. (unter Begutachtung); Bornkessel-Schlesewsky/Schlesewsky 2008a) resultiert aus den Merkmalen und Eigenschaften des Arguments, d.h. den overten Kasusmerkmalen und prototypischen Actoreigenschaften, und der Position eines Argumentes in der linearen Sequenz eine Vorhersage für einen bestimmten Verbtyp. Die Abfolge Nominativ vor Akkusativ (und damit Actor vor Undergoer) erzeugt im Türkischen eine Erwartung für ein satzfinales Aktivverb. Erscheint an dessen Stelle ein Experiencerverb, so zeigen sich wieder einmal erhöhte Aktivierungen in Form einer N400, die wiederum als Kosten einer Erwartungsverletzung an der Syntax-Semantik-Schnittstelle interpretiert werden können. Invertiert man die Abfolge der Handlungsteilnehmer, so verändert sich auch die Erwartung des Verbtyps und man beobachtet nun eine N400 für das Aktivverb. Die Möglichkeit, aus der präverbalen Argumentrelation generalisierte Verbtypenschemata vorherzusagen, hat den klaren Vorteil einer schnelleren Integration der Information und damit einer effizienteren Kommunikation in Echtzeit.

\section{Argumentidentifizierung}

In den bisherigen Ausführungen haben wir stets vorausgesetzt, dass das menschliche Sprachverstehenssystem bei der Verarbeitung von Informationen in Echtzeit unmittelbar erkennt, wann ein lautliches Segment als Argument verarbeitet werden kann. Wir haben dabei implizit vorausgesetzt, dass ein Argument auch ohne Probleme als solches erkannt wird. Diese Annahme kann man machen und sie scheint empirisch, folgt man Clifton/ Spear/Abney (1991), Friederici (2002) und Bornkessel/Schlesewsky (2006a), auch richtig. Sie ist aber keineswegs trivial. Zwei Präliminarien sind dazu nämlich unabdingbar. Um etwas als ein Argument zu interpretieren, muss man diesem zuerst die potenzielle Fähigkeit für ein solches zuschreiben, d.h. es muss inhärente Merkmale besitzen, die nominal interpretierbar sind, und man muss es darüber hinaus auch noch als ein solches interpretieren können, d.h. es muss in einer potenziell nominal verträglichen Umgebung stehen. Der Einfachheit halber beschränken wir unsere folgenden Betrachtungen auf nominale Argumente und lassen Präpositionalphrasen, für die natürlich Gleiches gilt, hier unbeachtet. Erkennen wir innerhalb eines Textes bzw. eines Lautstroms eine segmentierbare und damit wahrscheinlich wortfähige Einheit, dann muss man diese in einem ersten Schritt hinsichtlich ihrer Wortkategorie klassifizieren. Das scheint notwendig und unabding- 
bar, da z.B. sowohl Nomen als auch Verben das Merkmal [+belebt] besitzen können, dieses aber in beiden Fällen unterschiedliche Konsequenzen besitzt. ${ }^{11}$ Entscheidend für die Zuweisung eines Wortkategoriemerkmals scheint, wie die experimentellen Daten suggerieren, die Erwartung zu sein, die aus den bisher verarbeiteten Informationen resultiert, bzw. wenn keine Erwartung vorhanden ist, die Position, in der das vermeintliche Wort auftritt. Dabei scheint Letzteres, wie wir zum Englischen argumentiert haben, nur eine Ausnahme zu sein, da wir ja nur in Sprachen mit freier Wortstellung in der ersten Position auf diese allein vertrauen müssen. Ansonsten weist uns die vorherige sprachliche Information den Weg. Das wohl bekannteste Experiment zu der Frage der Zuweisung von Wortkategorieinformation stammt aus der Arbeitsgruppe von Angela Friederici und beinhaltet zwei Arten von unerwarteten Weiterführungen einer Sequenz (Hahne/ Friederici 1999). In ungrammatischen Sätzen wie Das Hubn wurde im gehalten beobachteten die Autoren eine bei ca. 120 Millisekunden beginnende Negativierung, die damit nicht nur früher als die hier bisher berichtete N400 auftrat, sondern auch eine andere Verteilung auf der Kopfoberfläche zeigte. Diese Komponente (Early Left Anterior Negativity - ELAN ${ }^{12}$ ) steht nun in einem direkten Zusammenhang mit der Wortkategorieverarbeitung. Man

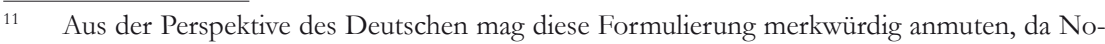
men und Verben ja i.d.R. eindeutig voneinander dissoziierbar sind (und das nicht nur allein durch die orthografische Konvention). Aus einer typologischen Sicht ist es aber keineswegs trivial, da Transkategorialität nicht nur allein Gegenstand deskriptiver Beschreibungen ist, wie man sie aus dem Chinesischen kennt (Bisang 2008), sondern generell ein Problem der linguistischen Theoriebildung darstellt. Hale/Marantz (1993) und andere Autoren haben im Kontext der Distributed Morphology (z.B. Harley/Noyer 1999) dieses Problem der Transkategorialität versucht zu modellieren. Danach besitzt ein lexikalischer Eintrag nur interpretierbare/formale Merkmale aber keine Wortkategorie. Letztere bekommt das Element nur im Kontext der Satzumgebung. So ist das Merkmal [+belebt] einem Item inhärent, wird aber durch die Umgebung entweder nominal charakterisierend oder subkategorisierend verstanden. Auch aus einer Verarbeitungsperspektive ist diese Idee attraktiv, da sie die initiale Verarbeitungsphase in Modellen wie Friederici (2002) oder Bornkessel/Schlesewsky (2006a) motiviert.

12 Die ELAN, eine früh auftretende Komponente mit topografischem Fokus auf der linken vorderen Schädeloberfläche, ist eigentlich eine Glaubenskomponente, d.h. die empirische Validierbarkeit ist so beschränkt, dass sie nur in derart ausgesuchten Kontexten erscheint, dass sie sich eigentlich jeder systematischen Untersuchung entzieht. Erstmals veröffentlicht wurde sie in einem Aufsatz von Neville et al. (1991). Die Studie berichtete eine frühe Negativierung zwischen 100 und 200 ms nach Stimulusonset, wenn die lokale Erwartung für eine Wortkategorie verletzt wird (*What did the scientist criticize Max's proof of?). In mehreren Studien zum Deutschen wurde diese Komponente in ähnlicher experimenteller Umgebung repliziert (Hahne/Friederici 1999). Kritik gab es aber stets aus zwei Richtungen. Während die eine argumentierte, dass der Satz potenziell weitergeführt werden kann (Die Gans wurde im gefüttert vorgefübrtem Zustand ausgestellt), kritisierte die andere, dass die Umgebung des Vergleichs, d.h. der prästimulus Bereich, keine seriöse physiologisch-empirische Auswertung erlaubt. Trotz aller Probleme zeigt die sprachübergreifende Transkategorialität (siehe Fußnote 11), dass es - wenn auch nicht diese Komponente - so doch einen frühen Schritt der Wortkategorisierung innerhalb des Sprachverstehens geben muss. 
kann argumentieren, dass die Komponente die Erwartungsverletzung widerspiegelt, die dann eintritt, wenn nach dem Präpositions-DeterminerKomplex das erwartete Nomen (z.B. Stall) nicht auftritt, sondern ein Wort verarbeitet werden muss, welches verbale Eigenschaften (Partizip) besitzt. Doch während die absolute Zeit der Komponenten zueinander nur einen ersten Hinweis auf einen frühen Prozess der Wortkategorieidentifizierung gibt, zeigte eine weitere experimentelle Manipulation die wahre Bedeutung des dort ablaufenden Prozesses. Friederici und Kollegen (Hahne/Friederici 2002) untersuchten darüber hinaus ungrammatische Sätze, die nicht nur eine Wortkategorieverletzung enthielten, sondern bei denen das Partizip, wie in Das Hubn wurde im gebügelt, auch noch einen Verstoß gegen unser Weltwissen enthielt, denn wir besitzen kein Alltagsszenario, in dem gebügelte Hühner vorkommen. Betrachtet man beide Verletzungen separat, dann zeigen sich - wie oben bereits erwähnt - zeitlich und räumlich (topographisch) unterschiedliche Effekte. Aus der Annahme, dass Sprachverstehensprozesse interagieren und/oder zeitgleich alle Informationsarten verarbeiten, leitete sich die Hypothese ab, dass in der gekoppelten Verletzungsbedingung beide Komponenten zu beobachten sein sollten. Doch das war genau nicht der Fall: Friederici und Kollegen beobachteten bei diesen Sätzen exakt das gleiche neurophysiologische Aktivierungsmuster wie bei einer einfachen Wortkategorieverletzung. Daraus kann man nicht nur schließen, dass der Prozess der Wortkategorie anderen Sprachverstehensprozessen vorangeht, sondern dass er auch in der Lage zu sein scheint, spätere Prozesse zu blockieren, d.h. nach Erkennung der Verletzung die Verarbeitung abzubrechen. Diese Eigenschaft der Blockierung und der Einzigartigkeit dieser im Kontext der Wortkategorieerkennung wurde für das Niederländische durch van den Brink und Hagoort (2004) bestätigt (für eine ausführliche Diskussion siehe Bornkessel-Schlesewsky/Schlesewsky 2009b).

Hat man also einen nominalen Ausdruck als einen solchen identifiziert, so gibt es nun wiederum zwei Möglichkeiten zur Interpretation. Auch dazu wieder ein Beispiel. Bei der inkrementellen Verarbeitung des sich aufbauenden Satzes Vielleicht hatte der Vater der Sängerin ... muss man im Moment des Erscheinens der ambigen Nominalphrase „entscheiden“, ob man diese als Dativ und damit als Argument eines möglicherweise folgenden Verbs interpretiert, oder ob man sie als Genitiv interpretiert und damit zum Adjunkt und Modifizierer des vorangehenden Nomens macht. Für den ersten Fall würde man erwarten, dass mit dem Auftreten eines intransitiven Verbs (geschlafen) eine neurophysiologische Aktivierungsveränderung zu beobachten sein sollte. Wäre die Entscheidung hingegen für den postnominalen Genitiv gefallen, dann sollte ein transitives Verb (folgen) aktivierungsverändernd sein. Bereits in früheren psycholinguistischen Studien (Abney 1989; Clifton/Spear/Abney 1991; Boland/Boehm-Jernigan 1998; Schütze/Gibson 1999) beobachtet man hier ein relativ klares Muster (siehe Augurzky/Schle- 
sewsky 2011). Auch wenn die durch Vater induzierte mögliche Verwandtschaftsrelation der Dativlesart entgegen wirkt, gibt es trotzdem eine klare Tendenz, die ambige NP als Argument und nicht als Adjunkt zu interpretieren. Gleiches gilt übrigens auch für Präpositionalphrasen, die, wie Juranek (2005) mit Hilfe von EEG-Messungen zeigen konnte, ebenfalls einer Argument-über-Adjunkt-Präferenz folgen.

Zusammenfassend kann man also aus einer Sprachverstehensperspektive argumentieren, dass bedeutungstragende Einheiten, die beispielsweise aus einem Lautstrom segmentiert und als nicht-verbfähige Information eingeordnet werden, mit hoher Wahrscheinlichkeit als Argument interpretiert werden. Diese Vorgehensweise hat dabei sowohl den Vorteil, dass Ereignisse im verbnahen engeren Sinne (d.h. wie zum Beispiel über lexikalische Dekomposition beschreibbar) schnell und effizient aufgebaut werden können, als auch die Konsequenz des Aufbaus maximal einfacher Strukturen. ${ }^{13}$ Auf diesen ersten Schritten bauen dann die in den Abschnitten 2-4 geschilderten relationalen und dependenzbildenden Prozesse auf.

\section{Ausblick}

Auch wenn die hier präsentierte Datenlage relativ stabil und klar erscheint und die vorgeschlagenen Annahmen stützt, bleiben natürlich doch noch einige offene Fragen. Die wohl einfachste betrifft die weitere Validierung mit Hilfe von bisher nicht untersuchten Sprachen. Dazu gehört mit Sicherheit die Hinzunahme weiterer Ergativsprachen (z.B. Baskisch) aber auch die Hinwendung zu verbinitialen Sprachen (z.B. Irisch). Auch gilt es natürlich zu untersuchen, wie die Interaktion der Prominenzskalen entsteht und wie bzw. ob überhaupt die in Experimenten zu beobachtende Hierarchisierung zwischen diesen Skalen Widerspiegelung in absoluten Häufigkeiten und Übergangswahrscheinlichkeiten findet. Der aber vielleicht wichtigste Schritt für ein umfassenderes Verständnis der dynamischen Interpretation liegt in der Untersuchung von Argumentrelationen, bei denen eine oder mehrere

13 Unter der hier vertretenen Annahme der maximalen Einfachheit (Bornkessel/Schlesewsky 2006; Bornkessel-Schlesewsky/Schlesewsky 2009c) werden andere Wortkategorien nur dann angenommen, wenn explizite Information für diese vorliegt. In Abwesenheit dieser werden alle segmentierbaren Einheiten entweder als Verb oder als nominales Argument präferiert interpretiert. Das Prinzip der Einfachheit („,Minimality principle: In the absence of explicit information to the contrary, the human language comprehension system assigns minimal structures. This entails that only required dependencies and relations are created“, Bornkessel/Schlesewsky 2006, S. 790), welches in seiner Philosophie dem „minimal everything principle“ (Fodor 1998; Inoue/Fodor 1995) ähnelt, appliziert auf allen Ebenen des Sprachverstehens. Im Kontext unseres eigenen Modells (Bornkessel 2002; Schlesewsky/Bornkessel 2004; Bornkessel/Schlesewsky 2006a; Bornkessel-Schlesewsky/Schlesewsky 2008b) fokussieren wir insbesondere auf die syntaktische, semantische Dimension und auf das Interface zwischen beiden. 
Argumente Quantoren enthalten. Hier besteht derzeit sowohl Forschungsals auch Modellierungsbedarf. Interessant ist der Punkt deshalb, da beispielsweise bei doppelt quantifizierenden Strukturen zwar theoretisch mehrere Lesarten bestehen, in der Echtzeitkommunikation diese Ambiguitäten aber nicht vorzukommen scheinen. Darüber hinaus sind, wenn man diese Strukturen in Reaktionszeitexperimenten (z.B. Bott/Schlotterbeck 2010) untersucht, die Reaktionszeiten mit einem Mittel von über 2 Sekunden in einem Bereich, der jenseits dessen ist, was man von Studien in anderen Domänen der Argumentverarbeitung kennt. Hier werden i.d.R. 1000 ms deutlich unterschritten. Diese Beobachtung deutet darauf hin, dass derartige Strukturen eher mit Hilfe allgemeinkognitiver Problemlösungsstrategien gelöst werden, als mit denjenigen Strategien, die wir in der Echtzeitkommunikation finden. Da es sich bei diesen aber nichtsdestoweniger um sprachbasierte Phänomene handelt, die die Relation der Argumente zum Teil mitbestimmen, verlangen diese Eigenschaften daher auch eine Integration in den in diesem Aufsatz vorgestellten Rahmen.

Offen bleibt natürlich, in welcher Relation die hier vorgestellten Daten zu existierenden Grammatiktheorien stehen, die ja alle keine zeitliche Dimension besitzen. Ob diese, wenn man Daten der Art, wie sie hier vorgestellt wurden, wirklich ernst nimmt, mit dieser Dimension umgehen können, oder ob es einer völlig neuen Art der grammatischen Theoriebildung bedarf, wird die Zukunft zeigen.

\section{Anhang}
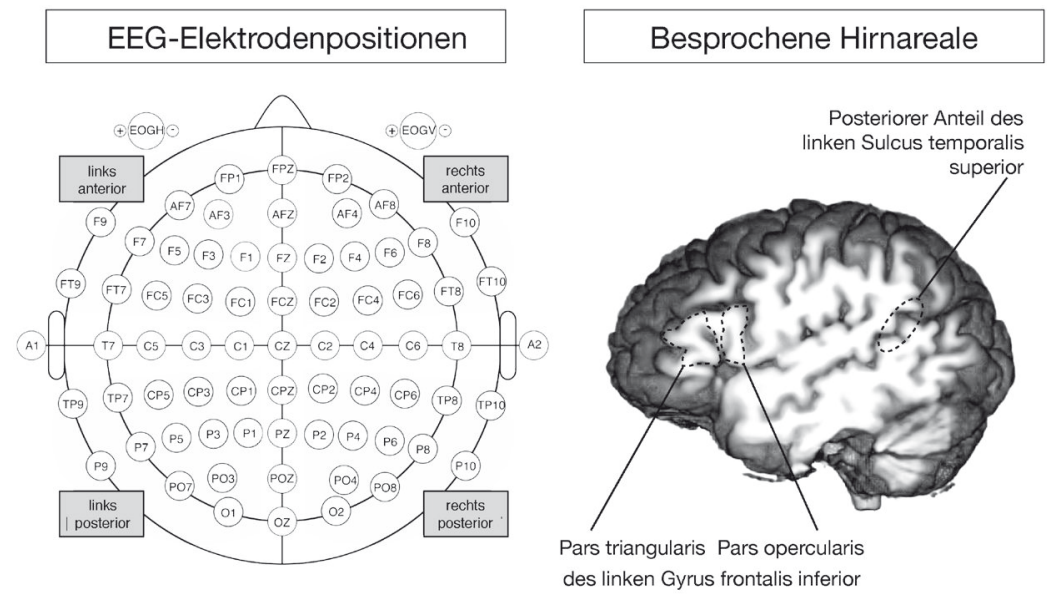

Abb. 6 
Abbildung 6 zeigt auf der linken Seite die Elektrodenpositionen, die standardmäßig in EEG-Experimenten verwendet werden (adaptiert aus Bornkessel-Schlesewsky/Schlesewsky 2009a). Sie geben auch die in den anderen Abbildungen genannten Elektrodenpositionen wieder. Auf der rechten Seite werden die ungefähren neuroanatomischen Positionen genannt und bezeichnet, die im vorliegenden Aufsatz erwähnt werden.

\section{Literatur}

Abney, Steven (1989): A computational model of human parsing. In: Journal of Psycholinguistic Research 18, S. 129-144.

Aissen, Judith (2003): Differential object marking: iconicity vs. economy. In: Natural Language and Linguistic Theory 21, S. 435-483.

Augurzky, Petra/Schlesewsky, Matthias (2011): Prosodic phrasing and transitivity in head-final sentence comprehension: ERP evidence from German ambiguous DPs. In: Yamashita, Hiroko et al. (Hg.): Processing and producing head-final structures. (= Studies in Theoretical Psycholinguistics 38). Berlin, S. 69-91.

Berger, Hans (1929): Über das Elrektrenkephalogramm des Menschen. In: Archiv für Psychiatrie und Nervenkrankheiten 87, S. 527-570.

Bergmann, Christopher (2010): Konstruktionen oder Strukturen? Zum Einfluss der Wortfrequenz auf die Sprachverarbeitung von Subjekt/Objekt-Ambiguitäten im Deutschen. Unveröff. Magisterarbeit. Mainz.

Bickel, Balthasar (2006): Clause-level vs. predicate-level linking. In: Bornkessel et al. (Hg.), S. 155-190.

Bisang, Walter (2006): From meaning to syntax - semantic roles and beyond. In: Bornkessel et al. (Hg.), S. 191-236.

Bisang, Walter (2008): Precategoriality and syntax-based parts of speech: the case of Late Archaic Chinese. In: Studies in Language 32, S. 568-589.

Boland, Julie/Boehm-Jernigan, Heather (1998): Lexical constraints and prepositional phrase attachment. In: Journal of Memory and Language 39, S. 684-719.

Bornkessel, Ina (2002): The Argument Dependency Model: a neurocognitive approach to incremental interpretation. (= MPI Series in Cognitive Neuroscience 28). Leipzig.

Bornkessel, Ina/Schlesewsky, Matthias (2006a): The Extended Argument Dependency Model: a neurocognitive approach to sentence comprehension across languages. In: Psychological Review 113, S. 787-821.

Bornkessel, Ina/Schlesewsky, Matthias (2006b): Generalised semantic roles and syntactic templates: a new framework for language comprehension. In: Bornkessel et al. (Hg.), S. 327-353.

Bornkessel, Ina/Fiebach, Christian J./Friederici, Angela D. (2004): On the cost of syntactic ambiguity in human language comprehension: an individual differences approach. In: Cognitive Brain Research 21, S. 11-21. 
Bornkessel, Ina/Schlesewsky, Matthias/Friederici, Angela D. (2003): Eliciting thematic reanalysis effects: the role of syntax-independent information during parsing. In: Language and Cognitive Processes 18, S. 268-298.

Bornkessel, Ina et al. (2004): Multi-dimensional contributions to garden path strength: dissociating phrase structure from case marking. In: Journal of Memory and Language 51, S. 495-522.

Bornkessel, Ina et al. (2005): Who did what to whom? The neural basis of argument hierarchies during language comprehension. In: NeuroImage 26, S. 221-233.

Bornkessel, Ina et al. (Hg.) (2006): Semantic role universals and argument linking: theoretical, typological, and psycholinguistic perspectives. (= Trends in Linguistics, Studies and Monographs 165). Berlin/New York.

Bornkessel-Schlesewsky, Ina/Schlesewsky, Matthias (2008a): Unmarked transitivity: a processing constraint on linking. In: van Valin, Robert D. (Hg.): Investigations of the syntax-semantics-pragmatics interface. (= Studies in Language Companion Series 105). Amsterdam u.a., S. 413-434.

Bornkessel-Schlesewsky, Ina/Schlesewsky, Matthias (2008b): An alternative perspective on „semantic P600“ effects in language comprehension. In: Brain Research Reviews 59, S. 55-73.

Bornkessel-Schlesewsky, Ina/Schlesewsky, Matthias (2009a): Processing syntax and morphology: a neurocognitive perspective. (= Oxford Surveys in Syntax and Morphology 6). Oxford u.a.

Bornkessel-Schlesewsky, Ina/Schlesewsky, Matthias (2009b): The role of prominence information in the real time comprehension of transitive constructions: a crosslinguistic approach. In: Language and Linguistics Compass 3, S. 19-58.

Bornkessel-Schlesewsky, Ina/Schlesewsky, Matthias (2009c): Minimality as vacuous distinctness: evidence from cross-linguistic sentence comprehension. In: Lingua 119, S. 1541-1559.

Bornkessel-Schlesewsky, Ina/Grewe, Tanja/Schlesewsky, Matthias (2010): Prominence vs. aboutness in sequencing: a functional distinction within the left inferior frontal gyrus. In: Brain and Language. [doi: 10.1016/j.bandl.2010.06.004].

Bornkessel-Schlesewsky, Ina/Schlesewsky, Matthias/von Cramon, D. Yves (2009): Word order and Broca's region: evidence for a supra-syntactic perspective. In: Brain and Language 111, S. 125-139.

Bornkessel-Schlesewsky, Ina et al. (eingereicht): Think globally: cross-linguistic variation in electrophysiological activity during sentence comprehension. In: Brain and Language.

Bott, Oliver/Schlotterbeck, Fabian (2010): An investigation into the computational complexity of quantifying expressions. Paperpräsentation bei der 23rd Annual Meeting of the CUNY Conference on Human Sentence Processing, New York 18.-21. März 2010.

Chomsky, Noam/Miller, George A. (1963): Introduction to the formal analysis of natural languages. In: Luce, R. Duncan/Bush, Robert R./Galanter, Eugene (Hg.): Handbook of mathematical psychology. New York u.a., S. 269-321. 
Choudhary, Kamal K. (2010): Incremental argument interpretation in a split ergative language: neurophysiological evidence from Hindi. Diss. Leipzig.

Choudhary, Kamal K. et al. (2009): The N400 as a correlate of interpretively-relevant linguistic rules: evidence from Hindi. In: Neuropsychologia 47, S. 3012-3022.

Choudhary, Kamal K. et al. (2010): An actor-preference in a split-ergative language: electrophysiological evidence from Hindi. Posterpräsentation bei der 23rd Annual CUNY Conference on Human Sentence Processing, New York 18.-21. März 2010.

Clifton Jr., Charles/Speer, Shari/Abney, Steven P. (1991): Parsing arguments: phrase structure and argument structure as determinants of initial parsing decisions. In: Journal of Memory and Language 30, S. 251-271.

Comrie, Bernard (1989): Linguistic universals and language typology: syntax and morphology. 2. Aufl. Oxford.

Crocker, Matthew W. (1994): On the nature of the principle-based sentence processor. In: Clifton Jr., Charles/Frazier, Lyn/Rayner, Keith (Hg.): Perspectives on sentence processing. Hillsdale, S. 245-266.

Croft, William (2003): Typology and universals. 2. Aufl. Cambridge u.a.

DeLancey, Scott (1981): An interpretation of split ergativity and related patterns. In: Language 57, S. 626-657.

Demiral, Şükrü Barış/Schlesewsky, Matthias/Bornkessel-Schlesewsky, Ina (2008): On the universality of language comprehension strategies: evidence from Turkish. In: Cognition 106, S. 484-500.

Demiral, Şükrü Barış/Schlesewsky, Matthias/Bornkessel-Schlesewsky, Ina (unter Begutachtung): Anticipating clause-final verbs: the interaction of prominence hierarchies in Turkish sentence comprehension. [Zur Veröffentlichung eingereichtes Manuskript].

de Swart, Peter (2007): Cross-linguistic variation in object marking. Diss. Utrecht.

Dowty, David (1991): Thematic proto-roles and argument selection. In: Language 67, S. 547-619.

Fanselow, Gisbert (2000): Optimal exceptions. In: Stiebels, Barbara/Wunderlich, Dieter (Hg.): Lexicon in Focus. (= Studia grammatica 45). Berlin, S. 173-209.

Farke, Hildegard (1994): Grammatik und Sprachverarbeitung. Zur Verarbeitung syntaktischer Ambiguitäten. Opladen.

Fodor, Janet D. (1998): Learning to parse. In: Journal of Psycholinguistic Research 27, S. 285-319.

Fodor, Jerry A. (1983): Modularity of mind. An essay on faculty psychology. Cambridge, MA.

Foley, William A./van Valin, Robert D. (1984): Functional syntax and universal grammar. (= Cambridge Studies in Linguistics 38). Cambridge u.a.

Frazier, Lyn/Fodor, Janet D. (1978): The sausage machine: a new two-stage parsing model. In: Cognition 6, S. 291-326. 
Frazier, Lyn/Flores d'Arcais, Giovanni B. (1989): Filler-driven parsing: a study of gap filling in Dutch. In: Journal of Memory and Language 28, S. 331-344.

Friederici, Angela D. (2002): Towards a neural basis of auditory sentence processing. In: Trends in Cognitive Sciences 6, S. 78-84.

Friederici, Angela D. et al. (2006): Processing linguistic complexity and grammaticality in the left frontal cortex. In: Cerebral Cortex 16, S. 1709-1717.

Frisch, Stefan/Schlesewsky, Matthias (2001): The N400 indicates problems of thematic hierarchizing. In: Neuroreport 12, S. 3391-3394.

Garnsey, Susan M. (1993): Event-related brain potentials in the study of language: an introduction. In: Language and Cognitive Processes 8, S. 337-356.

Gordon, Peter C./Hendrick, Randall/Johnson, Marcus (2001): Memory interference during language processing. In: Journal of Experimental Psychology: Learning, Memory and Cognition 27, S. 1411-1423.

Grewe, Tanja et al. (2005): The emergence of the unmarked: a new perspective on the language-specific function of Broca's area. In: Human Brain Mapping 26, S. 178190.

Grewe, Tanja et al. (2006): Linguistic prominence and Broca's area: the influence of animacy as a linearization principle. In: Neuroimage 32, S. 1395-1402.

Grewe, Tanja et al. (2007): The role of the posterior superior temporal sulcus in the processing of unmarked transitivity. In: Neuroimage 35, S. 343-352.

Grimshaw, Jane B. (1990): Argument structure. (= Linguistic Inquiry Monographs 18). Cambridge, MA u.a.

Hahne, Anja/Friederici, Angela D. (1999): Electrophysiological evidence for two steps in syntactic analysis: early automatic and late controlled processes. In: Journal of Cognitive Neuroscience 11, S. 194-205.

Hahne, Anja/Friederici, Angela D. (2002): Differential task effects on semantic and syntactic processes as revealed by ERPs. In: Cognitive Brain Research 13, S. 339-356.

Halle, Morris/Marantz, Alec (1993): Distributed Morphology and the pieces of inflection. In: Hale, Kenneth/Keyser, Samuel Jay (Hg.) The view from building 20. Essays in linguistics in honor of Sylvain Bromberger. (= Current Studies in Linguistics Series 24). Cambridge, MA, S. 111-176.

Harley, Heidi/Noyer, Rolf (1999): State-of-the-article: distributed morphology. In: Glot International 4, S. 3-9.

Haupt, Friederike S. et al. (2008): The status of subject-object reanalyses in the language comprehension architecture. In: Journal of Memory and Language 59, S. 54-96.

Hemforth, Barbara/Konieczny, Lars/Strube, Gerhard (1993): Incremental syntax processing and parsing strategies. In: Proceedings of the 15th Annual Conference of the Cognitive Science Society. Hillsdale, S. 539-545.

Hopper, Paul/Thompson, Sandra A. (1980): Transitivity in grammar and discourse. In: Language 56, S. 251-299. 
Inoue, Atsu/Fodor, Janet D. (1995): Information-paced parsing of Japanese. In: Mazuka, Reiko/Nagai, Noriko (Hg.): Japanese sentence processing. Hillsdale, S. 9-63.

Jackendoff, Ray (1972): Semantic interpretation in generative grammar. (= Studies in Linguistics Series 2). Cambridge, MA.

Janssen, Ulrike/Wiese, Richard/Schlesewsky, Matthias (2006): Electrophysiological responses to violations of morphosyntactic and prosodic features in derived German nouns. In: Journal of Neurolinguistics 19, S. 466-482.

Juranek, Katja (2005): Relationale Aspekte des Sprachverstehens: Die Verarbeitung von Argumenten und Adjunkten. Magisterarb. Leipzig.

Kolk, Herman H.J. et al. (2003): Structure and limited capacity in verbal working memory: a study with event-related potentials. In: Brain and Language 85, S. 1-36.

Konieczny, Lars et al. (1997): The role of lexical heads in parsing: evidence from German. In: Language and Cognitive Processes 12, S. 307-348.

Kuperberg, Gina R. et al. (2003): Electrophysiological distinctions in processing conceptual relationships within simple sentences. In: Cognitive Brain Research 17, S. $117-129$.

Kutas, Marta/Hillyard, Steven A. (1980): Reading senseless sentences: brain potentials reflect semantic incongruity. In: Science 207, S. 203-205.

Lenerz, Jürgen (1977): Zur Abfolge nominaler Satzglieder im Deutschen. (= Studien zur deutschen Grammatik 5). Tübingen.

Lewis, Richard L./Vasishth, Shravan (2005): An activation-based model of sentence processing as skilled memory retrieval. In: Cognitive Science 29, S. 1-45.

Li, Charles N./Thompson, Sandra A. (1981): Mandarin Chinese: a functional reference grammar. Berkeley u.a.

MacDonald, Maryellen C./Pearlmutter, Neal J./Seidenberg, Mark S. (1994): The lexical nature of syntactic ambiguity resolution. In: Psychological Review 101, S. 676703.

MacWhinney, Brian/Bates, Elizabeth/Kliegl, Reinhold (1984): Cue validity and sentence interpretation in English, German and Italian. In: Journal of Verbal Learning and Verbal Behavior 23, S. 127-150.

Marslen-Wilson, William (1973): Linguistic structure and speech shadowing at very short latencies. In: Nature 244, S. 522-533.

Müller, Gereon (1999): Optimality, markedness and word order in German. In: Linguistics 37, S. 777-818.

Muralikrishnan, R./Schlesewsky, Matthias/Bornkessel-Schlesewsky, Ina (2008): Universal and cross-linguistic influences on the processing of word order and animacy: neurophysiological evidence from Tamil. Posterpräsentation bei der 21st Annual CUNY Conference on Human Sentence Processing, Chapel Hill, NC.

Neville, Helen J. et al. (1991): Syntactically based sentence processing classes: evidence from event-related potentials. In: Journal of Cognitive Neuroscience 6, S. 233-255. 
Philipp, Markus et al. (2008): The role of animacy in the real time comprehension of Mandarin Chinese: evidence from auditory event-related brain potentials. In: Brain and Language 105, S. 112-133.

Primus, Beatrice (1999): Cases and thematic roles: ergative, accusative and active. (= Linguistische Arbeiten 393). Tübingen.

Pritchett, Bradley L. (1988): Garden path phenomena and the grammatical basis of language processing. In: Language 64, S. 539-576.

Pritchett, Bradley L. (1992): Grammatical competence and parsing performance. Chicago u.a.

Röder, Brigitte et al. (2002): Brain activation modulated by the comprehension of normal and pseudo-word sentences of different processing demands: a functional magnetic resonance imaging study. In: Neuroimage 15, S. 1003-1014.

Roehm, Dietmar et al. (2004): Fractionating language comprehension via frequency characteristics of the human EEG. In: Neuroreport 15, S. 409-412.

Roehm, Dietmar et al. (2007): To predict or not to predict: influences of task and strategy on the processing of semantic relations. In: Journal of Cognitive Neuroscience 19, S. $1259-1274$.

Schlesewsky, Matthias/Bornkessel, Ina (2003): Ungrammaticality detection and garden path strength: a commentary on Meng and Bader's (2000) evidence for serial parsing. In: Language and Cognitive Processes 18, S. 299-311.

Schlesewsky, Matthias/Bornkessel, Ina (2004): On incremental interpretation: degrees of meaning accessed during sentence comprehension. In: Lingua 114, S. $1213-$ 1234.

Schlesewsky, Matthias/Choudhary, Kamal K./Bornkessel-Schlesewsky, Ina (ersch.): Grammatical transitivity vs. interpretive distinctness: The case for a separation of two levels of representation that are often conflated. In: Brandt, Patrick/García García, Marco (Hg.): Transitivity. Form, meaning, acquisition, and processing. Amsterdam.

Schriefers, Herbert/Friederici, Angela D./Kühn, Katja (1995): The processing of locally ambiguous relative clauses in German. In: Journal of Memory and Language 34, S. 499-520.

Schütze, Carson T./Gibson, Edward (1999): Argumenthood and English prepositional phrase attachment. In: Journal of Memory and Language 40, S. 409-431.

Scott, Graham (1978): The Fore language of Papua New Guinea. (= Pacific Linguistics, Series B, 47). Canberra.

Silverstein, Michael (1976): Hierarchy of features and ergativity. In: Dixon, Robert M. W. (Hg.): Grammatical categories in Australian languages. (= Linguistic Series 22). Canberra, S. 112-171.

Sturt, Patrick/Crocker, Matthew W. (1996): Monotonic syntactic processing: a crosslinguistic study of attachment and reanalysis. In: Language and Cognitive Processes 11, S. 449-494. 
Townsend, David J./Bever, Thomas G. (2001): Sentence comprehension: the integration of habits and rules. Cambridge, MA u.a.

Urbach, Thomas P./Kutas, Marta (2010): Quantifiers more or less quantify on-line: ERP evidence for partial incremental interpretation. In: Journal of Memory and Language 2, S. 158-179.

Uszkoreit, Hans (1986): Constraints on order. In: Linguistics 24, S. 883-906.

van den Brink, Daniëlle/Hagoort, Peter (2004): The influence of semantic and syntactic context constraints on lexical selection and integration in spoken-word comprehension as revealed by ERPs. In: Journal of Cognitive Neuroscience 16, S. 1068-1084.

van Dyke, Julie A./McElree, Brian (2006): Retrieval interference in sentence comprehension. In: Journal of Memory and Language 55, S. 157-166.

Vosse, Thea/Kempen, Gerard (2000): Syntactic assembly in human parsing: a computational model based on competitive inhibition and lexicalist grammar. In: Cognition 75 , S. 105-143.

Wang, Luming et al. (2009): Exploring the nature of the subject'-preference: evidence from the online comprehension of simple sentences in Mandarin Chinese. In: Language and Cognitive Processes 24, S. 1180-1226.

Weckerly, Jill/Kutas, Marta (1999): An electrophysiological analysis of animacy effects in the processing of object relative sentences. In: Psychophysiology 36, S. 559570.

Wolff, Susann/Schlesewsky, Matthias/Bornkessel-Schlesewsky, Ina (2007): The interaction of universal and language-specific properties in the neurocognition of language comprehension: evidence from the processing of word order permutations in Japanese. In: Journal of Cognitive Neuroscience (Supplement) 107, S. 133-157.

Wolff, Susann et al. (2008): The neural mechanisms of word order processing revisited: electrophysiological evidence from Japanese. In: Brain and Language 107, S. 133157.

Wunderlich, Dieter (1997): Cause and the structure of verbs. In: Linguistic Inquiry 28, S. 27-68. 

Korpora und lexikalische Resourcen 

Christiane Fellbaum

\title{
Klassifikation des Verblexikons in WordNet und Abgleichung mit FrameNet ${ }^{1}$
}

\begin{abstract}
WordNet und FrameNet sind zwei umfangreiche lexikalische Ressourcen. Obwohl sie auf unterschiedlichen Theorien der lexikalischen Organisation basieren und die semantischen und syntaktischen Eigenschaften von Verben in beiden Ressourcen unterschiedlich dargestellt werden, wird mit beiden das Ziel verfolgt, die Regelmäßigkeit des Lexikons hervorzuheben. Seit Levin (1993) ist bekannt, dass die Selektion und Projektion von Argumenten eng mit den semantischen Eigenschaften von Verben zusammenhängt, und dass eine syntaktisch basierte Klassifikation des Verblexikons semantisch homogene Klassen ergibt. In diesem Beitrag werden die unterschiedlichen Ansätze von WordNet und FrameNet, das Verblexikon einheitlich zu klassifizieren, bewertet. Es wird ein laufender Versuch beschrieben, beide Ressourcen teilweise miteinander abzugleichen, indem ausgewählte Verben in einem großen Korpus zunächst manuell mit Bezug auf Einträge in WordNet und FrameNet annotiert werden. Anschließend werden verschiedene Ansätze zur automatischen Abgleichung, wie z.B. der des deutschen SALSA-Projekts, im Überblick dargestellt.
\end{abstract}

\section{Einleitung}

Es besteht allgemeine Übereinstimmung darin, dass der lexikalische Engpass in der maschinellen Sprachverarbeitung durch bessere lexikalische Ressourcen behoben werden kann und muss. Aber was sind „bessere“ Ressourcen? Eine mögliche Antwort setzt voraus, dass lexikalische Ressourcen sich auf unser Verstehen von menschlichem Sprachwissen stützen und dieses für maschinelle Anwendungen kodieren sollen. Manuell konstruierte Ressourcen basieren auf Introspektion und expliziten Regeln, die oft im Rahmen bestimmter, von diesen Ressourcen unabhängigen Theorien formuliert worden sind. Dies kann leicht zu inkompatiblen Analysen und Bedeutungsinventaren führen. Automatisch erstellte Ressourcen (vgl. Pantel/Lin 2002; Schulte im Walde ersch.), die auf Korpusextraktion basieren und nicht theoretisch vorbelastet sind, bringen zwar oft Regeln und Muster zum Vorschein, bergen aber die Gefahr in sich, unvollständig oder unausgewogen zu sein.

1 Die Arbeit wurde von der U.S. National Science Foundation (Grant IIS 0705155) unterstützt. Ich danke Collin Baker und Charles Fillmore für Kommentare zu einer früheren Version dieses Aufsatzes und den Bandherausgebern für ihr sorgfältiges Lektorat. 
Sprecherintuitionen zu Wortbedeutungen sind erstaunlich verschieden. Diskrepanzen zeigen sich deutlich in traditionellen Wörterbüchern sowie in modernen elektronischen Ressourcen, die den semantischen Raum, den ein Wort einnimmt, oft sehr unterschiedlich in zählbare und separate Lesarten aufteilen. Die Annotationen unterschiedlicher Annotierer, die die Tokens in einem Korpus mit entsprechenden Wörterbucheinträgen verknüpfen, stimmen oft nicht miteinander überein, da ein und dasselbe Token in einem bestimmten Kontext von unterschiedlichen Annotierern unterschiedlich interpretiert werden kann (vgl. Fellbaum/Grabowski/Landes 1997). Daher ist es nicht nur wichtig, verschiedene Ressourcen miteinander abzugleichen und somit eventuelle Ausreißer zu korrigieren, sondern auch die Ressourcen selbst mit einem Korpus abzugleichen. Im Folgenden wird zunächst über die Abgleichung von zwei, auf komplementären Modellen der lexikalischen Organisation basierenden Ressourcen, WordNet (Miller et al. 1990; Fellbaum (Hg.) 1998) und FrameNet (Baker/Fillmore/Cronin 2003), berichtet, die mittels der Annotation des American National Corpus (Ide/ Sudermann 2004) vorgenommen wird. Diese beiden Ressourcen werden schwerpunktmäßig im Hinblick auf die Struktur und die Alternationen der Argumente englischer Verben abgeglichen.

\section{WordNet}

WordNet ist eine lexikalische Datenbank in Form eines semantischen Netzes, die ursprünglich auf eine in den sechziger Jahren entwickelte und sowohl in der Psychologie als auch der Künstlichen Intelligenz gängige Theorie der Organisation des mentalen Lexikons gestützt war (Collins/Quillian 1969). Semantisch verwandte Wortformen sind durch lexikalische Relationen wie Synonymie, Antonymie und morphologische Ableitung miteinander verbunden. Kognitive Synonyme (im Sinne von Cruse 1986) sind in ungeordneten Mengen, so genannten Synsets, gruppiert. Die Lesarten polysemer Wortformen sind somit an die Zugehörigkeit zu verschiedenen Synsets gebunden; jede Kombination von Wortform und Synsetzugehörigkeit entspricht einer einmaligen Lesart (Miller et al. 1990; Fellbaum (Hg.) 1998). Zwei Lesarten des Verbs read sind z.B. in den zwei Synsets (1) und (2) (mit Beispielsätzen) dargestellt:

(1) learn, study, read, take („She is reading law at Oxford“)

(2) read, register, show, record (,The gauge read empty“)

Synsetzugehörigkeit in WordNet ist als Austauschbarkeit in vielen, aber nicht unbedingt allen Kontexten definiert. In vielen Kontexten ist eine bestimmte Wortform einer semantisch äquivalenten vorzuziehen: 
(3) The robbers tried to leave/\# exit the bank when the teller pressed the alarm button.

(4) Passengers are requested to exit/\#leave the bus through the rear door.

WordNets Verbsynsets sind nach rein semantischen Kriterien zusammengestellt. Folglich zeigen Verben, die in WordNet dem gleichen Synset zugeordnet sind, nicht immer dieselben syntaktischen Eigenschaften, wie in den Beispielen (5-7):

(5) My cats hate/detest/?loathe you.

(6) Lindsey hates/detests/loathes being known as a party girl.

(7) He will hate/??detest/??loathe to live in Milwaukee.

Auch Levins (1993) syntaktisch-basierte Klassifikation ordnet diese Verben ein und derselben Klasse (den Psych-Verben) zu; argumentstrukturelle Unterschiede wie die in den obigen Beispielen werden in Levins Klassifikation nicht berücksichtigt.

Synsets sind wiederum mittels konzeptuell-semantischer Relationen wie Hyperonymie, Meronymie und Kausalität verbunden; diese Relationen sind für alle Mitglieder eines Synsets identisch. Die meisten Relationen zwischen WordNets Verbsynsets basieren auf „Troponymie“, einer analogen Relation zu Hyperonymie im Nomenlexikon (Fellbaum 1990, 2002). Mehr oder weniger semantisch spezifische Verben werden mit Hilfe der Formel „X-en ist eine Art und Weise zu Y-en" in Baumstrukturen geordnet. Ein Beispiel ist der Baumausschnitt sich_fortbewegen, gehen, schleichen: schleichen ist eine Art zu gehen, gehen ist eine Art sich fortzubewegen, usw.

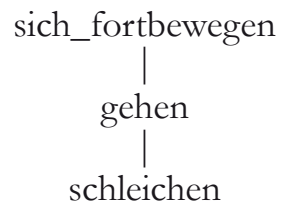

Die Zuordnung der Verben zu einem gegebenen Baum beruht auf der Intuition der Lexikografen, die sich dieser „Testformel“ bedienen. Fellbaum $(1990,2002)$ zeigt, dass der Großteil des englischen Verblexikons so vernetzt werden kann; die für andere Sprachen entworfenen WordNets, einschließlich GermaNet (Kunze 2000), können die Troponymierelation ebenfalls als primäre Relation zur Verbverknüpfung anwenden. Neologismen sind prinzipiell Troponyme von schon etablierten Verben. Zum Beispiel kann das Verb e-mail einer Baumstruktur mit Kommunikationsverben als Troponym von write zugeordnet werden. 
Schließlich zeigen die experimentell gewonnenen Daten von Fellbaum/ Chaffin (1990), Boyd-Graber et al. (2006) und Nikolova/Boyd-Graber/ Fellbaum (ersch.), dass Troponymie im mentalen Lexikon von Sprechern eine wichtige Rolle spielt. Als Relationen zwischen Verben und Verbsynsets kommen außerdem die folgenden Variationen von Entailment vor: „Cause“ (give-have), „Proper Inclusion“ (buy-pay), „Backward Presupposition" (succeed-try).

Fellbaum (1990) untersucht, ob sich aus der rein semantischen Organisation des Verblexikons in WordNet auch syntaktisch homogene Klassen ergeben. Dies ist oft, aber keineswegs immer, der Fall. Die semantische Unterklasse $\{g o$, travel, locomote $\}$ der Bewegungsverben bildet beispielsweise in syntaktischer Hinsicht eine homogene Klasse: Es sind alle intransitive Verben mit einer Präpositionalphrasenergänzung. In Bezug auf die Transitiv/ Intransitiv-Alternation verhält sich diese Klasse der Bewegungsverben aber uneinheitlich, vgl.:

(8) Kim walked/marched/promenaded her new dog this morning.

(9) *Kim strolled/ambled her new dog this morning.

In vielen Fällen zeigen die Verben in einer gegebenen Hierarchie unterschiedliche Argumentstrukturen. Ein bekanntes Beispiel ist die semantisch homogene Klasse der Transferverben, von denen nur eine Untermenge, die nicht semantisch, etymologisch oder morphologisch klar zu definieren ist, die Dativ-Alternation zeigt (Green 1974 inter alia):

(10) a. The professor gave an A to the best student.

b. Aunt Molly bequeathed her silver to Evelyne.

c. Mary donated $\$ 1,000$ to her favorite charity.

d. Peter conveyed his best wishes to the young couple.

(11) a. The professor gave the best student an A.

b. Aunt Molly bequeathed Evelyne her silver.

c. *Mary donated her favorite charity $\$ 1,000$.

d. *Peter conveyed the young couple his best wishes.

Die unterschiedlichen Argumentalternationen dieser intuitiv semantisch einheitlichen Verben sind in WordNet nicht berücksichtigt.

Umgekehrt gibt es Argumentalternationen, die scheinbar mit keiner semantisch kohärenten Verbklasse einhergehen. Die vielfach untersuchte Mittelkonstruktion (Keyser/Roeper 1984; Fagan 1992; Fellbaum/Zribi-Hertz 1990 inter alia) z.B. kann zwar grundsätzlich mit Verben, die eine Zustandsveränderung ausdrücken, auftreten (vgl. 12a-b und 13a-b), darüber hinaus erlauben aber auch manche Verben, deren Bedeutung keine Zustandsveränderung oder Affiziertheit involviert, und die keine eigenständige semantische Klasse bilden, diese Alternation (vgl. 12c-e und 13c-e): 
(12) a. The toddler broke the Chinese vase.

b. The tailor cut the silk.

c. My student sold his car in an hour.

d. The father photographed the triplets on Santa's lap.

e. The real estate agent showed us this attractive house.

(13) a. Chinese ceramics break easily.

b. The silk cut easily.

c. My student's car sold in an hour.

d. Triplets photograph nicely.

e. This attractive house shows nicely.

In manchen Fällen hängt die Verteilung von Argumentalternationen nicht nur von der Semantik der Verben, sondern auch von der der Argumente ab. Dies scheint bei der Instrument-Subjekt-Alternation der Fall zu sein, die bisher keine Erklärung gefunden hat (aber siehe Woicjik 1976), obwohl Sprecher robuste Intuitionen zur Akzeptabilität von Sätzen wie (14-15) zeigen:

(14) a. The key opened the door.

b. The knife cut the sausage.

c. The poison killed the rat.

(15) a. *The spoon stirred the soup.

b. *The pen signed the letter.

c. *The gun killed the prisoner.

Eine erste Konsequenz der hierarchischen Organisation von WordNet ist, dass manche Verben, die gemeinsame Argumentalternationen zeigen, wie z.B. die, die mit der Mittel- oder Instrument-Subjekt-Alternation vorkommen, verschiedenen Baumstrukturen zugewiesen sind.

Eine zweite Konsequenz von WordNets hierarchischer Organisation ist, dass verschiedene syntaktische Projektionen der Argumente eines Verbs als verschiedene Lesarten in separaten Bäumen erscheinen. Als Beispiel dienen hier die Verben, die die so genannte Lokativ-Alternation zeigen (Anderson 1971 inter alia). In (16) ist der Ort das direkte Objekt, und das Locatum wird als Präpositionalphrase realisiert. In (17) vertauschen diese beiden Argumente ihre syntaktischen Positionen:

(16) a. Load the truck with hay.

b. Spray the wall with paint.

a. Load hay onto the truck.

b. Spray paint onto the wall.

Die Verben, die diese Argumentalternation zeigen, sind in WordNet je zwei verschiedenen Bäumen zugeordnet. Der Baum, der die Lesart in (16) repräsentiert, hat die Wurzel fill; die Lesart in (17) wird durch einen Baum mit der 
Wurzel place dargestellt. Alle zu letzterem Baum gehörigen Verben sind Troponyme von place. Obwohl die Beziehung zwischen diesen beiden Lesarten insofern systematisch ist, als sie eine große Verbklasse betrifft, ist sie in WordNet nicht kodiert. Das trifft auch auf andere Alternationsmuster wie etwa die Mittel-Konstruktion zu. Die Mittel-Lesarten in (13) (vgl. 13a: Chinese ceramics break easily) sind alle einem Baum mit der Wurzel be (sein) zugeordnet, da sie eine Eigenschaft des strukturellen Subjekts ausdrücken; die transitiven Varianten in (12) (vgl. 12a: The toddler broke the Chinese vase) sind in einer Reihe von semantisch verschiedenen Bäumen vertreten.

Eine Ausnahme sind kausativ-inchoative Verben (break, move, grow usw.), deren transitiv-kausative und intransitiv-inchoative Lesarten (vgl. die Beispiele in (18) bzw. (19)) zwar in verschiedenen Bäumen repräsentiert, aber durch eine Cause-Relation verlinkt sind:

(18) a. The child broke the plate during dinner.

b. Farmers grow corn in New Jersey.

(19) a. The plate broke at dinner.

b. Corn grows in New Jersey.

Nicht alle Verben in einem gegebenen WordNet-Baum zeigen dasselbe syntaktische Verhalten. In manchen Fällen ist das syntaktische Potenzial bei den semantisch spezifischeren „Blätterknoten“ stärker eingeschränkt als bei den übergeordneten Verben; in anderen Fällen sind die durch solche Knoten repräsentierten Verben syntaktisch flexibler als die Wurzelverben; eine generell gültige Korrelation zwischen semantischer Spezifizität und Argumentalternationen scheint es nicht zu geben.

Zusammenfassend kann festgestellt werden, dass einerseits eine auf Argumentalternationen basierende Organisation des Verblexikons wie in Levin (1993) nicht immer semantisch homogene Klassen ergibt; umgekehrt ergibt WordNets rein semantische Klassifikation Verbgruppen mit unterschiedlichen syntaktischen Eigenschaften bzw. erfasst nicht immer die syntaktisch regelmäßigen Beziehungen zwischen Unterklassen in separaten Bäumen. ${ }^{2}$

Gross (1975) stellt eine andere semantisch-syntaktische Verbklassifikation dar, die feinkörniger als Levins (1993) ist und wenig Einsicht in die Regelmäßigkeit des Lexikons gewährt. VerbNet (Kipper et al. 2008) stellt einen Versuch dar, WordNet mit Levins Klassifikation auf automatischem Wege miteinander in Einklang zu bringen. Darüber hinaus verfeinert und ergänzt VerbNet die Levinsche Klassifizierung mit dem Ziel, semantische und syntaktische Klassen besser aufeinander abzustimmen. 


\section{FrameNet}

FrameNet (Baker/Fillmore/Cronin 2003, http://framenet.icsi.berkeley.edu/), eine maschinell lesbare lexikalische Ressource für das Englische, basiert auf Fillmores Theorie der Frame-Semantik (Fillmore 1982). Durch die einzelne Wörter übergreifende Perspektive der Darstellung in FrameNet wird die Bedeutung der Wörter nicht isoliert beschrieben, sondern eingebettet in als „Frames“ bezeichnete kognitive Schemata - sprachlich beschriebene Situationen oder Kontexte. Der Ansatz von FrameNet basiert auf der Annahme, dass die Bedeutung vieler Wörter am besten im Zusammenhang mit einem Frame sowie dessen Mitspielern und Requisiten - so genannten „FrameElementen " - verstanden werden kann. Der Unterschied zwischen WordNet und FrameNet kann daher grob als paradigmatisch vs. syntagmatisch dargestellt werden.

Lesarten in WordNet entsprechen in FrameNet sog. „Lexical Units“, d.h., Wortformen (Lemmata), die einem bestimmten Frame angehören. Ein Frame wird von einem Nomen, Verb oder Adjektiv evoziert und kann „Lexical Units“ aus allen Wortklassen enthalten. Wenn eine Wortform mehrere Frames evoziert, ist sie polysem und wird auf verschiedene „Lexical Units" abgebildet. Zum Beispiel wird das Verb tie up auf Basis der Kontexte in (20) und (21) zwei unterschiedlichen Frames und somit zwei Lesarten zugewiesen:

(20) tie up the deal (Activity-Finish Frame)

(21) tie up the prisoners (Immobilization Frame)

Frame-Elemente ähneln semantischen Rollen und können „Core“- oder „Non-Core“-Status haben; dies entspricht ungefähr dem Unterschied zwischen streng subkategorisierten und optionalen Argumenten (oder Adjunkten) von Verben. Das „Cutting“-Frame zum Beispiel umfasst das Verb cut und die „Core“-Frame-Elemente „Agens“, „Item“ (die Entität, die (ab)geschnitten oder zerschnitten wird) und „Pieces“ (die aus dem Schneiden resultierenden Teile). „Non-Core“-Frame-Elemente sind „Instrument" und „Manner“ (die Art und Weise des Schneidens). Letztere müssen nicht sprachlich ausgedrückt werden, obwohl unser Weltwissen sie als notwendige Elemente des Ereignisses voraussetzt.

Wortformen werden einem Frame zugewiesen, wenn „Core“-FrameElemente Wörtern in einer auf Korpusdaten basierenden Konstruktion zugewiesen werden können, und wenn sie dieselbe oder eine ähnliche Situation beschreiben. Grundlegend für den Ansatz von FrameNet ist die Annahme, dass das Sprecherwissen über ein Wort Wissen über dessen Frame-Elemente einschließt, und dass dieses Wissen nicht separat, sondern zusammen mit der lexikalischen Repräsentation des jeweiligen Wortes dargestellt werden soll. 
Ein Beispiel ist der „Claim_ownership“-Frame, den FrameNet folgendermaßen definiert:

(22) A Claimant asserts his or her right to possession of a piece of Property (either real, such as land, or private). The Claimant may be acting on the behalf of a Beneficiary.

Korpusbeispiele, die diesen Frame illustrieren, sind (23)-(25):

(23) The King claims this land and surrounding waters.

(24) Hoping to strike it rich, Thaddeus Gulch staked his claim by the river bed.

(25) I have dibs on the last piece of cake.

Zum „Claim_ownership“-Frame gehören die „Core“-Frame-Elemente "Claimant" (instanziiert durch King, Thaddeus Gulch, I) und „Property" (instanziiert durch this land and surrounding waters, his claim by the river bed, dibs on the last piece of cake). Frames werden typischerweise von Verben evoziert, im Fall vom „Claim_ownership“-Frame sind es u.a. die Verben call und claim.

Ein Frame schließt oft Wörter ein, die in WordNet als Synonyme (shut, close), Antonyme (love, hate), Troponyme (walk-stroll), Subevents (buy-pay) kodiert sind. Das bedeutet, dass die Zugehörigkeit zu einem Frame nicht durch syntaktische Eigenschaften wie Subkategorisierung und Argumentalternationen bestimmt wird. Ein Beispiel sind Emotionsverben, von denen sich viele im „Experiencer Subject“-Frame finden, darunter love und hate. Envy (,beneiden') ist ebenfalls diesem Frame zugewiesen, obwohl die syntaktischen Eigenschaften dieses Verbs sich von denen von love und hate unterscheiden: envy hat nicht nur ein zusätzliches obligatorisches FrameElement (die Entität, um die man jemanden beneidet), sondern es erscheint im Gegensatz zu den beiden anderen Verben auch in der Doppelobjektkonstruktion, wie (26) zeigt:

a. I envy you your talent.

b. *I love you your sense of humor.

c. *I hate you your rudeness.

Das derzeitige Bedeutungsinventar von FrameNet ist im Allgemeinen grobkörniger als das von WordNet, was zum Teil darauf zurückzuführen ist, dass FrameNet eine neuere Ressource ist und sich noch im Aufbau befindet. Kleinere Bedeutungsinventare sind vom Standpunkt der maschinellen Sprachverarbeitung, bei der die Disambiguierung von Lesarten eine beachtliche Herausforderung darstellt, wünschenswert. Grobkörnige Unterscheidungen sind jedoch nur dann vorteilhaft, wenn der richtige Grad an Unterspezifikation getroffen wird, so dass alle Lesarten klar unterschieden werden können und sich nicht überlappen. 
Wenn große Verbgruppen wie in FrameNet zusammengeführt werden, ist damit immer die Gefahr verbunden, dass die Möglichkeit feinerer linguistischer Analysen, die wichtige Generalisierungen erlauben, verloren geht. Zu FrameNets „Cutting“-Frame gehören beispielsweise sowohl Verben, die unterschiedliche Arten des Schneidens bezeichnen (carve, chop, mince, pare) als auch denominale Verben, die die durch das Schneideereignis geschaffenen Formen bezeichnen (cube, fillet, dice, slice). In WordNet befinden sich diese beiden Verbklassen in verschiedenen Bäumen mit den Wurzelverben separate bzw. dem Synset \{make, create\}. Die Argumentprojektionen der Verben werden in jedem Baum widergespiegelt: Im Gegensatz zu den Verben, die dem Synset \{separate\} zugeordnet sind, lassen die, die dem Synset $\{$ make, create $\}$ untergeordnet sind, keine Präpositionalphrase zu, in der das „Pieces“-Argument (die aus dem Schneiden resultierenden Teile) wiederholt wird:

(27) The chef carved/minced/chopped the vegetables into pieces/cubes/ slices/dice.

(28) The chef cubed/diced/sliced the vegetables (*into pieces/chunks).

FrameNet verzichtet auch auf die Unterscheidung von Verben mit verwandten aber unterschiedlichen transitiven und intransitiven Lesarten. Dass dadurch eine semantische Unterscheidung verloren gehen mag, zeigt z.B. eine Analyse des Verbs eat (Fellbaum/Kegl 1990). Das intransitive Verb eat weist zwei Lesarten auf, die sich in zwei verschiedenen Unterklassen von Verben widerspiegeln. Troponyme wie munch und nibble gehören zur inergativen Lesart (29). Die zweite intransitive Lesart bezieht ein Argument ein, welches sich auf eine Mahlzeit bezieht; diese Unterklasse schließt die Verben breakfast, lunch, dine und snack ein (30):

(29) The kids are eating/munching/nibbling all day.

(30) Did you eat/breakfast/dine already?

FrameNet kodiert „cross-Frame“-Relationen wie Entailment und Hyperonymie, die den Relationen zwischen Synsets in WordNet ähneln. Der Frame „Crime scenario" hat die Subframes „Committing Crime“, „Criminal Investigation“ und „Criminal Process“. Gemeinsame Frame-Elemente sind Crime and Perpetrator. Weiterhin ist ein „Perspective on“-Frame kodiert, der z.B. die Frames „Commerce_buy“ und „Commerce_sell“ verbindet: kaufen und verkaufen sind zwei unterschiedliche Perspektiven ein und desselben Ereignisses. 


\section{Verben in WordNet und FrameNet: Das Beispiel play}

Ein gutes Beispiel für die beiden unterschiedlichen Ansätze zur lexikalischsemantischen Analyse ist das Verb play in seiner Bedeutung ,Musik spielen (eine hier nicht ausgeführte Analyse des entsprechenden deutschen Verbs spielen fiele wahrscheinlich sehr ähnlich aus). WordNet unterscheidet die in den vier Synsets (31-34) angeführten Lesarten, von denen jede einer anderen Baumstruktur zugeordnet ist:

(31) play an instrument (play the flute) $\Rightarrow$ sound (cause to produce sound)

(32) play a piece of music/kind or piece of music (play Beethoven's Ninth; play Bach; play a waltz) $\Rightarrow$ perform, render

(33) play a location (he's playing Carnegie Hall/New York) $\Rightarrow$ appear

(34) play, make music (the band played all night) $\Rightarrow$ perform

Die Lesarten in (31-33) sind transitiv, die in (34) ist intransitiv (unergativ). Diese Lesarten sind alle verwandt: der Gebrauch von play in einer dieser Lesarten schließt die anderen Lesarten nicht aus. Durch die Verwendung von play mit Bezug auf ein bestimmtes Ereignis kann jede dieser vier Lesarten evoziert werden; jede von ihnen wird aber von einem jeweils anderen Aspekt ein und desselben Ereignisses evoziert. Die Unterscheidung der vier Lesarten von play in WordNet beruht auf der Zuordnung zu unterschiedlichen semantischen Hierarchien, spiegelt sich aber auch in unterschiedlichen syntaktischen Eigenschaften, insbesondere Argumentprojektionen, wider. Erstens können nur das Instrument- und das Ortsargument, aber nicht das Musikstück-Argument der transitiven Lesarten als Adjunkte ausgedrückt werden:

(35) She is still playing on the old piano.

(36) He played in Carnegie Hall/New York.

(37) *She played $\{$ on $/$ in $\}\{$ Bach/a waltz $\}$.

Zweitens kann die Lesart, cause to produce sound“ im Gegensatz zu der als ,play a location' paraphrasierten in einer Mittel-Konstruktion erscheinen:

(38) This old piano still plays well.

(39) *Carnegie Hall plays easily/well.

Drittens können die Instrument- und die Musikstück-Argumente im Gegensatz zum Ortsargument passiviert werden:

(40) The piano/waltz was played at midnight.

(41) *Carnegie Hall was played at midnight. 
Viertens können die Instrument- und die Musikstück/Musik/Komponisten-Argumente im Gegensatz zum Ortsargument als Subjekte des Verbs erscheinen:

(42) The piano played all night.

(43) The waltz/Strauss played all night.

(44) *Carnegie Hall played all night.

Diese Daten scheinen zunächst anzudeuten, dass die durch (31) und (32) exemplifizierten Lesarten (,sound' bzw. ,perform/render') zu einer Lesart zusammengeführt werden könnten. Dagegen spricht aber die Beobachtung, dass die benefaktive Dativ-Konstruktion nur das Musikstück oder den Komponisten, nicht aber das Instrument, als zweites Argument erlaubt:

She played a waltz/some Bach/the oboe for me.

Tabelle 1 fasst die Eigenschaften der verschiedenen Argumente von play mit Bezug auf Alternationen zusammen, die aus WordNets semantisch basierter Klassifikation der drei transitiven Lesarten hervorgehen. Die Tabelle zeigt, dass die auf der Zuordnung zu verschiedenen Überbegriffen beruhende Erstellung von drei Lesarten syntaktisch gerechtfertigt scheint.

\begin{tabular}{|l|c|c|c|c|c|}
\hline Argument/Syntax & Adjunkt & Mittel & Passiv & Subjekt & Benefaktiv \\
\hline Instrument & + & + & + & + & - \\
\hline Musikstück & - & $?$ & + & + & + \\
\hline Ort & + & - & - & - & - \\
\hline
\end{tabular}

Tab. 1: Eigenschaften der Argumente von play mit Bezug auf Alternationen

In FrameNet ist play mehreren miteinander verlinkten Frames zugeordnet. Jedes Frame ist mit einem deskriptiven Etikett bezeichnet: „Music“, „Instrumental_music“, „Play_instrument“, „Play_tune“, „Tune_plays“, „Instrument_plays“, „Cause-To_make_sound“, „Perform_Location“. „Core“Frame-Elemente sind „Performer“, „Tune“, „Instrument“, „Agent/Cause“, „Sound_maker“, „Venue“, „Score“. Die verschiedenen Frames sind mit je einer Untermenge von Frame-Elementen verbunden, die wiederum durch bestimmte „Lexical Units“ instanziiert werden können. In FrameNet werden die „Lexical Units“ nicht syntaktisch beschrieben oder klassifiziert; Frames und die dazugehörenden Frame-Elemente werden intuitiv und semantisch unterschieden.

Die Einträge von FrameNet basieren ausschließlich auf Belegen, die aus dem British National Corpus und dem American National Corpus gewonnen wurden. Da es nicht immer Korpusbelege für alle möglichen Argu- 
mentbelegungen eines gegebenen Verbs gibt, enthält FrameNet für viele Verben, die einem Frame zugeordnet sind, keine Beispiele, und die verschiedenen Valenzen sind somit nicht erschöpfend erläutert. Ebenso werden aufgrund unvollständiger Korpusdaten nicht alle Frame-Elemente, die Argumente oder Adjunkte eines Verbs sein können, in FrameNet mit Beispielen belegt. WordNets hierarchisch angeordnete Lexeme sind hier von Nutzen, da man davon ausgehen kann, dass die meisten Lexeme in einer gegeben Baumstruktur dasselbe Frame-Element instanziieren können.

\section{Abgleichung von WordNet und FrameNet}

Was können die beiden lexikalischen Ressourcen, die das englische Verblexikon großflächig und zum Teil komplementär in Bezug auf die erfassten Eigenschaften abdecken, über semantische und syntaktische Klassen aussagen? Levins (1993) Studie hat die Hypothese, dass syntaktische Eigenschaften von Verben semantisch intuitiv homogene Verbklassen widerspiegeln, größtenteils bestätigt. WordNet klassifiziert und unterscheidet Verben in mehr oder weniger spezifische lexikalisierte Abbildungen von Ereignissen; dieser Ansatz korreliert nur schwach mit einer syntaktischen Klassifikation, vor allem, wenn feinkörnige syntaktische Unterschiede beachtet werden. FrameNet zeigt, dass semantisch ähnliche Verben dieselben oder verwandte Frames und Frame-Elemente evozieren. Trotz einiger Ausnahmen und Unregelmäßigkeiten zeigen die drei verschiedenen Ansätze klare Muster und bestätigen die weitgehende Systematik im Verblexikon. Die unterschiedlichen Klassifikationen können daher nicht als imkompatibel angesehen, sondern sollten vielmehr als komplementär betrachtet werden; jeder Ansatz hebt unterschiedliche Aspekte einzelner Verben und des gesamten Verblexikons hervor. Damit erhebt sich die Frage, ob und wie die umfassende Klassifikation von FrameNet mit der feinkörnigeren von WordNet abgeglichen werden kann.

Ein zweiter potenzieller Gewinn der Abgleichung ist ein besseres Inventar von semantischen Rollen. Textverstehen setzt voraus, dass Satzkonstituenten nicht nur mit syntaktischen Funktionen wie Subjekt und Objekt, sondern darüber hinaus mit semantischen Rollen assoziiert werden können. Beim semantischen Parsing wird versucht, Rollen wie Agens, Patiens, Instrument, Benefizient, Zeit und Ort voneinander zu unterscheiden. Die automatische Abbildung von Satzkonstituenten mit einer größeren Anzahl von semantischen Rollen und Frame-Elementen, die feinkörniger voneinander unterschieden werden, ist eine bisher noch ungelöste Aufgabe. Die Abgleichung von WordNet und FrameNet kann eine optimale Anzahl von semantischen Rollen identifizieren, die zum einen ein tiefes semantisches Verstehen ermöglichen, zum anderen differenziert genug sind, damit ein 
automatisches System lernen kann, sie zu klassifizieren und zu erkennen. Zentral damit verbunden ist die Frage, ob das semantische Rolleninventar auf gewisse Verben und Verbklassen abgestimmt werden sollte.

Die Frame-Elemente von FrameNet können als framespezifische semantische Rollen angesehen werden. Obwohl die Anzahl der Frame-Elemente bereits sehr groß ist und mit fortschreitender Entwicklung der Ressource und der neuen Frames noch wächst, können manche Frame-Elemente in ein unterspezifiziertes verschmolzen werden. Zum Beispiel unterscheiden sich der Rezipient im „Sending“-Frame (47a) und der Benefizient im „Creating“-Frame (47b) nur semantisch aber nicht syntaktisch:
a. I sent Bill a poem.
b. I wrote Bill a poem.

Das Frame-Element „Benefizient“ könnte mit dem Rezipienten verschmolzen werden; beide sind intendierte Empfänger des Themenarguments. Eine kleinere Anzahl von Frame-Elementen erleichtert sowohl die manuelle als auch die automatische Annotation. Umgekehrt können zukünftige Analysen von Frame-Elementen und dazugehörigen Lexemklassen zu feineren Differenzierungen führen.

Ein mit Frame-Elementen annotiertes Korpus kann die Trainings- und Testdaten für das Lernen von semantischem Parsing liefern, und automatische Systeme können verschiedene Granularitäten von Frame-Elementen lernen.

\section{Was kann abgeglichen werden?}

WordNet und FrameNet eignen sich gut für eine Verknüpfung: „Lexical Units", die in FrameNet bestimmte FrameElemente instanziieren, können mit spezifischen Lesarten (Synsets) in WordNet verlinkt werden. So sollte zum Beispiel das „Core“-Frame-Element „Text“ im „Reading“-Frame, für das das „Lexical Unit“ paper angegeben ist, nur mit den entsprechenden Lesarten von paper in WordNet, $(48 \mathrm{a}, \mathrm{b})$, annotiert werden, aber nicht mit den Lesarten in (48c, d):
a. $\{$ composition, report, paper, theme (an essay)\}
b. \{newspaper, paper (a daily or weekly publication on folded sheets)\}
c. $\{$ paper (a material made of cellulose pulp)\}
d. \{newspaper, paper, newspaper publisher (a business firm that publishes newspapers) $\}$

Ein anderes Beispiel ist das polyseme Verb devour (,verschlingen'), das in je einer WordNet-Lesart mit dem „Reading“-Frame und dem „Ingestion“Frame verbunden ist. 
Die Organisation von WordNet erlaubt es, die Abgleichungen automatisch zu erweitern, indem z.B. die Synset-Mitglieder und die Hyperonyme der WordNet-Lesarten, die mit Frame-Elementen verknüpft sind, ebenfalls mit dem entsprechenden Frame-Element verlinkt werden.

\section{Annotation des American National Corpus mit WordNet und FrameNet}

Solange objektive Maßstäbe für ein optimales Bedeutungsinventar fehlen, können Klassifikationen dadurch miteinander verglichen werden, dass derselbe Text mit den unterschiedlichen Bedeutungsinventaren von WordNet und FrameNet annotiert wird. So können nicht nur äquivalente Einträge, sondern auch redundante und obskure oder seltene Lesarten einer polysemen Wortform identifiziert werden. Ein zusätzlicher Gewinn dieser doppelten Annotation besteht darin, dass maschinelle Anwendungen die verschiedenen Informationen, die mit dem jeweiligen Eintrag in WordNet und FrameNet verbunden sind und sich oft gegenseitig bereichern, kombiniert nutzen können.

Ide et al. (2008) annotieren zurzeit ausgewählte Zielwörter (Wortformen) in einem Teil des American National Corpus (www.americannationalcorpus.org; Ide/Suderman 2004) mit „Lexical Units“ in FrameNet und Synsets in WordNet. Kriterien für die Auswahl der 100 Zielwörter zur Annotation in diesem Manually Annotated Sub-Corpus (MASC) sind Wortklasse, Häufigkeit und Polysemie bzw. Homonymie. Die jeweilige Anzahl der als Zielwörter ausgewählten Nomen, Verben und Adjektive reflektiert grob die Proportionen im gesamten Lexikon (ca. 6:3:1). Da zum einen vermieden werden sollte, dass sich durch dieses Auswahlverfahren eine nicht zu bewältigende Anzahl von Tokens ergeben würde, zum anderen aber auch sichergestellt werden sollte, dass die Annotationen für diverse Anwendungen von maximalem Nutzen sind, werden nur solche Wortformen als Zielwörter ausgewählt, die weder extrem häufig noch extrem selten im Korpus vorkommen. Darüber hinaus wird eine ausgeglichene Mischung von homonymen und polysemen Lesarten angestrebt. Zu den bereits annotierten Wortformen gehören die Nomen window, date, part, rate, success, time, work, rule, die Verben lose, win, read, write, serve, try, forget, say, tell und die Adjektive warm, poor, rapid, curious, long, quiet, fair.

Nach der manuellen Bestimmung der Zielwörter annotieren speziell ausgebildete Studenten fünfzig beliebig ausgewählte Tokens pro Zielwort und liefern den Lexikografen detaillierte kritische Kommentare. Dabei werden offensichtliche Fehler, wie doppelte oder fehlende Einträge, entdeckt und verbessert. Die daraufhin revidierten Einträge dienen als Basis für die großflächige Annotation - bis zu 1.000 Tokens pro Zielwort - im Korpus. 
Unser Ziel ist nicht, die Überlegenheit einer lexikalischen Ressource gegenüber der anderen zu beweisen. Vielmehr dienen die Annotationsdaten der Korrektur fehlender oder doppelter Einträge in beiden Ressourcen. Ein weiteres Ziel besteht darin, WordNet und FrameNet so weit wie möglich aufeinander abzustimmen.

Da das Bedeutungsinventar von WordNet eher feinkörnig und das von FrameNet eher grobkörnig ist, ergeben sich viele mehrfach-zu-einfachAbbildungen. Fellbaum/Baker (ersch.) und Baker/Fellbaum (2009) erstellen eine Typologie der Nichtübereinstimmungen. In dem seltenen Fall, dass ein Synset und ein Frame genau dieselben Lexeme enthalten, wird diese Korrespondenz einfach festgehalten werden. Suspicious zum Beispiel hat zwei Bedeutungen in WordNet:

a. \{fishy, funny, shady, suspect, suspicious\}

b. \{leery, mistrustful, suspicious, untrusting, wary\}

Diese beiden Lesarten entsprechen den „Lexical Units“ in zwei Frames, „Suspiciousness", ein Subframe von „Social Behavior Evaluation“, und „Awareness“, ein Subframe von „Mental Activity“.

Häufig sind die Wörter in einem Frame eine Untermenge der Wörter in einem Synset. Dann werden die in WordNet mit dem Zielwort verlinkten Wörter als Synonyme (leery) und Antonyme (trustful) und oft auch als Hyperonyme den entsprechenden Frames als „Lexical Units“ hinzugefügt, was zu einer effizienten Erweiterung von FrameNet führt.

WordNet hat oft mehr Lesarten als FrameNet, insbesondere technische oder seltenere Lesarten. So ist z.B. die Lesart des Adjektivs long, die mit ,holding securities or commodities in expectation of a rise in prices' paraphrasiert werden kann, nicht in FrameNet vertreten. Wenn keine entsprechenden Korpusbelege gefunden werden, werden solche Lesarten auch nicht in FrameNet eingegliedert; die Diskrepanz zwischen den Bedeutungsinventaren der beiden Ressourcen scheint hier unproblematisch.

Zwei Synsets, die Untermengen der „Lexical Units“ in einem Frame sind, werden auf eine mögliche Zusammenlegung hin untersucht. Z.B. wurden die zwei Synsets in (50), die einem Frame entsprechen, zusammengeführt; FrameNet kodiert die metaphorische Lesart des Adjektivs legendary nicht gesondert von der wörtlichen.

(50) a. \{legendary (so celebrated as to having taken on the nature of a legend) $\}$

b. $\{$ fabled, legendary (celebrated in fable or legend) $\}$

Der umgekehrte Fall, dass die „Lexical Units“ zweier Frames Untermengen eines Synsets sind, was die Zusammenlegung der Frames nahelegen würde, ist seltener. Zum Beispiel gibt WordNet nur eine Lesart des Adjektivs successful an: 
(51) \{successful (having succeeded or being marked by a favorable outcome: ,a successful architect; a successful business venture“) $\}$

FrameNet unterscheidet zwei „Lexical Units“ successful; das eine, das einen Agens impliziert, ist dem Frame „Successful_action“ zugewiesen; ein zweites, ohne implizierten Agens, gehört zum Frame „Success_or_failure“.

In den meisten Fällen erfolgt die Abgleichung mittels der Über- bzw. Unterordnungsrelation zwischen Synsets, die der ,generalization“-Relation in FrameNet entspricht. So entspricht z.B. die Beziehung zwischen den zwei Frames „Inhibit Movement" und „Imprisonment“ dem Troponympaar detain - incarcerate in WordNet, und die „Lexical Units“ in dem übergeordneten Frame („Inhibit_Movement“) werden vom untergordneten Frame „Imprisonment" automatisch aufgenommen.

\section{Automatische Abgleichung}

Für viele der ausgewählten Zielwörter befinden sich mehr als 1.000 Tokens - unsere Obergrenze für manuelle Annotation - im Korpus. Automatische Annotation der zusätzlichen Tokens ist geplant; dabei werden die manuellen Tags als Trainings- und Testdaten für den Lernalgorithmus dienen. Verschiedene Versuche zur automatischen Abgleichung wurden bereits unternommen; sie sind zum Teil unbeaufsichtigt und nicht auf manuelle Annotationen gestützt. Wir fassen kurz einige Ansätze zusammen.

Shi/Mihalcea (2005) gleichen Verben in FrameNet und WordNet halbautomatisch ab. Zuerst wurden für 3.000 „Lexical Units“ in FrameNet die entsprechenden WordNet-Lesarten manuell identifiziert. Shi und Mihalcea erweitern die manuelle Abgleichung sodann automatisch und stützen sich dabei auf die Annahme, dass Synonyme und Antonyme eines Verbs aufgrund ihrer in WordNet kodierten semantischen Ähnlichkeit Frame-Elemente im selben Frame sind.

Chow und Websters (2007) Abgleichung erfolgt indirekt über die Suggested Upper Merged Ontology (SUMO), eine formale Ontologie, die manuell auf WordNet abgebildet wurde (Niles/Pease 2003). Ausgangspunkt für die Abgleichungen von Chow und Webster sind die Abgleichungen von Shi/Mihalcea (2005) von „Lexical Units“ in Frames mit Wörtern in einem Synset. Diesen Abgleichungen fügen Chow/Webster alle Synset-Mitglieder sowie alle in WordNet verlinkten Wörter hinzu. Diese Menge von Wörtern ergibt eine semantische Domäne unter der Annahme, dass die in WordNet miteinander verknüpften Wörter ein und derselben semantischen Domäne angehören.

Zum Beispiel werden die WordNet Synsets in (52) der Domäne „Statement" zugewiesen, da sie alle in WordNet mit dem Verb address in Synset (53) vernetzt sind und dieses Verb manuell von Shi/Mihalcea auf die entsprechende „Lexical Unit“ abgebildet wurde: 
(52) \{communicate, intercommunicate\}

$\{$ betray

\{inform $\}$

\{disclaim\}

(53) $\{$ address, turn to $\}$

Um die Abgleichung so fehlerfrei wie möglich zu gestalten, beziehen Chow/ Webster sich auf Domänen in SUMO und die unabhängig durchgeführten SUMO-WordNet-Abgleichungen (Niles/Pease 2003).

Wie andere Abgleichungsansätze basiert der von Chow/Webster auf der nicht immer korrekten Annahme, dass in WordNet verlinkte Lesarten den „Lexical Units“ in einem gegebenen Frame entsprechen. Diese Annahme trifft zwar relativ systematisch für alle Mitglieder eines Synsets zu, ist aber weniger verlässlich für andere semantische Relationen. Zum Beispiel sind die Verben cook und heat in WordNet durch Entailment verbunden. Cook ist ein Kreationsverb, aber heat drückt eine Zustandsveränderung aus. Es folgt also nicht zwingend, dass die in WordNet vernetzten Lesarten dieser Verben derselben Domäne angehören und dass sie die entsprechenden semantisch verwandten Lesarten der „Lexical Units“ in einem Frame sind.

Ein weiteres Problem mit Chows und Websters Ansatz ist, dass Frames sehr feinkörnig sind, und die in WordNet verwandten Verben in fast allen Fällen nicht nur einem, sondern einer Anzahl von Frames zugewiesen werden müssen. WordNets Relationen sind nicht dazu geeignet, diese feinkörnigen Zuteilungen automatisch zu erstellen.

Schließlich sind die Zuteilungen zu Domänen in WordNet oft arbiträr und beruhen nicht auf semantischer Ähnlichkeit; diese störenden Ungenauigkeiten sind auch in der Abgleichung mit den Domänen von SUMO übernommen.

Das Ziel von Burchardt/Erk/Frank (2005) ist, die Anzahl der „Lexical Units“ in FrameNet zu erhöhen. Die Methode, ein so genannter „Umweg“ über WordNet, macht Gebrauch von den Relationen in WordNet und erreicht gleichzeitig eine Abgleichung der beiden Ressourcen. In einem ersten Schritt werden ausgewählte Zielwörter mittels der auf WordNets Ähnlichkeitsmaßen basierten Methode von Banerjee/Pedersen (2003) disambiguiert. Diese Maße beruhen auf der Annahme, dass diejenigen Lesarten polysemer Wörter, die in WordNet miteinander verlinkt sind und sich in gegenseitiger „Nähe“ im Netz befinden, semantisch ähnlich sind; Relationen können dabei unterschiedlich gewichtet werden. Burchardt/Erk/ Frank (2005) fügen sodann alle in WordNet verlinkten Wörter den disambiguierten Zielwörtern hinzu (Hyperonyme, Troponyme, Antonyme, Meronyme, etc.). Der Frame, in dem ein Großteil dieser Wörter als „Lexical Units" vorkommt, wird identifiziert, und das Zielwort wird ihm zu- 
gewiesen. Multiple Kandidaten-Frames werden nach einem Gewichtsschema evaluiert. Ein einfaches Beispiel ist das Adjektiv new, welches aufgrund der Antonymierelation zu old dem „Age“-Frame zugewiesen werden konnte.

Wie Chow/Webster stützen sich Burchardt/Erk/Frank (2005) auf die nicht immer verlässliche Annahme, dass die semantische Ähnlichkeit von Wörtern in WordNet mit gemeinsamer Frame-Zugehörigkeit in FrameNet korrespondiert. Oltramari (2006) verfeinert die Methode von Burchardt/ Erk/Frank (2005) mit einer statistischen Analyse der Verteilung der Frames unter WordNets Hyponymen; dadurch werden die rein semantischen Ähnlichkeitsmaße durch ein quantitatives $\mathrm{Maß}$ ergänzt.

Eine Reihe anderer Ansätze zur automatischen Abgleichung von Lesarten in WordNet und „Lexical Units“ in FrameNet verlassen sich nicht nur auf die in WordNet kodierten Relationen, sondern ziehen zusätzlich die Wörter, die in den Definitionen der Synsets vorkommen, in Betracht. Tonelli/Pianta (2009) gleichen die „Lexical Units“ von FrameNet mit den Synsets von WordNet ab, indem sie die Anzahl der gemeinsamen Wortformen in der Definition eines WordNet-Synsets und der korrespondierenden lexikalischen Einheit in FrameNet messen. Ferrández et al. (2010) verfeinern diese Methode und erzielen etwas genauere Resultate, indem sie die Ähnlichkeit der lexikalischen Umgebung von Paaren von „Lexical Units“ in FrameNet und Synsets in WordNet messen.

Ides (2006) Abgleichung geht in die umgekehrte Richtung, von den „Lexical Units“ in FrameNet zu den Synset-Mitgliedern in WordNet. Ide disambiguiert je zwei „Lexical Units“ in einem Frame, indem sie die Ähnlichkeitsmaße für die Disambiguierung von Patwardhan/Pedersen (2006) anwendet. Diese Maße berechnen und gewichten die semantische Ähnlichkeit zweier Wortformen, indem sie alle Lesarten dieser Wortformen untersuchen und ein Ranking erstellen. Die Maße beruhen wie die von Banerjee/ Pedersen (2003) auf WordNets Struktur (diversen WordNet-Relationen und lexikalischer Überlappung der „Lexical Units“ mit Lemmata in den Definitionen und Beispielsätzen). Ides Grundannahme dabei ist, dass zwei Wortformen, die beide einem Frame angehören, semantisch verwandt sind, und dass diese Verwandtschaft in WordNet erschlossen werden kann. Die Lesarten der „Lexical Units“, die von Patwardhan/Pedersens (2006) Ähnlichkeitsmaßen am höchsten gerankt werden, werden als die für den Frame zutreffenden angesehen.

Ein Beispiel sind die Wortformen cast und pitch, die beide zum „Cause Motion"-Frame gehören, aber mehrfache Lesarten haben, welche anderen Frames (einschließlich „Create Physical Artwork“" und „Encoding“) zugewiesen sind. Patwardhan und Pedersens (2006) Ähnlichkeitsmaße disambiguieren dieses Wortpaar und identifizieren die in WordNet verwandten „Cause Motion“-Lesarten in WordNet. 
Automatische Abgleichungen können manuelle Annotation nicht ersetzen. Sie beruhen auf Ressourcen wie WordNet, die nicht für Abgleichungszwecke konstruiert wurden und deren interne Verknüpfung verschiedene Arten und Grade von semantischer Ähnlichkeit kodieren, die nicht unbedingt der Ähnlichkeit von „Lexical Units“ in einem Frame entsprechen. Wir erwarten, dass das Vorgehen von Baker/Fellbaum (2009) und Fellbaum/ Baker (ersch.), das die Abgleichung von WordNet und FrameNet gezielter mittels manueller Annotation von WordNet-Lesarten und FrameNet-,Lexical Units" vornimmt, verlässlichere Daten als Input für eine umfangreiche automatische Abgleichung liefert.

\section{Annotation eines deutschen Korpus mit Frames: SALSA}

Das SALSA Projekt (Saarbruecken Lexical Semantics Annotation and Analysis; Burchardt et al. 2009) hat sich, ähnlich wie MASC, zum Ziel gesetzt, ein großes deutsches Korpus mit Frames und Frame-Elementen zu annotieren, auf Grund der Annotationen ein framebasiertes Lexikon zu generieren und schließlich ein datenbasiertes Modell für die automatische Frame-Analyse zu entwickeln. Das Projekt geht von der Annahme aus, dass Frames und Frame-Elemente universalen Status haben. Es ist intuitiv einleuchtend, dass zum Beispiel ein Transfer-Ereignis folgende Argumente hat: Ursprung, Ziel und Thema (die überreichte Entität), unabhängig davon, wie diese FrameElemente syntaktisch und morphologisch realisiert werden. Bisher konnte gezeigt werden, dass sich Frames, die für das Englische entwickelt wurden, gut auf das Deutsche übertragen lassen. Boas (2009) diskutiert das Potenzial sowie die Grenzen der Anwendung von Frames als sprachunabhängige Repräsentationen für übereinzelsprachliche lexikalische Ressourcen.

Systematische Unterschiede zwischen entsprechenden deutschen und englischen Frames sind größtenteils auf sprachspezifische lexikalische und morphosyntaktische Eigenschaften zurückzuführen. So fordert z.B. der Gebrauch des Dativs im Deutschen bei untrennbarem Besitz ein zusätzliches strukturelles Argument, das im Englischen nicht als Nomen oder Pronomen erscheint:

a. Hans hat sich den Arm gebrochen.

b. Hans broke his arm.

a. Der Dieb hat mir die Handtasche gestohlen.

b. The thief stole my handbag.

Die deutschen Dativpronomen und die entsprechenden englischen Possessiva können mit einem „Extra-Frame“-Element „Possessor" etikettiert werden (Burchardt et al. 2009). Eine einheitliche Etikettierung mit einem englischen Äquivalent kann nicht für den so genannten „ethischen“ Dativ vorgenommen werden, der im Englischen nicht existiert: 
(56) a. Der Patient ist mir gestorben.

b. The patient died $\varnothing$.

In diesen Fällen müsste ein zusätzliches Frame-Element für die deutschen Frames angenommen werden oder - alternativ - das Argument ohne Bezeichnung bleiben. Da die Bedeutung dieses Elements unterspezifiziert und kontextspezifisch ist, wäre die erste Lösung problematisch und würde mehrere Frame-Elemente erfordern. Der ethische Dativ trägt nur wenig zur Satzbedeutung bei und verändert den Wahrheitswert des Satzes nicht. Es wäre schwer, zu argumentieren, dass ein entsprechendes Frame-Element im Englischen existiert, aber nicht auf der Satzoberfläche realisiert wird.

Ein anderer, recht systematischer Unterschied ist, dass Substantivableitungen von Verben im Englischen höchst produktiv, aber im Deutschen weniger häufig sind. Viele englische Verben wie pencil, bookmark und carpet enthalten ein Frame-Element („Instrument“, „Material“", usw.), das im Deutschen als eigenständiges Argument und Frame-Element des Verbs ausgedrückt wird.

Die in den SALSA-Frames bestimmten „Lexical Units“ werden nicht auf eine unabhängige Ressource wie das deutsche WordNet (GermaNet) abgebildet. Interessant ist der von Burchardt et al. (2009) durchgeführte Vergleich der durchschnittlichen Anzahl der „Lexical Units“ für eine Wortform mit der durchschnittlichen Anzahl der Lesarten in GermaNet: Letztere ist höher, d.h., die Frame-Analyse ergibt ähnlich wie bei den korrespondierenden englischen Ressourcen durchschnittlich mehr Lesarten pro Wortform.

\section{Schlussfolgerung}

WordNet und FrameNet stellen unterschiedliche Ansätze zur Bestimmung von Lesarten polysemer Wortformen und zur Erschließung des Lexikons dar. Beide sind semantisch ausgerichtet, lassen jedoch Regelmäßigkeiten erkennen, die mit einem syntaktischen Ansatz wie Levins Klassifikation des Verblexikons (1993) kompatibel sind. Eine Abgleichung von WordNet und FrameNet im Rahmen einer umfangreichen Korpusannotation zeigt klar, dass beide Ressourcen überlappende Lesarteninventare haben und miteinander in Einklang gebracht werden können. Einander entsprechende Lesarten zeigen die Übereinstimmung von Argumentstrukturen und Alternationen von Verben in WordNet mit Frame-Elementen in FrameNet. 


\section{Literatur}

Anderson, Stephen (1971): On the role of deep structure in semantic interpretation. In: Foundations of Language 7, S. 387-396.

Baker, Collin F./Fellbaum, Christiane (2009): WordNet and FrameNet as complementary resources for annotation. In: Association for Computational Linguistics ( $\mathrm{Hg})$ : Proceedings of the Third Linguistic Annotation Workshop. Singapur, S. 125-129.

Baker, Collin F./Fillmore, Charles J./Cronin, Beau (2003): The structure of the Framenet database. In: International Journal of Lexicography 16, 3, S. 281-296.

Banerjee, Satanjeev/Pedersen, Ted (2003): Extended gloss overlaps as a measure of semantic relatedness. Proceedings in the International Joint Conference on Artificial Intelligence (IJCAI). Acapulco, S. 805-810.

Boas, Hans C. (2009): Semantic frames as interlingual representations for multilingual lexical databases. In: Boas (Hg.), S. 59-100.

Boas, Hans C. (Hg.) (2009): Multilingual FrameNets in Computational Lexicography: Methods and applications. (= Trends in Linguistics: Studies and Monographs 200). Berlin.

Boyd-Graber, Jordan et al. (2006): Adding dense, weighted connections to WordNet. In: Sojka, Peter et al. (Hg.): Proceedings of the Third Global WordNet Conference. Jeju Island, Republik Korea, S. 29-35.

Burchardt Aljoscha/Erk, Katrin/Frank, Anette (2005): A WordNet Detour to FrameNet. In: Fisseni, Bernhard et al. $(\mathrm{Hg})$ : Sprachtechnologie, mobile Kommunikation und linguistische Ressourcen: Beiträge zur GLDV-Tagung 2005 in Bonn. (= Computer Studies in Langauge and Speech 8). Frankfurt a.M., S. 408-421.

Burchardt, Aljoscha/Erk, Katrin/Frank, Anette et al. (2009): Using FrameNet for the semantic analysis of German: Annotation, representation and automation. In: Boas (Hg.), S. 209-244.

Chow, Ian/Webster, Jonathan (2007): Integration of linguistic resources for verb classification: FrameNet frame, WordNet verb and suggested upper merged ontology. In: Gelbukh, Alexander (Hg.): Proceedings of the $8^{\text {th }}$ Conference on Computational Linguistics and Intelligent Text Processing (CICLing 2007). Mexico City, S. 1-11.

Collins, Allan M./Quillian, M. Ross (1969): Retrieval time from Semantic Memory. In: Journal of Verbal Learning and Verbal Behavior 8, S. 240-247.

Cruse, Alan D. (1986): Lexical semantics. Cambridge.

Fagan, Sarah (1992): The syntax and semantics of middle constructions: A study with special reference to German. (= Cambridge Studies in Linguistics 60). Cambridge.

Fellbaum, Christiane (1990): The English verb lexicon as a semantic net. In: International Journal of Lexicography 3, 4, S. 278-301.

Fellbaum, Christiane (Hg.) (1998): WordNet: An electronic lexical database. Cambridge, MA. 
Fellbaum, Christiane (2002): The semantics of troponymy. In: Green, Rebecca/Bean, Carol/Myaeng, Sung Hyon (Hg.): The semantics of relationships: An interdisciplinary perspective. (= Information Science and Knowledge Management 3). Dordrecht, S. 23-34.

Fellbaum, Christiane/Baker, Collin F. (ersch.): Aligning verbs in WordNet and FrameNet. In: Linguistics.

Fellbaum, Christiane/Chaffin, Roger (1990): Some principles of the organization of the verb lexicon. In: Proceedings of the $12^{\text {th }}$ Annual Conference of the Cognitive Science Society. Hillsdale, NJ, S. 420-428.

Fellbaum, Christiane/Grabowski, Joachim/Landes, Shari (1997): Analysis of a handtagging task. In: Association for Computational Linguistics (Hg.): Proceedings of the ACL/Siglex workshop. Washington, DC, S. 34-40.

Fellbaum, Christiane/Kegl, Judy (1990): Taxonomic structure and object deletion in the English verbal system. In: deJong, Ken/No, Yongkyoon (Hg.): Proceedings of the Sixth Eastern States Conference on Linguistics. Columbus, OH, S. 94-103.

Fellbaum, Christiane/Zribi-Hertz, Anne (1990): La construction moyenne en français et en anglais: Etude de syntaxe et de sémantique comparée. In: Recherches Linguistiques à Vincennes 18, S. 19-57.

Ferrández, Óscar et al. (2010): Aligning FrameNet and WordNet based on semantic neighborhoods. In: Calzolari, Nicoletta et al. (Hg.): Proceedings of the Seventh Conference on Language Resources and Evaluation (LREC 10). Valetta, S. 310-314.

Fillmore, Charles J. (1982): Frame semantics. In: Linguistic Society of Korea (Hg.): Linguistics in the morning calm. Seoul, S. 111-137.

Green, Georgia (1974): Semantics and syntactic regularity. Bloomington, IN.

Gross, Maurice (1975): Méthodes en syntaxe: Régime des constructions complétives. (= Actualités Scientifiques et Industrielles 1365). Paris.

Ide, Nancy (2006): Making senses: Bootstrapping sense-tagged lists of semantically related words. In: Gelbukh, Alexander (Hg.): Computational Linguistics and Intelligent Text, Lecture Notes in Computer Science Vol. 3878. Heidelberg, S. 13-27.

Ide, Nancy/Suderman, Keith (2004): The American National Corpus first release. In: Proceedings of the Fourth Language Resources and Evalation Conference (LREC). Lissabon, S. 1681-1684.

Ide, Nancy et al. (2008): MASC: A Manually Annotated Subcorpus of American English. In: Proceedings of the Sixth Language Resources and Evaluation Conference (LREC). Marrakesch, S. 617-620.

Keyser, Sam J./Roeper, Thomas (1984): On the middle and ergative construction in English. In: Linguistic Inquiry 15, S. 381-416.

Kipper, Karin et al. (2008): A large-scale classification of English verbs. In: Language Resources and Evaluation 42, S. 21-40.

Kunze, Claudia (2000): Extension and use of GermaNet, a lexical-semantic database. In: Proceedings of the Second International Conference on Language Resources and Evaluation. Athen, S. 999-1002. 
Levin, Beth (1993): English verb classes and alternations: A preliminary analysis. Chicago u.a.

Miller, George A./Beckwith, Richard/Fellbaum, Christiane et al. (1990): Introduction to WordNet: An on-line lexical database. In: International Journal of Lexicography 3, 4, S. 235-244.

Nikolova, Sonja/Boyd-Graber, Jordan/Fellbaum, Christiane (ersch.): Collecting semantic similarity ratings to connect concepts in assistive communication tools. In: Mehler, Alexander et al. (Hg.): Modeling, learning and processing of text technological data structures. Studies in computational intelligence. Heidelberg/New York.

Niles, Ian/Pease, Adam (2003): Linking lexicons and ontologies: Mapping WordNet to the suggested upper merged ontology. In: Arabnia, Hamid (Hg.): Proceedings of the 2003 International Conference on Information and Knowledge Engineering. Las Vegas, S. 412-416.

Oltramari, Alessandro (2006): LexiPass methodology: A conceptual path from frames to senses and back. In: Proceedings of the Fifth International Conference on Lexical Resources in the European Community (LREC). Genua, S. 2311-2314.

Pantel, Patrick/Lin, Dekang (2002): Discovering word senses from text. In: Proceedings of ACM Conference on Knowledge Discovery and Data Mining (KDD-02). Edmonton, S. 613-619.

Patwardhan, Siddhartha/Pedersen, Ted (2006): Using WordNet-based context vectors to estimate the semantic relatedness of concepts. In: Proceedings of the European Association of Computational Linguistics (EACL) 2006 Workshop Making Sense of Sense-Bringing Computational Linguistics and Psycholinguistics Together. Trient, S. $1-8$.

Schulte im Walde, Sabine (ersch.): The induction of verb frames and verb classes from corpora. In: Lüdeling, Anke/Kytö, Merja (Hg.): Corpus linguistics: An international handbook. Berlin.

Shi, Lei/Mihalcea, Rada (2005): Putting pieces together: Combining FrameNet, VerbNet and WordNet for robust semantic parsing. In: Gelbukh, Alexander (Hg.): Proceedings of the Sixth International Conference on Intelligent Text Processing and Computational Linguistics. Mexico City, S. 100-111.

Tonelli, Sara/Pianta, Emanuele (2009): A novel approach to mapping FrameNet lexical units to WordNet synsets. In: Proceedings of Eighth International Conference on Computational Semantics (IWCS-8). Tilburg, S. 342-345.

Woicjik, Richard (1976): Where do instrumental NPs come from? In: Shibatani, Masayoshi (Hg.): Syntax and semantics 6: The grammar of causative constructions. New York, S. 165-180. 



\title{
Patrick Hanks
}

\section{Wie man aus Wörtern Bedeutungen macht: Semantische Typen treffen Valenzen ${ }^{1}$}

\begin{abstract}
Wie versteht ein Hörer oder Leser die von einem Sprecher oder Schreiber beabsichtigte Bedeutung? Syntaktische Strukturen sind zu allgemein, um feine Bedeutungsunterscheidungen auszudrücken. Wörter sind oft sehr mehrdeutig, und aufgrund dessen unzuverlässig als „Bedeutungsleitfaden“. Im Gegensatz dazu zeigt die Korpusmusteranalyse, dass die meisten Äußerungen aus Mustern von vergleichsweise geringer Mehrdeutigkeit aufgebaut sind. Daher stellt sich die Frage: Was ist ein Muster? Muster sind häufig verwendete Sprachbausteine, die aus zwei Elementen bestehen: Valenzen und Kollokationen. Während Valenzen relativ stabil sind, sind Kollokationen extrem variabel. In der Korpusmusteranalyse wird eine große Anzahl von Gebrauchsbelegen jedes Wortes studiert, und seine Kollokationen werden, ihren semantischen Typen entsprechend, lexikalischen Sets zugeordnet.

Jedes Wort einer Sprache ist Bestandteil von mindestens einem Muster. Wenn es Teil von mehr als einem Muster ist, können die Bedeutungen seiner Muster meist durch unterschiedliche Kollokations-Präferenzen unterschieden werden.

Kreative Benutzungen sind Abweichungen von normalen Nutzmustern, aber Abweichungen sind selbst regelgeleitet. Daher benötigt man eine Theorie von Normen und Abweichungen. Da die zwei Regelsysteme interagieren, können wir die Theorie als eine „Doppelhelix“ beschreiben.
\end{abstract}

\section{Einführung}

Es ist weithin bekannt, dass Textbedeutung kontextabhängig ist. Aber was ist „Kontext“, und woran erkennt man (relevanten) Kontext? In diesem Beitrag untersuche ich den Einfluss von Kollokationen und Valenz auf Bedeutung und Bedeutungsunterscheidungen. Relevante Valenztheorien wurden von Tesnière (1959) und Halliday (1961) formuliert. Für das Englische sind Wortvalenzen weitgehend und intensiv von Herbst et al. (2004), und Kollokationen von Sinclair (Sinclair et al. 1987; Sinclair 1991, 1998), untersucht worden. Im Folgenden werde ich beide Stränge zusammenführen.

Der Ansatz hier ist korpusgesteuert, nicht nur korpusbasiert. „Korpusgesteuert" heißt, wir betrachten unaufbereitete Korpusdaten, fragen, wie sie auszuwerten sind, und versuchen dann, auf dieser Grundlage theoretische

Diese Arbeit wurde von dem tschechischen Bildungsministerium (MSM 0021620838) und der Grantagentur der tschechischen Republik (P406/2010/0875) als Teil einer korpusgesteuerten Untersuchung von lexikalischen Mustern am Institut für Formale und Angewandte Linguistik, Karls-Universität zu Prag, unterstützt. 
Schlüsse zu ziehen. „Korpusbasiert“ bedeutet, dass man von einigen a prioriAnnahmen ausgeht und diese dann mit Korpus- oder Internetdaten vergleicht, um stützende Beweise für die Hypothesen zu finden.

\section{Valenzen}

Oft entstehen durch Valenzen semantische Unterschiede. Das englische Verb shower beispielsweise hat zwei Bedeutungen: ,duschen' und ,überschütten'. Von den Verben in den folgenden Beispielen ist das erste intransitiv, mit nur einem Argument, ${ }^{2}$ dem Subjekt ((1); A). Das zweite hat drei Argumente, und ist mit zwei verschiedenen Mustern ((2), (3); B, C) assoziiert.

(1) He showered and dressed quickly.

(2) He showered her with kisses.

(3) He showered kisses on her. ${ }^{3}$

Zwischen (2) und (3) gibt es fast keinen Bedeutungsunterschied, sondern höchstens einen unterschiedlichen Fokus. Die unterliegenden Valenzmuster von (1)-(3) sind:

A. $[\mathrm{NP}]$ shower.

B. $\left[\mathrm{NP}_{1}\right]$ shower $\left[\mathrm{NP}_{2}\right]$ w with $\left[\mathrm{NP}_{3}\right]$ \}.

C. $\left[\mathrm{NP}_{1}\right]$ shower $\left[\mathrm{NP}_{3}\right]$ on $\left.\left[\mathrm{NP}_{2}\right]\right\}$.

Wenn man die Häufigkeiten vergleicht, mit der die jeweiligen Muster auftreten, so fällt auf, dass die große Mehrheit der Korpusbeispiele den Sinn ,überschütten' hat: In einer Stichprobe von 100 Zufallsbeispielen aus dem British National Corpus (BNC) finden sich lediglich 16 mit der Bedeutung ,duschen'.

Das Verb shower lässt jedoch noch ein weiteres Valenzmuster zu, und zwar D:

D. $\left[\mathrm{NP}_{1}\right]$ shower $\left[\mathrm{NP}_{2}\right]$.

Obwohl intuitiv sofort nachvollziehbar, kommt die Variante D sehr selten vor, im BNC (einem Korpus von immerhin einhundert Millionen Wörtern) sogar überhaupt nicht. Käme diese Version tatsächlich vor, würde man (im Englischen) erwarten, dass das direkte Objekt ein Kind oder ein Hund ist, wie in (4).

(4) She showers the dog every Sunday.

In diesem Beitrag bedeutet, Valenz' die gesamte Argumentstruktur und ,Argument' ein Valenzelement, d.h. eine Satzrolle wie Subjekt, Objekt, oder Adverbial.

Erfundene Beispiele sind, wie hier, kursiv gedruckt, echte (aus einem Korpus entnommene) hingegen nicht. Wenn nicht anders angegeben, ist das Korpus das BNC. 
Bedeutsam ist bei diesem Verb, dass durch das Vorhandensein bzw. Nichtvorhandensein eines Adverbials (,with $\left[\mathrm{NP}_{3}\right]$ “ in (2) oder „on [ $\left.\mathrm{NP}_{2}\right]$ “ in (3)) ein Bedeutungsunterschied erzeugt wird.

\section{Einführung: Kollokationen und semantische Typen}

Soweit, so gut. Es muss jedoch angemerkt werden, dass sehr viele semantische Unterscheidungen nicht durch Valenzen, sondern durch Kollokationen entstehen. Valenzen allein genügen nicht, Bedeutungen mit Sicherheit auseinander zu halten bzw. zu charakterisieren.

Für jedes Argument eines jeden Musters jedes Wortes gibt es eine ganze Menge von Kollokationen. Die Kollokationen können, ihren semantischen Typen entsprechend, lexikalischen Paradigmen zugeordnet werden. Der theoretische Hintergrund ist die „Generative Lexicon Theory“ (GL; Pustejovsky 1995). Ein kurzer Abriss:

Die GL wurde als ein theoretischer Rahmen zur Kodierung von Wissen über selektionale Präferenzen von Wörtern und ihren Bedeutungen entwickelt. Gemäß dieser Theorie gibt es vier Arten von Ressourcen für jedes Wort:

i. Event Structure/Ereignisstruktur: definiert den „Event Type“ (Ereignistyp) des Satzes oder des Ausdrucks, z.B. Action, Process, State.

ii. Argument Structure/Ereignisstruktur: spezifiziert die Anzahl und die Art der Argumente eines Prädikats.

iii. Lexical-Type Structure/Struktur Lexikalischer Typen: definiert den semantischen Typ eines Wortes in einer hierarchischen Ontologie von Konzepten, z.B. [[Human]], [[Artifact]], [[Vehicle]], [[Concept]]

iv. Qualia Structure/Qualia-Struktur: liefert eine Basis zur strukturellen Differenzierung der prädikativen Kraft eines lexikalischen Elements. Siehe Abschnitt 10 unten.

Sehen wir uns ein Beispiel an. Fragt man, was der „Event Type“ (Ereignistyp) von shower (Verb) mit drei Argumenten ist, erhält man die Antwort: „Es kommt darauf an ...". Typischerweise findet man Geschenke ((5), (6), (9)), Lob ((7), (10), (11)) oder Tadel ((8), (12)) als direktes Objekt oder präpositionales Objekt und einen Menschen als drittes Argument (indirektes Objekt).

(5) Boris showered the woman with presents.

(6) Rather than the hoped-for cash, they were showered with snuffboxes and other trinkets, to Leopold's disgust.

(7) Lauren Bacall, Bianca Jagger ... and Lionel Blair were among the stars who showered him with praise. 
(8) if they ignore the remark or reply negatively they may be accused of rudeness and/or showered with abuse.

(9) You long to shower gifts on everyone.

(10) Chinese parents do, of course, shower love and attention on their children.

(11) European heads of government ... showered telegrams of congratulation on Clinton.

(12) Despite all the criticisms showered on this model during the past forty years, it still occupies the center of the stage.

Selbstverständlich sind typische Argumente etwas ganz anderes als notwendige Argumente. Eine Korpusanalyse zeigt, dass eine Typifikation sehr oft möglich ist, notwendige Konditionen jedoch selten vorhanden sind. Obwohl Geschenke, Lob und Tadel typisch sind, findet man in diesen Musterargumenten auch Nomen von anderen semantischen Typen, insbesondere dingliche Objekte ((13)-(15)).

(13) whistling and swearing offends them and they will shower the guilty person with pebbles and gravel until he stops.

(15) The eruption showered debris on Pompeii.

In (13) ist das Subjekt menschlich, in (15) ist es ein Ereignis. In (14) ist unklar, ob das Subjekt von „showering“" der Gegenstand (,the DC-10") ist, oder ob es ein Ereignis ist, also die Explosion. Es wäre folgerichtig anzunehmen, dass das Ereignis der „Täter" ist. Die Beispiele 14 und 15 sind interessant, da in beiden Fällen die Argumente 2 und 3 (,them“ - vermutlich Menschen; „debris“ - Fragmente; „Pompeii“ - ein Ort) mit einem Ereignis als Subjekt (Argument 1) korrelieren. Es wäre unwahrscheinlich, wenn ein Ereignis jemanden mit Lob oder Tadel überschüttete. Das Verb shower mit unbelebtem Subjekt korreliert normalerweise mit einem dinglichen Objekt. Aber die Verwandtschaft ist nicht symmetrisch, weil ein direktes dingliches Objekt sowohl mit belebten als auch mit unbelebten Subjekten korreliert. Solche Korrelationen unter Argumenten, in Church/Hanks (1990, S. 28-29) als „Triangulation“ bezeichnet, spielen eine große Rolle in der Bedeutungsbestimmung:

Despite the fact that a concordance is indexed by a single word, often lexicographers actually use a second word such as from or an equally common semantic concept such as a time adverbial to decide how to categorize concordance lines. In other words, they use two words to triangulate in on a word sense. This triangulation approach clusters concordance lines together into word senses based primarily on usage (distributional evidence), as opposed to intuitive notions of meaning. Thus, the question of what is a word sense can be addressed with syntactic methods (symbol pushing), and need not address semantics (interpretation), even though the inventory of tags may appear to have semantic values. 
The triangulation approach requires „art.“ How does the lexicographer decide which potential cut points are ,interesting“ and which are merely due to chance? The proposed association ratio score provides a practical and objective measure that is often a fairly good approximation to the ,art.“ Since the proposed measure is objective, it can be applied in a systematic way over a large body of material, steadily improving consistency and productivity.

Die beschriebenen Zusammenhänge können als Muster mit semantischen Typen wie folgt formalisiert werden:

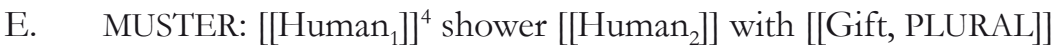
IMPLIKATUR: [[Human 1$]]$ gibt [[Human 2$]]$ viele [[Gift, PLURAL]] - Ereignistyp: Geben - [[Gift $]$ heißt allerlei Geschenke

F. MUSTER: [[Human 1$]$ shower [[Human $]$ ] with [[Physical Object, PLURAL]]

IMPLIKATUR: [[Human 1$]]$ gibt [[Human 2$]]$ viele [[Physical Object, PLURAL]]

- Ereignistyp: Werfen

G. MUSTER: [[Human 1$]$ shower [[Human 2$]]$ with [[Speech Act, MASS]] IMPLIKATUR: [[Human 1$]$ spricht [[Human $]$ ]] viel [[Speech Act, MASS]] aus.

- Ereignistyp: Sprechen

- [[Speech Act]] ist oft Lob oder Tadel

Die semantischen Typen repräsentieren „lexical sets“ - paradigmatische Wortgruppen bzw. Gruppen von lexikalischen Elementen -, die eine intrinsische semantische Eigenschaft gemeinsam haben. Im vorliegenden Kontext (als Argument des Verbs shower) ist der semantische Typ [[Speech Act]] u.a. den folgenden Substantiven gemeinsam:

praise, accolades, applause, plaudits, abuse, comments, compliments, questions, mail, blessings, telegrams, congratulations, bids, oratorical bouquets, tiny verbal blows.

Paradigmatische Wortgruppen sind nicht begrenzt: Sobald man glaubt, alle Mitglieder einer Gruppe entdeckt zu haben, gibt es immer die Möglichkeit, dass man auf ein weiteres stoßen wird. Ein lexikalisches Element darf aus mehr als einem Wort bestehen, und ein Wort darf in mehr als einer paradigmatischen Gruppe erscheinen.

Semantische Typen werden in einer „flachen Ontologie“ gespeichert (vgl. Pustejovsky/Rumshisky/Hanks 2004). Jeder semantische Typ in der Ontologie wird mit einer Gruppe bzw. einem Set von lexikalischen Elementen ausgestattet sein, basierend auf den im Korpus für jedes Muster gefundenen Beispielen.

${ }_{4} \quad$ Semantische Typen sind in doppelten eckigen Klammern angegeben. 


\section{Semantische Typen vs. Semantische Rollen in der Korpusmusteranalyse}

Die Korpusmusteranalyse (Corpus Pattern Analysis; CPA) ist eine Methode, Bedeutung auf Wortverwendungen abzubilden. Die Methode ermöglicht die systematische Analyse aller üblichen Gebrauchsmuster einer Sprache. Sie ermöglicht eine Beschreibung aller Wortmuster, die aus Valenzen und semantischen Typen bestehen, mit Verb oder Adjektiv als Angelpunkt. Bedeutungen sind eher mit Mustern als mit Wörtern assoziiert. Basierend auf dieser Methodologie ist für das Englische bereits „The Pattern Dictionary of English Verbs“" (PDEV; http://nlp.fi.muni.cz/projects/cpa) entstanden.

Für die Analyse der 700 bisher vollständig analysierten Verben braucht das Projekt „Corpus Pattern Analysis“ eine Ontologie von nur 200 semantischen Typen. Dies ist teilweise durch eine Unterscheidung zwischen Typ und Rolle möglich. Um diese Unterscheidung zu erklären, betrachten wir das englische Verb sentence. Dies hat nur ein Muster $(\mathrm{H})$.

H. MUSTER: [[Human 1$]]$ sentence $\left[\left[\right.\right.$ Human $\left.\left._{2}\right]\right]$ to $[[$ Event $=$ Punishment $]]$ IMPLIKATUR: [[Human ${ }_{1}=$ Richter] $]$ verurteilt $\left[\left[\right.\right.$ Human $_{2}=$ Verbrecher] $\mathrm{zu}[[$ Event $=\{$ Gefängnisstrafe $\mid \operatorname{Tod}\}]]$ BEISPIEL: $\{M r W o o d s\}$ sentenced $\{$ Bailey\} to $\{$ seven years $\}$.

Nichts in der intrinsischen Semantik der NPs $M r$ Woods und Bailey bedeutet ,Richter' oder ,Verbrecher', und nichts in der intrinsischen Semantik der NP seven years bedeutet, Gefängnisstrafe'. Diese Ausdeutungen sind semantische Rollen, die vom Kontext zugewiesen werden. Insbesondere die semantischen Rollen der Nomen werden in einem Satz vom Verb zugewiesen.

Semantische Typen sind in doppelten eckigen Klammern geschrieben. Semantische Rollen, wenn vorhanden, sind nach einem Gleichheitszeichen geschrieben.

\section{Das Idiomprinzip}

Nach Sinclair $(1991,1998)$ gibt es im Wortgebrauch eine Spannung zwischen einer „terminological tendency“ (terminologischen Tendenz) und einer „phraseological tendency“ (phraseologischen Tendenz). Die terminologische Tendenz ist die Tendenz von Wörtern, in Isolation eine Bedeutung zu tragen. Die phraseologische Tendenz ist die Tendenz von Wortbedeutungen, im Gebrauchskontext aktiviert zu werden. Diese Spannung nennt Sinclair „the idiom principle“ (das Idiomprinzip). Er stellt sich vor, dass zwei grundlegende semantische Tatsachen („two basic facts") durch die traditionelle Struktur eines Wörterbuchs (Eintrag - Erklärung - Beispiel) verdeckt werden (1998): 
(a) many, if not most, meanings require the presence of more than one word for their normal realization;

(b) patterns of co-selection among words, which are much stronger than any description has yet allowed for, have a direct connection with meaning.

Diese Spannung ist in vielen alltäglichen Verben und Adjektiven zu beobachten. Im Folgenden werde ich dies am Beispiel des Verbs admit nachvollziehen. Terminologisch betrachtet hat dieses Verb nur zwei Hauptbedeutungen: ,zulassen' und ,eingestehen'. Zusätzlich gibt es eine Vielzahl verschiedener Implikaturen. Die Hauptbedeutungen von ,admit a child into care“ ((16)) und ,admit a country to the United Nations“ ((17)) entsprechen einander (,aufnehmen', d.h. eine Art von ,zulassen'), aber die Nebenbedeutungen gleichen sich nur teilweise; in einigen Hinsichten sind sie sogar sehr verschieden. In beiden Fällen gibt es Implikaturen von bürokratischen Verfahren, Amtsschimmel und Schreiberei, aber in (16) ist impliziert, dass die Kinder krank oder in Gefahr sind, und „care“ heißt in diesem Falle Jugendamtsaufsicht, nicht etwa Sorgsamkeit. In (17) dagegen darf man annehmen, dass die in die Vereinten Nationen eintretenden Baltischen Staaten nach langen Jahren der Unterdrückung ,gesund“ und stabil geworden sind.

... the proportion of older children admitted into care.

The Baltic nations were admitted to the United Nations [in 1991].

Es stellt sich hier die Frage, wie viele lexikografische Unterscheidungen man treffen sollte, und wie fein diese gegliedert sein sollten. Auf diese Frage gibt es keine einzelne einfache Antwort. Die Entscheidung bleibt dem Lexikografen überlassen, der alle Umstände, einschließlich Zweck und Ziel der Arbeit, beachten muss.

Ich zeige hier die prinzipiellen Unterscheidungen in PDEV, mit Häufigkeiten, Implikaturen und Beispielen.

\section{HAUPTBEDEUTUNG: zulassen}

A. $10 \%$

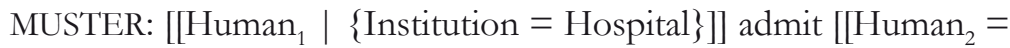
Patient $]]$ (to $[[$ Institution $=$ Hospital $]] \mid$ into $\{$ care $\})$ for $[[$ Activity $=\{$ Care $\mid$ Treatment $\}]]\}$

IMPLIKATUR: [[Human $]$ ] führt bürokratische Maßnahmen zur Zulassung von [[Human $]$ ] ins [[Institution = Hospital]] aus

BEISPIEL: The hospital admitted 35 people, four of whom had serious injuries. 
B. $18 \%$

MUSTER: [[Human 1 | Institution 2]] admit [[Human 2 | Institution 2]]

IMPLIKATUR: [[Human 1 | Institution]] führt bürokratische Maßnahmen zur Zulassung von [[Human 2]] als Mitglied von [[Institution]] aus.

BEISPIEL: The number of working-class boys admitted to grammar schools was disproportionately small | Spain and Portugal were admitted as new members of the DAC.

C. $3 \%$

MUSTER: $[[\{$ Human $=$ Judge $\} \mid\{$ Institution $=$ Court $\}]]$ admit $[[$ Anything $=$ Evidence]]

IMPLIKATUR: Law. [[Human = Judge]] lässt zu, dass [[Anything]] offiziell als Beweismaterial betrachtet wird.

BEISPIEL: Israel's Supreme Court refused to admit evidence allegedly showing that Demjanjuk had been the victim of mistaken identity.

\section{HAUPTBEDEUTUNG: eingestehen}

D. $44 \%$

MUSTER: [[Human 1]] admit [NO OBJ] (to [[Human 2]]) \{[thatCLAUSE $\mid$ QUOTE $=$ Bad] $]\}$

IMPLIKATUR: [[Human 1]] äußert widerstrebend, dass [[Eventuality $=$ Bad]] geschehen ist.

BEISPIEL: General Hakim admitted that the real problem will be fuel.

E. $8 \%$

MUSTER: [[Human]] admit [[Eventuality]]

IMPLIKATUR: [[Human]] äußert widerstrebend, dass [[Eventuality]] wahr ist

BEISPIEL: Some people never do admit defeat.

F. $8 \%$

MUSTER: [[Human]] admit $\{-$ ING $\}$

IMPLIKATUR: [[Human]] äußert widerstrebend, dass [[Human]]

$[[$ Action $=$ Bad $]]$ begangen hat.

BEISPIEL: She admitted causing the dog unnecessary suffering.

G. $4 \%$

MUSTER: [[Human] $]$ admit [[Action $=$ Bad $]]$

IMPLIKATUR: [[Human]] äußert widerstrebend, dass er/sie [[Action

$=\mathrm{Bad}]]$ begangen hat.

BEISPIEL: He admitted eight robberies. 
H. $2 \%$

MUSTER: [[Human | Institution]] admit [NO OBJ] \{to [-ING]\}

IMPLIKATUR: [[Human | Institution]] äußert widerstrebend, dass er, sie oder es [[Action = Bad] ] begangen hat.

BEISPIEL: Will people admit to having committed criminal offences, even if they are guaranteed confidentiality?

An den Mustern D-H kann man erkennen, dass ein und dieselbe Implikatur mit mehreren unterschiedlichen Mustern verknüpft sein kann.

Sehen wir uns nun ein Beispiel einer ,terminologischen“ Bedeutung an. Auf den ersten Blick erscheint es vielleicht sinnvoll, noch ein weiteres Muster unter der Hauptbedeutung, zulassen`zu subsumieren, nämlich I.

I. MUSTER: [[Aperture]] admit [[Physical Object | Stuff]] IMPLIKATUR: [[Aperture]] lässt [[Physical Object | Stuff]] in [[Location]]

Ohne Zweifel ist I idiomatisch wohlgeformt, aber es repräsentiert kein phraseologisches Muster, sondern eine terminologische Bedeutung. Warum? Weil diese Verwendung sowohl selten ist als auch einen hohen Grad semantischer Allgemeinheit aufweist. Obwohl selten, lassen sich doch einige Beispiele mit dieser Struktur finden (z.B. (18)). Solche Beispiele repräsentieren eine terminologische Verwendung des Verbs, nicht eine phraseologische Norm, vgl.:

(18) The skylights in the galleries admit light through angled screens.

\section{CPA-Vorgehensweise}

Die CPA (Corpus Pattern Analysis; ,Korpusmusteranalyse) ist eine empirische Methode zur semantischen Analyse, die methodische Grundlage von PDEV und ähnlichen Projekten für andere Sprachen. CPA konzentriert sich auf Verben, denn das Verb ist der Angelpunkt des Satzes.

Zur effizienten Korpusmusteranalyse empfiehlt es sich, zuerst in „Sketch Engine“ (Kilgarriff et al. 2004) die statistisch signifikanten Kollokate eines Verbs aufzurufen und zu untersuchen. Wenn ein Kollokat eine bestimmte Bedeutung des Verbs aktiviert, werden alle Korpuszeilen mit dieser Bedeutung einem Muster zugeordnet, d.h. mit der Nummer des entsprechenden Musters getaggt. Aktiviert ein Kollokat mehr als eine Bedeutung, so wird das Verb mit so vielen Musternummern wie nötig getaggt.

Als zweiten Schritt erstellt man zufallsgeneratorisch ein Subkorpus von Beispielzeilen und taggt jede Zeile im Subkorpus nach einer der folgenden Kategorien: 
- Musternummer ${ }^{5}$ ODER

- Abweichung (Exploitation ${ }^{6}$ ) von einem vorhandenen Muster ODER

- Auszuschließen (Zitat, Namensteil, Tagging-Fehler, oder einfach unklar).

Wenn man eine Konkordanz das erste Mal öffnet, springen sofort verschiedene Muster ins Auge. Je länger man hinsieht, desto mehr Muster erkennt man. Die Muster basieren auf Kollokationen. Um zu sehen, wie die Kookkurenzen von Wörtern Bedeutungen kreieren, müssen wir Kollokationen analysieren. Kollokationen neigen jedoch zur Variabilität, und wenn man versucht, diese Muster zu formalisieren, erkennt man mehr und mehr Ausnahmen. Dabei sind die Grenzen zwischen Mustern und Ausnahmen verwischt. Die meisten Verwendungen eines Wortes können einem Muster zugewiesen werden, aber es gibt fast immer einige (ca. 10\%), die ungewöhnlich sind.

\section{Was ist ein Muster?}

Ein Muster ist eine Festschreibung der Struktur eines Satzes (Valenz) zusammen mit den typischen semantischen Werten (Typen und Rollen) jedes Arguments, realisiert von salienten Kollokaten. Oft aktivieren verschiedene semantische Werte von Argumenten verschiedene Bedeutungen jedes Verbs.

Muster sind kontrastiv, d.h., dass zwei verschiedene Muster eines Verbs in ihrer Argumentstruktur identisch sein und dennoch verschiedene Bedeutungen tragen können. Der Unterschied hängt in solchen Fällen von den semantischen Typen eines oder mehrerer der Argumente ab.

Das Verb fire, zum Beispiel, kommt in mehr als 15 Mustern vor. Die fünf häufigsten im PDEV sind die folgenden:

i. $\quad[[$ Human $]]$ fire [[Firearm] $]$ (at [[Phys Obj $=$ Target $])$

ii. [[Human]] fire [[Projectile]] (from [[Firearm]]) (at [[Phys Obj = Target]])

iii. [[Human 1 | Institution]] fire [[Human 2]]

iv. [[Anything]] fire [[Human]] \{with enthusiasm\}

v. [[Human]] fire [NO OBJ] .... (= i oder ii, aber nicht iii oder iv)

Neue Muster - und ihre Nummern - werden nach Bedarf hinzugefügt.

Der Begriff „Exploitation“ (Abweichung) wurde zuerst von dem Philosophen H. Paul Grice (1975) benutzt. Er bezeichnet eine Methode der Bedeutungsvermittlung, bei der man darauf verzichtet, explizit zu sagen, was man meint. Ich verwende den Begriff „Abweichung“ im Zusammenhang mit Wortwahl und Musterbedeutung. Sprachbenutzer weichen zu verschiedenen Zwecken von den Regeln normalen Sprachgebrauchs ab, und zwar aus folgenden Gründen:

a) Um sprachlich schnell und sparsam zu kommunizieren, denn Unterhaltungen werden schnell geführt, und Zuhörer (oder Leser) sind leicht gelangweilt.

b) Um Neues auszudrücken, z.B. Entdeckungsberichte, neue Hypothesen und dergleichen mehr. 
Muster $i$ und ii beschreiben den gleichen Ereignistyp, der Unterschied besteht lediglich darin, dass die Schusswaffe in i bleibt, wo sie ist, während in ii das Projektil in Bewegung gesetzt wird. In FrameNet (http://framenet.icsi. berkeley.edu; Baker/Fillmore/Cronin 2003) konstituieren diese zwei Muster ein einziges Frame mit variablen Arten, die verschiedenen Frame-Elemente zu realisieren.

Diese zwei Muster stehen in semantischem Kontrast zu iii, dessen Bedeutung „(aus einem Arbeitsverhältnis) entlassen“ ist. Muster iv stellt einen weiteren Kontrast dar, da das Subjekt nicht auf [[Human]] begrenzt ist, sondern fast alles Mögliche sein kann, z.B. kann eine Einstellung in einen Job jemanden begeistern, aber auch ein philosophischer Gedanke oder ein schönes Lied.

Muster $\mathrm{v}$ ist besonders interessant, weil die Bedeutung des Musters von der Abwesenheit eines der Argumente abhängt. Das Nichtvorhandensein des Arguments bezeichnet man als Ellipse. Die Ellipse ist nur unter bestimmten, bislang leider noch unbekannten Umständen als Alternation zuzulassen. Den Gebrauch eines Verbs mit einem elliptischen oder abwesenden Argument könnte man als ein eigenes Muster bezeichnen. He fired kann beispielsweise nur bedeuten: ,He caused [[Firearm]] to discharge [[Projectile]]'; und nicht: ,Er entließ einen/mehrere Arbeitnehmer.

\section{Die Hypothese von der linguistischen ,Doppelhelix ${ }^{6}$}

Nach Hanks (1994) haben Wörter strenggenommen keine Bedeutung, sondern Bedeutungspotenzial. Verschiedene Aspekte dieses Potenzials werden in verschiedenen Kontexten realisiert, entweder durch regelgeleitete Verwendung oder durch Regelverletzung. Die Regelverletzung ist selbst auch regelgeleitet.

Daran kann man erkennen, dass eine Sprache ein System von regelgeleitetem Verhalten ist. In diesem System gibt es nicht ein, sondern zwei miteinander verbundene, interaktive Sets von Regeln:

a) Regeln, die den Normalgebrauch von Wörtern regulieren;

b) Regeln, die alle Abweichungen von der Norm (also Regelverletzungen) regulieren.

Beide Arten von Regeln stellen Wahrscheinlichkeiten, nicht Notwendigkeiten, fest.

Jedes Wort wird in einem oder mehreren Gebrauchsmustern (Valenz + Kollokationen) verwendet. Jedes Muster ist mit einer Bedeutung verbunden, wobei eine Bedeutung ein Set von prototypischen Annahmen ist. In der CPA werden Bedeutungen als, verankerte Implikaturen' ausgedrückt. Die Verankerung wird durch Wiedergabe eines oder mehrerer der seman- 
tischen Typen in der Implikatur bewirkt. Um dies zu erklären, betrachten wir noch einmal das Muster und die Implikatur für das Verb fire. Wie man erkennen kann, ist die Implikatur eine metasprachliche ${ }^{7}$ Paraphrase der Bedeutung des Musters.

MUSTER: [[Human]] fire [[Firearm]] (at [[Phys Obj = Target]]) IMPLIKATUR: [[Human]] bedingt [[Firearm]], ein [[Projektil]] in Richtung [[Phys $\mathrm{Obj}=$ Target] $\mathrm{zu}$ bewegen.

Dieses Muster ist durch Wiedergabe der drei Argumente - [[Human]], [[Firearm]], und [[Phys Obj]] - in seiner Implikatur verankert. Die Argumente erscheinen in der Implikatur und im Muster. Es kann auch Argumente geben (hier: [[Projektil]]), die im Muster nicht ausdrücklich realisiert sind, sondern nur insinuiert. Umgekehrt gibt es auch Muster, die ein Argument enthalten, das in der Implikatur nicht erscheint. Dies ist insbesondere für idiomatische Ausdrücke wie grasp the nettle der Fall. Eine nettle (Nessel) ist eine Pflanze, aber wenn man von ,grasping the nettle“ spricht, redet man nicht von Pflanzen.

MUSTER: [[Human | Institution]] grasp $\{\text { nettle }\}^{8}$

IMPLIKATUR: [[Human | Institution]] fasst einen kühnen Entschluss

Folglich kann man in der CPA einen idiomatischen Ausdruck als ein schlecht verankertes Muster definieren.

Nur wenige Verbmuster sind mit mehr als einer Bedeutung verbunden. Umgekehrt jedoch sind viele Bedeutungen manchmal durch ein ganze Menge von Mustern realisiert, wie schon am Verb admit festgestellt werden konnte.

Ein ähnlicher Analyseapparat ist für Adjektive benutzbar. Für prädikative Adjektive ist der Apparat derselbe (mit Einführung einer Kopula) wie für Verben.

MUSTER: [[Human]] be afraid \{of [[Anything]]\}

IMPLIKATUR: [[Human]] fürchtet [[Anything]]

Für attributive Adjektive muss man Kopfnomen kategorisieren, um verschiedene Bedeutungen zu realisieren:

MUSTER: green [[Phys Obj]]

IMPLIKATUR: [[Phys Obj]] ist grün(farben)

MUSTER: green [[Human]]

IMPLIKATUR: [[Human]] ist unerfahren

Für alle Fälle - Verben wie Adjektive - gilt, dass die Verbindung zwischen Muster und Bedeutung stets prototypisch und statistisch messbar ist.

Die Metasprache kann jede beliebige Sprache sein (hier: Deutsch).

Geschweifte Klammern enthalten lexikalische Elemente. Sie sind auch als einfache gruppierende Methode benutzt. 


\section{Korpusanalyse von Nomen}

Der Apparat, den man für die Analyse von Nomen braucht, unterscheidet sich von dem, der für Verben und Adjektive benutzt wird, genauso wie sich ein elektrischer Anschluss von einer Steckdose unterscheidet. Verben sind eng mit Kontext verbunden, aber Nomen sind typischerweise vielmehr auf die Welt bezogen. Daher ist für Nomen die Analyse von Eigenschaften nötig, während Verben Ereignistypen und Argumentstrukturen benötigen.

Many thousands of species of spiders are known (funnel-web, web-building, orb-weaving, bird-eating, ground-dwelling, giant, buge, large, tiny, poisonous, black widow, camel, redback, trapdoor, wolf, whitetail, crab. tarantula, etc.).

Some species of spiders bunt prey.

Spiders bite.

Some species of spiders are poisonous.

Many species of spiders spin webs, with threads of strong silk.

Spiders lurk in the centre of their webs.

Spiders control what is going on in their webs.

Spiders have eight legs.

Their legs are thin, hairy, and long in proportion to body size.

Spiders have eight eyes.

Spiders spend a lot of time being motionless.

Spiders' movement is sudden.

Spiders crawl.

Spiders scuttle.

Spiders are swift and agile.

Spiders can run up walls.

Many people have a dread of (hate) spiders.

People kill spiders.

In folk taxonomy, scorpions and cockroaches are often classified together with spiders as creepy-crawly creatures.

Abb. 1: Ein korpusbasiertes kognitives Profil für das englische Nomen spider

Nehmen wir das Nomen spider (Spinne). Eine statistische Analyse zeigt, dass scorpion (Skorpion) und cockroach (Kakerlake) zwei der signifikantesten Kollokate von spider sind. Als Kollokate stehen sie jedoch in keinerlei besonderer grammatischer Verwandtschaft zu spider. Sie finden sich an allen möglichen Stellen in einem Fenster von fünf Wörtern links und rechts von 
spider. Solche Korpusbelege gestatten es, für jedes Nomen, das eine Objektklasse denotiert, ein „kognitives Profil““ (oder kognitiven Prototyp; Abb. 1) zu konstruieren. Die Kollokate schließen auch Verben ein, die typischerweise mit spider auftreten, sind aber nicht auf diese beschränkt. Das Profil in Abbildung 1 basiert auf Korpusdaten aus dem BNC (British National Corpus) und dem OEC (Oxford English Corpus; 1,5 Milliarden Wörter).

Ein Profil wie dieses hat natürlich nichts mit der wissenschaftlichen Klassifikation von Konzepten zu tun; in gewissem Maße können die zwei Arten der Analyse sogar inkompatibel sein. Andererseits ist ein Profil wie dieses hilfreicher für das Verständnis der Mentalität/Geisteshaltung von Englischsprechern in Bezug auf Spinnen als Informationen, die in traditionellen englischen Wörterbüchern Spinne als ,arachnid“ definieren.

Ein kognitives Profil wie Abbildung 1 ist mit dem so genannten „lexical conceptual paradigm“ (lcp) kompatibel. Nach Pustejovsky (1995) ist ein lcp

useful for capturing the systematic ambiguities that are so pervasive in language. [...] Nouns such as newspaper appear in many semantically distinct contexts, able to function sometimes as an organization, a physical object, or the information contained in the articles within the newspaper.

Für jedes Argument eines Verbs sind nur einige Teile des gesamten lcp irgendeines Nomens relevant. Und in den meisten Fällen wird das Argument eines Verbs von irgendeinem einer großen Anzahl von Nomen realisiert; zusammen konstituieren diese Nomen ein paradigmatisches lexikalisches Set.

\section{Qualia}

Ein weiterer relevanter Aspekt der Nomenbedeutung wird von Pustejovsky als Qualia (Mehrzahl; Einzahl quale) bezeichnet. Dies sind aristotelische Eigenschaften von Konzepten, insbesondere von Nomenkonzepten. Es gibt die folgenden Qualia:

a) Formales Quale: Was für eine Art von Ding ist es?

b) Telisches Quale: Wofür ist es?

c) Konstitutives Quale: Woraus besteht es?

d) Agentives Quale: Was ist sein Ursprung?

UND (hiermit von mir vorgestellt):

e) das Axiologische Quale: Ist es gut oder schlecht? (und für wen?)

Das axiologische Quale bedarf der Erklärung. Viele Wörter haben positive/ neutrale/negative Implikationen; incite, z.B., hat normalerweise eine negative Konnotation. Obgleich encourage ein Fast-Synonym von incite ist, stellt sich bei einem Vergleich der beiden Verben heraus, dass encourage axiologisch neutral bis positiv ist, während incite axiologisch negativ ist. 
- You incite people to do bad things.

- You can encourage someone to write a dictionary (neutral value judgement), but if you say that I incited someone to write a dictionary, I'm implying that writing dictionaries is a bad thing to do.

Louw (1993) nennt dieses Phänomen „semantische Prosodie“.

Die semantisch-prosodischen Eigenschaften können etwas kompliziert werden. Ein Beispiel ist das Verb pander:

MUSTER: [[Human 1]] pander to [[Emotion = Bad | Human 2]]

IMPLIKATUR: [[Human 1]] gibt [[Emotion = Bad]] von [[Human 2]] nach

BEISPIELE: newspapers are pandering to people's baser instincts. ... we pander to his every whim ... we pander to our superiors while neglecting our subordinates.

ETYMOLOGIE: von Pandarus, Name eines Charakters in Chaucers Troilus and Criseyde

Daher sehen wir, dass dieses Verb eine besondere axiologische Komplexität zeigt. Der Gebrauch des Verbs pander to (im Vergleich zu gratify, please, oder indulge) impliziert:

- aus Sicht des SPRECHERS: pandering ist eine schlechte Handlung.

- aus Sicht des SPRECHERS: die Einstellung oder Emotion des PATIENTEN ist schlecht.

- aus Sicht des AGENS (the panderer): pandering kann gute Konsequenzen haben, obwohl der AGENS glaubt, dass die Einstellung/Emotion des PATIENTEN schlecht ist.

- PATIENT (the pandered-to) hat keine Meinung diesbezüglich.

Das präpositionale Objekt (nach to) kann zwei semantische Typen haben: [[Human]] oder [[Emotion]]. Beide sind jedoch immer impliziert; Selektion ist eine Sache des Fokus. Eine solche Alternation kommt in der Argumentstruktur sehr häufig vor.

Auch die Etymologie ist in diesem Falle relevant, weil jemand, der „pandert", sich wie der Charakter bei Chaucer verhält und nicht wie ein griechischer Held.

\section{Abweichungen}

Nicht alle Korpuszeilen können einem Muster zugeordnet werden. Eine Sprache bietet ihren Nutzern immer die Gelegenheit, kreativ zu sein. Betrachten wir zuerst das Idiom to clutch at straws. Sein Ursprung ist das Sprichwort, a drowning man will clutch at a straw. Es bedeutet, dass jemand verzweifelt nach Lösungen für ein Problem sucht, obwohl diese höchstwahrscheinlich nicht effektiv sein werden. 
(19) Ms Magee said: „Keith is clutching at straws in an effort to win the argument".

(20) Lebanese members of parliament clutched at the threads of a peace plan yesterday after a brief period when agreement to end 14 years of civil war seemed within their grasp.

(19) repräsentiert einen normalen Gebrauch des Idioms in seiner kanonischen Form. In (20) wird von dieser kanonischen Form abgewichen - es findet sich in diesem Beispiel keine explizite Realisierung des Wortes straw, dennoch ist das Idiom im Hintergrund versteckt; dafür sprechen das konative at und die Tatsache, dass die libanesischen Parlamentarier nicht im wörtlichen Sinne versuchen, ein dingliches Objekt zu ergreifen. Sie werden als ertrinkende Männer dargestellt, und ,the threads of a peace plan' sind ihre unwirksamen Strohhalme. Die Resonanz des Idioms wird gründlich genutzt, ${ }^{9}$ obgleich eines der wichtigsten lexikalischen Elemente nicht genannt wird. Die Bedeutung des Satzes wird dennoch klar genug, sogar für jemanden, der das Idiom nicht kennt.

Eine Abweichung ist eine absichtliche Abänderung eines etablierten Musters. Sie wird verwendet, um entweder über neue oder ungewöhnliche Dinge/Ereignisse zu sprechen, oder um Bekanntes auf neue, interessante oder unkonventionelle Art auszudrücken. Abweichungen sind Teil der natürlichen Tendenz von Menschen, mit Sprache zu spielen, aber sie sind auch in anderen Situationen zweckdienlich, insbesondere in solchen, in denen es keine etablierte Art und Weise gibt, über eine bestimmte Situation, ein bestimmtes Ereignis oder einen bestimmten Gegenstand zu sprechen.

Man kann im Hinblick auf Abweichungen mindestens vier Punkte anmerken:

i. Abweichungen sind selten im Vergleich zu Mustern. Der Gedanke an einen Politiker, der nach dem sprichwörtlichen Strohhalm greift, ist viel einprägsamer als der Gedanke an einen Politiker, der eine Rede hält.

ii. Abweichungen sind kognitiv salient. Soziale (oder statistische) Salienz lässt sich als häufige Benutzung definieren (oder ist als solche erkennbar). Ist ein Ausdruck kognitiv salient, so lässt er sich leicht ins Bewusstsein/ Gedächtnis zurückrufen.

iii. Aus diesem Grund sind Abweichungen rhetorisch effektiv. Wie Quintilian und andere Rhetoriker der klassischen Antike vor langer Zeit feststellten, sind Metaphern und andere Figuren (von denen viele phanta-

\footnotetext{
Resonanz gilt in der Metapherntheorie als eines der Kriterien für Metaphorizität, vgl. Hanks (2010, S. 140-141): , ,...] if one sense of an expression resonates semantically with another sense, it is metaphorical, and if there is no such resonance, it is literal" sowie Hanks (2006, S. 20): ,[.... secondary senses that are classed as metaphors ,resonate“ (Black 1962) with some other term (the primary subject) in the immediate context in a text".
} 
sievolle Abweichungen von Normen sind) viel einprägsamer als der alltägliche Sprachgebrauch, daher ermutigten sie ihre Schüler, diese Figuren zu nutzen.

iv. Sie machen den Terminus ,selektionale ,Restriktionen“ unsinnig. So etwas wie selektionale Restriktionen gibt es nicht; es gibt nur selektionale Präferenzen. Abweichungen sind völlig wohlgeformte, bedeutungsvolle und beabsichtigte Benutzungen von Sprache, obwohl sie außerhalb der selektionalen Präferenzen eines Wortes liegen. Sie werden nicht durch Restriktionen unmöglich gemacht. Ein Problem der Wortanalyse ist die unscharfe Abgrenzung zwischen Normen und Abweichungen; einige Wortverwendungen sind üblicher - normaler - als andere.

Das Bild wird verkompliziert durch verschiedene andere Faktoren, insbesondere:

v. Manchmal wird eine gelungene Abweichung von anderen Sprachbenutzern aufgegriffen und etabliert sich als Norm. Eine Abweichung von heute könnte eine Norm von morgen sein.

vi. Domänenspezifische Normen (z.B. der Jargon eines bestimmten Sportes oder von Sportjournalisten) finden sich in allgemeinen Korpora und sind selbst exploitierbar, sobald sie sich etabliert haben. Ausdrücke wie climb above the defenders und fire the ball into the back of the net sind nicht nur in Fußballjournalismus und Sportreportagen großer Zeitungen zu finden; sie könnten aufgegriffen und in Kontexten außerhalb des Sports metaphorisch genutzt werden.

vii. Es gibt keine klare Grenze zwischen Normen und Abweichungen. Manche Äußerungen sind extrem normal, andere sind Abweichungsextreme. Es gibt eine große Grauzone, in der einige Äußerungen weniger normal sind als andere. Eine Abweichung von einer Norm kann ein Einzelfall sein, oder sie kann sich als text- oder domänenspezifische Abweichung etablieren.

Das Ausmaß, in dem eine lexikalische Norm offen ist für Abweichungen, variiert von semantischem Typ zu semantischem Typ und von Wort zu Wort. Das Phänomen der Abweichung ist ein zentraler Aspekt der kreativen, dynamischen Natur von Sprache, so dass es nicht überrascht, dass es sich schwer festmachen lässt. Das Nichterkennen des Phänomens aber hat in der Vergangenheit dazu geführt, dass der Untersuchungsgegenstand Sprache noch komplizierter erschien als er ohnehin schon ist.

Eine Sprache besteht aus einem System von sich stets bewegenden und entwickelnden Regeln, die das linguistische Verhalten steuern: normaler Sprachgebrauch auf der einen Seite, Abweichungen von normalem Sprachgebrauch auf der anderen. 
Ein Hörer oder Leser kann zwischen einer echten neuen Abweichung und einer seltenen Norm letztendlich nicht unterscheiden. Ein Ausdruck, der dem Hörer unerhört neu und anschaulich vorkommt, kann in diesem Moment das erste Mal geprägt worden sein. Andererseits könnte der Sprecher diesen Ausdruck schon lange verwendet haben, er könnte ihn vor kurzem aufgeschnappt haben, oder der Ausdruck könnte Teil eines konventionellen Vokabulars einer (dem Hörer) unvertrauten Domäne sein. Dies ändert jedoch nichts daran, dass von den Normen einer Sprache dynamisch abgewichen werden kann und abgewichen wird. Es wäre vielleicht sinnvoll, von Sprecher-/Schreibernormen und Zuhörer-/Leserabweichungen zu sprechen.

\section{Verschwommenheit}

Die Grenzen aller linguistischen und lexikalischen Kategorien sind verschwommen. Es gibt daher zahllose schwierige Grenzfälle. Anstatt über Grenzen zu streiten, sollten wir jedoch Prototypen identifizieren. AnschlieBend wird man in der Lage sein, zu entscheiden, was mit was korreliert. Viele Entscheidungen werden offensichtlich sein, während man einige Entscheidungen - insbesondere bezüglich der Grenzfälle - willkürlich treffen müssen wird.

Die Korpuslinguistik hat gezeigt, dass natürliches linguistisches Verhalten stark geregelt ist - und trotzdem verschwommen. Viele Muster sind entdeckt worden; andere werden ohne Zweifel noch entdeckt werden. Alle bisherigen theoretischen Spekulationen müssen im Licht der neuen Korpusdaten neu bewertet werden. Gibt es dennoch einen Daseinszweck für spekulative Linguistik? Meine Antwort: Ja, sofern man sich auf die Interpretation von Daten beschränkt.

- Kontrastive negative Beispiele („Man darf nicht X sagen“) können durch korpuslinguistische Methoden nicht entdeckt werden.

- Sprache ist ein analoges System - oder eine verwirrende Mischung von Analogie und Prädikatenlogik.

- Außerhalb mathematischer Logik gibt es keine scharfen Grenzen.

- Kollokationen sind analoge Sets.

- Kollokationen können - und müssen - gemessen werden, wenn wir die Grundlagen von Bedeutung verstehen wollen.

- Bedeutungen sind holistisch - der Feind ist geistlose Kompositionalität (bzw. grammatikalischer Reduktionismus).

Die meisten lexikalischen Sets sind nicht stabil. Hanks/Jezek (2008) und Jezek/Hanks (2010) bezeichnen sie als schimmernde lexikalische Sets. Ein Beispiel:

MUSTER: [[Human]] attend [[Event]] 
In diesem Beispiel ist [[Event]] ein schimmerndes lexikalisches Set. Das „Event“ bzw. Ereignis könnte z.B. meeting (Treffen), wedding (Hochzeit), funeral (Beerdigung) usw. sein. Aber nicht alle Ereignisse gehören zu diesem Set in diesem Kontext. Ereignisse, die nicht zum lexikalischen Set des Verbs attend gehören, sind z.B. thunderstorm (Gewitter) und suicide (Selbstmord) - um nur zwei zu nennen. Auf der anderen Seite passen nicht nur Ereignisse in dieses Paradigma, sondern auch Orte, wo relevante Ereignisse stattfinden; vgl. attend school, attend a clinic. School und clinic bezeichnen Institutionen oder Orte, nicht Ereignisse. Allerdings: Man ,attends“ a school or clinic, um an den Ereignissen, die dort stattfinden, teilzunehmen.

\section{Schlussbemerkung}

Die Korpusmusteranalyse (CPA) ist eine Forschungsmethode zur Untersuchung von Valenzen und Kollokationen. Ein Ziel des CPA-Projektes ist es, die Analyse von Valenzen und semantischen Typen von Verbargumenten zusammenzubringen, um eine Ressource zu schaffen, mit Hilfe derer man Textbedeutung auf Wortverwendung abbilden kann. Diese Ressource besteht aus einem „Muster-Wörterbuch“, zunächst von englischen Verben. Derzeit wird an entsprechenden Projekten für italienische (Pavia) sowie spanische Verben (Universitat Pompeu Fabra, Barcelona) gearbeitet.

Ein weiteres Ziel der Korpusmusteranalyse ist es, Bedeutungstheorie empirisch besser zu begründen. Korpusbelege zeigen, dass die meisten Benutzungen eines Wortes sich an ein Muster anpassen. Die Sprachregularitäten sind viel regelmäßiger als man vor Anfang der Korpusanalyse vorhergesehen hatte. Einige Wortverwendungen sind jedoch sehr unregelmäßig - viel unregelmäßiger als man vor Anfang der Korpusanalyse vorhergesehen hatte: Es handelt sich um Abweichungen.

Bedeutungen sind eher mit Mustern assoziiert als mit einzelnen Wörtern. Ein „Muster-Wörterbuch“ beschreibt das Set von Mustern für jedes Verb, und jedes Muster ist mit einer Implikatur verbunden, die die Bedeutung des Musters als ganzes wiedergibt. Die folgenden Elemente konstituieren ein Muster:

- das Verb selbst;

- die Argumente, mit denen es normalerweise auftritt.

Jedes Argument wird entweder als ein lexikalisches Element realisiert oder als ein Paradigma von lexikalischen Elementen. Solche Paradigmen haben gewöhnlich eine semantische Eigenschaft gemeinsam, die als semantischer Typ dargestellt werden kann. In Bezug auf die Qualia-Struktur handelt es sich meist um das „Formale Quale“. 
Semantische Typen werden in einer hierarchischen Ontologie gespeichert. Für die Analyse von 700 englischen Verben hat sich eine flache Ontologie von 200 Elementen als ausreichend herausgestellt. Diese kleine Anzahl ist aus zwei Gründen möglich:

1) Es ist möglich, die lexikalischen Elemente aufzulisten, ohne semantische Typen zuzuordnen, und diese Möglichkeit wird oft genutzt.

2) Semantische Eigenschaften von Nomen, die ihm von dem Verb zugewiesen werden, mit dem es kookkuriert, werden als semantische Rollen ausgedrückt (z.B. ,Judge“).

Es gibt keine Einschränkung bezüglich dessen, was als semantische Rolle benutzt werden kann. Der Unterschied ist, dass semantische Typen intrinsische Eigenschaften eines Nomens ausdrücken, während semantische Rollen vom Kontext zugewiesen werden.

Man muss unterscheiden zwischen kanonischen Elementen in einem lexikalischen Set und ad hoc-Elementen, wie z.B. metaphorischen Wortverwendungen. Die gesamte Vorgehensweise ist analog, statistisch und probabilistisch, daher ist es keine Überraschung, dass es unerwartete und seltene Mitglieder von semantischen Typen gibt.

Die ersten Resultate deuten darauf hin, dass die Muster einen großen Einfluss auf die Bestimmung von Wortbedeutung im Text haben. Die Ergebnisse wurden von Cinkova et al. (2010) auf der Tagung „Text, Speech and Dialogue 2010“ in Brno präsentiert.

\section{Literatur}

Baker, Collin F./Fillmore, Charles J./Cronin, Beau (2003): The structure of the Framenet database. In: International Journal of Lexicography 16, 3, S. 281-296.

Black, Max (1962): Models and metaphors: studies in language and philosophy. Ithaca.

Church, Kenneth W./Hanks, Patrick (1990): Word association norms, mutual information, and lexicography. In: Computational Linguistics 16, 1. S. 22-29. [Wieder erschienen in Fontenelle (Hg.) (2008), S. 285-295 und Hanks (Hg.) (2008)].

Cinkova, Silvie et al. (2010): Can corpus pattern analysis be used in NLP? In: Sojka, Petr et al. (Hg.): Text, speech and dialogue. 13th International Conference, TSD 2010, Brno, Czech Republic, September 6-10, 2010. Proceedings. (= Lecture Notes in Computer Science/Subseries Lecture Notes in Artificial Science 6231). Berlin u.a., S. 67-74.

Fontenelle, Thierry (Hg.) (2008): Practical lexicography. A reader. Oxford/New York.

Grice, H. Paul (1975): Logic and conversation. In: Cole, Peter/Morgan, Jerry L. (Hg.): Syntax and semantics, Bd. 3: Speech acts. New York u.a., S. 41-58.

Halliday, Michael (1961): Categories of the theory of grammar. In: Word 17, 3, S. $241-$ 292. 
Hanks, Patrick (1994): Linguistic norms and pragmatic exploitations, or why lexicographers need prototype theory and vice versa. In: Kiefer, Ferenc/Kiss, Gábor/Pajzs, Júlia (Hg.): Papers in computational lexicography. Proceedings of the 3rd International Conference on Computational Lexicography (COMPLEX '94). Budapest, S. 89-113. [Wieder erschienen in Hanks (Hg.) (2008)].

Hanks, Patrick (2006): Metaphoricity is gradable. In: Stefanowitsch, Anatol/Gries, Stefan Th. (Hg.): Corpus-based approaches to metaphor and metonymy. (= Trends in Linguistics: Studies and Monographs 171). Berlin/New York, S. 17-35.

Hanks, Patrick (Hg.) (2008): Lexicology: critical concepts in linguistics. 6 Bde. London u.a.

Hanks, Patrick (2010): Nine issues in metaphor theory and analysis. In: International Journal of Corpus Linguistics 15, 1, S. 133-150.

Hanks, Patrick/Jezek, Elisabetta (2008): Shimmering lexical sets. In: Bernal, Elisenda/ De Cesaris, Janet (Hg.): Proceedings of the XIII EURALEX International Congress. Barcelona, S. 391-402.

Herbst, Thomas et al. (2004): A Valency Dictionary of English: a corpus-based analysis of the complementation patterns of English verbs, nouns and adjectives. (= Topics in English linguistics 40). Berlin/New York.

Jezek, Elisabetta/Hanks, Patrick (2010): What lexical sets tell us about conceptual categories. In: Lexis - E-Journal in English Lexicography 4 (Corpus Linguistics and the Lexicon), S. 7-22. Internet: http://screcherche.univ-lyon3.fr/lexis/IMG/pdf/Lexis_4_Jezek_ Hanks.pdf (Stand: 10.12.2010).

Kilgarriff, Adam et al. (2004): The Sketch Engine. In: Williams, Geoffrey/Vessier, Sandra (Hg.): Proceedings of the 11th Euralex International Congress, Lorient, France, July 6-10, 2004. Bd. 1. Lorient, S. 105-115.

Louw, Bill (1993): Irony in the text or insincerity in the writer? - The diagnostic potential of semantic prosodies. In: Baker, Mona/Francis, Gill/Tognini-Bonelli, Elena (Hg.): Text and technology: in honour of John Sinclair. Philadelphia u.a., S. 157-176.

Pustejovsky, James (1995): The generative lexicon. Cambridge, MA u.a.

Pustejovsky, James/Rumshisky, Anna/Hanks, Patrick (2004): Automated induction of sense in context. In: Proceedings of the 20th International Conference on Computational Linguistics. Geneva, S. 55-58.

Sinclair, John (1991): Corpus, concordance, collocation. Oxford u.a.

Sinclair, John (1998): The lexical item. In: Weigand, Edda (Hg.): Contrastive lexical semantics. (= Amsterdam Studies in the Theory and History of Linguistic Science: Series 4, Current Issues in Linguistic Theory 171). Amsterdam/New York, S. $1-$ 24. [Wieder erschienen in Hanks (Hg.) (2008)].

Sinclair, John et al. (1987): Collins COBUILD English Language Dictionary. London u.a. Tesnière, Lucien (1959): Éléments de syntaxe structurale. Paris. 



\title{
Lexikografie der Kollokationen zwischen Anforderungen der Theorie und der Praxis
}

\begin{abstract}
Kollokationen stellen einen noch zu wenig beachteten Teilbereich der Phraseologie dar. Sie sind bisher in den Wörterbüchern unzureichend erfasst und werden auch nicht systematisch gelehrt und gelernt. Es lassen sich zwei Typen von Kollokationen unterscheiden, die sowohl eine strukturelle als auch eine statistische Herleitung nutzen und beide für die unauffällige und kompetente Sprachproduktion im Alltag von Bedeutung sind. Angesichts der großen Zahl der auffindbaren Kollokationen ist zu differenzieren und zu gewichten: a) Es steht lexikografisch zunächst die Unterstützung bei der Sprachproduktion im Vordergrund sowie b) der Grundwortschatz bzw. Basiswortschatz und es sind c) die Unterscheidung von typischen Wortverbindungen und gebräuchlichen Wortverbindungen (den Hund loslassen vs. den Hund anleinen/ an die Leine nehmen) vorzunehmen.
\end{abstract}

\section{Einführung}

\subsection{Ausgangslage}

Kollokationen sind in der Sprachwissenschaft inzwischen sehr präsent, nicht jedoch außerhalb des engeren wissenschaftlichen Umfeldes. Wer an einem Kollokationswörterbuch arbeitet, muss in seinem weiteren sozialen Umfeld fast täglich erklären, worum es sich dabei handelt.

In den Lehrmitteln und im Schulunterricht werden Kollokationen kaum behandelt, obwohl sie - wenn man die Größe des Bereichs, die unsichere Sprachpraxis sowie den Lehr- und Lernbedarf mit Bezug auf Kollokationen nicht nur im Zweitspracherwerb, sondern auch im Erstspracherwerb berücksichtigt - einen prominenten Stellenwert haben müssten (vgl. Burger 2010, S. 52).

Dabei ist es intuitiv immer einsichtiger, mit Kollokationen zu argumentieren, die weder in sich variiert werden können, noch alternative Ausdrucksmöglichkeiten kennen, die man also „richtig“" verwenden muss, um keinen Fehler zu machen: *eine Entscheidung nehmen (vs. die korrekte Form eine Entscheidung fällen bzw. treffen): Wortverbindungen dieser Art sind in einer bestimmten Form bzw. in bestimmten Varianten einer Form gebräuchlich (und in einer anderen Form falsch). Als nicht oder schwach idiomatische Wortverbindungen gehören sie unzweifelhaft zu den festen Wortverbindungen im phraseologischen Sinn (vgl. ebd., S. 11). 
Kollokationen einer Sprache, wie „leise Töne“ „Abschied nehmen“, „zu vorgerückter Stunde“, ,schweren Herzens“, „in Strömen regnen“, sind ein fester und in der Regel nicht aktiv wahrgenommener Bestandteil der alläglichen Kommunikation. Sie werden beim Sprechen bzw. Schreiben nicht ad hoc gebildet, sondern als zusammengehörige Syntagmen abgerufen. Das hat zur Folge, dass alternative, frei kombinierte Konstruktionen (beispielsweise ,ruhige Töne") als unpassend empfunden werden, obwohl sie semantisch und syntaktisch ebenso korrekt wären.

Es gibt aber auch weitere Kollokationstypen, die weniger vor dem Hintergrund der sprachlichen Norm und der Fehlervermeidung als im Rahmen der Stilistik als bevorzugte Ausdrucksweisen identifizierbar sind: Es sind typische Wortverbindungen, die über die Ermittlung von Kookkurrenzen gefunden werden, die sich nicht einfach aus den Selektionsrestriktionen der Semantik der Wörter ergeben (wie die Kombination von Holz. und brennen in einem Satz das Holz brennt, bei dem Brennbarkeit zu den Merkmalen von Holz gehört). Für die Textproduktion sind die typischen Kollokationen ebenso hilfreich. Beide Arten von Kollokationen bilden die usuellen Wortverbindungen im Sinne von Kathrin Steyer passim (vgl. Steyer/ Brunner 2009).

Kollokationen ...

a) sind ebenso wie idiomatische Phraseologismen auf der zeichentheoretischen Ebene von Wortverbindungen interessant;

b) haben sprachgeschichtlich mit der Ökonomie der Sprachproduktion zu tun (vgl. von Polenz 2009). Die Ausbildung von Kollokationen stellt für die Sprachproduktion eine Formulierungserleichterung durch Vorprägung dar und geschieht durch die Einengung der Kombinationsmöglichkeiten;

c) können textgeschichtlich als Musterentwicklungen erfasst werden, wobei „Muster“ sowohl die durch den Sprachgebrauch erreichte Vorgeprägtheit von Wortkombinationen als auch die Struktur von Wortverbindungen im Sinne einer Konstruktion meint (Zwillingsformel als Struktur, Funktionsverbgefüge als Struktur etc.);

d) haben mit Wissensorganisation und Wissensstrukturierung zu tun (vgl. Filatkina/Hanauska i.Dr.);

e) bilden sich ontogenetisch in der Erstsprache als feste Einheiten heraus, werden aber nicht notwendigerweise so gelernt, sondern können auch als vermeintlich produzierbare Wortverbindungen erworben werden (vgl. Häcki Buhofer 1997). Sie werden jedoch in der Zweitsprache oft bewusst und in bewusstem Kontrast von der Erstsprache gelernt (vor allem für den Erwerb des Englischen in Form von Listen von „phrasal verbs“ üblich). 


\subsection{Hintergrund: Forschungsprojekte}

Die vorliegende Auseinandersetzung ist vor dem Hintergrund von verschiedenen Forschungsprojekten entstanden, die in verschieden ausgerichteten lexikografischen Projekten, zum Teil auch Datenbankprojekten, Kollokationen erfassen, nämlich:

- „Wörterbuch der festen Wendungen und Wortverbindungen der deutschen Sprache“ (www.kollokationenwoerterbuch.ch) (Prof. Dr. Harald Burger, Prof. Dr. Brigit Eriksson, Dr. Hans Bickel, M.A. Marcel Dräger, lic. phil. Tobias Roth);

- „Das Neue Baseldeutsch-Wörterbuch“ (www.baseldeutsch.ch) (Dr. Lorenz Hofer, Dr. Markus Gasser, lic. phil. Emilie Buri, Stefanie Meier);

- „DWDS - Schweizer Textkorpus“ (www.chtk.unibas.ch) (Dr. Lorenz Hofer, Dr. Markus Gasser, lic. phil Tobias Roth, lic. phil. Christoph Schön);

- „Hyperhamlet“ (www.hyperhamlet.unibas.ch) (M.A. Sixta Quassdorf);

- Pro*Doc „Sprache als soziale und kulturelle Praxis“, Forschungsmodul „Typisierte Sprache - in Geschichte, Diskurs und Ontogenese“ (Doktoranden u.a. Andreas Bürki); „Zum Erwerb von Kollokationen“ (Qualifikationsarbeit: Stefanie Meier).

Die folgenden Ausführungen bewegen sich insbesondere vor dem Hintergrund des Projektes „Wörterbuch der festen Wendungen und Wortverbindungen der deutschen Sprache“ bzw. abgekürzt „Kollokationenwörterbuch".

Wir gehen von einer Lemmabasis von 2.000 Wörtern des Grundwortschatzes aus, die den Rahmen des Wörterbuches bilden sollen. Dafür haben wir einige Grund- und Lernwortschätze des Deutschen ausgewertet und mit den Frequenzlisten auf dem DWDS Schweizer Textkorpus, dem Wortschatzportal Leipzig und dem Deutschen Referenz-Korpus in Mannheim verglichen. Dazu wurde das Variantenwörterbuch ausgewertet. Gerade bei den im Vergleich zu Idiomen unauffälligen Kollokationen scheinen viele regionale oder nationale Varianten zu existieren. Zu den 2.000 Wörtern des Grundwortschatzes werden in den oben genannten Korpora die kookkurrenten Wortverbindungen gesucht und daraus potenzielle Kollokationen, die im Kontext des Alltags nützlich sein können, ausgewählt. Nun gibt es natürlich nicht zu jedem Lemma des Grundwortschatzes eine Kollokation, dafür jedoch zu einigen Lemmata mehrere Kollokationen. Am häufigsten sind Substantive an Kollokationen beteiligt; sie machen im Basiswortschatz etwa 2/3 aus. Sie sind also wichtig, aber man kann sich nicht auf Substantivkombinationen beschränken. Dafür werden u.a. vor dem Hintergrund des Sprachvergleichs Komposita berücksichtigt, wo man es in anderen Sprachen mit Kollokationen zu tun hat. 


\subsection{Thesen}

Die systematische lexikografische Erfassung und Beschreibung von deutschen Kollokationen steht noch aus, auch wenn die Wörterbücher der deutschen Sprache, insbesondere das Stilwörterbuch des Dudenverlags, viele Kollokationen als Lemmavarianten in Beispielsätzen und Paraphrasen - ohne spezifische Kennzeichnung - enthalten.

Eine separate Lexikografie der Kollokationen der deutschen Standardsprache wird zeigen, dass systematische Korpusanalysen der Standardsprache auf der Basis von Kookkurrenzanalysen viel zusätzliches Material zu Tage fördern.

Bezüglich der Festigkeit von Kollokationen ist die Unterscheidung von gebräuchlichen und typischen Wortverbindungen sinnvoll: den Hund anleinen/ an die Leine nebmen (normative Varianten) vs. den Hund loslassen (typische Formulierung, stilistisch erfassbar). Mit der Benennung der unterschiedlichen Typen von Kollokationen kann man sich schwer tun, die unterschiedlichen Kriterien können jedoch aufgeführt werden und stellen normative Gebräuchlichkeit, die die Falschheit von Alternativen impliziert, und häufige Ausdrucksweise im Sinne von typischer Üblichkeit gegeneinander.

Bei der Arbeit im Rahmen der deutschschweizerischen Dialektlexikografie lassen sich auch zusätzliche Kollokationen finden, die jedoch - wohl in erster Linie der relativ kleinen Korpora wegen - quantitativ weniger ausgeprägt sind. Hier sind ergänzende Datenerhebungen über Umfragen hilfreich.

Die bisherige Lexikografie von Kollokationen insgesamt - das gilt für Standardsprachwörterbücher und Dialektwörterbücher gleichermaßen - verzeichnet Kollokationen, wenn überhaupt - ohne metasprachliche Kennzeichnung in Beispielsätzen. Auf diese Weise wird den BenutzerInnen weder klar, dass es sich dabei um Kollokationen handelt, noch können - ohne Angabe einer Nennform - die Möglichkeiten der formalen Verwendungen beurteilt werden. Es ist aber wünschenswert, dass die BenutzerInnen von Wörterbüchern auch über Kollokationen informiert werden: Kollokationen sind grundsätzlich nicht nur im Hinblick auf die Sprachrichtigkeit lexikografisch zu erfassen, sondern auch im Hinblick auf die Ausbildung von Sprachwissen lexikografisch angemessen darzustellen.

\subsection{Definitionen}

Kollokationen stellen im deutschsprachigen Forschungsbereich einen Teilbereich der Phraseologie dar (vgl. Burger 2010, S. 57). Als prototypisch gelten bei Kollokationen Substantiv-Verb-Verbindungen mit normativ festgelegtem Verb: Anzeige erstatten (nicht Anzeige stellen o.Ä.). Die Bandbreite der 
Strukturtypen ist jedoch viel größer und reicht von eine Rolle spielen bis zu vielfältigen adverbialen Kollokationen wie in Kürze, in diesem Augenblick oder aus persönlichen Gründen. Kollokationen werden grundsätzlich eher von der normativen Gebräuchlichkeit, der Üblichkeit und Usualität, der Typizität her als von der Semantik aus definiert. In semantischer Hinsicht gelten Kollokationen als „normal“, transparent, weil vollmotiviert wie freudiges Ereignis, eventuell auch als teilmotiviert (wie der blinde Passagier) (vgl. Barz 2007, S. 27; Burger 2010, S. 38 und S. 70). Die Üblichkeit wird oft durch die Häufigkeit des Miteinandervorkommens der Teile bestimmt, so beim DWDS, wo es auf der Titelseite der Homepage heißt - „Wortverbindungen, die besonders häufig gemeinsam auftreten“, wobei gemeint ist, deren Teile bzw. Elemente besonders häufig miteinander auftreten. Man kann zwei Operationalisierungsrichtungen der Kollokationsdefinition unterscheiden, nämlich die Korpuslinguistische Operationalisierung vs. Fehlerlinguistische Operationalisierung. Die Korpuslinguistische Operationalisierung sucht Kookkurrenzen in Korpora mit Hilfe mathematischer Verfahren und scheidet Wortkombinationen aus, die keine semantisch sinnvollen Wortverbindungen darstellen (wie ,und der“, die zwar häufig nacheinander vorkommen, aber semantisch keine Wortverbindung bilden). Die Fehlerlinguistische Operationalisierung geht von Varianten aus, die als fehlerhaft empfunden bzw. eingestuft werden (müssen). Diesen falschen Gebrauchsweisen, die auch in Texten wie Zeitungsartikeln häufig vorkommen, welche von professionellen Schreibenden verfasst werden, werden die richtigen Varianten gegenübergestellt. Die Fehlerlinguistische Operationalisierung kann ergänzt werden durch die Überlegung, wie eine Übersetzung in eine andere bekannte Sprache lauten müsste: Wenn sie nicht Wort für Wort erfolgen kann, ist die Wahrscheinlichkeit, dass eine Kollokation vorliegt, relativ hoch. Tener admiracion kann man mit Bewunderung haben (wörtlich) übersetzen, besser aber mit Bewunderung hegen, was es laut DWDS (bzw. seiner Kollokationsberechnung vom 12.01.2010) als Kollokation nicht gibt, wo man Bewunderung nur erregt. Bewnnderung hegen ist hingegen laut Wörterbuch der Deutschen Gegenwartssprache (WDG) möglich. Die Kollokationsberechnung ergibt hingegen Wünsche, Befürchtungen, Zweifel, die man hegt. Entsprechende Abklärungen sind oft durch Unvollständigkeiten aus der Korpusabfrage ebenso wie der lexikografischen Verzeichnung erschwert.

Varianten zu Kollokationen sind falsch im Sinne der Sprachnorm, z.B. in Fällen wie *eine Entscheidung nehmen oder *eine Notiz, lassen oder *eine Anzeige stellen (vgl. „Die XY-Immobilien AG bedauert den Vorgang und stellt als betroffene Eigentümerin Strafanzeige." Aus: Neue Zürcher Zeitung, 10.02.2010, S. 17). Anzeige muss man erstatten. Die automatische Kollokationenanzeige aus dem DWDS zeigt für Anzeige, dass erstatten zum Kollokationengefüge gehört, neben ganæseitig etc., und dass erstatten zu Anzeige 
führt, daneben auch zu Bericht und Meldung etc., die man auch noch erstatten kann. Stellen bringt demgegenüber bei der DWDS-Kollokationenanzeige feststellen, eine Frage stellen oder zur Verfügung stellen und bildet einen häufigen Kollokator, aber eben nicht den richtigen, wenn es um die Anzeige geht.

Man spielt eine Rolle, man gibt sie oder macht sie nicht, auch wenn man die Ophelia geben kann. Diese Fälle sind vergleichsweise einfach, die über das Fehlerempfinden operationalisiert werden können. Sie entstehen ebenso häufig bei L1 SprecherInnen, die nicht die richtige Verbindung finden bzw. ein Verbindungselement aus einem anderen Kontext verwenden, als auch bei L2 SprecherInnen, die eine Kollokation aus der Erstsprache übertragen und damit die Kollokation für native speakers erst so richtig deutlich machen. Schaden hingegen muss man nicht unbedingt anrichten, auch wenn das eine unauffällig professionelle Ausdrucksweise ist, man kann ihn auch verursachen.

Kookkurrenzen, also statistisch festgestellte feste Wortverbindungen (verschieden berechenbar, beispielsweise entsprechend Steyer/Brunner 2009, S. 2) bilden ein Reservoir für Kollokationen, sind aber nicht samt und sonders Kollokationen in einem lexikogrammatischen normativen oder stilistischen Sinn (siehe unten). Kollokationen sind gemäß ihrem Charakter als Wortverbindung jedenfalls nicht als beliebige bzw. als „freie Wortverbindungen“ einzustufen. Diese Nicht-Beliebigkeit besteht allenfalls nur in einer Vorprägung bzw. Formelhaftigkeit im nicht-semantischen Sinn.

$\mathrm{Ob}$ man solche kollokativen Mehrworteinheiten „Formeln“ nennen soll, ist eine offene Frage. Laut dem Handbuch zur Sprach- und Kommunikationswissenschaft zur Phraseologie (Burger et al. (Hg.) 2007) bilden Kollokationen zusammen mit Idiomen als Phraseme nur den engeren Bereich der Phraseologie. Es wird empfohlen, bei einer weiten Konzeption auf andere Termini wie formelhafte Sprache oder Texte oder feste Wortverbindungen „auszuweichen“ (ebd., S. 3). Kollokationen können Routineformeln enthalten oder auch nicht (ebd., S. 4). Es besteht auch die Möglichkeit, Routineformeln als kommunikative Phraseologismen von referenziellen Phraseologismen abzutrennen, die ihrerseits als nominative Phraseologismen in Kollokationen, Teil-Idiome und Idiome aufgeteilt werden können (vgl. Burger 2010, S. 36 f.).

Referenzielle Phraseologismen beziehen sich auf Objekte, Vorgänge oder Sachverhalte der Wirklichkeit, während kommunikative Phraseologismen bestimmte Aufgaben bei der Herstellung, Definition, dem Vollzug und der Beendigung kommunikativer Handlungen haben. Referenzielle Phraseologismen können als satzgliedwertige Phraseologismen (im Unterschied zu den satzwertigen) semantisch untergliedert werden: als Kollokationen, TeilIdiome und Idiome sind sie im Wesentlichen semantisch bestimmt.

Man macht nicht notwendigerweise einen sprachlichen Fehler, wenn man sich „,nicht-kollokativ“ ausdrückt, aber es tönt oft nicht professionell für den Empfänger und ist keine ökonomische Ausdrucksweise, mit der 
man sich aus dem Wortverbindungsreservoir und -repertoire bedient. Kollokationen sind in der festen Form in der Alltagskommunikation unauffällig und unmarkiert und - was ebenso wichtig ist - in der fachnahen oder fachinternen Kommunikation für den Eindruck der Professionalität wesentlich. Als fachnahe Kommunikation müssen weite Bereiche der Alltagskommunikation verstanden werden, in der beispielsweise medizinische, psychologische, schulische oder administrative Dinge besprochen werden, ohne dass die Beteiligten als Fachleute ausgebildet sind. Weil Kollokationen unauffällig und unmarkiert sind, ist es oft schwierig, sie sich bzw. Anderen bewusst zu machen. Jmdn. mit Verdacht auf einen Herzinfarkt ins Spital einliefern ist die professionelle Ausdrucksweise, die eine verbale und eine präpositionale Kollokation enthält. Wer davon spricht, dass ein Bekannter ins Spital gebracht werden musste, weil der Arzt vermutete, dass er einen Herzinfarkt gehabt haben könnte - die kollokative Ausdrucksweise wäre einen Her乏infarkt erlitten haben könnte - wird genauso verstanden, drückt sich aber mit freien Paraphrasen, oft etwas länger, oft weniger genau im professionellen Sinne oder einfach weniger professionell aus.

Während Kollokationen in Schulbüchern und im Schulunterricht eine gewisse Präsenz und Aufmerksamkeit erfahren, liegen Kollokationen der Erstsprache (den Tisch decken, die Nase putzen) unter der Aufmerksamkeitsund Bewusstseinsschwelle.

Franz Josef Hausmann hat das basisbezogene Kollokationskonzept eingeführt (Hausmann 2004, S. 321, vgl. auch 1989, S. 1010). Dabei geht er in vielen diskutierten Fällen von einer möglichen Unterscheidung richtig/ falsch in Bezug auf die Substantiv-Verb-Verbindung aus. Danach stellt sich wesentlich das Problem, welches Verb korrekterweise zu einem bestimmten Substantiv gehört. Das Substantiv ist die Basis, das Verb der Kollokator (Abschied nebmen). Nach diesem Konzept sind Basisartikel im Wörterbuch gefragt, Kollokatorenartikel im Wörterbuch jedoch überflüssig. Wenn und weil die Kombinationen Substantiv-Verb arbiträr und unvorhersehbar sind, soll, um der Ausdrucksnot zu begegnen, ein Ausdruckswörterbuch bzw. ein Produktionswörterbuch geschaffen werden.

Kollokatorenartikel können jedoch ebenso hilfreich sein wie Artikel zum (oft substantivischen) Basiswort. Dafür sollen vier Gründe angeführt werden:

1) Die Richtung, in der eine passende Ergänzung gesucht wird, ist nicht immer eindeutig und man merkt oft, wenn man sich überlegt, ob eine Wortverbindung gebräuchlich oder typisch ist, dass man nicht vom Substantiv her argumentiert: So kann der Hund mit allen möglichen Verben kombiniert werden, aber wedeln bzw. mit dem Schwanz wedeln wird typischerweise von einem Hund gesagt. 
2) Auch syntaktisch komplexere Wortverbindungen bilden Kollokationen, wie beispielsweise Substantiv-Verbindungen unter Einbezug von Präpositionen wie vom Stubl (herunter) fallen, Substantiv-Adjektivverbindungen (mit oder ohne Präposition) wie leise Töne, werdende Mutter, schweren Herzens, himmelweiter Unterschied, zu vorgerückter Stunde. Sie bilden Strukturen, die je nach grammatischer Ausrichtung auf einer Skala von grammatisch vielseitig variabel bis formelhaft oder in typischen Ausdrucksweisen vorgeprägt angeordnet werden können (vgl. Eichinger 2004: Skala „Redewendungen bis zu syntaktischen Schematisierungen“). Dazu kommt schließlich, dass für die Unterstützung der Entwicklung von Sprachwissen auch Kollokatorenartikel hilfreich sind.

3) Relativ allgemeine Verben mit vielfältiger und wenig spezifizierter Semantik wie stellen, legen, setzen, machen etc. können sehr viele Basiswörter „bedienen“: stellen hat vergleichsweise diejenige breite Palette von möglichen Verbindungen, die man im Auge hat, wenn man sagt, dass es „nichts bringt“" einen Artikel ₹u stellen (zu verfassen) (,überflüssige Kollokatorenartikel“, vgl. Hausmann 2004, S. 312). Wenn man jedoch ein Wort wie stellen hat und sich fragt, was man damit noch so „machen“ kann, hat auch diese Fragerichtung ihre Berechtigung, allerdings weniger für die Hausmannsche „Momo-Situation“, in der jemand schon ein Wort hat und die angemessene Ergänzung sucht, als für Fragen des Sprachwissens.

4) Zudem gibt es auch eine recht große Zahl seltener, veraltender oder unikaler Kollokatoren, die man wegen ihrer spezifischen Gebrauchsweise als Lemmata aufführen sollte, beispielsweise bei jmdm. das Leben vergällen; vergällen steht auf einer Vorstufe der Unikalität, indem das Verb fast nur noch gebunden an Freude oder eben Leben vorkommt und sollte entsprechend - je nach Häufigkeit der Wortverbindung als Ganzer und Ausrichtung des Wörterbuches - als eigenes Lemma aufgeführt werden; ebenso kommt bezichtigen mit wenigen Substantiven wie Lüge, Untreue oder Plagiat vor.

Die Heterogenität der verbalen Kollokatoren stellt bereits einen Hinweis darauf dar, dass alle Bestandteile von Kollokationen in die Analyse einzubeziehen sind und lexikografisch adäquat zu behandeln sind.

Eine Sprachtheorie muss nicht nur erklären, wie man aus wenigen syntaktischen Regeln praktisch unendlichen Nutzen ziehen kann,

sondern auch, wie uns Kollokationen und Kookkurrenzen helfen, Strukturen auf der lexikalischen Ebene schneller und effizienter zu durchdringen. Wenn lexikalische Einheiten nicht völlig unabhängig voneinander auftreten, bedeutet das einen wesentlichen Schritt zur Reduktion von Komplexität: wir werden auf Erwartbares, in diesem Sinn Normales verwiesen. (Eichinger 2004, S. 5) 
Bei Eichinger werden Kollokationen im Kontext wiederholter Rede gesehen: Für die Lernerlexikografie, aber auch für die Lexikografie überhaupt ist die Darstellung von wiederholter Rede, die nicht unmittelbar grammatische Regularitäten betrifft, aber die für die Akzeptabilität von Äußerungen zentral ist, eine wichtige Aufgabe (ebd., S. 5.). Auch für die Professionalität des Sprechens sind Kollokationen wichtig - und zwar nicht nur die berufliche, sondern auch die Alltagsprofessionalität, die ja mehr und mehr auch von Fachdiskursen mitgeprägt ist.

Als Phänomene wiederholter Rede gehören Kollokationen in den Kontext der historischen und ontogenetischen Musterentwicklung und des Musterlernens - dazu wird in Basel und Zürich an einigen Dissertationen gearbeitet (im Rahmen des Pro*Doc-Forschungsmoduls „Typisierte Sprache - in Geschichte, Diskurs und Ontogenese"), die Muster nicht als passive Imitationsübung, sondern als Einheiten der sprachgeschichtlichen Entwicklung im Kontext aktiver kognitiver Organisations- und Strukturierungsform sehen. Andreas Bürki (i.Dr.) untersucht am Schweizer Textkorpus Mehrworteinheiten, zu denen die Kollokationen gehören, und stellt die Entwicklung, sprachgeschichtliche Prägung und Varianz von Mehrworteinheiten in Jahrhundertvierteln dar.

Die folgende Tragetaschen-Werbung macht Gebrauch von einem solchen Muster, indem potenzielle Nichtraucher wie zukünftige Mütter durch

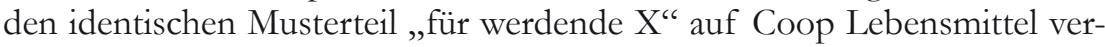
wiesen werden, die als Alternative zu Zigaretten genossen werden können.

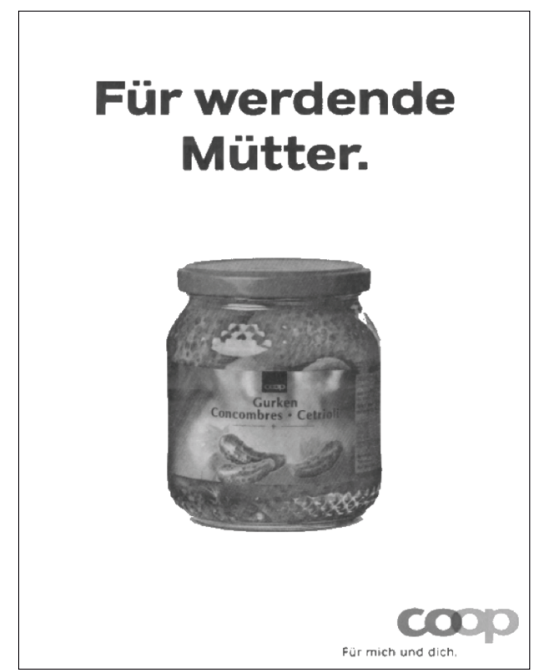

Abb. 1: Werbung „Für werdende Mütter“

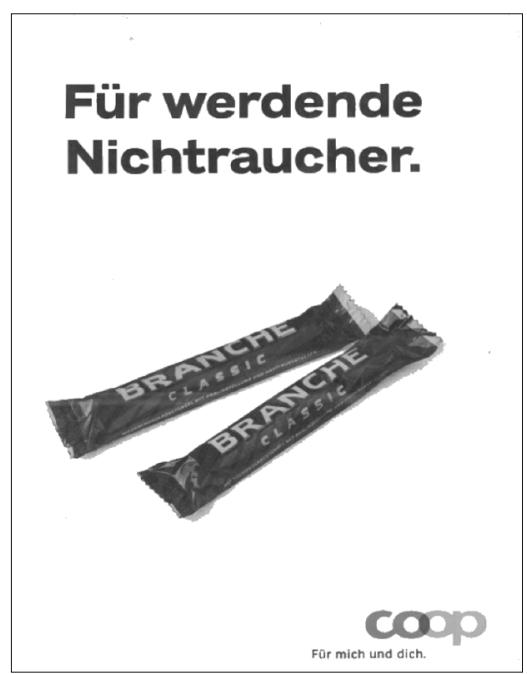

Abb. 2: „Für werdende Nichtraucher“ 


\section{Lexikografische Situation und Methoden der Darstellung und Auffindung}

\subsection{Problematik der lexikografischen Darstellung von Kollokationen}

Es gibt einen beachtlichen Stand der Lexikografie von Kollokationen, wie er im Duden 2 zur Stilistik und in anderen Wörterbüchern eher implizit, für das Englische im Oxford Dictionary zu „collocations“ explizit realisiert ist. Kollokationen haben aber in der deutschsprachigen Lexikografie noch keinen festen Platz.

Es ist nicht klar, wie die Kollokationen, die bisher in Wörterbüchern behandelt wurden, ermittelt wurden; ob statistische Verfahren, die bloße Häufigkeit, Autorenintuition oder vielleicht doch Kriterien der Festigkeit eine Rolle spielen. Sie stehen im Wörterbuch häufig ohne metakommunikative Kennzeichnung als typische Verwendungen, als Phrasen oder als Beispielformulierungen, seltener als Lemmata (vgl. Steyer 2008; Steyer/Brunner 2009 sowie Burger 2010).

Den Wörterbuchbenutzern auf jeden Fall wird nicht klar, was Kollokationen und was einfach typische Beispiele für Wortverwendungen sind. Das gilt auch für VerfasserInnen von Wörterbüchern, wenn sie als NutzerInnen versuchen, diese Wörterbuchartikel zu analysieren.

\subsection{Vergleichender Ertrag systematischer Korpusabfragen}

Die begonnenen Arbeiten zeigen schon bisher: Korpora liefern viel zusätzliches Material. Einerseits erhält man über Kookkurrenzanalysen (neben gröBeren Mengen auszusortierenden Materials) Belege mit ergänzenden Formen oder anderem Schwerpunkt als aus dem Wörterbuch hervorzugehen scheinen. Die Abfragen stellen also Bewertungshilfen dar. Andererseits liefern Korpora aber auch bisher lexikografisch nicht oder allenfalls als Beispielsätze verzeichnete Kollokationen.

Korpusabfragen sind aber - wie wir auch aus anderen Zusammenhängen wissen - keine Allheilmittel für alle lexikografischen Fragen, die man an den Sprachgebrauch hat. Manchmal ergeben sie auch einfach „,nichts“, obwohl man als kompetenter Sprecher oder kompetente Sprecherin sozusagen sicher ist, dass eine bestimmte Ausdrucksweise fest vorgeprägt, usuell, gebräuchlich oder typisch ist. Das ist an einem Vergleich der Abfragen beim DWDS (www.dwds.de) mit dem Stilwörterbuch des Duden Verlags (2010) konkret an einzelnen Beispielen zu zeigen.

Wenn man den Vergleich Wörterbuch - Korpus quantitativ abzuhandeln versucht, was zugegebenermaßen einige Probleme der Operationalisierung der Zählung der Nennungen mit sich bringt, so kann das Resultat in der Größenordnung ungefähr und mit Vorsicht zu genießen etwa folgender- 
maßen aussehen, wobei eine solche im Wörterbuch gezählte Nennung beispielsweise lauten kann: streunender Hund. Die Korpusanalyse bringt beispielsweise zusätzlich bissiger Hund.

\begin{tabular}{|l|c|c|c|}
\hline & Schnittmenge & Stilwörterbuch & Korpusanalyse \\
\hline Hund & 16 & 16 & 46 \\
\hline Wetter & 32 & 37 & 60 \\
\hline Ehre & 26 & 62 & 33 \\
\hline Laune & 11 & 14 & 15 \\
\hline Summe & 85 & 129 & 154 \\
\hline
\end{tabular}

Tab. 1: Anzahl der Kollokationen mit Hund, Wetter, Ehre und Laune im Vergleich Duden Stilwörterbuch - DWDS

Man ersieht aus diesem versuchsweisen quantitativen Vergleich (Tab. 1), dass es jeweils große Schnittmengen der Kollokationen zu Hund, Wetter, Ehre und Laune gibt. Die Kookkurrenzanalyse in einem Korpus fördert zusätzliche Kollokationen zu Tage, findet aber auch nicht alle Kollokationen, die das Stilwörterbuch verzeichnet. Die Differenzen sind zu erwarten, die Ergebnisse der Kookkurrenzanalysen aus angemessen großen und systematisch aufgebauten Korpora jedoch unbedingt einzubeziehen.

Im Folgenden werden drei Wörterbuchartikel zu den Lemmata Hund, Laune und Ehre aus dem Duden Stilwörterbuch aufgeführt und diskutiert. Die in diesen Artikeln aufgeführten Kollokatoren zu den jeweiligen Lemmata wurden um weitere, durch Korpusrecherchen im DWDS ermittelte Kollokatoren ergänzt. Die korpusbasiert ermittelten Kollokatoren sind mit einem Pfeil markiert. Dabei wird keine Aussage darüber gemacht, ob die mit Pfeil markierten Lemmata nicht eigenständig oder andernorts aufgeführt sind.

Das Stilwörterbuch spricht von „festen Verbindungen und Wendungen“ und meint damit alle Phraseologismen (vgl. Duden 2010, S. 11 f.). Die „festen Wortverbindungen und Wendungen" stehen im Druck abgehoben (farbig gedruckt) und mit einem Sternchen versehen am Ende des Artikels. Es werden folgende Arten unterschieden:

1) Feste Attribuierungen: schwarzer Markt,

2) Feste Verbindungen: einen zwitschern,

3) Funktionsverbgefüge: zur Verteilung kommen/gelangen,

4) Wortpaare (Zwillingsformeln): ab und zu; bei Nacbt und Nebel,

5) Feste Wendungen: etw. auf die lange Bank schieben. 
Die jeweilige Verbindung oder Wendung ist beim (ersten) Substantiv oder, wenn kein Substantiv vorkommt, beim ersten sinntragenden Wort aufgeführt (ebd.).

„Wörter, die nur in einer festen Verbindung oder in bestimmten Wendungen vorkommen, werden in der ihnen eigenen Form der Verwendung vorgeführt" (Duden 2010, S. 13):

z.B. ausfindig: (in der Verbindung) jmdn., etw. ausfindig machen

z.B. Kreuafeuer, das: (in den Wendungen): ins Kreuafener geraten; im Kreuəfener stehen.

\section{Hund, der}

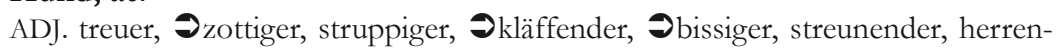
loser

SUBST. $\rightarrow$ Hund und Katze

H. (subj.) + VERB der H. bellt, Oेbellt jmdn. an, kläfft Kleine Hunde kläffen häufiger als große. schlägt an, winselt Nachbars Hund war alleine und winselte den ganzen Abend. jault, heult, $\boldsymbol{\nabla}$ knurrt, $\boldsymbol{O}$ fletscht, $\boldsymbol{O}$ schnappt, beisst (jmdn.) Beisst ihr Hund? Nein, der ist ganz brav. | $\boldsymbol{\vartheta}$ der H. beisst jmdn. in etw. Erst fletschte der Hund die Zähne, dann biss er dem Postboten in die Hose. wedelt mit dem Schwanz, Ohechelt,

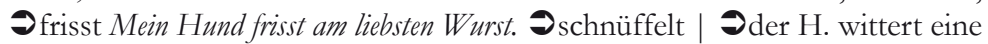
Fährte, $\boldsymbol{\vartheta}$ jagt, $\boldsymbol{O}$ holt ein Stöckchen/einen Ball/... Immer und immer wieder holte der Hund das Stöckchen. Oेbewacht etw. Der große Hund bewacht das ganze Gründstück. Hunde verbeissen sich ineinander Die Hunde haben sich so ineinander verbissen, dass nur die Polizei sie trennen konnte. $-\mathcal{d e r} \mathrm{H}$. hört (,gut erzogen sein?) Der Hund bört aber (gut). Oे folgt (,gut erzogen sein?)

H. (DAT) + VERB $כ$ dem H. pfeifen Der Halter pfeift seinem Hund, doch er kommt nicht.

H. (AKK.) + VERB Teinen H. halten, den H. anleinen Im Park, muss man Hunde anleinen. den H. an die Leine nehmen, den H. loslassen, כิden H. rufen, כen H. streicheln, den $\mathrm{H}$. auf jmdn. hetzen

PRÄP. „MIT“ $Э$ mit dem H. (raus) gehen Mit einem Hund muss man jeden Abend raus (gehen). $\boldsymbol{\vartheta}$ mit dem H. spazieren gehen, $\boldsymbol{\vartheta}$ mit dem H. spielen $\boldsymbol{\vartheta}$ mit dem H. Gassi gehen

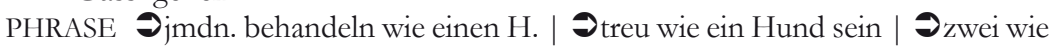
Hund und Katze zwei, die ständig gegenseitig ärgern bzw. überhaupt nicht ausstehen können Sie waren Geschwister aber auch zwei wie Hund und Katze.

Einige der Kollokatoren kommen im Stilduden als Lemmata nicht vor wie zottig, herrenlos, fletschen, hecheln, streunen. Einige der Kollokatoren kommen mit anderen Basiswörtern vor: so struppig im Beispiel struppiges Fell, kläffen (als Lemma) mit Köter, Dackel; spielen kommt mit einem Katzenbeispiel vor. In einigen Fällen ist das Basiswort Hund nicht mit dem adjektivischen Kollokator knurrend verbunden, sondern mit dem Verb knurren, ebenso bei bellen.

In manchen Fällen ist die Kollokation unter dem Adjektiv bzw. dem Verb - oft als Beispielsatz - angeführt: bissiger Hund unter bissig, der Hund frisst unter fressen. Der Hund schnüffelt ist unter schnüffeln aufgeführt, unter wittern wird als Beispielsatz der Hund wittert Wild angegeben, analog unter bewachen der Beispielsatz der Hund bewacht das Haus, analog unter pfeifen der 
Satz der Jäger pfeift seinem Hund, unter halten das Beispiel ich halte mir einen Hund, unter folgen der Satz der Hund folgt mir aufs Wort, unter spazieren die Wendung jmdn. spazieren fübren.

Den Hund rufen wird als Kookkurrenz in unseren Materialien sichtbar, ist aber für ein Wörterbuch zu unspezifisch, um unter rufen entweder als Kollokation oder als Beispielsatz aufgeführt zu werden. Es handelt sich also aus der Sicht der Intuition der BearbeiterInnen um eine unspezifische Kookkurrenz, die in einem Wörterbuch der Kollokationen nicht aufgeführt werden muss. Gassi gehen stellt wohl eine regional- bzw. nationalspezifische Variation dar. Treu kommt weder mit einer Hunde-Wendung noch mit einem Beispielsatz mit Hund vor (sondern in einem Beispielsatz mit Katze).

Laune, die

ADJ. Эgute L. (haben), (in) guter/bester L. (sein) Nach all dem Ärger bin ich nun wieder guter Laune. schlechte L. (haben), Эüble L. (haben), (in) übler/übelster L. sein

SUBST. $\boldsymbol{\nabla} \cdot$ Laune(n) der Natur Schnee im Juni ist eine Laune der Natur.

H. (subs.) + VERB die L. (ver-)bessert sich Mit dem Abendessen warten wir, bis sich deine Laune wieder gebessert hat.

H. (AKK.) + VERB jmdm. die (gute) L. verderben | seine (schlechte) L./seine Launen an jmdm. auslassen Musst du deine Launen immer an deinem kleinen Bruder auslassen? | jmds. (schlechte/miserable/...) Laune ertragen Tagtäglich musste das Kind die schlechte Laune seines Vaters ertragen.

H. (DAT.) + VERB $Э$ die (gute) L. verlieren Wenn man ständig getadelt wird, kann man schnell seine gute Laune verlieren.

PRÄP. „AUS“ Эaus einer Laune heraus (,plötzlich, ohne langes Überlegen') Er entschied sich ganz, spontan, aus einer Laune heraus, zu verreisen.

„BEI“ jmdn. bei L. halten (,jmdn. ablenken, indem man für gute Stimmung sorgt") „UNTER“ unter jmds. L. leiden

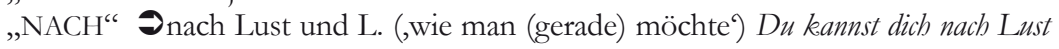
und Laune entscheiden.

PHRASE •Der/die hat (aber) eine Laune!

Die Kollokation gute Laune ist in der Variante gut gelaunt unter dem Adjektiv gut aufgeführt, ebenso analog üble Laune unter übel bzw. schlechte Laune als Beispielsatz unter schlecht; unter jmds. Launen leiden (ohne Relativierung des Plural-n-s) unter leiden. Die Kollokation nach Lust und Laune findet man unter dem Lemma Lust.

\section{Ehre, die}

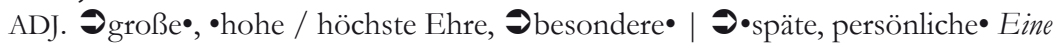
Beleidigung ist eine Verletzung der persönlichen Ehre eines anderen. $\boldsymbol{\nabla} \cdot$ bürgerliche (fachspr.: ,Anerkennung und Achtung gemäss den Regeln einer Gesellschaft') | Ogekränkte• Hass und gekränkte Ehre seien das Motiv des schrecklichen Mordes. $\boldsymbol{\partial} \cdot$ verletzte | $\boldsymbol{\partial} \bullet$ die (jmdm.) gebührende E. (,angemessene und jmdm. zustehende Anerkennung;) Beim Applaus wurde auch dem Regisseur des Theaterstücks die ihm gebührende Ehre zuteil. $\boldsymbol{\nabla} \cdot$ verdiente Ehre(n) | $\boldsymbol{\partial} \cdot$ zweifelhafte (ironisch: ,Anerkennung oder Auszeichnung, für eine wenig lobenswerte Handlung oder 
Tatsache) Die Auszeichnung als peinlichster Politiker ist eine zweifelhafte Ehre. I - $\cdot j m d m$. die letzte E. (erweisen) (,an jmds. Trauerfeier teilnehmen) Tausende erwiesen dem verstorbenen Fussballspieler die letəte Ehre. | - militärische (,repräsentatives militärisches (Begrüßungs-)Ritual') Der Staatsgast wird mit militärischen Ehren empfangen.

SUBST. $\rightarrow \cdot R u h m$ und E. (,besondere Anerkennung) Sein Sieg bracbte dem Tennisspieler Rubm und Ehre. | ein Mann von E. (,anständiger und vornehmer Herr') - $\odot$ die E. des Mannes (veralt.)

E. (sUB].) + VERB $\bullet \vartheta_{j m d m}$ gebührt E. (für etw.) (,jmdm. steht eine angemessene Anerkennung zu') $\mid \bullet \boldsymbol{O}_{\mathrm{jmdm}}$. widerfährt E. | die/seine/ihre E. verbietet jmdm. etw. (zu tun)• Kinder zu schlagen, verbietet mir meine Ehre.

E. (DAT.) + VERB seiner (eigenen)/ihrer/... E. etw. schuldig sein• Eine Entschuldigung nach einem Streit bin ich meiner eigenen Ehre schuldig.

E. (AKK.) + VERB • $\boldsymbol{\partial}$ die E. verdienen, $\boldsymbol{\vartheta}_{j m d m}$. eine E. • erweisen, • jmdm./etw. [alle] Ehre(n) machen (jmdm./etw. Lob und Anerkennung verschaffen') | jmds. Ehre (be)wahren• Durch eine Entschuldigung bei seinen Mitarbeitern konnte der Abteilungsleiter seine Ehre wahren. • jmds. E. verteidigen, Oseine/jmds. E. retten• Durch zablreiche Ausreden versuchte der Betrïger seine Ehre zu retten. $\mid \bullet j m d s$. E. verletzen, $\bullet$ jimds. E. beschmutzen Durch die Verfehlungen seiner Tochter sei die Ehre seiner Familie beschmutzt worden. | $\boldsymbol{\nabla}$ die (besondere/seltene/...) E. genießen, -die E. (miteinander) haben $\mid \bullet$ sich. die E. geben (,sich durch eine Handlung, einen Besuch, eine Aussage selbst Anerkennung und Geltung verschaffen') Der Startenor gab sich die Ehre, in London aufzutreten.

PRÄP. „ZU“ $\boldsymbol{\nabla} •(j m d m$.) zu Ehren Es gibt eine Gedenkveranstaltung zu Ehren der Opfer des zpweiten Weltkriegs. | •wieder zu Ehren kommen, $\boldsymbol{\nabla} \cdot$ zu neuen Ehren kommen (,wieder gewürdigt werden), $\boldsymbol{\nabla} \bullet$ etw. zu neuen Ehren verhelfen Die Katze verhalf Opas altem Sessel zu neuen Ehren, indem sie ibn als Schlafplatz, wählte.

„BEI“ •jmdn. bei der/seiner/... E. packen

„AN" $\boldsymbol{O} \bullet$ jmdn. an der/seiner/... E. packen

„IN“ $\boldsymbol{\vartheta} \bullet$ etw. in E. halten (,etw. wertschätzen und pflegen) $\mid \boldsymbol{\vartheta} \cdot$ jmdn. in seiner E. kränken, $\boldsymbol{\vartheta} \bullet$ sich in seiner E. angegriffen fühlen,

„ZUTEIL“ $\boldsymbol{\nabla} \cdot j \mathrm{mdm}$. wird E. zuteil Mit der Auszeichnung für sein Lebenswerk wurde dem Musiker späte Ehre zuteil.

„ZUVIEL“ 2 -zuviel (der) Ehre (Ausdruck der Bescheidenheit) Vielen Dank. für die Geschenke, aber das ist zuviel der Ehre.

„MIT“ $\boldsymbol{\vartheta} \bullet$ etw. mit Ehren bestehen (,sehr gut und mit Auszeichnungen abschneiden $) \mid \partial \cdot j m d n$. mit (großen/militärischen/...) Ehren empfangen

„UM““ •jmdn. um seine E. bringen Der Ausrutscher in der letəten Kurve bätte die Eisschnelläuferin fast um ibre Ehre gebracht. | • etw. [nur] um der E. willen tun

„AUF“ $כ \cdot$ •ein Angriff auf jmds. E. sein

PHRASE •Es ist/war mir eine E. (Ausdruck zur Erwiederung eines Dankes) Vielen Dank, dass Sie mir die Tür aufgehalten haben - Es war mir eine Ehre. | -Was verschafft mir die E.? (höflich bis ironische Frage nach dem Grund für einen

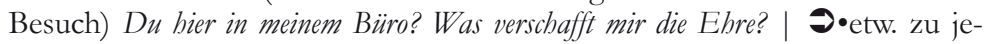
mandes E. sagen $\mid \bullet$ Ehre, wem E. gebührt. $\mid \bullet j$ imds. Wort in Ehren (höflicher Ausdruck zur Einleitung einer Widerrede) Dein Wort in Ehren, aber dieser Vorschlag ist einfach unakzeptabel. - • seinem Namen alle E. machen Herr Schlecht macht seinem Namen alle Ehre. 
Hier gibt es keine Kollokatoren, die als Lemmata im Stilduden nicht vorkommen. Hingegen gibt es Kollokatoren mit sehr weiter Bedeutung und großer Häufigkeit wie groß, geben, verdienen oder widerfahren, die kookkurrent mit Ehre vorkommen, aber deren Verbindung mit Ehre weder in einer Kollokationsdarstellung noch als Beispielsatz sichtbar werden. Unter hoch, bürgerlich, kränken, verletżen, Rubm, erweisen, genießen wird Ehre im Beispielsatz mit dem Lemma verbunden. Verdienen, widerfahren und geben, die als Kookkurrenzen aufscheinen, kommen im Wörterbuch auch als Beispielsätze nicht vor, was bei geben als „Allerweltswort" wenig erstaunlich ist. Im Unterschied $\mathrm{zu}$ den Hund rufen ist jmdm. die Ehre geben auch einfach falsch bzw. Ausweichstrategie bei Nicht-Erinnern des Verbs erweisen. Die präpositionalen Kollokationen zu Ehren kommen bzw. jmdn. bei der Ehre packen sind entweder nur als Beispielsatz (im ersten Beispiel) bzw. gar nicht im Wörterbuch (im zweiten Beispiel) zu finden. Die kollokativen Adjektive groß etc. sind zu unspezifisch, als dass darunter Ehre-Kollokationen - wenn auch als Beispielsätze aufgeführt wären.

Insgesamt ist also ein beachtlicher Teil der in Korpora zu findenden Kookkurrenzen bzw. Kollokationskandidaten in Beispielsätze verpackt bzw. unter anderen als den substantivischen Bestandteilen verzeichnet. Gegen letztere Praxis ist nichts einzuwenden, sie sollte allerdings systematisch und transparent gehandhabt werden. Die Aufführung von Kollokationen in Beispielsätzen ist Ausdruck des Kollokationsempfindens und der Kollokationskenntnis derjenigen, die den Artikel verfasst haben, bzw. kann auch auf die Beleglage zurückgehen, ist aber für diejenigen, die das Wörterbuch benutzen, in keiner Weise transparent. Ein ebenso beachtlicher Teil ist jedoch auch im Duden Stilwörterbuch, das den Kollokationen des Deutschen wohl am besten oder jedenfalls vergleichsweise gut Rechnung trägt, ohne den Begriff explizit zu verwenden bzw. die Kollokationen systematisch lexikografisch aufzuführen, nicht enthalten.

Der Ertrag der Arbeit durch Korporaabfragen ist von der Zusammensetzung, Strukturierung und Größe des Korpus abhängig, sowie von Lemmatisierungsprogrammen und der Berechnung der Kollokationen. Weiter hängt der Ertrag auch davon ab, welchen Stellenwert das abzufragende Element im Wortschatz insgesamt bzw. in den abgefragten Texten hat (vgl. Durĉo i.Dr.).

Kollokationen mit semantisch spezifischen, veraltenden oder veralteten oder unikalen Elementen, also eher seltenen Elementen des Wortschatzes, findet man in der Korpusabfrage leichter - nicht häufiger - als allgemeine Verben oder Adjektive wie legen, stellen, setzen, geben, viel etc. als Teile von Kollokationen. Das ist ein Problem der Bearbeitbarkeit der Ergebnisse einer Kookkurrenzanalyse. Damit diese Verben oder Adjektive etc. als signifikant oben in der (möglicherweise recht langen) Liste erscheinen, müssen sie wirk- 
lich sehr eng mit der Basis verbunden sein und recht häufig in dieser Kombination vorkommen. Sonst stehen sie weiter hinten in der Liste, wo der Aufwand der Unterscheidung von Spreu und Weizen zu groß wird. Präfigierte Verben, die gerade oft mit semantisch wenig spezifischen Wörtern wie stellen, legen, setzen etc. gebildet werden, werden von den Lemmatisierungsprogrammen aber nicht immer richtig aufgelöst. Das heißt, wenn Er stellt ihm ein Bein im Korpus vorkommt, kann es sein, dass das Verb einstellen erkannt wird, genauso aber auch anders herum, dass bei er stellt ibn nach längeren Gesprächen und reiflicher Überlegung letatendlich noch nicht ein nur stellen lemmatisiert wird.

Es ist wichtig und nötig, die Tradition der Wörterbucharbeit miteinzubeziehen, um die Schwächen der Korpusanalyse beim Auffinden bzw. mit Bezug auf die Bearbeitbarkeit der Ergebnisse auszugleichen.

$\mathrm{Zu}$ diesen Schwächen der Korporaabfrage gehört, dass sich mit Bezug auf seltene Elemente des Wortschatzes, wie sie gewisse Idiome und eventuell auch Kollokationen bei gleichzeitiger Salienz und Auffälligkeit darstellen, oft einfach keine Suchergebnisse einstellen, obwohl man als kompetenter Sprecher/kompetente Sprecherin weiß bzw. zu wissen meint, dass es die Verbindung gibt und geben muss. In solchen Fällen kann die oft überreichliche und schlecht bzw. gar nicht sortierte Beleglage der Internetabfrage weiterhelfen - wenn es nicht um Phänomene der gesprochenen Sprache, syntaxorientierte Phänomene mit diskontinuierlicher Repräsentation oder ähnliche geht - beides ist bei Internetrecherchen nicht so leicht zu finden. Ein Beispiel dafür: In einem Zeitungstext spricht Kaspar Villiger von einem Beispiel erster Sorte. Die Kompetenzvermutung ist, dass Erster Güte gemeint sein müsste - beides weder im DWDS noch im CHTK. ${ }^{1}$ Die Suche im Internet gibt 521.000 Treffer an (12.02.2010) und enthält sicherlich viel in diesem Zusammenhang Unbrauchbares, jedoch auch in Spitzenstellungen Beispiele vom Typ Diplomatischer Febltritt erster Güte bzw. ein Multimediamonitor erster Güte, während die Suche nach erster Sorte auf ganz andere Schienen führt, wie die erste und die zweite Sorte.

\subsection{Die Funktionen eines Kollokationenwörterbuches}

Weil Kollokationen semantisch transparent sind, haben entsprechende Wörterbücher weniger eine Funktion für das Verstehen als für das Produzieren. Das ist auch das Anliegen von Hausmann (2004) bzw. seines Momo: Hilfe für den armen Momo, der nicht weiß, wie das richtige Verb zum gewünschten Substantiv lautet. Um die Ausdrucksnot des bedauernswerten Momo zu lindern, müssen alle Kollokationen unter dem Substantiv zu finden sein, wenn Momo vom Substantiv ausgeht.

CHTK $=$ Schweizer Text Korpus, ein Projekt im Rahmen des Digitalen Wörterbuchs der deutschen Sprache. 
Aus der Sicht eines lernenden Sprachbenutzers ist es richtig, dass Substantiv-Verb-Kollokationen unter dem Substantiv aufgeführt werden. Aus der Sicht der Sprachbeschreibung und Sprachreflexion und im Hinblick auf das zu entwickelnde Sprachwissen ist nicht zu vergessen, dass es weitere nichtsubstantivische Kollokationsstrukturen gibt und dass für die Bewusstheit von sprachlichen Einheiten im Allgemeinen und Kollokationen im Besonderen jedes kollokative Element als Ausgangspunkt für das Nachschlagen von Bedeutung ist.

An welches Element man sich nämlich bei gefühlter Eingebundenheit in die Festigkeit von festen Wortverbindungen erinnert, ist nicht vorauszusagen. Es kann durchaus interessant sein, unter stellen nachzuschlagen, welche typischen kollokativen Leistungen das Wort übernimmt, ebenso, wie es interessant sein kann, nachzuschlagen, was man jemandem üblicherweise genau vergällen kann.

Das ist auch für native speakers von Bedeutung, weil sich in journalistischen und anderen Gebrauchstexten sehr viele fragliche Kollokationen bzw. falsch verwendete Kollokationen finden, wonach beispielsweise Hoffnungen auf jemandem lasten. Eine solche Äußerung könnte bei entsprechender Absicht eine aussagekräftige Variante zu Hoffnungen, die auf jmdm. ruhen bzw. eine ebenso aussagekräftige intendierte Kontamination von Hoffnung, die rubt, und Verantwortung, die lastet, darstellen. Analoges gilt für einen Beleg, wonach jmdm. die Teilnahme vergällt wird.

\section{Differenzierung der Sichten vor dem Hintergrund verschiedener lexikografischer Projekte}

Verschiedene Wörterbücher setzen unterschiedliche lexikografische Arbeitsmöglichkeiten voraus. Kollokationen in einem Wörterbuch zum deutschen Grundwortschatz für Sprachlernende der Erst- und Zweitsprache, insbesondere Schülerinnen und Schüler, mit ca. 2.000 Lemmata stellen eine vollkommen andere Aufgabe dar als Kollokationen in einem Dialektwörterbuch des Baseldeutschen mit ca. 10.000 Lemmata oder als Kollokationen in einem Variantenwörterbuch des Deutschen.

Wenn man Kollokationen versteht als nicht oder nur schwach idiomatische Wortverbindungen (vgl. Burger et al. 2007, S. 7), so verlangt dies eine Abgrenzung in zwei Richtungen. Zum einen sind sie von Wortkombinationen abzugrenzen, deren Elemente weder durch Realien, noch durch die Semantik noch durch den Usus überdurchschnittlich prädestiniert sind, miteinander vorzukommen, also von freien Wortverbindungen. Zum anderen sind sie von den übertragenen (idiomatischen) Wortverbindungen (Phrasemen) abzugrenzen, deren Bedeutung sich nicht wörtlich entschlüsseln lässt (beispielsweise starker Tobak, kalter Kaffee). 
Von einer kollokativen Wortverbindung kann man dann sprechen, wenn ein Sachverhalt durch mehrere Wortkombinationen ausgedrückt werden kann, aber von den SprecherInnen/SchreiberInnen häufig ein und dieselbe gewählt wird bzw. beim Sprechen oder Schreiben von fremdsprachigen SprecherInnen auch bei Berücksichtigung der Bedeutungen der Einzelwörter eine unübliche Wortverbindung entsteht, weil sie die kollokative Wortverbindung nicht kennen. Analoges gilt auch in der Erstsprache, wenn es um professionelle oder professionalisierte Ausdrucksweisen geht. Nicht routinisierten Umgang mit Situationen, Laien bzw. Outsidern erkennt man nicht zuletzt an ihrem Sprachgebrauch.

Oft lässt sich bei den Kollokationen eine einseitige, eventuell auch gegenseitige Evozierbarkeit feststellen, also dass ein Ausdruck seinen Kollokationspartner gewissermaßen zwangsläufig fordert, wie es bei den ungleichgewichtigen folgenden Beispielen Abbilfe schaffen oder Amok laufen der Fall ist. Diese Prädisposition, dass für einen bestimmten Sachverhalt häufig die gleiche Wortverbindung aus mehreren möglichen Wortkombinationen ausgewählt wird, lässt sich in Textkorpora mittels der Kookkurrenzanalyse statistisch nachweisen. Wissenschaftlich kann man sich Kollokationen sowohl von sprachsystematischer Seite als auch von pragmatischer Seite nähern. In der angewandten Sprachwissenschaft führt der sprachsystematische Ansatz in seiner konsequenten Umsetzung zur korpusanalytischen Ermittlung von Kookkurrenzen, also solchen Wortpaaren die - ungeachtet der Syntax und Semantik - rein statistisch überdurchschnittlich oft in einem definierten maximalen Abstand (meist fünf Wortzeichen) miteinander vorkommen.

\subsection{Das Kollokationenwörterbuch: Wörterbuch der typischen und gebräuchlichen Wortverbindungen der deutschen Sprache}

Mit dem Wörterbuch der typischen und gebräuchlichen Wortverbindungen der Deutschen Sprache soll eine Lücke in der Deutschen Lerner-Lexikografie geschlossen werden, die beispielsweise im Englischen längst mit dem vielgenutzten „Oxford collocations dictionary for students of English“ (2009), dem „Dictionary of selected collocations“ (1999) und dem „Collins Cobuild English collocations“ (1995) geschlossen wurde, wenngleich das Kollokationenwörterbuch von viel bescheidenerem Umfang als die genannten englischsprachigen Wörterbücher ist.

Kollokationen sind im Zweitspracherwerb noch nach sieben Jahren Unterricht auch in einfachen Texten schwierig, das Können der LernerInnen ist unberechenbarer als beim kindlichen Erstspracherwerb und von der Lehrperson abhängig bzw. von der Übereinstimmung von Erstsprache und unterrichteter Sprache (vgl. Meier 2010). 
Die Kenntnis der Kollokationen einer Sprache ist ein unverzichtbarer Baustein für ein unmarkiertes Sprechen und Schreiben und sollte dementsprechend systematisch in den Sprachunterricht einbezogen werden. Das auf einen Basiswortschatz beschränkte Wörterbuch der typischen und gebräuchlichen Wortverbindungen des Deutschen soll einen systematischeren und reflexiven Umgang mit Kollokationen in Lehr- und Lernmitteln ermöglichen.

Es wird ein korpusbasiertes Kollokationenwörterbuch für die Zielgruppe der fortgeschrittenen Sprachlerner für den Erst- und Zweitsprachunterricht angestrebt. In der Praxis sollte es zu einem nützlichen Nachschlagewerk für Sprachlernende werden, die über den Anfängerstatus hinaus sind, insofern als schon etwas fortgeschritten betrachtet werden können und in Termini des Europäischen Referenzrahmens auf Stufe A2, B1 und B2 anzusiedeln sind.

Das erste Ziel, eine Lemmabasis von 2.000 Wörtern zu erarbeiten, welche als Grund- oder Basiswortschatz den Rahmen des Kollokationenwörterbuchs vorgeben, wurde mit folgenden Quellen und Korpora bearbeitet.

Lern-/Grundwortschätze:

- Lübke, Diethard (2001): Lernwortschatz Deutsch: Deutsch - Englisch. [ca. 3.100 Lemmata];

- Bock, Heiko (Hg.) (1999): Basic German Vocabulary. [ca. 4.000 Lemmata];

- Wiktionary-Grundwortschatz-Liste: http://de.wiktionary.org/wiki/Wiktionary: Projekt:Grundwortschatz_plus_Wiktionary_minimum [ca. 1.400 Lemmata];

- Remanofsky, Ulrich (2000): Zertifikatstraining Deutsch. Wortschatz. [ca. 2.000 Lemmata];

- Schnörch, Ulrich (2002): Der zentrale Wortschatz des Deutschen: Strategien zu seiner Ermittlung, Analyse und lexikografischen Aufarbeitung. Mannheim, Institut für Deutsche Sprache.

Korpora:

- Lemmaliste des Schweizer Text Korpus (CHTK) und des DWDS nach Häufigkeit;

- Wortlisten der häufigsten Wörter des Wortschatzportals Leipzig: http:// wortschatz.uni-leipzig.de/html/wliste.html;

- IDS Mannheim: Korpusbasierte Wortgrundformenliste DeReWo, v30000g-2007-12-31-0.1, mit Benutzerdokumentation, www.ids-mannheim .de/kl/derewo/.

Speziell zur Berücksichtigung der nationalen Varianten wurde bei der Erstellung des Basiswortschatzes das Variantenwörterbuch (Ammon et al. 2004) mit einbezogen und der Basiswortschatz um die nationalen Varianten ergänzt. 
Da sich auch Lehr- und Lernmaterialien an Basiswortschätzen orientieren, wird auf diese Weise zu gewährleisten versucht, dass der Wörterbuchinhalt und der Kenntnisstand von Sprachlernenden möglichst große Übereinstimmungen aufweisen. Es hat sich gezeigt, dass Substantive bei der statistischen Ermittlung des Basiswortschatzes deutlich übergewichtet sind - etwa zwei Drittel der Lemmata sind substantivisch.

Nur eines der mehreren Lemmata der gesuchten Kollokationen muss zum Basiswortschatz gehören, weil Kollokationen oft Basiswortschatzlemmata in Kombination mit sehr allgemeinen und sehr spezifischen, veralteten, eventuell auch unikalen Elementen enthalten. Zudem spielt die Frage der Zugehörigkeit der Bedeutung zum Basiswortschatz eine Rolle: sich etwas nicht nehmen lassen besteht zweifellos aus Elementen des Basiswortschatz, ob auch die Bedeutung dazugehört, ist eine andere Frage, die nicht zuletzt mit Bezug auf die Rolle der Kollokation in Alltagstextsorten der besprochenen Art entschieden werden soll.

Der relevante Basiswortschatz wurde im Redaktionssystem erfasst und als Ausgangsbasis für die Korpusabfragen zur empirischen Datengewinnung verwendet. In der Bearbeitung spielen neben den Kriterien des Basiswortschatzes und der Frequenz die Kriterien der Bedeutung(en) des Lemmas und der darauf aufbauenden Kollokation, sowie das Vorkommen in Textsorten des Alltags, zu dem auch Antragsformulare für die Subventionierung der Krankenkassenprämien, die Steuererklärung sowie RoutineGespräche an Schaltern aller Art gehören, Kommunikationssituationen, die über den routinisierten Charakter hinaus zumindest für die eine der beiden Kommunikationsseiten auch einen professionellen Charakter haben.

Die Kookkurrenzenanalyse von Korpora bringt zu viele nicht wörterbuchrelevante Daten hervor und die rein kognitive und pragmatische Ermittlung der Kollokationen ist neben dem Arbeitsaufwand problematisch hinsichtlich der Repräsentativität des gefundenen Materials. Die Lösung besteht in der Kombination der beiden Verfahren, der Datenerhebung mittels korpusanalytischer Methoden und der anschließenden Auswertung der Daten unter pragmatisch-semantischen Gesichtspunkten.

Ein Beispielartikel rechnen: Es wird versucht, zwischen typischen und gebräuchlichen Wortverbindungen im obigen Sinn zu differenzieren und die Unterscheidung durch fette Initialen (typische Wortverbindungen) bzw. Fettdruck (gebräuchliche Wortverbindungen) visuell sichtbar zu machen. Weiter werden Bedeutungsangaben nur im Fall von polysemen Bedeutungen gemacht. Hingegen werden in jedem Fall auf der Basis empirisch erhobener Textausschnitte möglichst aussagekräftige Beispiele gegeben. 
rechnen

1 eine mathematische Rechnung durchführen

ADV. genau Genau rechnen vor dem Immobilienkauf. I richtig, falsch I schriftlich, im Kopf, mit dem Taschenrechner Einfache Rechnungen lassen sich schneller im Kopf als mit dem Taschenrechner rechnen.

ADV. + R. (PART.) rund Ein Fußballer verdient rund gerechnet 3 Millionen Euro im Jahr.

knapp, hoch Hoch gerechnet bleiben dir von deinem Einkommen noch 30.Franken am Monatsende.

2 ,mit etw. / jmdm. rechnen“: planen

ADV. fest Ich habe fest mit deinem Kommen gerechnet. I vorläufig nicht Die Meteorologen rechnen vorläufig nicht mit einer Wetterverbesserung. I frühestens in einer Woche/zwei Tagen/... Mit einer Antwort auf Ihre Bewerbung

3 sparsam sein können Sie frühestens in einem Monat rechnen.

R. (INF.) + VERB müssen Am Monatsende bleibt ibnen nicht viel Geld, da müssen sie bei jedem Einkauf rechnen. I nicht zu r. brauchen Er verdient genug, dass er nicht bei jedem Restaurantbesuch zu rechnen braucht.

PHRASE mit jedem Cent r. müssen (,wenig Geld (übrig) haben?) Eine Familie mit vier Kindern muss mit jedem Cent rechnen.

\subsection{Das Neue Baseldeutsch Wörterbuch}

Noch einmal andere Fragen stellen sich beim Neuen Baseldeutschen Wörterbuch (vgl. Christoph Merian Stiftung (Hg.) 2010, www.baseldeutsch.ch). Für relativ kleine Dialekte gibt es oft nur ein vorliegendes Wörterbuch, von dem man ausgehen kann. Dazu kommt als Informationsquelle die wertvolle Intuition der Lexikografen, die man weder ausschalten kann noch soll, auf die man sich aber auch nicht beschränken sollte. Deswegen wurden die Baseldeutsch-Sprechenden durch die lokalen Radio- und Zeitungsmedien auf eine Online-Umfrage zum Baseldeutsch-Wörterbuch aufmerksam gemacht, in deren Kontext sie Sprachgebrauchsdaten zu schlecht belegten Wörtern beisteuern konnten. Sie konnten Angaben machen zur persönlichen Gebräuchlichkeit und Kenntnis sowie typische Kontexte angeben. Die folgenden Daten beziehen sich auf die Ergebnisse dieser Umfrage.

Mit Korpora ist es bei Dialekten insofern schwieriger, als zwar Einiges an Texten schriftlich verfügbar ist, die Texte jedoch großmehrheitlich nicht digitalisiert und in vielen Fällen (bei Theaterstücken für Laiengruppen etc.) auch nicht gedruckt und nur als Schreibmaschinen-Manuskript vorhanden sind. Dazu kommt, dass die nicht normierte Dialektschreibung, auch wenn die Texte digitalisiert werden, die elektronische Suche nicht erleichtert. Dialektwörterbücher, die sich an ein Laienpublikum richten und regional vermarktet werden, haben zudem eine Tendenz, sich rückwärtsgewandt auszurichten. Laien suchen in einem kleinräumig ausgerichteten Dialektwörterbuch 
ein Nachschlagewerk zu sprachlichen Fragen der regionalen Identität. Regionale Identität ergibt sich in Zeiten, in denen Kleinräumigkeit durch tendenzielle Globalisierung bedroht ist, in viel stärkerem Ausmaß durch die Geschichte als durch ungewisse Zukunftsfunktionen oder die Modernität eines Dialekts, für die Kriterien und damit die Erkennbarkeit fehlen. Zudem gilt eine beschränkte Auswahl historischer Sprachformen, die man sich als Schibboleth merken kann, die man vielleicht noch vom Hören oder sogar vom Hörensagen kennt, als interessant. Als Alternative der Datenerhebung wurden hier Fragen an den Sprachgebrauch in einem Internetportal gestellt.

Das Baseldeutsche Wörterbuch wird daher nicht mehr aktuelle Wortformen, die einmal typisch für das Baseldeutsche waren, bei voller deskriptiver Ausrichtung nicht einfach entfernen, sondern als historische Formen, die im Sprachwissen und in der Sprachbewusstheit angesiedelt werden können, kennzeichnen. Es stellt sich sogar die Frage, ob man historische Schreibweisen, die zu ihrer Zeit die Aussprache abgebildet haben und heute noch gut bekannt und geschätzt sind, als orthographische Schreibweisen aufnehmen soll, obwohl sie nicht mehr gesprochen werden und in der Dialektschreibung normalerweise ein aktuelles Transkriptionsprinzip gilt. So beispielsweise mit Bezug auf die Entrundung: Historisch gesehen wurde im Baseldeutschen ein -ö- entrundet als -e- ausgesprochen sowie ein - ̈ü- entrundet als -i- realisiert. Da diese historische Aussprache das höhere Prestige hatte und teilweise noch hat, wurde die entsprechende Schreibweise für die ebenfalls prestigeträchtige und - wie in einer protestantischen Region üblich - streng reglementierte Fasnacht bzw. Fasnachtsschreibung vorschriftsmäßig verwendet.

Das baseldeutsche Adjektiv scheen (hdt.: schön) gilt vielen SprecherInnen und SchreiberInnen heute weniger als artikulatorische Abbildung, die sie nicht mehr ist, sondern als Rechtschreibnorm. Jedoch wird die Entrundung in keiner Weise systematisch gesprochen und geschrieben, sondern nur mit Bezug auf wichtige Fasnachtswörter fallweise realisiert: Niemandem würde es daher einfallen, analog Bürger als Bïrger zu schreiben.

Es gibt also bei der Aktualisierung eines Wörterbuchs viele Fragen zum Umgang mit dem bisher erfassten lexikografischen Material und seiner aktuellen Beurteilung hinsichtlich Bekanntheit und Gebräuchlichkeit, die normalerweise durch ein großes strukturiertes Korpus angegangen werden. Die folgenden Beispiele sollen das illustrieren.

E zrfrìideni Sach (hdt.: *Eine zufriedene Sache, jedoch auf Hochdeutsch nicht phraseologisch und (daher) ohne die Bedeutung ,eine gute Sache, mit der man zufrieden sein kann"). Eingebettet in einen Kontext wurde der Ausdruck in der Online-Umfrage beispielsweise folgendermaßen gebraucht:

$$
\begin{aligned}
& \text { a. Am Sùnntig usschloofe find ich e zriìdeni Sach (hdt.: „Am Sonntag } \\
& \text { ausschlafen finde ich *eine zufriedene Sache“). }
\end{aligned}
$$


b. Die Fè̀rie sinn e zfrìdeni Sach gsii (hdt.: „Die Ferien waren *eine zufriedene Sache ${ }^{6}$ ).

E glùngene Thüp (hdt. wörtlich: *Ein gelungener Typ, im Hochdeutschen nicht gebräuchlich, ohne die baseldeutsche Bedeutung „ein spezieller Typ“). Der Ausdruck war zehnmal in der Online-Umfrage, wurde neunmal bewertet und von allen neun Personen mit ,schon gehört und weiß, was es bedeutet“ gekennzeichnet. Vier Personen gebrauchten den Ausdruck „manchmal“, zwei „oft“, eine „nie“. Eine Person betitelte den Ausdruck als ,altmodisch“.

Interessant an dieser Auswahl von Beispielsätzen ist die Einbettung des Ausdrucks hinsichtlich der Palette von Partikeln, die im Kontext des Ausdrucks oft gebraucht werden wie halt e biz, no, äifach, denn, die in diesen Beispielsätzen von Laien sehr gut die typische sprachliche Vorsicht bei Qualifizierungen von Mitmenschen zum Ausdruck bringen.

(2) a. Wäisch är isch halt e biz e glùngene Thüp und nit wie alli andere (hdt. wörtlich: „,Weißt Du, er ist halt ein bisschen *ein gelungener Typ und nicht wie alle anderen").

b. Der Feelix isch nòn e glùngene Thüp (hdt.: „Der Felix ist noch *ein gelungener Typ").

c. Das isch äifach e glùngene Thüp (hdt.: „Das ist einfach *ein gelungener Typ").

d. Dasch mer nòn e glùngene Thüp (hdt.: „Das ist mir noch *ein gelungener Typ").

e. Kènnsch der Walti? Das isch dènn e glìngene Thüp (hdt.: „Kennst Du den Walter? Das ist vielleicht *ein gelungener Ty $\left.p^{\circ}\right)$.

f. Är isch biz. e glìngene Thüp (hdt.: „Er ist ein bisschen *ein gelungener Typ").

Nit ganz bache sii (hdt.: * nicht ganz gebacken sein im Sinne von „nicht ganz normal sein"). Der Ausdruck war siebenmal in der Umfrage und wurde fünfmal mit ,,schon gehört und weiß, was es bedeutet“ bewertet. Eine Person verwendete den Ausdruck „manchmal“, zwei „oft“.

Die Beispielsätze bringen im ersten Fall die Semantik und Pragmatik von nit gan z bache sii wiederum gut zum Ausdruck, im ersten Fall durch die syntagmatische Anfügung mit seiner Bieridee, im zweiten und dritten Fall durch die Verwendung in der Anrede als „,mäßig schlimme“ Beschimpfung.

(3) a. Dä isch dòch nì ganz bache mit sine Bieridee'e (hdt.: „Der ist doch * nicht ganz gebacken mit seinen Bierideen").

b. Du bisch dòch nit ganz bache (hdt.: „Du bist doch *nicht gan gebacken"r).

c. Bisch nit ganz bache, bi dääre Kélti nùr im Pulloower ùff d Strooss ₹.goo? (hdt.: „Bist Du *nicht ganz.gebacken, bei der Kälte nur im Pullover auf die Strasse zu gehen?"). 
Öpperem äini bache (hdt.: *Jemandem eine backen im Sinne von „,jmdm. eine runterhauen"). Der Ausdruck war zwölfmal in der Umfrage, wurde achtmal bewertet, siebenmal mit "schon gehört und weiß, was es bedeutet“, einmal mit „noch nie gehört“. Der Ausdruck wurde dreimal als „oft gebraucht“, zweimal als „,manchmal gebraucht", einmal als „,nie gebraucht“ taxiert. Als Beispielsätze wurden die prototypische konditionale Verwendung als Rede über Dritte und die Drohung gegenüber einem nervenden Gegenüber angegeben:

Interessant ist, dass die Beispielsätze wie im Fall der obigen Drohung oft - bis auf einen Partikel oder ähnlich - identisch vorgeschlagen werden und dementsprechend in der aufgeführten Verwendung sehr gebräuchlich sein müssen.

(4) a. I bät em äini könne bache (hdt.: „Ich hätte ihm eine runterhauen können“ im Sinne von „mögen“ oder „wollen“).

b. Däre blööde Kue söt men emòl ä̈ni bache (hdt.: „Dieser blöden Kuh sollte man mal eine runterhauen").

c. Wènn d jètz nit sòfort üffhö̈rsch, dènn bach der ä̈ni (hdt.: „Wenn du jetzt nicht sofort aufhörst, haue ich dir eine runter").

Ùff äi Dätsch (hdt.: ,auf einen Schlag ${ }^{\circ}$ ). Der Ausdruck war elfmal in der Umfrage, wurde zehnmal bewertet und neunmal mit ,schon gehört und weiß, was es bedeutet“" beurteilt. Eine Person meinte, der Ausdruck wäre „veraltet“. Drei Personen verwendeten den Ausdruck „oft“, vier „manchmal“.
a. Si bän ùff äi Dätsch drei Kinder bikoo: Drilling! (hdt.: „Sie haben auf einen Schlag drei Kinder bekommen: Drillinge!").
b. Üff äi Dätsch sinn alli wach gsì̀ (hdt.: „Auf einen Schlag waren alle wach").
c. Si hèt üff äi Dätsch 10000 Frangge gwùnne (hdt.: „Sie hat auf einen Schlag 10.000 Franken gewonnen").
d. Es kìnnt alles ìff äi Dätsch (hdt.: ,Es kommt alles auf einen Schlag").
e. Sisch alles îff äi Dätsch koo (hdt.: „Es ist alles auf einen Schlag gekommen").

\section{Fazit}

Insgesamt kann festgehalten werden: Erste Arbeiten an einer separaten Lexikografie der Kollokationen zeigen, dass systematische Korpusanalysen viel zusätzliches Material zu Tage fördern. Es stellt sich die Frage, wie dieses Material aufbereitet werden soll bzw. für wen welche Kenntnisse über Kollokationen nützlich sind. Kollokationen sind grundsätzlich nicht nur im Hin- 
blick auf die Förderung der Sprachrichtigkeit lexikografisch zu erfassen, sondern auch im Hinblick auf Sprachwissen zur Ökonomie der Sprachentwicklung und Sprachwissen zur Musterbildung und zum Mustererwerb wissenschaftlich zu beschreiben und lexikografisch zu verzeichnen.

Angesichts der großen Zahl der auffindbaren Kollokationen ist zu differenzieren und zu gewichten: a) Es steht lexikografisch zunächst die Unterstützung bei der Sprachproduktion im Vordergrund sowie b) der Grundwortschatz bzw. Basiswortschatz, und es sind c) die Unterscheidung von typischen Wortverbindungen und gebräuchlichen Wortverbindungen (den Hund loslassen vs. den Hund anleinen/an die Leine nehmen) vorzunehmen.

Bei der Dialektlexikografie (mit vergleichsweise kleinen Korpora im Hintergrund) findet man auch zusätzliche Kollokationen, jedoch quantitativ weniger ausgeprägt. Hier sind ergänzende Datenerhebungen über Umfragen hilfreich und liegen die Unterschiede zum Bisherigen vor allem auch in der Artikelstruktur, in der Herausnahme der Kollokationen aus dem inadäquaten Beispielsatzformat und in den Aktualisierungen der Form.

\section{Literatur}

\section{Wörterbücher}

Ammon, Ulrich et al. (2004): Variantenwörterbuch des Deutschen: die Standardsprache in Österreich, der Schweiz und Deutschland sowie in Liechtenstein, Luxemburg, Ostbelgien und Südtirol. Berlin/New York.

Bock, Heiko (Hg.) (1999): Basic German vocabulary. Überarb. Aufl. Berlin/München.

Christoph Merian Stiftung (Hg.) (2010): Neues Baseldeutsch Wörterbuch. Basel.

Collins Cobuild English collocations (1995) = Sinclair, John et al. (Hg.) (1995): Collins Cobuild English collocations on CD-ROM: a comprehensive database of common word patterns from the Bank of English. London.

Dictionary of selected collocations (1999) = Hill, Jimmie/Lewis, Michael (Hg.): Dictionary of selected collocations. Hove.

Duden (2010): Duden. Bd. 2: Das Stilwörterbuch: Grundlegend für gutes Deutsch. 9., völlig neu bearb. Aufl. Hrsg. v. Wissenschaftlichen Rat der Dudenredaktion. Mannheim u.a.

DWDS: Das digitale Wörterbuch der deutschen Sprache des 20. Jahrhunderts. Internet: www.dwds.de (Stand: 24.01.2011).

Lübke, Diethard (2001): Lernwortschatz Deutsch: Deutsch - Englisch: [Wortschatz für das neue Zertifikat Deutsch]. Ismaning.

Oxford collocations dictionary for students of English (2009). Hrsg. v. Colin McIntosh et al. 2. Aufl. Oxford u.a. 
Remanofsky, Ulrich (2000): Wortschatz: überarbeitet für das neue Zertifikat Deutsch. 3., bearb. u. erg. Aufl. Ismaning.

Schnörch, Ulrich (2002): Der zentrale Wortschatz des Deutschen: Strategien zu seiner Ermittlung, Analyse und lexikografischen Aufarbeitung. (= Studien zur Deutschen Sprache 26). Tübingen.

WDG $=$ Wörterbuch der deutschen Gegenwartssprache (1961-1977). Hrsg. v. Ruth Klappenbach und Wolfgang Steinitz. 6 Bde. Berlin.

Wiktionary-Grundwortschatz-Liste: http://de.wiktionary.org/wiki/Wiktionary:Projekt:Grund wortschatz_plus_Wiktionary_minimum (Stand: 16.03.2010)

\section{Sekundärliteratur}

Barz, Irmhild (2007): Wortbildung und Phraseologie. In: Burger et al. (Hg.), S. 27-36.

Bürki, Andreas (i.Dr.): Lexis that rings a bell: on the influence of auditory support in vocabulary acquisition. In: International Journal of Applied Linguistics.

Burger, Harald (2010): Phraseologie: eine Einführung am Beispiel des Deutschen. 4., neu bearb. Aufl. (= Grundlagen der Germanistik 36). Berlin.

Burger, Harald et al. (2007): Phraseologie: Objektbereich, Terminologie und Forschungsschwerpunkte. In: Burger, Harald et al. (Hg.), S. 1-10.

Burger, Harald et al. (Hg.) (2007): Phraseologie: ein internationales Handbuch zeitgenössischer Forschung. (= Handbücher zur Sprach- und Kommunikationswissenschaft (HSK) 28, Teilbd. I). Berlin u.a.

Durĉ́, Peter (i.Dr.): Extracting data from corpora statistically - pros and cons.

Eichinger, Ludwig M. (2004): Von Köpfen, Nägeln und anderen guten Bekannten. In: Steyer (Hg.), S. 1-18.

Filatkina, Natalia/Hanauska, Monika (i.Dr.): Wissensstrukturierung und Wissensvermittlung durch Routineformeln: Am Beispiel ausgewählter althochdeutscher Texte. In: Yearbook der europäischen Gesellschaft für Phraseologie.

Häcki Buhofer, Annelies (1997): Phraseologismen im Spracherwerb. In: Wimmer, Rainer/Berens, Franz Josef (Hg.): Wortbildung und Phraseologie. (= Studien zur deutschen Sprache 9). Tübingen, S. 209-232.

Hausmann, Franz Josef (2004): Was sind eigentlich Kollokationen? In: Steyer (Hg.), S. 309-334.

Hausmann, Franz Josef (1989): Le dictionnaire de collocations. In: Hausmann, Franz Josef et al. (Hg.): Dictionaries. An International Encyclopedia of Lexicography. (= Handbücher zur Sprach- und Kommunikationswissenschaft (HSK) 5). Berlin u.a., S. 1010-1019.

Meier, Stefanie (2010): Kollokationen im Zweitspracherwerb. Masterarb. Basel.

Polenz, Peter von (2009): Geschichte der deutschen Sprache. 10., völlig neu bearb. Aufl. Berlin u.a. 
Steyer, Kathrin (Hg.) (2004): Wortverbindungen - mehr oder weniger fest. (= Jahrbuch des Instituts für Deutsche Sprache 2003). Berlin u.a.

Steyer, Kathrin/Brunner, Annelen (2009): Das UWV-Analysemodell. Eine korpusgesteuerte Methode zur linguistischen Systematisierung von Wortverbindungen. (= OPAL 1/2009). Mannheim. Internet: http://pub.ids-mannheim.de/laufend/opal/pdf/ opal2009-1.pdf (Stand: 24.01.2011).

Steyer, Kathrin (2008): Kollokationen in deutschen Wörterbüchern und in der deutschen Wörterbuchforschung. In: Lexicographica 24. Berlin/New York, S. 185-207. 



\title{
Ulrich Heid
}

\section{Korpusbasierte Beschreibung der Variation bei Kollokationen: Deutschland - Österreich - Schweiz - Südtirol}

\begin{abstract}
Kollokationen sind nach unserem Verständnis phraseologische Kombinationen aus zwei Lexemen, die in einer Sprachgemeinschaft konventionalisiert und deren Elemente semantisch ungleichgewichtig sind: Eines wird in derselben Weise verwendet wie in kompositionellen Wortkombinationen (Basis), das andere (Kollokator) erhält seine spezifische Bedeutung nur innerhalb der Kollokation.

Der Artikel diskutiert Möglichkeiten, Kollokationen in Texten zu identifizieren, die für die deutschen Nationalvarietäten aus Deutschland, Österreich, der Schweiz und Südtirol typisch sind. Der Schwerpunkt liegt dabei auf methodischen Fragen: Lassen sich mit einfachen, im Wesentlichen frequenzbasierten Werkzeugen Kollokationsunterschiede zwischen Österreich und Deutschland, der Schweiz und Deutschland, usw. aufweisen? Wie können die Spezifika der Kollokationswahl in den einzelnen Nationalvarietäten anhand von Korpora genauer dokumentiert werden? Sind Varietäten nur in Bezug auf die Kollokatorenwahl spezifisch, oder auch bezüglich morphosyntaktischer Präferenzen (z.B. Artikelgebrauch etc.)?
\end{abstract}

\section{Einleitung}

In diesem Beitrag wird über Methoden und Werkzeuge berichtet, mit denen aus Textkorpora Kollokationskandidaten extrahiert werden können, die man als typisch für die Zeitungssprache von Deutschland (D) bzw. von Österreich (AT), der Schweiz (CH) oder von Südtirol (ST) auffassen kann. Der Schwerpunkt liegt auf Mehrwortausdrücken, die für Österreich, die Schweiz oder Südtirol typisch sind, jedoch in dieser Form im Deutschen von Deutschland selten. Wir ${ }^{1}$ gehen dabei von Zeitungstexten aus, die zum Teil aus einer Vorversion des „Deutschen Referenzkorpus“ des Instituts für Deutsche Sprache stammen. Unsere Materialien sind länderweise geordnet und weisen zwischen ca. 60 Millionen Wörter (für Südtirol) und über 360 Millionen Wörter (für Österreich) auf.

Die hier beschriebenen Arbeiten sind am Institut für maschinelle Sprachverarbeitung der Universität Stuttgart entstanden. Sie sind das Ergebnis der Zusammenarbeit mit Fabienne Cap, Marion Weller und Nadine Siegmund, denen ich für alle Diskussionen und für die praktische Arbeit an den Textdaten herzlich danken möchte. Die Interpretation der Daten (und alle eventuellen Fehlinterpretationen) stammen von mir. 
Als Kollokationen fassen wir phraseologische Kombinationen aus zwei Lexemen auf, die in einer (ggf. regionalen) Sprechergemeinschaft konventionalisiert sind, und die daher in Korpora „auffällig üblich“ sind (Hausmann 1984); ihre Elemente (Hausmann 1979: Basis vs. Kollokator) sind semantisch ungleichgewichtig: Basen haben in der Kollokation dieselbe Bedeutung wie außerhalb, während Kollokatoren eine kollokationstypische Bedeutung haben. Viele Kollokationen haben eine prädikationsartige Struktur (der Kollokator modifiziert die Basis), und fast alle lassen sich einem regelhaften syntaktischen Muster zuordnen; wir untersuchen insbesondere Verb + Objekt-Kollokationen (z.B. CH: Einsitz nehmen, Entscheid fällen) und Adjektiv + Nomen-Kollokationen (z.B. ST: fixe Stelle, fixer Professor).

Als typisch für eine nationale Varietät fassen wir Wortkombinationen auf, die in Texten einer bestimmten Region proportional häufiger auftreten als in denen einer anderen. Im Sinne von Ammons Polyzentrizitätshypothese (vgl. Ammon 1995 und Ammon/Bickel et al. 2004) nehmen wir stark vereinfachend an, dass die verschiedenen in unserem Korpus enthaltenen Zeitungen aus Deutschland, Österreich, der Schweiz und Südtirol jeweils zusammen so etwas wie eine "nationale Zeitungssprache“ repräsentieren. Bei der Kollokationsextraktion interessieren uns allerdings nicht diejenigen Kollokationskandidaten, deren Basen landestypische „Realien“ bezeichnen (CH: Kanton, AT: Bundesheer); ebenso nur am Rande diejenigen, die lexikalische Regionalismen enthalten (AT: betrïgerische Krida („Bankrott"), ST: Erhebungen („Ermittlungen“) aufnehmen): Hier ist nur die Kollokatorenwahl interessant (z.B. CH: Zustupf leisten vs. D: Zuschuss geben). Wir zielen stattdessen insbesondere auf „lexikalisch unauffällige“ Kollokationskandidaten: In der Schweiz ist z.B. tiefer Preis prominenter als niedriger Preis.

\section{Fragestellungen - Quellen}

Beim Lesen deutscher Texte aus Österreich, der Schweiz oder Südtirol fallen immer wieder Mehrwortausdrücke auf, die in dieser Form in deutschen Texten aus Deutschland unerwartet sind (vgl. die Beispiele oben in der Einleitung). Wenn es Unterschiede zwischen den einzelnen nationalen Varietäten des Deutschen hinsichtlich der Kollokationsbildung und des Kollokationsgebrauchs gibt, dann sollten diese Unterschiede mit den Mitteln korpuslinguistischer Datenextraktion erhoben und auf diese Weise einer detaillierteren linguistischen Untersuchung zugänglich gemacht werden können.

Eine erste Aufgabenstellung besteht also darin, Kollokationskandidaten aus Textmaterial aus Deutschland, Österreich, der Schweiz und Südtirol zu extrahieren. Für diese Aufgabe stehen uns lediglich Zeitungstexte zur Verfügung. Dabei ist keineswegs ausgemacht, dass der Zeitungstext für die Fragestellung eine besonders geeignete Quelle ist: Viele Meldungen werden von 
internationalen Presseagenturen verbreitet und gegebenenfalls in verschiedenen Ländern ohne Änderungen übernommen; dabei ist es möglich, dass im Sinne der Norm von Deutschland, unauffällige' Wendungen auch in Österreich, der Schweiz oder Südtirol verbreitet werden. Außerdem haben Journalisten zum Teil ein Interesse daran, eher unmarkierte Texte zu produzieren: Regionalismen, die dem Autor als solche bewusst sind, werden daher unter Umständen eher vermieden. Umgekehrt kann man argumentieren, dass Spezifika, die sich trotzdem in den Zeitungstexten auffinden lassen, unter Umständen vom Autor gar nicht als Regionalismen bemerkt worden sind.

Außerdem ist ein Korpus, welches nur aus Zeitungstexten besteht, natürlich in hohem Maße spezialisiert: Zeitungstext stammt aus einer spezifischen Kommunikationssituation, stellt einen (oder mehrere) spezifische Texttypen und damit auch Sprachverwendungen dar. Vermutlich wäre es sinnvoll, das verwendete Korpus weiter zu untergliedern: Wir berücksichtigen beispielsweise nicht, dass nationale Zeitungen sich vielleicht von regionalen Zeitungen unterscheiden, und dass innerhalb der Schweiz und Österreichs unter Umständen regionale Variation anhand einzelner Regionalzeitungen hätte beobachtet werden können. In dem von uns verwendeten Korpus fehlen die Metadaten, anhand derer eine solche feinkörnigere Analyse angestellt werden könnte.

Ebenso hätte es sich gelohnt, die Zeitungstexte nach Gegenstandsbereichen zu sortieren: Politik, Wirtschaft, Kultur, Sport, Regionalteil. Auch hier wurde keine detaillierte Untergliederung vorgenommen. Eine sorgfältige Studie müsste auch diese Dimension berücksichtigen.

Insgesamt wurden die Zeitungskorpora eher als „Experimentierfeld“ für die entwickelten Werkzeuge benutzt denn als Grundlage für detaillierte Aussagen über die Eigenschaften von Kollokationen des Deutschen von Österreich, der Schweiz oder Südtirol. Auch die Auswertung von Ergebnisdaten ist allenfalls exemplarisch. Geeignete Korpora vorausgesetzt, sollte sich die Methodik aber auf solche Detailuntersuchungen übertragen lassen.

Tabelle 1 stellt die verwendeten Quellen zusammen; die österreichischen und Schweizer Zeitungen aus dem „Deutschen Referenzkorpus“ decken die journalistische Produktion der beiden Länder relativ breit ab, sind aber der Übersichtlichkeit halber nicht einzeln aufgeschlüsselt.

Für Südtirol ist zu bemerken, dass lediglich die Tageszeitung „Dolomiten“ zur Verfügung stand. Wenn wir also Beispiele für „südtiroler Zeitungsprache" geben, so sind diese vielleicht korrekter als Spezifika der Zeitung „Dolomiten“ aufzufassen. ${ }^{2}$

An der Europäischen Akademie Bozen (EURAC) wird im Projekt „Korpus Südtirol“ derzeit ein breiteres und ausgewogeneres Korpus von südtiroler deutschen Texten zusammengestellt und computerlinguistisch erschlossen. Für die „Dolomiten“-Texte danken wir dem Athesia-Verlag, der uns das Material zugänglich gemacht hat, sowie der EURAC, Bozen. 


\begin{tabular}{|l|l|r|}
\hline Land & \multicolumn{1}{|c|}{ Quellen } & Umfang \\
\hline Deutschland & insg. & 258 Mio. \\
& FAZ (1997-1998) & Mio. \\
& Frankfurter Rundschau (1993/1994) & Mio. \\
& Die Zeit (1996-2001) & 51 Mio. \\
& Stuttgarter Zeitung (1993/1994) & 36 Mio. \\
& Handelsblatt (1995/1996) & Mio. \\
\hline Österreich & DeReKo (div. Zeitungen) & 360 Mio. \\
\hline Schweiz & DeReKo (div. Zeitungen) & 183 Mio. \\
\hline Südtirol & Dolomiten (Athesia, EURAC) & 65 Mio. \\
\hline
\end{tabular}

Tab. 1: Datensammlung: verwendete Korpora

Sehr viele Untersuchungen zu Kollokationen konzentrieren sich auf Probleme der Wortkombination: Welche Adjektive oder Verben werden typischerweise mit einem gegebenen Substantiv kombiniert, welche Adverbien mit einem Verb oder Adjektiv usw. Wie unten (Kapitel 3, speziell 3.1.2) gezeigt werden wird, stellen Kollokationen aber insbesondere auch ein interessantes Phänomen im Grenzbereich zwischen Lexik und Grammatik dar, insofern sehr viele Kollokationen spezifische morphosyntaktische Eigenschaften aufweisen, etwa hinsichtlich des Artikelgebrauchs, der Modifizierbarkeit durch Adjektive oder Adverbien, bezüglich Präferenzen für das Auftreten im Aktiv oder im Passiv usw. Wenn man nationale Varietäten untersucht, dann stellt sich auch die Frage, ob sich diese Varietäten primär oder vielleicht ausschließlich auf der lexikalischen Ebene unterscheiden, oder ob es bei gleichem lexikalischem Material auch Unterschiede hinsichtlich solcher morphosyntaktischer Präferenzen gibt. Dieser Frage wird ansatzweise nachgegangen (Abschnitt 4.5); das verfügbare Material reicht allerdings nicht aus, um hier flächendeckende Untersuchungen anzustellen.

Eine weitere Frage betrifft die Methodik des Vergleichs: Stellt sich heraus, dass ein bestimmtes Wortpaar in einer Nationalvarietät typisch und häufig, in einer anderen selten ist, so muss man fragen, durch welches alternative Konstrukt die in der einen Varietät vorhandene Kollokation alternativ in der anderen Varietät ausgedrückt wird. Dies können Kollokationen mit Synonymen sein, wie etwa im Fall von tiefer Preis, welches im Deutschen der Schweiz üblich, im Deutschen von Deutschland eher selten ist.

Es kann aber auch der Fall sein, dass eine Kollokation der einen Varietät durch einen nicht-kollokatorischen Ausdruck der anderen wiedergegeben wird. $\mathrm{Zu}$ fragen ist also, mit welchen Mitteln äquivalente Ausdrücke aus verschiedenen Varietäten miteinander verglichen werden können. Hierfür können bisher lediglich halbautomatische Verfahren auf der Basis einfacher korpuslinguistischer Werkzeuge angeboten werden, aber es ist denk- 
bar, dass Verfahren, die auf die Suche nach Äquivalenten in vergleichbaren Korpora (comparable corpora) angewendet werden, auch hier zum Einsatz kommen können (vgl. Abschnitt 4).

In Abschnitt 3.2 werden einfache Werkzeuge zum Vergleich von Mehrwortdaten in verschiedenen Korpora präsentiert. Diese Werkzeuge sind nicht neu; sie sind in ähnlicher Form für die Extraktion von Fachterminologie aus Fachtexten angewendet worden. Die Frage, die hier diesbezüglich diskutiert werden soll, zielt lediglich darauf, festzustellen, wie weit solche sehr einfachen Werkzeuge für den Varietätenvergleich tragen.

Zusammenfassend lassen sich also die folgenden Fragestellungen festhalten:

- Die lexikalische Selektion in Kollokationen ist präferenziell: Gibt es Präferenzen für die lexikalische Selektion, die als typisch für eine der Nationalvarietäten des Deutschen betrachtet werden können, und wie lassen sich „äquivalente“ Ausdrucksweisen in anderen Nationalvarietäten identifizieren?

- Kollokationen weisen über die lexikalische Selektion hinaus weitere Präferenzen auf, insbesondere im Bereich der morphosyntaktischen Form; untersucht werden soll, ob die Nationalvarietäten auch hier Spezifika aufweisen.

- Aus der Sicht von korpuslinguistischen Datenextraktionswerkzeugen ist zu fragen, inwieweit einfache, im Wesentlichen aus anderen Bereichen der Korpuslinguistik bekannte Extraktionsverfahren auf die Fragestellungen der Variation bei Kollokationen angewendet werden können.

\section{Kollokationen und Kollokationsextraktion aus Texten}

Dieser Abschnitt situiert die vorliegende Studie innerhalb der Kollokationstheorie und stellt dar, mit welchen Verfahren Kollokationskandidaten aus deutschen Texten extrahiert werden.

\subsection{Kollokationsbegriff}

In der Linguistik, Lexikografie und Computerlinguistik ist sehr ausführlich über den Begriff der ,Kollokation“ diskutiert worden. Erst 2004 hat das Institut für Deutsche Sprache seine Jahrestagung und den zugehörigen Sammelband dem Thema der Mehrwortausdrücke gewidmet, mit ausführlichen Diskussionen über Kollokationen (vgl. Steyer (Hg.) 2004).

Für die hier zur Diskussion stehenden Arbeiten ist daher eher eine Positionsbestimmung nötig als eine komplette Aufarbeitung der linguistischen Details (die den vorliegenden Rahmen sprengen würde, vgl. dazu neu z.B. Forkl 2010 und Tutin 2010). 


\subsubsection{Die Arbeitsdefinition von Bartsch}

Ausgangspunkt für die hier diskutierten Arbeiten ist eine computerlinguistische Interpretation des von Hausmann so genannten lexikografischdidaktischen Kollokationsbegriffs (vgl. Hausmann 2004); dieser ist in einer der Computerlinguistik nahen Form von Bartsch (2004, S. 76) zusammengefasst worden: „Collocations are lexically and/or pragmatically constrained recurrent cooccurrences of at least two lexical items which are in a direct syntactic relation with each other."

Diese „Arbeitsdefinition“ (Bartsch) enthält eine Reihe von definitionsrelevanten Aspekten, die in den folgenden Abschnitten kurz aufgelistet und interpretiert werden.

Wir bedienen uns dabei der Hausmann'schen Terminologie, die Basis und Kollokator unterscheidet (Hausmann 1979, 2004): In einer Kombination wie (einen) Hund ausfübren, (eine) Frage stellen oder scharfe Kritik, sind Hund, Frage und Kritik als (im Sinne von Hausmann autosemantische) Basen zu verstehen: Ihre Bedeutung ist innerhalb und außerhalb der Kollokation dieselbe. Die Verben ausfübren und stellen, bzw. das Adjektiv scharf sind Kollokatoren: Sie erhalten ihre Bedeutung erst innerhalb der Kollokation: stellen in Frage stellen dient dazu, eine allgemeine „Realisierung“ auszudrücken (Mel'čuk würde hier die Lexikalische Funktion, oper(ari)' benutzen, vgl. Mel'čuk et al. 1984); scharf in scharfe Kritik dient als Ausdruck eines hohen Grads an Kritik, ähnlich wie heftig oder harsch in heftige Kritik, harsche Kritik. Die Bedeutungen von stellen oder scharf sind also kollokationsspezifisch und nicht notwendig aus den Bedeutungen dieser Lexeme außerhalb der Kollokationen ableitbar; bei Hund ausfübren liegt der Fall ähnlich, auch wenn ausfübren spezifischer ist als die eher allgemeinen Bedeutungen von scharf oder stellen.

\subsubsection{Morphosyntaktische Idiomatisierung in Kollokationen}

Wenn Bartsch sagt, dass Kollokationen „lexically and/or pragmatically constrained" sind, dann verweist sie damit auf die u.a. auch von Hausmann (2004) hervorgehobene lexikalische Auswahl: Zur sprachlichen Realisierung des Konzepts des Spazierenführens eines Hunds gehört das Verb ausfübren, nicht z.B. (?) Hund führen, (?) Hund spazierenführen.

Diese lexikalische Auswahl interpretieren wir als ein Stück Idiomatisierung. Hierzu gehört auch die Festlegung morphosyntaktischer Präferenzen: Bei Frage + stellen kann Frage in beliebigen Nominalphrasen vorkommen (eine wichtige Frage stellen; die Fragen stellen, die keiner hören will; Fragen stellen, auf die es keine Antwort gibt; usw.); bei der Kollokation in + Kraft + treten ist dagegen die Morphosyntax der Nominal- bzw. Präpositionalphrase sehr eingeschränkt: Man hat die Kollokation nur im Singular und ohne Artikel, also in 
Kraft treten, aber nicht (?) in die Kräfte treten usw. (Wo wir bewusst die Determination in einer Kollokation unberücksichtigt lassen wollen, schreiben wir „+“ zwischen Basis, Kollokator und ggf. Präposition.)

Die morphosyntaktische Idiomatisierung (oder: formale Fixiertheit) ist für die Interpretation von Kollokationen konstitutiv, ähnlich wie für idiomatische Wendungen. Dementsprechend gibt es auch Fälle, wo zwei Lesarten sich durch die Morphosyntax, nicht aber durch die am Mehrwortausdruck beteiligten Lexeme unterscheiden (in Gang kommen vs. in die Gänge kommen), oder wo der Unterschied zwischen wörtlicher und idiomatischer Interpretation mit den morphosyntaktischen Eigenschaften einhergeht (in die Hand nebmen (,übernehmen, lenken') vs. (etw.) in die Hand/in die Hände nehmen (,anfassen')). Die morphosyntaktische Fixiertheit (bzw. die ggf. eingeschränkte morphosyntaktische Variabilität) von Kollokationen ist ein Phänomen an der Schnittstelle von Lexikon und Syntax; das (ggf. teilweise) Fehlen der üblichen syntaktischen Veränderungsmöglichkeiten von Phrasen ist Teil der Idiomatisierung. Im Hinblick auf die Analyse der Unterschiede zwischen den Varietäten des Deutschen ist zu fragen, wie stabil diese morphosyntaktischen Spezifika quer durch die untersuchten Varietäten sind.

Nicht alle Kollokationen weisen eine starke morphosyntaktische Fixiertheit auf. Die Kollokation Frage + stellen kann gerade in allen Formen verwendet werden, die für beliebige Nominalphrasen möglich sind; hier ist nur die Selektion von stellen (neben z.B. aufwerfen) typisch und idiomatisch. Die Kollokatorenwahl ist aber wiederum aus semantischen Prinzipien nicht vorhersagbar (und sprachspezifisch, vgl. ask a question, poser une question usw.), d.h. „lexically determined“ im Sinne von Bartsch (2004).

\subsubsection{Rekurrenz von Kollokationen}

Bartsch spricht davon, dass Kollokationen „recurrent" seien; die auch schon von Hausmann (1984) beobachtete ,auffällige Üblichkeit' der Kollokationen hat in der Computerlinguistik dazu geführt, dass statistische Assoziationsmaße dazu eingesetzt wurden, Kollokationskandidaten aus Texten zu extrahieren. Wenn zwei Wörter proportional häufiger miteinander vorkommen als statistisch erwartbar, dann werden sie von den Assoziationsmaßen identifiziert und extrahiert. Evert (2005) hat die Statistik dieser Maße untersucht und analysiert, inwieweit sie mit der linguistisch-lexikografischen Intuition über Kollokationen korrelieren. Als erster Anhaltspunkt sind einige der Maße sehr nützlich; ihr relativ enger Zusammenhang mit der Wortfrequenz kann allerdings mitunter zu Problemen führen. Wir benutzen auch für die vorliegende Studie Assoziationsmaße, insbesondere die Werte des „Log Likelihood Ratio Tests“ (Dunning 1993). 
Der Test vergleicht die beobachtete Häufigkeit der Kollokationen mit der statistisch erwartbaren Häufigkeit, unter Einbezug von Daten zu anderen Kombinationen der beiden Kollokationsbausteine. Es handelt sich um einen statistischen Hypothesentest, der ermittelt, wieviel Evidenz gegen die Annahme vorliegt, der jeweilige Kollokationskandidat sei eine Zufallskombination.

\subsubsection{Binarität und syntaktische Relation in Kollokationen}

Bartsch sagt, dass Kollokationen aus mindestens zwei Elementen bestehen; diese Annahme ist konsistent mit der Unterscheidung in Basis und Kollokator, wie sie Hausmann trifft; viel diskutiert wurde in den letzten Jahren über die Frage, welche Länge Kollokationen annehmen können. Dabei wurden Mehrwortausdrücke als Kollokationen diskutiert, die mehr als zwei Lexeme enthalten. Deutlich ist auf jeden Fall, dass Mehrwortausdrücke selbst Teil von Kollokationen sein sowie mehrere Kollokationen kombiniert auftreten können (z.B. scharf + Kritik, kombiniert mit Kritik + üben in scharfe Kritik üben, vgl. Heid 1994). Für die Untersuchungen im vorliegenden Rahmen zielen wir primär auf Kollokationskandidaten aus zwei Lexemen.

Kollokationen unterliegen den Regeln der Syntax; Bartsch folgt hier dem von Evert (2005) eingeführten Begriff der ,relationalen Kookkurrenz: Zwischen den Elementen der Kollokation gibt es eine grammatische Relation; diese kann parallel zu einer Modifikationsrelation im Sinne der Phrasenstrukturgrammatik liegen (z.B. bei Adjektiv + Nomen-Kollokationen, scharfe Kritik, oder bei Adverb + Adjektiv-Kollokationen, tief rot), sie kann aber auch über Phrasengrenzen hinweggehen (z.B. bei Subjekt + Verb-Kollokationen, die Frage stellt sich, Angst kommt auf, usw.).

Die Anzahl der grammatischen Relationen, die kollokationsträchtig sind, ist relativ begrenzt; die Relationen werden in unserer Extraktionsarbeit als Modell für die Suche nach Lexempaaren genommen; wir suchen z.B. nach Paaren aus Verb und Objekt, aus Verb und Präpositionalobjekt, aus Adjektiv und Nomen usw. Anders als in der Idiomatik, wo zum Teil idiosynkratische grammatische Strukturen auftreten (welche Art Phrase ist z.B. EN by and large?), halten wir die Festlegung auf ein vorab definiertes Inventar von grammatischen Typen bei der Suche nach Kollokationen nicht für problematisch; ausgehend von den einfachen Paar-Modellen kann in einem weiteren Schritt nach Kombinationen von Kollokationen gesucht werden (vgl. Heid/Weller 2010).

\subsubsection{Aktuelle Forschungen zum Kollokationsbegriff}

Die Arbeit von Bartsch (2004) wurde hier zusammen mit den Publikationen von Hausmann (insbesondere Hausmann 2004) als Grundlage für die Einordnung unseres Kollokationsbegriffs verwendet. Bei Bartsch und Haus- 
mann finden sich die folgenden definitionsrelevanten Elemente: (i) phraseologischer Charakter (,lexically and/or pragmatically determined“), (ii) Konventionalisiertheit (,recurrent"), (iii) Binarität (,at least two elements"), (iv) Asymmetrie und Semi-Kompositionalität (Hausmann: Basis vs. Kollokator) und (v) syntaktische Relation.

Neueste Arbeiten von Tutin (2010) und von Forkl (2010) gehen in eine ähnliche Richtung; statt von Semi-Kompositionalität spricht Forkl davon, dass Kollokationen unidirektional kompositionell sind: Man versteht sie, kann sie aber ohne Vorwissen nicht korrekt bilden. Diese Eigenschaft unterscheidet Kollokationen von idiomatischen Wendungen, die sowohl in der Sprachproduktion, als auch in der Rezeption ohne Vorwissen nicht verarbeitbar sind. Tutin und Forkl ergänzen das obige Inventar von Definitionsbausteinen um die Tatsache, dass in Kollokationen eine prädikationsartige Relation existiert: Der Kollokator fungiert in der Regel als Prädikat, die Basis als Argument. Tutin spricht von ,structure prédicat/argument", Forkl sagt, dass Kollokationen relationsprädikativ sind.

Dieser kurze Überblick zeigt, dass mehrere Faktoren bei der Definition von Kollokationen eine Rolle spielen; diese Faktoren sind genauso für die Datenextraktion aus Textkorpora relevant. Wie sie in Suchverfahren umgesetzt werden, wird im folgenden Abschnitt dargestellt.

\subsection{Kollokationsextraktion}

\subsubsection{Ansätze}

Hier kann aus Platzgründen kein vollständiger Überblick über Verfahren der Kollokationsextraktion gegeben werden (vgl. dazu z.B. Heid 2009; Evert 2005 usw.). In starker Vergröberung kann man zwischen symbolischen und statistischen Verfahren unterscheiden.

Die statistischen Verfahren nehmen insbesondere das Kriterium der Rekurrenz (der ,auffälligen Üblichkeit') der Kollokationen als Suchkriterium; extrahiert werden Wortpaare, die häufiger als statistisch erwartet miteinander auftreten, sei es adjazent oder in einem bestimmten ,Textfenster', z.B. im Abstand von drei Wörtern. Solche ,positionelle‘ Kookkurrenz (Evert 2005) ist z.B. im Werkzeug „Wordsmyth“ in reiner Form implementiert.

Symbolische Verfahren setzen auf dem Aspekt der relationalen Kookkurrenz auf: Gesucht werden z.B. alle Paare aus Adjektiven und (Bezugs-) Nomina, oder aus Verben und ihren Objektsnomina. Extrahiert man in der Tat alle derartigen Paare, dann erhält man natürlich auch sehr viele, die nicht rekurrent und auch nicht idiomatisch sind.

Folglich wurde vielfach mit Kombinationen beider Verfahrenstypen gearbeitet. Smadja (1993) hat als erster statistische und symbolische Ansätze kombiniert: erst eine statistische Suche nach allen signifikanten WortpaarAssoziationen, dann ein Filter nach Wortartkombinationen. Für das Deut- 
sche wurde der umgekehrte Weg vorgeschlagen: erst Extraktion aller Paare einer bestimmten grammatischen Relation (z.B. Verben und ihre Objektsnomina), dann eine Sortierung dieser Kandidaten nach der Stärke der Assoziation (Heid 1998; Krenn 2000; Evert 2005). Dieses letztere Verfahren ist z.B. auch in der „Sketch Engine“ von Kilgarriff et al. (2004) für das Englische implementiert. „Sketch Engine“ ist ein kommerzielles Werkzeug zur Analyse von Korpora, das die Funktionen einer Konkordanz mit denen der Suche nach Wortpaaren kombiniert. Der Benutzer kann zwischen beidem hin- und herspringen, z.B. um sich Konkordanzzeilen zu einer Wortkombination anzeigen zu lassen (vgl. http://sketchengine.co.uk/ (Stand: 13.12.2010) zu Details und eine Möglichkeit zum Ausprobieren des Werkzeugs).

Neuere Verfahren kombinieren die letztgenannte Strategie mit einer Suche nach denjenigen Paaren, die morphosyntaktische Fixiertheit zeigen (Fazly/Stevenson 2006); damit werden idiomatische Wendungen extrahiert, sowie derjenige Teil der Kollokationen, der starke morphosyntaktische Präferenzen aufweist; weniger geeignet ist dieses (im Kern eher auf Idiomatik ausgerichtete) Verfahren für Kollokationen vom Typ Frage + stellen, die keine morphosyntaktischen Präferenzen aufweisen.

\subsubsection{Spezifische Probleme der Extraktion deutscher Kollokationen}

Für das Englische erzielt die „Sketch Engine“ (Kilgarriff et al. 2004) Resultate, die mit der lexikografischen Intuition über Kollokationen (,welche der gefundenen Kombinationen sind potenziell wörterbuchrelevant?', vgl. Kilgarriff et al. 2010) relativ gut übereinstimmen. Die Extraktion in „Sketch Engine" erfolgt mit einer relativ einfachen flachen Grammatik, die mit Wortart- und Lemmaangaben annotierte Texte als Eingabe nimmt. Diese flache Analyse genügt für das Englische, weil eine statistisch relevante Menge von Belegen in der für das Englische normalen konfigurationalen Abfolge von Subjekt, Verb und Objekt vorkommt. Sucht das System also Nomina links vom flektierten Verb, so findet es typische Subjekt + VerbKollokationen, sucht es vom flektierten Verb ausgehend nach rechts, findet es Verb + Objekt-Kollokationen.

Weitere, relativ erfolgreiche Experimente mit „Sketch Engine“ wurden für das Slowenische und das Japanische durchgeführt (vgl. Kilgarriff et al. 2010); beide Sprachen haben relativ explizite und wenig ambige Markierungen der grammatischen Relationen (Subjekt, Objekt, usw.), entweder durch morphologische Kasusendungen (im Slowenischen) oder durch Partikeln (im Japanischen).

Die Extraktion aus deutschen Texten macht mehr Schwierigkeiten. Eine flache Analyse nach dem Muster der „Sketch Engine“ muss mindestens die drei Modelle der Konstituentenstellung des Deutschen berücksichtigen (Verb-erst, Verb-zweit, Verb-letzt): Es müssen also mehr Suchmuster 
bereitgestellt werden, als für das Englische. Die bloße Permutation der Suchmuster genügt aber nicht für eine qualitativ hochwertige Extraktion: Aufgrund des weit verbreiteten Kasus-Synkretismus lässt sich nicht ohne Weiteres feststellen, welche Nominalphrasen Subjekte und welche Objekte sind; selbst Dativobjekte sind bei artikellosen Nominalphrasen (z.B. das genügt Anforderungen von Arbeitgebern wie Arbeitnehmern) oft nicht kasusmarkiert.

Die Kombination von Kasussynkretismus, alternativen Wortstellungsmodellen und relativ freier Wortstellung im deutschen Mittelfeld führt dazu, dass ein flacher Ansatz auf der Basis von Korpusdaten mit Wortart- und Lemmaannotation allein für die Extraktion deutscher Kollokationen eher wenig überzeugende Resultate liefert (vgl. Ivanova et al. 2008). Evert (2004) hat anhand der Negra-Baumbank ermittelt, dass nur knapp über 20\% der Nominalphrasen eines Zeitungstexts kasus-eindeutig sind.

\subsubsection{Kollokationsextraktion auf der Basis tiefer Analyse}

Die oben dargestellte Problemlage suggeriert die Suche nach Alternativen zur flachen Kollokationsextraktion im Sinne von „Sketch Engine“. Wir benutzen daher Korpora, die mit einer tiefen syntaktischen Analyse annotiert sind, die von einem dependenzgrammatischen Analysewerkzeug geliefert wird. Wir verwenden Schiehlens (2003) Parser FSPar („Finite State Parser"), ein Werkzeug, das grammatische Funktionen, aber auch morphosyntaktische Merkmale ausgibt. Wenn ein Satz Wörter oder Wortfolgen enthält, deren grammatische Funktion nicht eindeutig identifiziert werden kann (Etiketten-Ambiguität, z.B. Subjekt vs. direktes Objekt), so wird die Mehrdeutigkeit markiert; ebenso bei Zuordnungsambiguitäten (z.B. PP-Attachment). Die Ausgabe des Parsers ist also (durch Angabe von Alternativen) explizit unterspezifiziert.

Für die Kollokationsextraktion hat die unterspezifizierte Ausgabe den Vorteil, dass z.B. Sätze mit Etikettenambiguitäten (Subjekt vs. Objekt) gezielt aus der Menge der Rohdaten für die Extraktion ausgeschlossen werden können; im Fall von Attachment-Ambiguitäten gehen wir davon aus, dass die Datenmenge eventuell vorhandene Fehlzuordnungen ausgleicht: Sie werden bei der Extraktion als unkritisch angesehen und ,überlesen'.

Tabelle 2 und die zugehörige Abbildung stellen die Analyse eines einfachen Satzes dar (die zweite Studie lieferte ähnliche Ergebnisse), und zwar einmal als Dependenzstrukturgraf sowie zweitens als Tabelle; die Tabellenform kann mit einfachen Mitteln automatisch durchsucht werden, und zwar nach grammatischen Relationen (Adjektiv + Nomen: ähnliche Ergebnisse, Verb + Objekt: Ergebnis + liefern, usw.), aber auch nach den morphosyntaktischen Merkmalen der einzelnen Kollokationsbausteine; in der fünften Spalte der Tabelle sind die vom Analysewerkzeug gefundenen morphosyntaktischen Eigenschaften explizit angegeben: Sie brauchen nur von dort extrahiert zu 
werden. Spalte 1 der Tabelle enthält die fortlaufende Position der Wörter im Satz (beginnend mit der Nummerierung ,0), Spalte 2 die Wortformen, Spalte 3 die Wortart-Etiketten des Stuttgart-Tübingen-Tagsets (dabei sind ADJA: Attributives Adjektiv, ART: Artikel, NN: Nomen, VVFIN: finites Vollverb). In Spalte 4 sind Lemmaangaben zu finden (dabei wird ,d- ' für alle Formen des bestimmten Artikels gewählt), in Spalte 5 morphosyntaktische Merkmale (hier in Auswahl dargestellt). Die grammatischen Relationen zwischen den Wörtern sind in der letzten Spalte notiert: Beim Nomen Ergebnisse (Zeile 5, d.h. das Wort, das an sechster Stelle im Satz steht) ist z.B. die Funktion des Objekts (,NP:akk' vermerkt; in der zweitletzten Spalte findet sich das Bezugswort für die grammatische Relation, im Fall von Ergebnisse das Wort mit der Positionsnummer 3 (d.h. das Verb liefern); die Positionsnummer , -1 ' wird für den Wurzelknoten des Dependenzbaums, für Satzendezeichen und für Teilstrukturen vergeben, die das Analysewerkzeug nicht in der Dependenzstruktur verorten kann.

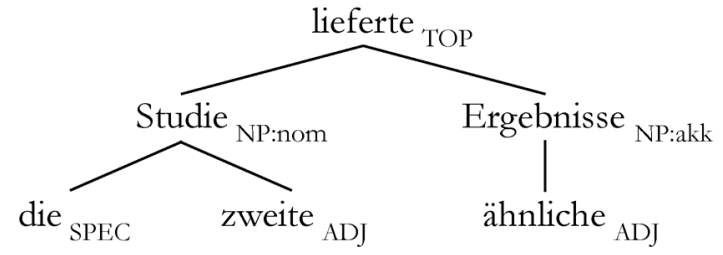

\begin{tabular}{|c|l|l|l|l|c|l|}
\hline Position & Wortform & POS & Lemma & Morphol. & Bezugswort & $\begin{array}{c}\text { gramm. } \\
\text { Funktion }\end{array}$ \\
\hline 0 & Die & ART & d- & & 2 & SPEC \\
\hline 1 & zweite & ADJA & zweite. & & 2 & ADJ \\
\hline 2 & Studie & NN & Studie & Nom:F:Sg & 3 & NP:nom \\
\hline 3 & lieferte & VVFIN & liefern & 3:Sg: Past:Ind & -1 & TOP \\
\hline 4 & ähnliche & ADJA & ähnlich & & 5 & ADJ \\
\hline 5 & Ergebnisse & NN & Ergebnis & Akk:N:Pl & 3 & NP:akk \\
\hline 6 & \$. & \$. &. & & -1 & TOP \\
\hline
\end{tabular}

Tab. 2: Dependenzbaum für die zweite Studie lieferte ähnliche Ergebnisse und tabellarische Darstellung der Dependenzstruktur

Zusammenfassend lassen sich folgende Merkmale (und gleichzeitig Vorteile) der dependenzbasierten Datenextraktion festhalten: Der Dependenzparser verwendet wesentlich mehr grammatisches Wissen als der „Sketch Engine“-Ansatz; er liefert daher auch detailliertere Ergebnisse; diese enthalten u.a. eine vollständige morphosyntaktische Beschreibung. Die Ergebnisrepräsentation ist unterspezifiziert, so dass Kasusambiguitäten als potenzielle Fehlerquelle bei der Kollokationsextraktion identifiziert und ausgefiltert 
werden können; das erhöht die Präzision der Extraktionsergebnisse. Da alle deutschen Wortstellungsmuster abgedeckt sind, erhöht sich auch der Recall (die Ausbeute) gegenüber einer flachen, pattern-basierten Grammatik (vgl. Heid et al. 2008).

\subsubsection{Linguistische Klassifikation der extrahierten Kollokationskandidaten}

Die oben diskutierten Extraktionsverfahren nutzen den Definitionsaspekt der relationalen Kookkurrenz, indem sie z.B. Verb + Objekt-Paare identifizieren. Außerdem wird der Aspekt der Rekurrenz überprüft, insofern die extrahierten Kandidatenmengen nach dem Log-Likelihood-Wert (absteigend) sortiert werden.

Darüber hinaus nutzen wir auch den Aspekt der morphosyntaktischen Fixiertheit: Die morphosyntaktischen Parameter jedes zur Extraktion benutzten Satzes werden einzeln notiert. Anders als Fazly/Stevenson (2006) verwenden wir aber die morphosyntaktischen Präferenzen der extrahierten Daten nicht als Entscheidungskriterium über idiomatische vs. nicht-idiomatische Kookkurrenzen, sondern wir bewahren die Daten auf, um sie nachfolgend statistisch auszuwerten: Wie stabil sind einzelne Präferenzen? Wieviel Flexibilität findet sich in den analysierten Texten?

\begin{tabular}{|l|l|}
\hline v_lemma & vor\#nehmen \\
reflexiv & - \\
negation & - \\
subjekt & Pharmakologe,Arzt \\
subjekt_num & Pl,Pl \\
akk_objekt_n_lemma & Bewertung \\
akk_objekt_determiner & indef \\
akk_objekt_adjektiv & kritisch \\
akk_objekt_num & Sg \\
prep & in \\
prep_nn & in:Bericht \\
fusion & - \\
satzbeleg & In dem seit 1984 jährlich erscheinenden \\
& Bericht nehmen Pharmakologen und \\
& Ärzte eine kritische Bewertung der Arz- \\
korpus & neimittel vor. \\
\hline
\end{tabular}

Tab. 3: Auszug aus der Kollokationsdatenbank: ein Satz und die daraus extrahierten Merkmale

Tabelle 3 stellt einen Auszug aus der Datenbank dar, in der die einzelnen Belegsätze gesammelt und mit den automatisch annotierten morphosyntaktischen Kriterien zusammen abgelegt werden. In der Datenbank können 
auch Adjektive, Adverbien und andere Kontextpartner der Kollokationen aufgesammelt und ausgewertet werden; eine mögliche Beispielanfrage könnte lauten: Bezogen auf alle Okkurrenzen von Bewertung + $(A D J)$ ? + vornehmen, wie häufig ist das Adjektiv kritisch, d.h die Kollokationskombination eine kritische Bewertung (von ...) vornehmen?

Die Auswertung der Datenbankeinträge erfolgt in der Regel nach Basis + Kollokator-Paaren; so kann z.B. festgestellt werden, dass Bewertung + vornehmen primär im Singular, häufiger mit indefinitem als mit definitem Artikel und oft mit Adjektiven wie endgültig, abschließend, kritisch vorkommt.

Für die Untersuchung der Kollokationen aus Texten aus Deutschland, Österreich, der Schweiz und Südtirol werden die Korpora einzeln nach den oben genannten Verfahren analysiert; für jedes Korpus werden die Daten in der Datenbank abgelegt; im nächsten Schritt müssen sie untereinander, nach den vier Sprachräumen, verglichen werden.

\section{Methoden und Probleme des Vergleichs von Varietäten bezüglich Kollokationen}

Wie oben angedeutet, sollen einfache Werkzeuge für den Vergleich von Kollokationsunterschieden zwischen den deutschen Varietäten benutzt werden. Die Frage, die hier ansatzweise mitbeantwortet werden soll, ist, wie weitgehend sich mit solchen einfachen Werkzeugen ein solcher Vergleich überhaupt realisieren lässt.

\subsection{Ein einfaches frequenzbasiertes Verfahren für den Varietätenvergleich}

Ein besonders einfaches Hilfsmittel für den lexikalischen Vergleich von Texten ist der Vergleich der (relativen) Frequenz von Wörtern in den verschiedenen Texten. Ein solcher Vergleich erlaubt es, Wörter (oder, in unserer Erweiterung, Wortkombinationen) zu identifizieren, die in einem gegebenen Text häufig, in einem anderen seltener sind. Ahmad et al. (1992) haben solche Berechnungen dazu eingesetzt, Fachtermini aus Fachtexten zu extrahieren.

Die zu Grunde liegende Idee ist sehr einfach: Wörter, die in einem Fachtext wichtig sind, kommen dort öfter vor als in einem Text oder Textkorpus, das keine fachliche Bindung aufweist. Man zählt also die Okkurrenzen jedes Wortes aus dem Fachtext und bestimmt außerdem die Häufigkeit aller Fachtext-Wörter im Vergleichstext. Da beide Texte unterschiedlich umfangreich sein können, dividiert man jeweils durch die Gesamtzahl der Wörter des betreffenden Texts (ähnlich wie beim Berechnen von parts-permillion). Dies ergibt zwei Zahlen, die zueinander in Relation gesetzt wer- 
den (dividiert: Fachtext-Frequenz/Gemeinsprachtext-Frequenz). Es ergibt sich ein Quotient, der angibt, wievielmal häufiger das betreffende Wort im Fachtext vorkommt als im Gemeinsprachtext.

Ahmad et al. (ebd.) nennen den Quotienten die „weirdness ratio“, weil er angibt, wie stark die Verteilung einzelner Wörter in einem Fachtext gegenüber der Frequenz in einem nicht-fachlichen Text „verschoben“ (weird) ist. Kommt das untersuchte Fachwort im Gemeinsprachtext überhaupt nicht vor, so entsteht ein unendlich großer Quotient (inf, man kann nicht durch Null dividieren), d.h. man bekommt für jedes Textpaar als Ergebnis des Verfahrens zwei Listen: diejenigen der Wörter, die nur Teil des Fachtexts sind, und zweitens eine Liste derjenigen, die in beiden Texten auftreten, aber im Fachtext häufiger sind. Die Elemente beider Listen sind, mindestens im oberen Frequenz- bzw. Quotientenbereich, relativ gute Fachwort-Kandidaten. Die Tabellen 4 und 5 zeigen die Ergebnisse der Analyse eines Korpus, das die Europäische Pharmabehörde EMEA als Sammlung von Medikamentenprüfberichten bereitgestellt hat. In unseren Experimenten wurde dieser Text mit einem Ausschnitt aus der Frankfurter Rundschau verglichen, den wir vereinfachend als nicht fachspezifisch betrachten.

In Tabelle 4 sind die zehn häufigsten Wörter vermerkt, die nur in den EMEA-Texten vorkommen. Tabelle 5 enthält zehn Wörter, die zwar auch im Vergleichstext (hier: die Jahrgänge 1993/94 der „Frankfurter Rundschau“, 40 Millionen Wörter) vorkommen, aber im EMEA-Korpus (10 Millionen Wörter) proportional viel häufiger sind (auch hier: top-10 nach Quotient).

Das dargestellte Suchverfahren kann (mit gewissen Einschränkungen) auf Texte beliebiger Größe angewendet werden; die Texte sollten nicht von allzu unterschiedlicher Größe sein. Außerdem lässt es sich statt auf einzelne Wortformen auf Lemmazahlen (aufsummiert aus den einzelnen Okkurrenzen der Wortformen) und auf Wort- bzw. Lemma-Paare anwenden (auch hier ist aus Sicht der Lexikonstatistik zum Teil allerdings Vorsicht geboten).

Da es sich um ein rein rechnerisches Verfahren handelt, ist es auf beliebige Fragestellungen anwendbar: Wir haben daher die Ahmad'schen „weirdness"-Zahlen für Schweizer bzw. für österreichische Texte ermittelt, jeweils mit den Zeitungstexten aus Deutschland als Vergleichstext. Das Verfahren wenden wir sowohl für Einzelwörter, als auch für Kollokationen an.

Für Adjektiv + Nomen-Kollokationen ergeben sich so z.B. die Kandidaten heurig + Jahr (heuriges Jahr, im heurigen Jahr, ...) als hochfrequent in den österreichischen Zeitungen, aber nicht vertreten im Material aus Deutschland. In ähnlicher Weise finden sich regierender Meister oder regierender Weltmeister in unseren österreichischen Texten 207-mal bzw. 193-mal häufiger als in unseren Daten aus Deutschland, wo die Wendung extrem selten ist. Die in Deutschland geläufige Kollokation amtierender (Welt-)Meister ist in den österreichischen Texten nur unerheblich häufiger (1,7 bzw. 2,9-mal) als in den deutschen Texten aus der Bundesrepublik. Diese kleinen Unterschiede 
gehen darauf zurück, dass die Proportionen von Sport-Texten gegenüber Texten über Wirtschaft, Kultur, Politik, etc. für die nationalen Teilkorpora nicht exakt gleich (und auch nicht im Detail bekannt) sind. Aus methodischer Sicht würde eine detailliertere Studie voraussetzen, dass auch die Verteilung von Kollokationen über die Domänen (d.h. thematischen Teile) der Zeitungen mit erhoben und in den Berechnungen berücksichtigt wird.

\begin{tabular}{|l|c|}
\hline \multicolumn{1}{|c|}{ Termkandidat } & Frequenz \\
\hline Durchstechflasche & 5638 \\
Injektionsstelle & 3489 \\
Pharmakokinetik & 3426 \\
Hämoglobinwert & 3395 \\
Fertigspritze & 3271 \\
Ribavirin & 3234 \\
Gebrauchsinformation & 2801 \\
Dosisanpassung & 2580 \\
Epoetin & 2302 \\
Hydrochlorothiazid & 2128 \\
\hline
\end{tabular}

Tab. 4: Fachausdrücke, die nur im EMEA-Korpus gefunden wurden (nach Frequenz, absteigend, top-10)

\begin{tabular}{|l|c|c|}
\hline Termkandidat & Quotient & Frequenz \\
\hline Filmtablette & 25522 & 6389 \\
Injektionslösung & 19854 & 4970 \\
Packungsbeilage & 14710 & 7365 \\
Niereninsuffizienz & 14233 & 3563 \\
Verkehrstüchtigkeit & 13558 & 3394 \\
Leberfunktion & 8385 & 2099 \\
Hypoglykämie & 8353 & 2091 \\
Toxizität & 7957 & 1992 \\
Einnehmen & 7035 & 7045 \\
Hypotonie & 6823 & 1708 \\
\hline
\end{tabular}

Tab. 5: Fachausdrücke aus dem EMEA-Korpus, die auch im gemeinsprachlichen Vergleichskorpus (Frankfurter Rundschau, 1993/1994) vorkommen (nach der ,weirdness-ratio“, absteigend, top-10)

Eine Prüfung unserer Texte aus der Schweiz und aus Südtirol ergibt übrigens, dass amtierender (Welt-)Meister in beiden Gebieten üblich und häufig ist; allerdings findet sich regierender (Welt-)Meister (erwarteterweise) auch in Südtirol (allerdings viel weniger prominent als in Österreich), in den Schweizer Texten dagegen überhaupt nicht. Also handelt es sich wohl um einen Austriazismus. 
Das oben beschriebene Verfahren erlaubt es also, Frequenzhelvetismen, -Austriazismen usw. zu identifizieren, und zwar für Einzelwörter und für Kollokationen. Wir berechnen für letztere die Häufigkeiten der lemmatisierten Paare.

\subsection{Klassifizierung von varietätenspezifischen Kollokationen}

Das oben diskutierte Verfahren wirft alle Einzelwörter und Wortpaare auf, deren Frequenz in einem der verglichenen Texte deutlich höher ist als im anderen. Will man diese Daten aus varietätenlinguistischer Sicht interpretieren, so muss eine Klassifizierung in verschiedene Subtypen vorgenommen werden.

Ein Grund für eine Klassifizierung liegt darin, dass bestimmte Lexeme, die in den Kollokationen vorkommen, auch alleine, ohne die jeweilige Kollokation, für einen bestimmten geographischen Raum spezifisch sind. Beispiele sind „regionale Realien“, wie z.B. CH Franken, AT Bundesheer, ST Landesrat. Dass diese Wörter in Schweizer, österreichischen oder südtiroler Texten eine proportional erheblich höhere Häufigkeit haben als in Texten aus Deutschland, ist trivialerweise durch die Regionen gegeben, über die die jeweiligen Zeitungen schreiben. Analoges gilt für regionale idiomatische Ausdrücke (z.B. CH ein Nein in die Urne legen), oder für regionale MehrwortTermini (z.B. des Rechts oder der Verwaltung, vgl. ST landwirtschaftliches Grün, $\mathrm{CH}$ blaue Zone). Solche Kombinationen und auch Kollokationen mit Wörtern, die Realien bezeichnen, sind nicht Ziel der Extraktion.

Eine zweite Kategorie von Kombinationen ist ebenfalls nur bedingt interessant, auch wenn es sich dabei um Kollokationen im Sinne der obigen Definition handelt. Beispiele sind AT betrügerische Krida, $\mathrm{CH}$ bängiges Verfahren. In diesen Fällen ist die Basis (z.B. Krida) oder der Kollokator (z.B. bängig) ein spezifisches Lexem der jeweiligen Nationalvarietät (Krida für Insolven₹, hängig für anhängig).

Dass wiederum solche Fälle vom statistischen Verfahren als massiv vom Gebrauch in Deutschland abweichend identifiziert werden, liegt auf der Hand: Krida und bängig kommen in den Texten aus Deutschland kaum vor. Aus Kollokationssicht sind diese Fälle aber nur bedingt interessant; in betrügerische Krida wird der Kollokator betrügerisch (der keine Varietätenprägung zeigt) von betrügerischer Bankrott, betrïgerische Insolvenz übernommen: Die österreichische Varietät zeichnet sich also nicht durch eine spezifische Kollokatorenwahl aus. Bei bängiges Verfahren ist zwar die Schweizer Kollokatorenwahl spezifisch, aber sie greift direkt auf einen Helvetismus zurück.

Der dritte Typ von Kollokationen ist schließlich derjenige, der für uns am interessantesten ist: Kollokationen, deren Bausteine in zwei oder mehr Varietäten vorkommen, aber nur in einer davon in kollokatorischer Kombination. Typisch hierfür ist AT regierender Weltmeister, $\mathrm{CH}$ einen Kredit sprechen 
(„,einen Kredit bewilligen“), usw. In diesen Kollokationen erfährt (typischerweise) der Kollokator eine varietätenspezifische Bedeutungserweiterung: regierend im Sinne von ,amtierend“, sprechen im Sinne von „bewilligen“ usw.

Die ersten beiden Typen von Kollokationen können mit dem oben in Abschnitt 4.1 beschriebenen Verfahren zur Suche nach Frequenzunterschieden relativ gut identifiziert und ,abgeschöpft" werden: Sie enthalten jeweils mindestens ein Element, das in der betreffenden Varietät signifikant viel häufiger ist als in derjenigen von Deutschland. Wir vergleichen also sowohl die Kollokationen, als auch die in den Kollokationen vorkommenden Einzelwörter nach der Frequenz. Kollokationen, deren eines Element in hohem $\mathrm{Maß}$ varietätentypisch ist, werden separat abgelegt von solchen, wo keiner der Kollokationspartner spezifisch ist. Die Trennung in Realien vs. varietätenspezifische Synonyme zu Wörtern aus dem Gebrauch in Deutschland (Typ AT Krida „Bankrott") muss manuell durchgeführt werden.

\subsection{Korpusbasierte Beschreibung von varietätentypischen Kollokationen}

Fälle von Wörtern, deren Form nur in einer der deutschen Varietäten vorkommt (CH Zustupf „Zuschuss“, AT Krida „Bankrott") sind relativ selten. Die oben genannten Verfahren liefern deswegen in der dritten Teilmenge, d.h. derjenigen der formal ,unauffälligen“ Kollokationen immer noch Kandidaten, deren Basen oder Kollokatoren regionale oder nationale Synonyme von Wörtern sind, die in Deutschland üblich sind. Beispiele sind AT bobe Abfertigung (,,hohe Abfindung“), ST fixer Professor (,,fest angestellter Professor") usw. Hier sind Abfertigung und fix in Lesarten benutzt, die regionaltypisch sind. Solche Lesarten bilden in der Regel Reihen innerhalb der jeweiligen Varietät: hohe/ niedrige/ ungerechtfertigte/... Abfertigung, fixer Professor/fixe Stelle/fixe Anstellung/.... Jeweils hat die Basis oder der Kollokator eine relativ stabile varietätentypische Bedeutung. Diese Bedeutung lässt sich für Basen anhand einer geeigneten Kollokationsliste (d.h. der Menge signifikanter Kollokationen mit derselben Basis) manuell ermitteln. Aus dem varietätenspezifischen Korpus können hierzu die häufigsten oder signifikantesten Kollokationskandidaten extrahiert werden; zu CH Anlass („Veranstaltung“) finden sich so in unseren Daten (in Reihenfolge abnehmender Varietätenspezifizität (= Quotient der relativen Frequenz)): spezieller Anlass, großer Anlass, kirchlicher Anlass, nationaler Anlass, etc. Die Kollokation sportlicher Anlass ist zwar in unseren Schweizer Texten sehr häufig, aber sie taucht auch in Texten aus Deutschland auf, so dass der „weirdness"-Wert eher gering ist.

Die Ermittlung varietätenspezifischer Kollokatorenbedeutungen setzt einen weiteren Schritt voraus. Ein typisches Beispiel ist eine Schweizer Verwendung des Adjektivs markant. Die von der Frequenz her am stärksten 
vom Gebrauch in Deutschland abweichenden Verwendungen in Schweizer Texten sind in Tabelle 6 angegeben, mit der Absolutfrequenz (größer 7) und dem ,weirdness"-Wert.

\begin{tabular}{|l|c|c|}
\hline \multicolumn{1}{|c|}{ Kollokation } & $\mathbf{f}_{\text {CH }}$ & RF-REL \\
\hline markante Zunahme & 62 & 59,5 \\
markanter Anstieg & 35 & 39,6 \\
markante Steigerung & 31 & 19,8 \\
markante Erhöhung & 9 & 17,3 \\
markante Verbesserung & 40 & inf \\
markante Gewinnsteigerung & 15 & inf \\
\hline markanter Rückgang & 45 & 43,2 \\
markanter Einbruch & 9 & 17,3 \\
markante Abnahme & 10 & inf \\
\hline markante Änderung & 17 & 16,3 \\
\hline markanter Temperatursturz & 8 & inf \\
\hline
\end{tabular}

Tab. 6: Regionale Lesarten von Kollokatoren: markant $_{\mathrm{CH}}$

Die Liste in Tabelle 6 weist Kollokationen auf, in denen markant eine starke (ggf. quantifizierbare) Veränderung eines Werts bezeichnet, etwa einer Lesart des Adjektivs erbeblich entsprechend. Dabei sind jeweils die Absolutfrequenz in unseren Schweizer Texten $\left(\mathrm{f}_{\mathrm{ch}}\right.$ ) und der ,weirdness"-Quotient (RFREL) im Verhältnis zu den Zeitungstexten aus Deutschland angegeben. Ein Vergleich mit den Kollokationen von markant in Texten aus Deutschland, sortiert nach dem Log-Likelihood-Wert (Tabelle 7, zusätzlich mit Angabe der Absolutfrequenz) zeigt, dass die Schweizer Lesart nicht oder nicht prominent vorhanden ist; markant wird mit Nomina kombiniert, die Formen oder Stellen in der Landschaft (Stelle, Gebäude, Bawwerk) bezeichnen.

\begin{tabular}{|l|c|c|}
\hline \multicolumn{1}{|c|}{ Kollokation } & $\mathbf{f}_{\mathrm{DE}}$ & $\mathbf{L L}_{\mathrm{DE}}$ \\
\hline markanter Punkt & 35 & 207,6 \\
markantes Profil & 19 & 145,8 \\
markantes Gesicht & 23 & 133,3 \\
markantes Kinn & 11 & 131,5 \\
markantes Beispiel & 23 & 120,7 \\
markantes Gebäude & 21 & 110,1 \\
markante Stelle & 24 & 103,9 \\
markantes Zeichen & 16 & 93,9 \\
markantes Bauwerk & 9 & 61,9 \\
\hline
\end{tabular}

Tab. 7: Lesarten von Kollokatoren: markant $_{\mathrm{DE}}$ in Texten aus Deutschland 
Ein ähnliches Beispiel stellen die Kollokatoren von Preis in Schweizer und deutschen Texten dar. In Tabelle 8 sind die häufigsten Adjektive mit Preis aufgelistet: in der Reihenfolge abnehmender Häufigkeit in Schweizer Texten, hier angegeben in ,Parts per Million' (rf: in ppm). Neben den Zahlen für das Schweizer Zeitungskorpus sind die analogen Zahlen für ein Teilkorpus des deutschen Zeitungskorpus angegeben, und zwar für 104 Millionen Wörter aus der Zeitung die tageszeitung (Berlin: taz) aus dem Zeitraum 1987 bis 1993.

\begin{tabular}{|l|r|r|}
\hline \multicolumn{1}{|c|}{ Kollokation } & $\mathbf{r f}_{\mathrm{CH}}$ & \multicolumn{1}{c|}{$\mathbf{r f}_{\mathrm{TAZ}}$} \\
\hline hoher Preis & 6,065 & 5,019 \\
günstiger Preis & 4,792 & 0,923 \\
attraktiver Preis & 3,650 & $<0,260$ \\
tiefer Preis & 3,328 & 0,019 \\
erschwinglicher Preis & 0,628 & 0,317 \\
niedriger Preis & 0,546 & 1,740 \\
stolzer Preis & 0,492 & 0,288 \\
\hline
\end{tabular}

Tab. 8: Die Kollokatoren von Preis in Texten aus der Schweiz und aus Deutschland (taz)

Ein Blick auf die Zahlenangaben zeigt erhebliche Unterschiede: Die Schweizer Texte haben proportional häufiger die Kollokationen günstiger Preis, attraktiver Preis und vor allem tiefer Preis. Dagegen ist das aus deutschen Texten vertraute niedriger Preis in den Schweizer Texten seltener als in den deutschen.

Ein Vergleich mit weiteren Kollokationen mit dem Kollokator tief zeigt eine Reihenbildung in den Schweizer Texten: Kombinationen wie tiefe Inflation, tiefer Preis, tiefer Zins/Zinssatz/Zinsniveau sind 40-mal bis 120-mal häufiger in Schweizer Texten als in Texten aus der Bundesrepublik. Zwar gibt es Tief(st)preis(garantie) in der Werbesprache, aber die Kollokation tiefer Preis ist sehr selten: Wir haben nur zwei Belege in unserem Korpus von 258 Millionen Wörtern. Das Adjektiv tief kollokiert in Texten aus Deutschland viel weniger mit Nomina, die den Stand oder Wert von Quantifizierbarem angeben; stattdessen finden sich prominent räumliche Dimensionen (tiefer Graben, tiefes Loch) oder deren übertragene Verwendungsweisen (tiefer Einschnitt, tiefe Spur, tiefe Rezession).

Die detaillierte Interpretation solcher Daten muss interaktiv erfolgen: Ob bei markant und tief spezifische Schweizer Lesarten vorliegen, oder ob die varietätentypischen Kollokationspräferenzen (noch) nicht Anlass geben, eine spezifische Lesart anzusetzen, liegt in der Entscheidung von Lexikografen und Varietätenlinguisten. Aus den Korpusdaten lässt sich jedenfalls für die genannten Fälle mit relativ wenig Aufwand Material extrahieren, welches die Identifikation solcher varietätentypischer Kollokationen auf einfache Weise möglich macht. 
4.4 Das ,tertium comparationis‘: Was bedeuten varietätentypische Kollokationen?

Die oben angegebenen Beispiele für Kollokationen der österreichischen und der Schweizer Nationalvarietät bzw. der Varietät von Südtirol sind intuitiv mit Kollokationen des Deutschen von Deutschland verglichen worden. Wir haben ohne Methodendiskussion, Äquivalente in Klammern angegeben und sind davon ausgegangen, dass den Schweizer oder österreichischen Kollokationen auch deutsche Kollokationen, z.B. mit einem anderen Kollokator, entsprechen. Wenn das der Fall ist, kann man sich vorstellen, die Ähnlichkeit (oder: „Äquivalenz") halbautomatisch mit Mitteln der distributionellen Semantik aufzuzeigen (ähnliche Kontexte als Anzeichen für ähnliche Bedeutungen).

Dass in den verschiedenen Varietäten parallel Kollokationen vorliegen, muss aber nicht der Fall sein. Die Kollokationsdaten zum Nomen Information in Texten aus Deutschland liefern folgende Kollokatoren als prominent (in der Reihenfolge absteigender Absolutfrequenz in unserem Korpus): Information sammeln, Information erbalten, Information liefern, Information haben/geben/ austauschen usw. Eine vergleichbare Liste für die Texte der südtiroler Tageszeitung „Dolomiten“ enthält die folgenden Kollokationen, ebenfalls nach Absolutfrequenz absteigend sortiert: Information einholen, Information erteilen, Information geben, Information austauschen, Information erbalten, usw. Bemerkenswert sind Information einholen und Information erteilen. Beide kommen auch in Texten aus Deutschland vor, aber viel seltener: Die Kollokationen sind in den Südtiroler Texten ca. 30-mal häufiger. Zu fragen ist, welcher Ausdruck in Texten aus Deutschland statt des in Südtirol häufigen Information einholen steht.

Eine Detailanalyse der Kontexte von Information einholen zeigt, dass es sich nahezu ausschließlich um Angaben dazu handelt, wo sich der Bürger bei Interesse Informationen verschaffen kann:

Informationen können unter Telefon 0471/63.55.82 eingeholt werden.

Informationen können beim Sekretariat des Skimarathons unter Telefonnummer 0474/97.21.32 eingeholt werden.

Um ,äquivalente‘, d.h. texttypologisch und pragmatisch gleichwertige Textstücke aus deutschen Zeitungen zu finden, wurde nach Telefonnummernangaben gesucht. In der „Frankfurter Allgemeinen Zeitung“ finden sich z.B. die folgenden Formulierungen:

Informationen unter Telefon 07541/38.560.

Nähere Informationen gibt es bei der Vertriebsgesellschaft von Aprilia unter der Telefonnummer...

Nähere Informationen sind unter der Telefonnummer ... zu erbalten. Informationen rund um das Luftbrücken-Jubiläum können unter der Telefonnummer ... abgerufen werden. 
Die Texte aus der FAZ weisen also eine ganz andere grammatische Struktur (z.T. ohne Verb) auf, oder sie verwenden andere Konstruktionen, wie z.B. es gibt. Bei der Untersuchung solcher Unterschiede stößt die automatische Analyse an ihre Grenzen: Hier müssen Parameter berücksichtigt werden, die nicht auf automatischem Wege aus den Texten extrahiert werden können.

\subsection{Varietätenunterschiede bei morphosyntaktischen Präferenzen?}

Oben war darauf hingewiesen worden (vgl. Abschnitt 3.1.2), dass manche Kollokationen deutliche morphosyntaktische Präferenzen aufweisen, d.h. formal weitgehend fixiert sind. Wenn die Kollokatorenwahl varietätenspezifisch sein kann (wie dies im vorigen Abschnitt an Beispielen gezeigt worden ist), dann stellt sich die Frage, ob es zwischen den deutschen Varietäten auch Unterschiede hinsichtlich der morphosyntaktischen Präferenzen einzelner Kollokationen gibt.

Deutlich ist, dass varietätentypische Kollokationen mit regionalspezifischen Basen (z.B. AT das Auslangen finden, ,(s)ein Auskommen finden ${ }^{6}$ oder $\mathrm{CH}$ Einsitz nehmen, Abgeordneter sein) auch spezifische Präferenzen hinsichtlich der Determination haben (z.B. die Präferenz für den definiten Artikel bei das Auslangen finden). Viel weniger deutlich ist, ob ein und dieselbe Kollokation je nach Varietät mit unterschiedlichen morphosyntaktischen Präferenzen auftritt. Ein solches Beispiel wurde für den Unterschied zwischen portugiesischem und brasilianischem Portugiesisch bei ter ciumes (,neidig sein', wörtlich ,Neid haben', Plural, PT/PT) vs. ter ciume (Singular, PT/BR) in den Textkorpora der Linguateca gefunden.

Aus unseren Daten ergeben sich keine signifikanten Unterschiede hinsichtlich der morphosyntaktischen Präferenzen. Einzelfälle ändern hier wenig am Gesamtbild; die Kollokation Comeback + geben findet sich z.B. in unseren Texten mit wechselndem Determinierer: mit indefinitem Artikel, ein Comeback geben, Possessivum, sein/ibr Comeback geben und in Sonderfällen (in der Regel mit Relativsatz oder einer anderen Ergänzung, die Definitheit erzwingt) mit definitem Artikel, das Comeback geben. Die Texte aus Deutschland und Südtirol weisen eine parallele Verteilung auf: Etwas mehr als die Hälfte der Belege sind indefinit (53-55\%), etwas weniger als die Hälfte haben ein Possessivum (43-47\%). In unseren Schweizer Texten ist demgegenüber der Possessiv-Fall überrepräsentiert: Er macht 63\% der 418 beobachteten Okkurrenzen aus, während nur 33\% indefinit sind. Es mag also sein, dass die Schweizer Autoren eher sein Comeback geben sagen, als ein Comeback geben.

Wir haben aber in unseren Texten ansonsten kaum Fälle gefunden, wo sich solche Präferenzen für einzelne Varietäten aufweisen lassen. Vermutlich gehört die morphosyntaktische Präferenz so eng zum idiomatischen Charakter der Kollokationen, dass sie keinen oder nur sehr geringen Variationen unterliegt. Die Untersuchung weiterer Materialien wäre hier sicher 
angebracht: Erst eine systematische Untersuchung auf der Grundlage sehr großer Textmengen kann hier genügend Daten liefern, so dass eindeutige Aussagen gemacht werden könnten.

\section{Aktueller Stand und weiterer Forschungsbedarf}

In diesem Artikel haben wir versucht, Fragestellungen zu varietätentypischen Kollokationen zu untersuchen. Eine grundlegende Frage war, ob sehr einfache, auf einem Vergleich der relativen Frequenz von Wörtern und Wortkombinationen beruhende Werkzeuge bereits Anhaltspunkte für varietätentypische Kollokationen geben. Die hier referierten Zwischenergebnisse legen die Annahme nahe, dass sich tatsächlich mit solchen einfachen Werkzeugen Material für eine Beschreibung der Kollokationen der deutschen Varietäten extrahieren lässt.

Dabei werden die Kollokationen einer Basis zwischen den zu untersuchenden Varietäten verglichen; ergeben sich Unterschiede bei der Kollokatorenwahl, so lohnt es sich, auch weitere typische Basen eines gegebenen varietätenspezifischen Kollokators zu identifizieren, um eine eventuelle Reihenbildung (z.B. bei CH markant) festzustellen. Die Datenbank, die mit unseren Extraktionsergebnissen befüllt wird, eignet sich für die teilweise Automatisierung solcher Vergleiche relativ gut.

Aus den ersten Experimenten ist deutlich geworden, dass die deutschen Nationalvarietäten zum Teil unterschiedliche Präferenzen hinsichtlich der Kollokatoren haben: Was in Deutschland niedriger Preis heißt, findet sich in Schweizer Texten eher als tiefer Preis. Gleichzeitig legen die hier beschriebenen Experimente die Annahme nahe, dass die morphosyntaktischen Präferenzen bei Kollokationen (d.h. Numerus, Determination usw.) quer durch die Varietäten relativ stabil sind - vielleicht weil sie Teil der Idiomatisiertheit der Kollokationen sind. Allerdings sollten hier mehr Untersuchungen vorgenommen werden, an größeren und texttypologisch sauberer klassifizierten Texten; erst auf der Basis breiterer Untersuchungen kann hier ein abschließendes Urteil gefällt werden.

Der Gegenstandsbereich ist mit den hier vorgelegten Untersuchungen noch keineswegs zufriedenstellend behandelt: Unsere bisherigen Arbeiten konnten noch nicht ausreichend in die Tiefe und in die Breite gehen, sondern stellen eher Stichproben und Experimente zum Test der Werkzeuge dar. Eine systematische Untersuchung wäre sicher sinnvoll. Hierbei ist festzustellen, dass nicht nur die deutschen Nationalvarietäten, sondern auch andere pragmatische Aspekte der Sprache zu untersuchen wären: auch über registerspezifische Kollokationen, über die Texttypgebundenheit bestimmter Kollokationen (z.B. ,Amtssprache) usw. ist wenig bekannt. Die Werkzeuge sind für diese Art Fragestellungen gleichermaßen einsetzbar. 
Längerfristig hoffen wir, dass sich auf der Grundlage großer Textkorpora und effektiver Extraktionsmethoden weitere Details untersuchen lassen; eine Erweiterung der Abdeckung bestehender Variantenwörterbücher im Bereich der Kollokationen wäre sicher für die Dokumentation des Deutschen in seinen vielen verschiedenen Erscheinungsformen nützlich.

\section{Literatur}

Ahmad, Khurshid et al. (1992): What is a term? The semi-automatic extraction of terms from text. In: Snell-Hornby, Mary et al. (Hg.): Translation studies - an interdiscipline. (= Benjamins Translation Library 2). Amsterdam/Philadelphia, S. 267-278.

Ammon, Ulrich (1995): Die deutsche Sprache in Deutschland, Österreich und der Schweiz: das Problem der nationalen Varietät. Berlin/New York.

Ammon, Ulrich et al. (2004): Variantenwörterbuch des Deutschen. Die Standardsprache in Österreich, der Schweiz und Deutschland sowie in Liechtenstein, Luxemburg, Ostbelgien und Südtirol. Berlin u.a.

Bartsch, Sabine (2004): Structural and functional properties of collocations in English. A corpus study of lexical and pragmatic constraints on lexical co-occurence. Tübingen.

Dunning, Ted (1993): Accurate methods for the statistics of surprise and coincidence. In: Computational Linguistics 19, 1, S. 61-74.

Dykstra, Anne/Schoonheim, Tanneke (Hg.) (2010): Proceedings of the XIVth EURALEX International Congress (Leeuwarden, 6-10 July 2010). Leeuwarden. [CD-ROM].

Evert, Stefan (2004): The statistical analysis of morphosyntactic distributions. In: Lino, Maria Teresa et al. (Hg.): Proceedings of the $4^{\text {th }}$ International Conference on Language Resources and Evaluation (LREC), Lisbon, Portugal 2004. Paris, S. 15391542.

Evert, Stefan (2005): The statistics of word cooccurrences - word pairs and collocations. Stuttgart. Internet: www.collocations.de (Stand: 15.12.2010).

Fazly, Afsaneh/Stevenson, Suzanne (2006): Automatically constructing a lexicon of verb phrase idiomatic combinations. In: Proceedings of the Workshop on Multiwords in a Multilingual Context, $11^{\text {th }}$ Conference of the European Chapter of the Association for Computational Linguistics, Trento, Italy, April 2006. Trient, S. 337-344.

Forkl, Yves (2010): Zur digitalen Zukunft der Kollokationslexikographie, Perspektiven der Präsentation von Wissen über usuelle französische und deutsche Wortverbindungen in gedruckten und elektronischen Wörterbüchern. Münster.

Hausmann, Franz Josef (1979): Un dictionnaire des collocations est-il possible? In: Travaux de Linguistique et de Littérature XVII, 1, S. 187-195.

Hausmann, Franz Josef (1984): Wortschatzlernen ist Kollokationslernen. In: Praxis des neusprachlichen Unterrichts 31, 4, S. 395-406. 
Hausmann, Franz Josef (2004): Was sind eigentlich Kollokationen? In: Steyer (Hg.), S. 309-334.

Heid, Ulrich (1994): On ways words work together - topics in lexical combinatorics. In: Martin, Willy et al. (Hg.): Proceedings of the VIth Euralex International Congress. Amsterdam, S. 226-257.

Heid, Ulrich (1998): Towards a corpus-based dictionary of German noun-verb collocations. In: Proceedings of the Euralex International Congress 1998. Liège, S. $301-$ 312.

Heid, Ulrich (2009): La saisie des collocations: objectifs et approaches. In: Cahiers de Lexicologie 94, S. 71-89. [Sonderband: Le traitement automatique du lexique, hrsg. v. Blumenthal, Peter/Petit, Gerard].

Heid, Ulrich et al. (2008): Providing corpus data for a dictionary for German juridical phraseology. In: Storrer, Angelika et al. (Hg.): Text resources and lexical knowledge. Selected papers from the $9^{\text {th }}$ Conference of Natural Language Processing, KONVENS 2008. (= Text, Translation, Computational Processing 8). Berlin u.a., S. 131-144.

Heid, Ulrich/Weller, Marion (2010): Corpus-derived data on German multiword expressions for lexicography. In: Dykstra/Schoonheim (Hg.), S. 331-340. [CD-ROM].

Ivanova, Kremena et al. (2008): Evaluating a German sketch grammar: a case study on noun phrase case. In: Proceedings of LREC-2008, Linguistic Resources and Evaluation Conference. Marrakesch. [CD-ROM].

Kilgarriff, Adam et al. (2004): The Sketch Engine. In: Williams, Geoffrey/Vessier, Sandra (Hg.): Proceedings of the 11th Euralex International Congress, Lorient, France, July 6-10, 2004. Bd. 1. Lorient, S. 105-115.

Kilgarriff, Adam et al. (2010): A quantitative evaluation of word sketches. In: Dykstra/ Schoonheim (Hg.), S. 372-379. [CD-ROM].

Krenn, Brigitte (2000): The usual suspects: data-oriented models for the identification and representation of lexical collocations. Diss. Saarbrücken.

Mel'čuk, Igor A. et al. (1984): Dictionnaire explicatif et combinatoire du français contemporain. Bd. I-IV. (= Recherches Lexico-Sémantiques 1). Montréal.

Schiehlen, Michael (2003): A cascaded finite-state parser for German. In: Proceedings of EACL 2003. Bd. 2. Budapest, S. 163-166.

Smadja, Frank (1993): Retrieving collocations from text. In: Computational Linguistics 19, 1, S. 143-177.

Steyer, Kathrin (Hg.) (2004): Wortverbindungen - mehr oder weniger fest. (= Jahrbuch des Instituts für Deutsche Sprache 2003). Berlin/New York.

Tutin, Agnès (2010): Sens et combinatoire lexicale: de la langue au discours. Habil. Grenoble. 



\title{
Carolin Müller-Spitzer / Stefan Engelberg
}

\section{Elektronische Lexikografie zwischen Grammatik und Lexikon}

\begin{abstract}
Der Artikel stellt die Projekte vor, die sich im Rahmen der Projektmesse zur „Elektronischen Lexikografie" präsentiert haben. Diese Messe wurde begleitend zur 46. Jahrestagung des Instituts für Deutsche Sprache veranstaltet. Es wird in diesem Beitrag auf der Basis der Messepräsentationen dargelegt, inwiefern Entwicklungen der Korpuslexikografie und der Internetlexikografie die lexikografische Erfassung syntagmatischer Aspekte des deutschen Wortschatzes befördern und welche lexikografischen Internetressourcen dazu verfügbar sind.
\end{abstract}

\section{Korpuslexikografie und Internetlexikografie}

Die Entwicklung der Lexikografie in der vergangenen Dekade ist zum einen gekennzeichnet durch den Einsatz immer größerer elektronischer Textkorpora und der Verfeinerung der Methoden zu ihrer automatischen Analyse (Korpuslexikografie) und zum anderen durch eine Hinwendung der elektronischen Lexikografie zum Internet (Internetlexikografie).

Sowohl die Fortschritte der Korpuslexikografie wie auch die neuen medienspezifischen Möglichkeiten der Internetlexikografie haben dazu geführt, dass Phänomene im Übergangsbereich zwischen Lexikon und Grammatik in den Fokus der lexikografischen Theorie und Praxis gerückt sind. Korpusanalysemethoden wie Kookkurrenzanalysen, N-Gramm-Analysen und Frequenzanalysen gehören mittlerweile zum Standard der lexikografischen Praxis (vgl. z.B. Klosa 2007; Prinsloo 2009). Kookkurrenzanalysen berechnen das überzufällig häufige gemeinsame Auftreten von Wörtern und ermöglichen so die (semi-)automatische Ermittlung von Kollokationen, Redewendungen, Funktionsverbgefügen und anderen Arten von Phrasemen. N-Gramm-Analysen ermitteln die Häufigkeit von Wortkombinationen und damit die Gebräuchlichkeit von Mehrwortausdrücken. Über andere Arten von Frequenzanalysen wird die Häufigkeit lexikalischer Ausdrücke in bestimmten Typen von Konstruktionen errechnet. All diese Verfahren haben zu der Erkenntnis geführt, dass die Anzahl von Mehrwortausdrücken, die über nicht regelhafte formale oder nicht-kompositionelle inhaltliche Idiosynkrasien verfügen oder die aufgrund von Frequenzauffälligkeiten einen sprachsystematisch nicht zu erklärenden Usualisierungsgrad aufweisen, um ein Vielfaches größer ist als in präkorpuslinguistischen Zeiten angenommen wurde. 
Die Hinwendung der Lexikografen zur elektronischen und neuerdings besonders zur Internetlexikografie hat wiederum die für die Printlexikografie typischen Platzbeschränkungen hinfällig werden lassen, die eine ausführliche Darstellung der Syntagmatik des Wortschatzes oft verhindern. Die großen gegenwartssprachlichen internetlexikografischen Projekte wie das „Digitale Wörterbuch der Deutschen Sprache“ (siehe unten Projekt 4) und ,elexiko" (siehe unten Projekt 14) sowie der vorwiegend automatisch erstellte „Deutsche Wortschatz“ (siehe unten Projekt 3) zeichnen sich insofern durch eine besondere Berücksichtigung syntagmatischer Aspekte von Wörtern aus.

\section{Projektmesse „Elektronische Lexikografie“}

Auf der 46. Jahrestagung des Instituts für Deutsche Sprache, die sich mit methodisch-empirischen und theoretischen Fragen des sprachlichen Wissens zwischen Lexikon und Grammatik befasste, wurde im Zusammenhang mit den oben dargestellten Entwicklungen in der Lexikografie eine von Carolin Müller-Spitzer organisierte Projektmesse „Elektronische Lexikografie“ veranstaltet (vgl. auch Müller-Spitzer/Vachková 2010): Die Projektmesse war dabei nicht beschränkt auf den oben beschriebenen zentralen Themenbereich der Syntagmatik, beinhaltete aber eine Vielzahl von Präsentationen, die in der einen oder anderen Weise dafür relevant sind. Insgesamt stellten sich 16 Projekte vor, von denen einige spezifisch dem Bereich der Syntagmatik zuzuordnen sind (Projekt 11 „E-VALBU“, Projekt 12 „Erlanger Valency Patternbank“, Projekt 13 „Usuelle Wortverbindungen“ bzw. „SprichWort"), die zum Teil aber auch in den Bereich der automatischen Sprachverarbeitung gehören wie „GermaNet“ (Projekt 6, 7) oder „SALSA“ (Projekt 8), internationale internetlexikografische Projekte wie das „Algemeen Nederlands Woordenboek“ (Projekt 1) oder das „Große akademische Wörterbuch Deutsch-Tschechisch“ (Projekt 2), Forschungsprojekte im Bereich der Internetlexikografie wie „Kognitiv Saliente Semantische Relationen“ (Projekt 10) oder „Benutzeradaptive Zugänge und Vernetzungen in elexiko" (Projekt 14). Darüber hinaus waren Projekte eingeladen, die entweder im Bereich der Korpuserstellung Besonderes zu berichten hatten, wie das Projekt 5 im Bereich der Deutschen Gebärdensprache, oder hinsichtlich der Darstellung innovative Formen wählen, wie das „Kicktionary“ (Projekt 9). Die Messe diente damit zum einen dazu, den Tagungsteilnehmern Projekte aus der Praxis zu zeigen, die einige der in dem Programm der Jahrestagung theoretisch beleuchteten Aspekte lexikografisch umsetzen, und zum anderen dazu, den Austausch der Forscher und Lexikografen aus dem Bereich Internetlexikografie untereinander zu fördern. 
- Projekt 1 (Algemeen Nederlands Woordenboek): Das ANW ist die Neuerarbeitung eines allgemeinen, einsprachigen Wörterbuchs gezielt für das elektronische Medium. Das Besondere hier ist u.a. der Ansatz einer formalisierten Bedeutungsbeschreibung durch so genannte Semagramme, die interessante und neuartige erweiterte Suchen im elektronischen Wörterbuch ermöglichen sollen. Semagramme sind semantische Merkmalskarten, in denen eine Vielzahl semantischer Eigenschaften zu einer Lesart möglichst formalisiert in festen Kategorien festgehalten werden (Moerdijk 2008; Moerdijk/Tiberius/Niestadt 2008).

- Projekt 2 (Das Große akademische Wörterbuch Deutsch-Tschechisch): In dem Prager Wörterbuchprojekt werden Kookurrenzanalysen sowie darauf basierende „Self-Organizing Lexical Feature Maps“ als korpuslinguistische Methoden eingesetzt, insbesondere zur Ermittlung von semantischen Relationen (Vachková/Belica 2009).

- Projekt 3 (Deutscher Wortschatz der Universität Leipzig): Der induktiv aus Korpora gewonnene „Deutsche Wortschatz“ zeichnet sich unter anderem durch automatisch berechnete Kollokationen und Hyponymierelationen aus (Langanke 2004).

- Projekt 4 (Digitales Wörterbuch zur deutschen Sprache - DWDS): Das DWDS basiert auf dem „Wörterbuch der deutschen Gegenwartssprache“ (Klappenbach/Steinitz (Hg.) 1967-1977), das mittlerweile durch eine Vielzahl weiterer Angaben und Funktionen erweitert wurde. Unter anderem können automatisch berechnete Kollokationen - auch grafisch visualisiert - als Ergänzung zu den Wortartikeln rezipiert werden (Klein 2004).

- Projekt 5 (Entwicklung eines korpusbasierten, elektronischen Wörterbuchs Deutsche Gebärdensprache - Deutsch): Das „Wörterbuch Deutsche Gebärdensprache" ist u.a. mit den Fragen konfrontiert, wie man ein Korpus zur Gebärdensprache aufbaut, welche Datenschutzprobleme dabei auftreten und wie man mit gebärdensprachlichen Varietäten umgeht.

- Projekt 6 (GermaNet - ein lexikalisch semantisches Wortnetz): GermaNet ist ein computerlexikografisch ausgerichtetes Projekt, welches eher die automatische Sprachverarbeitung als menschliche Nutzer anvisiert. GermaNet fügt sich in die Reihe nationaler WordNet-Vorhaben ein (vgl. Fellbaum in diesem Band) und ist mittlerweile in das EuroWordNet integriert. Es fokussiert wie alle WordNet-Projekte auf die lexikalische Vernetzung durch semantische Relationen (Kunze/Lemnitzer 2010).

- Projekt 7 (HyTex - Werkzenge zur Exploration von GermaNet): Im Dortmunder „HyTex“-Projekt werden Werkzeuge zur Exploration von GermaNet entwickelt, z.B. eine grafische Sicht auf das lexikalische Netz. $\mathrm{Da}$ für GermaNet im akademischen Kontext mittlerweile kostenfrei Lizenzen vergeben werden, gewinnen solche Werkzeuge an Bedeutung. 
- Projekt 8 (Saarbrücken Lexical Semantics Acquisition Project - SALSA): Ziel des „SALSA“-Projektes (Burchardt et al. 2009) ist es, angeschlossen an die internationalen FrameNet-Projekte (vgl. Ruppenhofer et al. 2010), ein großes framebasiertes Lexikon für das Deutsche korpusbasiert zu erstellen, das auf semantisch und syntaktisch detailliert annotierte Sätze zugreift. Im Gegensatz zu GermaNet basiert es also auf syntagmatischen und nicht auf paradigmatischen Relationen. Auch SALSA ist primär als Ressource für linguistische und computerlinguistische Forschungen gedacht.

- Projekt 9 (Kicktionary): Auch das multilinguale elektronische Wörterbuch zur Fußballsprache beruht auf einem framebasierten semantischen Ansatz. Es ermöglicht etwa einen Blick auf das sprachliche Inventar unterschiedlicher „Scenes“ wie z.B. „Shot“, den Schuss. Die einzelnen lexikalischen Einheiten werden nach Frames eingeordnet und sind über Konzepthierarchien zugreifbar (Schmidt 2009).

- Projekt 10 (Kognitiv Saliente Semantische Relationen): Im Kontext des „ELDIT“-Projektes (Abel et al. 2005; Abel 2008), eines digitalen bilingualen Online-Wörterbuchs Deutsch-Italienisch, ist die Dissertation von Gerald Kremer zu „Kognitiv salienten semantischen Relationen“ angesiedelt. In dem Projekt geht es um Hilfen zur Erweiterung von „ELDIT“ im Bereich der Anzeige von semantischen Relationen zu einem gesuchten Lexikoneintrag in der Lerner-Spache.

- Projekt 11 (E-VALBU): Das am IDS beheimatete Projekt „E-VALBU“ ist eine für das elektronische Medium aufbereitete Version des „Valenzwörterbuchs deutscher Verben“ (Schumacher et al. 2004). Das auch auf den DaF-Bereich ausgerichtete Wörterbuch zeichnet sich in der Internet-Version durch viele erweiterte Suchmöglichkeiten aus (Kubczak 2009).

- Projekt 12 (Erlanger Valency Patternbank): In der „Erlanger Valency Patternbank“, die auf dem „Valency Dictionary of English“ (Herbst et al. 2004) basiert, können lexikalische Einheiten nach unterschiedlichen Kriterien durchsucht und sortiert werden; z.B. sind einzelne Muster („Patterns") über Listen von „Pattern elements“ wie z.B. „about_ NP_V-ing" geordnet und die entsprechenden Verben somit als Menge zugreifbar.

- Projekte 13a (Usuelle Wortverbindungen) und 13b (SpricbWort): Die beiden in der Abteilung Lexik am Institut für Deutsche Sprache angesiedelten Projekte befassen sich mit Mehrwortausdrücken. In dem Wörterbuch zu „Usuellen Wortverbindungen“ werden Mehrwortausdrücke korpusbasiert in ihrer Varianz dargestellt (Steyer 2009). In dem daran angebundenen EU-Projekt „SprichWort“ wird eine mehrsprachige Sprichwort-Datenbank erstellt. 
- Projekt 14 (elexiko): In „elexiko“ - einem am Institut für Deutsche Sprache beheimateten Onlinewörterbuch zum Gegenwartsdeutschen werden alle Artikel neu auf Basis des elexiko-Korpus und gezielt für das elektronische Medium erarbeitet. Besonders interessant für den Kontext der Jahrestagung sind die umfangreichen Angaben zu „Lexikalischen Mitspielern“ sowie zu „Typischen Verwendungen“ (Storjohann/ Möhrs 2007).

- Projekt 15 (Benutzeradaptive Zugänge und Vernetzungen in elexiko): Einer der drei Schwerpunkte dieses Projektes ist die Benutzungsforschung in Bezug auf Online-Wörterbücher. Im zweiten Schwerpunkt geht es um die Kodierung und Verwaltung sowie um Möglichkeiten der Darstellung von Vernetzungen im lexikografischen Kontext. Der dritte Schwerpunkt hat die automatische Gewinnung morphologischer Angaben für „elexiko" zum Gegenstand.

- Projekt 16 (Online-Wortschatz-Informationssystem Deutsch - OWID): Das „Online-Wortschatz-Informationssystem Deutsch“ ist das lexikografische Internet-Portal des Instituts für Deutsche Sprache (MüllerSpitzer 2010; Engelberg/Klosa/Müller-Spitzer 2009). Hier werden alle internetlexikografischen Arbeiten des IDS in einem Portal zusammengeführt. Insbesondere die dort enthaltenen Wörterbücher „elexiko", „Usuelle Wortverbindungen“" und in naher Zukunft auch „E-VALBU“, das Sprichwörterbuch und das „Handbuch deutscher Kommunikationsverben" befassen sich mit Phänomenen im Bereich des Tagungsthemas.

\section{Internet-Ressourcen zur Syntagmatik des Lexikons der deutschen Gegenwartssprache}

Der obige Überblick zeigt, dass viele der auf der Tagung angesprochenen Phänomenbereiche auch zum Gegenstand lexikografischer Projekte geworden sind, insbesondere Kollokationen, feste Wendungen, Valenzen und Argumentstrukturen. Auch andere, nicht auf der Tagung diskutierte Phänomene wie Redewendungen und Sprichwörter sind mittlerweile über elektronische Ressourcen zu erschließen (Projekt 13).

Die am häufigsten diskutierten Phänomene in dem vorliegenden Band sind solche im Umfeld von Valenz und Argumentstruktur. Das sind Phänomene, die gewöhnlich in Wörterbüchern, die Verben verzeichnen, auch als Angabeklassen repräsentiert sind. Große allgemeinsprachliche Internetwörterbücher zum Gegenwartsdeutschen bieten daher im Allgemeinen auf die eine oder andere Weise Informationen zur Rektion bzw. Valenz von Wörtern (siehe Abbildungen 1 und 2). 


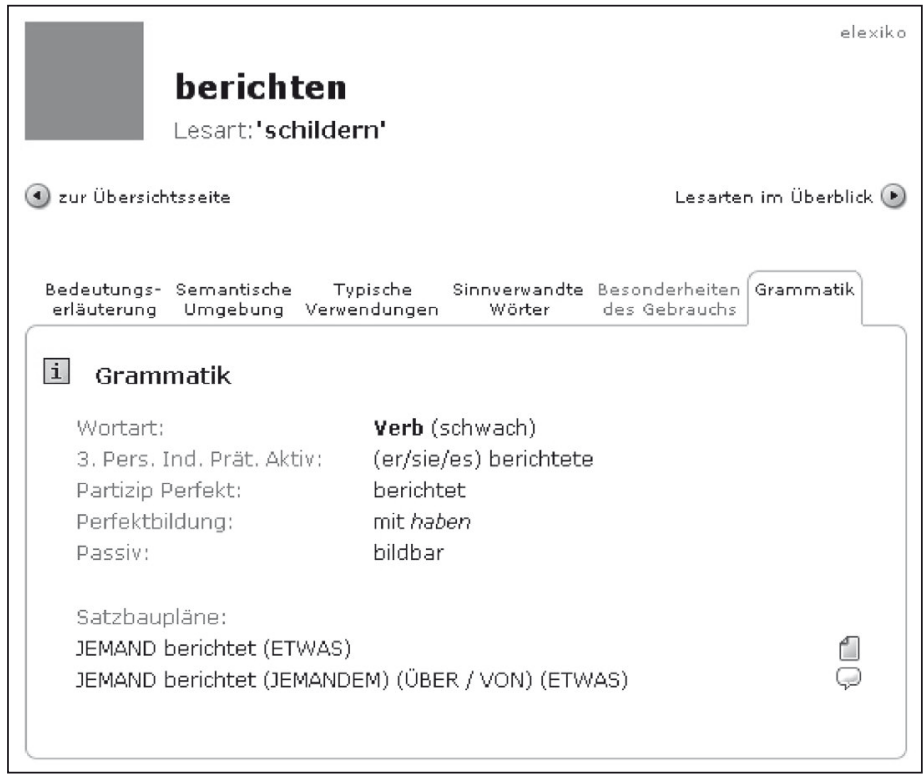

Abb. 1: Satzbaupläne zu berichten in ,elexiko“

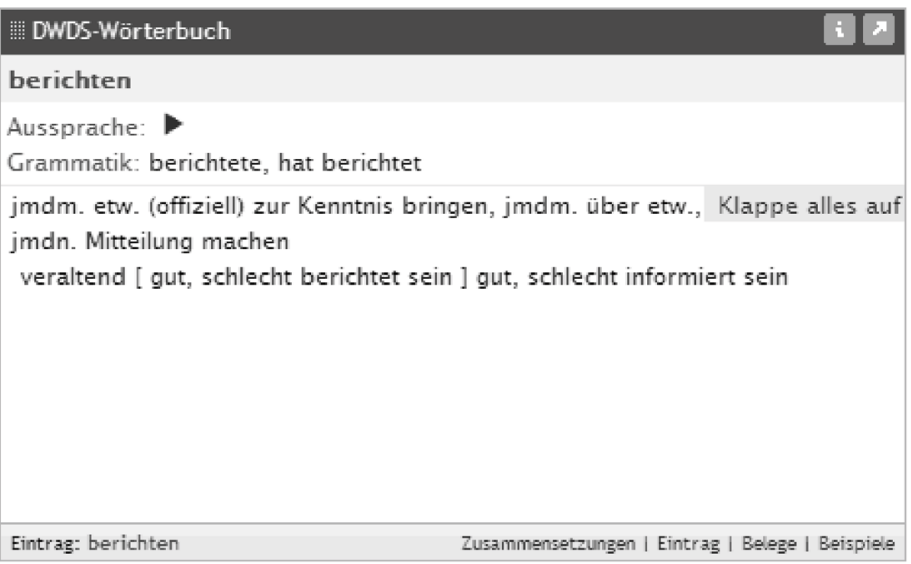

Abb. 2: Valenzangaben zu berichten im „DWDS“

Dabei ist es aber für externe Benutzer nicht möglich, Suchen nach Verben mit bestimmten Valenzen/Satzbauplänen/Argumentstrukturen durchzuführen. Dies können etwa in „elexiko“ nur die Lexikografen über einen internen Zugriff. Diese erweiterten Suchen sollen allerdings auch für externe Benutzer deutlich ausgebaut werden. Das „E-VALBU“ (Projekt 11) erlaubt dagegen jetzt schon Suchen über bestimmte formale Valenzmuster. In Abbil- 
dung 3 sieht man die Suchmaske und Ergebnisdarstellung zu einer Abfrage nach allen Verben, die außer dem Subjektargument eine NP im Dativ und eine im Akkusativ fordern.

\begin{tabular}{|c|c|c|}
\hline Komplemente: & $\nabla K_{\text {sub }} \nabla K_{\text {skk }} \square K_{\text {skk2 } 2} \square K_{\text {gen }} \nabla K_{\text {dat }} \square K_{\text {prp }} \square K_{\text {prp2 } 2} \square K_{\text {sdv }} \square K_{\text {save } 2} \square K_{\text {prd }} \square K_{\text {vrt }}$ & \multirow{7}{*}{$\begin{array}{l}\text { Ergebnisliste: } \\
\text { abgeben } 4 \\
\text { abnehmen 7 } \\
\text { abnehmenen } 8 \\
\text { abnehmen 9 } 9 \\
\text { abnehmen } 10 \\
\text { abnehmenen } 11 \\
\text { abnehmen } 12 \\
\text { anbieten 1 } \\
\text { anbieten 2 } \\
\text { anbieten 3 } \\
\text { anbleten 4 } \\
\text { ansehen 2 } \\
\text { antworten 1 } \\
\text { anzziehen 2 }\end{array}$} \\
\hline Satzbauplan: & belichig & \\
\hline Passiv: & beliebig & \\
\hline Pertinenzelemente: & beliebig & \\
\hline Volltextsuche: & 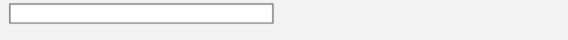 & \\
\hline Euche.sinterter & Fomular leeren & \\
\hline \multicolumn{2}{|c|}{ Bitte per Mausklick einen Eintrag aus der Ergebnisliste auswählen! } & \\
\hline
\end{tabular}

Abb. 3: Abfrage zu dreistelligen Nominativ-Akkusativ-Dativ-Verben im „E-VALBU“

Nicht valenz-, sondern frametheoretisch orientiert ist das Lexikon syntagmatischer Angaben zur Syntax und Semantik lexikalischer Einheiten des Deutschen, das in dem Projekt SALSA erstellt wird. Es basiert auf einem detailliert phrasenstrukturell und framesemantisch annotierten Korpus (Projekt 8, siehe Abbildung 4).

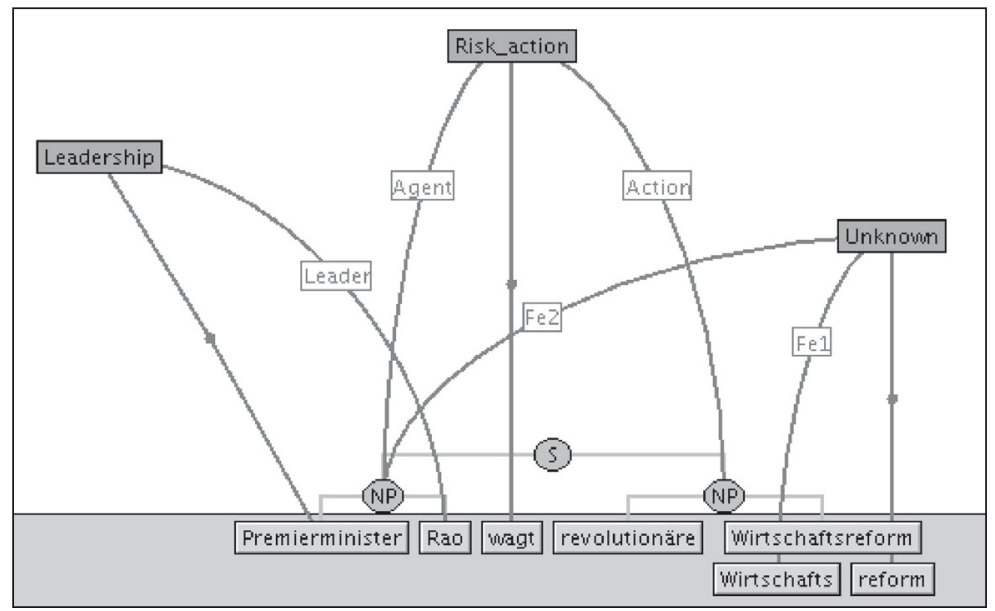

Abb. 4: Phrasenstrukturell und framesemantisch annotierter Satz im SALSA/TIGER-Korpus (www.coli.uni-saarland.de/projects/salsa/gold/page.php?id=s52 (Stand: 24.08.2010))

Im Bereich der lexikografischen Erschließung von Phänomenen im Umfeld von Valenz und Argumentstruktur gibt es allerdings durchaus noch Desiderata. So enthalten allgemeine, einsprachige Internetwörterbücher zum Gegenwartsdeutschen keine Angaben zur Inhaltsvalenz (semantische Rollen, Selektionsrestriktionen), die als Suchkriterien fungieren können. Angaben zu lexikalischen Mitspielern finden sich allerdings in ,elexiko". Sie werden dabei aber nicht zu Rollen oder Selektionsrestriktionen generalisiert. 
Insbesondere aber sind solche Argumentstrukturmuster, die mit stabilen Bedeutungen einhergehen (Resultativkonstruktionen, Mittelkonstruktionen, Applikativkonstruktionen und viele mehr) nicht nur nicht suchbar, sondern gewöhnlich bezüglich ihres Vorkommens mit bestimmten Verben in Wörterbüchern gar nicht verzeichnet, und das gilt nicht nur für Wörterbücher des Internets (vgl. Engelberg 2010). E-VALBU ermöglicht aber immerhin schon das Suchen nach Verben, die in bestimmten Passiv- oder Pertinenzkonstruktionen auftreten.

Eine Ressource, die Argumentstrukturmuster bzw. -konstruktionen im Zusammenspiel mit idiosynkratischen Eigenschaften von Verben erfasst, etwa auf der Basis von empirischen Verfahren wie sie von Stefanowitsch und Gries (Gries/Stefanowitsch 2010; Stefanowitsch in diesem Band) angewendet werden, bleibt allerdings ein Desideratum.

Die Lexikografie von Phrasemen, also etwa Kollokationen, festen Wortverbindungen, Funktionsverbgefügen, Redewendungen und Sprichwörtern, hat von der Entwicklung korpuslinguistischer Methoden schon deutlich mehr profitiert als der Argumentstrukturbereich. Insbesondere die Kookkurrenzanalyse hat dieses Gebiet vorangebracht. Lexikografische Informationssysteme präsentieren entweder vollautomatisch gewonnene typische Wortverbindungen wie im „Deutschen Wortschatz“ (Projekt 3; Abbildung 5) oder lexikografisch überarbeitete, semi-automatisch gewonnene Verbindungen (,elexiko", Projekt 14; Abbildung 6).

Signifikante Kookkurrenzen für trinken:

Bier (3505), essen (3098), Kaffee (2522), Alkohol (1405), Tee (1129), Wein (1026), Wasser (1022), zu (828), , (803), rauchen $(740)$, und (617), viel (537), sitzen (487), Liter (423), oder (364), Glas (351), man (332), sie (325), gehen (320), Cola (317), Bierchen (304), Flasche (290), Rotwein (282), etwas (278), mal (267), wir (242), Sie (232), Tasse (230), Wodka (217), Flussigkeit (187), ich (182), Mineralwasser (181), Sekt (179), Milch (166), Leute (163), Männer (161), Schnaps (160), reden (157), abends (156), Durst (155), dann (155), kann (146), predigen (144), tanzen (141), was (135), einen (134), darf (133), dürfen (121), Kneipe (121), täglich (120), Wenn (116), Bar (115), Tag (115), Viel (113), rät (110), Glühwein (110), Menschen (110), Cocktails (109), Schluck (109), keinen (107), Champagner (104), Espresso (103), Cappuccino (102), Café (100), lieber (99), ein (97), Leitungswasser (95), Gläschen (95), : (94), gerne (93), schlafen (92): Hitze (92), quatschen (91): Essen (89), sollte (88), Getränke (87), Frauen (87), mehr (86); Kölsch (85), gern (85)

Mehrwortkookkurenzen:

Kaffee trinken (2467), viel trinken (761), Tee trinken (502), zu viel (154), am Tag (139), zu wenig (126), Abwarten und Tee trinken (113), in Ruhe (94), ein Glas Wein (81), abwarten und Tee trinken (80), unter den Tisch trinken (80)

Signifikante linke Nachbarn von trinken:

zu (3252), Bier (2320), Kaffee (2171), Alkohol (991), Wein (625), Teee (604), Wasser (514), viel (366), und (327), Viel (236), Sie (187), Bierchen (180), Rotwein (174), Espresso (103), etwas (101), Champagner (101), Schnaps (96), was (90), Cola (88), mehr (84), Wodka (81), Wir (80), Cappuccino (78), am Tag (75), ein Glas Wein (74), Sekt (73), genügend (68), noch ein Bier (65), zu viel (64), eine Tasse Kaffee (58), wir (57), Kakao (56), Leute (56), ein Glas Wasser (56), Tag (55), Milch (53), um zu (50), Kölsch (50), Viertele (49), Gluhwein (48), Liter (47), eine Tasse Tee (46), noch etwas (43), Warmes (43), Deutschen (42), Durst (41), Saft (40), Caipirinha (40), Nektar (38), Apfelwein (38), Gin (38), einen (37), ausreichend (37), pro Tag (37), genug (36), Männer (36), Biere (36), Strohhalm (35), im Freien (35), Pils (34), regelmäßig (34), heiße Schokolade (33), Pilger (33), Cocktails (31), so viel (31), täglich (30), Ebbelwei (30), Obstler (30), zu wenig (29), Heißes (28), Britinnen (27), Apfelschorle (26), Freien (26), Schlafengehen (26), oder (26), Flüssigkeit (25), Merlot (25), Tisch (24), Cognac (24), Alcopops (23)

Abb. 5: Verschiedene Angaben zu Kookkurrenzen zu trinken im „Deutschen Wortschatz“ 


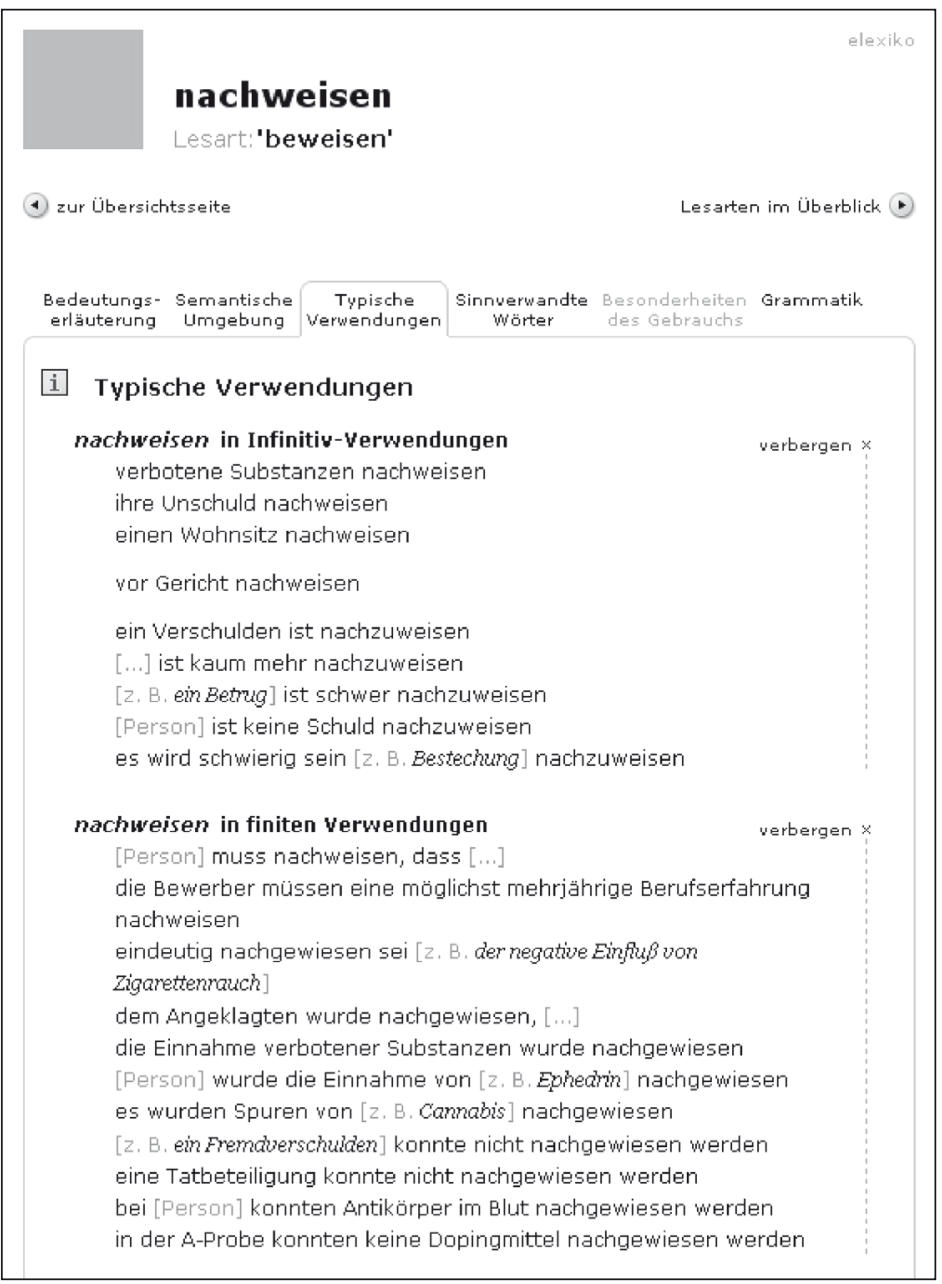

Abb. 6: Typische Verwendungen des Verbs nachweisen in „elexiko“

Dabei werden die Darstellungen häufiger durch netzbasierte oder typografisch gestaltete grafische Darstellungen ergänzt (siehe Abbildungen 7 und 8).

Mittlerweile sind aber auch spezifische neue Ressourcen für Phraseme ins Netz gegangen. Die im Aufbau befindliche Internetressource „Wortverbindungsfelder Online“ des Projekts „Usuelle Wortverbindungen“ (Projekt 13) dokumentiert detailliert auf der Basis von Korpusanalysen den Gebrauch und die Varianz von Wortverbindungen (siehe Abbildungen 9 und 10). 


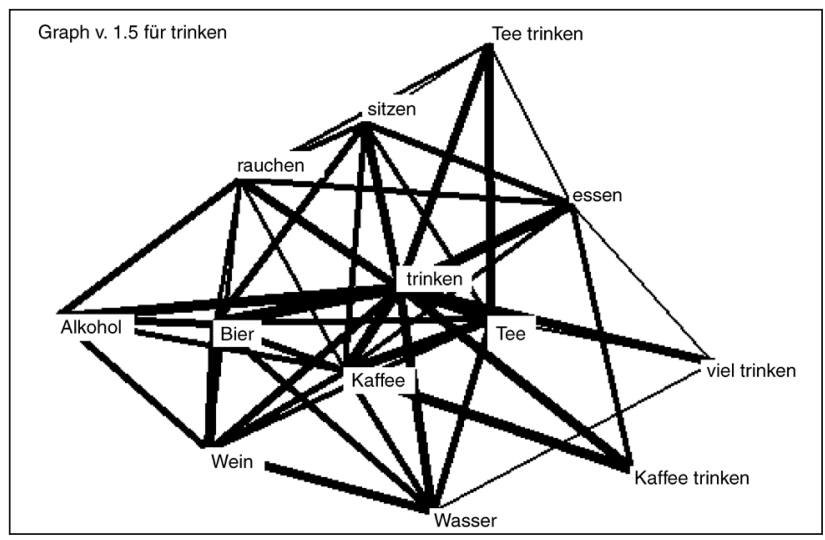

Abb. 7: Netzbasierte Darstellung der Kookkurrenzen zu trinken im „Deutschen Wortschatz“

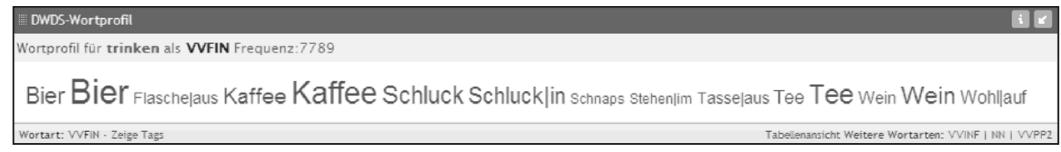

Abb. 8: Typografische Darstellung der Kookkurrenzen zu trinken im „DWDS“

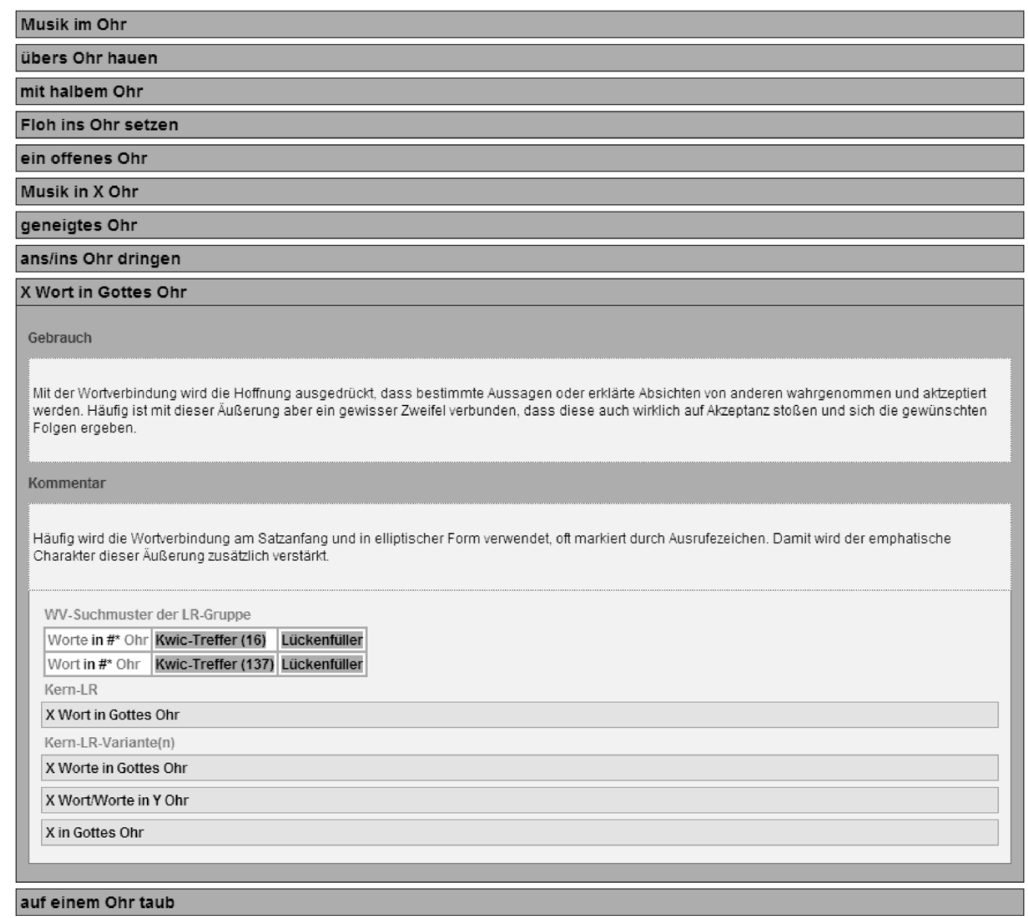

Abb. 9: Ausschnitt aus dem Wortverbindungsfeld Obr in „Wortverbindungsfelder online“ 


\begin{tabular}{|c|c|c|c|}
\hline \multicolumn{4}{|l|}{ Füller für Suchmuster } \\
\hline \multicolumn{4}{|l|}{ 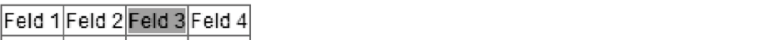 } \\
\hline Wort in & & & \\
\hline \multicolumn{4}{|c|}{$\begin{array}{l}\text { Die Füller können durch Klicken auf den Namen der Tabellenspalten sortiert werden. } \\
\text { Füllertabelle für Feld } 3\left(\#^{*}\right)\end{array}$} \\
\hline Lückenfüller & Anzahl & Prozentanteil & \\
\hline Menschen & 1 & 0,73 & \\
\hline der Amerikaner & 1 & 0,73 & \\
\hline (Fußball)-Gottes & 1 & 0,73 & \\
\hline des Wettergotts & 1 & 0,73 & \\
\hline des Theatergottes & 1 & 0,73 & \\
\hline Kabilas & 1 & 0,73 & \\
\hline Tschubais & 1 & 0,73 & \\
\hline Orffens & 1 & 0,73 & \\
\hline Wachters & 1 & 0,73 & \\
\hline der heimischen Pistenartisten & 1 & 0,73 & \\
\hline des Kanzlers & 1 & 0,73 & \\
\hline Gottes & 73 & 53,28 & \\
\hline Luizaos & 1 & 0,73 & \\
\hline Diebes & 1 & 0,73 & \\
\hline Levys & 1 & 0,73 & \\
\hline der Rechtsgelehrten & 1 & 0,73 & \\
\hline Bacchus & 1 & 0,73 & \\
\hline des Täters & 1 & 0,73 & \\
\hline Konsels und Prohaska & 1 & 0,73 & \\
\hline des Sachbearbeiters & 1 & 0,73 & \\
\hline des Fußball-Gottes & 2 & 1,46 & \\
\hline Handys & 1 & 0,73 & \\
\hline
\end{tabular}

Abb. 10: Füller zu der Wendung X Wort in [Gottes] Obr in „Wortverbindungfelder online“

Doch auch in diesem Bereich gibt es noch viele Lücken zu füllen. Dazu gehört ein großes lexikografisch bearbeitetes Internetkollokationswörterbuch des Deutschen. Auch ein beschreibendes Werk „klassischer Konstruktionen", also schwer regelhaft herleitbarer, partiell lexikalisch gefüllter Ausdrücke wie etwa die in diesem Band besprochenen Was-in-aller-WeltKonstruktion, X-hat-seinen-Preis-Konstruktion (vgl. Stefanowitsch in diesem Band), die verblosen Direktiva und sequenziellen Nominalreduplikationen (vgl. Müller in diesem Band) ist noch nicht erarbeitet.

Die Zugänglichkeit zu lexikografischen Daten wird sich aber nicht nur durch die Entwicklung spezifischer lexikografischer Produkte verbessern, sondern auch dadurch, dass bestehende lexikografische Ressourcen zusammengeführt werden. Das kann in Form von Projekten geschehen, die ganz spezifische lexikografische Ressourcen aufeinander abbilden, wie zum Beispiel paradigmatisch organisierte WordNets auf syntagmatisch ausgerichtete FrameNets (siehe Fellbaum in diesem Band). Die Zusammenführung kann aber auch durch die Implementierung von Wörterbuchportalen erfolgen. 
Wörterbuchportale integrieren eigenständige sprachlexikografische Referenzwerke, die im Falle von Wörterbuchnetzen wie etwa „OWID“ (Projekt 16) wörterbuchübergreifende Verlinkungen und Zugriffsmöglichkeiten bieten (vgl. Müller-Spitzer/Engelberg, ersch.). Auch aus diesen Bereichen sind in Zukunft für den Bereich der Syntagmatik des deutschen Wortschatzes wichtige Impulse sowohl für die wortschatzbezogene Forschung als auch für die lexikografische Praxis zu erwarten.

\section{Literatur}

Abel, Andrea (2008): ELDIT - Elektronisches Lernerwörterbuch Deutsch-Italienisch und elexiko: ein Vergleich. In: Klosa (Hg.), S. 175-189. Internet: www.ids-mannheim. de/pub/laufend/opal/privat/pdf/opal08-1_abel.pdf (Stand: 12.10.2010).

Abel, Andrea et al. (2005): ELDIT - Elektronisches Lernerwörterbuch Deutsch-Italienisch: einige Aspekte der syntagmatischen und paradigmatischen Ebene. In: Di Meola, Claudio/Hornung, Antonie/Rega, Lorenza (Hg.): Perspektiven Eins. Akten der ersten Tagung Deutsche Sprachwissenschaft in Italien (Rom, 6.-7. Februar 2004). (= Italienische Studien zur deutschen Sprache 1). Rom, S. 589-606.

Boas, Hans C. (Hg.) (2009): Multilingual FrameNets in computational lexicography: methods and applications. (= Trends in Linguistics: Studies and Monographs 200). Berlin/New York.

Burchardt, Aloscha et al. (2009): FrameNet for the semantic analysis of German: Annotation, representation and automation. In: Boas (Hg.), S. 209-244.

Engelberg, Stefan (2010): Die lexikographische Behandlung von Argumentstrukturvarianten in Valenz- und Lernerwörterbüchern. In: Fischer, Klaus/Fobbe, Eilika/ Schierholz, Stefan J. (Hg.): Valenz und Deutsch als Fremdsprache. (= Deutsche Sprachwissenschaft international 6). Frankfurt a.M., S. 113-141.

Engelberg, Stefan/Klosa, Annette/Müller-Spitzer, Carolin (2009): Internet lexicography as a challenge: The Internet dictionary portal at the Institute for German Language. In: Kernerman Dictionary News 17, S. 16-25.

Gries, Stefan T./Stefanowitsch, Anatol (2010): Cluster analysis and the identification of collexeme classes. In: Newman, John/Rice, Sally (Hg.): Empirical and experimental methods in cognitive/functional research. Stanford, S. 59-71.

Herbst, Thomas et al. (2004): A valency dictionary of English. A corpus-based analysis of the complementation patterns of English verbs, nouns and adjectives. (= Topics in English Linguistics 40). Berlin/New York.

Klappenbach, Ruth/Steinitz, Wolfgang (Hg.) (1967-1977): Wörterbuch der deutschen Gegenwartssprache. Bd. 1-6: A-Zytologie. Berlin.

Klein, Wolfgang (2004): Das Digitale Wörterbuch der deutschen Sprache des 20. Jahrhunderts (DWDS). In: Scharnhorst, Jürgen (Hg.): Sprachkultur und Lexikographie. Von der Forschung zur Nutzung von Wörterbüchern. (= Sprache 50). Frankfurt a.M., S. 281-309. 
Klosa, Annette (2007): Korpusgestützte Lexikographie: besser, schneller, umfangreicher? In: Kallmeyer, Werner/Zifonun, Gisela (Hg.): Sprachkorpora - Datenmengen und Erkenntnisfortschritt. (= Jahrbuch des Instituts für Deutsche Sprache 2006). Berlin/New York, S. 105-122.

Klosa, Annette (Hg.) (2008): Lexikografische Portale im Internet. (= OPAL - Online publizierte Arbeiten zur Linguistik 1/2008). Mannheim. Internet: www.ids-mannheim. de/pub/laufend/opal/privat/opal08-1.html (Stand: 12.10.2010).

Kubczak, Jacqueline (2009): Hier wird Ihnen geholfen! E-VALBU - Das elektronische Valenzwörterbuch deutscher Verben. In: Sprachreport 4/2009, S. 17-23.

Kunze, Claudia/Lemnitzer, Lothar (2010): Lexical-semantic and conceptual relations in GermaNet. In: Storjohann, Petra (Hg.): Lexical-semantic relations. Theoretical and practical perspectives. (= Linguisticae investigationes: Supplementa 28). Amsterdam/Philadelphia, S. 163-183.

Langanke, Ulrich (2004): Das Hypermedia-Online-Wörterbuch an der Schnittstelle zwischen Philologie, Kognition und Informatik. Am Beispiel des „Wortschatz-Lexikons“" (http://www.wortschatz.uni-leipzig.de). In: Brdar-Szabó, Rita/Knipf-Komlósi, Elisabeth (Hg.): Lexikalische Semantik, Phraseologie und Lexikographie. Abgründe und Brücken. Festgabe für Regina Hessky. (= Duisburger Arbeiten zur Sprach- und Kulturwissenschaft 57). Frankfurt a.M. u.a., S. 379-393.

Moerdijk, Fons (2008): Das „Algemeen Nederlandse Woordenboek“ (ANW) und elexiko - ein Vergleich. In: Klosa (Hg.), S. 143-151. Internet: www.ids-mannheim.de/pub/ laufend/opal/privat/pdf/opal08-1_moerdijk.pdf (Stand: 12.10.2010).

Moerdijk, Fons/Tiberius, Carole/Niestadt, Jan (2008): Accessing the ANW dictionary. In: Zock, Michael/Huang, Chu-Ren (Hg.): Coling 2008: Proceedings of the workshop on Cognitive Aspects on the Lexicon (COGALEX 2008), Manchester, August 2008, S. 18-24. Internet: www.aclweb.org/anthology/W08-1903 (Stand: 13.10.2010).

Müller-Spitzer, Carolin (2010): OWID - A dictionary net for corpus-based lexicography of contemporary german. In: Dykstra, Anne/Schoonheim, Tanneke (Hg.): Proceedings of the XIVth EURALEX International Congress (Leeuwarden, 6-10 July 2010). Leeuwarden, S. 445-452.

Müller-Spitzer, Carolin/Engelberg, Stefan (ersch.): Dictionary portals. In: Gouws, Rufus H. et al. (Hg.): Dictionaries. An international encyclopedia of lexicography. Supplementary volume: Recent developments with special focus on computational lexicography. Berlin/New York.

Müller-Spitzer, Carolin/Vachková, Marie (2010): Projektmesse „Elektronische Lexikographie“. In: Sprachreport 2/2010, S. 20-23.

Prinsloo, Daniel J. (2009): The role of corpora in future dictionaries. In: Nielsen, Sandro/Tarp, Sven (Hg.): Lexicography in the 21st century. In honour of Henning Bergenholtz. (= Terminology and Lexicography Research and Practice 12). Amsterdam/Philadelphia, S. 181-206.

Ruppenhofer, Josef et al. (2010): FrameNet II: extended theory and practice. Internet: http://framenet.icsi.berkeley.edu/index.php?option=com_wrapper\&Itemid=126 (Stand: 13.08.2010). 
Schmidt, Thomas (2009): The Kicktionary - A multilingual lexical resource of football language. In: Boas (Hg.), S. 101-134.

Schumacher, Helmut et al. (2004): VALBU - Valenzwörterbuch deutscher Verben. (= Studien zur Deutschen Sprache 31). Tübingen.

Steyer, Kathrin (2009): Zwischen theoretischer Modellierung und praxisnaher Anwendung. Zur korpusgesteuerten Beschreibung usueller Wortverbindungen. In: Mellado Blanco, Carmen (Hg.): Theorie und Praxis der idiomatischen Wörterbücher. (= Lexicographica, Series maior 135). Tübingen, S. 119-145.

Storjohann, Petra/Möhrs, Christine (2007): Das elexiko-Wörterbuch im Wandel - Typische Verwendungen neu beleuchtet. In: Sprachreport 3/2007, S. 12-16.

Vachková, Marie/Belica, Cyril (2009): Self-organizing lexical feature maps. Semiotic interpretation and possible application in lexicography. In: Interdisciplinary Journal for Germanic Linguistics and Semiotic Analysis 13, 2, S. 223-260.

\section{Links zu den vorgestellten Projekten}

Projekt 1 (Algemeen Nederlands Woordenboek): http://anw.inl.nl/search

Projekt 2 (Das Große akademische Wörterbuch Deutsch-Tschechisch): https://lexarchiv.ff.cuni.cz/ lexikograficka-sekce/_spravce/

Projekt 3 (Deutscher Wortschatz, der Universität Leipzig): http://wortschatz.uni-leipzig.de/

Projekt 4 (Digitales Wörterbuch zur deutschen Sprache - DWDS): www.dwds.de

Projekt 5 (Entwicklung eines korpusbasierten, elektronischen Wörterbuchs Deutsche Gebärdensprache - Deutsch): www.sign-lang.uni-hamburg.de/dgs-korpus/

Projekt 6 (GermaNet - ein lexikalisch semantisches Wortnetz): www.sfs.uni-tuebingen.de/lsd/

Projekt 7 (HyTex - Werkzenge zur Exploration von GermaNet): www.hytex.tu-dortmund.de/ ressourcen.html\#wortnetze

Projekt 8 (Saarbrücken Lexical Semantics Acquisition Project - SALSA): www.coli.uni-saarland. de/projects/salsa

Projekt 9 (Kicktionary): www.kicktionary.de

Projekt 11 (E-VALBU): http://hypermedia2.ids-mannheim.de/evalbu/index.html

Projekt 12 (Erlanger Valency Patternbank): www.patternbank.uni-erlangen.de

Projekt 13a (Usuelle Wortverbindungen): http://wvonline.ids-mannheim.de/

Projekt 13b (SprichWort): www.sprichwort-plattform.org/

Projekt 14 (elexiko): www.elexiko.de

Projekt 15 (Benutzeradaptive Zugänge und Vernetzungen in elexiko): www.ids-mannheim.de/ lexik/BZVelexiko/

Projekt 16 (Online-Wortschatz-Informationssystem Deutsch-OWID): www.owid.de 


\section{Sachindex}

A

(Argumentstruktur)alternation 19, 47, 221, 228, 378, 379, 460, 462-464, 466, 469, 478, 493, 497

Absolutiv 221, 441

Abstraktion(sgrad) 5, 7, 10, 13-15, 27, 28, 37, 38-40, 54, 57, 58, 60, 63, 71, 72, 93, 121, 129, 137-140, 181, 183, 184, 208, 375, 378, 382, 419

Activity 465, 473, 489

Actor, s. Agens

Addressee, s. Adressat

Adjektivierung 17, 286, 318-323, 326$328,336-340,357,359,367-368$

Adjektivmodifikation 55-56

Adjunkt 24, 57, 165, 166, 187, 195, 262, 337-338, 410, 446, 447, 465, 468470

Adjunktion 166, 337, 338, 410

Adressat (als semantische Rolle) 48, 86, 298, 305, 308

Adverbial-Konstruktion 75

Adverb-mit-Direktiv-Konstruktion, s. Direktiv, verbloses

Agens 193, 264, 286, 299-301, 305$309,318,321,323,325,329,330$, $332,336,340,379,381,384,390$, 431, 441-444, 465, 470, 474, 497

Agensdemotion, referenzielle, s. Argumentdemotion

Agentivität 286, 299-301, 303-309

Aktionsart 286, 290

Akzeptabilität 21-22, 25-26, 135, 258, 285-286, 289, 291-294, 297-299, 303-304, 306-308, 359, 360, 430, 432, 434, 463, 513

Algemeen Nederlands Woordenboek $560,561,572$

Ambiguität 125, 290, 291, 294, 299, 306, $308,397,417,434,448,543,544$

Annotation 21, 23, 24, 27, 262, 266, 460, 472, 474, 477

Annotation Mining 24, 27, 29, 251, 253 , 261, 262, 266, 267, 281
Antipassiv 14, 18, 58, 161-165, 169, 170, 174

Antonymie 460, 476

Apposition 194, 195

Arbeitsgedächtnis/Arbeitsspeicher 387, 430

Argumentabsenkung/-demotion 221, 294, 297, 298

Argumentinterpretation 429, 430, 435, 439,440

Argumentrealisierung 24, 377, 382, 389, 390, 391

Argumentrolle, s. Rolle, semantische

Argumentstruktur 3, 15, 19, 23, 28-30, 71-96, 101, 102, 104, 105, 107, 150, $151,176,177,188,337,375,376-$ 380, 382, 384, 390, 402, 420, 462, 478, 484, 492, 495, 497, 563-566

Argumentstrukturkonstruktion 15, 23, 71, 73-77, 80, 188

Argumentstrukturmuster 15, 19, 23, 28, 71-73, 75, 77-93, 95, 96, 101, 102, 104, 105, 484, 564, 566

Artikelwegfall 24, 253, 267, 269-281

Artikulation, doppelte 213

Atelizität, s. Telizität

Attribut 78, 79, 165, 273

Ausdrucksstufe 346-370

Auxiliar 38, 156-158, 286, 290-292, $306,308,320,321,386,388,406$

B

Baseldeutsch 507, 521, 525-527

Basis vs. Kollokator, s. Kollokation

Basisbedeutung, s. Grundbedeutung

Basiswortschatz, s. Grundwortschatz

Bedeutungsidentität 76

Bedeutungspotenzial 128, 493

Belebtheit 286, 299, 300, 307, 429, 436443

benefaktive Dativ-Konstruktion 18, 469

Benefizient 470, 471 
Benutzeradaptive Zugänge und Vernetzungen in elexiko 560, 563, 572

Berkeley Construction Grammar 149, 176, 177

Bewegungslesarten 73, 93

Bewegungsverb 19, 82, 83, 93, 95, 96, 379,462

Binarität IX, 540, 541

BNC (= British National Corpus) 46, 47, 469, 484, 496

Bootstrap validation 276, 277

Bootstrapping 378-380, 400, 416

C

CAUSE-BECOME 42, 57

Caused-Motion Construction 39, 47

CHILDES (= Child Language Data Exchange System) 386, 387, 395

Cleft-Relativsatz, s. Relativsatz

coercion, s. Uminterpretation

competition model 389, 392

Computerlinguistik 37,537-539

Computersimulation 387

Construction Grammar, s. Konstruktionsgrammatik

CPA (= Corpus Pattern Analysis) 488, 491, 493, 494, 501

\section{D}

Daten, elizitierte/spontane 21, 22, 25-27

Daten, qualitative/quantitative 21, 23, 24

Daten, sprachliche vs. metasprachliche 21

Datenelizitation, s. Elizitation

Dativ, ethischer 477, 478

Dativ-Alternation 19, 462

Default-Kategorie 29, 345, 364, 368,

Dekomposition 71, 73, 238, 239, 447

Dekonstruktion(sprozess) 398, 407, 416, 423

Demotion, s. Argumentdemotion

DeReKo (= Deutsches Referenzkorpus) 205, 206, 208, 536

Derivation 24, 153, 185, 214, 215, 235, $241,243,244,262,348,357-359$, $367,368,411$
Deutscher Wortschatz (Universität Leipzig) 561,572

Dialektlexikografie $508,521,525,529$

Dichotomie 1, 4-6, 17

Direktional(ausdruck) 93-95, 216, 223, 264,337

Direktiv 19, 28, 187, 188, 211, 214, 216222, 224-229, 233, 237, 238, 243, 244

Diskursfunktion $183,375,381$

Diskursmarker/-partikel 16,17, 118, 119 , 123-126, 137-139

Distributed Morphology 3, 214, 445

Ditransitivkonstruktion 74-77, 225, 382

Divisivität 287, 289, 295, 296

Doppel-Objekt-Konstruktion, s. Ditransitivkonstruktion

DP, possessive, s. Possessivum

$d$-Relativsatz, s. Relativsatz

Duplikationsdomäne, s. Nominalreduplikation, sequenzielle

Durativangabe 288, 289, 291, 296, 298

DWDS (= Digitales Wörterbuch der deutschen Sprache) 507, 509, 510, 514, 515, 520, 523, 529, 561, 564, 568,572

E

Echofrage 414

Echtzeit 26, 429, 444, 448

EEG (= Elektroenzephalogramm) 25, $30,432,438,447$

Einwortäußerung 383, 403

EKP (= ereigniskorrelierte Potenziale) 25,432

ELAN (= Early Left Anterior Negativity) 445

ELDIT (= Elektronisches Lernwörterbuch Deutsch-Italienisch) 462,470

elexiko X, 560, 563-567, 570

Elizitation 21, 26, 253

Ellipse 217, 218, 220, 228, 232, 233, 296-298, 307, 377, 493

EMEA 547,548

Entailment 462, 467, 475

Ereignis-Frame 35, 51-54, 56, 59, 60-63

Ereignisstruktur 286, 287, 289, 291, 294 298, 307, 337, 485 
Ergativ-Absolutiv-System 221-223, 441

Erlanger Valency Pattern Bank 560, 562, 572

Erzeugungsregel, s. Regel

E-VALBU (= Elektronisches Valenzwörterbuch deutscher Verben) XI, 560, 562-66, 571-572

Experiencer 466

Experiencerverb 432, 444

Extraktionsinsel 171,175

\section{$\mathbf{F}$}

Familienähnlichkeiten, s. Konstruktionsfamilie

Festigkeit, s. Kompositionalität

Finitheit 340, 385, 403, 406

Fixiertheit, s. Kompositionalität

Flexionsmorphologie 24, 224, 262, 357

Flexionsverbot 231, 233, 234, 241

fMRT (= funktionelle Magnetresonanztomographie) 438

Fokus(partikel) 328, 401, 403, 407, 408

for-Adverbial-Konstruktion 75

Fragepartikel 407

Frame-Element 465-467, 469-472, 474, 477, 478, 493

FrameNet 26, 30, 48-50, 459, 460, 465467, 469-478, 562, 569

Frame-Semantik 41, 48, 50, 465

Frequenz(analyse) 9, 10, 21, 22, 27, 28, 39, 53, 56, 378, 384, 392, 507, 524, $546,547,549,550,551,553,555$, 559

Funktionsverbgefüge 16, 268, 506, 515, 559,565

Fusionierung, s. Konstruktionsfusion

Fuß 352, 405, 421

G

Gebärdensprache 560, 561

Generalisierungsprozess 376, 380

Generative Semantik 3

Generative Syntax 175

Generatives Lexikon 485

Geräuschverben $14,73,93,95,96,102$, 105
GermaNet 24, 262, 263, 272, 275, 461, $478,560-562,572$

Gesprächsanalyse 113,117, 119, 138

Gesprächsforschung 13,113, 114, 117, 137

Gesprächspartikel, s. Diskursmarker

Gesprochene-Sprache-Forschung 113$115,121,128$

Getrennt-/Zusammenschreibung 353, $354,367,368$

Goal 42, 43, 51, 52, 57

Gradpartikel 127, 190

Grammatikalisierung 59, 114, 305, 306, 308, 309, 407

Granularität $7,13,20,28,53,113,128-$ 130, 133, 134, 137-141, 370, 471

Graphematik 357, 362

Großes akademisches Wörterbuch Deutsch-Tschechisch 560, 561, 572

Grundbedeutung 41, 43, 54, 61

Grundwortschatz 505, 507, 521, 523, 524,529

\section{H}

HaGenLex (= Hagen German Lexicon) $24,263,275,276$

Handlungsverursacher 436, 438, 441, 443

Herstellungsverb 82

Hintergrundwissen 55, 128, 324

Homonymie $74,75,92,472$

Homophonie 169, 170, 174-176

HPSG (= Head-Driven Phrase Structure

Grammar) 11, 12, 14, 38, 153, 176, 186, 187, 199, 208, 378

Hyperonymie 461, 467, 472, 473, 475

\section{I}

ich mein(e) 16, 17, 118, 124, 125, 137

Idiomatizität 16

Idiome 11, 15-20, 507, 510, 520

Idiomprinzip 488

Idiosynkrasie, s. Regel

Implikatur 305, 306, 308, 309, 333, 487 491, 493, 494, 497, 501

Inchoativ 288, 464 
Infinitiv $170,186,268,355,386,388-$ $390,401,421$

INFL 155,422

Informativitätsverstärkung 303, 305, 308, 309

Inkorporation 357,368

Inkrementalität 430

Insel, syntaktische $13,171,175,231-$ $233,241,242,384$

Instrumental(adverbial) 157, 279, 318, 321, 330, 331, 469

Instrument-Subjekt-Alternation 19, 463

Intentionalität $300,302,305,307$

Interaktionale Linguistik, s. Gesprächsforschung

Interjektion 119, 410

Internetlexikografie 559, 560

Interpretation, maximale 529

Interrogativsatz 161, 164, 170, 190-192, 194-196, 227, 401, 406, 407

Intransitivität, s. Transitivität

Intransitivkonstruktion 18

Inversion 157, 158, 410, 415

J

jetzt 20, 114, 123-124, 130-131, 135-138

\section{K}

Kasus(zuweisung) 72, 89, 215, 218, 221-223, 225, 231, 233, 239, 241, 264, 285, 304, 305, 378, 403, 431, $436,438,441,444,542-544$

Kategorienbildung 125, 130, 383

Kausalangabe 336

Kausativ-Inchoativ-Alternation 19

Kerngrammatik vs. Peripherie 38, 182, 189, 198, 208

Kicktionary 560, 562, 572

Klitikon/Klitisierung 353, 388, 417

Koerzion, s. Uminterpretation

Kognitiv Saliente Semantische Relationen 560,562

Kollokation X, 16, 22-24, 26, 28, 30, $39,47,52,53,55,56,62,100,113$, 117, 186, 483, 485, 492, 493, 500,
501, 505-517, 519-525, 527-529, 533-557, 559, 561, 563, 566, 569

Kommunikationsverb 44, 461, 563

Komplement 18, 24, 57, 78, 92, 150$152,158,191,220,233,234,238$, 240-242, 252, 254, 260, 262, 267, $272,273,275,278,280,281,348$, $360,362,393,399,402,403,407$, 408, 415

Komplementierer 19, 163, 165-169, 174, 393, 401, 403, 408, 409, 415

Kompositionalität IX, 9, 11, 16, 17, 184, 299, 419, 500, 508, 514, 521, 439, $541,542,545$

Kompositum 16, 17, 24, 185, 262, 275, $332,351,353,355,363,365,368$, 369,507

Konditionalsatz 157, 401

Kongruenz 23, 173, 175, 205-207, 215, 218, 239, 243, 349, 401, 403, 417, 420-422, 431

Konnektor XI

Konstrukt 134

Konstruktikon 4-5, 183-184, 197-198, 204-205

Konstruktion(sbegriff) $1,6,7,9,12,14$, $19,28,38,40,74,113,115,116,127$, $128,134,184,185,187,188,190$, 195, 197, 211, 290, 309, 375

Konstruktionsbedeutung 8, 11, 57, 128, 193, 290, 299, 306

Konstruktionsfamilie 13, 81, 87, 93, 95, 108, 109

Konstruktionsfusion 39, 42-45, 47, 50, 51,53

Konstruktionsgrammatik VII, 4-6, 811, 17, 19, 27, 28, 37-39, 60, 63, 73, 74, 76, 113, 116, 140, 157, 181-184, 187, 188, 190, 197, 198, 202, 204, 207, 208, 211, 290, 297, 300, 303, $310,375,381-383,385,392,423$

Konstruktionsidentität 27, 75, 76

Konstruktionsnetzwerk 14, 39, 57-60

Konstruktionsvarianz 27, 28

Kontrolle 302, 304, 305

Kookkurrenz(analyse) X, 21-24, 27, 28, 71-73, 93, 100, 102, 104, 223, 225, 238, 367, 368, 506, 508-510, 514, 
$515,517,519,522,524,540,541$, $545,559,566,568$

Kopf, (morpho)syntaktischer 24, 151, 152, 156, 162-165, 167-170, 174177, 214-215, 218-219, 221, 223$224,226,228,234,239,251,267$, 275, 279, 337-403, 407-408, 410, 411, 415, 419, 420, 422

Kopf-Komplement-Relation 399

Kopula 318, 319, 320-321, 323, 325, 326, 336, 340, 385-386, 404-407, 417, 494

Korngröße, s. Granularität

Korpusanalyse 25, 486, 495, 501, 508, $515,520,528,559,567$

Korpuslexikografie 559

Korpusmusteranalyse, s. CPA

Kotext 119, 123

Kovorkommen, s. Kookkurrenz

Kumulativität 289, 295, 296

$\mathbf{L}$

lcp (= lexical conceptual paradigm) 496 Lemmata 98, 100, 465, 476, 507, 512, 514-516, 519, 521, 523, 524

Lernerlexikografie 513

Lernmechanismen 381, 385

Lesarten, s. Polysemie

Lesezeitexperiment 21, 25, 321

Lexikalisierung 114, 151, 176

Lexikalismus 149, 153, 154, 157, 162, 176

Lexikografie, elektronische 31,559-572

Lexikoneintrag 39, 41-45, 47, 49, 51-53, $55,56,149,150-154,164,165,204$, $214,218,290,339,562$

Lexikonregel, s. Regel

Lexikontheorie 5

LFG (= Lexical-Functional Grammar) 153

Linking 3, 50, 52, 73, 200, 377-380

Log Likelihood Ratio 539, 545, 551

Lokal-Angabe 318, 321

Lokativ-Alternation 19, 463

Longitudinalstudie 21, 22, 24, 400

\section{M}

Majuskel(kriterium) 348-350, 355

Makrokonstruktion 59, 134-136
Massenterm, s. Zählbarkeit

Matrixsatz 118, 119, 124, 125, 137, 138, 191-193, 197, 199

Mehrsprachigkeit 414

Mehrwortlexem IX, X

Merkmale, strukturaufbauende, s. Strukturaufbau

Merkmalskookkurrenzbeschränkung $223,225,238$

Mesokonstruktion 59, 134, 135

Message (als semantische Rolle) 48

Minikonstruktion 14, 47, 50-55, 59, 60, 62

Minimalismus $157,176,392$

Minimalität(sbedingung) 224, 225, 230, $233,234,241,348,352$

Mittelfeld 114, 126, 140, 388, 401, 403, 439,543

Mittelkonstruktion 19, 462

Modalverb 385, 386, 406, 417

Modifikator, adverbialer 25, 318, 319, 321, 322, 327, 330, 334

Modifikator-Kopf-Konstruktion 184

Modifizierbarkeit 362, 536

Morphem 11, 20, 41, 185, 186, 211, 213-215, 221, 228, 235, 237, 240, 244, 255, 352, 354, 381, 405

Morphemgrenze 352

MOSAIC (= Model of Syntax Acquisition in Children) 387,398

Muster(begriff) XI, 8-10, 13-15, 17, $18,22-24,54,72,115,121,126-$ 127, 159, 174, 184-186, 202-207, 418, 492, 493

Muster, propositionales 73,101-105

Musterbildung XI, 23, 529

Musterentwicklung 506, 513

Musterlernen 513

Muster-Wörterbuch 501

$\mathbf{N}$

N400 433, 440, 442, 444, 445

Nachfeld 114, 172, 175, 401

Nachzustand 324, 325

Nationalvarietät, s. Varietät

Nebensatz 19, 125, 167, 385, 393, 401, 403, 408, 409, 413-415, 422 
Negationspartikel 422

Negativierung 25, 433, 436, 437, 442, 445

Netzwerkmodell, s. Konstruktionsnetzwerk

N-Gramm-Analyse 21, 559

Nominalreduplikation, sequenzielle 18, 28, 211, 214, 216, 229-244, 569

NP-hat-seinen-Preis-Konstruktion 19, 23 , 203-207, 569

N-P-N-Verbindungen 19

Numerus 173, 175, 215, 239, 405, 433, 434, 555

\section{O}

Objekt, inneres 88, 90

Objektellipse, s. Ellipse

OEC (= Oxford English Corpus) 496

Online-Umfrage, s. Umfrage

Ontologie 30, 263, 474, 485, 487, 488, 502

Operationalisierung 509, 514

Ortsargument 468, 469

OWID (= Online-Wortschatz-Informationssystem Deutsch) 563, 570, 572

\section{$\mathbf{P}$}

P600 433

Paarformel 16, 229, 515

Parser 24, 543, 544

Partikel 4, 118-119, 123, 127, 190, 350, 401, 403, 407-408, 416, 422, $527-528,542$

Partikelverb XI, 17, 29, 98, 268, 353, $355,357-360,363-366,368,369$, $386,388,401-402$

Partition 78, 129, 130-134, 137, 139-141

Partizip 153, 292, 317-321, 323, 326$330,332,334-338,355,381,382$, 386, 388, 401, 446

Partizipantenrolle, s. Rolle, semantische

PAS (= Preferred Argument Structure) 377

Passiv 286, 287, 290, 299, 300, 303-305, 308

Passiv, unpersönliches 19, 25, 303, 304
Path 51, 52

Patiens 48, 91, 381, 384, 431, 470, 444

Patternbank 560, 562, 572

PDEV (= Pattern Dictionary of English Verbs) 488-492

Perfekt 286, 287, 291, 306, 321, 324, 325,381

Perfektauxiliar 321

Perfektkonstruktion 386, 387

Peripherie, linke 170, 398, 404, 407, 408, 419

Phonotaktik 351

Phrasem 181, 212-214, 241, 510, 521, $599,566,567$

Phrasenstruktur 15, 38, 149, 150-153, $176,225,339,540,565$

Phrasenstrukturgrammatik 38, 540

Phrasenstrukturregel, s. Regel

Phraseologie IX, 202, 345-347, 368$370,505,508,510$

PNK, s. Präposition-Nomen-Kombination

Polysemie VIII, 15, 28, 48, 50, 71, 73-77, $95,105,123,232,260,263-265,472$, 486, 498

Polysemy links, s. WordNet

Possessivpronomen 203-207

Possessivum 400, 477, 554

PP-mit-Direktiv-Konstruktion, s. Direktiv, verbloses

Prädikativ 323, 336

Prädiktabilität, s. Kompositionalität

Präferenz, morphosyntaktische 24, 542, $545,554,555$

Präferenz, selektionale 485, 499

Pragmatikalisierung 114

Präposition-Nomen-Kombination 16, 234, 235, 251-256, 258-262, 264$269,272,274,275,280,281$

Priming 21, 25, 334, 335, 384, 385, 422

Prinzipien-und-Parametertheorie 154 $157,176,392$

Pro drop 155, 441

Produktionsdaten 21, 404

Produktivität 10, 24, 203, 243, 252, 253, $258,320,327,375,385,389,391$

Projektionismus/Projektionsprinzip 72, 176, 153

Prominenz(skala) 30, 378, 429, 439, 447 
Prosodie 114, 117, 119-121, 123, 328, $329,335,497$

Protorolle 431

Prototyp 309, 363, 440

Q

Qualia 485, 496, 501

Quantelung 295, 298

\section{$\mathbf{R}$}

Rattenfängereffekt 171,175

Reaktionszeit 25, 291, 334, 335, 448

Reanalyse 28, 113, 126, 135, 137, 139, 143, 405-407, 430-432, 434, 441

Redewendung, s. Wortverbindung, feste

Redundanzregel, s. Regel(begriff)

Reduplikation, s. Nominalreduplikation, sequenzielle

Regel(begriff) VII, 1, 3, 5-7, 27, 29, 60, $71,74,81,86,87,93,96,104,105$, 115, 199, 207, 208, 244, 254, 266, 346, 397, 493, 559

Regelverletzung, s. Regel(begriff)

Regionalismus 534, 535

Regression, binäre logistische 22, 26, 267-269

Reihenbildung 349, 552, 555

Rekonstruktion(sprozess) 398, 406, 407, $416,417,422$

Rektion 220, 349, 563

Relativelement 163,170

Relativkomplementierer, s. Relativelement

Relativpronomen, s. Relativelement

Relativsatz(typen) 19, 28, 149, 159-179, 187, 196, 401, 413-414, 554

Relativsatzkonstruktion $14,18,58,161-$ $165,169,170,174$

Reparatur 236, 237, 240, 291, 296-299, 306-308, 405, 409, 412, 416

Restriktion, selektionale, s. Selektion, lexikalische/semantische

Resultativkonstruktion 14, 18, 19, 29, $37,39,40-44,47,49,50,51,53$, 55-63, 72, 73, 95-98, 100, 101, $105,187,188,566$

Resultativphrase $40,45,47,52,53,57$
Resultatzustand $42,52,53,322,323$, 324

Rezeptionsdaten 21

Rezipient 471

Rolle, semantische 42-44, 286, 295, 296, $431,471,488,502,565$

\section{$\mathbf{S}$}

Salienz 378, 498

SALSA (= Saarbrücken Lexical Semantics Annotation and Analysis) 459, 477, $478,560,562,565,572$

Satzbauplan 150, 419-420, 564

Satzklammer 19, 24, 126, 348-349, 353, 363, 366, 376, 385, 392, 397, 401409

Satzmuster 18, 113, 150

Satzperipherie 398, 407, 419

Satztypoperator 160-162, 164

Schemakonstruktion, s. Konstruktion(sbegriff)

Schematisierung $7,10,54,512$

Scrambling 60, 330-331

Selektion, lexikalische/semantische 52, 71, 105, 201, 219, 259, 260, 265, 273, 275, 286, 290, 306, 308, 459, 485, 497, 499, 506, 537, 539, 565

senses, s. Polysemie

Sentience 301, 303, 305, 307

Serialisierung 415,416

set, lexical 483, 487, 496, 500-502

Sign-Based Construction Grammar 63

Sketch Engine 23, 491, 542-544

Source 51, 52

Sparsamkeitsprinzip 182, 201

Spatium 353

Speaker (als semantische Rolle), s. Sprecher

Spezifikator 216, 223, 233, 234, 402, 407, 408

Spontandaten, s. Daten, spontane

Sprachentwicklung 376, 383, 386, 529,

Spracherwerb 13, 21, 22, 24, 28, 30, 37, 59, 115, 117, 121, 202, 207, 208, 375-379, 381-383, 385, 387, 392, 393, 397-400, 402, 403, 409, 411, 416-423, 505, 522 
Sprachgebrauch $27,30,39,56,93,115$, $377,382,383,385,392,402,421$, 423, 492, 499, 505, 506, 514, 522, 525,526

Sprachnorm 493, 509

Sprachpraxis, s. Sprachgebrauch

Sprachproduktion 51, 208, 400, 505, $506,529,541$

Sprachtypologie 377

Sprachverarbeitung(sprozess/-mechanismen), s. Verarbeitung

Sprachverarbeitung, maschinelle/automatische 459, 466, 533, 560, 561

Sprecher (als semantische Rolle) 44, 48, 132, 157

Sprichwort 4, 16, 497, 562, 563, 566

SprichWort-Projekt 560, 562, 563, 572

Steigerungspartikel, s. Gradpartikel

Strukturaufbau, regulärer $25,212,215$, 217-219, 224, 233, 239, 384, 385, 391, 415, 419

Strukturausbau 317, 328

Stufenkategorie, s. (Ausdrucks)stufe

Subjekt-Auxiliar-Inversion-Konstruktion 157, 158

Subjekt-Prädikat-Konstruktion 184, 188

Subjektspräferenz 432

Subkategorisierung, s. Valenz

Substantiv-Verb-Verbindung/SubstantivVerb-Kollokation 508, 511, 521

Such-Argumentstrukturmuster 19, 73, 80-87, 93, 107, 109, 110

Syllabierung 348, 351-352, 364, 367-368

Synonymie 76, 255, 460

Synsets, s. WordNet

Syntaxerwerb 376, 378, 379, 383

Syntax-Lexikon-Kontinuum 40

T

Tagger 24, 262

Telizität(sbegriff) 286-288, 290, 294 299, 304-308

Tempus 136, 215, 325, 386, 401

Tendenz, phraseologische vs. terminologische 488

Textproduktion 506

Textsorte 26, 120, 524, 535, 553, 555
Thema 48, 217, 325, 339, 340, 477

Thema, inkrementelles 295, 296

Theorie dynamischer Systeme 19, 398

Topikalisierung 18, 184, 225, 330

Transfer(bedeutung) $\quad 74,381,382,477$

Transferverb 462

Transformation(sregel) 2, 3, 150, 153, 154, 175, 176, 197, 214

Transitivität 79, 437, 441

Transitivkonstruktion 18, 92

Transkription(stheorie), s. Nominalreduplikation, sequenzielle

Triangulation 486, 487

Troponymie 461, 462

Typ, semantischer 30, 484-488, 492-494, 497, 499, 501, 502

Typenhierarchie, s. Vererbung

Typifikation 486

$\mathbf{U}$

Übergeneralisierung 379

Übergenerierung 39, 43, 47, 52, 322

Umfrage 508, 525-529

Uminterpretation 4, 25, 39, 291, 294, 382,430

un-Affigierung 319, 322

Unakkusativa 286-287, 289, 290, 292, 294, 297-300, 304, 307

Undergoer, s. Patiens

Universalgrammatik VIII, 154, 170, 189, 377, 380, 419

Universalien 377

unter+Substantiv, s. Präposition-NomenKombination

Unterspezifizierung 4

Usualisierung 5, 27, 159, 174, 559

Usuelle Wortverbindungen 560, 562, $563,567,572$

\section{V}

V2-Frage 413

V2-Relativsatz, s. Relativsatz

V2-Satz 139, 140

Vagheit 122, 130, 140, 397

Valenz XI, 3, 5, 19, 23, 28, 30, 37, 40, 54, 59, 63, 71-73, 92, 105, 150, 187, 
215, 218, 219, 222-224, 229, 239, $257,286,375-378,382,384,466$, 470, 483-485, 488, 492, 493, 501, $562-565$

Valenzalternation, s. Argumentstrukturalternation

Valenzgrammatik, s. Valenz

Valenzmuster, s. Argumentstrukturmuster

Variantenwörterbuch 507, 521, 523

Variationsmuster 303, 308, 309

Varietät $23,30,228,533-534,536-537$, $539,546,549,550,552-556,561$

Verarbeitung 25, 26, 29, 105, 182, 184, 189, 201, 208, 262, 291, 309, 321, 389, 400, 418, 420, 429-448, 459, $466,533,560,561$

Verb des Anstrengens und Bemühens 82

Verb des Suchens 14

Verb mit innerem Objekt 14, 19

Verb, intransitiv 41, 89, 286, 288, 294, 446, 462, 467

Verb, kausativ-inchoatives 464

Verb, statisches 386

Verbalkomplex 328, 331, 332, 336, 337

Verbeintrag 3, 55, 72, 165

Verberst-Konstruktion 188

Verb-Erwerb 383

Verbklasse/Verbklassifikation 14, 96, 101, 156, 304, 462, 464, 467, 470, 471

Verblassung, semantische 305, 308, 309

Verbletzt- $d$-Relativsatz, s. Relativsatz

Verbletzt- $w$-Frage, s. $w$-Frage

Verbpartikel, s. Partikelverb

Verbzweitrelativsatz, s. Relativsatz

Verbzweitsatz, s. V2-Satz

Vererbung 7, 12, 14, 19, 63, 76, 104, 134, 149, 154, 158, 174, 220, 309, 364, 365

Vergewisserungssignal $16,119,120$

Verkettung 215, 218, 223, 227, 239

Versprecher 204, 409, 411, 412

Verstärkung, pragmatische 306

Verursachung 42, 301, 302

Volitionalität 300-302, 305, 307

Vorfeld 17, 114, 119, 172, 175, 196, 230, 330, 348, 349, 359-362, 366, 368, 401, 408
Vorfeldpartikel 362

Vorgangspassiv 17, 317, 318, 321, 324, 327-336, 339

W

Wahrnehmungsverb 82, 83

Was-In-Aller-Welt-Konstruktion 19, 190 200, 569

Way-Construction 18, 43, 44

Weglassbarkeit des Artikels, s. Artikelwegfall

weirdness $547,548,550,551$

weißt du 16, 119, 120

Weltwissen 48, 49, 51, 52, 99, 104, $197-$ 198, 323-325, 329, 340, 446, 465

$w$-Frage/ $w$-Phrase 19, 407, 409, 412-415

WhIAW, s. Was-In-Aller-Welt-Konstruktion

Wh-Konstruktion 14, 199

Wiktionary 523, 530

Wissen, enzyklopädisches 197

WordNet 26, 30, 76, 263, 272, 459-481, 561,569

Wort(begriff) 1, 4-5, 17, 29, 116, 229, 241, 243, 317, 328, 332, 338-339, 345-372, 376, 444, 445

Wort, graphematisches 345,354

Wort, phonologisches 243, 345, 351, 353-356

Wortbildung XI, 317-320

Wörterbuch Deutsche Gebärdensprache 561,572

Wortkategorieerkennung 446

Wortschatzportal Leipzig 507

Wortstellung 24, 58, 156, 158, 161, 162, 203, 224, 292, 376, 384, 389, 392, 393, 415, 440, 441, 445, 543, 545

Wortverbindung, feste/usuelle IX-X, $17,505-515,521-524,529,560$, 562-563, 566-569

Wortwiedererkennungsaufgabe 25, 334

w-Relativsatz, weiterführender, s. Relativsatz

\footnotetext{
$\mathbf{X}$

X-bar-Theorie 5, 18, 151, 153-155, 165, 176, 399, 402
} 
X-bat-seinen-Preis-Konstruktion, s. NP-hatseinen-Preis-Konstruktion 19, 23, 183, 202-207, 569

\section{Z}

Zählbarkeit 24, 253-258, 262, 263, 268, 273

Zeitdauerangabe, s. Durativangabe
Zeitspannenangabe 288, 296

Zooming, s. Granularität

Zustandsargument 325, 340

Zustandspassiv 17, 25, 29, 317-343

Zustandsveränderung 297, 462, 475

Zwei-Ebenen-Semantik 4

Zweitspracherwerb 505, 522

Zweiwortäußerung 383, 403

Zwillingsform, s. Paarformel 


\section{Namensindex}

A

Aarts, Bas 364

Abbot-Smith, Kirsten 381

Abeillé, Anne 186

Abel, Andrea 562

Abney, Steven 444, 446

Abraham, Werner 286, 287

Ackerman, Farrell 58, 60, 212

Adger, David 215, 224

Ágel, Vilmos XI, 364, 369

Ahmad, Khurshid 546, 547

Aikhenvald, Alexandra Y. 350

Aissen, Judith 305, 439

Akmajian, Adrian 410

Alexiadou, Artemis 321

Allan, Keith 255

Allen, Shanley 377, 382

Altmann, Hans 126

Ammon, Ulrich 523, 534

Anagnostopoulou, Elena 321

Anderson, Stephen 463

Anderson, Sven-Gunnar 171

Aronoff, Mark 3

Aslin, Richard N. 387

Atkins, Beryl T.S. 48

Auer, Peter 118, 126, 139, 140

Augurzky, Petra 446

\section{B}

Baayen, R. Harald 258, 259, 268

Baker, Collin 48, 460, 465, 473, 477, 493

Baker, Mark C. 154, 221, 223, 225, 337

Baldridge, Jason 163

Baldwin, Timothy 252, 253, 259, 260, 261

Bale, Alan 255, 273

Banerjee, Satanjeev 475, 476

Bannard, Colin 392

Barden, Birgit 118

Barlow, Michael 56

Barner, David 255, 256, 273

Baron, Naomi 88

Barsalou, Lawrence W. 336

Bartlett, Frederic Ch. 423
Bartsch, Sabine 538-540

Barz, Irmhild 509

Bechert, Johannes 150

Beck, Sigrid 229, 233

Behrens, Heike $8,12,13,19,22,24,29$, 30, 37, 59, 181, 202, 375, 377, 381, 382, 386-388, 398, 399, 402, 419

Belica, Cyril 100, 561

Bergen, Benjamin 37

Berger, Hans 432

Bergmann, Christopher 433

Bergs, Alexander 37

Bernardini, Petra 415

Bever, Thomas G. 440

Bickel, Balthasar 440, 507, 534

Bierwisch, Manfred 4, 234, 238, 337

Bisang, Walter 440, 445

Bittner, Maria 221

Bittner, Thomas 113, 115, 129-134, 137-141

Black, Max 498

Blom, Elma 386

Blume, Kerstin 3

Boas, Hans C. 8, 10, 12, 14, 19, 22, 23, 28, 37-39, 41, 43-53, 55, 56, 59-63, 477

Boehm-Jernigan, Heather 446

Borer, Hagit 3, 255

Bornkessel, Ina 19, 25, 30, 429, 431439, 442, 444-447, 449

Bott, Oliver 448

Bouillon, Pierrette 291

Bowerman, Melissa 377, 379, 380

Boyd, Jeremy K. 382

Boyd-Graber, Jordan 462

Braine, Martin D.S. 418

Brandt, Patrick 165, 166

Brandt, Silke 393

Brée, David 263, 264

Bresnan, Joan 153, 306, 309

Brinton, Laurel J. 118

Brooks, Patricia J. 384

Brown, Penelope 377, 378, 380

Bruening, Benjamin 216 
Brunner, Annelen 506, 510, 514

Bücker, Jörg 139

Bühler, Karl 123, 130, 135

Burchardt, Aljoscha 475-478, 562

Burger, Harald 16, 229, 505, 507-510, 514,521

Bürki, Andreas 507, 513

Burzio, Luigi 223, 286

Buscha, Joachim 263

Bybee, Joan 56, 57

C

Call, Josep 400

Carlson, Gregory N. 295, 298

Carnie, Andreas 286, 287

Cenoz, Jasone 415

Cernyševa, Irina I. 214

Chafe, Wallace 16

Chaffin, Roger 462

Chang, Nancy 37

Chiarcos, Christian 253

Choi, Soonja 383

Chomsky, Noam 6, 15, 37, 43, 149-151, 153-155, 157, 163, 175, 176, 189, 212$214,223,224,238,398,399,440$

Choudhary, Kamal K. 433, 441, 442

Chow, Ian 474-476

Church, Kenneth W. 486

Cinkova, Silvie 502

Cinque, Guglielmo 156, 157

Clahsen, Harald 403

Clifton, Charles 444, 446

Collins, Allan M. 460

Comrie, Bernard 437, 439

Couper-Kuhlen, Elizabeth 126, 127

Cowie, Anthony P. 16

Crocker, Matthew W. 430, 432

Croft, William 9-13, 18, 20, 37-40, 309, 346, 439

Cronin, Beau 460, 465, 493

Cruse, Alan D. 460

Culicover, Peter 160, 212

Culy, Christopher 242, 243

\section{D}

Dąbrowska, Ewa 392
D'Avis, Franz-Joseph 408

de Swart, Henriëtte 437

DeLancey, Scott 437, 439

Demiral, Şükrü Barış 441, 442, 444

Demuth, Katherine 421, 422

Deppermann, Arnulf VIII, 13, 41, 114, 117,128

Diessel, Holger 37, 188, 393

Diewald, Gabriele $37,54,59,140$

Dimroth, Christine 402, 403, 407

Dixon, Robert M.W. 350

Dobrovol'skij, Dmitrij 16

Dömges, Florian 252, 258, 259

Döpke, Susanne 415

Dowty, David R. 3, 52, 287, 300-303, 307, 431

Duden 119, 252, 254, 258, 263, 299, 300, 514-516, 519

Dunning, Ted 539

Durĉo, Peter 519

E

Eberle, Kurt 272

Echols, Catharine H. 418

Eco, Umberto 121, 122

Eichinger, Ludwig M. VII, X-XII, 512, 513

Eisenbeiß, Sonja 398

Eisenberg, Peter XII, 213, 229

Elman, Jeffrey L. 387, 398, 400, 403, 415, 419, 421

Elstermann, Mechthild 118, 128

Embick, David 214, 321

Engelberg, Stefan VIII, 1, 3, 4, 8, 14, 15, 19, 22, 23, 26, 28, 31, 71, 73, 93-95, 102, 103, 287, 305, 306, 559, 563, 566,570

Erk, Katrin 475, 476

Espinal, M. Teresa 253

Everaert, Martin 26, 213

Evert, Stefan 258, 539

\section{F}

Fagan, Sarah 462

Fanselow, Gisbert 224, 225, 432

Farke, Hildegard 432 
Fazly, Afsaneh 542, 545

Feldhaus, Anke 199

Feldman, Jacob 303

Fellbaum, Christiane 19, 26, 30, 459$462,467,473,477,561,569$

Ferrández, Óscar 476

Fiebach, Christian J. 436

Fiehler, Reinhard 118

Filatkina, Natalia 506

Fillmore, Charles J. 8, 9, 17-19, 38, 40, 41, 48-50, 63, 115-117, 134, 157, 158, 176, 188-190, 199, 309, 460, 465, 493

Fischer, Julia 400

Fischer, Kerstin 7, 37, 113, 115, 116, 183, 184, 218, 220

Fleischer, Wolfgang 229

Flores d'Arcais, Giovanni B. 432

Fodor, Janet D. 429, 440, 447

Foley, William A. 431

Forkl, Yves 537, 541

Frajzyngier, Zygmunt 300

Frampton, John 235, 236

Frank, Annette 475, 476

Fraser, Bruce 16, 118, 214

Frazier, Lyn 429, 432, 440

Freudenthal, Daniel 387, 391

Frey, Werner 330, 337, 339

Fried, Mirjam 37, 40, 113

Friederici, Angela D. 213, 431, 432, 436, 439, 444-446

Frisch, Stefan 443

Fritzenschaft, Agnes 403, 408

Fuhrhop, Nanna 353-355, 364

G

Gallmann, Peter 231

Garnsey, Susan M. 433

Gärtner, Hans-Martin 159, 160, 167, 168

Gawlitzek-Maiwald, Ira 403, 410, 415417,421

Gazdar, Gerald 213, 223, 238

Gelman, Rochel 303

Genesee, Fred 415, 417

Gentner, Dedre 377

Georgi, Doreen 216

Gese, Helga 25, 321, 332, 334, 335
Gibson, Edward 446

Gillon, Brendan S. 256

Gilquin, Gaëtanelle 22, 26

Ginzburg, Jonathan 153

Gleitman, Lila R. 380

Gohl, Christine 118

Goldberg, Adele E. 4, 8-12, 14, 15, 18, $20,28,37,39-45,47,49-53,55-57$, $60,63,71,73-77,80,82,83,92,93$, 95, 96, 104, 115-117, 182-184, 188, 212, 229, 290, 291, 294, 297, 298, $305,306,309,346,365,381,382$, 398, 399, 402, 423

Goldin, Philippe R. 303

Gordon, Peter 438

Gottschalk, Erin A. 382

Goulden, Robin 201

Grabowski, Joachim 460

Green, Georgia 462

Gretsch, Petra 408

Grewe, Tanja 438, 439

Grewendorf, Günther 167, 238, 286

Grice, H. Paul 492

Gries, Stefan Th. 22, 26, 37, 96, 566

Grimm, Jacob 190

Grimm, Wilhelm 190

Grimshaw, Jane 431

Gropen, Jess 378, 379

Gross, James J. 303

Gross, Maurice 464

Günthner, Susanne 1, 41, 114, 115, 118, 119, 121, 124, 128, 139

Gurevich, Olga 104

\section{$\mathbf{H}$}

Haberzettl, Stefanie 409

Häcki Buhofer, Annelies 16, 22, 26, 30, 505, 506

Hagoort, Peter 446

Hahne, Anja 445, 446

Haider, Hubert 337

Hale, Kenneth 3, 221, 445

Hall, Tracy Alan 352, 353

Halle, Morris 214, 224

Halliday, Michael 483

Hanauska, Monika 506

Handwerker, Brigitte 73 
Hanks, Patrick 16, 19, 22, 23, 26, 30, 483, 486, 487, 493, 498, 500

Harley, Heidi 3, 73, 214, 286, 287, 445

Harnisch, Rüdiger 407

Harris, Roy 122

Harris, Zellig 151

Hartrumpf, Sven 263, 275

Haupt, Friederike S. 430, 442

Hauser, Marc 213

Hausmann, Franz Josef 16, 511, 512, 520, 534, 538-541

Hawkins, John 62, 306, 309

Hay, Jennifer 295

Heck, Fabian 215

Heid, Ulrich 16, 22-24, 30, 533, 540, 541

Heine, Antje 360, 363, 369

Helbig, Gerhard 127, 263

Hemforth, Barbara 432

Hennig, Mathilde 113, 135

Heny, Frank 410

Herbst, Thomas 483, 562

Hillyard, Steven A. 433

Hilpert, Martin 37, 39

Himmelmann, Nikolaus 252, 261

Hobbs, Jerry J. 326

Höche, Silke 88

Hocket, Charles F. 16, 350

Hoekstra, Teun 406, 410

Hoffmann, Ludger 401

Hohenberger, Annette 377, 379, 398, 400, 408

Höhle, Barbara 379, 380, 400, 404

Höhle, Tilmann N. 3, 153, 401

Holler, Anke VIII, 1, 159, 168, 171, 191, 194

Holmberg, Anders 225

Hopper, Paul J. 115, 127, 437

Hughes, Mary 377, 382

Hyams, Nina 417, 421

\section{I}

Iatridou, Sabine 156

Ide, Nancy 460, 472, 476

Imo, Wolfgang $7,8,11,13,16,17,20$, 22, 28, 37, 55, 113-115, 118-120, $123,124,127,128,130,140$

Inoue, Atsu 447
Ivanova, Kremena 543

Iwata, Seizi 37-39, 47, 50

J

Jackendoff, Ray 3, 15, 16, 18, 37, 38, 53, $55,56,80,95,96,151,152,212,229$, $230,232,234,235,241,431$

Jacobs, Joachim 4, 6, 7, 11, 17-20, 25, 26, 29, 60, 63, 71-73, 104, 116, 187, 188, 202, 216-220, 226-229, 232, $328,337,338,353,360,363,367$, 399

Jespersen, Otto 255

Jezek, Elisabetta 500

Johnson, Christopher R. 48

Johnson, Matthew A. 104

Jordens, Peter 385, 386, 402, 406, 410

Juranek, Katja 447

K

Kallmeyer, Werner X

Kaltenbacher, Erika 404

Kaminski, Juliane 400

Kaufmann, Ingrid 95, 286, 300

Kay, Paul 8-10, 12, 14, 17, 18, 38, 40, 44, 47, 50, 116, 117, 134, 157, 176, 184, 188, 199, 309

Kegl, Judy 467

Keller, Frank 287, 292, 300, 305

Kemmer, Suzanne 56

Kempen, Gerhard A.M. 438

Kennedy, Christopher 287, 295

Keßelmeier, Katja 234, 251

Keyser, Samuel Jay 462

Kilgarriff, Adam 23, 491, 542

Kipper, Karin 464

Kiss, Tibor 7, 16, 22, 24, 27, 29, 234, 251, 252, 274

Klappenbach, Ruth 561

Klein, Wolfgang 561

Kliegl, Reinhold 440

Klosa, Anette 559, 563

Knobloch, Clemens 7,137

Kolk, Herman H.J. 433

Konieczny, Lars 430

Kornai, András 151 
Kratzer, Angelika 299, 318, 321, 322, $325,327,335,336$

Kremers, Joost 285, 365

Krenn, Brigitte 542

Krifka, Manfred 295, 296

Kubczak, Jacqueline XI, 562

Kühn, Katja 432

Külpmann, Robert 360

Kunze, Claudia 263, 272, 461, 561

Kuperberg, Gina R. 432

Kutas, Marta 433, 436, 440

Kvam, Sigmund 171

$\mathbf{L}$

Lakoff, George 8, 12, 13, 182, 183, 202, 301

Landau, Barbara 380

Landes, Shari 460

Lang, Ewald 320

Langacker, Ronald W. 9-13, 39, 56, 57, $60,115,116,182,184,186,188,375$, 381

Langanke, Ulrich 561

Larson, Richard 225

Lasser, Ingeborg 386, 387

Lattey, Elsa 402

Laws, Florian 262

Le Bruyn, Bert 252, 253

Lee-Goldman, Russell 38, 63

Lees, Robert 2

Legendre, Géraldine 305

Lehmann, Christian 22

Leisi, Ernst 62

Leiss, Elisabeth 183, 286, 287

Lemnitzer, Lothar 26, 263, 272, 561

Lemoine, Kevin 31

Lenerz, Jürgen 439

Lenz, Barbara 319

Levin, Beth 3, 91, 287, 295, 305, 306, $378,379,460,461,464,470,478$

Levinson, Steven C. 305, 306

Lewis, Richard L. 438

Lezius, Wolfgang 262

Libet, Benjamin 302

Lieven, Elena 383, 384, 391, 392

Lindner, Katrin 379

Linell, Per 117, 118, 128
Litvinov, Viktor P. 318

Louw, Bill 497

Lübbe, Anja 320

\section{M}

MacDonald, Maryellen C. 440

Mackin, Roland 16

MacWhinney, Brian 386-389, 392, 440

Maienborn, Claudia 4, 7, 17, 22, 25, 29, $95,317-319,321,323,325,330$, 337,340

Mair, Christian 62

Makkai, Adam 16

Malkiel, Yakov 229, 232

Mandler, Jean M. 383

Marantz, Alec 213, 214, 224, 445

Marcus, Gary 397

Marslen-Wilson, William 430

Martinet, André 213

Matsumoto, Masumi 88

Mauss, Iris B. 303

McCawley, James D. 3

McCullough, Elizabeth 421, 422

McDonough, Laraine 383

McElree, Brian 291, 438

McKoon, Gail 334

McNally, Louise 253

Meibauer, Jörg 359

Meier, Stefanie 507, 522

Meisel, Jürgen M. 402, 415

Mel'čuk, Igor A. 538

Menyuk, Paula 411

Michaelis, Laura A. 47,63

Mihalcea, Rada 474

Miller, George A. 440, 460

Minkoff, Seth A. 305

Mitchell, Sandra 129

Mittwoch, Anita 295, 296

Moerdijk, Fons 561

Möhrs, Christine 563

Morrill, Glyn 163

Motsch, Wolfgang XI

Müller, Christoph 262, 263

Müller, Gereon 28, 187, 208, 212, 214 216, 225, 439

Müller, Natascha 408, 415, 416 
Müller, Stefan 3, 7, 19, 20, 60, 61, 72, 73, 159, 163, 171, 173, 187, 188, 227, 229, 364

Müller-Spitzer, Carolin 26, 31, 559, 560, 563,570

Muralikrishnan, Ramasamy 441, 443

$\mathbf{N}$

Naigles, Letitia R. 380

Nederstigt, Ulrike 403, 407

Nedjalkov, Vladimir P. 318

Nemoto, Noriko 44, 47, 50

Nespor, Marina 353

Neville, Helen J. 445

Newmeyer, Frederick J. 156, 157, 309, 399

Newport, Elisabeth L. 387, 403

Nicoladis, Elena 415, 417

Niestadt, Jan 561

Nikolova, Sonja 462

Niles, Ian 474, 475

Norén, Kerstin 117, 128

Noyer, Rolf 3, 73, 214, 445

Nübling, Damaris 353

Nunberg, Geoffrey 17, 213, 214

O

O'Connor, Mary Catherine $8,9,17,38$, 40, 116, 117, 157, 176

Ochsner, Kevin N. 303

Oltramari, Alessandro 476

Ono, Tsuyoshi Ono 126, 127

Opfer, John E. 303

Östman, Jan Ola 11, 37, 40, 41, 113

Özkaragöz, Ýnci 289

\section{P}

Pantel, Patrick 459

Paradis, Johanne 415, 417

Patwardhan, Siddhartha 476

Pease, Adam 474, 475

Pedersen, Ted 475, 476

Peltzer-Karpf, Annemarie 398, 400

Penke, Martina 22, 26, 403
Penner, Zvi 402, 403, 407, 408

Pérez-Leroux, Ana T. 400

Perlmutter, David M. 286, 287, 289

Pesetsky, David 200, 213

Peters, Ann M. 288, 404, 408

Petruck, Miriam R.L. 48

Pianta, Emanuele 476

Pike, Kenneth L. 218

Pine, Julian M. 383, 384, 387

Pinker, Steven $376,378,380,400$

Polenz, Peter von 506

Poletto, Cecilia 157, 167

Pollard, Carl J. 38, 153, 163

Pollock, Jean-Yves 156

Postal, Paul M. 286, 287, 289

Primus, Beatrice 4, 7, 19, 22, 25, 29, 181, 211, 285, 294, 298, 301, 304, 355, 431,432

Prince, Alan 304

Prinsloo, Daniel J. 559

Pritchett, Bradley L. 430, 440

Proost, Kristel VIII, 1, 14, 23, 28, 71, 77,83

Pullum, Geoffrey K. 151

Pustejovsky, James 291, 485, 487, 496

Pylkkänen, Liina 291

Q

Quillian, M. Ross 460

\section{$\mathbf{R}$}

Radford, Andrew 402, 410, 411

Raffelsiefen, Renate 353

Randall, Janet 415

Rapp, Irene 289, 295, 300, 318, 319, 322, 323

Rappaport-Hovav, Malka 3, 378, 379

Ratcliff, Roger 334

Rhodes, Russell 38, 63

Riedl, Rupert 421

Rizzi, Luigi 157

Roberts, Ian 215

Röder, Brigitte 439

Roehm, Dietmar 433, 437, 443

Roeper, Thomas 213, 400, 410, 415, 462

Rosenbach, Anette 22, 26 
Ross, John Robert 176, 202

Rothstein, Susan 295, 297, 298

Rothweiler, Monika 403, 408, 409

Roussou, Anna 215

Rowland, Caroline F. 383, 384, 391

Ruhl, Charles 213

Rumshisky, Anna 487

Ruppenhofer, Joseph 47, 48, 562

\section{S}

Sag, Ivan A. 17, 38, 63, 153, 170

Sailer, Manfred 186, 213

Šanskij, Nikolaj M. 214

Savage, Ceri 385

Schabes, Yves 186

Schegloff, Emanuel A. 115, 128, 129, 134

Schiehlen, Michael 543

Schiffrin, Deborah 118

Schlesewsky, Matthias 19, 25, 30, 429, 431-435, 437-439, 442-447, 449

Schlotterbeck, Fabian 448

Schlücker, Barbara 319, 322, 336

Schlyter, Suzanne 415

Schmid, Helmut 262, 562

Schmidt, Thomas 562

Scholl, Brian J. 303

Schriefers, Herbert 432

Schröder, Jochen 263, 264

Schulte im Walde, Sabine 459

Schulz, Petra 402, 403

Schumacher, Helmut XI, 562

Schütze, Garson T. 446

Scott, Graham 440

Selkirk, Elisabeth 3

Shi, Lei 474

Shibatani, Masayoshi 300

Siewierska, Anna 300

Silverstein, Michael 439

Sinclair, John 16, 483, 488

Skarabela, Barbora 377, 382

Slobin, Dan I. 402, 418

Smadja, Frank 541

Smith, Barry 113, 115, 129-134, 137141

Smith, Linda B. 398

Smolensky, Paul 304
Snedeker, Jesse 256

Solstad, Torgrim 317, 331, 336

Sorace, Antonella 287, 292, 300, 305

Staudinger, Bernd 57

Stechow, Arnim von 171, 222, 229, 233 , 319, 321

Steedman, Mark J. 163

Stefanowitsch, Anatol 7-10, 14, 19, 22, 23, 26, 28, 37, 96, 113, 115, 116, 181, $183,184,186,202,212,220,566,569$

Steinitz, Renate 337

Steinitz, Wolfgang 561

Steinkrauss, Rasmus 202

Stern, Clara 408, 409

Stern, William 408, 409

Sternefeld, Wolfgang 168, 171, 215

Stevenson, Suzanne 542, 545

Steyer, Kathrin VIII, IX, 506, 510, 514, 537,562

Stolterfoht, Britta 25, 321, 332, 334, 335

Stolz, Thomas 235

Storjohann, Petra 563

Storrer, Angelika 16

Strecker, Bruno 401

Strube, Michael 262

Sturt, Patrick 432

Stvan, Laurel S. 252, 253, 259-261

Suderman, Keith 460, 472

Szabolcsi, Anna 200

Szczek, Joanna 369

T

Talmy, Len 51

Tappe, Hans Thilo 337, 339

Taylor, John R. 115, 116

Tenny, Carol 295

Tesnière, Lucien 483

Thelen, Esther 398

Thoma, Dieter 403, 404

Thompson, Sandra, A. 437, 440

Tiberius, Carole 561

Todorova, Marina 291

Tomasello, Michael 13, 37, 54, 115, 121 , 202, 212, 302, 375, 381-385, 393, 398-400, 402, 404, 415, 418, 419

Tonelli, Sara 476

Townsend, David J. 440 
Tracy, Rosemarie 19, 22, 24, 29, 30, 385, 397, 400, 402-404, 406-408, 410412, 414-418, 420, 421

Traugott, Elisabeth 54, 59, 134, 135

Tremoulet, Patrice D. 303

Trommer, Jochen 211, 224

Tutin, Agnès 537, 541

$\mathbf{U}$

Urbach, Thomas P. 433

Uszkoreit, Hans 439

\section{V}

Vaagland, Erling M. 318

Vachková, Marie 560, 561

van de Craats, Ineke 409, 412

van den Brink, Daniëlle 446

van Dyke, Julie A. 438

Van Pottelberge, Jeroen 16

van Schaik-Rădulescu, Mara 289

van Trijp, Remi 37

van Valin, Robert D. 431

Vasishth, Shravan 438

Vendler, Zeno 287

Verkuyl, Henk J. 295

Vogel, Irene 353

Vosse, Thea 438

W

Wahrig-Burfeind, Renate 190

Wang, Luming 441, 442

Wasow, Thomas 17

Webelhuth, Gerd 7, 8, 14, 19, 28, 58, 60, 149, 187, 202, 212, 378

Webster, Jonathan 474-476

Weckerly, Jill 436, 440
Weinrich, Harald 119

Weissenborn, Jürgen 402-404, 407, 408

Welke, Klaus 54, 56, 59, 60, 63, 73, 93, 95

Weller, Marion 540

Wenzel, Ramona 403

Wiese, Bernd 218

Wilder, Christopher 221

Williams, Edwin 5, 18, 154, 212, 213, 229, 232, 234, 239

Wiltschko, Martina 200

Winkler, Edeltraud 14, 23, 28, 71, 88

Winkler, Steffi 403, 407

Wittgenstein, Ludwig 77, 79, 80

Woicjik, Richard 463

Wolff, Susann 441, 442

Wong-Fillmore, Lily 115

Woolford, Ellen 305

Wulff, Stefanie 37, 202

Wunderlich, Dieter 3, 73, 213, 222, 319, 320, 432

Wurzel, Wolfgang Ulrich 357

Wymann, Karin 402, 403, 407, 408

Y

Yang, Charles 400

\section{$\mathbf{Z}$}

Zaenen, Annie 289, 290, 297, 298

Zeller, Jochen 365

Ziem, Alexander 48

Zifonun, Gisela X, XI, 119, 123, 286, 319, 320, 401

Zinsmeister, Heike 26

Zribi-Hertz, Anne 462

Zwarts, Frans 200

Zwicky, Arnold 218 